\title{
Transition Metal Catalyzed Linchpin-Based Strategies in Natural Product Synthesis: Synthesis of Asteriscunolide D, Aspergillide B and the Core of Labillarides E-H
}

\author{
by \\ Mark J. Bartlett \\ A thesis submitted to Victoria University of Wellington \\ for the degree of Doctor of Philosophy

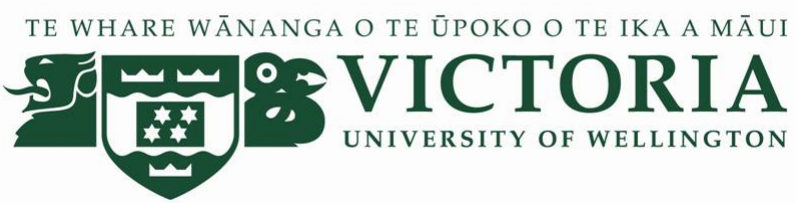

School of Chemical and Physical Sciences

February 2013

(C)Mark J. Bartlett, 2013 
"Act as if what you do makes a difference. It does."

— William James 
Dedicated to my Sister, Monique Bartlett (1990 - 2008).

You remind me to make the most of every opportunity. 


\section{Abstract}

The construction of complex molecular architectures in a facile and efficient manner remains an overarching goal for the chemical sciences. The development of synthetic linchpins, simple compounds that are used to join complex molecular fragments together, represents a powerful method for improving synthetic efficiency. A major challenge associated with linchpin-based synthetic strategies is the development of chemoselective and atom economic reactions that enable late-stage introduction of the linchpin compound.

Chiral propargylic alcohols are versatile synthetic intermediates and their preparation enables entry into a variety of alkyne-based linchpin strategies. Continued optimization of Zn-ProPhenol-catalyzed alkyne addition has led to the development of practical and general conditions for the asymmetric alkynylation of aldehydes. This methodology operates with relatively low catalyst loading and can avoid the use of excess alkyne and dialkylzinc reagents.

The total synthesis of asteriscunolide D was completed in just nine steps using a ZnProPhenol-catalyzed asymmetric alkynylation and Ru-catalyzed alkene-alkyne coupling. Other highlights of this protecting-group-free synthesis include the use of a thionium-induced cyclization to form the challenging 11-membered humulene ring. At the core of this succinct and selective synthetic strategy is the use of methyl propiolate as a strategic linchpin. In just two steps, three new bonds were formed to atoms derived from methyl propiolate and this ultimately enabled the construction of this structurally challenging natural product.

An enantioselective formal total synthesis of aspergillide B has been accomplished using sequential Zn-catalyzed alkyne additions to a masked butane dialdehyde linchpin. This synthesis has led to the development of new conditions for Zn-ProPhenol-catalyzed alkynylation that provide excellent yield and enantioselectivity using just a single equivalent of alkyne. Ru-catalyzed trans-hydrosilylation provides the desired $E$ alkene geometry and also allows chemoselective differentiation of the two double bonds in a subsequent hydrogenation step. This synthetic route provides access to aspergillide B in just 15 steps, using six highly efficient transition metal-catalyzed reactions.

Labillarides E-H are a group of diastereomeric oxylipin natural products with very interesting structural features and spectroscopic properties. The development of a $\mathrm{Pd}$ catalyzed allylic alkylation cascade has enabled the rapid construction of the furanopyrone core and alkenyl side chain of these compounds. The use of density functional calculations, in conjunction with spectroscopic data obtained from the truncated labillaride E-H structure, has provided good evidence that labillarides E and $\mathrm{G}$ have a 3,6-syn configuration, whereas labillarides $\mathrm{F}$ and $\mathrm{H}$ have a 3,6-anti configuration. Subsequent development of the Pd-AA cascade methodology has led to the discovery of a highly regioselective Pd-AA cascade with non-symmetric dihydropyran substrates. The combination of allylic carbonate and anomeric siloxy leaving groups in the dihydropyran substrate enables control of the many regiochemical possibilities in this reaction. Ultimately, annulation proceeds stereoconvergently to give a cisfused furopyran from either cis- or trans-substituted starting material. During the course of this 
research, the assignment of the remote relative stereochemistry of a number of 3,6-dihydro- $2 \mathrm{H}$ pyran starting materials and side products was achieved through a novel NMR-based analysis of axial shielding magnitudes.

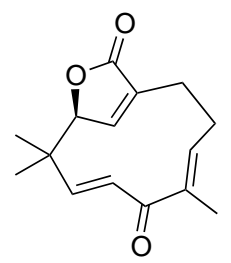

Asteriscunolide $D$

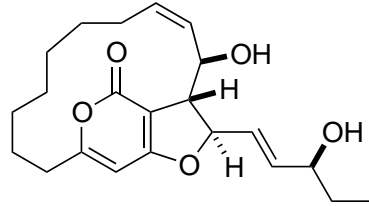

Labillarides $\mathrm{E}-\mathrm{H}$<smiles>CC1CCC/C=C\[C@H]2CC[C@@H](O)[C@@H](CC(=O)O1)O2</smiles>

Aspergillide B 


\section{Acknowledgements}

Graduate school has been an amazing experience - full of challenges and triumphs that have been rewarding in ways that extend far beyond the standard definition of 'school'. So many people have helped me along the way and contributed to this experience.

First and foremost I must thank my doctoral advisor, Dr. Joanne Harvey. The past three years have been enjoyable, exciting, educational, enlightening, and ultimately empowering having learnt so much about not only organic chemistry but also myself, I am now equipped with many of the tools needed to progress beyond the realm of the student. This is in large part thanks to you. Hopefully, some of your meticulous attention to detail has rubbed off on me. Whether interpreting an NMR spectrum, drawing a structure or editing a paper, your careful analysis of EVERY detail always impressed me. In fact, during the early stages of my $\mathrm{PhD}$ I took special care to make sure I'd eaten and used the bathroom before taking a set of NMR spectra into your office!! I have so many fond memories from my time as a graduate student - thanks once again.

I am extremely grateful to Professor Barry Trost, who has taught me so much and been a truly inspiring mentor. As an undergrad I was first introduced to the rigors of synthetic organic chemistry while in the Trost group, and this foundation has been instrumental in guiding me into a career that I am truly passionate about. Returning to the group as a visiting scholar during my $\mathrm{PhD}$ was an incredible roller coaster-like experience, culminating with the completion of the aspergillide synthesis just days before my flight back to NZ. I find myself amazed to think not only about the depth and diverse range of chemistry that I was exposed to in the Trost group, but also the amount of time Professor Trost took to discuss chemistry with me, given the small army of post-docs in the group - all eager to discuss their latest results.

Andrew Weiss has been a huge positive influence throughout my life as a student. As an undergraduate I found myself in admiration of Andrew's techno pump up music, oversized throne-like office chair, and witty one-liners - which ultimately led me to believe that, given Andrew's success, these were prerequisites to the completion of a total synthesis. It wasn't until my tenure as a graduate student that I began to really appreciate how much effort and care had been put into my training as a synthetic chemist. I can still remember being told, "Imagine you dropped your toothbrush in the toilet and you have to clean it before using it again - that's how clean your glassware should be!!!" Fun times aside, there aren't many people out there that apply for jobs with letters of recommendation from Trost, Evans, and Jacobsen, and I am incredibly grateful to have been trained and befriended by such a brilliant chemist.

I am extremely fortunate to have worked along side so many extremely smart and talented chemists over the years. All of whom rubbed off on me in one way or another. At Stanford: Sushant Malhotra, David Lupton, J.P. Lumb, Mike O'Keefe, David Thaisrivongs, Eric Ferreira, Alicia Gutierrez, Wei Quan, Nicolai Cramer, Brendon O'Boyle, Rajiv Dhawan, Keiichi Hirano, Xinjun Luan, Lara Czabaniuk, Melissa Herbage, Mike Ryan, Adrien Quintard, Guangbin Dong, Max Osipov, Andreas Buckl, Dustin Bringley, Tom Lam and Aaron Burns, among others. At Victoria: Hemi Cumming, Russell 'Hulk smash' Hewitt, Peter Moore, Sam Ting, Xuyu Lu, 
Thomas Bevan, Kalpani Somarathne, Jing Jing Wang, Claire Turner, Dylan Davies, Scott Riordan and Febly Tho. An additional thanks goes to Hemi and Jing Jing for proof reading parts of my thesis.

Overall, this thesis is the culmination of a number of collaborations, and I am very lucky to have worked with such a talented group of chemists on the alkynylation methodology and also the synthesis of asteriscunolide D. Namely, Andrew Weiss, Aaron Burns, Thomas Tautz, Axel von Wangelin, and Vincent Chan. Specific credit has been given to the people that contributed to this work in the text.

The School of Chemical and Physical Sciences at Victoria University is an amazing place to study and learn. I don't know of another place where collaboration across different chemical and scientific disciplines occurs with such enthusiasm. Paul Teesdale-Spittle has been a fantastic source of knowledge and advice in regards to both synthesis and medicinal chemistry. Rob Keyzers, Peter Northcote and their respective natural products groups (Peter Clark, Jackie, Jono) have been an amazing resource for spectroscopy and structure elucidation - and were always willing to help with $2 \mathrm{D}$ experiments on the 600 . I am very grateful to Matthias Lein for taking the time to talk me through some of the basics of computational chemistry. John Miller and his students, Dora and Arun, were always willing to test new furopyrone samples in the HL-60 assay.

There are a number of SCPS support staff that have been a huge help over the past 3 years. Sally, Lisa, Helen, Kara, Dan, Alan, Manu and Nick have helped make the administrative tasks as painless as possible. A special thanks must go to Ian Vorster, who on many occasions went out of his way to help me obtain NMR and MS data. I'd also like to thank Victoria University of Wellington for the provision of a PhD scholarship, the VUW Science Faculty for a strategic grant to attend Pacifichem in 2010 and ICOS in 2012. Also, the NZ Institute of Chemistry for a student travel award.

Completing a $\mathrm{PhD}$ in chemistry is challenging on so many levels and I could not have made it to this point without the support of my family. With two kids under two there is never a dull moment, or a full night of sleep. If there's one person who has accumulated more sleep debt over the past year than me, it would have to be my wife, Melissa. I am incredibly lucky to have found such a devoted and caring person to share my life with. In addition, the love and support of my parents, and also Melissa's parents, over the past few years has been invaluable. Over the years my parents have worked scrupulously to allow me to have educational opportunities that weren't afforded to them, so in many ways this thesis is their achievement too. 


\section{Table of Contents}

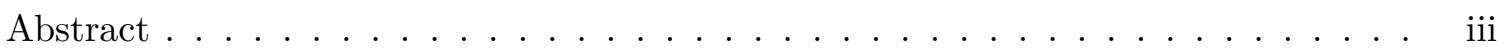

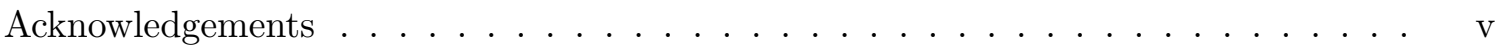

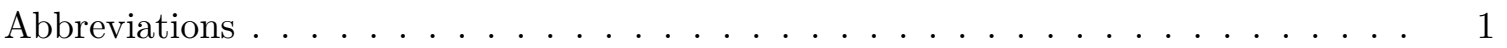

1 Introduction 1

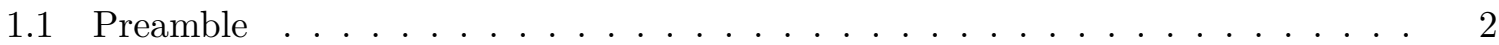

1.2 Total Synthesis and Natural Product Analogues

in the Drug Discovery Process . . . . . . . . . . . . . . . . . . 2

1.3 The Pursuit of Atom Economy in Total Synthesis . . . . . . . . . . . . . . . 4

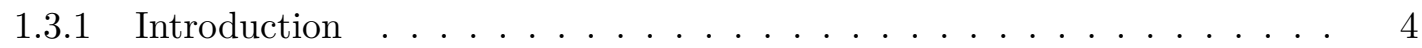

1.3.2 Pd-Catalyzed Asymmetric Allylic Alkylation . . . . . . . . . . . . . . 6

1.3.3 Ruthenium-Catalyzed Alkene-Alkyne Coupling . . . . . . . . . . . . . . 10

1.3.4 Zn-Catalyzed Direct Asymmetric Aldol Reaction . . . . . . . . . . . . . 12

1.3.5 Concluding Remarks . . . . . . . . . . . . . . . . . . 16

1.4 Linchpin-Based Strategies in Total Synthesis _ . . . . . . . . . . . . . 17

1.4 .1 Introduction . . . . . . . . . . . . . . . . 17

1.4.2 The Strategic Application of Synthetic Linchpins . . . . . . . . . . . . . 17

1.4.3 Catalytic Applications of Linchpins in Total Synthesis . . . . . . . . . . 21

1.4 .4 Summary . . . . . . . . . . . . . . . . . . . 29

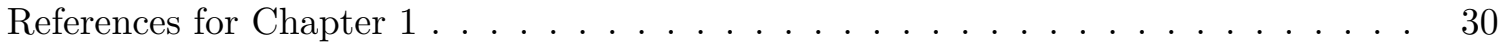

2 Zn-ProPhenol Catalyzed Asymmetric Alkyne Addition $\quad 34$

2.1 Introduction to Alkyne Addition . . . . . . . . . . . . . . . . 35

2.1 .1 Metal Alkynylides . . . . . . . . . . . . . . . . . . 35

2.1 .2 Propargylic Alcohols . . . . . . . . . . . . . . . . . . . 36

2.2 Zinc-ProPhenol Catalyzed Alkynylation

of Aldehydes . . . . . . . . . . . . . . . . . . . . 38

2.2 .1 Introduction and Prior Work . . . . . . . . . . . . . . 38

2.2.2 Alkyne Addition to Aliphatic Aldehydes . . . . . . . . . . . . . . . . . 42

2.2.3 Mechanistic Inferences and Reoptimization . . . . . . . . . . . . . . . 44

2.2 .4 Synthetic Applications . . . . . . . . . . . . . . . . . . . 52

2.2 .5 Summary . . . . . . . . . . . . . . . . . . 54

2.3 Experimental Data for Chapter $2 \ldots \ldots \ldots \ldots \ldots$

2.3.1 General Experimental Methods . . . . . . . . . . . . . . . . 55

2.3.2 Experimental Details and Characterization Data . . . . . . . . . . 56

2.3 .3 Spectra . . . . . . . . . . . . . . . 66

References for Chapter $2 \ldots \ldots \ldots \ldots \ldots \ldots$. . . . . . . . . . . . . . . . . . 
3 Total Synthesis of Asteriscunolide D:

A Ru-Catalyzed Alkene-Alkyne Coupling Strategy $\quad \mathbf{7 6}$

3.1 Introduction . . . . . . . . . . . . . . . . . . . . 77

3.2 Total Synthesis of Asteriscunolide D . . . . . . . . . . . . . . . 79

3.2.1 Synthetic Strategy and Retrosynthetic Analysis . . . . . . . . . . . . . . 79

3.2 .2 Linchpin-Based Butenolide Construction . . . . . . . . . . . . . . . . . . 81

3.2 .3 End Game . . . . . . . . . . . . . . . . . . . . . . 84

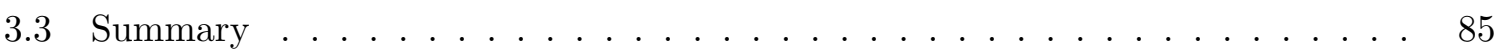

3.4 Experimental Data for Chapter $3 \ldots \ldots \ldots$. . . . . . . . . . 87

3.4.1 General Experimental Methods . . . . . . . . . . . . . . . . . 87

3.4.2 Experimental Details and Characterization Data . . . . . . . . . 88

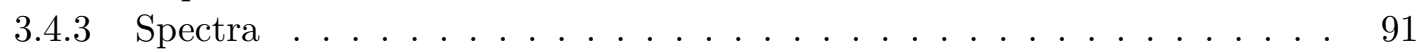

References for Chapter $3 \ldots \ldots \ldots \ldots \ldots \ldots$

4 Formal Total Synthesis of Aspergillide B:

$\begin{array}{ll}\text { A Bis-Alkynylation Linchpin Strategy } & 97\end{array}$

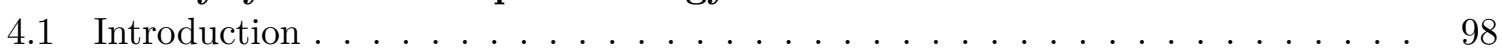

4.1 .1 Isolation and Bioactivity . . . . . . . . . . . . . . 98

4.1 .2 Previous Syntheses . . . . . . . . . . . . . . . . . . . . . . . 99

4.2 Formal Total Synthesis of Aspergillide B . . . . . . . . . . . . . . . . . . . 104

4.2.1 Synthetic Strategy and Retrosynthetic Analysis . . . . . . . . . . . . . . 104

4.2 .2 Alkynylation Optimization . . . . . . . . . . . . . . 106

4.2.3 Linchpin-Based Synthesis of Aspergillide B . . . . . . . . . . . . . . . 108

4.3 Summary . . . . . . . . . . . . . . . . . . . . . . . . . . . . . 114

4.4 Experimental Data for Chapter $4 \ldots \ldots \ldots \ldots$. . . . . . . . . . 115

4.4 .1 General Experimental Methods . . . . . . . . . . . . . . . . . 115

4.4.2 Experimental Details and Characterization Data . . . . . . . . . . 116

4.4 .3 Spectra . . . . . . . . . . . . . . . . . . . . . 134

References for Chapter $4 \ldots \ldots \ldots \ldots \ldots \ldots \ldots$

5 Pd-Catalyzed Allylic Alkylation: First and Second Generation

$\begin{array}{lr}\text { Approaches to the Synthesis of Labillarides E-H } & 157\end{array}$

5.1 Labillarides E-H: Target Driven Reaction Development . . . . . . . . . . . . . . 158

5.1.1 Isolation and Structure of Labillarides E-H . . . . . . . . . . . . . . 158

5.1 .2 Strategy and Major Disconnections . . . . . . . . . . . . . . . . . . 159

5.2 Palladium-Catalyzed Allylic Alkylations . . . . . . . . . . . . . . . . . . 161

5.2 .1 Mechanistic Introduction . . . . . . . . . . . . . . . . . . . 161

5.2 .2 Pd-AA with 4-Hydroxy- $\alpha$-Pyrone Nucleophiles . . . . . . . . . . . . 165

5.3 First Generation Approach to Labillarides E-H . . . . . . . . . . . . . . . . . 167

5.4 Second Generation Approach to Labillarides E-H . . . . . . . . . . . . . . . 171

5.5 Summary . . . . . . . . . . . . . . . . . . . . . 178

5.6 Experimental Data for Chapter $5 \ldots \ldots \ldots$. . . . . . . . . . . 179

5.6.1 General Experimental Methods . . . . . . . . . . . . . . . . 179

5.6.2 Experimental Details and Characterization Data . . . . . . . . . . 179

5.6 .3 Spectral Data . . . . . . . . . . . . . . . . . . 192

References for Chapter 5 . . . . . . . . . . . . . . . . 205 
6 Pd-AA Cascade I: Synthesis of the Core of Labillarides E-H 208

6.1 Alkylation Cascade in the Synthesis of Labillarides E-H . . . . . . . . . . . . . 209

6.2 Introduction . . . . . . . . . . . . . . . . . . . . . . 209

6.2.1 Pd-AA Cascades with Acyclic Bis-Electrophiles . . . . . . . . . . . . . . 209

6.2 .2 Pd-AA Cascade Reactions in Total Synthesis . . . . . . . . . . . . . . . 211

6.3 Development of a Pd-AA Cascade with a 4-Hydroxy- $\alpha$-pyrone. . . . . . . . . . . 212

6.4 Synthesis of the Core of Labillarides E-H . . . . . . . . . . . . . . . . 216

6.4.1 A Linchpin-Based Approach to the Core of Labillaride E-H . . . . . . . 216

6.4.2 Biological Testing of Labillaride Analogues syn- and anti-323 . . . . . . 217

6.4.3 NMR Analysis of the Labillaride E-H Side Chain . . . . . . . . . . . . . 218

6.5 Computational Investigations into Labillaride E-H

Atropisomerism . . . . . . . . . . . . . . . . . . . . 222

6.6 Experimental Data for Chapter $6 \ldots \ldots \ldots \ldots \ldots \ldots$

6.6.1 General Experimental Methods . . . . . . . . . . . . . . . . . 225

6.6.2 Experimental Details and Characterization Data . . . . . . . . . . 225

6.6 .3 Spectra . . . . . . . . . . . . . . . . . . . 228

References for Chapter $6 \ldots \ldots \ldots \ldots \ldots \ldots \ldots$

7 Pd-AA Cascade II: Regioselective Dihydropyran Alkylation in a Third Generation Approach to Labillarides E-H

7.1 Initial Investigations Into The Use of Substituted Bis-Electrophiles in Pd-AA

Cascades. . . . . . . . . . . . . . . . . . . 239

7.2 Development of a Non-Symmetric, Regioselective Pd-AA Cascade . . . . . . . . 240

7.2 .1 Reaction Optimization . . . . . . . . . . . . . . . . . . 241

7.2.2 Stereoconvergence and Mechanistic Inferences . . . . . . . . . . . . . . 242

7.2 .3 Substrate Scope . . . . . . . . . . . . . . . . . . . . . . . . . . 244

7.3 Biological Testing of the Furopyrones Generated via a Pd-AA Cascade . . . . . 249

7.4 Synthetic Utility of Furopyrones: Third Generation Approach to the Synthesis of Labillarides E-H . . . . . . . . . . . . . . . . . . . . . . . . . . . 252

7.5 Experimental Data for Chapter $7 \ldots \ldots \ldots \ldots \ldots$. . . . . . . . . 254

7.5.1 General Experimental Methods . . . . . . . . . . . . . . . . . 254

7.5.2 Experimental Details and Characterization Data . . . . . . . . . . . 254

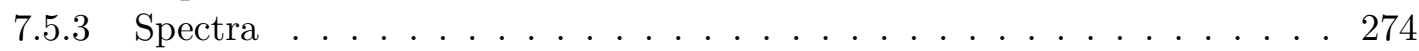

References for Chapter $7 \ldots \ldots \ldots \ldots \ldots$. . . . . . . . . . . . . 297

$8{ }^{13} \mathrm{C}$ NMR Analysis of 3,6-Dihydro-2 $H$-pyrans:

Assignment of Remote Stereochemistry using Axial Shielding Effects $\quad 299$

8.1 Introduction . . . . . . . . . . . . . . . . . . . . . . . 300

8.2 Conformational Analysis and ${ }^{13} \mathrm{C}$ NMR Shielding Effects . . . . . . . . . . . . 301

8.3 Assignment of Relative Stereochemistry using a ${ }^{13} \mathrm{C}$ NMR Shielding Template . 304

8.4 Complimentary Calculations of Dihydro- $2 \mathrm{H}$-pyran ${ }^{13} \mathrm{C}$ NMR Resonances . . . 309

8.5 Summary . . . . . . . . . . . . . . . . . . . . 314

8.6 Experimental Data for Chapter $8 \ldots \ldots \ldots \ldots \ldots \ldots \ldots$

8.6.1 General Experimental Methods . . . . . . . . . . . . . . . . . 315

8.6 .2 Experimental Data . . . . . . . . . . . . . . . . . . 315

8.6.3 NMR Data and Assignments . . . . . . . . . . . . . . . . . . 323

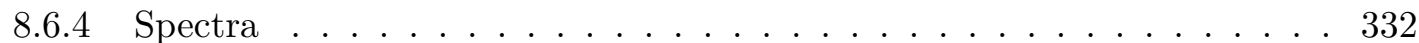

References for Chapter $8 \ldots \ldots \ldots$. . . . . . . . . . . . . . 341 
A Computational Data $\quad \mathbf{3 4 4}$

A.1 Computational Investigations of Labillarides E-H . . . . . . . . . . . . . 345

A.2 ${ }^{13} \mathrm{C}$ NMR Analysis of 3,6-Dihydro- $2 H$-pyrans . . . . . . . . . . . . . 348

B Crystallographic Information $\quad \mathbf{3 6 7}$

B.1 Furopyrone X-Ray Crystal Structure . . . . . . . . . . . . . . . . . . . 368 


\section{List of Schemes}

1.1 Therapeutic Drugs Inspired by Natural Products . . . . . . . . . . . . . . . 3

1.2 Considering Atom Economy in Bond Construction. . . . . . . . . . . . . 5

1.3 Atom Economy in the Conjugate Reduction of Citral. . . . . . . . . . . . . 6

1.4 The Evolution of Pd-Catalyzed Allylic Alkylation. . . . . . . . . . . . . 7

1.5 Retrosynthetic Analysis of Hamigeran B. . . . . . . . . . . . . . 8

1.6 Total Synthesis of Hamigeran B. . . . . . . . . . . . . . . . . . 8

1.7 Decarboxylative Pd-AAA Reaction in the Synthesis of Hamigeran B. . . . . . . 9

1.8 Ruthenium-Catalyzed Alkene-Alkyne Coupling. . . . . . . . . . . . . . . . . . . 10

1.9 Retrosynthetic Analysis of Mycalamide A. . . . . . . . . . . . . . . . 11

1.10 Synthesis of $(-)-7-$ Benzoylpederic Acid. . . . . . . . . . . . . . . . 11

1.11 Differentiating Traditional Enolate Aldol and Direct Aldol Reactions. . . . . . 12

1.12 Zinc Prophenol Catalyst and Proton Shuttle Turnover Mechanism. . . . . . . . 13

1.13 Direct Asymmetric Aldol Reaction with the Zn-ProPhenol Catalyst. . . . . . . 13

1.14 Retrosynthetic Analysis of Laulimalide. . . . . . . . . . . . . . . . . . . . 14

1.15 Synthesis of the Northern Fragment 38. . . . . . . . . . . . . . . . . 15

1.16 Total Synthesis of Laulimalide. . . . . . . . . . . . . . . . . . 15

1.17 A Linchpin Strategy Using the Bestmann Ylide. . . . . . . . . . . . . . . . . 17

1.18 Convergence and Strategic Linchpin Sequences. . . . . . . . . . . . . . . . 18

1.19 A Pd-Catalyzed Cross Coupling Linchpin Strategy . . . . . . . . . . . . . . 22

1.20 Halogen Selective Suzuki Linchpin in the Synthesis of Dragmacidin D . . . . . 23

1.21 Alkyne Cross Coupling Linchpin Strategy . . . . . . . . . . . . . . . . . 24

1.22 Alkene Cross Metathesis Linchpin Strategy . . . . . . . . . . . . . . . 25

1.23 Cyclobutane C-H Arylation Linchpin Strategy . . . . . . . . . . . . . 26

1.24 TMM [3+2] Cycloaddition in the Synthesis of Marcfortine B. . . . . . . . . . . 27

1.25 Reaction and Strategy Development in the Synthesis of Bryostatin 7. . . . . . 28

1.26 Strategic Linchpin Coupling in the Synthesis of Bryostatin 7. . . . . . . . . . 28

2.1 Mild Metal Alkynylide Formation in the Castro-Stephens Reaction. . . . . . . . 36

2.2 Formation and Reactivity of Propargylic Alcohols. . . . . . . . . . . . . . . . 36

2.3 Selected Alkynylation Examples. . . . . . . . . . . . . . . . . . . . . 37

2.4 Zn-ProPhenol Catalyzed Alkyne Addition. . . . . . . . . . . . . . . . . . 38

2.5 Alkynylation of Unsaturated Aldehydes. . . . . . . . . . . . . . . . . . . . 40

2.6 Proposed Mechanism for ProPhenol-Catalyzed Alkynylation of Aldehydes. . . . 44

2.7 Competing Reaction Pathways: A Methyl Addition Side Product. . . . . . . . . 48

2.8 Evaluating ProPhenol-Catalyzed Asymmetric Alkylation. . . . . . . . . . . . . 48

2.9 Scope and Evaluation of Alkynylation with Reduced Stoichiometry. . . . . . . . 51

2.10 Total Synthesis of Adociacetylene B. . . . . . . . . . . . . . . . . 52

2.11 Alkyne Additions in the Total Synthesis of Spirolaxine Methyl Ether. . . . . . . 53

2.12 Asymmetric Alkyne Addition in the Total Synthesis of Ushikulide A. . . . . . . 54 
3.1 Previous Syntheses of Humulene Macrocycles. . . . . . . . . . . . . . . . . . 79

3.2 Ru-Catalyzed Alkene-Alkyne Coupling with $\gamma$-Hydroxy- $\alpha, \beta$-Acetylenic Esters. . 79

3.3 DMTSF-mediated Cyclization and Thioether Elimination. . . . . . . . . . . . 79

3.4 Retrosynthetic Analysis of Asteriscunolide D. . . . . . . . . . . . . . . . 80

3.5 Aldol Reaction of Isobutyraldehyde and Formalin. . . . . . . . . . . . . . . . . 81

3.6 Horner-Wadsworth-Emmons Olefination and Oxidation to Prepare 140. . . . . 81

3.7 Tishchenko Disproportionation: An Unwanted Side Reaction. . . . . . . . . . . 82

3.8 Asymmetric Alkyne Addition in the Synthesis of Asteriscunolide D. . . . . . . 82

3.9 Ru-Catalyzed Alkene-Alkyne Coupling with Allyl Alcohol. . . . . . . . . . . . . . 83

3.10 Alkene-Alkyne Coupling Mechanism and Selectivity. . . . . . . . . . . . . . 83

3.11 Installation of the Cyclization Functionality. . . . . . . . . . . . . . . . 84

3.12 Thionium-Induced Macrocyclization. . . . . . . . . . . . . . . . 84

3.13 Stereospecific Thioether Elimination to Complete the Total Synthesis. . . . . . 85

3.14 Enantioselective Total Synthesis of Asteriscunolide D. . . . . . . . . . . . 86

4.1 Yamaguchi Macrolactonization of Aspergillides A, B, and C. . . . . . . . . . 99

4.2 Transannular Michael Addition in the Synthesis of Aspergillide A and B. . . . . 100

4.3 Direct Conversion of Aspergillide A to Aspergillide B. . . . . . . . . . . . . . . 100

4.4 The First Total Synthesis of Aspergillide B. . . . . . . . . . . . . . . . . 101

4.5 A Divergent Synthesis of Aspergillide A and B. . . . . . . . . . . . . . . 102

4.6 Fuwa's Transition Metal Catalyzed Synthesis of Aspergillide B. . . . . . . . . . 103

4.7 Synthesis of ent-Aspergillide B from Chiral Pool Starting Materials. . . . . . . . 104

4.8 Sequential Application of Asymmetric Alkynylation and Ru-Catalyzed Hydrosilylation. . . . . . . . . . . . . . . . . . 105

4.9 Retrosynthetic Analysis of Aspergillide B. . . . . . . . . . . . . . . 105

4.10 Synthesis of Alkyne $( \pm)-111 \ldots \ldots \ldots \ldots$. . . . . . . . . . . . 106

4.11 Competing Cross Aldol Side Reactions. . . . . . . . . . . . . . . . . . 106

4.12 Attempted Addition of Methyl Propiolate to Fumaraldehyde Dimethyl Acetal. . 108

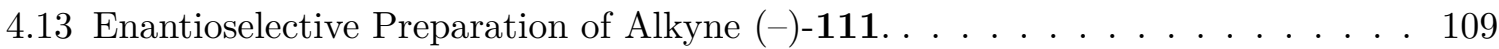

4.14 Alkynylation of Fumaraldehyde using a Single Equivalent of $(-)-111$. . . . . . . 109

4.15 Optimization of Enal Conjugate Reduction. . . . . . . . . . . . . . . . . . 110

$4.16 \mathrm{Ru}$-Catalyzed Cyclic Siloxane Formation. . . . . . . . . . . . . . . . . 111

$4.17 \mathrm{Ru}$-Catalyzed Hydrosilylation with Benzyldimethylsilane. . . . . . . . . . . . 112

4.18 First Alkyne Addition in the Synthesis of Aspergillide B. . . . . . . . . . . . . . 113

4.19 Formal Total Synthesis of Aspergillide B. . . . . . . . . . . . . . . . . . . . . . 114

5.1 Challenging Structural Elements of Labillarides E-H. . . . . . . . . . . . . . . . 160

5.2 Disconnection of Common Bonds Shared by A, B and C. . . . . . . . . . . 160

5.3 First and Second Generation Pd-AA Linchpin Strategies. . . . . . . . . . . . . 161

5.4 Seminal Pd-AA Reported by Tsuji. . . . . . . . . . . . . . . . . . . . . . . . . . 161

5.5 Pd-AA Mechanism with Soft and Hard Nucleophiles. . . . . . . . . . . . . . . 162

5.6 Chirality Transfer in Pd-AA Reactions. . . . . . . . . . . . . . . . . . . . . 162

$5.7 \pi-\sigma-\pi$ Isomerization Mechanisms. . . . . . . . . . . . . . . . . . 163

5.8 Regiocontrol in Pd-AA using Different Reaction Conditions. . . . . . . . . . . . 163

5.9 Regioselectivity and Proposed 'Distorted' $\pi$-Allyl-Pd Complexes. . . . . . . . . 164

5.10 Selected Pd-AA Examples in Natural Product Synthesis. . . . . . . . . . . . . . 165

5.11 Regioselectivity in Pd-AA with 4-Hydroxy- $\alpha$-pyrones. . . . . . . . . . . 166

5.12 Reversible $O$-Alkylation in the Presence of $\operatorname{Pd}(0) \ldots \ldots \ldots \ldots$

5.13 Pd-AA Reactions with 4 -Hydroxy- $\alpha$-pyrones. . . . . . . . . . . . 166 
5.14 Labillarides E-H First Generation Retrosynthesis. . . . . . . . . . . . . . . . . 167

5.15 Initial Pd-AA with 6-Methyl-4-hydroxy- $\alpha$-pyrone. . . . . . . . . . . . . . 168

5.16 Attempted Pd-AA with Vinyl Silane 251b. . . . . . . . . . . . . . . . 168

5.17 Bromoetherification of $\mathbf{2 5 2}$ to Form a Furanopyrone. . . . . . . . . . . . . . . . 169

5.18 Structural Requirements for a Diastereoselective Halocyclization. . . . . . . . . 170

5.19 Attempted Incorporation of Directing Groups for Haloetherification. . . . . . . 170

5.20 Labillarides E-H Second Generation Retrosynthesis. . . . . . . . . . . . . . 171

5.21 Synthesis of $\beta$-hydroxy Amide 265 . . . . . . . . . . . . . . . . . . 172

5.22 Synthesis of Aldehyde 272 from (L)-Ascorbic Acid. . . . . . . . . . . . . . 173

5.23 Synthesis of $\alpha$-Bromo- $\alpha$ - $\beta$-unsaturated Carboxylic Acid 264. . . . . . . . . . 173

5.24 Coupling of Carboxylic Acid 264 and Alcohol 265. . . . . . . . . . . . . . . 174

5.25 Sorensen's Intramolecular Acylation in the Synthesis of FR182877. . . . . . . . 174

5.26 Attempted Intramolecular Acylation of 268. . . . . . . . . . . . . . 175

5.27 Attempted Formation of Carboxylic Acid 283. . . . . . . . . . . . . . . 175

5.28 Synthesis of the $\alpha$-Bromophosphonate Linchpin 287 . . . . . . . . . . . 176

5.29 Synthesis of Alternative Substrates for Intramolecular Acylation. . . . . . . . . 176

5.30 Attempted Intramolecular Acylation with 291 and 292 . . . . . . . . . . . 177

5.31 Attempted Knovenagel Condensation with 4-Hydroxy-6-methyl- $\alpha$-pyrone. . . . 177

6.1 Pd-AA Cascade with a 4-Hydroxy- $\alpha$-pyrone. . . . . . . . . . . . . . . . . . 209

6.2 Generic Pd-AA Cascade. . . . . . . . . . . . . . . . . . . . . . . . 209

6.3 Regiochemical Possibilities of a Pd-AA casade with a Symmetric Bis-Electrophile.210

6.4 Product Diversity in Pd-AA Cascades. . . . . . . . . . . . . . . . . . 210

6.5 Enantioselective Synthesis of Morpholines and Dihydrofurans using Pd-AA

Cascades. . . . . . . . . . . . . . . . . . . . 210

6.6 Synthesis of the Core Structure of Neosarpagine using Pd-AA cascades. . . . . 211

6.7 Pd-AA Cascade in the Synthesis of Huperzine A . . . . . . . . . . . . . . . 212

6.8 Pd-AA Cascade in the Synthesis of Agelastatin A. . . . . . . . . . . . . . 212

6.94 -Hydroxy- $\alpha$-pyrone Pd-AA Cascade. . . . . . . . . . . . . . . . . . . 213

6.10 Potential Palladacycle Formation with $\mathrm{P}(o \mathrm{Tol})_{3}$. . . . . . . . . . . . . . . . 215

6.11 Literature Example of a Stable Palladacyclic Intermediate. . . . . . . . . . . . 215

6.12 A Linchpin-Based Approach to the Core of Labillaride E-H. . . . . . . . . . . . 216

6.13 Installation of the Labillaride Side Chain using Alkene Cross Metathesis. . . . . 217

7.1 Synthesis of a Mannitol-Derived, Substituted Bis-Electrophile. . . . . . . . . . . 239

7.2 Attempted Pd-AA Cascade with the Substituted Bis-Electrophile, 324. . . . . 239

7.3 A Cyclohexene-Based Pd-AA Cascade. . . . . . . . . . . . . . . . . . . . 240

7.4 Regiochemical Permutations for a Non-Symmetric Pd-AA Cascade. . . . . . . . 241

7.5 Synthesis of Pyran Substrates, cis-332 and trans-332. . . . . . . . . . . . . . 241

7.6 Inversion of Configuration in the Pd-AA Cascade with Dihydropyran trans-332. 243

7.7 Stereochemical Assignment of $\mathbf{3 3 1}$ and Mechanistic Inferences. . . . . . . . . . 243

7.8 Proposed Mechanism for the Pd-AA Cascade. . . . . . . . . . . . . . . . . . 244

7.9 Bis-Nucleophile Scope in the Pd-AA Cascade. . . . . . . . . . . . . . . . . . 245

7.10 Formation of $\alpha$-Disubstituted $\beta$-Dicarbonyl Side Products. . . . . . . . . . . . 246

7.11 Pd-AA Cascade with Meldrum's Acid: $\gamma$-Lactone Formation. . . . . . . . . . . 247

7.12 Pd-AA Cascade with Enantioenriched C2-substituted Bis-Electrophiles. . . . . 249

7.13 Attempted Dihydropyran Hydrolysis. . . . . . . . . . . . . . . . . 252

7.14 Bromohydroxylation and an Unexpected Epoxide Rearrangement. . . . . . . . 253 
7.15 Potential Application of a Pd-AA Cascade in the Synthesis of Canadensolide and Related Analogues. . . . . . . . . . . . . . . . . . . . 253

8.1 Preparation of Dihydropyran 332. . . . . . . . . . . . . . 301 


\section{List of Figures}

1.1 The Synthetic Evolution of Epothilones as Therapeutic Agents. . . . . . . . . . 4

1.2 Classification of Linchpin Strategies. . . . . . . . . . . . . . . . . . . . 19

1.3 Privileged Linchpins and their Application in Total Synthesis. . . . . . . . . . 20

2.1 Acidity, Bonding and Reactivity of Terminal Alkynes. . . . . . . . . . . . . . 35

2.2 Zn-ProPhenol Crystal Structure and Proposed Interaction with Lewis Basic Additives. . . . . . . . . . . . . . . . . . 45

$2.3{ }^{1} \mathrm{H}$ NMR Analysis of the Alkyne Premix. . . . . . . . . . . . . . . . . 47

3.1 The Asteriscunolide Family of Natural Products. . . . . . . . . . . . . . . . . . 77

3.2 Cyclic Conformers of Humulene. . . . . . . . . . . . . . . . . . . . 77

3.3 X-Ray Crystal Structures of Asteriscunolides A, B, C, and D. . . . . . . . 78

4.1 The Aspergillide Family of Natural Products. . . . . . . . . . . . . . . . . 98

4.2 X-Ray Crystal Structures of the $m$-Bromobenzoates of Aspergillides A and B. . 98

4.3 Examples of $\beta$-Selectivity in Hydrosilylations with $\mathrm{CpRu}\left(\mathrm{CH}_{3} \mathrm{CN}\right)_{3} \mathrm{PF}_{6}$ and

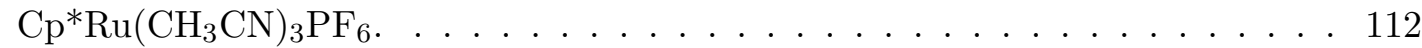

5.1 Natural Products from Phacelocarpus labillardieri: Labillarides E-H . . . . . 158

5.2 NMR Database Approach to the Structural Assignment of Oasomycin A: Typical ${ }^{13} \mathrm{C}$ NMR Differences Between Diastereomeric Compounds. . . . . . . . 159

5.3 Atropisomerism in the Neurymenolide Natural Products. . . . . . . . . . . . . . 159

6.1 LC-MS Analysis of the Furopyrones syn-, and anti-323. . . . . . . . . . . . 217

6.2 Comparison of the ${ }^{1} \mathrm{H}$ NMR Spectra of a Mixture of $s y n$ - and anti-323 in $\mathrm{CDCl}_{3}$ and Benzene- $d_{6} \ldots \ldots \ldots \ldots \ldots \ldots \ldots \ldots \ldots \ldots \ldots$

6.3 Selected ${ }^{13} \mathrm{C}$ NMR Resonances of syn- and -anti-323 Diastereomers. . . . . . . 219

6.4 Tentative Assignment of the Relative C3/C6 Configuration of Labillarides E-H

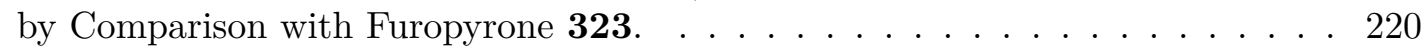

6.5 Axial Chirality in Labillarides E-H: Calculated Atropisomeric Transition State and Intrinsic Reaction Coordinate. . . . . . . . . . . . . . . . . . . . 223

6.6 Comparison of Experimental and Calculated Coupling Constants for Different Configurations of the Labillaride Scaffold. . . . . . . . . . . . . . . . . . . . 224

6.7 Structural Similarities Resulting from Inversion of Axial and C8 Point Chirality. 224

7.1 Attempted Pd-AA Cascades with Cyclic Bis-Electrophiles. . . . . . . . . . . . . 240

$7.2 \quad$ X-Ray Crystal Structure of $334 . \quad \ldots \ldots \ldots$. . . . . . . . . . . . 243

7.3 Bioactive Furopyrone and Pyrone-Containing Compounds. . . . . . . . . . . . . 250

7.4 Third Generation Approach to Labillarides E-H: Dihydropyran Fragmentation. 252 
8.1 Synthesis and Utility of 3,6 -dihydro- $2 H$-pyrans. . . . . . . . . . . . . . . 300

8.2 Challenges in the Structural Assignment of Dihydropyran 332. . . . . . . . . . 302

8.3 Axial Shielding Effects in Inositol and 3,6-Dihydro-2 $H$-pyran Diastereomers. . . 302

8.4 Anomeric and Allylic Effects in the Conformational Preference of 3,6-Dihydro$2 H$-pyrans. . . . . . . . . . . . . . . . . . 303

8.5 Conformational Insights from ${ }^{1} \mathrm{H}-\mathrm{NMR} . \ldots \ldots 303$

8.6 A Diagnostic Axial Shielding Pattern in 3,6-Dihydro- $2 H$-pyrans: $\delta_{\mathrm{C}}($ cis-332 $)-$ $\delta_{\mathrm{C}}($ trans-332). . . . . . . . . . . . . . . . . . . . 304

8.7 Assignment of Relative Stereochemistry using the Axial Shielding Template: A Worked Example. . . . . . . . . . . . . . . . . . . 306

8.8 Newman Projections for the Lowest Energy Conformers of cis-332 . . . . . . . . 312 


\section{List of Tables}

2.1 Initial Optimization. . . . . . . . . . . . . . . . . . . . . 39

2.2 Addition of Methyl Propiolate to $\alpha$ - $\beta$-Unsaturated Aldehydes. . . . . . . . . . . 41

2.3 Enantioselective Alkynylation of Aliphatic Aldehydes. . . . . . . . . . . . . . 43

2.4 Enantioselective Alkynylation with TPPO . . . . . . . . . . . . . . 45

2.5 Alkynylation with Reduced Stoichiometry. . . . . . . . . . . . . . . 46

2.6 Optimization of Alkyne Addition with an Enolizable Substrate. . . . . . . . . . 49

2.7 Optimization of Methyl Propiolate Addition to Octanal. . . . . . . . . . . . . 50

4.1 Biological Activity of the Aspergillides and Related Analogues. . . . . . . . . . 99

4.2 Alkynylation Substrate and Reaction Optimization. . . . . . . . . . . . . 107

5.1 Stereochemical Assignment of Bromosilanes 254 and 255. . . . . . . . . . . 169

6.1 Pd-AA Cascade Optimization: Annulation of 4-Hydroxy-6-methyl- $\alpha$-pyrone. . . 214

6.2 Comparative Analysis of the ${ }^{13} \mathrm{C}$ NMR Resonances of the Labillaride Core and Side Chain. . . . . . . . . . . . . . . . . . . . . . . . . . 221

7.1 Optimization of a Pd-AA Cascade with Dihydropyran cis-332 . . . . . . . . 242

7.2 Optimization of a Pd-AA Cascade with Meldrum's Acid. . . . . . . . . . . . . . 248

7.3 Bioactivity of Select Pd-AA Cascade Products. . . . . . . . . . . . . . 251

8.1 Development of a ${ }^{13} \mathrm{C}$ NMR Shielding Template. . . . . . . . . . . . . . . . 305

8.2 Using RMS Error to Evaluate Template Matching. . . . . . . . . . . . . . . . . 307

$8.3{ }^{13} \mathrm{C}$ NMR Shielding Effects in C3-Hydroxy Compounds. . . . . . . . . . . . . . 307

8.4 Scope and Limitations of the ${ }^{13} \mathrm{C}$ NMR Shielding Template. . . . . . . . . . . . . 308

8.5 Evaluating Calculated ${ }^{13} \mathrm{C}$ NMR Chemical Shifts for Dihydro- $2 H$-pyrans. . . . 310

8.6 Assignment of Relative Stereochemistry with NMR Data from a Single Diastereomer. . . . . . . . . . . . . . . . . . . . . 313

B.1 Crystal Data and Structure Refinement for MJBA . . . . . . . . . . . . . . 368

B.2 Fractional Atomic Coordinates (x $10^{4}$ ) and Equivalent Isotropic Displacement Parameters $\left(\AA^{2} \times 10^{3}\right)$ for MJBA. . . . . . . . . . . . . . . . 369

B.3 Hydrogen Atom Coordinates $\left(\AA \times 10^{4}\right)$ and Isotropic Displacement Parameters $\left(\AA^{2} \times 10^{3}\right)$ for MJBA. . . . . . . . . . . . . . . . . . . 369

B.4 Anisotropic Displacement Parameters $\left(\AA^{2} \times 10^{3}\right)$ for MJBA. . . . . . . . . . . 370

B.5 Bond Lengths for MJBA. . . . . . . . . . . . . . . . . . . . . 370

B.6 Bond Angles for MJBA. . . . . . . . . . . . . . . . . . 370 


\section{Abbreviations}

\begin{tabular}{|c|c|}
\hline AAA & Asymmetric Allylic Alkylation \\
\hline $\mathrm{AA}$ & Allylic Alkylation \\
\hline Ac & Acetyl \\
\hline $\mathrm{AChE}$ & Acetylcholinesterase \\
\hline $\mathrm{Am}$ & Amyl \\
\hline aq. & Aqueous \\
\hline $\mathrm{Ar}$ & Aryl \\
\hline BAIB & Bis-Acetoxyiodobenzene \\
\hline $9-\mathrm{BBN}$ & 9-Borabicyclo[3.3.1]nonane \\
\hline BDMS & Benzyldimethylsilyl \\
\hline BDP & 1,2-Bis(diphenylphosphino)benzene \\
\hline BINOL & 2,2'-Dihydroxy-1,1'-binaphthyl \\
\hline $\mathrm{Bn}$ & Benzyl \\
\hline Boc & tert-Butyloxycarbonyl \\
\hline BRSM & Based on Recovered Starting Material \\
\hline $\mathrm{Bu}$ & Butyl \\
\hline $\mathrm{Bz}$ & Benzoyl \\
\hline CDI & 1,1'-Carbonyldiimidazole \\
\hline CSA & Camphor-10-sulfonic Acid \\
\hline $\operatorname{cod}$ & Cyclooctadiene \\
\hline $\mathrm{Cp}$ & Cyclopentadienyl \\
\hline $\mathrm{Cp}^{*}$ & Pentamethylcyclopentadienyl \\
\hline $\mathrm{Cy}$ & Cyclohexyl \\
\hline $\mathrm{DABCO}$ & 1,4-Diazabicyclo[2.2.2] octane \\
\hline $\mathrm{DACH}$ & 1,2-Diaminocyclohexane \\
\hline dba & Dibenzylidene acetone \\
\hline DBU & 1,8-Diazabicyclo[5.4.0]undec-7-ene \\
\hline $\mathrm{DCE}$ & 1,2-Dichloroethane \\
\hline DCM & Dichloromethane \\
\hline DDQ & 2,3-Dichloro-5,6-dicyano-1,4- benzoquinone \\
\hline DEAD & Diethyl azodicarboxylate \\
\hline DEMS & Diethoxymethylsilane \\
\hline DET & Diethyl Tartrate \\
\hline DIAD & Diisopropyl Azodicarboxylate \\
\hline DIBAL-H & Diisobutylaluminum Hydride \\
\hline DIPEA & Diisopropylethylamine \\
\hline DMA & $N, N$-Dimethylacetamide \\
\hline DMAP & 4- $(N, N)$-Dimethylaminopyridine \\
\hline DME & Dimethoxyethane \\
\hline DMF & $N, N$-Dimethylformamide \\
\hline DMP & Dess-Martin periodinane \\
\hline DMSO & Dimethylsulfoxide \\
\hline DMTSF & Dimethyl(methylthio)sulfonium Tetrafluoroborate \\
\hline DPEphos & Bis[2-(diphenylphosphino)phenyl] ether \\
\hline dppb & 1,4-Bis(diphenylphosphino)butane \\
\hline dppe & 1,2 -Bis(diphenylphosphino)ethane \\
\hline dppf & 1,1 '-Bis(diphenylphosphino)ferrocene \\
\hline $\mathrm{dr}$ & Diastereomeric ratio \\
\hline EDCI & $N$-(3-Dimethylaminopropyl)- $N$ '-ethylcarbodiimide Hydrochloride \\
\hline ee & Enantiomeric excess \\
\hline Et & Ethyl \\
\hline gem- & Geminal \\
\hline $\mathrm{h}$ & $\operatorname{Hour}(\mathrm{s})$ \\
\hline $\mathrm{HF}$ & Hydrofluoric acid \\
\hline HFIP & Hexafluoroisopropyl alcohol \\
\hline
\end{tabular}




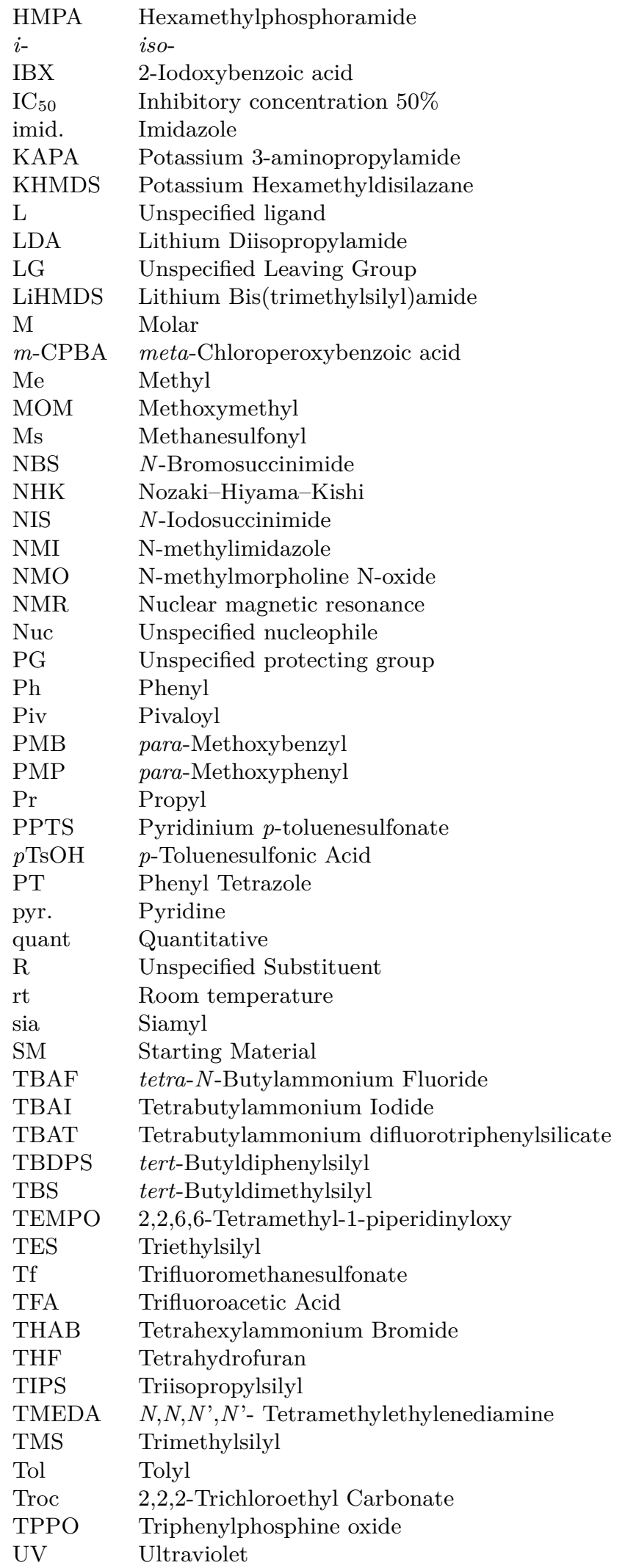


Chapter 1

\section{Introduction}




\section{$1.1 \quad$ Preamble}

The purpose of this introduction is to provide background and context to the research that follows, while also conveying a sense of why I have chosen to address specific research questions and why synthetic problems were approached in a certain way. Specifically, it is designed to address three main questions that underpin the field of synthetic organic chemistry:

Target - What compounds should be synthesized and why?

Methodology - What reactions should be used to construct bonds in a molecule?

Strategy - In what order should these reactions be performed to maximize efficiency?

Each of these relatively simple questions has enormous implications for the way in which one performs research in synthetic organic chemistry and spans topics such as pharmacodynamics, atom economy, chemoselectivity, stereoselectivity, and retrosynthetic analysis. The following three sections aim to provide an awareness of the implications that the research contained within this thesis has in regard to target, methodology and strategy.

\subsection{Total Synthesis and Natural Product Analogues in the Drug Discovery Process}

Natural products have inspired the development of many life saving medical treatments. ${ }^{1}$ Ailments such as bacterial infection, inflammatory diseases and cancer can be treated with natural products or natural product derivatives. Pharmacophores found in nature represent an unparalleled source of lead structures in the discovery of new therapeutic drugs. For example, the natural products Taxol ${ }^{\circledR}$ (paclitaxel, $\mathbf{1}$ ) and erythromycin $(\mathbf{2})$ are currently used to treat cancer and bacterial infection, respectively (Scheme 1.1). ${ }^{2}$ In addition to their direct use, the structures of Taxol ${ }^{\circledR}$ and erythromycin have inspired the development of the superior semisynthetic drugs, docetaxel (Taxotere ${ }^{\circledR}, 3$ ) and azithromycin (Zithromax ${ }^{\circledR}, 4$ ). ${ }^{3,4}$ Scheme 1.1 highlights the structural modifications made to these natural products to provide improved medicinal properties. Docetaxel has a better aqueous solubility than Taxol ${ }^{\circledR}$ and this results in an improved pharmacokinetic profile. The structural modifications present in azithromycin result in improved bioavailability and potency against gram-negative bacteria compared to erythromycin. These examples highlight the power of chemical synthesis in discovering novel medicinal compounds.

The total synthesis of a natural product is a process that often provides a wealth of knowledge and scientific bounty along the way. The essence of total synthesis has been linked to a quotation from Nobel Laureate Richard Feynman, "What I cannot create, I do not understand". ${ }^{5}$ Among other things, total synthesis provides a means to prepare substantial quantities of scarce, biologically active natural products. These compounds are present in the host organism in minute quantities, making biological evaluation and medical application very 


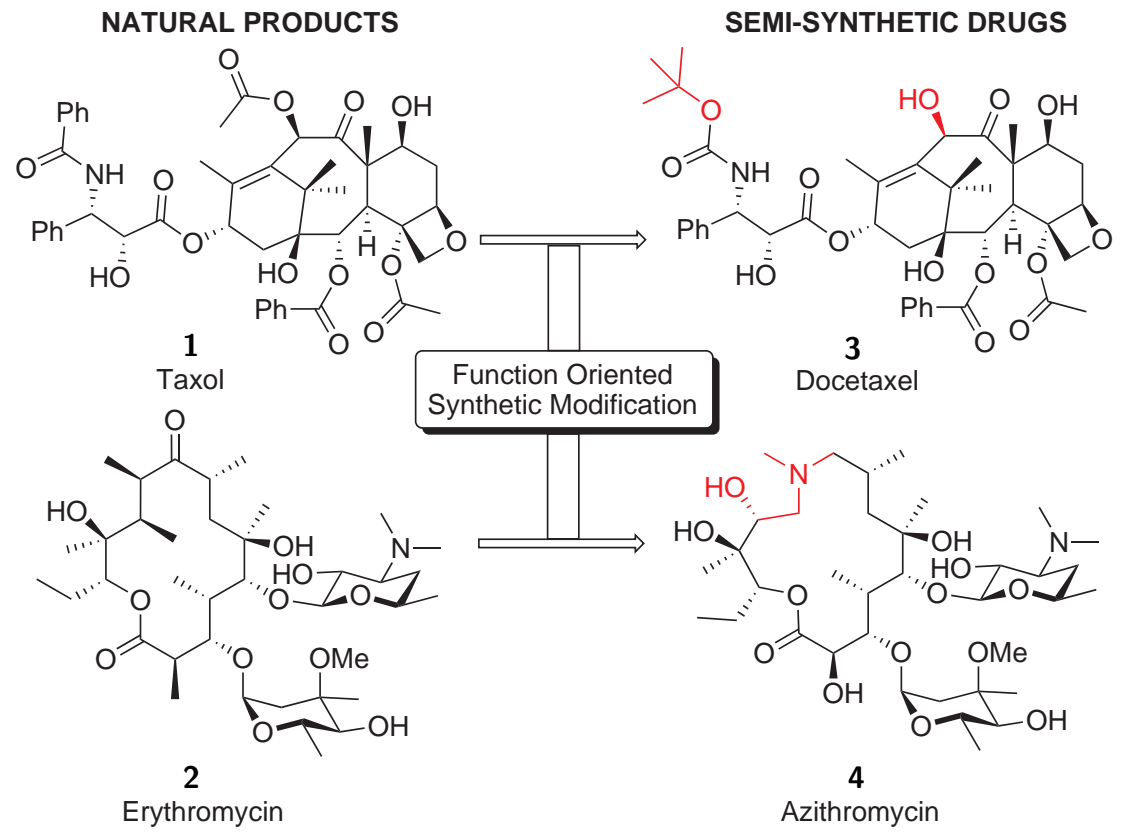

Scheme 1.1 Therapeutic Drugs Inspired by Natural Products

difficult when relying solely on natural sources. The synthetic preparation of a natural product allows further research into its therapeutic properties and is also a useful tool for confirming the structure and absolute configuration of the molecule. A number of structural revisions have been reported in the literature as a result of new insights gained from total synthesis. ${ }^{6}$

Total synthesis provides an excellent platform to investigate the medicinal properties of natural product analogues. Advanced synthetic intermediates provide exciting opportunities to explore derivatives that aren't accessible from the natural product itself. These structural modifications provide valuable insight into structure-activity relationships and the binding interactions that elicit a specific biological response. ${ }^{7}$ The epothilones are an excellent example of the synergistic application of total synthesis and rational drug design. Epothilone A $(\mathbf{5}, \mathrm{R}=\mathrm{H})$ and $\mathrm{B}\left(\mathbf{5}, \mathrm{R}=\mathrm{CH}_{3}\right)$ were first isolated by Hölfe from Sorangium cellulosum, a myxobacteria (Figure 1.1). ${ }^{8}$ Danishefsky and coworkers reported the first total synthesis of epothilone $\mathrm{A}$ in $1996 .{ }^{9}$ Despite the exciting in vitro anticancer activity of the epothilones, their clinical use is precluded by significant toxicity, even at subtherapeutic doses. ${ }^{10}$ Using diverted total synthesis, a number of advanced synthetic intermediates were converted into natural product analogues. ${ }^{11}$ This ultimately led to the development of a number of superior therapeutic drugs, two of which have progressed into clinical trials (Figure 1.1). 


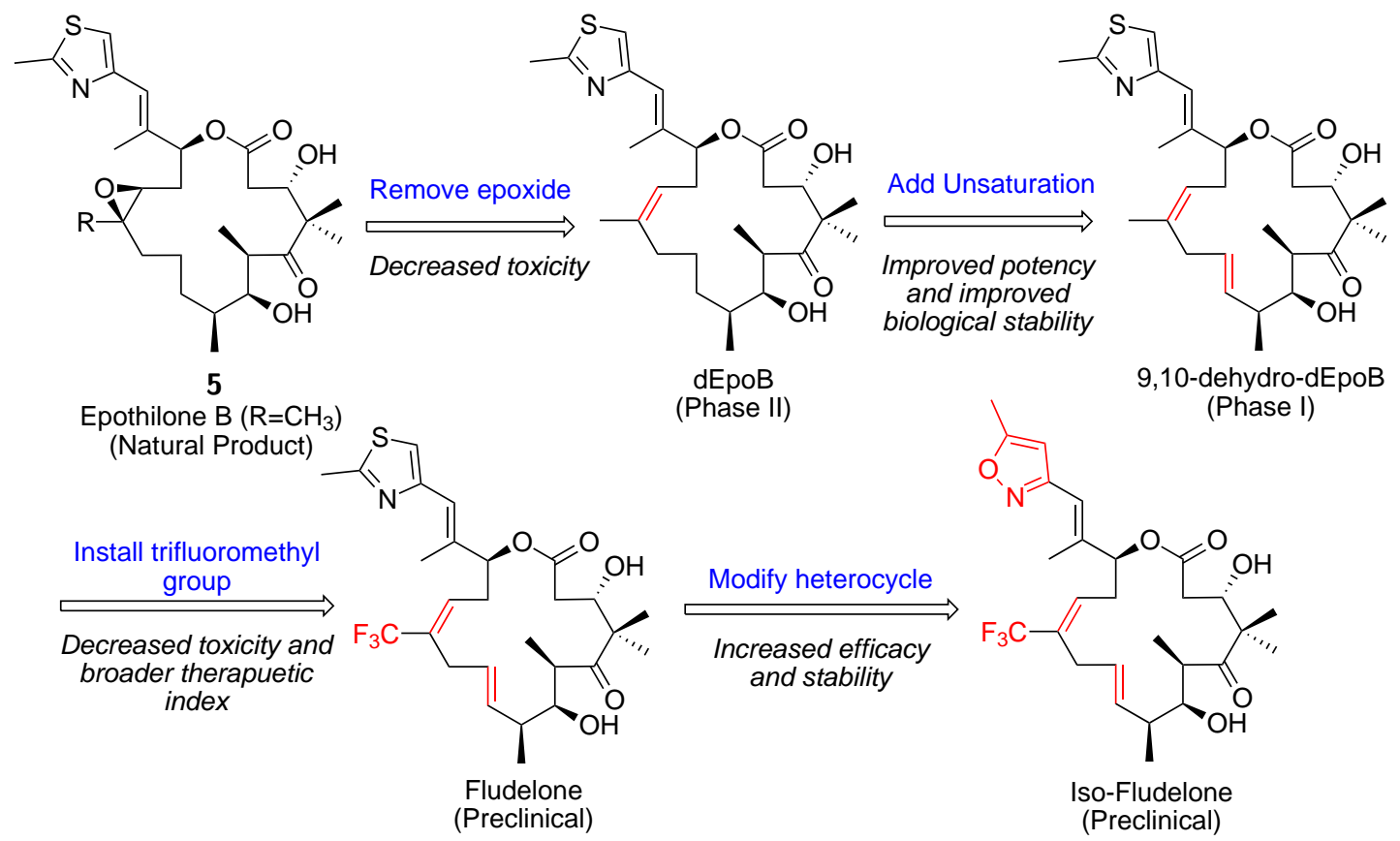

Figure 1.1 The Synthetic Evolution of Epothilones as Therapeutic Agents.

\subsection{The Pursuit of Atom Economy in Total Synthesis}

\subsubsection{Introduction}

Improving the efficiency of chemical synthesis has long been a fundamental goal for the chemical sciences. This goal has been pursued primarily through the development of new chemical transformations that generate molecular complexity in a facile and selective manner. ${ }^{12}$ The efficiency of a chemical reaction can be measured using a variety of metrics, one of the most prominent being atom economy. ${ }^{13} \mathrm{~A}$ number of landmark achievements in atom economic synthesis have been a direct result of the development of transition metal-catalyzed reactions, such as the Pd-catalyzed asymmetric allylic alkylation, ruthenium-catalyzed alkene-alkyne coupling, and zinc-catalyzed direct asymmetric aldol reactions (vide infra). The application of these methodologies in the synthesis of complex natural products provides a paradigm for further advancements in atom economic synthesis.

The goal of atom economy is to maximize the mass efficiency of a reaction; ideally, all the atoms of the starting materials are incorporated into the final product, using all other reagents in a catalytic manner. On a fundamental level, atom economy is enabled by the efficient activation of reagents, where control over the selectivity of bond-forming processes is paramount. In some instances the adjacent functional groups make bond forming inherently efficient, as is the case for the highly atom economic Diels-Alder reaction. This reaction can often occur in a stereoselective manner by simply heating the appropriate diene and dienophile, such as 6 and 7 (Scheme 1.2). ${ }^{14}$ However, the majority of cases are not that simple, and additional atoms are required to activate the reacting centers and control selectivity. The 
conjugate addition shown in Scheme 1.2 highlights the necessity of additional atoms to activate the starting materials and direct the reaction to occur in a stereoselective manner. ${ }^{15}$ In this case, a nucleophilic carbon atom is created by the presence of an adjacent magnesium atom. Nucleophilic addition is then directed via the magnesium alkoxide 'ate' complex $\mathbf{8}$, which is formed by prior deprotonation of the hydroxyl group using lithium diisopropylamide (LDA) and manipulation of the Schlenk equilibrium using a crown ether. Intramolecular delivery of the nucleophile ultimately results in a stereoselective conjugate addition, albeit with considerable lithium and magnesium byproducts.

Thermal Diels-Alder Reaction: Excellent Atom Economy

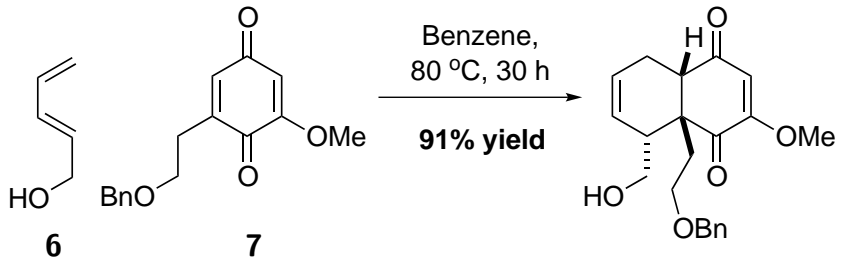

Conjugate Addition using Magnesium: Poor Atom Economy

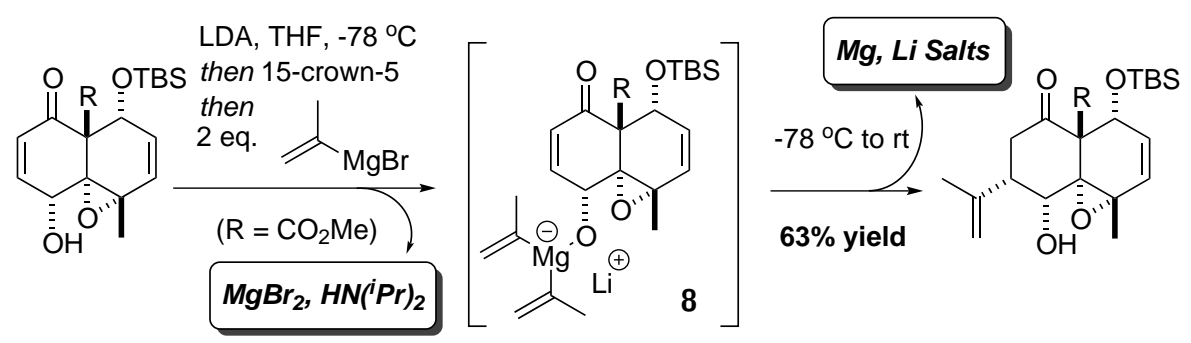

Scheme 1.2 Considering Atom Economy in Bond Construction.

Maximizing atom economy while maintaining high levels of selectivity remains challenging. The conjugate reduction of the $\alpha, \beta$-unsaturated aldehyde, citral (9) serves to highlight the extensive efforts that are required to evolve an atom-economic solution (Scheme 1.3). This transformation poses three major challenges: avoidance of 1,2-reduction of the aldehyde, selectivity for the enone double bond in the presence of an electron-rich trisubstituted alkene and avoiding the generation of a reactive enolate capable of participating in an unwanted aldol reaction. A variety of conditions have been used to overcome these challenges and perform this selective transformation in high yield (Scheme 1.3). However, the efficiency of these reactions, in terms of atom economy, varies considerably and in a number of cases the mass of the reaction byproducts is greater than that of the desired product 10. For example, the use of Stryker's reagent, $\left[\left(\mathrm{Ph}_{3} \mathrm{P}\right) \mathrm{CuH}\right]_{6}$, provides a very mild and selective hydride source for 1,4reduction. ${ }^{16}$ The resulting copper enolate is trapped as a silyl enol ether, which is subsequently hydrolyzed to produce the desired aldehyde 10. However, this method does not use mass economically, producing the stoichiometric reaction byproducts $\left[\left(\mathrm{Ph}_{3} \mathrm{P}\right) \mathrm{CuCl}\right]_{4}$ and $\mathrm{TBS}-\mathrm{OH}$ with a combined molecular weight of $1577 \mathrm{~g} / \mathrm{mol} .{ }^{17}$ The remaining methods in Scheme 1.3 contain increasing levels of atom economy, and include: Pd-catalyzed conjugate reduction using $\mathrm{Bu}_{3} \mathrm{SnH}$ and hydrolysis of the resulting tin enolate, ${ }^{18}$ organocatalytic transfer hydrogenation 


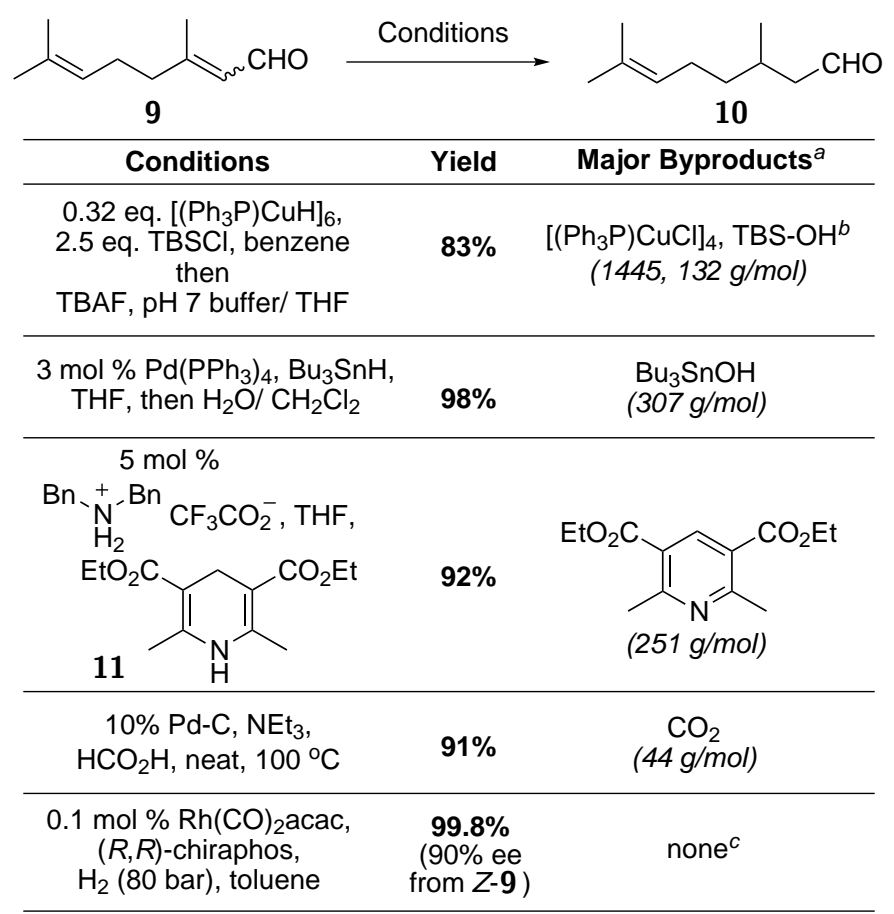

\begin{abstract}
a Starting materials used in excess and solvents were not taken into account when considering atom economy as these can, in theory, be recycled. ${ }^{b}$ While TBS-F is initially formed, under these conditions it is converted to TBS-OH and TBAF is regenerated. ${ }^{c}$ While no byproducts are produced, a large excess of hydrogen gas is needed to facilitate this reaction. $(R, R)$-chiraphos $=$ $(2 R, 3 R)$-2,3-bis(diphenylphosphino)butane
\end{abstract}

Scheme 1.3 Atom Economy in the Conjugate Reduction of Citral.

using the Hantzsch ester (11), ${ }^{19} \mathrm{Pd}$-catalyzed transfer hydrogenation using formic acid and triethylamine,${ }^{20}$ and lastly, an enantioselective rhodium-catalyzed hydrogenation. ${ }^{21}$ While an excellent yield of the desired aldehyde $\mathbf{1 0}$ is obtained in each case, the mass efficiency of most of these reactions is poor and therefore limits the broader application of this chemistry. Consequently, the highly atom-economic rhodium-catalyzed hydrogenation is part of a patented process used by BASF for the industrial preparation of (-)-menthol. The rhodium catalyst used to facilitate this asymmetric transformation can be recovered after the reaction and reused multiple times.

\title{
1.3.2 Pd-Catalyzed Asymmetric Allylic Alkylation
}

Palladium-catalyzed allylic alkylation, often referred to as the Tsuji-Trost reaction, has become a powerful synthetic tool and is capable of high levels of chemo-, regio-, and stereoselectivity. ${ }^{22}$ The first report of this reaction was limited to the addition of enamine or malonate-based nucleophiles to stoichiometric $\pi$-allyl palladium chloride dimer. ${ }^{23}$ Soon after this initial report, Trost and coworkers discovered that the addition of phosphine ligands dramatically enhanced the electrophilicity of $\pi$-allyl palladium complexes (Scheme 1.4). ${ }^{24}$ This discovery greatly expanded the substrate scope of these reactions and ultimately led to the development of chiral phosphine ligands for Pd-catalyzed asymmetric allylic alkylation (Pd-AAA). ${ }^{25}$ Scheme 1.4 

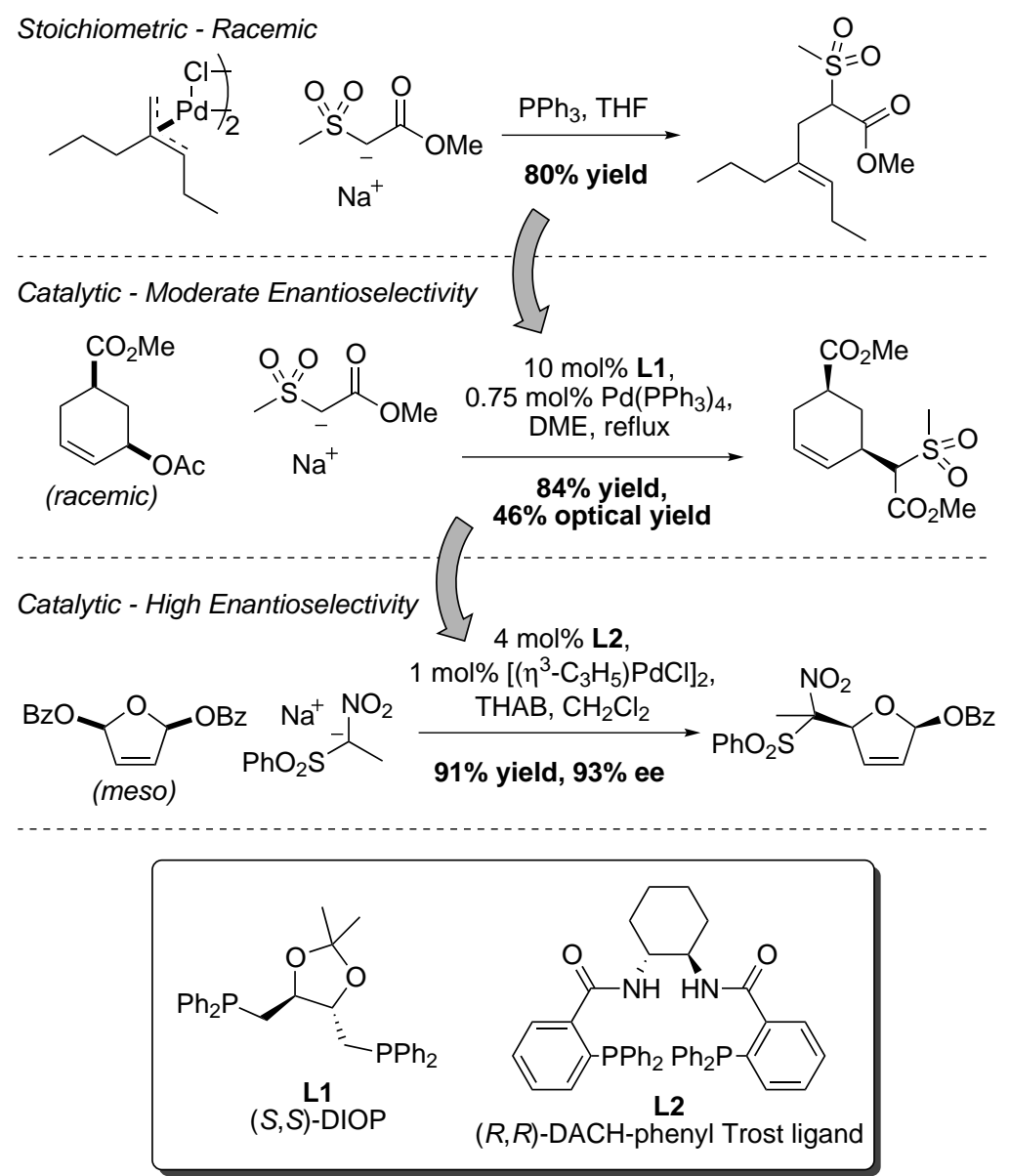

Scheme 1.4 The Evolution of Pd-Catalyzed Allylic Alkylation.

highlights the development of this methodology from the original stoichiometric racemic reaction to the modern catalytic enantioselective variants. Interestingly, some of the earliest research in the Trost group on the alkylation of stoichiometric $\pi$-allyl palladium complexes was performed by Terry Fullerton, a New Zealand Fulbright Scholar conducting post-doctoral research at the University of Wisconsin - Madison. ${ }^{26}$ Some of the most elegant applications of Pd-AAA reactions have been in the generation of chiral quaternary centers, a formidable task that often warrants special consideration in the planning of a synthesis. ${ }^{27}$

The synthetic strategy for the total synthesis of hamigeran B (12) was based on the use of a Pd-AAA reaction to form a challenging chiral quaternary center. ${ }^{28}$ Hamigeran $\mathrm{B}$ is a secondary metabolite originally isolated from the poecilosclerid sponge Hamigera tarangaensis collected from the Hen and Chicken Islands in New Zealand by Bergquist and Fromont (family Anchinoidae, syn. Phorbasidae). ${ }^{29}$ This compound displays potent anti-viral activity against both polio and herpes viruses, with only slight cytotoxicity to host cells. Moderate anticancer activity against P-388 leukemia cells was also observed $\left(\mathrm{IC}_{50}=13.5 \mu \mathrm{M}\right)$. The principal disconnections in the retrosynthesis of hamigeran B are shown in Scheme 1.5. The carbocyclic core of $\mathbf{1 2}$ was envisioned to arise from the intramolecular Heck reaction of aryl triflate $\mathbf{1 3}$. 


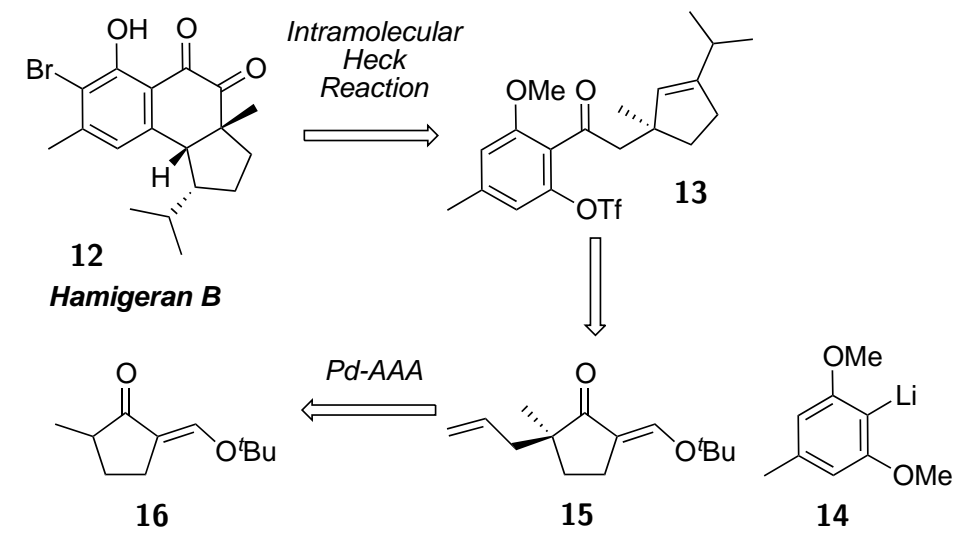

Scheme 1.5 Retrosynthetic Analysis of Hamigeran B.

This intermediate can be traced back to the aryl lithium reagent 14 and the aldehyde produced from ozonolysis of the terminal alkene 15. Alkene $\mathbf{1 5}$ could be generated using a Pd-AAA with a ketone enolate derived from 16. This class of nucleophiles has proven to be much more challenging to employ in AAA reactions than stabilized enolates derived from $\beta$-dicarbonyl compounds. ${ }^{30}$

The synthesis began with the formation of racemic ketone $\mathbf{1 6}$ from 2-methylcyclopentanone using a tandem formylation/vinylogous etherification sequence (Scheme 1.6). The prochiral tin enolate derived from $\mathbf{1 6}$ provided excellent yield and enantiomeric excess in the Pd-AAA reaction with only $1 \mathrm{~mol} \%$ of the active palladium catalyst. It was discovered that when fresh $n \mathrm{BuLi}$ was used to generate LDA for this reaction the ee of the product, 15, dropped dramatically. It was hypothesized that the presence of lithium alkoxides in older $n$ BuLi sources aids the stereoselectivity of this process. It was ultimately discovered that the addition of seven equivalents of $t \mathrm{BuOH}$ provides a reliable and scalable transformation. Lithium dimethylcuprate was then used to convert the tert-butyl enol ether into an isopropyl group. This was followed

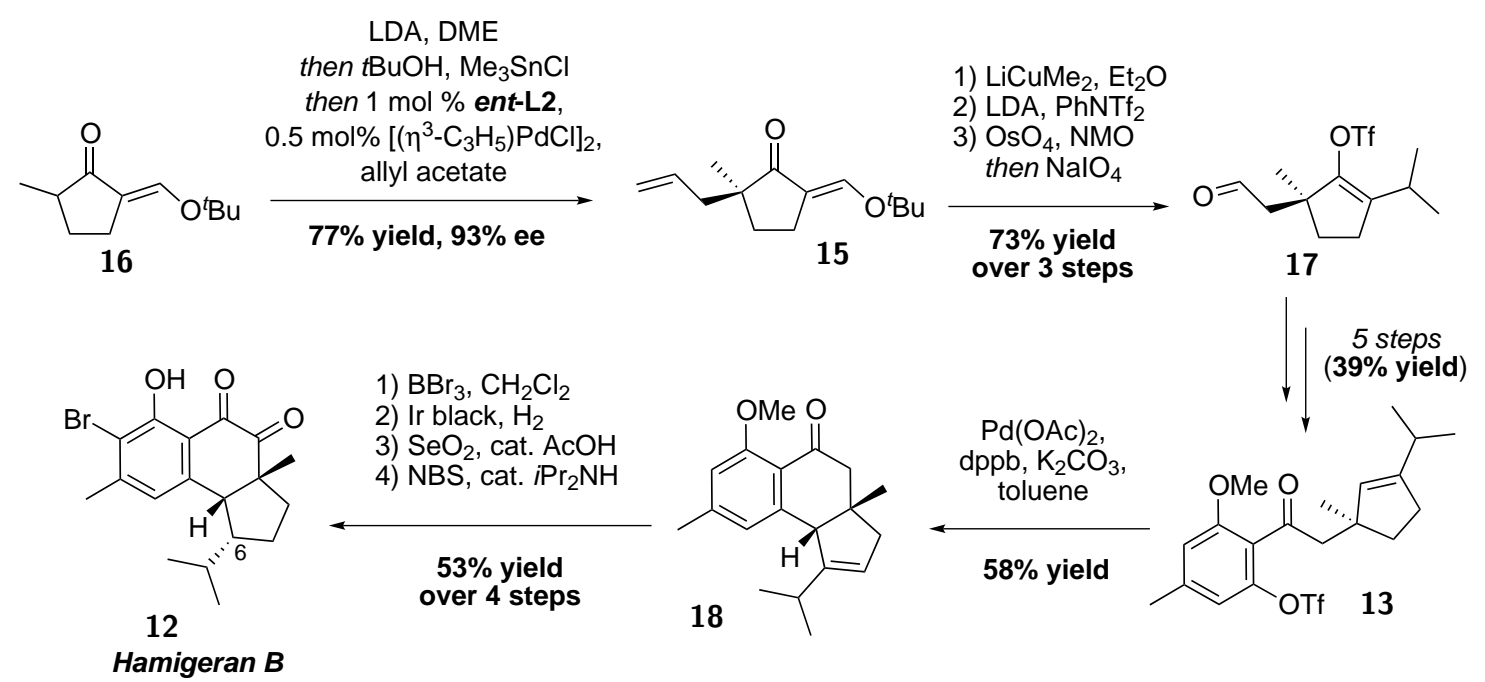

Scheme 1.6 Total Synthesis of Hamigeran B. 
by formation of an enol triflate and a one-pot dihydroxylation-periodate cleavage to produce aldehyde 17. In five additional steps aldehyde $\mathbf{1 7}$ was converted to the aryl ketone $\mathbf{1 3}$ required for the intramolecular Heck reaction. The intramolecular Heck reaction provided the desired product $\mathbf{1 8}$ in an optimized yield of $58 \%$, along with two isomeric alkene side products in a combined yield of $29 \%$. The use of a carbonate base rather than a tertiary amine base proved essential to avoid hydrogenolysis of the aryl triflate. Compound $\mathbf{1 8}$ was then deprotected using $\mathrm{BBr}_{3}$, and the trisubstituted double bond reduced using an iridium-catalyzed hydrogenation to give the kinetically favoured product with the isopropyl group on the concave face of the molecule. The corresponding palladium-catalyzed hydrogenation gave the thermodynamically favoured C6-epimer, which is presumably due to the equilibration of a semi-hydrogenated intermediate. Lastly, oxidation using selenium dioxide and careful monobromination provided the natural product 12. Ultimately, hamigeran B (12) was prepared using a 16-step longest linear sequence.

The decarboxylative Pd-AAA reaction has recently emerged as an attractive alternative to the traditional Pd-AAA with preformed metal enolates. ${ }^{31}$ Allyl enol carbonates, such as 19, can be used to prepare $\alpha$-chiral ketones in excellent yield and enantiomeric excess, while also avoiding the use of stoichiometric additives, such as $\mathrm{Me}_{3} \mathrm{SnCl}$ (Scheme 1.7). ${ }^{32}$ Ionization of the allyl carbonate and extrusion of carbon dioxide forms a cationic $\pi$-allyl palladium complex and an enolate anion. This variant of the Pd-AAA reaction enables the use of milder reaction conditions and a wider variety of substrates. Stoltz and coworkers have recently applied this strategy in a formal total synthesis of hamigeran B, as shown in Scheme 1.7. ${ }^{32}$ In this case, the trifluoromethyl substituted phosphinooxazole (PHOX) ligand, L3, was used to facilitate a Pd-catalyzed decarboxylative AAA reaction and provide the desired product $\mathbf{2 0}$ in excellent yield and enantioselectivity.

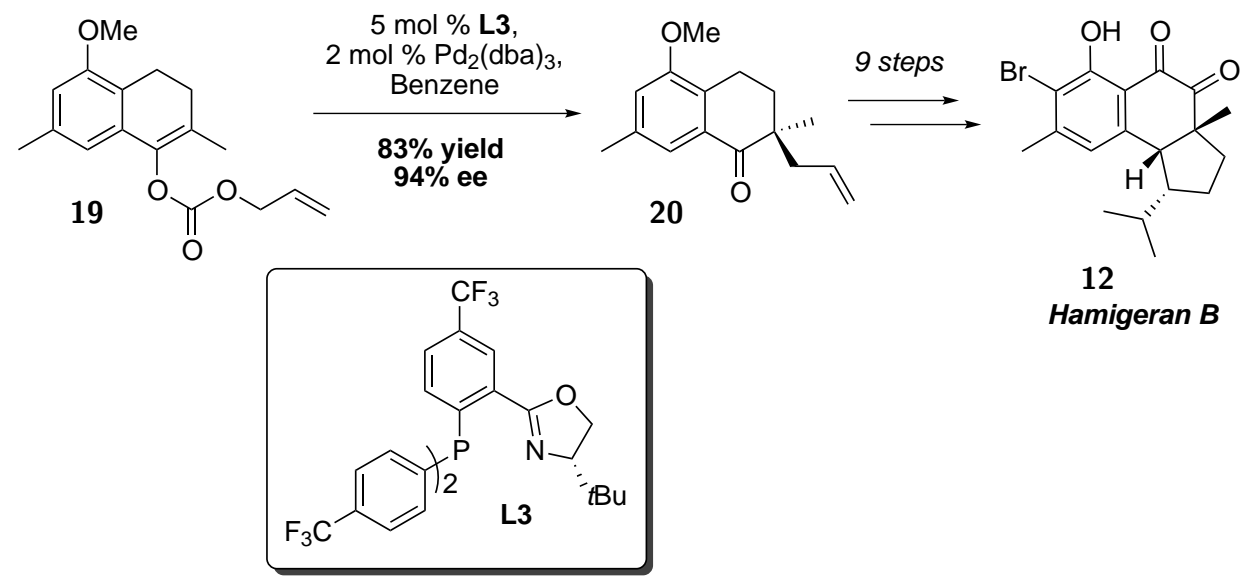

Scheme 1.7 Decarboxylative Pd-AAA Reaction in the Synthesis of Hamigeran B. 


\subsubsection{Ruthenium-Catalyzed Alkene-Alkyne Coupling}

The formation of carbon-carbon bonds is fundamental to chemical synthesis, and yet many syntheses still remain dependent on the use of activating groups or the presence of adjacent polarizing functionality to selectively construct these bonds. The ruthenium-catalyzed alkenealkyne coupling provides a tool by which simple unsaturated carbons can be coupled in a highly atom-economic fashion (Scheme 1.8). ${ }^{33}$ Using this reaction, the coupling of 1-octyne (21) and 1-octene (22) produces a ca. 5:1 mixture of branched (23) and linear (24) products. ${ }^{34}$ Additionally, no homocoupling products of either alkyne or alkene were observed.
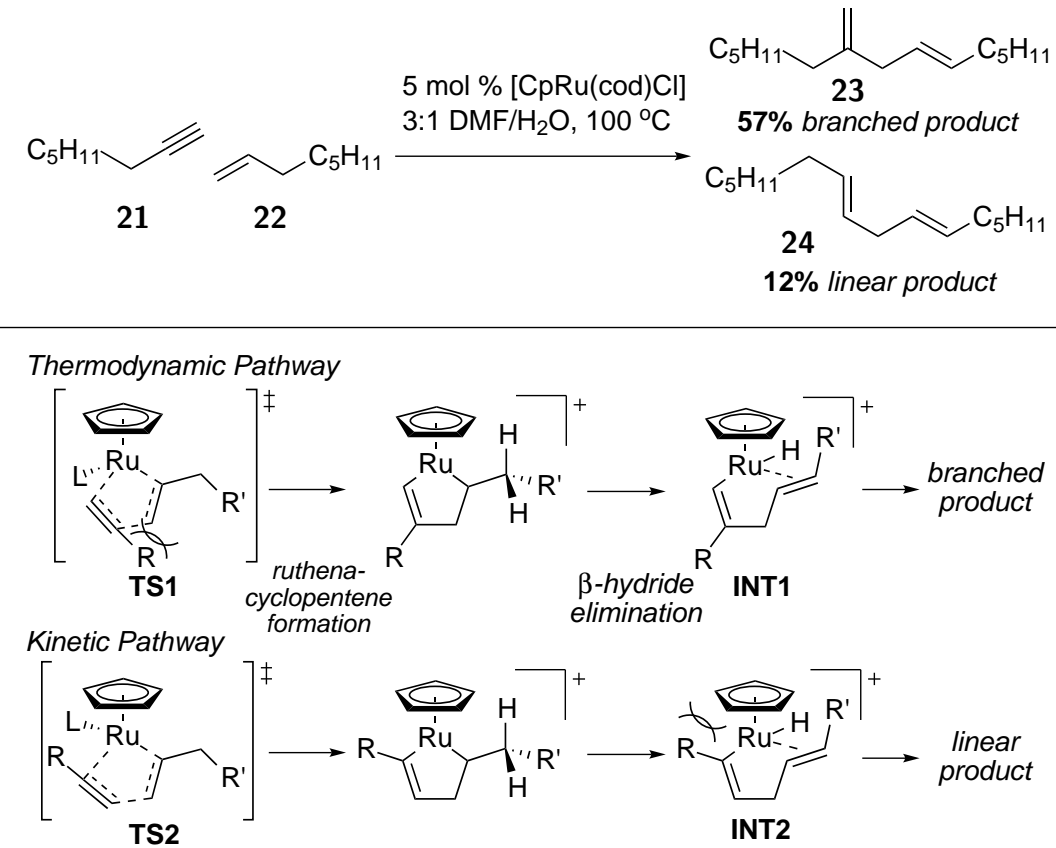

Scheme 1.8 Ruthenium-Catalyzed Alkene-Alkyne Coupling.

Two major mechanistic pathways have been proposed: one operates under thermodynamic control and produces the branched product; the other is under kinetic control and leads to the linear product. In reactions where $\beta$-hydride elimination is slower than ruthenacyclopentane formation (i.e. coupling of $\mathbf{2 1}$ and $\mathbf{2 2} ; \mathrm{R}, \mathrm{R}^{\prime}=$ alkyl), the steric interaction between the alkynyl substituent and the $[\mathrm{CpRu}]^{+}$moiety becomes differential and the thermodynamic product is formed on the basis of INT1 being favoured over INT2. Conversely, formation of the kinetic product is often observed with hindered alkynes, such as TMS-acetylene (see Scheme 1.10). In this case, regioselectivity is governed by the minimization of steric interactions in the transition state, and therefore TS2, where the alkynyl substituent points away from the alkene terminus, is favoured over TS1. With these mechanistic considerations in mind, the use of certain alkyne substitution patterns and reaction conditions can lead to regioselective alkene-alkyne coupling, providing a powerful tool for chemical synthesis.

The Ru-catalyzed alkene-alkyne coupling was used to great effect in the synthesis of mycalamide A (25), ${ }^{35}$ a highly potent antitumor agent originally isolated from the New Zealand 
marine sponge Mycale sp. ${ }^{36}$ Mycalamide A has been shown to inhibit protein synthesis and induce apoptosis in cancerous cells. ${ }^{37}$ Retrosynthetic disconnection of the central amide bond provides (-)-7-benzoylpederic acid $\mathbf{2 6}$ and azide $\mathbf{2 7}$, two fragments that have been utilized in a previous synthesis reported by Nakata and co-workers (Scheme 1.9). ${ }^{38}$ Compound 26 was envisioned to arise from the 1,4-diene 28, which in turn comes from the ruthenium-catalyzed alkene-alkyne coupling of $\mathbf{2 9}$ and the commercially available alkene $\mathbf{3 0}$.

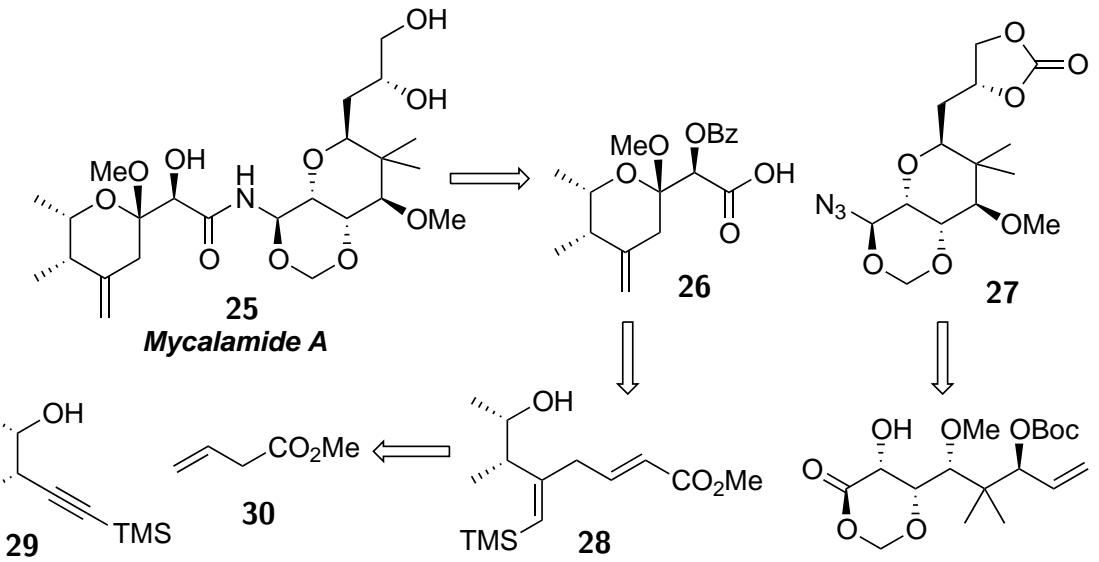

Scheme 1.9 Retrosynthetic Analysis of Mycalamide A.

The homopropargylic alcohol $\mathbf{2 9}$ was prepared in a single step by the addition of TMSacetylene to $(2 S, 3 S)-2,3$-epoxybutane (31, Scheme 1.10). The steric bulk of the trimethylsilyl group serves to direct the subsequent Ru-catalyzed alkene-alkyne coupling, producing the branched product $\mathbf{2 8}$ regioselectively. TBS-protection, dihydroxylation and selective mono-

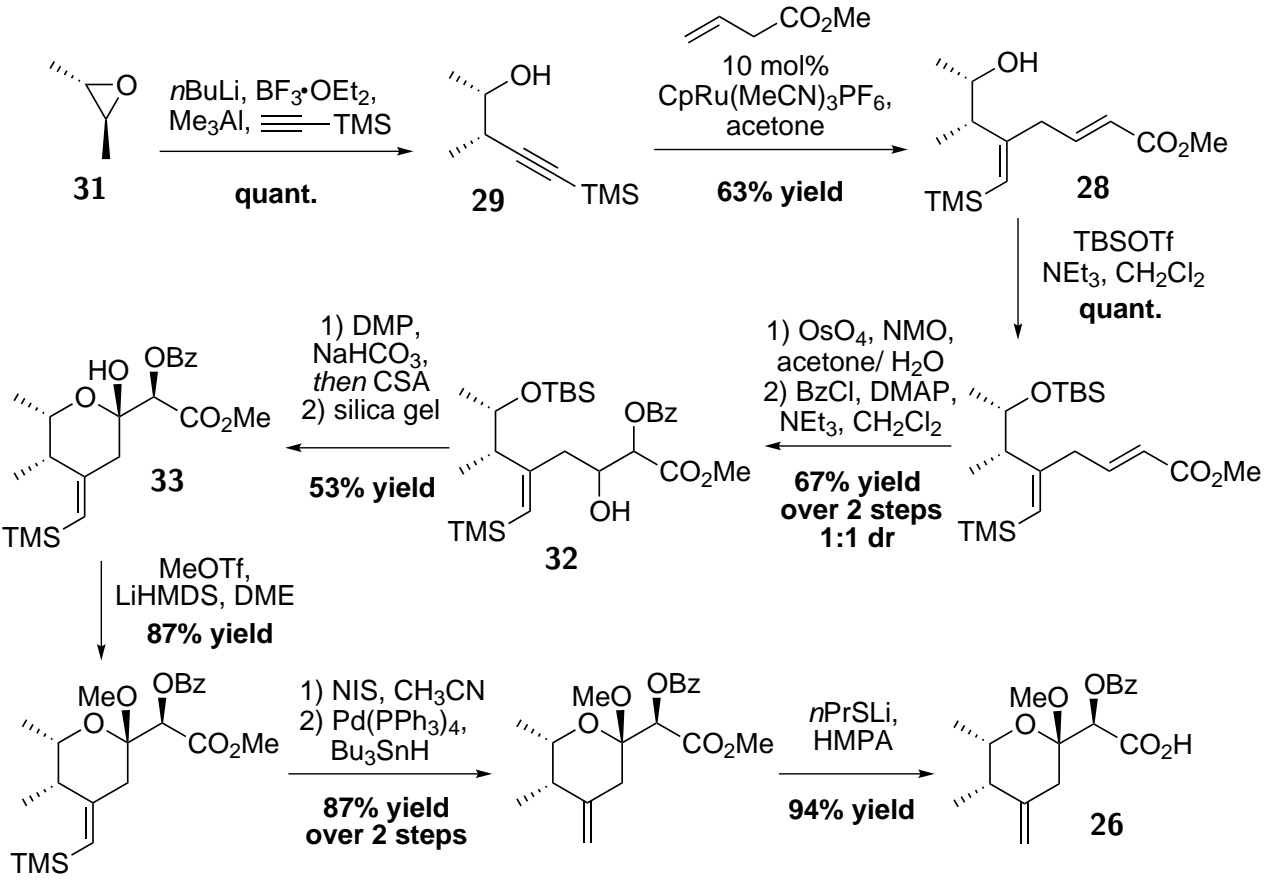

Scheme 1.10 Synthesis of (-)-7-Benzoylpederic Acid. 
esterification provided 32 as a 1:1 mixture of syn diastereomers. The poor diastereoselectivity in this transformation proves to be inconsequential, as the oxidative cyclization of alcohol 32 produces a mixture of diastereomeric hemiketals that ultimately equilibrate to the desired compound $\mathbf{3 3}$ on silica gel. Methylation of $\mathbf{3 3}$ was followed by a two-step removal of the silyl group and a dealkylative saponification to provide (-)-7-benzoylpederic acid $\mathbf{2 6}$. Azide $\mathbf{2 7}$ was prepared in 18 steps and together the two fragments constitute a formal total synthesis of mycalamide A.

\subsubsection{Zn-Catalyzed Direct Asymmetric Aldol Reaction}

The aldol reaction has proven to be an incredibly powerful synthetic tool in the preparation of complex molecular targets. ${ }^{39}$ The development of this reaction has enabled highly efficient transformations that produce $\beta$-hydroxy carbonyl compounds with excellent control of chemo-, regio-, and stereoselectivity. The traditional aldol reaction involves the step-wise formation of an enolate by addition of a stoichiometric amount of base to a carbonyl donor, followed by addition of an aldehyde (Scheme 1.11). Control of enantioselectivity in this process has typically been achieved through the use of a chiral auxiliary on the donor, such as Evans' chiral oxazolidinone. ${ }^{40}$ The direct aldol reaction provides an atom-economic variant of the traditional aldol reaction, avoiding the production of stoichiometric byproducts and the use of chiral auxiliaries. ${ }^{41}$ The major challenge of the catalytic direct aldol reaction with transition

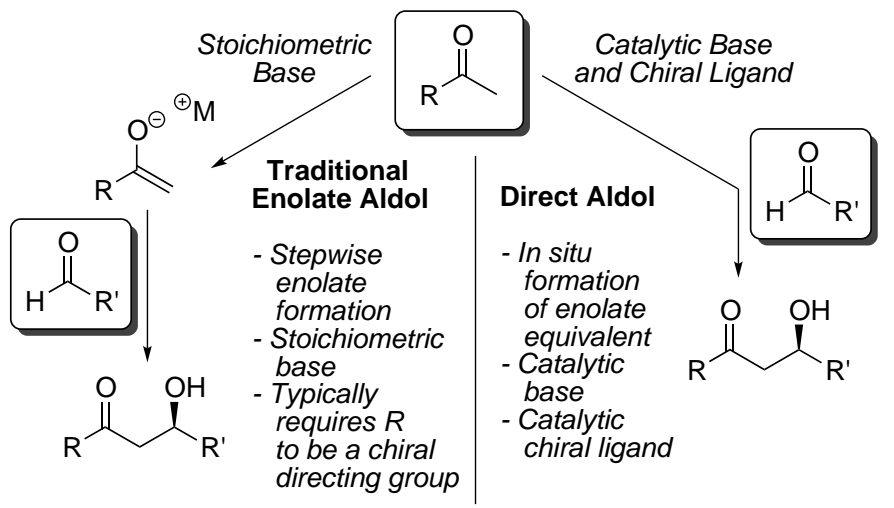

Scheme 1.11 Differentiating Traditional Enolate Aldol and Direct Aldol Reactions.

metal complexes is creating reaction conditions that enable catalyst turnover. The metal alkoxide that results from addition of an enolate to an aldehyde is often less basic than the starting enolate, which prevents catalyst dissociation and turnover. Chemoselectivity can also be a significant problem as the acceptor, an aldehyde, is often more acidic than the ketone or an ester equivalent used as the donor. Failure to address these issues often results in low reactivity or the generation of a number of different aldehyde self-aldol side products. The dinuclear zinc ProPhenol catalyst 34 provides an elegant solution to these problems (Scheme 1.12). ${ }^{42}$ This bifunctional Lewis acid/Brönsted base system serves to activate both reactants, create a chiral pocket for enantioselective addition, and act as a proton shuttle to 
release the product from the catalyst. The acetophenone-based donors shown in Scheme 1.13
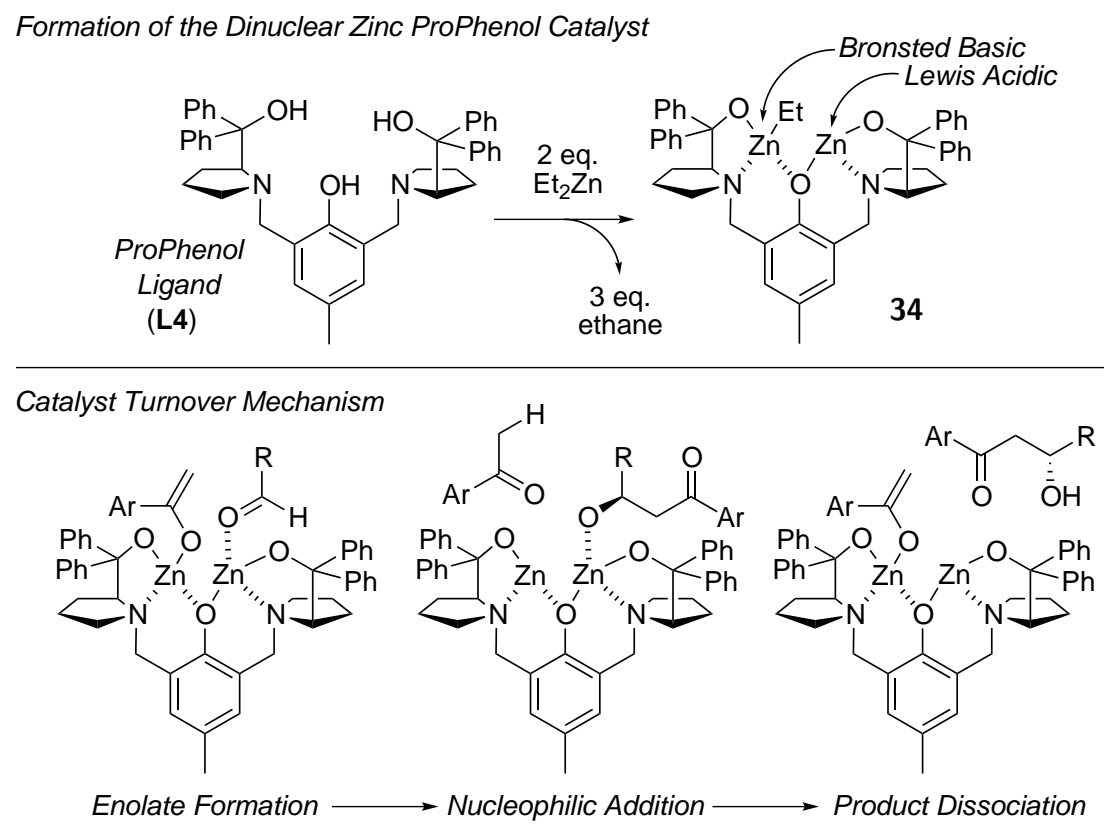

Scheme 1.12 Zinc Prophenol Catalyst and Proton Shuttle Turnover Mechanism.

afford $\beta$-hydroxy ketone products $\mathbf{3 5}$ and $\mathbf{3 6}$ with excellent levels of enantioselectivity and good yields. Triphenylphosphine sulfide was used as an additive to improve catalyst turnover; however, it was later discovered that the beneficial effects of this additive were limited to certain substrates. ${ }^{43} \mathrm{~A}$ variety of $\alpha$-hydroxy ketones have been shown to provide excellent yield, diastereoselectivity and enantiomeric excess with this methodology, creating two stereocenters in a single reaction. Interestingly, the aldehyde-derived stereocenter of $\mathbf{3 6}$ has the opposite absolute configuration to that in the product, 35, obtained when acetophenone is used as the donor . This phenomenon is rationalized by a proposed bidentate coordination of the $\alpha$ hydroxy ketone donor, bridging the two zinc atoms and altering the approach of the aldehyde to favour the opposite facial selectivity.

The excellent atom economy and stereoselectivity of the Zn-ProPhenol aldol reaction was used to great effect in the total synthesis of the complex macrocyclic natural product,

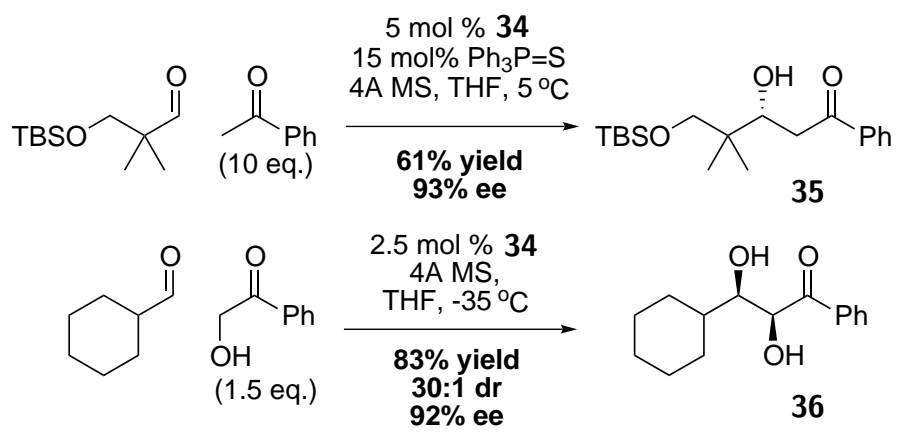

Scheme 1.13 Direct Asymmetric Aldol Reaction with the Zn-ProPhenol Catalyst. 
laulimalide (37) ${ }^{44}$ Laulimalide displays microtubule stabilizing activity similar to that of peloruside A and, as a result, is highly cytotoxic towards a number of cancer cell lines. ${ }^{45}$ The major retrosynthetic disconnections of this synthesis include macrocyclization via a rutheniumcatalyzed alkene-alkyne coupling and a $Z$-selective Still-Gennari olefination to tether the two major fragments 38 and 39 (Scheme 1.14). The northern fragment 38 was envisioned to arise from Julia olefination to connect dihydropyran $\mathbf{4 0}$ and the protected polyol $\mathbf{4 1}$, which in turn arises from the direct asymmetric aldol reaction of hydroxy 2-ethylacylpyrrole (42) and aldehyde 43. The forward synthesis commenced with the preparation of the donor and

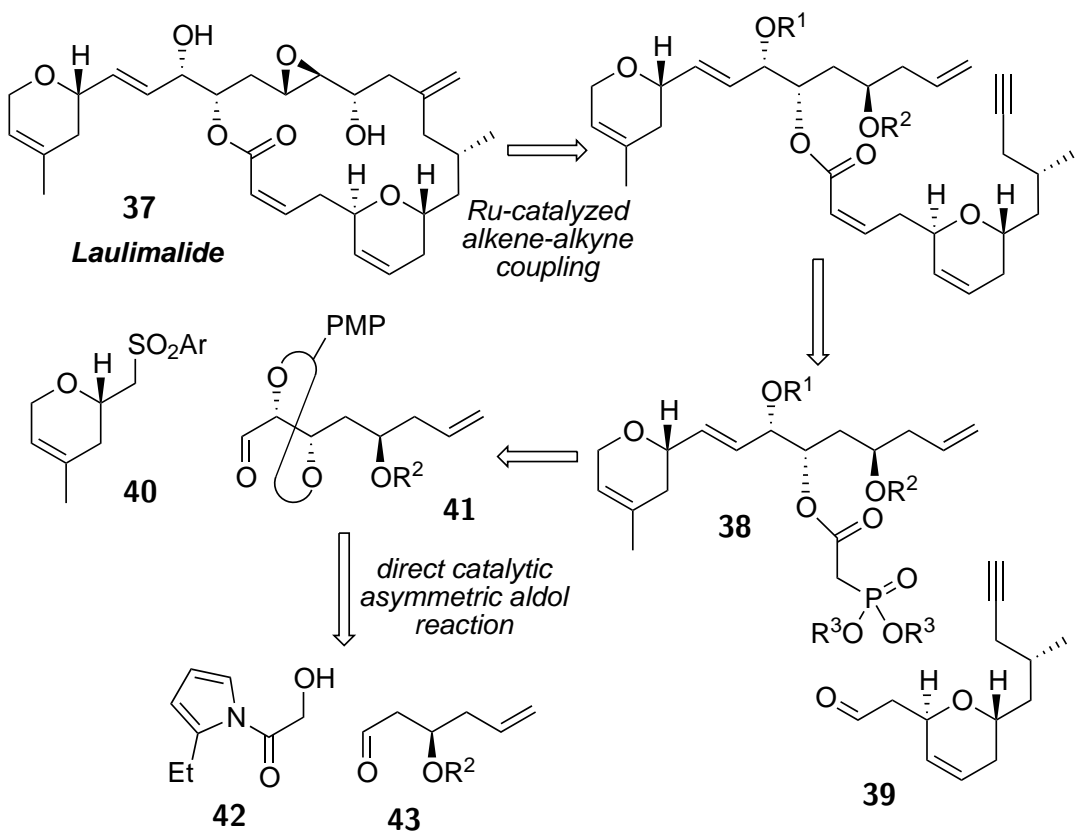

Scheme 1.14 Retrosynthetic Analysis of Laulimalide.

acceptor for the direct asymmetric aldol reaction. Aldehyde $\mathbf{4 3}$ was prepared in four steps from $(S)$-glycidyl tosylate via functional group interconversion and epoxide opening with a vinyl cuprate. The zinc ProPhenol catalyst ent-34 facilitated the direct asymmetric aldol reaction of 42 and $\mathbf{4 3}$, providing the desired product $\mathbf{4 4}$ in 53\% yield and 10:1 diastereomeric ratio (Scheme 1.15). Formation of a $p$-methoxyphenyl (PMP) acetal followed by reduction of the $\mathrm{N}$-acylpyrrole with $\mathrm{NaBH}_{4}$ leads to alcohol 45. Oxidation with Dess-Martin periodinane (DMP) and Julia-Kocienski olefination with phenyltetrazole sulfone $\mathbf{4 0}$ provided the desired $E$-alkene 46 selectively. The cyclic PMP-acetal was then selectively opened with DIBAL-H and the resulting alcohol was esterified with phosphonoacetic acid $\mathbf{4 7}$ under Yamaguchi conditions to complete the northern fragment $\mathbf{3 8}$.

The Southern fragment, 39, was prepared in 15 steps from D-aspartic acid and coupled to 38 using a Still-Gennari olefination to produce a $62 \%$ yield of the desired product as a 5:1 mixture of $Z / E$-geometric isomers (Scheme 1.16). Macrocyclization was then achieved using an intramolecular ruthenium-catalyzed alkene-alkyne coupling. Under highly dilute reaction conditions this reaction provided near quantitative yield of the desired macrocycle 48. MOM- 
<smiles>CCc1cccn1C(=O)CO</smiles>

42<smiles>C=CC[C@@H](CC=O)OC</smiles>
43

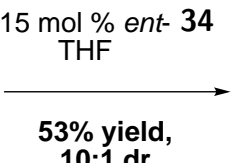

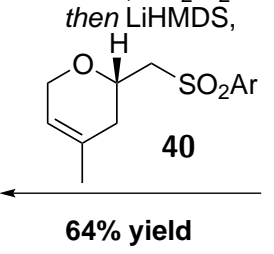<smiles>C=CC[C@@H](C[C@@H](O)[C@H](O)C(=O)n1cccc1CC)OC</smiles>

44

1) $\mathrm{PMPCH}(\mathrm{OMe})_{2}$, $\mathrm{CSA}, \mathrm{CH}_{2} \mathrm{Cl}_{2}$

2) $\mathrm{NaBH}_{4}, \mathrm{THF}$

$70 \%$ yield over 2 steps<smiles>C=CC[C@@H](C[C@@](O)(CO)[C@H](O)CO)OC</smiles>

45

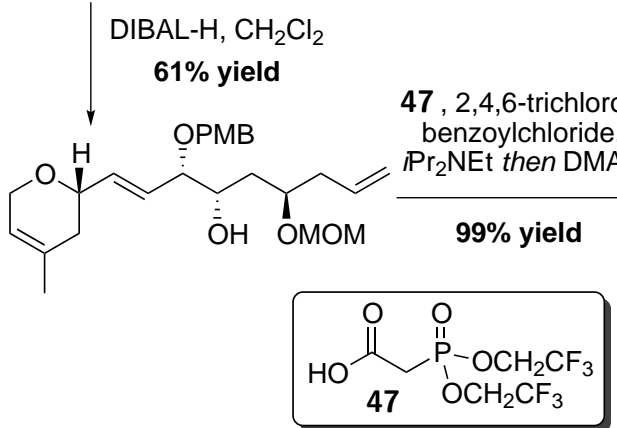

Scheme 1.15 Synthesis of the Northern Fragment 38.<smiles>C=CC[C@@H](C[C@@H](OC(=O)CP=O)[C@@H](/C=C/[C@H]1CC(C)=CCO1)OC(C)=O)OC</smiles>

$\mathrm{F}_{3} \mathrm{CH}_{2} \mathrm{CO}^{\prime} \mathrm{OCH}_{2} \mathrm{CF}_{3}$

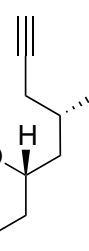

39
KHMDS, 18-crown-6, THF

\section{$62 \%$ yield}

$Z / E=5: 1$<smiles>C=CCC(C[C@@H](OC(=O)CP=O)[C@@H](/C=C/C1CC(C)=CCO1)OCCC)OC</smiles>

$\mathrm{F}_{3} \mathrm{CH}_{2} \mathrm{CO}^{\prime} \mathrm{OCH}_{2} \mathrm{CF}_{3}$

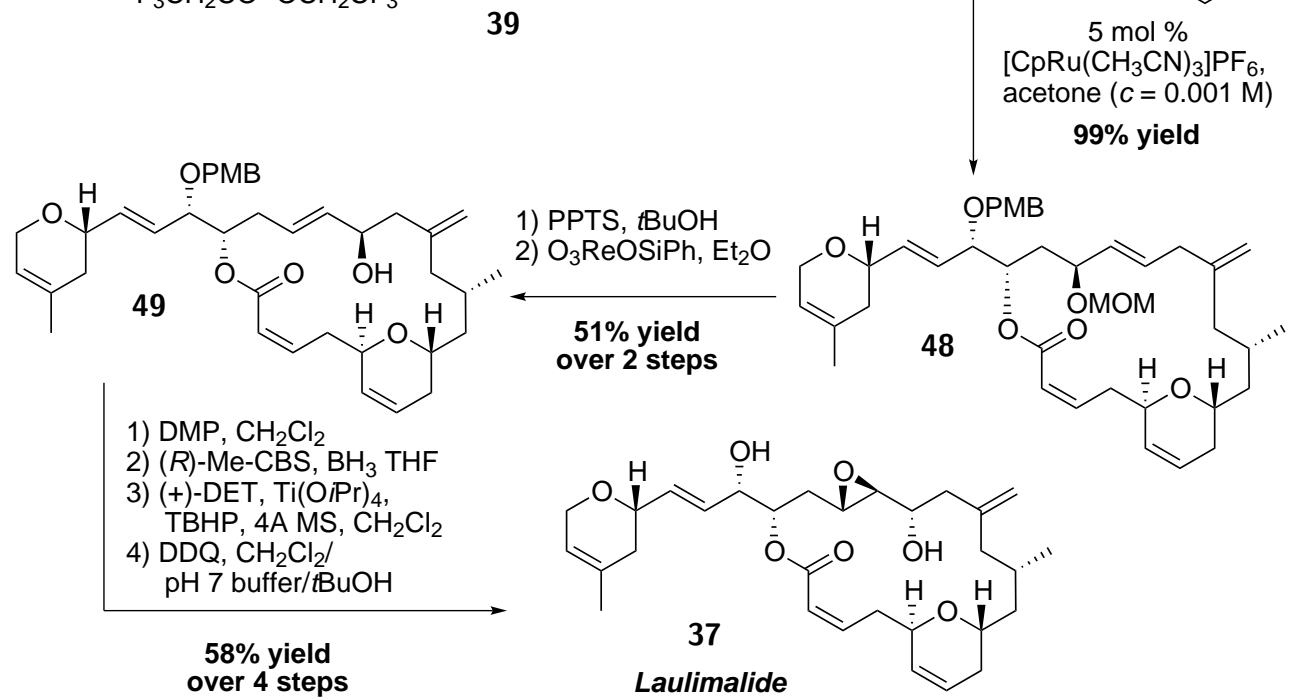

Scheme 1.16 Total Synthesis of Laulimalide. 
deprotection under acidic conditions was then followed by allylic transposition using a highly active perrhenate catalyst, $\mathrm{O}_{3} \mathrm{ReOSiPh}$. The desired rearranged product 49 was formed in $78 \%$ yield with complete retention of stereochemistry. The stereochemistry of the allylic alcohol was then inverted by oxidation with Dess-Martin periodinane (DMP) followed by CoreyBakshi-Shibata (CBS) reduction. Subsequent Sharpless asymmetric epoxidation and PMBdeprotection furnished the natural product, laulimalide (37). The final step was performed in the presence of a $\mathrm{pH} 7$ buffer to prevent the known acid-catalyzed rearrangement of laulimalide to isolaulimalide. ${ }^{46}$

\subsubsection{Concluding Remarks}

The palladium-catalyzed asymmetric allylic alkylation, Ru-catalyzed alkene-alkyne coupling and the Zn-ProPhenol-catalyzed asymmetric aldol reaction all form carbon-carbon bonds in a highly atom economic manner. The total syntheses of hamigeran B, mycalamide A and laulimalide clearly highlight the power and utility of these transition metal-catalyzed reactions. Although all of these syntheses rely on transformations with poor atom economy at some stage, the merit of each approach lies in the pursuit of atom-economic synthesis and the development of tools and synthetic strategies for this goal. Striving to adhere to the principles of atom economy necessitates an innovative and invention-based approach to synthesis and aids in expanding the frontiers of chemical synthesis. 


\subsection{Linchpin-Based Strategies in Total Synthesis}

\subsubsection{Introduction}

The development of linchpin-based methods represents a powerful strategy for efficient synthesis. A linchpin is literally described as "One that serves to hold together parts or elements that exist or function as a unit," 47 however, to define this term within the context of chemical synthesis, the following definition is proposed;

$A$ relatively simple compound that serves to join and hold together molecular fragments that ultimately exist together in a target molecule. ${ }^{\dagger}$

The Bestmann ylide (50) is an excellent example of a linchpin and in many instances the dual reactivity of this compound can be used in a single multicomponent reaction (Scheme 1.17). ${ }^{48}$ In this case, nucleophilic addition of the amine, $\mathbf{5 1}$, to the ketene is followed by a Wittig reaction between the resulting phosphorous ylide and piperonal, ultimately producing the $\alpha, \beta$ unsaturated amide $\mathbf{5 2}$.

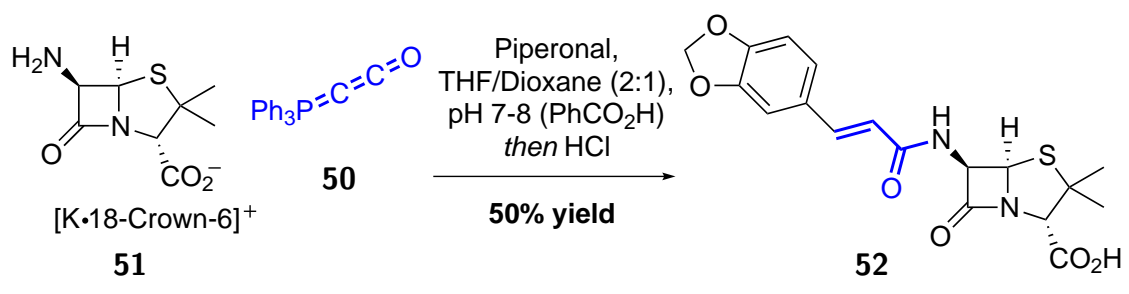

Scheme 1.17 A Linchpin Strategy Using the Bestmann Ylide.

\subsubsection{The Strategic Application of Synthetic Linchpins}

The ultimate aim of a linchpin-based strategy is to achieve improved synthetic efficiency through the use of convergent synthesis. This approach involves the union of multiple compound fragments, and therefore aids in the design of a route with a high degree of convergence. However, the efficiency imparted by this tactic is dependent on the methodology and disconnections used to implement it. Ideally, the key linchpin sequence would introduce a high level of structural complexity (i.e. chiral centers, cyclic connectivity, functional group content, etc.) and occur at a late stage in the synthesis to maximize convergence. Scheme 1.18 illustrates a formylation-alkyne addition linchpin strategy used in the total synthesis of petrocortyne A (53). ${ }^{49}$ In this synthesis, dimethylformamide (54) was used as a highly effective linchpin; reacting with a lithium acetylide to provide, after acidic workup, an ynal which was subsequently reacted with a second lithium acetylide to furnish the desired propargylic alcohol as a mixture of diastereoisomers that could be separated by chiral HPLC. This late stage linchpin sequence creates a highly convergent, and therefore efficient, synthesis. Using the

\footnotetext{
†The words 'relatively simple' provide a qualitative means of distinguishing a linchpin-based strategy from a standard fragment coupling, whereby three or more large fragments are joined together at the end of a synthesis.
} 


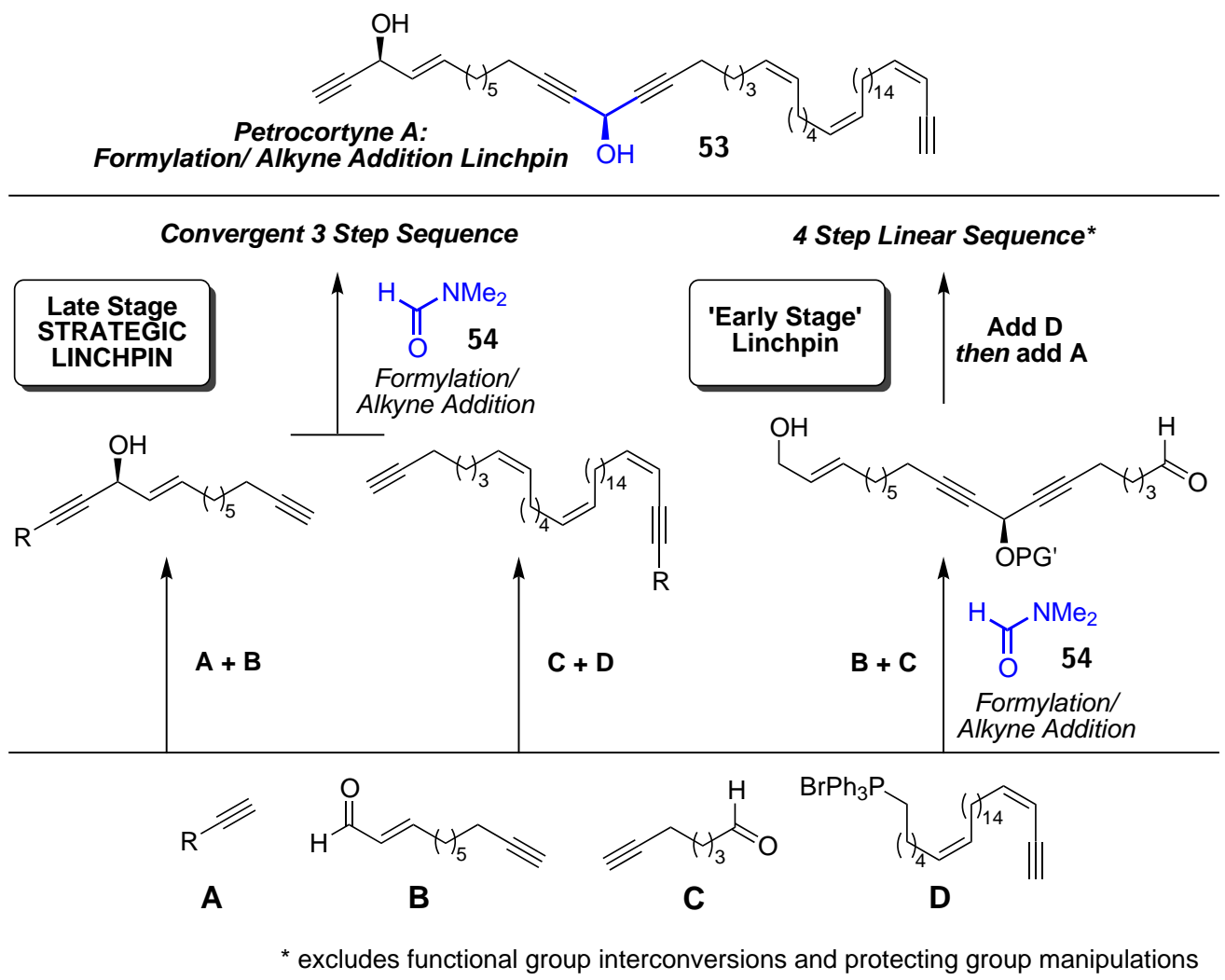

Scheme 1.18 Convergence and Strategic Linchpin Sequences.

same disconnections, a hypothetical 'early stage' linchpin sequence would ultimately produce petrocortyne $\mathrm{A}$ in a longer, four step, linear sequence. ${ }^{50}$ Comparison of these two routes illustrates an important distinction between linchpin-based strategies: the implementation of a linchpin sequence late in a synthetic route, with advanced intermediates, provides a highly convergent and more efficient approach to a target molecule. Additionally, a late stage linchpin sequence typically contains more functional groups and molecular complexity and therefore demands higher levels of chemoselectivity and efficiency (i.e. reagent stoichiometry and yield). Consequently, the term strategic linchpin has been coined to highlight the importance of this distinction when evaluating similar linchpin-based strategies. Inherent to this definition is also the requirement that a linchpin be a simple compound relative to the fragments being coupled - this serves to differentiate a linchpin sequence from simple chain elongation or three-fragment coupling in a synthesis.

The utility of linchpin-based strategies in total synthesis is a direct result of two inherent characteristics: firstly, the retrosynthetic disconnections that accompany this strategy result in multiple fragments and therefore impart convergence to a proposed route; secondly, the isolated preparation of a linchpin molecule often enables the introduction of potentially sensitive functional groups without a reliance on protecting group manipulations and redox operations to achieve chemoselectively. The application of linchpin sequences can be classified into three subgroups: linear, cyclic, and multi-tether linchpin strategies (Figure 1.2). A linear 


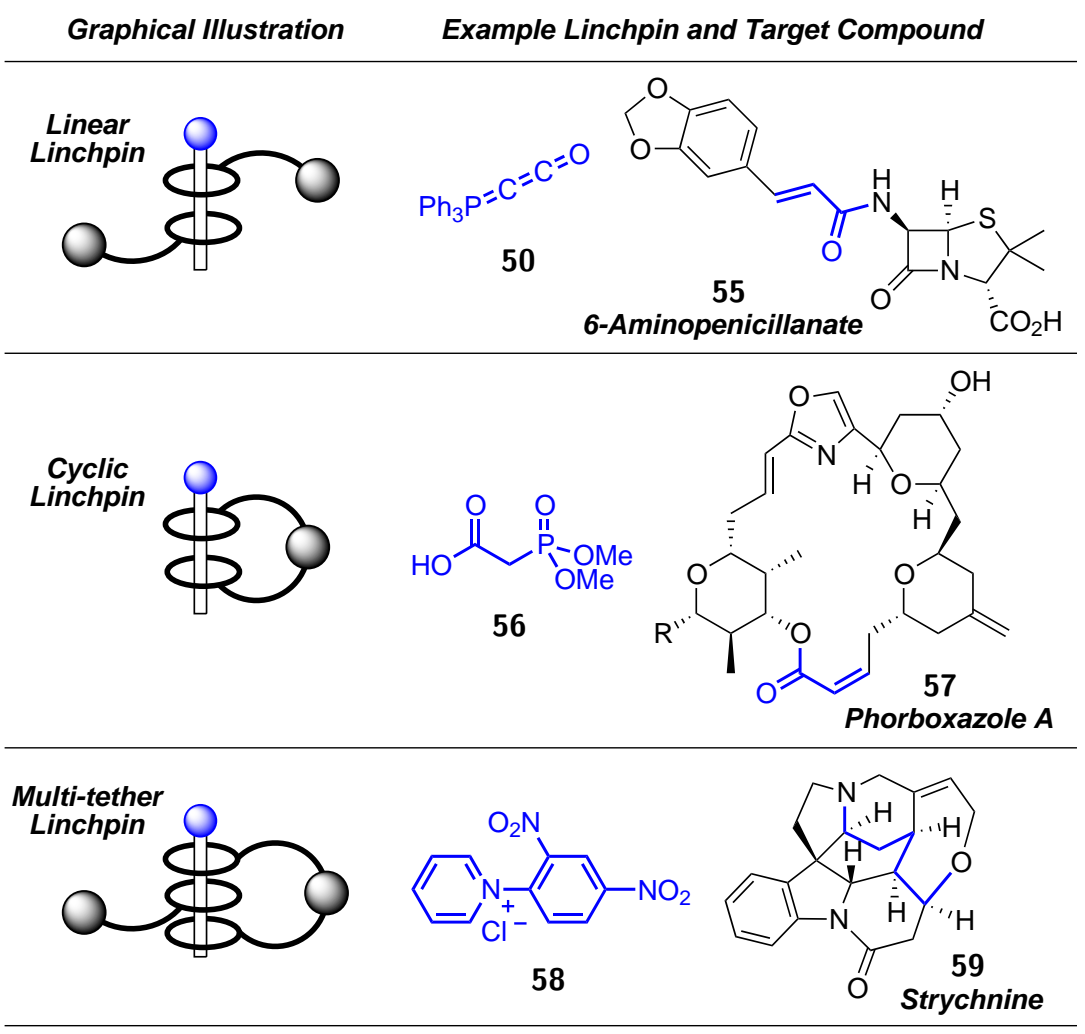

Figure 1.2 Classification of Linchpin Strategies.

linchpin strategy serves to couple two discrete molecular fragments via a series of intermolecular reactions. An example of this can be seen in the use of the Bestmann ylide (50) in the synthesis of 6-aminopenicillanates (i.e. 55). ${ }^{48}$ Alternatively, a cyclic linchpin strategy consists of an initial intermolecular reaction, followed by a subsequent intramolecular reaction to ultimately form a ring system. Phorboxazole A (57) has been prepared using this strategy; first coupling the carboxylic acid $\mathbf{5 6}$ and then using a Horner-Wadsworth-Emmons olefination to form the macrocyclic ring. ${ }^{51}$ Lastly, a multi-tether linchpin strategy forms three or more inter- or intramolecular bonds and, as a result, the linchpin often becomes embedded in the target molecule. A highly efficient six-step synthesis of strychnine (59) was enabled by the use of a multi-tether linchpin strategy. ${ }^{52}$ In this total synthesis, the pyridinium salt $\mathbf{5 8}$ was used to form a tryptamine-derived Zincke aldehyde, which subsequently underwent an intramolecular Diels-Alder reaction, conjugate addition and intramolecular hemiacetal formation. Ultimately, four $\mathrm{C}-\mathrm{C}$ bonds and one $\mathrm{C}-\mathrm{O}$ bond were formed to the original five carbons derived from the pyridine ring in $\mathbf{5 8}$.

A number of structural motifs have found frequent use as linchpins in total synthesis. Some of these privileged linchpin structures are shown in Figure 1.3. The key to the utility of these reagents lies in the high levels of chemoselectivity that can be obtained despite the dual reactivity contained within the linchpin moiety. Epoxy halide or alcohol derivatives (class I) provide a very useful three-carbon linchpin with two differentiated electrophilic sites. 

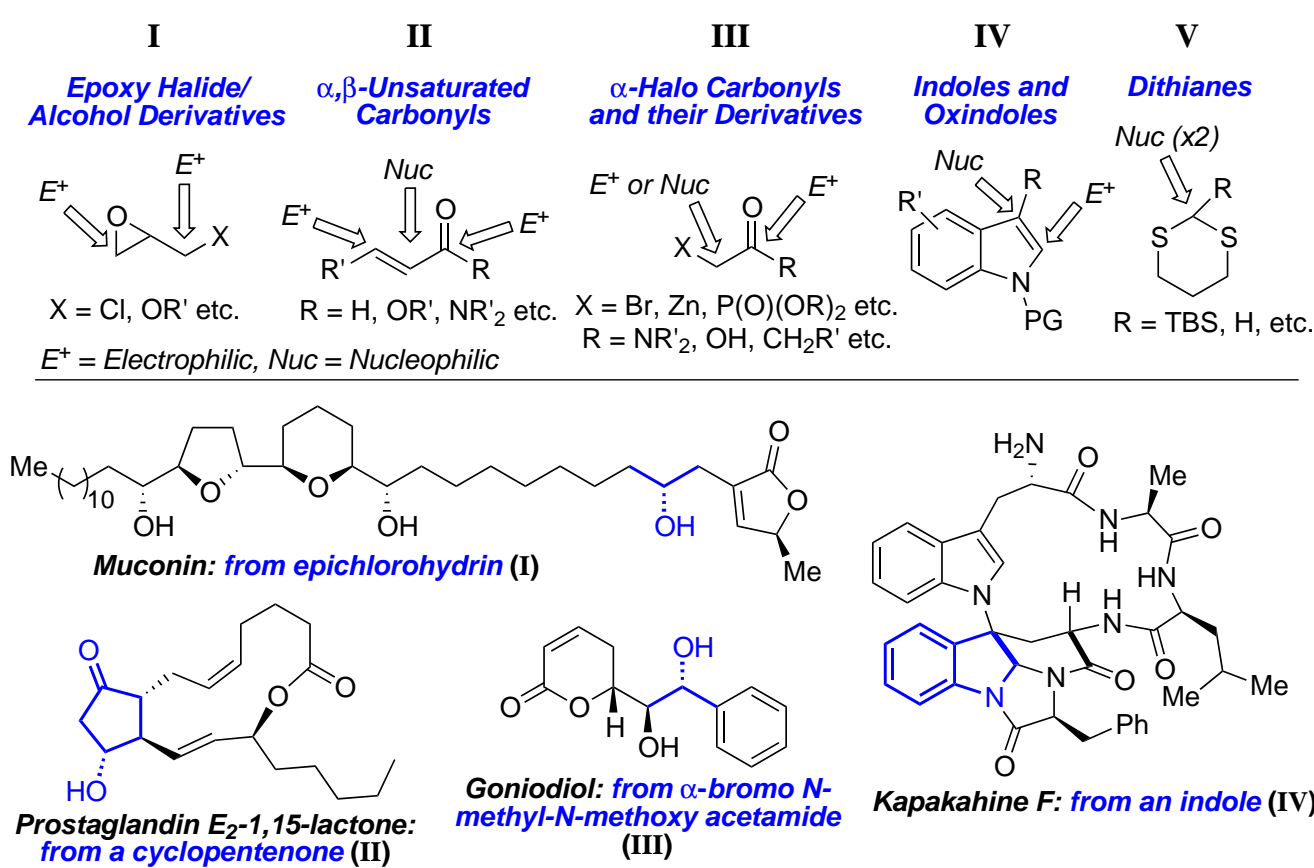

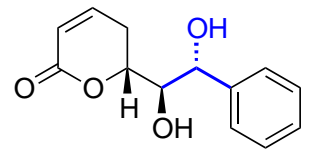

Goniodiol: from $\alpha$-bromo $N$ methyl-N-methoxy acetamide (III)

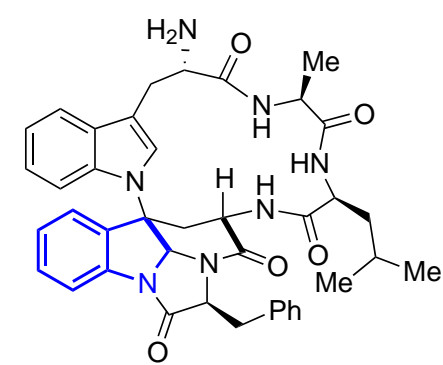

Kapakahine F: from an indole (IV) from a cyclopentenone (II)

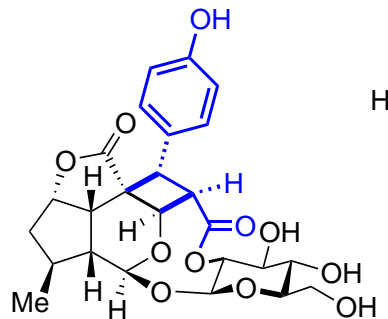

Littoralisone: from a p-hydroxycinnamate (II)

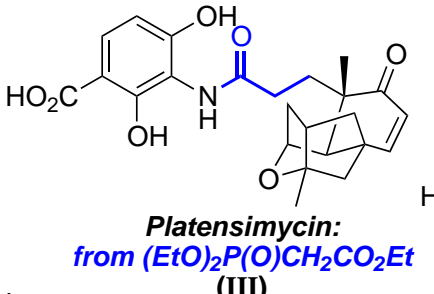
(III)

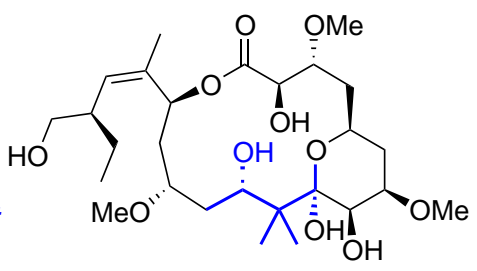

2-epi-Peloruside A: from a dithiane (V)

Figure 1.3 Privileged Linchpins and their Application in Total Synthesis.

Epichlorohydrin ( $\mathrm{I}: \mathrm{X}=\mathrm{Cl}$ ) was used to couple two advanced fragments in a total synthesis of muconin reported by Jacobsen and coworkers. ${ }^{53} \alpha, \beta$-Unsaturated carbonyls (class II) display a wide range of reactivity and, under certain conditions, can function as either a nucleophile or electrophile. ${ }^{54}$ Prostaglandin $\mathrm{E}_{2}$-1,15-lactone was synthesized by Fürstner and coworkers using a cyclopentenone linchpin (II: $\mathrm{R}=$ alkyl, $\mathrm{R}^{\prime}=$ alkyl). ${ }^{55}$ In this case, conjugate addition of a vinylzinc nucleophile was followed by trapping of the resulting enolate with an electrophile, allowing functionalization of both the $\alpha$ and $\beta$ positions in a single pot. The macrocyclic structure of littoralisone has also been constructed using this class of privileged linchpins a cinnamic acid derivative (II: $\mathrm{R}=\mathrm{OH}, \mathrm{R}^{\prime}=\mathrm{Ar}$ ) was first coupled via ester formation and then underwent a $[2+2]$ cycloaddition to form the desired macrocyclic ring system. ${ }^{56} \alpha$-Halo carbonyls and their derivatives (class III) provide a versatile two-carbon linchpin that have been used in the synthesis of both goniodiol and platensimycin. ${ }^{57}$ In the synthesis of goniodiol, an alkoxide nucleophile is added to the $\alpha$ position of $\alpha$-bromo $N$-methyl- $N$-methoxy acetamide (III: $\mathrm{R}=\mathrm{N}(\mathrm{OMe}) \mathrm{Me}, \mathrm{X}=\mathrm{Br})$ and the product was subsequently reacted with $\mathrm{PhMgBr}$ to give an $\alpha$-alkoxy acetophenone. Conversely, the $\alpha$-position of ethyl 2-(diethoxyphosphoryl)acetate 
(III: $\mathrm{R}=\mathrm{CO}_{2} \mathrm{Et}, \mathrm{X}=\mathrm{P}(\mathrm{O})(\mathrm{OEt})_{2}$ ) acts as a nucleophile in a Horner-Wadsworth-Emmons olefination used to tether the aliphatic portion of platensimycin. Subsequent saponification and amide bond formation were used to introduce the aryl fragment of platensimycin. Indole and oxindole-based heterocycles (class IV) are often inherently nucleophilic at the 3-position and electrophilic at the 2-position. This bifunctional reactivity makes these heterocycles excellent linchpins in the synthesis of indole alkaloid natural products. ${ }^{58}$ Baran and coworkers recently utilized a tryptamine derivative (IV: Boc-Trp-Phe-OMe) as a linchpin in a direct indole-aniline coupling en route to the natural product kapakahine F. ${ }^{59}$ In this case, $\mathrm{N}$ iodosuccinimide was used to facilitate an oxidative indole coupling with $o$-iodoaniline along with spontaneous intramolecular addition of an amine to C2. The resulting indole-fused pyrrolidine was isomerized to give the six-membered lactam present in kapakahine F. Lastly, dithianes (class V) serve as a powerful one-carbon nucleophilic linchpin and, through the use of a Brook rearrangement $(\mathrm{V}: \mathrm{R}=\mathrm{TBS})$, can often add to two different electrophiles in a single pot. ${ }^{60}$ Nucleophilic additions to epoxides and carbonyls have enabled the rapid and convergent assembly of various polyketide natural products and analogues thereof. Such a strategy was utilized by Smith and coworkers in an attempted synthesis of peloruside A, which instead led to the $\mathrm{C} 2$-epimer. ${ }^{61}$ Additionally, the conversion of a dithiane into a ketone means that dithianes serve as acyl anion equivalents in an umpolung-based approach. Thus, the dithiane-derived carbon atom can ultimately serve as an electrophile and can be utilized in the formation of cyclic ketals and hemiketals, as in the 2-epi-peloruside synthesis.

Overall, the privileged linchpin structures shown in Figure 1.3 and their application in synthesis provides an instructive model for the further development and improvement of linchpin-based synthetic strategies.

\subsubsection{Catalytic Applications of Linchpins in Total Synthesis}

The pursuit of convergent synthetic routes has led to the discovery of a variety of highly efficient linchpin-based total syntheses. However, the design elements of such strategies are often understated, despite the efficiency they impart to a synthesis. To illustrate the efficiency and application of strategic linchpin couplings, the following section highlights recent applications of late stage linchpin sequences in the synthesis of complex natural products. An emphasis has been placed on natural product syntheses that use catalytic methodology to couple the linchpin.

Transition metal catalysis has provided some of the highest impact chemical research of recent times, and as a result three Nobel prizes have been recently awarded for discoveries in transition metal catalysis. ${ }^{62}$ The mild reaction conditions and high levels of chemoselectivity afforded by many transition metal-catalyzed reactions make them well suited to the coupling of a linchpin with an advanced synthetic fragment. Additionally, high catalyst turnover numbers and possible regio- and enantioselectivity has the potential to enable linchpin sequences that not only unite major synthetic fragments, but do so in a way that simultaneously introduces 
a high level of structural complexity.

Conjugated polyene systems are versatile synthetic intermediates and common in a range of biologically active natural products. ${ }^{63}$ Coleman and coworkers recently utilized diene 64 as a linchpin for the synthesis of $E, E$ polyene systems and to ultimately achieve the total synthesis of lucilactaene (Scheme 1.19). ${ }^{64}$ The bis-metallated 1,3-butadiene 64 enabled an orchestrated
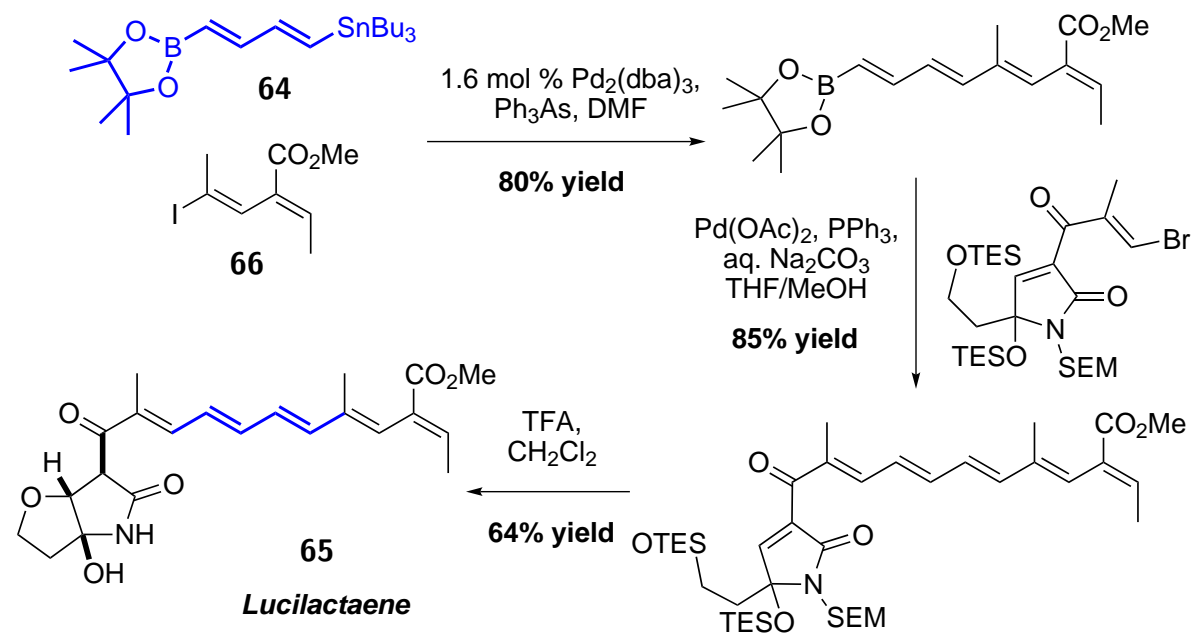

Scheme 1.19 A Pd-Catalyzed Cross Coupling Linchpin Strategy

sequence of $\mathrm{C}-\mathrm{C}$ bond forming events, namely a chemoselective Stille cross coupling followed by Suzuki-Miyuara cross coupling. Both reactions are high yielding and butadiene $\mathbf{6 4}$ can be prepared in just three steps by stannyl-cupration of propargaldehyde diethyl acetal, followed by acetal hydrolysis and Takai olefination with dichloromethylborinate. ${ }^{65}$ The orthogonal reactivity of the Stille cross coupling arises from the need for basic reaction conditions to effect transmetallation of palladium with boron, therefore base-free conditions and the use of triphenylarsine as an ancillary ligand enable selective Pd-cross coupling with vinyl iodide 66 . In certain cases, a one-pot sequential cross coupling can be achieved, functionalizing both ends of the diene in a single process.

Halogen-selective Suzuki-Miyaura cross couplings have been used very effectively to enable aromatic rings to function as synthetic linchpins (Scheme 1.20). 5-Bromo-2-iodo-3methoxypyrazine (67) was used as a highly effective linchpin in the total synthesis of the bis-indole natural product, dragmacidin D (68). ${ }^{66}$ In this case, chemoselective coupling of heteroaryl iodide 67 and boronic acid 69 was achieved using precise temperature control. Performing the first Suzuki-Miyaura cross coupling at $23^{\circ} \mathrm{C}$ provided bromopyrazine $\mathbf{7 0}$. The second cross coupling was performed with a more reactive boronate ester $\mathbf{7 1}$ and a reaction temperature of $50^{\circ} \mathrm{C}$. Interestingly, the aryl bromide present in $\mathbf{7 0}$ is unaffected during these reactions, as oxidative insertion into $\mathrm{C}-\mathrm{X}$ bonds on the pyrazine ring is much faster than an ordinary phenyl halide. With both indoles in place, installation of the aminoimidazolium ring and deprotection were all that was required to synthesize the natural product. This was ultimately achieved through silyl deprotection, oxidation, nitromethane addition, and a 
<smiles>COc1nc(Br)cnc1I</smiles>

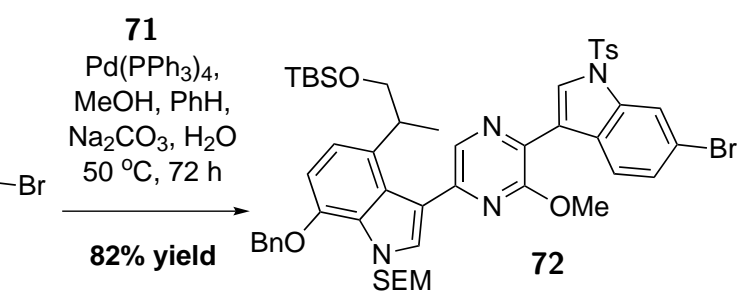

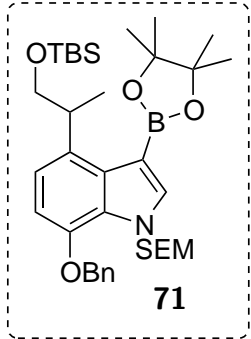

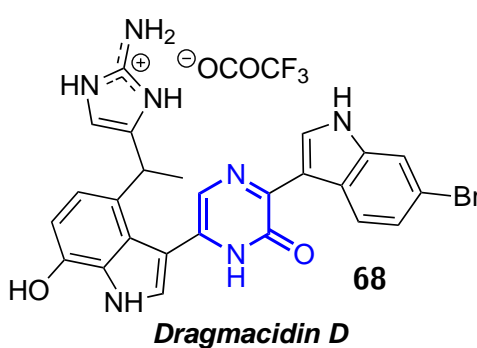
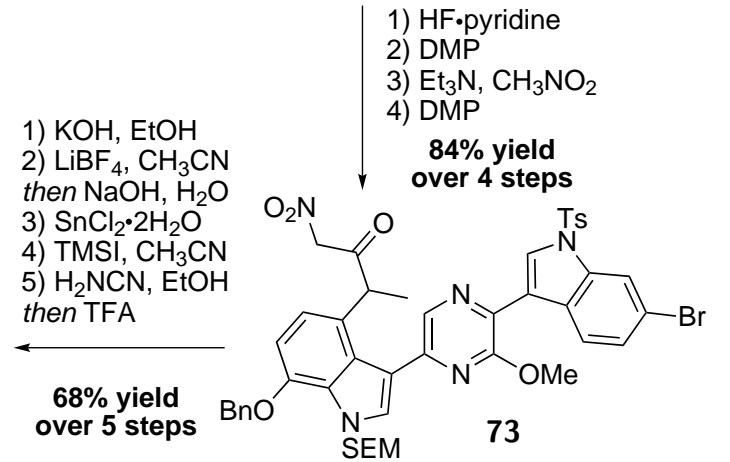

Scheme 1.20 Halogen Selective Suzuki Linchpin in the Synthesis of Dragmacidin D

second oxidation to provide nitromethylketone 73. The two indole nitrogen protecting groups were subsequently removed, and the use of stannous chloride provided mild conditions for the reduction of the nitro group. Removal of the benzyl and methyl ethers was effected by iodotrimethylsilane (TMSI) and the aminoimidazolium unit was installed by addition of cyanamide followed by treatment with trifluoroacetic acid (TFA). Interestingly, the order of this step-intensive sequence was crucial and the use of hydrogenative conditions was precluded by the heteroaryl bromide functionality.

Sonogashira cross coupling with silylated acetylenes, such as TMS-acetylene, can also be utilized to achieve linchpin-based synthetic strategies through the use of sequential cross coupling with each end of the ethylene motif. This approach was used to great effect in the total synthesis of the polycyclic natural product kibdelone C (74). ${ }^{67}$ The linchpin sequence began with the coupling of isoquinoline $\mathbf{7 5}$ with TIPS-acetylene (Scheme 1.21). Fluoride-mediated silyl deprotection was then used to reveal a second terminal acetylene $\mathbf{7 6}$ which was used in a copper-free Sonogashira cross coupling with xanthone $\mathbf{7 7}$, to provide $\mathbf{7 8}$, which contains all the carbons of the natural product. Interestingly, the $\mathrm{Cu}$-free conditions and slow addition of the alkyne were necessary to prevent oxidative dimerization. ${ }^{68}$ The aryl alkyne $\mathbf{7 8}$ was then subjected to hydrogenation, copper-catalyzed iodination and Boc protection to provide iodide 79. Compound $\mathbf{7 9}$ was then subjected to highly optimized palladium-catalyzed $\mathrm{C}-\mathrm{H}$ arylation conditions to generate the hexacyclic ring system of the kibdelones. Lastly, chlorination and careful removal of the various protecting groups provided the natural product kibdelone $\mathrm{C}$ (74). 

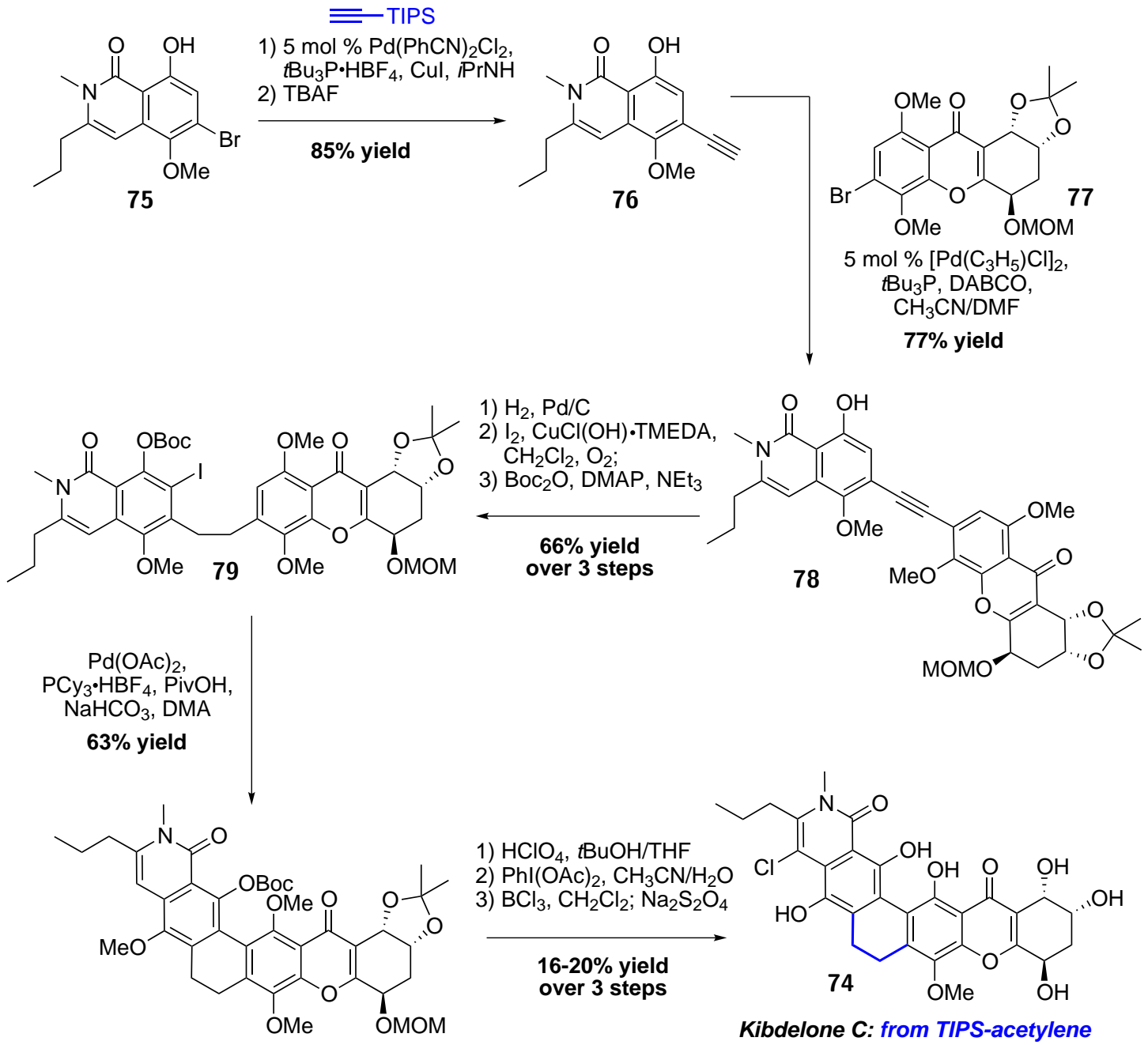

Scheme 1.21 Alkyne Cross Coupling Linchpin Strategy 
The mild and selective nature of ruthenium-catalyzed alkene metathesis is also well suited to a variety of linchpin-based synthetic strategies. The well established methodology of ringclosing metathesis means that the use of a late-stage allylation or vinylation, followed by intramolecular alkene metathesis constitutes a relatively straightforward linchpin strategy for the synthesis of macrocyclic targets. ${ }^{69}$ Alkene cross metathesis represents a much more challenging task, as a number of substrates are prone to statistical homo-coupling. A partial solution to this problem can be found in the use of a substantial excess of a readily available alkene. However, the use of a strategic linchpin sequence late in a synthesis typically involves advanced fragments that are too valuable to be used in excess. A recent total synthesis of the potent anticancer natural product FR901464 utilized a late-stage strategic linchpin based around the use of Ru-catalyzed alkene cross metathesis (Scheme 1.22). ${ }^{70}$ In this case, methacrolein served as a linchpin for the coupling of two advanced tetrahydropyran-based fragments. The linchpin sequence began with a ruthenium-catalyzed alkene cross metathesis between methacrolein and $\mathbf{8 0}$. To maximize the efficiency of this reaction and avoid undesired<smiles>C=CCC1OC(C)C(NC(=O)/C=C\C(C)OC)CC1C</smiles>

80

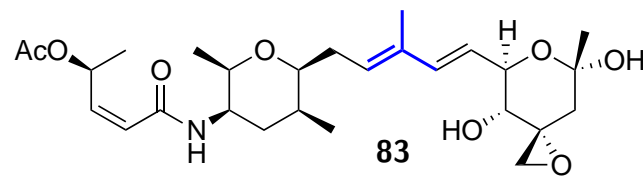

FR901464: from methacrolein

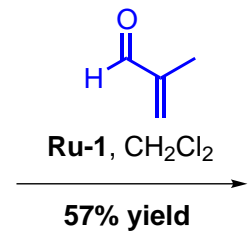

$57 \%$ yield<smiles>CC(=O)/C=C/C1OC(C)C(NC(=O)/C=C\C(C)C(C)=O)CC1C</smiles>

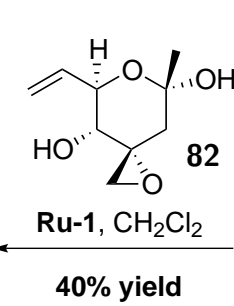

$40 \%$ yield

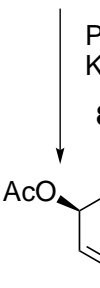

$\mathrm{Ph}_{3} \mathrm{PCH}_{3} \mathrm{Br}$ $\mathrm{KOtBu}, \mathrm{THF}$ $86 \%$ yield

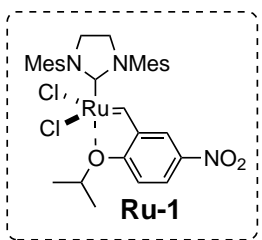

Scheme 1.22 Alkene Cross Metathesis Linchpin Strategy

homocoupling products, 20 equivalents of methacrolein were used along with the nitrosubstituted variant of the Hoveyda-Grubbs catalyst (Ru-1). This catalyst has increased activity relative to the traditional catalysts for alkene metathesis and facilitates alkene cross metathesis with electron-deficient alkenes (i.e. methacrolein) particularly well. ${ }^{71}$ Subsequent Wittig olefination provided the 1,3-diene $\mathbf{8 1}$ needed for the key fragment coupling reaction. Alkene cross metathesis of $\mathbf{8 2}$ and $\mathbf{8 1}$, catalyzed by $\mathbf{R u - 1}$, provided the natural product FR901464 (83), albeit in modest yield. However, this final transformation is particularly challenging as FR901464 is highly sensitive to $\mathrm{pH}$ - decomposition has been reported on silica gel and also in slightly basic aqueous buffer solutions.

Catalytic $\mathrm{C}-\mathrm{H}$ bond functionalization represents one of the most exciting areas in organic synthesis, and has been the focus of many research efforts. ${ }^{72} \mathrm{C}-\mathrm{H}$ bonds are often ubiquitous in intermediates used for synthetic organic chemistry, and this presents both a daunting challenge, in terms of selectivity, and an outstanding opportunity for synthetic efficiency. 
Palladium-catalyzed $\mathrm{C}-\mathrm{H}$ arylation was recently applied by Baran and Gutekunst to achieve a linchpin-based synthesis of two piperarborenine natural products (Scheme 1.23). ${ }^{73}$ In this case a simple disubstituted cyclobutane serves as a highly efficient linchpin for the construction of the bis-arylated tetrasubstituted cyclobutane 84. A key to this strategy was the use of a 2-

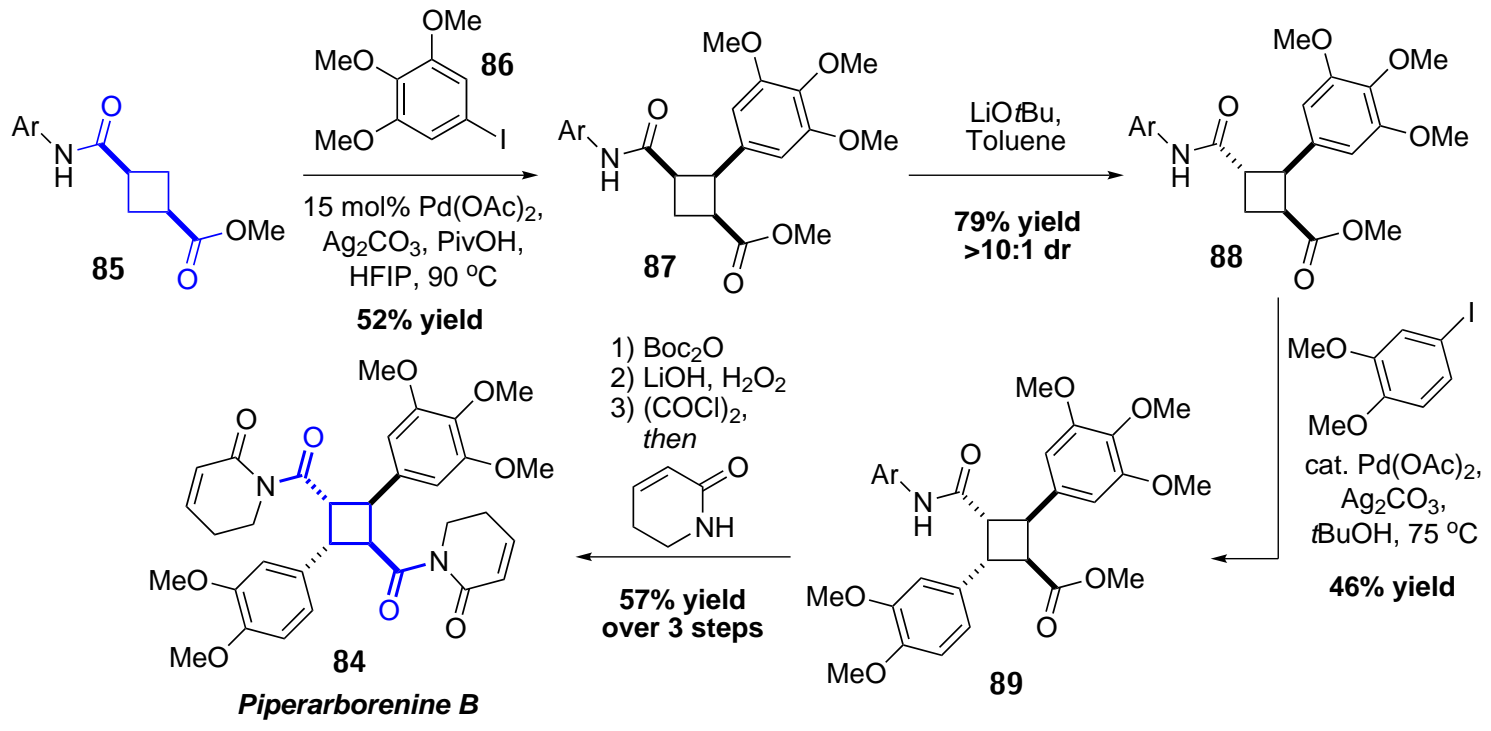

Scheme 1.23 Cyclobutane C-H Arylation Linchpin Strategy

aminothioanisole directing group ( $\mathrm{Ar}$ ) to promote $\mathrm{C}-\mathrm{H}$ bond cleavage and enable stereoselective arylation. The coupling of aryl iodide $\mathbf{8 6}$ to cyclobutane $\mathbf{8 5}$ required extensive optimization and it was ultimately found that hexafluoroisopropanol (HFIP) and pivalic acid were critical to the success of the reaction. Ultimately, the desired product $\mathbf{8 7}$ was obtained in $52 \%$ yield with only traces of bis-arylated side products. Selective epimerization of the C1 stereocenter provided 88 in good yield with less than $10 \%$ undesired epimerization. The second palladiumcatalyzed $\mathrm{C}-\mathrm{H}$ arylation proceeded in good yield, and again installed the aryl group cis to the amide-based directing group. Piperarborenine B (84) was prepared from 89 in three additional steps: the directing group was carbamoylated and both the amide and ester bonds were cleaved using lithium hydroperoxide. This was followed by conversion of the bis-carboxylic acid to the bis-acid chloride and nucleophilic addition of 5,6-dihydropyridinone. Ultimately, piperarborenine B was prepared in just seven steps using this succinct linchpin-based strategy.

Annulative linchpin coupling represents a highly efficient method of connecting advanced synthetic intermediates and can be used to achieve multi-tether linchpin strategies (see Figure 1.2). The Pd-catalyzed trimethylenemethane (TMM) $[3+2]$ cycloaddition is a powerful catalytic method by which this can be achieved and is the focal point of a recent synthesis of marcfortine B. ${ }^{74}$ In this case, the TMM donor $\mathbf{9 0}$ is used in a carboxylative TMM cycloaddition with 91. ${ }^{75}$ The proposed mechanism of this reaction proceeds via formation of a $\pi$-allyl $\mathrm{Pd}$ complex and ionization of the vinyl silane to generate Pd-TMM complex I which traps the carbon dioxide generated during $\pi$-allyl-Pd formation. The resulting intermediate (II), bearing a single silyl group, undergoes a rearrangement to generate a second Pd-TMM complex, III, 
which participates in a cycloaddition with the electron deficient alkene $\mathbf{9 1}$. This transformation introduces high levels of structural complexity, creating two $\mathrm{C}-\mathrm{C}$ bonds in the formation of a spirocyclic five-membered ring. Additionally, the exocyclic alkene and carboxylic acid generated in this reaction serve as handles for further synthetic manipulation. Ultimately, five bonds were formed to carbons derived from the linchpin compound 90, enabling a highly convergent total synthesis of marcfortine B.
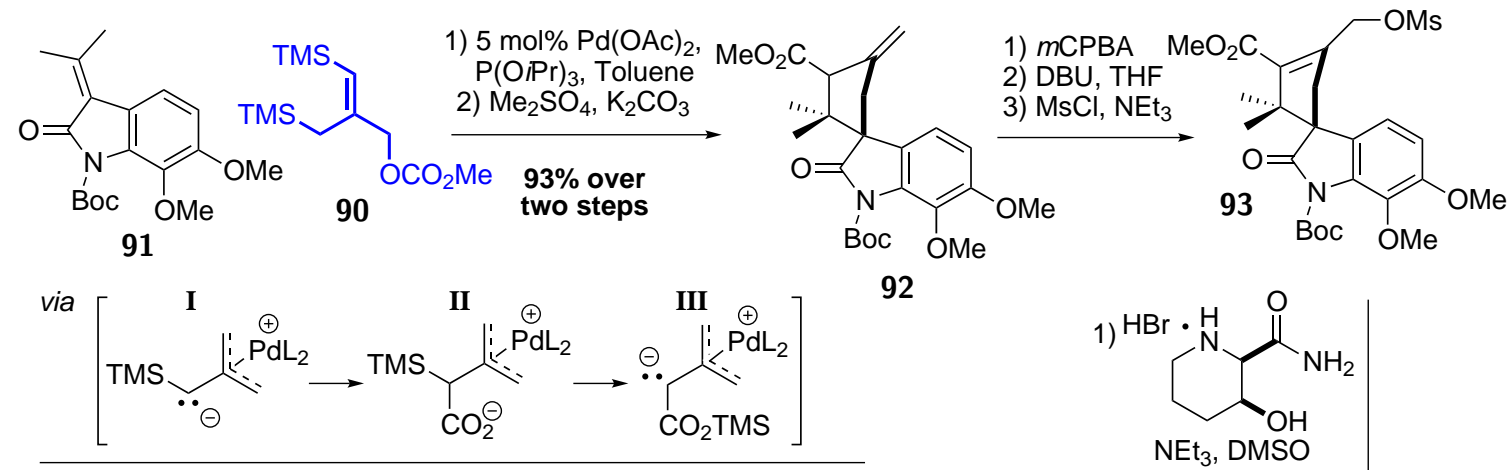

92
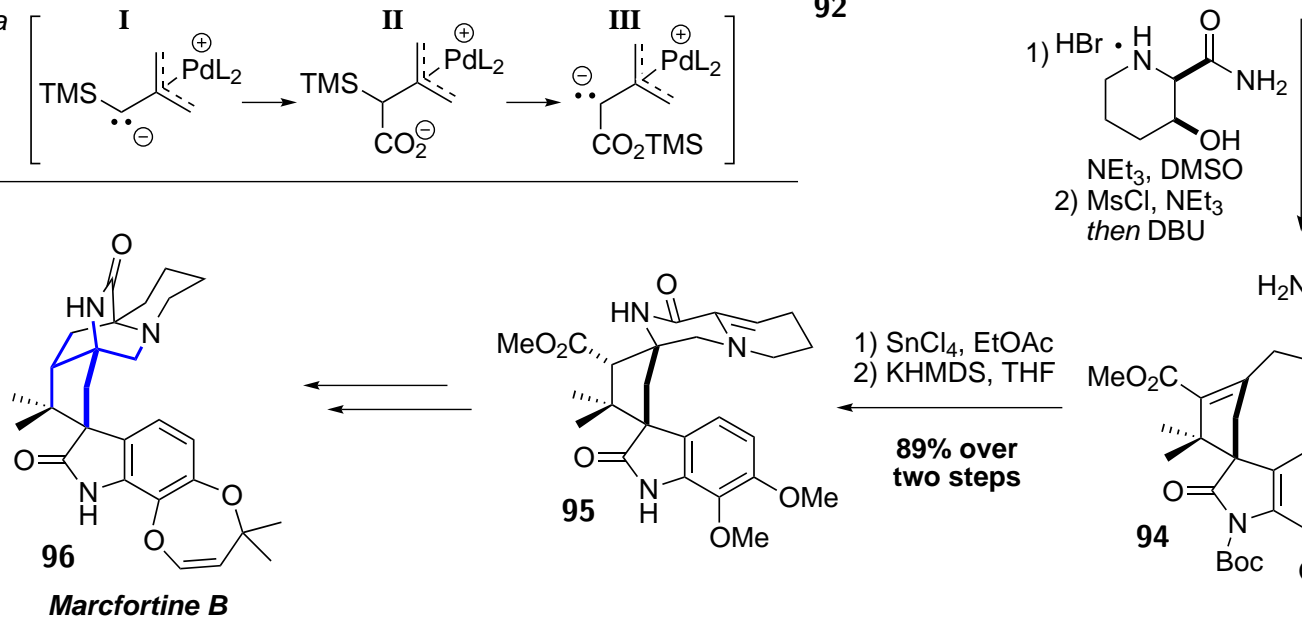
then DBU

Scheme 1.24 TMM [3+2] Cycloaddition in the Synthesis of Marcfortine B.

The development of hydrogen-mediated $\mathrm{C}-\mathrm{C}$ bond formation represents a significant breakthrough in redox economic synthesis and a departure from the use of stoichiometric metallated nucleophiles. ${ }^{76}$ Pioneering work from the Krische group has established a wide range of hydrogen-mediated $\mathrm{C}-\mathrm{C}$ bond forming variants, ${ }^{77}$ while also developing this methodology within the context of total synthesis. ${ }^{78}$ Utilizing this methodology as part of a linchpin-based synthetic strategy has led to the most concise synthesis of a bryostatin natural product to date (Scheme 1.25). ${ }^{79}$ Bryostatin 7 (97) was prepared in just 20 linear steps. The synthesis is comprised of five separate hydrogen-mediated $\mathrm{C}-\mathrm{C}$ bond forming events, two of which are shown in Scheme 1.25. In both cases, Ir-catalyzed dehydrogenation of an alcohol provides the hydrogen source. Allyl acetate and 1,1-dimethylallene (98) react with the respective aldehydes to ultimately provide chiral allylic alcohols. The key linchpin sequence is shown in Scheme 1.26 and begins with the iridium-catalyzed allylation of aldehyde 99. The enantioselectivity of this allylation is irrelevant as the following steps, selective mono-desilylation and oxidation, result in the conversion of the alcohol into ketone 101. Acid-catalyzed trans-esterification and ketal formation stereoselectively generate the desired tetrahydropyran. The allyl group introduced 


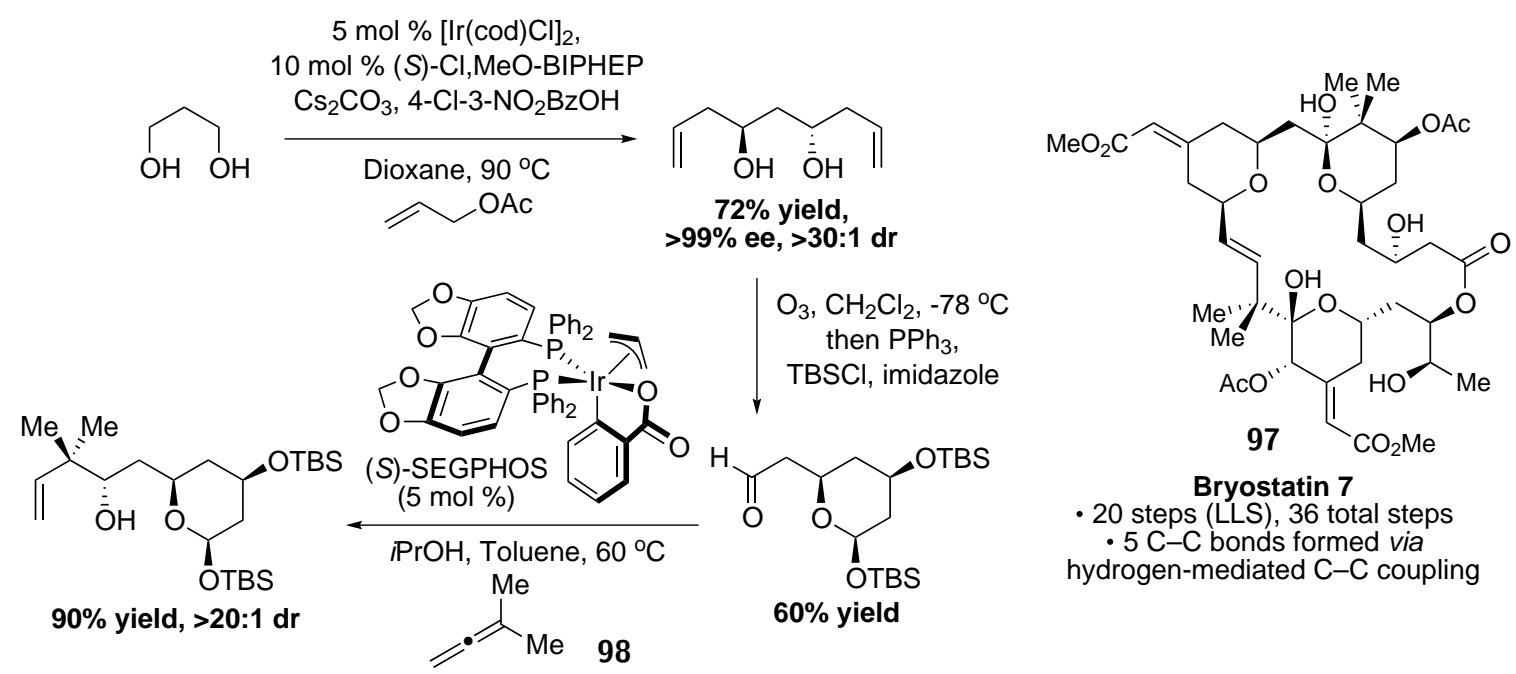

Scheme 1.25 Reaction and Strategy Development in the Synthesis of Bryostatin 7.

in the first step of the linchpin sequence could be ozonized to provide aldehyde 102, now primed for coupling with the other major bryostatin fragment. Compounds 102 and 103 were coupled using a stereoselective Prins-type annulation developed by Keck and $\mathrm{Yu} .{ }^{80}$ The desired methylene tetrahydropyran 104 was obtained in good yield along with $22 \%$ of the side product that results from elimination of methanol. However, both the desired product

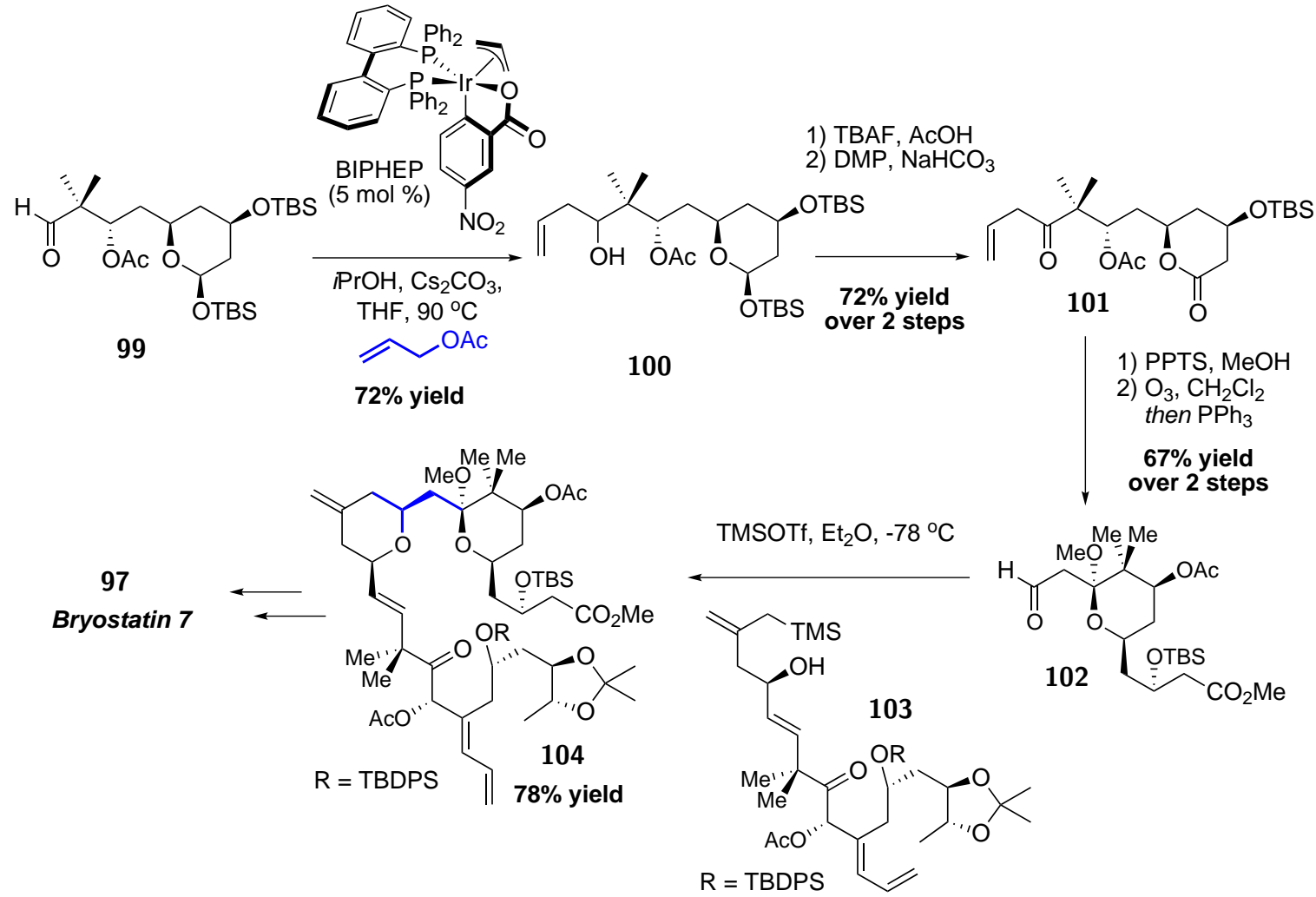

Scheme 1.26 Strategic Linchpin Coupling in the Synthesis of Bryostatin 7. 
and side product participate in acidic methanolysis to yield the same desired product with the TBS-ether and acetonide protecting groups removed. Bryostatin 7 was prepared from compound 104 in 8 additional steps. The application of merged redox and $\mathrm{C}-\mathrm{C}$ bond forming events central to this synthesis represents a new and highly efficient paradigm for synthetic strategy. ${ }^{77}$ Additionally, it could be argued that a number of reaction sequences throughout this synthesis contain linchpins, and perhaps this is part of the reason why this strategy leads to such an efficient synthesis.

\subsubsection{Summary}

The development of linchpin-based synthetic strategies represents an exciting and powerful approach to natural product synthesis. The merit of this tactic stems from the highly convergent nature of a late-stage linchpin coupling and the use of a linchpin molecule to introduce reactive functional groups that would be incompatible with conditions used earlier in the route. However, the efficiency imparted by a linchpin sequence is dependent on the methodology and disconnections used to implement it. Thus, the major challenge of this chemistry is the development and use of chemoselective reactions that introduce high levels of structural complexity when coupling the linchpin compound. 


\section{References for Chapter 1}

[1] Newman, D.; Cragg, G. J. Nat. Prod. 2012, 75, 311. Newman, D.; Cragg, G. J. Nat. Prod. $2007,70,461$.

[2] Nicolaou, K. C.; Montagnon, T. Molecules That Changed the World; Wiley-VCH: Weinheim, 2008.

[3] Lyseng-Williamson, K.; Fenton, C. Drugs 2005, 65, 2513.

[4] Kirst, H,; Sides, G.; Antimicrob. Agents Chemother. 1989, 33, 1419

[5] Nicolaou, K. C.; Snyder, S. Classics in Total Synthesis II; Wiley-VCH: Weinheim, 2003; pp 1.

[6] For examples of structural reassignments as a result of synthetic studies, see: Lin, D.; Masuda, T.; Biskup, M. B.; Nelson, J. D.; Baran, P. S. J. Org. Chem. 2011, 76, 1013. Evans, D.; Kværnø, L.; Dunn, T.; Beauchemin, A.; Raymer, B.; Mulder, J.; Olhava, E.; Juhl, M.; Kagechika, K.; Favor, D. J. Am. Chem. Soc., 2008, 130, 16295. Nicolaou, K. C.; Snyder, S. A. Angew. Chem. Int. Ed. 2005, 44, 1012.

[7] Silverman, R.; The Organic Chemistry of Drug Design and Drug Action; Elsevier Academic Press: San Diego, 2nd Edition, 2004

[8] Hölfe, G.; Bedorf, N.; Steinmetz, H.; Schomburg, D.; Gerth, K.; Reichenbach, H. Angew. Chem. Int. Ed. 1996, $35,1567$.

[9] Balog, A.; Meng, D.; Kamenecka, T.; Bertinato, P.; Su, D.; Sorensen, E.; Danishefsky, S Angew. Chem. Int. Ed. 1996, 35, 2801.

[10] Danishefsky, S. Nat. Prod. Rep. 2010 27, 1114

[11] Rivkin, A.; Chou, T.; Danishefsky, S. Angew. Chem. Int. Ed. 2005, 44, 2838.

[12] For a themed issue on the rapid formation of molecular complexity in organic synthesis, see: Davies, H. M. L.; Sorensen, E. J. Chem. Soc. Rev. 2009, 38, 2981 and articles therein.

[13] Trost, B. M. Science 1991, 254, 1471. Trost, B. M Angew. Chem. Int. Ed. 1995, 34, 259. Trost, B. M. Acc. Chem. Res. 2002, 35, 695.

[14] Corey, E.J.; Danheiser, R. L.; Chandrasekaran, S.; Siret, P.; Keck, G. E.; Gras, J.-L. J. Am. Chem. Soc. 1978, 100, 8031. Corey, E.J.; Danheiser, R. L.; Chandrasekaran, S.; Keck, G. E.; Gopalan, B.; Larsen, S. D.; Siret, P.; Gras, J.-L. J. Am. Chem. Soc. 1978, 100, 8034.

[15] White, J. D.; Shin, H.; Kim, T.-S.; Cutshall, N. S. J. Am. Chem. Soc. 1997, 119, 2404.

[16] Brestensky, D. M.; Stryker, J. M. Tetrahedron Lett. 1989, 30, 5677.

[17] For a detailed study of the formation of TBS-OH during fluoride-mediated silyl deprotections, see: DiLauro, A. M.; Seo, W.; Phillips, S. T. J. Org. Chem 2011, 76, 7352

[18] Keinan, E.; Gleize, P. A.; Tetrahedron Lett. 1982, 23, 477.

[19] Yang, J. W.; Fonseca, M. T. H, List, B Angew. Chem. Int. Ed. 2004, 43, 6660.

[20] Cortese, N. A.; Heck, R. F. J. Org. Chem. 1978, 43, 3985.

[21] Heydrich, G.; Gralla, G.; Rauls, M.; Schmidt-Leithoff, J.; Ebel, K.; Krause, W.; Oehlenschläger, S.; Jäkel, C.; Friedrich, M.; Bergner, E. J.; Kashani-Shirazi, N.; Paciello, R. Method for Producing Optically Active, Racemic Menthol US Patent US 2010/0249467 A1, Sept. 30, 2010.

[22] For selected reviews on Pd-AA see: Trost, B. M; Crawley, M. L. Chem. Rev. 2003, 103, 2921. Trost, B. M.; Van Vranken, D. L. Chem. Rev. 1996, 96, 395. Trost, B. M. Acc. Chem. Res. 1980, 13, 385. Tsuji, J. Palladium Reagents and Catalysts: Innovations in Organic Synthesis; Wiley \& Sons: New York, 1996; pp 290-399.

[23] Tsuji, J.; Takahashi, H.; Morikawa, M. Tetrahedron Lett. 1965, 49, 4387. 
[24] Trost, B. M.; Fullerton, T. J. J. Am. Chem. Soc. 1972, 95, 292. Trost, B. M.; Weber, L.; Strege, P. E.; Fullerton, T. J.; Dietsche, T. J. J. Am. Chem. Soc. 1978, 100, 3416.

[25] Trost, B. M.; Dietsche, T. J. J. Am. Chem. Soc. 1973, 95, 8200. Trost, B. M.; Strege, P. E. J. Am. Chem. Soc. 1977, 99, 1649.

[26] Based on personal correspondence with Dr. T. J. Fullerton.

[27] For reviews on the formation of chiral quaternary centres, see: Corey, E. J.; Guzman-Perez, A. Angew. Chem. Int. Ed. 1998, 37, 388. Christoffers, J.; Baro, A. Acc. Chem. Res. 2005, 347, 1473.

[28] Trost, B. M.; Pissot-Soldermann, C.; Chen, I.; Schroeder, G. M. J. Am. Chem. Soc. 2004, 126, 4480. Trost, B. M.; Pissot-Soldermann, C.; Chen, I. Chem. Eur. J., 2005, 11, 951.

[29] Wellington, K. D.; Cambie, R. C.; Rutledge, P. S.; Bergquist, P. R. J. Nat. Prod. 2000, 63, 79.

[30] Braun, M.; Meier, T. Angew. Chem. Int. Ed. 2006, 45, 6952.

[31] For a review on the decarboxylative Pd-AAA reaction, see: You, S.-L.; Dai, L-. X. Angew. Chem. Int. Ed. 2006, 45, 5246.

[32] Mukherjee, H.; McDougal, N. T.; Virgil, S. C.; Stoltz, B. M. Org. Lett., 2011, 13, 825.

[33] Trost, B. M.; Frederiksen, M. U.; Rudd, M. T. Angew. Chem. Int. Ed. 2005, 44, 6630.

[34] Trost, B. M.; Indolese, A. J. Am. Chem. Soc. 1993, 115, 4361.

[35] Trost, B. M.; Yang, H.; Probst, G. D. J. Am. Chem. Soc. 2004, 126, 48.

[36] Perry, N. B.; Blunt, J. W.; Munro, M. H. G. J. Am. Chem. Soc. 1988, 110, 4850. Perry, N. B.; Blunt, J. W.; Munro, M. H. G.; Thompson, A. M. J. Org. Chem. 1990, 55, 223.

[37] Hood, K. A.; West, L. M.; Northcote, P. T.; Berridge, M. V.; Miller, J. H. Apoptosis 2001, 6, 207.

[38] Nakata, B.; Fukui, H.; Nakagawa, T.; Matsukura, H. Heterocycles 1996, 42, 869.

[39] Mahrwald, R. Modern Aldol Reactions Wiley-VCH: Weinheim, 2004.

[40] Evans, D. A. Aldrichimica Acta 1982, 15, 23.

[41] Trost, B. M.; Brindle, C. S. Chem. Soc. Rev. 2010, 39, 1600.

[42] Trost, B. M.; Ito, H. J. Am. Chem. Soc. 2000, 122, 12003.

[43] Trost, B. M.; Ito, H.; Silcoff, E. R. J. Am. Chem. Soc. 2001, 123, 3367.

[44] Trost, B. M.; Amans, D.; Seganish, W. M.; Chung, C. K. J. Am. Chem. Soc. 2009, 131, 17087.

[45] Field, J. J.; Singh, A. J.; Kanakkanthara, A.; Halafihi, T.; Northcote, P. T.; Miller, J. H. J. Med. Chem. 2009, 52, 7328. Hamel, E.; Day, B. W.; Miller, J. H.; Jung, K. J.; Northcote, P. T.; Ghosh, A. K.; Curran, D. P.; Cushman, M.; Nicolaou, K. C.; Paterson, I.; Sorensen, E. J. Mol. Pharmacol. 2006, 70, 1555. Gaitanos, T. N.; Buey, R. B.; Díaz, J. F.; Northcote, P. T.; Teesdale-Spittle, P.; Andreu, J. M.; Miller, J. H. Cancer Res. 2004, 64, 5063.

[46] Corley, D. G.; Herb, R.; Moore, R. E.; Scheuer, P. J.; Paul, V. J. J. Org. Chem. 1988, 53, 3644.

[47] “linchpin.” Merriam-Webster.com. 2012. http://www.merriam-webster.com (18 May 2012).

[48] Schobert, R.; Stangl, A. Tetrahedron Lett. 2005, 46, 1127.

[49] Sui, B.; Yeh, E. A.-H.; Curran, D. P. J. Org. Chem. 2010, 75, 2942. Curran, D. P.; Sui, B. J. Am. Chem. Soc. 2009, 131, 5411.

[50] An enantioselective zinc-catalyzed alkyne addition method was used as an alternative to the lithium acetylide method. This asymmetric route places the DMF linchpin sequence at an earlier stage in the synthesis and is somewhat similar to the hypothetical early stage linchpin sequence shown in Scheme 1.18. 
[51] White, J. D.; Lee, T. H.; Kuntiyong, P. Org. Lett. 2006, 8, 6043.

[52] Martin, D. B. C.; Vanderwal, C. D. Chem. Sci. 2011, 2, 649.

[53] Schaus, S. E.; Branalt, J.; Jacobsen, E. N. J. Org. Chem. 1998, 63, 4876. For additional examples, see: Keum, G.; Hwang, C. H.; Kang, S. B.; Kim, Y.; Lee, E. J. Am. Chem. Soc. 2005, 127, 10396. Zurwerra, D.; Gertsch, J.; Altmann, K.-H. Org. Lett. 2010, 12, 2302. Gudla, V.; Balamurugan, R. J. Org. Chem. 2011, 76, 9919. Elliott, M. C.; El Sayad, N. N. E.; Paine, J. S. Org. Biomol. Chem. 2008, 6, 2611.

[54] For selected examples, see; Reiter, M.; Torssell, S.; Lee, S.; MacMillan, D. W. C. Chem. Sci. 2010, $1,37$. Jones, S. B.; Simmons, B.; MacMillan, D. W. C. J. Am. Chem. Soc. 2009, 131, 13606. Chackalamannil, S.; Davies, R. J.; Wang, Y.; Asberom, T.; Doller, D.; Wong, J.; Leone, D.; McPhail, A. T. J. Org. Chem. 1999, 64, 1932. Jones, S. B.; Simmons, B.; Mastracchio, A. MacMillan, D. W. C. Nature, 2011, 475, 183. Knowles, R. R.; Carpenter, J.; Blakey, S. B.; Kayano, A.; Mangion, I. K.; Sinz, C. J.; MacMillan, D. W. C. Chem. Sci. 2011, 2, 308

[55] Furstner, A.; Grela, K.; Mathes, C.; Lehmann, C. W. J. Am. Chem. Soc. 2000, 122, 11799.

[56] Mangion, I. K.; MacMillan, D. W. C. J. Am. Chem. Soc. 2005, 127, 3696.

[57] Goniodiol: Tate, E. W.; Dixon, D. J.; Ley, S. V. Org. Biomol. Chem. 2006, 4, 1698. Platensimycin: Ghosh, A. K.; Xi, K. J. Org. Chem. 2009, 74, 1163. For additional examples, see: Hoye, T. R.; Jeon, J.; Kopel, L. C.; Ryba, T. D.; Tennakoon, M. A.; Wang, Y. Angew. Chem. Int. Ed. 2010, 49, 6151.

[58] Baran, P. S.; Richter, J. M. J. Am. Chem. Soc. 2005, 127, 15394. Liu, C.; Masuno, M. N.; MacMillan, J. B.; Molinski, T. F. Angew. Chem. Int. Ed. 2004, 43, 5951. Kim, J.; Movassaghi, M. J. Am. Chem. Soc. 2011, 133, 14940.

[59] Newhouse, T.; Lewis, C. A.; Eastman, K. J.; Baran, P. S. J. Am. Chem. Soc. 2010, 132, 7119.

[60] Smith, A. B., III; Adams, C. M. Acc. Chem. Res. 2004, 37, 365.

[61] Smith, A. B., III; Cox, J. M.; Furuichi, N.; Kenesky, C. S.; Zheng, J.; Atasoylu, O.; Wuest, W. M. Org. Lett. 2008, 10, 5501.

[62] Official Website of the Nobel Prize. www.nobelprize.org (accessed July 20, 2012).

[63] Thirsk, C.; Whiting, A. J. Chem. Soc., Perkin Trans. 1, 2002, 999.

[64] Coleman, R. S.; Walczak, M. C.; Campbell, E. L. J. Am. Chem. Soc. 2005, 127, 16038.

[65] Coleman, R. S.; Walczak, M. C. Org. Lett. 2005, 7, 2289.

[66] Garg, N. K.; Sarpong, R.; Stoltz, B. M. J. Am. Chem. Soc. 2002, $124,13179$.

[67] Butler, J. R.; Wang, C.; Bian, J.; Ready, J. M. J. Am. Chem. Soc. 2011, 133, 9956.

[68] Soheili, A.; Albaneze-Walker, J.; Murry, J. A.; Dormer, P. G.; Hughes, D. L. Org. Lett. 2003, 5, 4191.

[69] Metathesis in Natural Product Synthesis: Strategies, Substrates and Catalysts; Cossy, J.; Arseniyadis, S.; Meyer, C., Eds.; Wiley-VCH: Weinheim, 2010.

[70] Albert, B. J.; Sivaramakrishnan, A.; Naka, T.; Koide, K. J. Am. Chem. Soc. 2006, 128, 2792.

[71] Michrowska, A.; Bujok, R.; Harutyunyan, S.; Sashuk, V.; Dolgonos, G.; Grela, K. J. Am. Chem. Soc. 2004, 126, 9318 .

[72] Godula, K.; Sames, D. Science 2006, 312, 67. Chen, D. Y.-K.; Youn, S. W. Chem. Eur. J. $2012,18,9452$.

[73] Gutekunst, W. R.; Baran, P. S. J. Am. Chem. Soc. 2011, 133, 19076.

[74] Trost, B. M.; Cramer, N.; Bernsmann, H. J. Am. Chem. Soc. 2007, 129, 3086.

[75] Trost, B. M.; Mignani, S. M.; Nanninga, T. N. J. Am. Chem. Soc. 1986, 108, 6051. 
[76] For a recent review of redox economy, see: Baran, P. S.; Hoffmann, R. W.; Burns, N. Z. Angew. Chem. Int. Ed. 2009, 48, 2854.

[77] Ngai, M.-Y.; Kong, J.-R.; Krische, M. J. J. Org. Chem. 2007, 72, 1063. Bower, J. F.; Kim, I. S.; Patman, R. L.; Krische, M. J. Angew. Chem. Int. Ed. 2009, 48, 34.

[78] Han, S. B.; Hassan, A.; Kim, I. S.; Krische, M. J. J. Am. Chem. Soc. 2010, 132, 15559.

[79] Lu, Y.; Woo, S. K.; Krische, M. J. J. Am. Chem. Soc. 2011, 133, 13876.

[80] Keck, G. E.; Covel, J. A.; Schiff, T.; Yu, T. Org. Lett. 2002, 4, 1189. 
Chapter 2

Zn-ProPhenol Catalyzed

Asymmetric Alkyne Addition 


\subsection{Introduction to Alkyne Addition}

\subsubsection{Metal Alkynylides}

The unique properties of terminal alkynes make them versatile and highly efficient scaffolds for the construction of $\mathrm{C}-\mathrm{C}$ bonds in organic synthesis. ${ }^{1}$ The high $s$ character of the alkynyl carbon enables the stabilization of negative charge, making terminal alkynes much more acidic than their alkenyl or alkyl counterparts (Figure 2.1). ${ }^{2}$ Deprotonation with an alkali metal base (i.e. $n \mathrm{BuLi}, \mathrm{NaNH}_{2}$ etc.) gives rise to various alkali metal alkynylides $(\mathrm{M}=\mathrm{Li}, \mathrm{Na}, \mathrm{K})$. These nucleophiles have been used extensively in organic synthesis, and have been shown to react efficiently with a wide variety of electrophiles, including epoxides, ketones, amides, imines and alkyl halides. Additionally, alkynes can function as $\pi$-acids and coordinate to transition

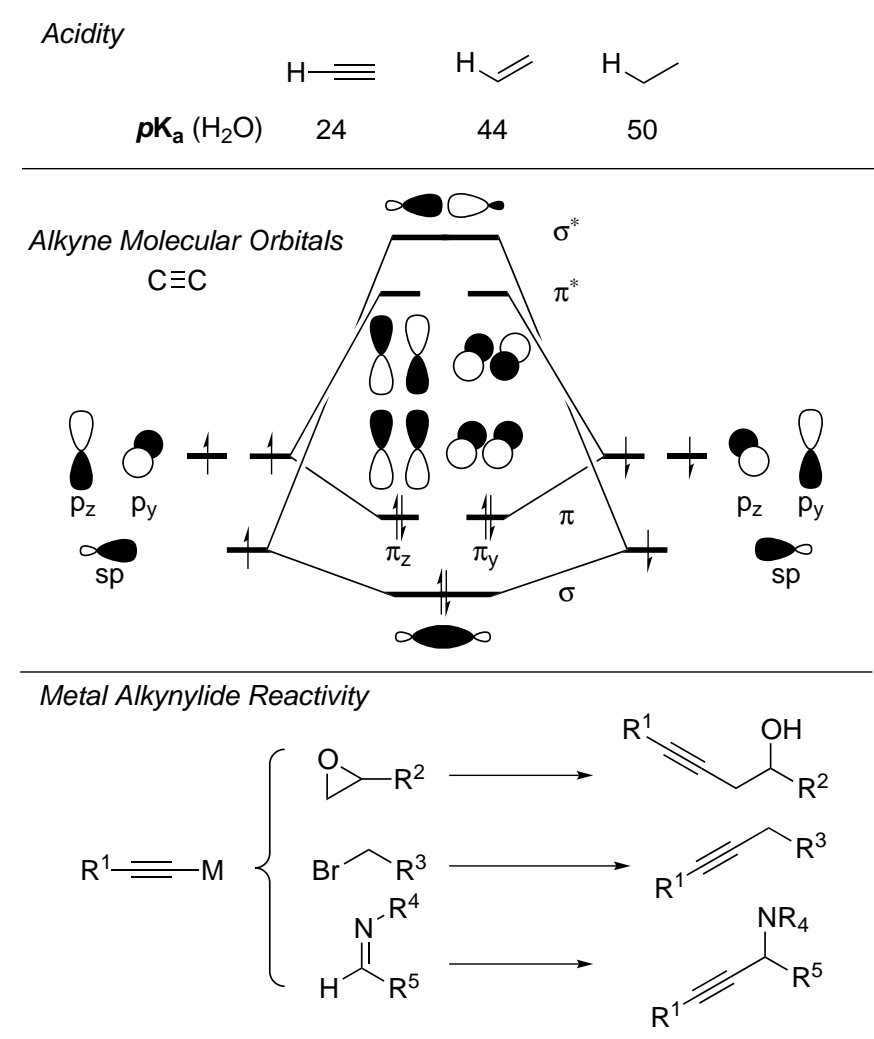

Figure 2.1 Acidity, Bonding and Reactivity of Terminal Alkynes.

metals, which increases the acidity of the terminal proton further and enables deprotonation under particularly mild conditions. Since the use of strong bases is often incompatible with highly functionalized compounds, this property makes alkynes extremely useful in the synthesis of complex molecules. A range of transition metals have been used in this capacity, with the most well known examples being the use of $\mathrm{Cu}(\mathrm{I})$ in Castro-Stephens and Sonogashira cross coupling reactions. ${ }^{3}$ Scheme 2.1 highlights the use of these mild reaction conditions in the synthesis of epothilone B. ${ }^{4}$ In general, the broad synthetic utility of alkynes lies in their bifunctional reactivity. A terminal alkyne can act as a nucleophile via deprotonation and 


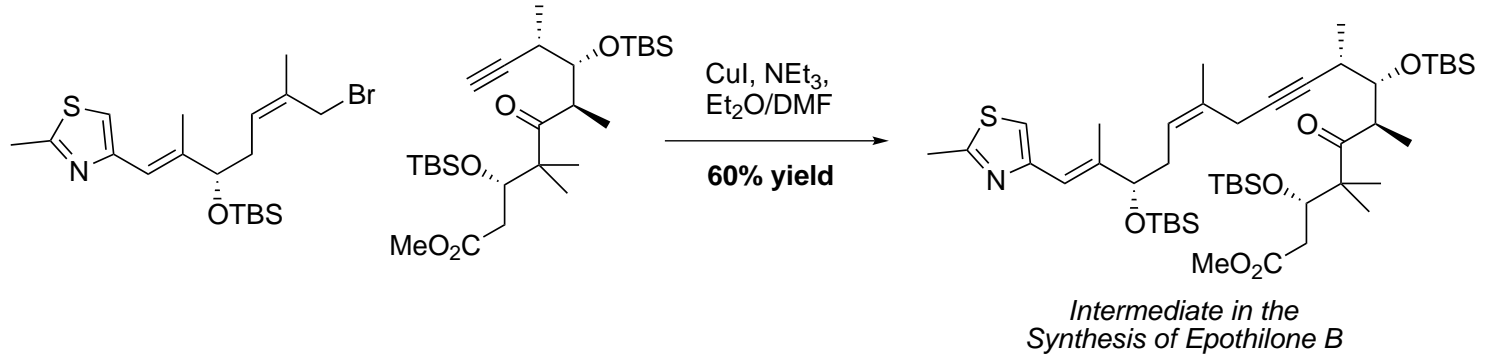

Scheme 2.1 Mild Metal Alkynylide Formation in the Castro-Stephens Reaction.

subsequent alkylation or metal-catalyzed cross coupling (vide supra). Conversely, the latent electrophilicity of alkynes can be chemoselectively activated by complexation with a transition metal. Furthermore, the reactivity of propargyl alcohols toward $\mathrm{S}_{\mathrm{N}} 2$ displacement extends the activation to the propargylic position as well. This synthetic versatility makes the catalytic enantioselective preparation of propargylic alcohols especially valuable.

\subsubsection{Propargylic Alcohols}

Propargylic alcohols serve as robust and versatile intermediates in the synthesis of fine chemicals, natural products and therapeutic agents (Scheme 2.2). ${ }^{5}$ Three general approaches have been utilized for the synthesis of secondary propargyl alcohols: enantioselective ynone reduction (A), ${ }^{6}$ asymmetric alkyne addition to aldehydes (B), ${ }^{7}$ and enantioselective ynal alkylation $(\mathbf{C}) .{ }^{8}$ Although a number of catalysts have been developed to facilitate both ynone

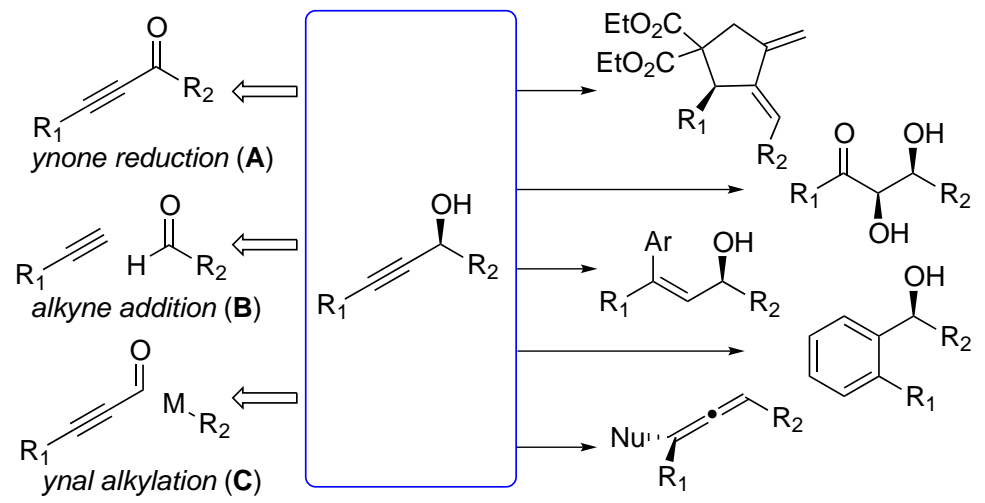

Scheme 2.2 Formation and Reactivity of Propargylic Alcohols.

reduction and ynal alkylation, the application of these methods is limited by the propensity of the alkynyl substrates to decompose, isomerize, and act as Michael acceptors. The addition of a terminal acetylene to an aldehyde avoids these problems and provides a convergent approach to the desired propargylic alcohol.

The mild reactivity of organozinc reagents has enabled the enantioselective addition of alkyl, vinyl, and alkynyl groups to a variety of carbonyl compounds with excellent functional group tolerance. ${ }^{9}$ The asymmetric addition of alkynylzinc nucleophiles to aldehydes has 
recently generated a large amount of interest in the chemical community. ${ }^{10}$ Early reports by Carreira demonstrated that stoichiometric (+)- $N$-methylephedrine (L5), $\mathrm{Zn}(\mathrm{OTf})_{2}$, and triethylamine could be used to achieve alkyne metalation and addition to aliphatic aldehydes under particularly mild conditions. ${ }^{11}$ High enantioselectivity and yield were obtained with a variety of alkynes, although aryl and $\alpha, \beta$-unsaturated aldehydes typically gave lower yields. The initial conditions requiring stoichiometric zinc and $N$-methylephedrine were ultimately rendered catalytic by increasing the reaction temperature to $60{ }^{\circ} \mathrm{C} .{ }^{11 \mathrm{f}}$ Independent work by $\mathrm{Pu}$ and Chan demonstrated the utility of $(S)$-BINOL (L6) in conjunction with $\mathrm{Ti}(\mathrm{O} i \mathrm{Pr})_{4}$ and either $\mathrm{Et}_{2} \mathrm{Zn}$ or $\mathrm{Me}_{2} \mathrm{Zn}$ to facilitate nucleophilic addition of alkynes to aldehydes. ${ }^{12,13}$ These conditions require an excess of alkyne and dialkylzinc but ultimately provide good yield and enantioselectivity with a range of substrates. A number of other chiral zinc catalysts have

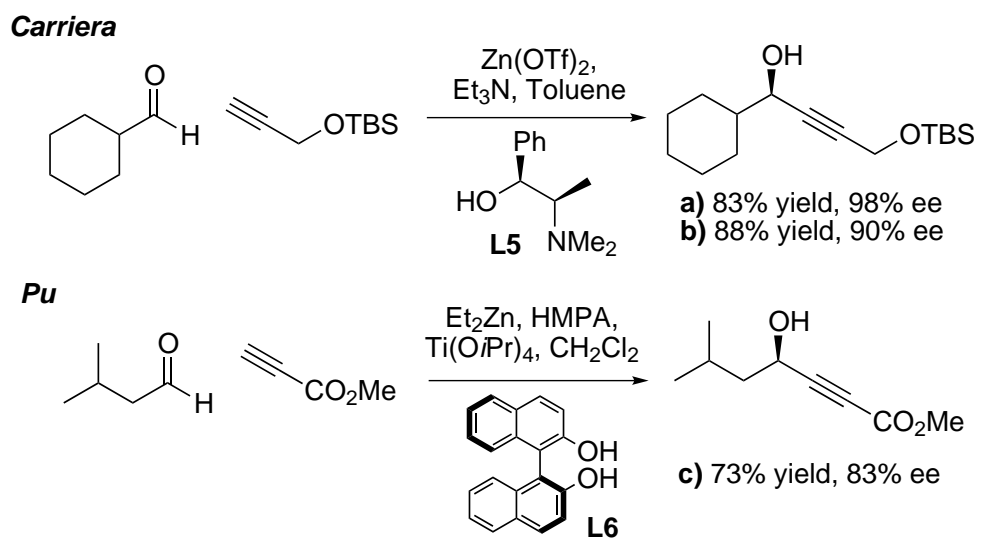

a) 1.2 eq. alkyne, 1.1 eq. $\mathrm{Zn}(\mathrm{OTf})_{2}, 1.2$ eq. (+)- $\mathrm{N}$-methylephedrine, 1.2 eq. $\mathrm{NEt}_{3}$, toluene $(0.3 \mathrm{M}), 23^{\circ} \mathrm{C}$.

b) 1.2 eq. alkyne, 0.2 eq. $\mathrm{Zn}(\mathrm{OTf})_{2}, 22 \mathrm{~mol} \%(+)-N$-methylephedrine, 0.5 eq. $\mathrm{NEt}_{3}$, toluene $(1 \mathrm{M}), 60^{\circ} \mathrm{C}$.

c) 4.0 eq. alkyne, 4.0 eq. $\mathrm{Et}_{2} \mathrm{Zn}, 40 \mathrm{~mol} \%$ (S)-BINOL, 2.0 eq. $\mathrm{HMPA}, \mathrm{CH}_{2} \mathrm{Cl}_{2}, 1.0$ eq. Ti(OPPr) 4 .

Scheme 2.3 Selected Alkynylation Examples.

also been reported to enable the enantioselective addition of alkynes to aldehydes. ${ }^{14}$ Efficient asymmetric alkyne addition often requires the use of relatively high catalyst loadings and an excess of alkyne and dialkylzinc reagents. ${ }^{15}$ An ongoing goal in this field is the development of an efficient chiral catalyst system capable of adding functionalized alkynes to a wide range of aldehydes while minimizing the use of excess reagents and stoichiometric additives. The development of such a catalyst system would ultimately enable facile access to chiral propargyl alcohols and entry into alkyne-based strategies in the synthesis of natural products. 


\subsection{Zinc-ProPhenol Catalyzed Alkynylation of Aldehydes}

\subsubsection{Introduction and Prior Work}

The design and development of the ProPhenol ligand, L4, ${ }^{16}$ as an enantioselective catalyst for base-mediated nucleophilic addition has led to the discovery of a number of highly efficient transformations. ${ }^{17}$ The combination of the ProPhenol ligand and a dialkylzinc reagent has been shown to catalyze the asymmetric Mannich and Henry reactions, ${ }^{18,19}$ the desymmetrization of meso 1,3 -diols ${ }^{20}$ and the direct aldol reaction (see Section 1.3.4). ${ }^{21}$ The success of this catalyst system with stabilized nucleophiles, such as enolates and nitronates, prompted the investigation of zinc alkynylides and their addition to aldehydes (Scheme 2.4). The ultimate goal of these investigations was to develop a practical and general methodology for zinc-catalyzed enantioselective alkynylation of aldehydes using the commercially available ProPhenol ligand, L4.

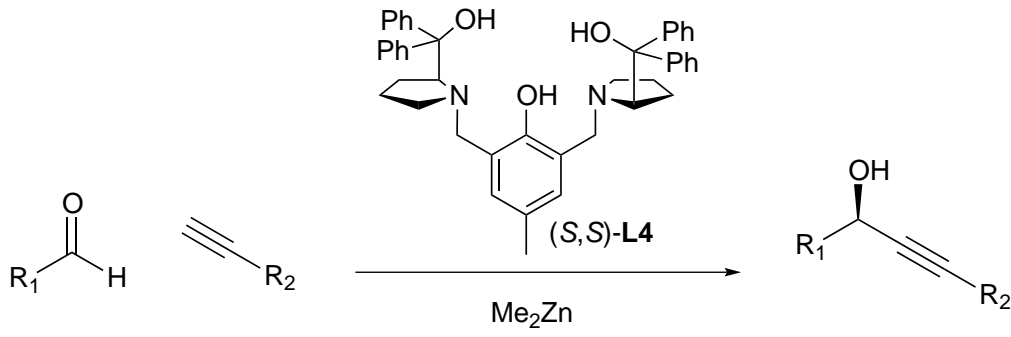

Scheme 2.4 Zn-ProPhenol Catalyzed Alkyne Addition.

\section{Initial optimization}

Optimization of the enantioselective addition of phenyl- and TMS-acetylene to $p$-anisaldehyde commenced with the screening of several $\mathrm{C}_{2}$ symmetric ligands, $(S, S)-\mathbf{L} \mathbf{4}, \mathbf{L} \mathbf{7}$ and $\mathbf{L} \mathbf{8}$, designed in the Trost group (Table 2.1) ${ }^{\dagger}{ }^{22,23}$ Stoichiometric zinc alkynylide was required for adequate enantioselectivity, and all optimization was initially carried out using nearly three equivalents of dialkylzinc and alkyne. Further experiments to improve the atom economy of this alkynylation methodology will be discussed in Section 2.2.3. Ligand screening revealed that the ProPhenol ligand, $(S, S)$-L4 , provided the best results in terms of both yield and enantioselectivity, with the desired propargyl alcohol being isolated in $78 \%$ yield and $80 \%$ ee (entry 1). Ligands L7 and L8, resembling a Salen ligand and the backbone of the Trost phosphine ligands for Pd-catalyzed asymmetric allylic alkylation, also provided the desired product, albeit with a lower enantioselectivity of $35 \%$ and $-66 \%$ ee, respectively (entries $2-3$ ). These results are in contrast to Cozzi's asymmetric alkyne addition to ketones, which utilizes a similar Salen ligand to obtain excellent results. ${ }^{25}$ Enantiomeric induction by $(S, S)-\mathbf{L} \mathbf{4}$ was found to be a robust process and provided excellent selectivity for the $(R)$-propargylic alcohol 106a, across a range of

\footnotetext{
${ }^{\dagger}$ The results in Table 2.1 were obtained by Andrew Weiss and Axel von Wangelin.
} 
Table 2.1 Initial Optimization.<smiles>COc1ccc(C=O)cc1</smiles>

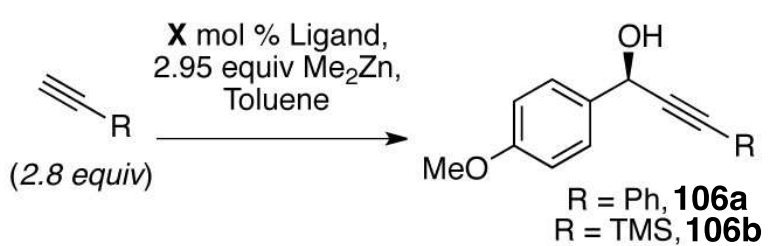

\begin{tabular}{|c|c|c|c|c|c|c|c|}
\hline & $\mathbf{R}$ & conc. $^{[\mathrm{a}]}$ & temp. & time & $\mathbf{X}$ & yield & ee \\
\hline \multicolumn{8}{|c|}{ Ligand Screening } \\
\hline 1 & $\mathrm{Ph}$ & 0.18 & $\mathrm{rt}$ & $22 \mathrm{~h}$ & $20(\mathbf{L} \mathbf{4})$ & $78 \%$ & $80 \%$ \\
\hline 2 & $\mathrm{Ph}$ & 0.18 & $0{ }^{\circ} \mathrm{C}$ & $16 \mathrm{~h}$ & 20 (L7) & $26 \%$ & $35 \%$ \\
\hline 3 & $\mathrm{Ph}$ & 0.18 & $0{ }^{\circ} \mathrm{C}$ & $16 \mathrm{~h}$ & $20(\mathbf{L 8})$ & $36 \%$ & $-66 \%$ \\
\hline
\end{tabular}

\begin{tabular}{|c|c|c|c|c|c|c|c|}
\hline 4 & $\mathrm{Ph}$ & 0.18 & $\mathrm{rt}$ & $72 \mathrm{~h}$ & 20 & $95 \%$ & $79 \%$ \\
\hline 5 & $\mathrm{Ph}$ & 0.18 & $\mathrm{rt}$ & $48 \mathrm{~h}$ & 10 & $77 \%$ & $83 \%$ \\
\hline 6 & $\mathrm{Ph}$ & 0.18 & $-20{ }^{\circ} \mathrm{C}$ & $45 \mathrm{~h}$ & 10 & $60 \%$ & $77 \%$ \\
\hline 7 & $\mathrm{Ph}$ & 0.18 & $-20^{\circ} \mathrm{C}$ & $45 \mathrm{~h}$ & 5 & $32 \%$ & $72 \%$ \\
\hline \multicolumn{8}{|c|}{ Reaction Concentration, $X=(S, S)-L 4$} \\
\hline 8 & TMS & 0.18 & $3{ }^{\circ} \mathrm{C}$ & $21 \mathrm{~h}$ & 10 & $35 \%$ & $85 \%$ \\
\hline 9 & TMS & 0.26 & $3{ }^{\circ} \mathrm{C}$ & $21 \mathrm{~h}$ & 10 & $50 \%$ & $85 \%$ \\
\hline 10 & TMS & 0.38 & $3{ }^{\circ} \mathrm{C}$ & $24 \mathrm{~h}$ & 10 & $74 \%$ & $85 \%$ \\
\hline 11 & TMS & 0.69 & $3{ }^{\circ} \mathrm{C}$ & $21 \mathrm{~h}$ & 10 & $87 \%$ & $75 \%$ \\
\hline
\end{tabular}

\begin{tabular}{c|c|c|c|c|c|c|c}
\hline \multicolumn{7}{c}{ Catalyst Loading at Higher Concentration, $X=(S, S)-L 4$} \\
\hline 12 & $\mathrm{Ph}$ & 0.38 & $3{ }^{\circ} \mathrm{C}$ & $24 \mathrm{~h}$ & 10 & $86 \%$ & $74 \%$ \\
13 & $\mathrm{Ph}$ & 0.38 & $3{ }^{\circ} \mathrm{C}$ & $24 \mathrm{~h}$ & 5 & $73 \%$ & $58 \%$ \\
14 & $\mathrm{Ph}$ & 0.38 & $3{ }^{\circ} \mathrm{C}$ & $21 \mathrm{~h}$ & 2.5 & $68 \%$ & $46 \%$ \\
\hline
\end{tabular}

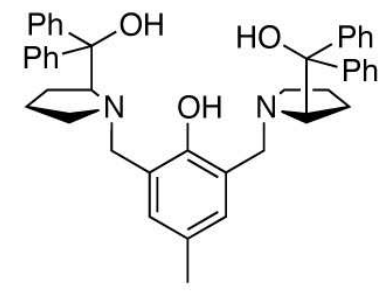

$(S, S)$-L4<smiles>O=C(N[C@H]1CCCC[C@H]1NC(=O)c1ccccn1)c1ccccn1</smiles>

L7<smiles>O=C(N[C@H]1CCCC[C@H]1NC(=O)c1ccccc1O)c1ccccc1O</smiles>

L8

[a] Reaction concentration (in molarity) is reported with respect to the alkyne and includes the toluene added as part of the dimethylzinc solution. 
temperatures and catalyst loadings (entries $4-7) .{ }^{26}$ Consequently, the majority of optimization experiments were focused primarily on improving reactivity and catalyst turnover. Reducing the catalyst loading to $10 \mathrm{~mol} \%$ and increasing the reaction time to $48 \mathrm{~h}$ produced the desired product in $77 \%$ yield and $83 \%$ ee (entry 5 ). These results are similar to those obtained in entry 1 with $20 \mathrm{~mol} \%(S, S)$-L4. In an attempt to obtain even better enantioselectivity the alkyne addition was performed at $-20{ }^{\circ} \mathrm{C}$ with both 5 and $10 \mathrm{~mol} \%(S, S)$-L4 (entries 67). These reactions provided similar levels of enantioselectivity but resulted in a substantial decrease in conversion over $c a$. two days. TMS-acetylene was found to be less reactive than phenyl acetylene in ProPhenol-catalyzed alkyne additions. However, by increasing the reaction concentration, improved reactivity could be obtained with TMS-acetylene to ultimately provide the desired product in $74 \%$ yield and $85 \%$ ee (entry 10 ). The optimal alkyne concentration was found to be $0.38 \mathrm{M}$ and a further increase in concentration, to $0.69 \mathrm{M}$, resulted in decreased enantioselectivity (entry 11). At higher reaction concentrations (ca. $2 \mathrm{M}$ ) the ligand-free background reaction proceeds readily, and is presumably the cause of the slight decrease in enantioselectivity. ${ }^{27}$ Unfortunately, decreasing the catalyst loading further $(<10 \mathrm{~mol} \%)$ provided lower yield and significantly lower ee (entries 13-14). The optimization of reaction temperature, time, concentration and catalyst loading has enabled the addition of either TMSacetylene or phenylacetylene to $p$-anisaldehyde in high yield $(>70 \%)$ and ee $(>70 \%)$ with a catalyst loading of just $10 \mathrm{~mol} \%$. Interestingly, similar results were generally observed for dimethyl- and diethylzinc; however, others have noted that alkyl transfer from dimethylzinc is significantly slower than from diethylzinc, and therefore dimethylzinc was chosen to avoid potential alkyl addition side products. ${ }^{28}$

\section{Alkynylation of Unsaturated Aldehydes}

A variety of aryl and $\alpha, \beta$-unsaturated aldehydes underwent efficient alkyne addition using the previously optimized conditions (Scheme 2.5). ${ }^{22,23}$ High yields and enantioselectivies were obtained in additions to benzaldehydes containing both electron-donating and electronwithdrawing substituents. Substitution on each aromatic carbon was tolerated and particularly

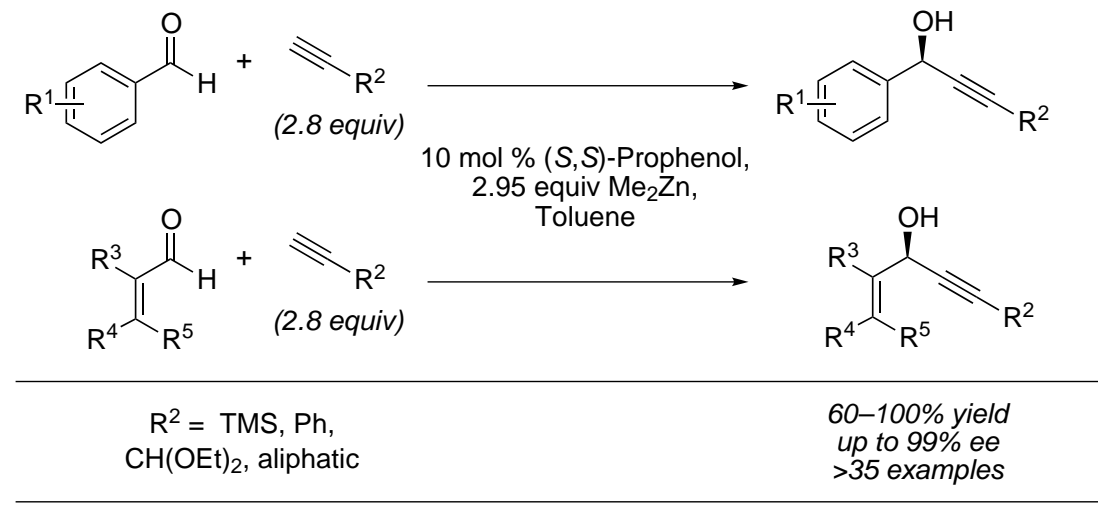

Scheme 2.5 Alkynylation of Unsaturated Aldehydes. 
good results were obtained with ortho-substituted benzaldehydes. Good results were also obtained with a variety of alkynes and only small variations in yield and enantioselectivity were observed. Alkyne addition to $\alpha, \beta$-unsaturated aldehydes provides a particularly valuable extension of substrate scope. The resulting alkenyl alkynyl carbinols contain three orthogonal functional groups primed for further synthetic manipulation.

Methyl propiolate represents a particularly attractive class of nucleophile for alkyne addition to aldehydes. ${ }^{29}$ The resulting $\gamma$-hydroxy- $\alpha, \beta$-acetylenic esters are extremely versatile synthetic intermediates and have been used in a number of total syntheses. ${ }^{30}$ Propiolate donors have traditionally been difficult substrates for asymmetric alkynylation due to their propensity to decompose in the presence of Lewis acids and nucleophiles. ${ }^{31}$ However, methyl propiolate ultimately proved to be one of the most effective alkynes under Zn-ProPhenol alkynylation conditions (Table 2.2) . Excellent results were obtained with a range of $\alpha, \beta$-unsaturated aldehydes, including $(E)$-non-2-enal, which provided $97 \%$ ee with methyl propiolate (entry 2), a major improvement from the $36 \%$ ee obtained with TMS-acetylene. The superior results are presumed to be a consequence of the inductive stabilization of the alkynylide along with potential coordination of the propiolate ester with the bimetallic catalyst.

Table 2.2 Addition of Methyl Propiolate to $\alpha$ - $\beta$-Unsaturated Aldehydes.

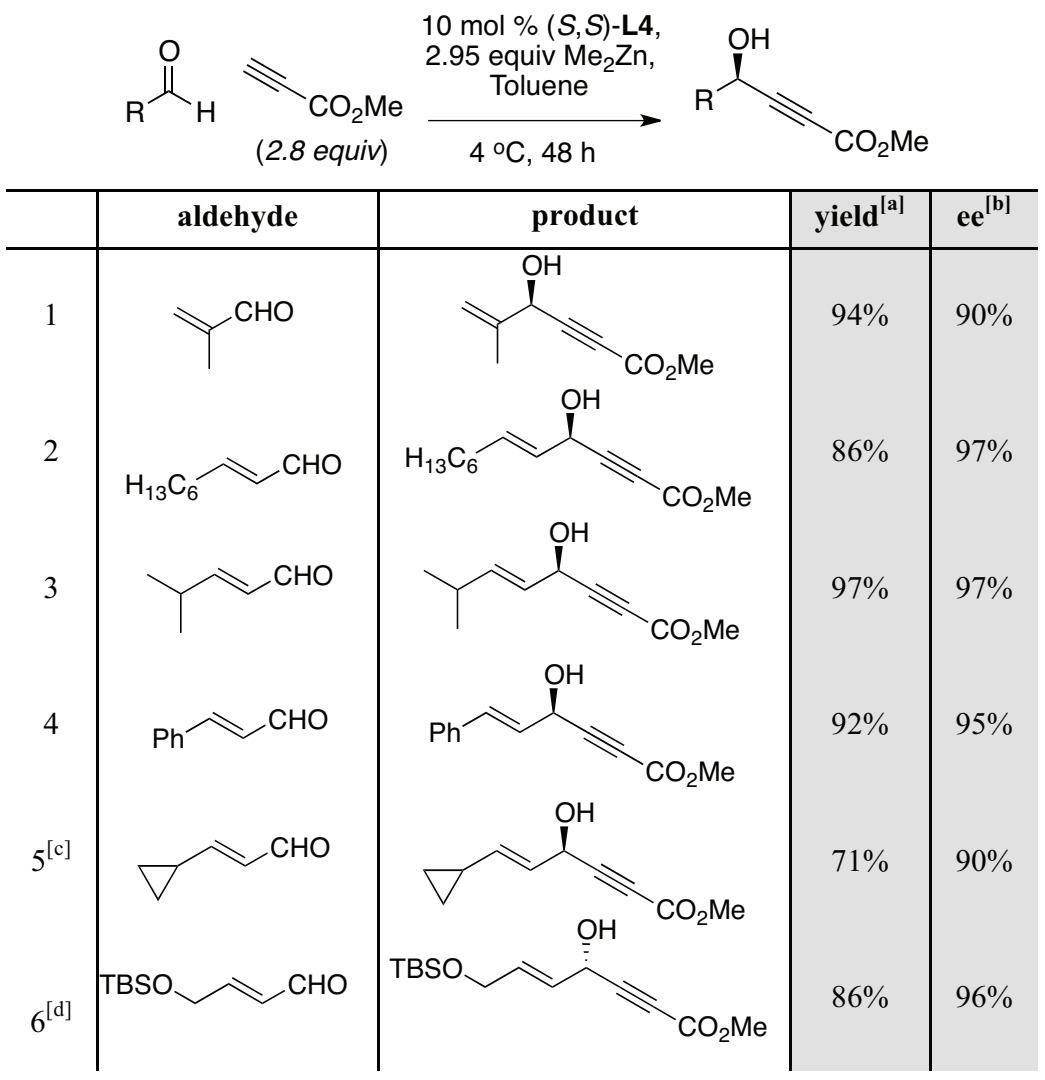

\footnotetext{
${ }^{\S}$ The results in Table 2.2 were obtained by Andrew Weiss, Mark Bartlett and Vincent Chan.
} 

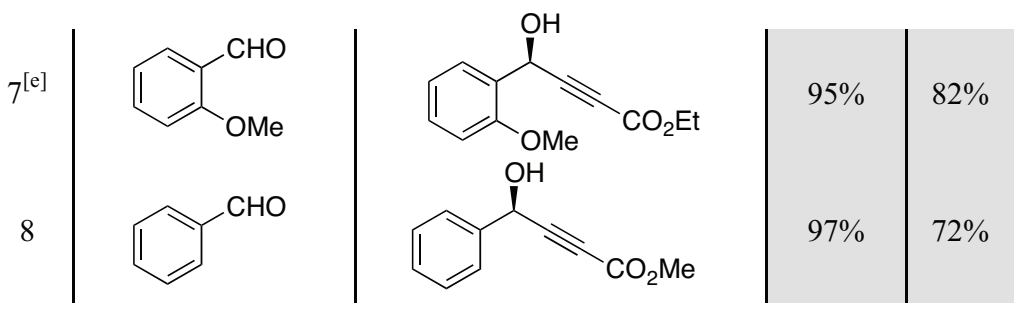

[a] Isolated yield. [b] Enantiomeric excess determined by chiral HPLC. [c] Reaction performed using 3 equiv alkyne and 3 equiv $\mathrm{Me}_{2} \mathrm{Zn}$. [d] Reaction performed using $(R, R)$-L4. [e] Reaction performed using ethyl propiolate.

\subsubsection{Alkyne Addition to Aliphatic Aldehydes}

n-Alkylcarboxaldehydes are particularly challenging substrates for alkynylation due to their propensity to enolize and undergo cross-aldol side reactions. ${ }^{32}$ Thus, initial research on ProPhenol-catalyzed alkyne additions only investigated the use of unsaturated aldehydes (Scheme 2.5). However, it was subsequently discovered that a variety of aliphatic aldehydes are also suitable electrophiles for asymmetric alkynylation (Table 2.3). The addition of methyl propiolate to $n$-Alkylcarboxaldehydes, such as octanal and dihydrocinnamaldehyde, typically provided moderate yields $(50-70 \%)$ but excellent enantioselectivity (>90\% ee). $\alpha$ and $\beta$-substituted aliphatic aldehydes, with a decrease propensity for enolization, proceeded in good yield and enantioselectivity. For example, the addition of methyl propiolate to cyclopropanecarboxaldehyde gave the desired propargylic alcohol in $88 \%$ yield and $94 \%$ ee. On the other hand, aliphatic aldehydes with an increased propensity to enolize, such as cyclopentane carboxaldehyde and 3-methylbut-3-enal, did not produce the desired products. Furthermore, the increased steric hindrance of 2,2-dimethyl-substituted aldehydes often resulted in decreased enantioselectivity, although addition of methyl propiolate to pivaldehyde provided the desired product in $88 \%$ ee. However, the analogous 3-chloroand 3-methoxycarbonyl substrates provided significantly lower enantioselectivity, generating the corresponding propargylic alcohols in $72 \%$ and $75 \%$ ee respectively. Unfortunately, 2,2diethoxypent-4-enal provided the desired propargyl alcohol as a racemic mixture, even at the lower reaction temperature of $-40{ }^{\circ} \mathrm{C}$. Presumably, the inductive effects of the gem-diethoxy group increase the reactivity of the aldehyde to the point where the uncatalyzed background reaction is facile. Extension of this methodology to aliphatic aldehydes further demonstrates the generality of the ProPhenol-catalyzed alkynylation with respect to both nucleophile and electrophile. 
Table 2.3 Enantioselective Alkynylation of Aliphatic Aldehydes.

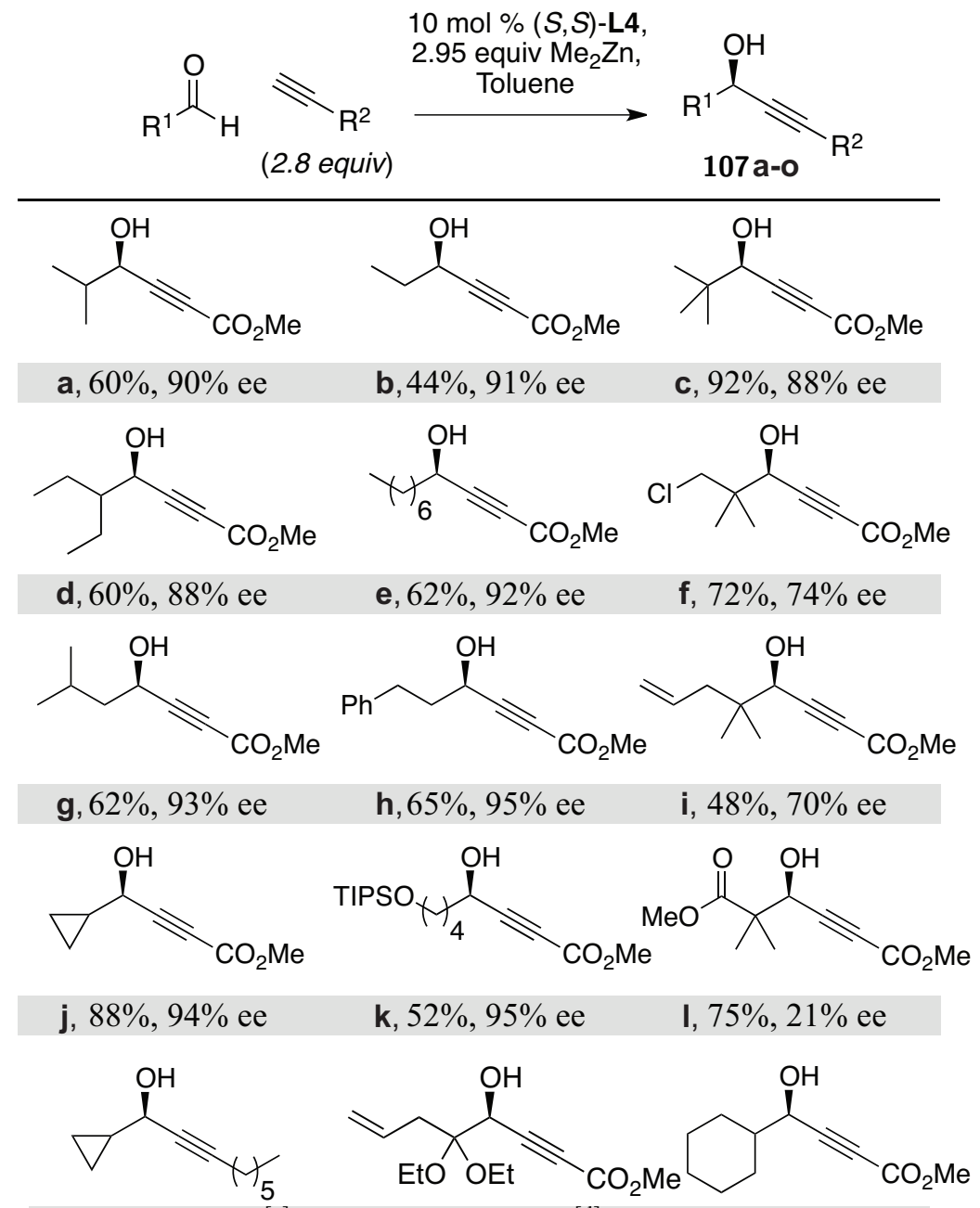

m, $94 \%, 69 \% \mathrm{ee}^{[\mathrm{c}\rfloor} \quad$ n, $87 \% .0 \% \mathrm{ee}^{[\mathrm{d}]} \quad$ o, $71 \%, 90 \%$ ee

[a] Isolated yield. [b] Enantiomeric excess determined by chiral HPLC analysis. [c] Enantiomeric excess determined by chiral HPLC analysis of the corresponding benzoate. [d] Reaction performed at $-40{ }^{\circ} \mathrm{C}$. 


\subsubsection{Mechanistic Inferences and Reoptimization}

\section{Proposed Mechanism}

The proposed mechanism for ProPhenol-catalyzed alkyne addition involves the formation of a dinuclear zinc species, A (Scheme 2.6). This complex contains both Brønsted basic and Lewis acidic sites, and can therefore act as a bifunctional catalyst, activating two reagents simultaneously. The relative acidity of a terminal alkyne (e.g. $\left.p \mathrm{~K}_{\mathrm{a}}(\mathrm{DMSO}) \mathrm{PhCCH}=28.7\right)^{33}$ enables the formation of an alkynylzinc nucleophile, which undergoes nucleophilic addition to the si face of an aldehyde. Product dissociation via metal exchange liberates a propargylic zinc alkoxide and regenerates the active catalyst. Turnover of the catalyst in this way necessitates the use of a stoichiometric amount of organozinc reagent. In contrast, the ProPhenol-catalyzed direct aldol reaction requires only a catalytic amount of dialkylzinc reagent and dissociation of the product alkoxide is postulated to occur via proton exchange. ${ }^{21}$

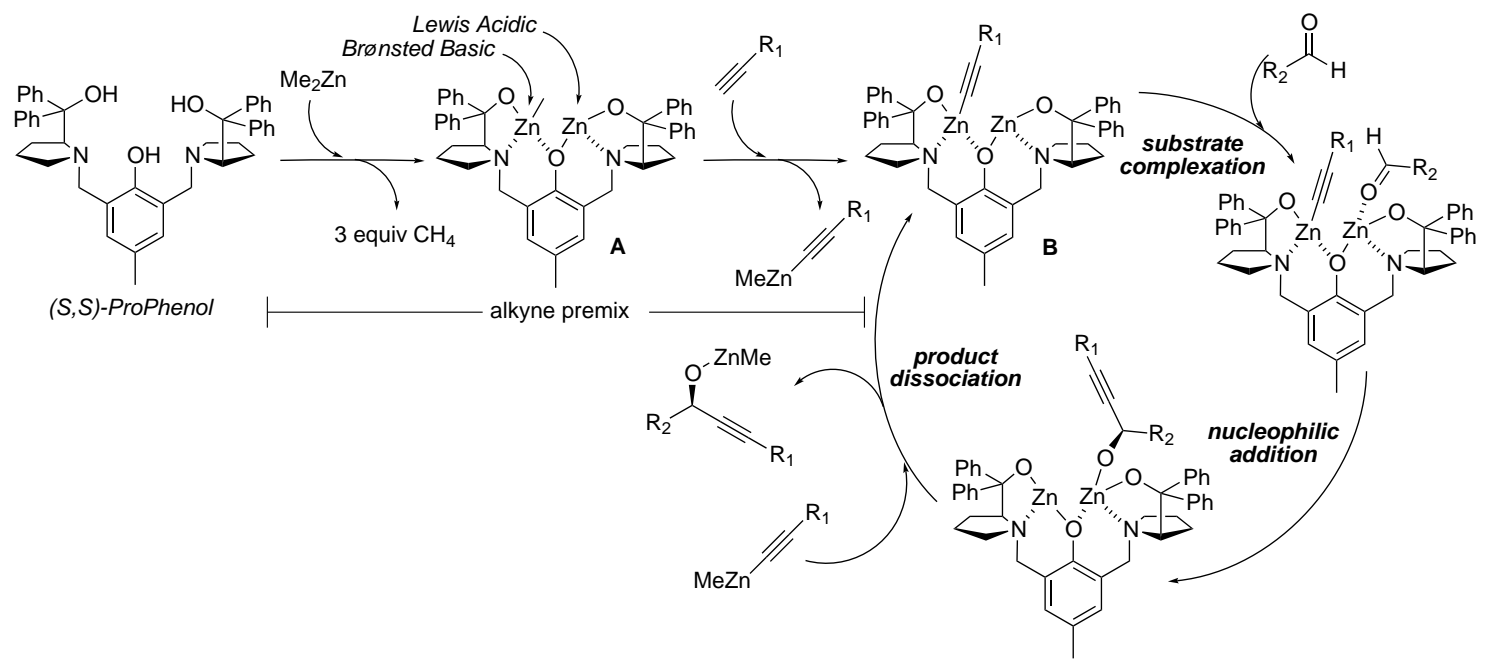

Scheme 2.6 Proposed Mechanism for ProPhenol-Catalyzed Alkynylation of Aldehydes.

\section{Additive Effects and TPPO}

During parallel research into the ProPhenol-catalyzed addition of diynes, Vincent Chan discovered that the presence of acetate groups in the substrate, far from the reacting alkyne terminus, resulted in significantly higher enantioselectivity. ${ }^{34}$ It was then hypothesized that this Lewis basic group interacts with the dinuclear zinc catalyst and reinforces the chiral pocket created by the ProPhenol ligand. The proposed coordination is supported by the X-ray crystal structure of the ProPhenol dinuclear zinc complex reported by Ding and coworkers (Figure 2.2). ${ }^{35}$ The crystal structure contains a molecule of THF (blue circle) coordinated to each of the zinc atoms, analogous to the postulated interaction with a Lewis base. Screening a variety of substoichiometric Lewis basic additives revealed that the addition of $20 \mathrm{~mol} \%$ triphenylphosphine oxide (TPPO) provided optimal results. The largest improvements were 

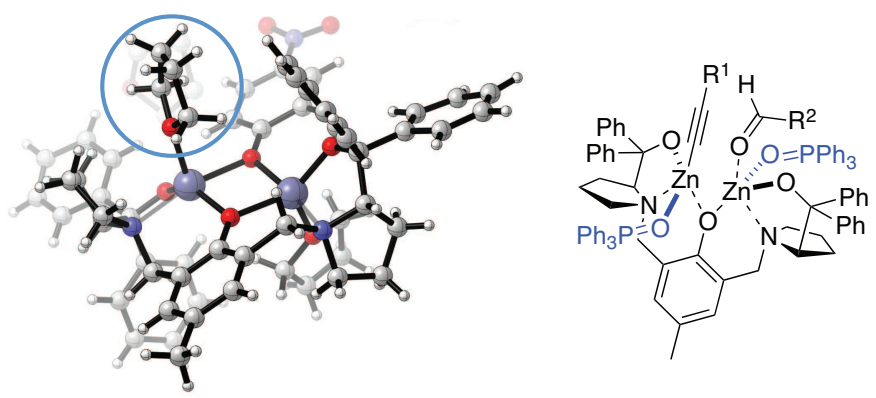

Figure 2.2 Zn-ProPhenol Crystal Structure and Proposed Interaction with Lewis Basic Additives.

observed in cases where the substrates lacked potential Lewis basic sites (Table 2.4). ${ }^{\dagger}$ Greatly increased enantioselectivity was observed for the addition of TMS-acetylene to $(E)$-nonenal upon addition of TPPO (entry 2).

Table 2.4 Enantioselective Alkynylation with TPPO.

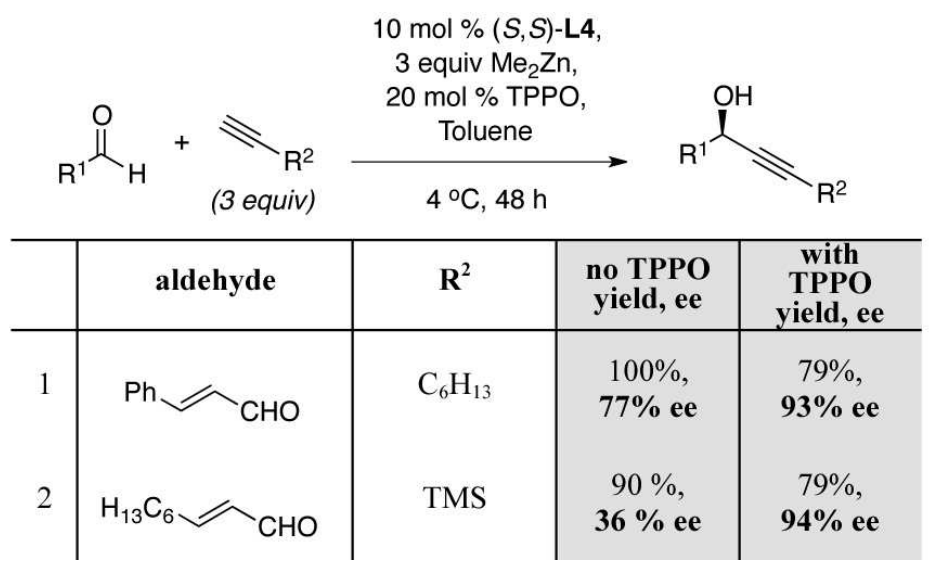

\section{Reagent Stoichiometry and Reoptimization}

To maximize the practicality and synthetic efficiency of this methodology we sought to reduce reagent stoichiometry to a minimum. Ideally, the alkyne addition could be catalytic in metal with the resultant zinc alkoxide serving as a base for subsequent alkyne deprotonations. Carreira and coworkers were able to achieve alkynylation that was catalytic with respect to zinc triflate by increasing the reaction temperature to $60{ }^{\circ} \mathrm{C}$. ${ }^{11 \mathrm{f}}$ Previous work in the Trost group by Andrew Weiss had revealed that the use a catalytic amount of dimethylzinc and elevated reaction temperatures in the ProPhenol-catalyzed alkynylation resulted in recovered starting material. ${ }^{36}$. Additionally, it was shown that the use of just a slight excess of alkyne and dimethylzinc, either 1.2 or 1.5 equivalents of each, caused a significant drop in enantioselectivity

\footnotetext{
${ }^{\dagger}$ The experiments in Table 2.4 were performed by Vincent Chan.
} 
Table 2.5 Alkynylation with Reduced Stoichiometry.

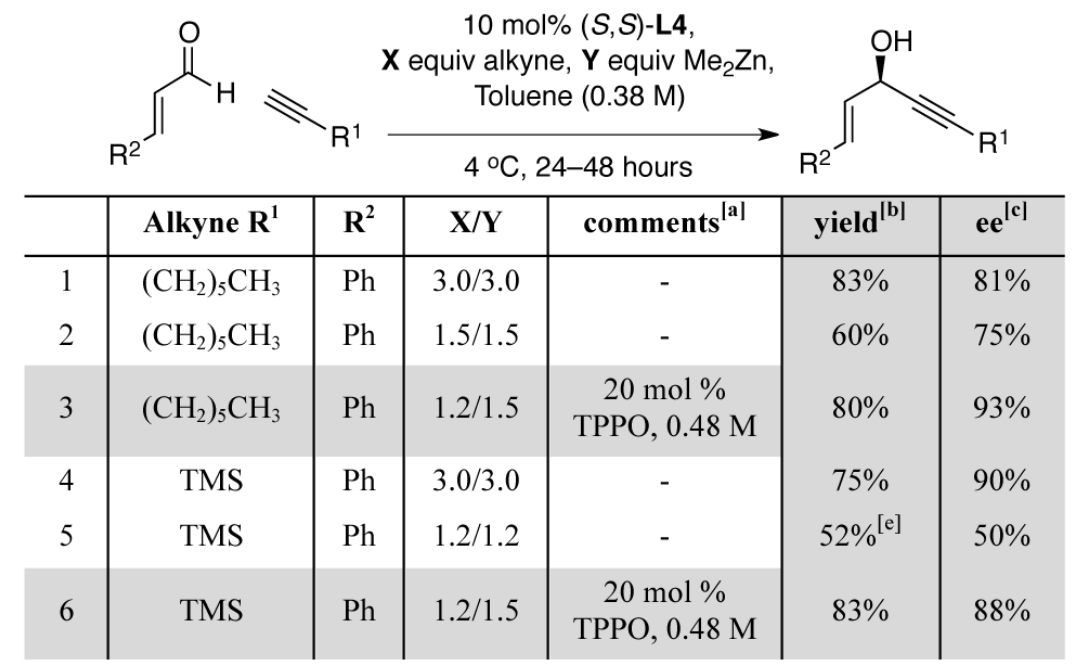

[a] Reaction concentration is reported with respect to alkyne. [b] Isolated yield. [c] Enantiomeric excess determined by chiral HPLC analysis. [d] Isolated along with $16 \%$ yield of the methyl addition side product. [e] $17 \%$ recovered starting material.

(Table 2.5,** entries 2 and 5). ${ }^{36}$ Zinc alkoxides are known to form aggregates, ${ }^{37}$ and it was hypothesized that the aggregation state of the reactive zinc species plays an important role in enantioselectivity. Since the aggregation state is likely to be concentration dependent, a number of experiments were performed with the concentration of alkyne and dimethylzinc held constant, while reducing the stoichiometry of each (as opposed to a ca. 2-fold dilution). Consequently, the ProPhenol-catalyzed addition of 1-octyne was found to give the same high enantioselectivity with half the amount of alkyne, although a drop in yield was observed (entry 2 ). The lower yield is presumably a consequence of decreased reactivity and to counter this, the reaction concentration was increased to $0.48 \mathrm{M}$. When this adjustment was made in conjunction with the use of $20 \mathrm{~mol} \%$ TPPO, the desired product was obtained in $80 \%$ yield and $93 \%$ ee using just 1.2 equivalents of alkyne (entry 3). TMS-acetylene also required higher reaction concentration and $20 \mathrm{~mol} \%$ TPPO to provide high yield and enantioselectivity (entry 6).

The concentration dependence of this reaction led to the possibility of a dimeric active catalyst. ${ }^{38}$ A number of dimeric zinc amino alcohol catalysts have been reported that display characteristic non-linear asymmetric induction in alkyl addition to aldehydes. ${ }^{39}$ In the case of the ProPhenol ligand, the enantiomeric excess of the product displayed a linear correlation with the enantiopurity of the ProPhenol ligand used to catalyze the reaction. ${ }^{\S}, 36$ Therefore, it is likely that a single ProPhenol ligand is present in the enantiodetermining step.

\footnotetext{
${ }^{* *}$ Entries 1, 2, 4 and 5 were performed by Andrew Weiss

$\S$ These experiments were performed by Andrew Weiss
} 


\section{Alkynylzinc Formation and Analysis of the Alkyne Premix}

Reducing the equivalents of alkyne used in additions to enolizable aldehydes represents a much more challenging prospect. These reactions produce undesired cross aldol side products and reducing the equivalents of alkyne exacerbates the problem (see Table 2.6, entries 1-3). It was hypothesized that this side reaction occurs as a result of incomplete alkynylzinc formation and the presence of unreacted dimethyl zinc in the reaction mixture. Formation of the alkynylzinc species is driven, in part, by the entropically favored release of methane gas. However, a number of additional factors also contribute to the overall composition of the alkyne premix. Non-polar solvents have been shown to disfavor formation of the alkynylzinc species. For example, in the case of phenylacetylene in heptane, no formation of the methylalkynylzinc species is observed. ${ }^{40}$ A number of other Zn-alkyne addition methodologies rely on the use of additives to aid in the formation of the alkynylzinc nucleophile. ${ }^{41}$ A stepwise ${ }^{1} \mathrm{H}$ NMR analysis of the methyl propiolate $/ \mathrm{Me}_{2} \mathrm{Zn} /(S, S)$-L4 premix in toluene- $d 8$ was used to evaluate alkyne deprotonation. Using a 1.2/1.5/0.1 ratio of methyl propiolate/ $\mathrm{Me}_{2} \mathrm{Zn} /(S, S)-\mathbf{L} \mathbf{4}$, integration of the peaks at $0.17\left(\mathrm{~s}, 3 \mathrm{H}, \mathrm{CH}_{3} \mathrm{ZnCC}\right)$ and $-0.69\left(\mathrm{~s}, 6 \mathrm{H},\left(\mathrm{CH}_{3}\right)_{2} \mathrm{Zn}\right) \mathrm{ppm}$ revealed that approximately $30 \%$ of the desired alkynylzinc species was being formed during the standard 1 hour premix at room temperature (Figure 2.3). ${ }^{42}$ Deprotonation of the terminal alkyne was not observed in the

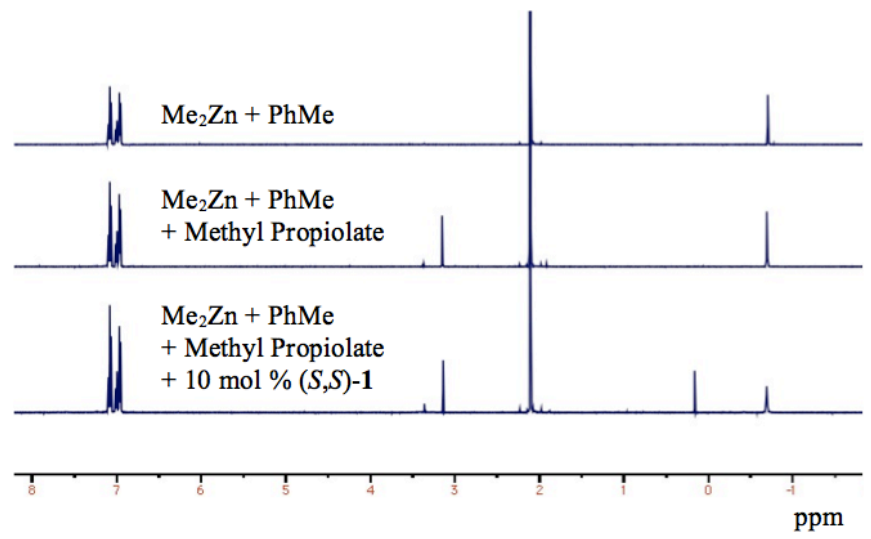

Figure $2.3{ }^{1} \mathrm{H}$ NMR Analysis of the Alkyne Premix.

absence of the ProPhenol ligand. Analysis of the alkyne premix by volumetric gas titration of methane revealed that the initial rate of deprotonation is rapid but tapers off after about 15 minutes. The volume of methane obtained over the standard premix time was in good agreement with the ${ }^{1} \mathrm{H}$ NMR results. In contrast to enolizable aldehydes, the presence of significant amounts of dimethylzinc has little effect on the outcome of alkyne additions to aryl and $\alpha, \beta$-unsaturated aldehydes. ${ }^{43}$ Minor amounts of methyl addition side products, such as compound 108, have been isolated in only a small number of cases (for example, see: Scheme 2.7). ProPhenol-catalyzed methyl addition is a relatively slow process and, in the case of 2-naphthaldehyde, required 3 days to react with approximately half of the starting material (Scheme 2.8). 


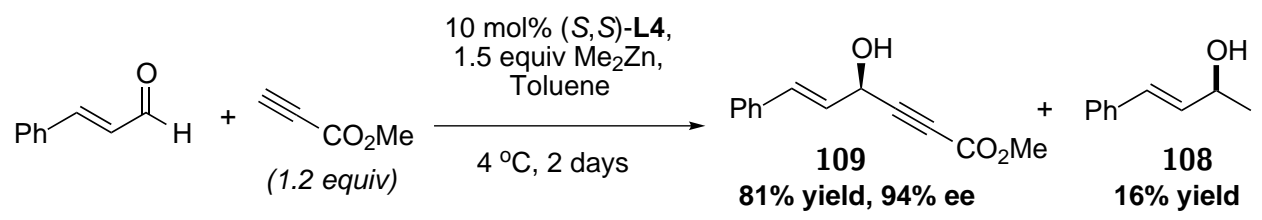

Scheme 2.7 Competing Reaction Pathways: A Methyl Addition Side Product.

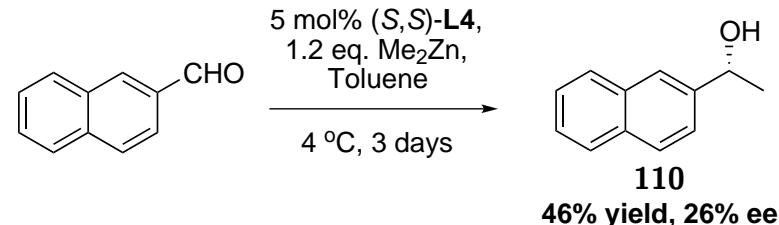

Scheme 2.8 Evaluating ProPhenol-Catalyzed Asymmetric Alkylation.

These observations prompted the investigation of methods to facilitate the formation of the alkynylzinc nucleophile and, therefore, reduce the amount of dimethylzinc present (Table 2.6). The addition of aliphatic alkyne 111 to the enolizable aldehyde 112 provided the desired propargylic alcohol 113 in low yield under the original super-stoichiometric conditions (entry 1). The low yield is a consequence of competing aldol side reactions, which provide a complex mixture of oligomers including compound 114, which was isolated as a mixture of diastereomers (19\% yield). The use of Lewis basic additives such as $N$-methylimidazole (NMI), DMSO and DMF to aid the formation of the desired alkynylzinc nucleophile provided a low yield of the desired product 113 (entries 4-6). ${ }^{41}$ However, extending the alkyne premix time from 1 hour to 24 hours provided 58\% yield of propargylic alcohol 116 (entry 7). Combining this discovery with a higher catalyst loading (20 mol \%) led to an optimized result of $69 \%$ yield and $67 \%$ ee for this challenging alkyne-aldehyde pairing (entry 8). Applying our mechanistic understanding led to a modest improvement in the addition of a non-stabilized alkyne nucleophile (111) to an enolizable aliphatic aldehyde (115), the most challenging combination for this methodology.

In contrast to the previous set of results, enolizable aliphatic aldehydes were shown to be excellent electrophiles in the ProPhenol-catalyzed alkynylation when more stabilized nucleophilic alkynes were employed. For example, the higher acidity of methyl propiolate provides improved results with enolizable aldehydes relative to other alkynes (Table 2.7). Addition of methyl propiolate to octanal under the initial conditions provides propargylic alcohol 117 in $62 \%$ yield. Lowering the reaction stoichiometry from 2.8 to 1.2 equivalents of alkyne results in a significantly decreased yield (entry 4). Longer alkyne premix time results in visible decomposition of methyl propiolate (entry 5). Therefore the use of higher catalyst loading was used to aid the consumption of dimethylzinc through alkynylzinc formation (Entries 6-7). Ultimately, 57\% yield and $90 \%$ ee of the desired propargyl alcohol were obtained using 1.2 equivalents of alkyne. 
Table 2.6 Optimization of Alkyne Addition with an Enolizable Substrate.

\begin{tabular}{|c|c|c|c|c|c|}
\hline \multirow{3}{*}{111} & \multicolumn{3}{|r|}{$\begin{array}{c}10 \text { mol\% }(S, S)-L \mathbf{4} \\
\mathbf{X} \text { equiv } \mathbf{1 1 1} \\
\text { Y equiv } \mathrm{Me}_{2} Z \mathrm{n}, \text { Toluene }\end{array}$} & \multirow{2}{*}{\multicolumn{2}{|c|}{$\begin{array}{l}113-R=\text { TBS } \\
116-R=P M B\end{array}$}} \\
\hline & \multicolumn{2}{|c|}{$\begin{array}{l}112-\mathrm{R}=\mathrm{TBS} \\
115-\mathrm{R}=\mathrm{PMB}\end{array}$} & & & \\
\hline & CHO & $\mathbf{X} / \mathbf{Y}$ & conditions & yield $^{[\mathrm{a}]}$ & $e e^{[b]}$ \\
\hline 1 & 112 & $2.8 / 2.95$ & - & $35 \%^{[\mathrm{c}]}$ & $45 \%$ \\
\hline 2 & 112 & $2.8 / 2.95$ & $20 \mathrm{~mol} \%$ ТРРО & $39 \%$ & $62 \%$ \\
\hline 3 & 112 & $1.2 / 1.5$ & $20 \mathrm{~mol} \%$ TPPO & $22 \%$ & $54 \%$ \\
\hline 4 & 112 & $1.2 / 1.3$ & $30 \mathrm{~mol} \% \mathrm{NMI}$ & $11 \%$ & $72 \%$ \\
\hline 5 & 112 & $1.2 / 1.4$ & 4 equiv DMSO & $4 \%$ & $14 \%$ \\
\hline 6 & 112 & $1.2 / 1.4$ & 4 equiv DMF & $10 \%$ & $17 \%$ \\
\hline 7 & 115 & $2.8 / 2.95$ & $\begin{array}{l}20 \mathrm{~mol} \% \text { TPPO, } 24 \\
\text { hour alkyne premix }\end{array}$ & $58 \%$ & $50 \%^{[\mathrm{d}]}$ \\
\hline 8 & 115 & $2.8 / 2.95$ & $\begin{array}{l}20 \mathrm{~mol} \%(S, S)-\mathbf{L} 4 \\
40 \mathrm{~mol} \% \mathrm{TPPO}, 24 \\
\text { hour alkyne premix }\end{array}$ & $69 \%$ & $67 \%{ }^{[\mathrm{d}]}$ \\
\hline
\end{tabular}

[a] Isolated yield. All reactions were run on a $0.1625 \mathrm{mmol}$ scale. [b] Enantiomeric excess determined by chiral HPLC analysis. [c] Isolated along with 19\% yield of the cross aldol side product 111 [d] Enantiomeric excess determined by ${ }^{1} \mathrm{H}-\mathrm{NMR}$ analysis of the corresponding $(S)$-methyl mandelate.

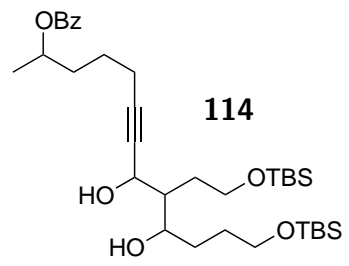


Table 2.7 Optimization of Methyl Propiolate Addition to Octanal.

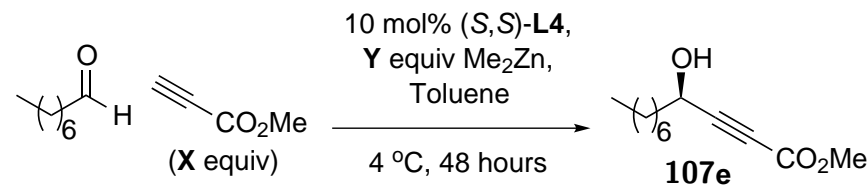

\begin{tabular}{c|c|c|c|c}
\hline entry & $\mathbf{X} / \mathbf{Y}$ & conditions & yield $^{[\mathbf{a}]}$ & $\mathbf{e e}^{[\mathbf{b}]}$ \\
\hline 1 & $2.8 / 2.95$ & - & $62 \%$ & $92 \%$ \\
2 & $1.5 / 1.5$ & - & $37 \%$ & $86 \%$ \\
3 & $1.5 / 1.5$ & $\begin{array}{c}20 \mathrm{~mol} \% \\
\text { TPPO }\end{array}$ & $35 \%$ & $90 \%$ \\
4 & $1.2 / 1.5$ & - & $42 \%$ & $91 \%$ \\
5 & $\begin{array}{c}2.8 / 2.95 \\
\left(\mathrm{Et} \mathrm{Zn}^{2}\right)\end{array}$ & $\begin{array}{c}4 \mathrm{~h} \text { alkyne } \\
\text { premix }\end{array}$ & $60 \%$ & $70 \%$ \\
6 & $1.2 / 1.5$ & $\begin{array}{c}20 \mathrm{~mol} \% \\
(S, S)-\mathbf{L 4}\end{array}$ & $57 \%$ & $90 \%$ \\
7 & $2.8 / 2.95$ & $\begin{array}{c}20 \mathrm{~mol} \% \\
(S, S)-\mathbf{L 4}\end{array}$ & $72 \%$ & $87 \%$
\end{tabular}

[a] All reactions were run on a $0.325 \mathrm{mmol}$ scale. Isolated yield. [b] Enantiomeric excess determined by chiral HPLC analysis.

\section{Alkynylation with Reduced Alkynylide Stoichiometry.}

The use of a stoichiometric amount of alkyne would facilitate the use of complex alkynes to access important synthetic targets, such as aspergillide B (see Chapter 4), in a highly efficient manner. Thus, we were pleased to observe that the newly developed reaction conditions allowed for the use of only 1.2 equivalents of alkyne with a variety of aldehydes (Scheme 2.9). In line with our previous results, the nature of the alkyne affected the results of the alkynylation. Methyl propiolate provided good enantioselectivity and yield with each class of aldehydes: aliphatic, $\alpha, \beta$-unsaturated and aryl. Higher catalyst loadings were used with enolizable aldehydes to disfavour aldol side reactions. More specifically, using $20 \mathrm{~mol} \%$ of the ProPhenol ligand enabled the addition of methyl propiolate to 3-phenylpropionaldehyde in $76 \%$ yield and $93 \%$ ee. Alkynylation with only 1.2 equivalents of a non-stabilized aliphatic alkyne proved much more challenging. However, increased reaction concentrations and the use of TPPO as an additive enabled these alkyne additions to proceed in good yield and enantioselectivity. Notably, the addition of an aliphatic alkyne to fumaraldehyde dimethyl acetal provided the desired product 118 in $88 \%$ yield and $90 \%$ ee using just a single equivalent of alkyne. The addition of aliphatic alkynes to enolizable aldehydes is particularly challenging and, in the example shown in Scheme 2.9, only moderate yield and enantioselectivity was obtained even when an excess of alkyne was used in conjunction with TPPO, increased catalyst loading and a 24 hour alkyne premix. 


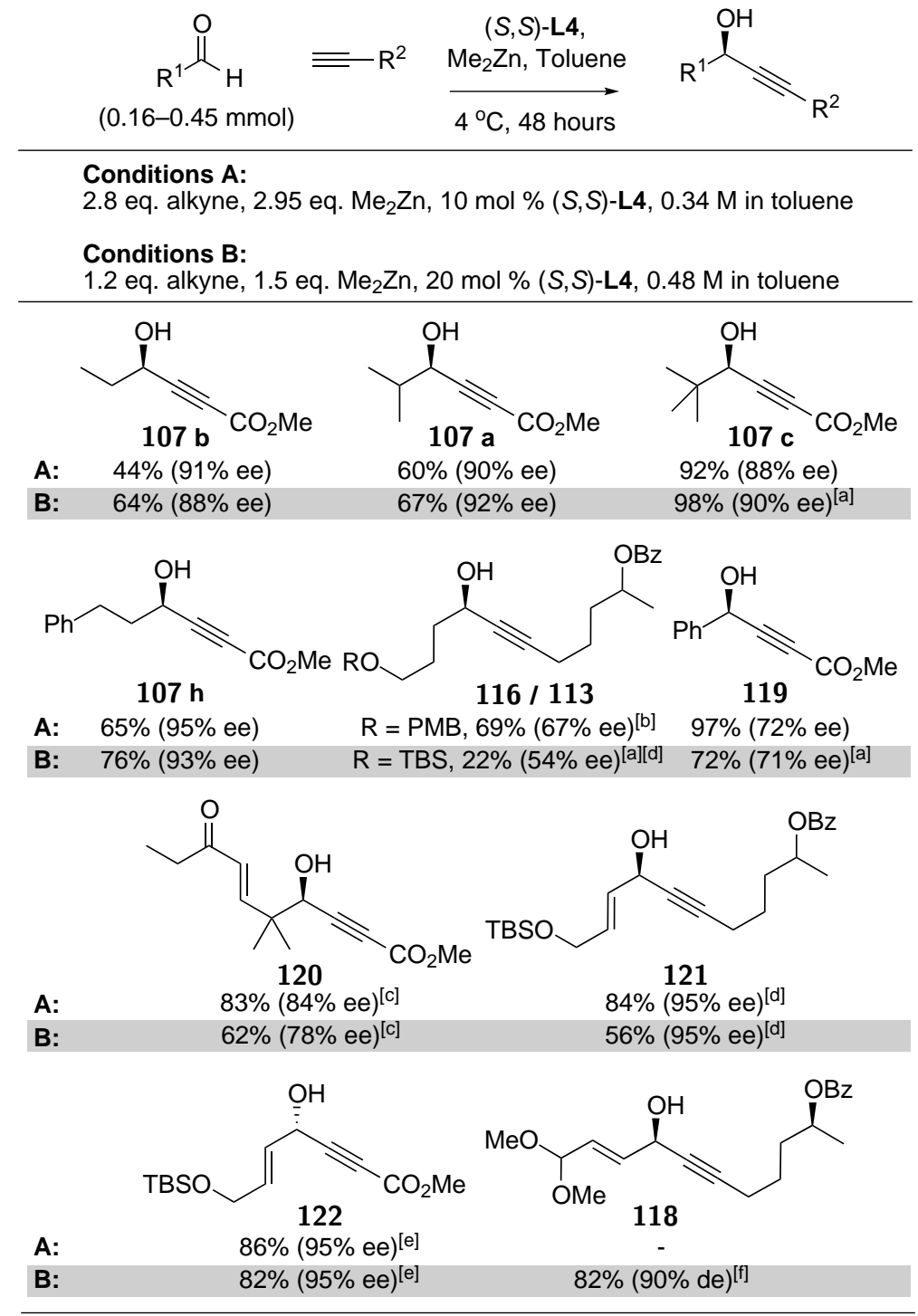

[a] $10 \mathrm{~mol} \%(S, S)$-L4 used in reaction. [b] $20 \mathrm{~mol} \%(S, S)$-L4, $40 \mathrm{~mol} \%$ TPPO used in conjunction with a $24 \mathrm{~h}$ premix. [c] $20 \mathrm{~mol} \%(S, S)$-L4 used in reaction. [d] $20 \mathrm{~mol} \%$ TPPO used in this reaction. [e] Reaction performed with $10 \mathrm{~mol} \%(R, R)$-L4. [f] $10 \mathrm{~mol}$ $\%(S, S)$-L4, 20 mol \% TPPO and 1 equiv of alkyne were used in this reaction.

Scheme 2.9 Scope and Evaluation of Alkynylation with Reduced Stoichiometry. 


\subsubsection{Synthetic Applications}

The development of the Zn-ProPhenol-catalyzed alkyne addition methodology has enabled the total synthesis of a wide range of complex natural products (vide infra). The synthetic utility of this transformation is a direct consequence of the mild reaction conditions and broad substrate scope that has become a focal point of this methodology. Propargylic alcohol moieties are present in a number of biologically and structurally interesting polyacetylenic natural products. The ProPhenol ligand enables the direct and convergent installation of this functionality, as shown in the total synthesis of adociacetylene B, 123 (Scheme 2.10). ${ }^{44}$ The key intermediate, bis-enal 124, was prepared from the symmetric propargylic alcohol 125 using a Ru-catalyzed redox-isomerization. ${ }^{45}$ This atom-economic transformation avoids the use of protecting groups and multiple redox operations often used with conventional olefination chemistry. Bis-alkynylation of $\mathbf{1 2 4}$ with TMS-acetylene provided the desired product $\mathbf{1 2 6}$ in excellent enantioselectivity and 9:1 mixture of $\mathrm{dl}$ and meso diastereomers. Alkynylation with the conditions from the original optimization ( 3 equiv $\mathrm{Me}_{2} \mathrm{Zn}, 3$ equiv alkyne and $10 \mathrm{~mol}$ $\%(S, S)$-L4) resulted in significant amounts of the mono-alkynylation product and starting material, even when extending the reaction time to 3 days. The superior performance of the methyl propiolate-derived alkynylzinc nucleophile was once again illustrated by the increased yield and dr obtained relative to TMS-acetylene. The resulting $\gamma$-hydroxy- $\alpha, \beta$-acetylenic ester
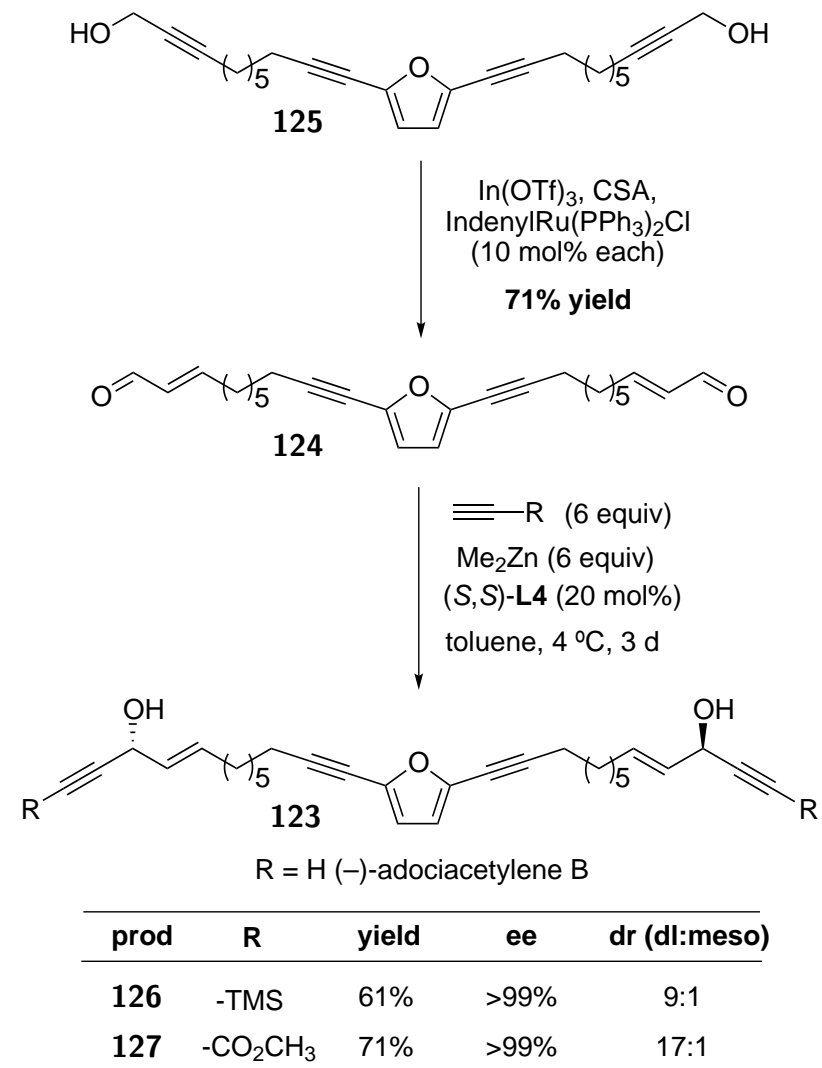

Scheme 2.10 Total Synthesis of Adociacetylene B. 
127 was saponified and decarboxylated to provide (-)-adociacetylene B (123) in a highly efficient manner. $(R, R)-\mathbf{L} \mathbf{4}$ was also used to facilitate this reaction, providing access to both enantiomers of the natural product.

The orthogonal reactivity of alkynes was utilized to great effect in the total synthesis of (+)-spirolaxine methyl ether, 128 (Scheme 2.11). ${ }^{46}$ ProPhenol-catalyzed addition of the aliphatic alkyne 129 to 3,5-dimethoxybenzaldehyde provided propargylic alcohol $\mathbf{1 3 0}$ in high ee and yield. In 5 steps this intermediate was transformed into $\alpha, \beta$-unsaturated aldehyde 131, the substrate required for the second key alkyne addition-hydrogenation sequence. The Zn-ProPhenol-catalyzed addition of a 4,4-diethoxybut-1-yne (132) was used to introduce a 4carbon linchpin that enabled the union of the phthalide fragment with the spiroketal fragment. This alkyne-based strategy ultimately led to the total synthesis of spirolaxine methyl ether in 13 linear steps and provided a first glimpse at the efficiency of alkyne-based linchpin strategies. This work also highlights the utility of asymmetric alkyne addition as a surrogate for a saturated alkyl group addition in the preparation of chiral alcohols.
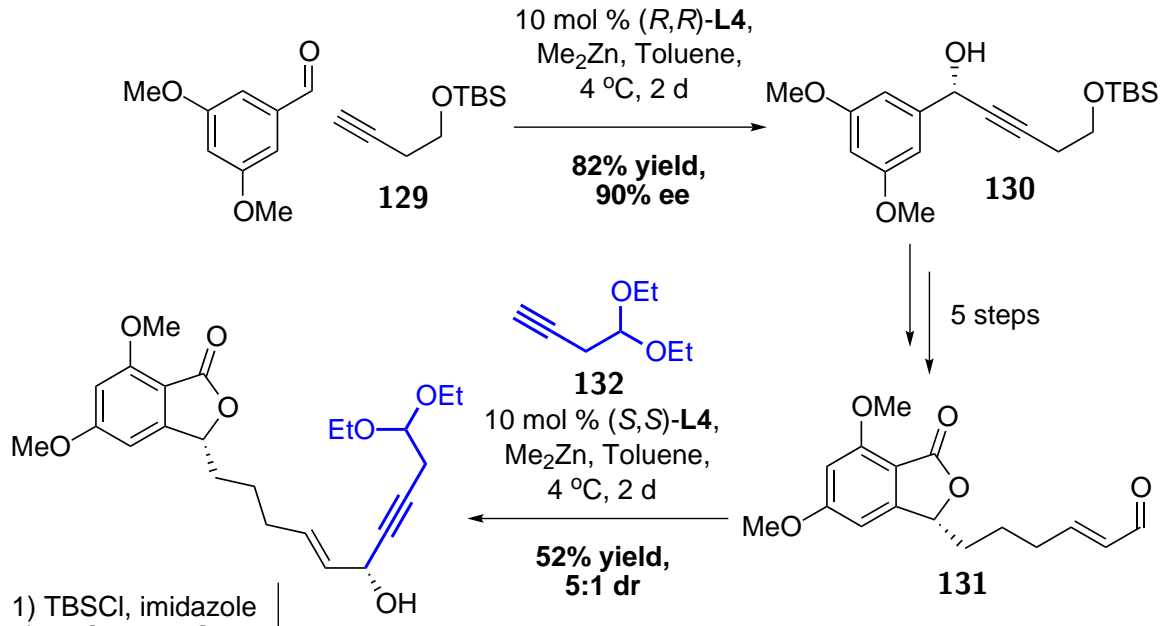

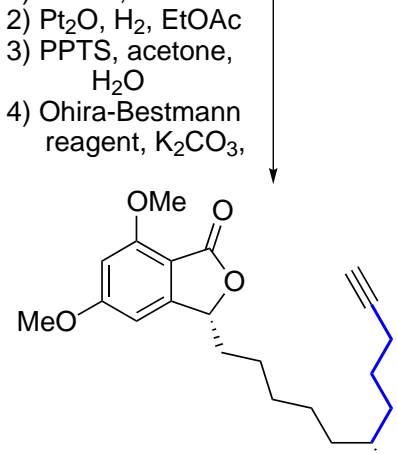

OTBS

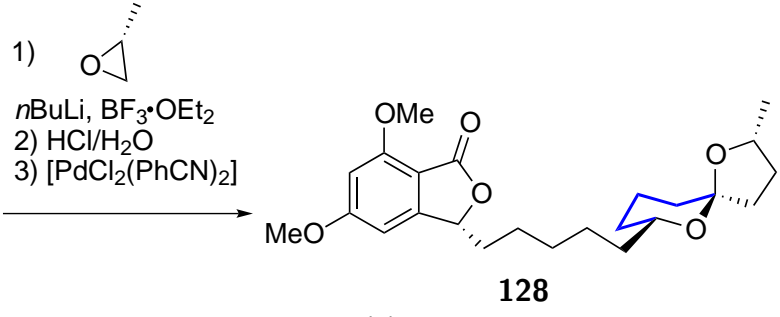

(+)-spirolaxine methyl ether

Scheme 2.11 Alkyne Additions in the Total Synthesis of Spirolaxine Methyl Ether.

The Zn-ProPhenol-catalyzed addition of methyl propiolate to 3-methylbutanal (133), initially shown in Table 2.3, was recently used to construct an enantioenriched propargyl mesylate used in the total synthesis of ushikulide A, 134 (Scheme 2.12). ${ }^{47}$ In this synthesis, the $\gamma$ hydroxy- $\alpha, \beta$-acetylenic ester 135 was subjected to a saponification/decarboxylation/mesylation 
sequence to provide propargyl mesylate 136. This intermediate was then used in a palladium-catalyzed Marshall-Tamaru propargylation. ${ }^{48}$ The terminal alkyne functionality was subsequently converted to an $(E)$-vinyl iodide and used in a $B$-alkyl Suzuki cross coupling to tether the two major fragments of ushikulide A. Ultimately, this alkyne-based linchpin strategy enabled the first total synthesis of (-)-ushikulide A in just 21 linear steps. ${ }^{47}$

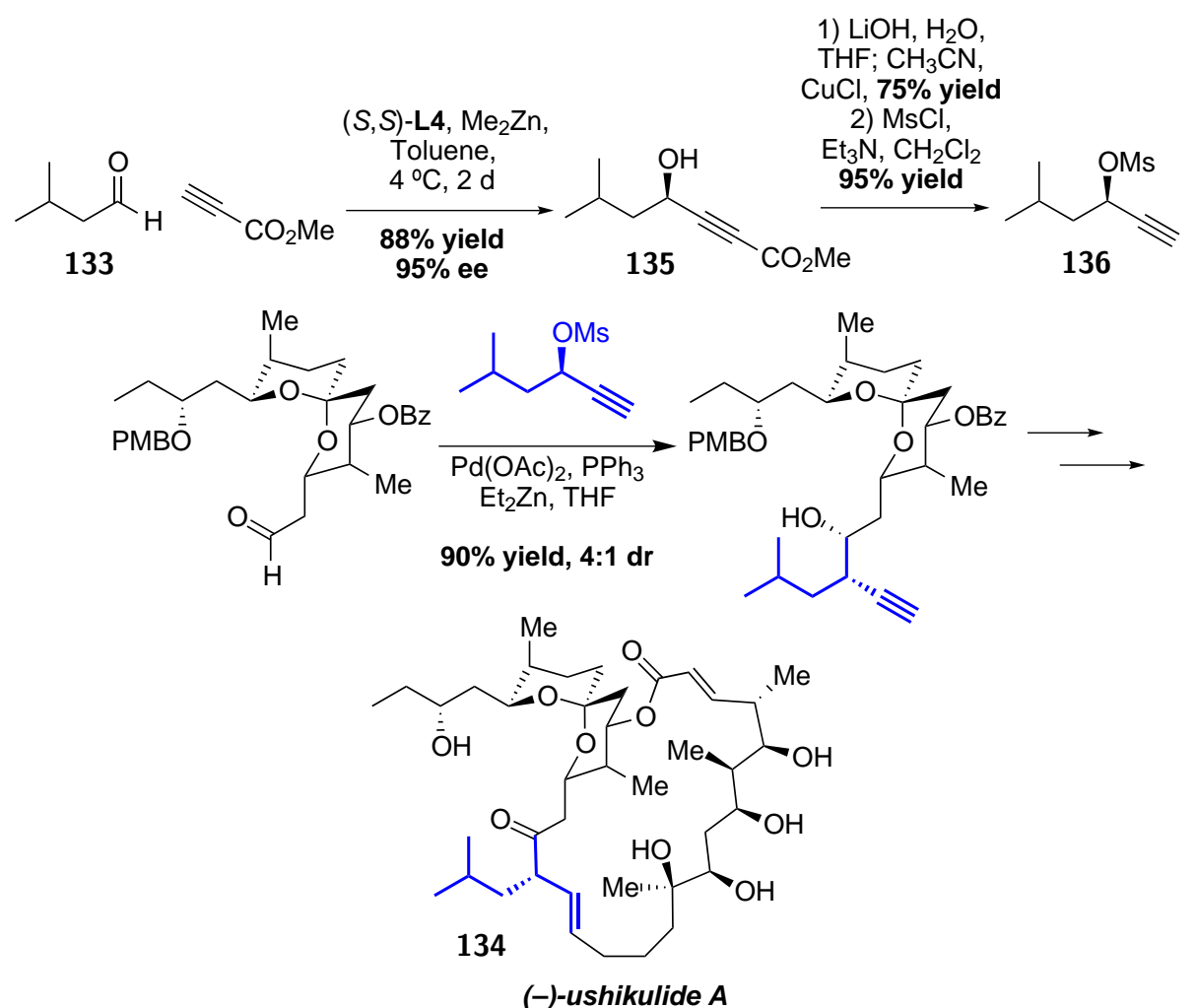

Scheme 2.12 Asymmetric Alkyne Addition in the Total Synthesis of Ushikulide A.

\subsubsection{Summary}

Continued optimization of Zn-ProPhenol-catalyzed alkyne addition has led to the development of practical and general conditions for the asymmetric alkynylation of aldehydes. ${ }^{23}$ This synthetically efficient methodology operates with relatively low catalyst loading and can avoid the use of excess alkyne and dialkylzinc reagents. The chiral propargylic alcohols produced in this reaction are versatile synthetic intermediates and have enabled the synthesis of various complex natural products. ${ }^{49}$ Further research into the asymmetric alkynylation of enolizable aldehydes is currently underway in the Trost group. 


\subsection{Experimental Data for Chapter 2}

\subsubsection{General Experimental Methods}

All reactions were performed under a nitrogen or argon atmosphere in flame- or oven-dried glassware unless reported otherwise. Solvents were purified on an alumina column solvent purification system. All reagents were purchased commercially and used without further purification unless stated otherwise. When possible, commercial aldehydes were distilled under nitrogen immediately prior to use. TLC was preformed with glass-backed plates coated with $0.2 \mathrm{~mm}$ silica (Merck, DC-Platten, Kieselgel, 60 F254). Visualization was performed by fluorescence quenching or staining with aqueous ceric ammonium molybdate, $p$-anisaldehyde or potassium permanganate. Organic solutions were concentrated on a rotary evaporator below $35{ }^{\circ} \mathrm{C}$. Flash column chromatography was preformed using silica gel (CM Science, Kieselgel 60, 230-400 mesh, ASTM). All isolated and characterized compounds were $>95 \%$ pure as judged by ${ }^{1} \mathrm{H}-\mathrm{NMR}$ spectroscopic analysis. ${ }^{1} \mathrm{H}$ and ${ }^{13} \mathrm{C}$ nuclear magnetic resonance (NMR) data were acquired on a Varian Inova $300(300 \mathrm{MHz})$, Varian Mecrury 400 (400 MHz), Varian Direct Drive $400(400 \mathrm{MHz})$ and Varian Unity $500(500 \mathrm{MHz})$ spectrometers as indicated. Data are listed as follows: chemical shift in ppm using chloroform as internal standard (7.26 ppm), multiplicity $(\mathrm{s}=$ singlet, $\mathrm{d}=$ doublet, $\mathrm{t}=$ triplet, $\mathrm{q}=$ quartet, quint $=$ quintet, $\mathrm{m}=$ multiplet or overlap of non-equivalent resonances, $\mathrm{br}=$ broad, app. $=$ apparent, obs. $=$ (partially) obscured), integration, peak assignment. Optical rotation was measured on a Jasco DIP-100 polarimeter in $5 \mathrm{~cm}$ cells with a sodium $589 \mathrm{~nm}$ filter. The reported specific rotations are an average of 10 measurements and are reported as $[\alpha]_{\mathrm{D}}{ }^{25}$, concentration $(\mathrm{g} / 100 \mathrm{~mL})$, and solvent. Infrared (IR) absorption data were acquired a a thin film on sodium chloride plates using a PerkinElmer Paragon 500 FT-IR spectrometer. High-resolution mass spectrometry (HRMS) was performed on a Waters Micromass Q-TOF API-US mass spectrometer. Chiral HPLC analysis was performed on a Thermo Separation Products Spectra Series P-100 and Agilent

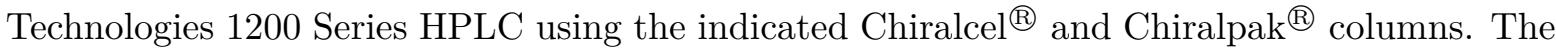
absolute stereochemistry of each alkyne addition product is proposed on the basis of analogy to previous results obtained with the ProPhenol ligand $(\mathbf{L} \mathbf{4})$. The use of $(S, S)$-L4 has been shown to provide the $(R)$-propargylic alcohol and vice versa. Additionally, the HPLC retention times, for a number of known propargylic alcohols, match those reported in the literature and support this assignment of absolute stereochemistry. The absolute configuration of $\mathbf{1 2 0}$ was also confirmed by the X-ray crystal structure of a subsequent synthetic intermediate, $\mathbf{1 3 7 .}$

\section{Representative Alkynylation Procedures}

\section{Alkynylation Procedure A: Super-Stoichiometric Conditions Methyl 4-cyclopropyl-4-hydroxybut-2-ynoate, 107j.}<smiles>COC(=CC(O)C1CC1)OC</smiles>

To a solution of $(S, S)$-ProPhenol ligand $(21 \mathrm{mg}, 0.033 \mathrm{mmol}, 10 \mathrm{~mol} \%)$ and methyl propiolate $(82 \mu \mathrm{L}, 0.92 \mathrm{mmol}, 2.8$ equiv) in anhydrous toluene $(2 \mathrm{~mL})$ was added dimethyl zinc $\left(800 \mu \mathrm{L}, 1.2 \mathrm{M}\right.$ solution in toluene, $0.96 \mathrm{mmol}, 2.95$ equiv) at $0{ }^{\circ} \mathrm{C}(2.73 \mathrm{~mL}$ total toluene, $0.34 \mathrm{M}$ alkyne concentration). The reaction mixture was warmed to room temperature and stirred for 60 minutes before addition of the cyclopropane carboxaldehyde ( $24 \mu \mathrm{L}, 0.325 \mathrm{mmol}$, 
1 equiv) at $0{ }^{\circ} \mathrm{C}$. The reaction was stirred for 48 hours at $4{ }^{\circ} \mathrm{C}$ before quenching with saturated, aqueous $\mathrm{NH}_{4} \mathrm{Cl}$. The organic phase was extracted three times with $\mathrm{Et}_{2} \mathrm{O}$ and the combined organics were concentrated in vacuo. The crude product was purified by flash column chromatography. The title compound was isolated as a clear yellow oil (44 $\mathrm{mg}, 88 \%$ yield). $\mathbf{R}_{\mathbf{f}}=0.22\left(2: 1, \mathrm{PE}_{\mathrm{Et}} \mathrm{O}\right) .{ }^{1} \mathbf{H}-\mathbf{N M R}\left(500 \mathrm{MHz}, \mathrm{CDCl}_{3}\right): \delta 4.26(\mathrm{t}, J=6.6 \mathrm{~Hz}, 1 \mathrm{H}), 3.81$ $(\mathrm{s}, 3 \mathrm{H}), 1.99(\mathrm{~d}, J=6.1 \mathrm{~Hz}, 1 \mathrm{H}), 1.32(\mathrm{~m}, 1 \mathrm{H}), 0.65(\mathrm{~m}, 2 \mathrm{H}), 0.52(\mathrm{~m}, 2 \mathrm{H}) .{ }^{13}$ C-NMR $(126$ $\mathrm{MHz}, \mathrm{CDCl}_{3}$ ): $\delta 154.1,86.7,76.3,65.6,53.1,17.0,3.7,2.2$. IR (film): 3411 (br, OH), 3088, 3011, 2957, 2886, 2237, 1715, 1436, 1256, 1034, $752 \mathrm{~cm}^{-1}$. HRMS - EI $(\mathrm{m} / z)$ : calculated for $\mathrm{C}_{8} \mathrm{H}_{10} \mathrm{O}_{3}$ : 154.0630, found: $154.0626,2.6 \mathrm{ppm}$. Chiral HPLC: Chiralcel ${ }^{\circledR}$ OD column, heptane $/ i \mathrm{PrOH}=90: 10,0.5 \mathrm{~mL} / \mathrm{min}, \lambda=220 \mathrm{~nm}: 15.8 / 17.3 \mathrm{~min}(94 \%$ ee $) \cdot[\alpha]_{\mathrm{D}}{ }^{25}=-51.9^{\circ}$ $\left(c=0.98, \mathrm{CHCl}_{3}\right)$.

\section{Alkynylation Procedure B: TPPO and Reduced Reagent Stoichiometry 1-Phenyl-5-trimethylsilanyl-pent-1-en-4-yn-3-ol, 138.}<smiles>CC#CC(O)C=Cc1ccccc1</smiles>

To a solution of (S,S)-ProPhenol ligand (20.8 mg, $0.0325 \mathrm{mmol}, 10 \mathrm{~mol} \%)$, triphenylphosphine oxide (18 mg, $0.065 \mathrm{mmol}, 20 \mathrm{~mol} \%$ ) and TMS-acetylene (56 $\mu \mathrm{L}, 0.39 \mathrm{mmol}, 1.2$ equiv) in anhydrous toluene $(0.44 \mathrm{~mL})$ was added dimethyl zinc $(406 \mu \mathrm{L}, 1.2 \mathrm{M}$ solution in toluene, $0.488 \mathrm{mmol}, 1.5$ equiv) at $0{ }^{\circ} \mathrm{C}(0.813 \mathrm{~mL}$ total toluene, $0.48 \mathrm{M}$ alkyne concentration $)$. The reaction was warmed to room temperature and stirred for 60 minutes before addition of the $E$ cinammaldehyde ( $43 \mathrm{mg}, 0.325 \mathrm{mmol}, 1$ equiv) at $0{ }^{\circ} \mathrm{C}$. The reaction was stirred for 48 hours at $4{ }^{\circ} \mathrm{C}$ before quenching with saturated, aqueous $\mathrm{NH}_{4} \mathrm{Cl}$. The organic phase was extracted three times with $\mathrm{Et}_{2} \mathrm{O}$ and the combined organics were concentrated in vacuo. The crude product was purified by flash column chromatography. The title compound was isolated as a white solid (67 mg, 83\% yield). ${ }^{1} \mathbf{H}-\mathbf{N M R}\left(400 \mathrm{MHz}, \mathrm{CDCl}_{3}\right): \delta 7.40-7.43(\mathrm{~m}, 2 \mathrm{H}), 7.32-7.36$ $(\mathrm{m}, 2 \mathrm{H}), 7.25-7.29(\mathrm{~m}, 1 \mathrm{H}), 6.77(\mathrm{dd}, J=16,1.2 \mathrm{~Hz}, 1 \mathrm{H}), 6.29(\mathrm{dd}, J=16,6 \mathrm{~Hz}, 1 \mathrm{H}), 5.05$ $(\mathrm{dt}, J=6,1.2 \mathrm{~Hz}, 1 \mathrm{H}), 1.96(\mathrm{t}, J=6 \mathrm{~Hz}, 1 \mathrm{H}), 0.21(\mathrm{~s}, 9 \mathrm{H}) .{ }^{\mathbf{1 3}} \mathbf{C}-\mathbf{N M R}\left(101 \mathrm{MHz}, \mathrm{CDCl}_{3}\right)$ : $\delta$ 136.0, 132.0, 128.6, 128.1, 127.8, 126.8, 104.1, 91.3, 66.3, -0.2. Chiral HPLC: Chiralcel ${ }^{\circledR}$ $\mathrm{AD}$ column, heptane $/ \mathrm{PrOH}=90 / 10,1.0 \mathrm{~mL} / \mathrm{min}, \lambda=254 \mathrm{~nm}: 6.97 / 8.79 \mathrm{~min}$ ( $88 \%$ ee). Characterization data matches literature. ${ }^{50}$

\subsubsection{Experimental Details and Characterization Data}

Alkyne Additions to Aliphatic Aldehydes

Methyl 4-hydroxy-5-methylhex-2-ynoate, 107a.<smiles>CC(=O)C#CC(C)C</smiles>

This reaction was run according to alkynylation procedure A with isobutyraldehyde (30 $\mu \mathrm{L}, 23 \mathrm{mg}, 0.325 \mathrm{mmol})$ and methyl propiolate $(82 \mu \mathrm{L}, 0.92 \mathrm{mmol}, 2.8$ equiv) in toluene $(2 \mathrm{~mL})$ at $4{ }^{\circ} \mathrm{C}$ for $48 \mathrm{~h}$. The title compound was isolated as a clear colorless oil (31 $\mathrm{mg}, 60 \%$ yield). $\mathbf{R}_{\mathbf{f}}=0.29\left(2: 1, \mathrm{PE}: \mathrm{Et}_{2} \mathrm{O}\right) .{ }^{1} \mathbf{H}-\mathbf{N M R}\left(500 \mathrm{MHz}, \mathrm{CDCl}_{3}\right): \delta 4.31(\mathrm{~d}, J=6 \mathrm{~Hz}, 1 \mathrm{H}), 3.81(\mathrm{~s}$, 
3H), 1.95 (sextet, $J=6 \mathrm{~Hz}, 1 \mathrm{H}), 1.06(\mathrm{dd}, J=6.5 \mathrm{~Hz}, 6 \mathrm{H}) .{ }^{\mathbf{1 3}} \mathbf{C}-\mathbf{N M R}\left(126 \mathrm{MHz}, \mathrm{CDCl}_{3}\right)$ : $\delta$ 154.0, 87.5, 67.8, 53.1, 34.4, 18.2, 17.7. IR (film): 3418, 2965, 2236, 1722, 1470, 1436, 1386, 1371, 1253, 1037, 953, $752 \mathrm{~cm}^{-1}$. Chiral HPLC: Chiralcel ${ }^{\circledR}$ AS column, heptane $/ i \operatorname{PrOH}$ $=98: 2,0.8 \mathrm{ml} / \mathrm{min}, 220 \mathrm{~nm}) 24.29 / 27.99 \mathrm{~min}, 90 \%$ ee. $[\alpha]_{\mathrm{D}}{ }^{25}=+6.85^{\circ}\left(c=0.216, \mathrm{CHCl}_{3}\right)$. Characterization data matches literature. ${ }^{51}$

Methyl 4-hydroxyhex-2-ynoate, 107b.<smiles>CCC(O)C#CC(C)=O</smiles>

This reaction was run according to alkynylation procedure A with propionaldehyde $(23 \mu \mathrm{L}$, $19 \mathrm{mg}, 0.325 \mathrm{mmol})$ and methyl propiolate $(82 \mu \mathrm{L}, 0.92 \mathrm{mmol}, 2.8$ equiv) in toluene $(2 \mathrm{~mL})$ at $4{ }^{\circ} \mathrm{C}$ for $48 \mathrm{~h}$. The title compound was isolated as a clear colorless oil (20.3 $\mathrm{mg}, 44 \%$ yield). $\mathbf{R}_{\mathbf{f}}=0.26\left(2: 1, \mathrm{PE}: \mathrm{Et}_{2} \mathrm{O}\right) .{ }^{1} \mathbf{H}-\mathbf{N M R}\left(400 \mathrm{MHz}, \mathrm{CDCl}_{3}\right): \delta 4.44(\mathrm{t}, J=6.4 \mathrm{~Hz}, 1 \mathrm{H}), 3.78(\mathrm{~s}$, $3 \mathrm{H}$ ), 1.80 (quintet, $J=7.2 \mathrm{~Hz}, \mathrm{H}), 1.04(\mathrm{t}, J=7.6 \mathrm{~Hz}, 2 \mathrm{H}) .{ }^{13} \mathrm{C}-\mathbf{N M R}\left(101 \mathrm{MHz}, \mathrm{CDCl}_{3}\right)$ : $\delta$ 154.1, 88.3, 76.5, 63.5, 53.1, 30.3, 9.5. IR (film): 3415, 2971, 2939, 2881, 2237, 1719, 1437, 1252, 1117, 1062, 1035, 1012, 972, 940, 878, $752 \mathrm{~cm}^{-1}$. Chiral HPLC: Chiralcel ${ }^{\circledR}$ OB-H column, heptane $/ \mathrm{PrOH}=95: 5,0.8 \mathrm{ml} / \mathrm{min}, \lambda=220 \mathrm{~nm}) 18.68 / 19.83 \mathrm{~min}, 91 \%$ ee. $[\alpha]_{\mathbf{D}}{ }^{25}$ $=+5.5^{\circ}\left(c=0.77, \mathrm{CHCl}_{3}\right)$. Characterization data matches literature. ${ }^{51}$

Methyl 4-hydroxy-5,5-dimethylhex-2-ynoate, 107c.<smiles>CC(=O)C#CC(O)C(C)(C)C</smiles>

This reaction was run according to alkynylation procedure A with pivaldehyde $(36 \mu \mathrm{L}, 28$ $\mathrm{mg}, 0.325 \mathrm{mmol})$ and methyl propiolate $(82 \mu \mathrm{L}, 0.92 \mathrm{mmol}, 2.8$ equiv) in toluene $(2 \mathrm{~mL})$ at $4{ }^{\circ} \mathrm{C}$ for $48 \mathrm{~h}$. The title compound was isolated as a clear colorless oil $(51.1 \mathrm{mg}, 92 \%$ yield $)$. $\mathbf{R}_{\mathbf{f}}=0.13\left(5: 1, \mathrm{PE}: \mathrm{Et}_{2} \mathrm{O}\right) .{ }^{\mathbf{1}} \mathrm{H}-\mathbf{N M R}\left(300 \mathrm{MHz}, \mathrm{CDCl}_{3}\right): \delta 4.16(\mathrm{~d}, J=6.1 \mathrm{~Hz}, 1 \mathrm{H}), 3.81(\mathrm{~s}$, $3 \mathrm{H}), 2.04(\mathrm{~d}, J=4.4 \mathrm{~Hz}, 1 \mathrm{H}), 1.05(\mathrm{~s}, 9 \mathrm{H}) .{ }^{13} \mathrm{C}-\mathbf{N M R}\left(76 \mathrm{MHz}, \mathrm{CDCl}_{3}\right): \delta 154.0,87.4,76.9$, 71.3, 53.1, 36.4, 25.5. IR (film): 3444, 2960, 2908, 2872, 2236, 1715, 1480, 1483, 1436, 1395, 1367, 1258, 1083, 1043, 1013, 954, 884, 828, 753, $655 \mathrm{~cm}^{-1}$. HRMS - EI $(\mathrm{m} / z)$ : calculated for $\mathrm{C}_{9} \mathrm{H}_{14} \mathrm{O}_{3}$ : 170.0943 , found: 170.0939, $2.2 \mathrm{ppm}$. Chiral HPLC: Chiralcel ${ }^{\circledR}$ AD column, heptane $/ \mathrm{PrOH}=95: 5,1.0 \mathrm{~mL} / \mathrm{min}, \lambda=220 \mathrm{~nm}: 10.35,11.4 \mathrm{~min}, 88 \%$ ee. $[\alpha]_{\mathrm{D}}{ }^{25}=+2.27^{\circ}$ $\left(c=0.89, \mathrm{CHCl}_{3}\right)$. Characterization data matches literature. ${ }^{29 \mathrm{~d}}$

Methyl 5-ethyl-4-hydroxyhept-2-ynoate, $107 \mathrm{~d}$.<smiles>CCC(CC)C(O)C#CC(C)=O</smiles>

This reaction was run according to alkynylation procedure A with 2-ethyl butyraldehyde (40 $\mu \mathrm{L}, 33 \mathrm{mg}, 0.325 \mathrm{mmol})$ and methyl propiolate $(82 \mu \mathrm{L}, 0.92 \mathrm{mmol}, 2.8$ equiv) in toluene $(2 \mathrm{~mL})$ at $4{ }^{\circ} \mathrm{C}$ for $48 \mathrm{~h}$. The title compound was isolated as a clear colorless oil $(36 \mathrm{mg}$, $60 \%$ yield). $\mathbf{R}_{\mathbf{f}}=0.43\left(2: 1, \mathrm{PE}: \mathrm{Et}_{2} \mathrm{O}\right) .{ }^{\mathbf{1}} \mathbf{H}-\mathbf{N M R}\left(300 \mathrm{MHz}, \mathrm{CDCl}_{3}\right): \delta 4.56(\mathrm{t}, J=5.5 \mathrm{~Hz}$, $1 \mathrm{H}), 3.81(\mathrm{~s}, 3 \mathrm{H}), 1.94(\mathrm{~d}, J=6 \mathrm{~Hz}, 1 \mathrm{H}), 1.65-1.39(\mathrm{~m}, 5 \mathrm{H}), 0.97(\mathrm{~m}, 6 \mathrm{H}) .{ }^{13} \mathbf{C}-\mathbf{N M R}(126$ 
$\left.\mathrm{MHz}, \mathrm{CDCl}_{3}\right): \delta 154.0,87.96,77.03,64.7,53.1,47.2,22.0,11.7$. IR (film): 3420, 2965, 2937, 2879, 2235, 1716, 1461, 1436, 1383, 1254, 1133, 1054, 1026, 968, 908, 830, $753 \mathrm{~cm}^{-1}$. Chiral HPLC: Chiralcel ${ }^{\circledR}$ AD column, heptane $/ i \mathrm{PrOH}=97: 3,0.8 \mathrm{ml} / \mathrm{min}, \lambda=220 \mathrm{~nm}: 12.74 / 15.67$ min, $88 \%$ ee. $[\alpha]_{\mathbf{D}}{ }^{25}=-1.83^{\circ}\left(c=0.78, \mathrm{CHCl}_{3}\right)$. Characterization data matches literature. ${ }^{52}$

Methyl 4-hydroxyhex-2-ynoate, 107e.<smiles>CCCCCCCC(O)C#CCOC</smiles>

This reaction was run according to alkynylation procedure A with octanal $(51 \mu \mathrm{L}, 41 \mathrm{mg}$, $0.325 \mathrm{mmol})$ and methyl propiolate $\left(82 \mu \mathrm{L}, 0.92 \mathrm{mmol}, 2.8\right.$ equiv) in toluene $(2 \mathrm{~mL})$ at $4{ }^{\circ} \mathrm{C}$ for $48 \mathrm{~h}$. The title compound was isolated as a slight yellow oil (41 mg, $62 \%$ yield). $\mathbf{R}_{\mathbf{f}}=$ 0.17 (5:1, PE:Et $\left.{ }_{2} \mathrm{O}\right) .{ }^{1} \mathbf{H}-\mathbf{N M R}\left(500 \mathrm{MHz}, \mathrm{CDCl}_{3}\right): \delta 4.5(\mathrm{q}, J=6.5 \mathrm{~Hz} 1 \mathrm{H}), 3.8(\mathrm{~s}, 3 \mathrm{H})$, $2.36($ br s, $1 \mathrm{H}), 1.78(\mathrm{~m}, 2 \mathrm{H}), 1.48(\mathrm{~m}, 2 \mathrm{H}), 1.33(\mathrm{~m}, 11 \mathrm{H}) \cdot{ }^{\mathbf{1 3}} \mathbf{C}-\mathbf{N M R}\left(126 \mathrm{MHz}, \mathrm{CDCl}_{3}\right): \delta$ 154.1, 88.6, 76.4, 62.3, 53.1, 37.1, 32.0, 29.4, 25.2, 22.9, 22.6, 14.3. IR (film): 3418, 2955, 2857, 2237, 1715, 1460, 1435, 1379, 1253, 1125, 1066, 987, 964, 752, $736 \mathrm{~cm}^{-1}$. Chiral HPLC: Chiralcel ${ }^{\circledR}$ OD column, heptane $/ i \operatorname{PrOH}=99: 1,0.8 \mathrm{ml} / \mathrm{min}, \lambda=220 \mathrm{~nm}: 36.5 / 41.08 \mathrm{~min}$, $92 \%$ ee. Characterization data matches literature. ${ }^{12 \mathrm{f}}$

Methyl 6-chloro-4-hydroxy-5,5-dimethylhex-2-ynoate, $107 \mathrm{f}$.<smiles>CC(=O)C#CC(O)C(C)(C)CCl</smiles>

This reaction was run according to alkynylation procedure A with 3-chloro-2,2-dimethylpropanal $(37 \mathrm{mg}, 0.325 \mathrm{mmol})$ and methyl propiolate $(82 \mu \mathrm{L}, 0.92 \mathrm{mmol}, 2.8$ equiv) in toluene $(2 \mathrm{~mL})$ at $4{ }^{\circ} \mathrm{C}$ for $48 \mathrm{~h}$. The title compound was isolated as a slight yellow oil $(48 \mathrm{mg}, 72 \%$ yield). $\mathbf{R}_{\mathbf{f}}=0.2\left(5: 1, \mathrm{PE}: \mathrm{Et}_{2} \mathrm{O}\right) .{ }^{1} \mathbf{H}-\mathrm{NMR}\left(400 \mathrm{MHz}, \mathrm{CDCl}_{3}\right): \delta 4.54(\mathrm{~s}, 1 \mathrm{H}), 3.79(\mathrm{~s}, 3 \mathrm{H})$, $3.65(\mathrm{~d}, J=10.8 \mathrm{~Hz}, 1 \mathrm{H}), 3.42(\mathrm{~d}, J=10.8 \mathrm{~Hz}, 1 \mathrm{H}), 1.11(\mathrm{~s}, 6 \mathrm{H}){ }^{13} \mathbf{C}-\mathbf{N M R}(101 \mathrm{MHz}$, $\mathrm{CDCl}_{3}$ ): $\delta 153.8,86.1,78.1,53.1,52.1,40.8,21.9,20.7$. IR (film): 3444, 2971, 2878, 2237, $1714,1470,1436,1389,1370,1253,1080,1039,1012,953,910,867,830,775,752,726,659$ $\mathrm{cm}^{-1}$. Chiral HPLC: Chiralcel ${ }^{\circledR}$ AD column, heptane $/ i \mathrm{PrOH}=90: 10,1.0 \mathrm{ml} / \mathrm{min}, \lambda=220$ nm: 7.35/8.19 $\min (77 \%$ ee).

Methyl 4-hydroxy-6-methylhept-2-ynoate, 107g.<smiles>COC#CC(O)CC(C)C</smiles>

This reaction was run according to alkynylation procedure A with 3-methylbutanal ( $36 \mu \mathrm{L}$, $28 \mathrm{mg}, 0.325 \mathrm{mmol})$ and methyl propiolate $(82 \mu \mathrm{L}, 0.92 \mathrm{mmol}, 2.8$ equiv) in toluene $(2 \mathrm{~mL})$ at $4{ }^{\circ} \mathrm{C}$ for $48 \mathrm{~h}$. The title compound was isolated as a slight yellow oil (35 mg, $62 \%$ yield). $\mathbf{R}_{\mathbf{f}}=0.29\left(2: 1, \mathrm{PE}: \mathrm{Et}_{2} \mathrm{O}\right) .{ }^{1} \mathbf{H}-\mathrm{NMR}\left(400 \mathrm{MHz}, \mathrm{CDCl}_{3}\right): \delta 4.5(\mathrm{q}, J=6.9 \mathrm{~Hz}, 1 \mathrm{H}), 3.76(\mathrm{~s}$, $3 \mathrm{H}), 2.7(\mathrm{~d}, J=5.2 \mathrm{~Hz}, 1 \mathrm{H}), 1.84(\mathrm{sept}, J=6.7 \mathrm{~Hz}, 1 \mathrm{H}), 1.64(\mathrm{~m}, J=7.4 \mathrm{~Hz}, 2 \mathrm{H}), 0.92$ $(\mathrm{dd}, J=6.6,3.4 \mathrm{~Hz}, 6 \mathrm{H}) .{ }^{13} \mathrm{C}-\mathrm{NMR}\left(101 \mathrm{MHz}, \mathrm{CDCl}_{3}\right): \delta 154.2,89.0,76.3,60.7,53.1,45.9$, 24.7, 22.7, 22.4. IR (film): 3415, 2958, 2936, 2872, 2237, 1721, 1469, 1436, 1388, 1370, 1254, 
1138, 1071, 1041, 997, 935, $752 \mathrm{~cm}^{-1}$. HRMS - EI $(\mathrm{m} / z):\left[\mathrm{MC}_{9} \mathrm{H}_{14} \mathrm{O}_{3}-\left(\mathrm{CH}_{3}\right)_{2} \mathrm{CHCH}_{2}\right]^{+}$ calculated for $\mathrm{C}_{5} \mathrm{H}_{6} \mathrm{O}_{3}$ : 114.0317 , found $114.0320,-2.8 \mathrm{ppm}$. Chiral HPLC: Chiralcel ${ }^{\circledR}$ OD column, heptane $/ i \operatorname{PrOH}=98: 2,0.8 \mathrm{~mL} / \mathrm{min}, \lambda=220 \mathrm{~nm}: 21.57 / 23.67 \mathrm{~min}(93 \%$ ee $) .[\alpha]_{\mathbf{D}}{ }^{25}$ $=+13.8^{\circ}\left(c=0.93, \mathrm{CHCl}_{3}\right)$. Characterization data matches literature. ${ }^{12 \mathrm{f}}$

Methyl 4-hydroxy-6-phenylhex-2-ynoate, $107 \mathrm{~h}$.<smiles>COC(C)=CC(O)CCc1ccccc1</smiles>

This reaction was run according to alkynylation procedure A with hydrocinnamaldehyde (43 $\mu \mathrm{L}, 44 \mathrm{mg}, 0.325 \mathrm{mmol}$ ) and methyl propiolate $(82 \mu \mathrm{L}, 0.92 \mathrm{mmol}, 2.8$ equiv) in toluene (2 $\mathrm{mL}$ ) at $4{ }^{\circ} \mathrm{C}$ for $48 \mathrm{~h}$. The title compound was isolated as a slight yellow oil (46 mg, $65 \%$ yield). $\mathbf{R}_{\mathbf{f}}=0.17\left(2: 1, \mathrm{PE}: \mathrm{Et}_{2} \mathrm{O}\right) .{ }^{1} \mathbf{H}-\mathbf{N M R}\left(500 \mathrm{MHz}, \mathrm{CDCl}_{3}\right): \delta 7.3(\mathrm{~m}, 2 \mathrm{H}), 7.2(\mathrm{~m}, 3 \mathrm{H}), 4.47(\mathrm{~s}$, 1H), $3.79(\mathrm{~s}, 3 \mathrm{H}), 2.95(\mathrm{~s}, 1 \mathrm{H}), 2.81(\mathrm{t}, 2 \mathrm{H}), 2.1(\mathrm{~m}, 2 \mathrm{H}) .{ }^{13} \mathrm{C}-\mathbf{N M R}\left(126 \mathrm{MHz}, \mathrm{CDCl}_{3}\right): \delta$ 154.2, 140.8, 128.8, 128.75, 126.4, 88.5, 76.6, 61.4, 53.1, 38.4, 31.3. IR (film): 3415, 3028, 2954, 2929, 2863, 2236, 1716, 1603, 1496, 1455, 1436, 1257, 1059, 1030, 947, 913, 751, $701 \mathrm{~cm}^{-1}$. HRMS - EI $(\mathrm{m} / z)$ : calculated for $\mathrm{C}_{13} \mathrm{H}_{14} \mathrm{O}_{3}: 218.0943$, found: 218.0934, $4.0 \mathrm{ppm}$. Chiral HPLC: Chiralcel ${ }^{\circledR}$ OD column, heptane $/ i \operatorname{PrOH}=95: 5,1.0 \mathrm{ml} / \mathrm{min}, \lambda=220 \mathrm{~nm}: 26.6 / 28.0$ $\min , 95 \%$ ee. $[\alpha]_{\mathbf{D}}{ }^{25}=-40.4^{\circ}\left(c=1.06, \mathrm{CHCl}_{3}\right)$. Characterization data matches literature. ${ }^{29 \mathrm{~d}}$

Methyl 4-hydroxy-5,5-dimethyloct-7-en-2-ynoate, $107 i$.<smiles>C=CCC(C)(C)C(O)C#CC(C)=O</smiles>

This reaction was run according to alkynylation procedure A with 2,2-dimethylpent-4-enal $(37 \mathrm{mg}, 0.325 \mathrm{mmol})$ and methyl propiolate $(82 \mu \mathrm{L}, 0.92 \mathrm{mmol}, 2.8$ equiv) in toluene $(2 \mathrm{~mL})$ at $4{ }^{\circ} \mathrm{C}$ for $48 \mathrm{~h}$. The title compound was isolated as a clear colorless oil (30 mg, $48 \%$ yield). $\mathbf{R}_{\mathbf{f}}=0.14\left(5: 1, \mathrm{PE}: \mathrm{Et}_{2} \mathrm{O}\right) .{ }^{1} \mathbf{H}-\mathbf{N M R}\left(300 \mathrm{MHz}, \mathrm{CDCl}_{3}\right): \delta 5.92-5.78(\mathrm{~m}, 1 \mathrm{H}), 5.16(\mathrm{~d}, J=$ $5.4 \mathrm{~Hz}, 1 \mathrm{H}), 5.10(\mathrm{~d}, 1 \mathrm{H}), 4.22(\mathrm{~d}, J=6.3 \mathrm{~Hz}, 1 \mathrm{H}), 3.81(\mathrm{~s}, 3 \mathrm{H}), 2.26-2.08(\mathrm{~m}, 2 \mathrm{H}), 1.03(\mathrm{~s}, 6 \mathrm{H})$. ${ }^{13}$ C-NMR $\left(101 \mathrm{MHz}, \mathrm{CDCl}_{3}\right): \delta 154.1,134.5,118.6,87.3,77.9,70.0,53.1,42.9,39.2,23.0$. IR (film): 3420, 3077, 2966, 2933, 2876, 2235, 1716, 1640, 1471, 1436, 1387, 1368, 1252, 1191, 1073, 1038, 1011, 918, $753 \mathrm{~cm}^{-1}$. Chiral HPLC: Chiralcel ${ }^{\circledR}$ OB-H column, hept $/$ PrOH $=97: 3,1.0 \mathrm{~mL} / \mathrm{min}, \lambda=220 \mathrm{~nm}, 9.41 / 10.33 \mathrm{~min}(70 \%$ ee$)$. Characterization data matches literature. ${ }^{53}$

Methyl 4-hydroxy-8-(triisopropylsilyloxy)oct-2-ynoate, 107k.<smiles>CC(=O)C#CC(O)CCCCOC(C)C</smiles>

This reaction was run according to alkynylation procedure A with 5-(triisopropylsilanyloxy)-pentanal ( $84 \mathrm{mg}, 0.325 \mathrm{mmol})$ and methyl propiolate $(82 \mu \mathrm{L}, 0.92 \mathrm{mmol}, 2.8$ equiv) in toluene $(2 \mathrm{~mL})$ at $4{ }^{\circ} \mathrm{C}$ for $48 \mathrm{~h}$. The title compound was isolated as a slight yellow oil (60 mg, $52 \%$ yield). $\mathbf{R}_{\mathbf{f}}=0.15$ (5:1, PE:Et $\left.{ }_{2} \mathrm{O}\right) .{ }^{1} \mathbf{H}-\mathbf{N M R}\left(400 \mathrm{MHz}, \mathrm{CDCl}_{3}\right): \delta 4.50$ (t, $J=$ $6.8 \mathrm{~Hz}, 1 \mathrm{H}), 3.77(\mathrm{~s}, 3 \mathrm{H}), 3.72(\mathrm{t}, J=6 \mathrm{~Hz}, 2 \mathrm{H}), 1.80(\mathrm{~m}, 2 \mathrm{H}), 1.57(\mathrm{~m}, 4 \mathrm{H}), 1.05(\mathrm{~m}, 21 \mathrm{H})$ 
${ }^{13}$ C-NMR $\left(101 \mathrm{MHz}, \mathrm{CDCl}_{3}\right): \delta 154.1,88.5,76.4,63.3,62.3,53.0,36.8,32.5,21.6,18.2$, 12.2. IR (film): 3418, 2944, 2867, 2237, 1722, 1463, 1436, 1384, 1251, 1107, 1070, 1040, 1014, 996, 919, 883, 797, 752, 721, 681, 658, $639 \mathrm{~cm}^{-1}$. Chiral HPLC: Chiralcel ${ }^{\circledR}$ OD column, heptane $/ i \operatorname{PrOH}=98: 2,0.8 \mathrm{ml} / \mathrm{min}, \lambda=220 \mathrm{~nm}: 17.64 / 20.75 \mathrm{~min}, 95 \%$ ee. $[\alpha]_{\mathbf{D}}{ }^{25}=-0.92^{\circ}$ $\left(c=1.13, \mathrm{CHCl}_{3}\right)$.

\section{Dimethyl 4-hydroxy-5,5-dimethylhex-2-ynedioate, 1071.}<smiles>COC(=O)C#CC(O)C(C)(C)C(=O)OC</smiles>

This reaction was run according to alkynylation procedure A with methyl 2,2-dimethyl-3oxopropanoate $(42 \mathrm{mg}, 0.325 \mathrm{mmol})$ and methyl propiolate $(82 \mu \mathrm{L}, 0.92 \mathrm{mmol}, 2.8$ equiv) in toluene $(2 \mathrm{~mL})$ at $4{ }^{\circ} \mathrm{C}$ for $48 \mathrm{~h}$. The title compound was isolated as a clear colorless oil (52 mg, $75 \%$ yield). $\mathbf{R}_{\mathbf{f}}=0.26\left(2: 1, \mathrm{PE}: \mathrm{Et}_{2} \mathrm{O}\right){ }^{\mathbf{1}} \mathbf{H}-\mathbf{N M R}\left(400 \mathrm{MHz}, \mathrm{CDCl}_{3}\right): \delta 4.55(\mathrm{~s}, 1 \mathrm{H}), 3.76$ $(\mathrm{s}, 3 \mathrm{H}), 3.73(\mathrm{~s}, 3 \mathrm{H}), 1.314(\mathrm{~s}, 3 \mathrm{H}), 1.29(\mathrm{~s}, 3 \mathrm{H}) .{ }^{\mathbf{1 3}} \mathbf{C}-\mathbf{N M R}\left(101 \mathrm{MHz}, \mathrm{CDCl}_{3}\right): \delta 176.7,153.8$, 94.7, 85.5, 68.4, 53.1, 52.7, 47.7, 22.9, 20.2. IR (film): 3474, 2955, 2238, 1716, 1436, 1390, 1250, 1136, 1082, 1041, 1014, 953, 916, $752 \mathrm{~cm}^{-1}$. Chiral HPLC: Chiralcel ${ }^{\circledR}$ AD column, heptane $/ i \mathrm{PrOH}=90: 10,0.8 \mathrm{~mL} / \mathrm{min}, \lambda=220 \mathrm{~nm}, 11.2 / 12.9 \mathrm{~min}(21 \%$ ee).

\section{1-Cyclopropyl-non-2-yn-1-ol, 107m.}

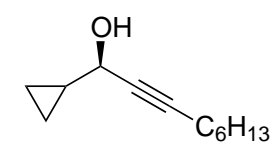

This reaction was run according to alkynylation procedure A with cyclopropane carboxaldehyde $(24 \mu \mathrm{L}, 0.325 \mathrm{mmol})$ and 1-octyne $(0.92 \mathrm{mmol}, 2.8$ equiv $)$ in toluene $(2 \mathrm{~mL})$ at $4{ }^{\circ} \mathrm{C}$ for $48 \mathrm{~h}$. The title compound was isolated as a clear colorless oil ( $54.8 \mathrm{mg}, 94 \%$ yield). $\mathbf{R}_{\mathbf{f}}=$ 0.185 (11:1, PE:Et $\left.{ }_{2} \mathrm{O}\right) .{ }^{1} \mathbf{H}-\mathbf{N M R}\left(500 \mathrm{MHz} \mathrm{CDCl}_{3}\right): \delta 4.25(\mathrm{tt}, J=6.5,1.5 \mathrm{~Hz}, 1 \mathrm{H}), 2.21$ $(\mathrm{td}, J=7,2 \mathrm{~Hz}, 2 \mathrm{H}), 1.52$ (quintet, $J=7 \mathrm{~Hz}, 2 \mathrm{H}), 1.42-1.23(\mathrm{~m}, 6 \mathrm{H}), 0.915(\mathrm{t}, J=7 \mathrm{~Hz}$, $3 \mathrm{H}), 0.59-0.41(\mathrm{~m}, 5 \mathrm{H}) .{ }^{13} \mathbf{C}-\mathbf{N M R}\left(126 \mathrm{MHz}, \mathrm{CDCl}_{3}\right): \delta 86.2,78.9,66.2,31.6,28.9,28.8$, 22.8, 18.9, 17.5, 14.3, 3.4, 1.6. IR (film): 3356 (br, OH), 3084, 3007, 2931, 2859, 2254, 1465, $1432,1328,1310,1272,1151,1131,1026,1009,917.8,832,723 \mathrm{~cm}^{-1}$. Enantiomeric excess determined from the corresponding p-nitrobenzoyl ester. To a solution of 1-cyclopropyl-non2-yn-1-ol from the previous reaction with DMAP $(1.34 \mathrm{mg}, 0.011 \mathrm{mmol}, 0.05$ equiv) in $1 \mathrm{~mL}$ of pyridine was added $p$-nitrobenzoyl chloride $(0.061 \mathrm{~g}, 0.33 \mathrm{mmol}, 3$ equiv). The reaction was stirred at room temperature for 3 hours before being quenched with water, washed with saturated aqueous $\mathrm{CuSO}_{4}$ and brine. The combined organics were dried over $\mathrm{MgSO}_{4}$ and the solvent was removed in vacuo. The product was purified using flash column chromatography to give a clear, colorless oil (7.3 mg, $20 \%$ yield). $\mathbf{R}_{\mathbf{f}}=0.43$ (11:1, PE:Et $\left.{ }_{2} \mathrm{O}\right) .{ }^{1} \mathbf{H}-\mathbf{N M R}(300$ $\left.\mathrm{MHz}, \mathrm{CDCl}_{3}\right): \delta 8.30(\mathrm{~m}, 4 \mathrm{H}), 5.58(\mathrm{dt}, J=6.6,1.8 \mathrm{~Hz}, 1 \mathrm{H}), 2.23(\mathrm{td}, J=6.9,2.1 \mathrm{~Hz}, 3 \mathrm{H})$, 1.53 (quintet, $J=6.9 \mathrm{~Hz}, 2 \mathrm{H}), 1.43-1.3(\mathrm{~m}, 6 \mathrm{H}), 0.70-0.55(\mathrm{~m}, 5 \mathrm{H})$. Chiral HPLC: Chiralcel ${ }^{\circledR}$ AD column, heptane $/ i \mathrm{PrOH}=99: 1,1.0 \mathrm{~mL} / \mathrm{min}, \lambda=254 \mathrm{~nm}: 8.56 / 7.43 \mathrm{~min}(69 \%$ ee).

\section{Methyl 4-hydroxy-5,5-dimethyloct-7-en-2-ynoate, 107n.}

This reaction was run according to alkynylation procedure A with methyl 5,5-diethoxy-4hydroxyoct-7-en-2-ynoate $(56.0 \mathrm{mg}, 0.325 \mathrm{mmol}, 1$ equiv) and methyl propiolate ( $82 \mu \mathrm{L}, 0.92$ mmol, 2.8 equiv) in toluene $(2 \mathrm{~mL})$ at $4{ }^{\circ} \mathrm{C}$ for $48 \mathrm{~h}$. The title compound was isolated as a 


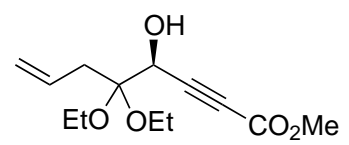

clear yellow oil $(67.7 \mathrm{mg}, 87 \%$ yield $) . \mathbf{R}_{\mathbf{f}}=0.16\left(5: 1, \mathrm{PE}: \mathrm{Et}_{2} \mathrm{O}\right) .{ }^{\mathbf{1}} \mathbf{H}-\mathbf{N M R}\left(400 \mathrm{MHz}, \mathrm{CDCl}_{3}\right)$ : $\delta 5.92-5.81(\mathrm{~m}, 1 \mathrm{H}), 5.25-5.19(\mathrm{~m}, 1 \mathrm{H}), 5.17-5.13(\mathrm{~m}, 1 \mathrm{H}), 4.56(\mathrm{~d}, J=6.4 \mathrm{~Hz}, 1 \mathrm{H}), 3.78(\mathrm{~s}$, $3 \mathrm{H}), 3.7-3.6(\mathrm{~m}, 4 \mathrm{H}), 2.61(\mathrm{~d}, 1 \mathrm{H}), 2.7-2.55(\mathrm{~m}, 2 \mathrm{H}), 1.2(\mathrm{dt}, J=2.4,6.8 \mathrm{~Hz}, 6 \mathrm{H}) .{ }^{13} \mathrm{C}-\mathbf{N M R}$ $\left(101 \mathrm{MHz}, \mathrm{CDCl}_{3}\right): \delta 153.9,132.9,119.4,100.9,85.5,77.8,66.1,57.6,53.0,38.9,15.6$. IR (film): 3484, 2979, 2932, 2898, 2239, 1719, 1437, 1252, 1086, $1053 \mathrm{~cm}^{-1}$. Chiral HPLC: Chiralcel ${ }^{\circledR}$ AD (heptane $/ \mathrm{PrOH}=90: 10,1.0 \mathrm{~mL} / \mathrm{min}, \lambda=220 \mathrm{~nm}$ ) 5.63/6.41 min, $0 \%$ ee.

Methyl 4-cyclohexyl-4-hydroxybut-2-ynoate, 107 o.<smiles>COC(=O)C#CC(O)C1CCCCC1</smiles>

This reaction was run according to alkynylation procedure A with cyclohexane carboxaldehyde $(39 \mu \mathrm{L}, 37 \mathrm{mg}, 0.325 \mathrm{mmol})$ and methyl propiolate $(82 \mu \mathrm{L}, 0.92 \mathrm{mmol}, 2.8$ equiv) in toluene $(2 \mathrm{~mL})$ at $4{ }^{\circ} \mathrm{C}$ for $48 \mathrm{~h}$. The title compound was isolated as a slight yellow oil $(45 \mathrm{mg}$, $71 \%$ yield $) . \mathbf{R}_{\mathbf{f}}=0.13\left(5: 1, \mathrm{PE}: \mathrm{Et}_{2} \mathrm{O}\right) .{ }^{\mathbf{1}} \mathbf{H}-\mathbf{N M R}\left(500 \mathrm{MHz}, \mathrm{CDCl}_{3}\right): \delta 4.29(\mathrm{~d}, 1 \mathrm{H}), 3.795(\mathrm{~s}$, $3 \mathrm{H}), 2.448(\mathrm{br} \mathrm{s}, 1 \mathrm{H}), 1.87(\mathrm{~m}, 2 \mathrm{H}), 1.805(\mathrm{~m}, 2 \mathrm{H}), 1.66(\mathrm{~m}, 2 \mathrm{H}), 1.25(\mathrm{~m}, 2 \mathrm{H}), 1.13(\mathrm{~m}, 2 \mathrm{H})$. ${ }^{13}$ C-NMR $\left(126 \mathrm{MHz}, \mathrm{CDCl}_{3}\right): \delta 154.2,88.0,67.1,53.1,43.8,28.6,28.3,26.4,25.9$. IR (film): 3417, 2928, 2855, 2236, 1722, 1451, 1435, 1258, 1085, 1068, 1050, 1017, 965, 914, 894,845, 821, 752, $734 \mathrm{~cm}^{-1}$. Chiral HPLC: Chiralcel ${ }^{\circledR}$ AD column, heptane $/ i \mathrm{PrOH}=99: 1,1.0 \mathrm{ml} / \mathrm{min}$, $\lambda=220 \mathrm{~nm}: 48.7 / 52.01 \mathrm{~min}(90 \% \mathrm{ee}) \cdot[\alpha]_{\mathbf{D}}{ }^{25}=+3.6^{\circ}\left(c=0.89, \mathrm{CHCl}_{3}\right)$. Characterization data matches literature. ${ }^{12 \mathrm{f}}$

See Chapter 3 for experimental details and characterization data on:

•( $R, E)$-methyl 4-hydroxy-5,5-dimethyl-8-oxodec-6-en-2-ynoate, $\mathbf{1 2 0 .}$

See Chapter 4 for experimental details and characterization data on:

- $(2 S / R, 8 R)$-11-((tert-Butyldimethylsilyl)oxy)-8-hydroxyundec-6-yn-2-yl benzoate, 113.

• $(2 S / R, 8 R)$-8-Hydroxy-11-((4-methoxybenzyl)oxy)undec-6-yn-2-yl benzoate, 116.

\section{Alkyne Additions with Unsaturated Aldehydes}

1-Phenyl-undec-1-en-4-yn-3-ol, 139.<smiles>CC#CC(O)C=Cc1ccccc1</smiles>

This reaction was run according to alkynylation procedure A with $(E)$-cinammaldehyde (43 mg, $0.325 \mathrm{mmol}$ ) and 1-octyne (101 mg, $0.92 \mathrm{mmol}, 2.8$ equiv) in toluene $(2 \mathrm{~mL})$ at $4{ }^{\circ} \mathrm{C}$ for $48 \mathrm{~h}$. The title compound was isolated as a clear yellow oil (79 $\mathrm{mg}, 100 \%$ yield). ${ }^{\mathbf{1}} \mathbf{H}-\mathbf{N M R}$ $\left(500 \mathrm{MHz}, \mathrm{CDCl}_{3}\right): \delta 7.38-7.41(\mathrm{~m}, 2 \mathrm{H}), 7.29-7.34(\mathrm{~m}, 2 \mathrm{H}), 7.23-7.28(\mathrm{~m}, 1 \mathrm{H}), 6.75(\mathrm{~d}, J=$ $16 \mathrm{~Hz}, 1 \mathrm{H}), 6.30(\mathrm{dd}, J=16,6 \mathrm{~Hz}, 1 \mathrm{H}), 5.04(\mathrm{~s}, 1 \mathrm{H}), 2.26(\mathrm{td}, J=7,2 \mathrm{~Hz}, 2 \mathrm{H}), 2.04(\mathrm{~s}, 1 \mathrm{H})$, 1.50-1.57 (m, 2H), 1.37-1.44 (m, 2H), 1.24-1.35 (m, 4H), $0.89(\mathrm{t}, J=7 \mathrm{~Hz}, 3 \mathrm{H}) .{ }^{13} \mathrm{C}-\mathbf{N M R}$ 
$\left(126 \mathrm{MHz}, \mathrm{CDCl}_{3}\right): \delta 136.2,131.4,128.8,128.5,127.9,126.7,87.5,79.1,63.2,31.3,28.55$, 28.52, 22.5, 18.8, 14.0. Chiral HPLC: Chiralcel ${ }^{\circledR}$ AD column, heptane $/ i \operatorname{PrOH}=90 / 10,1$ $\mathrm{mL} / \mathrm{min}, \lambda=254 \mathrm{~nm}$ : 9.4(major)/8.26(minor) $\min$ (77\% ee). Characterization data matches literature. ${ }^{22}$

(E)-Methyl 7-((tert-butyldimethylsilyl)oxy)-4-hydroxyhept-5-en-2-ynoate, 122.<smiles>COCC=CC(O)C#CCOC(C)=O</smiles>

This reaction was run according to alkynylation procedure A with (E)-4-((tert-butyldimethylsilyl)oxy)but-2-enal ( $33 \mathrm{mg}, 0.163 \mathrm{mmol}, 1.0$ equiv), methyl propiolate ( $41 \mu \mathrm{L}, 0.46$ mmol, 2.8 equiv), $(R, R)$-ProPhenol (11 mg, $0.0163 \mathrm{mmol}, 10 \mathrm{~mol} \%)$ and $\mathrm{Me}_{2} \mathrm{Zn}(399 \mu \mathrm{L}, 1.2$ $\mathrm{M}$ solution in toluene, $0.48 \mathrm{mmol}, 2.95$ equiv) in toluene $(1 \mathrm{~mL})$ at $4{ }^{\circ} \mathrm{C}$ for $48 \mathrm{~h}$. The title compound was isolated as a clear colorless oil ( $40 \mathrm{mg}, 86 \%$ yield). $\mathbf{R}_{\mathbf{f}}=0.39$ (15\% EtOAc/ pet. ether). ${ }^{1} \mathbf{H}-\mathbf{N M R}\left(500 \mathrm{MHz}, \mathrm{CDCl}_{3}\right): \delta 6.06(\mathrm{dtd}, J=15.3,4.1,1.3 \mathrm{~Hz}, 1 \mathrm{H}), 5.88$ $(\mathrm{ddt}, J=15.3,5.7,1.9 \mathrm{~Hz}, 1 \mathrm{H}), 5.07-5.04(\mathrm{~m}, 1 \mathrm{H}), 4.24(\mathrm{dt}, J=3.9,1.8 \mathrm{~Hz}, 2 \mathrm{H}), 3.82(\mathrm{~s}$, $3 \mathrm{H}), 2.12(\mathrm{~d}, J=6.6 \mathrm{~Hz}, 1 \mathrm{H}), 0.94(\mathrm{~s}, 9 \mathrm{H}), 0.10(\mathrm{~s}, 6 \mathrm{H}) .{ }^{13} \mathrm{C}-\mathrm{NMR}\left(126 \mathrm{MHz}, \mathrm{CDCl}_{3}\right): \delta$ 153.95, 134.02, 126.21, 86.18, 62.62, 62.42, 53.15, 26.17, 18.65, 0.26, -5.06. IR (film): 3406, 2955, 2857, 2238, 1721, 1471, 1436, 1255, 1128, 1004, $837 \mathrm{~cm}^{-1}$. Chiral HPLC: Chiralpak ${ }^{\circledR}$ IB column, heptane: $\mathrm{PrOH}=95: 5,0.4 \mathrm{~mL} / \mathrm{min}, \lambda=220 \mathrm{~nm}, \mathrm{t}_{\mathrm{R}}=18.1$ (minor), 18.9 (major) minutes $(95 \%$ ee $) .[\alpha]_{\mathbf{D}}{ }^{25}=+11.81^{\circ}\left(c=1.13, \mathrm{CHCl}_{3}\right)$.

Methyl 4-hydroxy-4-phenylbut-2-ynoate, 119.<smiles>COC(C)=CC(O)c1ccccc1</smiles>

This reaction was run according to alkynylation procedure A with benzaldehyde (34 $\mathrm{mg}$, $0.325 \mathrm{mmol})$ and methyl propiolate $\left(82 \mu \mathrm{L}, 0.92 \mathrm{mmol}, 2.8\right.$ equiv) in toluene $(2 \mathrm{~mL})$ at $4{ }^{\circ} \mathrm{C}$ for $48 \mathrm{~h}$. The title compound was isolated as as a clear colorless oil $(0.060 \mathrm{~g}, 97 \%$ yield $) . \mathbf{R}_{\mathbf{f}}=0.23$ $\left(15 \%\right.$ EtOAc/PE). ${ }^{1} \mathbf{H}-\mathbf{N M R}\left(500 \mathrm{MHz}, \mathrm{CDCl}_{3}\right): \delta 7.55-7.53(\mathrm{~m}, 2 \mathrm{H}), 7.45-7.38(\mathrm{~m}, 3 \mathrm{H}), 5.60$ $(\mathrm{d}, J=5.8 \mathrm{~Hz}, 1 \mathrm{H}), 3.82(\mathrm{~s}, 3 \mathrm{H}), 2.62(\mathrm{~d}, J=6.2 \mathrm{~Hz}, 1 \mathrm{H}) \cdot{ }^{13} \mathbf{C}-\mathbf{N M R}\left(126 \mathrm{MHz}, \mathrm{CDCl}_{3}\right): \delta$ 153.98, 138.70, 129.25, 129.14, 126.92, 86.70, 77.90, 64.57, 53.20. Chiral HPLC: Chiralcel ${ }^{\circledR}$ OD column, 95:5 heptane/ isopropanol, $0.8 \mathrm{~mL} / \mathrm{min}, 220 \mathrm{~nm}: 72 \%$ ee. Characterization data matches literature. ${ }^{29 \mathrm{~d}}$

See Chapter 4 for experimental details and characterization data on:

- $(2 S / R, 8 R, E)-11$-((tert-Butyldimethylsilyl)oxy)-8-hydroxyundec-9-en-6-yn-2-yl benzoate, 121.

• $(2 S, 8 R, E)-8$-Hydroxy-11,11-dimethoxyundec-9-en-6-yn-2-yl benzoate, 118. 


\section{Miscellaneous Experiments}

\section{Procedure for the Stepwise ${ }^{1} \mathrm{H}-\mathrm{NMR}$ Analysis of the Alkynylation Premix}

To an oven dried NMR tube was added toluene- $d 8(0.486 \mathrm{~mL})$ and dimethylzinc $(0.124$ $\mathrm{mL}$ of a $1.2 \mathrm{M}$ solution in PhMe, $0.149 \mathrm{mmol}, 15$ equiv). A ${ }^{1} \mathrm{H}-\mathrm{NMR}$ spectrum (500 MHz) was recorded, solvent suppression was not necessary. To the NMR tube was added methyl propiolate $\left(0.011 \mathrm{~mL}, 0.12 \mathrm{mmol}, 12\right.$ equiv). ${ }^{1} \mathrm{H}-\mathrm{NMR}$ spectrum recorded. Under a stream of nitrogen, $(S, S)$-ProPhenol ligand $\left(0.0069 \mathrm{~g}, 0.01 \mathrm{mmol}, 1\right.$ equiv) was added and ${ }^{1} \mathrm{H}-\mathrm{NMR}$ spectra were recorded at 10,30 and 50 minutes. Integration of the peaks at $0.17(\mathrm{~s}, 3 \mathrm{H}$, $\mathrm{CH}_{3} \mathrm{ZnCC}$ ) and $-0.69\left(\mathrm{~s}, 6 \mathrm{H},\left(\mathrm{CH}_{3}\right)_{2} \mathrm{Zn}\right)$ ppm was used to quantify alkynylzinc formation. Inherent to this calculation is the assumption that the deprotonation is clean and no side reactions occur. Extending the reaction time further resulted in visible decomposition.

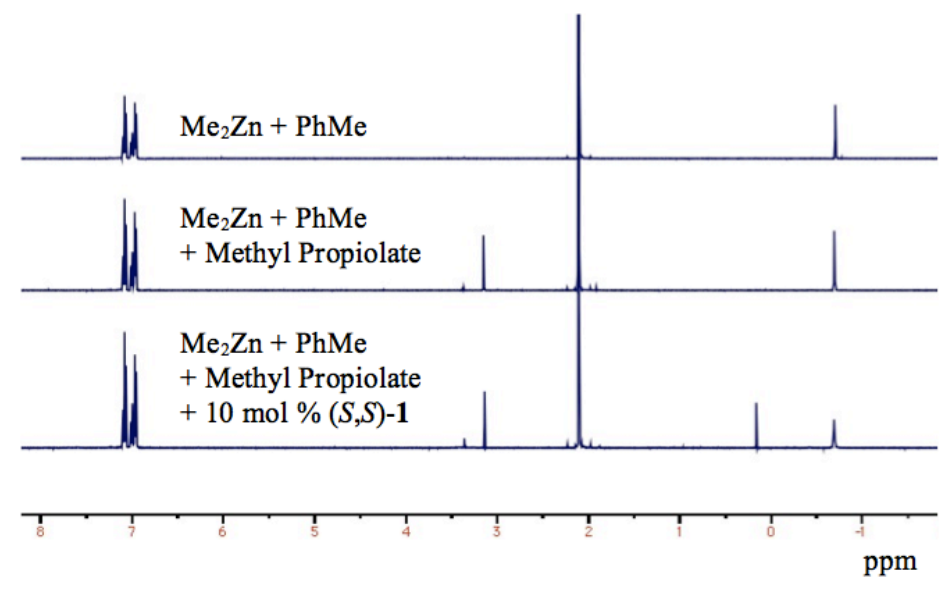

\begin{tabular}{ccc}
\hline Entry & Time & Conversion \\
\hline 1 & $10 \mathrm{~min}$ & $24 \%$ \\
2 & $30 \mathrm{~min}$ & $26 \%$ \\
3 & $50 \mathrm{~min}$ & $27 \%$ \\
\hline
\end{tabular}

1-Naphthalen-2-yl-ethanol, 110.<smiles>CC(O)c1ccc2ccccc2c1</smiles>

To a solution of $(S, S)$-Prophenol ligand $(0.038 \mathrm{~g})$ in toluene $(2.5 \mathrm{~mL})$ was added dimethylzinc (0.4). 2-Naphthaldehyde (0.183 g, $1.172 \mathrm{mmol}, 1$ equiv) was subsequently added and the reaction stirred for 3 days at $4{ }^{\circ} \mathrm{C}$. The title compound was isolated as a white solid $(0.92 \mathrm{~g}, 46 \%$ yield $) . \mathbf{R}_{\mathbf{f}}=0.31\left(2: 1, \mathrm{PE}: \mathrm{Et}_{2} \mathrm{O}\right) .{ }^{1} \mathbf{H}-\mathbf{N M R}\left(300 \mathrm{MHz}, \mathrm{CDCl}_{3}\right): \delta 7.87(\mathrm{~m}, 4 \mathrm{H})$, $7.5(\mathrm{~m}, 3 \mathrm{H}), 5.11(\mathrm{~m}, 1 \mathrm{H}), 1.9(\mathrm{~d}, J=3.3 \mathrm{~Hz}, 1 \mathrm{H}), 1.62(\mathrm{~d}, J=6.3 \mathrm{~Hz}, 3 \mathrm{H})$. Chiral HPLC: Chiralcel ${ }^{\circledR}$ OB-H column, hept $/ i \mathrm{PrOH} 90: 10,1.0 \mathrm{~mL} / \mathrm{min}, \lambda=254 \mathrm{~nm}: 9.49 / 10.79 \mathrm{~min}(37 \%$ ee). Characterization data matches literature. ${ }^{54}$ 


\section{Substrate Synthesis}

Methyl 2,2-dimethyl-3-oxopropanoate<smiles>COC(=O)C(C)(C)C=O</smiles>

To a solution of $3.08 \mathrm{~mL}$ of dimethylsulfoxide (DMSO, $36.3 \mathrm{mmol}, 2.82 \mathrm{~g}, 2.4$ equiv) in $120 \mathrm{~mL}$ of $\mathrm{CH}_{2} \mathrm{Cl}_{2}$ was added $1.43 \mathrm{~mL}$ of oxalyl chloride (16.6 mmol, $2.12 \mathrm{~g}, 1.1$ equiv) at $-78{ }^{\circ} \mathrm{C}$. The reaction mixture was stirred at $-78{ }^{\circ} \mathrm{C}$ for 10 minutes before addition of methyl 3 -hydroxy-2,2-dimethylpropanoate $(2.0 \mathrm{~g}, 15.1 \mathrm{mmol}, 1$ equiv). The reaction was stirred for 30 minutes before addition of $10.58 \mathrm{~mL}$ of triethylamine ( $75.7 \mathrm{mmol}, 7.68 \mathrm{~g}, 5$ equiv) via syringe. The reaction mixture was then slowly warmed to room temperature, quenched with water and the resulting mixture extracted with $\mathrm{CH}_{2} \mathrm{Cl}_{2}$. The combined organic layers were washed with brine, dried over $\mathrm{MgSO}_{4}$, filtered and concentrated in vacuo. The title compound was isolated as a clear, colorless oil (1.048 g, $53 \%$ yield). $\mathbf{R}_{\mathbf{f}}=0.28$ (5:1, PE:Et $\left.{ }_{2} \mathrm{O}\right) .{ }^{\mathbf{1}} \mathbf{H}-\mathbf{N M R}(400 \mathrm{MHz}$, $\left.\mathrm{CDCl}_{3}\right): \delta 9.66(\mathrm{~s}, 1 \mathrm{H}), 3.75(\mathrm{~s}, 3 \mathrm{H}), 1.35(\mathrm{~s}, 6 \mathrm{H})$. Characterization data matches literature. ${ }^{55}$

\section{3-Chloro-2,2-dimethyl-propionaldehyde}<smiles>CC(C)(C=O)CCl</smiles>

To a solution of $3.32 \mathrm{~mL}$ of dimethylsulfoxide (DMSO, $39.1 \mathrm{mmol}, 3.04 \mathrm{~g}, 2.4$ equiv) in $128 \mathrm{~mL}$ of $\mathrm{CH}_{2} \mathrm{Cl}_{2}$ was added $1.54 \mathrm{~mL}$ of oxalyl chloride $(17.9 \mathrm{mmol}, 2.27 \mathrm{~g}, 1.1$ equiv) at -78 ${ }^{\circ} \mathrm{C}$. The reaction mixture was stirred at $-78{ }^{\circ} \mathrm{C}$ for 10 minutes before addition of 3-chloro-2,2dimethyl-propanol ( $2.0 \mathrm{~g}, 16.3 \mathrm{mmol}$, 1equiv). The reaction was stirred for 30 minutes before addition of $11.4 \mathrm{~mL}$ of triethylamine ( $81.6 \mathrm{mmol}, 8.28 \mathrm{~g}, 5$ equiv) via syringe. The reaction mixture was then slowly warmed to room temperature, quenched with water and the resulting mixture extracted with $\mathrm{CH}_{2} \mathrm{Cl}_{2}$. The combined organic layers were washed with brine, dried over $\mathrm{MgSO}_{4}$, filtered and concentrated in vacuo. The title compound was isolated as a clear,

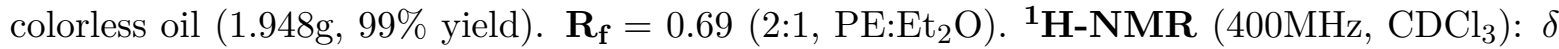
$9.50(\mathrm{~s}, 1 \mathrm{H}), 3.58(\mathrm{~s}, 2 \mathrm{H}), 1.17 \mathrm{ppm}(\mathrm{s}, 6 \mathrm{H}) \cdot{ }^{\mathbf{1 3}} \mathbf{C}-\mathbf{N M R}\left(100 \mathrm{MHz}, \mathrm{CDCl}_{3}\right): \delta 203.3,49.7,47.7$, 20.3. IR (film): 2976, 2875, 2811, 2714, 1730, 1470, 1397, 1368, 1285, 918, 875, 864, 776, 732 $\mathrm{cm}^{-1}$. Characterization data matches literature. ${ }^{56}$

\section{5-(Triisopropyl-silanyloxy)-pentanal}<smiles></smiles>

To a solution of $1.67 \mathrm{~g}$ of imidizole (49mmol, 2 equiv) and $1.13 \mathrm{~mL}$ of 5 -hydroxypentanal $(1.19 \mathrm{~g}, 24.5 \mathrm{mmol})$ in $25 \mathrm{~mL}$ of $\mathrm{CH}_{2} \mathrm{Cl}_{2}$ was added triisopropylsilyl chloride $(3.5 \mathrm{~mL}, 29.35$ mmol, 1.2 equiv). The reaction mixture was stirred for 4 hours before being quenched with saturated, aqueous $\mathrm{NH}_{4} \mathrm{Cl}$. The reaction mixture was extracted with $\mathrm{CH}_{2} \mathrm{Cl}_{2}$, and the combined organics washed with brine, dried over $\mathrm{MgSO}_{4}$, filtered, and concentrated in vacuo. The crude product was purified by flash column chromatography (3\% EtOAc/hexanes) to provide the title compound as a clear, colorless oil $\left(0.95 \mathrm{~g}, 15 \%\right.$ yield). $\mathbf{R}_{\mathbf{f}}=0.70(2: 1$, PE:Et $\left.{ }_{2} \mathrm{O}\right) .{ }^{1} \mathbf{H}-\mathrm{NMR}\left(400 \mathrm{MHz}, \mathrm{CDCl}_{3}\right): \delta 9.78(\mathrm{~s}, 1 \mathrm{H}), 3.72(\mathrm{t}, J=6 \mathrm{~Hz}, 2 \mathrm{H}), 2.47(\mathrm{td}, J$ 
$=6.8,2 \mathrm{~Hz}, 2 \mathrm{H}), 1.76$ (quintet, $J=6 \mathrm{~Hz}, 2 \mathrm{H}), 1.60(\mathrm{~m}, 2 \mathrm{H}), 1.07(\mathrm{~m}, 21 \mathrm{H})$. Characterization data matches literature. ${ }^{57}$

See Chapter 3 for experimental details and characterization data on:

-(E)-2,2-dimethyl-5-oxohept-3-enal, 140

See Chapter 4 for experimental details and characterization data on:

-(E)-4,4-Dimethoxybut-2-enal, 141

-4-((tert-Butyldimethylsilyl)oxy)butanal, 112

-4-((4-Methoxybenzyl)oxy)butanal, 115

- $(E)$-4-((tert-Butyldimethylsilyl)oxy)but-2-enal, 142

•(土)-Hept-6-yn-2-yl benzoate, 111 


\subsubsection{Spectra}

${ }^{1} \mathrm{H}-\mathrm{NMR}\left(500 \mathrm{MHz}, \mathrm{CDCl}_{3}\right)$

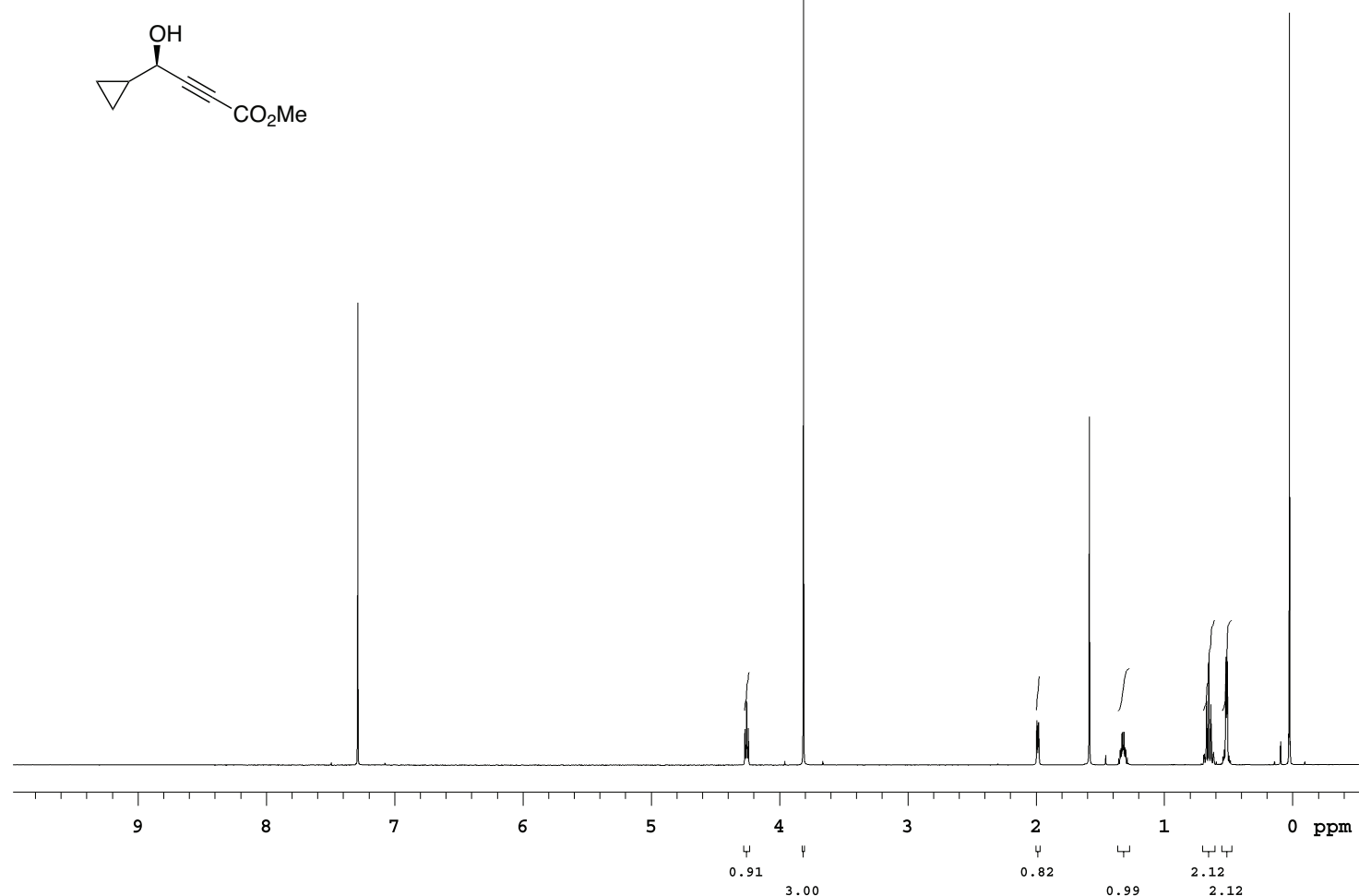

${ }^{13} \mathrm{C}-\mathrm{NMR}\left(126 \mathrm{MHz}, \mathrm{CDCl}_{3}\right)$<smiles>COC(=O)C#CC(O)C1CC1</smiles>

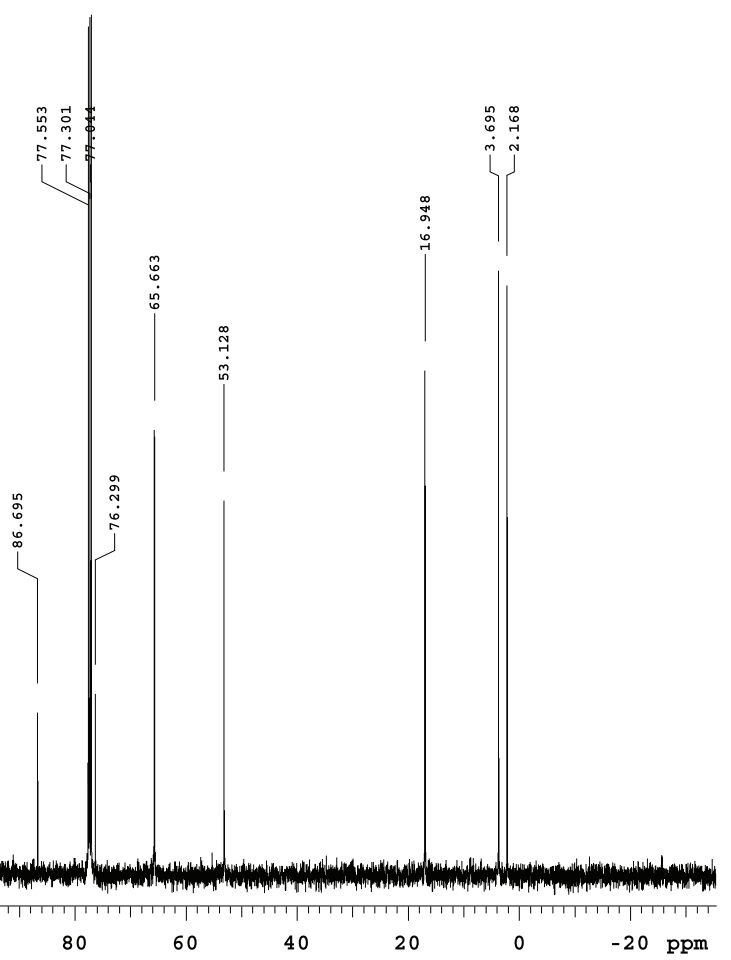




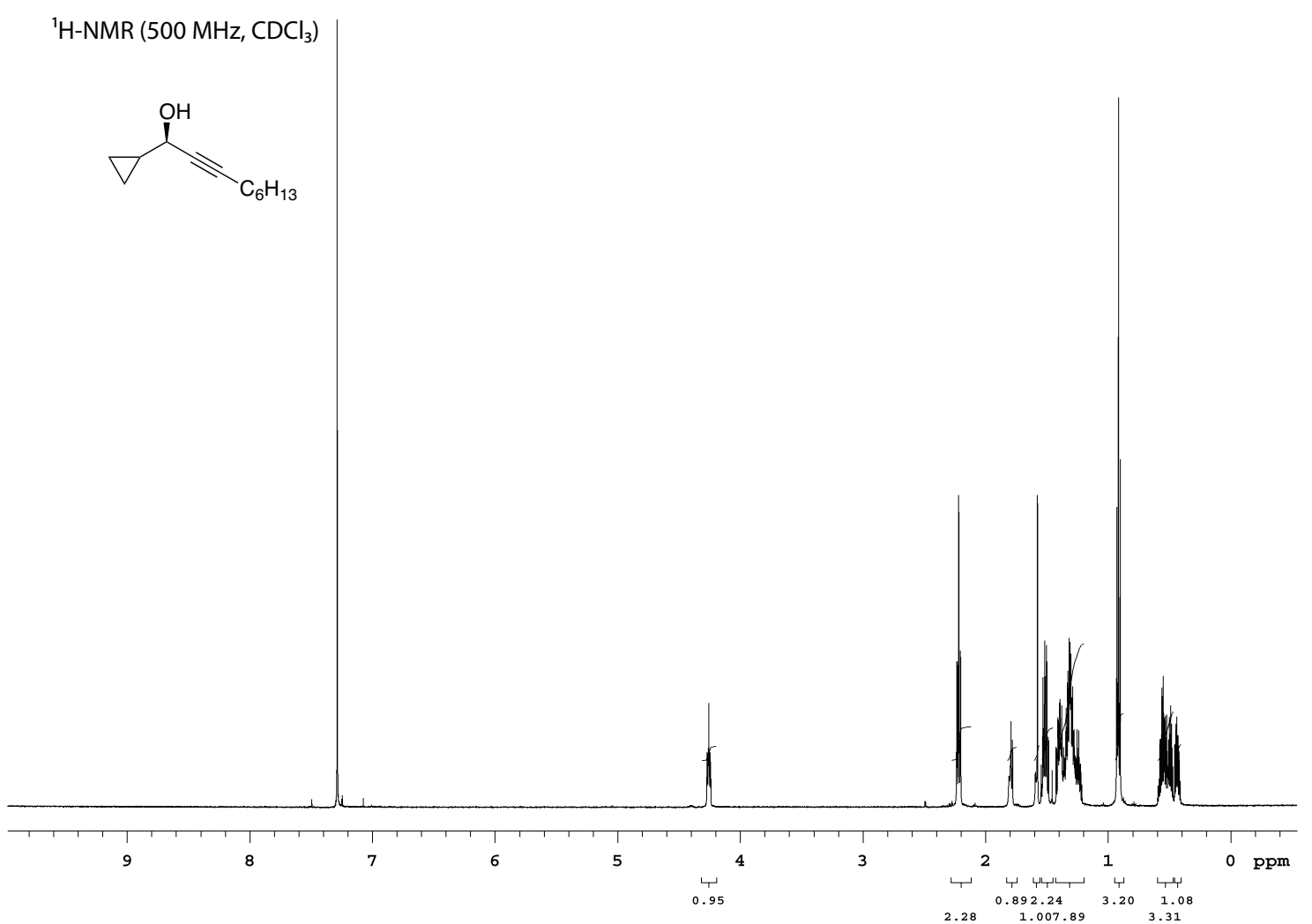

${ }^{13} \mathrm{C}-\mathrm{NMR}\left(126 \mathrm{MHz}, \mathrm{CDCl}_{3}\right)$<smiles>CCCCCCCCC#CC(O)C1CC1</smiles>

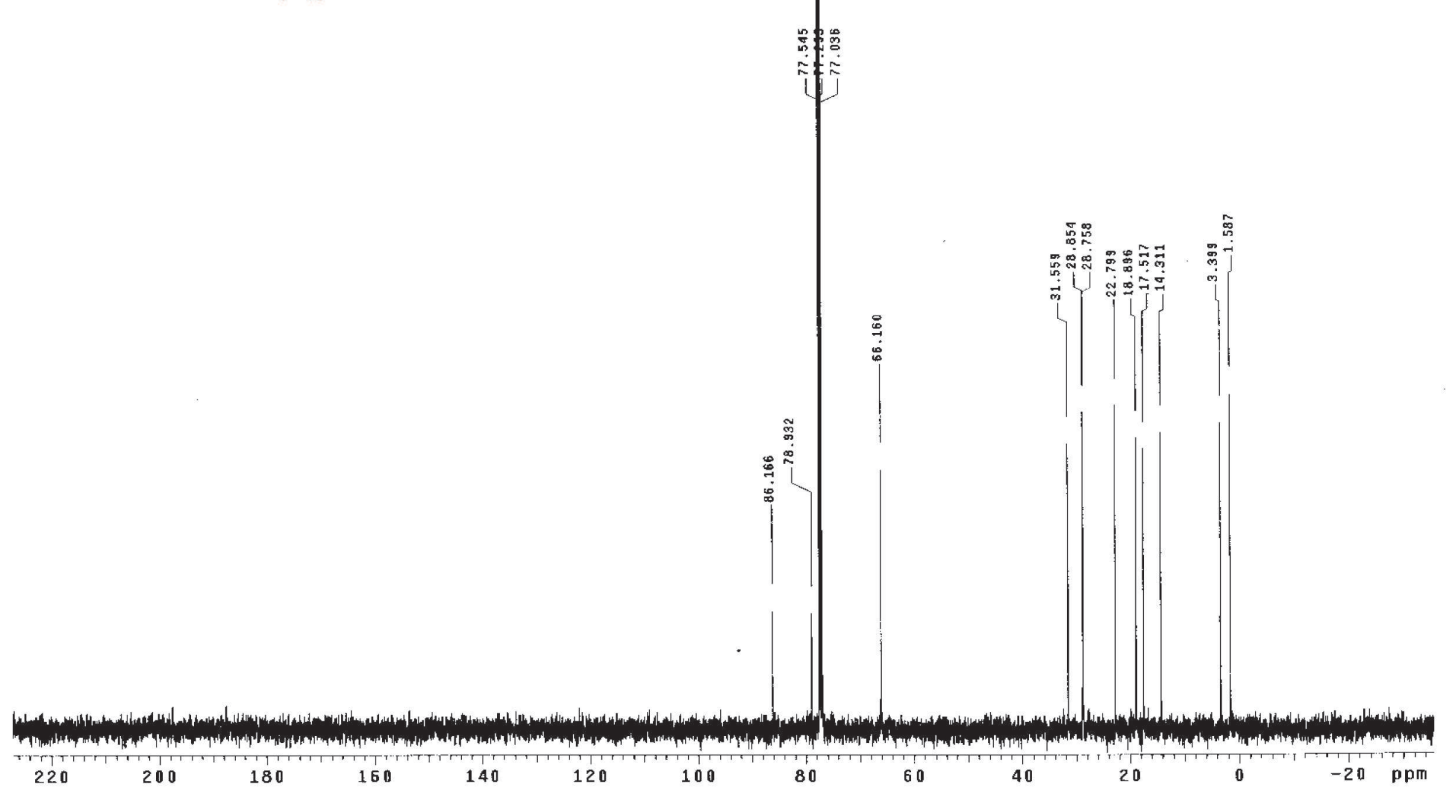



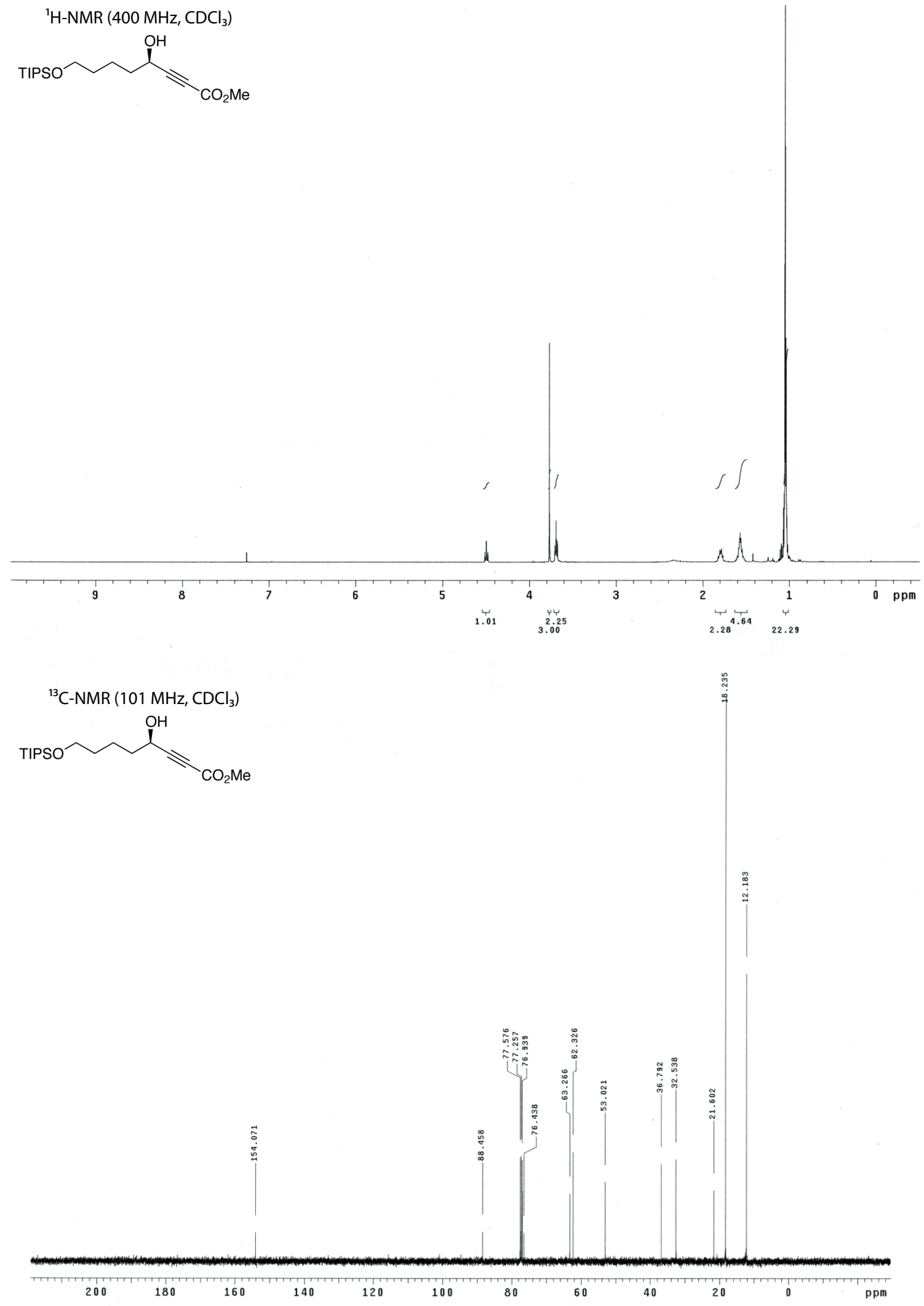
'H-NMR (400 MHz, CDCl $)$<smiles>CC(=O)C#CC(O)C(C)(C)CCl</smiles>
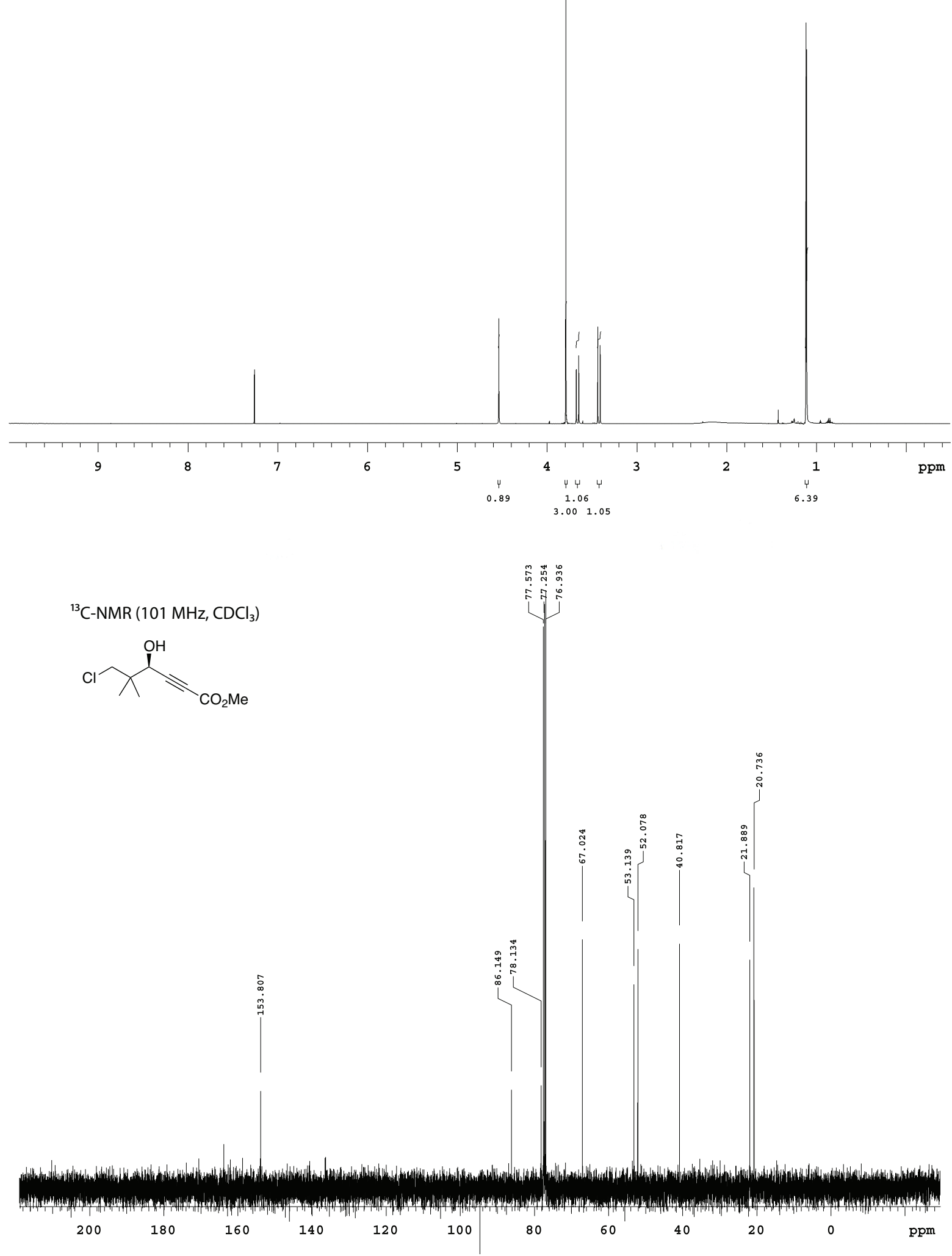

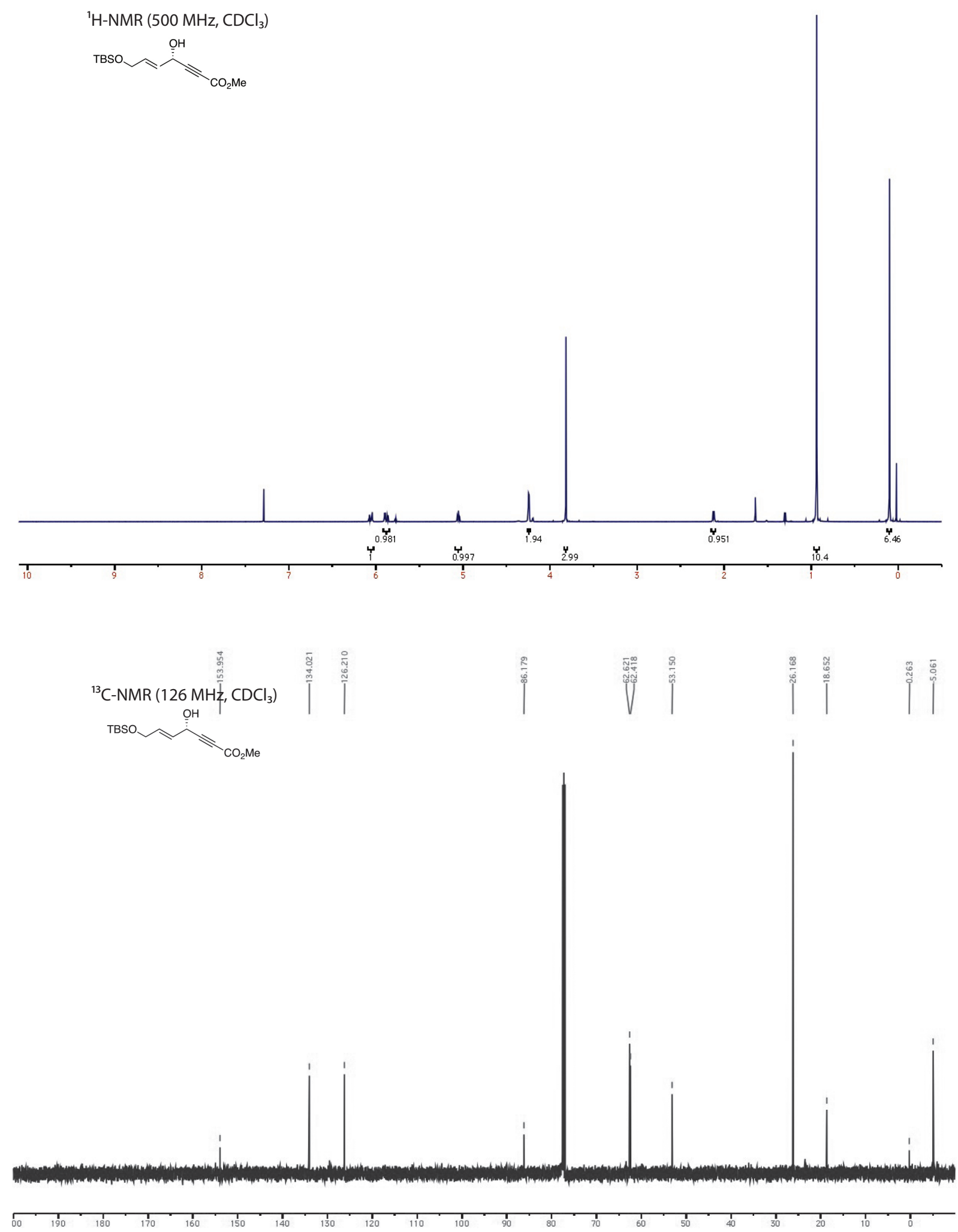

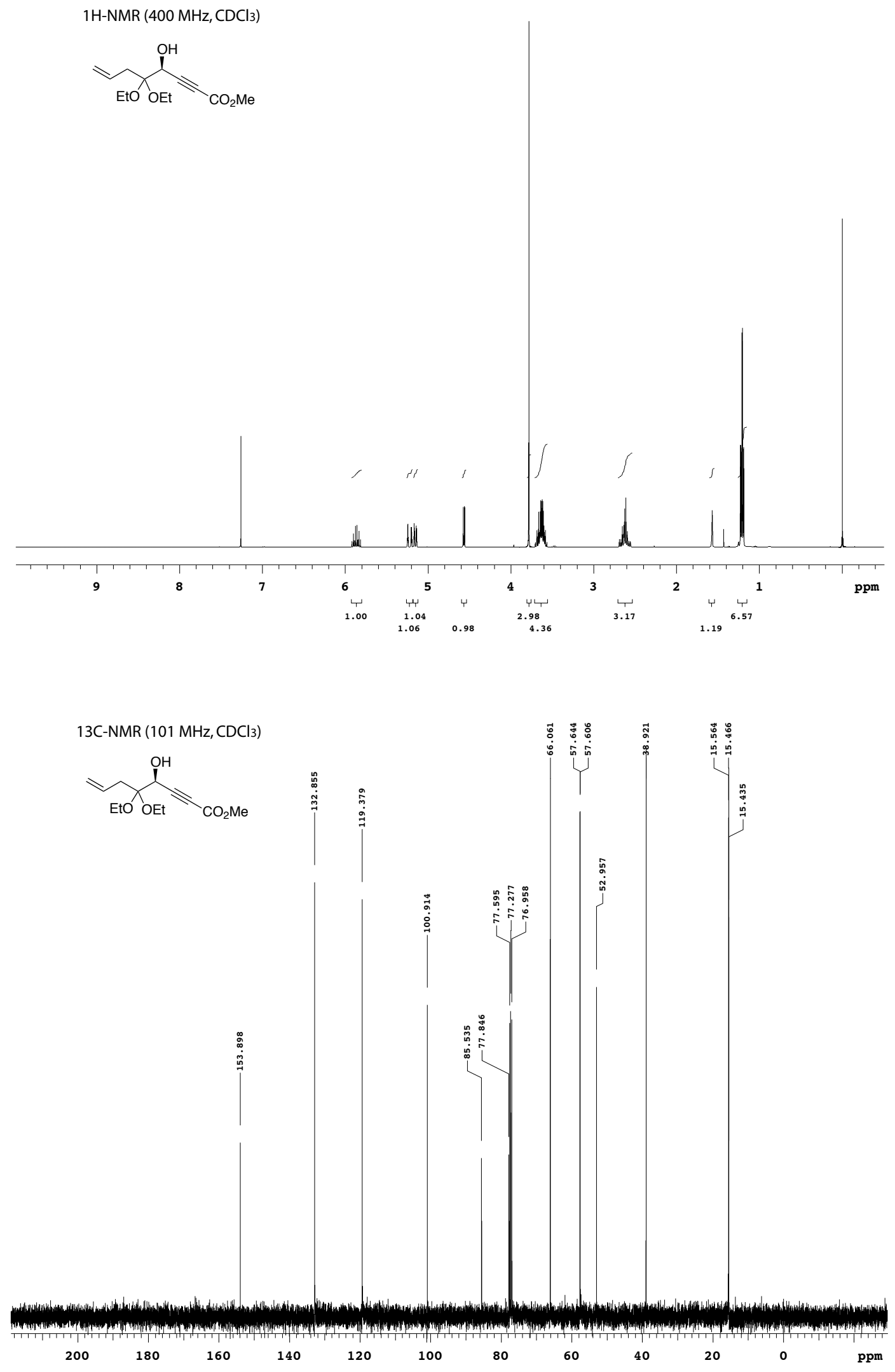


\section{References for Chapter 2}

[1] Modern Acetylene Chemistry; Stang, P. J., Diederich, F., Eds.; Wiley VCH: Weinheim, 2008.

[2] Ansyln, E. V.; Dougherty, D. A. Modern Physical Organic Chemistry; University Science Books: Sausalito, 2004, pp 259-292.

[3] Kürti, L.; Czakó, B. Strategic Applications of Named Reactions in Organic Synthesis; Elsevier Academic Press, 2005.

[4] White, J. D.; Carter, R. G.; Sundermann, K. F.; Wartmann, M. J. Am. Chem. Soc. 2001, $123,5407$.

[5] 1,2-Dialkylidenecyclopentane formation: see reference 22. Syn $\alpha, \beta$-dihydroxy ketone formation: Trost, B. M.; Ball, Z. T.; Laemmehold, K. M. J. Am. Chem. Soc. 2005, 127, 10028. $\beta$-arylation of propargylic alcohols: Trost, B. M.; Machacek, M. R.; Ball, Z. T. Org. Lett. 2003, 5, 1895 . $[2+2+2]$ cycloaddition: Witulski, B.; Zimmermann, A.; Gowans, N. D. Chem. Commun. 2002, 2984. Chiral allene formation: Pirkle, W. H.; Boeder, C. W. J. Org. Chem. 1978, 43, 1950.

[6] For selected examples of catalytic enantioselective ynone reduction, see: Helal, C. J.; Magriotis, P. A.; Corey, E. J. J. Am. Chem. Soc. 1996, 118, 10938. Matsumura, K.; Hashiguchi, S.; Ikariya, T.; Noyori, R. J. Am. Chem. Soc. 1997, 119, 8738. Parker, K. A.; Ledeboer, M. W. J. Org. Chem. 1996, 61, 3214.

[7] For a recent review on asymmetric alkyne addition to carbonyls, see: Trost, B. M.; Weiss, A. H. Adv. Synth. Catal. 2009, 351, 963.

[8] For selected examples of asymmetric ynal alkylation, see: Seebach, D.; Beck, A. K.; Schmidt, B.; Wang, Y. M. Tetrahedron 1994, 50, 4363. Fontes, M.; Verdaguer, X.; Sola, L.; Vidal-Ferran, A.; Reddy, K. S.; Riera, A.; Pericas, M. A. Org. Lett. 2002, 4, 2381. BouzBouz, S.; Pradaux, F.; Cossy, J.; Ferroud, C.; Falguieres, A. Tetrahedron Lett. 2000, 41, 8877. Uraguchi, D.; Nakamura, S.; Ooi, T. Angew. Chem. Int. Ed. 2010, 49, 7562. Belot, S.; Quintard, A.; Alexakis, A.; Krause, N. Adv. Synth, Cat. 2010, 352, 667.

[9] For reviews on asymmetric addition of organozinc nucleophiles, see: Soai, K.; Niwa, S. Chem. Rev. 1992, 92, 833. Pu, L.; Yu, H.-B. Chem. Rev. 2001, 101, 757. Noyori, R.; Kitamura, M. Angew. Chem. Int. Ed. Engl. 1991, 30, 49. Knochel, P.; Singer, R. D. Chem. Rev. 1993, 93, 2117.

[10] For reviews on zinc-mediated alkyne addition, see: Pu, L. Tetrahedron 2003, 59, 9873. Cozzi, P. G.; Hilgraf, R.; Zimmermann, N. Eur. J. Org. Chem. 2004, 4095. Lu, G.; Li, Y.-M.; Li, X.-S.; Chan, A. S. C. Coord. Chem. Rev. 2005, 249, 1736 and reference 7.

[11] a) Frantz, D.; Fässler, R.; Carreira, E. J. Am. Chem. Soc. 2000, 122, 1806. b) Boyall, D.; López, F.; Sasaki, H.; Frantz, D.; Carreira, E. Org. Lett. 2000, 2, 4233. c) Frantz, D.; Fässler, R.; Tomooka, C.; Carreira, E. Acc. Chem. Res. 2000, 33, 373. d) Sasaki, H.; Boyall, D.; Carreira, E. Helv. Chem. Acta. 2001, 84, 964. e) El-Sayed, E.; Anand, N. K.; Carreira, E. M. Org. Lett. 2001, 3, 3017. f) Anand, N.; Carreira, E. J. Am. Chem. Soc. 2001, 123, 9687. g) Diez, R. S.; Adger, B.; Carreira, E. M. Tetrahedron 2002, 58, 8341. h) Knopfel, T.; Boyall, D.; Carreira, E. Org. Lett. 2004, 6, 2281. i) Fässler, R.; Tomooka, C. S.; Frantz, D. E.; Carreira, E. M. Proc. Natl. Acad. Sci. U.S.A 2004, 101, 5843.

[12] a) Gao, G.; Moore, D.; Xie, R.; Pu. L. Org. Lett. 2002, 4, 4143. b) Moore, D.; Pu. L. Org. Lett. 2002, 4 , 1855. c) Xu, M.; Pu, L. Org. Lett. 2002, 4, 4555. d) Liu, L.; Pu, L. Tetrahedron, 2004, 60, 7427. e) Li, Z.; Pu, L. Org. Lett. 2004, 6, 1065. f) Rajaram, A.; Pu, L. Org. Lett. 2006, 8, 2019. g) Yue, Y.; Turlington, M.; $\mathrm{Yu}$, X.-Q.; Pu, L. J. Org. Chem. 2009, 74, 8681. h) Zhou, L.-H.; Yu, X.-Q.; Pu, L. J. Org. Chem. 2009, 74, 2013. i) Turlington, M.; Yue, Y.; Yu, X.-Q.; Pu, L. J. Org. Chem. 2010, 75, 6941. j) Turlington, M.; Du, Y.; Ostrum, S. G.; Santosh, V.; Wren, K.; Lin, T.; Sabat, M.; Pu, L. J. Am. Chem. Soc. 2011, 133, 11780 .

[13] Lu, G.; Li, X.; Zhou, Z.; Chan, W.; Chan, A. Tetrahedron: Asymmetry 2001, 12, 2147. Li, X.; Lu, G.; Kwok, W.; Chan, A. J. Am. Chem. Soc. 2002, 124, 12636. Lu, G.; Li, X.; Chen, G.; Chan, W.; Chan, A. Tetrahedron: Asymmetry 2003, 14, 449.

[14] Niwa, S.; Soai, K. J. Chem. Soc., Perkin Trans. 1 1990, 937. Tombo, G. M. R.; Didier, E.; Loubinoux, B. Synlett, 1990, 9, 547. Ishizaki, M.; Hoshino, O. Tetrahedron Asymmetry 1994, 5, 1901. Jiang, B.; Chen, Z.; Xiong, W. Chem. Commun. 2002, 1524. Lu, G.; Li, X.; Chan, W. L.; Chan, A. S. C. Chem. Commun. 
2002, 172. Chen, Z.; Xiong, W.; Jiang, B. Chem. Commun. 2002, 2098. Marshall, J. A.; Bourbeau, M. P. Org. Lett. 2003, 5, 3197. Xu, Z.; Wang, R.; Xu, J.; Da, C.-S.; Yan, W.-J.; Chen, C. Angew. Chem. Int. Ed. 2003, 42, 5747. Zhou, Y.-F.; Wang, R.; Xu, Z.-Q.; Yan, W.-J.; Lei, L.; Gao, Y.-F.; Da, C.-S. Tetrahedron: Asymmetry 2004, 15, 589. Kang, Y.-F.; Liu, L.; Wang, R.; Yan, W.-J.; Zhou, Y.-F. Tetrahedron: Asymmetry 2004, 15, 3155. Dahmen, S. Org. Lett. 2004, 6, 2113. Kang, Y.; Wang, R.; Liu, L.; Da, C.; Yan, W.; Xu, Z. Tetrahedron Lett. 2005, 46, 863. Ni, M.; Wang, R.; Han, Z.-J.; Mao, B.; Da, C.-S.; Liu, L.; Chen, C. Adv. Synth. Catal. 2005, 347, 1659. Wolf, C.; Liu, S. J. Am. Chem. Soc. 2006, 128, 10996. Emerson, D. P. G.; Hems, W. P.; Davis, B. G. Org. Lett. 2006, 8, 207. Li, Z. B.; Liu, T.-D.; Pu, L. J. Org. Chem. 2007, 72, 4340. Xu, J.; Mao, J.; Zhang, Y. Org. Biomol. Chem. 2008, 6, 1288. Hui, X. P.; Yin, C.; Chen, Z.-C.; Huang, L.-N.; Xu, P.-F.; Fan, G. F. Tetrahedron 2008, 64, 2553. Wang, M.-C.; Zhang, Q.-J.; Zhao, W.-X.; Wang, X.-D.; Ding, X.; Jing, T.-T.; Song, M.-P. J. Org. Chem. 2008, 73, 168. Li, H.; Huang, Y.; Jin, W.; Xue, F.; Wan, B. Tetrahedron Lett. 2008, 49, 1686. Blay, G.; Cardona, L.; Fernández, I.; Macro-Aleixandre, A.; Muñoz, M. C.; Pedro, J. R. Org. Biomol. Chem. 2009, 7, 4301. Niu, J.-L.; Wang, M.-C.; Lu, L.-J.; Ding, G.-L.; Lu, H.-J.; Chen, Q.-T.; Song, M.-P. Tetrahedron: Asymmetry 2009, 20, 2616. Li, Z.-Y.; Wang, M.; Bian, Q.-H.; Zheng, B.; Mao, J.-Y.; Li, S.-N.; Liu, S.-Z.; Wang, M.-A.; Zhong, J.-C.; Guo, H.-C. Chem. Eur. J. 2011, 17, 5782. Graham, E. R.; Tykwinski, R. R. J. Org. Chem. 2011, 76, 6574. Bauer, T.; Smolinski, S.; Gawel, P.; Jurczak, J. Tetrahedron Lett. 2011, 52, 4882. Lu, X.; Xie, G.; Li, T.; Qu, X.; Mao, J. Synthetic Commun. 2012, 42, 775. Boobalan, R.; Chen, C.; Lee, G.-H. Org. Biolmol. Chem. 2012, 10, 1625.

[15] For rare examples of zinc-catalyzed asymmetric alkynylation with 1 equivalent of alkyne, see: a) Kojima, N.; Nishijima, S.; Tsuge, K.; Tanaka, T. Org. Biomol. Chem. 2011, 9, 4425. b) reference 11f.

[16] Trost, B. M.; Ito, H. J. Am. Chem. Soc. 2000, 122, 12003.

[17] For subsequent applications of this ligand, see: Trost, B. M.; Malhotra, S.; Koschker, P.; Ellerbrock, P. J. Am. Chem. Soc. 2012, 134, 2075. Trost, B. M.; Hitce, J. J. Am. Chem. Soc. 2009, 131, 4572 and references cited therein.

[18] Trost, B. M.; Terrell, L. R.; J. Am. Chem. Soc. 2003, 125, 338. Trost, B. M.; Jaratjaroonphong, J.; Reutrakul, V. J. Am. Chem. Soc. 2006, 128, 2778.

[19] Trost, B. M.; Yeh, V. S. C. Angew. Chem., Int. Ed. 2002, 41, 861. Trost, B. M.; Yeh, V. S. C.; Ito, H.; Bremeyer, N. Org. Lett. 2002, 4, 2621. Trost, B. M.; Lupton, D. W. Org. Lett. 2007, 9, 2023.

[20] Trost, B. M.; Mino, T. J. Am. Chem. Soc. 2003, 125, 2410. Trost, B. M.; Malhotra, S.; Mino, T.; Rajapaksa, N. S. Chem. Eur. J. 2008, 14, 7648 .

[21] Trost, B. M.; Ito, H.; Silcoff, E. R. J. Am. Chem. Soc. 2001, 123, 3367. Trost, B. M.; Fettes, A.; Shireman, B. T. J. Am. Chem. Soc. 2004, 126, 2660. Trost, B. M.; Shin, S.; Sclafani, J. A. J. Am. Chem. Soc. 2005, 127,8602 .

[22] For the initial communication of this methodology, see: Trost, B. M.; Weiss, A. H.; von Wangelin, A. J. J. Am. Chem. Soc. 2006, 128, 8.

[23] Trost, B. M.; Bartlett, M. J.; Weiss, A. H.; von Wangelin, A. J; Chan, V. S. Chem. Eur. J. 2012, accepted.

[24] Trost, B. M. Science 1991, 254, 1471. Trost, B. M. Acc. Chem. Res. 2002, 35, 695.

[25] Cozzi, P. G. Angew. Chem. Int. Ed. 2003, 42, 2895.

[26] Absolute configuration $(R)$ was determined by comparison to the optical rotation reported in the literature.

[27] Cozzi, P. G.; Rudolph, J.; Bolm, C.; Norrby, P.; Tomasini, C. J. Org. Chem. 2005, 70, 5733

[28] Li, Z.; Upadhyay, V.; DeCamp, A. E.; DiMichele, L.; Reider, P. J. Synthesis, 1999, 1453.

[29] For successful asymmetric additions of propiolate nucleophiles to aldehydes, see: a) Gao, G.; Wang, Q.; Yu, X.-Q.; Xie, R.-G.; Pu, L. Angew. Chem. Int. Ed. 2006, 45, 122. b) Rajaram, A. R.; Pu, L. Org. Lett. 2006, 8, 2019. c) Lin, L.; Jiang, X.; Liu, W.; Qiu, L.; Xu, Z.; Xu, J.; Chan, A. S. C.; Wang, R. Org. Lett. 2007, 9, 2329. d) Turlington, M.; DeBerardinis, A. M.; Pu, L. Org. Lett. 2009, 11, 2441. e) Xu, T.; Liang, C.; Cai, Y.; Li, J.; Li, Y.-M.; Hui, X.-P. Tetrahedron: Asymmetry 2009, 20, 2733. Also, reference 15a. 
[30] For selected examples of $\gamma$-hydroxy- $\alpha, \beta$-acetylenic esters as synthetic intermediates, see: Midland, M. M.; Tramontano, A.; Cable, J. R. J. Org. Chem. 1980, 45, 28. Midland, M. M.; McDowell, D. C.; Hatch, R. L. Tramontano, A. J. Am. Chem. Soc. 1980, 102, 867. Trost, B. M. Crawley, M. L. J. Am. Chem. Soc. 2002, 124, 9328. Trost, B M.; Ball, Z. T. J. Am. Chem. Soc. 2004, 126, 13942. Molander G. A.; St. Jean, D. J., Jr. J. Org. Chem. 2002, 67, 3861. Meta C. T.; Koide, K. Org. Lett. 2004, 6, 1785. Albert, B. J. Sivaramakrishnan, A.; Naka, T.; Koide, K. J. Am. Chem. Soc. 2006, 128, 2792.

[31] Shahi, S.; Koide, K. Angew. Chem. Int. Ed. 2004, 43, 2525

[32] Others have also attributed the modest yield obtained with $n$-aliphatic aldehydes to competing selfcondensation aldol side reactions, see reference 11a.

[33] Bordwell, F. G.; Drucker, G. E.; Anderen, N. H.; Denniston, A. D. J. Am. Chem. Soc. 1986, $108,7310$.

[34] Trost, B. M.; Chan, V. S.; Yamamoto, D. J. Am. Chem. Soc. 2010, 132, 5186.

[35] Xiao, Y.; Wang, Z.; Ding, K. Chem. Eur. J. 2005, 11, 3668.

[36] Weiss, A. H. Dinuclear Zinc-Catalyzed Asymmetric Alkynylation: Development and Application to the Synthesis of Natural Products. Ph.D. Thesis, Stanford University, Stanford, CA, USA, 2008

[37] Coates, G. E.; Ridley, D. J. Chem. Soc. 1965, 1870. Shearer, H. M. M.; Spencer, C. B. Acta Crystallogr. 1980, B36, 2046. For a theoretical discussion on the structure and stability of various zinc alkoxide aggregates, see; Steudel, R.; Steudel, Y. J. Phys. Chem. A 2006, 110, 8912.

[38] For a review on nonlinear effects and polymeric chiral catalysts, see: Girard, C.; Kagan, H. B. Angew. Chem. Int. Ed. 1998, 37, 2922.

[39] For selected examples of chiral zinc catalysts that display nonlinear effects, see: Kitamura, M.; Okada, S.; Suga, S.; Noyori, R. J. Am. Chem. Soc. 1989, 111, 4028. Kitamura, M.; Suga, S.; Oka, H.; Noyori, R. J. Am. Chem. Soc. 1998, 120, 9800. Oguni, N.; Matsuda, Y.; Kaneko, T. J. Am. Chem. Soc. 1988, 110, 7877.

[40] Okhlobystin, O. Y.; Zakharkin, L. I. J. Organometal. Chem. 1965, 3, 257.

[41] For selected examples of the use of additives to facilitate the formation of alkynylzinc nucleophiles, see: (a) Gao, G.; Xie, R.-G.; Pu, L. Proc. Natl. Acad. Sci. U.S.A. 2004, 101, 5417. (b) Yang, F.; Xi, P.; Yang, L.; Lan, J.; Xie, R.; You, J. J. Org. Chem. 2007, 72, 5457. (c) Du, Y.; Turlington, M.; Zhou, X.; Pu, L. Tetrahedron Lett. 2010, 51, 5024.

[42] For related NMR analysis of alkynylzinc nucleophiles, see: Evans, D. F.; Fazakerley, G. V. J. Chem. Soc. 1971, 182. De Koning, A. J.; Van Rijn, P. E.; Boersma, J.; Van Der Kerk, G. J. M. J. Organometal. Chem. 1979, 174, 129. Fässler, R. The Metalation of Terminal Alkynes by $Z n^{\mathrm{II}}$ and Their Addition to Nitrones and Aldehydes. Ph.D. Thesis, ETH Zürich, Zürich, Switzerland, 2003 and reference 28.

[43] In contrast, alkynylation with $\mathrm{Me}_{2} \mathrm{Zn} / \mathrm{Ti}(\mathrm{O} i \mathrm{Pr})_{4} / \mathrm{BINOL}$ suffers from significant amounts of methyl addition if formation of the alkynylzinc nucleophile does not go to completion, see reference 41a.

[44] Trost, B. M.; Weiss, A. H. Org. Lett. 2006, 8, 4461.

[45] Trost, B. M.; Livingston, R. C. J. Am. Chem. Soc. 2008, 130, 11970.

[46] Trost, B. M.; Weiss, A. H. Angew. Chem. Int. Ed. 2007, 46, 7664.

[47] Trost, B. M.; O’Boyle, B. M. J. Am. Chem. Soc. 2008, 130, 16190. Trost, B. M.; O'Boyle, B. M.; Hund, D. J. Am. Chem. Soc. 2009, 131, 15061.

[48] Tamaru, Y. Eur. J. Org. Chem. 2005, 13, 2647. Marshall, J. A.; Schaaf, G. M. J. Org. Chem. 2001, 66, 7825 .

[49] For additional natural product syntheses that utilize the Zn-ProPhenol alkyne addition methodology, see: Trost, B. M.; Sieber, J. D.; Qian, W.; Dhawan, R.; Ball, Z. T. Angew. Chem. Int. Ed. 2009, 48, 1. Trost, B. M.; Quintard, A. Org. Lett. 2012 14, 4698. Trost, B. M.; Quintard, A. Angew. Chem. Int. Ed. 2012 51, 6704 . 
[50] Midland, M. M.; Tramontano, A.; Bazubski, A.; Graham, R. S.; Tsai, D. J. S.; Cardin, D. B. Tetrahedron 1984, 40, 1371.

[51] Trost, B. M.; Ball, Z. T. J. Am. Chem. Soc. 2005, 127, 17644.

[52] Gabriele, B.; Salerno, G.; De Pascali, F.; Costa, M.; Chiusoli, G. P. J. Chem. Soc., Perkin Trans. 1, 1997, 147.

[53] Trost, B. M.; Tanoury, G. J.; Lautens, M.; Chan, C.; MacPherson, D. T. J. Am. Chem. Soc. 1994, 116, 4255.

[54] Sokeirik, Y., Mori, H., Omote, M., Sato, K., Tarui, A., Kumadaki, I., Ando, A. Org. Lett. 2007, 9, 1927

[55] Ng, S.-S.; Jamison, T. J. Am. Chem. Soc. 2005, 12\%, 14194.

[56] Effenberger, F.; Eichhorn, J.; Roos, J. Tetrahedron: Asymmetry 1995, 6, 271.

[57] Brown, S. P.; Brochu, M. P.; Sinz, C. J.; MacMillan, D. W. C. J. Am. Chem. Soc. 2003, 125, 10808. 
Chapter 3

Total Synthesis of Asteriscunolide D:

A Ru-Catalyzed Alkene-Alkyne Coupling Strategy 


\subsection{Introduction}

The asteriscunolides are a family of four humulene natural products originally isolated from the Mediterranean plants Asteriscus aquaticus and Asteriscus graveolens. ${ }^{1}$ These sesquiterpene natural products contain an inherently strained 11-membered ring with an embedded butenolide heterocycle and two alkenes (Figure 3.1). Asteriscunolides A, B, C, and D represent all possible alkene isomers of this cyclic framework: $(Z, Z),(Z, E),(E, Z)$, and $(E, E)$, respectively. All four asteriscunolides display cytotoxicity towards a range of cancerous cells,

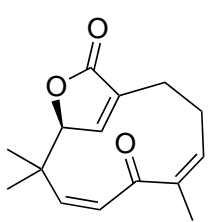

143

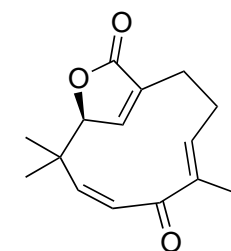

144

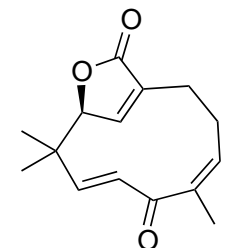

145

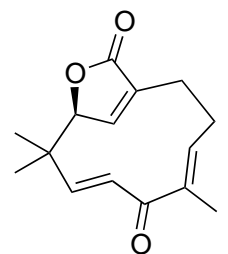

146

asteriscunolide $A$ asteriscunolide $B$ asteriscunolide $C$ asteriscunolide $D$

with asteriscunolide D being particularly cytotoxic towards MEL-28 (human melanoma), A-549 (human lung carcinoma), and HT-29 (human colon carcinoma) cell lines. ${ }^{2}$ Recent studies into the mode of action of these compounds has revealed that asteriscunolide $\mathrm{C}$ causes apoptosis associated with activation of mitogen-activated protein kinases. ${ }^{3}$

The unique macrocyclic structure of the asteriscunolides gives rise to a number of distorted bond angles and atypical conformations. As a result, the initial structures of asteriscunolide $\mathrm{A}$ and $\mathrm{B}$ were misassigned due to an unusually high $14 \mathrm{~Hz}^{3} J_{\mathrm{HH}}$ coupling across the cisdisubstituted alkene. The unfunctionalized humulene framework (147) has four macrocyclic conformations, which interconvert readily at room temperature (Figure 3.2). ${ }^{4}$ With these properties of humulene in mind, the initial report of the structures for the asteriscunolides proposed that asteriscunolide $\mathrm{A}$ and $\mathrm{B}$ were atropisomers of asteriscunolide $\mathrm{C}$ and $\mathrm{D}$, respectively. The true structure of these natural products was ultimately revealed by $\mathrm{X}$ -

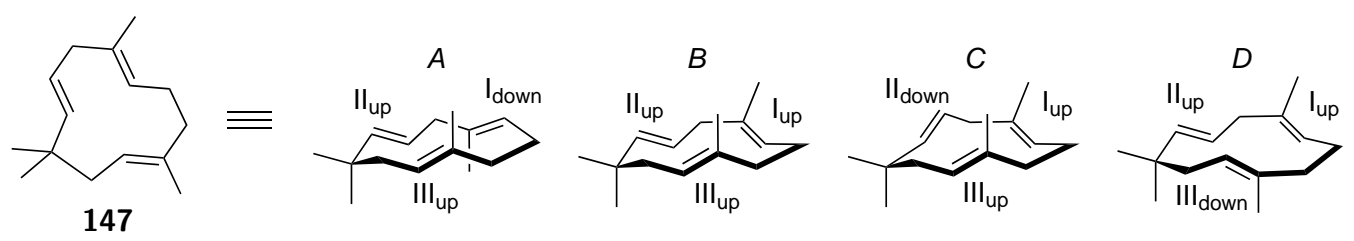

Figure 3.2 Cyclic Conformers of Humulene.

ray crystallography, ${ }^{5}$ which also provided detailed information on their atypical structure (Figure 3.3). The dihedral angles of the alkenes present in these natural products provide a good indication of the strained nature of each of the asteriscunolide macrocycles. In this case, ring strain leads to a distortion of the typical planar geometry of an alkene in a butenolide ring. Examination of the $\mathrm{H} 2-\mathrm{C} 2-\mathrm{C} 3-\mathrm{C} 4$ dihedral angle shows that asteriscunolide 


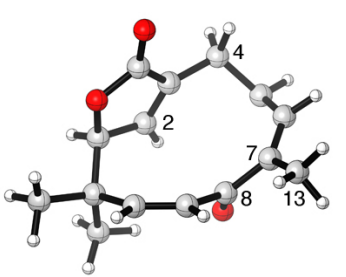

A

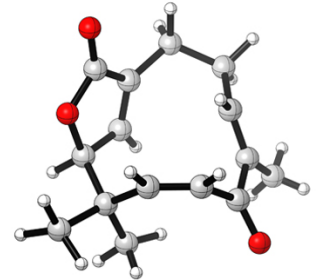

B

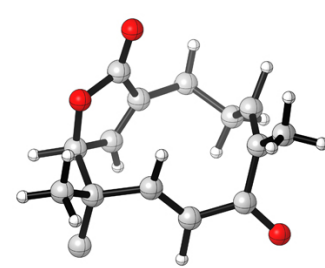

C

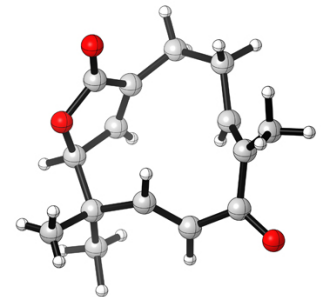

D

\begin{tabular}{ccccc}
\hline Dihedral & A & B & C & D \\
\hline H2-C2-C3-C4 & $0.1^{\circ}$ & $-14.0^{\circ}$ & $-9.0^{\circ}$ & $-10.3^{\circ}$ \\
C13-C7-C8-O8 & $-131.6^{\circ}$ & $14.5^{\circ}$ & $-71.9^{\circ}$ & $-39.6^{\circ}$ \\
O8-C8-C9-H9 & $132.9^{\circ}$ & $80.9^{\circ}$ & $-7.8^{\circ}$ & $-35.8^{\circ}$ \\
\hline
\end{tabular}

Figure 3.3 X-Ray Crystal Structures of Asteriscunolides A, B, C, and D.

B experiences the greatest distortion $\left(-14^{\circ}\right)$, followed by asteriscunolide $\mathrm{D}\left(-10.3^{\circ}\right), \mathrm{C}\left(-9.0^{\circ}\right)$, and $\mathrm{A}\left(0.1^{\circ}\right)$, respectively. Asteriscunolide A displays a much smaller deviation from the ideal planar geometry than the other asteriscunolides, which suggests that the $(Z, Z)$-macrocycle is less strained than the isomers that contain an $E$-alkene. Additionally, the dihedral angles C13-C7-C8-O8 and O8-C8-C9-H9 show that neither the C6 nor the C9 alkene resides in the same plane as the neigbouring ketone and, therefore, overlap of these adjacent $\pi$-systems is minimal.

The strained 11-membered ring of humulene and humulene-derived natural products represents a formidable challenge for synthetic chemists. ${ }^{6}$ The construction of this macrocycle has been described as a major impediment to synthesis and typically requires harsh reactions conditions to provide only modest yields (Scheme 3.1). ${ }^{7}$ The methods used previously to construct this ring system include: dibromide coupling, ${ }^{8}$ McMurry coupling, ${ }^{9}$ SuzukiMiyaura coupling, ${ }^{10}$ and a Horner-Wadsworth-Emmons (HWE) olefination. ${ }^{11}$ The use of superstoichiometric amounts of either $\mathrm{Ni}(\mathrm{CO})_{4}$ or $\mathrm{TiCl}_{3}$ at elevated temperatures is likely to tolerate only the most robust functional groups, whereas the use of milder Suzuki-Miyaura and HWE conditions provide low yields of the desired macrocycle. Interestingly, the intramolecular HWE olefination shown in Scheme 3.1 provides $55 \%$ yield of a dimeric 22-membered macrocyclic product, despite the highly dilute reaction conditions. Overall, this presents a daunting challenge for the synthesis of a functionalized humulene natural product such as asteriscunolide D (146), which contains potentially sensitive butenolide and carbonyl motifs in addition to the humulene ring. 


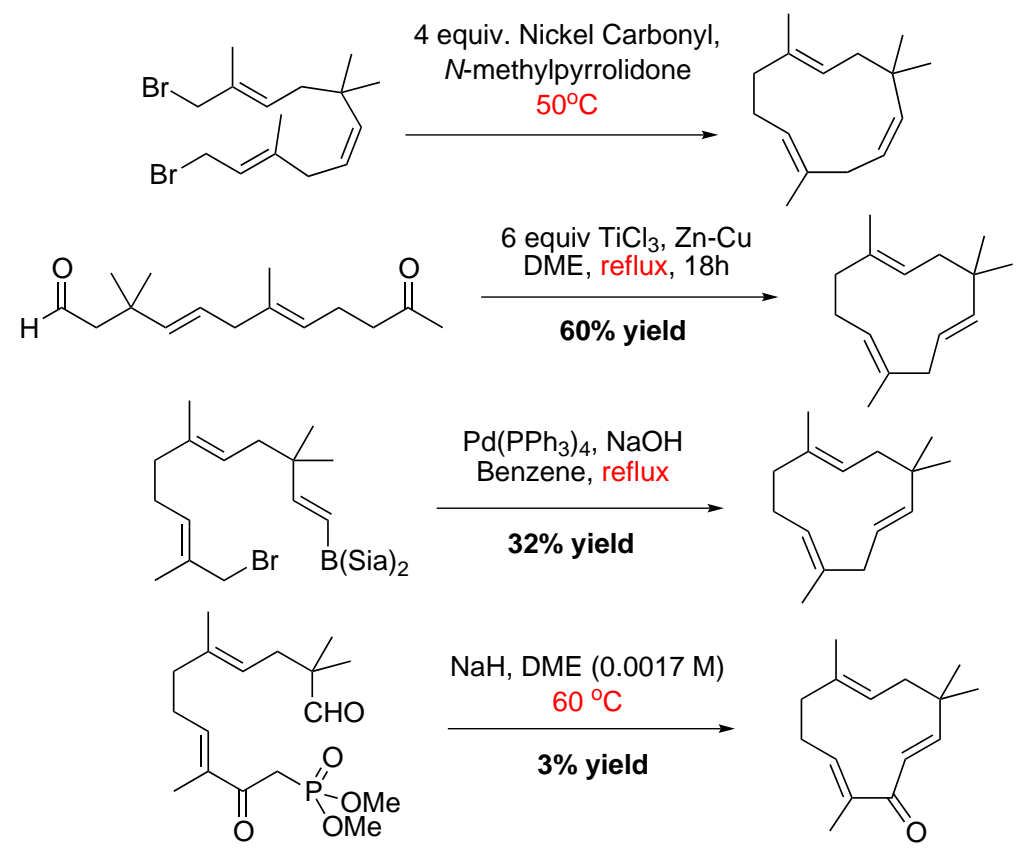

Scheme 3.1 Previous Syntheses of Humulene Macrocycles.

\subsection{Total Synthesis of Asteriscunolide D}

\subsubsection{Synthetic Strategy and Retrosynthetic Analysis}

Our interest in asteriscunolide D originated from the potential use of Zn-ProPhenol-catalyzed asymmetric alkyne addition and Ru-catalyzed alkene-alkyne coupling to form chiral butenolides in a highly efficient manner (i.e. Scheme 3.2). ${ }^{12}$ The appeal of this target was augmented by the realization that a thionium-induced cyclization could be utilized to provide a mild and efficient method of forming the challenging 11-membered ring system (i.e. Scheme 3.3). ${ }^{13}$

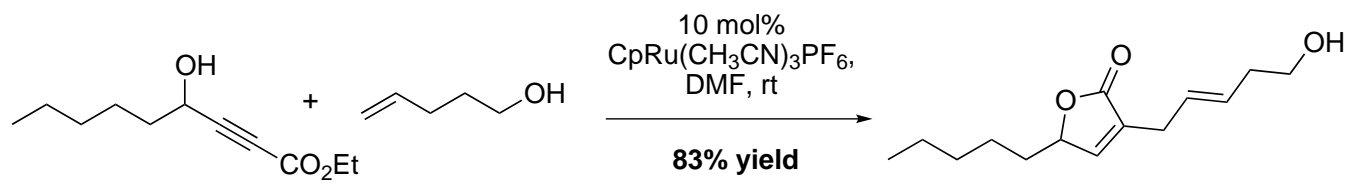

Scheme 3.2 Ru-Catalyzed Alkene-Alkyne Coupling with $\gamma$-Hydroxy- $\alpha, \beta$-Acetylenic Esters.

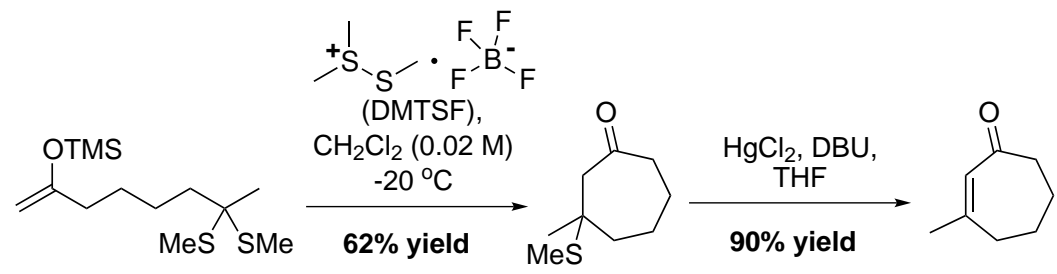

Scheme 3.3 DMTSF-mediated Cyclization and Thioether Elimination. 
This approach represents a formal aldol disconnection, however, the desired product would be formed irreversibly, and thus avoids problematic retro-aldol reactions that often prevent the use of aldol reactions in the formation of macrocyclic ring systems. ${ }^{14}$ Research in both the Trost and Overman groups has shown that thionium ions behave as 'super carbonyl' equivalents and react effectively with nucleophilic alkenes. ${ }^{15}$ This reactivity led to the hypothesis that this methodology would be an excellent candidate for the challenging task of forming the strained humulene-based macrocycle.

Retrosynthetic disconnection of asteriscunolide D (146) began with the aforementioned thionium-induced macrocyclization and stereospecific thioether elimination (Scheme 3.4). The
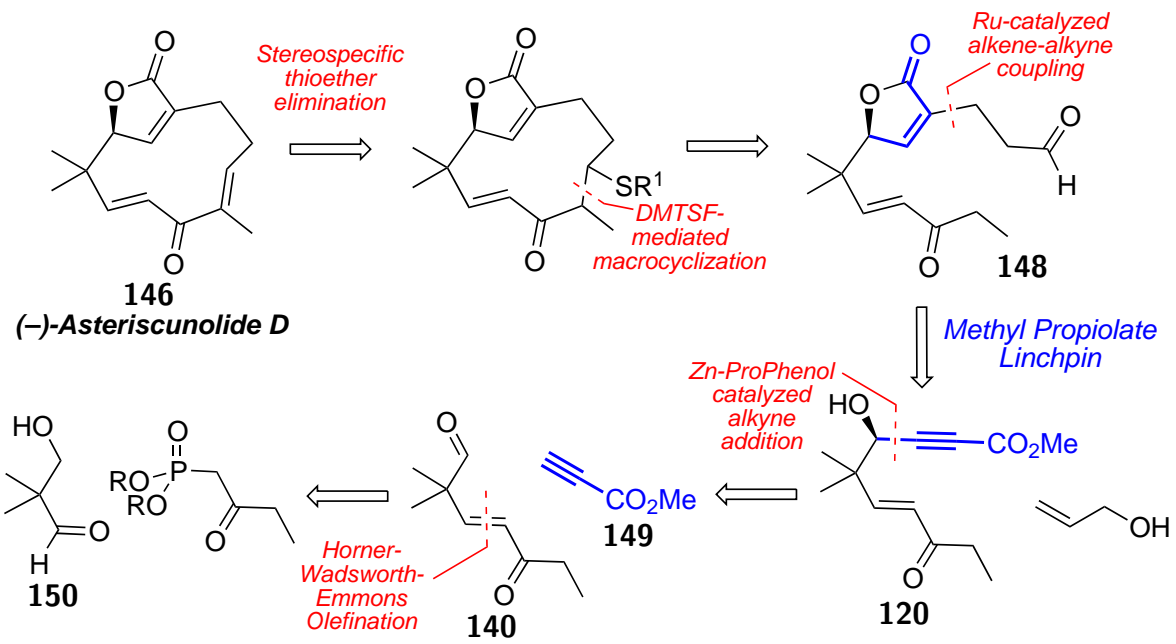

Scheme 3.4 Retrosynthetic Analysis of Asteriscunolide D.

thioacetal and silyl enol ether functional groups required for this transformation would be generated from the corresponding aldehyde and ketone, respectively. This sequence constitutes a formal aldol disconnection and leads back to keto aldehyde 148. This compound would in turn be prepared using a Ru-catalyzed addition of allyl alcohol to the $\gamma$-hydroxy- $\alpha, \beta$-acetylenic ester 120. The alkene-alkyne coupling reaction ultimately forms the required butenolide and occurs with alkene migration to produce an enol, which would tautomerize to form the desired aldehyde functionality. One potential problem was the racemization of the enantioenriched butenolide upon exposure to basic reaction conditions. However, the adjacent gem-dimethyl group and the macrocyclic ring strain are likely to help prevent the racemization of late-stage intermediates. The propargylic alcohol $\mathbf{1 2 0}$ was envisioned to arise from the Zn-ProPhenol catalyzed addition of methyl propiolate $(\mathbf{1 4 9})$ to the aliphatic aldehyde $\mathbf{1 4 0}$. The efficiency of this sequence is derived from the use of methyl propiolate as linchpin - three key bonds of the natural product are formed with atoms derived from methyl propiolate, ultimately forming the chiral butenolide and introducing all the carbons present in the natural product. Lastly, ketoaldehyde 140 would be prepared via Horner-Wadsworth-Emmons olefination of the $\beta$-hydroxy aldehyde 150. 


\subsubsection{Linchpin-Based Butenolide Construction}

The forward synthesis of asteriscunolide D commenced with the aldol reaction of isobutyraldehyde and formalin to provide the $\beta$-hydroxy aldehyde 150 in the dimeric 1,3-dioxane form 151 (Scheme 3.5). This highly efficient aldol reaction requires only a catalytic amount of

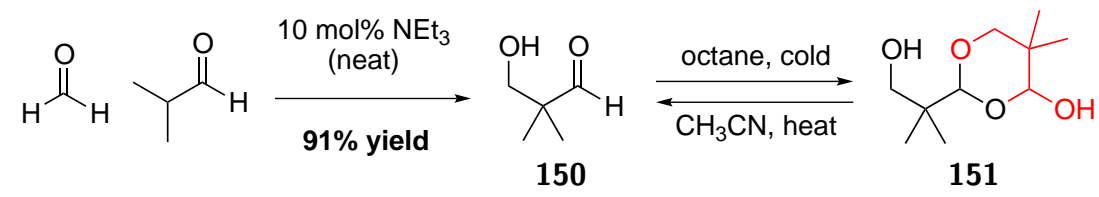

Scheme 3.5 Aldol Reaction of Isobutyraldehyde and Formalin.

triethylamine and provides the desired product $\mathbf{1 5 1}$ in $91 \%$ yield. The higher electrophilicity of formaldehyde relative to isobutyraldehyde and the product aldehyde, 150, means that homo aldol and other unwanted aldol reactions are kept to a minimum. In a non-polar solvent at $4{ }^{\circ} \mathrm{C}$, the product $\beta$-hydroxy aldehyde readily dimerizes to give the stable, crystalline 1,3 dioxan-4-ol 151 as an inconsequential mixture of diastereomers. The dimer, 151, can be converted back into the monomeric $\beta$-hydroxy aldehyde 150 by heating in a polar solvent such as acetonitrile or THF. This cracking procedure was performed in situ with a HornerWadsworth-Emmons olefination of the resulting aldehyde 150 (Scheme 3.6). ${ }^{\dagger}$ The use of mild Masamune-Roush conditions ( $\mathrm{LiCl}, \mathrm{DBU}$ ) with diethyl (2-oxobutyl)phosphonate in THF provided the desired $\alpha, \beta$-unsaturated ketone 152 in $35 \%$ yield. ${ }^{17}$ In contrast, the use of a strong base, $\mathrm{KO} t \mathrm{Bu}$, provided only the undesired Tishchenko esterification side product, $\mathbf{1 5 3}{ }^{18}$ The

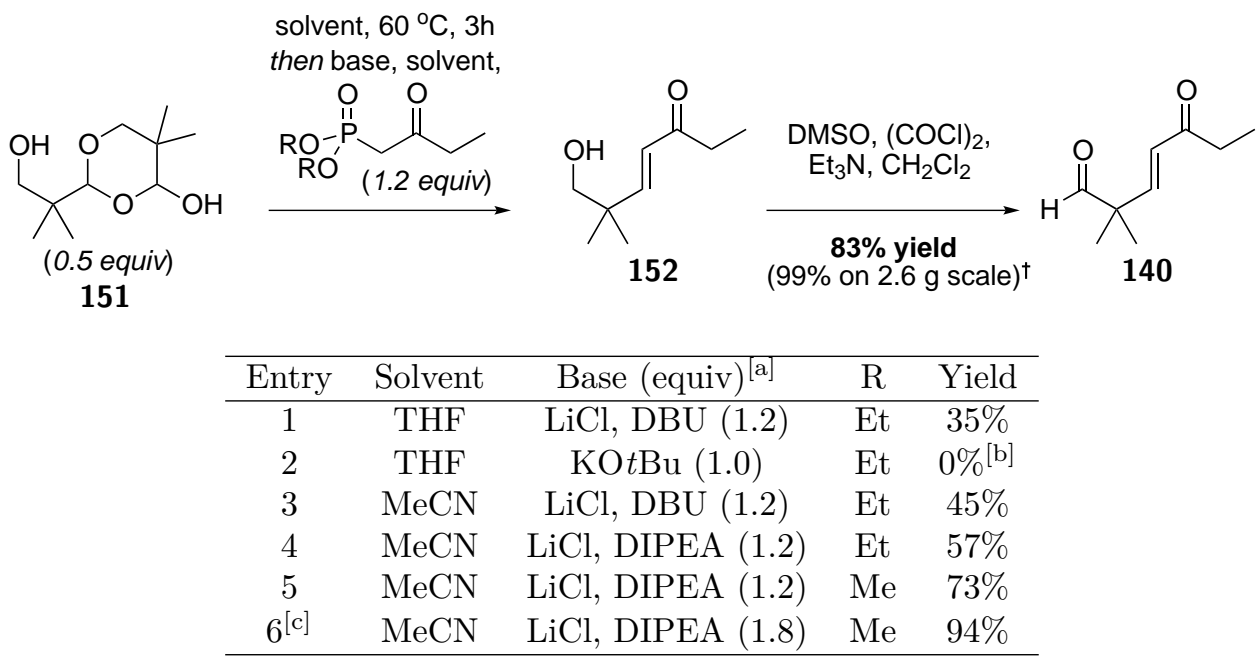

[a] equimolar amounts of $\mathrm{LiCl}$ and amine base were used in all cases. [b] The Tishchenko side product $\mathbf{1 5 3}$ was the major product of this reaction. [c] 1.8 equivalents of phosphonate was used, reaction performed on a 9.8 mmol scale ( $1 \mathrm{~g}$ of $\mathbf{1 5 1})$.

Scheme 3.6 Horner-Wadsworth-Emmons Olefination and Oxidation to Prepare 140.

\footnotetext{
${ }^{\dagger}$ Entries 5 and 6 , and the large scale oxidation were performed by Aaron Burns and Thomas Tautz.
} 
proposed mechanism of this side reaction is shown in Scheme 3.7 and involves an intramolecular hydride shift to provide the ester $\mathbf{1 5 3} .{ }^{19}$ This side reaction requires only a catalytic amount of metal alkoxide, and therefore the use of weakly basic reaction conditions became the focus of optimization experiments. Performing the reaction in acetonitrile under Masamune-Roush

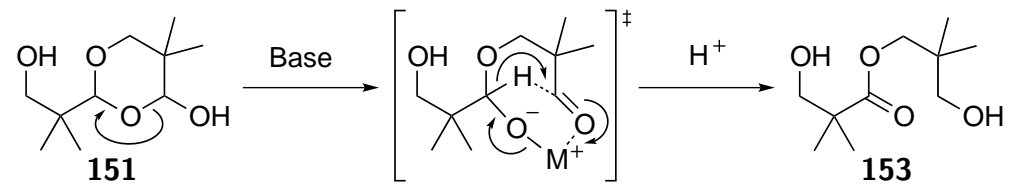

Scheme 3.7 Tishchenko Disproportionation: An Unwanted Side Reaction.

conditions gave a slight improvement in yield, as did the use of $i \mathrm{Pr}_{2} \mathrm{NEt}$ instead of DBU (Entries 3 and 4). Surprisingly, the use of dimethyl (2-oxobutyl)phosphonate gave higher yields than the corresponding diethyl phosphonate. Increasing the reagent stoichiometry to 1.8 equivalents of $\mathrm{LiCl}$, DIPEA, and phosphonate provided the desired $\alpha, \beta$-unsaturated ketone $\mathbf{1 5 2}$ in an excellent $94 \%$ yield. Subsequent Moffatt-Swern oxidation of $\mathbf{1 5 2}$ provided the aldehyde, 140, required for the key asymmetric alkynylation step.

The original super-stoichiometric reaction conditions with $10 \mathrm{~mol} \%$ of the ProPhenol ligand provided the desired propargylic alcohol in $31 \%$ yield and $73 \%$ ee (Scheme 3.8, Entry 1). ${ }^{20}$ The presence of an enolizable ketone, capable of producing aldol side products, ${ }^{21}$ demands a particularly chemoselective alkyne addition. Thus, we were delighted to discover that increasing the catalyst loading to 20 mol \% produced a marked increase in yield, providing propargylic alcohol $\mathbf{1 2 0}$ in $72 \%$ yield and $86 \%$ ee (Entry 3). As reported in the literature, moderate and large scale reactions required freeze-pump-thaw solvent degassing to ensure consistent results. ${ }^{22 \ddagger}$ Using just 1.2 equivalents of methyl propiolate in conjunction with

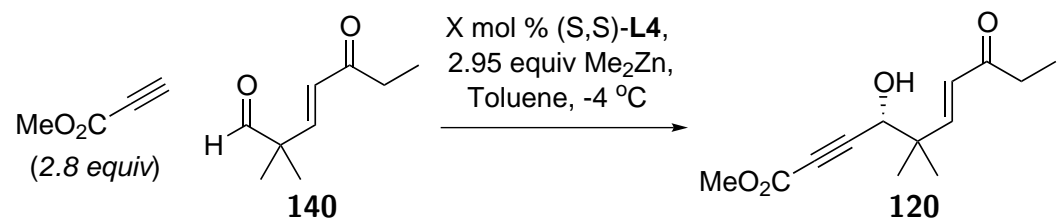

\begin{tabular}{ccccc}
\hline Entry & $\mathrm{X}$ & Conditions & Yield & ee \\
\hline 1 & 10 & - & $31 \%$ & $73 \%$ \\
$2^{[\mathrm{b}]}$ & 20 & Freeze-Pump-Thaw Degassing & $72 \%$ & $86 \%$ \\
$3^{[\mathrm{c}]}$ & 20 & Freeze-Pump-Thaw Degassing & $83 \%$ & $84 \%$ \\
4 & 20 & $1.2 / 1.5$ equiv alkyne/ $/ \mathrm{Me}_{2} \mathrm{Zn}$ & $62 \%$ & $78 \%$ \\
5 & 20 & $1.2 / 3.0$ equiv alkyne/ $\mathrm{Me}_{2} \mathrm{Zn}$ & $61 \%$ & $60 \%$ \\
\hline
\end{tabular}

[a] Enantiomeric excess determined by chiral HPLC analysis. Absolute stereochemistry was assigned by analogy to previous results with $(S, S)$-L4 but confirmed by the X-ray crystal structure of a subsequent synthetic intermediate, 137. [b] Reaction performed on a $6.16 \mathrm{mmol}$ scale $(0.95 \mathrm{~g}$ of 140) [c] Reaction performed on a $16.9 \mathrm{mmol}$ scale $(2.6 \mathrm{~g}$ of $\mathbf{1 4 0})$.

Scheme 3.8 Asymmetric Alkyne Addition in the Synthesis of Asteriscunolide D.

\footnotetext{
${ }^{\ddagger}$ Entry 3 was performed by Aaron Burns and Thomas Tautz.
} 
increased reaction concentration $(0.48 \mathrm{M})$ gave a respectable $62 \%$ yield and $78 \%$ ee (Entry 4 ). This result is particularly notable, as many of the alternative catalyst systems used for the enantioselective addition of methyl propiolate require a large excess of alkyne.

The $\gamma$-hydroxy- $\alpha, \beta$-acetylenic ester $\mathbf{1 2 0}$ was subsequently used in a ruthenium-catalyzed alkene-alkyne coupling with allyl alcohol (Scheme 3.9). ${ }^{23}$ Using just $5 \mathrm{~mol} \%$ of the cationic ruthenium catalyst $\mathrm{CpRu}(\mathrm{MeCN}){ }_{3} \mathrm{PF}_{6}$, the desired chiral butenolide, 148, was obtained in $51 \%$ yield. This reaction provides regioselective $\mathrm{C}-\mathrm{C}$ bond formation at the $\alpha$-position of

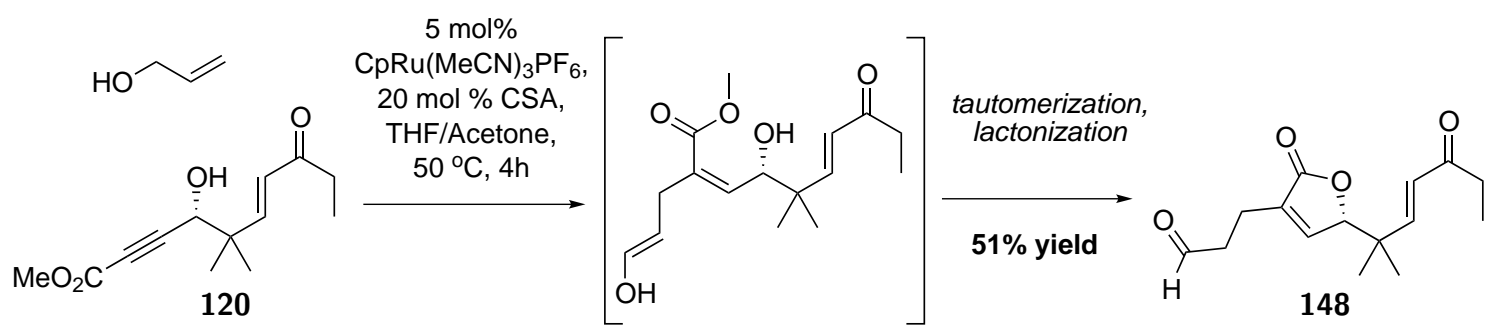

Scheme 3.9 Ru-Catalyzed Alkene-Alkyne Coupling with Allyl Alcohol.

the propiolate via a formal syn-addition process to give the desired $Z$-alkene geometry, which leads to spontaneous butenolide formation. The regioselectivity of alkene-alkyne coupling was confirmed by ${ }^{1} \mathrm{H} \mathrm{NMR}$ - specifically, the presence of a distinct doublet $(J=1.5 \mathrm{~Hz})$ at $7.06 \mathrm{ppm}$ corresponding to the alkenyl proton $\beta$ to the carbonyl of the butenolide ring. The proposed mechanism for this reaction is shown in Scheme 3.10 and the selectivity for attack at the $\alpha$-position is attributed to a preference for TS2 over TS1, due to reduced steric interactions between the alkyne substituents and the incoming alkene. Additionally, the regioselectivity of this reaction is complimentary to that observed in Michael additions to ynoates, which are driven by electronic factors.

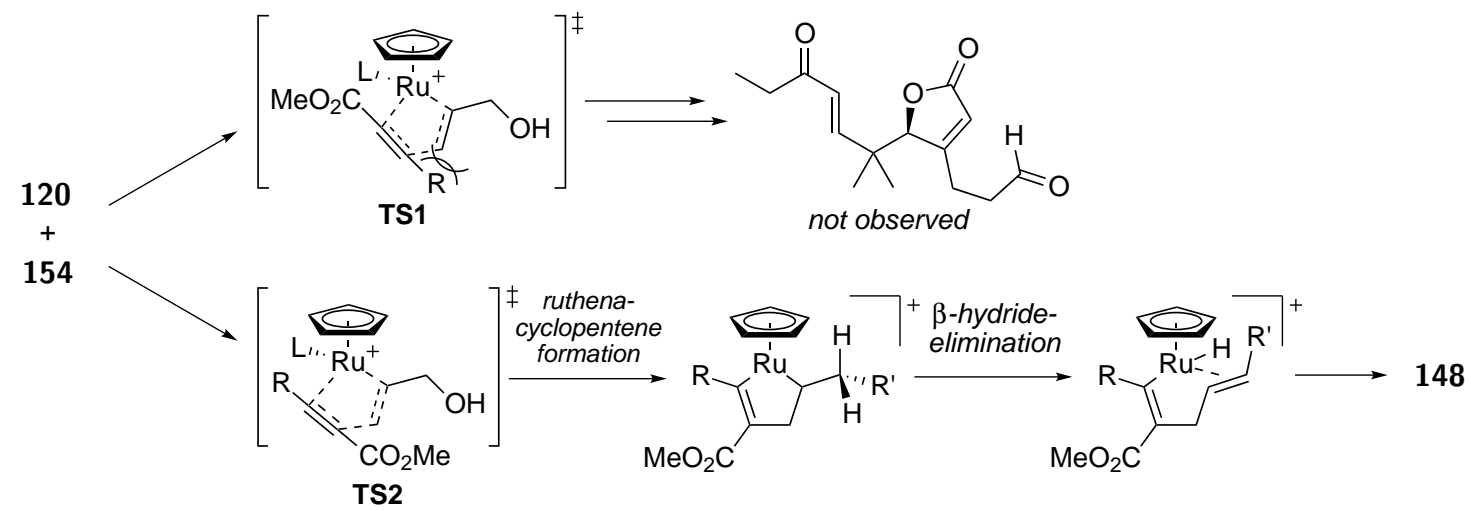

Scheme 3.10 Alkene-Alkyne Coupling Mechanism and Selectivity. 


\subsubsection{End Game}

With the carbons of the natural product in place, all that remained was the formation of the challenging 11-membered humulene ring. ${ }^{\S}$ Keto-aldehyde 148 was chemoselectively converted into dithiophenyl acetal $\mathbf{1 5 5}$ in $79 \%$ yield using $\mathrm{BF}_{3} \cdot \mathrm{Et}_{2} \mathrm{O}$ and thiophenol (Scheme 3.11). ${ }^{24}$ Subsequent silyl enol ether formation was achieved using TMSOTf and Hünig's base to provide compound 156. These soft enolization conditions generate the $Z$-TMS-enol ether $\mathbf{1 5 6}$ selectively, providing the substrate needed to perform the key thionium-induced cyclization. ${ }^{25}$

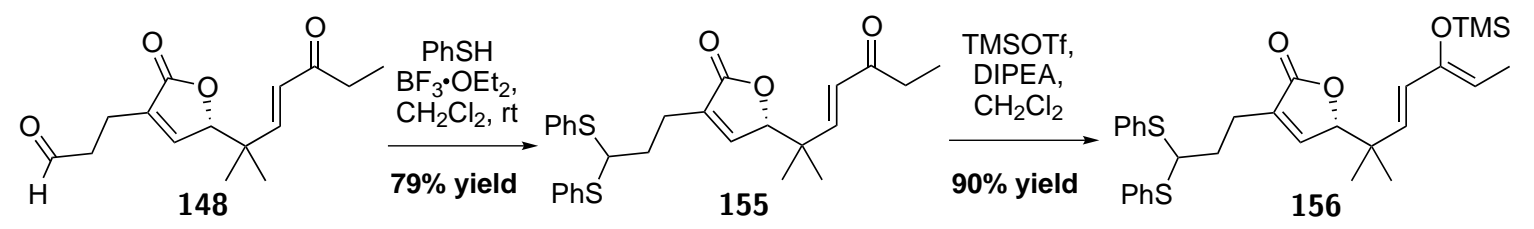

Scheme 3.11 Installation of the Cyclization Functionality.

Addition of 1.2 equivalents of dimethyl(methylthio)sulfonium tetrafluoroborate (DMTSF) to compound 156 at $-30{ }^{\circ} \mathrm{C}$ afforded the desired macrocyclic product, 137, in $41 \%$ yield (Scheme 3.12). The restricted geometry required for cyclization provided the product as a single diastereomer, which was recrystallized to improve the ee to $>98 \%$ and provide an X-ray crystal structure to confirm the structure and absolute stereochemistry.
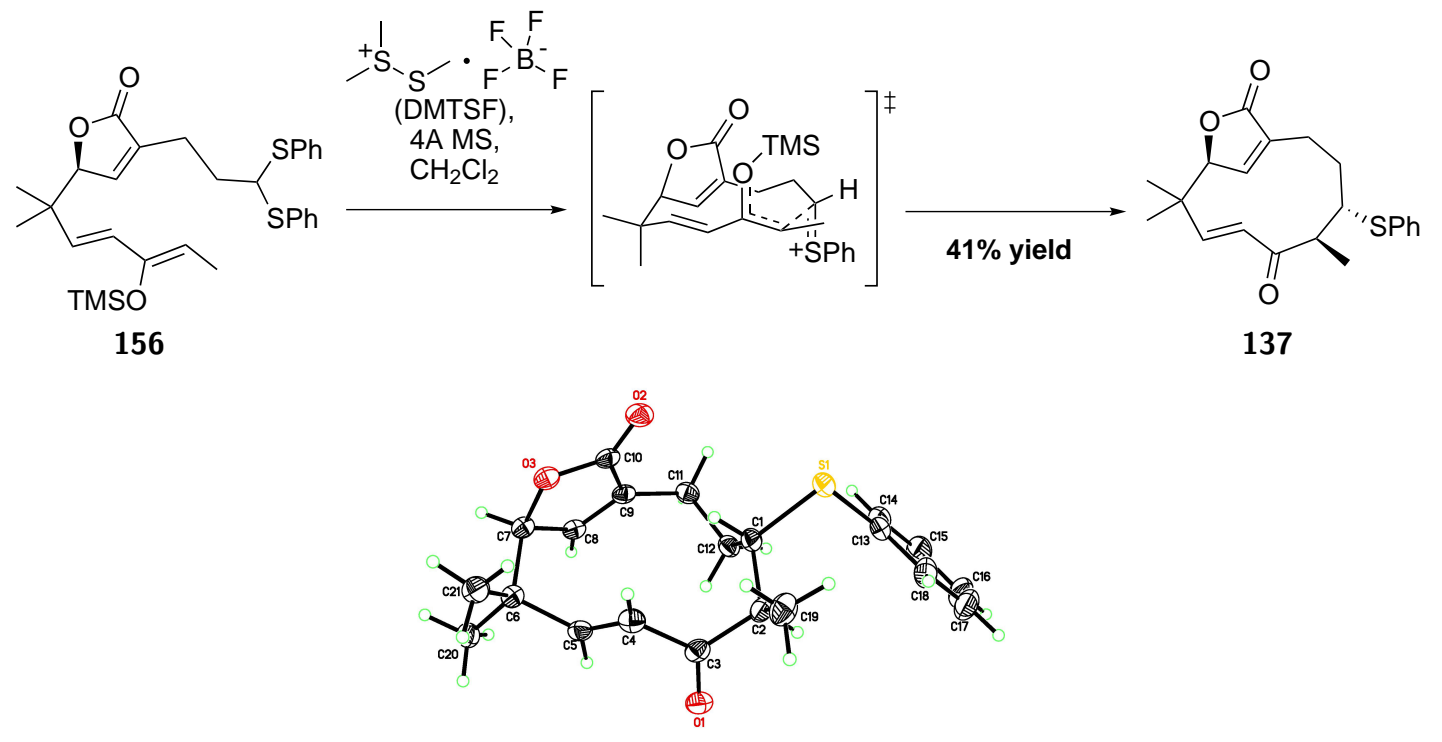

Scheme 3.12 Thionium-Induced Macrocyclization.

The final alkylative thioether elimination was performed using Meerwein's salt $\left(\mathrm{Me}_{3} \mathrm{OBF}_{4}\right)$ and Hünig's base, and generated the desired $E$-alkene stereospecifically, providing asteriscunolide D (146) in $83 \%$ yield. ${ }^{26}$ This Hoffmann-type elimination requires an antiperiplanar orientation, and therefore produces the $E$-alkene geometry despite the potential formation

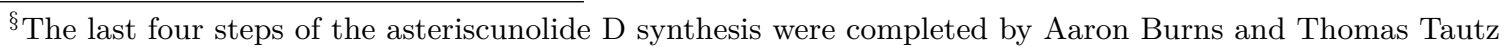




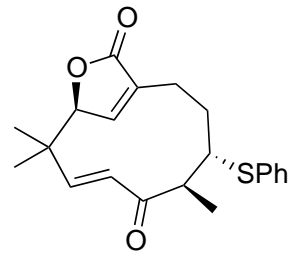

137

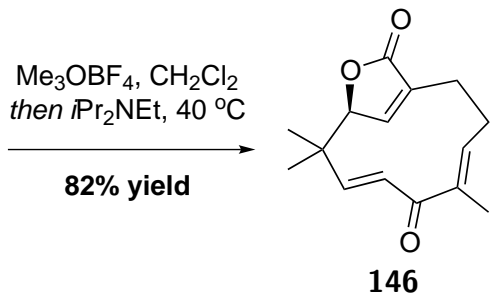

(-)-Asteriscunolide $D$

Scheme 3.13 Stereospecific Thioether Elimination to Complete the Total Synthesis.

of other alkenes. Racemization during the synthesis was excluded by performing chiral HPLC analysis on each intermediate and comparing the results with the corresponding racemate. Asteriscunolide D could also be used to access the other members of the asteriscunolide family. Thus, isomerization using $\mathrm{PhSeSePh}$ and UV light provided a $c a$. 3:3:1:1 mixture of asteriscunolides A, B, C, and D, respectively. This result suggests that all four natural products have similar thermodynamic stabilities and highlights the importance of strict stereochemical control during the synthesis.

\subsection{Summary}

In summary, the first total synthesis of asteriscunolide D was achieved in nine steps without the use of protecting groups, and is outlined in Scheme 3.14. ${ }^{27}$ The chiral butenolide motif was prepared enantioselectively through the sequential application of Zn-ProPhenol-catalyzed asymmetric alkynylation and Ru-catalyzed alkene-alkyne coupling. The challenging 11membered humulene ring was forged using a thionium-induced cyclization. This reaction serves as a highly efficient aldol surrogate, and avoids problems associated with competing retro-aldol processes. At the core of this succinct and selective synthetic strategy is the use of methyl propiolate as a strategic linchpin. In just two steps, three new bonds were formed to atoms derived from methyl propiolate and this ultimately enabled the construction of a synthetically challenging natural product almost 20 years after the orginal disclosure of its structure. 

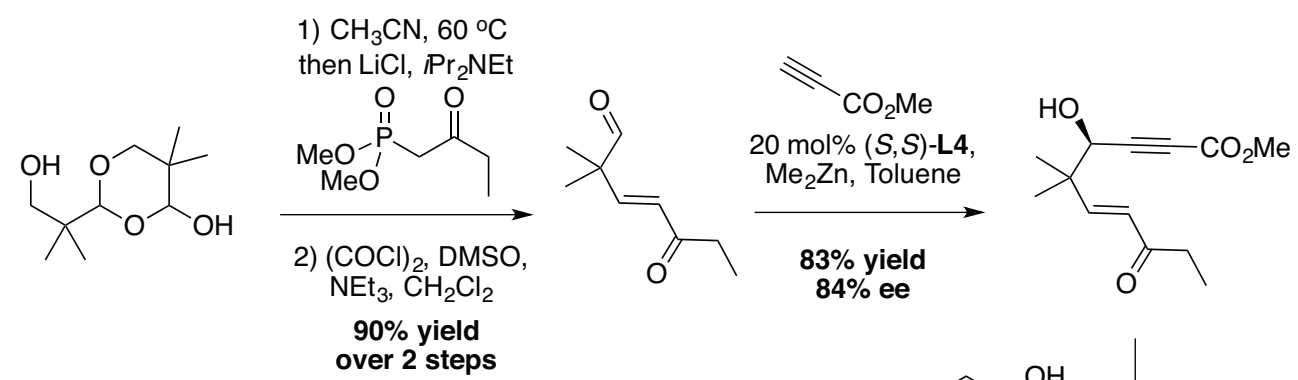

$$
\begin{gathered}
90 \% \text { yield } \\
\text { over } 2 \text { steps }
\end{gathered}
$$
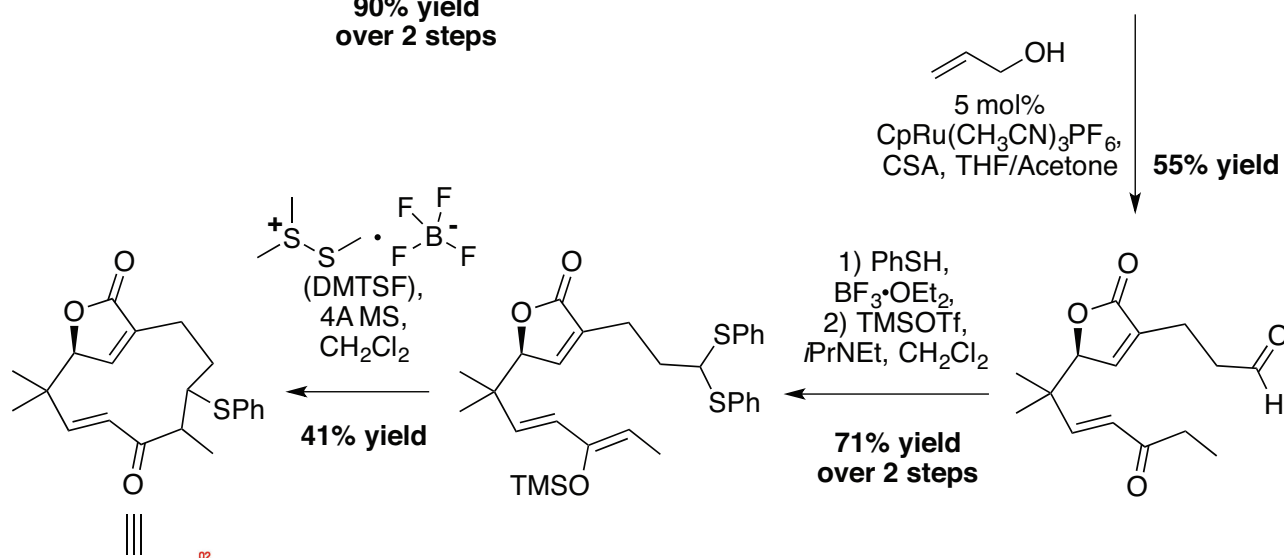

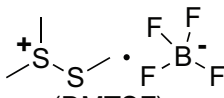
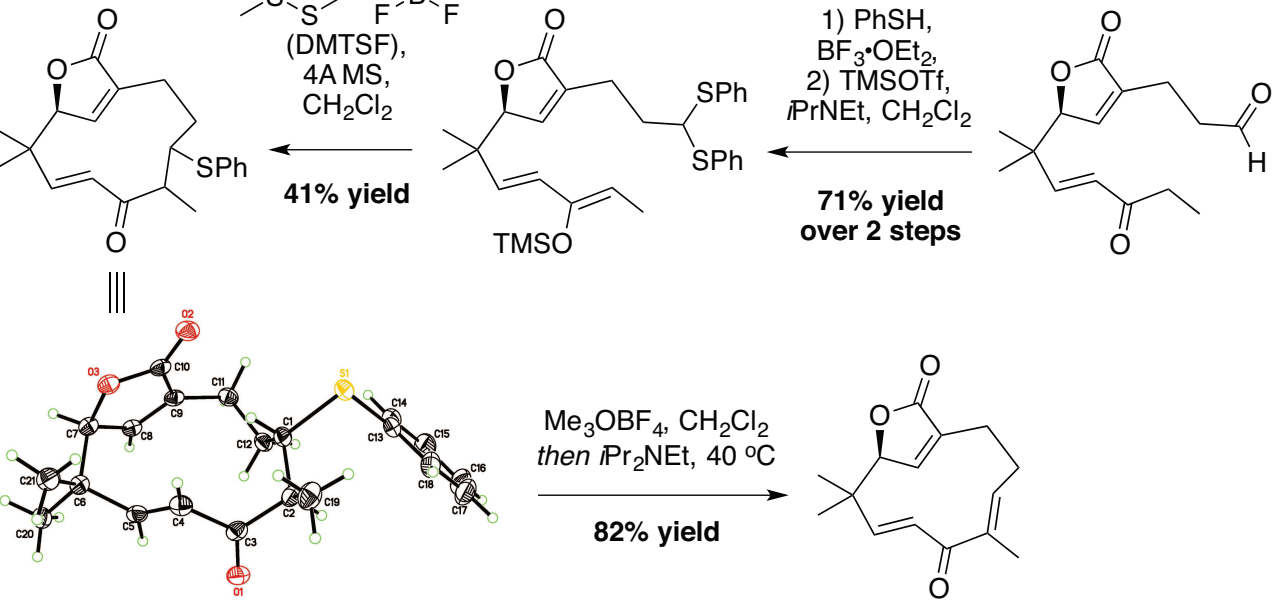

(-)-Asteriscunolide D

Scheme 3.14 Enantioselective Total Synthesis of Asteriscunolide D. 


\subsection{Experimental Data for Chapter 3}

\subsubsection{General Experimental Methods}

Unless otherwise noted, the following conditions apply. All reactions were performed in flame-dried septum sealed glassware with magnetic stirring under an atmosphere of argon or nitrogen. Moisture- and oxygen-sensitive liquids and solutions were transferred using an oven-dried stainless steel syringe or cannula. Toluene, tetrahydrofuran, acetonitrile, diethyl ether, dimethylsulfoxide (DMSO), and dichloromethane were purified with a Solv-Tek solvent purification system by passing through a column of activated alumina. Isobutyraldehyde was distilled immediately before use. $N, N$-Diisopropylethylamine were distilled from calcium hydride. Lithium chloride was dried at $120{ }^{\circ} \mathrm{C}$ for 24 hours before use. All other commercially available chemicals were used as received, without further purification. Organic solutions were concentrated by rotary evaporation below $40{ }^{\circ} \mathrm{C}$. All isolated compounds were $>90 \%$ pure as judged by ${ }^{1} \mathrm{H}-\mathrm{NMR}$. Analytical thin layer chromatography (TLC) was performed on EMD silica gel $60 \mathrm{~F}_{254}$ plates $(0.25 \mathrm{~mm})$. Visualization was achieved by UV irradiation $(254 \mathrm{~nm})$ or by heating after treatment with a potassium permanganate or ceric ammonium molybdate dip. Purification of products by flash column chromatography (FCC) was conducted using Silicycle silica gel (particle size 0.040-0.063 mm) with the solvent systems indicated. ${ }^{1} \mathrm{HNMR}$ spectra were recorded on either: a Varian Unity Inova 500 spectrometer at $500 \mathrm{MHz}$, a Varian Mercury NMR spectrometer at $400 \mathrm{MHz}$, Varian Direct Drive 400 spectrometer at $400 \mathrm{MHz}$ or a Varian Inova 300 at $300 \mathrm{MHz}$. Data are listed as follows: chemical shift in $\mathrm{ppm}$ using chloroform as internal standard $(7.26 \mathrm{ppm})$, multiplicity $(\mathrm{s}=$ singlet, $\mathrm{d}=$ doublet, $\mathrm{t}=$ triplet, $\mathrm{q}=$ quartet, quint $=$ quintet, $\mathrm{m}=$ multiplet or overlap of non-equivalent resonances, $\mathrm{br}=$ broad, app. $=$ apparent, obs. $=$ (partially) obscured), integration. ${ }^{13} \mathrm{C}-$ NMR spectra were recorded on a Varian Unity Inova 500 spectrometer at $126 \mathrm{MHz}$ or a Varian Mercury NMR spectrometer at $101 \mathrm{MHz}$ and the data are listed as chemical shift in ppm using chloroform as internal standard $(77 \mathrm{ppm})$. All ${ }^{13} \mathrm{C}-\mathrm{NMR}$ spectra were proton decoupled. Infrared spectroscopic data was recorded as a thin film on a sodium chloride plate using a Thermo Scientific Nicolet IR100 FT-IR spectrometer. High resolution mass spectra were acquired by the Vincent Coates Foundation Mass Spectrometry Laboratory, Stanford University (http://massspec.stanford.edu) on a Micromass Q-TOF API-US mass spectrometer (Waters Corporation, Milford, MA). Chiral HPLC analysis was performed on a Thermo Separation Products Spectra Series P-100 and Agilent Technologies 1200 Series HPLC using the indicated Chiralcel ${ }^{\circledR}$ and Chiralpak ${ }^{\circledR}$ columns. Optical rotations were measured using a Jasco DIP-1000 digital polarimeter using $5 \mathrm{~cm}$ glass cells with a Na $589 \mathrm{~nm}$ filter. Specific rotations are reported as $[\alpha]_{\mathrm{D}}^{25}$, concentration $(\mathrm{g} / 100 \mathrm{~mL})$, and solvent.

Note: The experimental data reported below reflects the initial work on this project carried out by the author. For details of larger scale experiments and the full synthesis, see: Trost, B. M.; Burns, A. C.; Bartlett, M. J.; Tautz, T.; Weiss, A. H. J. Am. Chem. Soc. 2012, 134, 1474 . 


\subsubsection{Experimental Details and Characterization Data}

Preparation of 2-(2-Hydroxy-1,1-dimethyl-ethyl)-5,5-dimethyl-[1,3]dioxan-4-ol, 151.

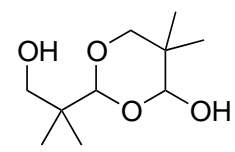

To a mixture of neat isobutyraldehyde $(1.28 \mathrm{~mL}, 14 \mathrm{mmol}, 1$ equiv) and triethylamine (0.195 mL, $1.4 \mathrm{mmol}, 0.1$ equiv) was added $37 \mathrm{wt} \%$ formaldehyde in $\mathrm{H}_{2} \mathrm{O}$ (formalin, $1.216 \mathrm{~g}$, $14.98 \mathrm{mmol}, 1.07$ equiv). The reaction mixture was heated to $60{ }^{\circ} \mathrm{C}$ and stirred for 1 hour, then cooled to room temperature and octane $(3.5 \mathrm{~mL})$ was added. The resulting suspension was stored at $4{ }^{\circ} \mathrm{C}$ overnight and the resulting precipitate was isolated by filteration and washed with cold octane. The title compound was isolated as a white crystalline solid (1.304 g, $91 \%$ yield, mixture of diastereomers). $\mathbf{R}_{\mathbf{f}}=0.44$ and 0.39 (2:1, Et $\left.{ }_{2} \mathrm{O}: \mathrm{PE}\right) .{ }^{\mathbf{1}} \mathbf{H}-\mathbf{N M R}(400 \mathrm{MHz}$, $\left.\mathrm{CDCl}_{3}\right): \delta 4.90(\mathrm{~s}, 1 \mathrm{H}), 4.82(\mathrm{~s}, 1 \mathrm{H}), 4.59(\mathrm{~s}, 1 \mathrm{H}), 4.41(\mathrm{~s}, 1 \mathrm{H}), 3.86(\mathrm{~d}, J=11.2 \mathrm{~Hz}, 1 \mathrm{H}), 3.62$ $(\mathrm{d}, J=11.2 \mathrm{~Hz}, 1 \mathrm{H}), 3.49(\mathrm{~s}, 2 \mathrm{H}), 3.46(\mathrm{~s}, 2 \mathrm{H}), 3.41(\mathrm{dd}, J=10.8,1.6 \mathrm{~Hz}, 1 \mathrm{H}), 3.35(\mathrm{dd}, J$ $=11.6,0.8 \mathrm{~Hz}, 1 \mathrm{H}), 1.17(\mathrm{~s}, 3 \mathrm{H}), 1.06(\mathrm{~s}, 3 \mathrm{H}), 0.96(\mathrm{~s}, 3 \mathrm{H}), 0.96(\mathrm{~s}, 3 \mathrm{H}), 0.94(\mathrm{~s}, 3 \mathrm{H}), 0.92(\mathrm{~s}$, $3 \mathrm{H}), 0.83(\mathrm{~s}, 3 \mathrm{H}), 0.82(\mathrm{~s}, 3 \mathrm{H})$. Characterization data matches those found in the literature. ${ }^{16}$

\section{Preparation of (E)-7-Hydroxy-6,6-dimethylhept-4-en-3-one, 152.}<smiles>CCC(=O)/C=C/C(C)(C)CO</smiles>

A solution of 2-(2-hydroxy-1,1-dimethyl-ethyl)-5,5-dimethyl-[1,3] dioxan-4-ol, 151 (0.1 g, $0.49 \mathrm{mmol}, 1$ equiv) in acetonitrile $(5 \mathrm{~mL})$ was heated to $65{ }^{\circ} \mathrm{C}$ and stirred for 3 hours. This solution was then cooled to room temperature and transferred via cannula to a preformed solution of lithium chloride $(0.025 \mathrm{~g}, 0.587 \mathrm{mmol}, 1.2 \mathrm{equiv}), N, N$-diisopropylethylamine $(0.101$ mL, $0.98 \mathrm{mmol}, 1.2$ equiv.) and diethyl 2-oxobutanephosphonate (0.114 mL, $0.587 \mathrm{mmol}, 1.2$ equiv.) in acetonitrile $(5 \mathrm{~mL})$. The reaction mixture was stirred for 1 hour at room temperature before being quenched with a $1 \mathrm{M}$ solution of aqueous $\mathrm{HCl}$ and subsequently extracted with $\mathrm{Et}_{2} \mathrm{O}$. The combined organics were dried over $\mathrm{MgSO}_{4}$, filtered, and concentrated in vacuo. The crude product was purified by silica gel chromatography $\left(2: 1, \mathrm{Et}_{2} \mathrm{O}: \mathrm{PE}\right)$ and the title compound was isolated as a clear colorless oil (0.088 g, $57 \%$ yield). $\mathbf{R}_{\mathbf{f}}=0.35$ (2:1, Et $\left.{ }_{2} \mathrm{O}: \mathrm{PE}\right)$. IR (film): 3439 (br), 2966, 2938, 1668, 1625, $1362 \mathrm{~cm}^{-1} \cdot{ }^{\mathbf{1}} \mathbf{H}-\mathbf{N M R}\left(500 \mathrm{MHz}, \mathrm{CDCl}_{3}\right): \delta$ $6.82(\mathrm{~d}, J=16.0 \mathrm{~Hz}, 1 \mathrm{H}), 6.12(\mathrm{~d}, J=16.5 \mathrm{~Hz}, 1 \mathrm{H}), 3.46(\mathrm{br} . \mathrm{s}, 2 \mathrm{H}), 2.60(\mathrm{q}, J=7.0 \mathrm{~Hz}$, $2 \mathrm{H}), 1.10(\mathrm{t}, J=7.5 \mathrm{~Hz}, 3 \mathrm{H}), 1.09$ (s, 6H). ${ }^{13} \mathrm{C}-\mathbf{N M R}\left(125 \mathrm{MHz}^{\mathrm{CDCl}} \mathrm{CD}_{3}\right): \delta 201.7,153.0$, 128.0, 71.2, 39.4, 33.9, 23.4, 8.3. HRMS-ESI $(m / z)$ : calculated for $\mathrm{C}_{9} \mathrm{H}_{16} \mathrm{O}_{2} \mathrm{Na}^{+},[\mathrm{M}+\mathrm{Na}]^{+}$, 179.1043, found 179.1053 . 


\section{Preparation of (E)-2,2-Dimethyl-5-oxohept-3-enal, 140.}<smiles>CCC(=O)/C=C/C(C)(C)C=O</smiles>

To a stirred solution of oxalyl chloride $\left(0.798 \mathrm{~mL}, 9.29 \mathrm{mmol}, 1.1\right.$ equiv) in $\mathrm{CH}_{2} \mathrm{Cl}_{2}(30$ $\mathrm{mL})$ at $-78{ }^{\circ} \mathrm{C}$ was added a solution of dimethylsulfoxide (1.72 mL, $20.28 \mathrm{mmol}, 2.4$ equiv), dropwise, in $\mathrm{CH}_{2} \mathrm{Cl}_{2}(30 \mathrm{~mL})$. (E)-7-hydroxy-6,6-dimethylhept-4-en-3-one, 152. (1.32 g, 8.45 mmol, 1.0 equiv) was then added dropwise to the reaction mixture followed by continued stirring for $1 \mathrm{~h}$. Triethylamine $(5.91 \mathrm{~mL}, 42.25 \mathrm{mmol}, 5.0$ equiv) was added dropwise and the temperature was maintained at $-78{ }^{\circ} \mathrm{C}$ for an additional 75 min before quenching with saturated aqueous $\mathrm{NH}_{4} \mathrm{Cl}$ and allowing the mixture to warm to room temperature. The reaction mixture was then diluted with water and extracted with $\mathrm{CH}_{2} \mathrm{Cl}_{2}$. The combined organic extracts were washed with brine, dried over $\mathrm{MgSO}_{4}$, filtered, and concentrated in vacuo. The crude product was purified by silica gel chromatography $\left(1: 1 \mathrm{PE} / \mathrm{Et}_{2} \mathrm{O}\right)$ affording the title compound as a pale yellow oil. (1.079 g, $83 \%$ yield). $\mathbf{R}_{\mathbf{f}}=0.65\left(1: 2, \mathrm{PE} / \mathrm{Et}_{2} \mathrm{O}\right) .{ }^{1} \mathbf{H}$ NMR $\left(400 \mathrm{MHz}, \mathrm{CDCl}_{3}\right): \delta 9.46(\mathrm{~s}, 1 \mathrm{H}), 6.82(\mathrm{~d}, J=16.5 \mathrm{~Hz}, 1 \mathrm{H}), 6.15(\mathrm{~d}, J=16.5 \mathrm{~Hz}$, $1 \mathrm{H}), 2.61(\mathrm{q}, J=7.0 \mathrm{~Hz}, 2 \mathrm{H}), 1.28(\mathrm{~s}, 6 \mathrm{H}), 1.11(\mathrm{t}, J=7.0 \mathrm{~Hz}, 3 \mathrm{H}) .{ }^{13} \mathrm{C}$ NMR $(100 \mathrm{MHz}$, $\mathrm{CDCl}_{3}$ ): $\delta 201.2,200.6,146.4,129.8,49.3,34.1,21.5,8.1$. IR (film): 2976, 2939, 2878, 1728, $1702,1677,1626,1461,1377,1364,1320,1289,1202,1123,1046,983,894,779 \mathrm{~cm}^{-1}$.

Preparation of $(R, E)$-Methyl 4-hydroxy-5,5-dimethyl-8-oxodec-6-en-2-ynoate, 120.

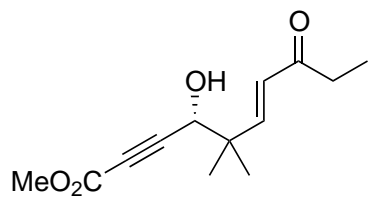

To a solution of $(S, S)$ Prophenol ligand (0.789 g, $1.23 \mathrm{mmol}, 20 \mathrm{~mol} \%)$ in $37.9 \mathrm{~mL}$ of freshly degassed toluene (freeze-pump-thaw) was added $1.54 \mathrm{~mL}$ of methyl propiolate $(17.25 \mathrm{mmol}, 2.8$ equiv) and $15.16 \mathrm{~mL}$ of dimethylzinc (1.2 $\mathrm{M}$ in toluene, $18.17 \mathrm{mmol}, 2.95$ equiv) at $0{ }^{\circ} \mathrm{C}$. The reaction mixture was subsequently stirred at room temperature for 60 minutes before addition of 2,2-dimethyl-5-oxo-hept-3-enal , 140. (0.95 g, $6.16 \mathrm{mmol}, 1$ equiv) at $0{ }^{\circ} \mathrm{C}$. The reaction was moved to a $4{ }^{\circ} \mathrm{C}$ cold room and stirred for 48 hours. After this time the reaction was quenched with saturated, aqueous $\mathrm{NH}_{4} \mathrm{Cl}(40 \mathrm{~mL})$ and stirred vigorously for 5 minutes. The reaction mixture was extracted with $\mathrm{Et}_{2} \mathrm{O}$. The combined organics were concentrated in vacuo and purified by flash column chromatography to provide the title compound as a yellow oil

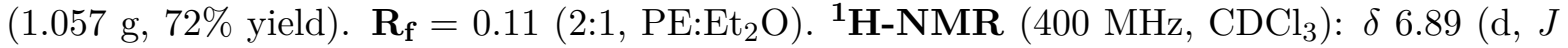
$=16.4 \mathrm{~Hz}, 1 \mathrm{H}), 6.16(\mathrm{~d}, J=16.4 \mathrm{~Hz}, 1 \mathrm{H}), 4.29(\mathrm{~s}, 1 \mathrm{H}), 3.78(\mathrm{~s}, 3 \mathrm{H}), 2.60(\mathrm{q}, J=7.2 \mathrm{~Hz}$, $2 \mathrm{H}), 1.42(\mathrm{~s}, 1 \mathrm{H}), 1.21(\mathrm{~s}, 3 \mathrm{H}), 1.20(\mathrm{~s}, 3 \mathrm{H}), 1.1(\mathrm{t}, J=7.6 \mathrm{~Hz}, 3 \mathrm{H}) .{ }^{\mathbf{1 3}} \mathbf{C}-\mathbf{N M R}\left(\mathrm{CDCl}_{3}, 101\right.$ $\mathrm{MHz}): \delta 201.4,153.7,150.0,129.3,85.7,78.2,69.6,53.1,42.3,33.9,23.2,22.5,8.2$. IR (film): 3418, 2975, 2879, 2237, 1715, 1668, 1627, 1462, 1436, 1412, 1380, 1366, 1254, 1204, 1126, 1081, 1044, 988, 951, 895, 872, 829, 774, $752 \mathrm{~cm}^{-1}$. HPLC: Chiralcel AD (hept/ $i \mathrm{PrOH}=90: 10,0.8$ $\mathrm{ml} / \mathrm{min}, 220 \mathrm{~nm}) 12.86 / 16.69 \mathrm{~min}, 86 \%$ ee. $[\alpha]_{\mathrm{D}}=+30.98^{\circ}\left(c=1.13, \mathrm{CHCl}_{3}\right)$. HRMS-ESI $(m / z)$ calculated for $\mathrm{C}_{13} \mathrm{H}_{18} \mathrm{O}_{4} \mathrm{Na}^{+}[\mathrm{M}+\mathrm{Na}]^{+} 261.1097$, found 261.1102 . 
Preparation of ( $S, E)-3-(5-(2-m e t h y l-5-o x o h e p t-3-e n-2-y l)-2-o x o-2,5-$ dihydrofuran-3-yl)propanal, 148.<smiles>CCC(=O)/C=C/C(C)(C)C1C=C(CCC=O)C(=O)O1</smiles>

To a solution of $8.6 \mu \mathrm{L}$ of freshly distilled allyl alcohol ( $0.126 \mathrm{mmol}, 0.0073 \mathrm{~g}, 1.5$ equiv), $0.020 \mathrm{~g}$ of 4-hydroxy-5,5- dimethyl-8-oxo-dec-6-en-2-ynoic acid methyl ester 157 (0.084 mmol, 1 eq.) and $0.049 \mathrm{~g}$ of camphor sulfonic acid $(0.021 \mathrm{mmol}, 25 \mathrm{~mol} \%$, dried under high vacuum prior to use) in THF/actetone $\left(2: 1 v / v\right.$ ratio) was added $1.8 \mathrm{mg}$ of $\mathrm{CpRu}(\mathrm{MeCN})_{3} \mathrm{PF}_{6}(0.0042 \mathrm{mmol}$, $5 \mathrm{~mol} \%$ ). The reaction was stirred at $50{ }^{\circ} \mathrm{C}$ for 2 hours. The solvent was subsequently removed in vacuo and the crude product purified by flash column chromatography (1:5, $\mathrm{PE}_{\mathrm{Et}} \mathrm{O}$ ). The title compound was isolated as a clear, colorless oil $\left(0.0114 \mathrm{~g}, 51 \%\right.$ yield). $\mathbf{R}_{\mathbf{f}}=0.18$ (1:5, PE:Et $\left.{ }_{2} \mathrm{O}\right) .{ }^{1} \mathbf{H}-\mathbf{N M R}\left(500 \mathrm{MHz}, \mathrm{CDCl}_{3}\right): \delta 9.79(\mathrm{t}, J=1 \mathrm{~Hz}, 1 \mathrm{H}), 7.06(\mathrm{~d}, J=1.5 \mathrm{~Hz}, 1 \mathrm{H})$, $4.29(\mathrm{~s}, 1 \mathrm{H}), 6.70(\mathrm{~d}, J=16.5 \mathrm{~Hz}, 1 \mathrm{H}), 6.14(\mathrm{~d}, J=16.5 \mathrm{~Hz}, 1 \mathrm{H}), 4.73(\mathrm{~d}, J=1.5 \mathrm{~Hz}, 1 \mathrm{H})$, $2.8(\mathrm{t}, J=7 \mathrm{~Hz}, 2 \mathrm{H}), 2.64(\mathrm{dt}, J=1.5,7 \mathrm{~Hz}, 2 \mathrm{H}), 2.58(\mathrm{q}, J=7 \mathrm{~Hz}, 2 \mathrm{H}), 1.16(\mathrm{~s}, 3 \mathrm{H}), 1.14$ $(\mathrm{s}, 3 \mathrm{H}), 1.1(\mathrm{t}, J=7 \mathrm{~Hz}, 3 \mathrm{H}) \cdot{ }^{13} \mathrm{C}-\mathrm{NMR}\left(\mathrm{CDCl}_{3}, 125 \mathrm{MHz}\right): \delta 200.9,200.5,173.1,148.8$, 146.9, 134.6, 129.0, 86.8, 41.4, 40.8, 34.3, 23.2, 22.5, 18.4, 8.2. IR (film): 2974, 2937, 1755, 1673, 1629, 1462, 1412, 1368, 1315, 1200, 1123, 1093, 1065, 988, $911 \mathrm{~cm}^{-1}$.

For experimental details on the last four steps in the synthesis of asteriscunolide D, X-Ray diffraction data for 137, and details on the isomerization of asteriscunolide $\mathrm{D}$ to asteriscunolides A, B, and C, see: Trost, B. M.; Burns, A. C.; Bartlett, M. J.; Tautz, T.; Weiss, A. H. J. Am. Chem. Soc. 2012, 134, 1474. 


\subsubsection{Spectra}
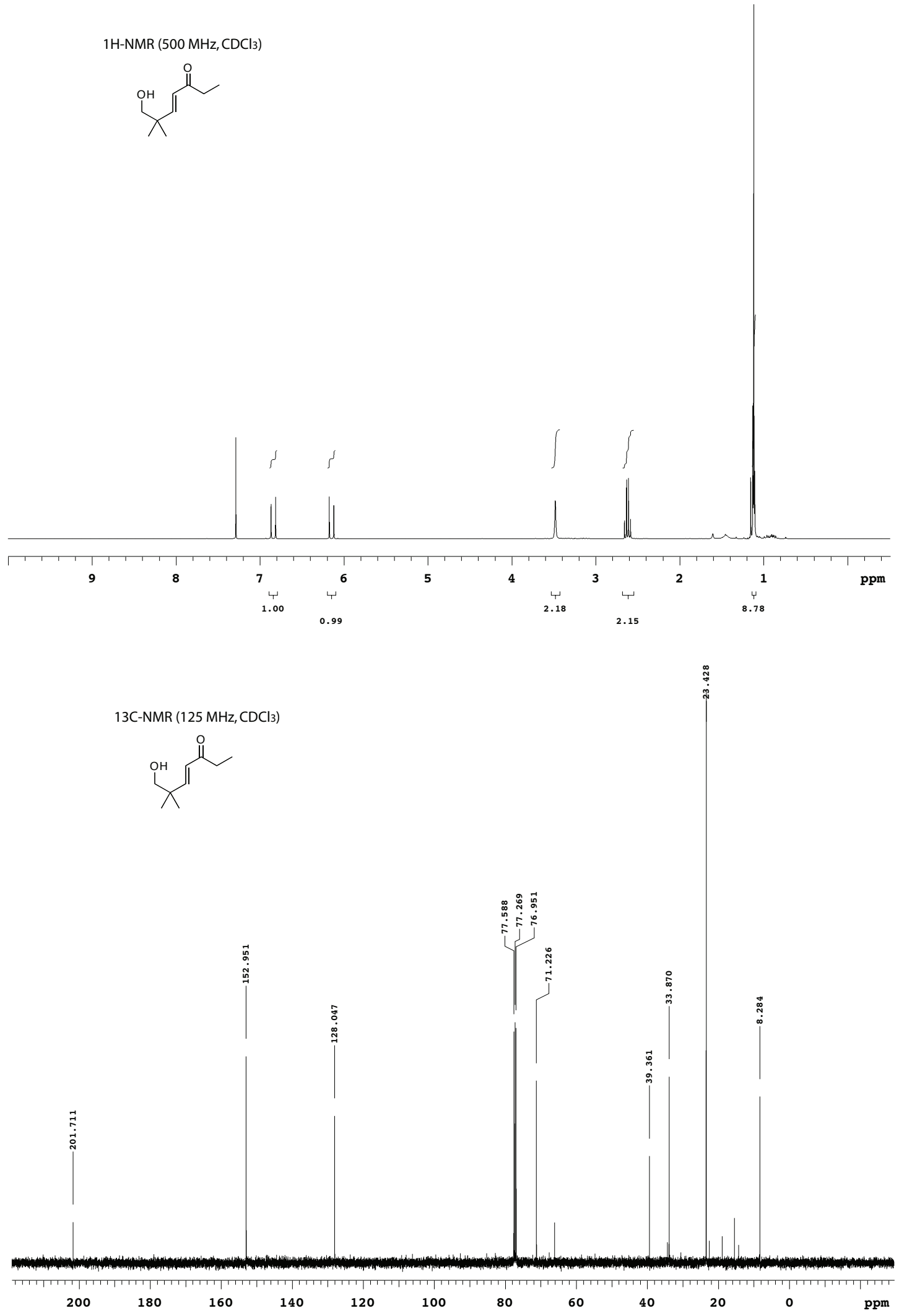
3.4. EXPERIMENTAL DATA FOR CHAPTER 3

92
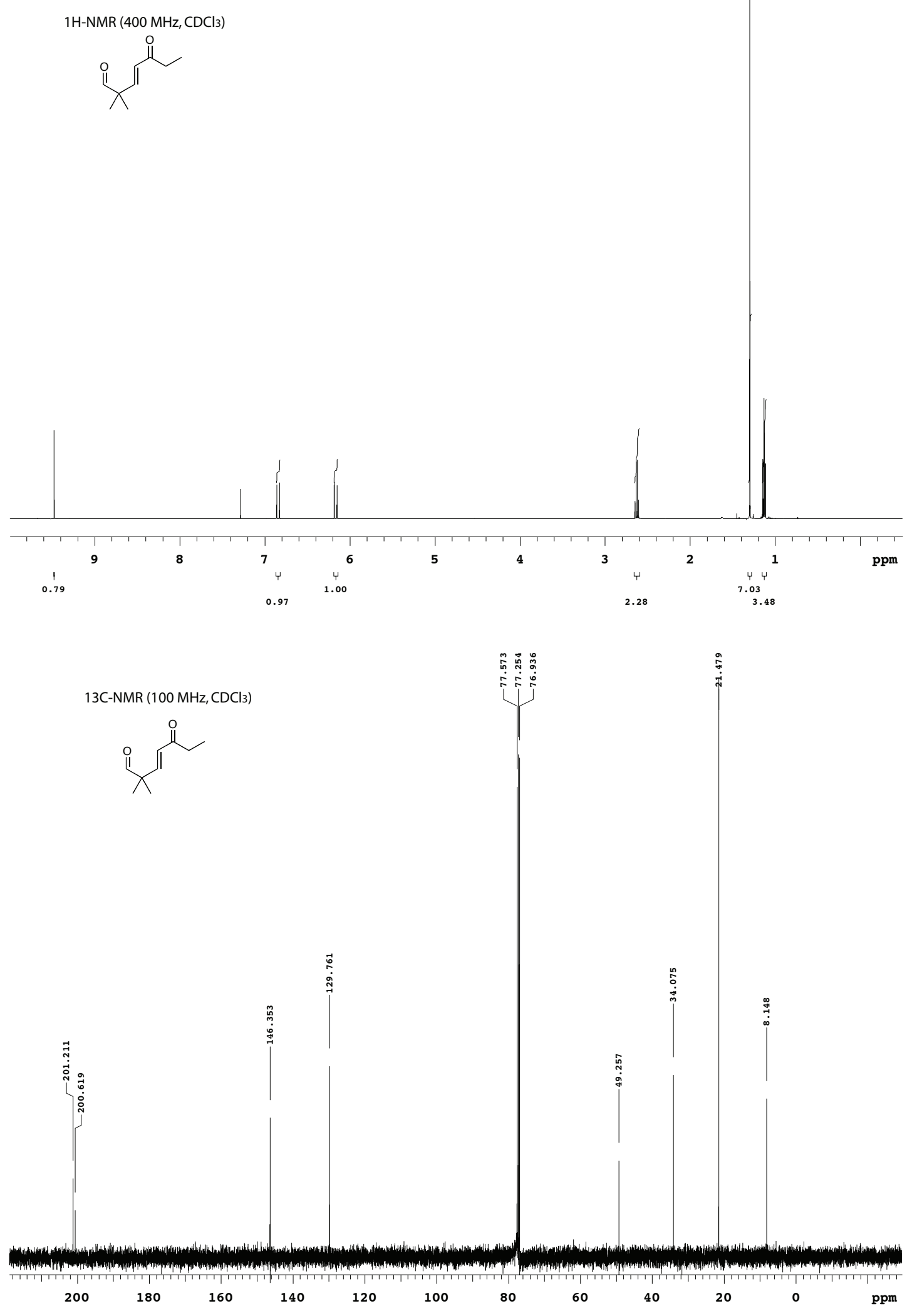

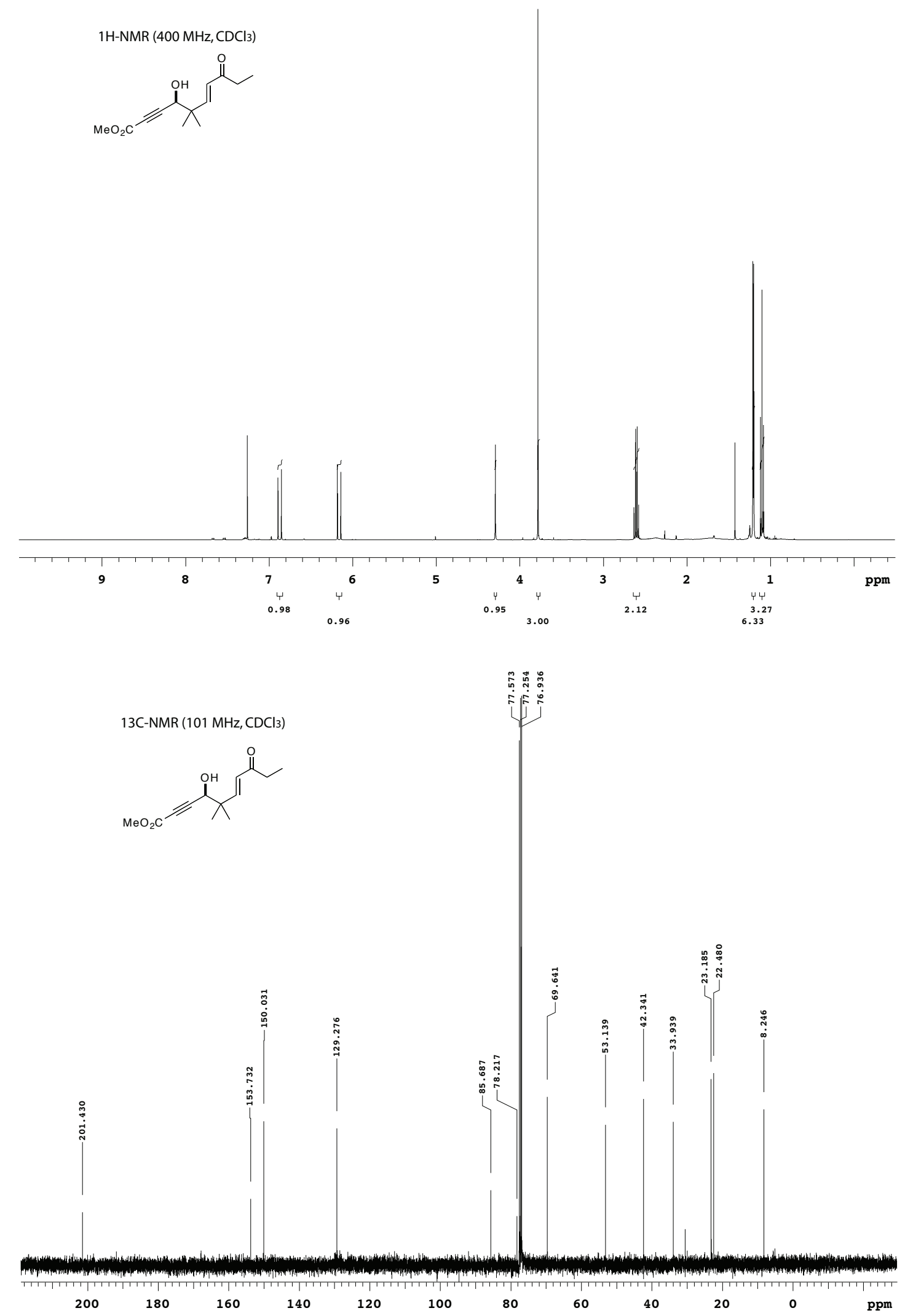
3.4. EXPERIMENTAL DATA FOR CHAPTER 3

94

1H-NMR (500 MHz, CDCl3)
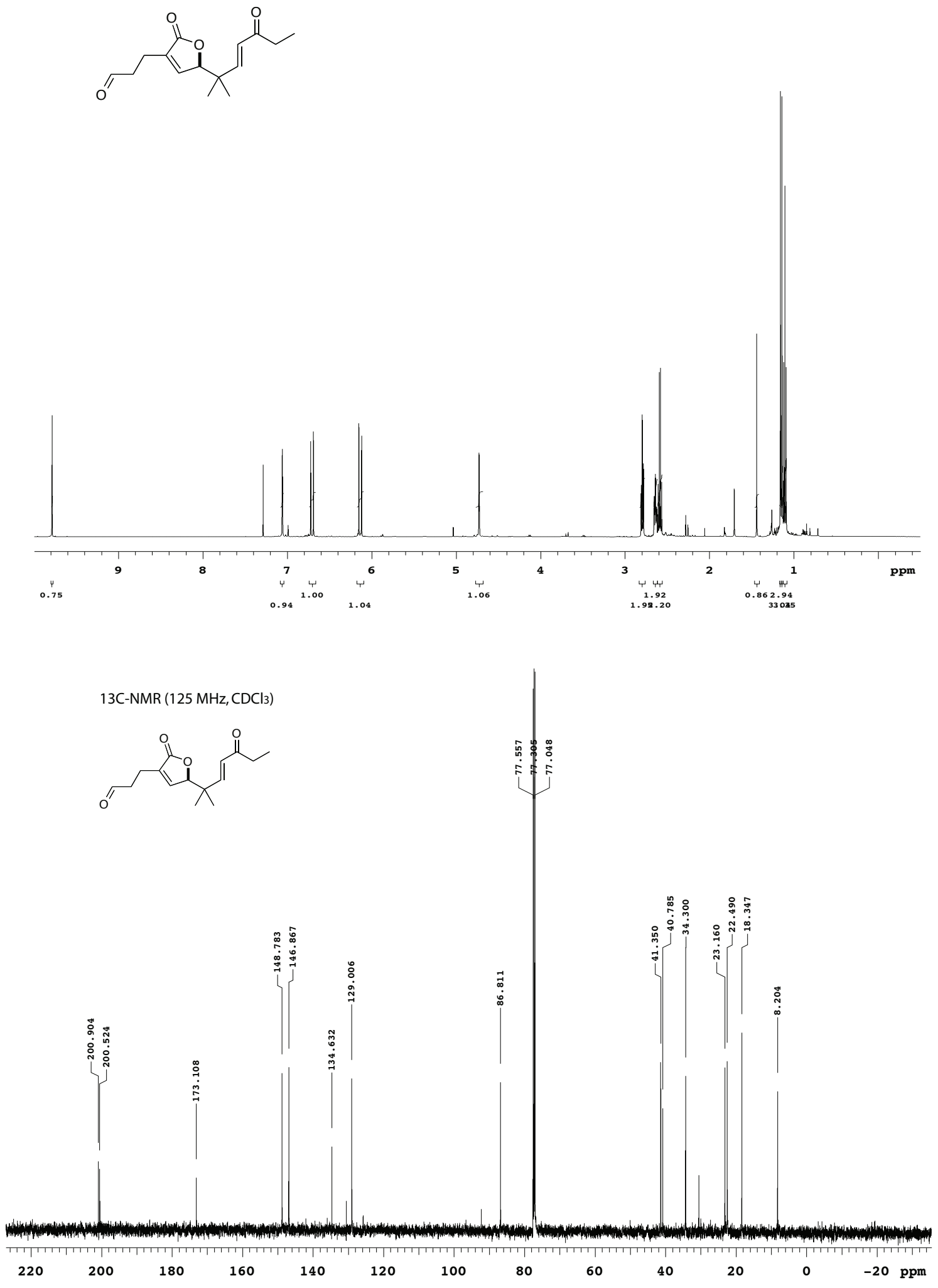


\section{References for Chapter 3}

[1] San Feliciano, A. S.; Barrero, A. F.; Medarde, M.; Miguel del Corral, J. M.; Ledesma, E. Tetrahedron Lett. 1982, 23, 3097. San Feliciano, A.; Barrero, A. F.; Medarde, M.; Miquel del Corral, J. M.; Aramburu Aizpiri, A.; S.-Ferrando, F. Tetrahedron 1984, 40, 873.

[2] Rauter, A. P., Branco, I.; Bermejo, J.; González, A. G.; G.- Grávalos, M. D.; San Feliciano, A. Phytochemistry 2001, 56, 167.

[3] Negrín, G.; Eiroa, J. L.; Morales, M.; Triana, J.; Quintana, J.; Estévez, F. Molecular Carcinogenesis 2010, 49, 488.

[4] Neuenschwander, U.; Czarniecki, B.; Hermans, I. J. Org. Chem. 2012, 7r, 2865.

[5] San Feliciano, A.; Barrero, A. F.; Medarde, M.; Miguel del Corral, J. M.; Aramburu, A.; Perales, A.; Fayos, J.; S.-Ferrando, F. Tetrahedron 1985, 41, 5711.

[6] Vig. O. P.; Ram, B.; Atwal, K. S.; Bari, S. S. Ind. J. Chem. 1976, 14B, 855. Kitagawa, Y.; Itoh, A.; Hashimoto, S.; Yamamoto, H.; Nozaki, H. J. Am. Chem. Soc. 1977, 99, 3864. Takahashi, T.; Kitamura, K.; Tsuji, J. Tetrahedron Lett. 1983, 24, 4695. Corey, E. J.; Daigneault, S.; Dixon, B. R. Tetrahedron Lett. 1993, 34, 3675. Hu, T.; Corey, E. J. Org. Lett. 2002, 4, 2441.

[7] Corey, E. J.; Cheng, X.-M. The Logic of Chemical Synthesis; John Wiley \& Sons, Inc.: New York, 1995; p. 159.

[8] Corey, E. J.; Hamanaka, E. J. Am. Chem. Soc. 1967, 89, 2758.

[9] McMurry, J. E.; Matz, J. R. Tetrahedron Lett. 1982, 23, 2723.

[10] Miyaura, N.; Suginome, H. Tetrahedron Lett. 1984, 25, 761.

[11] Kodama, M.; Shiobara, Y.; Sumitomo, H.; Mitani, K.; Ueno, K. Chem. Pharm. Bull. 1987, 35, 4039.

[12] Trost, B. M.; Toste, F. D. Tetrahedron Lett. 1999, 40, 7739. Trost, B. M.; Müller, T. J. J.; Martinez, J. J. Am. Chem. Soc. 1995, 117, 1888.

[13] Trost, B. M.; Murayama, E. J. Am. Chem. Soc. 1981, 103, 6529.

[14] For an example of the potential retro-aldol reaction of strained ring systems, see: Yang, W.; Digits, C. A.; Hatada, M.; Narula, S.; Rozamus, L. W.; Huestis, C. M.; Wong, J.; Dalgamo, D.; Holt, D. A. Org. Lett. 1999, 1, 2033. For rare examples of intramolecular aldol reactions, with non-enolizable acceptors, that form macrocyclic rings, see: Hayward, C. M.; Yohannes, D.; Danishefsky, S. J. J. Am. Chem. Soc. 1993, 115, 9345. Meng, D.; Bertinato, P.; Balog, A.; Su, D.-S.; Kamenecka, T.; Sorensen, E. J.; Danishefsky, S. J. J. Am. Chem. Soc. 1997, 119, 10073. Knowles, R. R.; Carpenter, J.; Blakey, S. B.; Kayano, A.; Mangion, I. K.; Sinz, C. J.; MacMillan, D. W. C. Chem. Sci. 2011, 2, 308.

[15] Trost, B. M.; Sato, T. J. Am. Chem. Soc. 1985, 107, 719. Lebsack, A. D.; Overman, L. E.; Valentekovich, R. J. J. Am. Chem. Soc. 2001, 123, 4851. Martin, C. L.; Nakamura, S.; Otte, R.; Overman, L. E. Org. Lett. 2011, 13, 138.

[16] Törmäkangas, O. P.; Saarenketo, P.; Koskinen, M. P. Org. Process Res. Dev. 2002, 6, 125.

[17] Blanchette, M. A.; Choy, W.; Davis, J. T.; Essenfeld, A. P.; Masamune, S.; Roush, W. R.; Sakai, T. Tetrahedron Lett. 1984, 25, 2183.

[18] Törmäkangas, O. P.; Koskinen, A. M. P. Tetrahedron Lett. 2001, 42, 2743.

[19] Fouquet, G.; Merger, F.; Platz, R. Liebigs Ann. Chem. 1979, 1591.

[20] Trost, B. M.; Weiss, A. H.; von Wangelin, A. J. J. Am. Chem. Soc. 2006, $128,8$.

[21] For a recent example of the Zn-ProPhenol-catalyzed aldol reaction, see: Trost, B. M.; Quintard, A. Org. Lett. 2012 14, 4698. 
[22] Trost, B. M.; Weiss, A. H. Angew. Chem. Int. Ed. 2007, 46, 7664.

[23] For an example of a related transformation, see: Roethle, P. A.; Hernandez, P. T.; Trauner, D. Org. Lett. 2006, 8, 5901 .

[24] Kametani, T.; Suzuki, Y.; Honda, T. Heterocycles 1985, 23, 305.

[25] For related examples of the selective conversion of $\alpha, \beta$-unsaturated ketones into $Z$-silyl enol ethers, see: Larson, E. R.; Danishefsky, S. J. Am. Chem. Soc. 1982 104, 6458. Li, L.-H.; Tius, M. A. Org. Lett. 2002, 4, 1637. Kanemasa, S.; Kumegawa, M.; Wada, E.; Nomura, M. Bull. Chem. Soc. Jpn. 1991, 64, 2990.

[26] For related transformations, see: Vedejs, E.; Engler, D. A. Tetrahedron Lett. 1976, 17, 3487. Cope, A. C.; Pike, R. A.; Spencer, C. F. J. Am. Chem. Soc. 1953, 75, 3212.

[27] Trost, B. M.; Burns, A. C.; Bartlett, M. J.; Tautz, T.; Weiss, A. H. J. Am. Chem. Soc. 2012, $134,1474$. 
Chapter 4

Formal Total Synthesis of

Aspergillide B:

A Bis-Alkynylation Linchpin

Strategy 


\subsection{Introduction}

\subsubsection{Isolation and Bioactivity}

The aspergillides are a family of bioactive natural products originally derived from the marine fungus aspergillus ostianus by Kusumi and coworkers in 2008. ${ }^{1}$ Aspergillides A, B and C, shown in Figure 4.1, share common macrolactone and pyran motifs but differ with respect to the stereochemistry at $\mathrm{C} 3$ and the unsaturation present in the pyran ring. Despite extensive NMR data and synthetic derivatization, the original report of these natural products incorrectly assigned the stereochemistry of aspergillide A (158) and B (159) at C3 and C13, respectively. The synthetic efforts of Hande and Uenishi identified structural discrepancies between the synthetic and natural material, ${ }^{2}$ prompting Kusumi and coworkers to re-examine these compounds and ultimately revise the previous structural assignments on the basis of X-ray crystal data from the corresponding $m$-bromobenzoates of aspergillides $\mathrm{A}$ and $\mathrm{B}$ (Figure 4.2). ${ }^{3}$
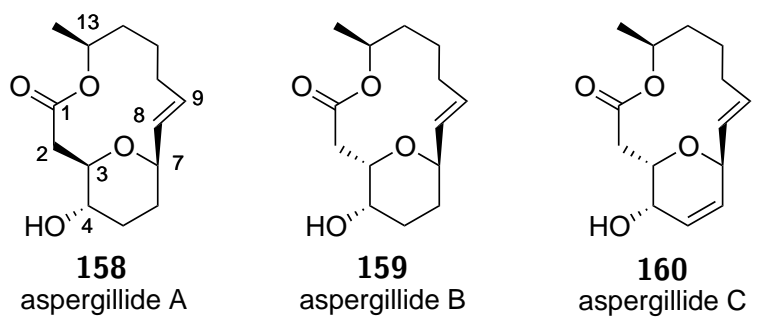

Figure 4.1 The Aspergillide Family of Natural Products.
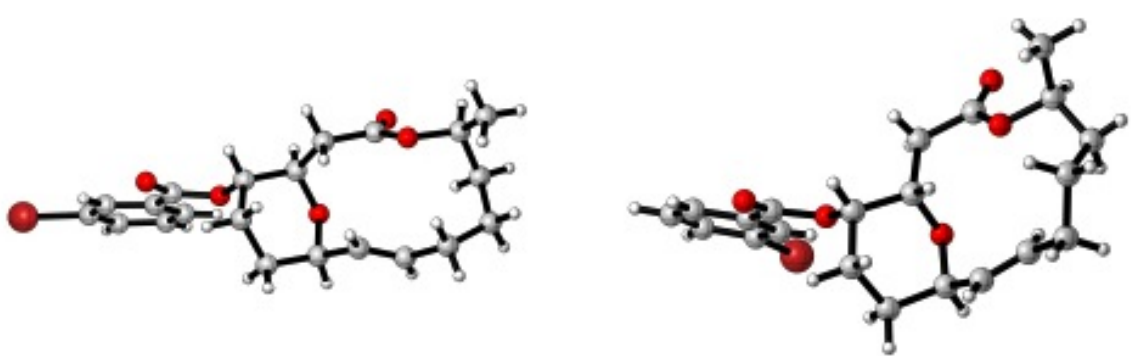

Figure 4.2 X-Ray Crystal Structures of the $m$-Bromobenzoates of Aspergillides A and B.

The aspergillides exhibit cytotoxicity towards a number of different cancer cell lines, including HL-60 (human promyelocytic leukemia), MDA-MB-231 (human breast carcinoma) and HT1080 (human fibrosarcoma) cell lines. ${ }^{4}$ Additionally, Marco and coworkers have prepared a number of aspergillide $\mathrm{A}$ and $\mathrm{B}$ analogues, including $(Z)$-aspergillide A (161) and the reduced form of aspergillide B (162). Compound 161 displayed increased cytotoxicity towards all the cancer cell lines tested, with potency comparable to that of the clinical drug fludarabine. ${ }^{5}$ 
Table 4.1 Biological Activity of the Aspergillides and Related Analogues.

\begin{tabular}{cccc}
\hline \multirow{2}{*}{ Aspergillide } & \multicolumn{3}{c}{$\mathrm{IC}_{50}(\mu \mathrm{g} / \mathrm{mL})$} \\
\cline { 2 - 4 } $\mathrm{A}(\mathbf{1 5 8})$ & $\mathrm{HL}-60$ & $\mathrm{MDA}-\mathrm{MB}-231$ & $\mathrm{HT}-1080$ \\
$\mathrm{~B}$ (159) & $32.8 \pm 7.6$ & $99.0 \pm 7.9$ & $84.3 \pm 13.7$ \\
$\mathbf{1 6 1}$ & $1.8 \pm 0.2$ & $2.9 \pm 0.2$ & $64.3 \pm 1.5$ \\
$\mathbf{1 6 2}$ & $62.8 \pm 10.9$ & $71.2 \pm 10.2$ & $92.3 \pm 0.4$ \\
& &
\end{tabular}

\subsubsection{Previous Syntheses}

The exciting biological activity of the aspergillides, along with the challenging structural features present, have motivated a number of groups to pursue the synthesis of aspergillides A-C. ${ }^{6,7}$ The majority of synthetic approaches to the aspergillides rely on the Yamaguchi lactonization to form the 12-membered macrocyclic ring (Scheme 4.1). However, this approach provides very different results for the different macrocyclic ring isomers of aspergillides $\mathrm{A}, \mathrm{B}$ and C. Formation of the aspergillide A macrocycle proceeded in poor yield under standard

Aspergillide A

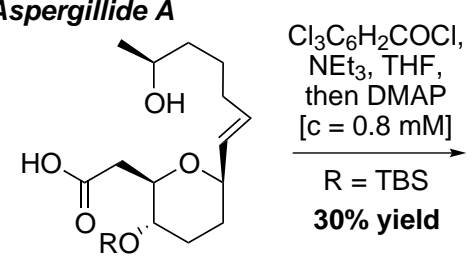

Aspergillide B<smiles>[R9]C1CCC(C=CCCCCCCC)OC1CC(=O)O</smiles>

Aspergillide C<smiles>[R9][C@@H]1C=C[C@H](/C=C/CCC[C@@H](C)O)O[C@@H]1CC(=O)O</smiles><smiles>[R9][C@@H]1CC[C@@H]2/C=C\CCCC(C)O[C@@H]1CC(=O)O2</smiles>

$\mathrm{RO}$

$\mathrm{Cl}_{3} \mathrm{C}_{6} \mathrm{H}_{2} \mathrm{COCl}$, $\mathrm{NEt}_{3}$, THF, then DMAP $[\mathrm{c}=1 \mathrm{mM}]$

$\mathrm{R}=\mathrm{MOM}$ $73 \%$ yield<smiles>C[C@@H]1CCC/C=C/[C@H]2CC[C@@H]([18O])[C@@H](CC(=O)O2)O1</smiles>

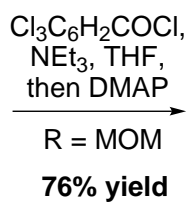

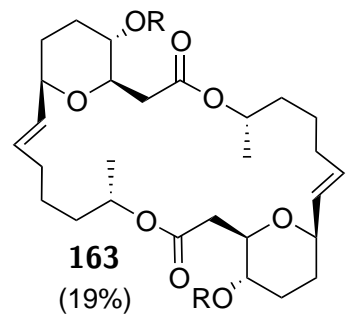

$(19 \%)$

Scheme 4.1 Yamaguchi Macrolactonization of Aspergillides A, B, and C.

Yamaguchi conditions - only $30 \%$ yield of the desired macrolactone and $19 \%$ of the undesired dimer 163 were obtained. In contrast, aspergillides B and C cyclize much more effectively, 
providing the desired macrocyclic products in $73 \%$ and $76 \%$, respectively. These results suggest that the macrolactone of aspergillide A (158) is much more strained and, therefore, harder to cyclize than the macrocycle of either aspergillide B (159) or C (160). Inspection of the X-ray crystal structures in Figure 4.2 also suggests a high degree of ring strain in the aspergillide A macrocycle. Alternative methods, such as Ru-catalyzed alkene metathesis, also struggle with this challenging macrocyclization. Attempts at ring-closing metathesis to form the aspergillide A macrocycle resulted in selective formation of the undesired $Z$-alkene (see Scheme 4.5). ${ }^{8}$ Ultimately, a novel macrocyclization strategy was designed by Shishido and coworkers to overcome the aforementioned challenges through formation of a larger 14membered ring followed by a transannular addition reaction to form the desired pyran and macrocycle (Scheme 4.2). ${ }^{9}$ An intramolecular Horner-Wadsworth-Emmons olefination was

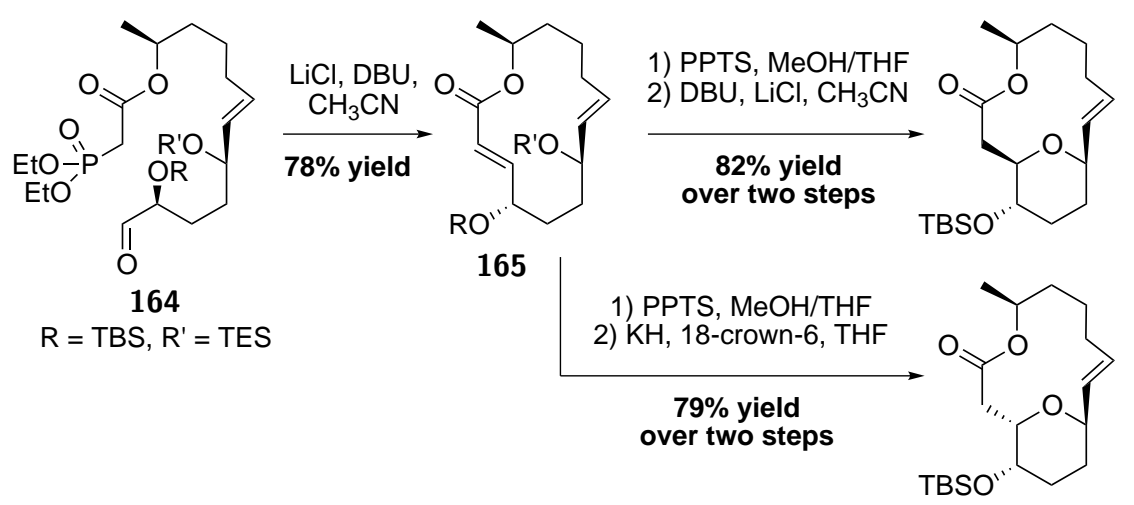

Scheme 4.2 Transannular Michael Addition in the Synthesis of Aspergillide A and B.

used to form the larger, 14-membered macrocycle in good yield. The desired 12-membered macrocycle of either aspergillide A or B could be formed selectively via a transannular oxyMichael reaction. Transannular addition under kinetic control (DBU, $\mathrm{LiCl}$ ) provides the aspergillide A ring system, whereas addition under thermodynamic control (KH, 18-crown6) produces the aspergillide B ring system. It was also shown that aspergillide A (166) can be readily converted into aspergillide B (159) via a retro-Michael/Michael addition process shown in Scheme 4.3. This observation prompted the investigation of whether the isolation conditions $\left(\mathrm{SiO}_{2}\right.$ in $\left.\mathrm{MeOH} / \mathrm{CHCl}_{3}\right)$ could have led to the formation of aspergillide $\mathrm{B}$. However, no reaction was observed, indicating that aspergillide $\mathrm{B}$ is, in fact, a natural product and not an artifact of the isolation process.

The first total synthesis of aspergillide B (then thought to be aspergillide A) was completed

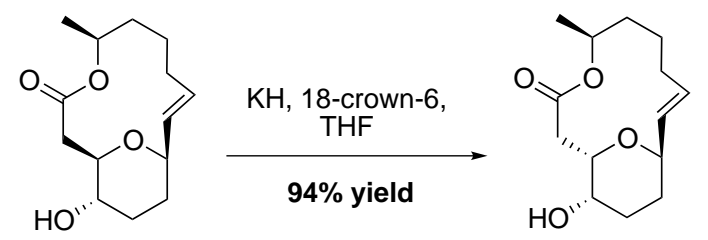

Scheme 4.3 Direct Conversion of Aspergillide A to Aspergillide B. 
by Hande and Uenishi in 2008 (Scheme 4.4). ${ }^{2}$ A Sharpless asymmetric dihydroxylation was used to install the two adjacent stereocenters of $\mathrm{C} 3$ and $\mathrm{C} 4$ (aspergillide numbering) and ultimately form the $\beta$-hydroxy- $\gamma$-lactone 167. Protection and functional group manipulations then gave rise to the terminal alkene $\mathbf{1 6 8}$ which was subjected to an alkene cross metathesis using the chiral allylic alcohol 169 and Grubbs' second generation Ru-catalyst (Grubbs II). This reaction produces compound $\mathbf{1 7 0}$, which was subsequently deprotected and treated with $\mathrm{PdCl}_{2}\left(\mathrm{CH}_{3} \mathrm{CN}\right)_{2}$ to facilitate an intramolecular Pd-catalyzed allylic alkylation to form the desired pyran ring stereospecifically. Opening of the $\gamma$-lactone with sodium methoxide and protection of the resulting alcohol as a silyl ether provided compound 171. A second Ru-catalyzed alkene cross metathesis was used to form compound $\mathbf{1 7 2}$ and append the functionalized alkyl chain needed to form the macrocycle. Double saponification of $\mathbf{1 7 2}$ produced the seco acid $\mathbf{1 7 3}$ which was cyclized using a Yamaguchi macrolactonization and deprotected to produce aspergillide B with a longest linear sequence (LLS) of 18 steps.

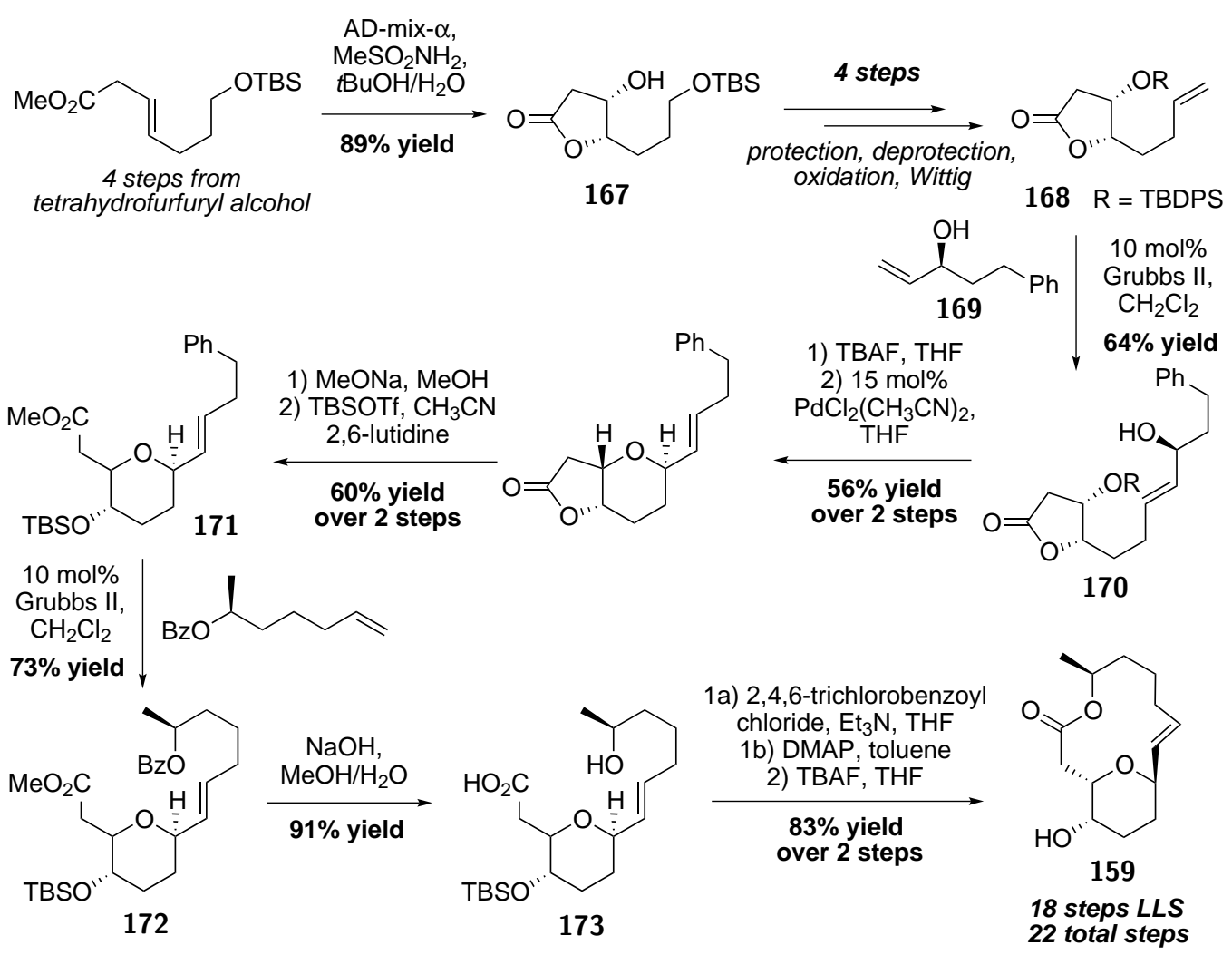

Scheme 4.4 The First Total Synthesis of Aspergillide B.

Murga, Marco and coworkers have also used alkene metathesis as a key step in the synthesis of aspergillides A and B (Scheme 4.5). ${ }^{8,10}$ In this case, asymmetric allylation, alkene isomerization, and oxidation provide access to compound 174, a common intermediate in the synthesis of both aspergillide A and B. At this point, nucleophilic addition of an ester enolate followed by reduction with triethylsilane provides compound 175. Saponification and esterification yields compound $\mathbf{1 7 6}$ and sets the stage for ring closing alkene metathesis. 
Grubbs first generation Ru-catalyst provided the initially undesired $Z$-alkene $\mathbf{1 7 7}$ in excellent yield. After deprotection, photoisomerization provides aspergillide A in a total of 16 linear steps. Alternatively, reduction of lactone $\mathbf{1 7 4}$ followed by acylation and a Mukaiyama-type nucleophilic addition provided a tert-butyl thioester which was saponified to produce carboxylic acid 178. Alkene cross metathesis was then used to install the functionalized alkyl chain needed for macrocyclization, producing the desired seco acid as a 7:3 mixture of $E$ - and $Z$ alkene isomers, respectively. Lastly, macrolactonization and deprotection produced the natural product, aspergillide B.

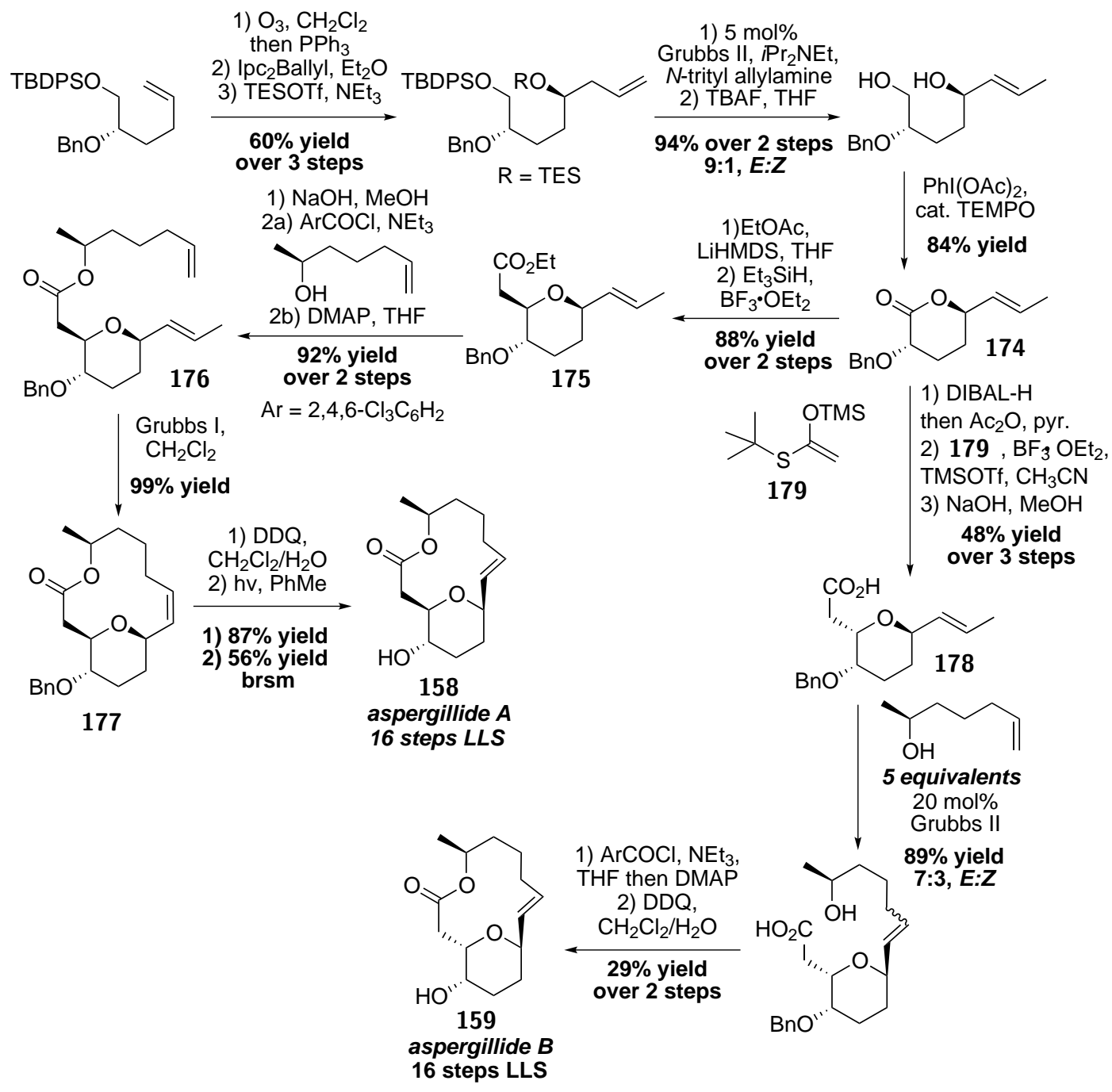

Scheme 4.5 A Divergent Synthesis of Aspergillide A and B.

Fuwa and coworkers reported a highly efficient transition metal catalyzed synthesis of both aspergillide A and B in 2010 (Scheme 4.6). ${ }^{11}$ This synthesis contains consistently high yields throughout and was the first to recognize the utility and efficiency of an intramolecular oxyMichael addition to stereoselectively form either a 2,6-cis or 2,6-trans tetrahydropyran ring. To begin the synthesis, the chiral allylic alcohol $\mathbf{1 8 0}$ was silyl-protected and subjected to a 
hydroboration/oxidation sequence. The resulting primary alcohol was then oxidized, reacted in a Wittig olefination, and reduced, providing allylic alcohol 181 in excellent yield. A Sharpless asymmetric epoxidation was followed by formation of a primary alkyl iodide and reductive elimination to ultimately provide allylic alchol 182. The slow rate at which methyl acrylate undergoes homodimerization in the presence of Grubbs II enables selective cross metathesis with compound 182. ${ }^{12} \mathrm{MOM}$-protection and silyl-deprotection were subsequently used to set the stage for the key intramolecular oxy-Michael addition. Using $\mathrm{KO} t \mathrm{Bu}$ at $-78{ }^{\circ} \mathrm{C}$ provided the 2,6-trans substituted tetrahydropyran $\mathbf{1 8 3}$ in excellent yield and diastereoselectivity. In contrast to these kinetically controlled conditions, the thermodynamic 2,6-cis substituted product can be obtained selectively using an excess of DBU in toluene at $135{ }^{\circ} \mathrm{C}$ for 36 hours. Next, ozonolysis of the styrenyl group and a Takai olefination provide the $E$-vinyl iodide $\mathbf{1 8 4}$. This compound was then used in a highly efficient $B$-alkyl Suzuki-Miyaura cross coupling. These reaction conditions form the alkylboron species in situ and use triphenylarsine, a weakly coordinating ancillary ligand that gives rise to coordinatively unsaturated Pd-complexes with high reactivity. In contrast to the use of alkene cross metathesis to install the vinyl side chain, this reaction provides the desired $E$-alkene 185 with complete selectivity. The endgame consists of bis-saponification, Yamaguchi macrolactonization and MOM-deprotection, providing aspergillide B (159) in an 18 step longest linear sequence.

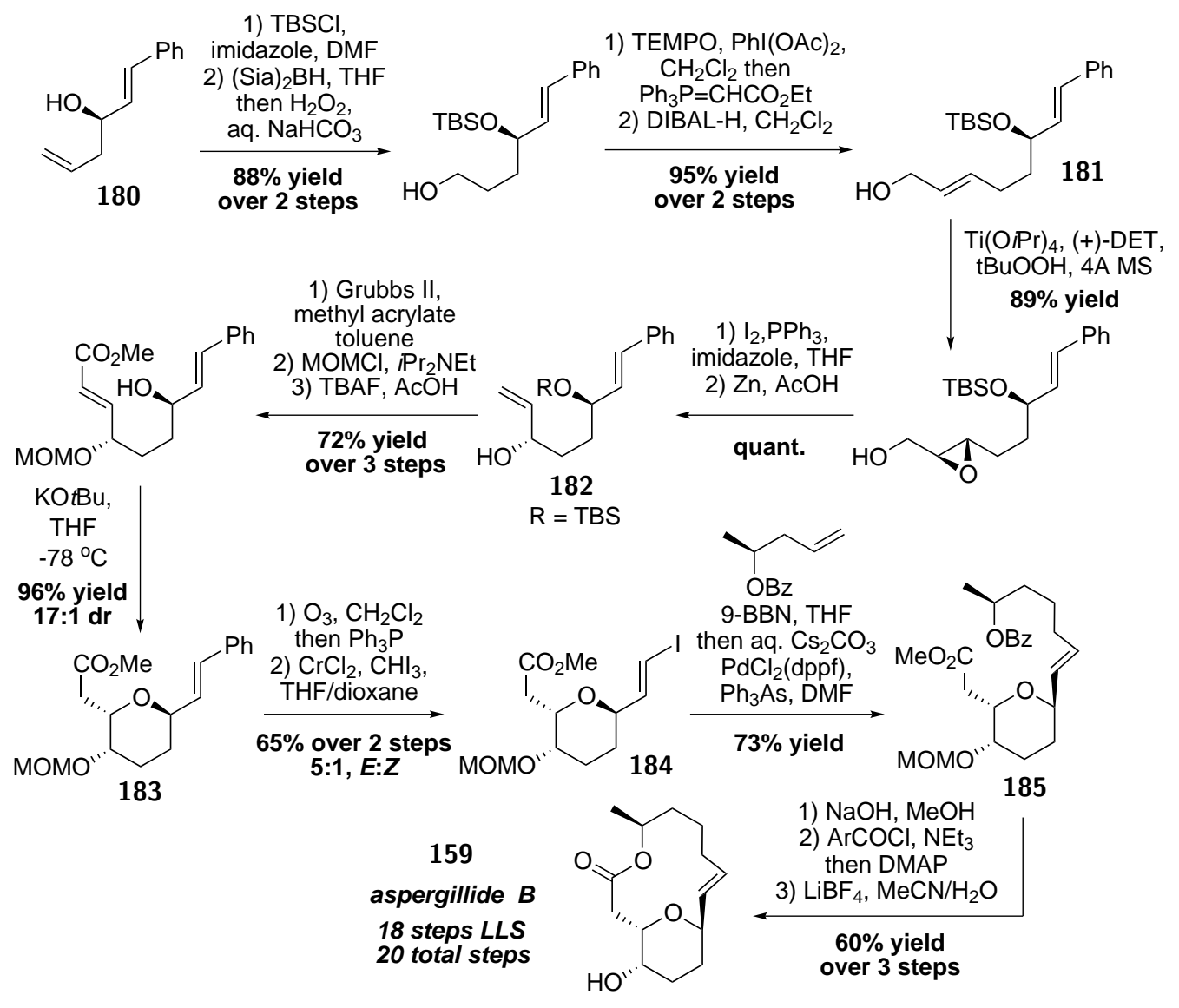

Scheme 4.6 Fuwa's Transition Metal Catalyzed Synthesis of Aspergillide B. 
Galactose pentaacetate (186) was recently used as a chiral pool starting material in a concise total synthesis of ent-aspergillide B reported by She and coworkers (Scheme 4.7). ${ }^{13}$ The protected sugar 186 was converted to the known tetrahydropyran $\mathbf{1 8 7}$ in 3 steps and was then subjected to a 4-step protection/orthoformate elimination sequence to provide the dihydropyran 188. Silyl deprotection, alkene hydrogenation, and oxidation gave rise to aldehyde 189, which was subjected to a Julia-Kocienski olefination to install all the carbons present in the natural product. Lastly, silyl deprotection and saponification provided the seco acid required for macrocyclization, which was achieved using Mitsunobu conditions. Removal of the MOM-protecting group produced the natural product antipode, (+)-aspergillide $\mathrm{B}$, in a longest linear sequence of 15 steps.

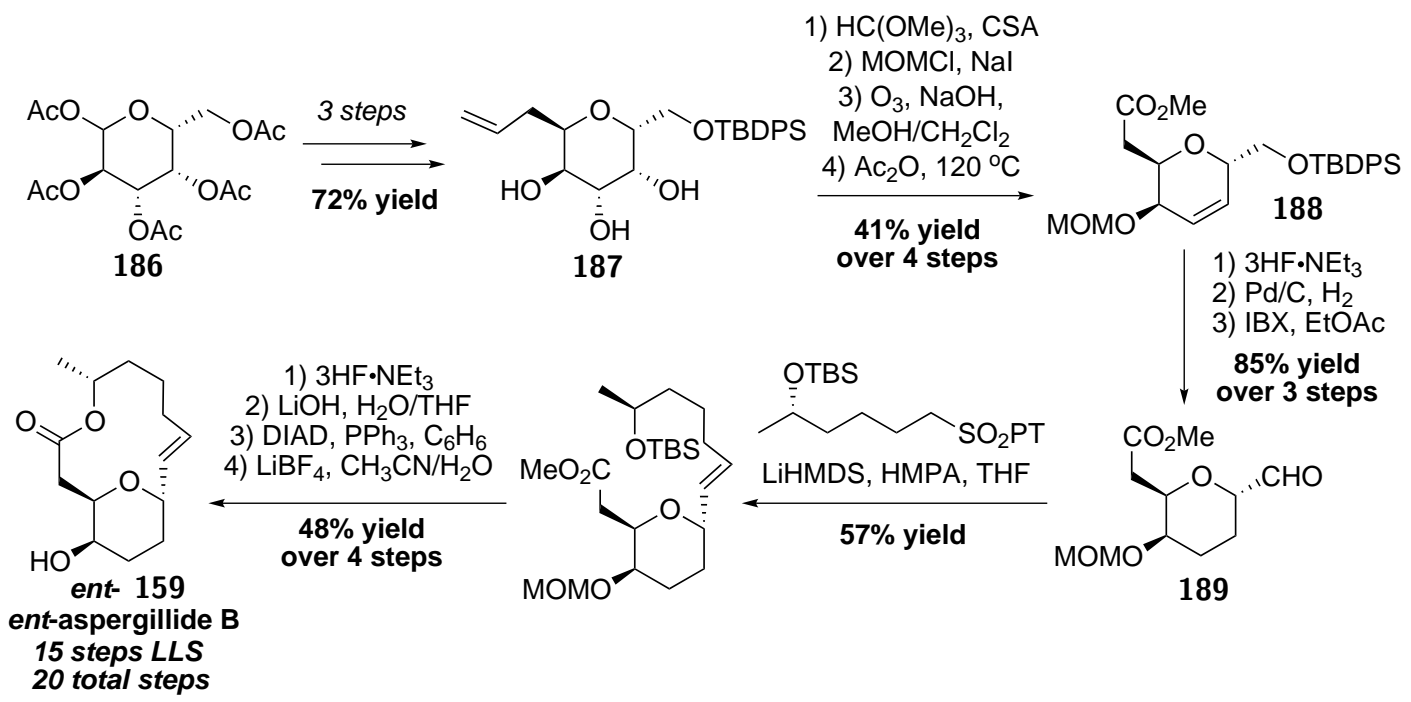

Scheme 4.7 Synthesis of ent-Aspergillide B from Chiral Pool Starting Materials.

The previous total syntheses of aspergillide A, B and C provide a number of highly valuable pieces of information that must be taken into consideration when attempting to design an improved synthesis of the aspergillides. This is especially true in regard to formation of the macrocyclic ring and the minimization of protecting group and redox manipulations, two areas that have limited the efficiency by which these bioactive natural products can be accessed.

\subsection{Formal Total Synthesis of Aspergillide B}

\subsubsection{Synthetic Strategy and Retrosynthetic Analysis}

Our interest in the aspergillides originated from the potential application of the zinc-catalyzed asymmetric alkynylation and ruthenium-catalyzed hydrosilylation methodologies (Scheme 4.8). The sequential application of these reactions would provide access to two chiral allylic alcohol moieties that could ultimately lead to a highly efficient synthesis of aspergillide B. Additionally, these disconnections would give rise to a convergent synthetic route based around alkyne 


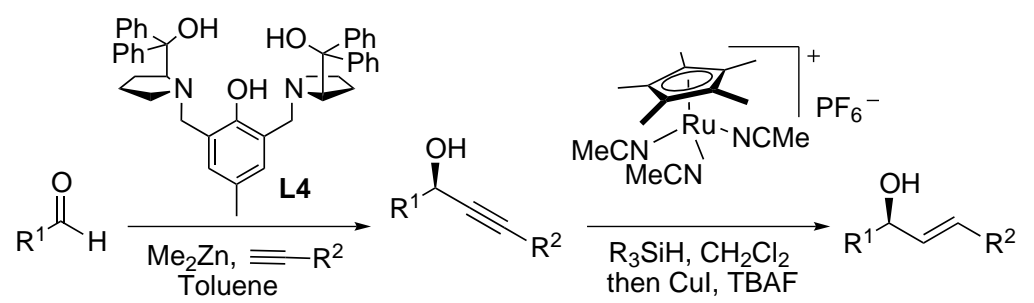

Scheme 4.8 Sequential Application of Asymmetric Alkynylation and Ru-Catalyzed Hydrosilylation.

additions to each end of a butane dialdehyde linchpin. This alkyne-based strategy envisioned for the synthesis of aspergillide B is outlined in Scheme 4.9.

Retrosynthetic disconnection of the macrolactone leads back to diester 190, a late-stage intermediate used in a previous synthesis. ${ }^{11}$ Diastereoselective formation of the pyran ring was expected to arise from intramolecular oxy-Michael addition of the $\mathrm{C} 7$ hydroxyl group and the pendant $\alpha, \beta$-unsaturated ester in 191. It was anticipated that the two chiral allylic alcohol moieties could be prepared by ruthenium-catalyzed hydrosilylation of the corresponding propargylic alcohols. Lastly, asymmetric alkyne addition would be used to access the aforementioned propargylic alcohols via separate addition of $(S)$-hept-6-yn-2-yl benzoate $((-)-\mathbf{1 1 1})$ and methyl propiolate (192) to each end of a butane dialdehyde equivalent 193 that serves as a linchpin.

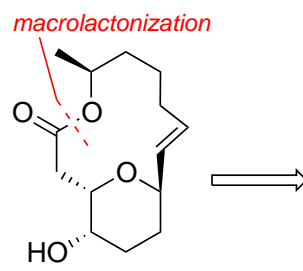

159
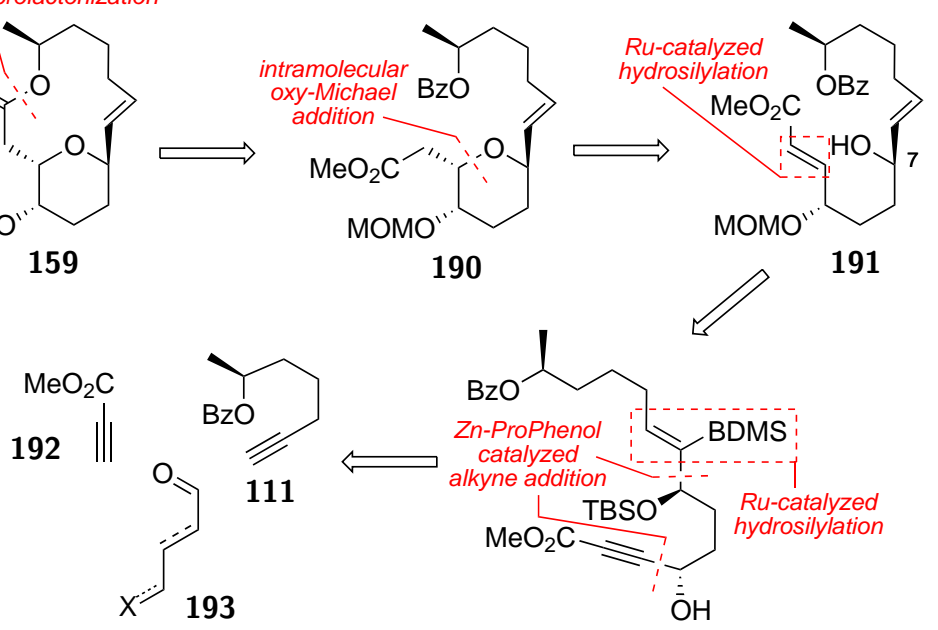

Scheme 4.9 Retrosynthetic Analysis of Aspergillide B.

The development of Zn-ProPhenol catalyzed alkyne addition has enabled the enantioselective synthesis of a wide range of propargylic alcohols (see Chapter 2). At the outset of this work the ProPhenol alkylation reaction required the use of 2.8 equivalents of alkyne and 2.95 equivalents of $\mathrm{Me}_{2} \mathrm{Zn}$ to obtain high levels of enantioselectivity and we quickly recognized that the use of a super-stoichiometric amount of alkyne (-)-111 would be particularly inefficient, given that this chiral intermediate would be prepared using a multistep 
synthesis. Consequently, the use of a stoichiometric quantity of alkyne in ProPhenol-catalyzed alkynylations was investigated.

\subsubsection{Alkynylation Optimization}

Alkyne 111 was prepared in racemic form from 3-methyl cyclohexenone (194) using an Eschenmoser fragmentation (Scheme 4.10). ${ }^{14}$ This sequence provided rapid access to alkyne $( \pm)-\mathbf{1 1 1}$, the pronucleophile needed to evaluate and optimize the reaction conditions and aldehyde substrate used in the first key alkyne addition.

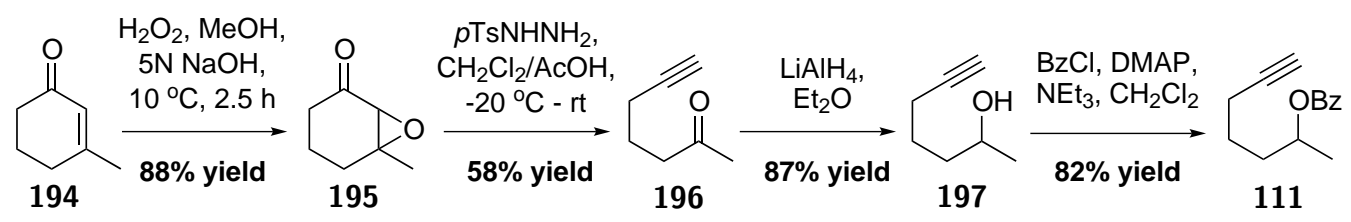

Scheme 4.10 Synthesis of Alkyne ( \pm$)-\mathbf{1 1 1}$.

Using 1.2 equivalents of alkyne ( \pm )-111, alkynylation of aliphatic aldehyde $\mathbf{1 1 2}$ resulted in only $22 \%$ yield of the desired product 113 in $54 \%$ ee (Entry 1, Table 4.2). The low isolated yield was a consequence of competing cross aldol side reactions, which ultimately produce a complex mixture of oligomers (Scheme 4.11). The aldol side product 114 was isolated in $19 \%$ yield as a mixture of diastereomers from Entry 1. The traditional superstoichiometric conditions provided only modest improvements in yield and enantioselectivity (Entries 2-3). Low yields of the desired product 113 were primarily a consequence of competing aldol reactions. The predominance of this side reaction led to the hypothesis that incomplete formation of the alkynylzinc nucleophile may be leaving significant amounts of basic dimethylzinc in the reaction mixture. Consequently, we examined a number of additives and methods that have been shown to facilitate the formation of alkynylzinc nucleophiles. The use of $N$-methylimidazole (NMI), DMSO and DMF all resulted in lower yields of the desired product, 113 (Entries 4-6). Returning to super-stoichiometric reagent loading, while also increasing the alkyne/ $\mathrm{Me}_{2} \mathrm{Zn} /(S, S)-\mathbf{L} \mathbf{4}$ premix time and the catalyst loading provided

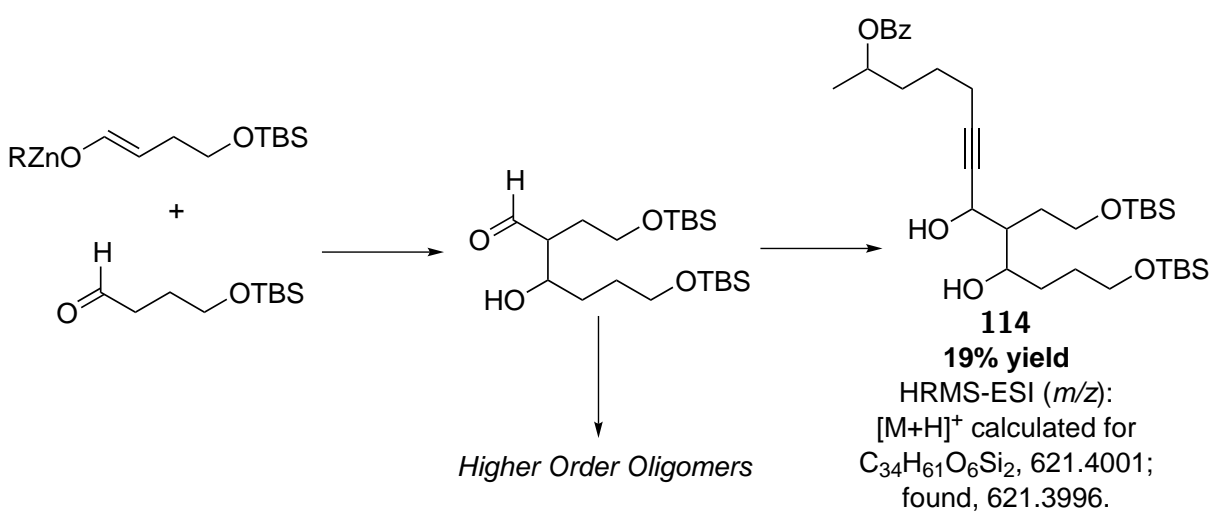

Scheme 4.11 Competing Cross Aldol Side Reactions. 
Table 4.2 Alkynylation Substrate and Reaction Optimization.<smiles>C=CCCC(C)O</smiles>

(士)- 111

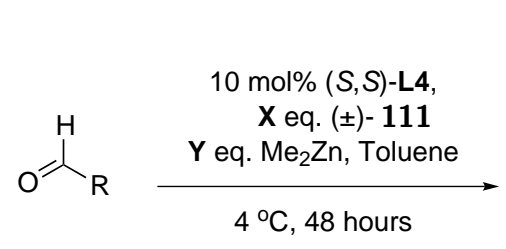

$\mathbf{R}$

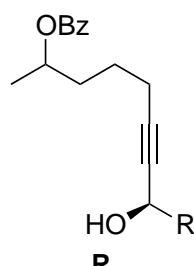

$113-\left(\mathrm{CH}_{2}\right)_{3} \mathrm{OTBS}$

$116-\left(\mathrm{CH}_{2}\right)_{3} \mathrm{OPMB}$

$121-(E)-\mathrm{CH}=\mathrm{CHCH}_{2} \mathrm{OTBS}$

$118-(E)-\mathrm{CH}=\mathrm{CHCH}(\mathrm{OMe})_{2}$

$141-(E)-\mathrm{CH}=\mathrm{CHCH}(\mathrm{OMe})_{2}$

\begin{tabular}{|c|c|c|c|c|c|}
\hline Entry & Aldehyde & $X / Y$ & Conditions $^{\mathrm{a}}$ & Yield $^{\mathrm{b}}$ & $e^{c}$ \\
\hline 1 & 112 & $1.2 / 1.5$ & $20 \mathrm{~mol} \%$ TPPO & $22 \%$ & $54 \%$ \\
\hline 2 & 112 & $2.8 / 2.95$ & $20 \mathrm{~mol} \%$ TPPO & $39 \%$ & $\begin{array}{l}62 \% \\
(57 \%)^{d}\end{array}$ \\
\hline 3 & 112 & $2.8 / 2.95$ & - & $35 \%$ & $45 \%$ \\
\hline 4 & 112 & $1.2 / 1.3$ & $30 \mathrm{~mol} \% \mathrm{NMI}$ & $11 \%$ & $72 \%$ \\
\hline 5 & 112 & $1.2 / 1.4$ & 4 eq. DMSO & $4 \%$ & $14 \%$ \\
\hline 6 & 112 & $1.2 / 1.4$ & 4 eq. DMF & $10 \%$ & $17 \%$ \\
\hline 7 & 115 & $2.8 / 2.95$ & $\begin{array}{c}20 \text { mol\% TPPO, } \\
24 \mathrm{~h} \text { alkyne premix }\end{array}$ & $58 \%$ & $50 \%^{d}$ \\
\hline 8 & 115 & $2.8 / 2.95$ & $\begin{array}{c}40 \text { mol\% TPPO, } \\
20 \text { mol\% L4, } \\
24 \text { h alkyne premix }\end{array}$ & $69 \%$ & $67 \% d$ \\
\hline 9 & 142 & $2.8 / 2.95$ & $\mathrm{c}=0.35 \mathrm{M}$ & $84 \%$ & $95 \%$ \\
\hline 10 & 142 & $1.2 / 1.5$ & $20 \mathrm{~mol} \%$ TPPO & $56 \%$ & $95 \%$ \\
\hline 11 & 142 & $1.0 / 1.3$ & $20 \mathrm{~mol} \%$ TPPO & $54 \%$ & $95 \%$ \\
\hline 12 & 141 & $1.0 / 1.3$ & $20 \mathrm{~mol} \% \mathrm{TPPO}^{\mathrm{e}}$ & $82 \%$ & $90 \%$ \\
\hline
\end{tabular}

All reactions were run on $0.1625 \mathrm{mmol}$ scale using the standard ProPhenol alkynylation procedure. ${ }^{\text {a }}$ All reactions were run at a concentration of $0.5 \mathrm{M}$ with respect to alkyne unless otherwise noted. ${ }^{\mathrm{b}}$ Isolated yield. ${ }^{\mathrm{c}}$ Enantiomeric excess determined by chiral HPLC analysis. ${ }^{d}$ Enantiomeric excess determined by ${ }^{1} \mathrm{H}-\mathrm{NMR}$ analysis of the corresponding $(S)$-methyl mandelate. e Reaction performed with (-) $\mathbf{1 1 1}$ on a $0.45 \mathrm{mmol}$ scale. TPPO = triphenylphosphine oxide, $\mathrm{NMI}=\mathrm{N}$-methylimidazole

improved yields of propargylic alcohol 116 (Entries 7-8). While the excess alkyne could be recovered quantitatively, this inefficiency along with the moderate yield and enantioselectivity prompted the investigation of the analogous unsaturated aldehyde, 142. The ProPhenolcatalyzed addition of $( \pm)-\mathbf{1 1 1}$ to $\mathbf{1 4 2}$ provided a much improved $84 \%$ yield and $95 \%$ ee (Entry 9). Reducing the stoichiometry of the alkyne to either 1.2 or 1.0 equivalents provided lower yield, although excellent ee was maintained in both cases (Entries 10-11). The moderate yield was presumably a consequence of poor reactivity and a solution to this problem was found in the use of fumaraldehyde dimethyl acetal (141). The inductive effects of the dimethyl acetal are presumed to create a more electrophilic aldehyde, and as a result, the desired propargylic alcohol 118 was obtained in $82 \%$ yield using a single equivalent of alkyne (Entry 12).

The results in Table 4.2 provide a number of new insights into the proposed reaction mechanism for ProPhenol-catalyzed alkyne addition (see Chapter 2, Section 2.2.3). Incomplete formation of the alkynylzinc nucleophile was confirmed by ${ }^{1} \mathrm{H}-\mathrm{NMR}$ analysis of a standard 
premix with 1-octyne, $\mathrm{Me}_{2} \mathrm{Zn}$ and $(S, S)-\mathbf{L} 4$ in toluene- $d 8$. Despite the entropically favored release of methane gas, deprotonation of the terminal alkyne was not observed in the absence of ProPhenol ligand. The presence of significant amounts of dimethylzinc in the reaction mixture appears to have little effect on the outcome of alkyne additions to $\alpha, \beta$-unsaturated aldehydes. However, enolizable aldehydes suffer from undesired aldol side reactions and typically only produce moderate yields of the desired propargylic alcohol. Methyl propiolate, a more acidic alkyne, has been shown to give significantly higher yields in additions to enolizable aldehydes under the standard conditions. As a result of this observation and the results in Table 4.2, the two alkyne additions in the planned synthesis of aspergillide B were ordered such that methyl propiolate would be added second, to an enolizable aldehyde, despite the reduced convergence imparted to the synthetic route. Additionally, it was discovered that the addition of methyl propiolate to fumaraldehyde dimethyl acetal (141) produces the unstable propargylic alcohol 198. Attempts to convert 198 into a more stable compound via silyl protection of the alcohol were unsuccessful and silyl ether 199 was not observed. Instead, an allenyl side product was observed, as evidenced by a characteristic ${ }^{13} \mathrm{C}$ NMR signal at $220 \mathrm{ppm}$. The formation of this functionality is presumably a consequence of the combined inductive effects of the propiolate ester and dimethyl acetal.

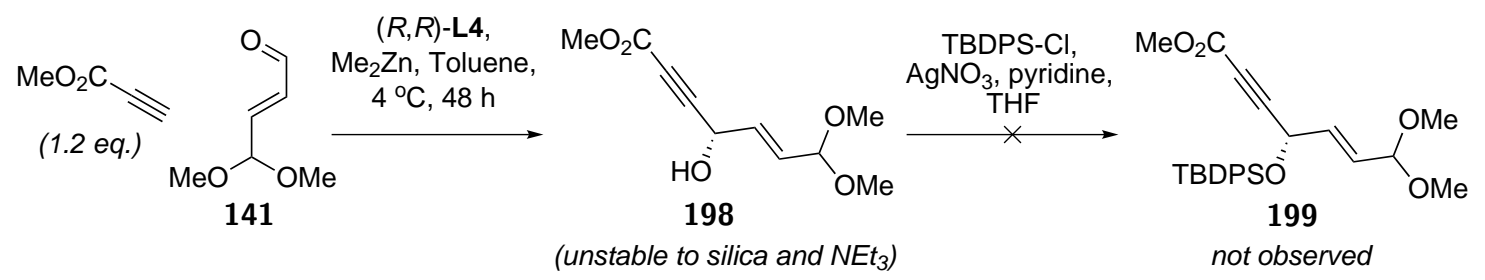

Scheme 4.12 Attempted Addition of Methyl Propiolate to Fumaraldehyde Dimethyl Acetal.

\subsubsection{Linchpin-Based Synthesis of Aspergillide B}

The synthesis of aspergillide B commenced with the preparation of chiral alkyne (-)-111 (Scheme 4.13). ${ }^{16}$ The challenging nature of the enantioselective reduction of ketone 196, the product of Eschenmoser fragmentation, prompted the use of a different route to synthesize (-)-111. While the enantioselective reduction of linear alkyl/alkyl ketones (i.e. 196) is notoriously difficult due to the relatively small steric difference between substituents, ${ }^{17}$ the reduction of alkyl/alkynyl ketones has been achieved with a number of chiral catalysts. ${ }^{18}$ The required ynone was prepared by nucleophilic addition of lithiated 1-pentyne (200) to $N, N$-dimethylacetamide (DMA), with acidic workup to liberate dimethylamine (Scheme 4.13). Enantioselective reduction of ynone 201 was achieved using Noyori's asymmetric transfer hydrogenation methodology. ${ }^{19}$ The desired propargylic alcohol was formed in $70 \%$ yield and $97 \%$ ee using just 1 mol\% of the chiral ruthenium catalyst 202. The ynone starting material was prone to decomposition and the best results were obtained when the ynone was used immediately. The literature yields for this exact transformation vary from 30-60\% yield, and 


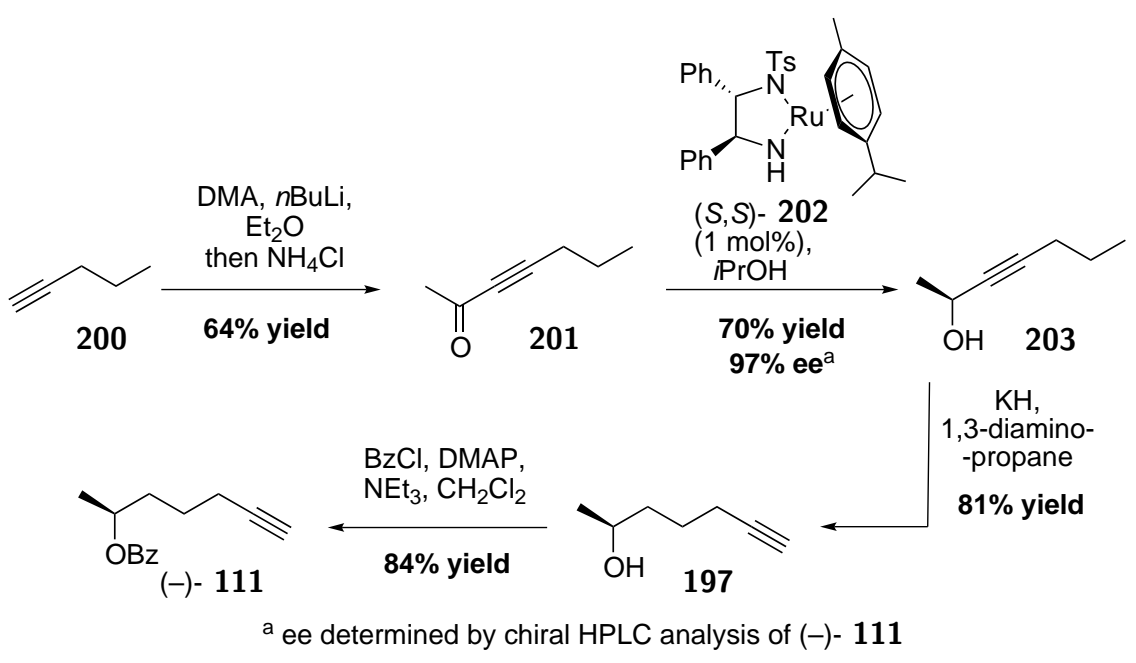

Scheme 4.13 Enantioselective Preparation of Alkyne (-)-111.

this is presumably a result of partial ynone decomposition. ${ }^{20}$ The acetylene zipper reaction was then used to carry out a multiposition isomerization of 203 to the terminal alkyne $197 .{ }^{21} \mathrm{In}$ this case, direct formation of the superbase, potassium 3-aminopropylamide (KAPA), from 1,3diaminopropane and $\mathrm{KH}$ provided a clean and high yielding reaction whereas the formation of KAPA via transmetallation of lithium aminopropylamide with $\mathrm{KO} t \mathrm{Bu}$ gave $30-55 \%$ yield and required multiple rounds of chromatography to separate the product from various undesired internal alkynes. ${ }^{22}$ Lastly, protection of the alcohol as a benzoyl ester provided enantiomerically pure material for use in the first key alkyne addition step. One of the benefits of using a Znalkynylide is that ester functional groups, such as the benzoate in 111, are typically inert to nucleophilic addition, whereas the corresponding $\mathrm{Mg}$ - or Li-alkynylide will often react with esters. Using the optimized conditions from Table 4.2, the desired enantioenriched propargylic alcohol 118 was prepared in excellent yield (Scheme 4.14). With fumaraldehyde identified as a potential linchpin in the synthesis of aspergillide $\mathrm{B}$, investigation of the required functional group interconversions ensued.

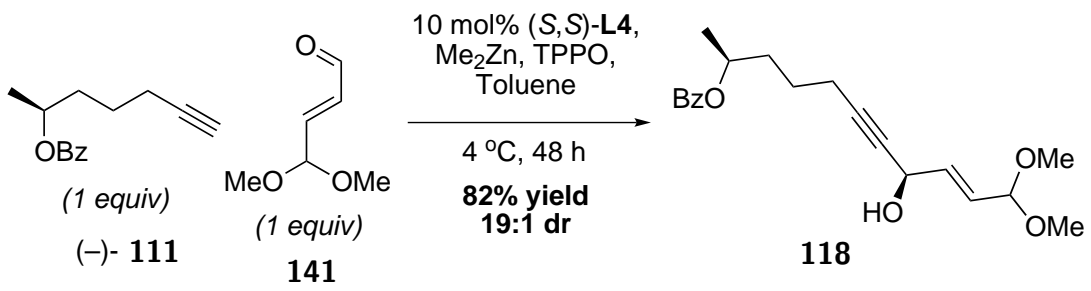

Scheme 4.14 Alkynylation of Fumaraldehyde using a Single Equivalent of (-)-111.

Initial attempts to convert the gem-dimethoxypropene moiety into a propanal group were based around the use of a protection, hydrolysis, conjugate reduction sequence (Scheme 4.15). Conversion of propargylic alcohol $\mathbf{1 1 8}$ into the corresponding acetate and hydrolysis of the resulting acetal provided the $\alpha, \beta$-unsaturated aldehyde 204 in excellent yield. Unfortunately, this compound ws found to be unstable and provided an early indication that the doubly 


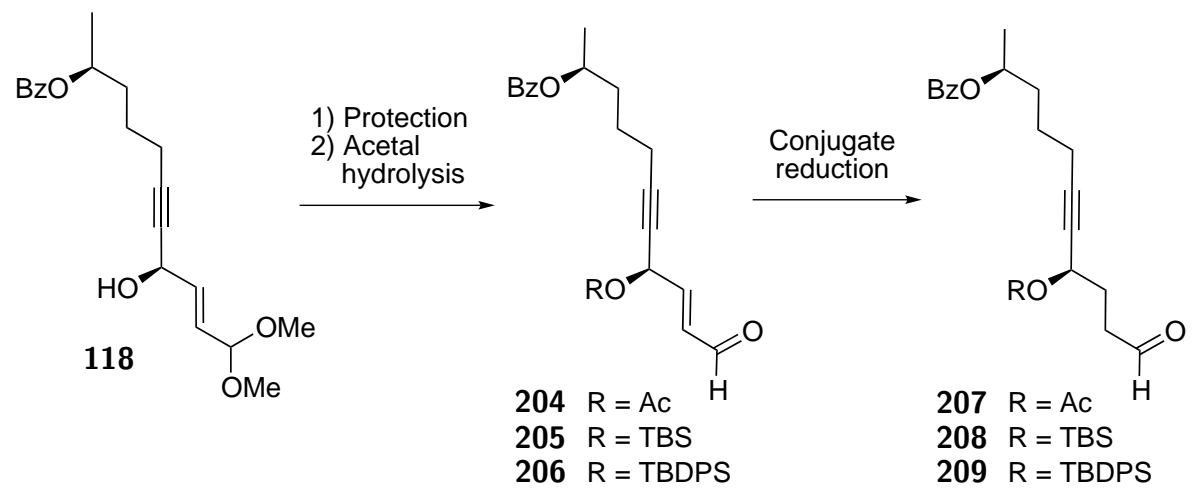

\begin{tabular}{|c|c|c|c|c|}
\hline $\mathbf{R}$ & Protection & $\begin{array}{c}\text { Acetal } \\
\text { Hydrolysis }\end{array}$ & $\begin{array}{c}\text { Conjugate } \\
\text { Reduction (CR) }\end{array}$ & $\begin{array}{c}\text { CR } \\
\text { Comments }\end{array}$ \\
\hline $\mathrm{Ac}$ & $\begin{array}{c}\mathrm{Ac}_{2} \mathrm{O}, \text { pyridine } \\
\mathbf{8 8 \%} \text { yield }\end{array}$ & $\begin{array}{c}\text { PPTS, acetone, } \\
\mathrm{H}_{2} \mathrm{O}, 65{ }^{\circ} \mathrm{C} \\
\mathbf{8 6 \%} \text { yield }\end{array}$ & & $\begin{array}{l}\text { acetate } \mathbf{2 0 4} \\
\text { decomposes }\end{array}$ \\
\hline TBS & $\begin{array}{c}\text { TBSCl, TBAI, } \\
\text { imidazole, DMF } \\
\mathbf{4 1 \%} \text { yield } \\
\\
\text { TBSCl, } \mathrm{AgNO}_{3}, \\
\text { pyridine, THF } \\
\mathbf{2 7 \%} \text { yield }\end{array}$ & $\begin{array}{c}\text { PPTS, acetone, } \\
\mathrm{H}_{2} \mathrm{O}, 65{ }^{\circ} \mathrm{C} \\
\mathbf{0 \%} \text { yield } \\
\\
\text { PPTS, acetone, } \\
\mathrm{H}_{2} \mathrm{O}, 45{ }^{\circ} \mathrm{C} \\
\mathbf{9 0 \%} \text { yield }\end{array}$ & $\begin{array}{c}\mathrm{Na}_{2} \mathrm{~S}_{2} \mathrm{O}_{4}, \mathrm{NaHCO}_{3}, \\
\text { dioxane } / \mathrm{H}_{2} \mathrm{O} \\
\\
\mathrm{Hantzch} \text { Ester, THF, } \\
\mathrm{Bn}_{2} \mathrm{NH}_{2}{ }^{+} \mathrm{CF}_{3} \mathrm{COO}^{-} \\
5 \mathrm{~mol} \% \mathrm{Cu}(\mathrm{OAc})_{2} \\
\mathrm{BDP}, \mathrm{DEMS} \\
\mathbf{1 5 \%} \text { yield }\end{array}$ & $\begin{array}{c}\text { no alkene, } \\
\text { trace aldehyde } \\
\text { trace SM, no } \\
\text { desired product } \\
\text { KOH } \\
\text { hydrolysis }\end{array}$ \\
\hline TBDPS & $\begin{array}{l}\text { TBDPSCl, TBAI, } \\
\text { imidazole, DMF } \\
\mathbf{4 2 \%} \text { yield }\end{array}$ & $\begin{array}{c}\text { PPTS, acetone, } \\
\mathrm{H}_{2} \mathrm{O}, 45{ }^{\circ} \mathrm{C} \\
\mathbf{8 4} \text { yield \% }\end{array}$ & $\begin{array}{c}10 \mathrm{~mol} \% \mathrm{Cu}(\mathrm{OAc})_{2} \\
\mathrm{BDP}, \mathrm{DEMS} \\
\mathbf{1 8 \%} \text { yield }\end{array}$ & $\begin{array}{c}1 \mathrm{M} \mathrm{HCl} \\
\text { hydrolysis }\end{array}$ \\
\hline
\end{tabular}

Reagents:<smiles>CCOC(=O)C1=C(C)NC(C)=C(C(=O)OCC)C1</smiles><smiles>Pc1cccc(P)c1P</smiles>

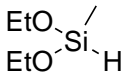
Hantzch BDP DEMS

Scheme 4.15 Optimization of Enal Conjugate Reduction. 
activated, propargylic and allylic, oxymethine would require special consideration. With this in mind, the less reactive TBS-ether 205 was formed. Careful control of the reaction temperature was required during the acetal hydrolysis step - performing the reaction at $65{ }^{\circ} \mathrm{C}$ resulted in removal of the TBS group, whereas performing the reaction at $45{ }^{\circ} \mathrm{C}$ provided $90 \%$ yield of the desired aldehyde 205. Initial attempts to chemoselectively reduce the alkene using either sodium dithionite $\left(\mathrm{Na}_{2} \mathrm{~S}_{2} \mathrm{O}_{4}\right)$ or an organocatalytic reduction using $5 \mathrm{~mol} \%$ of $\mathrm{Bn}_{2} \mathrm{NH}_{2}{ }^{+} \mathrm{CF}_{3} \mathrm{COO}^{-}$and the Hantzch ester were unsuccessfull. ${ }^{23,24}$ However, the use of the 'Hot' Strkyer's reagent reported by Lipshutz and coworkers, $5 \mathrm{~mol} \% \mathrm{Cu}(\mathrm{OAc})_{2}$, 1,2-bis(diphenylphosphino)benzene (BDP), and diethoxymethylsilane (DEMS), provided the desired aliphatic aldehyde $\mathbf{2 0 8}$ in $15 \%$ yield. ${ }^{25}$ The low yield of the desired product led to the hypothesis that the $\mathrm{KOH}$ workup conditions may be unecessarily harsh and, therefore, detrimental to yield. Consequently, the $(\mathrm{BDP}) \mathrm{CuH}$ conditions were used on a more robust silyl ether, TBDPS-ether 206, in conjuction with a mild $1 \mathrm{M} \mathrm{HCl}$ workup. Unfortunately, these conditions provided a similarly low yield, providing $18 \%$ yield of the aliphatic aldehyde 209. These poor results prompted the investigation of an alternative approach in which the alkene reduction is performed under hydrogenation conditions prior to hydrolysis of the acetal.

The use of a ruthenium-catalyzed hydrosilylation and subsequent protodesilylation provides a versatile method by which $E$-selective alkyne reduction can be achieved. ${ }^{27}$ However, by postponing protodesilylation to later in the synthesis and leaving the $\mathrm{C} 8$ - $\mathrm{C} 9$ alkene protected as a vinyl silane, an opportunity for chemoselective hydrogenation of the C5-C6 alkene was envisioned (aspergillide numbering). With this new strategy in mind, initial attempts were made to achieve concomitant alkyne hydrosilylation and alcohol protection through the formation of cyclic siloxane $\mathbf{2 1 0}$ (Scheme 4.16). Hydrosilylation of alkyne 211 with
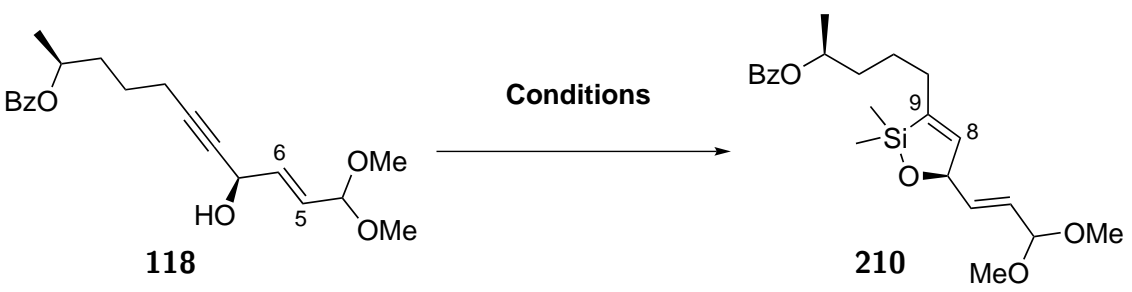

\begin{tabular}{|c|c|c|}
\hline Entry & Conditions & Results \\
\hline 1 & $\begin{array}{l}5 \mathrm{~mol} \% \mathrm{CpRu}\left(\mathrm{CH}_{3} \mathrm{CN}\right)_{3} \mathrm{PF}_{6}, \\
1.2 \text { equiv } \mathrm{Me}_{2}(\mathrm{EtO}) \mathrm{SiH}, \mathrm{CH}_{2} \mathrm{Cl}_{2} \\
\text { (0.5M), } 0{ }^{\circ} \mathrm{C}-\mathrm{rt}, 45 \text { minutes }\end{array}$ & $20 \%$ yield \\
\hline 2 & $\begin{array}{c}5 \mathrm{~mol} \% \mathrm{CpRu}\left(\mathrm{CH}_{3} \mathrm{CN}\right)_{3} \mathrm{PF}_{6} \\
\text { 3.0 equiv } \mathrm{Me}_{2}(\mathbf{E t O}) \mathbf{S i H}, \mathrm{CH}_{2} \mathrm{Cl}_{2} \\
(0.5 \mathrm{M}), 0^{\circ} \mathrm{C}-\mathrm{rt}, 60 \text { minutes }\end{array}$ & $0 \%$ yield \\
\hline 3 & $\begin{array}{c}5 \text { mol\% } \mathbf{C p}^{*} \mathbf{R u}\left(\mathbf{C H}_{\mathbf{3}} \mathbf{C N}\right)_{3} \mathbf{P F}_{\mathbf{6}} \\
1.5 \text { equiv } \mathbf{M e}_{\mathbf{2}}(\mathbf{E t O}) \mathrm{SiH}, \mathrm{CH}_{2} \mathrm{Cl}_{2} \\
(0.5 \mathrm{M}), 0{ }^{\circ} \mathrm{C}-\mathrm{rt}, 60 \text { minutes }\end{array}$ & $\begin{array}{l}40 \% \text { yield as a } \\
c a .1: 1 \text { mixture } \\
\text { of regioisomers }\end{array}$ \\
\hline
\end{tabular}

Scheme 4.16 Ru-Catalyzed Cyclic Siloxane Formation. 
$\mathrm{Me}_{2}(\mathrm{EtO}) \mathrm{SiH}$ and $5 \mathrm{~mol} \% \mathrm{CpRu}\left(\mathrm{CH}_{3} \mathrm{CN}\right)_{3} \mathrm{PF}_{6}$ provided the desired siloxane in $20 \%$ yield (Entry 1). The use of excess ethoxy(dimethyl)silane was found to be detrimental to the reaction (Entry 2). However, switching to the more reactive $\mathrm{Cp} * \mathrm{Ru}\left(\mathrm{CH}_{3} \mathrm{CN}\right)_{3} \mathrm{PF}_{6}$ catalyst provided 1:1 miture of regioisomers, one cyclic (210) and one acyclic, in $40 \%$ combined yield (Entry 3).

The modest yields obtained in these reactions prompted a switch to benzyldimethylsilane (BDMS-H), and it was discovered that by using just 1.05 equivalents of silane an excellent $89 \%$ yield of 212 could be obtained (Scheme 4.17). Performing the reaction under similar conditions with the $\mathrm{CpRu}\left(\mathrm{CH}_{3} \mathrm{CN}\right)_{3} \mathrm{PF}_{6}$ catalyst provided only $63 \%$ yield. The regioselectivity of this reaction was confirmed by analysis of the ${ }^{1} \mathrm{H} /{ }^{1} \mathrm{H}$ COSY NMR spectrum.
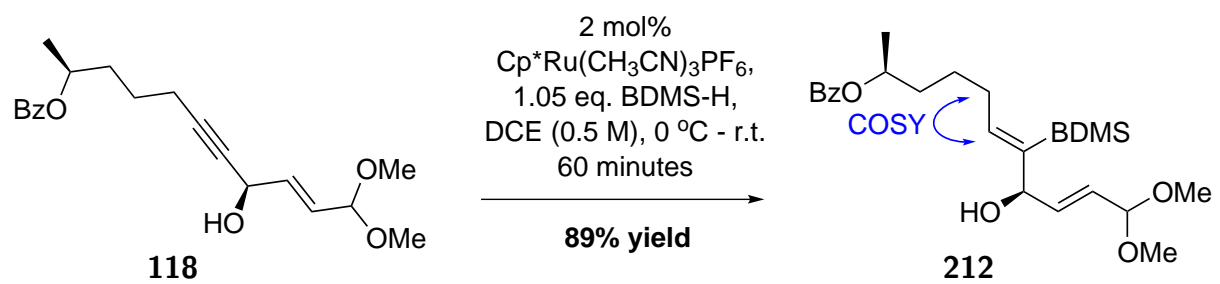

Scheme 4.17 Ru-Catalyzed Hydrosilylation with Benzyldimethylsilane.

Hydrosilylation of propargylic alcohols under these conditions typically results in silylation at the $\beta$-position, providing the products shown in Figure 4.3. ${ }^{27}$ The similar steric environment around these vinyl silanes and $\mathbf{2 1 2}$ suggests that electronic factors are responsible for the dramatic reversal in regioselectivity observed in Scheme 4.17. Thus, the regioselective formation of $\mathbf{2 1 2}$ is thought to be a consequence of a coordinative interaction between the ruthenium catalyst and electron poor alkene.

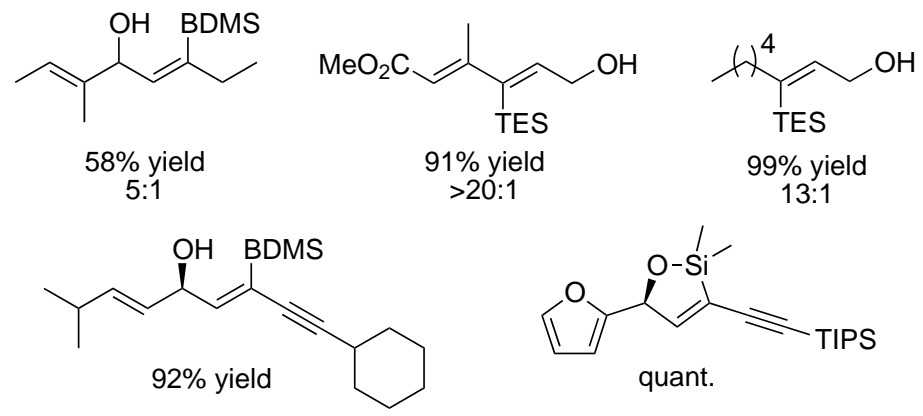

Figure 4.3 Examples of $\beta$-Selectivity in Hydrosilylations with $\mathrm{CpRu}\left(\mathrm{CH}_{3} \mathrm{CN}\right)_{3} \mathrm{PF}_{6}$ and $\mathrm{Cp} * \mathrm{Ru}\left(\mathrm{CH}_{3} \mathrm{CN}\right)_{3} \mathrm{PF}_{6}$.

To recap, alkynylation of fumaraldehyde dimethyl acetal (141) using just 1 equivalent of alkyne (-)-111 and 10 mol\% of the $(S, S)$-ProPhenol Ligand $(\mathbf{L} 4)$ provided $\mathbf{1 1 8}$ in $82 \%$ yield and $90 \%$ de (Scheme 4.18). Alkyne trans-hydrosilylation using benzyldimethylsilane (BDMS-H) and $2 \mathrm{~mol} \%$ of $\mathrm{Cp} * \mathrm{Ru}\left(\mathrm{CH}_{3} \mathrm{CN}\right)_{3} \mathrm{PF}_{6}$, provided vinyl silane 212 regioselectively. The presence of the silicon on one of the double bonds provides a key for their differentiation towards hydrogenation. Moving forward, the disubstituted olefin of allylic alcohol $\mathbf{2 1 2}$ was 


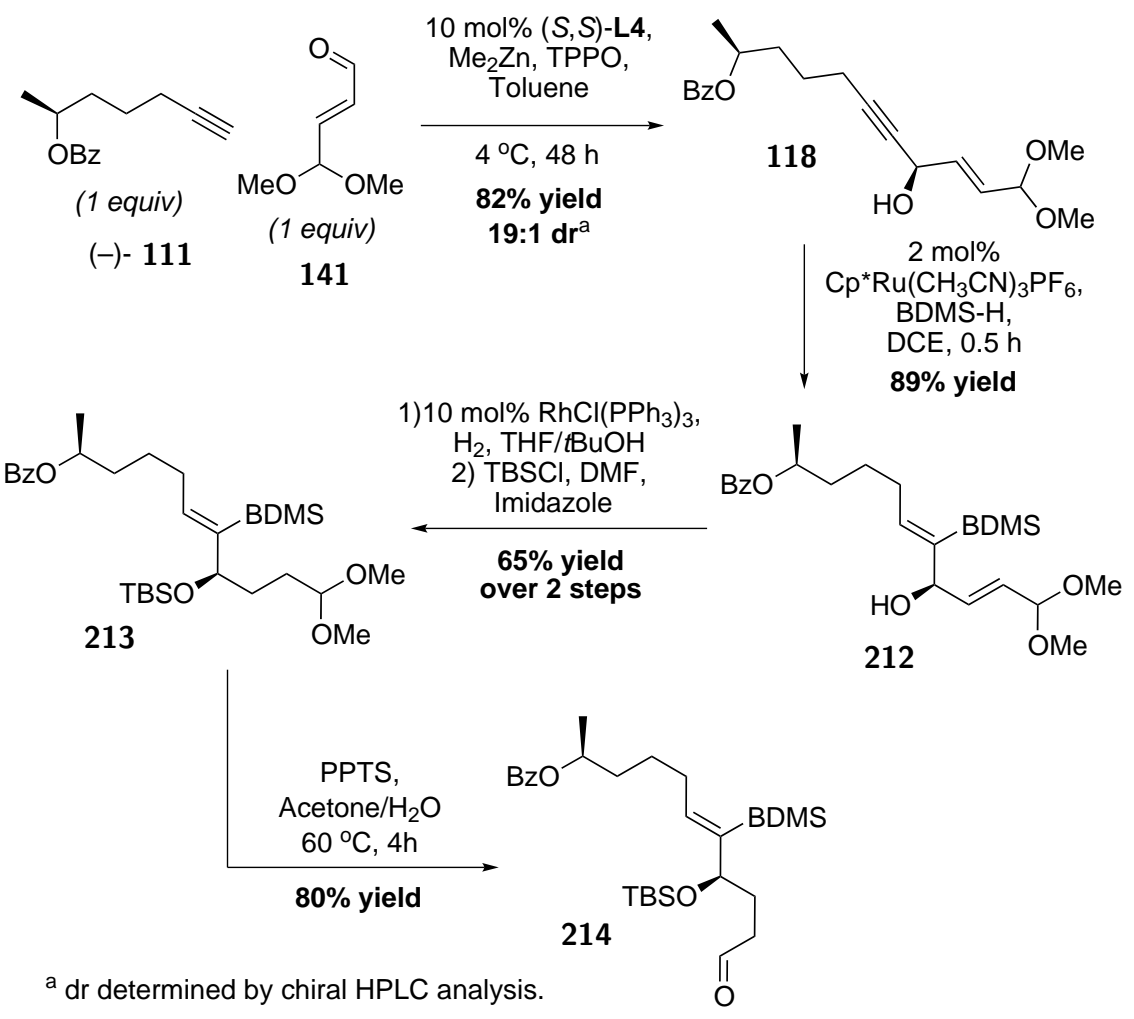

Scheme 4.18 First Alkyne Addition in the Synthesis of Aspergillide B.

chemoselectively hydrogenated using Wilkinson's catalyst, $\mathrm{RhCl}\left(\mathrm{PPh}_{3}\right)_{3}$, and the resulting alcohol was silyl protected to give 213. ${ }^{28}$ Hydrolysis of the dimethyl acetal was performed under mild acid-catalyzed conditions, providing the desired aldehyde $\mathbf{2 1 4}$ for the second alkyne addition.

ProPhenol-catalyzed addition of methyl propiolate (192) to aldehyde $\mathbf{2 1 4}$ provided the desired propargylic alcohol $\mathbf{2 1 5}$ in $71 \%$ yield as a 5.2:1 mixture of diastereomers (Scheme 4.19). ${ }^{29}$ MOM-protection of the propargylic alcohol was followed by a chemoselective alkyne reduction using the hydrosilylation/protodesilylation protocol for formation of E-double bonds. ${ }^{30}$ The basic reaction conditions used for this transformation resulted in spontaneous intramolecular oxy-Michael addition. Consequently, the desired 2,6-anti tetrahydropyran 190 was isolated in $38 \%$ yield $(77 \%$ brsm) over 2 steps. The preparation of compound 190 constitutes a formal total synthesis of aspergillide B. Compound $\mathbf{1 9 0}$ has been previously transformed into aspergillide B in 3 additional steps (bis-saponification, Yamaguchi macrolactonization and MOM-deprotection). ${ }^{11}$ 


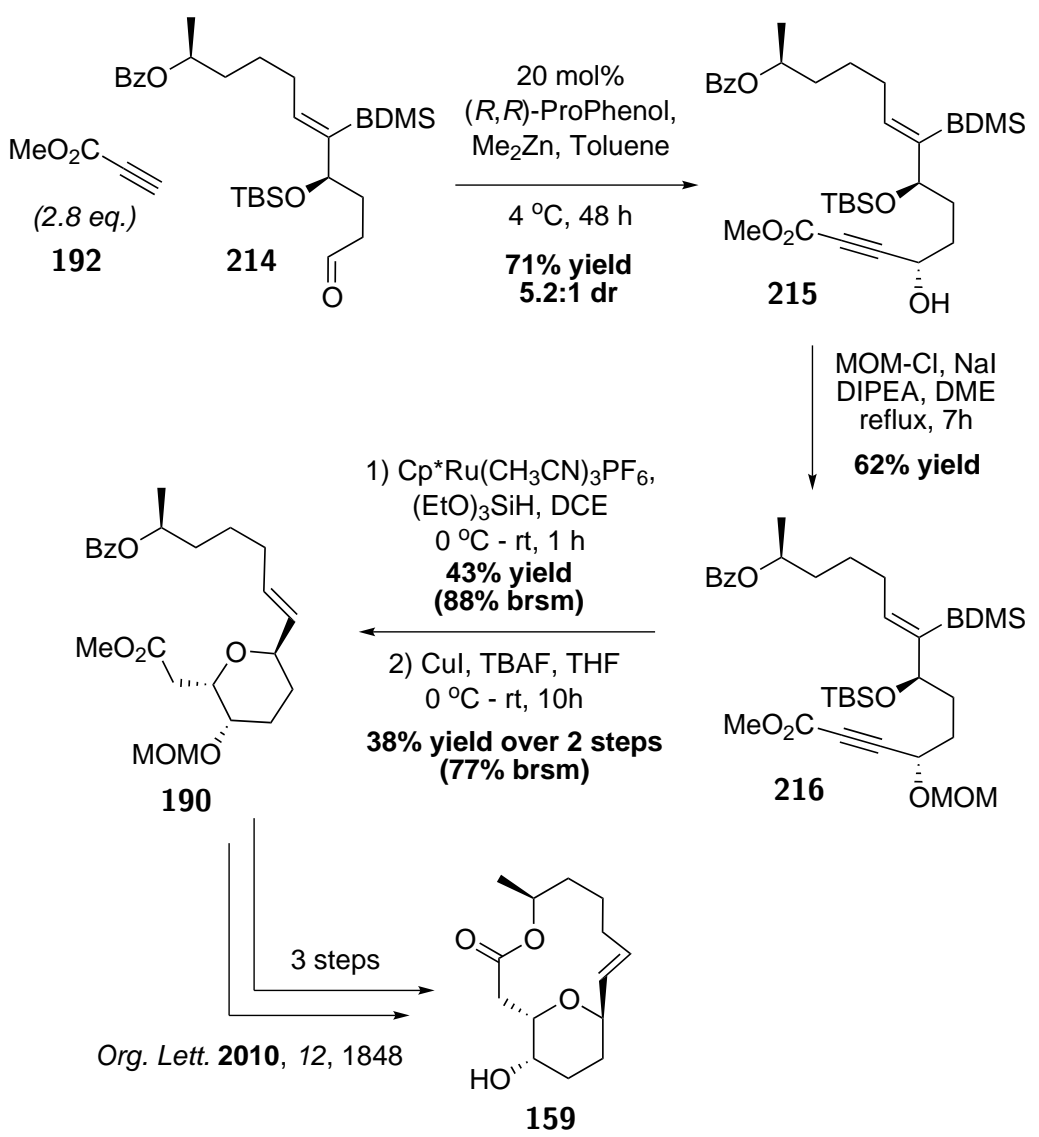

Scheme 4.19 Formal Total Synthesis of Aspergillide B.

\subsection{Summary}

In summary, an enantioselective formal total synthesis of aspergillide B has been accomplished using sequential Zn-catalyzed alkyne addition and Ru-catalyzed trans-hydrosilylationdesilylation to access $E$-alkenes. ${ }^{31}$ The hydrosilylation-desilylation protocol not only provides the $E$ geometry but also allows chemoselective differentiation of the two double bonds in a subsequent hydrogenation step. This synthesis of an aspergillide B precursor has led to the development of new conditions for Zn-ProPhenol catalyzed alkynylation that provide excellent yield and enantioselectivity using just a single equivalent of alkyne. This synthetic route provides access to aspergillide B from inexpensive and commercially available materials in just 16 total steps, with a longest linear sequence of 15 steps, and the use of 6 highly efficient transition metal-catalyzed reactions. 


\subsection{Experimental Data for Chapter 4}

\subsubsection{General Experimental Methods}

Unless otherwise noted, the following conditions apply. All reactions were performed in flamedried septumsealed glassware with magnetic stirring under an atmosphere of argon or nitrogen. Moisture- and oxygen-sensitive liquids and solutions were transferred using an oven-dried stainless steel syringe or cannula. Toluene, tetrahydrofuran, diethyl ether, dimethylformamide (DMF), dimethylsulfoxide (DMSO), dimethoxyethane (DME) and dichloromethane were purified with a Solv-Tek solvent purification system by passing through a column of activated alumina. 1,2-Dichloroethane and $N, N$-Diisopropylethylamine were distilled from calcium hydride. 1,3- Diaminopropane was distilled from barium oxide immediately before use. Benzyldimethylsilane, ethoxy(dimethyl)silane and triethoxysilane were distilled immediately before use. All other commercially available chemicals were used as received, without further purification. Organic solutions were concentrated by rotary evaporation below 40 ${ }^{\circ} \mathrm{C}$. All isolated compounds were $>90 \%$ pure as judged by ${ }^{1} \mathrm{H}-\mathrm{NMR}$. Analytical thin layer chromatography (TLC) was performed on EMD silica gel $60 \mathrm{~F}_{254}$ plates $(0.25 \mathrm{~mm})$. Visualization was achieved by UV irradiation $(254 \mathrm{~nm})$ or by heating after treatment with a potassium permanganate or ceric ammonium molybdate dip. Purification of products by flash column chromatography (FCC) was conducted using Silicycle silica gel (particle size 0.040-0.063 $\mathrm{mm}$ ) with the solvent systems indicated. ${ }^{1} \mathrm{HNMR}$ spectra were recorded on either: a Varian Unity Inova 600 spectrometer at $600 \mathrm{MHz}$, a Varian Unity Inova 500 spectrometer at $500 \mathrm{MHz}$, a Varian Mercury NMR spectrometer at $400 \mathrm{MHz}$, Varian Direct Drive 400 spectrometer at $400 \mathrm{MHz}$ or a Varian Inova 300 at $300 \mathrm{MHz}$. Data are listed as follows: chemical shift in ppm using chloroform as an internal standard $(7.26 \mathrm{ppm})$, multiplicity $(\mathrm{s}=$ singlet, $\mathrm{d}=$ doublet, $\mathrm{t}$ $=$ triplet, $\mathrm{q}=$ quartet, quint $=$ quintet, $\mathrm{m}=$ multiplet or overlap of non-equivalent resonances, $\mathrm{br}=$ broad, app. = apparent, obs. = (partially) obscured $)$, integration, peak assignment. ${ }^{13} \mathrm{C}-\mathrm{NMR}$ spectra were recorded on a Varian Unity Inova 500 spectrometer at $126 \mathrm{MHz}$ or a Varian Mercury NMR spectrometer at $101 \mathrm{MHz}$ and the data are listed as follows: chemical shift in ppm using chloroform as an internal standard $(77 \mathrm{ppm})$, peak assignment. All ${ }^{13} \mathrm{C}$ NMR spectra were proton decoupled. Infrared spectroscopic data was recorded as a thin film on a sodium chloride plate using a Thermo Scientific Nicolet IR100 FT-IR spectrometer. High resolution mass spectrometry (HRMS) was performed on a Waters Micromass Q-TOF API-US mass spectrometer. Chiral HPLC analysis was performed on a Thermo Separation Products Spectra Series P-100 and Agilent Technologies 1200 Series HPLC using the indicated Chiralcel ${ }^{\circledR}$ and Chiralpak ${ }^{\circledR}$ columns. Optical rotations were measured using a Jasco DIP1000 digital polarimeter using $5 \mathrm{~cm}$ glass cells with a $\mathrm{Na} 589 \mathrm{~nm}$ filter. The reported specific rotations are an average of 10 measurements and are reported as $[\alpha]_{\mathrm{D}}{ }^{25}$, concentration $(\mathrm{g} / 100$ $\mathrm{mL}$ ), and solvent.

\section{General Procedure for Zn-ProPhenol-Catalyzed Addition of Alkynes to Alde- hydes}

To a solution of $(S, S)$-ProPhenol ligand (10 or $20 \mathrm{~mol} \%$ ) and alkyne (1.2 equiv or 2.8 equiv) in toluene (0.48 $\mathrm{M}$ with respect to alkyne) was added dimethyl zinc (as a $1.2 \mathrm{M}$ solution in toluene, 1.5 equiv or 2.95 equiv) at $0{ }^{\circ} \mathrm{C}$. The reaction was warmed to room temperature and stirred for 60 minutes before addition of the aldehyde substrate ( 1 equiv) at $0{ }^{\circ} \mathrm{C}$. The reaction was stirred for 48 hours at $4{ }^{\circ} \mathrm{C}$ before quenching with saturated, aqueous $\mathrm{NH}_{4} \mathrm{Cl}$. The organic phase was extracted three times with $\mathrm{Et}_{2} \mathrm{O}$ and the combined organics were concentrated in vacuo. The crude product was purified by flash column chromatography. 
General Procedure for the Preparation of Racemic Propargylic Alcohols for the Determination of Enantiomeric Excess

To a solution of $( \pm)$-ProPhenol ligand (10 mol\%) and alkyne (1.2 equiv or 2.8 equiv) in toluene (0.25 $\mathrm{M}$ with respect to alkyne) was added dimethyl zinc (as a 1.2 $\mathrm{M}$ solution in toluene,1.5 equiv or 2.95 equiv) at $0{ }^{\circ} \mathrm{C}$. The reaction was warmed to room temperature and stirred for 60 minutes before addition of the aldehyde substrate $(1$ eq. $)$ at $0{ }^{\circ} \mathrm{C}$. The reaction was stirred overnight at room temperature before quenching with saturated, aqueous $\mathrm{NH}_{4} \mathrm{Cl}$. The organic phase was extracted three times with $\mathrm{Et}_{2} \mathrm{O}$ and the combined organics were concentrated in vacuo. The crude product was purified by flash column chromatography.

\subsubsection{Experimental Details and Characterization Data}

\section{Alkyne Synthesis}

\section{Preparation of Alkyne ( \pm$)-111$}

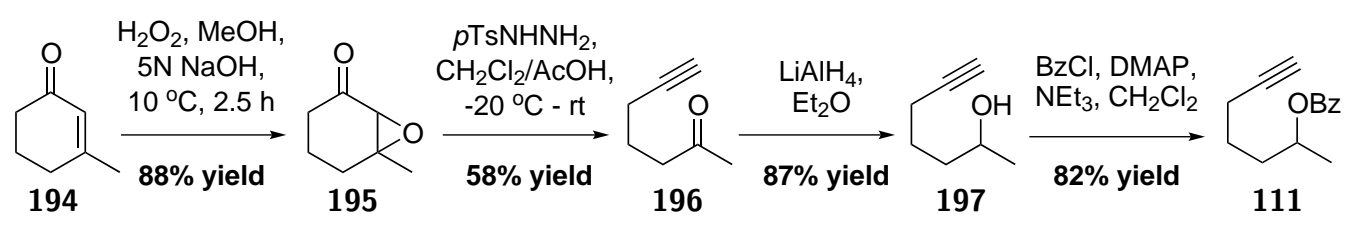

Preparation of 6-Methyl-7-oxabicyclo[4.1.0]heptan-2-one, 195.<smiles>CC12CCCC(=O)C1O2</smiles>

To a solution of 3-methylcyclohex-2-enone $(6.80 \mathrm{~mL}, 59.9 \mathrm{mmol}, 1.0$ equiv $)$ in methanol $(60 \mathrm{~mL})$ at $0{ }^{\circ} \mathrm{C}$ was added $30 \%$ aqueous $\mathrm{H}_{2} \mathrm{O}_{2}(18.3 \mathrm{~mL})$ over a 10 minute period. This was followed by the dropwise addition of $5 \mathrm{~N} \mathrm{NaOH}(0.4 \mathrm{~mL})$. After stirring at $c a .10{ }^{\circ} \mathrm{C}$ for 2.5 hours, the reaction was quenched with brine, extracted with $\mathrm{CH}_{2} \mathrm{Cl}_{2}$ and concentrated in vacuo. The crude product was purified by Kugelrohr distillation to yield the title compound as a clear, colorless oil $\left(6.64 \mathrm{~g}, 88 \%\right.$ yield). ${ }^{1} \mathbf{H}-\mathbf{N M R}\left(300 \mathrm{MHz} ; \mathrm{CDCl}_{3}\right): \delta 3.07(\mathrm{~s}, 1 \mathrm{H})$, 2.52-2.44 (m, 1H), 2.14-1.85 (m, 4H), 1.67-1.61 (m, 1H), $1.44(\mathrm{~s}, 3 \mathrm{H}) .{ }^{13}$ C-NMR $(75 \mathrm{MHz}$, $\left.\mathrm{CDCl}_{3}\right): \delta 207.1,62.7,62.2,35.9,28.6,22.4,17.4$. Characterization data matches that reported in the literature. ${ }^{14}$

Preparation of Hept-6-yn-2-one, 196.

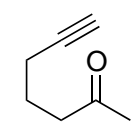

To a solution of 6-methyl-7-oxabicyclo[4.1.0] heptan-2-one 195 (6.5 g, $51.5 \mathrm{mmol}, 1.0$ eq.) in $\mathrm{CH}_{2} \mathrm{Cl}_{2} / \mathrm{AcOH}(2: 1$ mixture, $75 \mathrm{~mL}$ total $)$ at $-20{ }^{\circ} \mathrm{C}$ was added a solution of $p \mathrm{TsNHNH}_{2}(9.65$ $\mathrm{g}$ in $75 \mathrm{~mL}$ of $\left.2: 1 \mathrm{CH}_{2} \mathrm{Cl}_{2} / \mathrm{AcOH}\right)$. The reaction mixture was stirred at $-20{ }^{\circ} \mathrm{C}$ for 7 hours, $-15{ }^{\circ} \mathrm{C}$ for 2 hours, $0{ }^{\circ} \mathrm{C}$ for 3 hours and room temperature for 4 hours before quenching with $\mathrm{H}_{2} \mathrm{O}$. The reaction mixture was extracted twice with $\mathrm{Et}_{2} \mathrm{O}$ and the combined organic layers were dried over $\mathrm{MgSO}_{4}$, filtered and concentrated in vacuo. The crude product was purified by 
flash column chromatography $\left(10 \% \mathrm{Et}_{2} \mathrm{O} /\right.$ pet. ether). The final traces of $\mathrm{AcOH}$ were removed by dissolving the product in $\mathrm{Et}_{2} \mathrm{O}$ and washing with saturated aqueous $\mathrm{NaHCO}_{3}$. The title compound was isolated as a clear, colorless oil ( $3.28 \mathrm{~g}, 58 \%$ yield $) . \mathbf{R}_{\mathbf{f}}=0.26(5 \% \mathrm{EtOAc} /$ pet. ether). ${ }^{1} \mathbf{H}-\mathbf{N M R}\left(400 \mathrm{MHz} ; \mathrm{CDCl}_{3}\right): \delta 2.55(\mathrm{t}, J=7.2 \mathrm{~Hz}, 2 \mathrm{H}), 2.19(\mathrm{td}, J=6.9,2.6 \mathrm{~Hz}$, 2H), $2.12(\mathrm{~s}, 3 \mathrm{H}), 1.92(\mathrm{t}, J=2.7 \mathrm{~Hz}, 1 \mathrm{H}), 1.75$ (quintet, $J=7.1 \mathrm{~Hz}, 2 \mathrm{H}) .{ }^{13} \mathbf{C}-\mathbf{N M R}(101$ $\left.\mathrm{MHz}, \mathrm{CDCl}_{3}\right): \delta 208.4,83.7,69.2,42.2,30.2,22.4,17.9$. Characterization data matches that reported in the literature. ${ }^{14}$

Preparation of ( \pm )-Hept-6-yn-2-ol, 197.

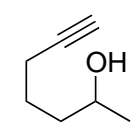

To a suspension of $\mathrm{LiAlH}_{4}(0.053 \mathrm{~g}, 1.40 \mathrm{mmol})$ in $\mathrm{Et}_{2} \mathrm{O}(7 \mathrm{~mL})$ at $0{ }^{\circ} \mathrm{C}$ was added a solution of hept-6-yn-2-one $\left(0.300 \mathrm{~g}, 2.72 \mathrm{mmol}, 1\right.$ equiv) in $\mathrm{Et}_{2} \mathrm{O}(0.7 \mathrm{~mL})$. The reaction was stirred at $0{ }^{\circ} \mathrm{C}$ for 30 minutes before quenching with water. The reaction mixture was extracted with $\mathrm{Et}_{2} \mathrm{O}$ and the combined organics were dried over $\mathrm{MgSO}_{4}$, filtered and concentrated in vacuo. The desired product was isolated as a clear colorless oil $(0.265 \mathrm{~g}, 87 \%$ yield $)$ and was used without further purification. ${ }^{1} \mathbf{H}-\mathbf{N M R}\left(400 \mathrm{MHz} ; \mathrm{CDCl}_{3}\right): \delta 8.03-8.01(\mathrm{~m}, 2 \mathrm{H}), 7.56-7.51$ $(\mathrm{m}, 1 \mathrm{H}), 7.44-7.40(\mathrm{~m}, 2 \mathrm{H}), 5.21-5.13(\mathrm{~m}, 1 \mathrm{H}), 2.23(\mathrm{td}, J=7.0,2.6 \mathrm{~Hz}, 2 \mathrm{H}), 1.94(\mathrm{t}, J=$ $2.5 \mathrm{~Hz}, 1 \mathrm{H}), 1.87-1.58(\mathrm{~m}, 4 \mathrm{H}), 1.34(\mathrm{~d}, J=6.3 \mathrm{~Hz}, 3 \mathrm{H})$. Characterization data matches that reported in the literature. ${ }^{14}$

\section{Preparation of $( \pm)$-Hept-6-yn-2-yl benzoate, 111.}

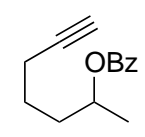

See below for experimental details and characterization data. Characterization data matches that reported in the literature. ${ }^{14}$

Preparation of Alkyne (-)-111

Preparation of Hept-3-yn-2-one, 201.

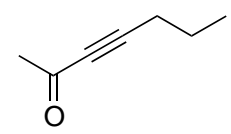

To a solution of 1-pentyne, $13(5.0 \mathrm{~g}, 73 \mathrm{mmol}, 1.0$ eq. $)$ in $\mathrm{Et}_{2} \mathrm{O}(200 \mathrm{~mL})$ at $-78{ }^{\circ} \mathrm{C}$ was added $n \mathrm{BuLi}(29.2 \mathrm{~mL}$ of a $2.5 \mathrm{M}$ solution in hexanes, $73 \mathrm{mmol}, 1.0$ eq.). The reaction was stirred for 30 minutes before addition of $N, N$-dimethylacetamide $(8.48 \mathrm{~mL}, 91.25 \mathrm{mmol}$, 1.25 eq.) in 7 portions over 35 minutes. The reaction was then warmed to $4{ }^{\circ} \mathrm{C}$ and stirred overnight before being quenching with water and acidified with saturated aqueous $\mathrm{NH}_{4} \mathrm{Cl}$. The reaction mixture was then extracted twice with $\mathrm{Et}_{2} \mathrm{O}$ and the combined organics were dried over $\mathrm{MgSO}_{4}$, filtered and concentrated in vacuo. The crude product was purified by flash column chromatography ( $5 \% \mathrm{Et}_{2} \mathrm{O} /$ pet. ether). The title compound was isolated as a yellow oil (5.138 g, $64 \%$ yield). $\mathbf{R}_{\mathbf{f}}=0.6\left(15 \% \mathrm{Et}_{2} \mathrm{O} /\right.$ pet. ether $) .{ }^{\mathbf{1}} \mathbf{H} \mathbf{~ N M R}\left(400 \mathrm{MHz} ; \mathrm{CDCl}_{3}\right)$ : $\delta 2.32(\mathrm{~m}, 2 \mathrm{H}), 2.30(\mathrm{~s}, 3 \mathrm{H}), 1.59(\mathrm{~m}, 2 \mathrm{H}), 0.99(\mathrm{t}, J=7.4 \mathrm{~Hz}, 3 \mathrm{H}) .{ }^{13} \mathbf{C}$ NMR $(101 \mathrm{MHz}$, 
$\left.\mathrm{CDCl}_{3}\right): \delta 185.19,94.15,81.73,32.99,21.43,21.06,13.68$. Characterization data matches literature. ${ }^{20}$

Preparation of $(S)$-Hept-3-yn-2-ol, 203.

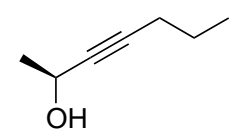

To a solution of freshly prepared hept-3-yn-2-one 201 (2.0 g, $18.16 \mathrm{mmol}, 1.0$ eq. $)$ in degassed $i \mathrm{PrOH}(51 \mathrm{~mL}$, freeze-pump-thaw degassing) was added a solution of $\mathrm{Ru}[(1 S, 2 S)$ $p \operatorname{TsNCH}(\mathrm{Ph}) \mathrm{CH}(\mathrm{Ph}) \mathrm{NH}]\left(\eta^{6}\right.$ - $p$-cymene) $(0.108 \mathrm{~g}, 1 \mathrm{~mol} \%)$ in degassed $i \mathrm{PrOH}(40 \mathrm{~mL})$ at room temperature. The reaction was stirred for 20 hours before careful removal of $i \mathrm{PrOH}$ in vacuo. The crude product was purified by flash column chromatography $\left(15 \% \mathrm{Et}_{2} \mathrm{O} /\right.$ pet. ether). The title compound was obtained as a yellow oil (1.435 g, $70 \%$ yield). $\mathbf{R}_{\mathbf{f}}=0.32(15 \%$ $\mathrm{Et}_{2} \mathrm{O} /$ pet. ether). ${ }^{1} \mathbf{H}$ NMR $\left(500 \mathrm{MHz} ; \mathrm{CDCl}_{3}\right): \delta 4.56-4.53(\mathrm{~m}, 1 \mathrm{H}), 2.22-2.18(\mathrm{~m}, 2 \mathrm{H}), 1.55$ (sextet, $J=7.2 \mathrm{~Hz}, 2 \mathrm{H}), 1.45(\mathrm{~d}, J=6.5 \mathrm{~Hz}, 3 \mathrm{H}), 1.00(\mathrm{t}, J=7.4 \mathrm{~Hz}, 3 \mathrm{H}) .{ }^{13} \mathrm{C}$ NMR $(101$ $\left.\mathrm{MHz}, \mathrm{CDCl}_{3}\right): \delta 84.75,82.57,58.81,24.97,22.26,20.82,13.66 .[\alpha]_{\mathrm{D}}{ }^{25}=-29.2^{\circ}(c=1.13$, $\mathrm{CHCl}_{3}$ ). Enantiomeric excess was determined by chiral HPLC analysis of the subsequently prepared hept-6-yn-2-yl benzoate (Chiral HPLC: Chiralcel ${ }^{\circledR}$ AD column, heptane: $i \mathrm{PrOH}$ $=150: 1,0.6 \mathrm{~mL} / \mathrm{min}, \lambda=254 \mathrm{~nm} ; \mathrm{t}_{\mathrm{R}}=14.86$ (minor), 16.85 (major) minutes: $97 \%$ ee). Characterization data matches literature. ${ }^{20}$

\section{Preparation of $(S)$-Hept-6-yn-2-ol, 197.}<smiles>C#CCCCC(C)O</smiles>

A $30 \%$ dispersion of potassium hydride in mineral oil (4.0 g, $30 \mathrm{mmol}, 4.0$ eq.) was washed twice with $\mathrm{Et}_{2} \mathrm{O}$ under an atmosphere of nitrogen, triturating the ether washings (for a detailed description of the safe use of KH, see: Brown, C. A. J. Org. Chem. 1974, 39, 3913.). Residual solvent was removed in vacuo. Freshly distilled 1,3-diaminopropane $(23 \mathrm{~mL})$ was added and the reaction was stirred at room temperature for an hour. (S)-Hept-3-yn-2-ol (0.839 g, $7.48 \mathrm{mmol}$, 1.0 eq.) was then added dropwise at $c a .15{ }^{\circ} \mathrm{C}$ (water bath). The reaction was subsequently warmed to room temperature and stirred for 2 hours. The reaction was then cooled to $0{ }^{\circ} \mathrm{C}$ and quenched carefully with water, while under an atmosphere of nitrogen. The reaction mixture was extracted with $\mathrm{Et}_{2} \mathrm{O}$, dried over $\mathrm{MgSO}_{4}$, filtered and concentrated in vacuo. The crude product was purified by flash column chromatography $\left(30 \% \mathrm{Et}_{2} \mathrm{O} /\right.$ pet. ether $)$. The title compound was isolated as a clear, colorless oil $(0.682 \mathrm{~g}, 81 \%$ yield $) . \mathbf{R}_{\mathbf{f}}=0.20\left(25 \% \mathrm{Et}_{2} \mathrm{O} /\right.$ pet. ether). ${ }^{1} \mathbf{H}$ NMR $\left(500 \mathrm{MHz} ; \mathrm{CDCl}_{3}\right): \delta 3.87(\mathrm{~m}, 1 \mathrm{H}), 2.25(\mathrm{~m}, 2 \mathrm{H}), 1.99(\mathrm{t}, J=2.6 \mathrm{~Hz}$, $1 \mathrm{H}), 1.71-1.58(\mathrm{~m}, 4 \mathrm{H}), 1.35(\mathrm{~s}, 1 \mathrm{H}), 1.24(\mathrm{~d}, J=6.2 \mathrm{~Hz}, 3 \mathrm{H}) .{ }^{13} \mathbf{C} \mathbf{N M R}\left(101 \mathrm{MHz}, \mathrm{CDCl}_{3}\right)$ : $\delta 84.55,68.71,67.83,38.40,24.88,23.81,18.58$. Characterization data matches literature. ${ }^{20}$

Preparation of $(S)$-Hept-6-yn-2-yl benzoate, 111.

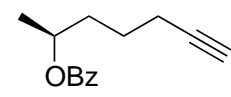

To a solution of $(S)$-hept-6-yn-2-ol, 17 (0.426 g, $3.80 \mathrm{mmol}, 1.0$ eq.) in $\mathrm{CH}_{2} \mathrm{Cl}_{2}$ (19 mL) was added $\mathrm{NEt}_{3}$ (0.95 mL, $6.83 \mathrm{mmol}, 1.8$ eq.), DMAP (0.232 g, $1.9 \mathrm{mmol}, 0.5$ eq.) and benzoyl chloride $(0.617 \mathrm{~mL}, 5.32 \mathrm{mmol}, 1.4$ eq.) at room temperature. The reaction was stirred for 
6 hours before being quenched with water and extracted twice with $\mathrm{CH}_{2} \mathrm{Cl}_{2}$. The combined organics were dried over $\mathrm{MgSO}_{4}$, filtered and concentrated in vacuo. The crude product was purified by flash column chromatography $\left(5 \% \mathrm{Et}_{2} \mathrm{O} /\right.$ pet. ether $)$ to produce the title compound as a colorless oil $(0.688 \mathrm{~g}, 84 \%$ yield $) . \mathbf{R}_{\mathbf{f}}=0.38$ (5\% Et ${ }_{2} \mathrm{O} /$ Pet. Ether). ${ }^{1} \mathbf{H}$ NMR $(400 \mathrm{MHz}$; $\left.\mathrm{CDCl}_{3}\right): \delta 8.03-8.01(\mathrm{~m}, 2 \mathrm{H}), 7.56-7.51(\mathrm{~m}, 1 \mathrm{H}), 7.44-7.40(\mathrm{~m}, 2 \mathrm{H}), 5.21-5.13(\mathrm{~m}, 1 \mathrm{H}), 2.23(\mathrm{td}$, $J=7.0,2.6 \mathrm{~Hz}, 2 \mathrm{H}), 1.94(\mathrm{t}, J=2.5 \mathrm{~Hz}, 1 \mathrm{H}), 1.87-1.58(\mathrm{~m}, 4 \mathrm{H}), 1.34(\mathrm{~d}, J=6.3 \mathrm{~Hz}, 3 \mathrm{H})$. ${ }^{13} \mathrm{C}$ NMR $\left(101 \mathrm{MHz}, \mathrm{CDCl}_{3}\right): \delta 166.37,133.01,130.93,129.74,128.52,84.21,71.32,68.92$, 35.27, 24.63, 20.35, 18.53. $[\alpha]_{\mathrm{D}}{ }^{25}=-39.4^{\circ}\left(c=1.10, \mathrm{CHCl}_{3}\right)$. Chiral HPLC: Chiralcel ${ }^{\circledR} \mathrm{AD}$ column, heptane: $i \mathrm{PrOH}=150: 1,0.6 \mathrm{~mL} / \mathrm{min}, \lambda=254 \mathrm{~nm}$; $\mathrm{t}_{\mathrm{R}}=14.86$ (minor), 16.85 (major) minutes: $97 \%$ ee. Characterization data matches literature. ${ }^{32}$

\section{Preparation of Aldehyde Substrates}

Preparation of 4-((tert-Butyldimethylsilyl)oxy)butan-1-ol.<smiles>OCCCC[SeH2]</smiles>

To a suspension of $\mathrm{NaH}$ (4.9 $\mathrm{g}$ as a $60 \%$ dispersion in mineral oil, $120 \mathrm{mmol}, 4.0$ equiv) in THF $(160 \mathrm{~mL})$ at $0{ }^{\circ} \mathrm{C}$ was added 1,4-butanediol $(10.03 \mathrm{~mL}, 120 \mathrm{mmol}, 4.0$ equiv). After stirring the reaction mixture for 15 minutes at $0{ }^{\circ} \mathrm{C}$, a solution of tert-butyldimethylsilyl chloride (4.5 g, $30 \mathrm{mmol}, 1.0$ equiv) in THF $(40 \mathrm{~mL})$ was added. The reaction mixture was slowly warmed to room temperature and stirred for 3 hours before quenching with $\mathrm{H}_{2} \mathrm{O}$. The reaction mixture was extracted with EtOAc and the combined organics were dried over $\mathrm{MgSO}_{4}$, filtered and concentrated in vacuo. The crude product was purified by silica gel chromatography (20\% EtOAc/ pet. ether). The title compound was isolated as a clear, colorless oil ( $5.3 \mathrm{~g}, 87 \%$ yield). $\mathbf{R}_{\mathbf{f}}=0.26$ (15\% EtOAc/pet. ether). ${ }^{\mathbf{1}} \mathbf{H}-\mathbf{N M R}(400 \mathrm{MHz}$ $\left.\mathrm{CDCl}_{3}\right): \delta 3.68-3.60(\mathrm{~m}, 4 \mathrm{H}), 2.44-2.41(\mathrm{~m}, 1 \mathrm{H}), 1.66-1.60(\mathrm{~m}, 4 \mathrm{H}), 0.88(\mathrm{~s}, 9 \mathrm{H}), 0.05(\mathrm{~s}, 6 \mathrm{H})$. ${ }^{13} \mathrm{C}-\mathrm{NMR}\left(101 \mathrm{MHz}, \mathrm{CDCl}_{3}\right): \delta 63.6,63.0,30.5,30.1,26.1,18.5,-5.2$. Characterization data matches that reported in the literature. ${ }^{33}$

\section{Preparation of 4-((tert-Butyldimethylsilyl)oxy)butanal, 112.}<smiles>O=CCCC[18OH]</smiles>

To a solution of 4-((tert-butyldimethylsilyl)oxy)butan-1-ol (3.0 g, $14.68 \mathrm{mmol}, 1.0$ equiv) in $\mathrm{CH}_{2} \mathrm{Cl}_{2}(70 \mathrm{~mL})$ was added $\mathrm{H}_{2} \mathrm{O}(70 \mathrm{~mL}), \mathrm{KBr}\left(0.176 \mathrm{~g}, 1.47 \mathrm{mmol}, 0.1\right.$ equiv), $\mathrm{NaHCO}_{3}(0.321$ $\mathrm{g}, 3.82 \mathrm{mmol}, 0.26$ equiv) and TEMPO $(0.046 \mathrm{~g}, 0.29 \mathrm{mmol}, 2 \mathrm{~mol} \%)$. The reaction mixture was cooled to $0{ }^{\circ} \mathrm{C}$ and $\mathrm{NaOCl}(16.18 \mathrm{~mL}$ of $10-15 \%$ aqueous solution, $16.15 \mathrm{mmol}, 1.1$ equiv) was added slowly over 10 minutes. The reaction was stirred at $0{ }^{\circ} \mathrm{C}$ for an hour, slowly warmed to room temperature and quenched with $10 \%$ aqueous $\mathrm{Na}_{2} \mathrm{~S}_{2} \mathrm{O}_{3}$. The reaction mixture was extracted twice with $\mathrm{CH}_{2} \mathrm{Cl}_{2}$ and the combined organic layers were dried over $\mathrm{MgSO}_{4}$, filtered and concentrated in vacuo. The crude product was purified by silica gel chromatography (10\% $\mathrm{Et}_{2} \mathrm{O} /$ pet. ether) followed by Kugelrohr distillation. The title compound was isolated as a clear, colorless oil (0.548 g, $18 \%$ yield - unoptimized). $\mathbf{R}_{\mathbf{f}}=0.59$ (5\% EtOAc/ pet. ether). ${ }^{1} \mathbf{H}-\mathbf{N M R}\left(400 \mathrm{MHz} ; \mathrm{CDCl}_{3}\right): \delta 9.78(\mathrm{t}, J=1.7 \mathrm{~Hz}, 1 \mathrm{H}), 3.64(\mathrm{t}, J=6.0 \mathrm{~Hz}, 2 \mathrm{H}), 2.50(\mathrm{td}$, $J=7.1,1.7 \mathrm{~Hz}, 2 \mathrm{H}), 1.88-1.82(\mathrm{~m}, 2 \mathrm{H}), 0.88(\mathrm{~s}, 9 \mathrm{H}), 0.03(\mathrm{~s}, 6 \mathrm{H}) .{ }^{\mathbf{1 3}} \mathbf{C}-\mathbf{N M R}(101 \mathrm{MHz}$, $\left.\mathrm{CDCl}_{3}\right): \delta 202.9,65.2,62.3,41.0,26.1,18.5,-5.2$. Characterization data matches that reported in the literature. ${ }^{34}$ 


\section{Preparation of 4-((4-Methoxybenzyl)oxy)butan-1-ol}<smiles>OCCCCO[18OH]</smiles>

To a suspension of $\mathrm{NaH}$ (4.9 g as a $60 \%$ dispersion in mineral oil, $120 \mathrm{mmol}, 4.0$ equiv) in THF $(200 \mathrm{~mL})$ at $0{ }^{\circ} \mathrm{C}$ was added 1,4-butanediol $(10.03 \mathrm{~mL}, 120 \mathrm{mmol}, 4.0$ equiv). After the reaction was warmed to room temperature and stirred for 15 minutes, $p$-methoxybenzyl chloride (2.31 g, $14.75 \mathrm{mmol}, 1.0$ equiv) was added and the reaction was stirred overnight. The reaction was then quenched with $\mathrm{H}_{2} \mathrm{O}$, extracted with $\mathrm{Et}_{2} \mathrm{O}$ and the combined organics were dried over $\mathrm{MgSO}_{4}$, filtered and concentrated in vacuo. The crude product was purified by silica gel chromatography (60\% EtOAc/ pet. ether). The title compound was isolated as a clear, colorless oil (0.412 g, $13 \%$ yield - unoptimized). $\mathbf{R}_{\mathbf{f}}=0.10$ ( $15 \%$ EtOAc/ pet. ether). ${ }^{1} \mathbf{H}-\mathbf{N M R}\left(400 \mathrm{MHz} ; \mathrm{CDCl}_{3}\right): \delta 7.24(\mathrm{~m}, 2 \mathrm{H}), 6.88-6.84(\mathrm{~m}, 2 \mathrm{H}), 4.43(\mathrm{~s}, 2 \mathrm{H}), 3.78(\mathrm{~s}, 3 \mathrm{H})$, $3.62(\mathrm{t}, J=5.7 \mathrm{~Hz}, 2 \mathrm{H}), 3.47(\mathrm{t}, J=5.7 \mathrm{~Hz}, 2 \mathrm{H}), 1.70-1.62(\mathrm{~m}, 4 \mathrm{H}) .{ }^{13}$ C-NMR $(101 \mathrm{MHz}$, $\left.\mathrm{CDCl}_{3}\right): \delta 159.4,130.4,129.4,114.0,73.0,70.3,62.8,55.5,30.7,27.0$. Characterization data matches that reported in the literature. ${ }^{35}$

\section{Preparation of 4-((4-Methoxybenzyl)oxy)butanal, 115.}

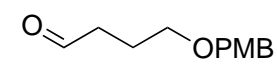

To a solution of 4-((4-methoxybenzyl)oxy)butan-1-ol (0.412 g, $1.96 \mathrm{mmol}, 1.0$ equiv) in $\mathrm{CH}_{2} \mathrm{Cl}_{2}(3.45 \mathrm{~mL})$ was added DMSO $(0.46 \mathrm{~mL})$ and $\mathrm{NEt}_{3}(0.45 \mathrm{~mL}, 3.23 \mathrm{mmol}, 1.6$ equiv). The reaction mixture was then cooled to $0{ }^{\circ} \mathrm{C}$ and $\mathrm{SO}_{3}$.pyridine $(0.518 \mathrm{~g}, 3.25 \mathrm{mmol}, 1.7$ equiv) was added in one portion. The reaction was warmed to room temperature and stirred for 4 hours before quenching with $\mathrm{H}_{2} \mathrm{O}$. The reaction mixture was subsequently extracted with $\mathrm{Et}_{2} \mathrm{O}$ and the combined organics were dried over $\mathrm{MgSO}_{4}$, filtered and concentrated in vacuo. The crude product was purified by flash column chromatography $\left(20 \rightarrow 30 \% \mathrm{Et}_{2} \mathrm{O} /\right.$ pet. ether $)$ to give the title compound as a clear, colorless oil $\left(0.2113 \mathrm{~g}, 52 \%\right.$ yield - unoptimized). $\mathbf{R}_{\mathbf{f}}=$ $0.10\left(15 \%\right.$ EtOAc/ pet. ether). ${ }^{1} \mathbf{H}-\mathbf{N M R}\left(300 \mathrm{MHz} ; \mathrm{CDCl}_{3}\right): \delta 9.80(\mathrm{t}, J=1.6 \mathrm{~Hz}, 1 \mathrm{H})$, 7.30-7.24 (m, 2H), 6.93-6.88 (m, 2H), $4.44(\mathrm{~s}, 2 \mathrm{H}), 3.83(\mathrm{~s}, 3 \mathrm{H}), 3.50(\mathrm{t}, J=6.1 \mathrm{~Hz}, 2 \mathrm{H}), 2.56$ $(\mathrm{td}, J=7.1,1.6 \mathrm{~Hz}, 2 \mathrm{H}), 1.96(\mathrm{tt}, J=7.1,6.1 \mathrm{~Hz}, 2 \mathrm{H}) .{ }^{13} \mathrm{C}-\mathrm{NMR}\left(126 \mathrm{MHz}, \mathrm{CDCl}_{3}\right): \delta$ 202.6, 159.4, 130.6, 129.5 (2C), 114.0 (2C), 72.9, 69.1, 55.5, 41.3, 22.8. Characterization data matches that reported in the literature. ${ }^{36}$

Preparation of (E)-4-((tert-Butyldimethylsilyl)oxy)but-2-enal, 142.
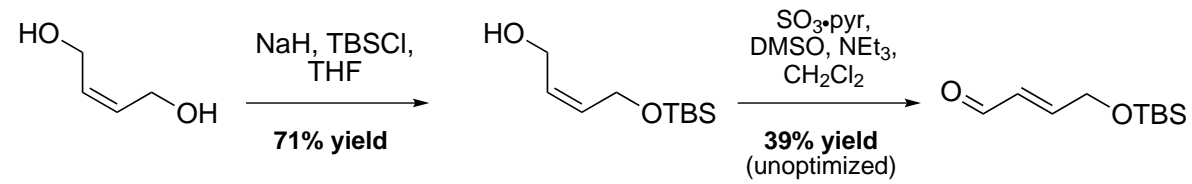

\section{(Z)-4-((tert-Butyldimethylsilyl)oxy)but-2-en-1-ol}

To a suspension of $\mathrm{NaH}(4.9 \mathrm{~g}$ as a $60 \%$ dispersion in mineral oil, $120 \mathrm{mmol}, 4.0$ equiv) in THF $(160 \mathrm{~mL})$ at $0{ }^{\circ} \mathrm{C}$ was added (Z)-but-2-ene-1,4-diol $(9.86 \mathrm{~mL}, 120 \mathrm{mmol}$, 4.0 equiv). The reaction mixture was stirred at $0{ }^{\circ} \mathrm{C}$ for 15 minutes before the addition of tert-butyldimethylsilyl chloride $(4.5 \mathrm{~g}, 30 \mathrm{mmol}, 1.0 \mathrm{equiv})$. The reaction was warmed to room temperature and stirred overnight before quenching with $\mathrm{H}_{2} \mathrm{O}$. The reaction mixture was extracted twice with $\mathrm{Et}_{2} \mathrm{O}$, the combined organics were dried over $\mathrm{MgSO}_{4}$, filtered and concentrated in vacuo. The crude product was purified via silica gel chromatography $(20 \%$ 


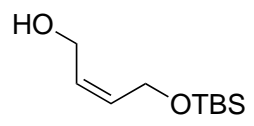

EtOAc/ pet. ether). The title compound was isolated as a clear, colorless oil (4.34 g, $71 \%$ yield). $\mathbf{R}_{\mathbf{f}}=0.34$ (20\% EtOAc/pet. ether). ${ }^{\mathbf{1}} \mathbf{H}-\mathbf{N M R}\left(400 \mathrm{MHz} ; \mathrm{CDCl}_{3}\right): \delta 5.72-5.61(\mathrm{~m}$, $2 \mathrm{H}), 4.23(\mathrm{~d}, J=4.9 \mathrm{~Hz}, 2 \mathrm{H}), 4.17(\mathrm{t}, J=4.9 \mathrm{~Hz}, 2 \mathrm{H}), 0.88(\mathrm{~s}, 9 \mathrm{H}), 0.06(\mathrm{~s}, 6 \mathrm{H}) .{ }^{13} \mathbf{C}-\mathbf{N M R}$ $\left(101 \mathrm{MHz}, \mathrm{CDCl}_{3}\right): \delta 131.5,130.3,59.8,59.0,26.1,18.5,-5.1$. Characterization data matches that reported in the literature. ${ }^{37}$

\section{Preparation of (E)-4-((tert-Butyldimethylsilyl)oxy)but-2-enal, 142.}

$$
\mathrm{O}_{\text {OTBS }}
$$

To a solution of (Z)-4-((tert-butyldimethylsilyl)oxy)but-2-en-1-ol (1.15 g, $5.68 \mathrm{mmol})$, DMSO $(0.67 \mathrm{~mL})$ and $\mathrm{NEt}_{3}(1.3 \mathrm{~mL})$ in $\mathrm{CH}_{2} \mathrm{Cl}_{2}(10 \mathrm{~mL})$ at $0{ }^{\circ} \mathrm{C}$ was added $\mathrm{SO}_{3} \cdot \operatorname{pyr}(1.5 \mathrm{~g})$. The reaction was warmed to room temperature and stirred for 5 hours before quenching with $\mathrm{H}_{2} \mathrm{O}$. The reaction mixture was extracted twice with $\mathrm{CH}_{2} \mathrm{Cl}_{2}$ and the combined organic layers were dried over $\mathrm{MgSO}_{4}$, filtered and concentrated in vacuo. The crude product was purified by silica gel chromatography $\left(5 \rightarrow 10 \% \mathrm{Et}_{2} \mathrm{O} /\right.$ pet. ether). The title compound was isolated as a clear, colorless oil $(0.44 \mathrm{~g}, 39 \%$ yield $) . \mathbf{R}_{\mathbf{f}}=0.24\left(5 \% \mathrm{Et}_{2} \mathrm{O} /\right.$ pet. ether $) .{ }^{1} \mathbf{H}-\mathbf{N M R}(300$ $\left.\mathrm{MHz} ; \mathrm{CDCl}_{3}\right): \delta 9.62(\mathrm{~d}, J=8.1 \mathrm{~Hz}, 1 \mathrm{H}), 6.91(\mathrm{dt}, J=15.5,3.3 \mathrm{~Hz}, 1 \mathrm{H}), 6.42(\mathrm{ddt}, J=$ 15.4, 8.1, 2.2 Hz, 1H), $4.47(\mathrm{dd}, J=3.3,2.2 \mathrm{~Hz}, 2 \mathrm{H}), 0.94(\mathrm{~s}, 9 \mathrm{H}), 0.11(\mathrm{~s}, 6 \mathrm{H}) .{ }^{13} \mathrm{C}-\mathrm{NMR}$ $\left(75 \mathrm{MHz}, \mathrm{CDCl}_{3}\right): \delta 193.7,156.8,130.8,62.5,26.1,18.6,-5.2$. Characterization data matches that reported in the literature. ${ }^{35}$

Preparation of (E)-4,4-Dimethoxybut-2-enal, 141.<smiles>COC(=O)/C=C/C(C)OC</smiles>

To a solution of fumaraldehyde bis(dimethyl acetal) $(2.0 \mathrm{~g}, 11.35 \mathrm{mmol}, 1.0$ equiv) in acetone $(45 \mathrm{~mL})$ was added water $(0.68 \mathrm{~mL})$, followed by Amberlyst-15 $(0.44 \mathrm{~g})$. The reaction mixture was stirred vigorously at room temperature for 5 minutes before removal of the acidic resin by filtration. The filtrate was concentrated in vacuo and prurified by silica gel chromatography $\left(15 \% \mathrm{Et}_{2} \mathrm{O} /\right.$ pet. ether). The title compound was isolated as a clear, colorless oil $(1.073 \mathrm{~g}, 73 \%$ yield $) . \mathbf{R}_{\mathbf{f}}=0.24\left(20 \% \mathrm{Et}_{2} \mathrm{O} /\right.$ pet. ether $) .{ }^{\mathbf{1}} \mathbf{H}-\mathbf{N M R}\left(400 \mathrm{MHz} ; \mathrm{CDCl}_{3}\right): \delta$ $9.60(\mathrm{~d}, J=7.8 \mathrm{~Hz}, 1 \mathrm{H}), 6.61(\mathrm{dd}, J=15.9,3.9 \mathrm{~Hz}, 1 \mathrm{H}), 6.35(\mathrm{ddd}, J=15.9,7.8,1.3 \mathrm{~Hz}$, $1 \mathrm{H}), 5.03(\mathrm{dd}, J=3.9,1.3 \mathrm{~Hz}, 1 \mathrm{H}), 3.34(\mathrm{~s}, 6 \mathrm{H}) .{ }^{13} \mathrm{C}-\mathbf{N M R}\left(126 \mathrm{MHz}, \mathrm{CDCl}_{3}\right): \delta 193.47$, $150.70,134.51,100.69,53.30$. Characterization data matches literature. ${ }^{38}$

\section{Alkynylation Products}

Preparation of $(2 S / R, 8 R)-11$-((tert-Butyldimethylsilyl)oxy)-8-hydroxyundec-6-yn2-yl benzoate, 113.

Using the standard procedure for Zn-ProPhenol-catalyzed addition of alkynes to aldehydes, the reaction was performed with $0.011 \mathrm{~g}(S, S)$-ProPhenol ligand $(0.01625 \mathrm{mmol}, 10 \mathrm{~mol} \%)$, 


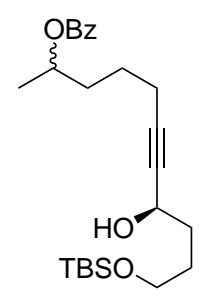

$0.009 \mathrm{~g}$ triphenylphosphine oxide $(0.0325 \mathrm{mmol}, 20 \mathrm{~mol} \%), 1 \mathrm{~mL}$ of toluene, $0.099 \mathrm{~g}$ of $( \pm)$ hept-6-yn-2-yl benzoate ( $0.455 \mathrm{mmol}, 2.8 \mathrm{equiv}), 0.399 \mathrm{~mL}$ of dimethyl zinc (as a $1.2 \mathrm{M}$ solution in toluene, $0.479 \mathrm{mmol}, 2.95 \mathrm{eq}$.$) and 0.033 \mathrm{~g}$ of 4 -((tert-butyldimethylsilyl)oxy)butanal $(0.1625$ mmol, 1.0 eq.). The crude product was purified by silica gel chromatography $(10 \rightarrow 15 \%$ EtOAc/Pet. Ether) and the title compound was obtained as a clear, colorless oil (0.0262 $\mathrm{g}$, $39 \%$ yield $) . \mathbf{R}_{\mathbf{f}}=0.38$ (20\% EtOAc/ pet. ether). ${ }^{1} \mathbf{H}-\mathbf{N M R}\left(400 \mathrm{MHz} ; \mathrm{CDCl}_{3}\right): \delta 8.02(\mathrm{~m}$, $2 \mathrm{H}), 7.53(\mathrm{~m}, 1 \mathrm{H}), 7.42(\mathrm{~m}, 2 \mathrm{H}), 5.18$ (sextet, $J=6.3 \mathrm{~Hz}, 1 \mathrm{H}), 4.37$ (app. s, $1 \mathrm{H}), 3.65(\mathrm{~m}, 2 \mathrm{H})$, $3.04(\mathrm{t}, J=5.4 \mathrm{~Hz}, 1 \mathrm{H}), 2.24(\mathrm{t}, J=7.0 \mathrm{~Hz}, 2 \mathrm{H}), 1.83-1.57(\mathrm{~m}, 8 \mathrm{H}), 1.33(\mathrm{~d}, J=6.3 \mathrm{~Hz}, 3 \mathrm{H})$, $0.87(\mathrm{~s}, 9 \mathrm{H}), 0.04(\mathrm{~s}, 6 \mathrm{H}) .{ }^{13} \mathrm{C}$ NMR $\left(101 \mathrm{MHz}, \mathrm{CDCl}_{3}\right): \delta 166.42,133.00,130.92,129.73$, $128.51,84.68,82.05,71.37,63.41,62.50,35.79,35.76,35.39,28.81,26.12,24.73,20.32,18.83$, 18.51, -5.16, -5.18. IR (film): 3415, 2930, 2857, 2236, 1717, 1451, 1385, 1359, 1315, 1275, 1176, 1108, 1027, 836, 777, $713 \mathrm{~cm}^{-1} \cdot[\alpha]_{\mathbf{D}}{ }^{25}=+5.85^{\circ}\left(c=2.5, \mathrm{CHCl}_{3}\right)$. Chiral HPLC: Chiralpak ${ }^{\circledR}$ IB column, heptane: $i \mathrm{PrOH}=92: 8,0.4 \mathrm{~mL} / \mathrm{min},=254 \mathrm{~nm} ; \mathrm{t}_{\mathrm{R}}=26.97$ (major), 27.76 (minor), 28.34 (ent-major + ent-minor) minutes: $62 \%$ ee $\left(57 \%\right.$ ee by ${ }^{1} \mathrm{H}$ NMR analysis of the corresponding $(S)$-methyl mandelate). HRMS-ESI $(m / z):[\mathrm{M}+\mathrm{Na}]^{+}$calculated for $\mathrm{C}_{24} \mathrm{H}_{38} \mathrm{O}_{4} \mathrm{SiNa}^{+}, 441.2432$; found, 441.2428 .

Representative Procedure for the Preparation ( $S$ )-Methyl Mandelate Derivatives for the Determination of Enantiomeric Excess

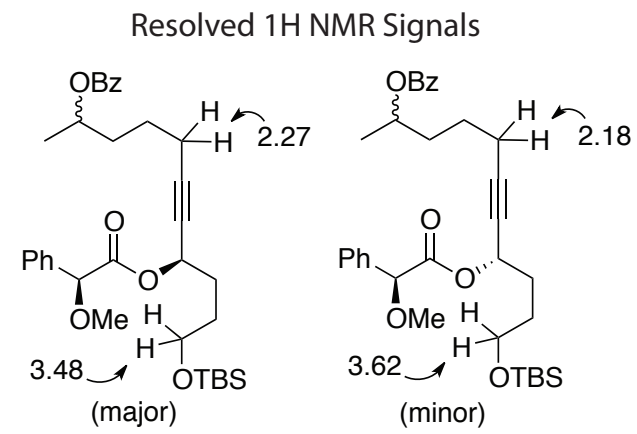

Anisotropic Shielding of the Propargylic Methylene

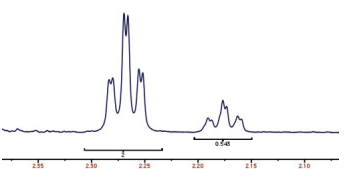

To a solution of 11-((tert-butyldimethylsilyl)oxy)-8-hydroxyundec-6-yn-2-yl benzoate $(0.0175$ g, $0.042 \mathrm{mmol}, 1.0$ eq.) in $\mathrm{CH}_{2} \mathrm{Cl}_{2}(1 \mathrm{~mL})$ was added $\mathrm{EDCI} \cdot \mathrm{HCl}(0.024 \mathrm{~g}, 0.126 \mathrm{mmol}, 3.0$ eq. $)$, $\mathrm{NEt}_{3}(0.035 \mathrm{~mL}, 0.252 \mathrm{mmol}, 6.0$ eq.), $(S)$-methoxyphenylacetic acid (0.0278 g, $0.167 \mathrm{mmol}$, 4.0 eq.) and DMAP (0.0005 g, $0.004 \mathrm{mmol}, 10 \mathrm{~mol} \%)$. The reaction was stirred overnight at room temperature before being quenched with saturated aqueous $\mathrm{NaHCO}_{3}$. The reaction mixture was then extracted with $\mathrm{CH}_{2} \mathrm{Cl}_{2}$ and the combined organic layers were dried over $\mathrm{MgSO}_{4}$, filtered and concentrated in vacuo. The crude product was purified by preparative TLC and the title compound was obtained as a clear, colorless oil ( $0.0179 \mathrm{~g}, 75 \%$ yield $)$. $\mathbf{R}_{\mathbf{f}}$ 
$=0.37\left(10 \%\right.$ EtOAc/Pet. Ether). ${ }^{1} \mathrm{H}$ NMR analysis shows a $1: 0.2715$ ratio of diastereomers, indicating $57 \%$ ee in the starting material.

(2S / R,8R)-8-Hydroxy-11-((4-methoxybenzyl)oxy)undec-6-yn-2-yl benzoate, 116.

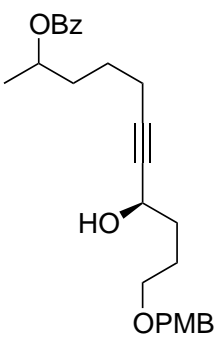

Standard procedure with 24 hour premix. To a solution of $(S, S)$-ProPhenol ligand $(0.021$ g, $0.0325 \mathrm{mmol}, 20 \mathrm{~mol} \%)$, triphenylphosphine oxide $(0.018 \mathrm{~g}, 0.065 \mathrm{mmol}, 40 \mathrm{~mol} \%)$ and ( \pm -hept-6-yn-2-yl benzoate $(0.099 \mathrm{~g}, 0.455 \mathrm{mmol}, 2.8$ eq.) in toluene $(1 \mathrm{~mL})$ was added dimethyl zinc $(0.399 \mathrm{~mL}$ as a $1.2 \mathrm{M}$ solution in toluene, $0.479 \mathrm{mmol}, 2.95$ eq. $)$ at $0{ }^{\circ} \mathrm{C}$. The reaction was warmed to room temperature and stirred for 24 hours before addition of 4-((4methoxybenzyl)oxy)butanal $\left(0.034 \mathrm{~g}, 0.1625 \mathrm{mmol}, 1 \mathrm{eq}\right.$.) at $0{ }^{\circ} \mathrm{C}$. The reaction was stirred for 48 hours at $4{ }^{\circ} \mathrm{C}$ before quenching with saturated, aqueous $\mathrm{NH}_{4} \mathrm{Cl}$. The organic phase was extracted three times with $\mathrm{Et}_{2} \mathrm{O}$ and the combined organics were concentrated in vacuo. The crude product was purified by flash column chromatography (25\% EtOAc/Pet. Ether) and the title compound was obtained as a clear, colorless oil $(0.047 \mathrm{~g}, 69 \%$ yield, $67 \%$ ee determined by ${ }^{1} \mathrm{H}$ NMR analysis of the corresponding $(S)$-methyl mandelate). $\mathbf{R}_{\mathbf{f}}=0.26$ (30\% EtOAc/ pet. ether). ${ }^{1} \mathbf{H}$ NMR $\left(400 \mathrm{MHz} ; \mathrm{CDCl}_{3}\right): \delta 8.02(\mathrm{~d}, J=7.9 \mathrm{~Hz}, 2 \mathrm{H}), 7.53(\mathrm{t}, J=7.4 \mathrm{~Hz}$, $1 \mathrm{H}), 7.41(\mathrm{t}, J=7.7 \mathrm{~Hz}, 2 \mathrm{H}), 7.23(\mathrm{~d}, J=8.5 \mathrm{~Hz}, 2 \mathrm{H}), 6.85(\mathrm{~d}, J=8.4 \mathrm{~Hz}, 2 \mathrm{H}), 5.18$ (sextet, $J=6.2 \mathrm{~Hz}, 1 \mathrm{H}), 4.42(\mathrm{~s}, 2 \mathrm{H}), 4.36$ (app. s, $1 \mathrm{H}), 3.78(\mathrm{~s}, 3 \mathrm{H}), 3.48(\mathrm{~m}, 2 \mathrm{H}), 2.80$ (br. s, $1 \mathrm{H})$, $2.24(\mathrm{t}, J=6.8 \mathrm{~Hz}, 2 \mathrm{H}), 1.83-1.57(\mathrm{~m}, 8 \mathrm{H}), 1.33(\mathrm{~d}, J=6.3 \mathrm{~Hz}, 3 \mathrm{H}) .{ }^{13} \mathbf{C}$ NMR $(101 \mathrm{MHz}$, $\left.\mathrm{CDCl}_{3}\right): \delta 166.42,159.40,133.01,130.91,130.39,129.73(2 \mathrm{C}), 128.52,114.01,84.84,81.96$, $72.84,71.37,70.06,62.52,55.48,35.75,35.38,25.82,24.70,20.32$, 18.81. IR (film): 3423,2936 , $2863,2232,1714,1612,1513,1451,1358,1275,1248,1115,1029,821 \mathrm{~cm}^{-1} \cdot[\alpha]_{\mathrm{D}}{ }^{25}=+2.51^{\circ}(c$ $\left.=0.78, \mathrm{CHCl}_{3}\right)$. HRMS-ESI $(\mathrm{m} / z):[\mathrm{M}+\mathrm{Na}]^{+}$calculated for $\mathrm{C}_{26} \mathrm{H}_{32} \mathrm{O}_{5} \mathrm{Na}, 447.2142$; found, 447.2134 .

$(2 S / R, 8 R, E)-11-(($ tert-Butyldimethylsilyl)oxy)-8-hydroxyundec-9-en-6-yn-2-yl benzoate, 217 .

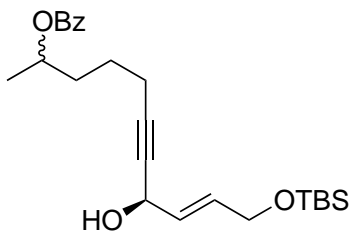

Using the standard procedure for Zn-ProPhenol-catalyzed addition of alkynes to aldehydes, the reaction was performed with $0.011 \mathrm{~g}(S, S)$-ProPhenol ligand $(0.01625 \mathrm{mmol}, 10 \mathrm{~mol} \%)$, $0.009 \mathrm{~g}$ triphenylphosphine oxide $(0.0325 \mathrm{mmol}, 20 \mathrm{~mol} \%), 0.149 \mathrm{~mL}$ of toluene, $0.035 \mathrm{~g}$ of $( \pm)$ hept-6-yn-2-yl benzoate ( $0.1625 \mathrm{mmol}, 1.0$ eq.), $0.176 \mathrm{~mL}$ of dimethyl zinc (as a $1.2 \mathrm{M}$ solution in toluene, $0.211 \mathrm{mmol}, 1.3 \mathrm{eq}$.$) and 0.033 \mathrm{~g}$ of $(E)-4$-((tert-butyldimethylsilyl)oxy)but-2-enal 
(0.1625 mmol, 1.0 eq.). The crude product was purified by flash column chromatography (15\% EtOAc/pet. ether) and the title compound was obtained as a clear, colorless oil (0.0365 g, $54 \%$ yield). $\mathbf{R}_{\mathbf{f}}=0.37\left(20 \% \mathrm{EtOAc} /\right.$ pet. ether). ${ }^{1} \mathbf{H} \mathbf{~ N M R}\left(400 \mathrm{MHz} ; \mathrm{CDCl}_{3}\right): \delta 8.02(\mathrm{~m}, 2 \mathrm{H})$, $7.53(\mathrm{tt}, J=7.4,1.6 \mathrm{~Hz}, 1 \mathrm{H}), 7.42(\mathrm{~m}, 2 \mathrm{H}), 5.92(\mathrm{~m}, 1 \mathrm{H}), 5.80$ (dddt, $J=15.2,5.5,3.7,1.8 \mathrm{~Hz}$, $1 \mathrm{H}), 5.20(\mathrm{~m}, 1 \mathrm{H}), 4.86(\mathrm{~m}, 1 \mathrm{H}), 4.17(\mathrm{ddt}, J=6.2,4.6,1.6 \mathrm{~Hz}, 2 \mathrm{H}), 2.27(\mathrm{tt}, J=6.9,1.7 \mathrm{~Hz}$, $2 \mathrm{H}), 1.96(\mathrm{t}, J=5.6 \mathrm{~Hz}, 1 \mathrm{H}), 1.84-1.59(\mathrm{~m}, 4 \mathrm{H}), 1.33(\mathrm{~d}, J=6.3 \mathrm{~Hz}, 3 \mathrm{H}), 0.88(\mathrm{~s}, 9 \mathrm{H}), 0.05$ $(\mathrm{s}, 3 \mathrm{H}), 0.04(\mathrm{~s}, 3 \mathrm{H}) .{ }^{13} \mathrm{C}$ NMR $\left(101 \mathrm{MHz}, \mathrm{CDCl}_{3}\right): \delta 166.45,133.03,131.88,130.89,129.74$, $129.27,128.52,86.49,80.12,71.33,62.93,62.86,35.40,26.15,24.59,20.32,18.86,18.61,-5.03$. IR (film): 3423, 2930, 2856, 2236, 1716, 1602, 1451, 1379, 1275, 1117, 1071, $836 \mathrm{~cm}^{-1} \cdot[\alpha]_{\mathrm{D}}{ }^{25}$ $=-3.85^{\circ}\left(c=1.09, \mathrm{CHCl}_{3}\right)$. Chiral HPLC: Chiralpak ${ }^{\circledR} \mathrm{IB}$ column, heptane:EtOAc $=98: 2$, $0.8 \mathrm{~mL} / \mathrm{min}, \lambda=254 \mathrm{~nm} ; \mathrm{t}_{\mathrm{R}}=65.90$ (major), 78.07 (minor) minutes: $95 \%$ ee. HRMS-ESI $(\mathrm{m} / z):[\mathrm{M}+\mathrm{Na}]^{+}$calculated for $\mathrm{C}_{24} \mathrm{H}_{36} \mathrm{O}_{4} \mathrm{SiNa}$, 439.2275; found, 439.2271.

\section{(2S,8R,E)-8-Hydroxy-11,11-dimethoxyundec-9-en-6-yn-2-yl benzoate, 118.}

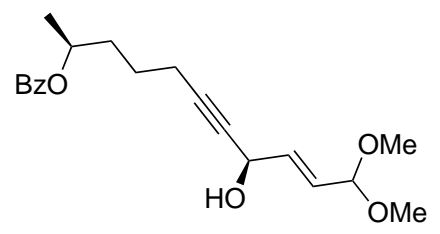

Standard procedure with buffered quench. To a solution of (S)-hept-6-yn-2-yl benzoate (0.097 g, $0.45 \mathrm{mmol}, 1.0$ eq.), (S,S)-ProPhenol ligand (0.029 g, $0.045 \mathrm{mmol}, 10 \mathrm{~mol} \%$ ) and triphenylphosphine oxide $(0.025 \mathrm{~g}, 0.090 \mathrm{mmol}, 20 \mathrm{~mol} \%)$ in toluene $(0.45 \mathrm{~mL})$ was added dimethyl zinc $\left(0.488 \mathrm{~mL}\right.$ of $1.2 \mathrm{M}$ solution in toluene, $0.585 \mathrm{mmol}, 1.3 \mathrm{eq}$.) at $0{ }^{\circ} \mathrm{C}$. The reaction mixture was warmed to room temperature and stirred for 1 hour before addition of (E)-4,4-dimethoxybut-2-enal (0.059 g, $0.45 \mathrm{mmol}, 1.0$ eq.) at $0{ }^{\circ} \mathrm{C}$. The reaction was then stirred at $4{ }^{\circ} \mathrm{C}$ for 48 hours before quenching with $\mathrm{pH} 7$ phosphate buffer. The reaction mixture was extracted with twice with $\mathrm{Et}_{2} \mathrm{O}$, the combined organics were dried over $\mathrm{MgSO}_{4}$, filtered and concentrated in vacuo. The crude product was purified by flash column chromatography $\left(30 \% \mathrm{EtOAc} /\right.$ pet. ether with $\left.1 \% \mathrm{NEt}_{3}\right)$. The title compound was isolated as a clear colorless oil (0.129 g, $82 \%$ yield). $\mathbf{R}_{\mathbf{f}}=0.31$ (30\% EtOAc/pet. ether). ${ }^{1} \mathbf{H} \mathbf{~ N M R}\left(400 \mathrm{MHz} ; \mathrm{CDCl}_{3}\right)$ : $\delta 8.04(\mathrm{~m}, 2 \mathrm{H}), 7.56(\mathrm{tt}, J=7.4,1.6 \mathrm{~Hz}, 1 \mathrm{H}), 7.44(\mathrm{~m}, 2 \mathrm{H}), 5.97(\mathrm{~m}, 1 \mathrm{H}), 5.84$ (app. dtd, $J$ $=15.6,4.4,1.3 \mathrm{~Hz}, 1 \mathrm{H}), 5.23(\mathrm{~m}, 1 \mathrm{H}), 4.90(\mathrm{~m}, 1 \mathrm{H}), 4.82(\mathrm{dd}, J=8.6,4.6 \mathrm{~Hz}, 1 \mathrm{H}), 3.31(\mathrm{~m}$, $6 \mathrm{H}), 2.29$ (tt, $J=6.9,2.0 \mathrm{~Hz}, 2 \mathrm{H}), 2.07$ (br. s, $1 \mathrm{H}), 1.88-1.55(\mathrm{~m}, 4 \mathrm{H}), 1.35(\mathrm{~d}, J=6.3 \mathrm{~Hz}$, $3 \mathrm{H}) .{ }^{13} \mathrm{C}$ NMR $\left(126 \mathrm{MHz}, \mathrm{CDCl}_{3}\right): \delta 166.53,134.28,133.10,130.88,129.79,128.58,128.32$, 102.24, 86.92, 79.61, 71.35, 62.43, 53.00, 35.44, 24.54, 20.36, 18.88. IR (film): 3430, 2939, $2831,2234,1714,1602,1584,1451,1356,1315,1276,1192,1129,1070,973 \mathrm{~cm}^{-1} \cdot[\alpha]_{\mathrm{D}}{ }^{25}=$ $+3.41^{\circ}\left(c=1.05, \mathrm{CHCl}_{3}\right)$. Chiral HPLC: Chiralpak ${ }^{\circledR} \mathrm{IB}$ column, heptane: $i \operatorname{PrOH}=90: 10$, $0.8 \mathrm{~mL} / \mathrm{min}, \lambda=254 \mathrm{~nm} ; \mathrm{t}_{\mathrm{R}}=10.2$ (minor), 11.7 (major) minutes: 19:1 dr. HRMS-ESI $(m / z):[\mathrm{M}+\mathrm{Na}]^{+}$calculated for $\mathrm{C}_{20} \mathrm{H}_{26} \mathrm{O}_{5} \mathrm{Na}, 369.1672$; found, 369.1669. 


\section{Chiral HPLC trace:}

racemic alkyne addition

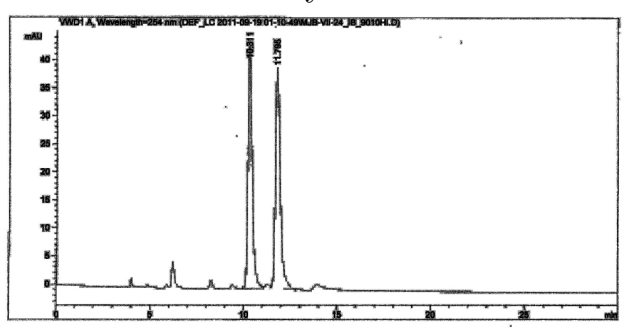

$19: 1 \mathrm{dr}$

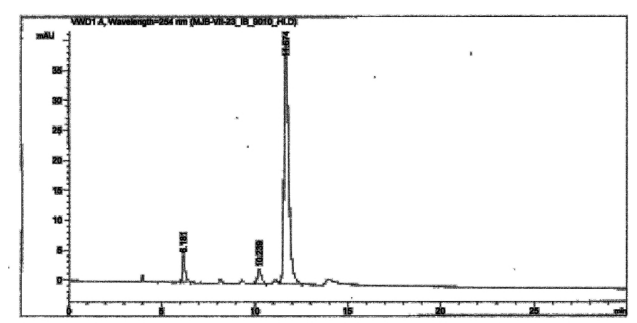

Conjugate Reduction of $\alpha$ - $\beta$-unsaturated Aldehydes

$(2 S, 8 R, E)-8$-Acetoxy-11,11-dimethoxyundec-9-en-6-yn-2-yl benzoate.

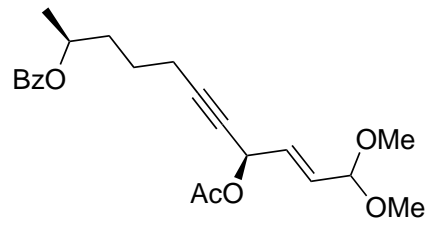

To a solution of $(2 S, 8 R, E)$-8-hydroxy-11,11-dimethoxyundec-9-en-6- yn-2-yl benzoate $(0.107 \mathrm{~g}, 0.307 \mathrm{mmol}, 1.0$ eq. $)$ in pyridine $(1 \mathrm{~mL})$ was added acetic anhydride $(1 \mathrm{~mL})$ at room temperature. The reaction was stirred for 1 hour before quenching with $\mathrm{pH} 7$ phosphate buffer at $0{ }^{\circ} \mathrm{C}$. The reaction mixture was extracted with $\mathrm{CH}_{2} \mathrm{Cl}_{2}$, the combined organics were washed with $1 \mathrm{M} \mathrm{HCl}$, dried over $\mathrm{MgSO}_{4}$, filtered and concentrated in vacuo. The title compound was isolated as a clear, colorless oil ( $0.105 \mathrm{~g}, 88 \%$ yield) and was used without further purification. $\mathbf{R}_{\mathbf{f}}=0.33$ (20\% EtOAc/Pet. Ether). ${ }^{1} \mathbf{H}$ NMR $\left(500 \mathrm{MHz} ; \mathrm{CDCl}_{3}\right): \delta 8.07-8.05(\mathrm{~m}, 2 \mathrm{H})$, 7.59-7.56 (m, 1H), 7.46 (t, $J=7.6 \mathrm{~Hz}, 2 \mathrm{H}), 5.94(\mathrm{~m}, 3 \mathrm{H}), 5.19(\mathrm{~m}, 1 \mathrm{H}), 4.86$ (app. s, 1H), 3.33 $(\mathrm{s}, 6 \mathrm{H}), 2.31(\mathrm{t}, J=7.0 \mathrm{~Hz}, 2 \mathrm{H}), 2.11(\mathrm{~s}, 3 \mathrm{H}), 1.83-1.63(\mathrm{~m}, 4 \mathrm{H}), 1.37(\mathrm{~d}, J=6.3 \mathrm{~Hz}, 3 \mathrm{H})$. ${ }^{13}$ C NMR $\left(126 \mathrm{MHz}, \mathrm{CDCl}_{3}\right): \delta$ 169.95, 166.39, 133.05, 130.91 (2C), 130.11, 129.77, 128.57, 101.71, 76.02, 71.31, 63.83, 52.96, 52.93, 35.40, 24.59, 21.33, 20.36, 18.93. IR (film): 2938, 2831, 2245, 1742, 1715, 1602, 1584, 1451, 1371, 1314, 1275, 1229, 1193, 1130, 1070, 1025, 963, $715 \mathrm{~cm}^{-1} \cdot[\alpha]_{\mathrm{D}}^{25}=+17.34^{\circ}\left(c=1.2, \mathrm{CHCl}_{3}\right)$. HRMS-ESI $(m / z):[\mathrm{M}+\mathrm{Na}]^{+}$calculated for C22H28O6Na, 411.1778; found, 411.1775.

(2S,8R,E)-8-Acetoxy-11-oxoundec-9-en-6-yn-2-yl Benzoate, 204.

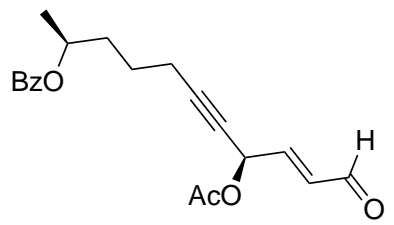

To a solution of $(2 S, 8 R, E)$-8-acetoxy-11,11-dimethoxyundec-9-en-6- yn-2-yl benzoate (0.105 $\mathrm{g}, 0.270 \mathrm{mmol}, 1.0$ eq. $)$ in acetone $(40 \mathrm{~mL})$ was added water $(1.25 \mathrm{~mL})$ and pyridinium $p$ toluenesulfonate $(0.040 \mathrm{~g})$ at room temperature. The reaction mixture was heated to reflux and stirred for 3 hours. The reaction mixture was cooled to room temperature and the acetone 
was removed in vacuo. The crude product was redissolved in $\mathrm{CH}_{2} \mathrm{Cl}_{2}, 20 \mathrm{~mL}$ of water was added before extracting twice with $\mathrm{CH}_{2} \mathrm{Cl}_{2}$. The combined organics were dried over $\mathrm{MgSO}_{4}$, filtered and concentrated in vacuo. The title compound was isolated as a yellow oil $(0.0793 \mathrm{~g}$, $86 \%$ yield) and was used without further purification. $\mathbf{R}_{\mathbf{f}}=0.34(20 \%$ EtOAc/ pet. ether) ${ }^{1} \mathbf{H}$ NMR $\left(400 \mathrm{MHz} ; \mathrm{CDCl}_{3}\right): \delta 9.59(\mathrm{dd}, J=7.7,1.5 \mathrm{~Hz}, 1 \mathrm{H}), 8.03-8.00(\mathrm{~m}, 2 \mathrm{H}), 7.56-7.52$ $(\mathrm{m}, 1 \mathrm{H}), 7.44-7.40(\mathrm{~m}, 2 \mathrm{H}), 6.69(\mathrm{ddd}, J=15.6,4.7,1.1 \mathrm{~Hz}, 1 \mathrm{H}), 6.40-6.34(\mathrm{~m}, 1 \mathrm{H}), 6.09-6.06$ $(\mathrm{m}, 1 \mathrm{H}), 5.17(\mathrm{~m}, 1 \mathrm{H}), 2.29$ (app. td, $J=7.0,2.0 \mathrm{~Hz}, 2 \mathrm{H}), 2.11(\mathrm{~s}, 3 \mathrm{H}), 1.84-1.58(\mathrm{~m}, 4 \mathrm{H})$, $1.34(\mathrm{~d}, J=6.3 \mathrm{~Hz}, 3 \mathrm{H}) .{ }^{13} \mathbf{C}$ NMR $\left(101 \mathrm{MHz}, \mathrm{CDCl}_{3}\right): \delta 192.94,169.59,166.34,149.46$, $133.11,133.06,130.85,129.73,128.55,89.18,74.28,71.13,62.75,35.34,24.39,21.04,20.31$, 18.85. IR (film): 2936, 2244, 1747, 1710, 1694, 1602, 1451, 1371, 1274, 1220, 1114, 1068, 1023, $974 \mathrm{~cm}^{-1} \cdot[\alpha]_{\mathrm{D}}{ }^{25}=+34.63^{\circ}\left(c=1.15, \mathrm{CHCl}_{3}\right)$. HRMS-ESI $(m / z):[\mathrm{M}+\mathrm{Na}]^{+}$calculated for $\mathrm{C}_{20} \mathrm{H}_{22} \mathrm{O}_{5} \mathrm{Na}$, 365.1359; found, 365.1355.

Note: This product was found to decompose at ambient temperatures.

$(2 S, 8 R, E)-8-(($ tert-Butyldimethylsilyl)oxy)-11,11-dimethoxyundec-9-en-6-yn-2yl Benzoate

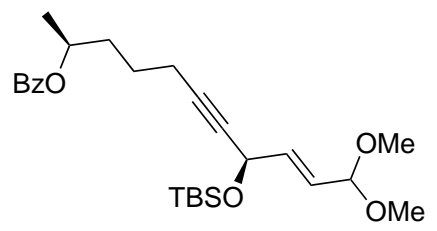

To a solution of $(2 S, 8 R, E)$-8-hydroxy-11,11-dimethoxyundec-9-en-6- yn-2-yl benzoate $(0.342 \mathrm{~g}, 0.987 \mathrm{mmol}, 1.0$ eq.), imidazole $(0.101 \mathrm{~g}, 1.48 \mathrm{mmol}, 1.5$ eq.) and tetra- $N$ butylammonium iodide $(0.109 \mathrm{~g}, 0.296 \mathrm{mmol}, 0.3 \mathrm{eq}$.$) in DMF (30 \mathrm{~mL})$ was added a solution of tert-butyldimethylsilyl chloride $(0.223 \mathrm{~g}, 1.48 \mathrm{mmol}, 1.5 \mathrm{eq}$.$) in DMF (5 \mathrm{~mL})$ at room temperature. The reaction mixture was heated to $60{ }^{\circ} \mathrm{C}$ and stirred for 3 hours. The reaction was cooled to room temperature, diluted with $\mathrm{H}_{2} \mathrm{O}$ and $\mathrm{CH}_{2} \mathrm{Cl}_{2}$, and extracted twice with $\mathrm{CH}_{2} \mathrm{Cl}_{2}$. The combined organics were washed with water, then brine, dried over $\mathrm{MgSO}_{4}$, filtered and concentrated in vacuo. The crude product was purified by flash column chromatography ( $5 \%$ EtOAc/pet. ether with $\left.1 \% \mathrm{NEt}_{3}\right)$. The title compound was isolated as a clear, colorless oil ( $0.1875 \mathrm{~g}, 41 \%$ yield). $\mathbf{R}_{\mathbf{f}}=0.35$ (5\% EtOAc/pet. ether). ${ }^{1} \mathbf{H}$ NMR (400 $\left.\mathrm{MHz} ; \mathrm{CDCl}_{3}\right): \delta 8.03-8.00(\mathrm{~m}, 2 \mathrm{H}), 7.55-7.51(\mathrm{~m}, 1 \mathrm{H}), 7.44-7.40(\mathrm{~m}, 2 \mathrm{H}), 5.88(\mathrm{ddd}, J=15.5$, $4.6,1.1 \mathrm{~Hz}, 1 \mathrm{H}), 5.75$ (ddd, $J=15.5,4.5,1.4 \mathrm{~Hz}, 1 \mathrm{H}), 5.19-5.11(\mathrm{~m}, 1 \mathrm{H}), 4.92-4.90(\mathrm{~m}, 1 \mathrm{H})$, $4.81(\mathrm{~m}, J=1.0 \mathrm{~Hz}, 1 \mathrm{H}), 3.29(\mathrm{~s}, 6 \mathrm{H}), 2.24(\mathrm{td}, J=6.9,2.0 \mathrm{~Hz}, 2 \mathrm{H}), 1.84-1.52(\mathrm{~m}, 4 \mathrm{H}), 1.33$ $(\mathrm{d}, J=6.3 \mathrm{~Hz}, 3 \mathrm{H}), 0.88(\mathrm{~s}, 9 \mathrm{H}), 0.10(\mathrm{~d}, J=2.6 \mathrm{~Hz}, 6 \mathrm{H}) .{ }^{13} \mathrm{C} \mathbf{N M R}\left(101 \mathrm{MHz}, \mathrm{CDCl}_{3}\right): \delta$ 166.35, 135.12, 132.97, 130.94, 129.73, 128.50, 126.71, 102.32, 85.68, 80.2, 71.35, 62.89, 52.80, 35.41, 26.0, 24.69, 20.34, 18.88, 18.56, -4.34, -4.63. IR (film): 2932, 2857, 2232, 1718, 1603, $1451,1274,1192,1116,1070,973,838,779,713 \mathrm{~cm}^{-1}$.

\section{$(2 S, 8 R, E)-8-(($ tert-Butyldimethylsilyl)oxy)-11-oxoundec-9-en-6-yn-2-yl Benzoate, 205.}

To a solution of (2S,8R,E)-8-((tert-butyldimethylsilyl)oxy)-11,11- dimethoxyundec-9-en-6yn-2-yl benzoate $(0.069 \mathrm{~g}, 0.150 \mathrm{mmol}, 1.0 \mathrm{eq}$.$) in acetone (22 \mathrm{~mL})$ was added water $(0.69$ $\mathrm{mL})$ and pyridinium $p$-toluenesulfonate $(0.022 \mathrm{~g})$ at room temperature. The reaction mixture stirred at $45{ }^{\circ} \mathrm{C}$ for 4.5 hours. The reaction mixture was cooled to room temperature and the acetone was removed in vacuo. The crude product was redissolved in $\mathrm{CH}_{2} \mathrm{Cl}_{2}, 20 \mathrm{~mL}$ 


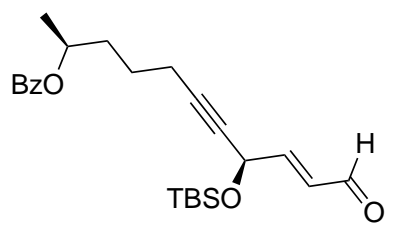

of water was added before extracting twice with $\mathrm{CH}_{2} \mathrm{Cl}_{2}$. The combined organics were dried over $\mathrm{MgSO}_{4}$, filtered and concentrated in vacuo. The title compound was isolated as a clear, colorless oil (0.056 g, $90 \%$ yield) and was used without further purification. $\mathbf{R}_{\mathbf{f}}=0.30(5 \%$ EtOAc/pet. ether). ${ }^{1} \mathbf{H}$ NMR $\left(500 \mathrm{MHz} ; \mathrm{CDCl}_{3}\right): \delta 9.65(\mathrm{dd}, J=7.9,0.5 \mathrm{~Hz}, 1 \mathrm{H}), 8.07-8.05$ $(\mathrm{m}, 2 \mathrm{H}), 7.60-7.56(\mathrm{~m}, 1 \mathrm{H}), 7.48-7.45(\mathrm{~m}, 2 \mathrm{H}), 6.78(\mathrm{dd}, J=15.4,4.0 \mathrm{~Hz}, 1 \mathrm{H}), 6.39$ (dddd, $J=15.4,8.0,1.8,0.5 \mathrm{~Hz}, 1 \mathrm{H}), 5.24-5.18(\mathrm{~m}, 1 \mathrm{H}), 5.15$ (app. dq, $J=3.9,1.9 \mathrm{~Hz}, 1 \mathrm{H}), 2.31$ $(\mathrm{td}, J=7.0,2.0 \mathrm{~Hz}, 2 \mathrm{H}), 1.88-1.61(\mathrm{~m}, 6 \mathrm{H}), 1.38(\mathrm{~d}, J=6.3 \mathrm{~Hz}, 3 \mathrm{H}), 0.94(\mathrm{~s}, 9 \mathrm{H}), 0.16(\mathrm{~d}$, $J=4.8 \mathrm{~Hz}, 6 \mathrm{H}) \cdot{ }^{13} \mathrm{C}$ NMR $\left(126 \mathrm{MHz}, \mathrm{CDCl}_{3}\right): \delta 193.74,166.39,155.65,133.09,130.90$, $130.72,129.76,128.57,87.20,78.26,71.24,62.36,35.43,25.97,24.56,20.39,18.86,-4.33,-4.77$. IR (film): 2931, 2857, 22220, 1716, 1694, 1602, 1462, 1314, 1275, 1102, 1071, 973, 838, 713 $\mathrm{cm}^{-1}$. HRMS-ESI $(\mathrm{m} / z):[\mathrm{M}+\mathrm{H}]^{+}$calculated for $\mathrm{C}_{24} \mathrm{H}_{34} \mathrm{O}_{4} \mathrm{SiNa}, 437.2119$; found, 437.2123.

$(2 S, 8 R, E)-8-(($ tert-Butyldiphenylsilyl)oxy)-11,11-dimethoxyundec-9-en-6-yn2-yl Benzoate

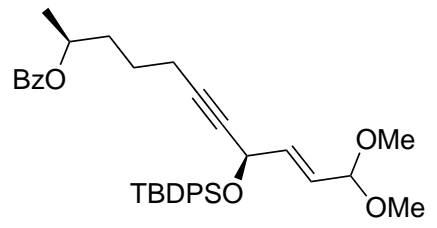

To a solution of $(2 S, 8 R, E)$-8-hydroxy-11,11-dimethoxyundec-9-en-6- yn-2-yl benzoate (0.2496 g, $0.72 \mathrm{mmol}, 1.0$ eq.), imidazole $(0.074 \mathrm{~g}, 1.08 \mathrm{mmol}, 1.5$ eq.) and tetra- $N$ butylammonium iodide $(0.080 \mathrm{~g}, 0.216 \mathrm{mmol}, 0.3 \mathrm{eq}$.$) in DMF (7.2 \mathrm{~mL})$ was added tertbutyldiphenylsilyl chloride $(0.297 \mathrm{~g}, 1.08 \mathrm{mmol}, 1.5 \mathrm{eq}$.$) at room temperature. The reaction$ mixture was heated to $50{ }^{\circ} \mathrm{C}$ and stirred for 9 hours. The reaction was cooled to room temperature and the DMF was removed in vacuo. The reactions mixture was redissolved in $\mathrm{Et}_{2} \mathrm{O}$ and water, and extracted twice with $\mathrm{Et}_{2} \mathrm{O}$. The combined organics were dried over $\mathrm{MgSO}_{4}$, filtered and concentrated in vacuo. The crude product was purified by flash column chromatography ( $5 \%$ EtOAc/pet. ether with $\left.1 \% \mathrm{NEt}_{3}\right)$. The title compound was isolated as a clear, colorless oil (0.1752 g, $42 \%$ yield). $\mathbf{R}_{\mathbf{f}}=0.27$ (5\% EtOAc/ pet. ether). ${ }^{\mathbf{1}} \mathbf{H} \mathbf{~ N M R}$ $\left(400 \mathrm{MHz} ; \mathrm{CDCl}_{3}\right): \delta$ 8.03-8.00 (m, 2H), 7.74-7.72 (m, 2H), 7.68-7.64 (m, 2H), 7.55-7.51 (m, $1 \mathrm{H}), 7.43-7.30(\mathrm{~m}, 8 \mathrm{H}), 5.88(\mathrm{dd}, J=15.5,4.9 \mathrm{~Hz}, 1 \mathrm{H}), 5.73-5.67(\mathrm{~m}, 1 \mathrm{H}), 5.12-5.07(\mathrm{~m}, 1 \mathrm{H})$, $4.87(\mathrm{~m}, J=0.7 \mathrm{~Hz}, 1 \mathrm{H}), 4.78(\mathrm{~d}, J=4.7 \mathrm{~Hz}, 1 \mathrm{H}), 3.27-3.26(\mathrm{~m}, 6 \mathrm{H}), 2.10(\mathrm{td}, J=7.0$, $1.9 \mathrm{~Hz}, 2 \mathrm{H}), 1.72-1.42(\mathrm{~m}, 4 \mathrm{H}), 1.29(\mathrm{dd}, J=6.2,2.1 \mathrm{~Hz}, 3 \mathrm{H}), 1.05(\mathrm{~s}, 9 \mathrm{H}) .{ }^{13} \mathbf{C}$ NMR $(101$ $\left.\mathrm{MHz}, \mathrm{CDCl}_{3}\right): \delta 166.34,136.19,135.98,135.72,134.80,133.75,132.98,130.95,129.93,129.83$, 129.80, 129.73, 128.51, 127.88, 127.76, 127.57, 126.98, 102.30, 86.40, 79.99, 71.38, 63.78, 52.78, 35.37, 27.07, 26.98, 24.56, 20.33, 19.52, 18.86. IR (film): 2933, 2858, 1716, 1587, 1451, 1428, 1359, 1314, 1274, 1191, 1112, 1070, 998, 973, 823, 741, $708 \mathrm{~cm}^{-1}$. HRMS-ESI $(\mathrm{m} / z):[\mathrm{M}+$ $\mathrm{H}]{ }^{+}$calculated for $\mathrm{C}_{36} \mathrm{H}_{44} \mathrm{O}_{5} \mathrm{SiNa}, 607.2850$; found, 607.2842. 
$(2 S, 8 R, E)-8-(($ tert-Butyldiphenylsilyl)oxy)-11-oxoundec-9-en-6-yn-2-yl Benzoate, 206.

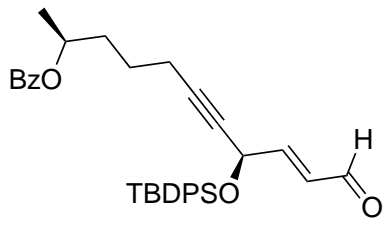

To a solution of (2S,8R,E)-8-((tert-butyldiphenylsilyl)oxy)-11,11- dimethoxyundec-9-en-6yn-2-yl benzoate $(0.175 \mathrm{~g}, 0.299 \mathrm{mmol}, 1.0$ eq. $)$ in acetone $(40 \mathrm{~mL})$ was added water $(1.37$ $\mathrm{mL})$ and pyridinium $p$-toluenesulfonate $(0.045 \mathrm{~g})$ at room temperature. The reaction mixture stirred at $45{ }^{\circ} \mathrm{C}$ for 5 hours. The reaction mixture was cooled to room temperature and the acetone was removed in vacuo. The crude product was redissolved in $\mathrm{CH}_{2} \mathrm{Cl}_{2}, 20 \mathrm{~mL}$ of water was added before extracting twice with $\mathrm{CH}_{2} \mathrm{Cl}_{2}$. The combined organics were dried over $\mathrm{MgSO}_{4}$, filtered and concentrated in vacuo. The title compound was isolated as a clear, colorless oil ( $0.120 \mathrm{~g}, 84 \%$ yield) and was used without further purification. ${ }^{1} \mathbf{H}$ NMR (400 $\left.\mathrm{MHz} ; \mathrm{CDCl}_{3}\right): \delta 9.56(\mathrm{dd}, J=7.9,4.0 \mathrm{~Hz}, 1 \mathrm{H}), 8.02-8.00(\mathrm{~m}, 2 \mathrm{H}), 7.72-7.62(\mathrm{~m}, 4 \mathrm{H}), 7.56-7.52$ $(\mathrm{m}, 1 \mathrm{H}), 7.44-7.31(\mathrm{~m}, 8 \mathrm{H}), 6.68(\mathrm{dt}, J=15.4,4.5 \mathrm{~Hz}, 1 \mathrm{H}), 6.33$ (dddd, $J=15.4,7.9,3.0$, $1.7 \mathrm{~Hz}, 1 \mathrm{H}), 5.12-5.08(\mathrm{~m}, 1 \mathrm{H}), 5.05(\mathrm{tt}, J=3.8,1.9 \mathrm{~Hz}, 1 \mathrm{H}), 2.10(\mathrm{td}, J=7.0,2.0 \mathrm{~Hz}, 2 \mathrm{H})$, $1.73-1.54(\mathrm{~m}, 2 \mathrm{H}), 1.50-1.40(\mathrm{~m}, 2 \mathrm{H}), 1.29(\mathrm{dd}, J=6.3,1.9 \mathrm{~Hz}, 3 \mathrm{H}), 1.07(\mathrm{~d}, J=1.4 \mathrm{~Hz}$, 9H). ${ }^{13}$ C NMR $\left(101 \mathrm{MHz}, \mathrm{CDCl}_{3}\right): \delta 193.67,155.13,136.15,135.90,133.04,130.79,130.26$, $130.08,129.72$, 128.54, 127.96, 127.69, 87.98, 71.22, 63.20, 35.34, 27.02, 26.97, 24.36, 20.32, 19.52, 18.78. IR (film): 2933, 2858, 1715, 1694, 1451, 1428, 1314, 1274, 1113, 972, 822, 741, 703 $\mathrm{cm}^{-1}$. HRMS-ESI $(\mathrm{m} / z):[\mathrm{M}+\mathrm{H}]^{+}$calculated for $\mathrm{C}_{34} \mathrm{H}_{36} \mathrm{O}_{4} \mathrm{SiNa}, 561.2432$; found, 561.2425.

\section{Hydrosilylation and Hydrogenation Experiments}

$(S)-5-((R)-5-((E)-3,3-D i m e t h o x y p r o p-1-e n-1-y l)-2,2-d i m e t h y l-2,5-d i h y d r o-$ 1,2-oxasilol-3-yl)pentan-2-yl Benzoate, 210.

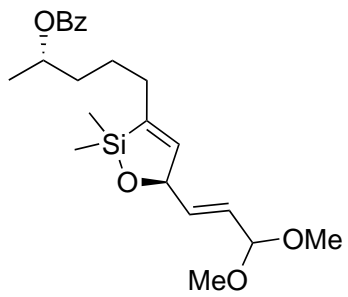

To a solution of $(2 S, 8 R, E)$-8-hydroxy-11,11-dimethoxyundec-9-en-6-yn-2- yl benzoate (0.055 g, $0.159 \mathrm{mmol}, 1.0$ eq.) in $\mathrm{CH}_{2} \mathrm{Cl}_{2}(0.25 \mathrm{~mL})$ at $0{ }^{\circ} \mathrm{C}$ was added freshly distilled dimethylethoxysilane $(0.026 \mathrm{~mL}, 0.020 \mathrm{~g}, 0.191 \mathrm{mmol}, 1.2 \mathrm{eq}$.$) , immediately followed by a$ solution of $\mathrm{CpRu}\left(\mathrm{CH}_{3} \mathrm{CN}\right)_{3} \mathrm{PF}_{6}(0.0035 \mathrm{~g}, 0.008 \mathrm{mmol}, 5 \mathrm{~mol} \%)$ in $\mathrm{CH}_{2} \mathrm{Cl}_{2}(0.1 \mathrm{~mL})$. The reaction was warmed to room temperature and stirred for 30 minutes before being diluted with pet. ether $(0.5 \mathrm{~mL})$ and passed through a florasil plug - rinsing with $30 \%$ EtOAc/pet. ether. The filtrate was concentrated in vacuo and the crude product was purified by flash column chromatography ( $5 \% \mathrm{EtOAc} /$ pet. ether with $\left.1 \% \mathrm{NEt}_{3}\right)$. The title compound was isolated as a clear, colorless oil $\left(0.013 \mathrm{~g}, 20 \%\right.$ yield). $\mathbf{R}_{\mathbf{f}}=0.74$ (30\% EtOAc/pet. ether). ${ }^{\mathbf{1}} \mathbf{H} \mathbf{~ N M R}$ (400 $\left.\mathrm{MHz} ; \mathrm{CDCl}_{3}\right): \delta 8.03-8.00(\mathrm{~m}, 2 \mathrm{H}), 7.53(\mathrm{tt}, J=7.4,1.6 \mathrm{~Hz}, 1 \mathrm{H}), 7.44-7.40(\mathrm{~m}, 2 \mathrm{H}), 6.26(\mathrm{~d}$, $J=1.5 \mathrm{~Hz}, 1 \mathrm{H}), 5.78(\mathrm{ddd}, J=15.6,5.9,1.0 \mathrm{~Hz}, 1 \mathrm{H}), 5.63(\mathrm{ddd}, J=15.6,4.6,1.3 \mathrm{~Hz}, 1 \mathrm{H})$, 
$5.20-5.12(\mathrm{~m}, 1 \mathrm{H}), 5.08(\mathrm{~d}, J=5.8 \mathrm{~Hz}, 1 \mathrm{H}), 4.78(\mathrm{~d}, J=4.6 \mathrm{~Hz}, 1 \mathrm{H}), 3.28(\mathrm{~s}, 6 \mathrm{H}), 2.26-2.22$ $(\mathrm{m}, 2 \mathrm{H}), 1.73-1.50(\mathrm{~m}, 4 \mathrm{H}), 1.32(\mathrm{~d}, J=6.3 \mathrm{~Hz}, 3 \mathrm{H}), 0.22-0.21(\mathrm{~m}, 6 \mathrm{H}) .{ }^{13} \mathrm{C}$ NMR $(101$ $\mathrm{MHz}, \mathrm{CDCl} 3): \delta 166.38,143.44,142.29,136.14,132.98,130.96,129.70,128.51,126.18,102.40$, $81.55,71.53,52.80,52.67,52.05,36.06,30.85,25.56,20.34,1.38,0.48,0.21$. IR (film): 2934 , $2831,1716,1451,1314,1276,1113,1070,1055,1027,864,785,713 \mathrm{~cm}^{-1} \cdot[\alpha]_{\mathrm{D}}{ }^{25}=-13.06^{\circ}$ $\left(c=1.3, \mathrm{CH}_{2} \mathrm{Cl}_{2}\right)$. HRMS-ESI $(m / z):[\mathrm{M}+\mathrm{H}]^{+}$calculated for $\mathrm{C}_{22} \mathrm{H}_{32} \mathrm{O}_{5} \mathrm{SiNa}, 427.1911$; found, 427.1915 .

Preparation of (2S,6Z,8R,9E)-7-(Benzyldimethylsilyl)-8-hydroxy-11,11dimethoxyundeca-6,9-dien-2-yl Benzoate, 212.<smiles>COC(=O)c1ccccc1</smiles>

To a solution of ( $2 S, 8 R, E)$-8-hydroxy-11,11-dimethoxyundec-9-en-6-yn-2-yl benzoate (0.229 g, $0.662 \mathrm{mmol}, 1.0$ equiv) in 1,2-dichloroethane $(1.0 \mathrm{~mL})$ at $0{ }^{\circ} \mathrm{C}$ was added benzyldimethylsilane $(0.110 \mathrm{~mL}, 0.104 \mathrm{~g}, 0.695 \mathrm{mmol}, 1.05$ equiv), immediately followed by a solution of $\mathrm{Cp} * \mathrm{Ru}\left(\mathrm{CH}_{3} \mathrm{CN}\right){ }_{3} \mathrm{PF}_{6}(0.0067 \mathrm{~g}, 0.013 \mathrm{mmol}, 2 \mathrm{~mol} \%)$ in 1,2-dichloroethane $(0.32 \mathrm{~mL})$. The reaction was warmed to room temperature and stirred for 30 minutes before being diluted with pet. ether $(0.5 \mathrm{~mL})$ and passed through a florasil plug, rinsing with $30 \%$ EtOAc/ pet. ether. The filtrate was concentrated in vacuo and the crude product was purified by silica gel chromatography ( $20 \% \mathrm{EtOAc} /$ pet. ether with $1 \% \mathrm{NEt}_{3}$ ). The title compound was isolated as a clear, colorless oil ( $0.2923 \mathrm{~g}, 89 \%$ yield). $\mathbf{R}_{\mathbf{f}}=0.41$ (30\% EtOAc/ pet. ether). ${ }^{\mathbf{1}} \mathbf{H}-\mathbf{N M R}$ $\left(400 \mathrm{MHz} ; \mathrm{CDCl}_{3}\right): \delta 8.04(\mathrm{~m}, 2 \mathrm{H}), 7.55(\mathrm{~m}, 1 \mathrm{H}), 7.43(\mathrm{~m}, 2 \mathrm{H}), 7.20(\mathrm{~m}, 2 \mathrm{H}), 7.07(\mathrm{~m}, 1 \mathrm{H})$, $6.98(\mathrm{~m}, 2 \mathrm{H}), 6.24(\mathrm{t}, J=7.4 \mathrm{~Hz}, 1 \mathrm{H}), 5.80(\mathrm{ddd}, J=15.8,5.0,1.0 \mathrm{~Hz}, 1 \mathrm{H}), 5.59$ (ddd, $J$ $=15.8,4.9,1.5 \mathrm{~Hz}, 1 \mathrm{H}), 5.17(\mathrm{~m}, 1 \mathrm{H}), 4.75(\mathrm{~d}, J=4.9 \mathrm{~Hz}, 1 \mathrm{H}), 4.62(\mathrm{~m}, 1 \mathrm{H}), 3.30-3.29(\mathrm{~m}$, $6 \mathrm{H}), 2.23(\mathrm{~s}, 2 \mathrm{H}), 2.16$ (app. q, $J=7.5 \mathrm{~Hz}, 2 \mathrm{H}), 1.77-1.45(\mathrm{~m}, 4 \mathrm{H}), 1.35(\mathrm{~d}, J=6.3 \mathrm{~Hz}, 3 \mathrm{H})$, $0.16(\mathrm{~s}, 6 \mathrm{H}) .{ }^{13} \mathrm{C}-\mathbf{N M R}\left(101 \mathrm{MHz}, \mathrm{CDCl}_{3}\right): \delta 166.39,145.42,140.08,139.92,137.38,133.00$, $130.93,129.72,128.51,128.47,128.39,126.91,124.43,102.81,76.60,71.55,52.91,52.88,36.05$, 31.95, 26.95, 25.75, 20.32, -1.04. IR (film): 3425, 2936, 1716, 1601, 1493, 1451, 1355, 1276 , $1114,1055,974,834,713 \mathrm{~cm}^{-1} \cdot[\alpha]_{\mathbf{D}}{ }^{25}=+21.46^{\circ}\left(c=1.4, \mathrm{CH}_{2} \mathrm{Cl}_{2}\right)$. HRMS-ESI $(\mathrm{m} / z):[\mathrm{M}$ $+\mathrm{Na}]^{+}$calculated for $\mathrm{C}_{29} \mathrm{H}_{40} \mathrm{O}_{5} \mathrm{SiNa}, 519.2537$; found, 519.2530.

Preparation of $(2 S, 8 R, Z)-7-($ Benzyldimethylsilyl)-8((tert-butyldimethylsilyl)oxy)-11,11-dimethoxyundec-6-en-2-yl Benzoate, 213.
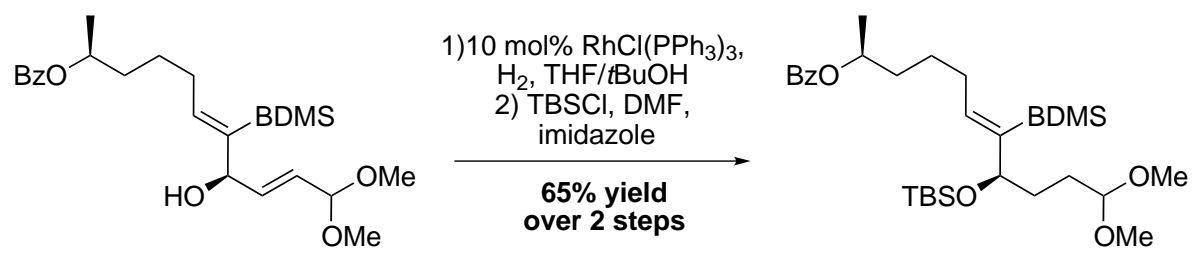

To a flask containing $(2 S, 6 Z, 8 R, 9 E)$-7-(benzyldimethylsilyl)-8-hydroxy-11,11-dimethoxyundeca-6,9-dien-2-yl benzoate $212(0.0615 \mathrm{~g}, 0.124 \mathrm{mmol}, 1.0$ eq. $)$ was added a $\mathrm{RhCl}\left(\mathrm{PPh}_{3}\right)_{3}$ $(0.012 \mathrm{~g}, 0.0124 \mathrm{mmol}, 10 \mathrm{~mol} \%)$ in degassed THF $/ \mathrm{BuOH}(1: 1,0.244 \mathrm{~mL}$, freeze-pump-thaw degassing). The reaction flask was then evacuated and backfilled with hydrogen (balloon) three 
times and the reaction was stirred for 10 hours at room temperature. The reaction mixture was then diluted with $\mathrm{Et}_{2} \mathrm{O}$ and passed through a florasil plug. The filtrate was concentrated in vacuo and used immediately in the next step. A pure analytical sample (purified by preparative TLC on basic alumina, $10 \%$ EtOAc/ pet. ether) gave the following characterization data: $\mathbf{R}_{\mathbf{f}}$ $=0.44\left(30 \%\right.$ EtOAc/ pet. ether). ${ }^{1} \mathbf{H}-\mathbf{N M R}\left(400 \mathrm{MHz} ; \mathrm{CDCl}_{3}\right): \delta 8.02(\mathrm{~m}, 2 \mathrm{H}), 7.53(\mathrm{~m}$, $1 \mathrm{H}), 7.40(\mathrm{t}, J=7.7 \mathrm{~Hz}, 2 \mathrm{H}), 7.17(\mathrm{t}, J=7.5 \mathrm{~Hz}, 2 \mathrm{H}), 7.04(\mathrm{t}, J=7.4 \mathrm{~Hz}, 1 \mathrm{H}), 6.96$ $(\mathrm{d}, J=7.1 \mathrm{~Hz}, 2 \mathrm{H}), 6.23(\mathrm{t}, J=7.3 \mathrm{~Hz}, 1 \mathrm{H}), 5.16(\mathrm{~m}, 1 \mathrm{H}), 4.30(\mathrm{t}, J=5.6 \mathrm{~Hz}, 1 \mathrm{H})$, $4.00(\mathrm{~m}, 1 \mathrm{H}), 3.27(\mathrm{~s}, 6 \mathrm{H}), 2.20(\mathrm{~s}, 2 \mathrm{H}), 2.15(\mathrm{q}, J=7.4 \mathrm{~Hz}, 2 \mathrm{H}), 1.75-1.38(\mathrm{~m}, 8 \mathrm{H}), 1.33$ $(\mathrm{d}, J=6.3 \mathrm{~Hz}, 3 \mathrm{H}), 0.13-0.11(\mathrm{~m}, 6 \mathrm{H}) .{ }^{13} \mathrm{C}-\mathrm{NMR}\left(101 \mathrm{MHz}, \mathrm{CDCl}_{3}\right): \delta 166.40,142.77$, $141.48,140.09,132.97,130.96,129.72,128.51,128.46,128.37,124.41,104.64,76.24,71.60$, $53.03,52.87,36.05,32.59,31.89,29.36,26.99,25.88,20.32,-1.11$. IR (film): 3447,2933 , $1716,1601,1493,1452,1315,1276,1205,1175,1125,1070,1027,831,713 \mathrm{~cm}^{-1} \cdot[\alpha]_{\mathrm{D}}{ }^{25}=$ $+23.11^{\circ}\left(c=1.0, \mathrm{CH}_{2} \mathrm{Cl}_{2}\right)$. HRMS-ESI $(\mathrm{m} / z):[\mathrm{M}+\mathrm{Na}]^{+}$calculated for $\mathrm{C}_{29} \mathrm{H}_{42} \mathrm{O}_{5} \mathrm{SiNa}$, 521.2694; found, 521.2688. The crude product, (2S,8R,Z)-7-(benzyldimethylsilyl)-8-hydroxy11,11-dimethoxyundec-6-en-2-yl benzoate), was dissolved in DMF $(1.2 \mathrm{~mL})$. Imidazole $(0.025$ $\mathrm{g}, 0.372 \mathrm{mmol}, 3.0$ equiv) and tert-butyldimethylsilyl chloride ( $0.049 \mathrm{~g}, 0.372 \mathrm{mmol}, 3.0$ equiv) were added at room temperature. The reaction was stirred at room temperature for 9 hours before being diluted with $\mathrm{Et}_{2} \mathrm{O}$ and water. The reaction mixture was then extracted with $\mathrm{Et}_{2} \mathrm{O}$, dried over $\mathrm{MgSO}_{4}$, filtered and concentrated in vacuo. The crude product was purified by silica gel chromatography $\left(2.5 \%\right.$ EtOAc/pet. ether with $\left.1 \% \mathrm{NEt}_{3}\right)$. The title compound was isolated as a clear, colorless oil $\left(0.049 \mathrm{~g}, 65 \%\right.$ yield over 2 steps.). $\mathbf{R}_{\mathbf{f}}=0.29$ (5\% EtOAc/ pet. ether). ${ }^{1} \mathbf{H}-\mathbf{N M R}\left(400 \mathrm{MHz} ; \mathrm{CDCl}_{3}\right): \delta 8.02(\mathrm{~m}, 2 \mathrm{H}), 7.52(\mathrm{tt}, J=7.4,1.5 \mathrm{~Hz}, 1 \mathrm{H})$, $7.40(\mathrm{~m}, 2 \mathrm{H}), 7.17(\mathrm{~m}, 2 \mathrm{H}), 7.04(\mathrm{~m}, 1 \mathrm{H}), 6.97(\mathrm{~m}, 2 \mathrm{H}), 6.18(\mathrm{t}, J=7.3 \mathrm{~Hz}, 1 \mathrm{H}), 5.14(\mathrm{~m}$, $1 \mathrm{H}), 4.25(\mathrm{t}, J=5.8 \mathrm{~Hz}, 1 \mathrm{H}), 4.04(\mathrm{t}, J=5.9 \mathrm{~Hz}, 1 \mathrm{H}), 3.26(\mathrm{~s}, 3 \mathrm{H}), 3.25(\mathrm{~s}, 3 \mathrm{H}), 2.23(\mathrm{~s}$, $1 \mathrm{H}), 2.21(\mathrm{~s}, 1 \mathrm{H}), 2.13(\mathrm{~m}, 2 \mathrm{H}), 1.76-1.43(\mathrm{~m}, 8 \mathrm{H}), 1.33(\mathrm{~d}, J=6.2 \mathrm{~Hz}, 3 \mathrm{H}), 0.86(\mathrm{~s}, 9 \mathrm{H}), 0.09$ $(\mathrm{s}, 6 \mathrm{H}),-0.02(\mathrm{~s}, 3 \mathrm{H}),-0.07(\mathrm{~s}, 3 \mathrm{H}) .{ }^{13} \mathrm{C}-\mathrm{NMR}(101 \mathrm{MHz}, \mathrm{CDCl} 3): \delta 166.39,142.87,141.38$, 140.16, 132.93, 131.01, 129.72, 128.55, 128.48, 128.33, 124.27, 104.54, 71.7, 71.67, 52.81, 52.39, $36.06,33.91,31.82,29.00,27.12,26.17,25.94,20.32,18.41,-1.08,-1.26,-3.93,-4.72$. IR (film): 2931, 2857, 1718, 1602, 1493, 1452, 1383, 1360, 1314, 1274, 1252, 1113, 1070, 1027, 833, 775, $712 \mathrm{~cm}^{-1} \cdot[\alpha]_{\mathrm{D}}{ }^{25}=+14.32^{\circ}\left(c=1.5, \mathrm{CH}_{2} \mathrm{Cl}_{2}\right)$. HRMS-ESI $(m / z):[\mathrm{M}+\mathrm{Na}]^{+}$calculated for $\mathrm{C}_{35} \mathrm{H}_{58} \mathrm{O}_{5} \mathrm{Si}_{2} \mathrm{Na}$, 635.3558; found, 635.3549.

\section{Formal Total Synthesis of Aspergillide B}

Preparation of ((2S,8R,Z)-7-(Benzyldimethylsilyl)-8-((tert-butyldimethylsilyl)oxy)11-oxoundec-6-en-2-yl Benzoate, 214.

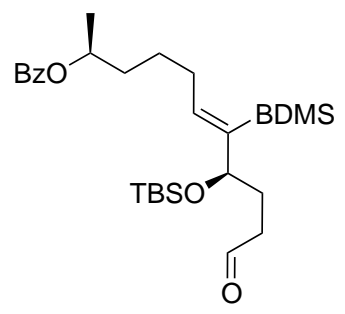

To a solution of (2S,8R,Z)-7-(benzyldimethylsilyl)-8-((tert-butyldimethylsilyl)oxy)-11,11dimethoxyundec-6-en-2-yl benzoate $213(0.0548 \mathrm{~g}, 0.089 \mathrm{mmol})$ in acetone $(10 \mathrm{~mL})$ was added $0.25 \mathrm{~mL}$ of water and $0.007 \mathrm{~g}$ of pyridiunium $p$-toluenesulfonate $(30 \mathrm{~mol} \%, 0.028 \mathrm{mmol})$. The reaction was stirred at $60{ }^{\circ} \mathrm{C}$ (bath temperature) for 4 hours. After cooling to ambient 
temperature, the reaction mixture was concentrated in vacuo, diluted with $\mathrm{CH}_{2} \mathrm{Cl}_{2}$ and $\mathrm{H}_{2} \mathrm{O}$, and extracted with $\mathrm{CH}_{2} \mathrm{Cl}_{2}$. The combined organic layers were dried over $\mathrm{MgSO}_{4}$, filtered and concentrated in vacuo. The title compound was isolated as a clear, colorless oil $(0.0405$ $\mathrm{g}, 80 \%$ yield) and was used without further purification. $\mathbf{R}_{\mathbf{f}}=0.26(5 \%$ EtOAc/pet. ether $)$. ${ }^{1} \mathbf{H}-\mathbf{N M R}\left(400 \mathrm{MHz} ; \mathrm{CDCl}_{3}\right): \delta 9.64(\mathrm{t}, J=1.7 \mathrm{~Hz}, 1 \mathrm{H}), 8.05-8.03(\mathrm{~m}, 2 \mathrm{H}), 7.57-7.53(\mathrm{~m}$, $1 \mathrm{H}), 7.42(\mathrm{t}, J=7.6 \mathrm{~Hz}, 2 \mathrm{H}), 7.18(\mathrm{t}, J=7.5 \mathrm{~Hz}, 2 \mathrm{H}), 7.05(\mathrm{t}, J=7.4 \mathrm{~Hz}, 1 \mathrm{H}), 6.98(\mathrm{~d}, J=$ $7.0 \mathrm{~Hz}, 2 \mathrm{H}), 6.23(\mathrm{t}, J=7.4 \mathrm{~Hz}, 1 \mathrm{H}), 5.17(\mathrm{~m}, 1 \mathrm{H}), 4.11(\mathrm{t}, J=5.6 \mathrm{~Hz}, 1 \mathrm{H}), 2.29(\mathrm{td}, J=7.3$, $1.6 \mathrm{~Hz}, 2 \mathrm{H}), 2.23(\mathrm{~m}, 2 \mathrm{H}), 2.17(\mathrm{t}, J=7.4 \mathrm{~Hz}, 2 \mathrm{H}), 1.80-1.43(\mathrm{~m}, 6 \mathrm{H}), 1.36(\mathrm{~d}, J=6.2 \mathrm{~Hz}$, 3H), $0.87(\mathrm{~s}, 9 \mathrm{H}), 0.14(\mathrm{~s}, 3 \mathrm{H}), 0.12(\mathrm{~s}, 3 \mathrm{H}),-0.02(\mathrm{~s}, 3 \mathrm{H}),-0.06(\mathrm{~s}, 3 \mathrm{H}) .{ }^{13} \mathrm{C}-\mathbf{N M R}(101 \mathrm{MHz}$, $\left.\mathrm{CDCl}_{3}\right): \delta 203.08,166.41,143.55,140.43,139.96,133.00,130.97,129.73,128.52,128.42(2 \mathrm{C})$, 124.42 , $71.66(2 \mathrm{C}), 40.11,36.08,31.93,31.06,27.09,26.14,25.92,20.36,18.41,-1.00,-1.31$, -4.00, -4.79. IR (film): 2953, 2930, 2856, 1718, 1601, 1493, 1452, 1274, 1253, 1112, 1070, 1026, $834 \mathrm{~cm}^{-1} \cdot[\alpha]_{\mathrm{D}}{ }^{25}=+1.09^{\circ}\left(c=2.1, \mathrm{CH}_{2} \mathrm{Cl}_{2}\right)$. HRMS-ESI $(\mathrm{m} / z):[\mathrm{M}+\mathrm{H}]^{+}$calculated for $\mathrm{C}_{33} \mathrm{H}_{50} \mathrm{O}_{4} \mathrm{Si}_{2} \mathrm{Na}$, 589.3140; found, 589.3133.

Preparation of (2S,8R,11S,Z)-7-(Benzyldimethylsilyl)-8-((tertbutyldimethylsilyl)oxy)-11-hydroxy-14-methoxy-14-oxotetradec-6-en-12-yn-2-yl Benzoate, 215.

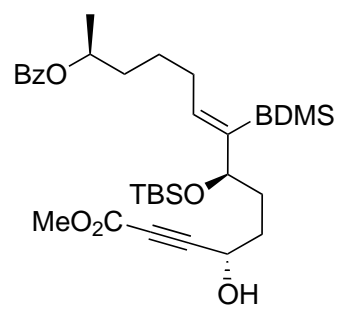

To a solution of $(R, R)$-ProPhenol ligand $(\mathbf{L} 4)(0.0091 \mathrm{~g}, 0.014 \mathrm{mmol}, 20 \mathrm{~mol} \%)$ and methyl propiolate $(0.0178 \mathrm{~mL}, 0.0168 \mathrm{~g}, 0.20 \mathrm{mmol}, 2.8$ equiv) in toluene $(0.15 \mathrm{~mL})$ was added dimethyl zinc $\left(0.176 \mathrm{~mL}\right.$ of a $1.2 \mathrm{M}$ solution in toluene, $0.211 \mathrm{mmol}, 2.95$ equiv) at $0{ }^{\circ} \mathrm{C}$. The reaction was warmed to room temperature and stirred for 60 minutes before addition of a solution of (2S,8R,Z)-7-(benzyldimethylsilyl)-8-((tert-butyldimethylsilyl)oxy)-11-oxoundec6-en-2-yl benzoate $214(0.0405 \mathrm{~g}, 0.0714 \mathrm{mmol}, 1$ equiv $)$ in toluene $(0.15 \mathrm{~mL})$ at $0{ }^{\circ} \mathrm{C}$. The reaction was stirred for 48 hours at $4{ }^{\circ} \mathrm{C}$ before quenching with saturated, aqueous $\mathrm{NH}_{4} \mathrm{Cl}$. The organic phase was extracted three times with $\mathrm{Et}_{2} \mathrm{O}$ and the combined organics were concentrated in vacuo. The crude product was purified by silica gel chromatography (5\% 15\% EtOAc/ pet. ether) to give the title compound as a clear, colorless oil ( $0.033 \mathrm{~g}, 71 \%$ yield $)$. $\mathbf{R}_{\mathbf{f}}=0.50\left(20 \% \mathrm{Et}_{2} \mathrm{O} /\right.$ pet. ether). ${ }^{1} \mathbf{H}$ NMR $\left(400 \mathrm{MHz} ; \mathrm{CDCl}_{3}\right): \delta 8.02(\mathrm{~d}, J=7.1 \mathrm{~Hz}, 2 \mathrm{H})$, $7.52(\mathrm{t}, J=7.4 \mathrm{~Hz}, 1 \mathrm{H}), 7.40(\mathrm{t}, J=7.6 \mathrm{~Hz}, 2 \mathrm{H}), 7.17(\mathrm{t}, J=7.5 \mathrm{~Hz}, 2 \mathrm{H}), 7.04(\mathrm{t}, J=7.3 \mathrm{~Hz}$, $1 \mathrm{H}), 6.96(\mathrm{~m}, 2 \mathrm{H}), 6.20(\mathrm{t}, J=7.4 \mathrm{~Hz}, 1 \mathrm{H}), 5.15(\mathrm{~m}, 1 \mathrm{H}), 4.34($ app. q, $J=6.2 \mathrm{~Hz}, 1 \mathrm{H}), 4.12$ $(\mathrm{m}, 1 \mathrm{H}), 3.74(\mathrm{~s}, 3 \mathrm{H}), 2.38(\mathrm{dd}, J=5.4,0.3 \mathrm{~Hz}, 1 \mathrm{H}), 2.21-2.14(\mathrm{~m}, 4 \mathrm{H}), 1.77-1.46(\mathrm{~m}, 8 \mathrm{H})$, $1.34(\mathrm{~d}, J=6.3 \mathrm{~Hz}, 3 \mathrm{H}), 0.85(\mathrm{~s}, 9 \mathrm{H}), 0.12(\mathrm{~s}, 3 \mathrm{H}), 0.09(\mathrm{~s}, 3 \mathrm{H}),-0.02(\mathrm{~s}, 3 \mathrm{H}),-0.06(\mathrm{~s}, 3 \mathrm{H})$. ${ }^{13} \mathrm{C}$ NMR $\left(101 \mathrm{MHz}, \mathrm{CDCl}_{3}\right): \delta 166.48,154.03,143.34,140.10(2 \mathrm{C}), 133.01,130.94,129.74$, $128.63,128.53,128.42,124.35,88.48,71.76,62.37$ (2C), 52.99, 35.98, 33.92, 33.11, 31.88, 27.10, 26.15, 26.12, 25.85, 20.28, 18.45, -1.02, -1.32, -4.00, -4.76. IR (film): 3450, 2930, 2856, 2236, $1717,1601,1493,1452,1435,1315,1274,1252,1113,1070,1027,834,775,713 \mathrm{~cm}^{-1} \cdot[\alpha]_{\mathrm{D}}{ }^{25}$ $=+9.05^{\circ}\left(c=1.44, \mathrm{CH}_{2} \mathrm{Cl}_{2}\right)$. HRMS-ESI $(m / z):[\mathrm{M}+\mathrm{Na}]^{+}$calculated for $\mathrm{C}_{37} \mathrm{H}_{54} \mathrm{O}_{6} \mathrm{Si}_{2} \mathrm{Na}$, 673.3351 ; found, 673.3341 . 
Preparation of $(2 S, 8 R, 11 S, Z)-7-($ Benzyldimethylsilyl)-8-((tertbutyldimethylsilyl)oxy)-14-methoxy-11-(methoxymethoxy)-14-oxotetradec6-en-12-yn-2-yl Benzoate, 216.

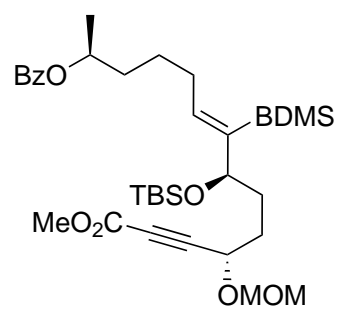

To a solution of (2S,8R,11S,Z)-7-(benzyldimethylsilyl)-8-((tert-butyldimethylsilyl)oxy)-11hydroxy-14-methoxy-14-oxotetradec-6-en-12-yn-2-yl benzoate 215 (0.0226 g, 0.0347 mmol, 1.0 eq.) in DME $(0.35 \mathrm{~mL})$ was added $N, N$-diisopropylethylamine $(0.021 \mathrm{~mL}, 0.016 \mathrm{~g}, 0.121 \mathrm{mmol}$, 3.5 eq.), sodium iodide $(0.002 \mathrm{~g}, 0.010 \mathrm{mmol}, 0.3 \mathrm{eq}$.$) and lastly, methyl chloromethyl ether$ (0.008 mL, $0.0084 \mathrm{~g}, 0.104 \mathrm{mmol}, 3.0$ eq.). The reaction was heated to $80{ }^{\circ} \mathrm{C}$ (bath temp.) in a sealed vessel and stirred for 7 hours. After cooling to room temperature, the reaction was diluted with $\mathrm{CH}_{2} \mathrm{Cl}_{2}$ and water, and was subsequently extracted with $\mathrm{CH}_{2} \mathrm{Cl}_{2}$. The combined organic layers were dried over $\mathrm{MgSO}_{4}$, filtered and concentrated in vacuo. The crude product was purified by flash column chromatography ( $5 \%$ EtOAc/pet. ether). The title compound was isolated as a clear, colorless oil $(0.0149 \mathrm{~g}, 62 \%$ yield $) . \mathbf{R}_{\mathbf{f}}=0.32(5 \%$ EtOAc/pet. ether). ${ }^{1} \mathbf{H}$ NMR $\left(500 \mathrm{MHz} ; \mathrm{CDCl}_{3}\right): \delta 8.07-8.05(\mathrm{~m}, 2 \mathrm{H}), 7.57(\mathrm{tt}, J=7.4,1.5 \mathrm{~Hz}, 1 \mathrm{H}), 7.45(\mathrm{t}, J=$ $7.8 \mathrm{~Hz}, 2 \mathrm{H}), 7.21(\mathrm{t}, J=7.6 \mathrm{~Hz}, 2 \mathrm{H}), 7.09(\mathrm{t}, J=7.4 \mathrm{~Hz}, 1 \mathrm{H}), 7.01(\mathrm{~d}, J=7.0 \mathrm{~Hz}, 2 \mathrm{H}), 6.24$ $(\mathrm{t}, J=7.5 \mathrm{~Hz}, 1 \mathrm{H}), 5.18(\mathrm{~m}, 1 \mathrm{H}), 4.88(\mathrm{~d}, J=6.9 \mathrm{~Hz}, 1 \mathrm{H}), 4.60(\mathrm{~d}, J=6.9 \mathrm{~Hz}, 1 \mathrm{H}), 4.38(\mathrm{t}$, $J=6.5 \mathrm{~Hz}, 1 \mathrm{H}), 4.13(\mathrm{t}, J=5.8 \mathrm{~Hz}, 1 \mathrm{H}), 3.78(\mathrm{~s}, 3 \mathrm{H}), 3.38(\mathrm{~s}, 3 \mathrm{H}), 2.27(\mathrm{~s}, 1 \mathrm{H}), 2.25(\mathrm{~s}, 1 \mathrm{H})$, 2.20-2.15 (m, 2H), 1.80-1.46 (m, 8H), $1.37(\mathrm{~d}, J=6.2 \mathrm{~Hz}, 3 \mathrm{H}), 0.90(\mathrm{~s}, 9 \mathrm{H}), 0.14(\mathrm{~m}, 6 \mathrm{H})$, $0.03(\mathrm{~s}, 3 \mathrm{H}),-0.02(\mathrm{~s}, 3 \mathrm{H}) .{ }^{13} \mathrm{C}$ NMR $\left(126 \mathrm{MHz}, \mathrm{CDCl}_{3}\right): \delta 166.43,153.90,143.13,140.11$ (2C), 132.99, 131.02, 129.76, 128.57, 128.54, 128.40, 124.37, 94.88, 86.70, 77.14, 71.73, 71.70, $65.62,56.05,52.98,36.10,34.20,31.86,31.43,27.09,26.19,25.97,20.37,18.46,-1.11,-1.28$, -3.93, -4.71. IR (film): 2953, 2930, 2856, 2235, 1719, 1601, 1451, 1253, 1152, 1111, 1070, 1028, $834 \mathrm{~cm}-1 .[\alpha]_{\mathrm{D}}{ }^{25}=-27.56^{\circ}\left(c=1.49, \mathrm{CH}_{2} \mathrm{Cl}_{2}\right)$. HRMS-ESI $(m / z):[\mathrm{M}+\mathrm{Na}]^{+}$calculated for $\mathrm{C}_{39} \mathrm{H}_{58} \mathrm{O}_{7} \mathrm{Si}_{2} \mathrm{Na}, 717.3613$; found, 717.3604 .

Preparation of $(S, E)-7-((2 R, 5 S, 6 S)-6$-(2-methoxy-2-oxoethyl)-5(methoxymethoxy)tetrahydro-2H-pyran-2-yl)hept-6-en-2-yl benzoate, 190.

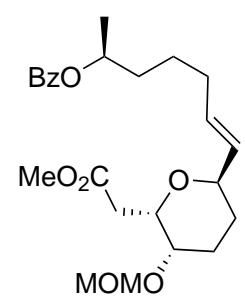

To a solution of (2S,8R,11S,Z)-7-(benzyldimethylsilyl)-8-((tert-butyldimethylsilyl)oxy)-14methoxy-11-(methoxymethoxy)-14-oxotetradec-6-en-12-yn-2-yl benzoate 216 (0.0177 g, 0.0255 mmol, 1.0 eq. $)$ in DCE $(0.1 \mathrm{~mL})$ at $0{ }^{\circ} \mathrm{C}$ was added freshly distilled triethoxysilane $(0.007 \mathrm{~mL}$, $0.0063 \mathrm{~g}, 0.0383 \mathrm{mmol}, 1.5$ eq.), immediately followed by $0.0013 \mathrm{~g}$ of $\mathrm{RuCp} *\left(\mathrm{CH}_{3} \mathrm{CN}\right)_{3} \mathrm{PF}_{6}$ (0.003 mmol, $10 \mathrm{~mol} \%)$. The reaction was allowed to warm to room temperature and stirred for 1 hour before being diluted with $\mathrm{Et}_{2} \mathrm{O}$ and passed through a plug of florasil. The desired 
vinylsilanes were isolated as a clear, colorless film $\left(0.018 \mathrm{~g}, 43 \%\right.$ yield based on ${ }^{1} \mathrm{H}$ NMR, $88 \%$ based on recovered starting material).

The crude reaction product was dissolved in $0.33 \mathrm{~mL}$ of THF and $0.017 \mathrm{~g}$ of $\mathrm{CuI}(0.089 \mathrm{mmol}$, 8 eq.) was added. The reaction mixture was cooled to $0{ }^{\circ} \mathrm{C}$ and TBAF $(0.179 \mathrm{~mL}$ as a $1 \mathrm{M}$ solution in THF, $0.179 \mathrm{mmol}, 16$ eq.) was added dropwise. The reaction was stirred at 0 ${ }^{\circ} \mathrm{C}$ for 2 hours before warming to room temperature and stirring for 8 additional hours. The reaction was then diluted with $\mathrm{Et}_{2} \mathrm{O}$ and passed through a silica plug. The crude product was purified by preparative TLC (25\% EtOAc/pet. ether) and the title compound was isolated as a clear, colorless film (0.0042 g, 88\% yield, $38 \%$ yield over 2 steps $) . \mathbf{R}_{\mathbf{f}}=0.25(20 \%$ EtOAc/pet. ether). ${ }^{1} \mathbf{H}$ NMR $\left(600 \mathrm{MHz} ; \mathrm{CDCl}_{3}\right): \delta 8.03(\mathrm{~d}, J=7.1 \mathrm{~Hz}, 2 \mathrm{H}), 7.54(\mathrm{t}, J=7.4 \mathrm{~Hz}, 1 \mathrm{H})$, $7.43(\mathrm{t}, J=7.7 \mathrm{~Hz}, 2 \mathrm{H}), 5.66(\mathrm{dt}, J=14.8,6.9 \mathrm{~Hz}, 1 \mathrm{H}), 5.48(\mathrm{dd}, J=15.7,5.1 \mathrm{~Hz}, 1 \mathrm{H})$, $5.16(\mathrm{~m}, 1 \mathrm{H}), 4.67(\mathrm{~d}, J=6.9 \mathrm{~Hz}, 1 \mathrm{H}), 4.61(\mathrm{~d}, J=6.8 \mathrm{~Hz}, 1 \mathrm{H}), 4.32(\mathrm{ddd}, J=8.7,5.0$, $3.6 \mathrm{~Hz}, 1 \mathrm{H}), 4.26(\mathrm{q}, J=4.9 \mathrm{~Hz}, 1 \mathrm{H}), 3.69(\mathrm{dt}, J=7.1,3.5 \mathrm{~Hz}, 1 \mathrm{H}), 3.67(\mathrm{~s}, 3 \mathrm{H}), 3.37(\mathrm{~s}$, $3 \mathrm{H}), 2.73(\mathrm{dd}, J=15.4,8.7 \mathrm{~Hz}, 1 \mathrm{H}), 2.58(\mathrm{dd}, J=15.4,5.2 \mathrm{~Hz}, 1 \mathrm{H}), 2.09(\mathrm{~m}, 2 \mathrm{H}), 1.95(\mathrm{~m}$, $1 \mathrm{H}), 1.80-1.62(\mathrm{~m}, 4 \mathrm{H}), 1.50-1.45(\mathrm{~m}, 3 \mathrm{H}), 1.34(\mathrm{~d}, J=6.3 \mathrm{~Hz}, 3 \mathrm{H}) . \mathbf{1 H}-\mathbf{N M R}(600 \mathrm{MHz}$; Benzene- $d 6): \delta 8.15(\mathrm{~d}, J=7.5 \mathrm{~Hz}, 2 \mathrm{H}), 7.07(\mathrm{t}, J=7.3 \mathrm{~Hz}, 1 \mathrm{H}), 7.02(\mathrm{t}, J=7.4 \mathrm{~Hz}, 2 \mathrm{H})$, $5.62(\mathrm{~m}, 1 \mathrm{H}), 5.44(\mathrm{~m}, 1 \mathrm{H}), 5.20(\mathrm{~m}, 1 \mathrm{H}), 4.53(\mathrm{dt}, J=9.0,4.4 \mathrm{~Hz}, 1 \mathrm{H}), 4.39(\mathrm{~d}, J=6.7 \mathrm{~Hz}$, $1 \mathrm{H}), 4.32(\mathrm{~d}, J=6.8 \mathrm{~Hz}, 1 \mathrm{H}), 4.16(\mathrm{~m}, 1 \mathrm{H}), 3.53(\mathrm{dt}, J=7.7,3.8 \mathrm{~Hz}, 1 \mathrm{H}), 3.31(\mathrm{~s}, 3 \mathrm{H}), 3.06$ $(\mathrm{s}, 3 \mathrm{H}), 2.82(\mathrm{dd}, J=15.2,9.2 \mathrm{~Hz}, 1 \mathrm{H}), 2.55(\mathrm{dd}, J=15.2,4.6 \mathrm{~Hz}, 1 \mathrm{H}), 1.89(\mathrm{q}, J=6.4$ $\mathrm{Hz}, 2 \mathrm{H}), 1.70(\mathrm{~m}, 1 \mathrm{H}), 1.60-1.22(\mathrm{~m}, 8 \mathrm{H}), 1.13(\mathrm{~d}, J=6.4 \mathrm{~Hz}, 3 \mathrm{H}) .{ }^{13} \mathrm{C}$ NMR $(150 \mathrm{MHz}$, $\left.\mathrm{CDCl}_{3}\right): \delta 172.17,166.33,132.88,132.73,130.96,129.72,129.66,128.43,95.38,72.00,71.65$, $71.38,70.40,55.83,51.83,35.67,34.80,32.37,26.26,25.07,24.01,20.23$. IR (film): 2917, 2849, $1740,1714,1575,1538,1463,1452,1276,1150,1111,1070,1037 \mathrm{~cm}^{-1} \cdot[\alpha]_{\mathrm{D}}{ }^{25}=-3.21^{\circ}(c=$ 0.42, $\left.\mathrm{CH}_{2} \mathrm{Cl}_{2}\right)$. LRMS-ESI $(\mathrm{m} / z):[\mathrm{M}+\mathrm{Na}]^{+}$calculated for $\mathrm{C}_{24} \mathrm{H}_{34} \mathrm{O}_{7} \mathrm{Na}, 457.22$; found, 457.17. Characterization Data matches that reported in the literature. ${ }^{11}$

Comparison of $\mathbf{1 9 0}$ to the Reported Literature NMR data. ${ }^{11}$

\begin{tabular}{|c|c|c|c|}
\hline$\delta_{\mathbf{H}}\left(600 \mathrm{MHz} ; \mathrm{CDCl}_{3}\right)$ & Lit. $\delta_{\mathbf{H}}\left(500 \mathrm{MHz} ; \mathrm{CDCl}_{3}\right)$ & $\delta_{\mathbf{C}}$ & Lit. $\delta_{\mathbf{C}}$ \\
\hline $8.03(\mathrm{~d}, J=7.1 \mathrm{~Hz}, 2 \mathrm{H})$ & 8.04-7.99 (m, 2H) & 172.17 & 172 \\
\hline $7.54(\mathrm{t}, J=7.4 \mathrm{~Hz}, 1 \mathrm{H})$ & $7.52(\mathrm{~m}, 1 \mathrm{H})$ & 166.33 & 166.1 \\
\hline $7.43(\mathrm{t}, J=7.7 \mathrm{~Hz}, 2 \mathrm{H})$ & 7.44-7.38 (m, 2H) & 132.88 & 132.7 \\
\hline $5.66(\mathrm{dt}, J=14.8,6.9 \mathrm{~Hz}, 1 \mathrm{H})$ & $5.64(\mathrm{~m}, 1 \mathrm{H})$ & 132.73 & 132.5 \\
\hline $5.48(\mathrm{dd}, J=15.7,5.1 \mathrm{~Hz}, 1 \mathrm{H})$ & $5.47(\mathrm{dd}, J=15.5,4.5 \mathrm{~Hz}, 1 \mathrm{H})$ & 130.96 & 130.8 \\
\hline $5.16(\mathrm{~m}, 1 \mathrm{H})$ & $5.14(\mathrm{~m}, 1 \mathrm{H})$ & 129.72 & 129.5 \\
\hline $4.67(\mathrm{~d}, J=6.9 \mathrm{~Hz}, 1 \mathrm{H})$ & $4.66(\mathrm{~d}, J=7 \mathrm{~Hz}, 1 \mathrm{H})$ & 129.66 & 129.5 \\
\hline $4.61(\mathrm{~d}, J=6.8 \mathrm{~Hz}, 1 \mathrm{H})$ & $4.59(\mathrm{~d}, J=7 \mathrm{~Hz}, 1 \mathrm{H})$ & 128.43 & 128.2 \\
\hline $4.32(\mathrm{ddd}, J=8.7,5.0,3.6 \mathrm{~Hz}, 1 \mathrm{H})$ & $4.30(\mathrm{ddd}, J=9,5.5,4 \mathrm{~Hz}, 1 \mathrm{H})$ & 95.38 & 95.2 \\
\hline $4.26(\mathrm{q}, J=4.9 \mathrm{~Hz}, 1 \mathrm{H})$ & $4.24(\mathrm{~m}, 1 \mathrm{H})$ & 72 & 71.8 \\
\hline $3.69(\mathrm{dt}, J=7.1,3.5 \mathrm{~Hz}, 1 \mathrm{H})$ & \multirow{2}{*}{$3.70-3.62(\mathrm{~m}, 4 \mathrm{H})$} & 71.65 & 71.4 \\
\hline $3.67(\mathrm{~s}, 3 \mathrm{H})$ & & 71.38 & 71.2 \\
\hline $3.37(\mathrm{~s}, 3 \mathrm{H})$ & $3.35(\mathrm{~s}, 3 \mathrm{H})$ & 70.4 & 70.2 \\
\hline $2.73(\mathrm{dd}, J=15.4,8.7 \mathrm{~Hz}, 1 \mathrm{H})$ & $2.71(\mathrm{dd}, J=15.5,9 \mathrm{~Hz}, 1 \mathrm{H})$ & 55.83 & 55.6 \\
\hline $2.58(\mathrm{dd}, J=15.4,5.2 \mathrm{~Hz}, 1 \mathrm{H})$ & $2.57(\mathrm{dd}, J=15,5 \mathrm{~Hz}, 1 \mathrm{H})$ & 51.83 & 51.6 \\
\hline $2.09(\mathrm{~m}, 2 \mathrm{H})$ & $2.12-2.03(\mathrm{~m}, 2 \mathrm{H})$ & 35.67 & 35.5 \\
\hline $1.95(\mathrm{~m}, 1 \mathrm{H})$ & $1.93(\mathrm{~m}, 1 \mathrm{H})$ & 34.8 & 34.6 \\
\hline $1.80-1.62(\mathrm{~m}, 4 \mathrm{H})$ & $1.82-1.56(\mathrm{~m}, 4 \mathrm{H})$ & 32.37 & 32.2 \\
\hline $1.50-1.45(\mathrm{~m}, 3 \mathrm{H})$ & $1.55-1.38(\mathrm{~m}, 3 \mathrm{H})$ & 26.26 & 26.1 \\
\hline \multirow[t]{3}{*}{$1.34(\mathrm{~d}, J=6.3 \mathrm{~Hz}, 3 \mathrm{H})$} & \multirow[t]{3}{*}{$1.31(\mathrm{~d}, J=6.5 \mathrm{~Hz}, 3 \mathrm{H})$} & 25.07 & 24.9 \\
\hline & & 24.01 & 23.8 \\
\hline & & 20.23 & 20 \\
\hline
\end{tabular}




\subsubsection{Spectra}
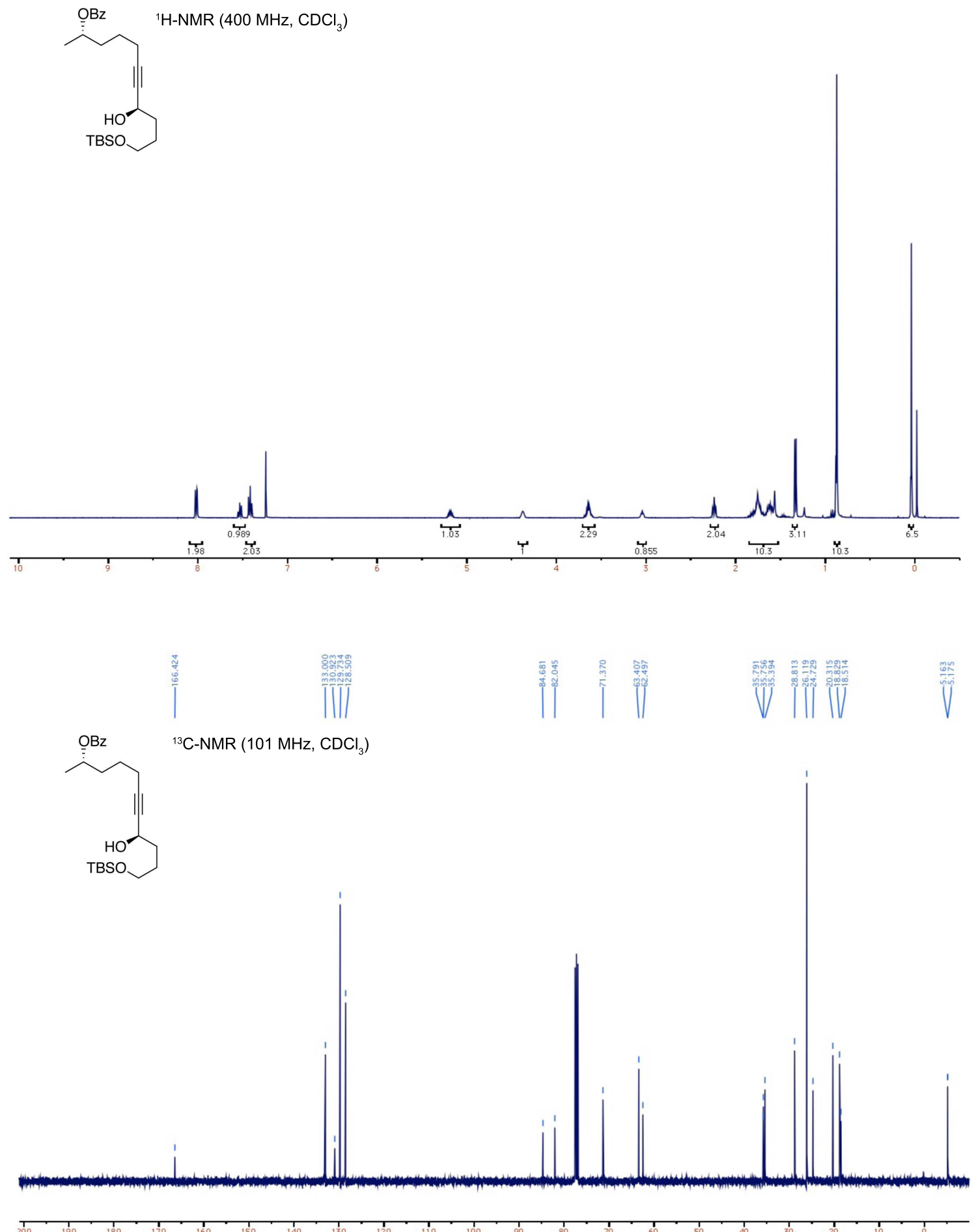

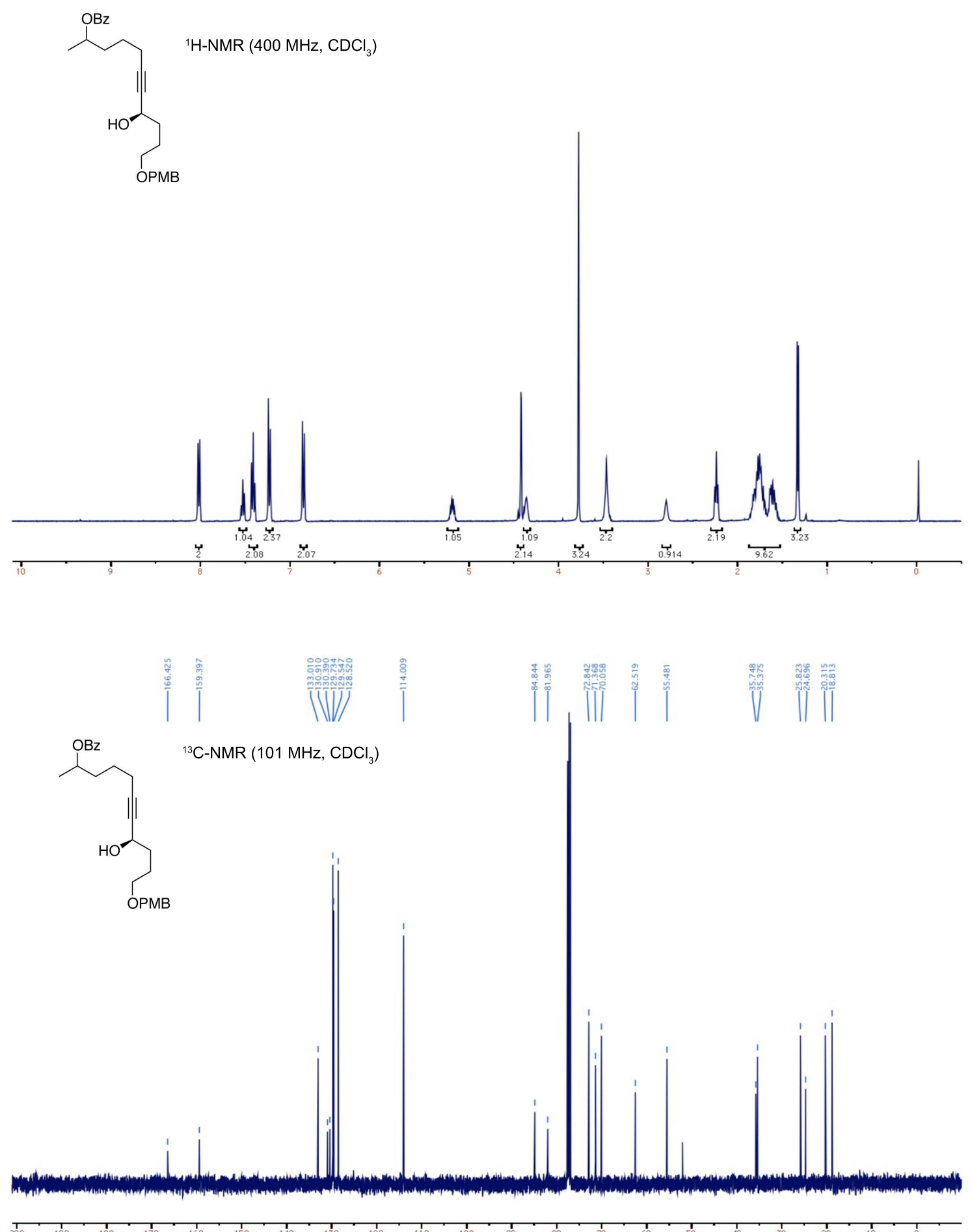

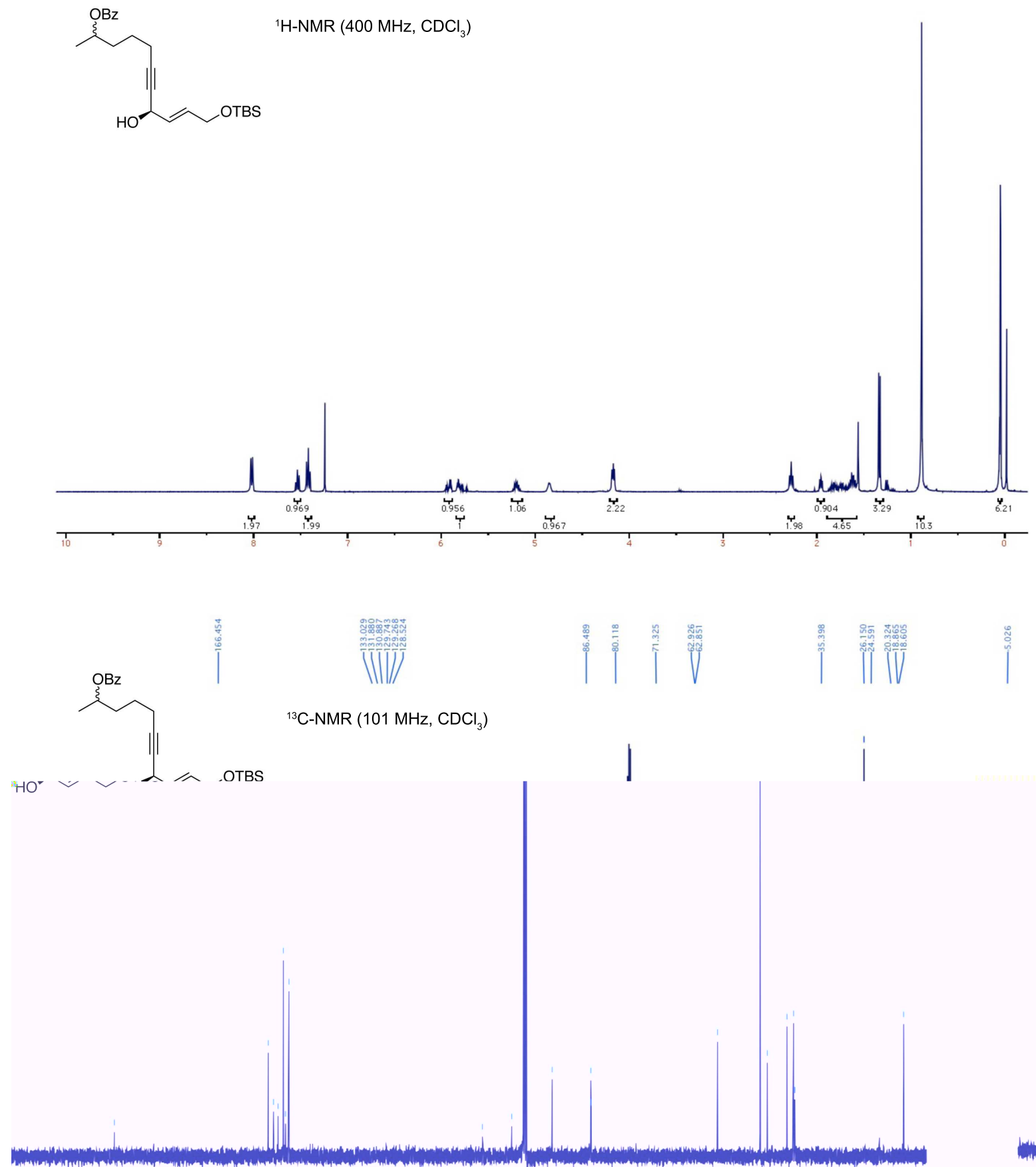

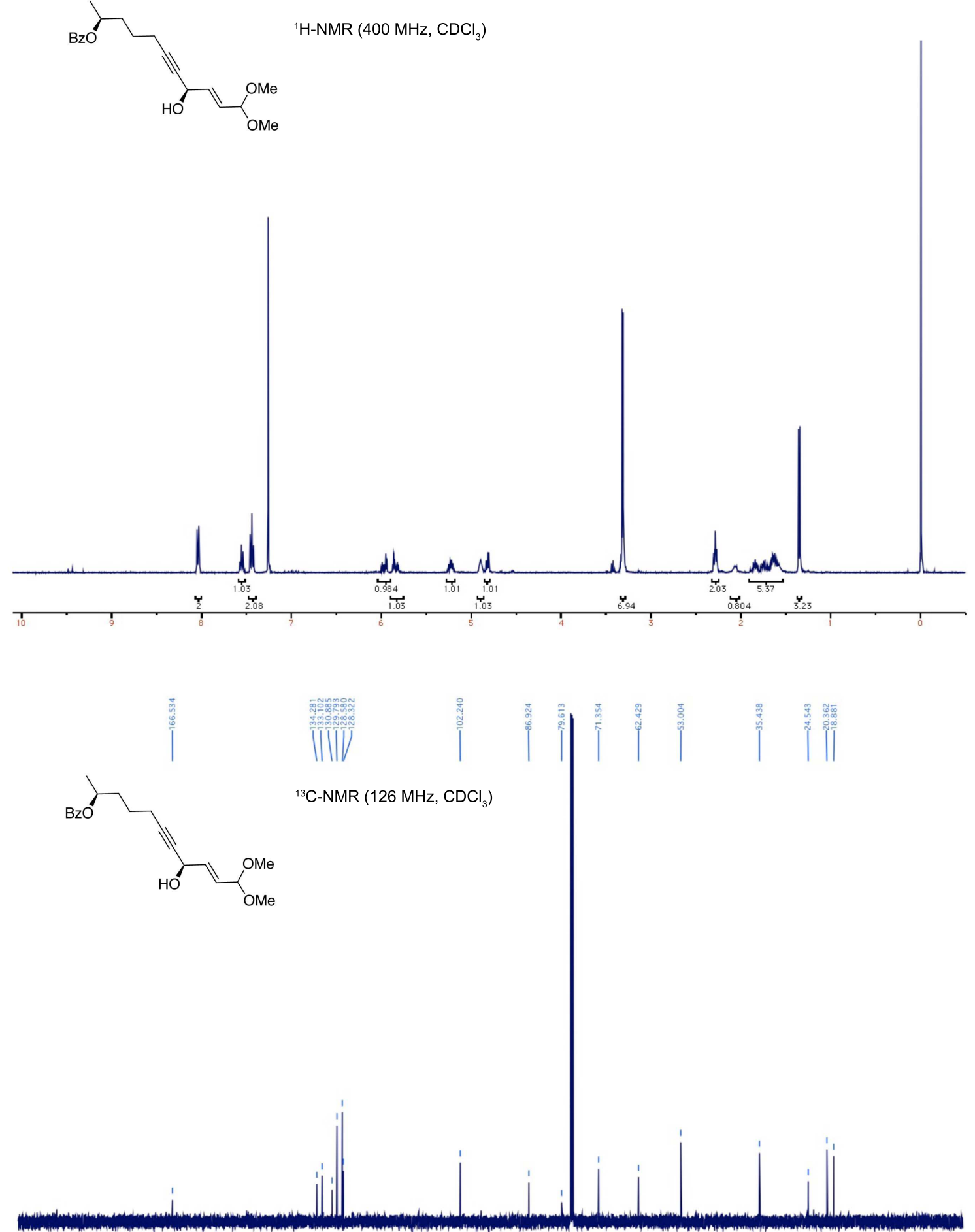

200 

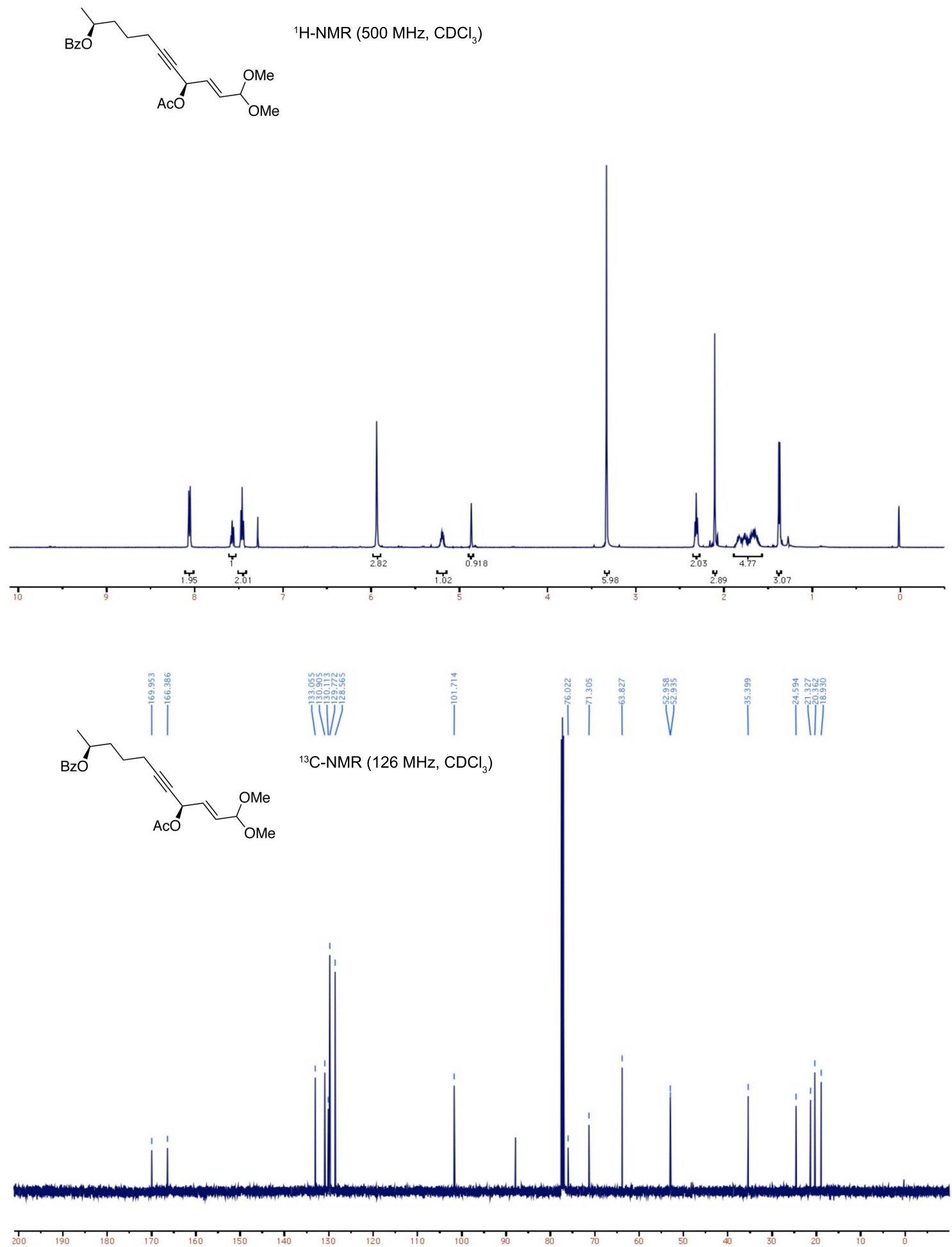

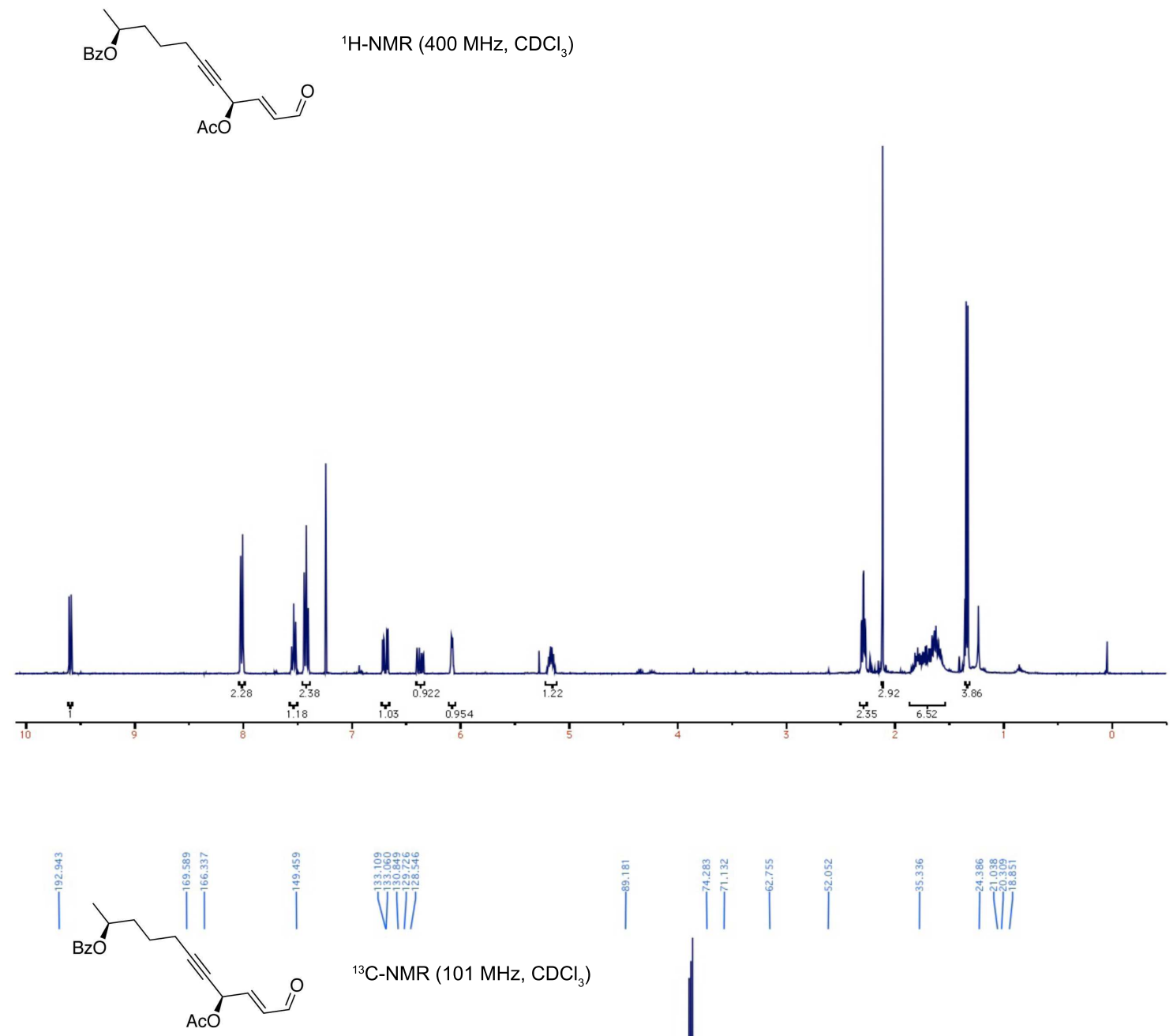

${ }^{13} \mathrm{C}-\mathrm{NMR}\left(101 \mathrm{MHz}, \mathrm{CDCl}_{3}\right)$
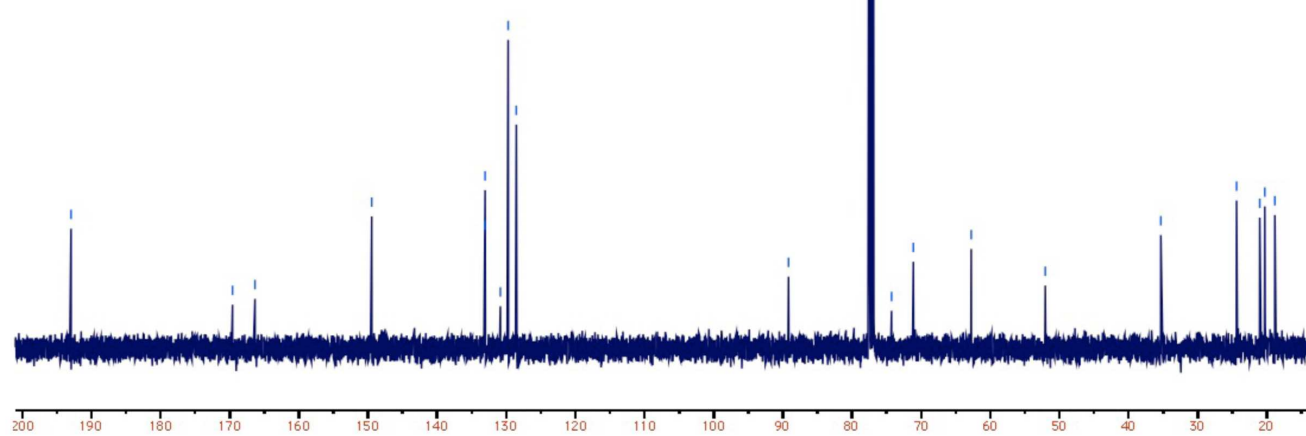

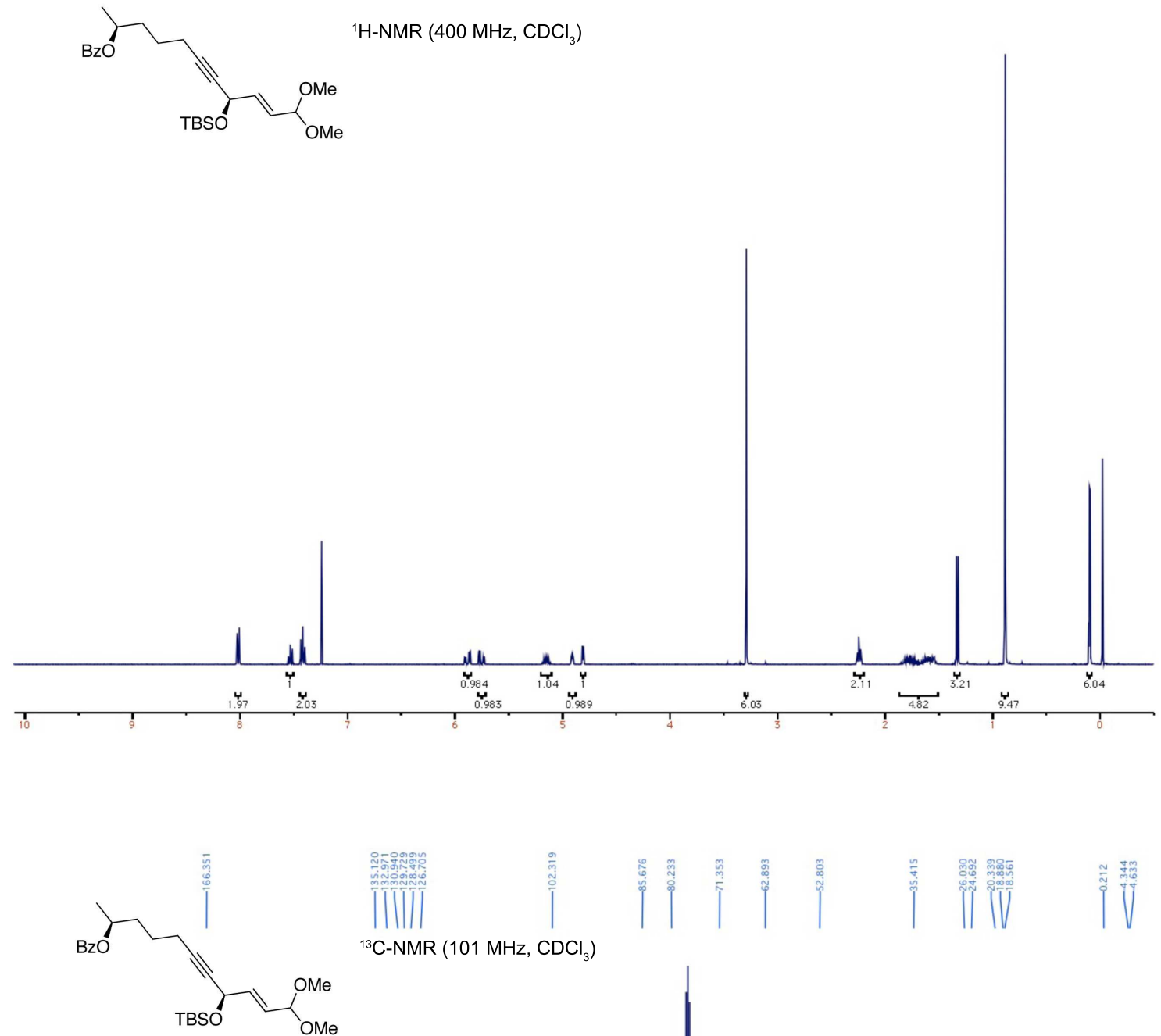

|

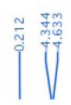

${ }^{13} \mathrm{C}-\mathrm{NMR}\left(101 \mathrm{MHz}, \mathrm{CDCl}_{3}\right)$
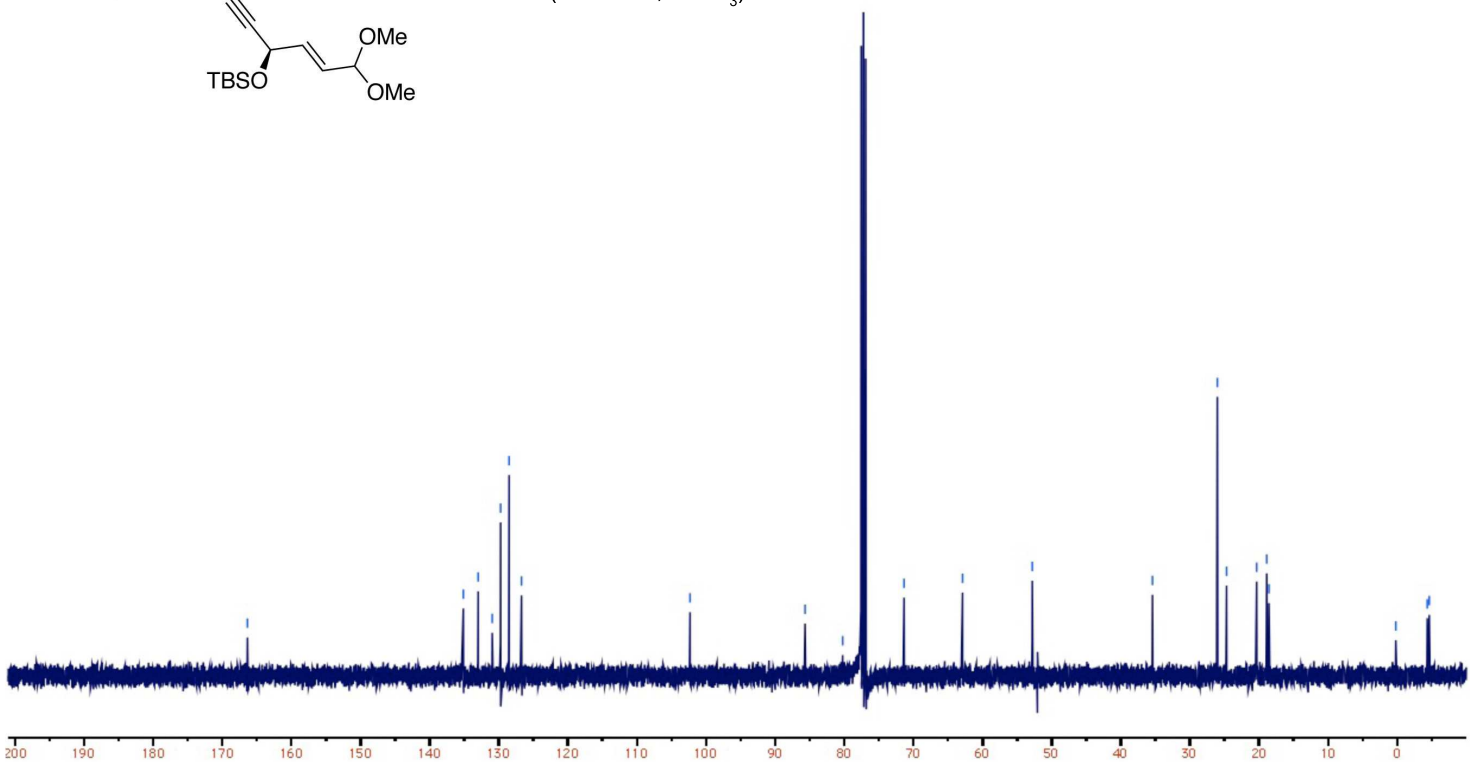

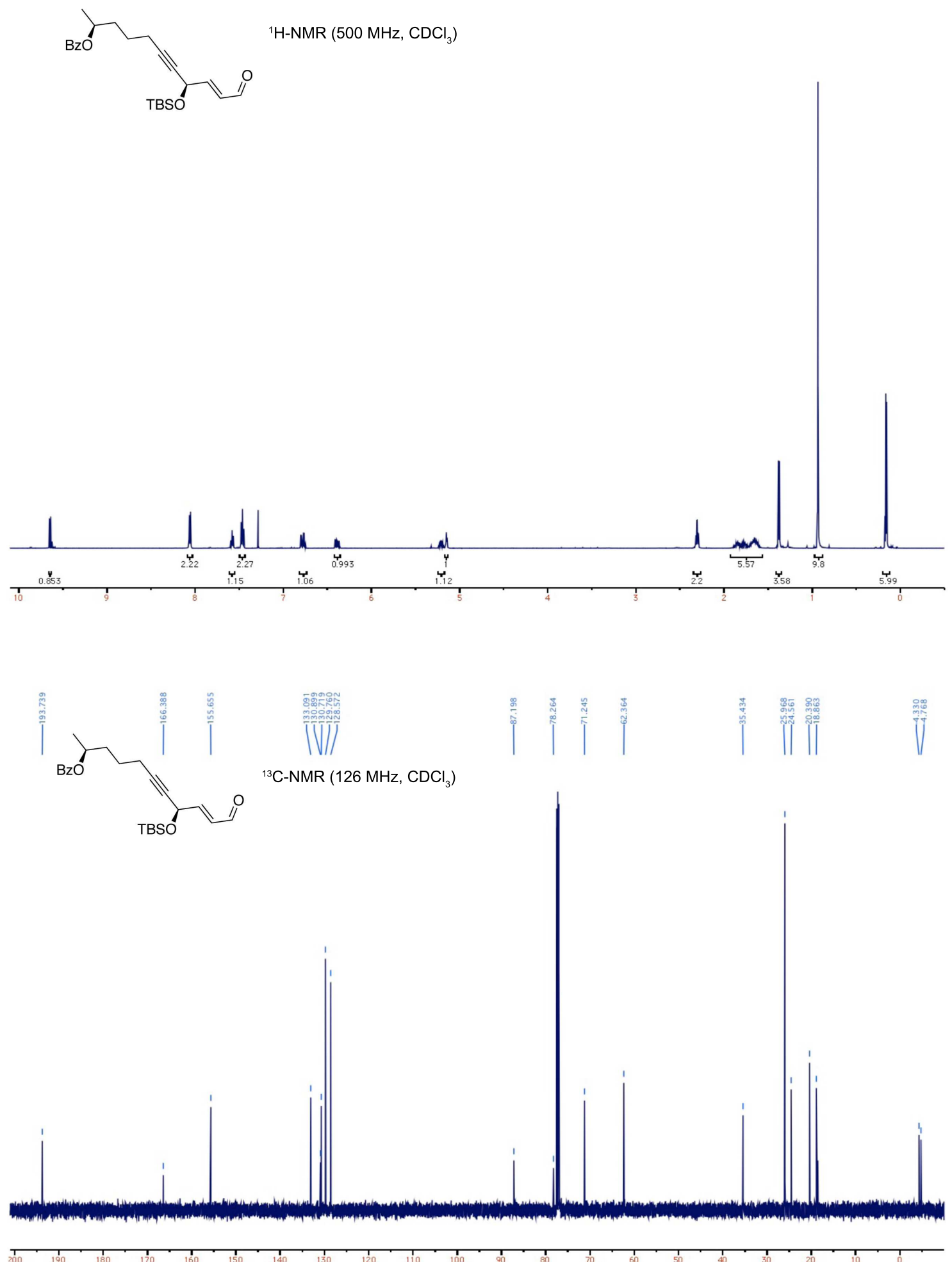

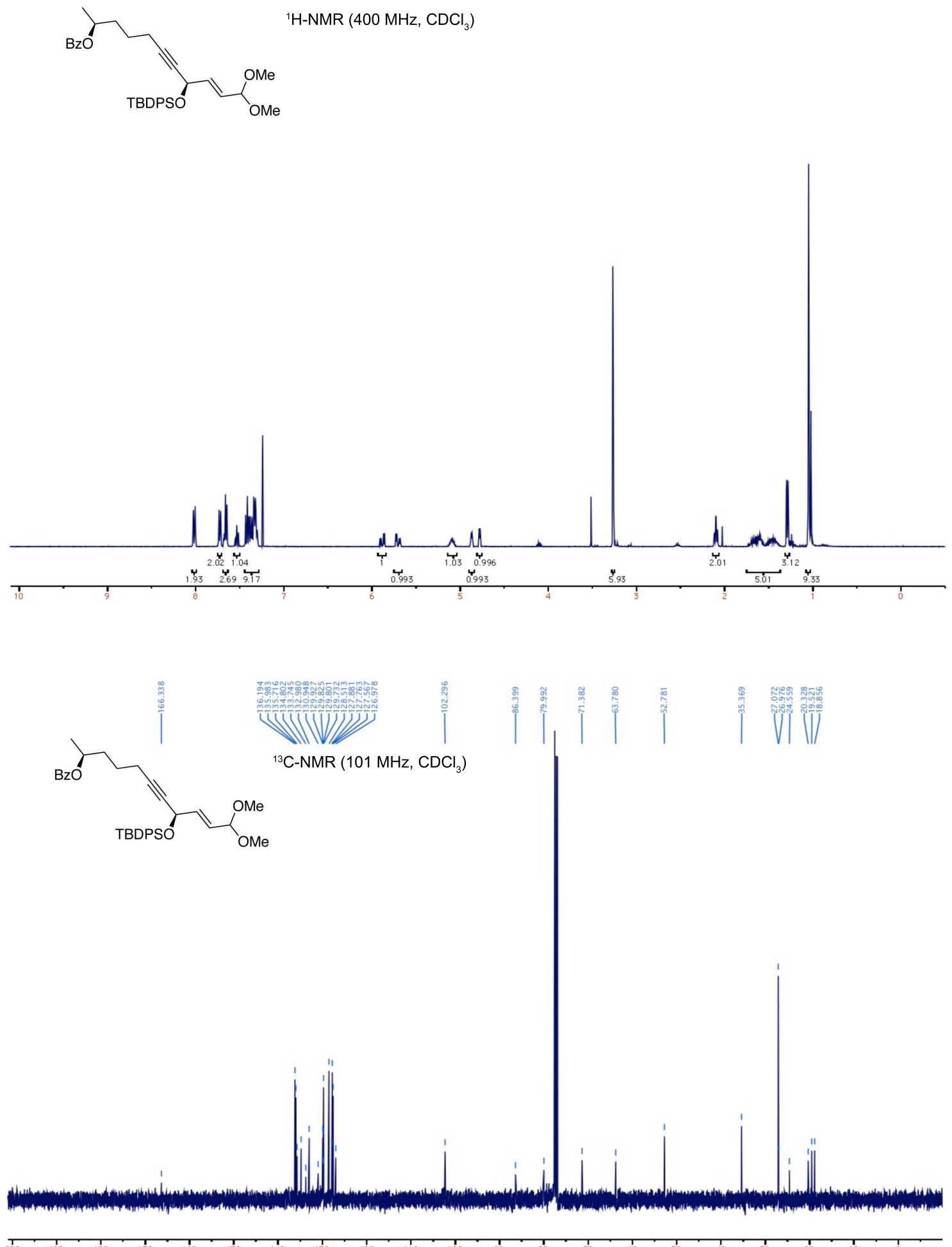

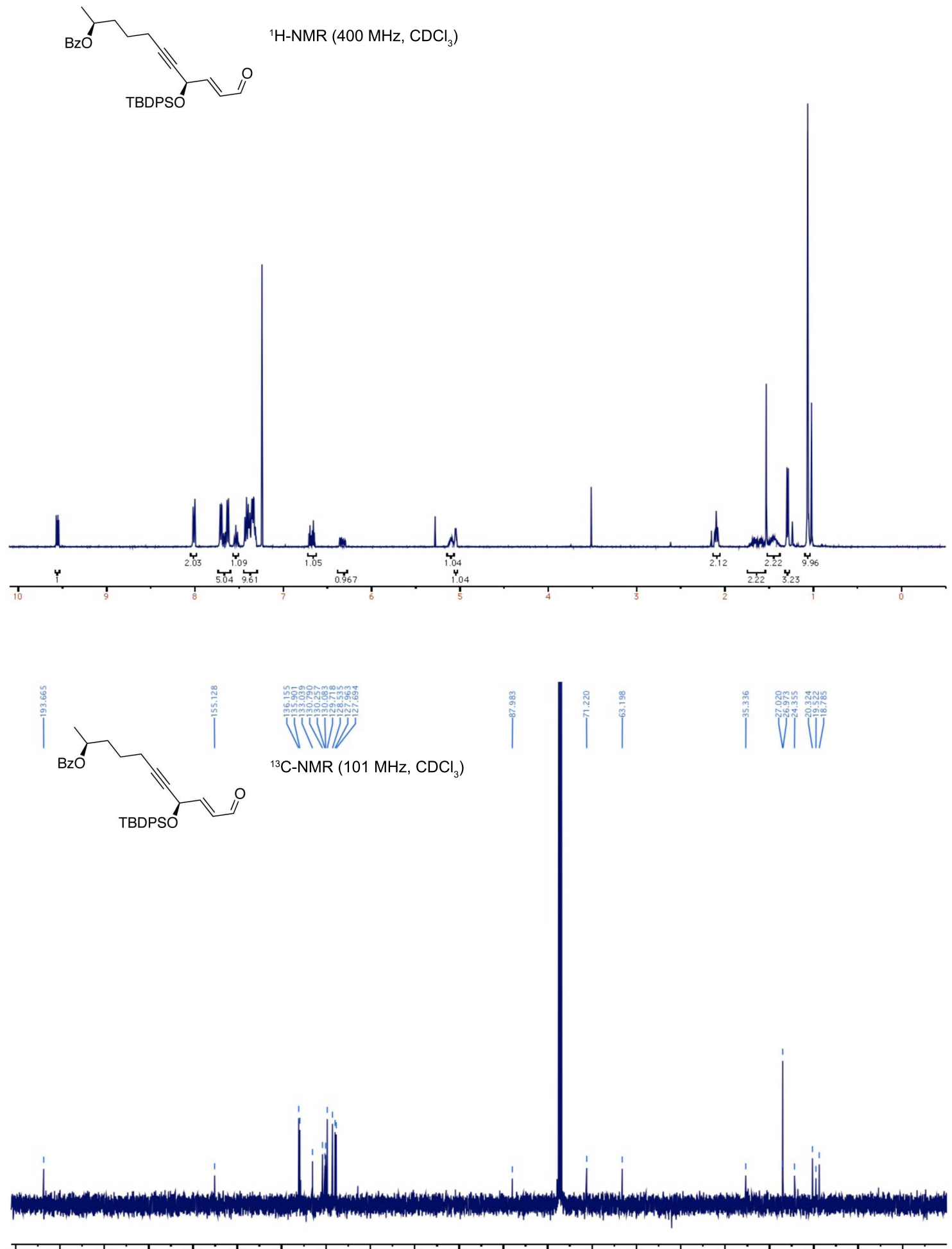

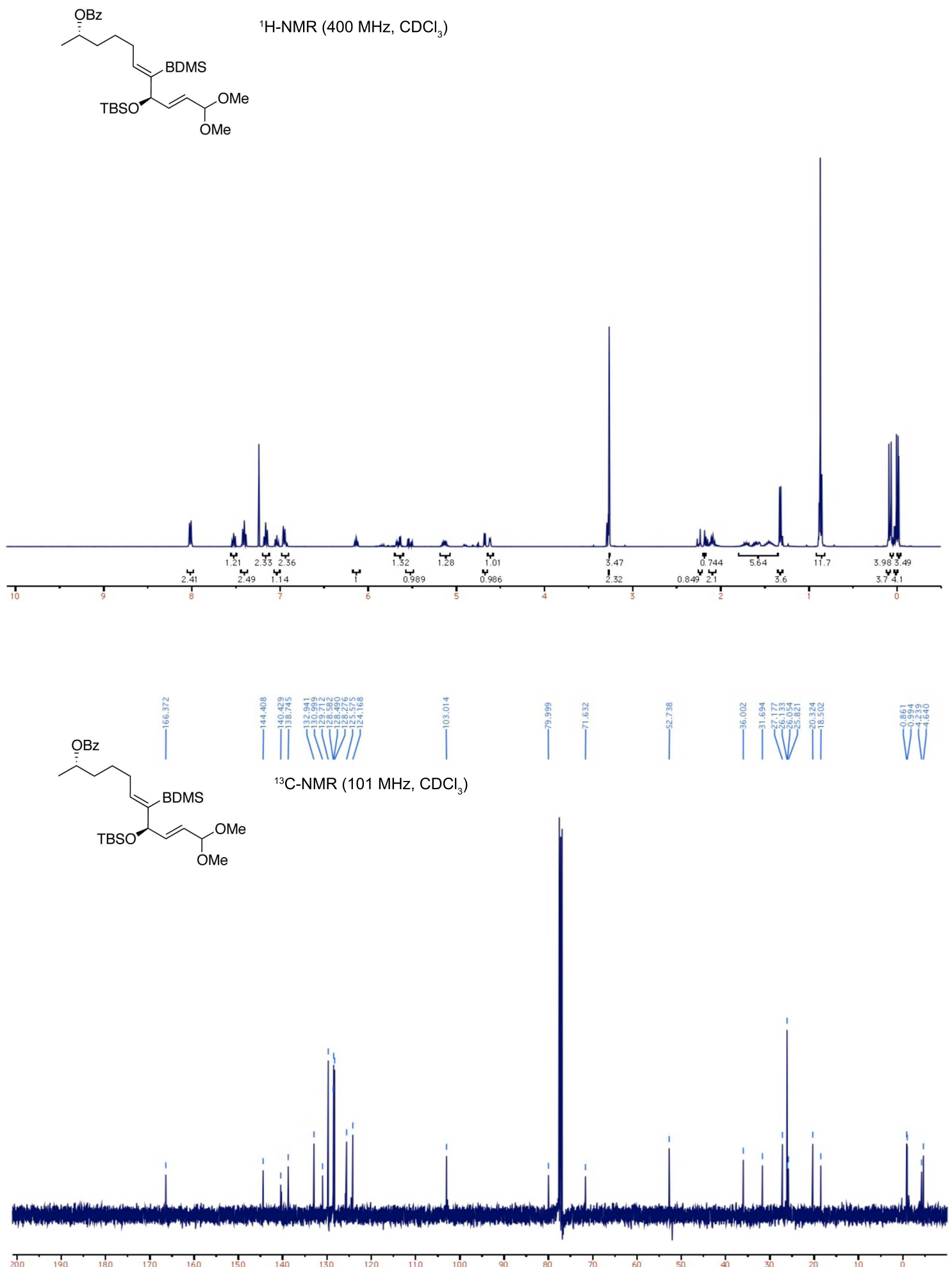

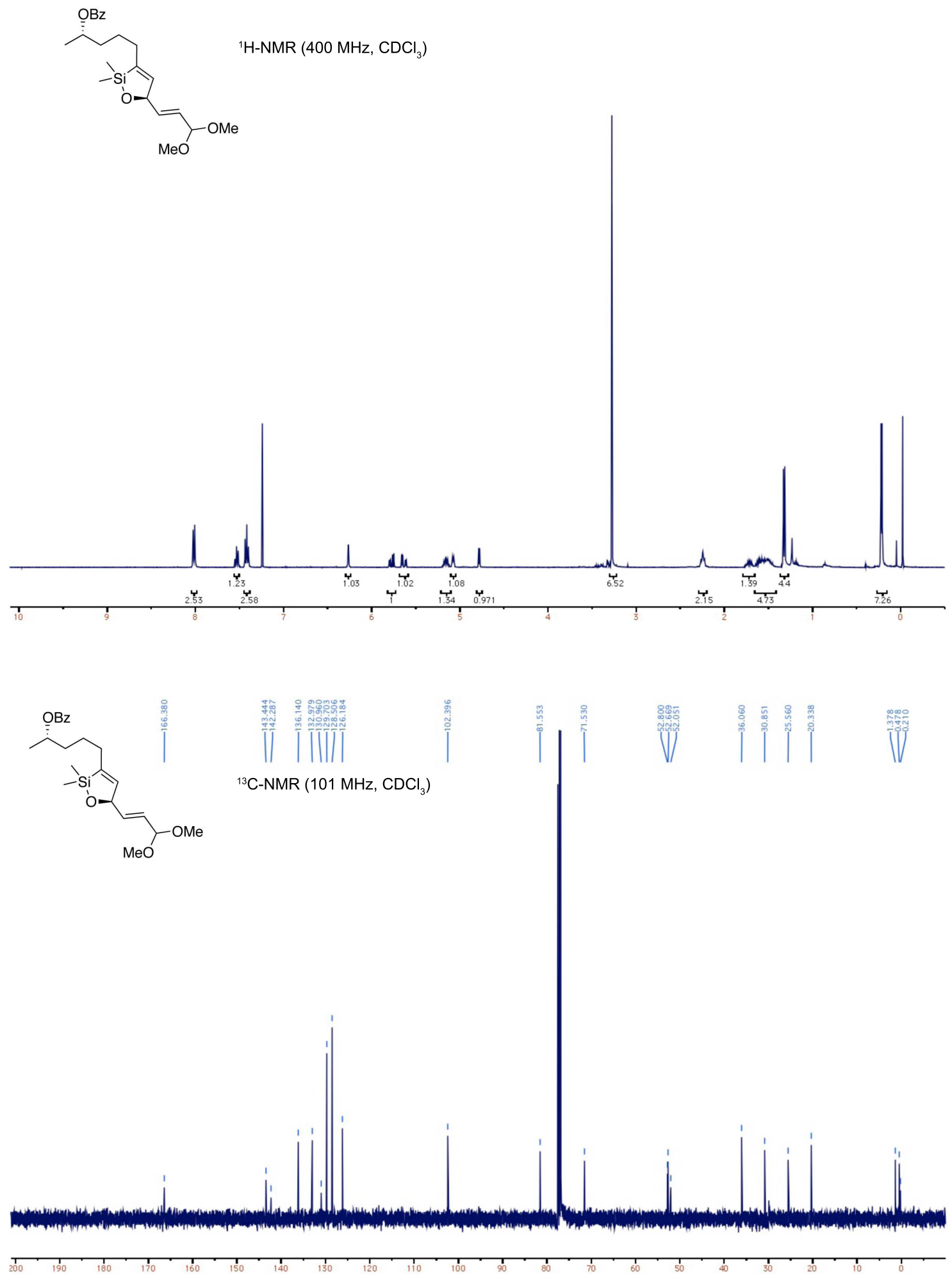

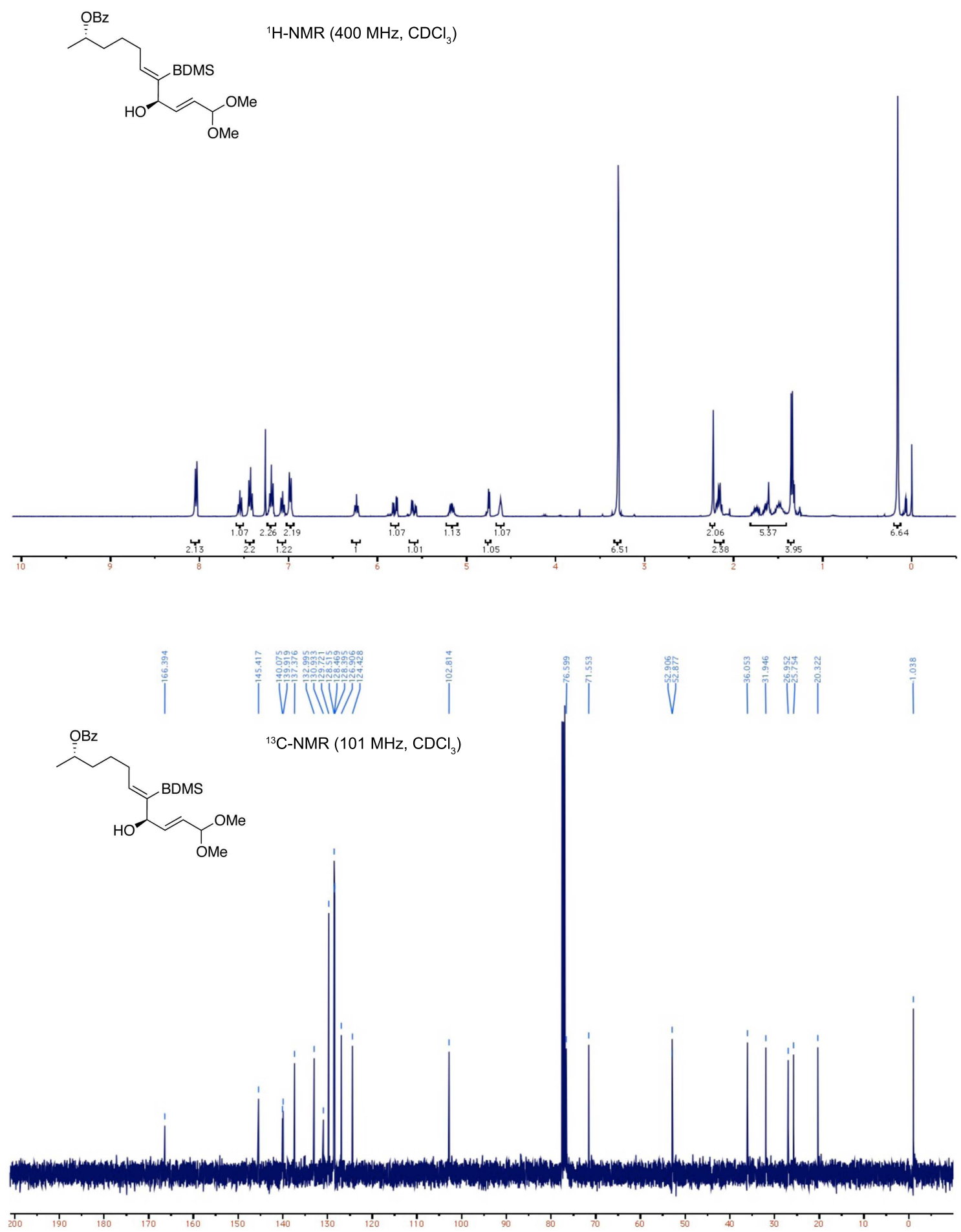


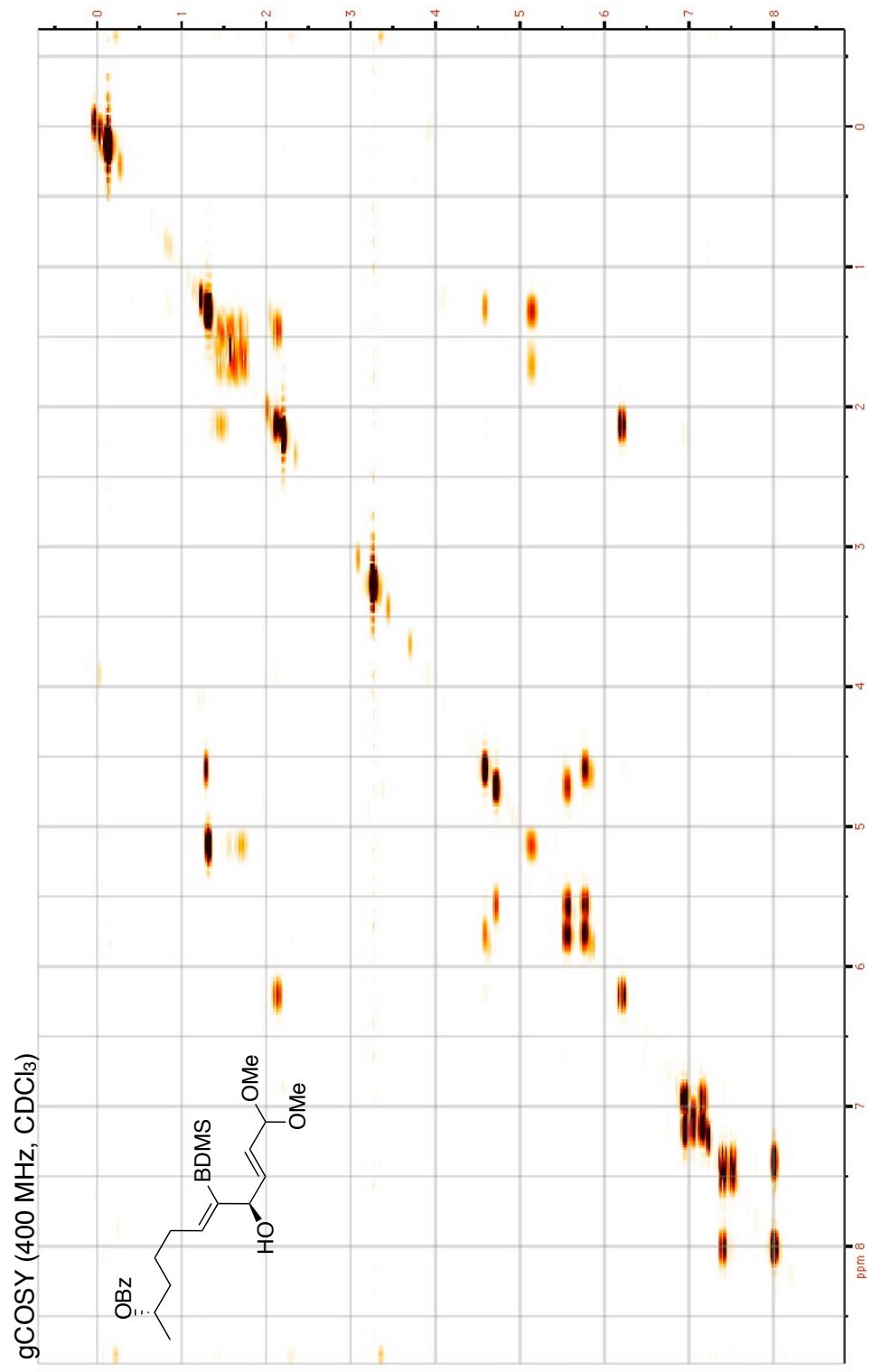




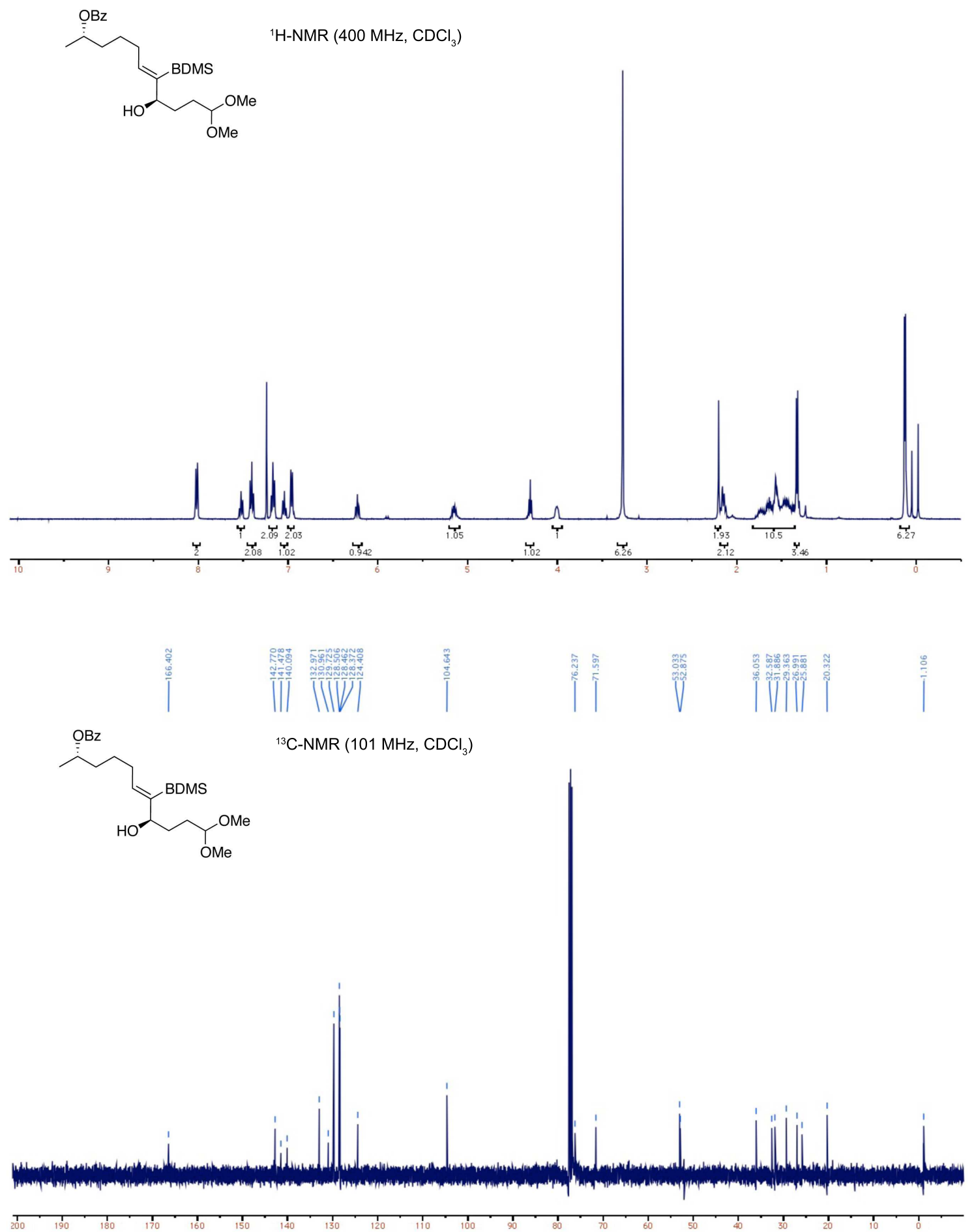



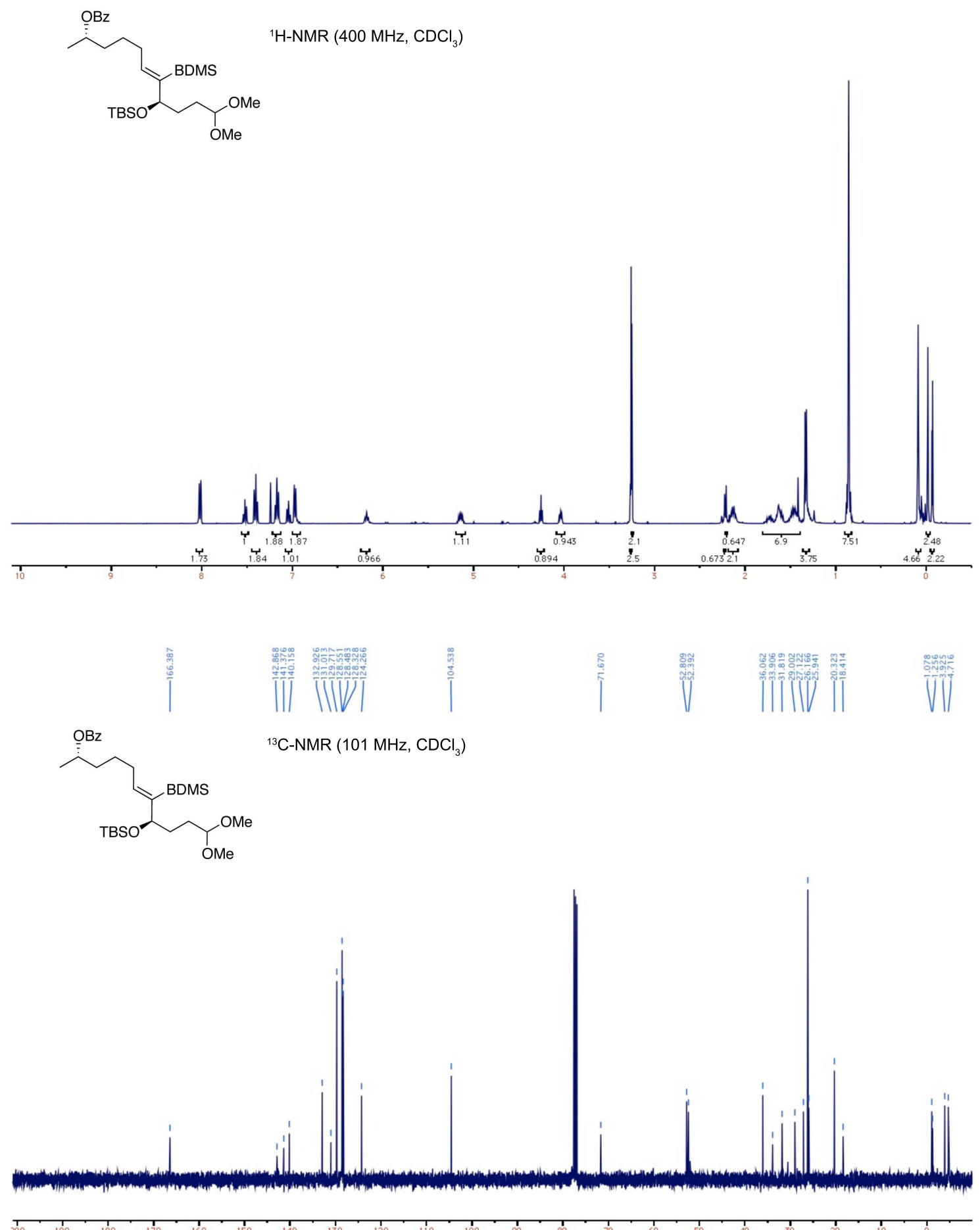

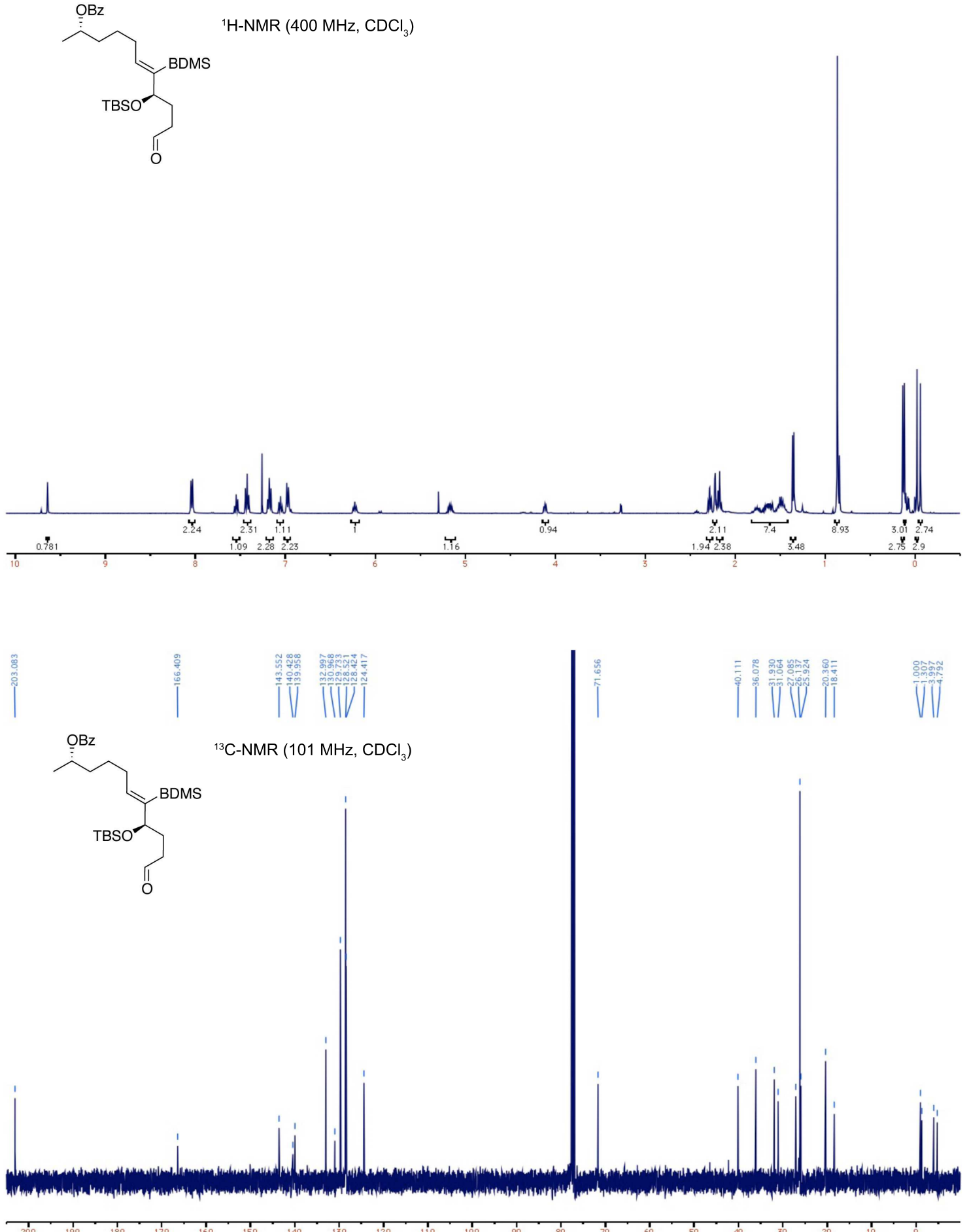

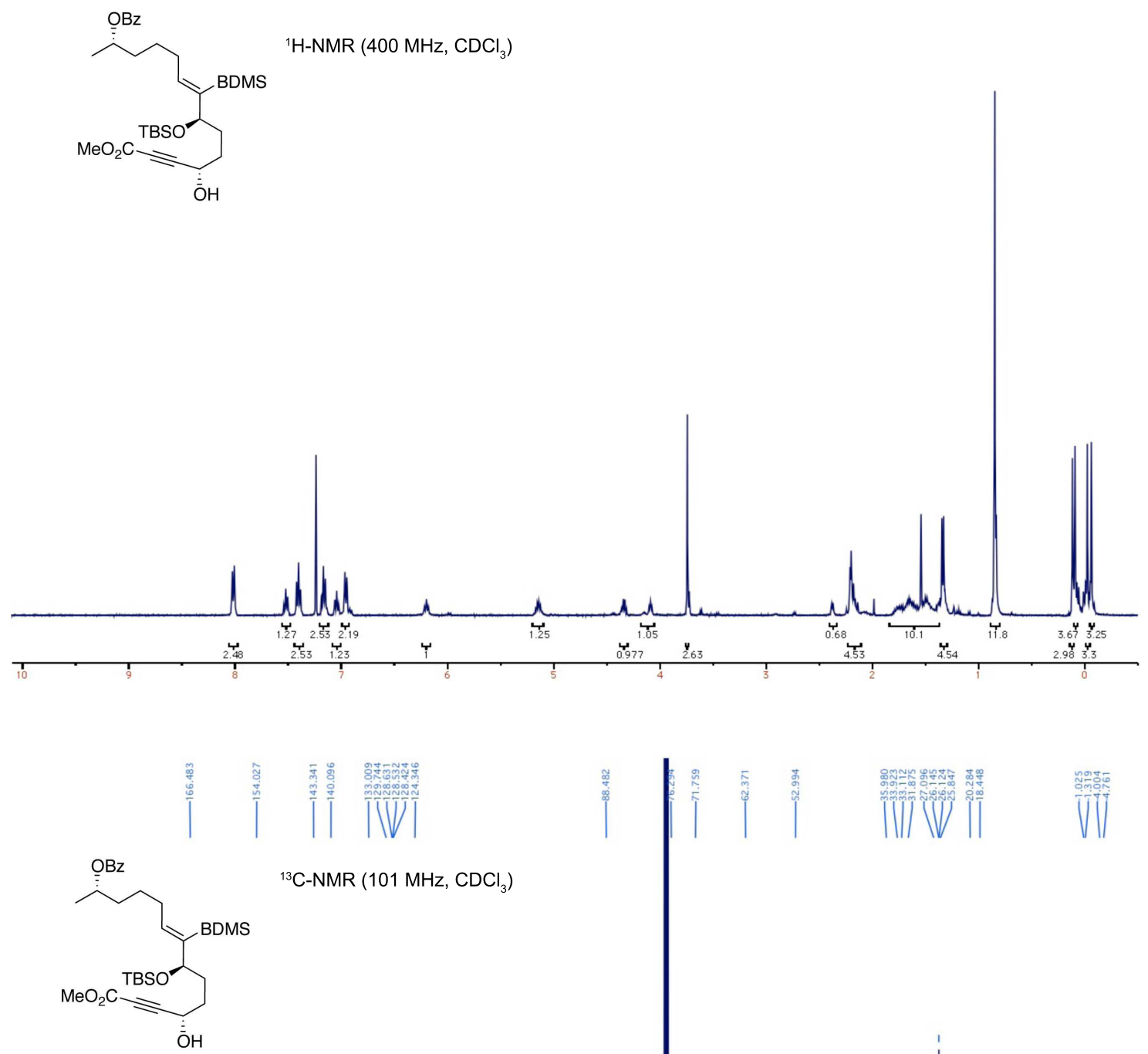

${ }^{13} \mathrm{C}-\mathrm{NMR}\left(101 \mathrm{MHz}, \mathrm{CDCl}_{3}\right.$ )
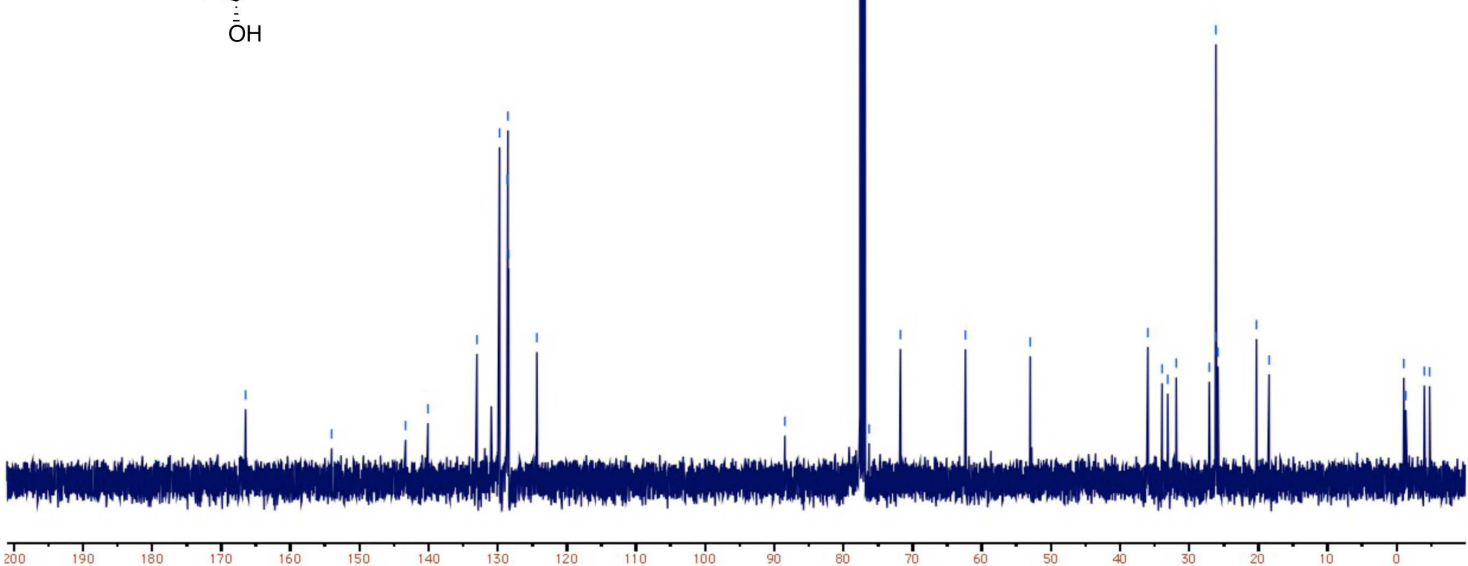

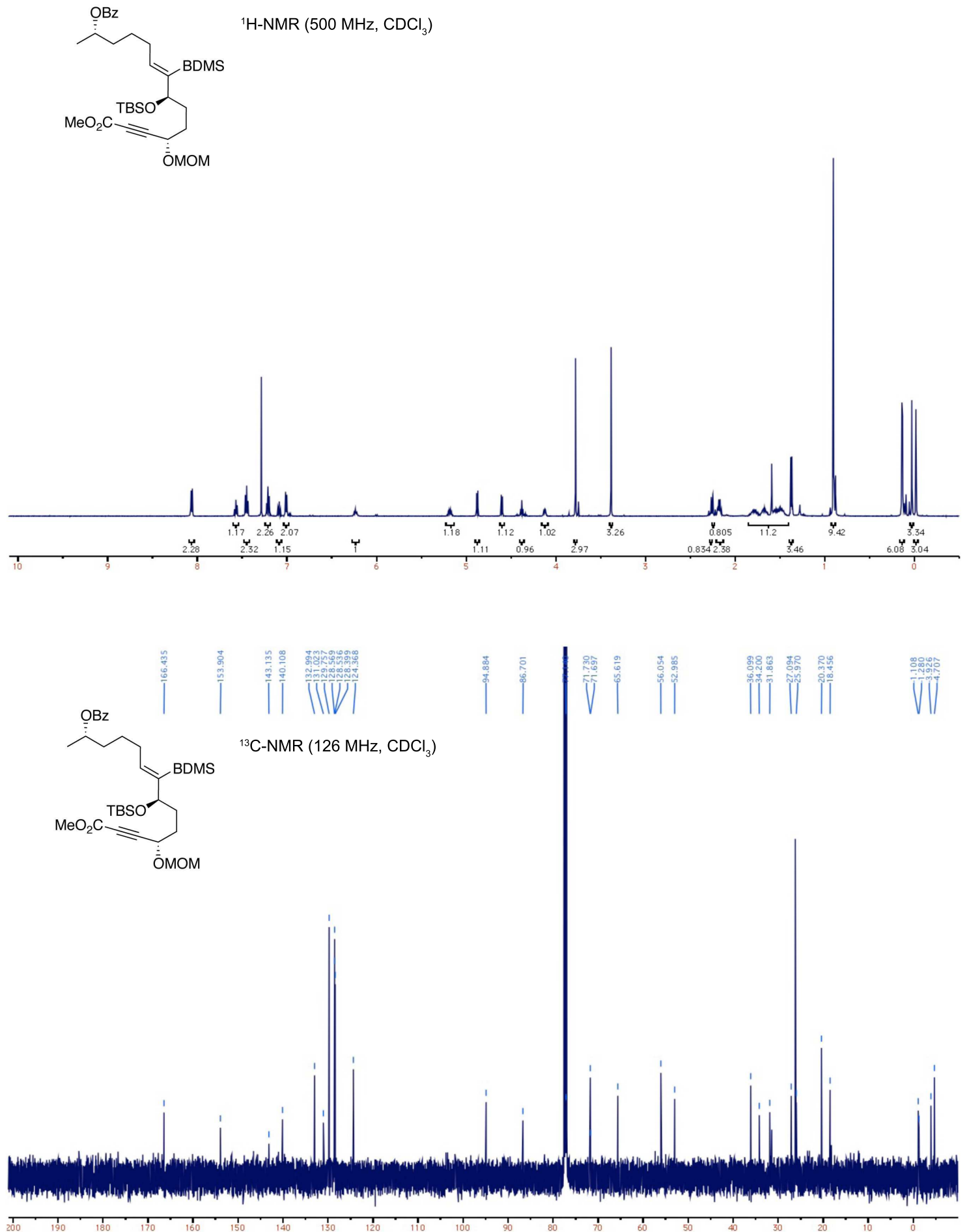


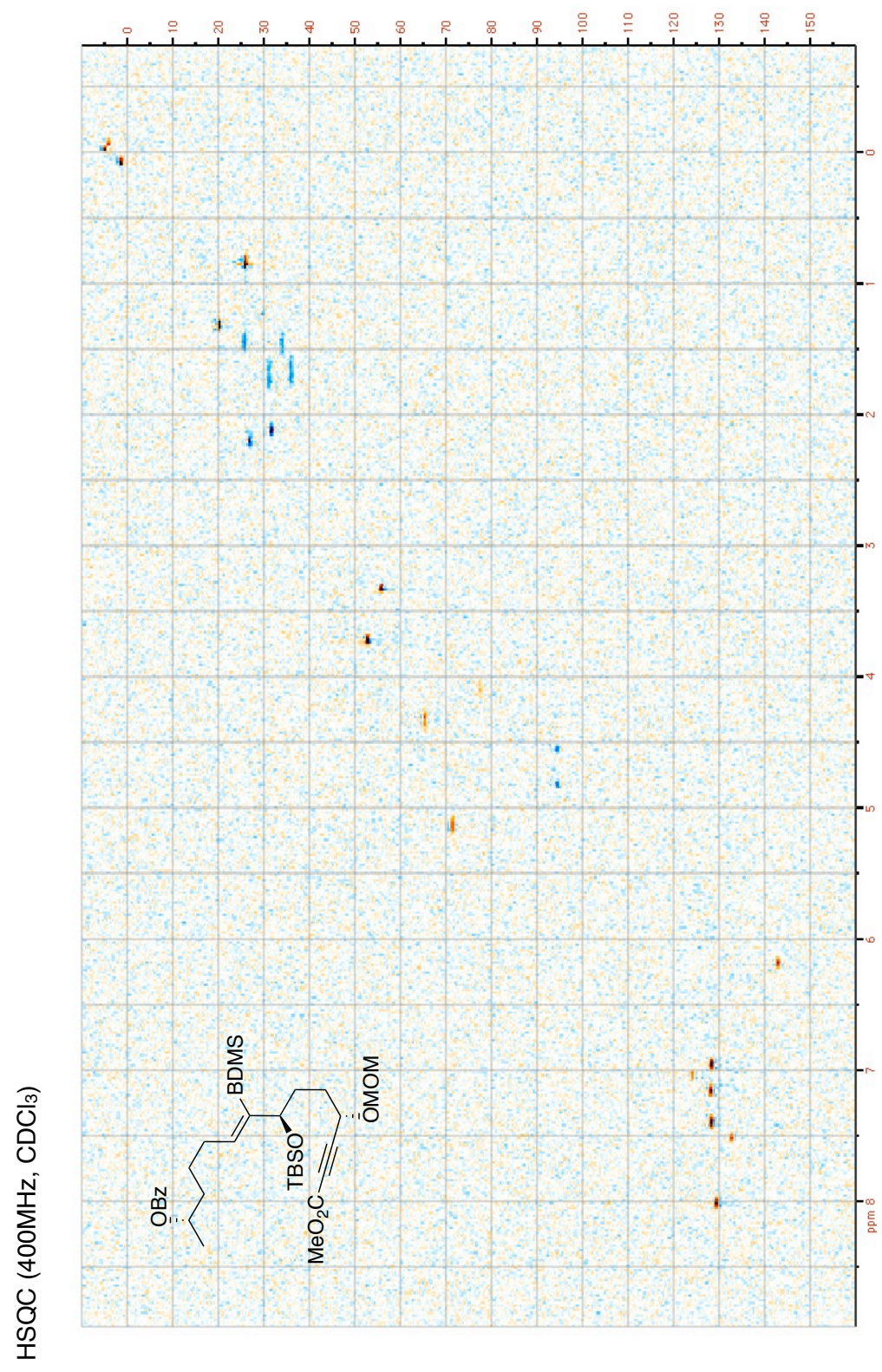



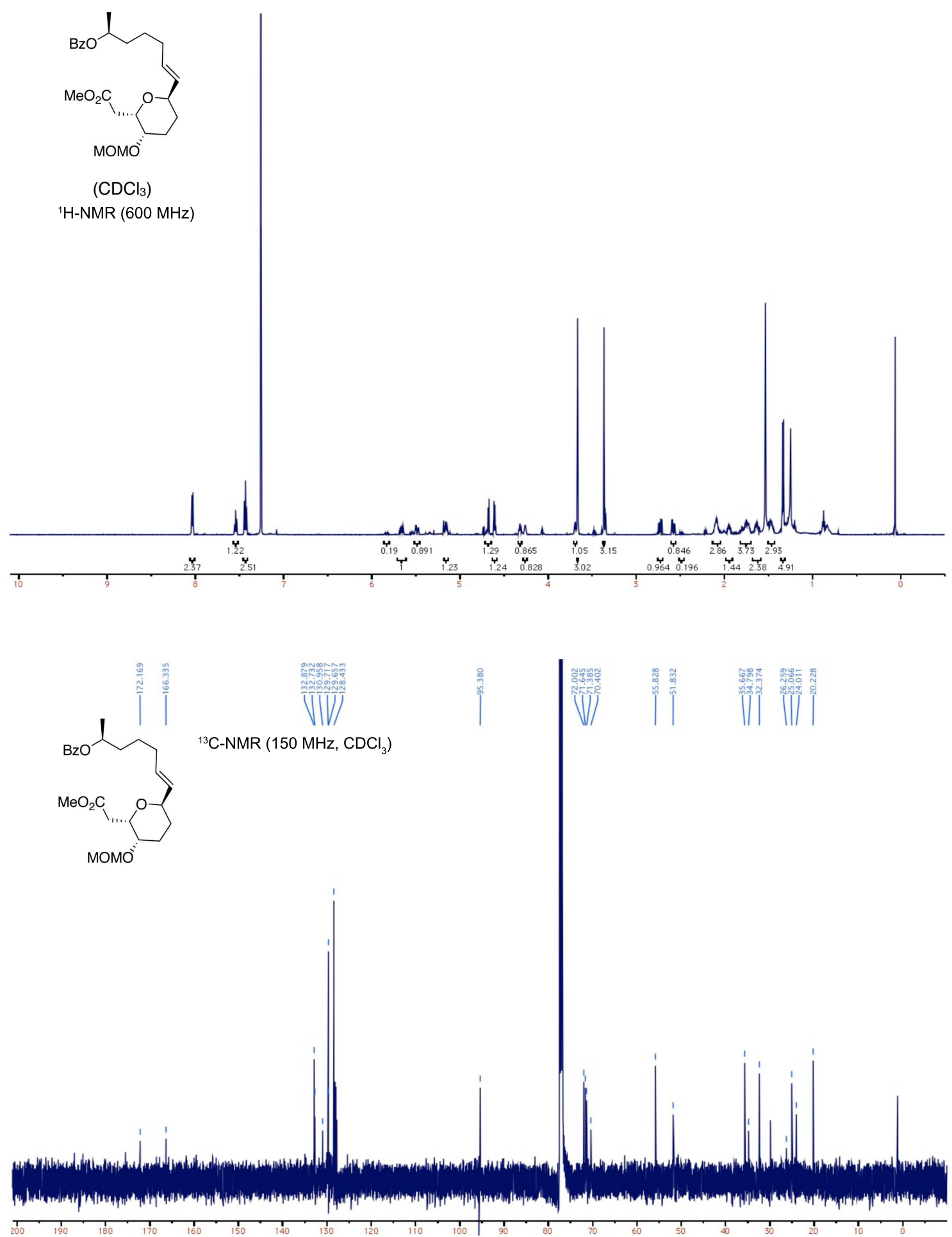


\section{References for Chapter 4}

[1] Kito, K.; Ookura, S. Y.; Namikoshi, M.; Ooi, T.; Kusumi, T. Org. Lett. 2008, 10, 225.

[2] Hande, S. M.; Uenishi, J. Tetrahedron Lett. 2009, 50, 189.

[3] Ookura, R.; Kito, K.; Saito, Y.; Kusumi, T.; Ooi, T. Chem. Lett. 2009, 38, 384.

[4] Díaz-Oltra, S.; Angulo-Pachón, C. A.; Murga, J.; Falomir, E.; Carda, M.; Marco, J. A. Chem. Eur. J. 2011, 17,675 .

[5] Doğan, A. L.; Doğan, A.; Canpinar, H.; Düzgünçinar, Ö.; Demirpençe, E. Chemotherapy 2004, 50, 283.

[6] For previous syntheses of aspergillide B, see: Nagasawa, T.; Kuwahara, S. Biosci. Biotechnol. Biochem. 2009, 73, 1893. Mueller Hendrix, A. J.; Jennings, M. P. Tetrahedron Lett. 2010, 51, 4260.

[7] For previous syntheses of aspergillides A and C, see: Nagasawa, T.; Kuwahara, S. Org. Lett. 2009, $11,761$. Panarese, J. D.; Waters, S. P. Org. Lett. 2009, 11, 5086. Sabitha, G.; Reddy, D. V.; Rao, A. S.; Yadav, J. S. Tetrahedron Lett. 2010, 51, 4195. Kobayashi, H.; Kanematsu, M.; Yoshida, M.; Shishido, K. Chem. Commun. 2011, 47, 7440. Kanematsu, M.; Yoshida, M.; Shishido, K. Tetrahedron Lett. 2011, 52, 1372. Izuchi, Y.; Kanomata, N.; Koshino, H.; Hongo, Y.; Nakata, T.; Takahashi, S. Tetrahedron: Asymmetry 2011, 22, 246. Nagasawa, T.; Nukada, T.; Kuwahara, S. Tetrahedron 2011, 67, 2882. Zúniga, A.; Pérez, M.; Gonález, M.; Gómez, G.; Fall, Y. Synthesis 2011, 20, 3301. Srihari, P.; Sridhar, Y. Eur. J. Org. Chem. 2011, 6690 .

[8] Díaz-Oltra, S.; Angulo-Pachón, C. A.; Murga, J.; Carda, M.; Marco, J. A. J. Org. Chem. 2010, 75, 1775.

[9] Kanematsu, M.; Yoshida, M.; Shishido, K. Angew. Chem. Int. Ed. 2011, 50, 2618. Kanematsu, M.; Yoshido, M.; Shishido, K. Tetrahedron Lett. 2011, 52, 1372

[10] Díaz-Oltra, S.; Angulo-Pachón, C. A.; Kneeteman, M. N.; Murga, J.; Carda, M.; Marco, J. A. Tetrahedron Lett. 2009, 50, 3783 .

[11] Fuwa, H.; Yamaguchi, H.; Sasaki, M. Org. Lett. 2010, 12, 1848. Fuwa, H.; Yamaguchi, H.; Sasaki, M. Tetrahedron 2010, 66, 7492.

[12] Chatterjee, A. K.; Choi, T.-L.; Sanders, D. P.; Grubbs, R. H. J. Am. Chem. Soc. 2003, 125, 11360.

[13] Liu, J.; Xu, K.; He, J.; Zhang, L.; Pan, X.; She, X. J. Org. Chem. 2009, 74, 5063.

[14] ( \pm )-9 was prepared in 4 steps from 3-methyl cyclohexenone. See Section 4.4 for experimental details. Le Drain, C.; Greene, A. E. J. Am. Chem. Soc. 1982, 104, 5473.

[15] The cross aldol side reaction produces a complex mixture of oligomers. The aldol side product A was isolated in $19 \%$ yield as a mixture of diastereomers from Entry 1 (Table 1$)$. HRMS-ESI $(m / z):[\mathrm{M}+\mathrm{H}]^{+}$ calculated for $\mathrm{C}_{34} \mathrm{H}_{61} \mathrm{O}_{6} \mathrm{Si}_{2}, 621.4001$; found, 621.3996 .

[16] Parenty, A.; Campagne, J.-M.; Aroulanda, C.; Lesot, P. Org. Lett. 2002, 4, 1663.

[17] Schlatter, A.; Kundu, M. K.; Woggon, W.-D. Angew. Chem. Int. Ed. 2004, 116, 6899. Midland, M. M.; Kazubski, A. J. Org. Chem. 1982, 47, 2496.

[18] For selected examples of catalytic enantioselective ynone reduction, see: Helal, C. J.; Magriotis, P. A.; Corey, E. J. J. Am. Chem. Soc. 1996, 118, 10938. Parker, K. A.; Ledeboer, M. W. J. Org. Chem. 1996, 61,3214

[19] Matsumura, K.; Hashiguchi, S.; Ikariya, T.; Noyori, R. J. Am. Chem. Soc. 1997, 119, 8738.

[20] Parenty, A.; Campagne, J.-M.; Aroulanda, C.; Lesot, P. Org. Lett. 2002, 4, 1663. Pétry, N.; Parenty, A.; Campagne, J.-M. Tetrahedron: Asymmetry 2004, 15, 1199. Oliveira, R. A.; Oliveira, J. M.; Rahmeier, L. H. S.; Comasseto, J. V.; Marino, J. P.; Menezes, P. H. Tetrahedron Lett. 2008, 49, 5759.

[21] Brown, C. A.; Yamashita, A. J. Am. Chem. Soc. 1975, 97, 891. Li, M.; O'Doherty, G. A. Org. Lett. 2006, 8,6087 . 
[22] Abrams, S. R.; Shaw, A. C. Org. Synth. 1993, 8, 146.

[23] Camps, F.; Coll, J.; Guitart, J.; Tetrahedron 1986, 16, 4603.

[24] Yang, J. W.; Fonseca, M. T. H.; List, B. Angew. Chem. Int. Ed 2004, 43, 6660.

[25] Baker, A. B.; Boskovic, Z. V.; Lipshutz, B. H. Org. Lett., 2008, 10, 289.

[26] Fumaraldehyde dimethyl acetal was prepared in one step from commercially available fumaraldehyde bis(dimethyl acetal). Coppola, G. M. Synthesis 1984, 1021.

[27] Trost, B. M.; Ball, Z. T.; Jöge, T. Angew. Chem. Int. Ed. 2003, 115, 3537. Trost, B. M.; Ball, Z. T. J. Am. Chem. Soc. 2005, 127, 17644.

[28] Young, J. F.; Osborn, J. A.; Jardine, F. H.; Wilkinson, G. Chem. Commun. 1965, 131. O'Connor, C.; Wilkinson, G. J. Chem. Soc. A 1968, 2665.

[29] The diastereoselectivity of the second alkyne addition was determined by ${ }^{1} \mathrm{H}$ NMR analysis of the cyclized compound 190.

[30] Trost, B. M.; Ball, Z. T.; Jöge, T. J. Am. Chem. Soc. 2002, 124, 7922.

[31] Trost, B. M.; Bartlett, M. J. Org. Lett. 2012, 14, 1322.

[32] Romanov, S. G.; Ivanov, I. V.; Shevchenko, V. P.; Nagaev, I. Y.; Pushkov, A. A.; Myasoedov, N. F.; Myagkova, G. I.; Kuhn, H. Chem. Phys. Lipids, 2004, 130, 117.

[33] Guéret, S. M.; O’Connor, P. D.; Brimble, M. A. Org. Lett. 2009, 11, 963.

[34] Taillier, C.; Gille, B.; Bellosta, V.; Cossy, J. J. Org. Chem. 2005, 70, 2097

[35] Ramachandran, P. V.; Liu, H.; Reddy, M. V. R.; Brown, H. C. Org. Lett. 2003, 5, 3755.

[36] Nielsen, L.; Lindsay, K. B.; Faber, J.; Nielsen, N. C.; Skrydstrup, T. J. Org. Chem. 2007, $72,10035$.

[37] Marshall, J. A.; Garofalo, A. W. J. Org. Chem. 1996, 61, 8732.

[38] Raap, J.; Nieuwenhuis, S.; Creemers, A.; Hexspoor, S.; Kragl, U.; Lugtenburg, J. Eur. J. Org. Chem. 1999, 2609. 
Chapter 5

Pd-Catalyzed Allylic Alkylation:

First and Second Generation

Approaches to the Synthesis of

Labillarides E-H 


\subsection{Labillarides E-H: Target Driven Reaction Development}

\subsubsection{Isolation and Structure of Labillarides E-H}

The labillarides are a family of recently discovered oxylipin natural products isolated from the New Zealand red algae, Phacelocarpus labillardieri (Figure 5.1). ${ }^{1}$ Labillarides E-H (218) are particulary interesting as these four compounds display virtually identical ${ }^{1} \mathrm{H}$ and ${ }^{13} \mathrm{C}$ NMR spectra. Analysis of the ${ }^{3} J_{\mathrm{H} 6-\mathrm{H} 7}$ coupling constant and NOE correlation between $\mathrm{H} 6$ and $\mathrm{H} 8$ provides strong evidence that all four compounds have a trans-relationship between substituents on the furan ring. This conclusion suggests that the structures of labillarides E-H differ with respect to the configuration of the C3 and C8 hydroxyl groups. The use of benzene- $d_{6}$ to generate solvent-induced shifts of proton resonances resulted in the tentative assignment of labillaride $\mathrm{F}$ as 3,8-bis-epi-labillaride $\mathrm{E}$, labillaride $\mathrm{H}$ as 3-epi-labillaride $\mathrm{E}$, and labillaride $\mathrm{G}$ as 8-epi-labillaride $\mathrm{E}$. The discovery of diastereomeric natural products that differ

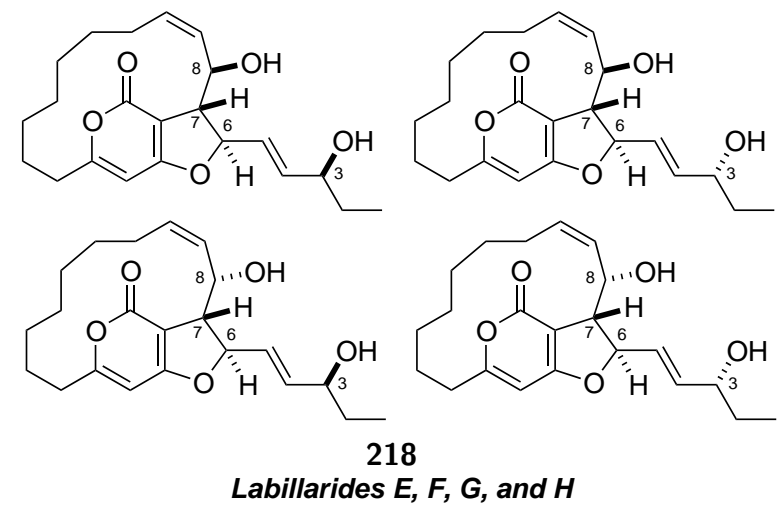

Figure 5.1 Natural Products from Phacelocarpus labillardieri: Labillarides E-H

with respect to the configuration of pairs of adjacent stereocenters but still exhibit virtually the same ${ }^{1} \mathrm{H}$ and ${ }^{13} \mathrm{C}$ NMR spectra is both highly unusual and very interesting. This is especially true when one considers that the use of NMR databases to assign 1,2- and 1,3stereochemical relationships depends on each stereochemical permutation having distinctly different ${ }^{1} \mathrm{H}$ and ${ }^{13} \mathrm{C}$ NMR shifts. ${ }^{2}$ The NMR database approach, which was pioneered by Kishi, has been successfully applied to a variety of acyclic natural product fragments, including oasomycin A, shown in Figure 5.2. ${ }^{3}$ This example highlights the typically significant differences in ${ }^{13} \mathrm{C}$ chemical shift observed between diastereomers varying at three adjacent stereocenters. The unusual spectroscopic properties of labillarides E-H are likely derived from a highly interesting macrocyclic structure. A structurally similar $\alpha$-pyrone macrolide, neurymenolide A (Figure 5.3), exists as two atropisomers due to a relatively high barrier to rotation about the $\alpha$-pyrone ring system, however the slightly larger macrocycle of neurymenolide B does not exhibit atropisomerism. ${ }^{4}$ Given the atropisomerism exhibited by neurymenolide $\mathrm{A}$, the 15-membered macrocyclic ring of labillarides E-H may also suffer from hindered rotation 

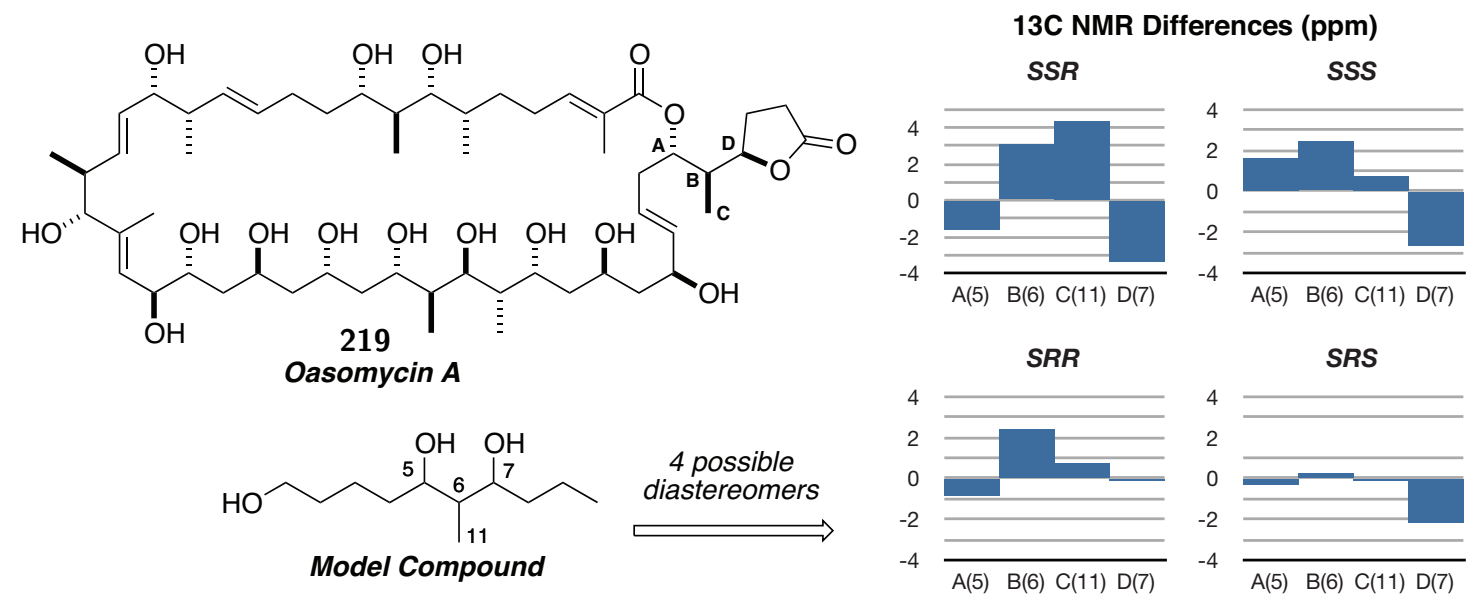

Figure 5.2 NMR Database Approach to the Structural Assignment of Oasomycin A: Typical ${ }^{13} \mathrm{C}$ NMR Differences Between Diastereomeric Compounds.

about the $\alpha$-pyrone ring system. However, the challenging task of confirming or refuting atropisomerism would likely require a scalable total synthesis of these natural products. A successful enantioselective synthesis of labillarides E-H would also provide the opportunity to deduce the absolute configuration of these compounds.

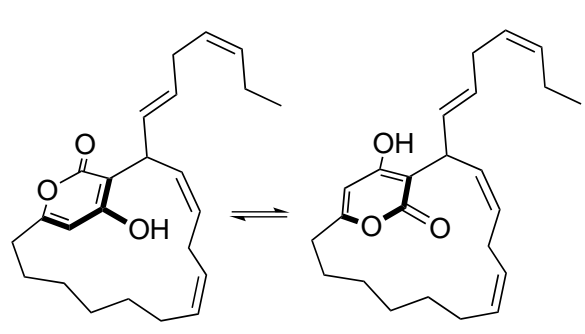

221

Neurymenolide $A$ exists as two atropisomers (16-membered macrocycle)

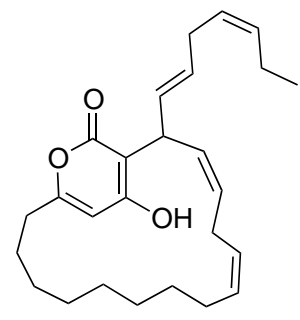

222

Neurymenolide B

not atropisomeric

(18-membered macrocycle)

Figure 5.3 Atropisomerism in the Neurymenolide Natural Products.

Biological testing of the original labillaride E (218) sample repeatedly showed potent cytotoxicity ( ca. $600 \mathrm{nM}$ ) against human leukaemia cells (HL-60). However, subsequent repurification by HPLC resulted in an inactive sample. Presumably, a very minor impurity was responsible for the potent cytotoxicity and it is believed that further synthetic research into labillarides E-H may provide additional clues to the identity of this highly potent compound.

\subsubsection{Strategy and Major Disconnections}

With the aim of designing a highly efficient and concise synthesis of labillarides E-H, we chose to investigate retrosynthetic disconnections that remove a large amount of structural complexity. Structural complexity can be defined by six general categories: ${ }^{5}$ 
- molecular size

- functional group content

- highly reactive centers
- cyclic connectivity

- stereochemistry

- centers of kinetic instability

These criteria led to the identification of three major structural features, which arguably represent the most challenging aspects of constructing labillarides E-H (Scheme 5.1). Namely, the 15-membered macrocyclic ring $(\mathbf{A})$, the bicyclic furanopyrone core $(\mathbf{B})$ and the four stereogenic centers $(\mathbf{C})$. Analysis of the common red areas of structures $\mathbf{A}, \mathbf{B}$ and $\mathbf{C}$ from

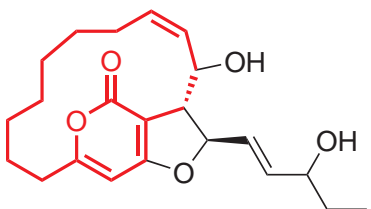

A

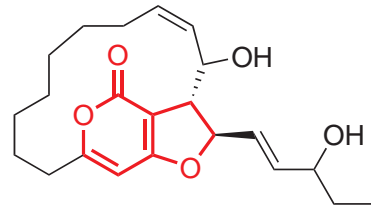

B

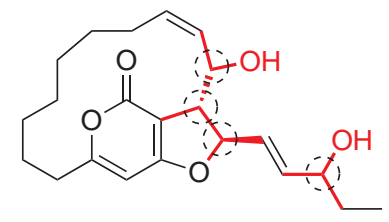

C

Scheme 5.1 Challenging Structural Elements of Labillarides E-H.

Scheme 5.1 reveals six bonds (shown in red) that would dramatically simplify the molecule. In each case bond cleavage would remove a stereocenter and break some form of cyclic connectivity (Scheme 5.2). Of the six possible disconnections identified, the three shown in boxes lead to synthons with attractive synthetic equivalents: synthon $\mathbf{2 2 3}$ represents the addition of a vinyl anion equivalent to an aldehyde, synthon $\mathbf{2 2 4}$ represents the displacement of a leaving group with a nucleophilic enolate equivalent, and synthon $\mathbf{2 2 5}$ represents the attack of an oxyanion on an allylic cation. These reactive modes can be achieved using a number of different synthetic methodologies and are good choices as preliminary disconnections. Disconnections II and III

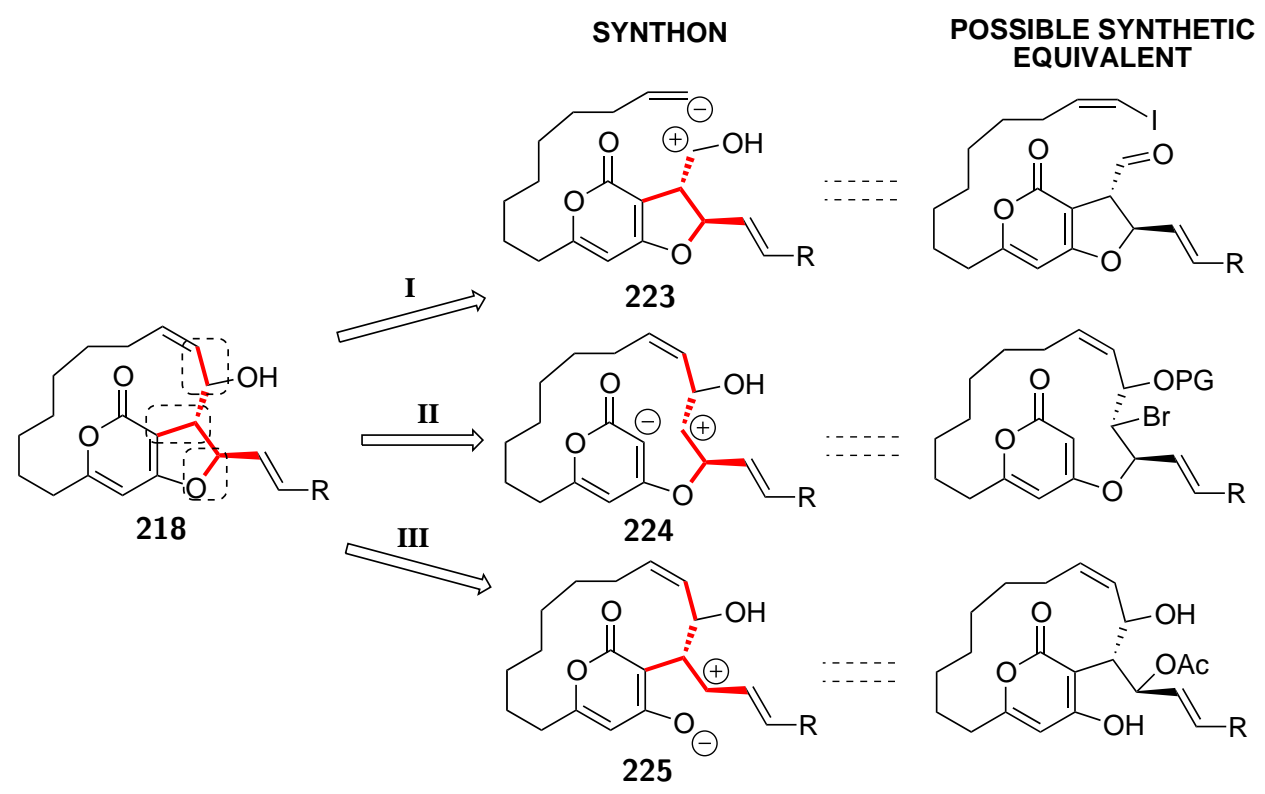

Scheme 5.2 Disconnection of Common Bonds Shared by A, B and C.

can be translated into the inter- and intramolecular palladium-catalyzed allylic alkylations (Pd-AA) shown in Scheme 5.3. These palladium-catalyzed reactions are key transformations 
in the first and second generation approaches to labillarides E-H, respectively, and would provide a highly efficient method of utilizing a three-carbon allyl linchpin in the construction of labillarides E-H.

First Generation Synthetic Strategy<smiles>[R]C=CC([R])c1c(O)cc(C)oc1=O</smiles><smiles>[R]C1c2c(cc(C)oc2=O)OC1/C=C/[3H]</smiles>

Second Generation Synthetic Strategy<smiles>[R]C(/C=C\C(=O)O)c1c([O-])cc(C)oc1=O</smiles><smiles>[R]C1c2c(cc(C)oc2=O)OC1C=C</smiles>

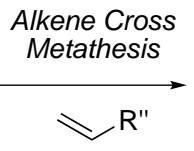<smiles>[R]C1c2c(cc(C)oc2=O)OC1/C=C/[P]</smiles>

Scheme 5.3 First and Second Generation Pd-AA Linchpin Strategies.

\subsection{Palladium-Catalyzed Allylic Alkylations}

\subsubsection{Mechanistic Introduction}

Palladium is recognized as one of the most useful and versatile metals in organic synthesis. ${ }^{6}$ The facile interconversion between $\operatorname{Pd}(0)$ and $\operatorname{Pd}(\mathrm{II})$ oxidation states has allowed palladium complexes to be used as catalysts for a variety of different reactions. ${ }^{7}$ The use of $\pi$-allyl palladium complexes as electrophiles in alkylation reactions was first reported by Jiro Tsuji in 1965 (Scheme 5.4). ${ }^{8}$ This methodology quickly evolved into the catalytic asymmetric methodology known today as the Tsuji-Trost reaction (see Section 1.3.2). ${ }^{9}$

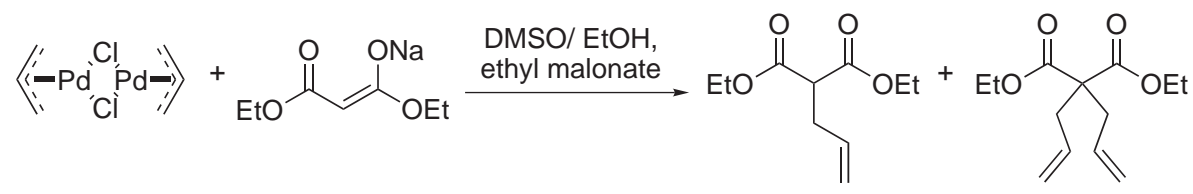

Scheme 5.4 Seminal Pd-AA Reported by Tsuji.

Some of the most interesting and unique aspects of Pd-AA reactions are the many mechanistic intricacies of this process. Two distinct mechanisms have been proposed for this reaction depending on whether hard or soft nucleophiles are used (Scheme 5.5). Soft nucleophiles, derived from conjugate acids with a $p \mathrm{~K}_{a}$ of less than 25 , add to the electrophilic carbon directly, resulting in a double inversion (net retention) of configuration. Examples of soft nucleophiles include: sodium dimethyl malonate, morpholide and sodium cyclopentadienide. ${ }^{10}$ Hard nucleophiles on the other hand, attack the metal center and the resulting complex undergoes reductive elimination to form the alkylated product with an overall inversion of configuration. Examples of hard nucleophiles include: phenylzinc chloride, (E)-(2-methylhex-1-enyl)dimethylalane and ammonium formate. ${ }^{11}$ However, stereospecificity is not always guaranteed, and varying degrees of chirality transfer have been observed in 


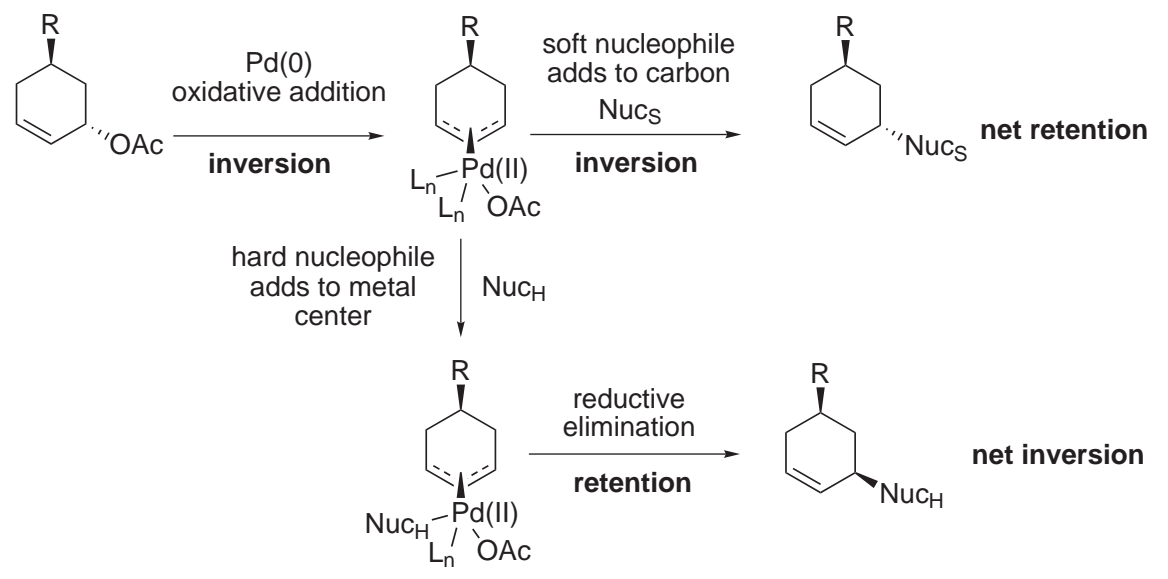

Scheme 5.5 Pd-AA Mechanism with Soft and Hard Nucleophiles.

certain cases (Scheme 5.6). The racemization of $\pi$-allyl palladium intermediates has been proposed to occur via two main mechanisms: attack of a $\operatorname{Pd}(0)$ species on the $\pi$-allyl palladium complex, and racemization of the starting material through cis-attack of the departed acetate anion via coordination with palladium. These two problems have be overcome by keeping the concentration of $\mathrm{Pd}(0)$ low (using low catalyst loading or high dilution) and through the use of a carbonate leaving group, respectively. The intramolecular Pd-AA reaction shown in Scheme 5.6 provides only $41 \%$ chirality transfer when $20 \mathrm{~mol} \% \mathrm{Pd}(0)$ is used. ${ }^{12}$ However, reducing the catalyst loading to $3 \mathrm{~mol} \%$ provides a dramatic increase in stereospecificity, delivering the desired product with $78 \%$ chirality transfer. Thus, a significant increase in chirality transfer was observed when the concentration of $\operatorname{Pd}(0)$ is reduced. Ultimately, the use of $5 \mathrm{~mol}$ $\% \mathrm{Pd}_{2}(\mathrm{dba})_{3} \cdot \mathrm{CHCl}_{3}$, THF and $\mathrm{NaH}$ was found to provide near perfect chirality transfer. Additionally, palladium-catalyzed allylic alkylations can lose stereospecificity through $\pi-\sigma$ -

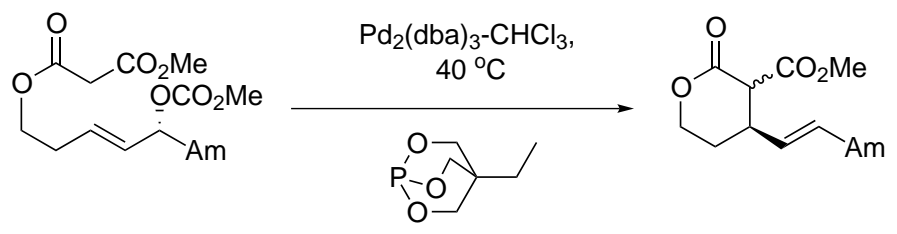

\begin{tabular}{ccccc}
\hline Entry & Pd(0) mol\% & Solvent/ Base & Yield & Chirality Transfer \\
\hline 1 & 20 & DMSO & $71 \%$ & $41 \%$ \\
2 & 10 & DMSO & $62 \%$ & $58 \%$ \\
3 & 3 & DMSO & $88 \%$ & $78 \%$ \\
4 & 10 & THF $/ \mathrm{NaH}$ & $53 \%$ & $96 \%$ \\
\hline
\end{tabular}

Scheme 5.6 Chirality Transfer in Pd-AA Reactions.

$\pi$ isomerization of the intermediate $\mathrm{Pd}(\mathrm{II})$ complex (Scheme 5.7). Thus, $\pi$-allyl-Pd complexes with identical substituents on one end quickly lose stereochemical information via reversible formation of the achiral $\sigma$-Pd complex 229. This process is also responsible for the $Z$-to- $E$ alkene isomerization observed during alkylations of $Z$-allyl acetates and $Z$-allyl carbonates. In 
this case, the initially formed syn-Pd complex $\mathbf{2 3 0}$ often converts to the thermodynamically favored anti-Pd complex 231 via $\pi-\sigma-\pi$ isomerization and $\sigma$-bond rotation.

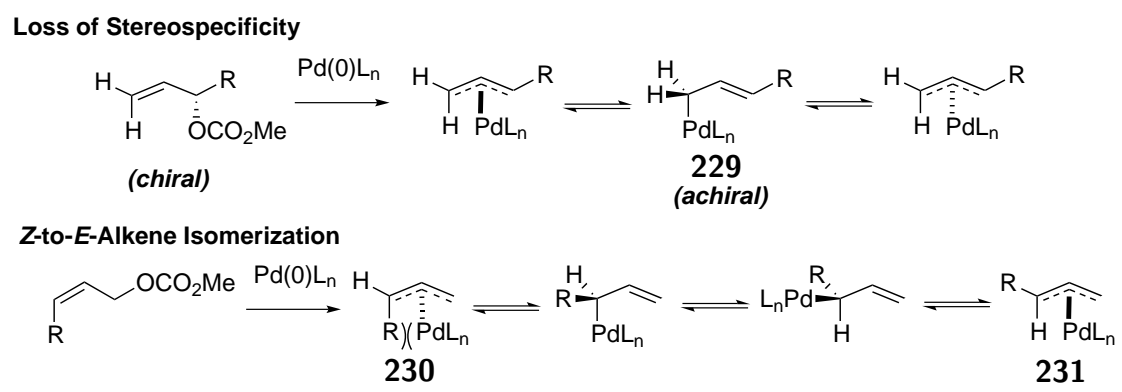

Scheme $5.7 \pi-\sigma-\pi$ Isomerization Mechanisms.

Regioselectivity in Pd-AA reactions can be driven by the steric or electronic properties of: the electrophile, the nucleophile, the palladium catalyst, or combinations of the aforementioned possibilities. Additionally, simple changes in the reaction conditions (solvents, additives etc.) have been shown to enable the complete reversal of regioselectivity (Scheme 5.8). ${ }^{13}$ Analysis of the steric and electronic properties associated with the $\pi$-allyl-Pd intermediate

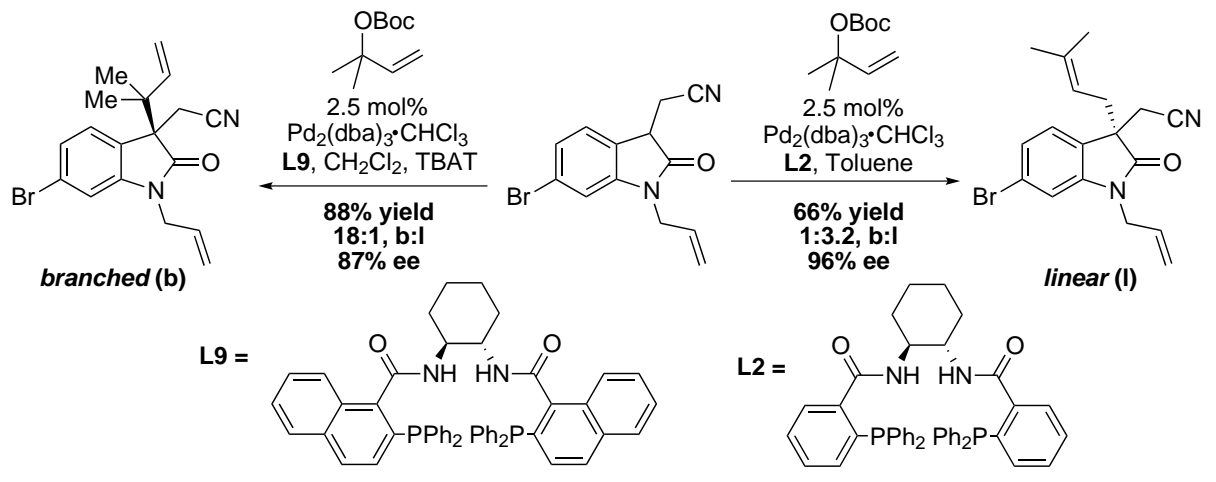

Scheme 5.8 Regiocontrol in Pd-AA using Different Reaction Conditions.

provides, arguably, the most reliable means of predicting and understanding the regiochemical outcome of these reactions. ${ }^{14}$ In many cases, ionization of an allyl acetate or carbonate provides a 'symmetric' $\eta^{3}$ complex, where the metal resides symmetrically over the allyl ligand (Scheme 5.9). ${ }^{14}$ This situation typically favors nucleophilic addition to the least hindered carbon, and therefore the 1,4-substituted regioisomer 232, shown in Scheme 5.9, is heavily favored over the 1,2-regioisomer 233. However, the formation of a 'distorted' $\pi$-allyl-Pd complex, that leads to very different regiochemical outcomes, has been proposed in situations where unfavorable steric interactions between palladium and neigbouring substituents are likely (i.e. 234 and 235). These 'distorted' $\pi$-allyl complexes can be thought of as a $\sigma, \eta^{2}$ species that undergo nucleophilic addition in an $\mathrm{S}_{\mathrm{N}} 2$ '-like manner, giving rise to the 1,2-regioisomer as the major product. Thus, the allyl benzoates $\mathbf{2 3 4}$ and $\mathbf{2 3 5}$ provided the 1,2-substituted regioisomer as the major product, in a regioisomeric ratio of 4:1 (Scheme 5.9). 


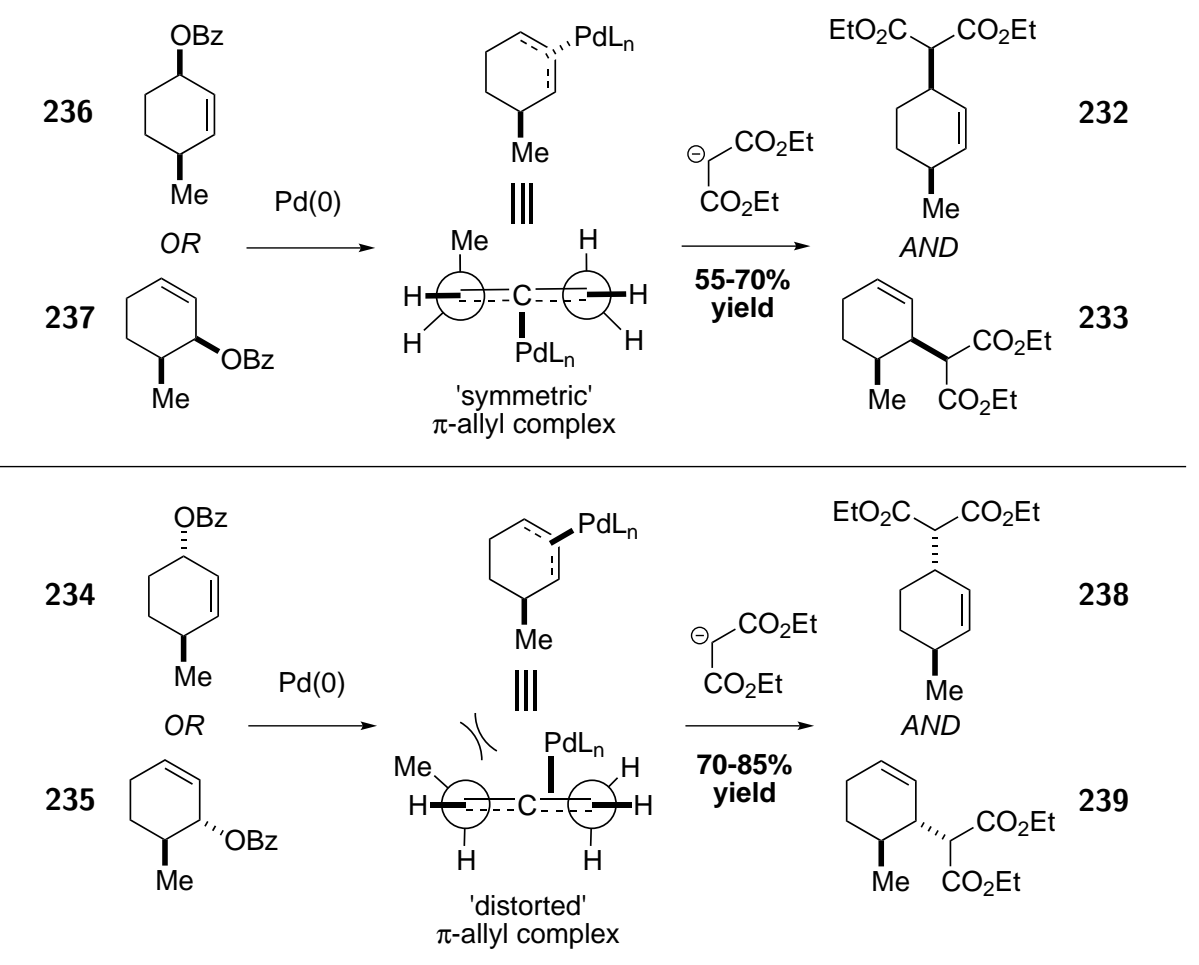

\begin{tabular}{|c|c|c|c|}
\hline \multirow{2}{*}{ Entry } & \multirow{2}{*}{ Starting Material } & \multicolumn{2}{|c|}{ Regioisomeric Ratio } \\
\hline & & 1,4-Regioisomer & 1,2-Regioisomer \\
\hline \multicolumn{4}{|c|}{ 'symmetric' $\pi$-allyl complex } \\
\hline 1 & 236 & $14(\mathbf{2 3 2})$ & $1(233)$ \\
\hline 2 & 237 & $20(\mathbf{2 3 2})$ & $1(233)$ \\
\hline \multicolumn{4}{|c|}{ 'distorted' $\pi$-allyl complex } \\
\hline 3 & 234 & $1(238)$ & $4(\mathbf{2 3 9})$ \\
\hline 4 & 235 & $1(238)$ & $4(239)$ \\
\hline
\end{tabular}

Scheme 5.9 Regioselectivity and Proposed 'Distorted' $\pi$-Allyl-Pd Complexes. 
Palladium-catalyzed allylic alkylations are extremely versatile and frequently used in natural product synthesis. ${ }^{9}$ Successful reactions have been reported with a variety of nucleophiles and $\pi$-allyl-Pd precursors. These nucleophiles include: enolates, alkoxides, carboxylates, nitronates, thiolates and amines. The different types of $\pi$-allyl-Pd precursors include: allylic acetates, allylic carbonates, vinyl epoxides, benzyl phosphonates, and propargylic acetates. Scheme 5.10 provides a small sample of what can be achieved in natural product synthesis using this reaction.

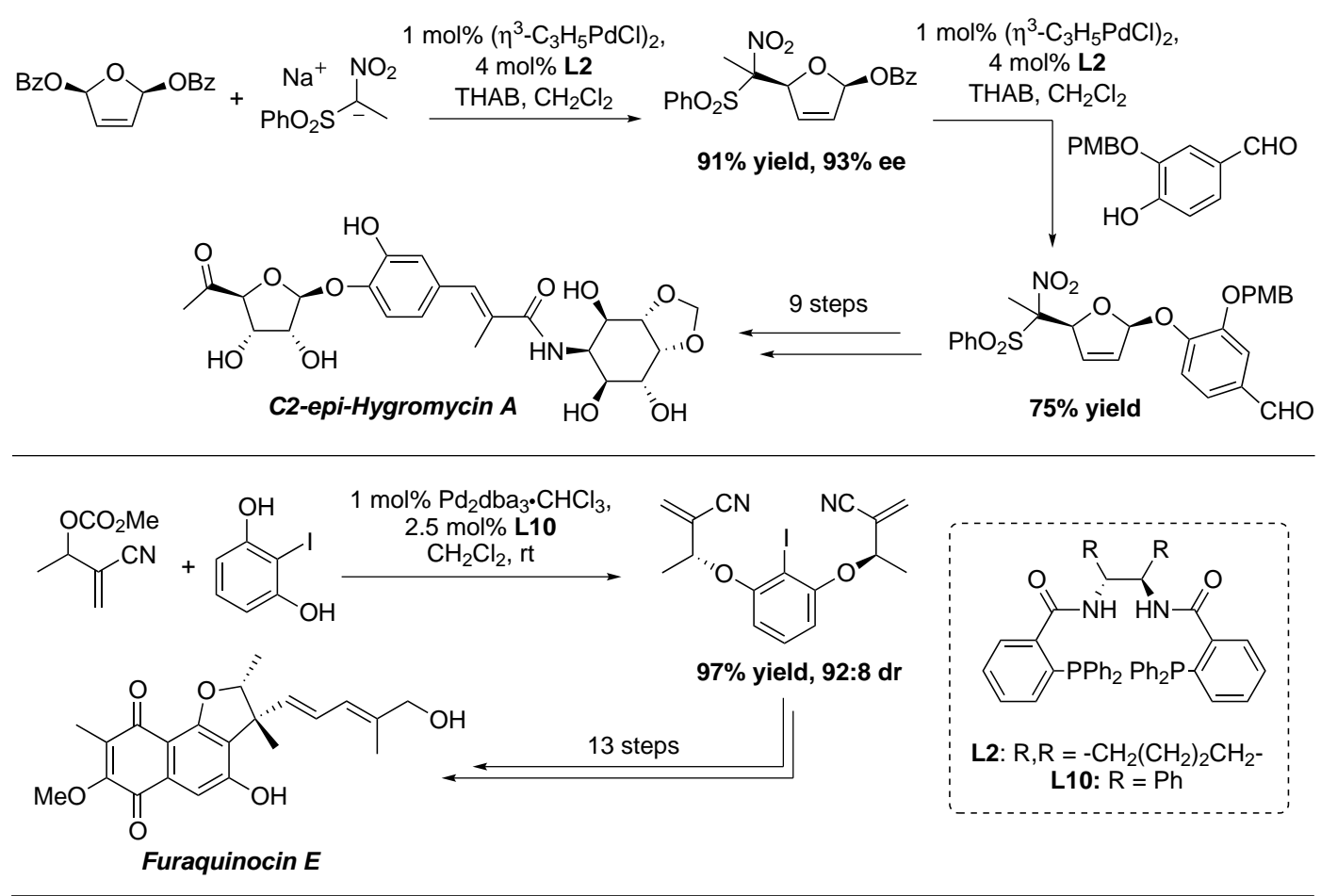

Scheme 5.10 Selected Pd-AA Examples in Natural Product Synthesis.

\subsubsection{Pd-AA with 4-Hydroxy- $\alpha$-Pyrone Nucleophiles}

The proposed synthesis of labillarides E-H aims to use a Pd-AA reaction to exploit the inherent nucleophilicity of a 4-hydroxy- $\alpha$-pyrone. Highly acidic 1,3-dicarbonyl compounds such as 4hydroxy- $\alpha$-pyrones $\left[p \mathrm{~K}_{\mathrm{a}}\right.$ (6-methyl-4-hydroxy- $\alpha$-pyrone $)=4.83\left(\mathrm{H}_{2} \mathrm{O}\right), 6.83(80 \% w / w \mathrm{DMSO}-$ $\left.\left.\mathrm{H}_{2} \mathrm{O}\right)\right]$ and tetronic acids, often exist primarily as the enol tautomer and can be difficult to selectively $C$-alkylate without undesired $O$-alkylation. However, in the presence of $\operatorname{Pd}(0), O$ alkylation products have been shown to undergo ionization to form $\pi$-allyl palladium complexes and this suggests that the formation of these products in a Pd-AA reaction is reversible (Scheme 5.11). In contrast, the corresponding $C$-alkylation is irreversible under these reaction conditions, and thus, the product distribution is expected shift to the desired $C$-alkylation product over time. Moreno-Mañas and coworkers have shown that, in the presence of a $\operatorname{Pd}(0)$ catalyst, the allyl ether $\mathbf{2 4 0}$ reacts to give the $C$-alkylated product, $\mathbf{2 4 1}$ (Scheme 5.12). ${ }^{15}$ 


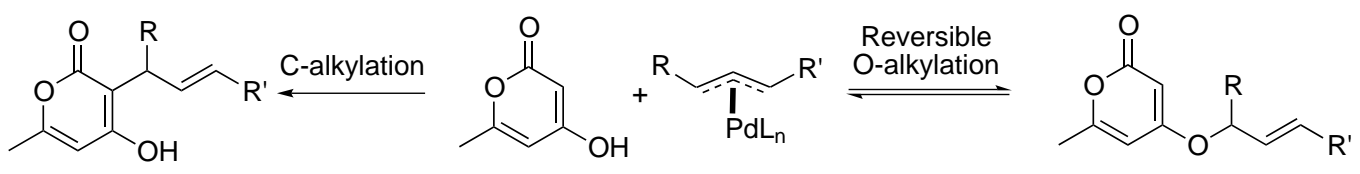

Scheme 5.11 Regioselectivity in Pd-AA with 4-Hydroxy- $\alpha$-pyrones.<smiles>CC=CCOc1cc(C)oc(=O)c1</smiles>

240

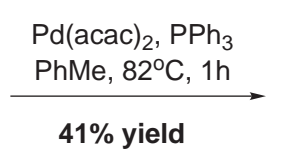

$41 \%$ yield<smiles>C/C=C/Cc1c(O)cc(C)oc1=O</smiles>

241

Scheme 5.12 Reversible $O$-Alkylation in the Presence of $\operatorname{Pd}(0)$.

Additionally, $O$-alkylation products can participate in a thermal Claisen rearrangements, which would result in formation of the alternative $C$-alkylation regioisomer. ${ }^{15} \mathrm{Pd}$-catalyzed allylic alkylations with 4-hydroxy- $\alpha$-pyrones are typically less facile than those with traditional $\beta$-dicarbonyl nucleophiles, and often require elevated temperatures. ${ }^{16}$ The relatively high activation barrier is likely to be a consequence of the temporary loss of aromaticity during the alkylation step. Additionally, the high energetic cost to breaking aromaticity disfavors the undesired bis- $C$-alkylation pathway, often prominent in Pd-AA reactions with Meldrum's acid and barbituric acid. ${ }^{19}$
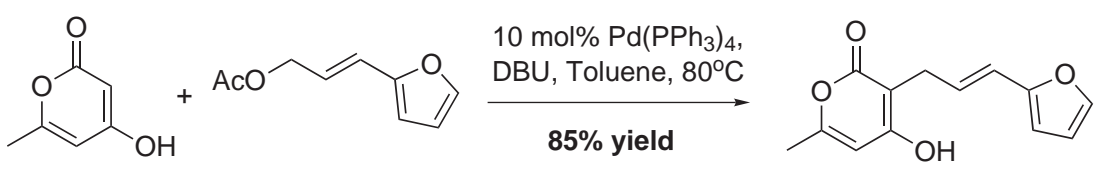<smiles>[R]C=CCOC(C)=[18O]</smiles>
$5 \mathrm{~mol} \% \mathrm{Pd}(\mathrm{acac})_{2}$ $20 \mathrm{~mol}^{2} \mathrm{PPh}_{3}$, $\stackrel{\mathrm{DBU}, \text { Toluene, } 70^{\circ} \mathrm{C}}{\longrightarrow}$

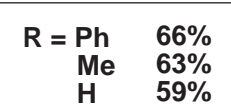<smiles>[R]C=CCc1c(O)cc(C)oc1=O</smiles>

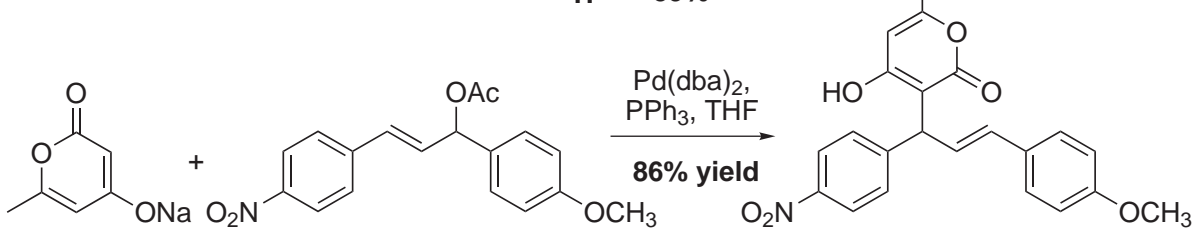

Scheme 5.13 Pd-AA Reactions with 4-Hydroxy- $\alpha$-pyrones. 


\subsection{First Generation Approach to Labillarides E-H}

The first generation approach to labillarides E-H centered around the use of a Pd-catalyzed allylic alkylation to selectively couple a 4 -hydroxy- $\alpha$-pyrone with an allylic carbonate linchpin 242 (Scheme 5.14). The functionality present in this 4-carbon linchpin was envisioned to enable formation of the macrocyclic ring and the embedded dihydrofuran, as well as provide a synthetic handle for installation of the side chain.

Retrosynthetic disconnection of labillarides E-H (218) began with removal of the side chain to enable late-stage stereochemical divergence. A Grignard addition to the optically active aldehyde $\mathbf{2 4 3}$ and Peterson olefination were envisioned, ${ }^{18}$ thus leading back to bromosilane 244. Compound $\mathbf{2 4 4}$ would, in turn, be formed through the intramolecular bromoetherification of vinyl silane 245. Formation of the 15-membered macrocyclic ring from $Z$ vinyl iodide $\mathbf{2 4 6}$ was envisioned using a Nozaki-Hiyama-Kishi reaction, after the necessary protecting group and redox manipulations. As mentioned previously, the key $\mathrm{C}-\mathrm{C}$ bond joining the heterocyclic nucleus would be forged using a stereospecific Pd-catalyzed allylic alkylation between allylic carbonate $\mathbf{2 4 2}$ and 4-hydroxy- $\alpha$-pyrone $\mathbf{2 4 7}$. The regioselectivity of this transformation would be controlled by the silyl group, which is known to direct nucleophilic addition to the distal end of the $\pi$-allyl-Pd complex. ${ }^{17}$ Lastly, formation of the $\alpha$-pyrone heterocycle was envisaged using the $\beta, \delta$-diketoester derived from addition of methyl acetoacetate (248) and carboxylic acid $\mathbf{2 4 9}$.

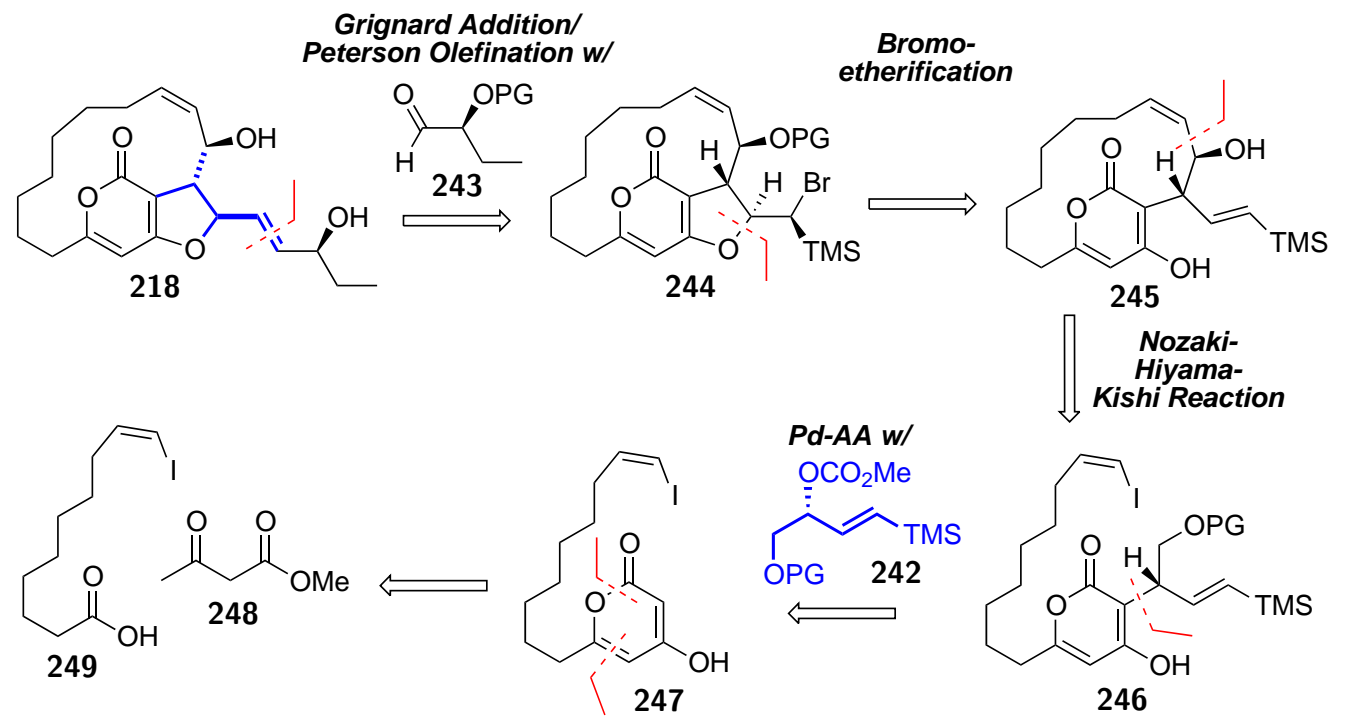

Scheme 5.14 Labillarides E-H First Generation Retrosynthesis.

Two different allylic carbonate substrates were used to evaluate this approach; one derived from benzaldehyde, the other from a protected $\alpha$-hydroxy aldehyde (Scheme 5.15). These Pd-AA substrates were prepared in three steps, commencing with the addition of TMSacetylene to the respective aldehydes to form propargylic alcohols 250a and 250b. A hydroxyldirected alkyne reduction, using Vitride, gave the desired $(E)$-vinyl silane selectively. Methyl 


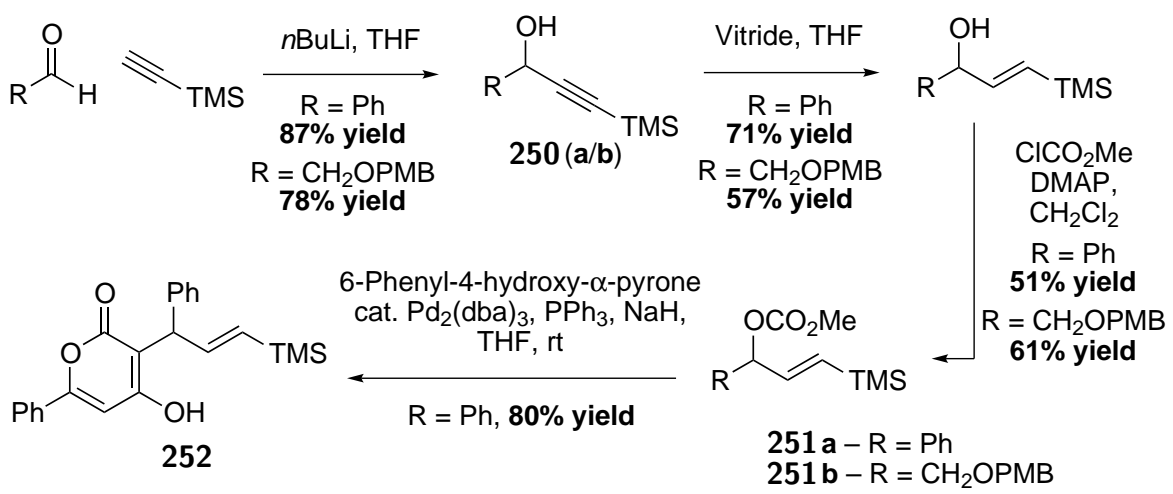

Scheme 5.15 Initial Pd-AA with 6-Methyl-4-hydroxy- $\alpha$-pyrone.

chloroformate was then used to convert the alcohol to a methyl carbonate and provide the key substrate for Pd-catalyzed allylic alkylation.

Pd-catalyzed allylic akylations with 4-hydroxy- $\alpha$-pyrones are typically less facile than those with traditional $\beta$-dicarbonyl nucleophiles, and often require elevated temperatures. ${ }^{16}$ Nonetheless, the model substrate 251a cleanly underwent allylic alkylation at room temperature to produce pyrone $\mathbf{2 5 2}$ selectively. Unfortunately the corresponding reaction on the real system, with allyl carbonate $\mathbf{2 5 1 b}$, resulted in no reaction under a variety of conditions (Scheme 5.16). The activating effect of the phenyl substituent in the ionization of the allyl carbonate appears critical for reactivity under these conditions. While the phenyl-substituted product 252 is unsuitable for the synthesis of the labillarides, it afforded an opportunity to evaluate the second key step in this sequence - bromo-etherification.

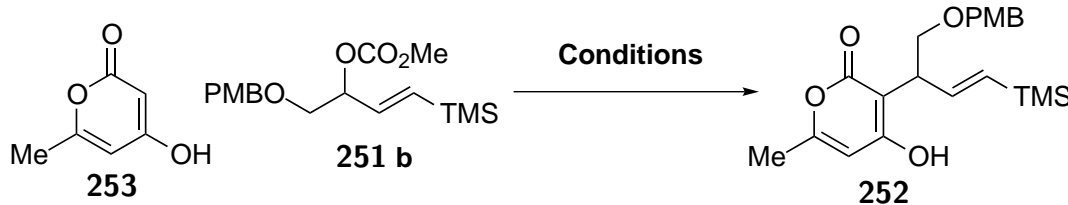

\begin{tabular}{ccc}
\hline Entry & Conditions & Results \\
\hline 1 & $\mathrm{Pd}_{2}\left(\mathrm{dba}_{3}, \mathrm{PPh}_{3}\right.$, THF, rt & Recovered SM \\
2 & $\mathrm{Pd}_{2}(\mathrm{dba})_{3}, \mathrm{PPh}_{3}$, NaOtBu , Toluene, $80{ }^{\circ} \mathrm{C}$ & Recovered SM \\
3 & $\mathrm{Pd}_{2}(\mathrm{dba})_{3}, \mathrm{PPh}_{3}$, dppe, DMSO, $120{ }^{\circ} \mathrm{C}$ & Recovered SM \\
\hline
\end{tabular}

Scheme 5.16 Attempted Pd-AA with Vinyl Silane 251b.

Cyclization of 252 using NBS provided the desired furopyrone in moderate yield as a 1.2:1 mixture of cis- and trans-substituted furan diastereomers $\mathbf{2 5 4}$ and 255 . The postulated mechanism for bromoetherification proceeds via a bromonium species, followed by attack of a nucleophile in a stereospecific $\mathrm{S}_{\mathrm{N}}$ 2-like fashion and therefore, the same relative stereochemical relationship between the $\mathrm{C} 2$ oxygen and $\mathrm{C} 1$ bromine atom is expected (Scheme 5.17 numbering). The relative stereochemistry of the two products obtained from the halo-cyclization reaction was assigned by analysis of the ${ }^{3} J_{\mathrm{HH}}$ coupling constants between the three contiguous stereocenters (Table 5.1). Using the Karplus equation to relate the 


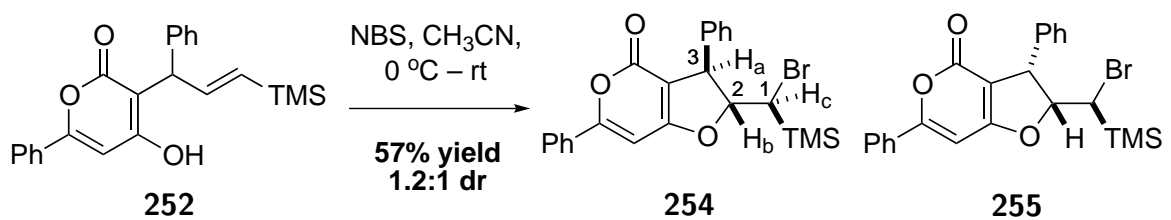

Scheme 5.17 Bromoetherification of 252 to Form a Furanopyrone.

observed ${ }^{3} J_{\mathrm{HH}}$ coupling constant to dihedral angle, a $4.1 \mathrm{~Hz}$ coupling corresponds to a $48^{\circ}$ dihedral angle and a $7.9 \mathrm{~Hz}$ coupling corresponds to a $9^{\circ}$ dihedral angle. ${ }^{20}$ This suggests that the major product is the trans-substituted furan 254. However, the configuration of five-membered rings can often be challenging to assign correctly, ${ }^{21}$ and therefore density functional calculations (B3LYP, 6-31G(d)) were performed to provide further evidence to support this assignment. ${ }^{22}$ Geometry optimization of a range of possible conformers for each diastereomer revealed that the $\left(R^{*}, R^{*}, R^{*}\right)$-diastereomer has three conformers of similar relative energy. In contrast, the $\left(S^{*}, R^{*}, R^{*}\right)$-diastereomer appears to suffer from an unfavorable steric interaction between the phenyl ring and the bromo-silane moiety and, as a result, exists predominantly as the conformer where both $\mathrm{Br}$ and TMS point away from the phenyl ring. These conformational differences explain the difference in the ${ }^{3} J_{\mathrm{HbHc}}$ coupling constants observed for each diastereomer. Additionally, the calculated GIAO ${ }^{1} \mathrm{H}$ NMR shifts for each diastereomer are in good agreement with the proposed assignment, providing a CP3 probability of $>99 \% .{ }^{23}$ Thus, the proposed $\left(R^{*}, R^{*}, R^{*}\right)$ - and $\left(S^{*}, R^{*}, R^{*}\right)$-diastereomers result from an approximately equal preference for bromonium formation from each face of the alkene, despite the potential directing effect of the adjacent stereocenter. Literature examples of

Table 5.1 Stereochemical Assignment of Bromosilanes 254 and 255.

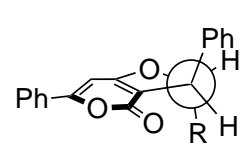

$\left(R^{\star}, R^{\star}, R^{\star}\right) \quad 254$

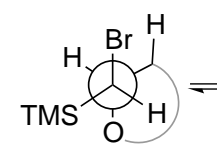

$E_{\text {rel }}=0.005$

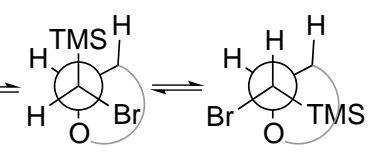

0

$1.69 \mathrm{kcal} / \mathrm{mol}$

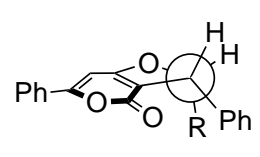

$\left(S^{\star}, R^{\star}, R^{\star}\right)$

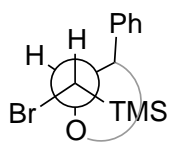

255

\begin{tabular}{ccccccc}
\hline \multirow{2}{*}{ Compound } & \multicolumn{3}{c}{${ }^{\mathbf{1}} \mathbf{H}$ NMR Shifts $(\delta, \mathbf{p p m})$} & \multicolumn{2}{c}{$\mathbf{H H}$ Coupling $(\mathbf{H z})$} \\
& $\mathbf{H}_{\mathbf{a}}$ & $\mathbf{H}_{\mathbf{b}}$ & $\mathbf{H}_{\mathbf{c}}$ & ${ }^{3} \boldsymbol{J}_{\mathrm{HaHb}}$ & ${ }^{3} \boldsymbol{J}_{\mathrm{HbHc}}$ \\
\hline Experimental & ${ }^{1} H$ & NMR & data & & & \\
Major & 4.55 & 5.10 & 3.54 & 4.1 & 7.1 \\
Minor & 4.68 & 5.25 & 2.84 & 7.9 & 11.7 \\
\hline Calculated ${ }^{1} H$ & $N M R$ & data & & & \\
$\boldsymbol{R}^{*} \boldsymbol{R}^{*} \boldsymbol{R}^{*}$ & 4.52 & 4.89 & 3.60 & 5.9 & 5.7 \\
$\boldsymbol{S}^{*} \boldsymbol{R}^{*} \boldsymbol{R}^{*}$ & 4.35 & 5.06 & 2.84 & 6.6 & 9.6 \\
\hline
\end{tabular}

diastereoselective intramolecular haloetherification typically rely on 1,3-allylic strain or the inside alkoxy effect to elicit stereoselectivity. ${ }^{24,25}$ The reaction shown in Scheme 5.18 illustrates the structural features that lead to a diastereoselective halocyclization. ${ }^{26} \mathrm{With}$ this in mind, the $\pi$-allyl-Pd precursors $\mathbf{2 5 6}$ and $\mathbf{2 5 7}$ were prepared with the aim of generating a C3-substituted 4-hydroxy- $\alpha$-pyrone that will undergo diastereoselective intramolecular halocyclization. 


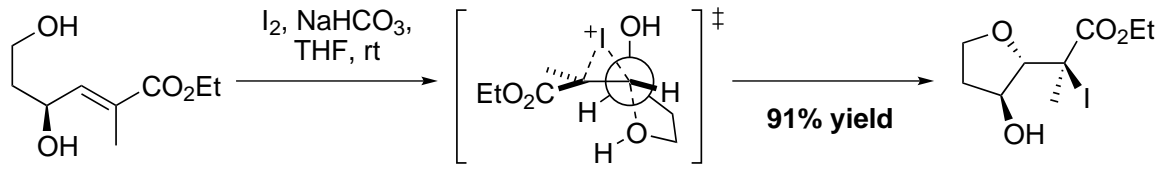

Scheme 5.18 Structural Requirements for a Diastereoselective Halocyclization.

The allylic alkylation of gem-diacetate 256 with 6-methyl-4-hydroxy- $\alpha$-pyrone (258) would provide access to a pyrone-based compound with a suitable hydroxyl directing group for subsequent cyclization. Unfortunately, this reaction did not produce the desired product 259 despite screening a variety of reaction conditions. ${ }^{27}$ With the same ultimate goal in mind, the use of bis-vinylsilane $\mathbf{2 5 7}$ was investigated. This trisubstituted alkene was designed to invoke $\mathrm{A}^{1,3}$-strain and stereoselectivity in the subsequent bromoetherification. Unfortunately, this substrate was also found to be unreactive in $\mathrm{Pd}$-AA reactions with 4-hydroxy- $\alpha$-pyrone 260. Since both of the aforementioned electrophiles have been shown to participate in Pd-AA reactions with malonate derivatives, the poor reactivity observed with 4-hydroxy- $\alpha$-pyrones is presumably a consequence of using a relatively inert nucleophile in combination with a relatively inert electrophile.
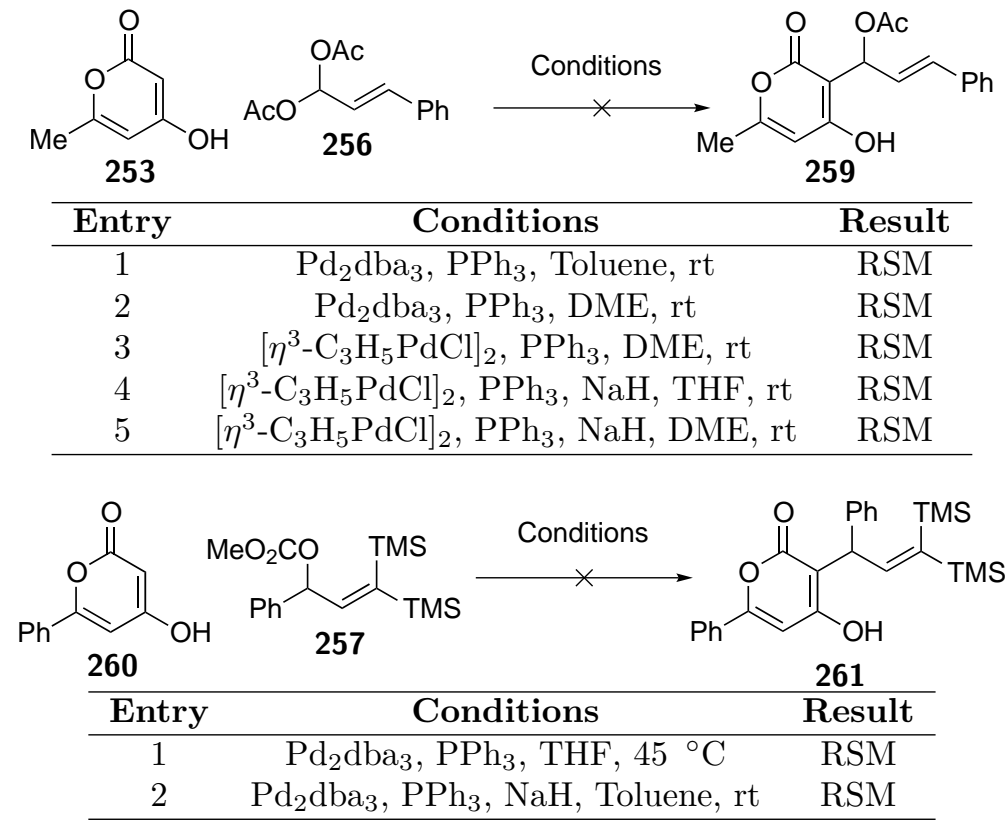

Scheme 5.19 Attempted Incorporation of Directing Groups for Haloetherification.

The characteristics required for reactivity in the $\mathrm{Pd}-\mathrm{AA}$ reaction and those that engender stereoselectivity in the intramolecular bromoetherification seemed to be somewhat in opposition to each other. The difficulty in designing a substrate that fits these narrow criteria prompted the investigation of an alternative approach to constructing a C3-substituted $\alpha$ pyrone. 


\subsection{Second Generation Approach to Labillarides E-H}

The short-comings of the first generation approach led to a revised synthetic strategy based around the use of an intramolecular palladium-catalyzed allylic alkylation to form the furanopyrone core of labillarides E-H (Scheme 5.20). This strategy utilizes the three-carbon linchpin 262 to fuse the labillaride fragments using a Rh-catalyzed conjugate addition, Pdcatalyzed allylic alkylation and Ru-catalyzed alkene metathesis.

Late-stage introduction of the chiral side chain was envisioned using Ru-catalyzed alkene cross metathesis. This would enable access to two of the labillaride E-H diastereomers, as either of the known compounds $(R)$ - or $(S)$-pent-1-en-3-ol could be used as the coupling partner. The prerequisite vinyl dihydrofuran intermediate would be formed using an intramolecular $\mathrm{Pd}$ catalyzed allylic alkylation of 4-hydroxy- $\alpha$-pyrone 263. The use of achiral phosphine ligands in this reaction is likely to lead to substrate controlled stereoselectivity. However, if poor selectivity for the desired trans-substituted dihydrofuran is observed, a chiral phosphine ligand could be used to provide improved stereoselectivity. To avoid the chemoselectivity issues

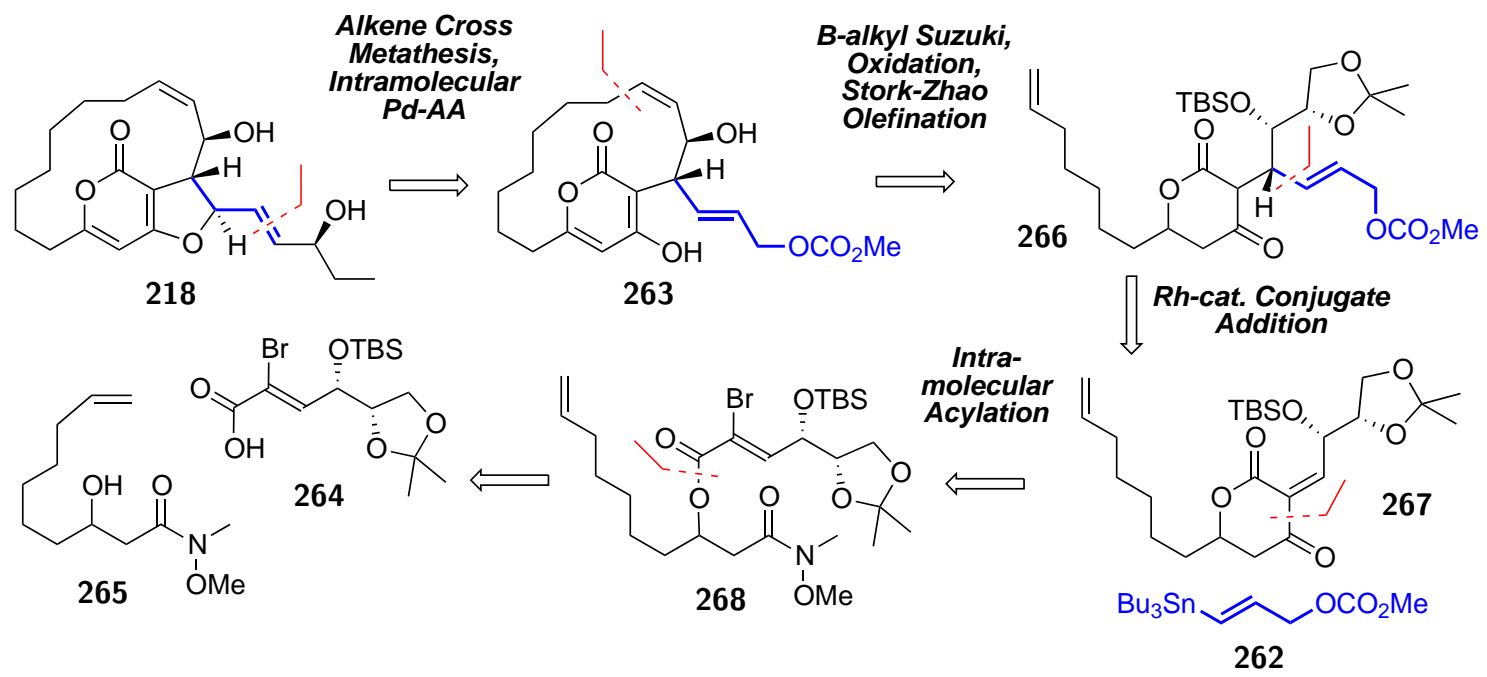

Scheme 5.20 Labillarides E-H Second Generation Retrosynthesis.

associated with selectively functionalizing one of two terminal alkenes later in the route, the $B$-alkyl Suzuki macrocyclization will be performed prior to the intramolecular Pd-AA. This reaction has been used successfully in the synthesis of a number of macrocyclic natural products including phomactin $\mathrm{A}$ and 4-hydroxydictyolactone. ${ }^{28}$ However, the use of $Z$-vinyl iodides in Pd-catalyzed cross coupling has been shown to be sensitive to steric hindrance at the allylic carbon and this may necessitate the use of a smaller protecting group in place of the TBSether shown in Scheme 5.20. ${ }^{29}$ The macrocyclization will be preceded by Stork-Zhao olefination and oxidation of the $\delta$-lactone to provide a 4 -hydroxy- $\alpha$-pyrone. ${ }^{30}$ These transformations lead back to the cyclic $\beta$-keto ester 266, which was envisioned to arise from the Rh-catalyzed conjugate addition of vinyl stannane $\mathbf{2 6 2}$ to the highly reactive alkylidene dicarbonyl $\mathbf{2 6 7}$. An intramolecular acylation, which occurs after lithium-halogen exchange, will give rise to 
alkylidene $\mathbf{2 6 7}$ from the vinyl bromide 268. This highly functionalized intermediate will be prepared by the coupling of carboxylic acid $\mathbf{2 6 4}$ and $\beta$-hydroxy amide $\mathbf{2 6 5}$.

The forward synthesis began with the preparation of $\beta$-hydroxy amide $\mathbf{2 6 5}$ in three steps from commercially available 1,7-octadiene (Scheme 5.21). Mono hydroboration-oxidation using an excess of 1,7-octadiene (269) provided the desired primary alcohol $\mathbf{2 7 0}$ in good yield with minimal bis-hydroboration side products. In this reaction, borane-dimethylsulfide complex provides three equivalents of hydride and the excess 1,7-octadiene could be recovered from the reaction, making this a highly efficient and atom economic transformation in comparison to the use of 9-BBN or (Sia) ${ }_{2} \mathrm{BH}$ as boron hydride sources. The primary alcohol $\mathbf{2 7 0}$ was then oxidized using either a TEMPO/BAIB or Moffat-Swern oxidation to provide aldehyde 271, which serves as the acceptor in the subsequent Reformatsky reaction. The donor component of this reaction,

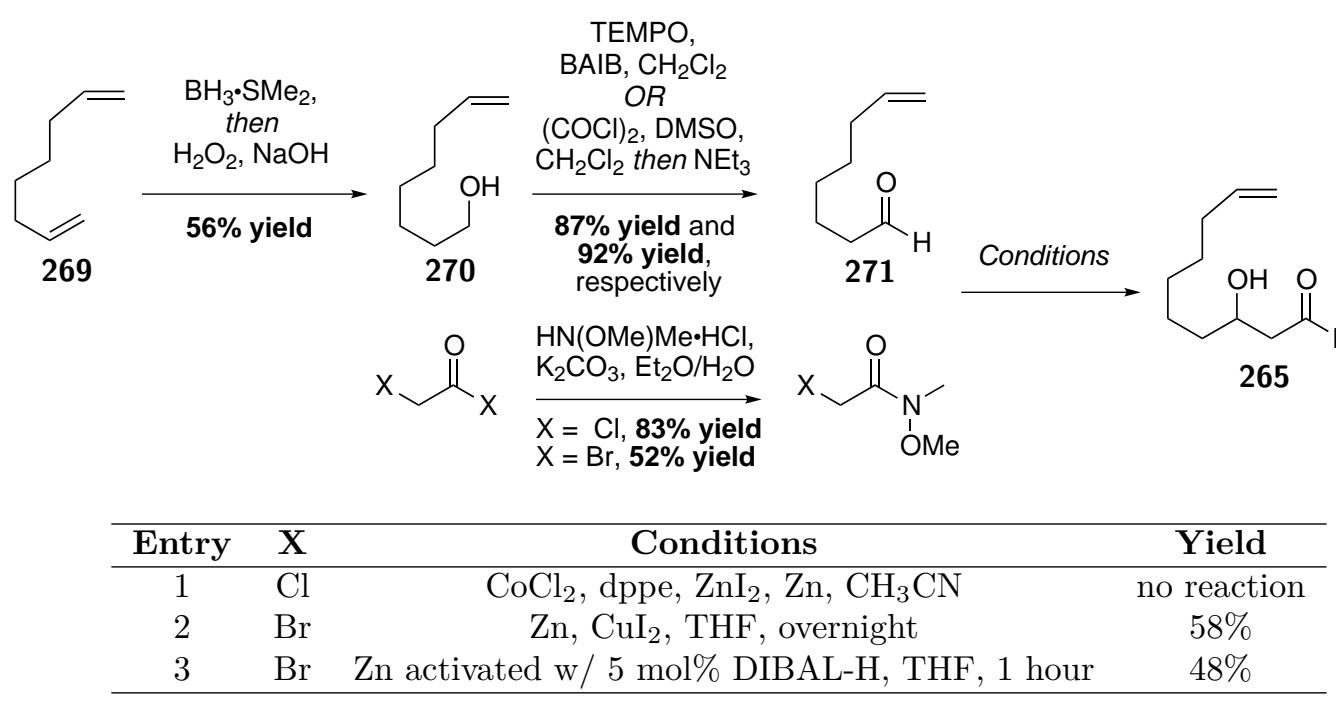

Scheme 5.21 Synthesis of $\beta$-hydroxy Amide 265.

an $\alpha$-halo amide, was prepared in a single step from either chloroacetyl chloride or bromoacetyl bromide. Initial attempts to use the $\alpha$-chloroamide, along with a catalytic amount of a Co(II)phosphine complex to facilitate formation of the organozinc halide nucleophile, resulted in recovered starting materials (Entry 1). ${ }^{31}$ Using the more reactive $\alpha$-bromo amide and $\mathrm{Zn}-\mathrm{Cu}$ couple, which was generated in situ from $\mathrm{Zn}$ and $\mathrm{CuI}_{2}$, provided the desired $\beta$-hydroxy amide in $58 \%$ yield. ${ }^{32}$ Unfortunately, this reaction suffered from a relatively long induction period, presumably due to the presence of passivated $\mathrm{Zn}$. This was overcome by activating the $\mathrm{Zn}$ dust with $5 \mathrm{~mol} \%$ DIBAL-H prior to addition of the aldehyde, which provided the desired product in $48 \%$ yield. ${ }^{33}$ Ultimately, this Reformatsky reaction proved somewhat capricious; however, the use of high purity aldehyde immediately after its preparation, and moderate to large amounts (>0.75 g) of $\alpha$-bromoamide, provided the desired product consistently. ${ }^{34}$ With $\beta$-hydroxyamide $\mathbf{2 6 5}$ in hand, the preparation of the $\alpha$-bromo- $\alpha$ - $\beta$-unsaturated carboxylic acid was undertaken.

The known aldehyde $\mathbf{2 7 2}$ was prepared in five steps from (L)-ascorbic acid, 273 
(Scheme 5.22) ${ }^{35}$ Conversion of the vicinal diol to an acetonide under acidic conditions provided 5,6-O-isopropylidene-L-ascorbic acid (274), which was subsequently transformed into the $\alpha$ hydroxy carboxylate salt $\mathbf{2 7 5}$ using a biomimetic oxidative degradation. This compound was transformed into the ethyl ester 276 upon exposure to ethyl iodide and $\mathrm{K}_{2} \mathrm{CO}_{3}$ in DMF. It is interesting to note that carboxylates are typically poor nucleophiles, however, the presence of an $\alpha$-hydroxy group polarizes the carboxylate group via an internal hydrogen bond and enables efficient addition to electrophiles such as alkyl iodides. TBS-protection of the $\alpha$-hydroxy ester $\mathbf{2 7 6}$ provided silyl ether $\mathbf{2 7 7}$, which was reduced to the corresponding aldehyde, $\mathbf{2 7 2}$, using DIBAL-H. In this case, the presence of a chelating substituent at the $\alpha$-position and low reaction temperature $\left(-100{ }^{\circ} \mathrm{C}\right)$ prevent the breakdown of the tetrahedral aluminum alkoxide intermediate which often leads to over-reduction.

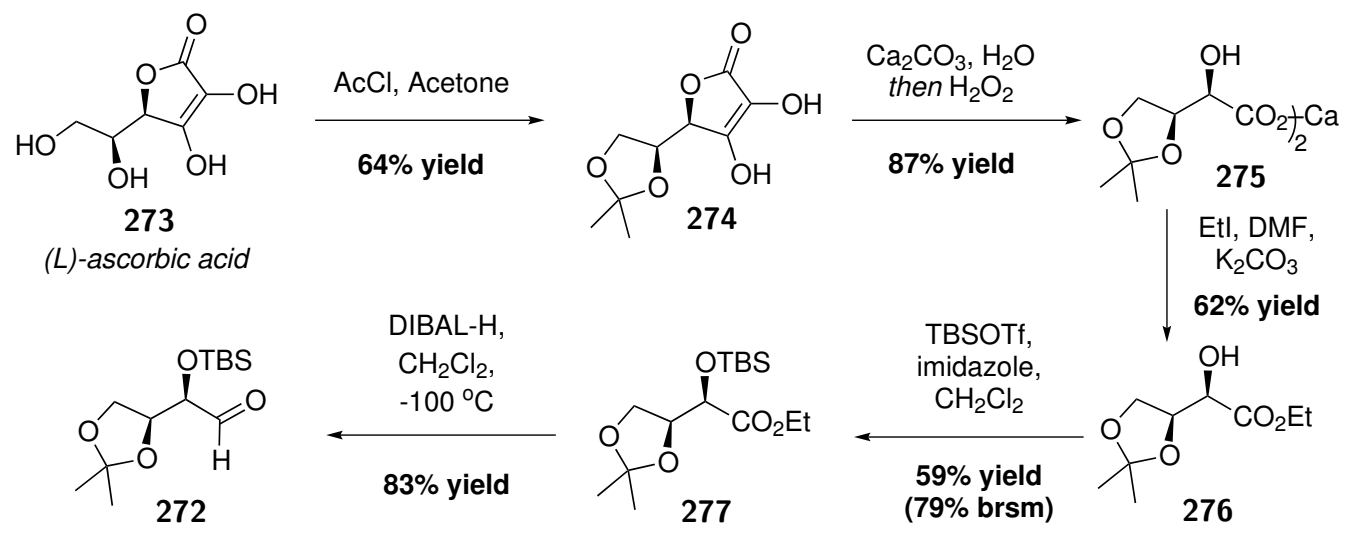

Scheme 5.22 Synthesis of Aldehyde 272 from (L)-Ascorbic Acid.

The optically active aldehyde $\mathbf{2 7 2}$ was then used in a Wittig olefination to provide vinyl bromide 278 (Scheme 5.23). In this reaction, ethyl triphenylphosphorylacetate (279), NBS and $\mathrm{K}_{2} \mathrm{CO}_{3}$ are combined in $\mathrm{CH}_{2} \mathrm{Cl}_{2}$ to generate the corresponding bromo-substituted ylide (X $=\mathrm{Br}$ ) in situ at $-20{ }^{\circ} \mathrm{C}$, followed by addition of aldehyde $\mathbf{2 7 2}$ at room temperature. Initially, only $25 \%$ yield of the desired vinyl bromide was obtained, along with a $25 \%$ recovery of aldehyde

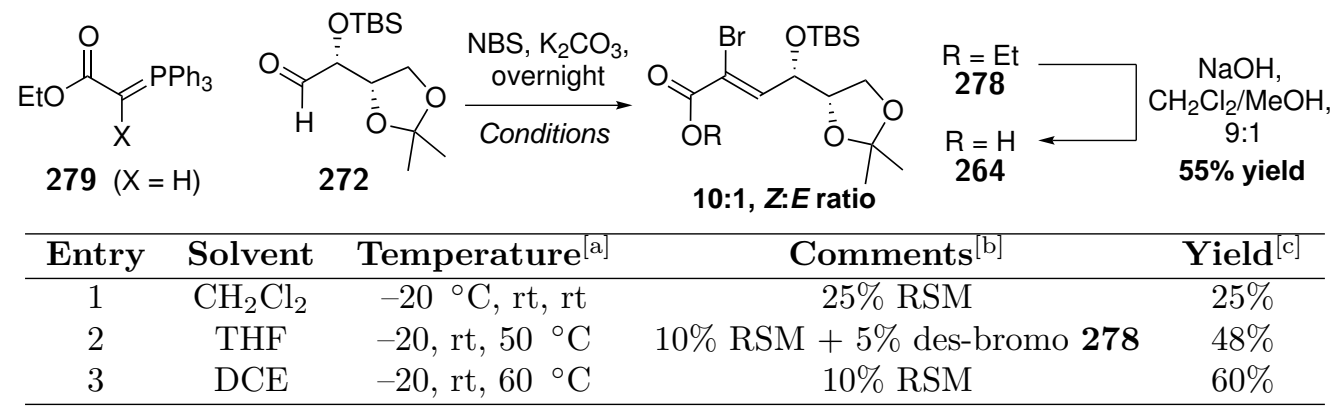

[a] The initial mixing of the phosphorus ylide and NBS was performed at $-20{ }^{\circ} \mathrm{C}$, the reaction was then stirred overnight at room temperature. The third temperature describes the 3 hour period at the end of the reaction. [b] Determined by ${ }^{1} \mathrm{H}$ NMR. [c] Isolated yield.

Scheme 5.23 Synthesis of $\alpha$-Bromo- $\alpha$ - $\beta$-unsaturated Carboxylic Acid 264. 
272 (Entry 1). By changing the reaction solvent to THF and heating the reaction mixture to $50{ }^{\circ} \mathrm{C}$ for 3 hours, an improved yield of $48 \%$ was obtained (Entry 2). Unfortunately, these conditions produced $5 \%$ yield of the des-bromo side product which could not be separated from the desired product. Finally, performing the reaction in 1,2-dichloroethane (DCE) produced vinyl bromide $\mathbf{2 7 8}$ in $60 \%$ yield without formation of the unwanted des-bromo side product (Entry 3).

Coupling of the two fragments, $\mathbf{2 6 5}$ and 264, was achieved in moderate yield using EDCI to activate the acid towards nucleophilic addition. The synthesis of the highly functionalized ester $\mathbf{2 6 8}$ provided the starting material for an intramolecular acylation to form the key intermediate, alkylidene dicarbonyl $\mathbf{2 6 7}$.

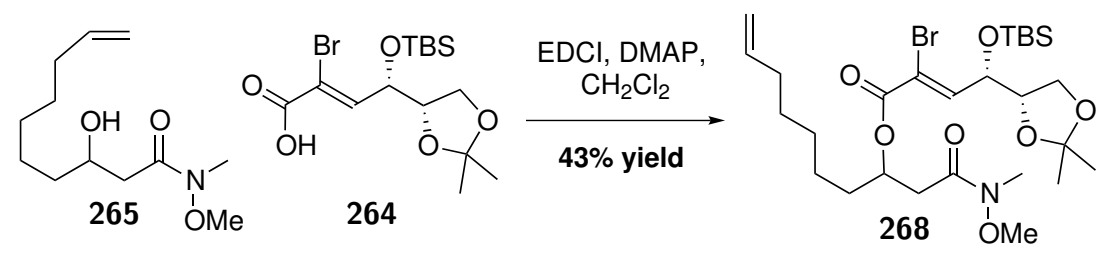

Scheme 5.24 Coupling of Carboxylic Acid 264 and Alcohol 265.

A similar intramolecular acylation was employed by Sorensen and coworkers in their synthesis of the natural product FR182877 (Scheme 5.25). ${ }^{36}$ Interestingly, they discovered that both the $Z$ - and $E$-bromoalkene starting materials $\mathbf{2 8 0}$ and $\mathbf{2 8 1}$ provide the same product, 282, stereoselectively. This stereoconvergence led to the postulate that the reaction proceeds through a common lithium allenolate intermediate, and the stereoselectivity is a consequence of the bulky hydrindene ring system blocking attack from one face. The resulting alkylidene dicarbonyl $\mathbf{2 8 2}$ was found to be highly reactive and decomposed under a number of subsequent reaction conditions and also on silica gel. The intramolecular acylation of compound $\mathbf{2 6 8}$ was

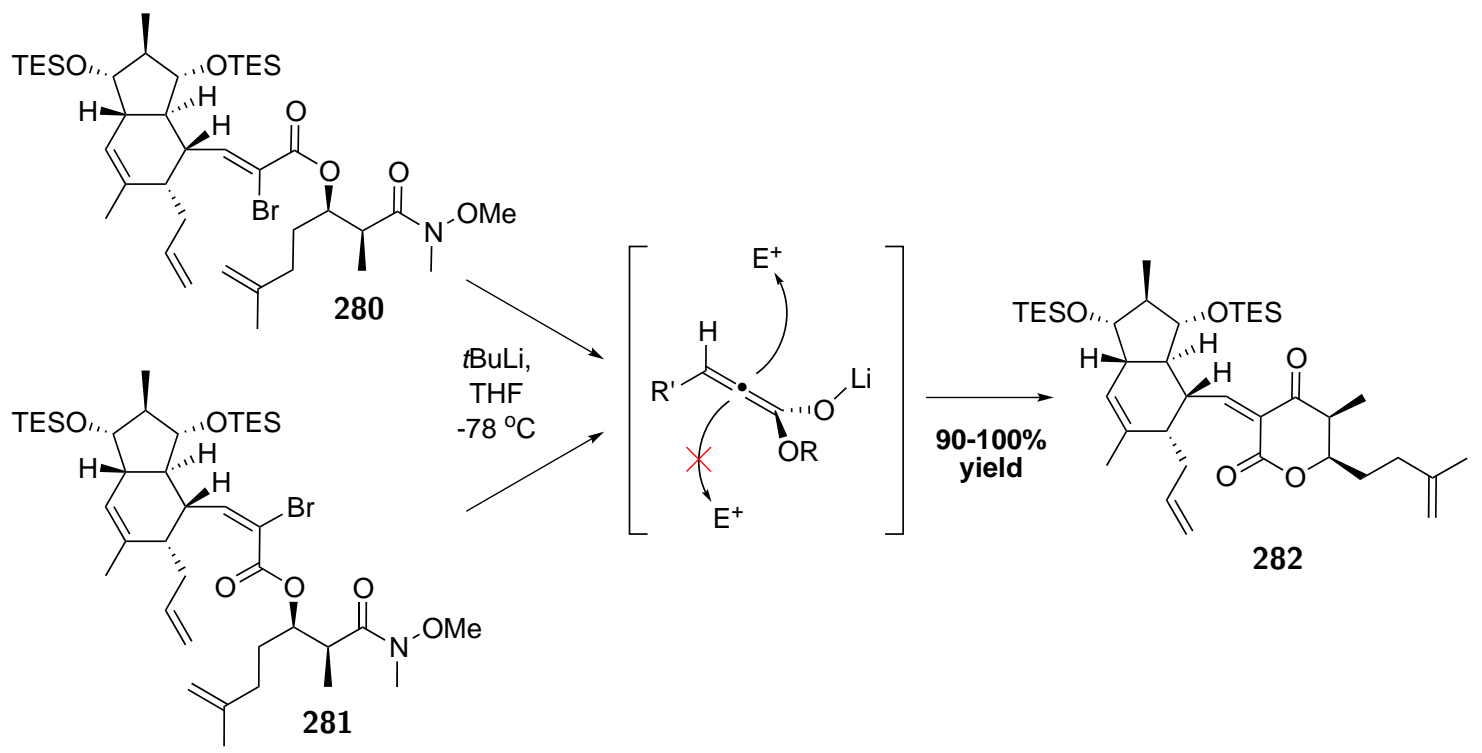

Scheme 5.25 Sorensen's Intramolecular Acylation in the Synthesis of FR182877. 
attempted with the knowledge that the product dicarbonyl alkylidene was likely to be relatively unstable. Thus, the reaction was quenched with $\mathrm{pH} 7$ phosphate buffer and ${ }^{1} \mathrm{H}$ NMR analysis of the crude reaction mixture was performed in benzene- $d 6$ to avoid the traces of acid often present in $\mathrm{CDCl}_{3}$. Rapid and complete consumption of the starting material occurred but unfortunately none of the desired product was observed. ${ }^{1} \mathrm{H}$ NMR analysis suggested that the major side product from this reaction was a trimer generated by sequential intermolecular nucleophilic additions. The predominance of this side reaction is surprising given that the reaction was performed at high dilution $(0.01 \mathrm{M})$. However, subsequent molecular modelling (semi-empirical, MM2) revealed that the combination of -OTBS and acetonide groups adjacent to the nucleophilic center, create unfavorable steric interactions in the conformation required for intramolecular nucleophilic addition. ${ }^{37}$ This insight led to the hypothesis that the bulky nature of the $\alpha, \beta$-unsaturated ester was, in part, responsible for the intermolecular side reactions and the absence of the desired product. Consequently, the intramolecular acylation of compounds with smaller $\beta$-substituents was investigated.
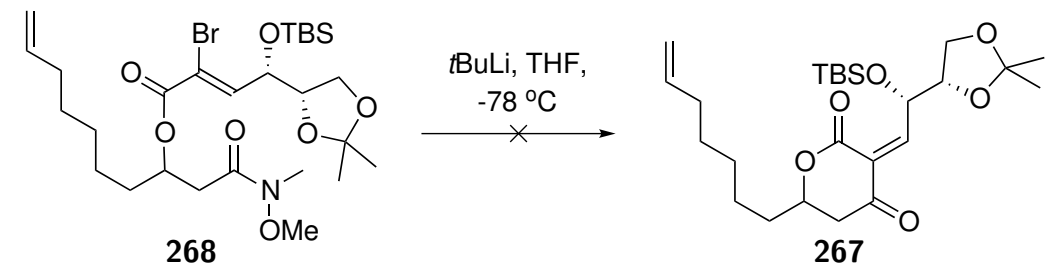

Scheme 5.26 Attempted Intramolecular Acylation of 268.

The same reaction sequence used to prepare the carboxylic acid 264 was used in the attempted preparation of an alternative carboxylic acid, 283 (Scheme 5.27). Interestingly, saponification of the $\alpha$-bromo- $\alpha, \beta$-unsaturated ester $\mathbf{2 8 4}$ did not provide the desired carboxylic acid 283, but instead provided enol ether 285 as a mixture of alkene isomers. This undesired product presumably arises from oxy-Michael addition followed by elimination to regenerate an $\alpha, \beta$-unsaturated carbonyl. Given that this side reaction was not observed in the formation of the previous carboxylic acid 264, this result suggests that this new substrate has considerably less steric hinderance around the $\beta$-position, and therefore is more reactive towards nucleophilic addition. To circumvent this problem, a new route, where coupling of the carboxylic acid occurs prior to olefination, was pursued.
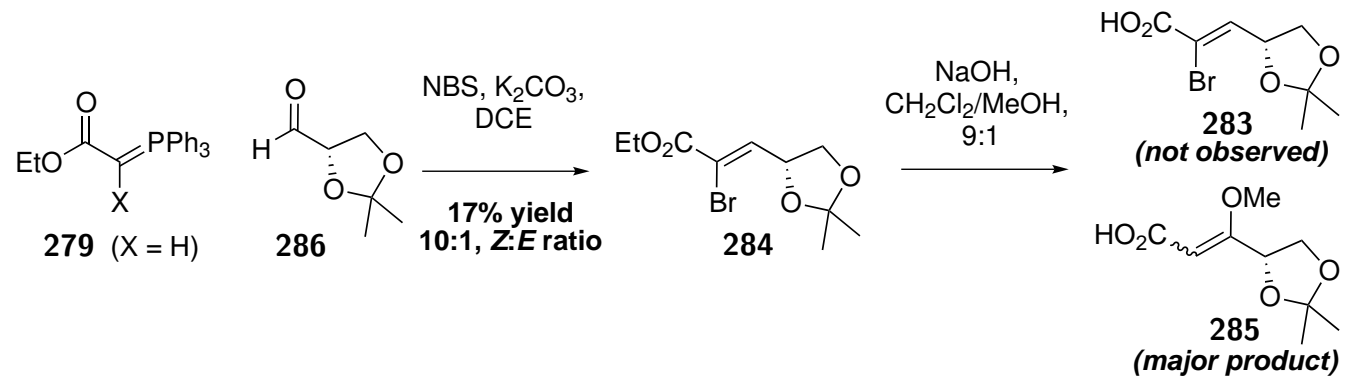

Scheme 5.27 Attempted Formation of Carboxylic Acid 283. 
The new route to alternative substrates for intramolecular acylation was centered around the use of the $\alpha$-bromophosphonate $\mathbf{2 8 7}$ as a linchpin to couple $\beta$-hydroxy amide $\mathbf{2 6 5}$ to various aldehydes. This phosphonate linchpin was prepared in 4 steps from $t$-butyl bromoacetate, $\mathbf{2 8 8}$ (Scheme 5.28). ${ }^{31} \mathrm{P}$ NMR was extremely useful in the characterization of various intermediates en route to compound 287 .

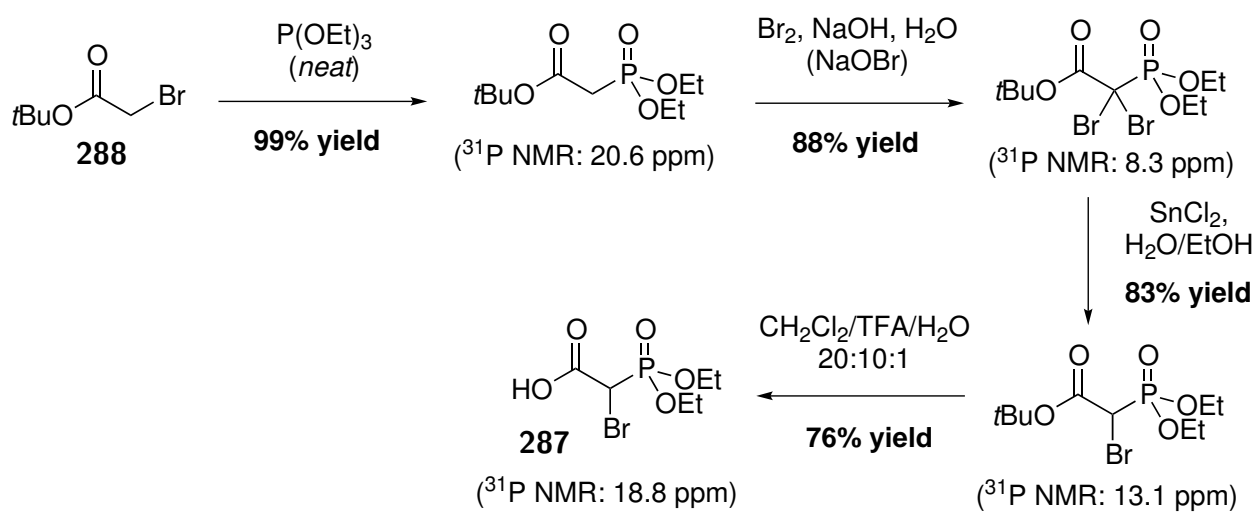

Scheme 5.28 Synthesis of the $\alpha$-Bromophosphonate Linchpin 287.

EDCI-mediated coupling of carboxylic acid 287 and $\beta$-hydroxy amide 265 provided phosphonate ester 289 (Scheme 5.29). This intermediate was used in Horner-WadsworthEmmons olefinations with either $(R)$-glyceraldehyde acetonide $(\mathbf{2 9 0})$ or benzaldehyde to provide two new substrates with which to investigate the key intramolecular acylation reaction. Unfortunately, attempts to promote the intramolecular acylation of either compound 291 or

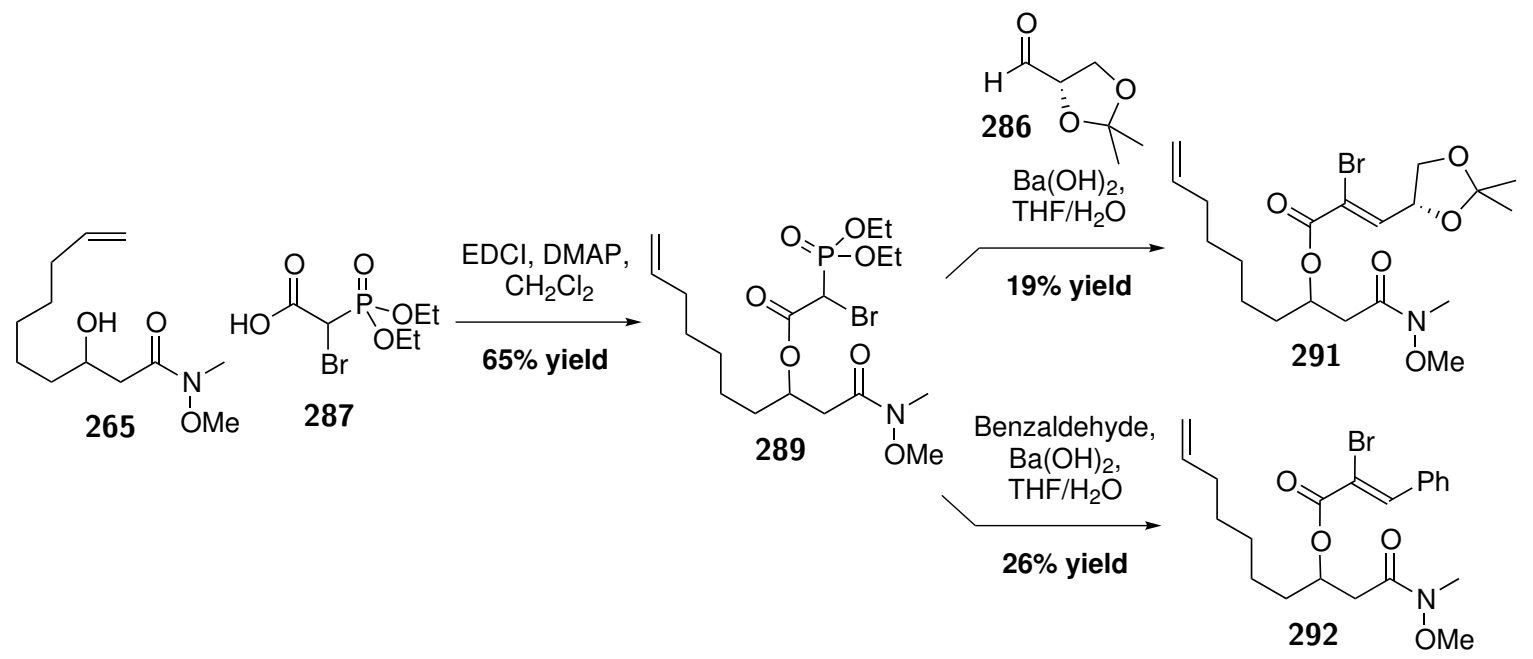

Scheme 5.29 Synthesis of Alternative Substrates for Intramolecular Acylation.

292 were unsuccessful (Scheme 5.30). In both cases complete consumption of the starting material occurred but ${ }^{1} \mathrm{H}$ NMR analysis of the crude reaction mixture showed no downfield peaks characteristic of the alkenyl protons of $\alpha, \beta$-unsaturated carbonyls. Additionally, the crude ${ }^{1} \mathrm{H}$ NMR spectra contained signals that correspond with the terminal alkene and aliphatic chain present in the starting material, however, no significant peaks matching the oxymethine 
( $\beta$ to the amide) or the methyl groups of the Weinreb amide were observed. The disappearance of ${ }^{1} \mathrm{H}$ NMR signals associated with specific functional groups led to the hypothesis that the structure of vinyl bromides 291 and 292 is incompatible with the aforementioned reaction conditions. Specifically, elimination of the $\beta$-acyloxy group is potentially problematic and Paquette and coworkers have reported this problem while attempting a similar transformation in studies towards the synthesis of cochleamycin A. ${ }^{37}$ Additionally, successful intramolecular acylations reported in the literature seem to contain a substituent (i.e. $-\mathrm{CH}_{3}$ or $-\mathrm{OMe}$ ) $\alpha$ to the carbonyl, which is likely to disfavor $\beta$-elimination under basic conditions. ${ }^{36,38}$<smiles>C=CCCCCCC(CC(=O)N(C)OC)OC(=O)/C(Br)=C/C1COC(C)(C)O1</smiles><smiles>C=CCCCCCC(CC(=O)N(C)C)OC(=O)/C(Br)=C/c1ccccc1</smiles>

292
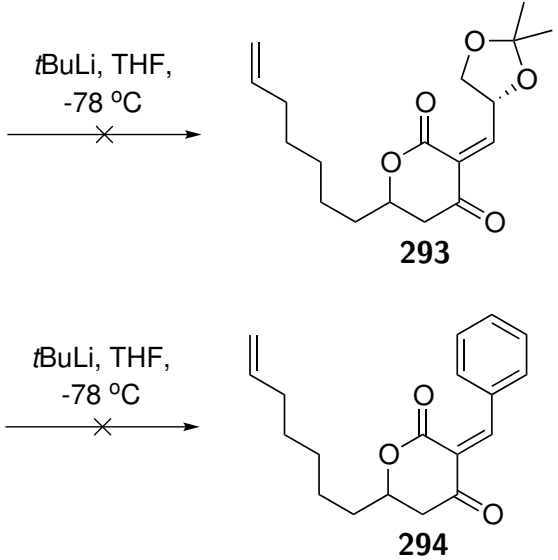

Scheme 5.30 Attempted Intramolecular Acylation with 291 and 292.

The direct preparation of the desired alkylidene dicarbonyl via a Knovenagel condensation was briefly examined using 4-hydroxy-6-methyl- $\alpha$-pyrone and aldehyde 272 (Scheme 5.31). Unfortunately, all of the attempted conditions provided complex mixtures from which the desired alkylidene dicarbonyl could not be isolated. These results are consistent with a number of literature reports that describe failed attempts to perform Knovenagel condensations with aliphatic aldehydes. ${ }^{36,39}$

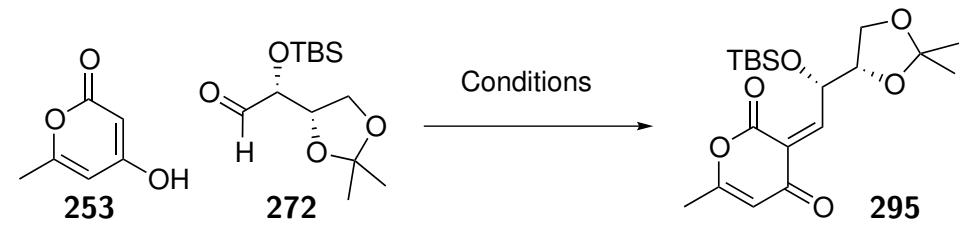

\begin{tabular}{ccc}
\hline Entry & Conditions & Result \\
\hline 1 & EtOH, rt & no product, some SM remains \\
2 & TEBAC, $\mathrm{H}_{2} \mathrm{O}, 95{ }^{\circ} \mathrm{C}$ & no product, complex mixture \\
3 & Pyridine, Toluene, $95{ }^{\circ} \mathrm{C}$ & no product, complex mixture \\
3 & Piperidine, EtOAc, reflux & no product, complex mixture \\
\hline
\end{tabular}

Scheme 5.31 Attempted Knovenagel Condensation with 4-Hydroxy-6-methyl- $\alpha$-pyrone. 


\subsection{Summary}

In summary, labillarides E-H are a group of diastereomeric oxylipin natural products with very interesting structural features and spectroscopy properties. The use of a Pd-catalyzed allylic alkylation to utilize the inherent nucleophilicity of a 4-hydroxy- $\alpha$-pyrone has been identified as a potentially powerful method of constructing the furopyrone core of these natural products. The first generation synthetic strategy was based on the use of an intermolecular $\mathrm{Pd}-\mathrm{AA}$ reaction to selectively couple a functionalized vinyl silane. While a model compound provided excellent yield in the key Pd-AA, the actual substrate displayed poor reactivity under a variety of reaction conditions. Additionally, the subsequent bromo-etherification provided poor diastereoselectivity with the model system. The second generation approach to labillarides E-H was centered around the use of vinylstannane $\mathbf{2 6 2}$ as a three-carbon linchpin. Coupling of the linchpin was envisioned using a Rh-catalyzed conjugate addition to the highly reactive alkylidene dicarbonyl 267. Unfortunately, attempts to utilize an intramolecular acylation to prepare the key alkylidene intermediate were unsuccessful. The shortcomings of this reaction were hypothesized to be a consequence of the reactive nature of the $\beta$-acyloxy moiety. 


\subsection{Experimental Data for Chapter 5}

\subsubsection{General Experimental Methods}

Unless otherwise noted, the following conditions apply. All reactions were performed in flamedried septum-sealed glassware with magnetic stirring under an atmosphere of argon. Moistureand oxygen-sensitive liquids and solutions were transferred using a stainless steel syringe or cannula. Before use, solvents were refluxed over the appropriate drying agent and distilled under argon: tetrahydrofuran from sodium benzophenone ketyl radical; dichloromethane, acetonitrile and triethylamine from calcium hydride; methanol and toluene from sodium. NBS was recrystallized from $\mathrm{H}_{2} \mathrm{O}$ and dried in a vacuum desiccator. All other commercially available chemicals were used as received, without further purification. Analytical thin layer chromatography (TLC) was performed using plastic-backed pre-coated silica TLC plates (Polygram SilG/UV 254 ). Visualization was achieved by UV irradiation $(254 \mathrm{~nm}$ ) or by heating after treatment with a potassium permanganate dip $\left(1.5 \mathrm{~g} \mathrm{KMnO}_{4}, 10 \mathrm{~g} \mathrm{~K}_{2} \mathrm{CO}_{3}, 1.25 \mathrm{~mL}\right.$ of $10 \%$ aqueous $\mathrm{NaOH}$ solution and $200 \mathrm{~mL}$ of water $)$ or $p$-anisaldehyde dip $(0.7 \mathrm{~mL} p$ anisaldehyde, $9.5 \mathrm{~mL}$ conc. $\mathrm{H}_{2} \mathrm{SO}_{4}, 2.7 \mathrm{~mL}$ of acetic acid and $250 \mathrm{~mL}$ of EtOH). The purification of products by flash column chromatography (FCC) was conducted using silica gel 60 (220-240 mesh) with the solvent systems indicated. ${ }^{1} \mathrm{H}$ NMR spectra were recorded on either: a Varian Unity Inova 500 spectrometer at $500 \mathrm{MHz}$, or a Varian Inova 300 at 300 $\mathrm{MHz}$. Data are listed as chemical shift in ppm using $\mathrm{CDCl}_{3}$ as internal standard (7.26 ppm). ${ }^{13} \mathrm{C}$ NMR spectra were recorded on a Varian Unity Inova 500 spectrometer at $125 \mathrm{MHz}$ and the data are listed as chemical shift in ppm using $\mathrm{CDCl}_{3}$ as internal standard (77 ppm). All ${ }^{13} \mathrm{C}$ experiments were ${ }^{1} \mathrm{H}$ decoupled. IR bands were measured as a thin film on a Bruker FTIR Tensor 27 spectrometer with ATR sampling accessory. High-resolution mass spectrometry (HRMS) was performed on a Waters QTOF Premier Tandem mass spectrometer.

\subsubsection{Experimental Details and Characterization Data}

\section{First Generation Approach to Labillarides E-H}

Preparation of 1-Phenyl-3-(trimethylsilyl)prop-2-yn-1-ol, 250a.<smiles>CC(C)(NC#CC(O)c1ccccc1)c1ccccc1</smiles>

To a solution of TMS-acetylene ( $1 \mathrm{~mL}, 7.08 \mathrm{mmol}, 1$ equiv) in THF $(5.3 \mathrm{~mL})$ at $-78{ }^{\circ} \mathrm{C}$ was added $n$-BuLi $(4.43 \mathrm{~mL}$ of a $1.6 \mathrm{M}$ solution, $7.08 \mathrm{mmol}, 1$ equiv). The reaction mixture was stirred at $-78{ }^{\circ} \mathrm{C}$ for 30 minutes before the addition of benzaldehyde $(0.72 \mathrm{~mL}, 7.08 \mathrm{mmol}, 1$ equiv). The reaction was slowly warmed to room temperature and stirred for 1 hour before quenching with sat. aq. $\mathrm{NH}_{4} \mathrm{Cl}$. The reaction mixture was then extracted twice with $\mathrm{Et}_{2} \mathrm{O}$, dried over $\mathrm{MgSO}_{4}$, filtered and concentrated in vacuo. The crude product was purified by silica gel chromatography (20\% EtOAc/pet. ether) and the desired product was isolated as a yellow oil $(1.26 \mathrm{~g}, 87 \%$ yield $) . \mathbf{R}_{\mathbf{f}}=0.45\left(25 \%\right.$ EtOAc/PE). ${ }^{\mathbf{1}} \mathbf{H}-\mathbf{N M R}\left(500 \mathrm{MHz}, \mathrm{CDCl}_{3}\right): \delta$ $7.56(\mathrm{~m}, 2 \mathrm{H}), 7.40(\mathrm{~m}, 2 \mathrm{H}), 7.34(\mathrm{~m}, 1 \mathrm{H}), 5.47(\mathrm{~s}, 1 \mathrm{H}), 0.22(\mathrm{~s}, 9 \mathrm{H}) .{ }^{13} \mathbf{C}-\mathrm{NMR}(126 \mathrm{MHz}$, $\left.\mathrm{CDCl}_{3}\right): \delta 140.3,128.6,128.4,126.8,104.9,91.6,65.0,-0.2$. Characterization data matches th reported in the literature. ${ }^{40}$ 


\section{Preparation of (E)-1-Phenyl-3-(trimethylsilyl)prop-2-en-1-ol.}<smiles>OC(/C=C/[As])c1ccccc1</smiles>

To a solution of 1-phenyl-3-(trimethylsilyl)prop-2-yn-1-ol (0.932 g, $4.56 \mathrm{mmol}, 1$ equiv) in THF $(24 \mathrm{~mL})$ at $0{ }^{\circ} \mathrm{C}$ was added Vitride ${ }^{\circledR}(1.95 \mathrm{~mL}$ of a $3.5 \mathrm{M}$ solution, $6.84 \mathrm{mmol}, 1.5$ equiv). The reaction was slowly warmed to room temperature and then quenched with sat. aq. Rochelle's salt after 4 hours. The reaction mixture was subsequently extracted twice with $\mathrm{Et}_{2} \mathrm{O}$, dried over $\mathrm{MgSO}_{4}$, filtered and concentrated in vacuo. The crude product was purified by column chromatography $(15 \% \mathrm{EtOAc} / \mathrm{PE})$ and the desired product was obtained as a yellow oil $(0.671 \mathrm{~g}, 71 \%$ yield $) . \mathbf{R}_{\mathbf{f}}=0.4\left(15 \%\right.$ EtOAc/PE). ${ }^{1} \mathbf{H}-\mathbf{N M R}\left(500 \mathrm{MHz}, \mathrm{CDCl}_{3}\right): \delta 7.37$ $(\mathrm{m}, 4 \mathrm{H}), 7.31(\mathrm{~m}, 1 \mathrm{H}), 6.21(\mathrm{dd}, J=18.7,5.2 \mathrm{~Hz}, 1 \mathrm{H}), 6.01(\mathrm{dd}, J=18.6,1.4 \mathrm{~Hz}, 1 \mathrm{H}), 5.20$ $(\mathrm{dd}, J=5.3,1.4 \mathrm{~Hz}, 1 \mathrm{H}), 0.08(\mathrm{~s}, 9 \mathrm{H}) .{ }^{13} \mathrm{C}-\mathbf{N M R}\left(126 \mathrm{MHz}, \mathrm{CDCl}_{3}\right): \delta 147.0,142.6,130.0$, 128.6, 127.7, 126.5, 76.9, -1.3. Characterization data matches that reported in the literature. ${ }^{41}$

Preparation of (E)-Methyl (1-Phenyl-3-(trimethylsilyl)allyl) Carbonate, 251a.<smiles>CC(=O)OC(C=CC(C)(C)C)c1ccccc1</smiles>

To a solution of (E)-1-phenyl-3-(trimethylsilyl)prop-2-en-1-ol (0.617 g, $3.25 \mathrm{mmol}, 1$ equiv) and DMAP $\left(0.397 \mathrm{~g}, 3.25 \mathrm{mmol}, 1\right.$ equiv) in $\mathrm{CH}_{2} \mathrm{Cl}_{2}(30 \mathrm{~mL})$ at $0{ }^{\circ} \mathrm{C}$ was added methyl chloroformate $(0.30 \mathrm{~mL}, 0.369 \mathrm{~g}, 3.9 \mathrm{mmol}, 1.2$ equiv). The reaction was slowly warmed to room temperature and stirred overnight. The reaction was quenched with sat. aq. $\mathrm{NH}_{4} \mathrm{Cl}$, extracted twice with $\mathrm{CH}_{2} \mathrm{Cl}_{2}$, dried over $\mathrm{MgSO}_{4}$, filtered and concentrated in vacuo. The crude product was purified by column chromatography (10\% EtOAc/PE) and the desired product was isolated as a clear colorless oil (0.442 g, $51 \%$ yield). $\mathbf{R}_{\mathbf{f}}=0.75\left(25 \%\right.$ EtOAC/PE). ${ }^{1} \mathbf{H}-\mathbf{N M R}$ $\left(500 \mathrm{MHz}, \mathrm{CDCl}_{3}\right): \delta 7.42-7.29(\mathrm{~m}, 5 \mathrm{H}), 6.16(\mathrm{dd}, J=18.6,5.2 \mathrm{~Hz}, 1 \mathrm{H}), 6.07(\mathrm{dd}, J=5.2$, $1.4 \mathrm{~Hz}, 1 \mathrm{H}), 5.98(\mathrm{dd}, J=1.87,1.4 \mathrm{~Hz}, 1 \mathrm{H}), 3.79(\mathrm{~s}, 3 \mathrm{H}), 0.07(\mathrm{~s}, 9 \mathrm{H}) .{ }^{\mathbf{1 3}} \mathbf{C}-\mathbf{N M R}(126 \mathrm{MHz}$, $\left.\mathrm{CDCl}_{3}\right): \delta 155.0,142.2,138.4,132.8,128.6,128.4,127.3,81.6,54.8,-1.5$. Characterization data matches that reported in the literature. ${ }^{42}$

\section{Preparation of 2-((4-Methoxybenzyl)oxy)ethanol.}<smiles>CCCCOCCO</smiles>

To a neat ethylene glycol $\left(11.87 \mathrm{~g}, 171.8 \mathrm{mmol}, 2\right.$ equiv) at $0{ }^{\circ} \mathrm{C}$ was added $\mathrm{NaH}(3.78 \mathrm{~g}$ of a $60 \%$ dispersion, $94.5 \mathrm{mmol}, 1$ equiv). A think grey suspension formed and the reaction was slowly warmed to room temperature. The reaction mixture was cooled back to $0{ }^{\circ} \mathrm{C}$ and TBAI (3.49 g, $9.5 \mathrm{mmol}, 0.11$ equiv) and freshly prepared 4-methoxybenzyl chloride (15 $\mathrm{mL}, 85.9 \mathrm{mmol}, 1$ equiv). The reaction was slowly warmed back to room temperature and stirred overnight before quenching with sat. aq. $\mathrm{NH}_{4} \mathrm{Cl}$. The reaction mixture was extracted with EtOAc, dried over $\mathrm{MgSO}_{4}$ and concentrated in vacuo. The crude product was purified by column chromatogarphy $(50 \% \mathrm{EtOAc} / \mathrm{PE})$ and the desired product was isolated as a yellow oil $(8.07 \mathrm{~g}, 52 \%$ yield $) . \mathbf{R}_{\mathbf{f}}=0.35(50 \% \mathrm{EtOAC} / \mathrm{PE}) .{ }^{1} \mathbf{H}-\mathbf{N M R}\left(500 \mathrm{MHz}, \mathrm{CDCl}_{3}\right): \delta 7.29(\mathrm{~m}$, $2 \mathrm{H}), 6.90(\mathrm{~m}, 2 \mathrm{H}), 4.50(\mathrm{~s}, 2 \mathrm{H}), 3.82(\mathrm{~s}, 3 \mathrm{H}), 3.79(\mathrm{~m}, 2 \mathrm{H}), 3.59(\mathrm{~m}, 2 \mathrm{H}), 1.98(\mathrm{~m}, 1 \mathrm{H}) .{ }^{13} \mathrm{C}-$ NMR $\left(126 \mathrm{MHz}, \mathrm{CDCl}_{3}\right): \delta 159.3,130.1,129.5,113.9,73.0,71.0,61.9$, 55.3. Characterization 
data matches that reported in the literature. ${ }^{43}$

Preparation of 1-((4-Methoxybenzyl)oxy)-4-(trimethylsilyl)but-3-yn-2-ol, 250b.<smiles>[13CH3]C#CC(O)CO[13CH3]</smiles>

To a solution of DMSO $\left(0.6 \mathrm{~mL}, 8.47 \mathrm{mmol}, 1\right.$ equiv) in $\mathrm{CH}_{2} \mathrm{Cl}_{2}(28 \mathrm{~mL})$ at $-78{ }^{\circ} \mathrm{C}$ was added oxalyl chloride $(0.35 \mathrm{~mL}, 3.88 \mathrm{mmol}, 1.1$ equiv). Vigorous gas evolution was observed. The reaction mixture was stirred at $-78{ }^{\circ} \mathrm{C}$ for 15 minutes before addition of 2((4-methoxybenzyl)oxy)ethanol ( $0.643 \mathrm{~g}, 3.53 \mathrm{mmol}, 1$ equiv). The reaction was stirred at -78 ${ }^{\circ} \mathrm{C}$ for 45 minutes before addition of $\mathrm{NEt}_{3}(2.46 \mathrm{~mL}, 17.64 \mathrm{mmol}, 5$ equiv). The reaction was then slowly warmed to room temperature before quenching with $\mathrm{H}_{2} \mathrm{O}$. The reaction mixture was extracted twice with $\mathrm{CH}_{2} \mathrm{Cl}_{2}$, dried over $\mathrm{MgSO}_{4}$, filtered, concentrated in vacuo. The crude product was used immediately in the next step. ${ }^{1} \mathbf{H}-\mathbf{N M R}\left(500 \mathrm{MHz}, \mathrm{CDCl}_{3}\right): \delta 9.69$ (app. s, 1H), $7.28(\mathrm{~m}, 2 \mathrm{H}), 6.89(\mathrm{~m}, 2 \mathrm{H}), 4.55(\mathrm{~s}, 2 \mathrm{H}), 4.06($ app. s, 2H) $3.80(\mathrm{~s}, 3 \mathrm{H})$. Characterization data matches that reported in the literature. ${ }^{44}$

To a solution of TMS-acetylene $\left(0.695 \mathrm{~mL}, 5.3 \mathrm{mmol}, 1.5\right.$ equiv) in THF $(10 \mathrm{~mL})$ at $-78{ }^{\circ} \mathrm{C}$ was added $n$-BuLi (3.31 mL of a $1.6 \mathrm{M}$ solution, $5.3 \mathrm{mmol}, 1.5$ equiv). The reaction mixture was stirred at $-78{ }^{\circ} \mathrm{C}$ for 30 minutes before the addition of aldehyde $(0.636 \mathrm{mmol}, 3.53 \mathrm{mmol}$, 1 equiv). The reaction was slowly warmed to room temperature and stirred for 1 hour before quenching with sat. aq. $\mathrm{NH}_{4} \mathrm{Cl}$. The reaction mixture was then extracted twice with $\mathrm{Et}_{2} \mathrm{O}$, dried over $\mathrm{MgSO}_{4}$, filtered and concentrated in vacuo. The crude product was purified by column chromatography $(30 \% \mathrm{EtOAc} / \mathrm{PE})$ and the desired product was isolated as a clear colorless oil ( $0.77 \mathrm{~g}, 78 \%$ yield over two steps). $\mathbf{R}_{\mathbf{f}}=0.27$ (25\% EtOAC/PE). ${ }^{\mathbf{1}} \mathbf{H}-\mathbf{N M R}(500$ $\left.\mathrm{MHz}, \mathrm{CDCl}_{3}\right): \delta 7.28(\mathrm{~m}, 2 \mathrm{H}), 6.90(\mathrm{~m}, 2 \mathrm{H}), 4.58-5.42(\mathrm{~m}, 3 \mathrm{H}), 3.82(\mathrm{~s}, 3 \mathrm{H}), 3.63(\mathrm{dd}, J=$ 9.85, 3.46 Hz, 1H), $3.53(\mathrm{dd}, J=9.83,7.79 \mathrm{~Hz}, 1 \mathrm{H}), 0.18(\mathrm{~s}, 9 \mathrm{H}) .{ }^{13} \mathrm{C}-\mathbf{N M R}(126 \mathrm{MHz}$, $\left.\mathrm{CDCl}_{3}\right): \delta 159.4,129.7,129.5,113.9,102.9,90.6,73.2,73.0,62.1,55.3-0.2$. IR (film): 3426, 2958, 2862, 2174, 1613, 1513, 1302, 1247, 1174, 1091, 1033, 839, $759 \mathrm{~cm}^{-1}$. HRMS - ESI $(\mathrm{m} / z):[\mathrm{M}+\mathrm{Na}]^{+}$calculated for $\mathrm{C}_{15} \mathrm{H}_{22} \mathrm{O}_{3} \mathrm{SiNa}^{+}, 301.1236$; found, 301.1230.

Preparation of (E)-1-((4-Methoxybenzyl)oxy)-4-(trimethylsilyl)but-3-en-2-ol.

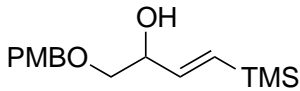

To a solution of 1-((4-methoxybenzyl)oxy)-4-(trimethylsilyl)but-3-yn-2-ol (0.77 g, 2.77 mmol, 1 equiv) in THF $(14 \mathrm{~mL})$ at $0{ }^{\circ} \mathrm{C}$ was added Vitride $(1.19 \mathrm{~mL}$ of a $3.5 \mathrm{M}$ solution, 4.15 mmol, 1.5 equiv). The reaction was slowly warmed to room temperature and then quenched with sat. aq. Rochelle's salt after 4 hours. The reaction mixture was subsequently extracted twice with $\mathrm{Et}_{2} \mathrm{O}$, dried over $\mathrm{MgSO}_{4}$, filtered and concentrated in vacuo. The crude product was purified by column chromatography $(30 \% \mathrm{EtOAc} / \mathrm{PE})$ and the desired product was obtained as a clear colorless oil $(0.44 \mathrm{~g}, 57 \%$ yield $) . \quad \mathbf{R}_{\mathbf{f}}=0.37\left(25 \%\right.$ EtOAC/PE). ${ }^{\mathbf{1}} \mathbf{H}-\mathbf{N M R}$ (500 $\left.\mathrm{MHz}, \mathrm{CDCl}_{3}\right): \delta 7.27(\mathrm{~m}, 2 \mathrm{H}), 6.90(\mathrm{~m}, 2 \mathrm{H}), 6.01-5.98(\mathrm{~m}, 2 \mathrm{H}), 4.33(\mathrm{dt}, J=8.31,3.39 \mathrm{~Hz}$, $1 \mathrm{H}), 3.82(\mathrm{~s}, 3 \mathrm{H}), 3.53(\mathrm{dd}, J=9.60,3.24 \mathrm{~Hz}, 1 \mathrm{H}), 3.32(\mathrm{dd}, J=9.50,8.46 \mathrm{~Hz}, 1 \mathrm{H}), 0.07(\mathrm{~s}$, 9H). ${ }^{13}$ C-NMR $\left(126 \mathrm{MHz}, \mathrm{CDCl}_{3}\right): \delta 159.3,143.5,131.7,130.0,129.4,113.9,73.6,73.0(2 \mathrm{C})$, 55.3, -1.4. IR (film): 3438, 2953, 2857, 1613, 1513, 1302, 1245, 1173, 1100, 1035, 990, 864, $834 \mathrm{~cm}^{-1}$. HRMS - ESI $(\mathrm{m} / z):[\mathrm{M}+\mathrm{Na}]^{+}$calculated for $\mathrm{C}_{15} \mathrm{H}_{24} \mathrm{O}_{3} \mathrm{SiNa}^{+}, 303.1392$; found, 
303.1386 .

Preparation of (E)-1-((4-Methoxybenzyl)oxy)-4-(trimethylsilyl)but-3-en-2-yl Methyl Carbonate, 251b.<smiles>CCOCC(C=CC(C)=O)OC(C)=O</smiles>

To a solution of (E)-1-((4-methoxybenzyl)oxy)-4-(trimethylsilyl)but-3-en-2-ol (0.44 g, 1.57 mmol, 1 equiv) and DMAP (0.19 g, $1.57 \mathrm{mmol}, 1$ equiv) in $\mathrm{CH}_{2} \mathrm{Cl}_{2}(13 \mathrm{~mL})$ was added methyl chloroformate $(0.146 \mathrm{~mL}, 1.88 \mathrm{mmol}, 1.2$ equiv). The reaction was stirred overnight at room temperature before being quenched with sat. aq. $\mathrm{NH}_{4} \mathrm{Cl}$. The reaction mixture was extracted twice with $\mathrm{CH}_{2} \mathrm{Cl}_{2}$, dried over $\mathrm{MgSO}_{4}$, filtered and concentrated in vacuo. The crude product was purified by column chromatography $(20 \%$ EtOAc/PE) and the desired product was isolated as a clear colorless oil $(0.322 \mathrm{~g}, 61 \%$ yield $) . \mathbf{R}_{\mathbf{f}}=0 .\left(25 \%\right.$ EtOAc/PE). ${ }^{1} \mathbf{H}-\mathbf{N M R}(500 \mathrm{MHz}$, $\left.\mathrm{CDCl}_{3}\right): \delta 7.26(\mathrm{~m}, 2 \mathrm{H}), 6.88(\mathrm{~m}, 2 \mathrm{H}), 6.01(\mathrm{dd}, J=18.9,0.7 \mathrm{~Hz}, 1 \mathrm{H}), 5.95(\mathrm{dd}, J=18.9$, $4.6 \mathrm{~Hz}, 1 \mathrm{H}), 5.32$ (tdd, $J=5.4,4.7,0.7 \mathrm{~Hz}, 1 \mathrm{H}), 4.50(\mathrm{~m}, 2 \mathrm{H}), 3.81(\mathrm{~s}, 3 \mathrm{H}), 3.80(\mathrm{~s}, 3 \mathrm{H}), 3.55$ $(\mathrm{d}, J=5.6 \mathrm{~Hz}, 2 \mathrm{H}), 0.07(\mathrm{~s}, 9 \mathrm{H}) .{ }^{13} \mathrm{C}-\mathrm{NMR}\left(126 \mathrm{MHz}, \mathrm{CDCl}_{3}\right): \delta 159.2,155.2,139.4,134.0$, 130.0, 129.3, 113.8, 78.6, 72.8, 70.8, 55.3, 54.8, -1.5. IR (film): 2956, 2860, 1748, 1613, 1513, 1442, 1246, 1090, 1034, 988, 837, $735 \mathrm{~cm}^{-1}$. HRMS - ESI $(m / z):[\mathrm{M}+\mathrm{Na}]^{+}$calculated for $\mathrm{C}_{17} \mathrm{H}_{26} \mathrm{O}_{5} \mathrm{SiNa}^{+}, 361.1447$; found, 361.1455 .

Preparation of (E)-4-Hydroxy-6-phenyl-3-(1-phenyl-3-(trimethylsilyl)allyl)$2 H$-pyran-2-one, 252.<smiles>CC=CC(c1ccccc1)c1c(O)cc(-c2ccccc2)oc1=O</smiles>

To a solution of 6-phenyl-4-hydroxy- $\alpha$-pyrone $(0.030 \mathrm{~g}, 0.161 \mathrm{mmol}, 1$ equiv) in THF (3 $\mathrm{mL})$ at $0{ }^{\circ} \mathrm{C}$ was added $\mathrm{NaH}(0.007 \mathrm{~g}$ of a $60 \%$ dispersion, $0.161 \mathrm{mmol}, 1$ equiv $)$. The reaction mixture was warmed to room temperature and $\mathrm{Pd}_{2}(\mathrm{dba})_{3}(0.015 \mathrm{~g}, 0.016 \mathrm{mmol}, 10 \mathrm{~mol} \%)$ and $\mathrm{PPh}_{3}(0.017 \mathrm{~g}, 0.064 \mathrm{mmol}, 40 \mathrm{~mol} \%)$ were added, followed by $E$ )-methyl (1-phenyl-3(trimethylsilyl)allyl) carbonate $(0.043 \mathrm{~g}, 0.161 \mathrm{mmol}, 1$ equiv) as a solution in THF $(2 \mathrm{~mL})$. The reaction was stirred overnight at room temperature before being quenched with sat. aq. $\mathrm{NH}_{4} \mathrm{Cl}$. The reaction mixture was extracted twice with EtOAc, dried over $\mathrm{MgSO}_{4}$, filtered and concentrated in vacuo. The crude product was purified by column chromatography ( $30 \%$ EtOAc/PE) and the desired product was isolated as an yellow solid $(0.049 \mathrm{~g}, 80 \%$ yield $)$. $\mathbf{R}_{\mathbf{f}}$ $=0.17\left(25 \%\right.$ EtOAC/PE). ${ }^{1} \mathbf{H}-\mathbf{N M R}\left(500 \mathrm{MHz}, \mathrm{CDCl}_{3}\right): \delta 7.80(\mathrm{~m}, 2 \mathrm{H}), 7.51-7.40(\mathrm{~m}, 2 \mathrm{H})$, $7.37(\mathrm{~m}, 2 \mathrm{H}), 7.33-7.27(\mathrm{~m}, 2 \mathrm{H}), 6.62(\mathrm{dd}, J=19.0,5.0 \mathrm{~Hz}, 1 \mathrm{H}), 6.48(\mathrm{~s}, 1 \mathrm{H}), 5.87(\mathrm{dd}, J=$ 19.0, $2.0 \mathrm{~Hz}, 1 \mathrm{H}), 5.23(\mathrm{dd}, J=5.0,2.1 \mathrm{~Hz}, 1 \mathrm{H}), 0.11(\mathrm{~s}, 9 \mathrm{H}) .{ }^{13} \mathbf{C}-\mathbf{N M R}\left(126 \mathrm{MHz}, \mathrm{CDCl}_{3}\right)$ : $\delta 165.96,164.62,159.28,145.33,139.70,134.65,130.98,130.86,128.90,128.88,128.24,127.17$, 125.52, 104.49, 98.44, 46.63, -1.26. IR (film): 3417, 2951, 1648, 1564, 1493, 1402, 1286, 1244, 1118, 1028, 992, 834, $763 \mathrm{~cm}^{-1}$. HRMS - ESI $(\mathrm{m} / z):[\mathrm{M}+\mathrm{H}]^{+}$calculated for $\mathrm{C}_{23} \mathrm{H}_{25} \mathrm{O}_{3} \mathrm{Si}^{+}$, 377.1573 ; found, 377.1568 . 
Preparation of $\left(2 S^{*} / R^{*}, 3 R^{*}\right)-2-\left(\left(S^{*} / R^{*}\right)\right.$-Bromo(trimethylsilyl)methyl)-6methyl-3-phenyl-2H-furo[3,2-c]pyran-4(3H)-one, 254 and 255.<smiles></smiles>

To a solution of $E$ )-4-hydroxy-6-phenyl-3-(1-phenyl-3-(trimethylsilyl)allyl)-2H-pyran-2-one ( $0.078 \mathrm{~g}, 0.207 \mathrm{mmol}, 1$ equiv) in $\mathrm{CH}_{2} \mathrm{Cl}_{2}(8 \mathrm{~mL})$ at $0{ }^{\circ} \mathrm{C}$ was added NBS (0.055 g, 0.311 mmol, 1.5 equiv). The reaction was slowly warmed to room temperature and stirred for 2 hours before quenching with $\mathrm{H}_{2} \mathrm{O}$. The reaction mixture was subsequently extracted twice with $\mathrm{CH}_{2} \mathrm{Cl}_{2}$, dried over $\mathrm{MgSO}_{4}$, filtered and concentrated in vacuo. The crude product was purified by silica gel chromatography $(10 \% \mathrm{EtOAc} / \mathrm{PE})$ and the desired product was isolated as a yellow oil (0.054 g, 57\% yield, 1.2:1 mixture of diastereomers). $\mathbf{R}_{\mathbf{f}}=0.68(15 \% \mathrm{EtOAc} / \mathrm{PE})$. MAJOR - ${ }^{1} \mathbf{H}-N M R\left(500 \mathrm{MHz}, \mathrm{CDCl}_{3}\right): \delta 7.86(\mathrm{~m}, 2 \mathrm{H}), 7.51-7.43(\mathrm{~m}, 2 \mathrm{H}), 7.40-7.25(\mathrm{~m}$, $6 \mathrm{H}), 6.63(\mathrm{~s}, 1 \mathrm{H}), 5.10(\mathrm{dd}, J=7.1,4.2 \mathrm{~Hz}, 1 \mathrm{H}), 4.55(\mathrm{~d}, J=4.1 \mathrm{~Hz}, 1 \mathrm{H}), 3.54(\mathrm{~d}, J=7.1$ $\mathrm{Hz}, 1 \mathrm{H}), 0.20(\mathrm{~s}, 9 \mathrm{H}) . \mathbf{M I N O R}-{ }^{1} \mathbf{H}-\mathbf{N M R}\left(500 \mathrm{MHz}, \mathrm{CDCl}_{3}\right): \delta 7.86(\mathrm{~m}, 2 \mathrm{H}), 7.51-7.43$ $(\mathrm{m}, 2 \mathrm{H}), 7.40-7.25(\mathrm{~m}, 2 \mathrm{H}), 7.20(\mathrm{~m}, 2 \mathrm{H}), 6.63(\mathrm{~s}, 1 \mathrm{H}), 5.25(\mathrm{dd}, J=11.7,7.9 \mathrm{~Hz}, 1 \mathrm{H}), 4.68$ $(\mathrm{d}, J=7.9 \mathrm{~Hz}, 1 \mathrm{H}), 2.84(\mathrm{~d}, J=11.7 \mathrm{~Hz}, 1 \mathrm{H}), 0.21(\mathrm{~s}, 9 \mathrm{H})$. HRMS - ESI $(m / z):[\mathrm{M}+\mathrm{H}]^{+}$ calculated for $\mathrm{C}_{23} \mathrm{H}_{24} \mathrm{O}_{3} \mathrm{SiBr}^{+}$, 455.0678; found, 455.0682.

\section{Preparation of E-3-Phenylprop-2-ene-1,1-diyl Diacetate, 256.}<smiles>CC(=O)OC(C=Cc1ccccc1)OC(C)=O</smiles>

To neat acetic anhydride at $0{ }^{\circ} \mathrm{C}$ was added $(E)$-cinnamaldehyde $(1.90 \mathrm{~mL}, 15.1 \mathrm{mmol}, 1$ equiv). The reaction mixture was stirred at $0{ }^{\circ} \mathrm{C}$ for 15 minutes before the addition of $\mathrm{FeCl}_{3}$ $(0.003 \mathrm{~g}, 0.015 \mathrm{mmol}, 0.1 \mathrm{~mol} \%)$. The reaction was stirred at $0{ }^{\circ} \mathrm{C}$ for 45 minutes before quenching with water. The reaction mixture was extracted twice with $\mathrm{Et}_{2} \mathrm{O}$, the combined organics were dried over $\mathrm{MgSO}_{4}$, filtered and concentrated in vacuo. The crude product was purified by silica gel chromatography $(15 \%$ EtOAc/PE). The desired product was isolated as a white solid (2.86 g, 81\% yield). ${ }^{1} \mathbf{H}-\mathbf{N M R}\left(500 \mathrm{MHz}, \mathrm{CDCl}_{3}\right): \delta 7.43(\mathrm{~m}, 2 \mathrm{H}), 7.39-7.28$ $(\mathrm{m}, 4 \mathrm{H}), 6.88(\mathrm{~d}, J=16.1 \mathrm{~Hz}, 1 \mathrm{H}), 6.22(\mathrm{dd}, J=16.0,6.5 \mathrm{~Hz}, 1 \mathrm{H}), 2.13(\mathrm{~s}, 6 \mathrm{H}) .{ }^{13} \mathbf{C}-\mathbf{N M R}$ $\left(126 \mathrm{MHz}, \mathrm{CDCl}_{3}\right): \delta 168.70,135.63,135.11,128.84,128.68,127.02,121.67,89.73,20.93$. Characterization data matches that reported in the literature. ${ }^{27}$

\section{Preparation of Z-3-Bromo-1-phenyl-3-(trimethylsilyl)prop-2-en-1-ol.}<smiles>[N+]=C(Br)C=C(O)c1ccccc1</smiles>

To a solution of 1-phenyl-3-(trimethylsilyl)prop-2-yn-1-ol (0.40 g, $1.96 \mathrm{mmol}, 1$ equiv) in THF $(10 \mathrm{~mL})$, at room temperature, was added Vitride $(0.95 \mathrm{~mL}$ of a $3.5 \mathrm{M}$ solution, 3.33 mmol, 1.7 equiv). The reaction mixture was stirred at room temperature for 45 minutes before cooling to $-78{ }^{\circ} \mathrm{C}$ and addition of NBS (0.628 g, $3.53 \mathrm{mmol}, 1.8$ equiv) as a solution in $2 \mathrm{~mL}$ 
of THF. The reaction was stirred at $-78{ }^{\circ} \mathrm{C}$ for 30 additional minutes before being quenched with saturated aqueous Rochelle's salt. The reaction mixture was subsequently extratced with $\mathrm{Et}_{2} \mathrm{O}$, the combined organics were dried over $\mathrm{MgSO}_{4}$, filtered and concentrated in vacuo. The crude product was purifiec by silica gel chromatography ( $5 \% \mathrm{EtOAc} / \mathrm{PE})$. The desired product was isolated as a yellow oil $(0.208 \mathrm{~g}, 37 \%$ yield $) .{ }^{1} \mathbf{H}-\mathbf{N M R}\left(500 \mathrm{MHz}, \mathrm{CDCl}_{3}\right): \delta 7.48(\mathrm{~m}$, $2 \mathrm{H}), 7.38(\mathrm{~m}, 2 \mathrm{H}), 7.32(\mathrm{~m}, 1 \mathrm{H}), 6.49(\mathrm{~d}, J=7.5 \mathrm{~Hz}, 1 \mathrm{H}), 5.81(\mathrm{~d}, J=7.5 \mathrm{~Hz}, 1 \mathrm{H}), 0.20(\mathrm{~s}$, 9H). ${ }^{13} \mathrm{C}-\mathrm{NMR}\left(126 \mathrm{MHz}, \mathrm{CDCl}_{3}\right): \delta 142.83,141.79,132.79,128.62,127.84,126.06,73.68$, -2.07. IR (film): 3333, 2958, 2899, 1601, 1493, 1451, 1249, 1018, 897, 838, $752 \mathrm{~cm}^{-1}$. HRMS - ESI $(m / z):[\mathrm{M}+\mathrm{H}]^{+}$calculated for $\mathrm{C}_{12} \mathrm{H}_{17} \mathrm{OSiBrNa}^{+}, 307.0130$; found, 307.0127.

\section{Preparation of 1-Phenyl-3,3-bis(trimethylsilyl)prop-2-en-1-ol.}<smiles>CC([As])=CC(O)c1ccccc1</smiles>

To a solution of Z-3-bromo-1-phenyl-3-(trimethylsilyl)prop-2-en-1-ol (0.208 g, $0.729 \mathrm{mmol}$, 1 equiv) and $\mathrm{NEt}_{3}\left(0.203 \mathrm{~mL}, 1.46 \mathrm{mmol}, 2.0\right.$ equiv) in $\mathrm{CH}_{2} \mathrm{Cl}_{2}(7 \mathrm{~mL})$ was added chlorotrimethylsilane $(0.185 \mathrm{~mL}, 1.46 \mathrm{mmol}, 2.0$ equiv). The reaction was stirred at room temperature overnight before beong diluted with additional $\mathrm{CH}_{2} \mathrm{Cl}_{2}$, filtered and concentrated in vacuo. The crude product was dissolved in THF $(5 \mathrm{~mL})$, cooled to $-78{ }^{\circ} \mathrm{C}$ and $n$-BuLi $(0.401$ $\mathrm{mL}, 0.802 \mathrm{mmol}, 1.1$ equiv) was added. The reaction was slowly warmed to room temperature and stirred overnight before being quenched with $\mathrm{H}_{2} \mathrm{O}$. The reaction mixture was extracted with $\mathrm{Et}_{2} \mathrm{O}$, the combined organics were dried over $\mathrm{MgSO}_{4}$, filtered and concentrated in vacuo. The desired product was isolated as a yellow oil ( $0.122 \mathrm{~g}, 60 \%$ yield over 2 steps $) .{ }^{1} \mathbf{H}-\mathbf{N M R}$ $\left(500 \mathrm{MHz}, \mathrm{CDCl}_{3}\right): \delta 7.44-7.34(\mathrm{~m}, 4 \mathrm{H}), 7.31(\mathrm{~m}, 1 \mathrm{H}), 6.69(\mathrm{~d}, J=8.9 \mathrm{~Hz}, 1 \mathrm{H}), 5.47(\mathrm{~d}, J=$ $8.9 \mathrm{~Hz}, 1 \mathrm{H}), 0.26(\mathrm{~s}, 9 \mathrm{H}), 0.13(\mathrm{~s}, 9 \mathrm{H}) .{ }^{13} \mathrm{C}-\mathrm{NMR}\left(126 \mathrm{MHz}, \mathrm{CDCl}_{3}\right): \delta 155.81,144.57,142.53$, 128.55, 127.59, 126.22, 74.16, 2.31, 0.22. IR (film): 3368, 2953, 2899, 1567, 1493, 1450, 1248, 1011, 884, 860, $832 \mathrm{~cm}^{-1}$. HRMS - ESI $(\mathrm{m} / z)$ : $[\mathrm{M}+\mathrm{Na}]^{+}$calculated for $\mathrm{C}_{15} \mathrm{H}_{26} \mathrm{OSi}_{2} \mathrm{Na}^{+}$, 301.1420; found, 301.1426 .

\section{Preparation of Methyl (1-Phenyl-3,3-bis(trimethylsilyl)allyl) Carbonate, 257.}<smiles>CC(=O)C=C(OC(C)=O)c1ccccc1</smiles>

To a solution of 1-phenyl-3,3-bis(trimethylsilyl)prop-2-en-1-ol $(0.120 \mathrm{~g}, 0.431 \mathrm{mmol}, 1.0$ equiv) and DMAP (0.079 g, $0.647 \mathrm{mmol}, 1.5$ equiv) in $\mathrm{CH}_{2} \mathrm{Cl}_{2}(5 \mathrm{~mL})$ was added methyl chloroformate $(0.050 \mathrm{~mL}, 0.647 \mathrm{mmol}, 1.5$ equiv). The reaction was stirred overnight at room temperature before being quenched with $\mathrm{H}_{2} \mathrm{O}$. The reaction mixture was extracted twice with $\mathrm{CH}_{2} \mathrm{Cl}_{2}$, the combined organics were dried over $\mathrm{MgSO}_{4}$, filtered and concentrated in vacuo. The crude product was purified by silica gel chromatography (5\% EtOAc/PE). The desired product was isolated as a yellow oil $(0.121 \mathrm{~g}, 83 \%$ yield $) .{ }^{\mathbf{1}} \mathbf{H}-\mathbf{N M R}\left(500 \mathrm{MHz}, \mathrm{CDCl}_{3}\right): \delta$ $7.43-7.35(\mathrm{~m}, 4 \mathrm{H}), 7.33(\mathrm{~m}, 1 \mathrm{H}), 6.78(\mathrm{~d}, J=9.0 \mathrm{~Hz}, 1 \mathrm{H}), 6.36(\mathrm{~d}, J=9.0 \mathrm{~Hz}, 1 \mathrm{H}), 3.78(\mathrm{~s}$, $3 \mathrm{H}), 0.24(\mathrm{~s}, 9 \mathrm{H}), 0.13(\mathrm{~s}, 9 \mathrm{H}) .{ }^{13} \mathrm{C}-\mathrm{NMR}\left(126 \mathrm{MHz}, \mathrm{CDCl}_{3}\right): \delta 154.92,150.31,146.91,138.97$, 128.64, 128.31, 127.14, 79.53, 54.79, 1.91, 0.19. IR (film): 2955, 2899, 1747, 1572, 1441, 1246, 939, 879, 861, $835 \mathrm{~cm}^{-1}$. HRMS - ESI $(\mathrm{m} / z)$ : $[\mathrm{M}+\mathrm{Na}]^{+}$calculated for $\mathrm{C}_{17} \mathrm{H}_{28} \mathrm{O}_{3} \mathrm{Si}_{2} \mathrm{Na}^{+}$, 359.1475 ; found, 359.1474 . 


\section{Second Generation Approach to Labillarides E-H}

Preparation of Oct-7-en-1-ol, 270.

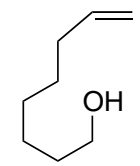

To a solution of octa-1,7-diene (30 mL, 22.38g, $203 \mathrm{mmol}, 6.0$ equiv) in $\mathrm{CH}_{2} \mathrm{Cl}_{2}$ (30.8 $\mathrm{mL}$ ) was added $\mathrm{BH}_{3} \cdot \mathrm{SMe}_{2}$ (16.92 $\mathrm{mL}$ of a $2 \mathrm{M}$ solution in THF, $33.8 \mathrm{mmol}, 1.0$ equiv) via syringe pump, at $0{ }^{\circ} \mathrm{C}$, over 30 minutes. The reaction was then warmed to room temperature and stirred for an additional hour before being cooled back to $0{ }^{\circ} \mathrm{C}$. A $3 \mathrm{~N}$ solution of aqueous $\mathrm{NaOH}(75 \mathrm{~mL}, 225 \mathrm{mmol}, 6.65$ equiv) was added dropwise via addition funnel. Ethanol $(10 \mathrm{~mL})$ was added to the reaction mixture as a cosolvent, followed by the addition of hydrogen peroxide ( $75 \mathrm{~mL}$ of a $30 \mathrm{wt} \%$ solution, $735 \mathrm{mmol}, 21.7 \mathrm{mmol}$ ) then added dropwise via addition funnel. The reaction was then allowed to warm to room temperature and stirred overnight. The reaction mixture was subsequently diluted with $\mathrm{H}_{2} \mathrm{O}$ and extracted with $\mathrm{CH}_{2} \mathrm{Cl}_{2}$. The combined organics were dried over $\mathrm{MgSO}_{4}$, filtered and concentrated in vacuo. The crude product was purified by silica gel chromatography (Pet. Ether then $20 \%$ EtOAc/Pet. Ether). The title compound was isolated as a clear colorless oil $\left(7.42 \mathrm{~g}, 57 \%\right.$ yield). $\mathbf{R}_{\mathbf{f}}=0.13$ (10\% EtOAc/Pet. Ether). ${ }^{1} \mathbf{H}$ NMR $\left(500 \mathrm{MHz}, \mathrm{CDCl}_{3}\right) \delta 5.81$ (ddt, $\left.J=16.9,10.2,6.7 \mathrm{~Hz}, 1 \mathrm{H}\right)$, 5.00 (ddt, $J=17.1,2.2,1.6 \mathrm{~Hz}, 1 \mathrm{H}), 4.94(\mathrm{ddt}, J=10.2,2.3,1.2 \mathrm{~Hz}, 1 \mathrm{H}), 3.65$ (t, $J=6.6 \mathrm{~Hz}$, $2 \mathrm{H}), 2.12-2.00(\mathrm{~m}, 2 \mathrm{H}), 1.57(\mathrm{ddt}, J=7.8,6.5,5.2 \mathrm{~Hz}, 2 \mathrm{H}), 1.47-1.30(\mathrm{~m}, 6 \mathrm{H}) .{ }^{13} \mathrm{C}$ NMR $\left(126 \mathrm{MHz}, \mathrm{CDCl}_{3}\right) \delta 139.05,114.25,63.03,33.70,32.72,28.88,28.85,25.58$. Characterization data matches those reported in the literature. ${ }^{45}$

\section{Preparation of Oct-7-enal, 271.}

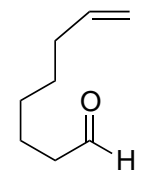

To a solution of oxalyl chloride $\left(0.512 \mathrm{~mL}, 5.85 \mathrm{mmol}, 1.5\right.$ equiv) in $\mathrm{CH}_{2} \mathrm{Cl}_{2}(39 \mathrm{~mL})$ at $-78{ }^{\circ} \mathrm{C}$ was added DMSO $(0.83 \mathrm{~mL}, 11.7 \mathrm{mmol}, 3.0$ equiv $)$. The reaction mixture was stirred at $-78{ }^{\circ} \mathrm{C}$ for 40 minutes before the addition of oct-7-en-1-ol $(0.5 \mathrm{~g}, 3.90 \mathrm{mmol}, 1.0$ equiv). The reaction was stirred for another 40 minutes before the addition of $\mathrm{NEt}_{3}$ (3.26 mL, 23.4 mmol, 6.0 equiv). The reaction was stirred at $-78{ }^{\circ} \mathrm{C}$ for an additional 30 minutes before quenching with $\mathrm{NH}_{4} \mathrm{Cl}$ and warming to room temperature. The reaction mixture was diluted with $\mathrm{H}_{2} \mathrm{O}$ and extracted with $\mathrm{CH}_{2} \mathrm{Cl}_{2}$. The combined organics were dried over $\mathrm{MgSO}_{4}$, filtered and concentrated in vacuo. The crude product was purified by silica gel chromatography $(5 \%$ EtOAc/Pet. Ether). The title compound was isolated as a clear colorless oil (0.452 g, 92\% yield). $\mathbf{R}_{\mathbf{f}}=0.28\left(5 \%\right.$ EtOAc/Pet. Ether). ${ }^{1} \mathbf{H N M R}\left(500 \mathrm{MHz}, \mathrm{CDCl}_{3}\right) \delta 9.77$ (t, $J=1.7$ $\mathrm{Hz}, 1 \mathrm{H}), 5.80(\mathrm{ddt}, J=16.9,10.2,6.7 \mathrm{~Hz}, 1 \mathrm{H}), 5.12-4.87(\mathrm{~m}, 2 \mathrm{H}), 2.44(\mathrm{td}, J=7.4,1.7$ $\mathrm{Hz}, 2 \mathrm{H}), 2.10-2.01(\mathrm{~m}, 2 \mathrm{H}), 1.65(\mathrm{p}, J=7.4 \mathrm{~Hz}, 2 \mathrm{H}), 1.48-1.22(\mathrm{~m}, 4 \mathrm{H}) .{ }^{13} \mathrm{C}$ NMR $(126$ $\left.\mathrm{MHz}, \mathrm{CDCl}_{3}\right) \delta 202.81,138.68,114.50,43.84,33.50,28.58(2 \mathrm{C}), 21.90$. Characterization data matches those reported in the literature. ${ }^{46}$ 


\section{Preparation of 2-Bromo- $N$-methoxy- $N$-methylacetamide.}<smiles>CON(C)C(=O)CBr</smiles>

To a solution of $N, O$-dimethylhydroxylamine hydrochloride $(2.0 \mathrm{~g}, 20.5 \mathrm{mmol}, 1.0$ equiv) in $\mathrm{Et}_{2} \mathrm{O} / \mathrm{H}_{2} \mathrm{O}(1: 1,50 \mathrm{~mL})$ at $0{ }^{\circ} \mathrm{C}$ was added $\mathrm{K}_{2} \mathrm{CO}_{3}(6.24 \mathrm{~g}, 45.2 \mathrm{mmol}, 2.2$ equiv), followed by 2-bromoacetyl chloride $(1.96 \mathrm{~mL}, 4.56 \mathrm{~g}, 22.57 \mathrm{mmol}, 1.2 \mathrm{equiv})$. The reaction was allowed to warm to room temperature and stirred for 3 hours before diluting with $\mathrm{H}_{2} \mathrm{O}$ and extracting with $\mathrm{Et}_{2} \mathrm{O}$. The combined organics were dried over $\mathrm{MgSO}_{4}$, filtered and concentrated in vacuo. The crude product was purified by silica gel chromatography ( $40 \%$ EtOAc/Pet. Ether). The title compound was isolated as a clear colorless oil $\left(1.95 \mathrm{~g}, 52 \%\right.$ yield). $\mathbf{R}_{\mathbf{f}}=0.31$ (40\% EtOAc/Pet. Ether). ${ }^{\mathbf{1}} \mathbf{H}$ NMR $\left(500 \mathrm{MHz}, \mathrm{CDCl}_{3}\right) \delta 4.02(\mathrm{~s}, 2 \mathrm{H}), 3.80(\mathrm{~s}, 3 \mathrm{H}), 3.24(\mathrm{~s}, 3 \mathrm{H})$. ${ }^{13} \mathrm{C}$ NMR $\left(126 \mathrm{MHz}, \mathrm{CDCl}_{3}\right) \delta 167.70,61.67,32.56,25.12$. Characterization data matches those reported in the literature. ${ }^{47}$

\section{Preparation of 2-Chloro- $N$-methoxy- $N$-methylacetamide.}<smiles>CON(I)C(=O)CCl</smiles>

To a solution of $N, O$-dimethylhydroxylamine hydrochloride $(0.7 \mathrm{~g}, 7.18 \mathrm{mmol}, 1.0$ equiv) in $\mathrm{Et}_{2} \mathrm{O} / \mathrm{H}_{2} \mathrm{O}(1: 1,18 \mathrm{~mL})$ at $0{ }^{\circ} \mathrm{C}$ was added $\mathrm{K}_{2} \mathrm{CO}_{3}(2.18 \mathrm{~g}, 15.79 \mathrm{mmol}, 2.2 \mathrm{equiv})$, followed by 2-chloroacetyl chloride $(0.685 \mathrm{~mL}, 0.973 \mathrm{~g}, 8.61 \mathrm{mmol}, 1.2$ equiv). The reaction was allowed to warm to room temperature and stirred for 3 hours before diluting with $\mathrm{H}_{2} \mathrm{O}$ and extracting with EtOAc. The combined organics were dried over $\mathrm{MgSO}_{4}$, filtered and concentrated in vacuo. The title compound was isolated as a faint yellow solid $(0.819 \mathrm{~g}, 83 \%$ yield $)$ and was used without further purification. ${ }^{1} \mathbf{H}$ NMR $\left(500 \mathrm{MHz}, \mathrm{CDCl}_{3}\right) \delta 4.26(\mathrm{~s}, 2 \mathrm{H}), 3.76(\mathrm{~s}, 3 \mathrm{H})$, $3.25(\mathrm{~s}, 3 \mathrm{H})$. Characterization data matches those reported in the literature. ${ }^{48}$

\section{Preparation of 3-Hydroxy- $N$-methoxy- $N$-methyldec-9-enamide, 265.}

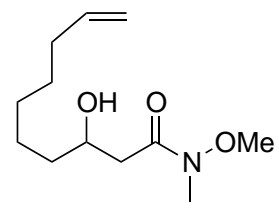

To a suspension of zinc dust ( $0.185 \mathrm{~g}, 2.83 \mathrm{mmol}, 3.57$ equiv) in THF $(2 \mathrm{~mL})$ was added $0.013 \mathrm{~g}$ of 2 -Bromo- $N$-methoxy- $N$-methylacetamide, followed by DIBAL-H $(28 \mu \mathrm{L}, 0.028 \mathrm{mmol}$, 0.04 equiv). The reaction was heated to $50{ }^{\circ} \mathrm{C}$ and the remaining 2 -Bromo- $N$-methoxy- $N$ methylacetamide $(0.26 \mathrm{~g}, 1.43 \mathrm{mmol}, 1.8 \mathrm{equiv})$ was added. The reaction was stirred at 50 ${ }^{\circ} \mathrm{C}$ for 1 hour before cooling to room temperature. Oct-7-enal ( $0.1 \mathrm{~g}, 0.792 \mathrm{mmol}$, 1 equiv) was added and the reaction stirred at room temperature. Complete conversion by TLC was observed after an hour and the reaction was quenched with saturated aqueous $\mathrm{NH}_{4} \mathrm{Cl}$. The reaction mixture was extracted with $\mathrm{Et}_{2} \mathrm{O}$, dried over $\mathrm{MgSO}_{4}$, filtered and concentrated in vacuo. The crude product was purified by silica gel chromatography ( $40 \%$ EtOAc/Pet. Ether). The title compound was isolated as a clear colorless oil $\left(0.087 \mathrm{~g}, 48 \%\right.$ yield). $\mathbf{R}_{\mathbf{f}}=0.19(40 \%$ EtOAc/Pet. Ether). ${ }^{1} \mathbf{H}$ NMR $\left(500 \mathrm{MHz}, \mathrm{CDCl}_{3}\right) \delta 5.81$ (ddt, $\left.J=16.9,10.2,6.7 \mathrm{~Hz}, 1 \mathrm{H}\right)$, 
$5.00(\mathrm{~d}, J=17.1 \mathrm{~Hz}, 1 \mathrm{H}), 4.94(\mathrm{dd}, J=10.2,1.0 \mathrm{~Hz}, 1 \mathrm{H}), 4.02($ app. s, $1 \mathrm{H}), 3.79(\mathrm{~d}, J=$ $3.1 \mathrm{~Hz}, 1 \mathrm{H}), 3.70(\mathrm{~s}, 3 \mathrm{H}), 3.20(\mathrm{~s}, 3 \mathrm{H}), 2.67(\mathrm{~d}, J=16.7 \mathrm{~Hz}, 1 \mathrm{H}), 2.45(\mathrm{dd}, J=16.9,9.6 \mathrm{~Hz}$, $1 \mathrm{H}), 2.05(\mathrm{q}, J=6.9 \mathrm{~Hz}, 2 \mathrm{H}), 1.67-1.27(\mathrm{~m}, 8 \mathrm{H}) .{ }^{13} \mathrm{C} \mathbf{N M R}\left(126 \mathrm{MHz}, \mathrm{CDCl}_{3}\right) \delta 173.98$, $139.09,114.22,67.84,61.24,38.15,36.47,33.73,31.83,29.07,28.85,25.40$. HRMS - ESI $(m / z):[\mathrm{M}+\mathrm{Na}]^{+}$calculated for $\mathrm{C}_{12} \mathrm{H}_{24} \mathrm{NO}_{3}{ }^{+}, 230.1751$; found, 230.1742 .

Preparation of $(S, Z)$-2-Bromo-4-((tert-butyldimethylsilyl)oxy)-4-(( $S)$-2,2-dimethyl1,3-dioxolan-4-yl)but-2-enoic Acid, 264.

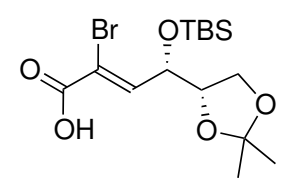

To a solution of ethyl-2-(triphenylphosphoranylidene)acetate $(0.459 \mathrm{~g}, 1.317 \mathrm{mmol}, 1.5$ equiv) in DCE $(2.9 \mathrm{~mL})$ at $-20{ }^{\circ} \mathrm{C}$ was added NBS $(0.266 \mathrm{~g}, 1.49 \mathrm{mmol}, 1.7$ equiv). The reaction was stirred at $-20{ }^{\circ} \mathrm{C}$ for an hour before being warmed to room temperature. $\mathrm{K}_{2} \mathrm{CO}_{3}$ $(0.303 \mathrm{~g}, 2.195 \mathrm{mmol}, 2.5$ equiv) was added, followed by $(R)-2$-((tert-butyldimethylsilyl)oxy)-2((S)-2,2-dimethyl-1,3-dioxolan-4-yl)acetaldehyde ( $0.241 \mathrm{~g}, 0.878 \mathrm{mmol}, 1$ equiv). The reaction was stirred overnight at room temperature before being heated to $60{ }^{\circ} \mathrm{C}$ for 3 hours, cooled back to room temperature and quenched with $\mathrm{H}_{2} \mathrm{O}$. The reaction mixture was extracted with $\mathrm{CH}_{2} \mathrm{Cl}_{2}$ and the combined organics dried over $\mathrm{MgSO}_{4}$, filtered and concentrated in vacuo. The crude product was purified by silica gel chromatography (10\% EtOAc/Pet. Ether). The desired ester was isolated as a clear colorless oil (0.223 g, 60\% yield). ${ }^{\mathbf{1}} \mathbf{H}$ NMR $(500 \mathrm{MHz}$, $\left.\mathrm{CDCl}_{3}\right) \delta 7.20(\mathrm{~d}, J=8.5 \mathrm{~Hz}, 1 \mathrm{H}), 4.67(\mathrm{dd}, J=8.5,4.6 \mathrm{~Hz}, 1 \mathrm{H}), 4.37-4.25(\mathrm{~m}, 2 \mathrm{H}), 4.21$ (ddd, $J=6.7,5.8,4.7 \mathrm{~Hz}, 1 \mathrm{H}), 4.09-3.93(\mathrm{~m}, 2 \mathrm{H}), 1.41(\mathrm{~d}, J=0.8 \mathrm{~Hz}, 3 \mathrm{H}), 1.39-1.32(\mathrm{~m}, 6 \mathrm{H}), 0.90$ $(\mathrm{s}, 9 \mathrm{H}), 0.11(\mathrm{~s}, 3 \mathrm{H}), 0.06(\mathrm{~s}, 3 \mathrm{H})$. This product was dissolved in $\mathrm{MeOH} / \mathrm{CH}_{2} \mathrm{Cl}_{2}(1: 9,5 \mathrm{~mL})$ and $\mathrm{NaOH}$ (0.084 g, $2.1 \mathrm{mmol}$, 4 equiv) was added at room temperature. The reaction was stirred overnight before being diluted with $\mathrm{H}_{2} \mathrm{O}$ and washed with $\mathrm{CH}_{2} \mathrm{Cl}_{2}$. The aqueous layer was then acidified by the dropwise addition of saturated aqueous $\mathrm{KHSO}_{4}$ and extracted with EtOAc. The combined organics were concentrated in vacuo and the title compound isolated as a clear oil $(0.114 \mathrm{~g}, 55 \%$ yield $) .{ }^{1} \mathbf{H}$ NMR $\left(500 \mathrm{MHz}, \mathrm{CDCl}_{3}\right) \delta 7.35(\mathrm{~d}, J=8.5 \mathrm{~Hz}, 1 \mathrm{H})$, $4.69(\mathrm{dd}, J=8.5,4.3 \mathrm{~Hz}, 1 \mathrm{H}), 4.25$ (ddd, $J=6.6,5.7,4.3 \mathrm{~Hz}, 1 \mathrm{H}), 4.03(\mathrm{qd}, J=8.7,6.1 \mathrm{~Hz}$, $2 \mathrm{H}), 1.42(\mathrm{~s}, 3 \mathrm{H}), 1.36(\mathrm{~s}, 3 \mathrm{H}), 0.90(\mathrm{~s}, 9 \mathrm{H}), 0.11(\mathrm{~s}, 3 \mathrm{H}), 0.07(\mathrm{~s}, 3 \mathrm{H}) .{ }^{\mathbf{1 3}} \mathrm{C}$ NMR $(126 \mathrm{MHz}$, $\left.\mathrm{CDCl}_{3}\right) \delta 165.73,146.52,115.44,110.10,77.92,72.60,64.94,26.09,25.68,25.20,18.08,-4.51$, -4.97. HRMS - ESI $(m / z)$ : $[\mathrm{M}-\mathrm{H}]^{-}$calculated for $\mathrm{C}_{15} \mathrm{H}_{26} \mathrm{BrO}_{5} \mathrm{Si}^{-}, 393.0738$; found, 393.0720.

Preparation of (4S,Z)-1-(methoxy(methyl)amino)-1-oxodec-9-en-3-yl 2-bromo4-((tert-butyldimethylsilyl)oxy)-4-((S)-2,2-dimethyl-1,3-dioxolan-4-yl)but-2-enoate, 268.

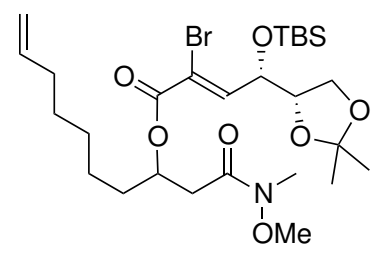

To a solution of (S,Z)-2-bromo-4-((tert-butyldimethylsilyl)oxy)-4-(( $S)$-2,2-dimethyl-1,3dioxolan-4-yl)but-2-enoic acid $(0.053 \mathrm{~g}, 0.134 \mathrm{mmol}, 1.0$ equiv), 3-Hydroxy- $N$-methoxy- $N$ methyldec-9-enamide (0.034 g, $0.147 \mathrm{mmol}, 1.1$ equiv) and DMAP (1.6 mg, $0.013 \mathrm{mmol}, 0.1$ 
equiv) in $\mathrm{CH}_{2} \mathrm{Cl}_{2}(0.67 \mathrm{~mL})$ at $0{ }^{\circ} \mathrm{C}$ was added $\mathrm{EDCI} \cdot \mathrm{HCl}(0.028 \mathrm{~g}, 0.147 \mathrm{mmol}, 1.1$ equiv). The reaction was allowed to warm to room temperature and stirred for 5 hours before concentration under a stream of nitrogen and purification using silica gel chromatography (30\% EtOAc/Pet. Ether). The title compound was isolated as a clear film (0.035 g, $43 \%$ yield). $\mathbf{R}_{\mathbf{f}}=0.38$ (30\% EtOAc/Pet. Ether). ${ }^{1} \mathbf{H}$ NMR $\left(600 \mathrm{MHz}, \mathrm{CDCl}_{3}\right) \delta 7.15(\mathrm{t}, J=8.9 \mathrm{~Hz}, 1 \mathrm{H}), 5.83-5.72(\mathrm{~m}$, $1 \mathrm{H}), 5.35(\mathrm{td}, \mathrm{J}=6.4,3.7 \mathrm{~Hz}, 1 \mathrm{H}), 5.01-4.94(\mathrm{~m}, 1 \mathrm{H}), 4.92(\mathrm{~d}, \mathrm{~J}=10.1 \mathrm{~Hz}, 1 \mathrm{H}), 4.65(\mathrm{td}, \mathrm{J}$ $=8.5,4.6 \mathrm{~Hz}, 1 \mathrm{H}), 4.22-4.16(\mathrm{~m}, 1 \mathrm{H}), 3.99(\mathrm{ddd}, \mathrm{J}=8.7,6.7,3.4 \mathrm{~Hz}, 1 \mathrm{H}), 3.94(\mathrm{ddd}, \mathrm{J}=$ 8.4, 5.8, $2.2 \mathrm{~Hz}, 1 \mathrm{H}), 3.68(\mathrm{~s}, 3 \mathrm{H}), 3.19-3.11(\mathrm{~m}, 3 \mathrm{H}), 2.92-2.81(\mathrm{~m}, 1 \mathrm{H}), 2.65(\mathrm{ddd}, \mathrm{J}=15.8$, $5.9,2.9 \mathrm{~Hz}, 1 \mathrm{H}), 2.02(\mathrm{tt}, \mathrm{J}=6.6,3.1 \mathrm{~Hz}, 3 \mathrm{H}), 1.77-1.67(\mathrm{~m}, 2 \mathrm{H}), 1.44-1.26(\mathrm{~m}, 14 \mathrm{H}), 0.88(\mathrm{~s}$, $11 \mathrm{H}) .{ }^{13} \mathrm{C}$ NMR $\left(151 \mathrm{MHz}, \mathrm{CDCl}_{3}\right) \delta 170.76,161.34,161.27,143.78,143.55,138.91,138.89$, 116.91, 116.83, 114.32, 114.31, 109.87, 109.86, 78.02, 73.92, 73.85, 72.75, 72.72, 65.03, 65.00, $61.33,36.50,36.43,33.98,33.93,33.62,33.60,32.07,28.76$, 28.70, 28.67, 26.18, 26.17, 25.74, $25.73,25.72,25.70,25.69,25.37,25.30,25.02,24.97,18.09,-4.50,-4.51,-4.93,-4.95$. HRMS - ESI $(m / z):[\mathrm{M}+\mathrm{Na}]^{+}$calculated for $\mathrm{C}_{27} \mathrm{H}_{48} \mathrm{BrNO}_{7} \mathrm{SiNa}^{+}, 628.2276$; found, 628.2244.

\section{Preparation of tert-butyl 2-(diethoxyphosphoryl)acetate.}<smiles>CCOC(=O)CP(=O)(O)OCC</smiles>

To neat tert-butyl 2-bromoacetate $(9.46 \mathrm{~mL}, 64.1 \mathrm{mmol}, 1.005$ equiv) was added triethyl phosphite (11.1 mL, $63.8 \mathrm{mmol}, 1$ equiv). The reaction was heated to $100{ }^{\circ} \mathrm{C}$ and refluxed for 3 hours before cooling to room temperature and removal of bromoethane, the reaction byproduct, in vacuo. The desired product was isolated as a clear, colorless oil (16 g, 99\% yield) and used without further purification. ${ }^{1} \mathbf{H}-\mathbf{N M R}\left(300 \mathrm{MHz}, \mathrm{CDCl}_{3}\right): \delta 4.15(\mathrm{~m}, 4 \mathrm{H})$, $2.87(\mathrm{dd}, J=21.4,5.0 \mathrm{~Hz}, 2 \mathrm{H}), 1.46(\mathrm{~s}, 9 \mathrm{H}), 1.33(\mathrm{~m}, 6 \mathrm{H}) .{ }^{13} \mathbf{C}-\mathbf{N M R}\left(126 \mathrm{MHz}, \mathrm{CDCl}_{3}\right): \delta$ $164.90(\mathrm{~d}, J=6.3 \mathrm{~Hz}), 81.99,62.46(\mathrm{~d}, J=6.2 \mathrm{~Hz}), 35.57(\mathrm{~d}, J=133.1 \mathrm{~Hz}), 16.34(\mathrm{~d}, J=$ $6.4 \mathrm{~Hz}) .{ }^{31} \mathbf{P}-\mathbf{N M R}\left(121 \mathrm{MHz}, \mathrm{CDCl}_{3}\right): \delta 20.56$. Characterization data matches that reported in the literature. ${ }^{49}$

\section{Preparation of tert-butyl 2,2-dibromo-2-(diethoxyphosphoryl)acetate.}<smiles>CCOC(=O)C(Br)(Br)P(=O)(Br)OCC</smiles>

To a solution of $\mathrm{NaOH}\left(18.8 \mathrm{~g}, 470 \mathrm{mmol}, 9.4\right.$ equiv) in water $(60 \mathrm{~mL})$ at $0{ }^{\circ} \mathrm{C}$ was added bromine (11.85 mL, $230 \mathrm{mmol}, 4.6$ equiv) dropwise ovre 30 minutes enusring the internal temperature does not exceed $10{ }^{\circ} \mathrm{C}$. Tert-butyl 2-(diethoxyphosphoryl)acetate $(12.61 \mathrm{~g}, 50$ mmol, 1 equiv) was then added to the reaction mixture dropwise over 10 minutes, once again ensuring the internal temperature does not exceed $10{ }^{\circ} \mathrm{C}$. The reaction was stirred for an additional 30 minutes at $0{ }^{\circ} \mathrm{C}$ before being extracted with chloroform three times. The combined organic layers were washed with water, dried over $\mathrm{MgSO}_{4}$, filtered and concentrated in vacuo. The desired product was isolated as a yellow oil (18.12 g, $88 \%$ yield) and was used without further purification. ${ }^{\mathbf{1}} \mathbf{H}-\mathbf{N M R}\left(300 \mathrm{MHz}, \mathrm{CDCl}_{3}\right): \delta 4.38(\mathrm{~m}, 4 \mathrm{H}), 1.53(\mathrm{~s}, 9 \mathrm{H}), 1.39$ $(\mathrm{m}, 6 \mathrm{H}) .{ }^{13} \mathrm{C}-\mathrm{NMR}\left(126 \mathrm{MHz}, \mathrm{CDCl}_{3}\right): \delta 162.21(\mathrm{~d}, J=3.6 \mathrm{~Hz}), 85.82,66.21(\mathrm{~d}, J=7.2$ $\mathrm{Hz}), 49.02(\mathrm{~d}, J=158.5 \mathrm{~Hz}), 27.45,16.42(\mathrm{~d}, J=6.0 \mathrm{~Hz}) .{ }^{31} \mathbf{P}-\mathbf{N M R}\left(121 \mathrm{MHz}, \mathrm{CDCl}_{3}\right)$ : $\delta$ 8.33. Characterization data matches that reported in the literature. ${ }^{50}$ 
<smiles>CCOC(=O)C(Br)P(=O)(OCC)OCC</smiles>

\section{Preparation of tert-butyl 2-bromo-2-(diethoxyphosphoryl)acetate.}

To a solution of tert-butyl 2,2-dibromo-2-(diethoxyphosphoryl)acetate (18.14 g, $44.2 \mathrm{mmol}$, 1 equiv) in ethanol $(50 \mathrm{~mL})$ at $0{ }^{\circ} \mathrm{C}$ was added a suspension of $\mathrm{SnCl}_{2}(7.97 \mathrm{~g}, 42.0 \mathrm{mmol}, 0.95$ equiv) in water $(70 \mathrm{~mL})$, dropwise using an addition funnel. The reaction mixture was stirred at $0{ }^{\circ} \mathrm{C}$ for 20 minutes before warming to room temperature and extracting twice with chloroform. The combined organics were dried over $\mathrm{MgSO}_{4}$, filtered and concentrated in vacuo. The desired product was isolated as a clear, colorless oil $(12.11,83 \%$ yield) and was used without further purification. ${ }^{1} \mathbf{H}-\mathbf{N M R}\left(300 \mathrm{MHz}, \mathrm{CDCl}_{3}\right): \delta 4.46-4.07(\mathrm{~m}, 5 \mathrm{H}), 1.49(\mathrm{~s}, 9 \mathrm{H}), 1.37$ (t, $J=$ $7.1 \mathrm{~Hz}, 6 \mathrm{H}) .{ }^{13} \mathbf{C}-\mathbf{N M R}\left(126 \mathrm{MHz}, \mathrm{CDCl}_{3}\right): \delta 163.79,84.05,64.51(\mathrm{~m}), 37.36(\mathrm{~d}, J=145.1$ $\mathrm{Hz}), 27.80(\mathrm{~d}, J=28.3 \mathrm{~Hz}), 16.37(\mathrm{~d}, J=6.1 \mathrm{~Hz}) .{ }^{31} \mathbf{P}-\mathbf{N M R}\left(121 \mathrm{MHz}, \mathrm{CDCl}_{3}\right): \delta 13.09$. Characterization data matches that reported in the literature. ${ }^{50}$

\section{Preparation of 2-Bromo-2-(diethoxyphosphoryl)acetic Acid, 287.}<smiles>CCOP(=O)(OC)C(Br)C(=O)O</smiles>

To tert-butyl 2-bromo-2-(diethoxyphosphoryl)acetate (4.0 g, $12.08 \mathrm{mmol}, 1$ equiv) was added a solution of $\mathrm{CH}_{2} \mathrm{Cl}_{2} / \mathrm{TFA} / \mathrm{H}_{2} \mathrm{O}(20: 10: 1,150 \mathrm{~mL})$. The reaction mixture was stirred at room temperature for 4 hours before being concentrated in vacuo $\left(<40{ }^{\circ} \mathrm{C}\right)$. The title compound was obtained as a slight yellow oil $\left(2.54 \mathrm{~g}, 76 \%\right.$ yield). ${ }^{\mathbf{1}} \mathbf{H}-\mathbf{N M R}(300 \mathrm{MHz}$, DMSO-d $\left._{6}\right): \delta 4.98(\mathrm{~d}, J=14.5 \mathrm{~Hz}, 1 \mathrm{H}), 4.22-3.92(\mathrm{~m}, 5 \mathrm{H}), 1.23(\mathrm{~m}, 6 \mathrm{H}) .{ }^{13}$ C-NMR $(126$ MHz, DMSO-d $\left.{ }_{6}\right): \delta 166.44,79.41(\mathrm{~d}, J=66.4 \mathrm{~Hz}), 64.02,16.57 .{ }^{31} \mathbf{P}-\mathrm{NMR}(121 \mathrm{MHz}$, DMSO- $\left.\mathrm{d}_{6}\right): \delta 18.78$. Characterization data matches that reported in the literature. ${ }^{51}$

Preparation of 1-(Methoxy(methyl)amino)-1-oxodec-9-en-3-yl 2-bromo-2(diethoxyphosphoryl)acetate, 289.

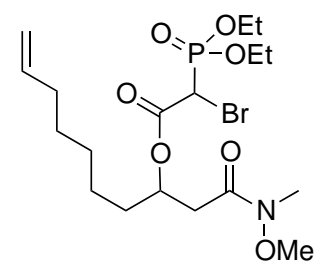

To a solution of 2-bromo-2-(diethoxyphosphoryl)acetic acid (0.148 g, $0.536 \mathrm{mmol}, 1.5$ equiv) and 3-hydroxy- $N$-methoxy- $N$-methyldec- 9 -enamide $(0.082 \mathrm{~g}, 0.358 \mathrm{mmol}, 1$ equiv) in $\mathrm{CH}_{2} \mathrm{Cl}_{2}(1.8 \mathrm{~mL})$ was added EDCI $\cdot \mathrm{HCl}(0.099 \mathrm{~g}, 0.518 \mathrm{mmol}, 1.45$ equiv) and DMAP $(4 \mathrm{mg}$, $0.036 \mathrm{mmol}, 0.1$ equiv). The reaction was sealed and stirred overnight before being diluted with $\mathrm{CH}_{2} \mathrm{Cl}_{2}$, filtered through a silica plug (EtOAc wash), and concentrated in vacuo. The crude product was purified by silica gel chromatography (60\% EtOAc/Pet. Ether), The title compound was isolated as a clear colorless oil (0.113 g, 65\% yield). $\mathbf{R}_{\mathbf{f}}=0.14$ (60\% EtOAc/Pet. Ether). ${ }^{1} \mathbf{H}$ NMR $\left(500 \mathrm{MHz}, \mathrm{CDCl}_{3}\right) \delta 5.80$ (ddt, $\left.J=16.9,10.3,6.8 \mathrm{~Hz}, 1 \mathrm{H}\right), 5.37$ (h, $J=$ $6.4 \mathrm{~Hz}, 1 \mathrm{H}), 4.99(\mathrm{dd}, J=17.3,2.2 \mathrm{~Hz}, 1 \mathrm{H}), 4.93(\mathrm{~d}, J=10.1 \mathrm{~Hz}, 1 \mathrm{H}), 4.46-4.21(\mathrm{~m}, 5 \mathrm{H})$, $3.71(\mathrm{~s}, 3 \mathrm{H}), 3.18(\mathrm{~s}, 3 \mathrm{H}), 2.86(\mathrm{ddd}, J=21.6,15.9,6.7 \mathrm{~Hz}, 1 \mathrm{H}), 2.71-2.59(\mathrm{~m}, 1 \mathrm{H}), 2.04(\mathrm{q}$, 
$J=7.0,5.6 \mathrm{~Hz}, 2 \mathrm{H}), 1.71(\mathrm{q}, J=6.9 \mathrm{~Hz}, 2 \mathrm{H}), 1.39(\mathrm{dt}, J=12.2,7.1 \mathrm{~Hz}, 12 \mathrm{H}) .{ }^{13} \mathbf{C} \mathbf{~ N M R}$ $\left(126 \mathrm{MHz}, \mathrm{CDCl}_{3}\right) \delta 170.57,165.49,164.23,138.91,114.32,74.07,73.99,65.67,65.61,64.89$, $64.83,64.81,64.75,64.68,61.36,36.69,36.41,36.31,35.53,35.16,35.10,33.94,33.66,32.11$, $28.78,28.72,24.85,16.38,16.33,16.31,16.29,16.26,16.24$. HRMS - ESI $(m / z):[\mathrm{M}+\mathrm{H}]^{+}$ calculated for $\mathrm{C}_{18} \mathrm{H}_{34} \mathrm{BrNO}_{7} \mathrm{P}^{+}, 486.1251$; found, 486.1257 .

Preparation of ( $Z$ )-1-(Methoxy(methyl)amino)-1-oxodec-9-en-3-yl 2-bromo-3((R)-2,2-dimethyl-1,3-dioxolan-4-yl)acrylate, 291.

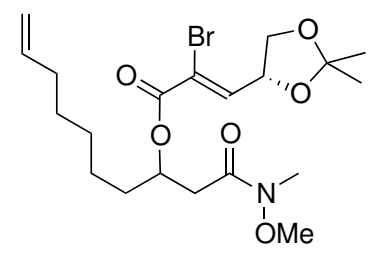

To a solution of 1-(methoxy(methyl)amino)-1-oxodec-9-en-3-yl 2-bromo-2-

(diethoxyphosphoryl)acetate $\left(0.060 \mathrm{~g}, 0.123 \mathrm{mmol}, 1\right.$ equiv) in $\mathrm{THF} / \mathrm{H}_{2} \mathrm{O}(40: 1,0.6 \mathrm{~mL})$ at $0{ }^{\circ} \mathrm{C}$ was added activated $\mathrm{Ba}(\mathrm{OH})_{2}(0.036 \mathrm{~g}, 0.21 \mathrm{mmol}, 1.7$ equiv). The reaction mixture was stirred for 30 minutes before the addition of $(S)$-2,2-dimethyl-1,3-dioxolane-4-carbaldehyde (0.032 g, $0.247 \mathrm{mmol}, 2$ equiv). The reaction was stirred overnight at room temperature before quenching with saturated aqueous $\mathrm{NaHCO}_{3}$. The reaction mixture was extracted with $\mathrm{Et}_{2} \mathrm{O}$ and the combined organics were dried over $\mathrm{MgSO}_{4}$, filtered and concentrated in vacuo. The crude product was purified by silica gel chromatography (30\% EtOAc/Pet. Ether). The title compound was isolated as a clear film $(0.011 \mathrm{~g}, 19 \%$ yield $) . \mathbf{R}_{\mathbf{f}}=0.3(30 \%$ EtOAc/Pet. Ether $)$. ${ }^{1} \mathbf{H}$ NMR $\left(600 \mathrm{MHz}, \mathrm{CDCl}_{3}\right)$ MIXTURE OF ALKENE ISOMERS: $\delta 7.34(\mathrm{~d}, J=6.7 \mathrm{~Hz}$, $1 \mathrm{H}), 6.77(\mathrm{~d}, J=7.0 \mathrm{~Hz}, 1 \mathrm{H}), 5.79(\mathrm{~m}, 2 \times 1 \mathrm{H}), 5.36(\mathrm{~m}, 2 \times 1 \mathrm{H}), 4.99(\mathrm{ddd}, J=17.2,3.4,1.7$ $\mathrm{Hz}, 2 \times 1 \mathrm{H}), 4.96-4.90(\mathrm{~m}, 2 \times 1 \mathrm{H}), 4.31$ (ddd, $J=8.1,6.7,1.3 \mathrm{~Hz}, 2 \times 1 \mathrm{H}), 3.70(\mathrm{~s}, 3 \mathrm{H}), 3.69(\mathrm{~s}$, $3 \mathrm{H}), 3.17(\mathrm{~s}, 3 \mathrm{H}), 3.16(\mathrm{~s}, 3 \mathrm{H}), 2.85(\mathrm{~m}, 2 \times 1 \mathrm{H}), 2.71-2.59(\mathrm{~m}, 2 \times 1 \mathrm{H}), 2.08-1.98(\mathrm{~m}, 2 \times 2 \mathrm{H})$, 1.76-1.65 (m, $2 \times 2 \mathrm{H}), 1.45$ (m, $2 \times 6 \mathrm{H}), 1.42-1.29(\mathrm{~m}, 2 \times 6 \mathrm{H}) .{ }^{\mathbf{1 3}} \mathbf{C} \mathbf{N M R}\left(151 \mathrm{MHz}, \mathrm{CDCl}_{3}\right)$ MIXTURE OF ALKENE ISOMERS: $\delta$ 170.72, 161.58, 160.99, 160.97, 148.43, 147.90, 143.93, 138.94, 138.91, 138.87, 118.60, 114.37, 114.34, 110.30, 110.04, 75.60, 75.58, 74.57, 73.85, 73.82, $73.66,73.64,69.10,69.07,68.08,61.66,61.38,61.34,36.45,36.36,34.00,33.67,33.63,32.44$, $32.11,28.77,28.75,28.67,28.64,28.14,26.53,26.40,25.52,25.37,25.01,24.99$. IR (film): 2984, 2930, 2858, 1729, 1666, 1640, 1461, 1373, 1219, 1154, $1062 \mathrm{~cm}^{-1}$. HRMS - ESI $(\mathrm{m} / z)$ : $[\mathrm{M}+\mathrm{H}]^{+}$calculated for $\mathrm{C}_{20} \mathrm{H}_{33} \mathrm{BrNO}_{6}{ }^{+}, 462.1486$; found, 462.1499 . 
Preparation of ( $Z)$-1-(methoxy(methyl)amino)-1-oxodec-9-en-3-yl 2-bromo-3phenylacrylate, 292.

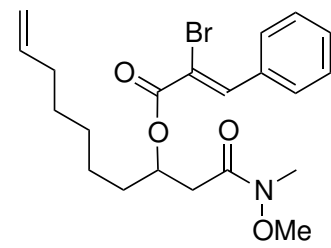

To a solution of 1-(methoxy(methyl)amino)-1-oxodec-9-en-3-yl 2-Bromo-2(diethoxyphosphoryl)acetate $\left(0.047 \mathrm{~g}, 0.097 \mathrm{mmol}, 1\right.$ equiv) in $\mathrm{THF} / \mathrm{H}_{2} \mathrm{O}(40: 1,0.48 \mathrm{~mL})$ at 0 ${ }^{\circ} \mathrm{C}$ was added activated $\mathrm{Ba}(\mathrm{OH})_{2}(0.028 \mathrm{~g}, 0.164 \mathrm{mmol}, 1.7$ equiv). The reaction mixture was stirred for 30 minutes before the addition of benzaldehyde $(0.021 \mathrm{~g}, 0.193 \mathrm{mmol}, 2$ equiv). The reaction was stirred overnight at room temperature before quenching with saturated aqueous $\mathrm{NaHCO}_{3}$. The reaction mixture was extracted with $\mathrm{Et}_{2} \mathrm{O}$ and the combined organics were dried over $\mathrm{MgSO}_{4}$, filtered and concentrated in vacuo. The crude product was purified by silica gel chromatography (20\% EtOAc/Pet. Ether). The title compound was isolated as a clear film (0.011 g, $26 \%$ yield). $\mathbf{R}_{\mathbf{f}}=0.32\left(20 \%\right.$ EtOAc/Pet. Ether). ${ }^{\mathbf{1}} \mathbf{H N M R}(500 \mathrm{MHz}$, $\left.\mathrm{CDCl}_{3}\right) \delta 8.20(\mathrm{~s}, 1 \mathrm{H}), 7.85(\mathrm{~m}, 2 \mathrm{H}), 7.43(\mathrm{~m}, 2 \mathrm{H}), 7.32($ app. s, $1 \mathrm{H}), 5.80$ (ddt, $J=12.9,10.2$, $6.7 \mathrm{~Hz}, 1 \mathrm{H}$ ), 5.44 (app. dt, $J=12.6,6.2 \mathrm{~Hz}, 1 \mathrm{H}$ ), 4.99 (app. d, $J=17.2 \mathrm{~Hz}, 1 \mathrm{H}$ ), 4.93 (app. d, $J=10.2 \mathrm{~Hz}, 1 \mathrm{H}), 3.72(\mathrm{~s}, 3 \mathrm{H}), 3.19(\mathrm{~s}, 3 \mathrm{H}), 2.93(\mathrm{dd}, J=15.5,7.0 \mathrm{~Hz}, 1 \mathrm{H}), 2.72(\mathrm{dd}, J=$ 15.6, $5.9 \mathrm{~Hz}, 1 \mathrm{H}), 2.03(\mathrm{~m}, 3 \mathrm{H}), 1.78(\mathrm{q}, J=7.3 \mathrm{~Hz}, 2 \mathrm{H}), 1.48-1.27(\mathrm{~m}, 6 \mathrm{H}) .{ }^{13} \mathbf{C}$ NMR $(126$ $\left.\mathrm{MHz}, \mathrm{CDCl}_{3}\right) \delta 170.91,162.72,140.79,138.95,133.79,130.29,130.12,128.80,128.41,128.38$, 128.23, 128.06, 114.33, 73.74, 61.37, 34.11, 33.65, 28.83, 28.71, 25.07. IR (film): 2929, 2857, 1720, 1665, 1611, 1447, 1388, 1256, 1198, $999 \mathrm{~cm}^{-1}$. HRMS - ESI $(\mathrm{m} / z):[\mathrm{M}+\mathrm{H}]^{+}$calculated for $\mathrm{C}_{21} \mathrm{H}_{29} \mathrm{BrNO}_{4}{ }^{+}$, 438.1274; found, 438.1292 . 


\subsubsection{Spectral Data}
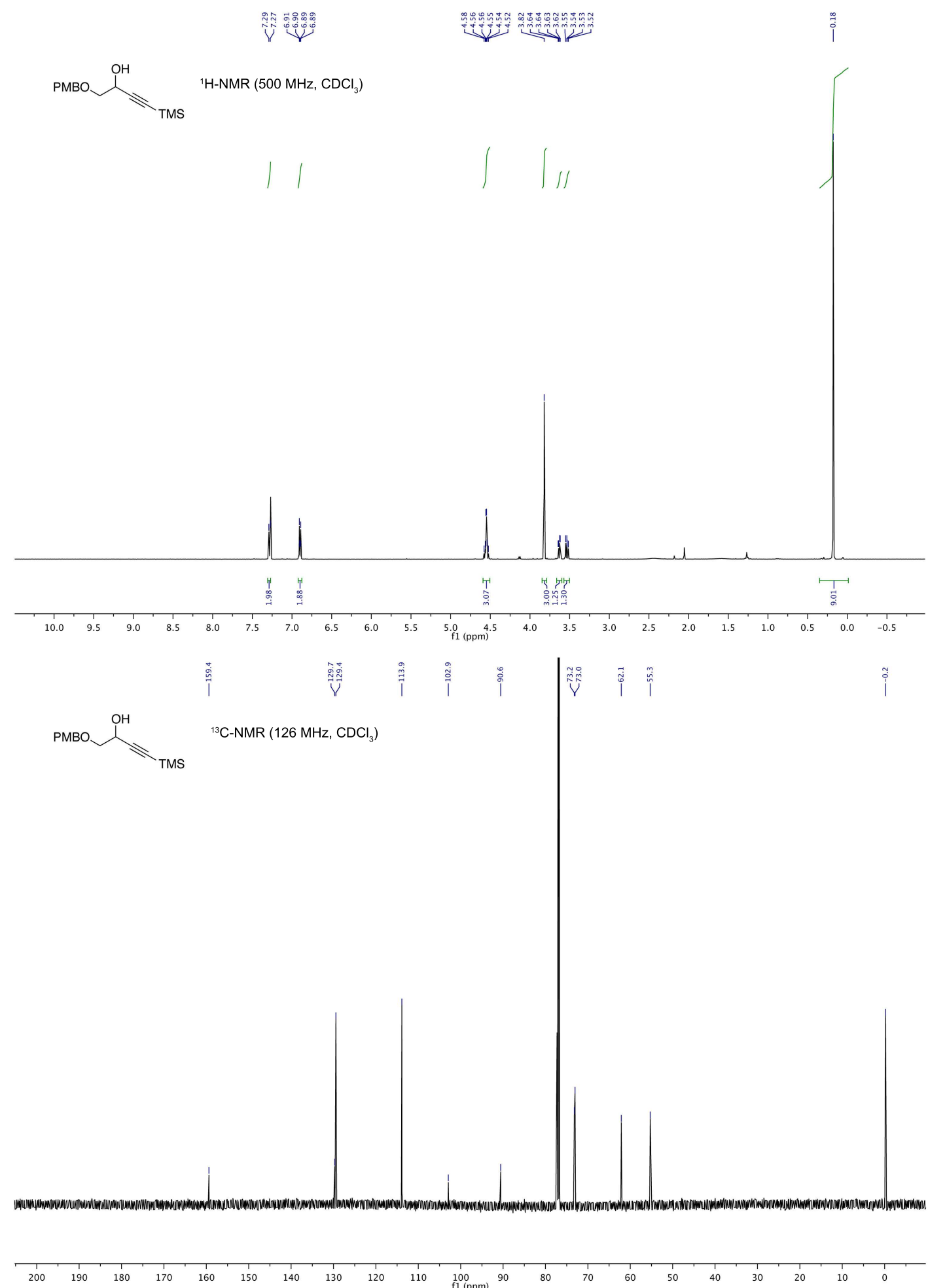
5.6. EXPERIMENTAL DATA FOR CHAPTER 5

193
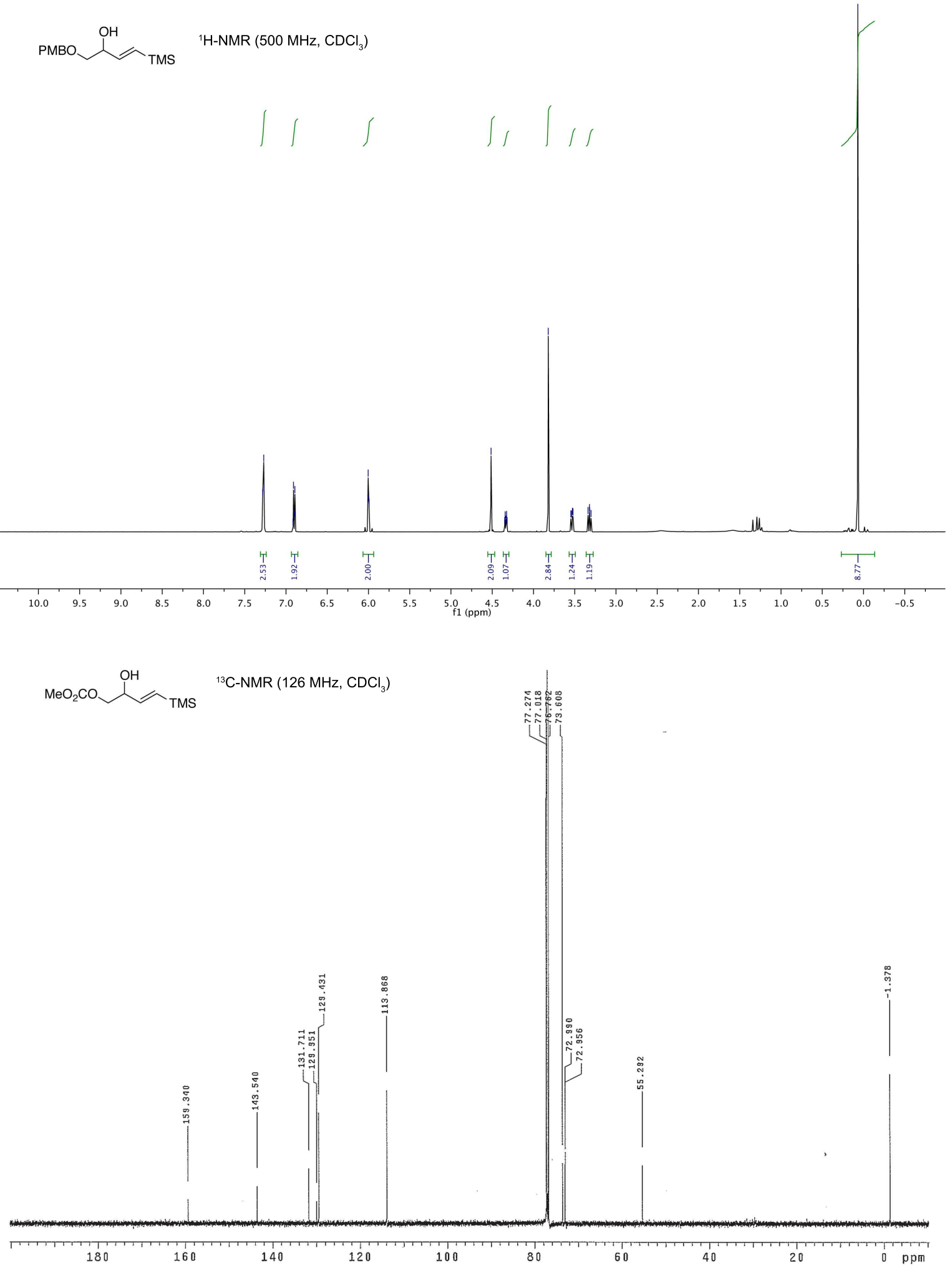


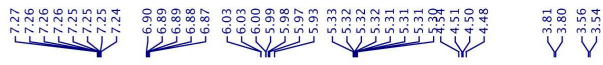

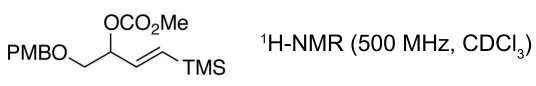

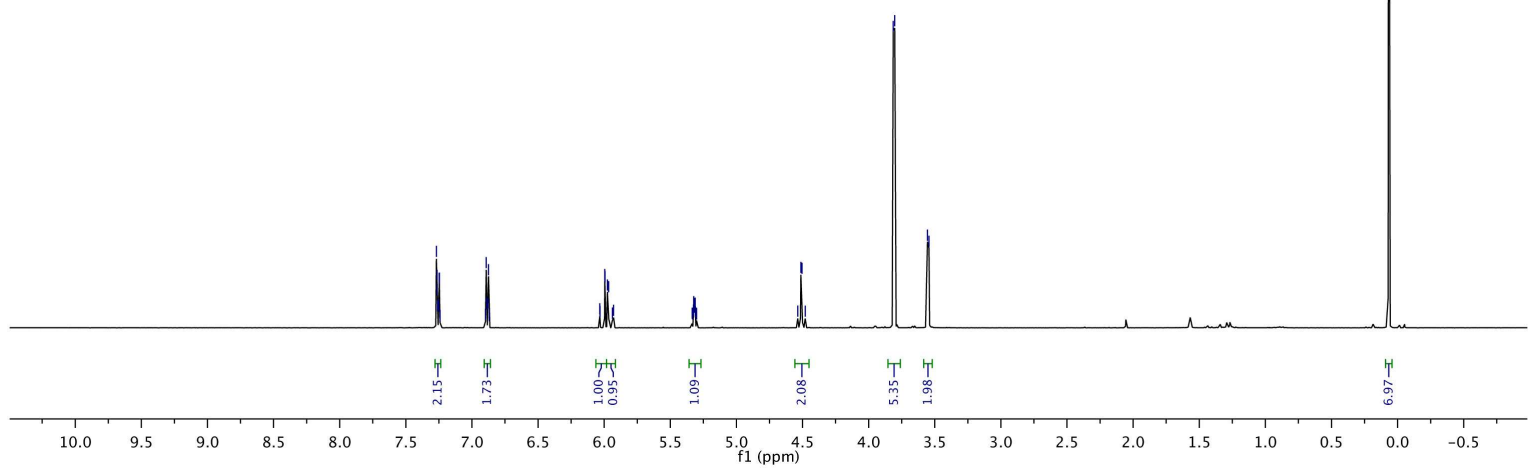

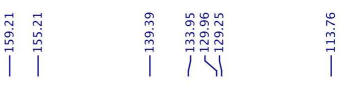

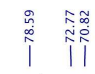

PMBO

${ }^{13} \mathrm{C}-\mathrm{NMR}\left(126 \mathrm{MHz}, \mathrm{CDCl}_{3}\right)$

ไู่
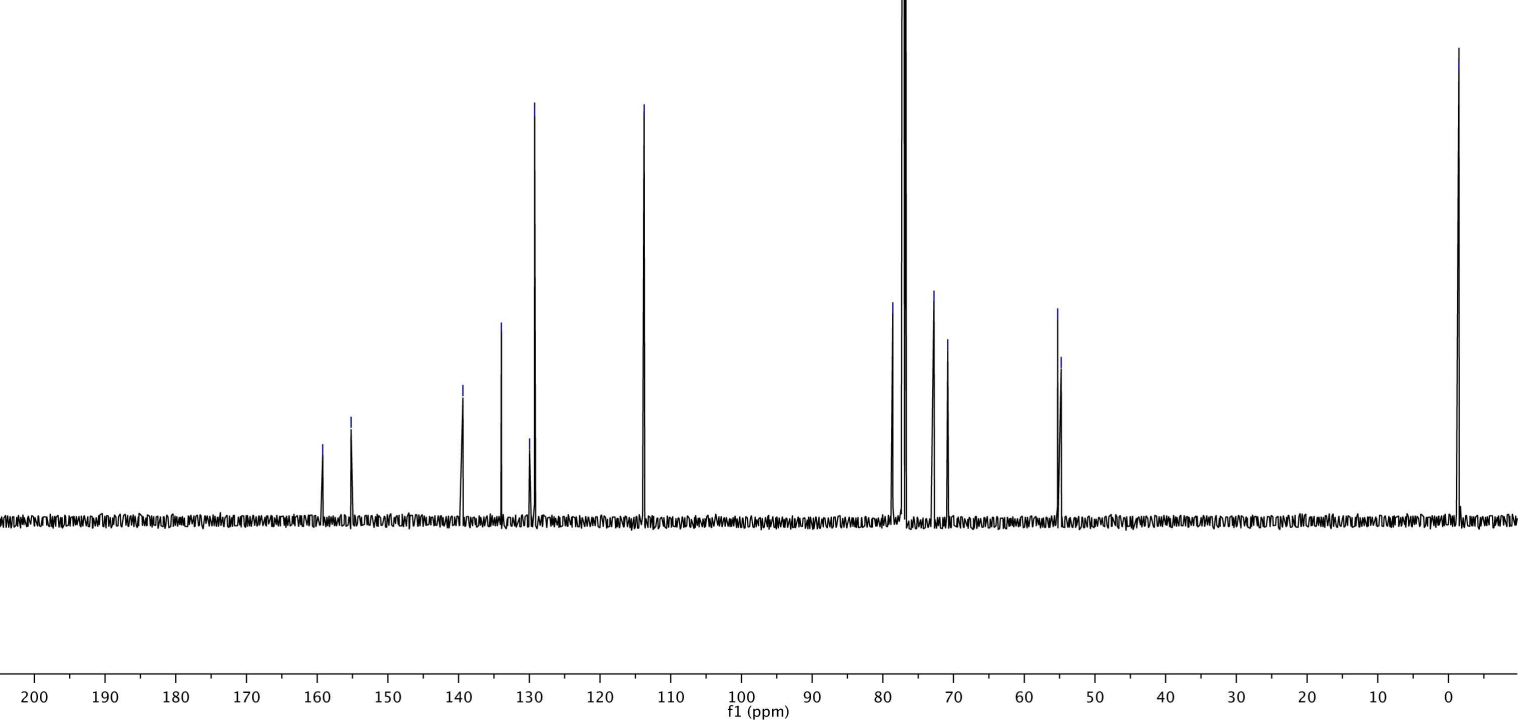

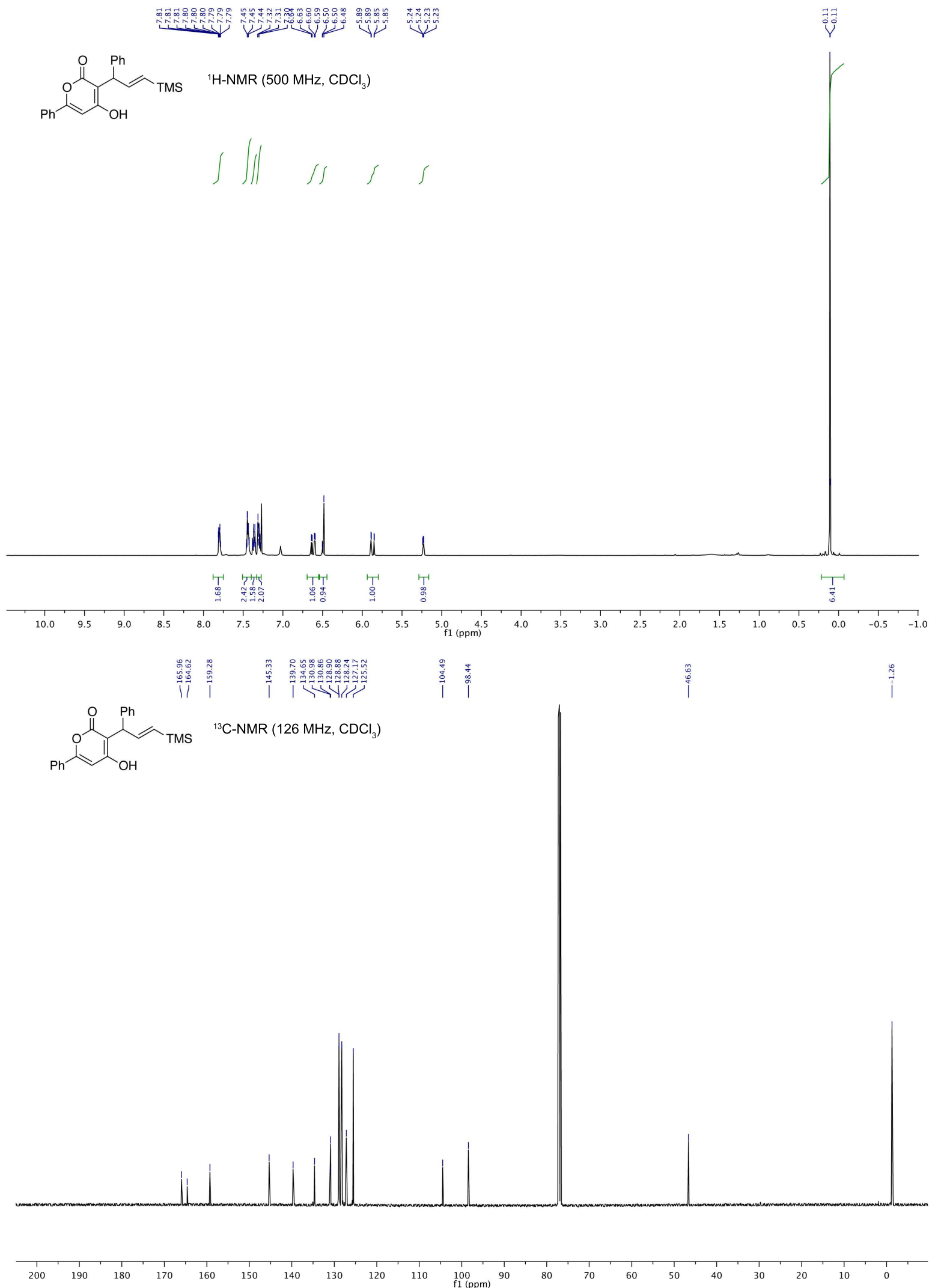
5.6. EXPERIMENTAL DATA FOR CHAPTER 5

196
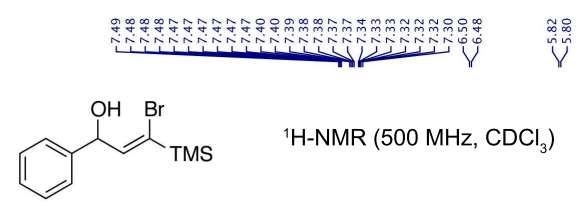

Ifs
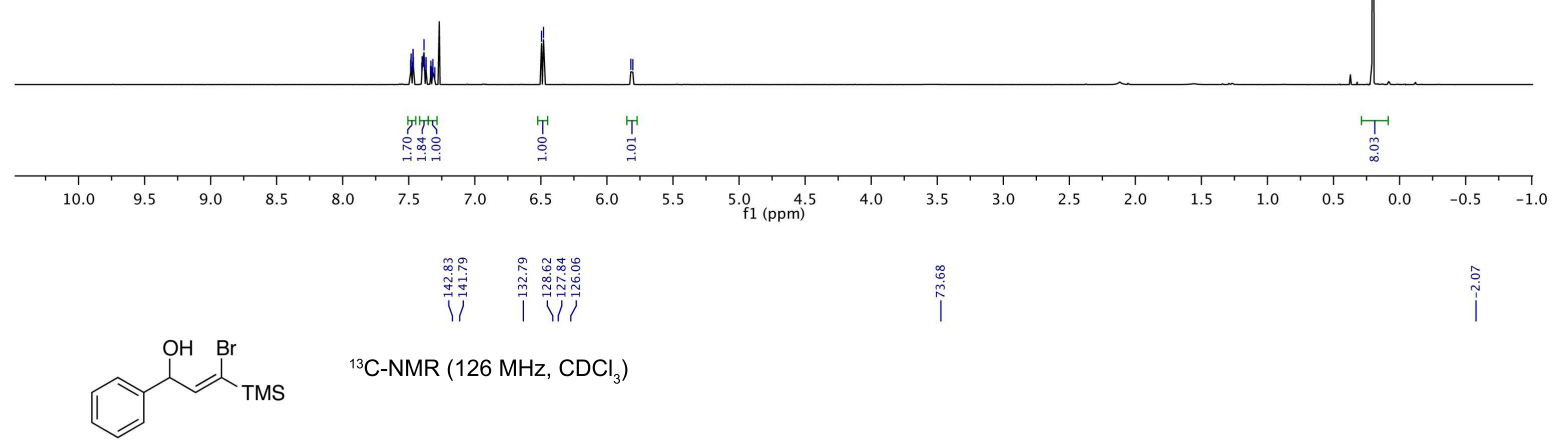

${ }^{13} \mathrm{C}-\mathrm{NMR}\left(126 \mathrm{MHz}, \mathrm{CDCl}_{3}\right)$

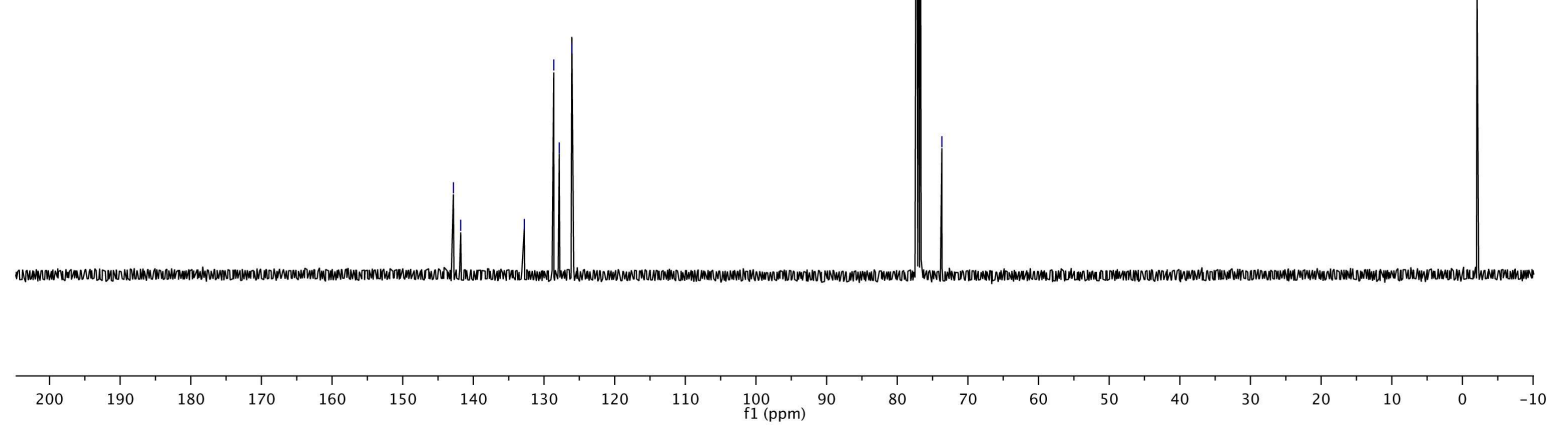



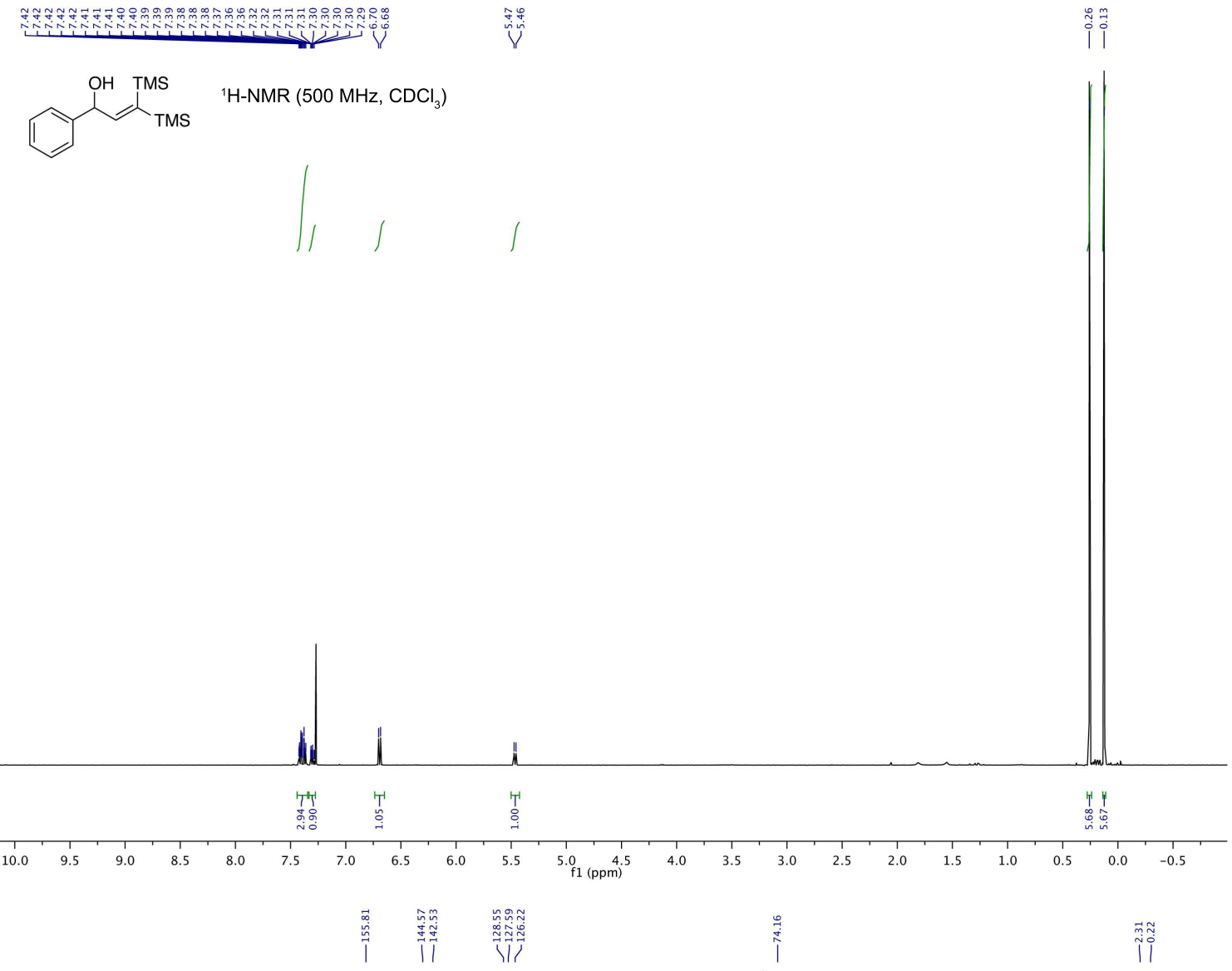

Iิ

$\mathrm{OH} \quad \mathrm{TMS} \quad{ }^{13} \mathrm{C}-\mathrm{NMR}\left(126 \mathrm{MHz}, \mathrm{CDCl}_{3}\right)$

TMS

(a)

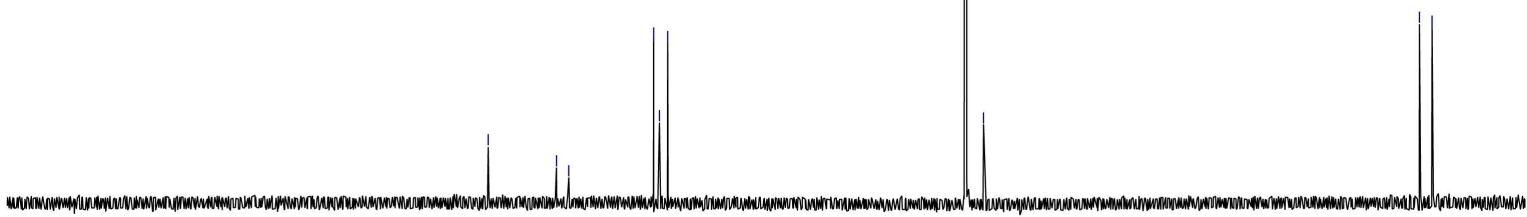

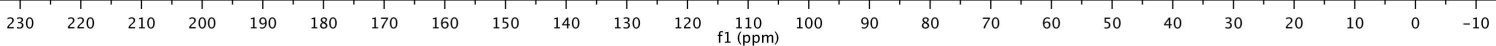




$$
\begin{aligned}
& =1 \\
& \\
&
\end{aligned}
$$




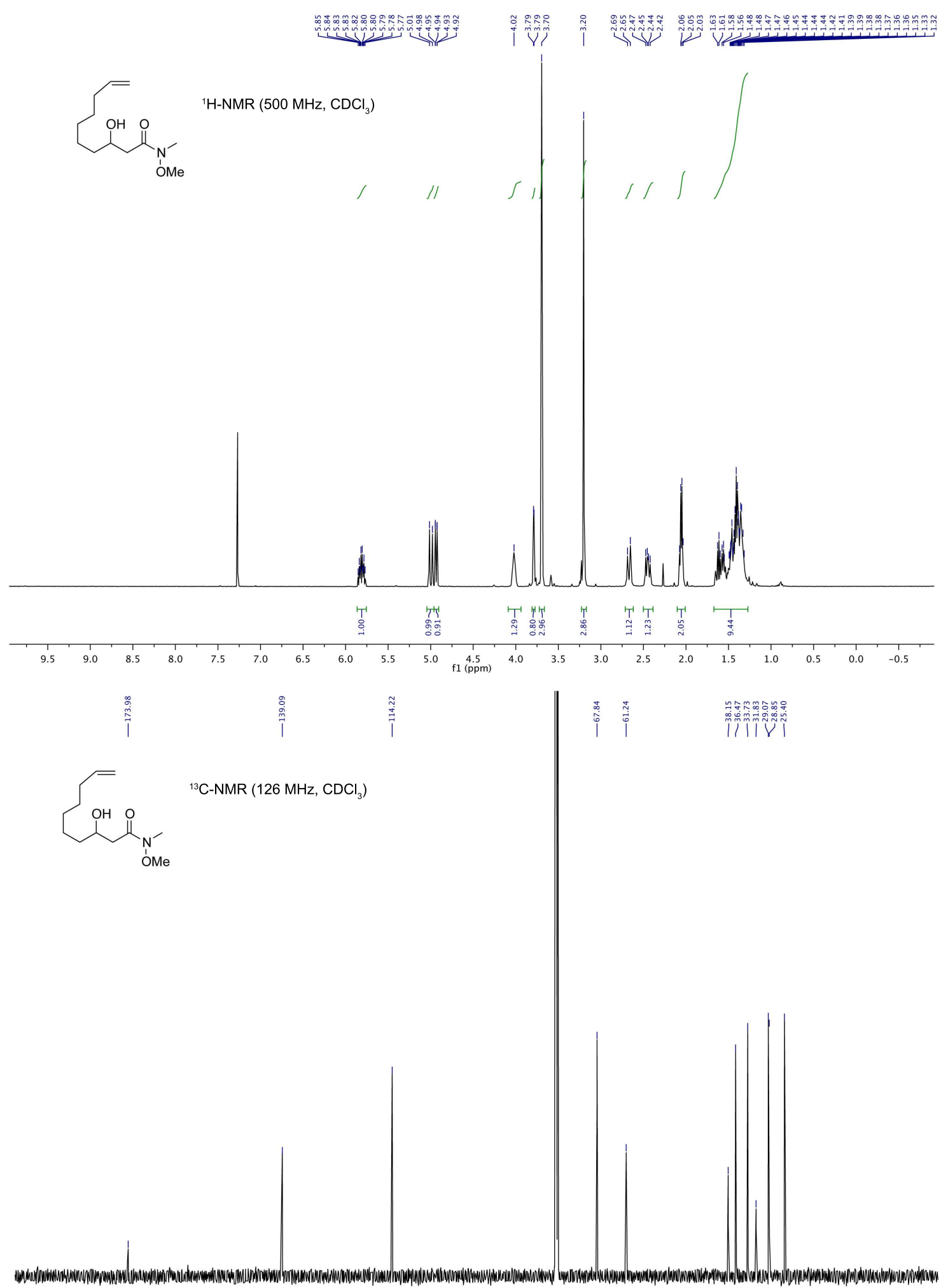

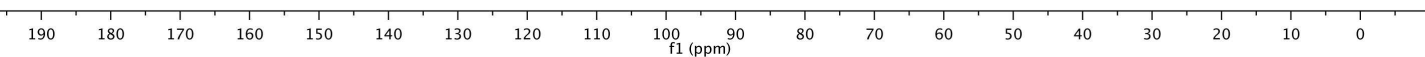




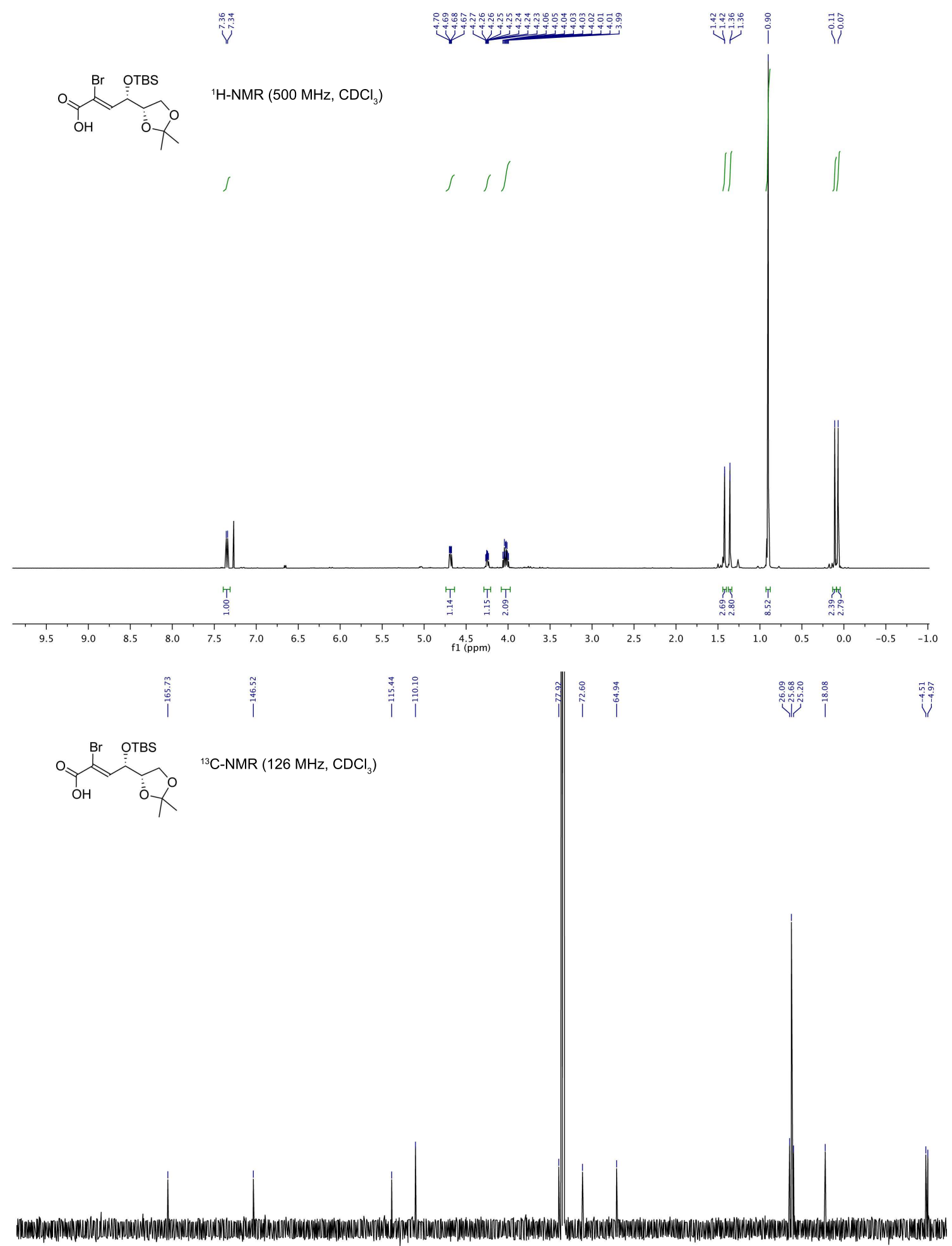

$\begin{array}{llllllllll}190 & 180 & 170 & 160 & 150 & 140 & 130 & 120 & 110 & \underset{f}{100}(\mathrm{fpm}) \\ \mathrm{f} 1 & 90\end{array}$ 


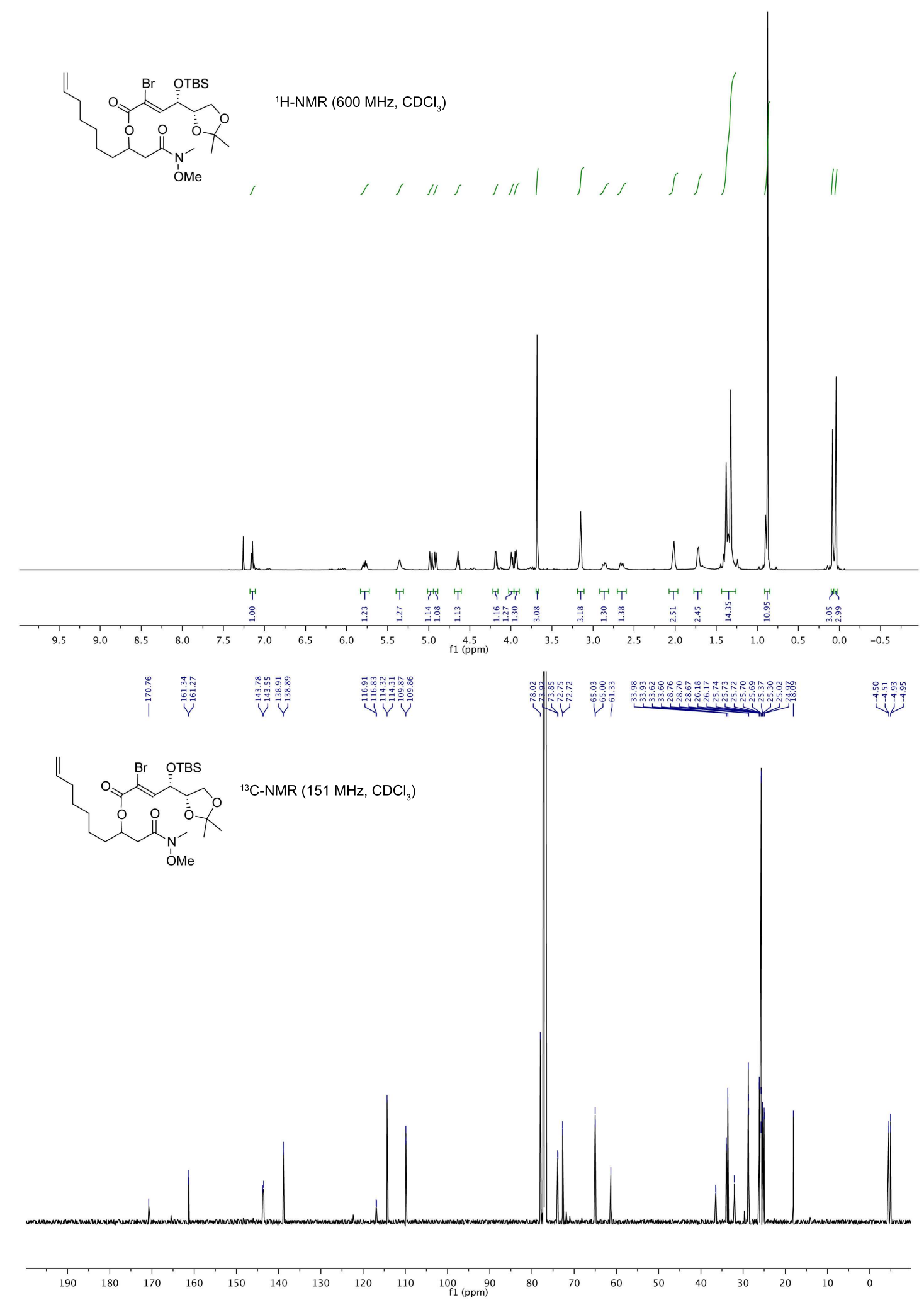




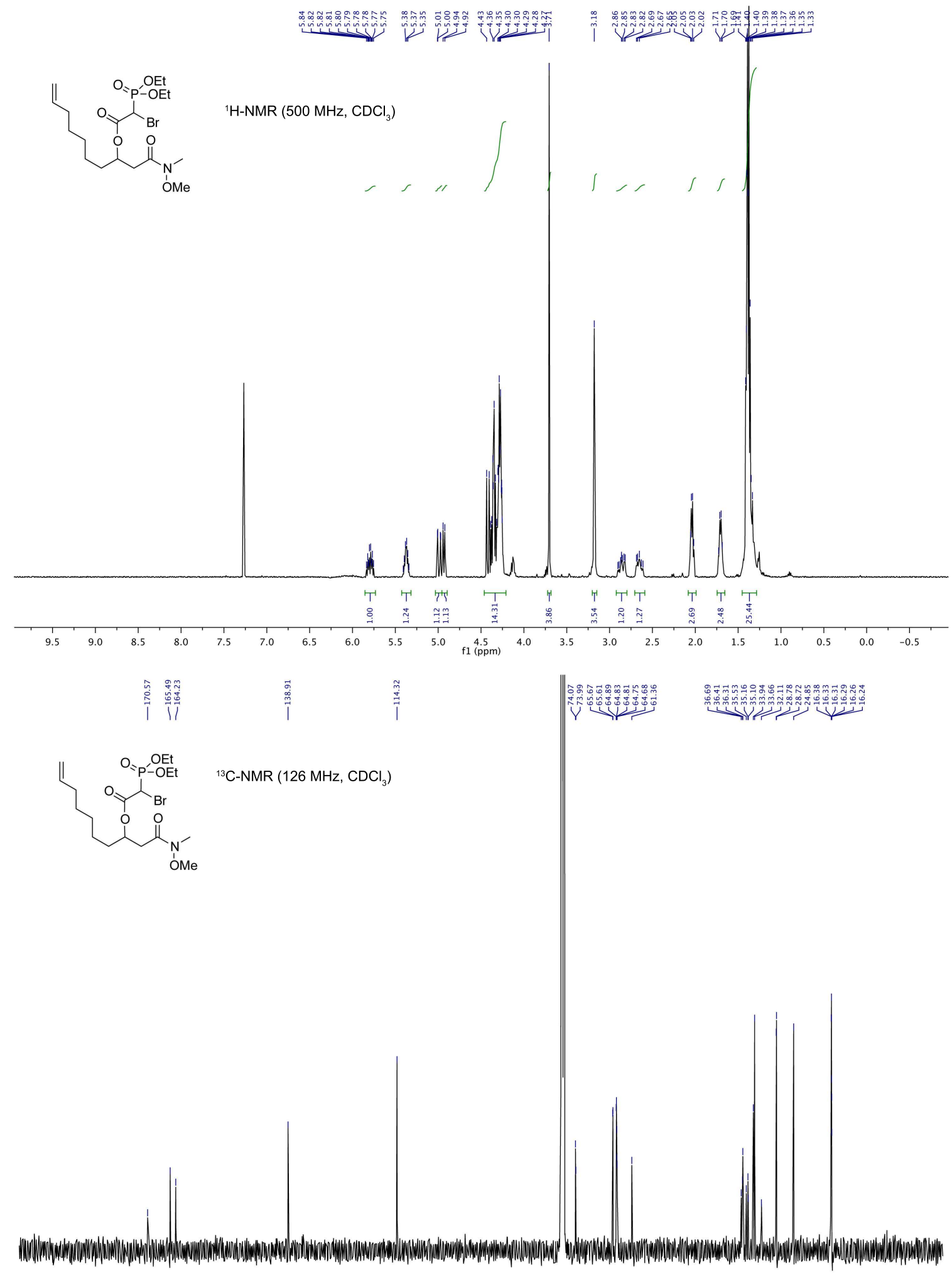

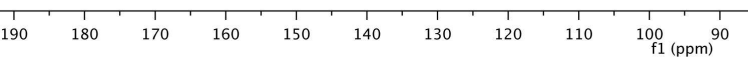



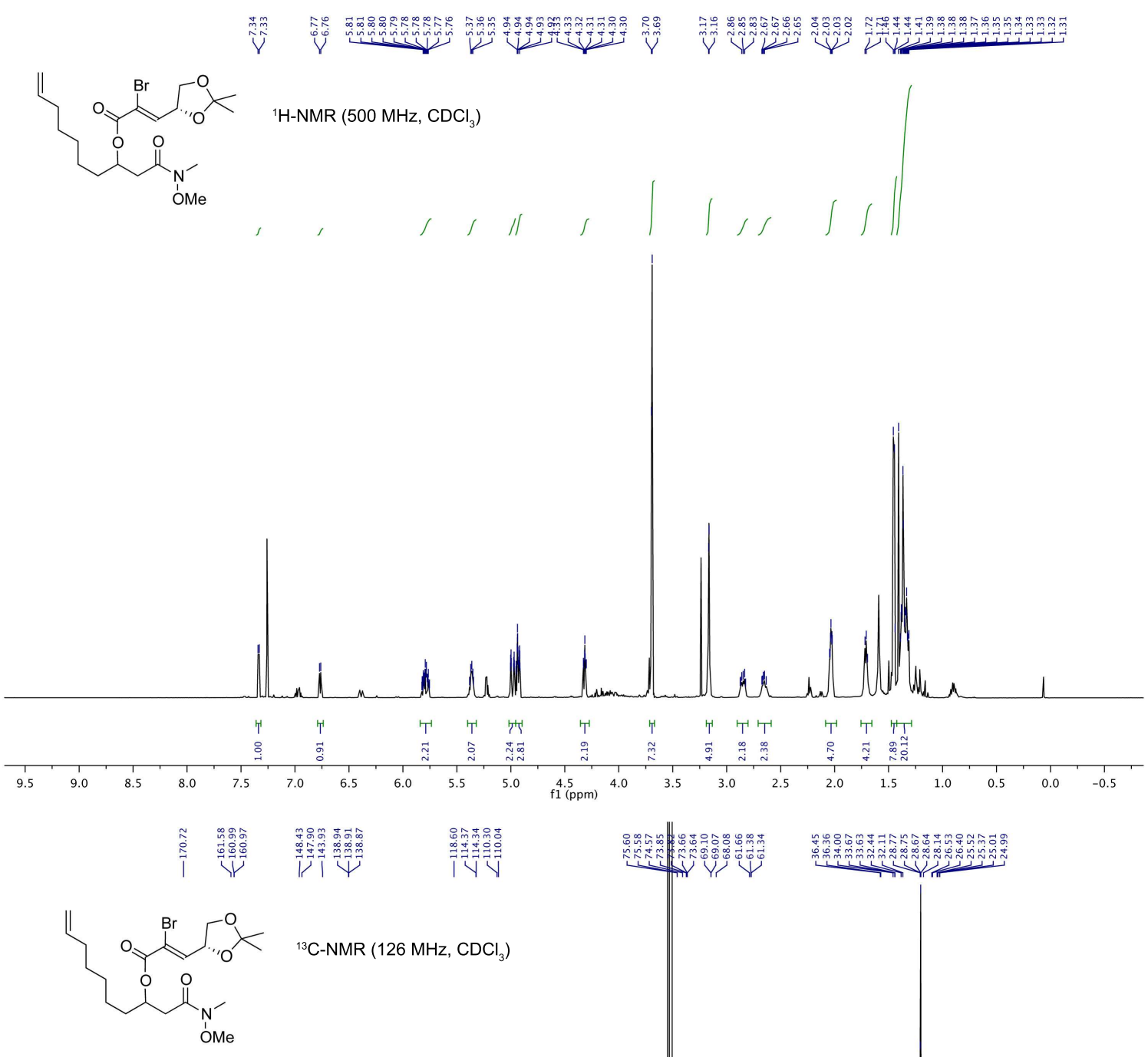

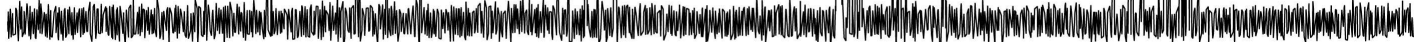

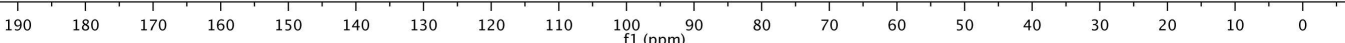



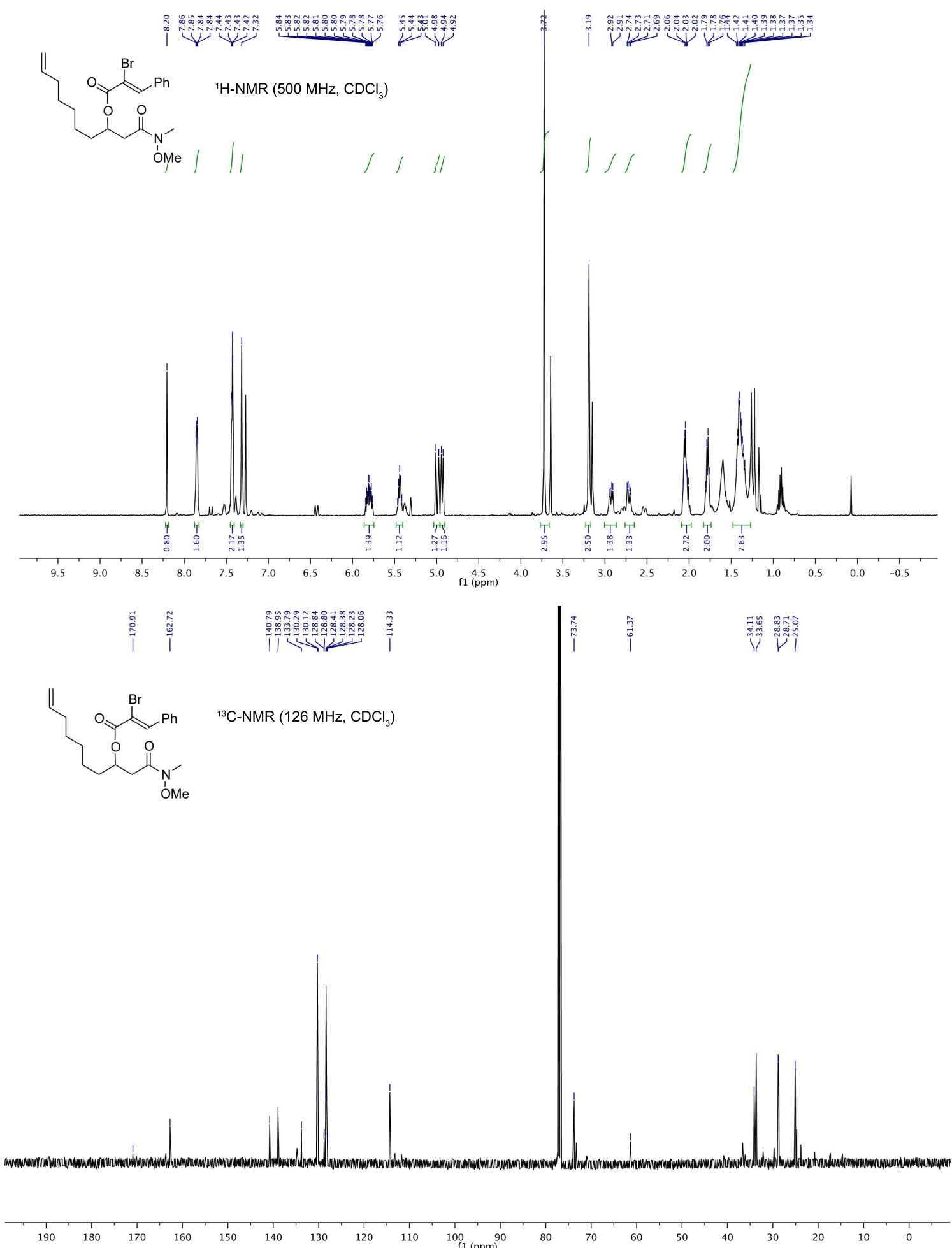


\section{References for Chapter 5}

[1] Popplewell, W. Isolation and Structure Elucidation of New Secondary Metabolites from New Zealand Marine Red Algae. Ph.D. Thesis, Victoria University of Wellington, 2008.

[2] Kobayashi, Y.; Lee, J.; Tezuka, K.; Kishi, Y. Org. Lett. 1999, 1, 2177. Kobayashi, Y.; Lee, J.; Tezuka, K.; Kishi, Y. Org. Lett. 1999, 1, 2181. Seike, H.; Ghosh, I.; Kishi, Y. Org. Lett. 2006, 8, 3861. Seike, H.; Ghosh, I.; Kishi, Y. Org. Lett. 2006, 8, 3865. Dambruoso, P.; Bassarello, C.; Bifulco, G.; Appendino, G.; Battaglia, A.; Fontana, G.; Gomez-Paloma, L. Org. Lett. 2005, 7, 983.

[3] The chemical shift differences shown in Figure 5.2 are uncorrected and intended to simply illustrate the typical differences observed between diastereomers of three adjacent stereocenters. For additional details, see: Kobayashi, Y.; Tan, C.-H.; Kishi, Y. J. Am. Chem. Soc. 2001, $123,2076$.

[4] Stout, E. P.; Hasemeyer, A. P.; Lane, A. L.; Davenport, T. M.; Engel, S.; Hay, M. E.; Fairchild, C. R.; Prudhomme, J.; Le Roch, K.; Aalbersbeger, Kubanek, J. Org. Lett. 2009, 11, 225.

[5] Corey, E. J.; Cheng, X. The Logic of Chemical Synthesis; John Wiley and Sons: New York, 1989

[6] Negishi, E. Handbook of Organopalladium Chemistry for Organic Synthesis; John Wiley and Sons: New York, 2002

[7] Tsuji, J. Palladium Reagents and Catalysts: Innovations in Organic Synthesis; John Wiley and Sons: Chichester, 1995.

[8] Tsuji, J.; Takahashi, H.; Morikawa, M. Tetrahedron Letters 1965, 49, 4387.

[9] Trost, B. M.; Van Vranken, D. Chem. Rev. 1996, 96, 395. Trost, B. M.; Crawley, M. Chem. Rev. 2003, 103, 2921.

[10] Fiaud, J.; Legros, J. J. Org. Chem. 1987, 52, 1907.

[11] Matsushita, H.; Negishi, E. Chem. Commun. 1982, 160.

[12] Takahashi, T.; Jinbo, Y.; Kitamura, K.; Tsuji, J. Tetrahedron Lett. 1984. 25, 5921.

[13] For a recent example of altering the reaction conditions to achieve regiocontrol in Pd-AA reactions, see: Trost, B. M.; Malhotra, S.; Chan, W. H. J. Am. Chem. Soc. 2011, 133, 7328.

[14] Hoke, M. E.; Brescia, M.-R.; Bogaczyk, S.; DeShong, P.; King, B. W.; Crimmins, M. T. J. Org. Chem. 2002, 67, 327. M. E.; Brescia, Shimshock, Y. C.; DeShong, P. J. Org. Chem. 1997, 62, 1257.

[15] Moreno-Mañas, M.; Ribas, J.; Virgili, A. J. Org. Chem. 1988, 53, 5328.

[16] Moreno-Mañas, M.; Prat, M.; Ribas, J.; Virgili, A. Tetrahedron Lett. 1988, 29, 581. Moreno-Mañas, M.; Ribas, J.; Virgili, A. J. Org. Chem. 1988, 53, 5328. Moreno-Manas, M.; Ribas, J. Tetrahedron Lett. 1989, 30, 3109. Prat, M.; Ribas, J.; Moreno-Mañas, M. Tetrahedron 1992, 48, 1695. Chase, C.; Bender, J.; West, F. Synlett 1996, 1173.

[17] Tsuji, T.; Yuhara, M.; Minato, M.; Yamada, H.; Sato, F.; Kobayashi, Y. Tetrahedron Lett. 1988, $29,343$.

[18] Barrett, A. G. M.; Flygare, J. A. J. Org. Chem. 1991, 56, 638. Barrett, A. G. M.; Flygare, J. A.; Hill, J. M.; Wallace, E. M. Org. Synth. 1996, 73, 50. Barrett, A. G. M.; Hill, J. M.; Wallace, E. M.; Flygare, J. A. Synlett 1991, 764 .

[19] Prat, M.; Moreno-Mañas, M.; Ribas, J. Tetrahedron 1988, 44, 7205.

[20] Balacco, G. J. Chem. Inf. Comput. Sci. 1996, 36 885. Haasnoot, C. A. G.; de Leeuw, F. A. A. M.; Altona, C. Tetrahedron, 1981, 36, 2783. Donders, L. A.; de Leeuw, Altona, C. Magn. Reson. Chem. 1989, $27,556$. Karplus, M. J. Am. Chem. Soc. 1963, 852870.

[21] Napolitano, J. G.; Gavìn, J. A.; García, C.; Norte, M.; Fernández, J. J.; Hernández Daranas, A. Chem. Eur. J. 2011, 17, 6338. 
[22] B3LYP functional: Becke, A. D. J. Chem. Phys. 1993, 98, 5648. Stephens, P. J.; Devlin, P. J.; Chabalowski, C. F.; Frisch, M. J. J. Phys. Chem. 1994, 98, 11623. 6-31G(d) basis set: Frisch, M. J.; Pople, J. A.; Binkley, J. S. J. Chem. Phys. 1984, 80, 3265. Ditchfield, R.; Hehre, W. J.; Pople, J. A. J. Chem. Phys. 1971, 54, 724.

[23] Smith, S. M.; Goodman, J. M. J. Org. Chem. 2009, 74, 4597.

[24] For related examples of the inside alkoxy effect, see: Houk, K. N.; Moses, S. R.; Wu, Y. D.; Rondan, N. G.; Jager, V.; Schohe, R.; Fronczek, F. R. J. Am. Chem. Soc. 1984, 106, 3880. Houk, K. N.; Duh, H.-Y.; Wu, Y.-D.; Moses, S. R. J. Am. Chem. Soc. 1986, 108, 2754. Raimondi, L.; Wu, Y.-D.; Brown, F. K.; Houk, K. N. Tetrahedron 1992, 31, 4409. Haller, J.; Strassner, T.; Houk, K. N. J. Am. Chem. Soc. 1997, $119,8031$. Haller, J.; Niwayama, S.; Duh, H.-Y.; Houk, K. N. J. Org. Chem. 1997, 62, 5728.

[25] Hoffmann, R. W. Chem. Rev. 1989, 89, 1841.

[26] Guindon, Y.; Soucy, F.; Yoakim, C.; Ogilvie, W. W.; Plamondon, L. J. Org. Chem. 2001, 66, 8992.

[27] Trost, B. M.; Lee, C. J. Am. Chem. Soc. 2001, 123, 12191.

[28] For examples of $B$-alkyl Suzuki macrocyclizations, see: Chemler, S. R.; Danishefsky, S. J. Org. Lett. 2000, 17, 2695. Kallan, N. C.; Halcomb, R. L. Org. Lett. 2000, 17, 2687. Mohr, P. J.; Halcomb, R. L. J. Am. Chem. Soc. 2003, 125, 1712. Kawada, H.; Iwamoto, M.; Utsugi, M.; Miyano, M.; Nakada, M. J. Am. Chem. Soc. 2004, 6, 4491. Williams, D. R.; Walsh, M. J.; Miller, N. A. J. Am. Chem. Soc. 2009, 131, 9038. Jia, Y.; Bois-Choussy, M.; Zhu, J. Org. Lett. 2007, 9, 2401.

[29] For an example of the difficulty associated with sterically hindered ( $Z$ )-vinyl iodides in Pd-catalyzed cross coupling, see: Gebhardt, B.; König, Schleth, C.; Dauber, M.; Koert, U. Chem. Eur. J. 2010, 16, 5934.

[30] A recent report by Baran and coworkers provides an instructive example of the development of conditions for the oxidation of a partially saturated ring to the corresponding fully unsaturated system. Burns, N. Z.; Krylova, I. N.; Hannoush, R. N.; Baran, P. S. J. Am. Chem. Soc. 2009, 131, 9172.

[31] Lombardo, M.; Gualandi, A.; Pasi, F.; Trombini, C. Adv. Synth. Catal. 2007, 349, 465.

[32] For a review on the preparation of activated zinc, see: Ender, E. Tetrahedron 1987, 43, 2203.

[33] Girgis, M. J.; Liang, J. K.; Du, Z.; Slade, J.; Prasad, K. Org. Proc. Res. Dev. 2009, 13, 1094. Loh, G.; Tanigawara, R.; Shaik, S. M.; Sa-ei, K.; Wong, L.; Sharratt, P. N. Org. Proc. Res. Dev. 2012, 16, 958.

[34] Aldehyde 304 was found to be unstable and did not store well, even at $-5{ }^{\circ} \mathrm{C}$. Aldehyde prepared using a Moffat-Swern oxidation was typically of higher purity than material prepared using a TEMPO/BAIB oxidation. The addition of moderate to large amounts of the $\beta$-hydroxy amide to activated zinc dust often resulted in a significant exotherm and spontaneous reflux of the reaction mixture.

[35] Lilly, M. J.; Miller, N. A.; Edwards, A. J.; Willis, A. C.; Turner, P.; Paddon-Row, M. N.; Sherburn, M. S. Chem. Eur. J. 2005, 8, 2525.

[36] Vanderwal, C. D.; Vosburg, D. A.; Weiler, S.; Sorensen, E. J. J. Am. Chem. Soc. 2003, 125, 5393.

[37] Paquette, L. A.; Chang, J.; Liu, Z. J. Org. Chem. 2004, 69, 6441.

[38] Flann, C. J.; Overman, L. E. J. Am. Chem. Soc. 1987, 109, 6115.

[39] Munakata, R.; Katakai, H.; Ueki, T.; Kurosaka, J. Takao, K.; Tadano, K. J. Am. Chem. Soc. 2004, 126, 11254. Dineen, T. A.; Roush, W. R. Org. Lett. 2004, 6, 2043.

[40] Denmark, S. E.; Yang, S.-M. J. Am. Chem. Soc. 2004, 126, 12432.

[41] Rane, A. M.; Vaquer, J.; Colberg, J. C.; Soderquist, J. A. Tetrahedron Lett. 1995, 36, 987.

[42] Thaisrivongs, S.; Romero, D. L.; Tommasi, R. A.; Janakiraman, M. N.; Strohbach, J. W.; et al. J. Med. Chem. 1996, 39, 4630 .

[43] Guéret, S. M.; O’Connor, P. D.; Brimble, M. A. Org. Lett. 2009, 11, 963. 
[44] Nicolal, S.; Erard, S.; Gonzalez, D. F.; Waser, J. Org. Lett. 2010, 12, 384.

[45] Stoltenberg, D.; Lüthje, S.; Winkelmann, O.; Näther, C.; Lüning, U. Eur. J. Org. Chem. 2011, 5845.

[46] Snider, B. B.; Zhou, J. Org. Lett. 2006, 8, 1283.

[47] Donohoe, T. J.; Fishlock, L. P.; Basutto, J. A.; Bower, J. F.; Thompson, A. L.; Procopiou, P. A. Chem. Commun. 2009, 21, 3008.

[48] Weidner, K.; Giroult, A.; Panchaud, P.; Renaud, P. J. Am. Chem. Soc. 2010, 132, 7511.

[49] Ma, Y.; Collum, D. B. J. Am. Chem. Soc. 2007, 129, 14818.

[50] Baron, B. M.; Cregge, R. J.; Farr, R. A.; Friedrich, D.; Gross, R. S.; Harrison, B. L.; Janowick, D. A.; Matthews, D.; McCloskey, T. C.; Meikrantz, S.; Nyce, P. L.; et al. J. Med. Chem. 2005, 48, 995.

[51] Zhao, S.; He, Y.-H.; Wu, D.; Guan, Z. J. Fluorine Chem. 2010, textit131, 597.

[52] Thaisrivongs, S.; Romero, D.; Tommasi, R.; Janakiraman, M.; Strohbach, J.; Turner, S.; Biles, C.; Morge, R.; Johnson, P.; Aristoff, P.; Tomich, P.; Lynn, J.; Horng, M.; Chong, K.; Hinshaw, R.; Howe, W.; Finzel, B.; Watenpaugh, K. J. Med. Chem. 1996, 39, 4630.

[53] Brodifacoum Factsheet; New Zealand Department of Conservation. [Online]2007, http://search.doc.govt.nz/

[54] Jung, M.; Min, S. Tetrahedron 2007, 63, 3682.

[55] Whitton, P.; Lau, A.; Salisbury, A.; Whitehouse, J.; Evans, C. Phytochemistry 2003, 64, 673.

[56] Vyvyan, J. Tetrahedron 2002, 58, 1631.

[57] Kraus, G.; Zhang, N. J. Org. Chem. 2000, 65, 5644.

[58] Ang, K.; Tan, S. J. Chem. Soc. Perkin Trans. 1 1979, 1525.

[59] Moreno-Mañas, M.; Ribas, J.; Virgili, A J. Org. Chem. 1988, 53, 5328.

[60] Omura, S.; Sunazuka, T.; Chem. Rev. 2005, 105, 4559.

[61] Jung, J.; Park, O. Molecules 2009, 14, 4790. 
Chapter 6

Pd-AA Cascade I: Synthesis of the Core of Labillarides E-H 


\subsection{Alkylation Cascade in the Synthesis of Labillarides E-H}

Building on the research described in Chapter 5, the use of a Pd-AA cascade reaction with a 4-hydroxy- $\alpha$-pyrone bis-nucleophile was investigated with the ultimate goal of preparing labillarides E-H (Scheme 6.1). This cascade reaction consists of two sequential allylic alkylations and passes through an initial $C$-alkylated product to ultimately form a substituted furopyrone. The ability of this reaction to enable rapid and stereospecific formation of substituted furopyrones has great potential in the development of a concise total synthesis of labillarides E-H.

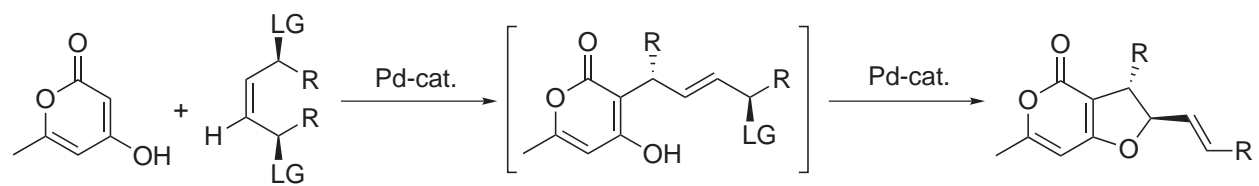

Scheme 6.1 Pd-AA Cascade with a 4-Hydroxy- $\alpha$-pyrone.

\subsection{Introduction}

\subsubsection{Pd-AA Cascades with Acyclic Bis-Electrophiles}

Palladium-catalyzed allylic alkylation (Pd-AA) cascades involve the sequential addition of a bis-nucleophile (B) to an allylic bis-electrophile (A) to ultimately achieve a net annulation (Scheme 6.2). ${ }^{1}$ Various combinations of nitrogen, oxygen and carbon bis-nucleophiles (B) have been used previously in highly efficient syntheses of vinyl-substituted ring systems (C) (vide infra). Given the numerous regiochemical possibilities that exist during the course of

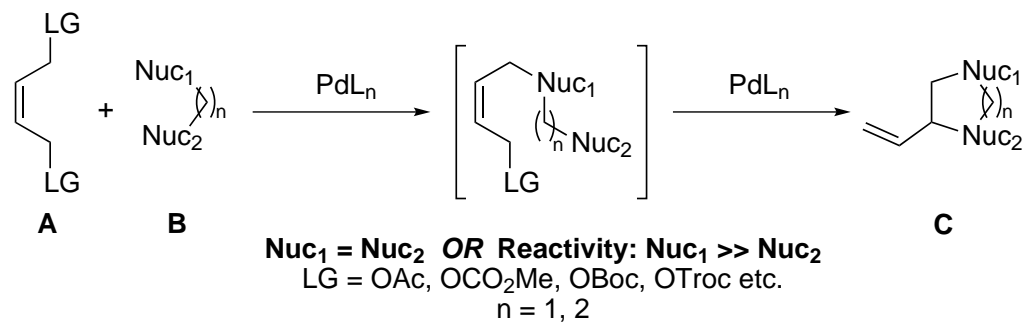

Scheme 6.2 Generic Pd-AA Cascade.

these reactions, strict regioselectivity is required to produce a single product (Scheme 6.3). Selectivity in the first alkylation is often driven by the preference for alkylation to occur at the less hindered terminal position of the $\pi$-allyl palladium complex. Regioselectivity in the second alkylation is due to 5(or 6)-exo cyclization being kinetically favoured over the competing 7 (or 8 )-endo cyclization. ${ }^{2}$ These reactions are typically performed with symmetric bis-electrophiles and therefore regioselectivity is, for the most part, dependent on one nucleophile being more reactive than the other (Scheme 6.2). 


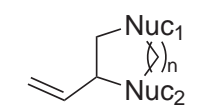

Nuc $_{1}$ attacks first

$$
\left\{\begin{array}{c}
\mathrm{Nuc}_{1} \\
()_{\mathrm{n}} \\
\mathrm{Nuc}_{2}
\end{array}\right.
$$

$\mathrm{Nuc}_{2}$ attacks first<smiles>O=C1C=CCN2CCN1C2</smiles>

second alkylation occurs at terminus

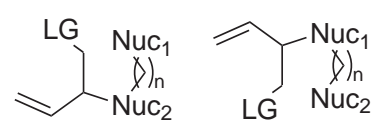

first alkylation gives branched product

Scheme 6.3 Regiochemical Possibilities of a Pd-AA casade with a Symmetric Bis-Electrophile.

Palladium allylic alkylation cascades have been successfully applied to the synthesis of substituted piperazines, ${ }^{3}$ piperidines,${ }^{4}$ morpholines, ${ }^{5}$ dioxanes,${ }^{6}$ oxazolidinones, ${ }^{7}$ dihydropyrans, ${ }^{8}$ dihydrofurans,${ }^{9}$ cyclohexanes, ${ }^{10,11}$ cyclopentanes, ${ }^{12}$ and cyclopropanes (Scheme 6.4). ${ }^{13}$ The majority of these methodologies use butene-1,4-dicarbonate substrates rather than the less reactive butene-1,4-diacetates. The enantioselective formation of substituted morpholines and dihydrofurans was achieved using the chiral phosphine ligands L10 and L11 Scheme 6.5.

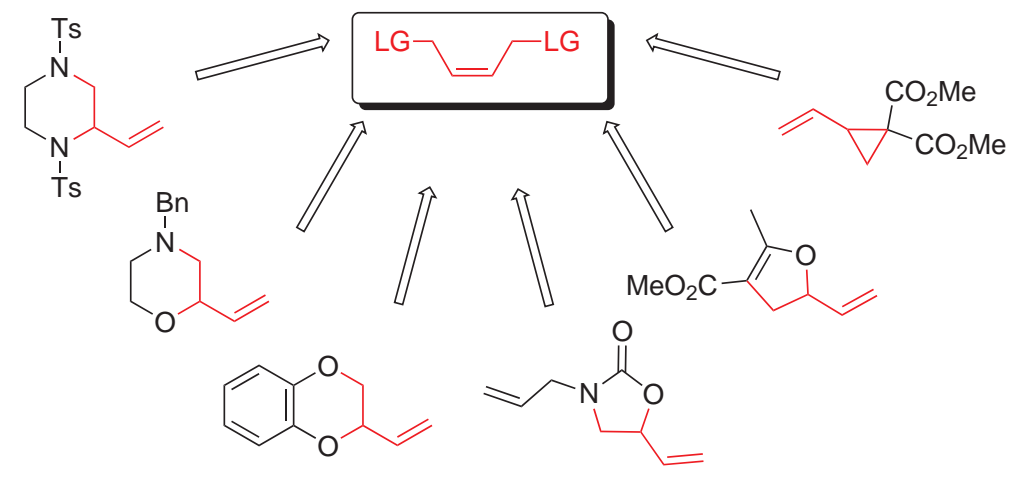

Scheme 6.4 Product Diversity in Pd-AA Cascades.

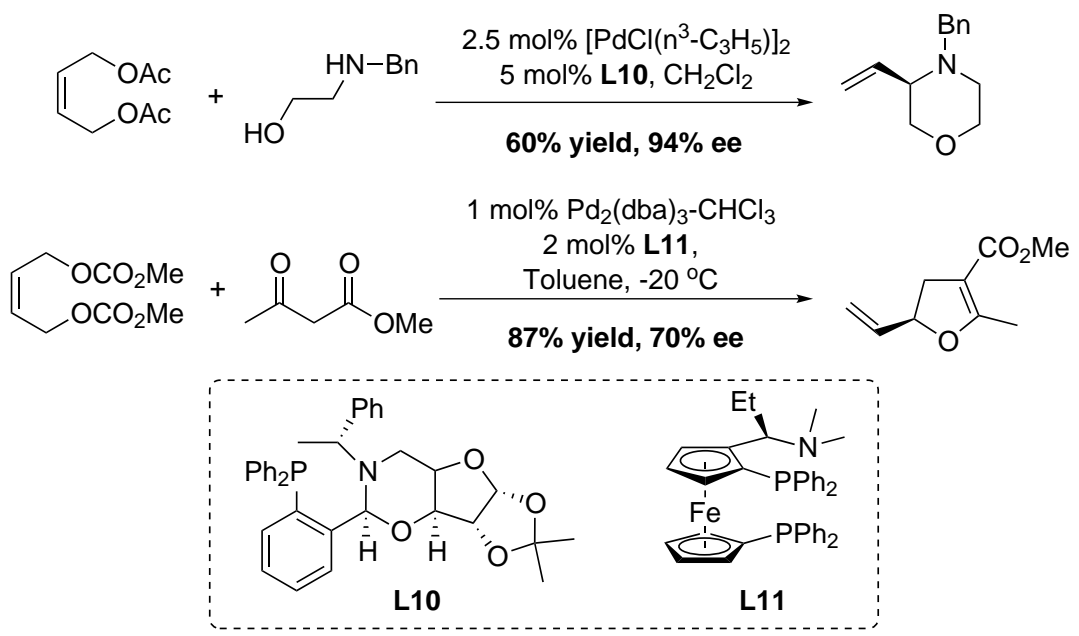

Scheme 6.5 Enantioselective Synthesis of Morpholines and Dihydrofurans using Pd-AA Cascades. 


\subsubsection{Pd-AA Cascade Reactions in Total Synthesis}

Palladium-catalyzed allylic alkylation cascades have been used in a number of elegant natural product syntheses. Cook and coworkers have reported a diastereoselective Pd-AA cascade to prepare the core of neosarpagine $(\mathbf{3 0 5}$, Scheme 6.6$) .{ }^{14}$ In this reaction, the amine nucleophile of $\mathbf{3 0 6}$ adds to the first $\pi$-allyl palladium complex, followed by intramolecular addition of the stabilized enolate nucleophile to the second $\pi$-allyl palladium complex. Notably, the use of a ketone substrate devoid of the unwanted ester functionality (307) resulted in a significant drop in reactivity. However, the desired annulation was eventually achieved through the use of a $\mathrm{Pd}(\mathrm{dba})_{2} / \mathrm{DPEphos}$ catalyst system, forming a zinc enolate and employing a more reactive dicarbonate electrophile.

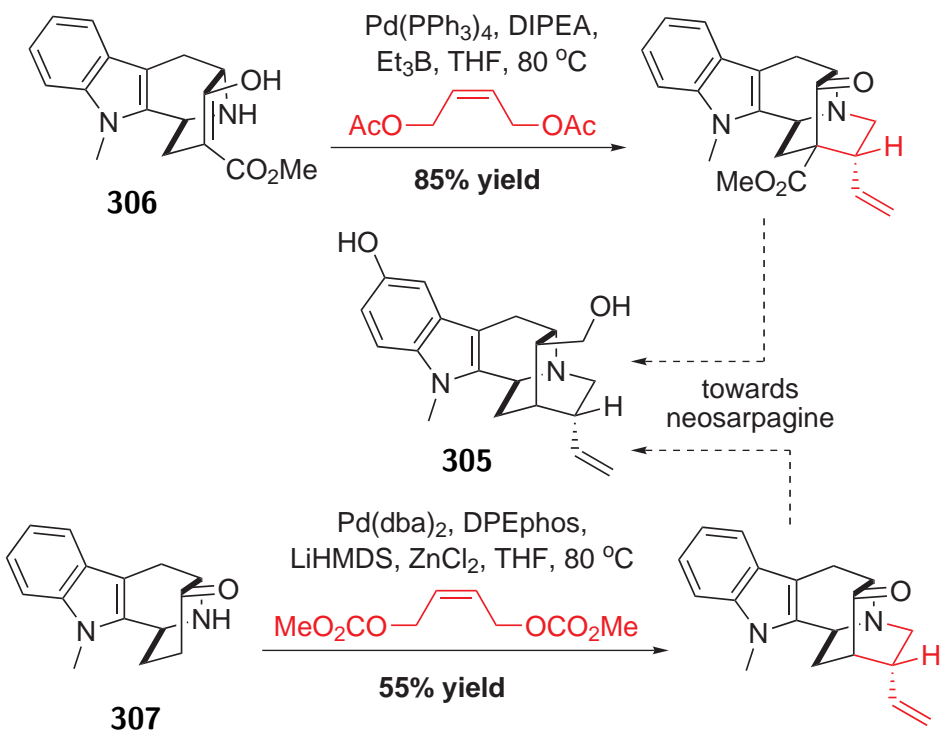

Scheme 6.6 Synthesis of the Core Structure of Neosarpagine using Pd-AA cascades.

Palladium-catalyzed allylic alkylation cascades have also been utilized in both racemic and enantioselective syntheses of huperzine A, a potent acetylcholinesterase (AChE) inhibitor used in the treatment of Alzheimer's disease. ${ }^{10}$ In the presence of a $\operatorname{Pd}(0)$ catalyst, the $\beta$-ketoester 308 reacts with 2-methylene-1,3-propanediol diacetate $(\mathbf{3 0 9})$ to form a 6 -membered ring (Scheme 6.7). After extensive optimization, Bai and coworkers achieved an $82 \%$ yield and $92 \%$ ee. ${ }^{11}$ Interestingly, the use of $-\mathrm{Cl},-\mathrm{OBz}$ or $-\mathrm{OCOCF}_{3}$ as allylic leaving groups was found to be detrimental to both yield and ee.

Trost and Dong use an impressive enantioselective Pd-AA cascade in a recent total synthesis of agelastatin A (311, Scheme 6.8). ${ }^{15}$ Initial attempts to form piperazinone $\mathbf{3 1 2}$ under basic conditions produced none of the desired product. It was hypothesized that the dinucleophile $\mathbf{3 1 3}$ could act as a bidentate ligand for palladium and therefore halt the reaction by forming 314. Acetic acid was added to the reaction to overcome this problem and the desired piperazinone was ultimately obtained in $82 \%$ yield and $98 \%$ ee. These optimized conditions required the addition of a second batch of $\mathrm{Pd}_{2}(\mathrm{dba})_{3} \cdot \mathrm{CHCl}_{3} /$ racemic- $\mathbf{L} \mathbf{1}$ after the 


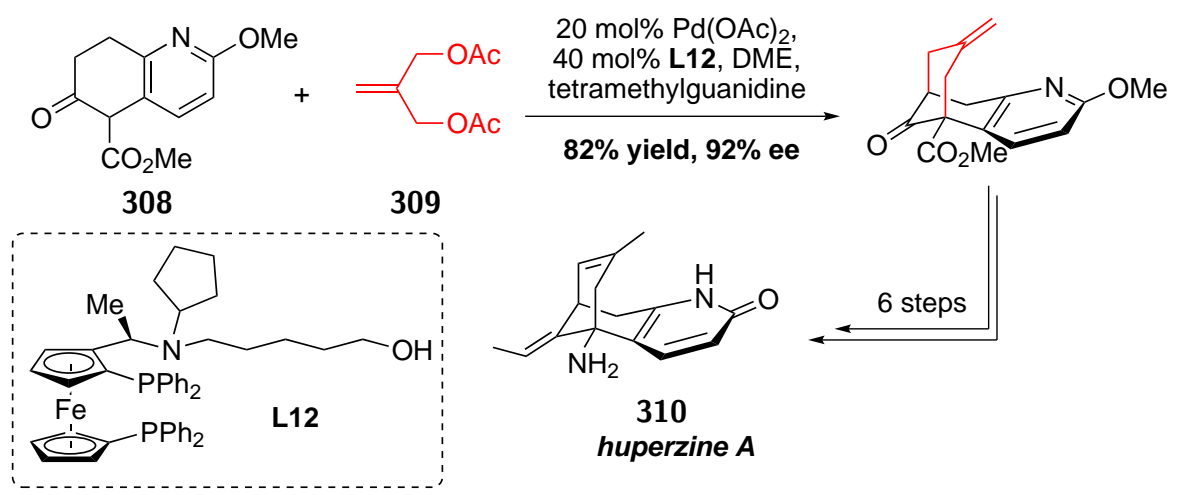

Scheme 6.7 Pd-AA Cascade in the Synthesis of Huperzine A.

first enantioselective allylic alkylation. This is attributed to the fact that ent-L1 is better at facilitating the second allylic alkylation. The chiral steric environment of the substrate and $e n t-\mathbf{L} \mathbf{1}$ are matched, whereas $\mathbf{L} \mathbf{1}$ that facilitated the first alkylation is mismatched. The relative stereochemistry is substrate dependent and the $c i s$-orientation of the starting materials ensures the second addition will occur from the same face as the first addition.

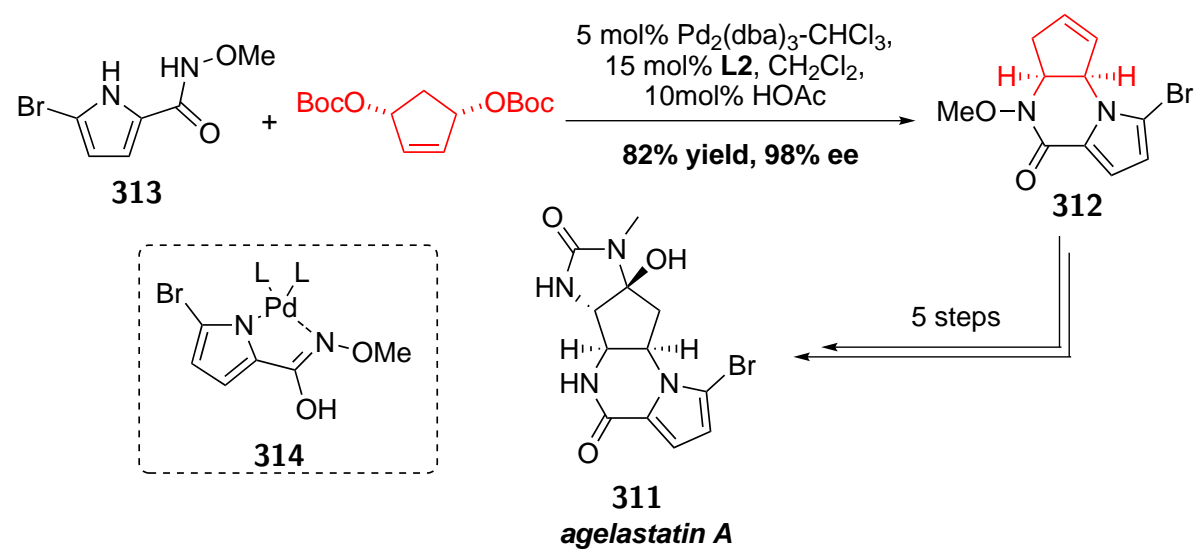

Scheme 6.8 Pd-AA Cascade in the Synthesis of Agelastatin A.

\subsection{Development of a Pd-AA Cascade with a 4-Hydroxy- $\alpha$ - pyrone.}

To examine the feasibility of a Pd-catalyzed allylic alkylation cascade in the synthesis of labillarides E-H, we first investigated the annulation of 6-methyl-4-hydroxy- $\alpha$-pyrone (253) with dicarbonate 315 (Scheme 6.9). This simple symmetric substrate was investigated in the hope of gaining valuable information about the conditions required to obtain the desired reactivity. Optimization experiments began with the screening of a variety of reaction solvents in conjunction with the use of $\mathrm{Pd}_{2}(\mathrm{dba})_{3} / \mathrm{PPh}_{3}$ to generate the active $\mathrm{Pd}(0)$ catalyst in situ (Table 6.1). The use of THF, $\mathrm{CH}_{3} \mathrm{CN}, \mathrm{CH}_{2} \mathrm{Cl}_{2}$, and DMF all provided modest yields of 


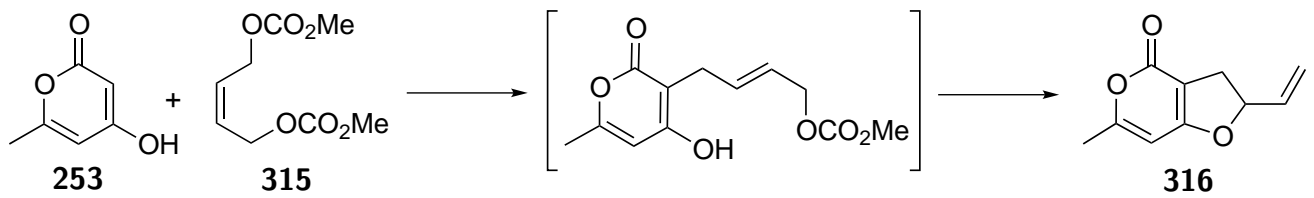

Scheme 6.9 4-Hydroxy- $\alpha$-pyrone Pd-AA Cascade.

furopyrone 316, ranging from 37-52\% (Entries 1 \& 4-6), while toluene, DME and DMSO provided poor yields (0-14\%, Entries 2, 5, and 7). Interestingly, 6 -methyl-4-hydroxy- $\alpha$-pyrone (253) was only sparingly soluble in some of the less polar solvents such as toluene and $\mathrm{CH}_{2} \mathrm{Cl}_{2}$. However, in the case of dichloromethane this did not seem to impact negatively on the yield of the desired product 316. Additionally, an excess of the 4-hydroxy- $\alpha$-pyrone proved essential for a successful reaction, and the use of excess bis-electrophile $\mathbf{3 1 5}$ did not provide the desired furanopyrone (Entry 8).

One of the major concerns borne out of the initial solvent screening experiments was the apparent inability of the palladium catalyst to exceed two or three turnovers, based on the yield obtained. This observation prompted the investigation of a number of alternative palladium precursors and phosphine ligands, including $\mathrm{Pd}(\mathrm{OAc})_{2}, \mathrm{Pd}\left(\mathrm{PPh}_{3}\right)_{4}$, dppe, $\mathrm{P}(o \mathrm{Tol})_{3}$ and (S,S)-DACH-phenyl Trost ligand, L2 (Entries 10-16). Unfortunately, the majority of these conditions gave significantly lower yields of the desired product $\mathbf{3 1 6}$ than the original $\mathrm{Pd}_{2}(\mathrm{dba})_{3} / \mathrm{PPh}_{3}$ conditions, with the exception of Entries 10 and 15. Entry 10 utilized the bidentate chiral phosphine ligand $(S, S)$-DACH-phenyl Trost ligand and produced the desired furopyrone in $49 \%$ yield.* This result did not prove to be general for bidentate ligands, as experiments with dppe showed a complete lack of reactivity (Entries 11\& 12). Interestingly, performing the reaction with $\mathrm{Pd}_{2}(\mathrm{dba})_{3} / \mathrm{P}(o \mathrm{Tol})_{3}$ provided none of the desired product, but instead generated a relatively stable organopalladium complex that was isolated using silica gel chromatography and appeared as a single spot by TLC (Entry 14). The presence of Pd in this side product was confirmed by the observation of a six-isotope pattern in the mass spectrum, characteristic of palladium. ${ }^{16}$ It is well known that $\mathrm{P}(o \mathrm{Tol})_{3}$ displays special intramolecular reactivity, forming the palladacyclic complex known as Hermann's catalyst (317) via a $\mathrm{C}-$ $\mathrm{H}$ insertion process (Scheme 6.10). ${ }^{17}$ With this in mind, palladacycle 318 is hypothesized to be the organopalladium side product generated under these conditions. ${ }^{18} \mathrm{NMR}$ analysis of the proposed palladacycle revealed the expected ${ }^{31} \mathrm{P}$ resonance, while also displaying ${ }^{1} \mathrm{H}$ NMR resonances indicative of the pyrone heterocycle and an alkenyl fragment. Unfortunately, the exact structure of this complex could not be elucidated and attempts at crystallization were unsuccessful. This result shed light on the previous issues associated with catalyst turnover and suggested the possibility that the $\mathrm{Pd}$ may be trapped as a relatively stable palladacyclic complex, which would ultimately halt the catalytic cycle. The plausibility of this hypothesis was augmented by the observation that performing the reaction with $10 \mathrm{~mol}$ $\% \mathrm{Pd}\left(\mathrm{PPh}_{3}\right)_{4}$ gave $31 \%$ yield, whereas the same reaction with $20 \mathrm{~mol} \% \mathrm{Pd}\left(\mathrm{PPh}_{3}\right)_{4}$ provided

*Unfortunately, the enantioselectivity of this reaction was not determined. 
Table 6.1 Pd-AA Cascade Optimization: Annulation of 4-Hydroxy-6-methyl- $\alpha$-pyrone.<smiles>C=CC1Cc2c(cc(C)oc2=O)OC1=O</smiles>

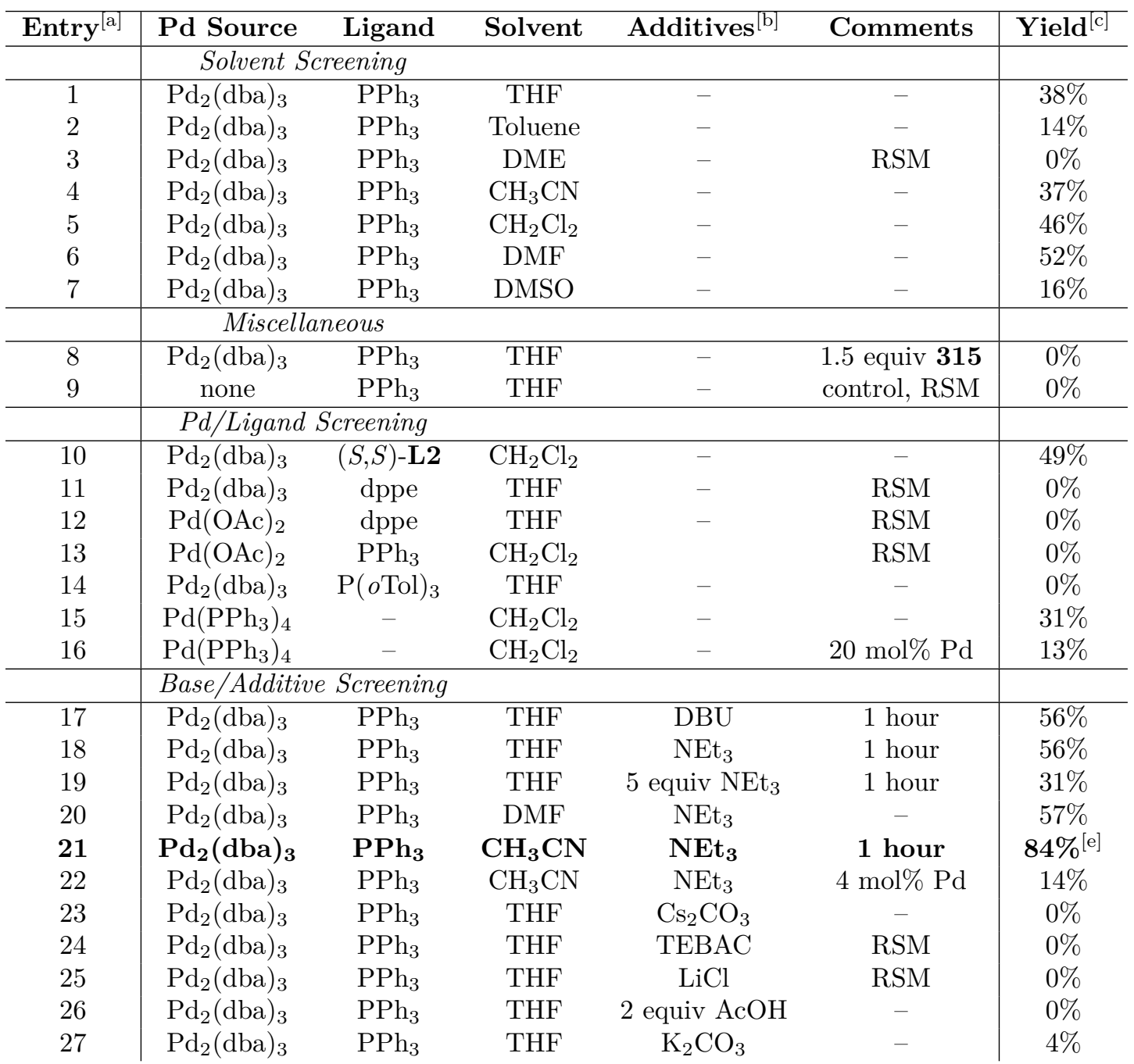

[a] Unless otherwise stated the following conditions apply: 2.2 equiv. of 253, $10 \mathrm{~mol} \% \mathrm{Pd}$ precursor complex, and a Pd:P ratio of 1:2 was used. Reactions were performed at room temperature on a $0.4 \mathrm{mmol}$ scale and were stirred overnight before workup. [b] Unless otherwise stated 1 equivalent of additive was used. [c] Isolated yields are reported. [d] An organopalladium side product was isolated from this reaction, see Scheme 6.10 for details. [e] This reaction proved somewhat capricious upon scale up. Nonetheless, with careful solvent degassing $60-75 \%$ yield could be obtained consistently on a 150-300 mg scale. RSM = Recovered Starting Material.

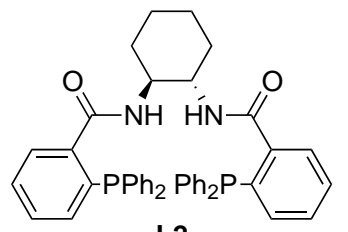

L2

$(S, S)$-DACH-phenyl Trost ligand 


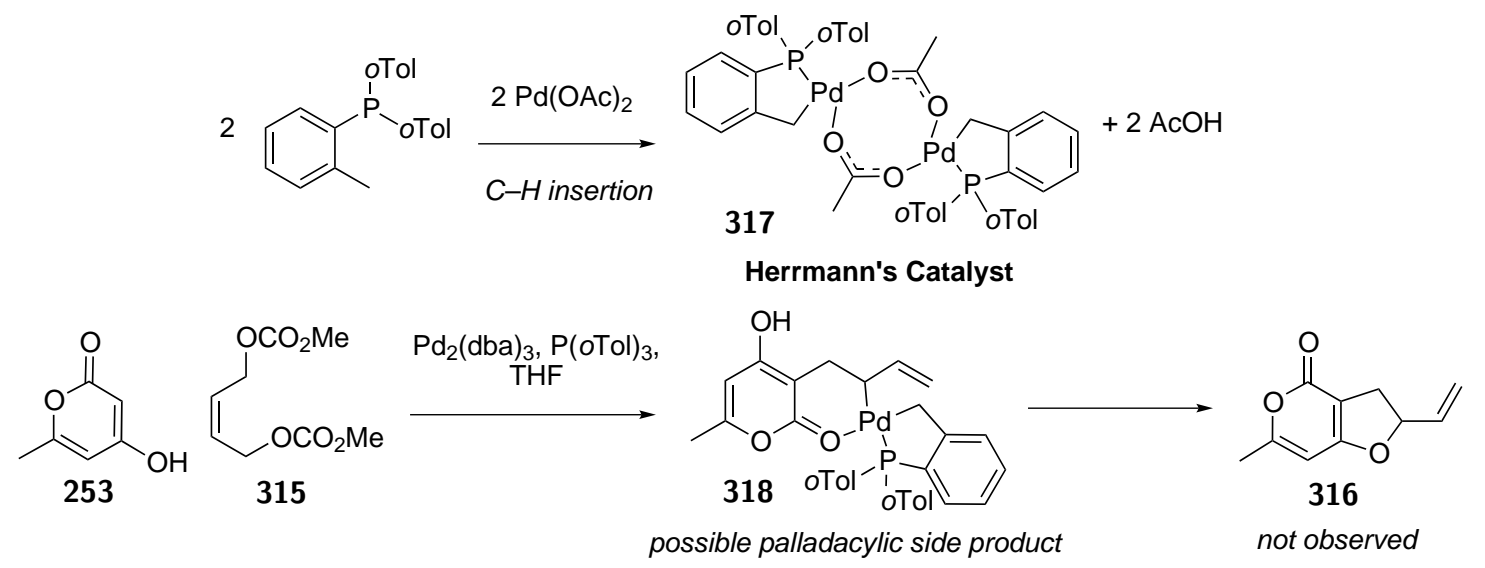

Scheme 6.10 Potential Palladacycle Formation with $\mathrm{P}(o \mathrm{Tol})_{3}$.

only $13 \%$ yield of furopyrone $\mathbf{3 1 6}$ along with another relatively stable organopalladium complex (Entry 16). Once again the structure of this complex could not be fully elucidated, although spectroscopic data once again suggests that both a pyrone and alkenyl moiety are present in this Pd-complex. A number of unexpected palladacyclic side products have been reported in the literature during attempted intramolecular Pd-catalyzed reactions. ${ }^{18}$ Broggini and coworkers reported the isolation of the palladacyclic compound 319 while attempting to convert vinyl iodide $\mathbf{3 2 0}$ into tetrahydroisoquinoline $\mathbf{3 2 1}$ (Scheme 6.11). ${ }^{19}$ In this case, the

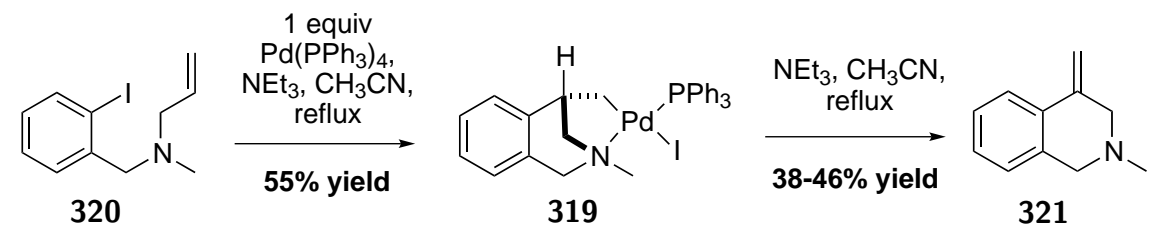

Scheme 6.11 Literature Example of a Stable Palladacyclic Intermediate.

stability of palladacycle 319 is problematic, preventing $\beta$-hydride elimination and catalyst turnover. However, prolonged exposure of $\mathbf{3 1 9}$ to triethylamine in refluxing acetonitrile caused the breakdown of the palladacyclic species and formation of the desired product 321. It is interesting to note that this problem was significantly less prominent when $\mathrm{Pd}(\mathrm{OAc})_{2} / \mathrm{PPh}_{3}$ or $\mathrm{Pd}_{2}(\mathrm{dba})_{3} / \mathrm{PPh}_{3}$ were used to generate the active palladium catalyst, which is consistent with the result obtained with $\mathrm{Pd}\left(\mathrm{PPh}_{3}\right)_{4}$ in this work (see Table 6.1, Entry 16).

The palladacycle hypothesis led to the use of an amine base as a stoichiometric additive, in the hope of improving both catalyst turnover and product yield. The use of either DBU or $\mathrm{NEt}_{3}$ provided a nice improvement in both yield and the rate of the reaction (Entries 17 \& 18). Under these conditions, the reaction showed complete consumption of starting material after just an hour, whereas the earlier solvent screening experiments contained significant amounts of starting material after an hour and often required stirring overnight to consume the majority of the bis-electrophile 315. Ultimately, it was discovered that performing the PdAA cascade reaction with one equivalent of $\mathrm{NEt}_{3}$ and catalytic $\mathrm{Pd}_{2}(\mathrm{dba})_{3} / \mathrm{PPh}_{3}$ in $\mathrm{CH}_{3} \mathrm{CN}$ 
provided a gratifying $84 \%$ yield (Entry 21). Unfortunately, the good yield obtained with 10 mol\% $\mathrm{Pd}_{2}(\mathrm{dba})_{3}$ did not enable the use of lower catalyst loadings, and only $14 \%$ yield was obtained when $2 \mathrm{~mol} \% \mathrm{Pd}_{2}(\mathrm{dba})_{3}$ was used. Additional additive screening experiments with inorganic bases or salts provided poor yields (Entries 23-27) and thus far, conditions which achieve higher Pd turnover numbers have yet to be discovered.

\subsection{Synthesis of the Core of Labillarides E-H}

\subsubsection{A Linchpin-Based Approach to the Core of Labillaride E-H}

Development of the Pd-AA cascade shown in Table 6.1 enabled a linchpin-based approach to the synthesis of the furanopyrone and alkenyl side chain of labillaride E-H (Scheme 6.12). ${ }^{20}$ This sequence constitutes a multi-tether linchpin strategy, forming three bonds to a four carbon

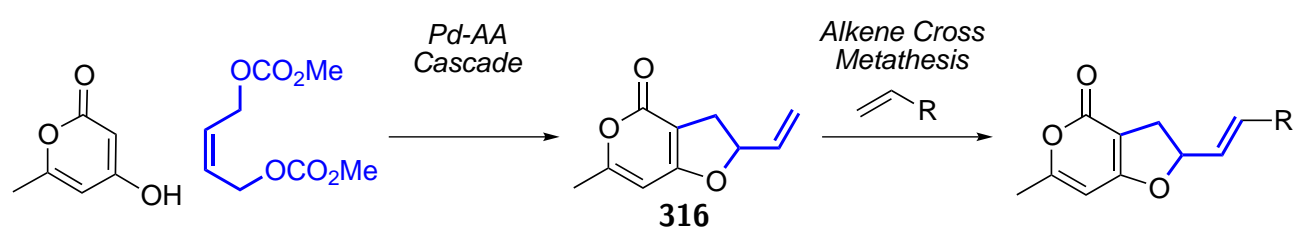

Scheme 6.12 A Linchpin-Based Approach to the Core of Labillaride E-H.

linchpin while also forming a heterocyclic ring. We sought to evaluate this strategy and also gain valuable insight into the spectroscopic challenges associated with the differentiation of labillarides E, F, G, and $\mathrm{H}$.

$\mathrm{Ru}$-catalyzed alkene cross metathesis between $\mathrm{Pd}-\mathrm{AA}$ cascade product 316 and pent-1-en3 -ol (322) provided the desired product $\mathbf{3 2 3}$ in $45 \%$ yield (Scheme 6.13 ). Initially, this reaction was performed using a large excess (7 equiv) of allylic alcohol $\mathbf{3 2 2}$ to minimize homo-coupling of the precious alkene 316. However, the large excess of alkene $\mathbf{3 2 2}$ proved detrimental to the conversion of $\mathbf{3 1 6}$ to $\mathbf{3 2 3}$, and $53 \%$ of the furopyrone starting material was recovered (91\% yield BRSM). The desired cross metathesis product was obtained as a mixture of syn323 and anti-323 diastereomers. A partial kinetic resolution was observed, providing a 66:34 ratio of diastereomers, as determined by LC-MS (Figure 6.1). The alkene cross metathesis was also performed with a smaller excess (2 equiv) of 1-penten-3-ol (322), which provided $\mathbf{3 2 3}$ in $40 \%$ yield as a 59:41 ratio of diastereomers. In this case, complete consumption of starting material was observed, however, the minor amounts of the furanopyrone homo-coupling product generated during the reaction were very challenging to separate from the desired product. In analogy with labillarides $\mathrm{E}-\mathrm{H}$, the syn- and anti-diastereomers of $\mathbf{3 2 3}$ displayed very similar ${ }^{1} \mathrm{H}$ and ${ }^{13} \mathrm{C}$ NMR spectra. As a result, structural assignment of the major and minor product from Scheme 6.13 was not possible with simple NMR analysis.

The sequential application of a Pd-catalyzed allylic alkylation cascade and rutheniumcatalyzed alkene cross metathesis enabled the rapid construction of the furanopyrone core and alkenyl side chain of labillarides E-H. In just two steps, over half of the natural product 


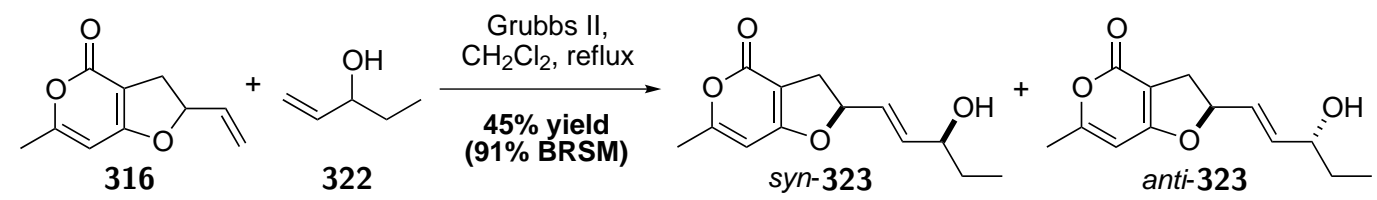

Scheme 6.13 Installation of the Labillaride Side Chain using Alkene Cross Metathesis.
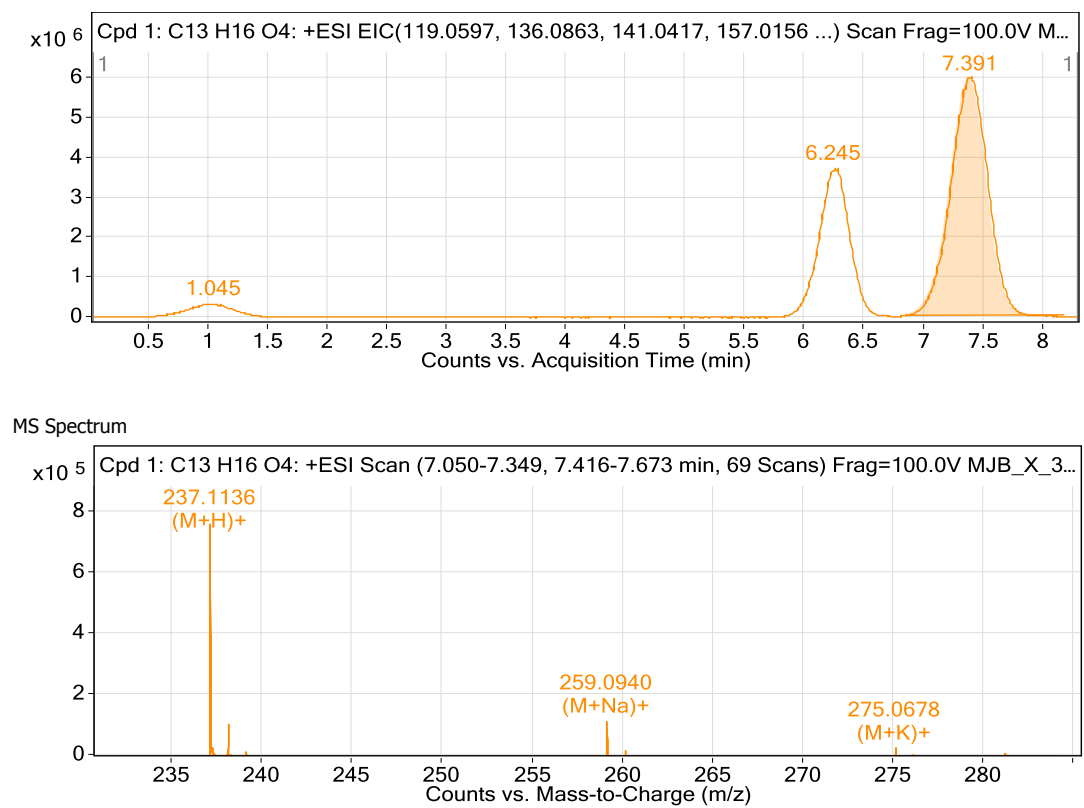

Figure 6.1 LC-MS Analysis of the Furopyrones syn-, and anti-323.

was assembled including the heterocyclic core and two stereogenic centers. Additionally, the ability to generate substantial quantities of the furanopyrone $\mathbf{3 2 3}$ affords the opportunity to investigate both the biological and spectroscopic properties of labillaride fragments and analogues.

\subsubsection{Biological Testing of Labillaride Analogues syn- and anti-323}

The two furopyrone diastereomers were separated using normal phase HPLC and the cytotoxicity of each compound was testing against an HL-60 (human promyelocytic leukemia) cancer cell line. ${ }^{\dagger}$ Unfortunately, the major diastereomer was inactive at all concentrations up to $250 \mu \mathrm{M}$. However, the minor diastereomer was biologically active and displayed cytotoxicity in the low $\mu \mathrm{M}$ range $\left(\mathrm{IC}_{50}=27 \mu \mathrm{M}\right)$. These results are consistent with the HL-60 results obtained with labillarides E, F, G, and H, which were all inactive at concentrations below $10 \mu \mathrm{M}-$ higher concentrations were not tested. ${ }^{21}$ The promising biological activity of the labillaride analogue $\mathbf{3 2 3}$ and the ability to generate and purify substantial quantities of material provides an excellent platform for future research into the medicinal properties of the labillarides and

\footnotetext{
${ }^{\dagger}$ Cell biology assays were performed by Arun Kanakkanthara
} 
related analogues.

\subsubsection{NMR Analysis of the Labillaride E-H Side Chain}

One of the most fascinating aspects of labillarides $\mathrm{E}, \mathrm{F}, \mathrm{G}$, and $\mathrm{H}$ is the almost identical ${ }^{1} \mathrm{H}$ and ${ }^{13} \mathrm{C}$ NMR spectra of these diastereomeric natural products. As discussed previously, the structure assigned to labillarides E-H seems to contradict a number of fairly common assumptions made in regards to the spectroscopic properties of diastereomers pertaining to a group of contiguous stereocenters (for additional details see Section 5.1). With furanopyrone 323 in hand, one of the two major structural uncertainties could be investigated - namely, how different configurations at $\mathrm{C} 3$ affect the spectroscopic properties of these compounds.

The ${ }^{1} \mathrm{H}$ NMR spectra obtained from a mixture of syn- and anti-323 shows that these compounds exhibit remarkably similar chemical shifts and coupling constants (Figure 6.2). In

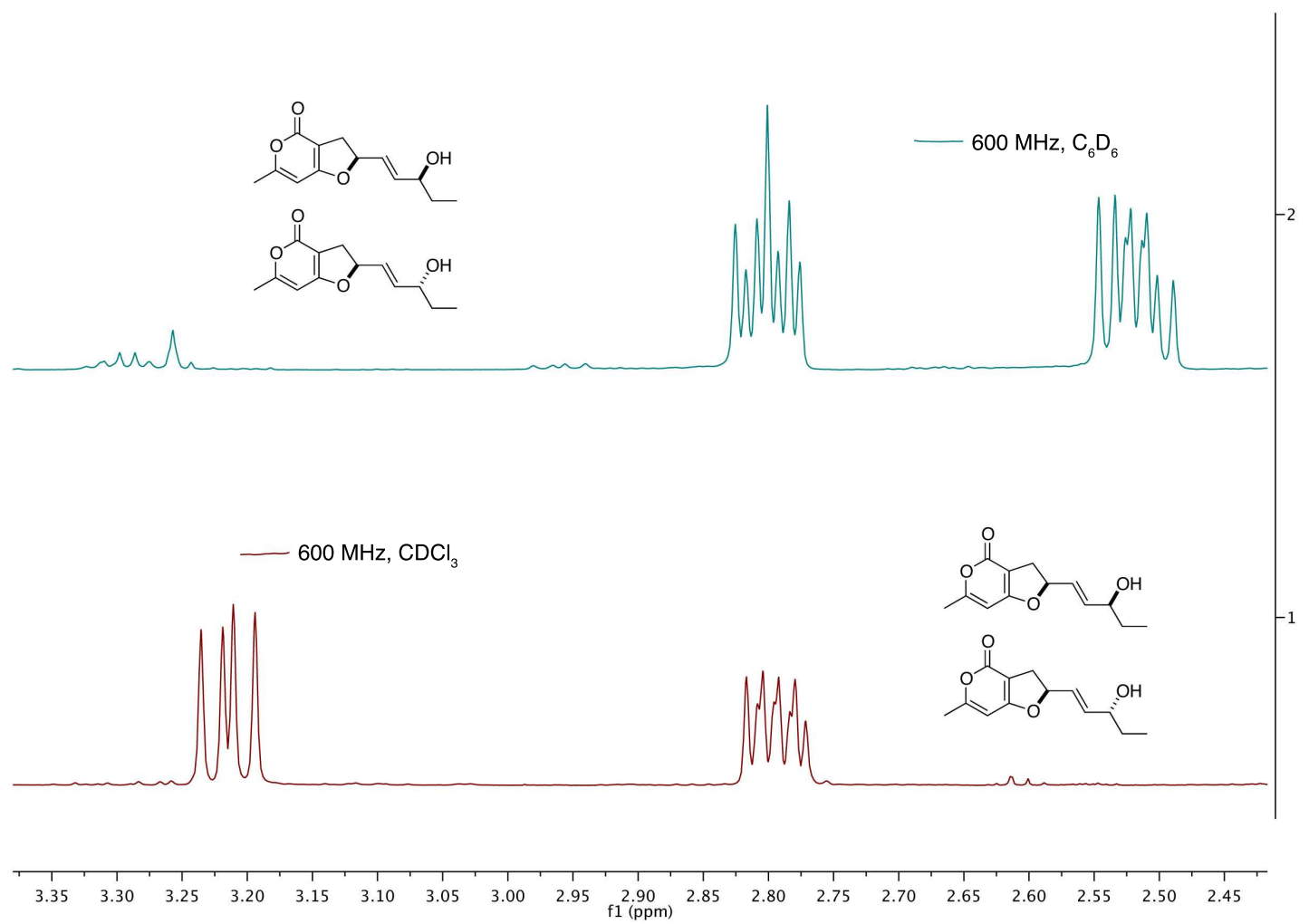

Figure 6.2 Comparison of the ${ }^{1} \mathrm{H}$ NMR Spectra of a Mixture of syn- and anti-323 in $\mathrm{CDCl}_{3}$ and Benzene- $d_{6}$.

$\mathrm{CDCl}_{3}$, subtle twinning of just two signals alluded to the presence of two diastereomers. However, the use of benzene- $d 6$ provided significantly improved separation of the diastereomeric resonances, and twinning was observed in numerous cases, including the oxymethines that differentiate the compounds structurally. The ${ }^{13} \mathrm{C}$ NMR spectra also show only very small differences between the two diastereomers - the largest differences were observed in the alkene carbons (C4 and $\mathrm{C} 5)$ of each diastereomer, which differed by 0.11 and $0.17 \mathrm{ppm}$, respectively 
(Figure 6.3). Overall, these results, and a full suite of 2D NMR experiments (see Section 7.5), agree with those reported for labillarides E-H and support the assignment of these natural products as two pairs of epimers, each differing in the configuration at C3.

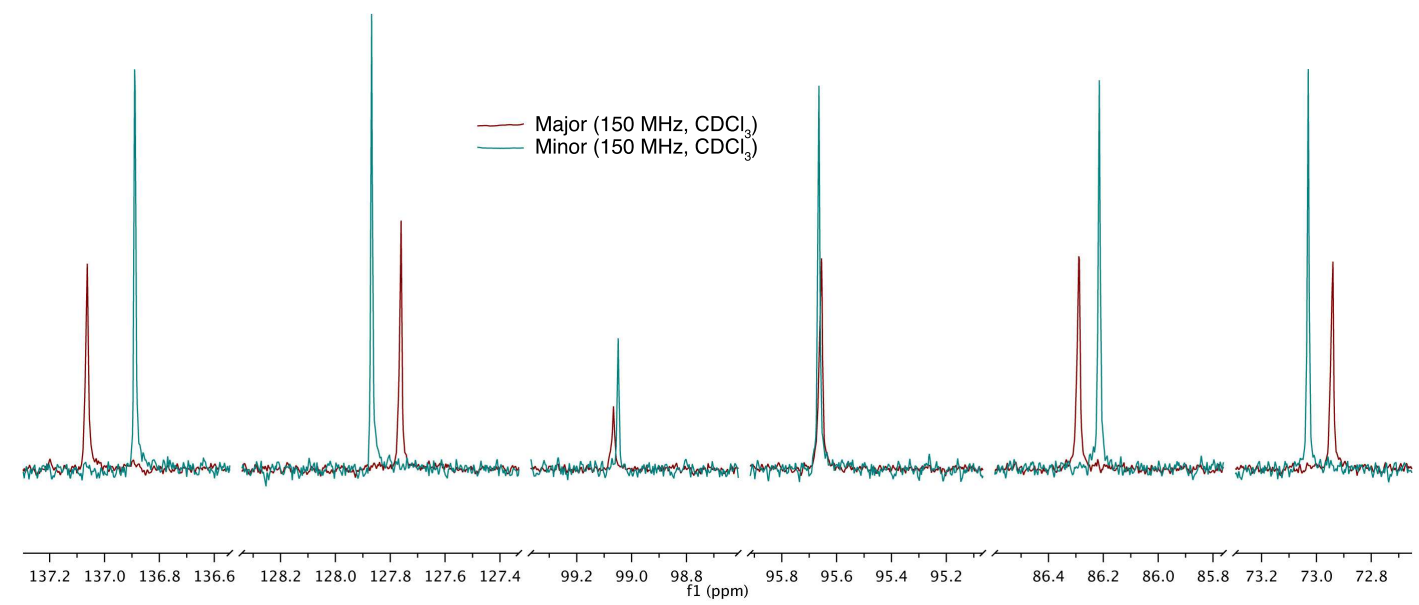

Figure 6.3 Selected ${ }^{13} \mathrm{C}$ NMR Resonances of syn- and -anti-323 Diastereomers.

The results shown in Figure 6.2 and Figure 6.3 provide useful insight into the difficulty faced in trying to assign the structures of labillarides E-H. Spectroscopic differences of $<0.02$ ppm in the ${ }^{1} \mathrm{H}$ spectra and $<0.2 \mathrm{ppm}$ in the ${ }^{13} \mathrm{C}$ spectra mean that, even with a successful total synthesis, isolated ${ }^{1} \mathrm{H}$ and ${ }^{13} \mathrm{C}$ NMR analysis is likely to provide only a speculative estimate as to the exact structure of these natural products. However, preparation of all possible labillaride $\mathrm{E}-\mathrm{H}$ diastereomers and analysis of the relative differences in ${ }^{13} \mathrm{C}$ chemical shifts should provide a reasonable means of differentiating each diastereomer. It is important that the NMR data for each diastereomer is obtained at approximately equal sample concentrations, as different concentrations can lead to slightly shifted NMR resonances. ${ }^{21}$ Analysis of the relative differences in ${ }^{13} \mathrm{C}$ NMR shifts of syn- and anti-323 show an excellent correlation with the differences reported for labillarides $\mathrm{E}$ and $\mathrm{F}$, particularly with respect to $\mathrm{C} 3$, $\mathrm{C} 4$, C5, and $\mathrm{C} 6$, which are the most significant for the differentiation of these compounds (Table 6.2). These results imply that the structure of the major diastereomer of $\mathbf{3 2 3}$ matches best with labillaride $\mathrm{E}$ and the structure of the minor diastereomer of $\mathbf{3 2 3}$ matches that of labillaride F. To gain additional insight into the relative stereochemistry of the diastereomers of $\mathbf{3 2 3}$, their spectroscopic properties were predicted using density functional calculations. ${ }^{22}$ Geometry optimization (B3LYP/6-31G(d)) and GIAO NMR calculation (B3LYP/6-31G(d), TMS referencing) of both syn- and anti-323 gave two data sets that were an excellent match to the experimental data from major and minor diastereomers, respectively. ${ }^{23}$ The relative differences between the calculated ${ }^{13} \mathrm{C}$ NMR shifts of the two diastereomers correlate well with the differences observed in the experimental data and the assignment of the major diastereomer as $s y n-\mathbf{3 2 3}$ and the minor diastereomer as anti-323 has a CP3 probability of $>99 \% .{ }^{24}$

Overall, the use of density functional calculations to assign the relative stereochemistry of syn-323 and anti-323 has provided good evidence that labillarides E and G have a 3,6-syn 


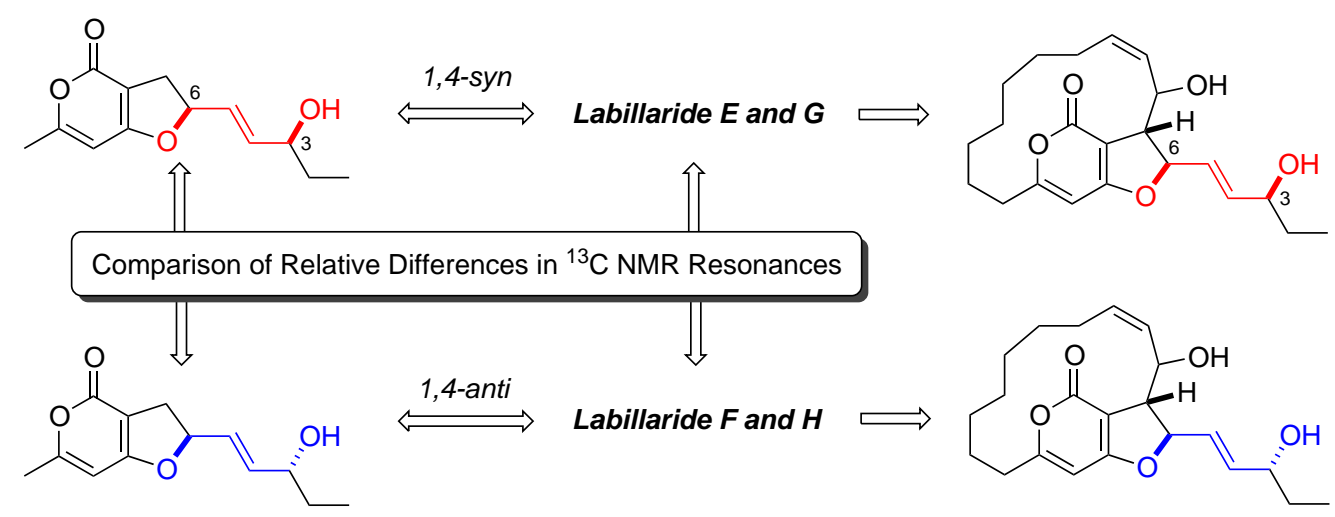

Figure 6.4 Tentative Assignment of the Relative C3/C6 Configuration of Labillarides E-H by Comparison with Furopyrone 323.

configuration, whereas labillarides $\mathrm{F}$ and $\mathrm{H}$ have a 3,6-anti configuration (Figure 6.4). However, the small difference in the NMR chemical shifts of these compounds leaves a very small margin for error, a margin that is smaller than the error typically associated with calculated NMR shifts. ${ }^{22}$ Thus, despite the excellent correlation between calculated and experimental data, the proposed assignment of the relative stereochemistry of labillarides E-H remains tentative. A definitive stereochemical assignment of this very challenging structural motif would likely require parallel spectroscopic analysis of both synthetic and naturally derived material along with an X-ray crystal structure of a synthetic intermediate or natural product derivative to provide a structural point of reference. ${ }^{25}$ 


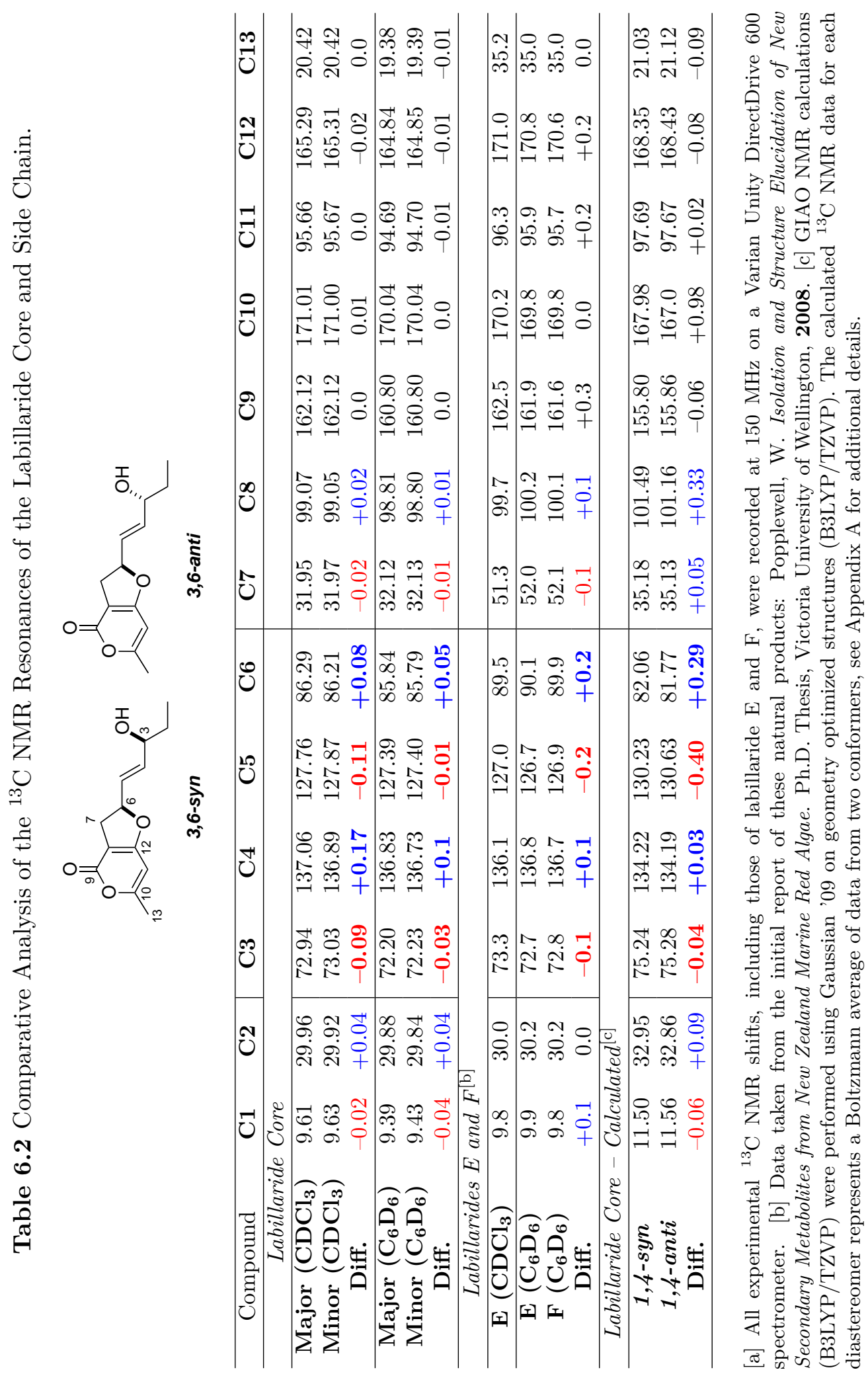




\subsection{Computational Investigations into Labillaride E-H Atropisomerism}

The successful computational analysis of furopyrone 323, prompted an in silico investigation of labillarides $\mathrm{E}-\mathrm{H}$ with the hope of gaining insight into how two different configurations in a set of three contiguous stereocenters (viz. C6, C7 and C8 in labillarides E-H) could result in virtually identical ${ }^{1} \mathrm{H}$ and ${ }^{13} \mathrm{C}$ NMR chemical shifts and coupling constants. At the outset, we recognized that the labillaride E-H macrocycle may have the potential to exist in two atropisomeric forms, based on literature precedent from the neurymenolides (see Figure 5.3). However, initial molecular modeling showed that the furopyrone core of the labillarides is fixed in a way such that rotation of the macrocyclic ring about the heterocyclic nucleus provides an awkward and highly unlikely 'pac-man' shaped macrocyclic conformation. Closer examination of the macrocyclic ring revealed surprisingly hindered rotation about the $Z$-alkene. Rotation in one direction engenders a large amount of macrocyclic ring strain, whereas rotation in the other direction encounters a significant steric interaction with the furopyrone ring. Density functional calculations (B3LYP/TZVP) modelling the rotation of the $Z$-alkene revealed a distinct transition state between two relatively low energy macrocyclic ring conformers, a defining characteristic of atropisomerism (Figure 6.5). These calculations suggest an activation energy of around $30 \mathrm{kcal} / \mathrm{mol}$ for conformer interconversion, which is in good agreement with values associated with stable atropisomers that experience very slow interconversion $\left(t_{1 / 2}=\right.$ years $)$. Adding an element of axial chirality to the proposed structure of labillarides E-H means that additional diastereomeric combinations need to be considered (Figure 6.6). Molecular modelling of the $\boldsymbol{R}_{\mathbf{a}}, \mathbf{8} \boldsymbol{S}, 7 R, 6 R, 3 R-, \boldsymbol{S}_{\mathbf{a}}, \mathbf{8} \boldsymbol{S}, 7 R, 6 R, 3 R-$, $\boldsymbol{R}_{\mathbf{a}}, \mathbf{8} \boldsymbol{R}, 7 R, 6 R, 3 R$-, and $\boldsymbol{S}_{\mathbf{a}}, \mathbf{8} \boldsymbol{R}, 7 R, 6 R, 3 R$-diastereomers of the labillaride E-H scaffold revealed a number of interesting structural relationships. ${ }^{26}$ Firstly, the key $\mathrm{H}-\mathrm{H}$ coupling constants, ${ }^{3} J_{\mathrm{HaHb}}$ and ${ }^{3} J_{\mathrm{HbHc}}$, reported for labillarides E-H match very well with those calculated for the $\boldsymbol{S}_{\mathrm{a}}, \mathbf{8} \boldsymbol{R}, 7 R, 6 R, 3 R$-diastereomer. ${ }^{27}$ Additionally, the calculated ${ }^{3} J_{\mathrm{HH}}$ values for the $\boldsymbol{S}_{\mathbf{a}}, \mathbf{8} \boldsymbol{S}, 7 R, 6 R, 3 R$ - and $\boldsymbol{R}_{\mathbf{a}}, \mathbf{8} \boldsymbol{R}, 7 R, 6 R, 3 R$-diastereomers were both a poor match with the experimental data, while the $\boldsymbol{R}_{\mathbf{a}}, \mathbf{8} \boldsymbol{S}, 7 R, 6 R, 3 R$-diastereomer had one good match and one poor match. Interestingly, the layout of Figure 6.6 resembles that typically used when defining stereochemical relationships between the stereoisomers of a compound containing two stereocenters, where the vertically and horizontally adjacent structures are related as diastereomers and the structures that lie on the same diagonal are related as enantiomers. To a certain extent, the diastereomers shown in Figure 6.6 mirror this relationship - by inverting both the axial and $\mathrm{C} 8$ point chirality, in conjunction with a reorientation of the macrocycle to minimize unfavourable gauche interactions, one observes a new structure that conserves many of the spatial relationships present in the original structure - these 'pseudo-enantiomers' are shown in Figure 6.7. 


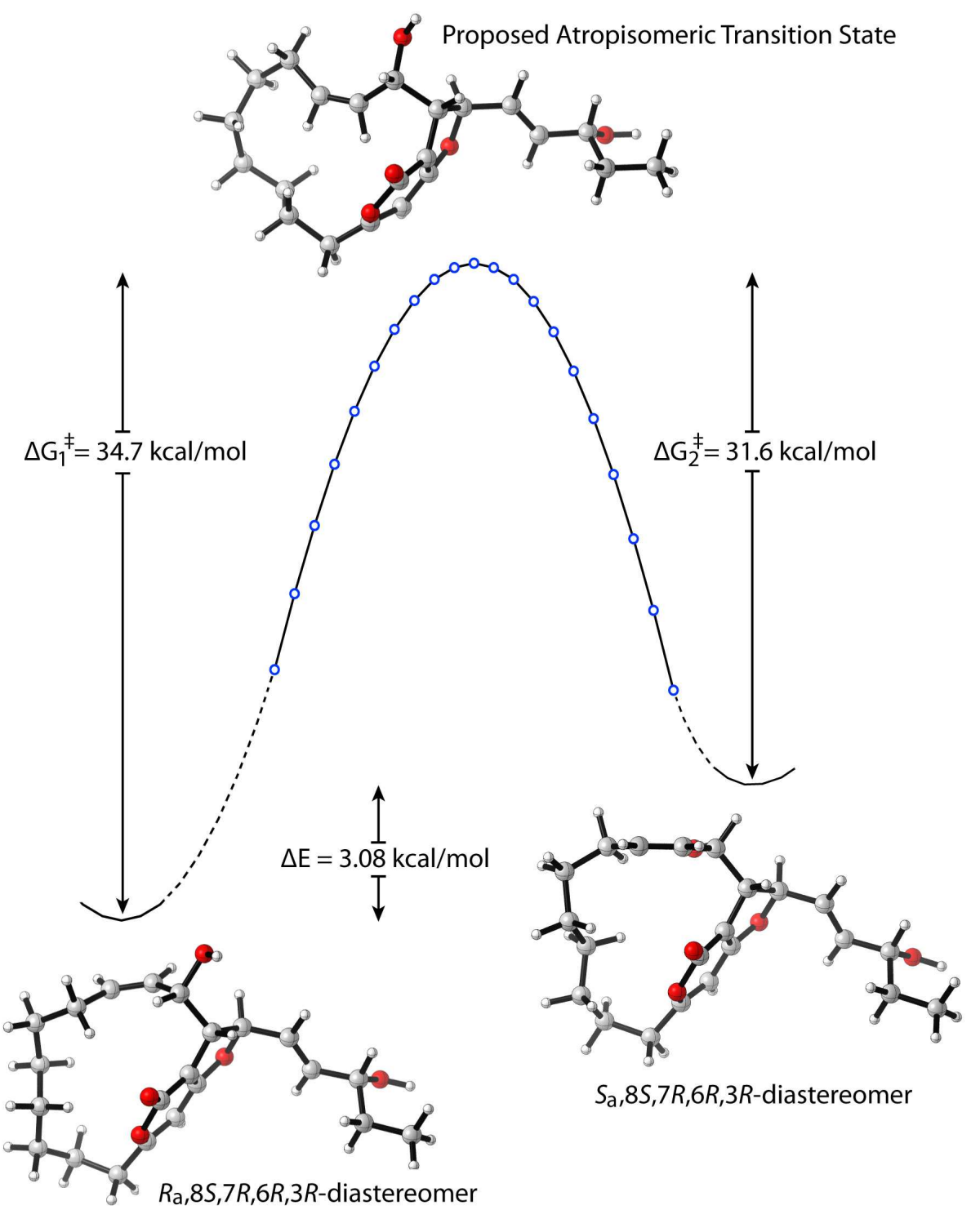

Figure 6.5 Axial Chirality in Labillarides E-H: Calculated Atropisomeric Transition State and Intrinsic Reaction Coordinate. 
Labillaride E-H Experimental ${ }^{3} J_{H H}$ Values $=\mathrm{H}_{\mathrm{a}} \backslash \mathrm{H}_{\mathrm{b}}: 9 \mathrm{~Hz}, \mathrm{H}_{\mathrm{b}} \backslash \mathrm{H}_{\mathrm{c}}: 9.5 \mathrm{~Hz}$

KEY $=\mathrm{Hx} \backslash \mathrm{Hy}$ : Dihedral Angle (Calculated $\left.{ }^{3} \mathrm{~J}_{\mathrm{HH}}\right)$

Match to Experimental Data: black $=$ good, red $=$ poor

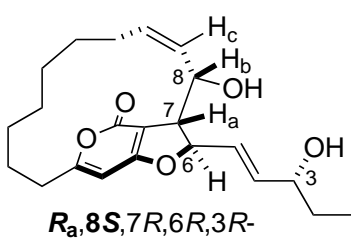

$\mathrm{H}_{\mathrm{a}} \backslash \mathrm{H}_{\mathrm{b}}:-74.0^{\circ}(1.7 \mathrm{~Hz})$

$\mathrm{H}_{\mathrm{b}} \backslash \mathrm{H}_{\mathrm{c}}: 150.1^{\circ}(8.2 \mathrm{~Hz})$
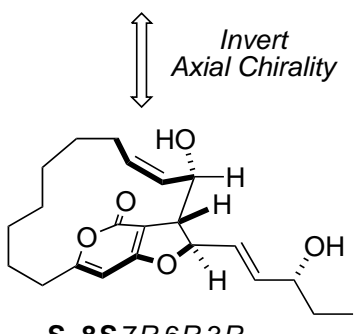

$S_{3}, 8 S, 7 R, 6 R, 3 R$

$\mathrm{H}_{\mathrm{a}} \mathrm{l} \mathrm{H}_{\mathrm{b}}: 57.4^{\circ}(3.1 \mathrm{~Hz})$

$\mathrm{H}_{\mathrm{b}} \backslash \mathrm{H}_{\mathrm{c}}:-60.8^{\circ}(2.7 \mathrm{~Hz})$
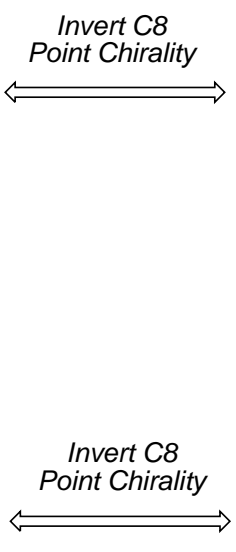

$\mathrm{H}_{\mathrm{a}} \backslash \mathrm{H}_{\mathrm{b}}: 179.6^{\circ}(10.3 \mathrm{~Hz})$

$\mathrm{H}_{\mathrm{b}} \backslash \mathrm{H}_{\mathrm{c}}: 167.7^{\circ}(9.9 \mathrm{~Hz})$

Figure 6.6 Comparison of Experimental and Calculated Coupling Constants for Different Configurations of the Labillaride Scaffold.

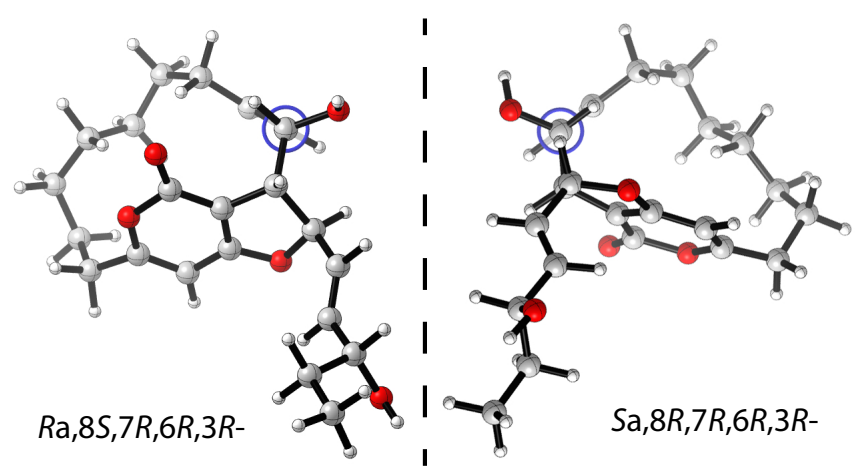

Figure 6.7 Structural Similarities Resulting from Inversion of Axial and C8 Point Chirality.

While this computational analysis does not provide a complete explanation of the unusual spectroscopic similarities displayed by labillarides E-H, it does shed light on how such similar ${ }^{1} \mathrm{H}$ and ${ }^{13} \mathrm{C}$ NMR spectra might result from these diastereomeric compounds. This new-found awareness of the potential structural nuances of labillarides E-H is likely to be valuable in research towards the total synthesis of these compounds. 


\subsection{Experimental Data for Chapter 6}

\subsubsection{General Experimental Methods}

Unless otherwise noted, the following conditions apply. All reactions were performed in flamedried septum-sealed glassware with magnetic stirring under an atmosphere of argon. Moistureand oxygen-sensitive liquids and solutions were transferred using a stainless steel syringe or cannula. Before use, solvents were refluxed over the appropriate drying agent and distilled under argon: tetrahydrofuran from sodium benzophenone ketyl radical; dichloromethane, acetonitrile and triethylamine from calcium hydride; toluene from sodium. All other commercially available chemicals were used as received, without further purification. Analytical thin layer chromatography (TLC) was performed using plastic-backed pre-coated silica TLC plates (Polygram SilG/UV 254 ). Visualization was achieved by UV irradiation $(254 \mathrm{~nm}$ ) or by heating after treatment with a potassium permanganate dip $\left(1.5 \mathrm{~g} \mathrm{KMnO}_{4}, 10 \mathrm{~g} \mathrm{~K} \mathrm{KO}_{3}\right.$, $1.25 \mathrm{~mL}$ of $10 \%$ aqueous $\mathrm{NaOH}$ solution and $200 \mathrm{~mL}$ of water) or $p$-anisaldehyde dip (0.7 $\mathrm{mL} p$-anisaldehyde, $9.5 \mathrm{~mL}$ conc. $\mathrm{H}_{2} \mathrm{SO}_{4}, 2.7 \mathrm{~mL}$ of acetic acid and $250 \mathrm{~mL}$ of EtOH). The purification of products by flash column chromatography (FCC) was conducted using silica gel 60 (220-240 mesh) with the solvent systems indicated. ${ }^{1} \mathrm{H}$ NMR spectra were recorded on either: a Varian Unity Inova 500 spectrometer at $500 \mathrm{MHz}$, or a Varian Inova 300 at 300 $\mathrm{MHz}$. Data are listed as chemical shift in ppm using $\mathrm{CDCl}_{3}$ as internal standard (7.26 ppm). ${ }^{13} \mathrm{C}$ NMR spectra were recorded on a Varian Unity Inova 500 spectrometer at $125 \mathrm{MHz}$ and the data are listed as chemical shift in ppm using $\mathrm{CDCl}_{3}$ as internal standard (77 ppm). All ${ }^{13} \mathrm{C}$ experiments were ${ }^{1} \mathrm{H}$ decoupled. IR bands were measured as a thin film on a Bruker FTIR Tensor 27 spectrometer with ATR sampling accessory. High-resolution mass spectrometry (HRMS) was performed on a Waters QTOF Premier Tandem mass spectrometer or an Agilent 6530 Accurate-Mass Q-TOF LC/MS.

\subsubsection{Experimental Details and Characterization Data}

\section{Preparation of $Z$-But-2-ene-1,4-diyl dimethyl dicarbonate, 315}<smiles>COOC/C=C\COC(C)=O</smiles>

To a solution of 1,4-butene diol (1.12 mL, $13.62 \mathrm{mmol}, 1.0$ equiv) and DMAP (4.16 g, 34.05 mmol, 2.5 equiv) in $\mathrm{CH}_{2} \mathrm{Cl}_{2}(30 \mathrm{~mL})$ was added methyl chloroformate $(2.63 \mathrm{~mL}, 34.05 \mathrm{mmol}$, 2.5 equiv), portionwise, at $0{ }^{\circ} \mathrm{C}$. The reaction was subsequently warmed to room temperature and stirred overnight before being quenched with $\mathrm{H}_{2} \mathrm{O}$. The reaction mixture was diluted with $\mathrm{CH}_{2} \mathrm{Cl}_{2}$ and washed with saturated aqueous $\mathrm{CuSO}_{4}$. The organic layer was subsequently dried with $\mathrm{MgSO}_{4}$, filtered and concentrated in vacuo. The crude product was purified by silica gel chromatography $(20 \%$ EtOAc/Pet. Ether). The title compound was isolated as a clear colorless oil (2.661 g, $96 \%$ yield). $\mathbf{R}_{\mathbf{f}}=0.49$ (20\% EtOAc/Pet. Ether). ${ }^{1} \mathbf{H}$ NMR $(500 \mathrm{MHz}$, $\left.\mathrm{CDCl}_{3}\right) \delta 5.82(\mathrm{ddd}, J=5.2,4.0,1.2 \mathrm{~Hz}, 1 \mathrm{H}), 4.76(\mathrm{~m}, 2 \mathrm{H}), 3.80(\mathrm{~s}, 3 \mathrm{H}) .{ }^{\mathbf{1 3}} \mathrm{C} \mathbf{N M R}(126$ $\left.\mathrm{MHz}_{2} \mathrm{CDCl}_{3}\right) \delta 155.54,127.98,63.18,54.92$. Characterization data matches the literature. ${ }^{28}$ 


\section{Preparation of 6-Methyl-2-vinyl-2H-furo[3,2-c]pyran-4(3H)-one, 316}<smiles>C=CC1Cc2c(cc(C)oc2=O)O1</smiles>

${ }^{1} \mathbf{H}-\mathbf{N M R}\left(500 \mathrm{MHz}, \mathrm{CDCl}_{3}\right): \delta 5.96(\mathrm{~m}, 1 \mathrm{H}, \mathrm{HC}=), 5.94(\mathrm{~d}, J=0.5 \mathrm{~Hz}, 1 \mathrm{H}, \mathrm{PyH}), 5.40$ - $5.32\left(\mathrm{~m}, 2 \mathrm{H}\right.$, one of $=\mathrm{CH}_{2}$ and $\left.\mathrm{PyOCH}\right), 5.29\left(\mathrm{dt}, J=10.4,1.0 \mathrm{~Hz}, 1 \mathrm{H}\right.$, one of $\left.=\mathrm{CH}_{2}\right), 3.22$ (dd, $J=14.9,10.1 \mathrm{~Hz}, 1 \mathrm{H}$, one of $\mathrm{PyCH}_{2}$ ), 2.81 (dd, $J=14.9,7.5 \mathrm{~Hz}, 1 \mathrm{H}$, one of $\mathrm{PyCH}_{2}$ ), 2.26 $\left(\mathrm{d}, J=0.7 \mathrm{~Hz}, 3 \mathrm{H}, \mathrm{PyCH}_{3}\right) .{ }^{13} \mathrm{C}-\mathbf{N M R}\left(126 \mathrm{MHz}, \mathrm{CDCl}_{3}\right): \delta 171.06,165.28,162.13,135.55$, 118.06, 99.03, 95.65, 86.75, 31.64, 20.39. IR (film): 3084, 2924, 1714, 1636, 1579, 1451, 1416, 1254, 1165, 1108, 976, $919 \mathrm{~cm}^{-1}$. HRMS - ESI $(\mathrm{m} / z):[\mathrm{M}+\mathrm{Na}]^{+}$calculated for $\mathrm{C}_{10} \mathrm{H}_{10} \mathrm{O}_{3}{ }^{+}$, 179.0708; found, 179.0711.

\section{Preparation of Pent-1-en-3-ol, 322}

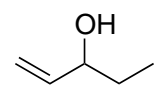

To a solution of propionaldehyde ( $1 \mathrm{~g}, 17.22 \mathrm{mmol}, 1.0$ equiv) in THF $(86 \mathrm{~mL})$ at $0{ }^{\circ} \mathrm{C}$ was added vinyl magnesium bromide $(22.38 \mathrm{~mL}$ of a $1 \mathrm{M}$ solution, $22.38 \mathrm{mmol}, 1.3$ equiv). The reaction was subsequently warmed to room temperature and stirred for 10 hours before being quenched with saturated aqueous $\mathrm{NH}_{4} \mathrm{Cl}$. The reaction mixture was diluted with $\mathrm{H}_{2} \mathrm{O}$ and $\mathrm{Et}_{2} \mathrm{O}$, extracted twice with $\mathrm{Et}_{2} \mathrm{O}$, and the combined organics were dried over $\mathrm{MgSO}_{4}$, filtered, and concentrated in vacuo. The crude product was purified by silica gel chromatography $(20 \%$ $\mathrm{Et}_{2} \mathrm{O} /$ Pet. Ether $)$. The title compound was isolated as a clear yellow oil ( $0.46 \mathrm{~g}, 31 \%$ yield $)$. $\mathbf{R}_{\mathbf{f}}=0.18\left(20 \% \mathrm{Et}_{2} \mathrm{O} /\right.$ Pet. Ether). ${ }^{1} \mathbf{H}$ NMR (500 MHz, $\left.\mathrm{CDCl}_{3}\right) \delta 5.87$ (ddd, $J=16.9,10.4$, $6.2 \mathrm{~Hz}, 1 \mathrm{H}), 5.23(\mathrm{~d}, J=17.2 \mathrm{~Hz}, 1 \mathrm{H}), 5.12(\mathrm{~d}, J=10.4 \mathrm{~Hz}, 1 \mathrm{H}), 4.04(\mathrm{q}, J=5.6 \mathrm{~Hz}, 1 \mathrm{H})$, $1.57(\mathrm{~m}, 2 \mathrm{H}), 0.94(\mathrm{t}, J=7.5 \mathrm{~Hz}, 3 \mathrm{H}) .{ }^{13} \mathrm{C} \mathbf{~ N M R}\left(126 \mathrm{MHz}, \mathrm{CDCl}_{3}\right) \delta 140.94,114.79,74.57$, 29.85, 9.59. Characterization data matches the literature. ${ }^{29}$

Preparation of $(E)-2-(3-h y d r o x y p e n t-1-e n-1-y l)-6$-methyl-2 $H$-furo[3,2-c]pyran$4(3 H)$-one, 323

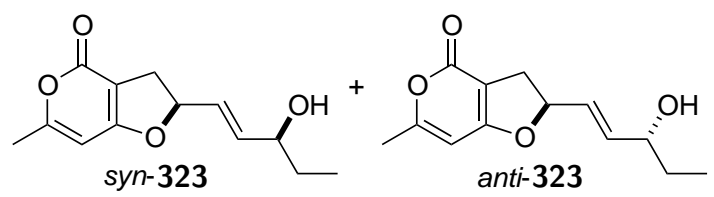

To a solution of Grubbs $2^{\text {nd }}$ generation catalyst $(0.014 \mathrm{~g}, 0.017 \mathrm{mmol}, 10 \mathrm{~mol} \%)$ in $\mathrm{CH}_{2} \mathrm{Cl}_{2}$ $(7.46 \mathrm{~mL})$ was added a solution of 6 -methyl-2-vinyl- $2 H$-furo[3,2-c]pyran- $4(3 H)$-one $(0.030 \mathrm{~g}$, $0.169 \mathrm{mmol}, 1.0$ equiv) and pent-1-en-3-ol (0.102 g, $1.184 \mathrm{mmol}, 7$ equiv) in $\mathrm{CH}_{2} \mathrm{Cl}_{2}(1 \mathrm{~mL})$. The reaction mixture was heated to $45{ }^{\circ} \mathrm{C}$ and was stirred overnight. The reaction was then cooled to room temperature, concentrated in vacuo (note: the title compound showed visible signs of decomposition when concentrated at temperatures above $45{ }^{\circ} \mathrm{C}$.) and purified by silica gel chromatography. The title compound was isolated as a clear colorless oil (0.018 g, 45\% yield, 66:34 ratio of syn- and anti-diastereomers, $0.016 \mathrm{~g} \mathrm{RSM}) . \mathbf{R}_{\mathbf{f}}=0.21$ (60\% EtOAc/Pet. Ether). IR (film): 3400, 2963, 2927, 1704, 1638, 1580, 1453, 1256, 978, $919 \mathrm{~cm}^{-1}$. The two diastereomers were separated using HPLC: Diol (Analytical, $250 \mathrm{~mm}$ x $4.6 \mathrm{~mm}, 5$ micron), $45 \% \mathrm{EtOAc} / n$ Hexane (isocratic), flow rate $=1 \mathrm{~mL} / \mathrm{min}, \lambda=230 / 300 \mathrm{~nm}, \mathrm{R}_{\mathrm{t}}($ major $)=10.6$ 
$\min , \mathrm{R}_{\mathrm{t}}$ (minor) $=11.4$ min. (Note: The order of elution of the major and minor diastereomer in the HPLC trace below is the opposite to that shown in the LC-MS trace (Figure 6.1) because the later uses a reverse phase column and the products were eluted using $12 \% \mathrm{MeCN} / \mathrm{H}_{2} \mathrm{O}$ (isocratic) with $0.05 \%$ ammonium formate.

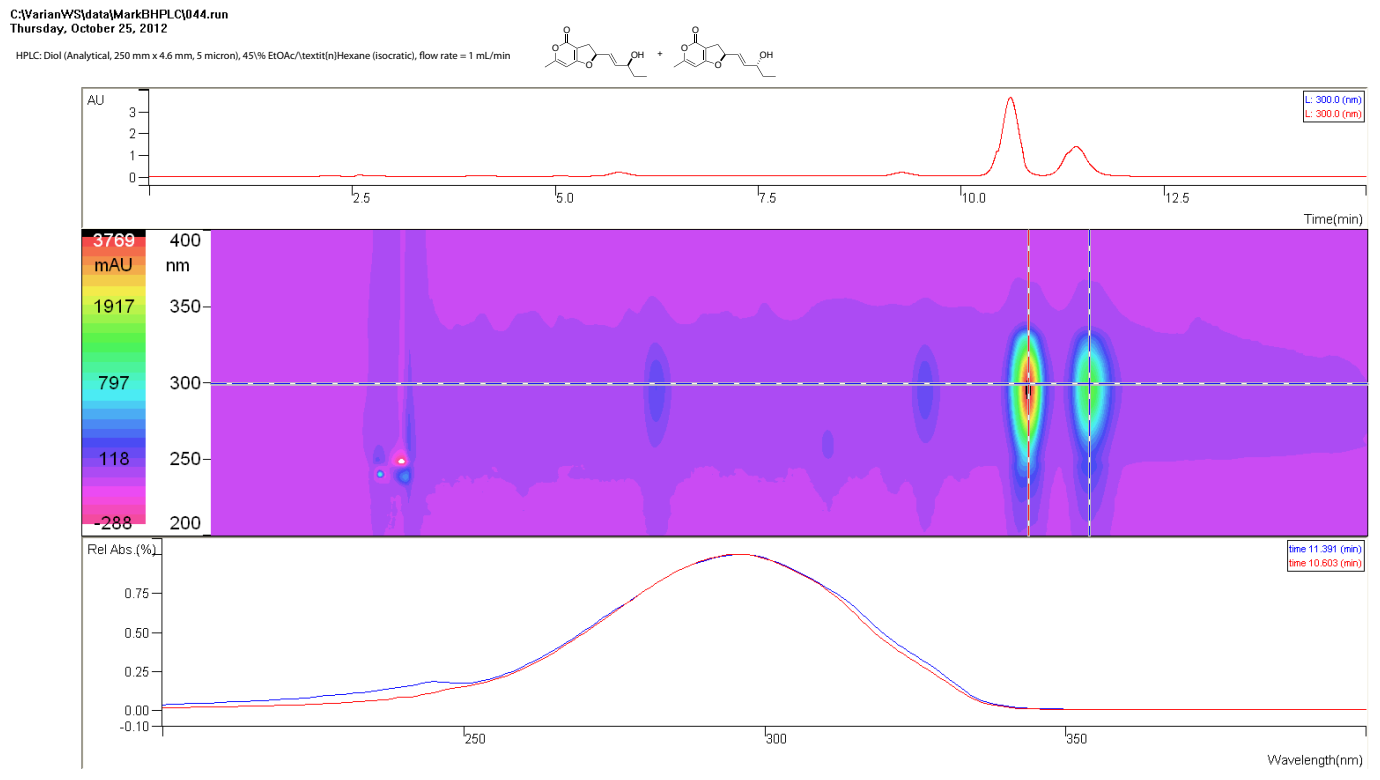

Major diastereomer (syn-323): ${ }^{\mathbf{1}} \mathbf{H}-\mathbf{N M R}\left(600 \mathrm{MHz}, \mathrm{CDCl}_{3}\right): \delta 5.92(\mathrm{~m}, 1 \mathrm{H}), 5.86$ $(\mathrm{dd}, J=18,6 \mathrm{~Hz}, 1 \mathrm{H}), 5.83(\mathrm{dd}, J=12,6 \mathrm{~Hz}, 1 \mathrm{H}), 5.37(\mathrm{~m}, 1 \mathrm{H}), 4.11(\mathrm{~m}, 1 \mathrm{H}), 3.22(\mathrm{dd}, J$ $=14.9,10.0 \mathrm{~Hz}, 1 \mathrm{H}), 2.80(\mathrm{dd}, J=14.9,7.6 \mathrm{~Hz}, 1 \mathrm{H}), 2.26$ (app. d, $J=0.7 \mathrm{~Hz}, 3 \mathrm{H}), 1.58(\mathrm{~m}$, $2 \mathrm{H}), 0.95$ (t, $J=7.5 \mathrm{~Hz}, 3 \mathrm{H}) .{ }^{13} \mathrm{C}$ NMR $\left(151 \mathrm{MHz}, \mathrm{CDCl}_{3}\right) \delta 171.01,165.29,162.12,137.06$, 127.76, 99.07, 95.66, 86.29, 72.94, 31.95, 29.96, 20.42, 9.61. HRMS - EI $(\mathrm{m} / z)$ calculated for $\mathrm{C}_{13} \mathrm{H}_{16} \mathrm{O}_{4}{ }^{+}[\mathrm{M}+\mathrm{H}]^{+}:$236.1049, found: 236.1059 .

Minor diastereomer (anti-323): ${ }^{1} \mathbf{H}-\mathbf{N M R}\left(600 \mathrm{MHz}, \mathrm{CDCl}_{3}\right): \delta 5.93(\mathrm{~s}, 1 \mathrm{H}), 5.85(\mathrm{dd}$, $J=18,6 \mathrm{~Hz}, 1 \mathrm{H}), 5.82(\mathrm{dd}, J=18,6 \mathrm{~Hz}, 1 \mathrm{H}), 5.38(\mathrm{~m}, 1 \mathrm{H}), 4.11(\mathrm{~m}, 1 \mathrm{H}), 3.22(\mathrm{dd}, J=$ $14.9,10.0 \mathrm{~Hz}, 1 \mathrm{H}), 2.79(\mathrm{dd}, J=14.9,7.5 \mathrm{~Hz}, 1 \mathrm{H}), 2.26(\mathrm{~s}, 3 \mathrm{H}), 1.58(\mathrm{~m}, 2 \mathrm{H}), 0.94(\mathrm{t}, J=7.5$ $\mathrm{Hz}, 3 \mathrm{H}) .{ }^{13} \mathrm{C}$ NMR $\left(151 \mathrm{MHz}, \mathrm{CDCl}_{3}\right) \delta 171.00,165.31,162.12,136.89,127.87,99.05,95.66$, 86.21, 73.03, 31.97, 29.92, 20.42, 9.63. HRMS - EI $(m / z)$ calculated for $\mathrm{C}_{13} \mathrm{H}_{16} \mathrm{O}_{4}{ }^{+}[\mathrm{M}+\mathrm{H}]^{+}$: 236.1049 , found: 236.1056 . 


\subsubsection{Spectra}

${ }^{1} \mathrm{H}-\mathrm{NMR}\left(500 \mathrm{MHz}, \mathrm{CDCl}_{3}\right)$<smiles>C/C=C/C1Cc2c(cc(C)oc2=O)O1</smiles>
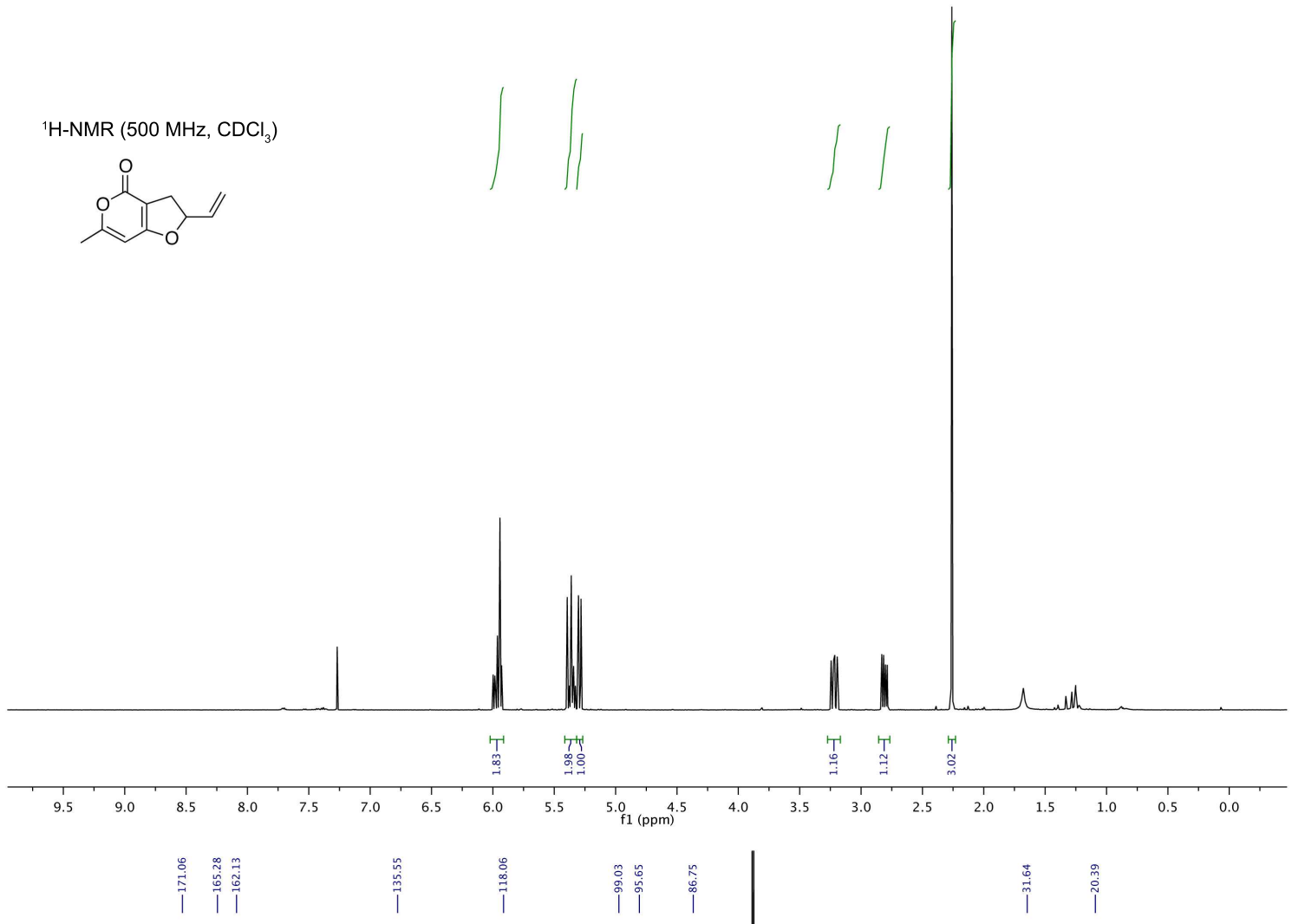

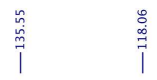

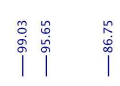

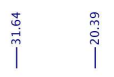

${ }^{13} \mathrm{C}-\mathrm{NMR}\left(126 \mathrm{MHz}, \mathrm{CDCl}_{3}\right)$

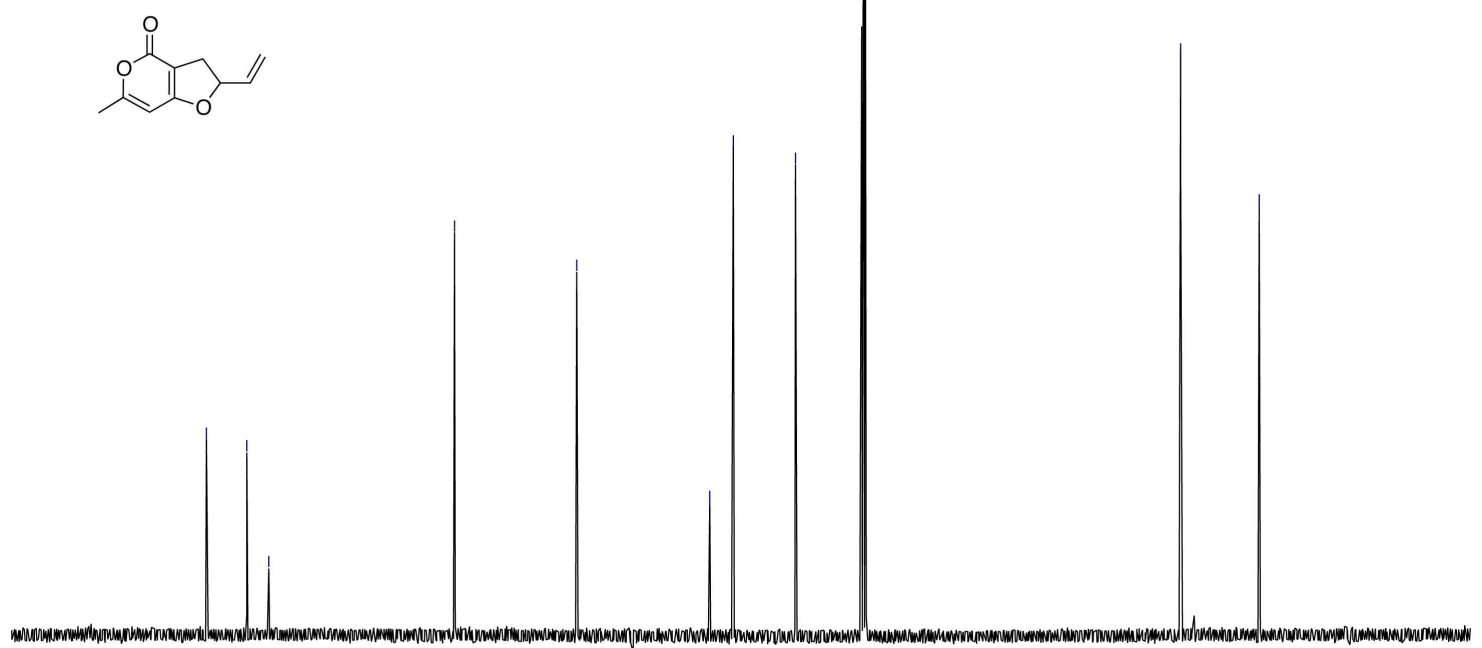

$\begin{array}{llllllllll}190 & 180 & 170 & 160 & 150 & 140 & 130 & 120 & 110 & 100 \\ \mathrm{f} 1(\mathrm{ppm}) & 90\end{array}$

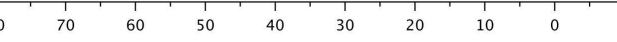



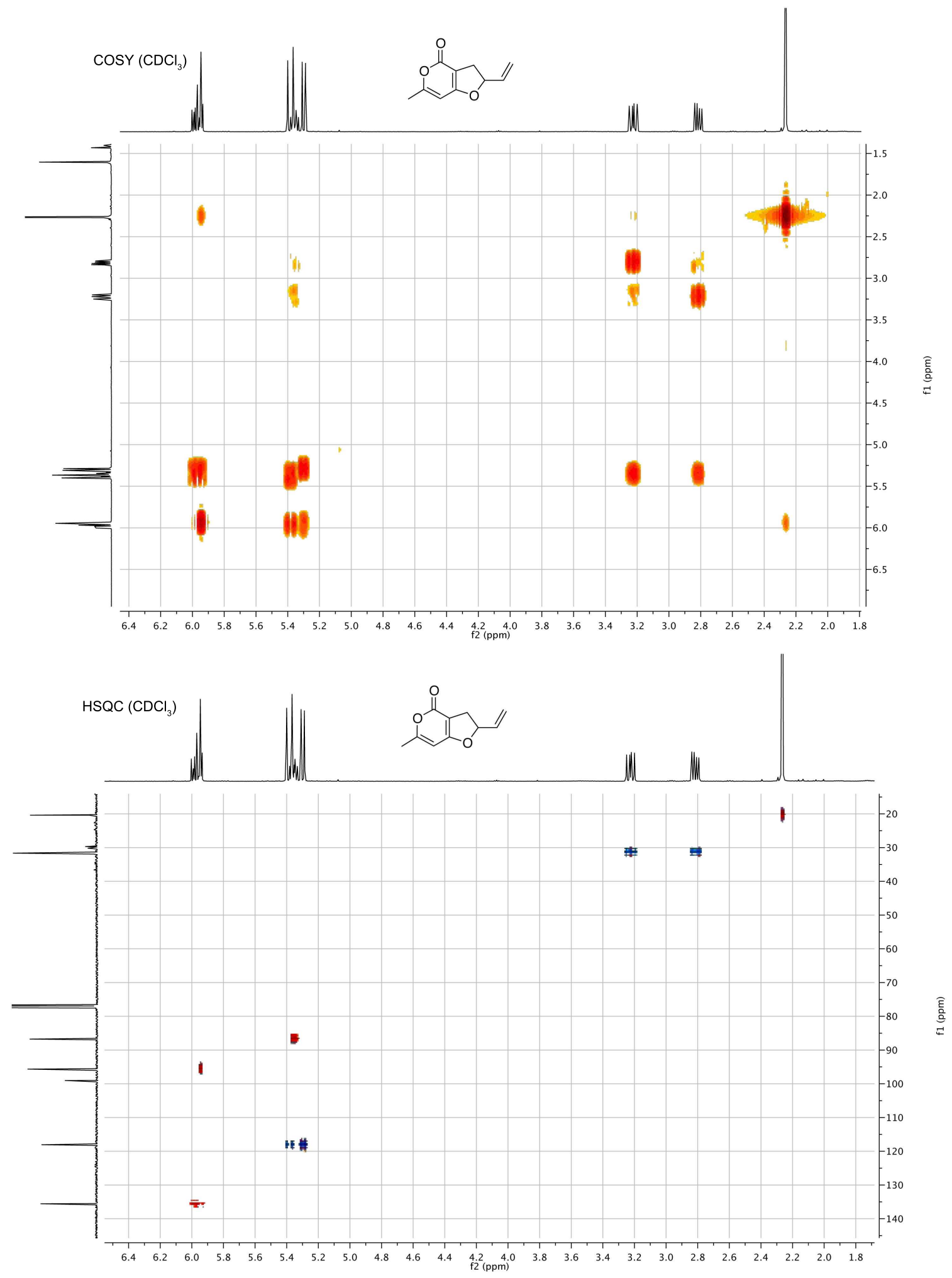


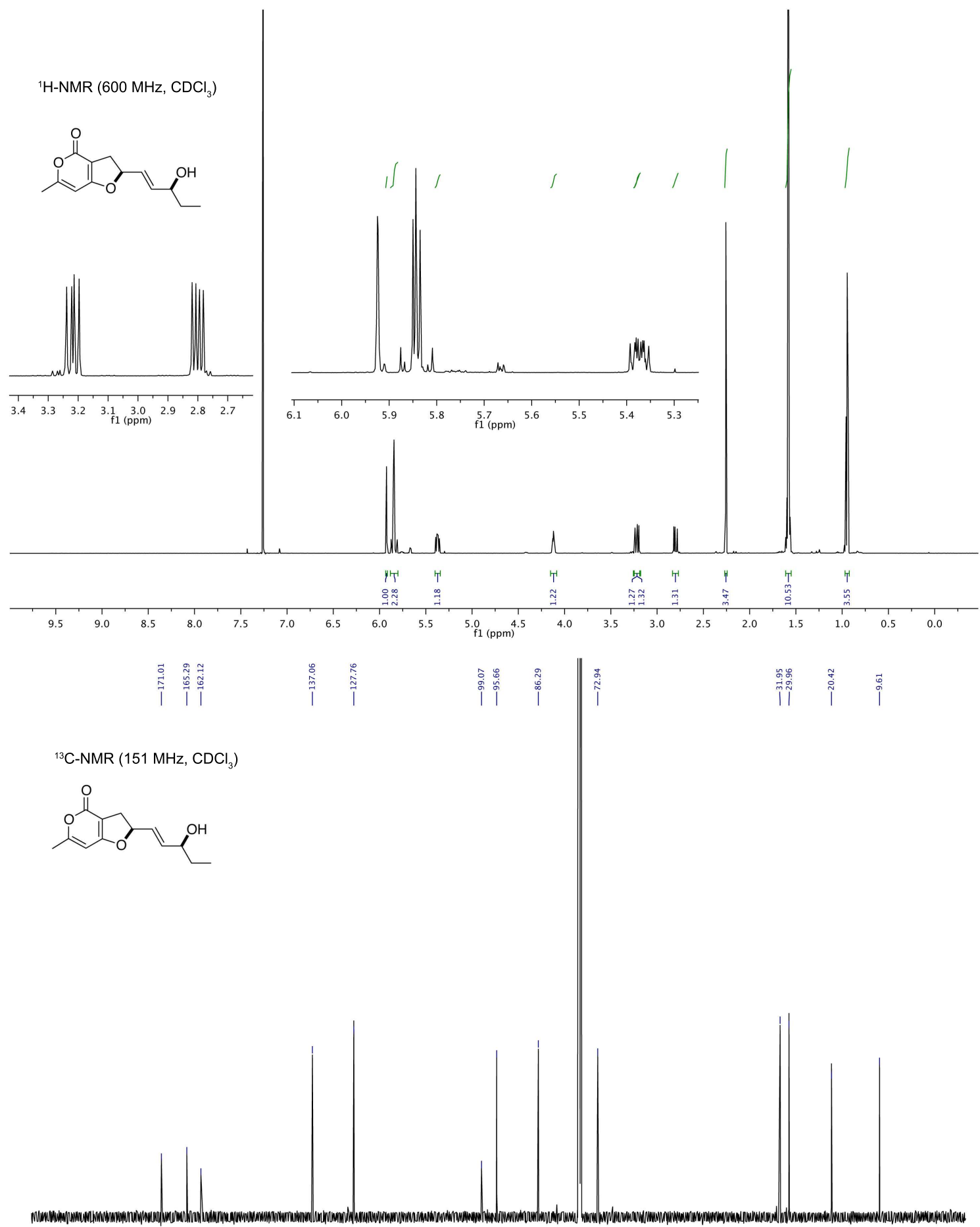

$\begin{array}{lllllllllllllllllllllll} & \\ 200 & 190 & 180 & 170 & 160 & 150 & 140 & 130 & 120 & 110 & \underset{\mathrm{f} 1(\mathrm{ppm})}{100} & 90 & 70 & 60 & 50 & 40 & 30 & 20 & 10 & 0\end{array}$ 

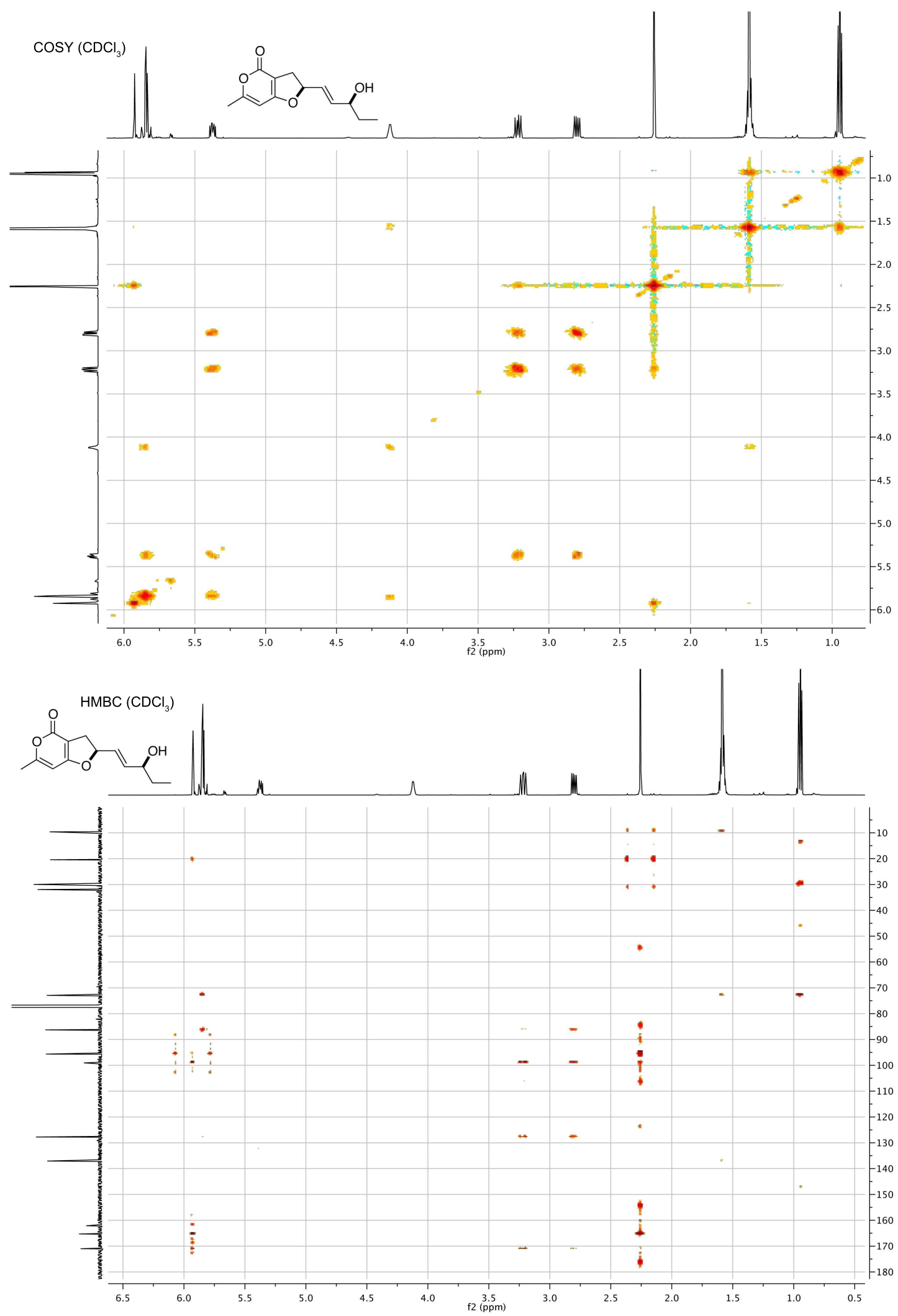
6.6. EXPERIMENTAL DATA FOR CHAPTER 6

232

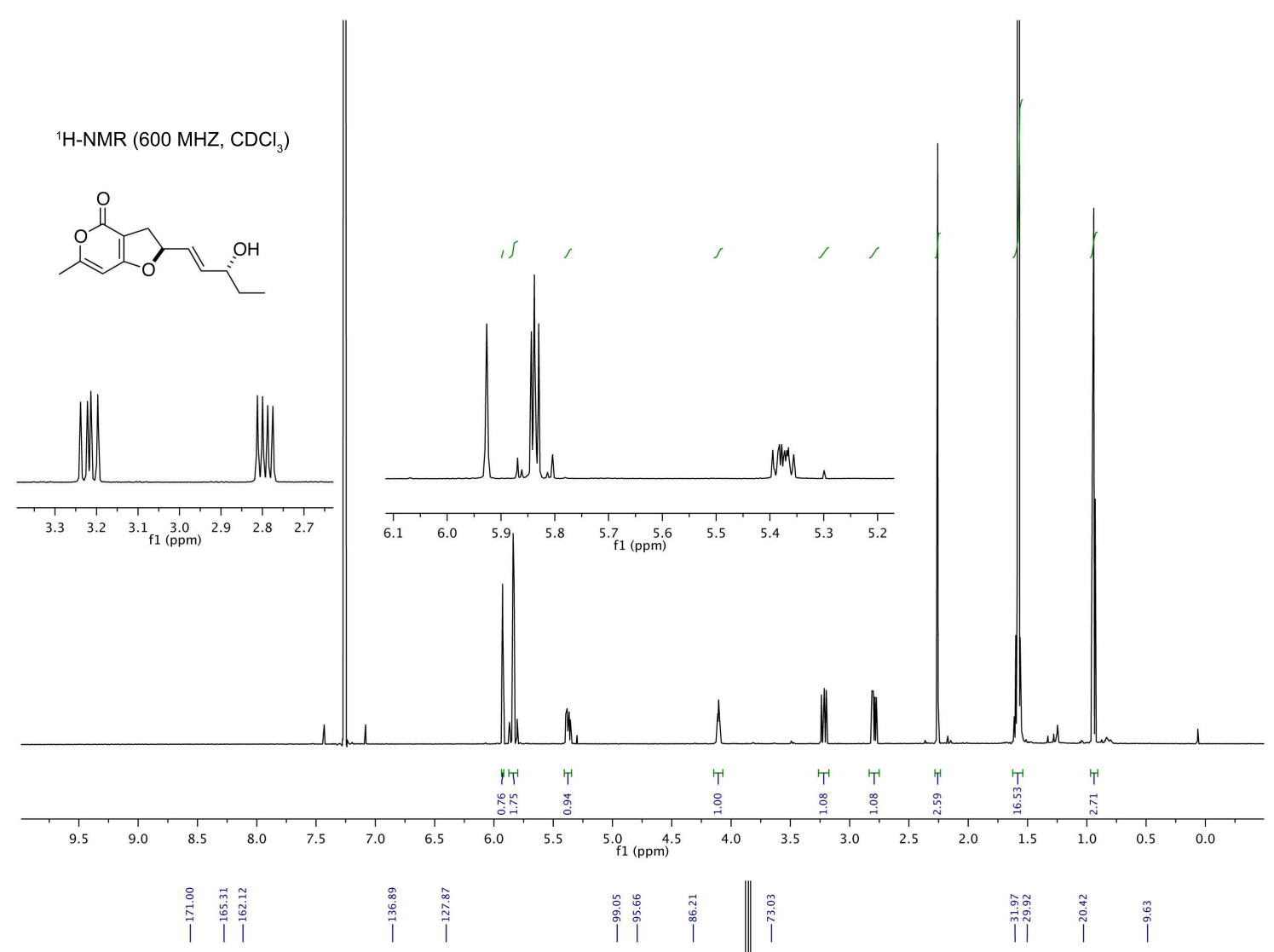

${ }^{13} \mathrm{C}-\mathrm{NMR}\left(151 \mathrm{MHz}, \mathrm{CDCl}_{3}\right)$

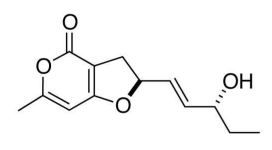



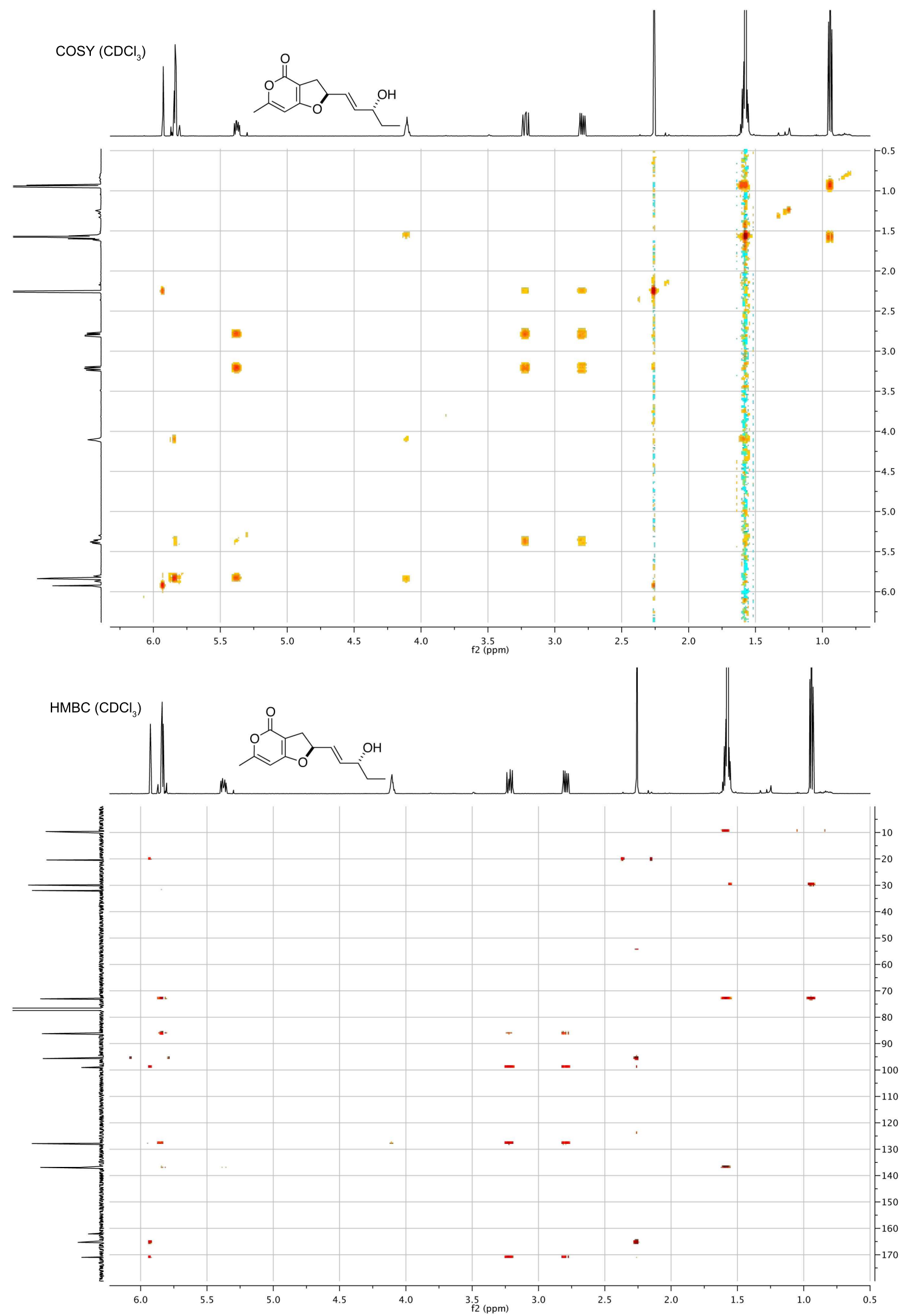

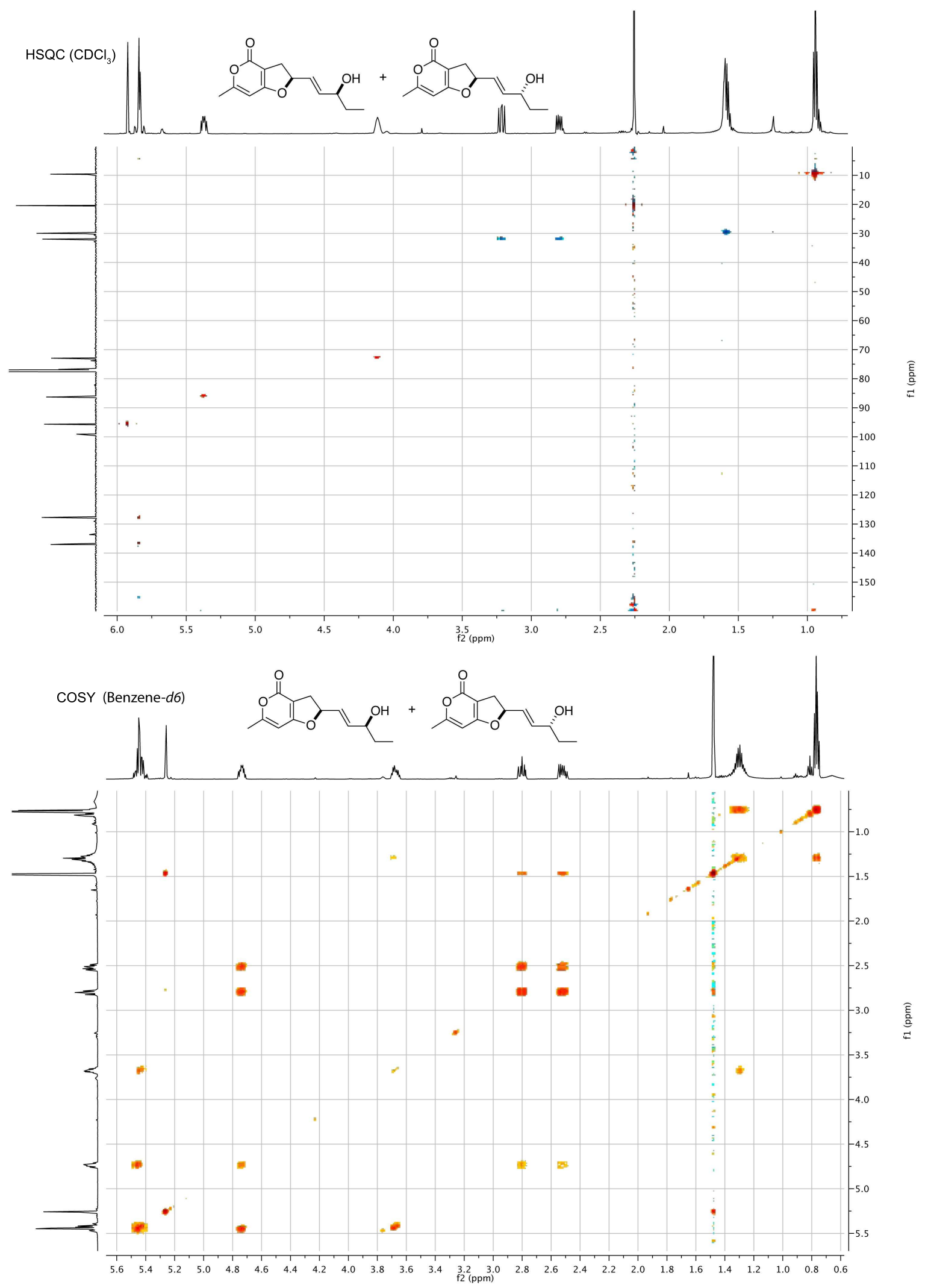
6.6. EXPERIMENTAL DATA FOR CHAPTER 6

235
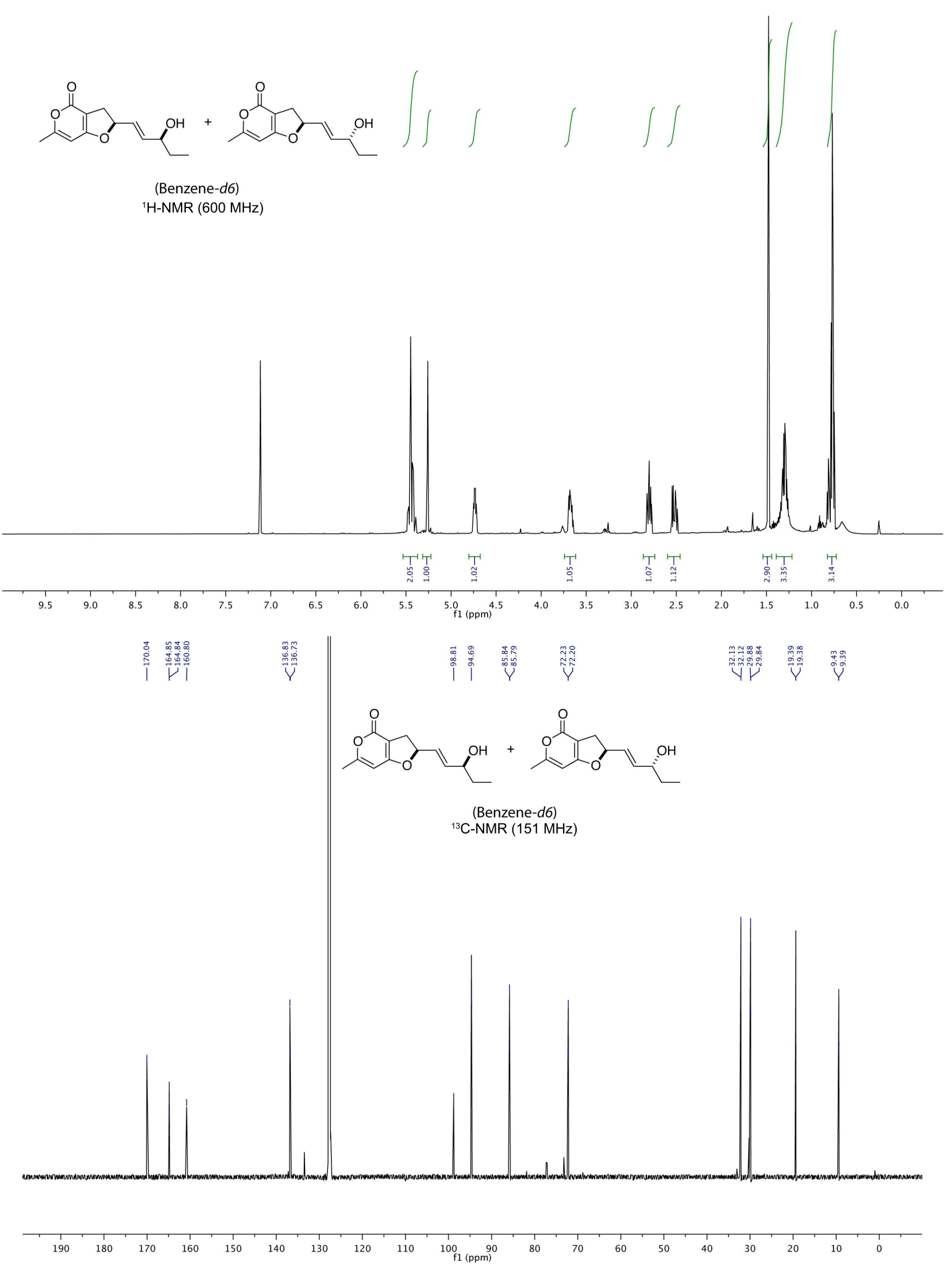


\section{References for Chapter 6}

[1] For leading references on palladium-catalyzed allylic alkylation, see: Trost, B. M. Acc. Chem. Res. 1980, 13, 385. Trost, B. M; Crawley, M. L. Chem. Rev. 2003, 103, 2921. Tsuji, J. Palladium Reagents and Catalysts: Innovations in Organic Synthesis; Wiley \& Sons: New York, 1996; pp 290-399.

[2] Trost, B. M.; Scalan, T. S. J. Am. Chem. Soc. 1989, 111, 4988.

[3] (a) Tsuda, T.; Kiyoi, T.; Saegusa, T. J. Org. Chem. 1990, 55, 3388. (b) Uozumi, Y.; Tanahashi, A.; Hayashi, T. J. Org. Chem. 1993, 58, 6826. (c) Massacret, M.; Lhoste, P.; Sinou, D. Eur. J. Org. Chem. 1999, 129. (d) Trost, B. M.; Dong, G. Chem. Eur. J. 2009, 15, 6910.

[4] Liao, X.; Huang, S.; Zhou, H.; Parrish, D.; Cook, J. Org. Lett. 2007, 9, 1469.

[5] a) Thorey, C.; Wilken, J.; Henin, F.; Martens, J.; Mehler, T.; Muzart, J. Tetrahedron Letters 1995, 36, 5527. b) Yamazaki, A.; Achiwa, I.; Achiwa, K. Tetrahedron Asymm. 1996, 7, 403. c) Massacret, M.; Lakhmiri, R.; Lhoste, P.; Nguefack, C.; Abdelouahab, F.; Fadel, R.; Sinou, D. Tetrahedron Asymm. 2000, $11,3561$. d) Nakano, H.; Yokoyama, J.; Fujita, R.; Hongo, H. Tetrahedron Lett. 2002, 43, 7761.

[6] a) Massacret, M.; Goux, C.; Lhoste, P.; Sinou, D. Tetrahedron Lett. 1994, 35, 6093. b) Massacret, M.; Lhoste, P.; Lakhmiri, R.; Parella, T.; Sinou, D. Eur. J. Org. Chem. 1999, 2665. c) Zawisza, A.; Toupet, L.; Sinuo, D. Letters in Organic Chemistry 2006, 3, 861.

[7] a) Hayashi, T.; Yamamoto, A.; Ito, Y. Tetrahedron Lett. 1988, 29, 99. b) Tanimori, S.; Kirihata, M. Tetrahedron Lett. 2000, 41, 6785. c) Tanimori, S.; Inaba, U.; Kato, Y.; Kirihata, M. Tetrahedron 2003, 59, 3745 .

[8] Huang, Y.; Lu, X. Tetrahedron Lett. 1987, 28, 6219.

[9] a) Hayashi, T.; Yamamoto, A.; Ito, Y. Tetrahedron Lett. 1988, 29, 669. b) Hayashi, T.; Ohno, A.; Lu, S.; Matsumoto, Y.; Fukuyo, E.; Yanagi, K. J. Am. Chem. Soc. 1994, 116, 4221. c) Ohno, A.; Yamane, M.; Hayashi, T.; Oguni, N.; Hayashi, M. Tetrahedron Assym. 1995, 6, 2495. d) Mori, M.; Nukui, S.; Shibasaki, M. Chem. Lett. 1991, 1797.

[10] Racemic syntheses: Kozikowski, A.; Campiani, G.; Aagaard, P.; McKinney, M. J. Chem. Soc. Chem. Commun. 1993, 860. Campiani, G.; Sun, L.; Kozikowski, A.; Aagaard, P.; McKinney, M. J. Org. Chem. 1993, 58, 7660. Enantioselective syntheses: Kaneko, S.; Yoshino, T.; Katoh, T.; Terashima, S. Tetrahedron, 1998, 54, 5471. Kaneko, S.; Yoshino, T.; Katoh, T.; Terashima, S. Tetrahedron: Asymmetry 1997, 8, 829. He, X.; Wang, B.; Bai, D. Tetrahedron Letters 1998, 39, 411.

[11] He, X.; Wang, B.; Yu, G.; Bai, D. Tetrahedron: Asymmetry 2001, 12, 3213.

[12] Lu, X.; Huang, Y. Tetrahedron Letters 1986, 27, 1615. Lu, X.; Huang, Y. Tetrahedron Letters 1988, 29, 5663.

[13] a) Shimizu, I.; Ohashi, Y.; Tsuji, J. Tetrahedron Lett. 1985, 26, 3049. b) Burgess, K. J. Org. Chem. 1987, 52, 2046. c) Gaucher, A.; Dorizon, P.; Ollivier, J.; Sala§n, J. Tetrahedron Lett. 1995, 36, 2979.

[14] Liao, X.; Huang, S.; Zhou, H.; Parrish, D.; Cook, J. 2007, 9, 1469.

[15] Trost, B. M.; Dong, G. Chem. Eur. J. 2009, 15, 6910. Trost, B. M.; Dong, G.; J. Am. Chem. Soc. 2006 , 128,6054 .

[16] The $\mathrm{Pd}$ isotope pattern and natural abundances are as follows; ${ }^{102} \mathrm{Pd}: 1.02 \%,{ }^{104} \mathrm{Pd}: 11.14 \%,{ }^{105} \mathrm{Pd}$ : $22.33 \%,{ }^{106} \mathrm{Pd}: 27.33 \%,{ }^{108} \mathrm{Pd}: 26.46 \%,{ }^{110} \mathrm{Pd}: 11.72 \%$. WebElements: the periodic table on the web. http://www.webelements.com/palladium/isotopes.html (accessed Oct 20, 2012).

[17] Herrmann, W. A.; Brossmer, C.; Ofele, K.; Reisinger, C.-P.; Priermeier, T.; Beller, M.; Fischer, H. Angew. Chem., Int. Ed. Engl. 1995, 34, 1844. Xiong, Z.; Wang, N.; Dai, M.; Li, A.; Chen, J.; Yang, Z. Org. Lett. 2004, 6, 3337. Blackmond, D. G.; Rosner, T.; Pfaltz, A. Org. Proc. Res. Dev. 1999, 3, 275. 
[18] For selected examples of the isolation of palladacyclic intermediates during intramolecular Pd-catalyzed reactions, see: Vicente, J.; Saura-Llamas, I.; García-López, J.-A. Organometallics 2010, $29,4320$. Danishefsky, S. J.; Masters, J. J.; Young, W. B.; Link, J. T.; Snyder, L. B.; Magee, T. V.; Jung, D. K.; Isaacs, R. C. A.; Bornmann, W. G.; Alaimo, C. A.; Coburn, C. A.; Di Grandi, M. J. J. Am. Chem. Soc. 1996, 118, 2843. Burke, B. J.; Overman, L. E. J. Am. Chem. Soc. 2004, 126, 16820. Oestreich, M.; Dennison, P. R.; Kodanko, J. J.; Overman, L. E. Angew. Chem., Int. Ed. 2001, 40, 1439.

[19] Beccalli, E. M.; Broggini, G.; Martinelli, M.; Masciocchi, N.; Sottocornola, S. Org. Lett. 2006, 8, 4521.

[20] Morimoto, S.; Shindo, M.; Yoshida, M.; Shishido, K. Tetrahedron Lett. 2006, 47, 7353.

[21] Popplewell, W. Isolation and Structure Elucidation of New Secondary Metabolites from New Zealand Marine Red Algae. Ph.D. Thesis, Victoria University of Wellington, 2008.

[22] For reviews on the computational prediction of NMR chemical shifts, see: Lodewyk, M. W.; Siebert, M. R.; Tantillo, D. J. Chem. Rev. 2012, 112, 1839. Bifulco, G.; Dambruoso, P.; Gomez-Paloma, L.; Riccio, R. Chem Rev. 2007, 107, 3744.

[23] B3LYP functional: Becke, A. D. J. Chem. Phys. 1993, 98, 5648. Stephens, P. J.; Devlin, P. J.; Chabalowski, C. F.; Frisch, M. J. J. Phys. Chem. 1994, 98, 11623. 6-31G(d) basis set: Frisch, M. J.; Pople, J. A.; Binkley, J. S. J. Chem. Phys. 1984, 80, 3265. Ditchfield, R.; Hehre, W. J.; Pople, J. A. J. Chem. Phys. 1971, 54, 724.

[24] Smith, S. G.; Goodman, J. M. J. Org. Chem. 2009, 74, 4597.

[25] For a related structure assignment invloving a 1,4-syn(anti)-relationship across an alkene, see: jiang, W.; Liu, D.; Deng, Z.; de Voogd, N. J.; Proksch, P.; Lin, W. Tetrahedron 2011, 67, 58.

[26] The absolute configuration of the proposed axial chirality was assigned based on the intersection of the furopyrone (FP) and $Z$-alkene planes (AP). The carbonyl side of the furopyrone plane (FP) was given the highest priority followed by the furan ring oxygen side of the FP, the alkene side of the AP, and, lastly, the alcohol side of the AP.

[27] The calculated ${ }^{3} J_{\mathrm{HH}}$ coupling constants were obtained using the Karplus equation and dihedral angles from the lowest energy geometry optimized structure for each diastereomer. Balacco, G. J. Chem. Inf. Comput. Sci. 1996, 36, 885. Haasnoot, C. A. G.; DeLeeuw, F. A. A. M.; Altona, C. Tetrahedron 1980, 36, 2783. Karplus, M. J. Am. Chem. Soc. 1963, 85, 2870.

[28] Paterson, I.; Anderson, E. A.; Dalby, S. M.; Lim, J. H.; Maltas, P. Org. Biomol. Chem. 2012, $10,5873$.

[29] Shen, Z.-L.; Goh, K. K. K.; Cheong, H.-L.; Wong, C. H. A.; Lai, Y.C.; Yang, Y.-S.; Loh, T.-P. J. Am. Chem. Soc. 2010, 132, 15852 . 
Chapter 7

Pd-AA Cascade II: Regioselective Dihydropyran Alkylation in a Third Generation Approach to Labillarides E-H 


\subsection{Initial Investigations Into The Use of Substituted Bis- Electrophiles in Pd-AA Cascades.}

The use of a Pd-AA cascade in the synthesis of labillarides E-H is contingent on the introduction of a synthetic handle at C7 (labillaride numbering) to enable the construction of the macrocyclic ring. One method by which this can be achieved is through the use of a substituted butene1,4-dicarbonate bis-electrophile in the Pd-AA cascade reaction. With this in mind, the use of the D-mannitol derived substrate $\mathbf{3 2 4}$ in a Pd-AA cascade with 4-hydroxy-6-methyl- $\alpha$-pyrone (253) was investigated. The aforementioned substituted bis-electrophile was prepared in five steps from D-mannitol (Scheme 7.1). Unfortunately, 324 was found to be unreactive under<smiles>OCC(O)C(O)C(O)CO</smiles>

325<smiles>CO[C@H](/C=C/[C@@H](CO[SnH3])OC(C)=O)COc1ccccc1</smiles>

324

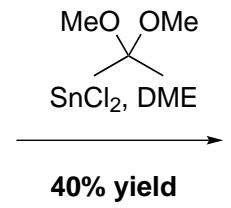<smiles>CC1(C)OC[C@@H]([C@H](O)[C@H]2COC3(CC3)O2)O1</smiles>

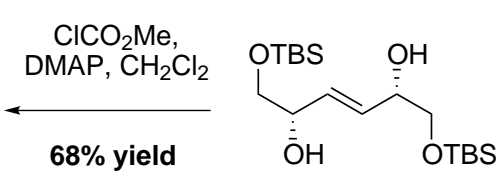

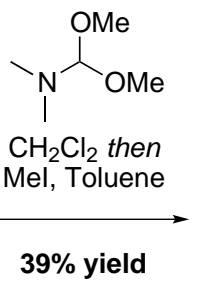
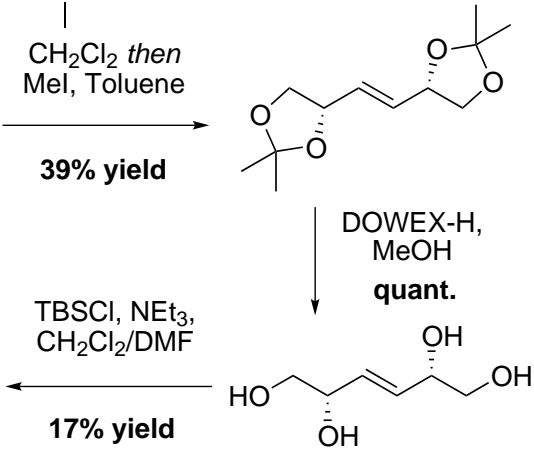

Scheme 7.1 Synthesis of a Mannitol-Derived, Substituted Bis-Electrophile.

the previously optimized Pd-AA conditions (Scheme 7.2). This observation is not dissimilar to previous results obtained during the first generation approach to labillarides E-H (see Section 5.3). The poor reactivity of this substrate is presumably a consequence of steric hinderance preventing the formation of the $\pi$-allyl-Pd intermediate required for alkylation to occur.

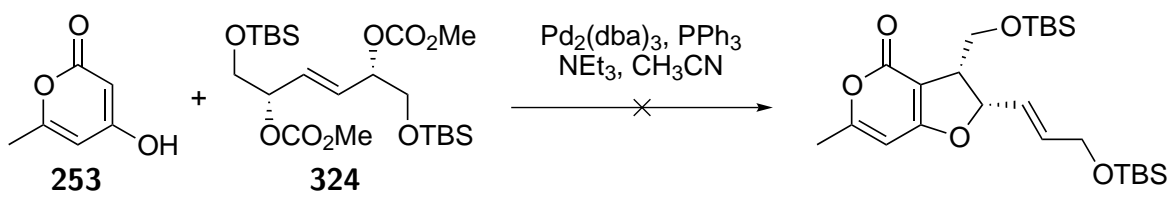

Scheme 7.2 Attempted Pd-AA Cascade with the Substituted Bis-Electrophile, 324.

In an attempt to overcome this problem, a number of cyclic bis-electrophiles were investigated with the hope that tying back some of the steric bulk of the substrate, in the form of a ring, would enable substituted bis-electrophiles to participate in the desired Pd-AA cascade. ${ }^{\S}$ Using the Pd-AA conditions shown in Scheme 7.2, the tetrasubstituted cyclohexenes 326 and 327 did not react with 4-hydroxy-6-methyl- $\alpha$-pyrone, despite reports in the literature describing Pd-AA reactions with very similar substrates. ${ }^{1}$ Use of the less sterically encumbered

\footnotetext{
${ }^{\S}$ See Section 7.5 for details on the synthesis of these substrates.
} 


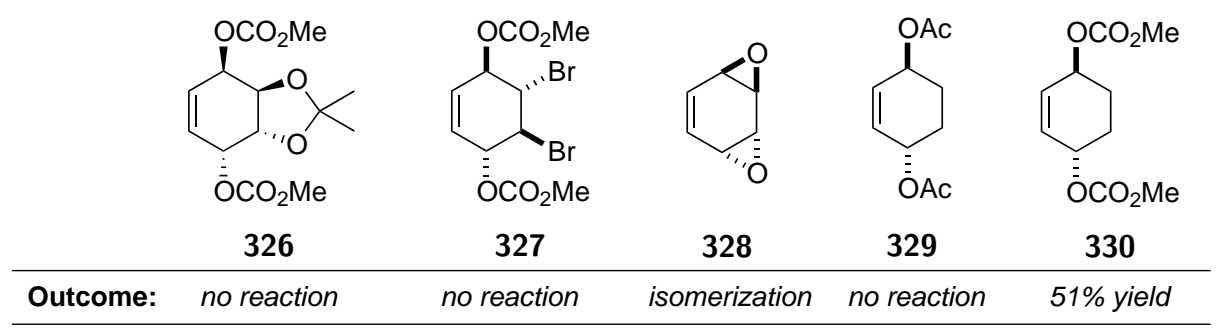

Figure 7.1 Attempted Pd-AA Cascades with Cyclic Bis-Electrophiles.

substrate, bis-epoxide $\mathbf{3 2 8}$ resulted in the isolation of an unwanted isomerization product, 1,2catechol. Moving on to a disubstituted cyclohexene, acetate 329, again gave no reaction. However, the corresponding carbonate, 330, provided the desired reactivity and produced tetrahydrobenzofuran $\mathbf{3 3 1}$ in $51 \%$ yield (Scheme 7.3 ).

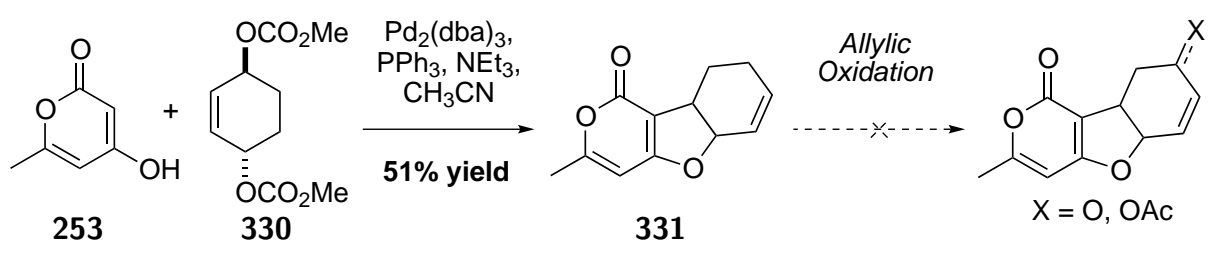

Scheme 7.3 A Cyclohexene-Based Pd-AA Cascade.

Unfortunately, tetrahydrobenzofuran $\mathbf{3 3 1}$ lacks the functionality necessary for the synthesis of labillarides E-H, and attempts to perform an allylic oxidation were unsuccessful. With a new found awareness of the substrate limitations inherent to the Pd-AA cascade, our focus shifted away from substituted cyclohexenes and towards finding effective non-symmetric heterocyclic bis-electrophiles for the Pd-AA cascade.

\subsection{Development of a Non-Symmetric, Regioselective Pd-AA Cascade}

The major challenge of $\mathrm{Pd}-\mathrm{AA}$ cascade reactions with non-symmetric substrates is the differentiation of the two nucleophilic and electrophilic centers so that a single product is formed regioselectively. Scheme 7.4 exemplifies the large number of regiochemical possibilities that exist in a Pd-AA of a non-symmetric allyl bis-electrophile with a non-symmetric bis-nucleophile. The initial permutations shown are further compounded by a second addition. Typically, this challenge has been avoided by employing symmetric electrophiles or nucleophiles, thereby obviating the need for regioselectivity. Thus, despite the synthetic efficiency of the Pd-AA cascade, it has found limited use in total synthesis (vide supra). During the course of our current research into pyrone-based natural products, we have discovered that certain dihydropyran substrates serve as non-symmetric bis-electrophiles for Pd-AA cascades with cyclic $\beta$-dicarbonyl compounds. This methodology provides rapid access to unsaturated furopyran ring systems with excellent regio- and diastereoselectivity. 


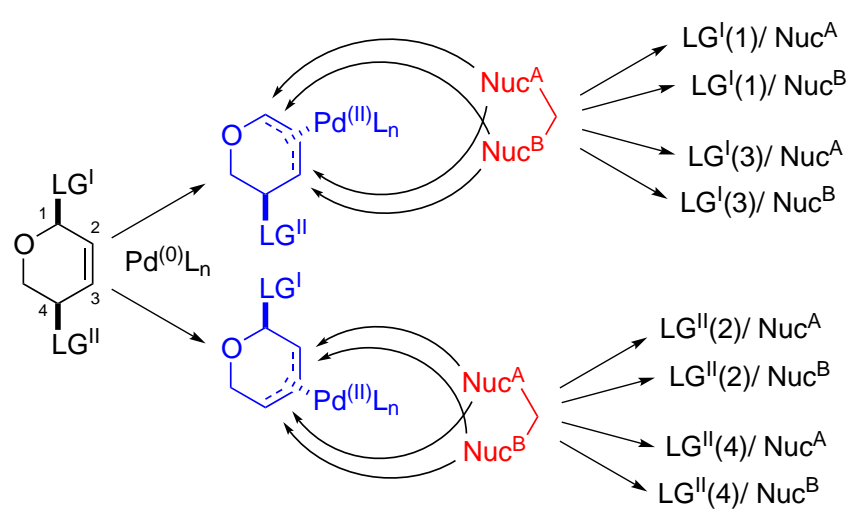

Scheme 7.4 Regiochemical Permutations for a Non-Symmetric Pd-AA Cascade.

\subsubsection{Reaction Optimization}

A non-symmetric Pd-AA cascade was attempted using cis-332, which was prepared in four steps from furfuryl alcohol via known alcohol 333 (Scheme 7.5). ${ }^{2}$ The relative stereochemistry of these 3,6-dihydro-2H-pyrans is discussed in detail in Chapter 8. Optimization of the Pd-

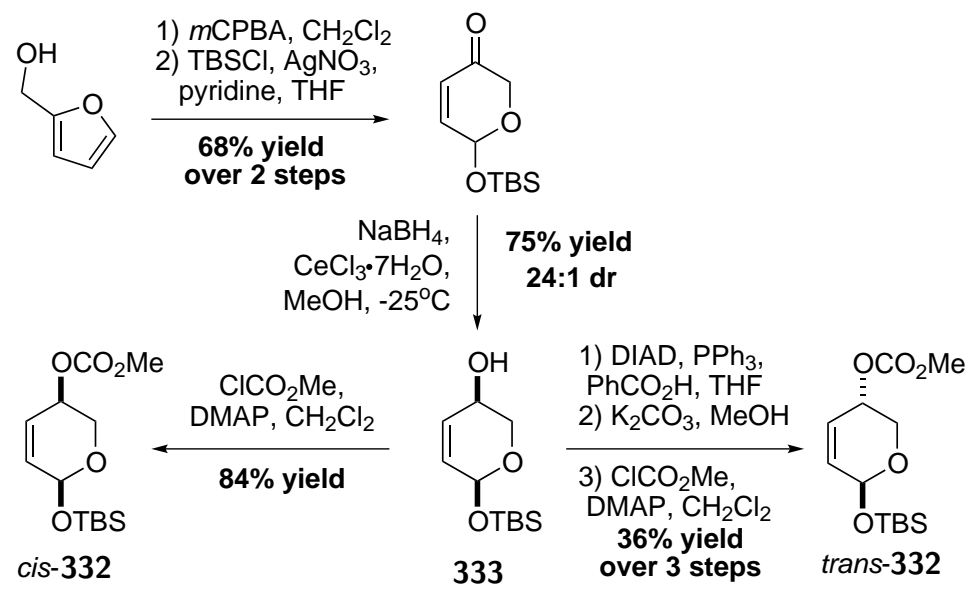

Scheme 7.5 Synthesis of Pyran Substrates, cis-332 and trans-332.

AA cascade reaction began with the conditions from the symmetric Pd-AA cascade (see 6.3), which provided the desired furopyran 334 in a modest $42 \%$ yield (Table 7.1). However, it soon became evident that a number of conclusions from the previous optimization were not consistent with the results from the dihydropyran-based substrate 332. Specifically, the use of 2.2 equivalents of 4 -hydroxy- $\alpha$-pyrone was not necessary and, in fact, the use of excess pyrone was detrimental in some cases (Entries 1-4), leaving unreacted starting material (cis-332) and producing the undesired $O$-alkylation side product, 335 (see Scheme 7.8). The disparity between the two sets of results meant that any assumptions that were carried over from earlier work needed to be treated lightly, and therefore a new set of solvent screening experiments was performed. Performing the reaction in THF or $\mathrm{CH}_{2} \mathrm{Cl}_{2}$ gave modest yields of $44 \%$ and $30 \%$ respectively (Entries $6 \& 3$ ). The use of $\mathrm{CH}_{3} \mathrm{CN}$, without any additives, provided a poor 
$12 \%$ yield (Entry 5), whereas toluene gave a very promising $65 \%$ yield (Entry 7 ). Changing to a more reactive palladium complex, $\mathrm{Pd}\left(\mathrm{PPh}_{3}\right)_{4},{ }^{4}$ provided a higher yield and enabled the the $\mathrm{Pd}$ loading to be significantly reduced. Ultimately, the use of $5 \mathrm{~mol} \%$ of $\mathrm{Pd}\left(\mathrm{PPh}_{3}\right)_{4}$ in toluene provided an optimized yield of $83 \%$ (Entry 9). Unfortunately, reducing the catalyst loading further $(1 \mathrm{~mol} \%)$ caused a significant decrease in yield. The addition of triethylamine to these optimized conditions provided no additional improvements in yield (Entry 10).

Table 7.1 Optimization of a Pd-AA Cascade with Dihydropyran cis-332

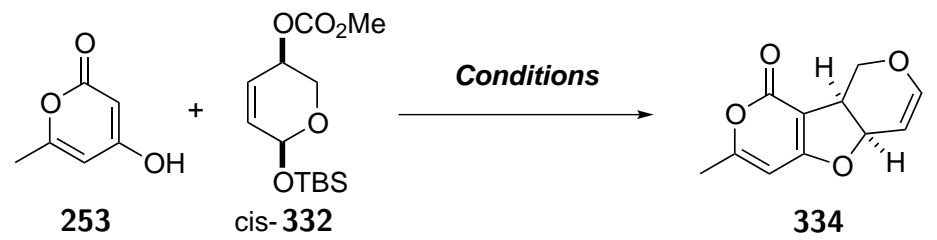

\begin{tabular}{|c|c|c|c|c|}
\hline Entry $^{\mid a\rfloor}$ & Catalyst $(\mathrm{mol} \%)^{[\mathrm{b}]}$ & Solvent & Comments & Yield \\
\hline 1 & $\mathrm{Pd}_{2}(\mathrm{dba})_{3} / \mathrm{PPh}_{3}$ (10) & $\mathrm{CH}_{3} \mathrm{CN}$ & $\begin{array}{c}2.2 \text { equiv } \mathbf{2 5 3}, \\
1 \text { equiv } \mathrm{NEt}_{3}\end{array}$ & $42 \%$ \\
\hline 2 & $\mathrm{Pd}_{2}(\mathrm{dba})_{3} / \mathrm{PPh}_{3}(10)$ & $\mathrm{CH}_{3} \mathrm{CN}$ & 1 equiv $\mathrm{NEt}_{3}$ & $40 \%$ \\
\hline 3 & $\mathrm{Pd}_{2}(\mathrm{dba})_{3} / \mathrm{PPh}_{3}$ (10) & $\mathrm{CH}_{2} \mathrm{Cl}_{2}$ & - & $30 \%$ \\
\hline 4 & $\mathrm{Pd}_{2}(\mathrm{dba})_{3} / \mathrm{PPh}_{3}(10)$ & $\mathrm{CH}_{2} \mathrm{Cl}_{2}$ & 2.2 equiv $\mathbf{2 5 3}$ & $5 \%[c]$ \\
\hline 5 & $\mathrm{Pd}_{2}(\mathrm{dba})_{3} / \mathrm{PPh}_{3}$ (10) & $\mathrm{CH}_{3} \mathrm{CN}$ & - & $12 \%$ \\
\hline 6 & $\mathrm{Pd}_{2}(\mathrm{dba})_{3} / \mathrm{PPh}_{3}(10)$ & $\mathrm{THF}$ & - & $44 \%$ \\
\hline 7 & $\mathrm{Pd}_{2}(\mathrm{dba})_{3} / \mathrm{PPh}_{3}$ (10) & Toluene & - & $65 \%$ \\
\hline 8 & $\mathrm{Pd}\left(\mathrm{PPh}_{3}\right)_{4}$ & Toluene & - & $71 \%$ \\
\hline 9 & $\mathrm{Pd}\left(\mathrm{PPh}_{3}\right)_{4}$ & Toluene & - & $83 \%$ \\
\hline 10 & $\mathrm{Pd}\left(\mathrm{PPh}_{3}\right)_{4}$ & Toluene & 1 equiv $\mathrm{NEt}_{3}$ & $71 \%$ \\
\hline 11 & $\mathrm{Pd}\left(\mathrm{PPh}_{3}\right)_{4}(1)$ & Toluene & - & $56 \%$ [d] \\
\hline
\end{tabular}

[a] Isolated yields are reported. [b] 2:1 ratio of P:Pd was used. [c] 39\% recovered $\mathbf{3 3 2}$ and $4 \%$ of the $O$-alkylation side product were also isolated.

The use of an anomeric siloxy group proved crucial to the success of this chemistry as the relatively low reactivity of the -OTBS group allows for preferential ionization of the allylic carbonate. ${ }^{3}$ Alkylation of the resulting $\pi$-allyl-Pd species occurs regioselectively at $\mathrm{C} 5$ as a result of both steric and electronic factors inherent to pyran-based substrates. ${ }^{5}$

\subsubsection{Stereoconvergence and Mechanistic Inferences}

Pd-AA with soft nucleophiles typically proceeds via double inversion of configuration, ${ }^{6}$ such that the product stereochemistry is dependent on that of the starting material. Interestingly, the Pd-AA cascade of trans-332 (see Scheme 7.5) with $\mathbf{2 5 3}$ also produced the cis-fused product, 334 (Scheme 7.6). The cis-stereochemistry of $\mathbf{3 3 4}$ was confirmed by X-ray crystallographic analysis (Figure 7.2, see Appendix B for crystal data).

The formation of the cis-fused product (334) from trans-332 is presumably a consequence of the prohibitively large amount of strain energy present in the unsaturated trans-fused furopyran structure of $\mathbf{3 3 6} .{ }^{8}$ The relative energy difference between the cis- and trans-fused 


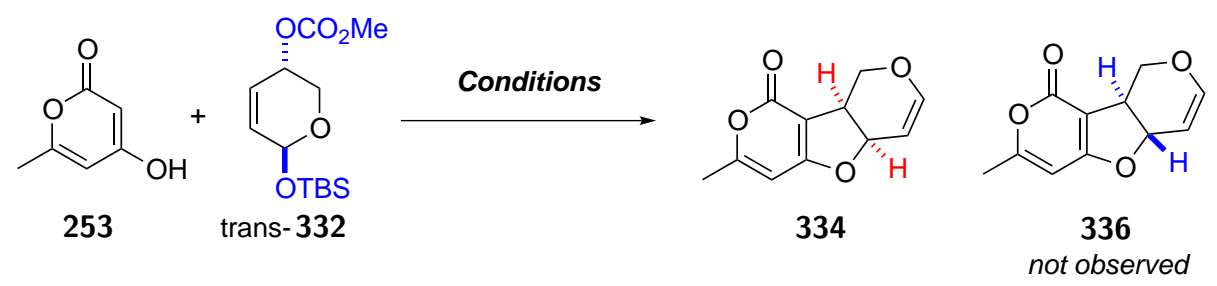

\begin{tabular}{ccccc}
\hline Entry & Catalyst $(\text { mol \% })^{[\mathrm{b}]}$ & Solvent & Comments & Yield of 334 ${ }^{[\mathrm{a}\rfloor}$ \\
\hline 1 & $\mathrm{Pd}_{2}(\mathrm{dba})_{3} / \mathrm{PPh}_{3}(10)$ & $\mathrm{CH}_{3} \mathrm{CN}$ & 1 equiv $\mathrm{NEt}_{3}$ & $38 \%$ \\
2 & $\mathrm{Pd}_{2}\left(\mathrm{PPh}_{3}\right)_{4}(5)$ & Toluene & - & $10 \%$ \\
3 & $\mathrm{Pd}\left(\mathrm{PPh}_{3}\right)_{4}(20)$ & Toluene & - & $74 \%$ \\
\hline
\end{tabular}

[a] Isolated yields are reported. [b] 2:1 ratio of P:Pd was used.

Scheme 7.6 Inversion of Configuration in the Pd-AA Cascade with Dihydropyran trans-332.

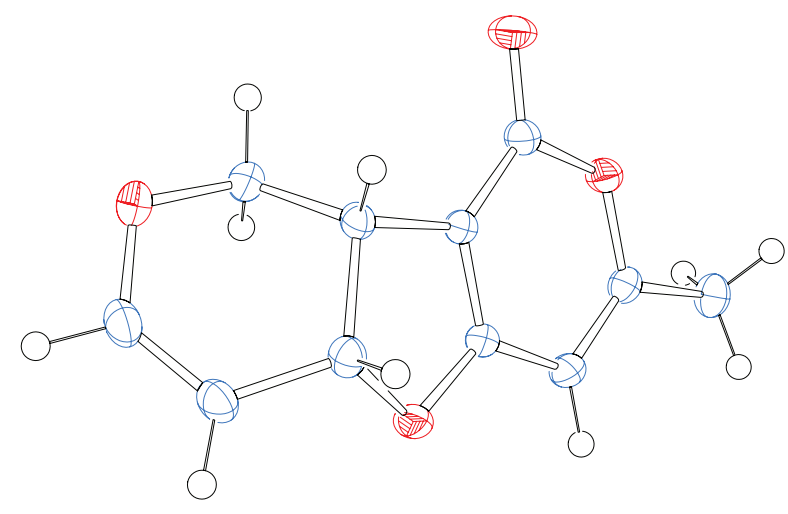

ORTEP drawing shows $50 \%$ thermal ellipsoids. ${ }^{7}$

Figure 7.2 X-Ray Crystal Structure of $\mathbf{3 3 4}$.

products was calculated to be $51 \mathrm{~kJ} / \mathrm{mol}$ (B3LYP/6-31G*). The higher catalyst loading required for efficient transformation of trans-332 (Scheme 7.6, Entry 3) suggests that the initial syn-palladium complex (337) may be isomerized by nucleophilic attack of a transient $\operatorname{Pd}(0)$ species (step VI, Scheme 5). ${ }^{9}$ Conveniently, the Pd-AA cascade of cyclohexene-based substrate, trans-330 (Scheme 7.7) can be used to validate this mechanistic hypothesis. This substrate provides an opportunity to evaluate the putative $\pi$-allyl-Pd isomerization without the possibility of the reaction proceeding through an oxonium intermediate, as may be the case for trans-332. Ultimately, 2D NOE spectroscopy and comparison of the ${ }^{3} J_{\mathrm{HH}}$ values of

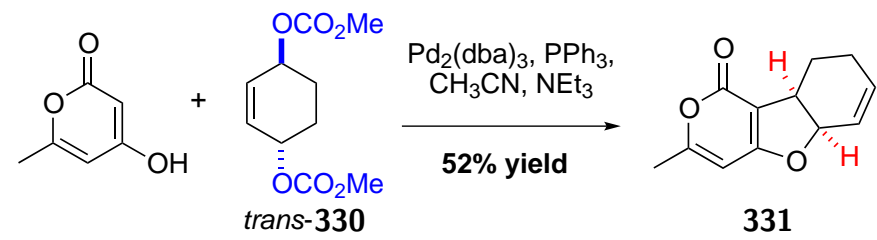

Scheme 7.7 Stereochemical Assignment of $\mathbf{3 3 1}$ and Mechanistic Inferences. 
the isosteric compounds $\mathbf{3 3 4}$ and $\mathbf{3 3 1}$ confirmed that trans-330 also provides the cis-fused product, $\mathbf{3 3 1}$ in a stereoconvergent manner.

Throughout these investigations, double alkylation was observed in almost all instances. Single $O$-alkylation was only observed in two cases where reactivity was impaired, either through low catalyst loading (Table 7.1, Entry 11) or by the presence of excess pyrone, 253 (Table 7.1, Entry 4). 4-Hydroxy-6-methyl- $\alpha$-pyrone (253) has a $p \mathrm{~K}_{\mathrm{a}}$ of 6.83 (80 $\left.w / w \% \mathrm{DMSO} / \mathrm{H}_{2} \mathrm{O}\right)^{10}$ and therefore, under effective reaction conditions, the $O$-alkylation product (335, Scheme 7.8) can act as an allyl acetate equivalent, reacting with zero-valent palladium to re-form the previous $\pi$-allyl complex (Scheme 7.8, step III). ${ }^{11}$ These results suggest that the excellent regioselectivity observed in most cases is a consequence of the first substitution operating under thermodynamic control. Based on the aforementioned experimental observations, a mechanism for the stereoconvergent Pd-AA cascade is proposed in Scheme 7.8.

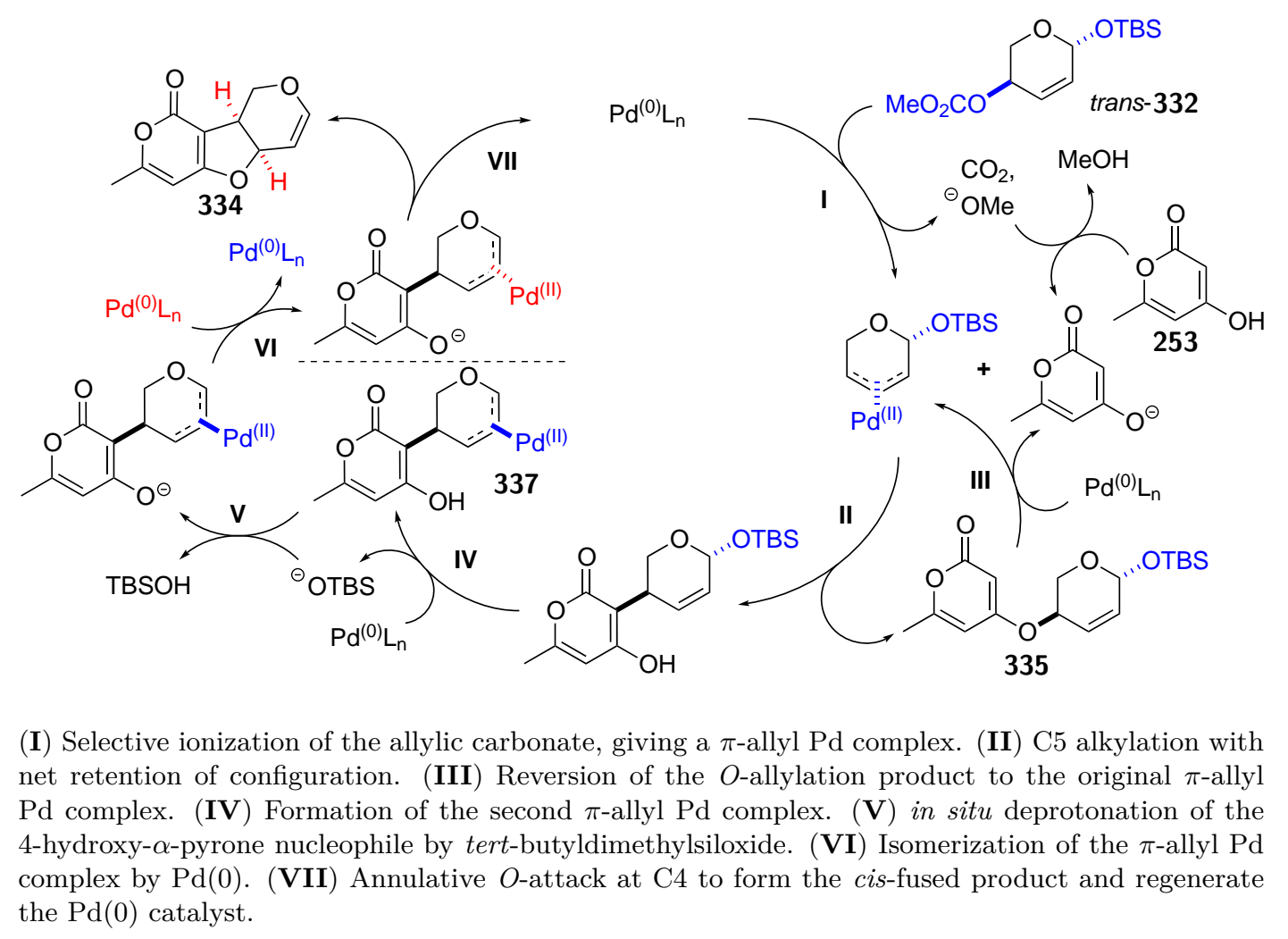

Scheme 7.8 Proposed Mechanism for the Pd-AA Cascade.

\subsubsection{Substrate Scope}

The scope of this reaction was explored using the optimized reaction conditions from Table 7.1 and a variety of $\beta$-dicarbonyl bis-nucleophiles (Scheme 7.9). In general, nucleophiles with high enol content, such as $\alpha$-pyrone 332 and coumarins $338 \mathbf{a}$ and $\mathbf{3 3 8} \mathbf{b}$, produced the highest 


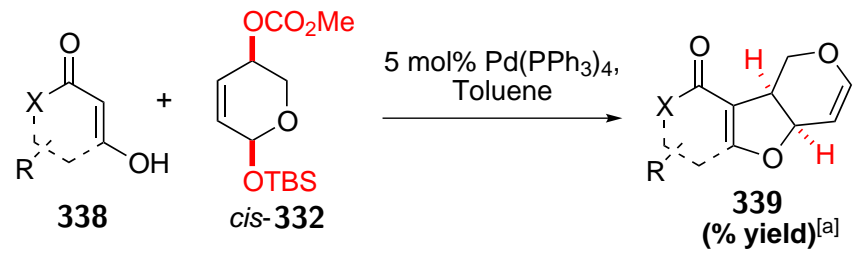<smiles>Cc1cc2c(c(=O)o1)[C@@H]1COC=C[C@@H]1O2</smiles>

334

(83\%)<smiles>O=c1oc2ccccc2c2c1C1COC=CC1O2</smiles>

$(78 \%)$<smiles>COc1ccc2c3c(c(=O)oc2c1)C1COC=CC1O3</smiles>

$(68 \%)^{[b]}$<smiles>O=C1OC2(CCCCC2)CC2=C1C1COC=CC1O2</smiles><smiles>O=C1OC(c2ccc(Br)cc2)CC2=C1C1COC=CC1O2</smiles>

(61\%)

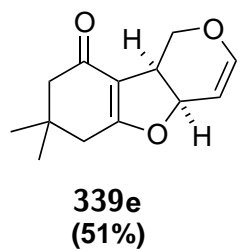

(51\%)<smiles>COC(=O)[C@H]1C(=O)O[C@H]2C=COCC12</smiles>

340

$(39 \%, 10: 1 \mathrm{dr})^{[\mathrm{c}]}$<smiles>Cc1cc2c(c(=O)n1Cc1ccccc1)C1COC=C[C@@H]1O2</smiles>

$339 \mathrm{~g}$ $(0 \%)^{[d]}$

[a] All reactions were run on a $0.27 \mathrm{mmol}$ scale and isolated yields are reported. [b] $10 \mathrm{~mol} \%$ $\mathrm{Pd}\left(\mathrm{PPh}_{3}\right)_{4}$ was used. [c] $53 \%$ of the $\alpha$-disubstituted Meldrum's acid side product 341 was also isolated. [d] $15 \%$ yield of the corresponding $O$-alkylation side product was isolated. [e] Reaction run using a 3:1 mixture of toluene:DMF.

\section{Unsuccessfull Bis-Nucleophiles:}<smiles>CN1C(=O)CC(=O)N([14CH3])C1=O</smiles><smiles>O=C1C=C(O)C(Cc2ccccc2)N1C(=O)OCc1ccccc1</smiles><smiles>CC(C)(C)c1ccccc1</smiles><smiles>O=C1CCC(=O)C1</smiles><smiles></smiles><smiles>Oc1ccccc1O</smiles><smiles>COC(=O)CC(=O)OC</smiles><smiles>COC(=O)CC(C)=O</smiles>

Scheme 7.9 Bis-Nucleophile Scope in the Pd-AA Cascade. 
yields (68-83\% yield). Overall, the parameters that define an effective nucleophile in these cascade reactions proved relatively complex, and high enol content alone does not seem to guarantee high yield. For example, the 4-hydroxy-pyridinone $338 \mathrm{~g}$ provided none of the desired product $339 \mathrm{~g}$ under the standard conditions, presumably due to poor solubility in toluene. However, by performing the reaction in a 3:1 mixture of toluene/DMF, $37 \%$ yield was obtained. Additionally, tetronic acid $\mathbf{3 4 4}$ and tetramic acid 343, which both exist predominantly in the enol form, provided a complex mixture that did not contain the desired product. Enolization does however play a crucial role in the intramolecular substitution, and substrates that are predisposed to the keto form, such as dimedone (338e), Meldrum's acid (351), and 1,3-indandione (346), form $\alpha$-disubstituted $\beta$-dicarbonyl side products, as shown in Scheme 7.10. Despite this competing side reaction, a moderate $51 \%$ yield was obtained with

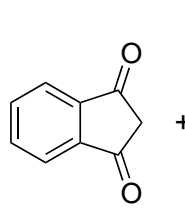

346

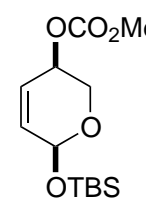

cis-332

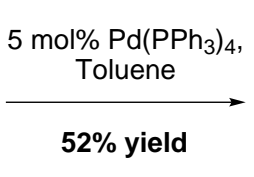

$52 \%$ yield

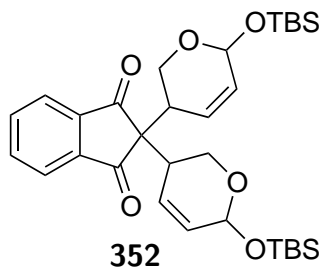

Scheme 7.10 Formation of $\alpha$-Disubstituted $\beta$-Dicarbonyl Side Products.

dimedone (338e). Additionally, the dihydro- $\alpha$-pyrone bis-nucleophiles $\mathbf{3 3 8 c}$ and $\mathbf{3 3 8 d}$ provide the desired furopyran products in $55 \%$ and $61 \%$, respectively. Unfortunately, both barbituric acid (347) and $N, N$-dimethyl barbituric acid did not provide the desired products. As with the other nitrogen-containing heterocycles, the solubility of bis-nucleophile was a concern in both of these reactions. It is also interesting to note that use of acyclic nucleophiles, dimethyl malonate (349) and methyl acetoacetate (350), resulted in a standard allylic alkylation and the anomeric-OTBS group remained unchanged.

The analogous Pd-AA cascade reaction with Meldrum's acid provides an atypical $\gamma$-lactone product, 340, in 39\% yield. This product is presumably formed through the extrusion of acetone from the initial dioxinone, followed by trapping of the resulting acylketene intermediate with methanol liberated from the carbonate, as shown in Scheme 7.11. ${ }^{12}$ This result is particularly exciting given the potential of $\gamma$-lactone $\mathbf{3 4 0}$ as a synthetic intermediate in the preparation of $\alpha$-methylene- $\gamma$-butyrolactone natural products. ${ }^{13}$ The potential utility of this transformation prompted a separate optimization to reduce the amount of the unwanted $\alpha$ disubstituted side product $\mathbf{3 4 1}$ and improve the yield of the desired $\gamma$-lactone. At the outset, two key steps in the reaction sequence were identified as targets for optimization - namely, improving the preference for intramolecular substitution as opposed to a second intermolecular alkylation (i.e. formation of $\mathbf{3 4 1}$ ), and a more effective trapping of the acylketene intermediate. Given that the Pd-AA cascade produces both $\mathrm{MeOH}$ and $\mathrm{TBSOH}$, it is likely that some acylketene is trapped by the silanol and subsequently hydrolyzed to give a carboxylic acid side product. In an attempt to improve the acylketene trapping with methanol, the reaction was performed in a 10:1 mixture of toluene/ $\mathrm{MeOH}$ - these conditions provided the desired product 


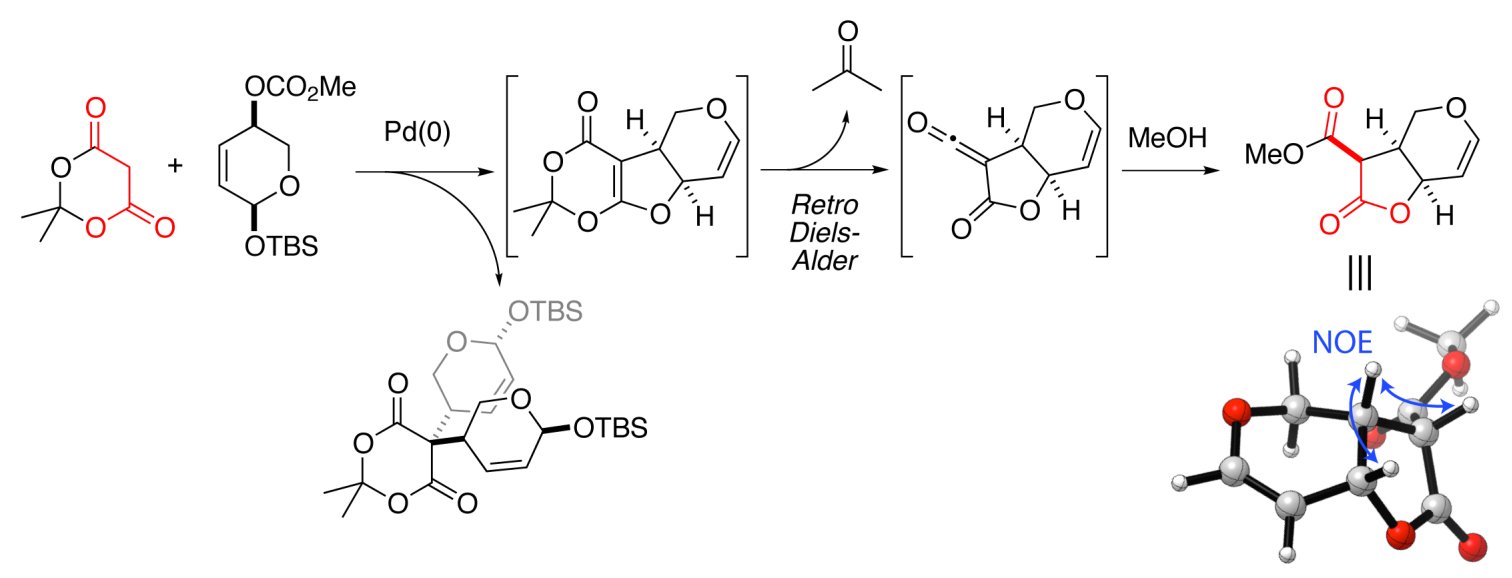

Scheme 7.11 Pd-AA Cascade with Meldrum's Acid: $\gamma$-Lactone Formation.

340 in an improved yield of $46 \%$ and supressed formation of the $\alpha$-disubstuted side product 341 (Entry 2). A number of literature examples show that the use of different solvents can greatly influence the regioselectivity of enolate alkylation, ${ }^{14}$ and therefore it's not surprizing that the use of methanol as a co-solvent could prevent the formation of the bis- $C$-alkylated side product altogether. A brief period of heating $\left(80^{\circ} \mathrm{C}, 2\right.$ hours $)$ was added at the end of the reaction to aid the retroaldol process that produces the acylketene, however, approximately the same yield was obtained suggesting that acylketene formation goes to completion at room temperature (Entry 3). While the use of methanol was beneficial in preventing the formation of the $\alpha$-disubstuted side product $\mathbf{3 4 1}$, it proved deterimental to the overall rate of the $\mathrm{Pd}-\mathrm{AA}$ cascade and the experiments performed in toluene/MeOH often contained unreacted starting material, even at solvent ratios as low as 100:1 (Entry 4). In a parallel series of experiments, the effect of concentration was investigated with the aim of disfavoring the intermolecular side reaction by performing the reaction at higher dilution. Unfortunately, performing the reaction at $0.017 \mathrm{M}$, instead of $0.05 \mathrm{M}$, had little effect on the isolated yields of $\mathbf{3 4 0}$ and $\mathbf{3 4 1}$ (Entries $5 \& 6$ ). The use of a less reactive acetate leaving group would also decrease the relative rate at which the side product $\mathbf{3 4 1}$ is formed, however, this electrophile was unreactive in initial experiments (Entries $8 \& 9$ ). Ultimately, it was discovered that the desired reactivity could be obtained by performing the reaction at $80^{\circ} \mathrm{C}$ using 5 equivalents of Meldrum's acid, $10 \mathrm{~mol} \%$ $\mathrm{Pd}\left(\mathrm{PPh}_{3}\right)_{4}, 1.5$ equivalents $\mathrm{MeOH}$, and THF as the reaction solvent (Entry 10). In this case, the desired product was obtained in $43 \%$ yield, making this experiment the first example of an allylic acetate participating in a Pd-AA cascade. However, the moderate yield obtained in this reaction prompted a return to the more reactive carbonate substrates. This decision also motivated by the observation of Meldrum's acid degradation products in reactions performed at elevated temperatures. In theory, the use of an excess of Meldrum's acid should disfavor the formation of 341, as Meldrum's acid is in direct competition with the mono-alkylated intermediate for electrophiles to react with. With the results from Table $7.1 \mathrm{in}$ mind, the Pd-AA 
Table 7.2 Optimization of a Pd-AA Cascade with Meldrum's Acid.

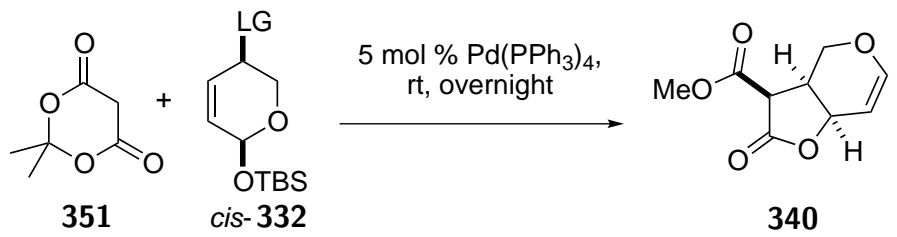

\begin{tabular}{|c|c|c|c|c|c|}
\hline Entry & LG & $\begin{array}{c}\text { Equiv } \\
351\end{array}$ & Solvent (conc.) & Comments $^{[\mathrm{a}]}$ & Yield $^{[\mathrm{b}]}$ \\
\hline 1 & $-\mathrm{OCO}_{2} \mathrm{Me}$ & 1.0 & Toluene $(0.05 \mathrm{M})$ & $53 \% \mathbf{3 4 1}$ & $39 \%$ \\
\hline 2 & $-\mathrm{OCO}_{2} \mathrm{Me}$ & 1.0 & Toluene/MeOH, 10:1 (0.05 M) & no 341 & $46 \%$ \\
\hline 3 & $-\mathrm{OCO}_{2} \mathrm{Me}$ & 1.0 & Toluene/MeOH, 10:1 (0.05 M) & $\mathrm{rt} / 80^{\circ} \mathrm{C}^{[\mathrm{c}]}$ & $50 \%$ \\
\hline 4 & $-\mathrm{OCO}_{2} \mathrm{Me}$ & 1.0 & Toluene/MeOH, 100:1 (0.05 M) & RSM & $49 \%$ \\
\hline 5 & $-\mathrm{OCO}_{2} \mathrm{Me}$ & 1.0 & Toluene $(0.017 \mathrm{M})$ & $50 \% \mathbf{3 4 1}$ & $48 \%$ \\
\hline 6 & $-\mathrm{OCO}_{2} \mathrm{Me}$ & 1.0 & Toluene $(0.017 \mathrm{M})$ & $15 \mathrm{~mol} \% \mathrm{Pd}$ & $40 \%$ \\
\hline 7 & $-\mathrm{OCO}_{2} \mathrm{Me}$ & 1.0 & Dioxane $(0.05 \mathrm{M})$ & - & $0 \%$ \\
\hline 8 & $-\mathrm{OAc}$ & 1.0 & Toluene $(0.05 \mathrm{M})$ & $\mathrm{NaOMe}, 80^{\circ} \mathrm{C}^{[\mathrm{d}]}$ & $0 \%$ \\
\hline 9 & $-\mathrm{OAc}$ & 5.0 & Toluene $(0.05 \mathrm{M})$ & $\begin{array}{c}10 \mathrm{~mol} \% \mathrm{Pd} \\
\mathrm{MeOH}, 80^{\circ} \mathrm{C}^{[\mathrm{e}]}\end{array}$ & $0 \%$ \\
\hline 10 & $-\mathrm{OAc}$ & 5.0 & THF $(0.05 M)$ & $\begin{array}{l}10 \mathrm{~mol} \% \mathrm{Pd} \\
\mathrm{MeOH}, 80^{\circ} \mathrm{C}^{[\mathrm{e}]}\end{array}$ & $43 \%$ \\
\hline 11 & $-\mathrm{OCO}_{2} \mathrm{Me}$ & 3.0 & Toluene $(0.05 \mathrm{M})$ & $\mathrm{NEt}_{3}[\mathrm{f}]$ & $0 \%$ \\
\hline 12 & $-\mathrm{OCO}_{2} \mathrm{Me}$ & 5.0 & Toluene $(0.05 \mathrm{M})$ & $80^{\circ} \mathrm{C}$ & $0 \%$ \\
\hline 13 & $-\mathrm{OCO}_{2} \mathrm{Me}$ & 5.0 & $\mathrm{THF}(0.05 \mathrm{M})$ & $80^{\circ} \mathrm{C}$ & $0 \%$ \\
\hline 14 & $-\mathrm{OCO}_{2} \mathrm{Me}$ & 5.0 & $\operatorname{THF}(0.1 \mathrm{M})$ & $10 \mathrm{~mol} \% \mathrm{Pd}$ & $29 \%$ \\
\hline 15 & $-\mathrm{OCO}_{2} \mathrm{Me}$ & 5.0 & $\mathrm{THF} / \mathrm{MeOH}, 25: 1(0.1 \mathrm{M})$ & $10 \mathrm{~mol} \% \mathrm{Pd}$ & $41 \%$ \\
\hline 16 & $-\mathrm{OCO}_{2} \mathrm{Me}$ & 5.0 & THF/MeOH, 10:1 (0.1 M) & $10 \mathrm{~mol} \% \mathrm{Pd}$ & $69 \%$ \\
\hline 17 & $-\mathrm{OCO}_{2} \mathrm{Me}$ & 5.0 & THF/MeOH, 2:1 (0.1 M) & $10 \mathrm{~mol} \% \mathrm{Pd}$ & $22 \%$ \\
\hline
\end{tabular}

[a] The $\alpha$-disubstituted Meldrum's acid side product, 341, was only isolated in select experiments. [b] Isolated yields are reported. $\mathrm{Pd}$ refers to $\mathrm{Pd}\left(\mathrm{PPh}_{[3]}\right)_{[4]}$ [c] The reaction was heated to $80^{\circ} \mathrm{C}$ for 2 hours after being stirred overnight at room temperature. [d] Reaction performed at $80^{\circ} \mathrm{C}$ using 1.1 equiv NaOMe. [e] 1.5 equiv $\mathrm{MeOH}$ were used. [f] 3 equiv $\mathrm{NEt}_{3}$ were used.

cascade was attempted using an excess of Meldrums acid and $\mathrm{NEt}_{3}$ (Entry 11). Unfortunately, these conditions gave none of the desired product, nor did those without NEt 3 (Entry 12). Surprisingly, it was discovered that using an excess of Meldrum's acid in conjunction with THF as the reaction solvent restored the desired reactivity (Entry 14). Combining this discovery with the use of $\mathrm{MeOH}$ as a co-solvent (10:1, THF:MeOH) provided the desired $\gamma$-lactone in a satisfying $69 \%$ yield (Entry 16). The ratio of $\mathrm{MeOH}$ proved important, presumably the use of too much $\mathrm{MeOH}$ inhibits the overall reactivity of the allylic alkylation cascade, whereas not enough methanol leads to poor trapping of the acylketene intermediate (Entries 15 \& 17).

The use of C2-substituted bis-electrophiles, such as $\mathbf{3 5 3},{ }^{15}$ also provides good yields of the desired furopyrone product (Scheme 7.12). In this case, compound 353 represents a mixture of C6 epimers, which, given the stereoconvergent nature of the Pd-AA cascade, ultimately form a single cis-fused product diastereoselectively. However, to facilitate this process a higher catalyst loading was used (15 mol \%). Thus, 6-methyl-4-hydroxy- $\alpha$-pyrone provided 
the furopyrone $\mathbf{3 5 4}$ in $77 \%$ yield. When the same reaction was performed with $5 \mathrm{~mol} \%$ $\mathrm{Pd}\left(\mathrm{PPh}_{3}\right)_{4}$, only $50 \%$ yield of $\mathbf{3 5 4}$ was obtained. Additionally, the use of 4-hydroxycoumarin as a bis-nucleophile provided furopyrone $\mathbf{3 5 5}$ in an excellent $90 \%$ yield.

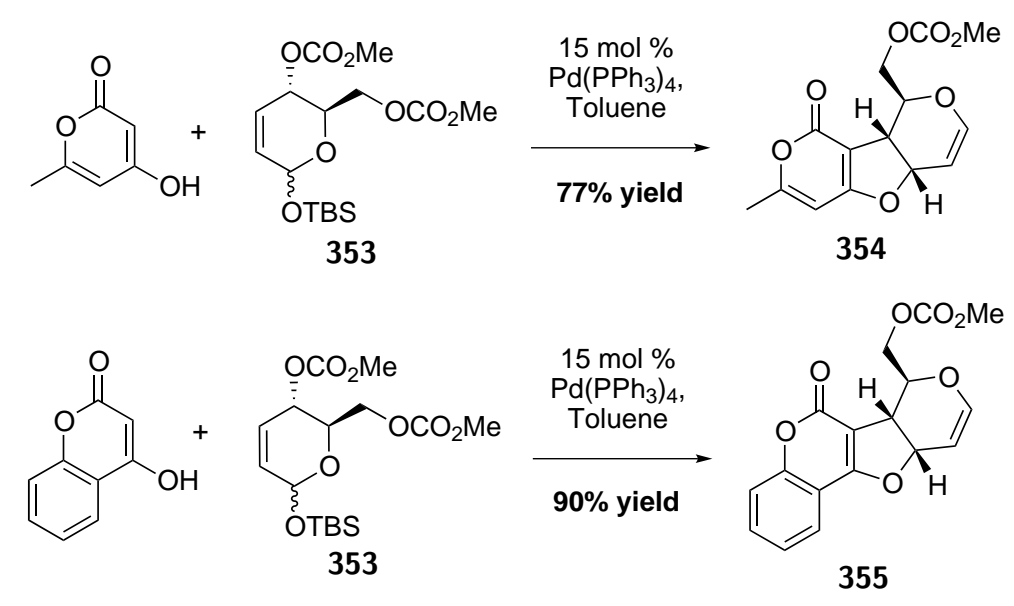

Scheme 7.12 Pd-AA Cascade with Enantioenriched C2-substituted Bis-Electrophiles.

In summary, a palladium-catalyzed allylic alkylation cascade with non-symmetric pyranbased substrates has been developed and provides rapid access to substituted cis-fused furopyrans. The use of an anomeric siloxy leaving group imparts regiochemical control, providing the desired products in a rapid, selective and stereoconvergent manner. This methodology leads to a number of versatile synthetic intermediates, whose exploitation in several synthetic endeavors is being pursued. Additionally, these heterocyclic products resemble a number of bioactive natural products and contain structural elements that are often conducive to interesting biological activity.

\subsection{Biological Testing of the Furopyrones Generated via a Pd- AA Cascade}

Pyrone and coumarin-based heterocycles are present in a wide range of bioactive natural products and pharmaceutical agents. These planar motifs enable a conformationally rigid structure containing both polar and lipophilic elements, and these characteristics are often essential for effective binding to a biological receptor. ${ }^{16} \mathrm{~A}$ wide range of biological activity has been reported for various $\alpha$-pyrone-containing compounds, some of which are shown in Figure 7.3. Warfarin (Coumadin ${ }^{\circledR}$ ) is an important anticoagulant used to prevent formation of potentially lethal blood clots. ${ }^{17}$ Pfizer developed U-96988 as a first generation clinical candidate for the treatment of HIV infection. ${ }^{18}$ Arisugacin A is a potent inhibitor of acetylcholinesterase (AChE), which has been linked to the treatment of Alzheimer's disease. ${ }^{19}$ TMC-69 is a potent antitumor agent that has served as the parent compound in a number of SAR studies. ${ }^{20}$ Brevione B is an allelochemical with potential application as a herbicide. ${ }^{21}$ Lastly, coumestrol is a potent phytoestrogen. ${ }^{22}$ 


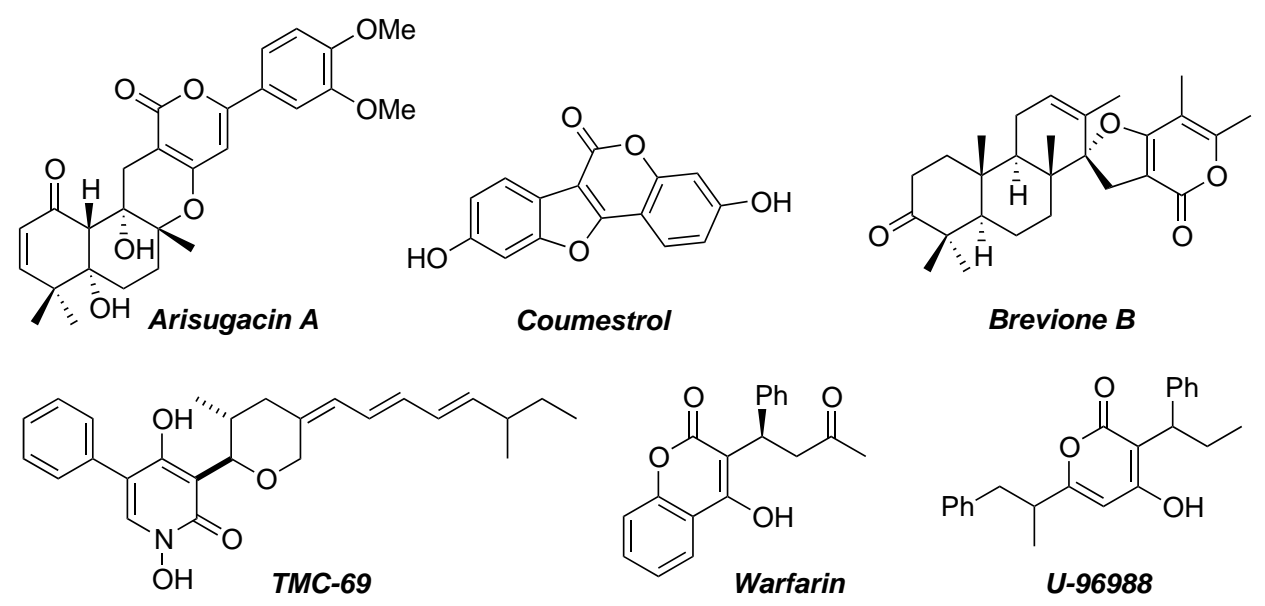

Figure 7.3 Bioactive Furopyrone and Pyrone-Containing Compounds.

The Pd-AA cascade products shown in Table 7.3 were found to have exciting cytotoxicity against HL-60, a human leukemia cell line. Surprisingly, the coumarin and pyrone-containing compounds 334, 339a, and 339b were the least active of all the furopyrones screened. The furopyrones containing a partially saturated pyrone ring displayed consistently low $\mathrm{IC}_{50}$ values, with aryl bromide 339d and spirocycle 339c having $\mathrm{IC}_{50}$ values of $97 \mu \mathrm{M}$ and $94 \mu \mathrm{M}$, respectively. Additionally, the dimedone-derived heterocycle 339e was found to be particularly active, with an $\mathrm{IC}_{50}$ of $29 \mu \mathrm{M}$. The introduction of a carbonate substituent on the dihydropyran ring produced compounds with significantly improved activity. Pyrone 354 and coumarin 355 provided HL-60 $\mathrm{IC}_{50}$ values of $21 \mu \mathrm{M}$ and $25 \mu \mathrm{M}$, respectively. This constitutes a ten-fold increase in cytotoxicity relative to the pyrone and coumarin-based compounds lacking the carbonate moiety. It is interesting to note that the low micro-molar potency observed for some of these compounds does not correlate with their relative lipophilicity $(c \log \mathrm{P})$. This suggests that the cytotoxic effects of these compounds may be a consequence of a specific binding interaction, as opposed to a general non-specific cytotoxicity.

In addition to anti-cancer activity, the anti-bacterial activity of these compounds was also investigated. Unfortunately, the three compounds, 339a, 339b, and 339c, that were screened against E. coli, S. aureus, and M. smegmatis bacterial cell lines showed poor activity.

The rapid preparation of furopyrones using a Pd-AA cascade affords the opportunity for continued investigations into the biological activities of this heterocyclic scaffold. The promising anti-cancer properties displayed by some of these compounds bodes well for further testing, and the combination of the dimedone-derived heterocycle with the carbonate moiety represents a particularly exciting lead. 
Table 7.3 Bioactivity of Select Pd-AA Cascade Products.<smiles>Cc1cc2c(c(=O)o1)C1COC=CC1O2</smiles>

334<smiles>O=c1oc2ccccc2c2c1[C@H]1COC=C[C@H]1O2</smiles>

339 a<smiles>COc1ccc2c3c(c(=O)oc2c1)C1COC=C[C@H]1O3</smiles>

$339 \mathrm{~b}$<smiles>CC(=O)OC[C@H]1OC=C[C@H]2Oc3cc(C)oc(=O)c3[C@@H]21</smiles>

354<smiles>O=C1OC(c2ccc(Br)cc2)CC2=C1C1COC=CC1O2</smiles><smiles></smiles><smiles>CC1(C)CC(=O)C2=C(C1)O[C@H]1C=COC[C@H]21</smiles><smiles>CC(=O)OC[C@H]1OC=C[C@H]2Oc3c(c(=O)oc4ccccc34)[C@@H]21</smiles>

\begin{tabular}{|c|c|c|c|c|c|}
\hline \multirow{2}{*}{ Compound } & \multirow{2}{*}{$c \log \mathbf{P}^{[\mathrm{a}]}$} & \multirow{2}{*}{ HL-60 $\left(\mathrm{IC}_{50}\right)^{[\mathrm{b}]}$} & \multicolumn{3}{|c|}{ Antibacterial $\mathbf{I C}_{90}[\mathrm{c}]$} \\
\hline & & & E. coli & S. aureus & M. smegmatis \\
\hline 334 & -1.43 & $>250 \mu \mathrm{M}$ & - & - & - \\
\hline $339 a$ & -0.54 & $>100 \mu \mathrm{M}$ & inactive & $1.35 \mathrm{mM}$ & inactive \\
\hline $339 b$ & -0.57 & $>100 \mu \mathrm{M}$ & inactive & inactive & inactive \\
\hline $339 c$ & 0.31 & $94 \mu \mathrm{M}$ & - & - & - \\
\hline $339 d$ & 0.74 & $97 \mu \mathrm{M}$ & inactive & inactive & inactive \\
\hline $339 \mathrm{e}$ & 0.08 & $29 \mu \mathrm{M}$ & - & - & - \\
\hline 354 & -1.40 & $21 \mu \mathrm{M}$ & - & - & - \\
\hline 355 & -0.52 & $25 \mu \mathrm{M}$ & - & - & - \\
\hline
\end{tabular}

[a] $c \log \mathrm{P}$ was calculated using CS ChemBioDraw Ultra 12. [b] HL-60 cytotoxicity testing was performed by Dora Leahy and Arun Kanakkanthara. [c] Antibacterial screening was performed by Nathaniel Dasyam. 


\subsection{Synthetic Utility of Furopyrones: Third Generation Ap- proach to the Synthesis of Labillarides E-H}

The furopyrone products generated from the Pd-AA cascade reactions contain a rich array of functionality, and it was envisioned that the potential synthetic utility of these functional groups could be utilized in the total synthesis of labillarides E-H. To this end, the development of an efficient method for the fragmentation of the appended dihydropyran ring was crucial, as this provides access to the two functionalized furan side chains needed to construct labillarides E-H (Figure 7.4). The second key requirement in this approach is the epimerization of one of the furan stereocenters, to ultimately provide the desired trans-substitution. Epimerizations of this general type, 1,2-cis to 1,2-trans on a 5-membered ring, have good literature precendents and an intermediate containing a $\mathrm{C} 7$ formyl substituent would be well suited to this transformation. ${ }^{23}$
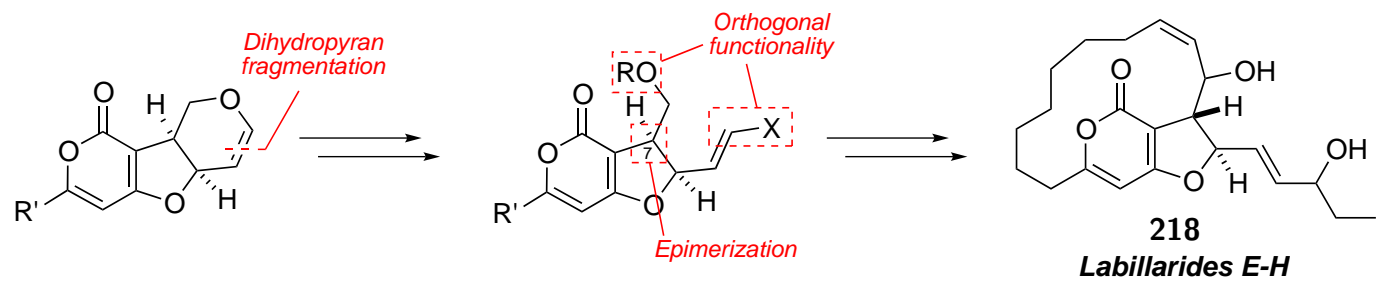

Figure 7.4 Third Generation Approach to Labillarides E-H: Dihydropyran Fragmentation.

Initial attempts to functionalize the 'glycal-like' cyclic enol ether were based around hydrolysis, to form a hemi acetal, followed by a chemoselective reaction with the aldehyde functionality present in the open form (356) of this compound (Scheme 7.13). Unfortunately, hydrolysis under acidic conditions provided a complex mixture of products. The failure of this seemingly simple reaction is attributed to the presence of a good leaving group (i.e. ring opening to give a 4-hydroxy- $\alpha$-pyrone) at the allylic position. A number of literature reports also mention the difficulties associated with this type of transformation. ${ }^{24}$ To circumvent these problems, a bromohydroxylation approach was investigated.

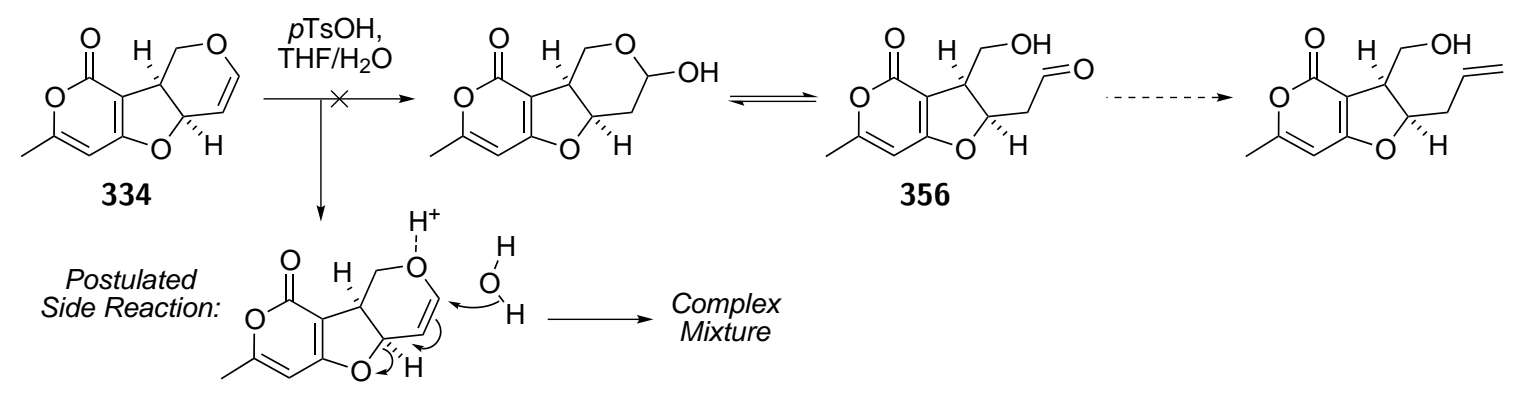

Scheme 7.13 Attempted Dihydropyran Hydrolysis.

Bromohydroxylation of dihydropyran 334, using NBS in $\mathrm{CH}_{3} \mathrm{CN} / \mathrm{H}_{2} \mathrm{O}$, provided bromohydrin $\mathbf{3 5 7}$ in $43 \%$ yield (Scheme 7.14). This product is a result of electrophilic attack on the 
more accessible convex face of the molecule and subsequent nucleophilic addition. A moderate $19 \%$ yield of another diastereomer was also isolated during silica gel chromatography, however this material was not taken forward. The reduction of $\mathbf{3 5 7}$ using sodium borohydride did not produce the expected epoxy alcohol $\mathbf{3 5 8}$, but instead formed the constitutionally isomeric furan, $\mathbf{3 5 9}$, in $72 \%$ yield. Confirming that the product of this reaction was furan $\mathbf{3 5 8}$, and not<smiles>Cc1cc2c(c(=O)o1)C1COC=CC1O2</smiles>

334

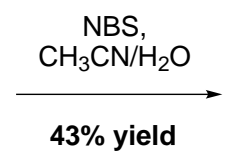

$43 \%$ yield

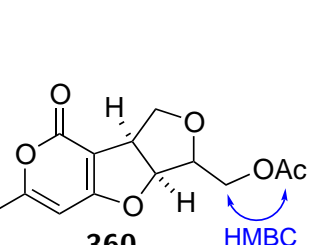
360<smiles>Cc1cc2c(c(=O)o1)C1COC(O)C(Br)C1O2</smiles>

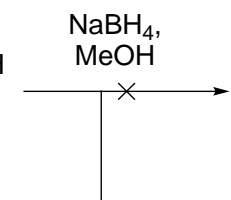

$74 \%$ yield<smiles></smiles>

not observed

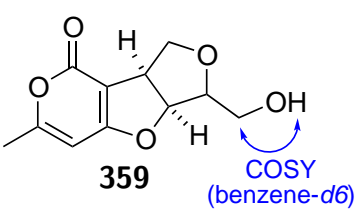

Scheme 7.14 Bromohydroxylation and an Unexpected Epoxide Rearrangement.

epoxide 358, proved challenging. Ultimately, the use of benzene- $d_{6}$ as an NMR solvent, various ${ }^{1} \mathrm{H} /{ }^{1} \mathrm{H}$ and ${ }^{1} \mathrm{H} /{ }^{13} \mathrm{C} 2 \mathrm{D}$ NMR experiments, and spectroscopic analysis of the corresponding acetate 360 were used to assign the structure of this product. Particularly strong evidence of this structure could be seen in the ${ }^{1} \mathrm{H} /{ }^{1} \mathrm{H}$ COSY NMR spectrum in benzene- $d_{6}$, which showed a correlation between the hydroxyl proton and the neighbouring oxymethylene, and also a correlation between the acetyl carbonyl carbon and the exocyclic oxymethylene in the ${ }^{1} \mathrm{H} /{ }^{13} \mathrm{C}$ HMBC spectrum.

While the unwanted formation of furan 359 prevents further progress towards the structures of labillarides E-H along this route, it also reveals some very interesting reactivity. Coincidently, the fused bis-furan structure of $\mathbf{3 5 9}$ closely resembles that of a fungicidal natural product, canadensolide (Scheme 7.15). ${ }^{25}$ The use of a Pd-catalyzed allylic alkylation cascade, along with the conditions used to form 359, could potentially be utilized in a total synthesis of this bioactive $\alpha$-methylene- $\gamma$-butyrolactone natural product and analogues thereof.

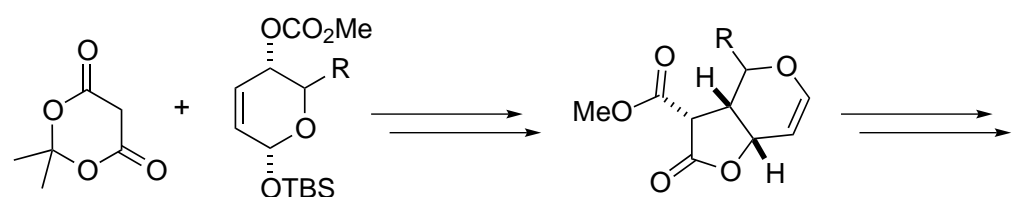

351

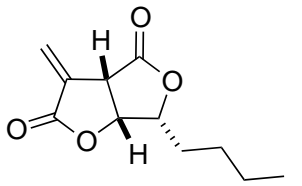

362

Canadensolide

Scheme 7.15 Potential Application of a Pd-AA Cascade in the Synthesis of Canadensolide and Related Analogues. 


\subsection{Experimental Data for Chapter 7}

\subsubsection{General Experimental Methods}

Unless otherwise noted, the following conditions apply. All reactions were performed in flamedried septum-sealed glassware with magnetic stirring under an atmosphere of argon. Moistureand oxygen-sensitive liquids and solutions were transferred using a stainless steel syringe or cannula. Before use, solvents were refluxed over the appropriate drying agent and distilled under argon: tetrahydrofuran from sodium benzophenone ketyl radical; dichloromethane, acetonitrile and triethylamine from calcium hydride; methanol and toluene from sodium. NBS was recrystallized from $\mathrm{H}_{2} \mathrm{O}$ and dried in a vacuum desiccator. All other commercially available chemicals were used as received, without further purification. Analytical thin layer chromatography (TLC) was performed using plastic-backed pre-coated silica TLC plates (Polygram SilG/UV 254 ). Visualization was achieved by UV irradiation $(254 \mathrm{~nm}$ ) or by heating after treatment with a potassium permanganate dip $\left(1.5 \mathrm{~g} \mathrm{KMnO}_{4}, 10 \mathrm{~g} \mathrm{~K}_{2} \mathrm{CO}_{3}, 1.25 \mathrm{~mL}\right.$ of $10 \%$ aqueous $\mathrm{NaOH}$ solution and $200 \mathrm{~mL}$ of water $)$ or $p$-anisaldehyde dip $(0.7 \mathrm{~mL} p$ anisaldehyde, $9.5 \mathrm{~mL}$ conc. $\mathrm{H}_{2} \mathrm{SO}_{4}, 2.7 \mathrm{~mL}$ of acetic acid and $250 \mathrm{~mL}$ of EtOH). The purification of products by flash column chromatography (FCC) was conducted using silica gel 60 (220-240 mesh) with the solvent systems indicated. ${ }^{1} \mathrm{H}$ NMR spectra were recorded on either: a Varian Unity Inova 500 spectrometer at $500 \mathrm{MHz}$, or a Varian Inova 300 at 300 $\mathrm{MHz}$. Data are listed as chemical shift in ppm using $\mathrm{CDCl}_{3}$ as internal standard (7.26 ppm). ${ }^{13} \mathrm{C}$ NMR spectra were recorded on a Varian Unity Inova 500 spectrometer at $125 \mathrm{MHz}$ and the data are listed as chemical shift in ppm using $\mathrm{CDCl}_{3}$ as internal standard (77 ppm). All ${ }^{13} \mathrm{C}$ experiments were ${ }^{1} \mathrm{H}$ decoupled. IR bands were measured as a thin film on a Bruker FTIR Tensor 27 spectrometer with ATR sampling accessory. High-resolution mass spectrometry (HRMS) was performed on a Waters QTOF Premier Tandem mass spectrometer.

\subsubsection{Experimental Details and Characterization Data}

\section{Experimental Data for 7.1}

Preparation of $(1 S, 2 S)-1,2-B i s((R)-2,2$-dimethyl-1,3-dioxolan-4-yl)ethane-1,2-diol

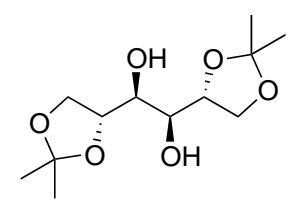

To a solution of D-mannitol (11.45 g, $62.9 \mathrm{mmol}, 1.0$ equiv) and 1,2-dimethoxy propane (18.51 mL, $151 \mathrm{mmol}, 2.4$ equiv) in DME $(27.5 \mathrm{~mL})$ was added catalytic $\mathrm{SnCl}_{2}(0.165 \mathrm{~g})$. The resulting white suspension was heated to $90{ }^{\circ} \mathrm{C}$ and stirred for 1.5 hours, during which time the reaction became a clear homogeneous solution. The reaction mixture was subsequently cooled to room temperature and two drops of pyridine were addded. The reaction solvent was removed in vacuo, $\mathrm{CH}_{2} \mathrm{Cl}_{2}$ was added to form a white slurry, which was then filtered to remove the insoluble mono-acetonide side product. The filtrate was then concentrated in vacuo and purified using silica gel chromatography (50\% EtOAc/Pet. Ether). The title compound was isolated as a white solid ( $6.58 \mathrm{~g}, 40 \%$ yield). $\mathbf{R}_{\mathbf{f}}=0.24$ (50\% EtOAc/Pet. Ether). ${ }^{\mathbf{1}} \mathbf{H}-\mathbf{N M R}$ $\left(500 \mathrm{MHz}, \mathrm{CDCl}_{3}\right) \delta 4.15(\mathrm{q}, J=6.3 \mathrm{~Hz}, 2 \times 1 \mathrm{H}), 4.10(\mathrm{dd}, J=8.5,6.3 \mathrm{~Hz}, 2 \times 1 \mathrm{H}), 3.97$ $(\mathrm{dd}, J=8.5,5.3 \mathrm{~Hz}, 2 \times 1 \mathrm{H}), 3.72(\mathrm{~d}, J=6.7 \mathrm{~Hz}, 2 \times 1 \mathrm{H}), 2.90$ (br. s, $2 \times 1 \mathrm{H}), 1.40(\mathrm{~s}, 2$ x $3 \mathrm{H}), 1.34(\mathrm{~s}, 2 \times 3 \mathrm{H}) \cdot{ }^{13} \mathrm{C}-\mathrm{NMR}\left(126 \mathrm{MHz}, \mathrm{CDCl}_{3}\right) \delta 109.56,79.38,76.33,66.26,26.51$, 
25.31. Characterization data matches the literature. ${ }^{33}$

\section{Preparation of $(E)-1,2-B i s((S)-2,2$-dimethyl-1,3-dioxolan-4-yl)ethene}

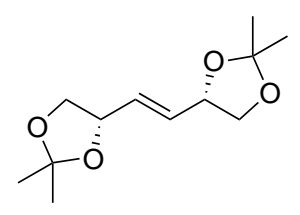

To a solution of $(1 S, 2 S)$-1,2-bis $((R)$-2,2-dimethyl-1,3-dioxolan-4-yl)ethane-1,2-diol (0.800 g, $3.05 \mathrm{mmol}, 1.0$ equiv) in $\mathrm{CH}_{2} \mathrm{Cl}_{2}(9 \mathrm{~mL})$ was added dimethylformamide dimethyl acetal $(0.813$ $\mathrm{mL}, 6.10 \mathrm{mmol}, 2.0$ equiv). The reaction mixture was stirred overnight at room temperature and then concentrated in vacuo and redissolved in toluene $(9 \mathrm{~mL})$. MeI $(1.52 \mathrm{~mL}, 24.4 \mathrm{mmol}, 8.0$ equiv) was added to the reaction mixture, which was subsequently stirred at room temperature for 2 hours, then heated to reflux and stirred for an additional hour. The reaction was then cooled to room temperature, hexanes was added and the resulting slurry was filtered through celite. The filtrate was concentrated in vacuo and purified by silica gel chromatography ( $15 \%$ EtOAc/Pet. Ether). The title compound was isolated as a white solid (0.273 g, 39\% yield). $\mathbf{R}_{\mathbf{f}}=0.39$ (20\% EtOAc/Pet. Ether). ${ }^{1} \mathbf{H}-\mathbf{N M R}\left(500 \mathrm{MHz}, \mathrm{CDCl}_{3}\right) \delta 5.81$ (dd, $J=3.8,1.8$ $\mathrm{Hz}, 2 \times 1 \mathrm{H}), 4.54$ (dddd, $J=7.9,5.9,3.7,1.9 \mathrm{~Hz}, 2 \times 1 \mathrm{H}), 4.10(\mathrm{dd}, J=8.1,6.2 \mathrm{~Hz}, 2 \times 1 \mathrm{H})$, $3.60(\mathrm{t}, J=7.9 \mathrm{~Hz}, 2 \times 1 \mathrm{H}), 1.44(\mathrm{~s}, 2 \times 3 \mathrm{H}), 1.40(\mathrm{~s}, 2 \times 3 \mathrm{H})$. Characterization data matches the literature.

\section{Preparation of $(2 S, 5 S, E)$-Hex-3-ene-1,2,5,6-tetraol}<smiles>OCC(O)C=CC(O)CO</smiles>

To a solution of $(E)$-1,2-bis $((S)$-2,2-dimethyl-1,3-dioxolan-4-yl)ethene (0.99 g, $0.434 \mathrm{mmol}$, 1.0 equiv) in methanol $(5 \mathrm{~mL})$ was added DOWEX-H $(0.49 \mathrm{~g})$. The reaction mixture was stirred overnight at room temperature before being filtered and concentrated in vacuo. The title compound was isolated as a clear viscous oil (0.064 g, quant.). ${ }^{\mathbf{1}} \mathbf{H}-\mathbf{N M R}(500 \mathrm{MHz}$, $\left.\mathrm{CD}_{3} \mathrm{OD}\right) \delta 5.77(\mathrm{dd}, J=3.2,1.5 \mathrm{~Hz}, 2 \times 1 \mathrm{H}), 4.12(\mathrm{~m}, 2 \times 1 \mathrm{H}), 3.50(\mathrm{dd}, J=11.2,4.9 \mathrm{~Hz}, 2$ x $1 \mathrm{H}), 3.46(\mathrm{dd}, J=11.1,6.7 \mathrm{~Hz}, 2 \times 1 \mathrm{H}) .{ }^{13} \mathbf{C} \mathbf{N M R}\left(126 \mathrm{MHz}, \mathrm{CD}_{3} \mathrm{OD}\right) \delta 135.20,76.27$, 69.69. Characterization data matches the literature.

\section{Preparation of $(2 S, 5 S, E)$-Hex-3-ene-1,6-di(tert-butyldimethylsilyloxy)-2,5-diol}<smiles>CCCCOCC(O)C=CCC(O)COCC</smiles>

To a solution of $(2 S, 5 S, E)$-hex-3-ene-1,2,5,6-tetraol (0.064 g, $0.432 \mathrm{mmol}, 1.0$ equiv) in $\mathrm{CH}_{2} \mathrm{Cl}_{2} / \mathrm{DMF}\left(3.9 \mathrm{~mL}\right.$ and $0.4 \mathrm{~mL}$, respectively) was added $\mathrm{NEt}_{3}(0.132 \mathrm{~mL}, 0.950 \mathrm{mmol}$, 2.2 equiv), DMAP (0.003 g, $0.026 \mathrm{mmol}, 6 \mathrm{~mol} \%)$ and TBS-Cl (0.130 g, $0.864 \mathrm{mmol}, 2.0$ equiv). The reaction was stirred at room temperature overnight before being diluted with $\mathrm{Et}_{2} \mathrm{O}$, washed with water, then brine, dried over $\mathrm{MgSO}_{4}$, filtered and concentrated in vacuo. The crude product was purified by silica gel chromatography (20\% EtOAc/Pet. Ether). The 
title compound was isolated as a white solid (0.027 g, $17 \%$ yield). $\mathbf{R}_{\mathbf{f}}=0.23(25 \%$ EtOAc/Pet. Ether). ${ }^{1} \mathbf{H}-N M R\left(500 \mathrm{MHz}, \mathrm{CDCl}_{3}\right) \delta 5.77(\mathrm{dt}, J=2.9,1.1 \mathrm{~Hz}, 2 \times 1 \mathrm{H}), 4.20(\mathrm{~m}, 2 \times 1 \mathrm{H})$, 3.65 (ddd, $J=10.1,3.6,1.5 \mathrm{~Hz}, 2 \times 1 \mathrm{H}$ ), 3.43 (ddd, $J=9.9,7.9,1.6 \mathrm{~Hz}, 2 \times 1 \mathrm{H}$ ), 2.31 (br. $\mathrm{s}, 2 \times 1 \mathrm{H}), 0.91(\mathrm{~s}, 2 \times 9 \mathrm{H}), 0.08(\mathrm{~s}, 2 \times 6 \mathrm{H})$. Characterization data matches the literature.

Preparation of (2S,5S,E)-Methyl Hex-3-ene-1,6-di(tert-butyldimethylsilyloxy)2,5-carbonate, 324.

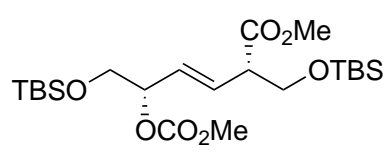

To a solution of (2S,5S,E)-hex-3-ene-1,6-di(tert-butyldimethylsilyloxy)-2,5-diol (0.027 g, $0.072 \mathrm{mmol}, 1.0$ equiv) and DMAP (0.027 g, $0.22 \mathrm{mmol}, 3.0$ equiv) in $\mathrm{CH}_{2} \mathrm{Cl}_{2}(0.75 \mathrm{~mL})$ was added methyl chloroformate $(0.017 \mathrm{~mL}, 0.22 \mathrm{mmol}, 3.0$ equiv). The reaction was stirred overnight at room temperature before being concentrated in vacuo and purified by silica gel chromatography (10\% EtOAc/Pet. Ether). The title compound was isolated as a clear colorless oil $\left(0.024 \mathrm{~g}, 68 \%\right.$ yield). $\mathbf{R}_{\mathbf{f}}=0.25$ (10\% EtOAc/Pet. Ether). ${ }^{\mathbf{1}} \mathbf{H}-\mathbf{N M R}\left(500 \mathrm{MHz}, \mathrm{CDCl}_{3}\right)$ $\delta 5.80(\mathrm{dd}, J=3.3,1.5 \mathrm{~Hz}, 2 \times 1 \mathrm{H}), 5.20(\mathrm{tdt}, J=5.4,3.3,1.4 \mathrm{~Hz}, 2 \times 1 \mathrm{H}), 3.78(\mathrm{~s}, 2 \times 3 \mathrm{H})$, $3.68(\mathrm{~d}, J=5.9 \mathrm{~Hz}, 2 \times 2 \mathrm{H}), 0.88(\mathrm{~s}, 2 \times 9 \mathrm{H}), 0.05(\mathrm{~d}, J=2.8 \mathrm{~Hz}, 2 \times 6 \mathrm{H})$.

\section{Experimental Data for Non-symmetric Pd-AA Cascade}

\section{Palladium-Catalyzed Allylic Alkylation Cascade General Procedures}

METHOD A: A reaction vessel was charged with $\beta$-dicarbonyl bis-nucleophile (1.0 equiv), evacuated and back-filled with argon. Toluene ( $c a$. $0.14 \mathrm{M}$, degassed by sparging with argon for approximately 20 minutes) was added to the reaction vessel. A separate reaction vessel was charged with $\mathrm{Pd}\left(\mathrm{PPh}_{3}\right)_{4}(5 \mathrm{~mol} \%)$, evacuated and back-filled with argon, then charged with degassed toluene (ca. $0.7 \mathrm{M}$ relative to substrate) followed by the substrate, cis- $( \pm)-6-[($ tertbutyldimethylsilyl)oxy]-3,6-dihydro-2 $H$-pyran-3-yl methyl carbonate (1.0 equiv). The contents of this flask were stirred for approximately 20 minutes before being added via syringe to the bis-nucleophile solution. The reaction was stirred overnight at room temperature before being concentrated in vacuo and purified by flash column chromatography.

METHOD B: A reaction vessel was charged with $\beta$-dicarbonyl bis-nucleophile (1.0 equiv) and $\mathrm{Pd}\left(\mathrm{PPh}_{3}\right)_{4}(5 \mathrm{~mol} \%)$, evacuated and back-filled with argon. Toluene (ca. $0.05 \mathrm{M}$, degassed by sparging with argon for approximately 20 minutes) was added to the reaction vessel, followed by the substrate, cis-( \pm )-6-[(tert-butyldimethylsilyl)oxy]-3,6-dihydro-2H-pyran-3-yl methyl carbonate $(1.0$ eq.). The reaction was stirred overnight at room temperature before being concentrated in vacuo and purified by flash column chromatography. 


\section{Pd-AA Cascade Products}

cis-( \pm )-3-Methyl-9,9a-dihydrofuro[3,2-c:4,5-c']dipyran-1(5aH)-one, 334.<smiles>Cc1cc2c(c(=O)o1)C1COC=CC1O2</smiles>

The reaction was performed with 4-hydroxy-6-methyl- $\alpha$-pyrone $(0.040 \mathrm{~g}, 0.32 \mathrm{mmol})$ according to Method A. The crude product was purified by flash column chromatography (30\% EtOAc / pet. ether) to give the title compound as a yellow solid ( $0.054 \mathrm{~g}, 83 \%$ yield). $\mathbf{R}_{\mathbf{f}}=0.21\left(30 \%\right.$ EtOAc /Pet. Ether). ${ }^{1} \mathbf{H}-\mathbf{N M R}\left(500 \mathrm{MHz}, \mathrm{CDCl}_{3}\right): \delta 6.78(\mathrm{~d}, J=6.2 \mathrm{~Hz}$, $1 \mathrm{H} \mathrm{HC}=), 5.93(\mathrm{~s}, 1 \mathrm{H}, \mathrm{PyH}), 5.19(\mathrm{dd}, J=6.2,4.4 \mathrm{~Hz}, 1 \mathrm{H}, \mathrm{HC}=), 5.10(\mathrm{dd}, J=7.6,4.6 \mathrm{~Hz}$, $1 \mathrm{H}, \mathrm{PyOCH}), 4.36\left(\mathrm{dd}, J=10.7,4.9 \mathrm{~Hz}, 1 \mathrm{H}\right.$, one of $\left.\mathrm{CH}_{2} \mathrm{OR}\right), 3.53(\mathrm{t}, J=10.6 \mathrm{~Hz}, 1 \mathrm{H}$, one of $\mathrm{CH}_{2} \mathrm{OR}$ ), 3.44 (ddd, $\left.J=10.4,7.6,4.8 \mathrm{~Hz}, 1 \mathrm{H}, \mathrm{PyCH}\right), 2.26$ (s, 3H, $\left.\mathrm{PyCH}_{3}\right) .{ }^{13} \mathbf{C}-\mathbf{N M R}(126$ $\left.\mathrm{MHz}, \mathrm{CDCl}_{3}\right): \delta 172.2,166.0,161.8,150.6,99.9,98.4,96.0,78.6,64.7,36.7,20.5$. IR (film): 3099, 2926, 1705, 1630, 1574, 1452, 1252, 975, 954, $838 \mathrm{~cm}^{-1}$. HRMS - ESI $(m / z):[\mathrm{M}+\mathrm{Na}]^{+}$ calculated for $\mathrm{C}_{11} \mathrm{H}_{10} \mathrm{O}_{4} \mathrm{Na}^{+}, 229.0477$; found, 229.0473. Melting point $=138-140{ }^{\circ} \mathrm{C}$.

4-[cis-( \pm$)-6$-[(tert-Butyldimethylsilyl)oxy]-3,6-dihydro- $2 H$-pyran-3-yl $]$ oxy-6methyl-2H-pyran-2-one, 335.<smiles>Cc1cc(OC2C=CC([18OH])OC2)cc(=O)o1</smiles>

The reaction was performed with 4-hydroxy-6-methyl- $\alpha$-pyrone $(0.040 \mathrm{~g}, 0.315 \mathrm{mmol})$ according to Method B. The crude reaction mixture was purified by flash column chromatography (30\% EtOAc /pet. ether) to give furopyran 4 (0.037 g, $56 \%$ yield $)$, along with the title compound as a yellow oil (0.009 g, $9 \%$ yield). $\mathbf{R}_{\mathbf{f}}=0.24$ (30\% EtOAc / Pet. Ether). ${ }^{\mathbf{1}} \mathbf{H}-$ NMR $\left(500 \mathrm{MHz}, \mathrm{CDCl}_{3}\right): \delta 5.95$ (app. dt, $J=10.3,0.9 \mathrm{~Hz}, 1 \mathrm{H}, \mathrm{HC}=$ ), 5.89 (app. dt, $J$ $=10.3,1.9 \mathrm{~Hz}, 1 \mathrm{H}, \mathrm{HC}=), 5.77(\mathrm{~m}, 1 \mathrm{H}, \mathrm{PyH}), 5.41(\mathrm{~d}, J=2.1 \mathrm{~Hz}, 1 \mathrm{H}, \mathrm{CHOTBS}), 5.30(\mathrm{~s}$, $1 \mathrm{H}, \mathrm{PyH}), 4.79(\mathrm{~m}, 1 \mathrm{H}, \mathrm{PyOCH}), 3.95$ (dd, $J=11.1,8.0 \mathrm{~Hz}, 1 \mathrm{H}$, one of $\mathrm{CH}_{2} \mathrm{OR}$ ), 3.88 (ddd,

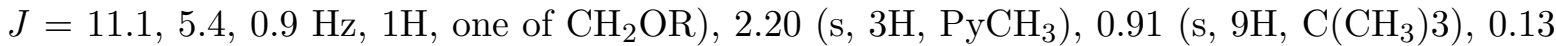
(s, 6H, Si $\left.\left(\mathrm{CH}_{3}\right) 2\right) .{ }^{13} \mathrm{C}-\mathrm{NMR}\left(126 \mathrm{MHz}, \mathrm{CDCl}_{3}\right): \delta 169.2,164.7,162.6,132.7,125.2,100.6$, 89.3, 88.4, 68.6, 59.8, 25.7, 19.9, 18.0, -4.4, -5.3. IR (film): 2929, 2857, 1731, 1711, 1650, 1563, 1248, 1035, 838, $781 \mathrm{~cm}^{-1}$. HRMS - ESI $(\mathrm{m} / z):[\mathrm{M}+\mathrm{Na}]^{+}$calculated for $\mathrm{C}_{17} \mathrm{H}_{26} \mathrm{O}_{5} \mathrm{SiNa}^{+}$, 361.1447 ; found, 361.1443 .

cis-( \pm )-3-Methyl-5a,8,9,9a-tetrahydro-1H-pyrano[4,3-b]benzofuran-1-one, 331.

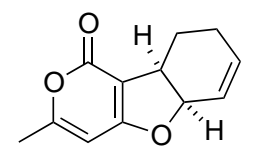

To the solid reagents 4-hydroxy-6-methyl- $\alpha$-pyranone (0.252 g, $1.997 \mathrm{mmol}, 2.2$ eq.), $\mathrm{Pd}_{2}(\mathrm{dba})_{3}(0.083 \mathrm{~g}, 0.091 \mathrm{mmol}, 10 \mathrm{~mol} \%)$, triphenylphosphine $(0.095 \mathrm{~g}, 0.364 \mathrm{mmol}, 40$ mol\%) and trans- $( \pm)-4$-[(methoxycarbonyl)oxy]cyclohex-2-en-1-yl methyl carbonate $(0.209 \mathrm{~g}$, $0.908 \mathrm{mmol}, 1.0$ eq.) was added $25 \mathrm{~mL}$ of anhydrous acetonitrile at room temperature. This was 
followed by the addition of triethylamine $(0.127 \mathrm{~mL}, 0.908 \mathrm{mmol}, 1$ eq. $)$. The reaction mixture was stirred at room temperature for 2 hours before concentration in vacuo and purification by flash column chromatography (20\% EtOAc / pet. ether). The desired product was isolated as a yellow solid (0.095 g, $51 \%$ yield). $\mathbf{R}_{\mathbf{f}}=0.21$ (20\% EtOAc /pet. ether). ${ }^{\mathbf{1}} \mathbf{H}-\mathbf{N M R}$ (500 $\left.\mathrm{MHz} \mathrm{CDCl}_{3}\right): \delta 6.21(\mathrm{~m}, 1 \mathrm{H}, \mathrm{HC}=), 5.91(\mathrm{~m}, 1 \mathrm{H}, \mathrm{HC}=), 5.89(\mathrm{~s}, 1 \mathrm{H}, \mathrm{PyH}), 5.08($ app. $\mathrm{d}, J$ $\left.=8.5 \mathrm{~Hz},{ }^{*} 1 \mathrm{H}, \mathrm{PyOCH}\right), 3.36$ (app. td, $\left.J=9.2,4.5 \mathrm{~Hz}, 1 \mathrm{H}, \mathrm{PyCH}\right), 2.23$ (s, 3H, $\mathrm{PyCH}_{3}$ ), $2.11\left(\mathrm{~m}, 2 \mathrm{H}, \mathrm{CH}_{2} \mathrm{C}=\right), 1.92\left(\mathrm{~m}, 1 \mathrm{H}\right.$, one of $\left.\mathrm{CH}_{2} \mathrm{CH}_{2} \mathrm{C}=\right), 1.53\left(\mathrm{~m}, 1 \mathrm{H}\right.$, one of $\left.\mathrm{CH}_{2} \mathrm{CH}_{2} \mathrm{C}=\right)$. ${ }^{13} \mathrm{C}-\mathrm{NMR}\left(126 \mathrm{MHz}, \mathrm{CDCl}_{3}\right): \delta 171.1,165.1,162.3,135.5,122.6,103.5,96.1,82.0,37.0,23.4$, 22.4, 20.4. IR (KBr): 2938, 1709, 1630, 1578, 1452, 1384, 1255, 1128, $979 \mathrm{~cm}^{-1}$. HRMS ESI $(m / z):[\mathrm{M}+\mathrm{H}]^{+}$calculated for $\mathrm{C}_{12} \mathrm{H}_{13} \mathrm{O}_{3}{ }^{+}, 205.0864$; found, 205.0865. Melting point $=$ $106-109{ }^{\circ} \mathrm{C}$.

* Homonuclear decoupling experiments were used to confirm the coupling constant for the ring-junction protons: $J=8.21 \mathrm{~Hz}$. The homonuclear decoupling spectra can be found in the Spectra section.

cis-( \pm$)-7,10 \mathrm{a}-D i h y d r o p y r a n o\left[3^{\prime}, 4^{\prime}: 4,5\right]$ furo[3,2-c]chromen-6(6bH)-one, 339a.<smiles>O=c1oc2ccccc2c2c1C1C=COCC1O2</smiles>

The reaction was performed with 4-hydroxycoumarin (0.045 g, $0.278 \mathrm{mmol})$ according to Method A. The crude product was purified by flash column chromatography (10\% EtOAc / pet. ether) to give the title compound as a yellow solid $\left(0.052 \mathrm{~g}, 77 \%\right.$ yield). $\mathbf{R}_{\mathbf{f}}=0.16(10 \%$ EtOAc / pet. ether). ${ }^{1} \mathbf{H}-\mathbf{N M R}\left(500 \mathrm{MHz}, \mathrm{CDCl}_{3}\right): \delta 7.68(\mathrm{dd}, J=7.8,1.6 \mathrm{~Hz}, 1 \mathrm{H}, \mathrm{ArH})$, 7.59 (ddd, $J=8.6,7.2,1.5 \mathrm{~Hz}, 1 \mathrm{H}, \mathrm{ArH}), 7.39$ (dd, $J=8.4,0.5 \mathrm{~Hz}, 1 \mathrm{H}, \mathrm{ArH}), 7.31(\mathrm{~m}, 1 \mathrm{H}$, $\mathrm{ArH}), 6.85(\mathrm{~d}, J=5.1 \mathrm{~Hz}, 1 \mathrm{H}, \mathrm{HC}=), 5.33-5.29($ complex $\mathrm{m}, 2 \mathrm{H}, \mathrm{HC}=$ and $\mathrm{PyOCH}), 4.45$ (dd, $J=10.2,4.3 \mathrm{~Hz}, 1 \mathrm{H}$, one of $\mathrm{CH}_{2} \mathrm{OR}$ ), 3.68-3.60 (complex m, 2H, one of $\mathrm{CH}_{2} \mathrm{OR}$ and PyCH). ${ }^{13}$ C-NMR $\left(126 \mathrm{MHz}, \mathrm{CDCl}_{3}\right): \delta 167.7,160.4,155.1,150.8,132.9,124.0,123.0,117.1$, 112.6, 102.6, 98.5, 79.2, 64.7, 37.8. IR (KBr): 2918, 1711, 1640, 1416, 1236, 1161, 1066, 1033, $834 \mathrm{~cm}^{-1}$. HRMS (ESI) calculated for $\mathrm{C}_{14} \mathrm{H}_{10} \mathrm{O}_{4} \mathrm{Na}^{+}[\mathrm{M}+\mathrm{Na}]^{+}:$265.0477, found 265.0470 . Melting point $=178-179{ }^{\circ} \mathrm{C}$.

cis-( \pm )-3-Methoxy-7,10a-dihydropyrano[3',4':4,5] furo[3,2-c] chromen-6(6bH)one, 339b.<smiles>COc1ccc2c3c(c(=O)oc2c1)C1COC=CC1O3</smiles>

The reaction was performed with 4-hydroxy-7-methoxycoumarin (0.040 g, $0.208 \mathrm{mmol})$ according to Method A. The crude product was purified by flash column chromatography (20\% EtOAc / pet. ether) to give the title compound as a yellow solid ( $0.038 \mathrm{~g}, 68 \%$ yield). $\mathbf{R}_{\mathbf{f}}=0.38\left(30 \%\right.$ EtOAc / pet. ether). ${ }^{\mathbf{1}} \mathbf{H}-\mathbf{N M R}\left(500 \mathrm{MHz}, \mathrm{CDCl}_{3}\right): \delta 7.57(\mathrm{~d}, J=9.4$ $\mathrm{Hz}, 1 \mathrm{H}, \mathrm{ArH}), 6.88-6.83(\mathrm{~m}, 3 \mathrm{H}, 2 \mathrm{x} \mathrm{ArH}$ and $\mathrm{HC}=), 5.31$ (dd, $J=5.9,4.6 \mathrm{~Hz}, 1 \mathrm{H}, \mathrm{HC}=)$, $5.25(\mathrm{dd}, J=6.1,5.0 \mathrm{~Hz}, 1 \mathrm{H}, \mathrm{ArOCH}), 4.44\left(\mathrm{~m}, 1 \mathrm{H}\right.$, one of $\left.\mathrm{CH}_{2} \mathrm{OR}\right), 3.89\left(\mathrm{~s}, 3 \mathrm{H}, \mathrm{OCH}_{3}\right)$, 3.63-3.57 (m, 2H, one of $\mathrm{CH}_{2} \mathrm{OR}$ and $\left.\mathrm{ArCH}\right) .{ }^{13} \mathbf{C}-\mathrm{NMR}\left(126 \mathrm{MHz}, \mathrm{CDCl}_{3}\right): \delta 168.0,163.7$, 
160.7, 157.1, 150.8, 124.0, 112.6, 105.8, 100.8, 99.7, 98.5, 79.1, 64.9, 55.8, 37.5. IR (KBr): 3002, 2968, 1702, 1638, 1290, 1154, 1106, 1071, 1030, $840 \mathrm{~cm}^{-1}$. HRMS (ESI) calculated for $\mathrm{C}_{15} \mathrm{H}_{12} \mathrm{O}_{5} \mathrm{Na}^{+}[\mathrm{M}+\mathrm{Na}]^{+}:$295.0582, found 295.0589. Melting point $=150-151{ }^{\circ} \mathrm{C}$.

cis-( \pm -1', 3',4',5a',9',9a'-Hexahydro-spiro[cyclohexane-1,3'-furo[3,2-c:4,5-c'] dipyran]1 '-one, 339c.<smiles>O=C1OC2(CCCCC2)CC2=C1C1COC=CC1O2</smiles>

The reaction was performed with 1-oxaspiro[5.5] undecane-2,4-dione $(0.050 \mathrm{~g}, 0.277 \mathrm{mmol})$ according to Method A. The crude product was purified by flash column chromatography $(20 \%$ EtOAc/pet. ether $)$ to give the title compound as a white foam $(0.040 \mathrm{~g}, 56 \%$ yield $) . \mathbf{R}_{\mathbf{f}}=$ $0.29\left(20 \%\right.$ EtOAc/pet. ether). ${ }^{1} \mathbf{H}-\mathbf{N M R}\left(500 \mathrm{MHz}, \mathrm{CDCl}_{3}\right): \delta 6.78(\mathrm{~d}, J=6.2 \mathrm{~Hz}, 1 \mathrm{H}$, $\mathrm{HC}=), 5.18(\mathrm{dd}, J=6.2,4.4 \mathrm{~Hz}, 1 \mathrm{H}, \mathrm{HC}=), 5.03(\mathrm{dd}, J=7.9,4.4 \mathrm{~Hz}, 1 \mathrm{H}, \mathrm{PyOCH}), 4.35$ $\left(\mathrm{dd}, J=10.8,5.0 \mathrm{~Hz}, 1 \mathrm{H}\right.$, one of $\left.\mathrm{CH}_{2} \mathrm{OR}\right), 3.47\left(\mathrm{t}, J=10.6 \mathrm{~Hz}, 1 \mathrm{H}\right.$, one of $\mathrm{CH}_{2} \mathrm{OR}$ ), 3.31 $\left(\mathrm{m}, 1 \mathrm{H}, \mathrm{CHCH}_{2} \mathrm{OR}\right), 2.50\left(\mathrm{t}, J=1.5 \mathrm{~Hz}, 2 \mathrm{H},=\mathrm{CCH}_{2}\right), 2.03-1.96\left(\right.$ complex m, $\left.2 \mathrm{H}, \mathrm{cy}-\mathrm{CH}_{2}\right)$, 1.83-1.75 (complex m, 2H, cy- $\mathrm{CH}_{2}$ ), 1.56 (complex m, 2H, cy-CH2), 1.49-1.45 (complex m, $2 \mathrm{H}$, cy- $\left.\mathrm{CH}_{2}\right), 1.35$ (complex m, $2 \mathrm{H}$, cy-CH 2$) .{ }^{13} \mathrm{C}-\mathrm{NMR}\left(126 \mathrm{MHz}, \mathrm{CDCl}_{3}\right): \delta 172.1,164.2$, 150.5, 101.9, 98.7, 80.9, 78.2, 65.5, 37.3, 36.7, 36.5, 25.2, 21.7. IR (film): 2935, 2863, 1762, 1673, 1407, 1210, $1035 \mathrm{~cm}^{-1}$. HRMS (ESI) calculated for $\mathrm{C}_{15} \mathrm{H}_{19} \mathrm{O}_{4}{ }^{+}[\mathrm{M}+\mathrm{H}]^{+}: 263.1283$, found 263.1292. Melting point $=70-75{ }^{\circ} \mathrm{C}$. 339e.

cis-( \pm )-7,7-Dimethyl-4a,6,7,8,9,9b-hexahydro-1H-pyrano[4,3-b]benzofuran-9-one,<smiles>CC1(C)CC(=O)C2=C(C1)OC1C=COCC21</smiles>

The reaction was performed with dimedone $(0.039 \mathrm{~g}, 0.278 \mathrm{mmol})$ according to Method B. The crude product was purified by flash column chromatography $(20 \%$ EtOAc/pet. ether) to give the title compound as a yellow oil $(0.035 \mathrm{~g}, 57 \%$ yield $) . \mathbf{R}_{\mathbf{f}}=0.24(20 \% \mathrm{EtOAc} /$ pet. ether). ${ }^{1} \mathbf{H}-\mathbf{N M R}\left(500 \mathrm{MHz}, \mathrm{CDCl}_{3}\right): \delta 6.77(\mathrm{~d}, J=6.1 \mathrm{~Hz}, 1 \mathrm{H}, \mathrm{HC}=), 5.20(\mathrm{~m}, 1 \mathrm{H}, \mathrm{HC}=)$, $4.93(\mathrm{t}, J=5.6 \mathrm{~Hz}, 1 \mathrm{H}, \mathrm{OCH}), 4.35\left(\mathrm{~m}, 1 \mathrm{H}\right.$, one of $\left.\mathrm{CH}_{2} \mathrm{OR}\right), 3.36(\mathrm{t}, J=10.6 \mathrm{~Hz}, 1 \mathrm{H}$, one of $\mathrm{CH}_{2} \mathrm{OR}$ ), 3.30 (m, $1 \mathrm{H}, \mathrm{CHCH}_{2} \mathrm{OR}$ ), 2.37-2.18 (complex m, $\left.2 \times 2 \mathrm{H},\left(\mathrm{CH}_{3}\right) 2 \mathrm{CCH}_{2}\right), 1.13(\mathrm{~s}, 3 \mathrm{H}$, $\left.\mathrm{CH}_{3}\right), 1.08\left(\mathrm{~s}, 3 \mathrm{H}, \mathrm{CH}_{3}\right) .{ }^{13} \mathrm{C}-\mathrm{NMR}\left(126 \mathrm{MHz}, \mathrm{CDCl}_{3}\right): \delta 194.6,177.7,150.3,112.8,98.8$, 77.6, 65.5, 51.0, 38.0, 36.8, 34.2, 29.3, 28.0. IR (film): 2958, 2926, 1724, 1614, 1404, 1215, 1033, 842, $733 \mathrm{~cm}^{-1}$. HRMS (ESI) calculated for $\mathrm{C}_{13} \mathrm{H}_{16} \mathrm{O}_{3} \mathrm{Na}^{+}[\mathrm{M}+\mathrm{Na}]^{+}: 243.0997$, found 243.0999 .

cis-( \pm )-3-(4-Bromophenyl)-3,4,9,9a-tetrahydrofuro[3,2-c:4,5-c']dipyran-1(1H,5aH)-one, 339d.

The reaction was performed with 6-(4-bromophenyl)oxane-2,4-dione (0.075 g, $0.279 \mathrm{mmol})$ according to Method B. The crude product was purified by flash column chromatography $(20 \%$ EtOAc / pet. ether.) to give the title compound as a yellow solid (0.059 g, $61 \%$ yield, 1:1 mixture of diastereomers). $\mathrm{Rf}=0.15$ (20\% EtOAc / pet. ether). ${ }^{1} \mathbf{H}-\mathbf{N M R}(500 \mathrm{MHz}$, 


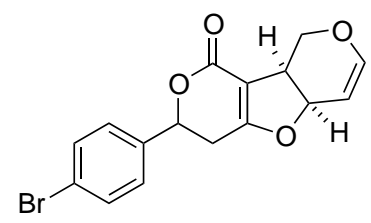

$\left.\mathrm{CDCl}_{3}\right): \delta 7.53(\mathrm{~d}, J=8.4 \mathrm{~Hz}, 2 \mathrm{H}, \mathrm{ArH}), 7.30(\mathrm{dd}, J=8.4,2.1 \mathrm{~Hz}, 2 \mathrm{H}, \mathrm{ArH}), 6.80(\mathrm{~d}, J=$ $5.5 \mathrm{~Hz}, 1 \mathrm{H}, \mathrm{HC}=), 5.44(\mathrm{dd}, J=11.2,5.0 \mathrm{~Hz}, 1 \mathrm{H}, \mathrm{ArCH}), 5.21(\mathrm{dd}, J=6.2,4.5 \mathrm{~Hz}, 0.5 \mathrm{H}$, $\mathrm{HC}=), 5.18-5.14(\mathrm{~m}, 2 \times 0.5 \mathrm{H}, \mathrm{HC}=$ and $\mathrm{OCH}), 5.09(\mathrm{dd}, J=7.5,4.5 \mathrm{~Hz}, 0.5 \mathrm{H}, \mathrm{OCH}), 4.47$ $\left(\mathrm{dd}, J=10.7,4.9 \mathrm{~Hz}, 0.5 \mathrm{H}\right.$, one of $\left.\mathrm{CH}_{2} \mathrm{OR}\right), 4.27\left(\mathrm{dd}, J=11.0,4.8 \mathrm{~Hz}, 0.5 \mathrm{H}\right.$, one of $\mathrm{CH}_{2} \mathrm{OR}$ ), $3.67\left(\mathrm{dd}, J=11.0,9.2 \mathrm{~Hz}, 0.5 \mathrm{H}\right.$, one of $\left.\mathrm{CH}_{2} \mathrm{OR}\right), 3.46-3.40\left(\mathrm{~m}, 2 \times 0.5 \mathrm{H}\right.$, one of $\mathrm{CH}_{2} \mathrm{OR}$ and $\left.\mathrm{CHCH}_{2} \mathrm{OR}\right), 3.34\left(\mathrm{~m}, 0.5 \mathrm{H}, \mathrm{CHCH}_{2} \mathrm{OR}\right), 2.82-2.69\left(\mathrm{~m}, 2 \mathrm{H}, \mathrm{ArCHCH}_{2}\right) .{ }^{13} \mathbf{C}-\mathbf{N M R}(126$ $\left.\mathrm{MHz}, \mathrm{CDCl}_{3}\right): \delta 173.0,164.2,150.7,137.1,131.9,127.7,122.7,103.2,98.9 / 98.2,79.2 / 78.8$, 76.8, 65.4/65.3, 37.5/37.4, 31.4/31.3. IR (KBr): 2926, 1701, 1641, 1400, 1384, 1237, 1107, 1067, $1037 \mathrm{~cm}^{-1}$. HRMS (ESI) calculated for $\mathrm{C}_{16} \mathrm{H}_{13} \mathrm{BrO}_{4} \mathrm{Na}^{+}[\mathrm{M}+\mathrm{Na}]^{+}: 370.9895$, found 370.9900. Melting point $=132-135{ }^{\circ} \mathrm{C}$.

(cis)-8-Benzyl-7-methyl-8,9b-dihydro-1 $H$-pyrano[3',4':4,5] furo[3,2-c]pyridin-9(4aH)-one, 339g.<smiles>Cc1cc2c(c(=O)n1Cc1ccccc1)C1COC=CC1O2</smiles>

The reaction was performed with 1-benzyl-4-hydroxy-6-methylpyridin-2(1H)-one (0.022 g, 0.104 mmol, 1.0 equiv) according to method B. The crude product was purified by flash column chromatography (30\% EtOAc/Pet. Ether) to give the title compound as a clear film $(0.0115$ g, $37 \%$ yield $) . \mathbf{R}_{\mathbf{f}}=0.36\left(50 \%\right.$ EtOAc/Pet. Ether). ${ }^{1} \mathbf{H} \mathbf{~ N M R}\left(500 \mathrm{MHz}, \mathrm{CDCl}_{3}\right) \delta 7.32(\mathrm{dd}$, $J=8.2,6.7 \mathrm{~Hz}, 2 \mathrm{H}), 7.17-7.12(\mathrm{~m}, 2 \mathrm{H}), 6.80(\mathrm{dd}, J=6.2,1.1 \mathrm{~Hz}, 1 \mathrm{H}), 5.88(\mathrm{~s}, 1 \mathrm{H}), 5.47$ $(\mathrm{d}, J=15.8 \mathrm{~Hz}, 1 \mathrm{H}), 5.23(\mathrm{dd}, J=6.2,4.4 \mathrm{~Hz}, 1 \mathrm{H}), 5.17(\mathrm{~d}, J=15.5 \mathrm{~Hz}, 1 \mathrm{H}), 5.08-5.01$ $(\mathrm{m}, 1 \mathrm{H}), 4.51-4.43(\mathrm{~m}, 1 \mathrm{H}), 3.64-3.52(\mathrm{~m}, 2 \mathrm{H}), 2.27(\mathrm{~s}, 2 \mathrm{H}) \cdot{ }^{\mathbf{1 3}} \mathbf{C} \mathbf{N M R}\left(126 \mathrm{MHz}, \mathrm{CDCl}_{3}\right) \delta$ 150.12 , 136.73, 128.81, 127.31, 126.41, 126.35, 98.92, 96.39, 90.30, 64.97, 46.66, 37.94, 29.70, 25.64, 21.35. IR (film): 2925, 2854, 1722, 1650, 1563, 1354, 1253, 1068, 1030, $843 \mathrm{~cm}^{-1}$.

( \pm )-Methyl $\left(3 R^{*}, 3 \mathrm{a} S^{*}, 7 \mathrm{a} R^{*}\right)-2 \mathrm{H}-2-O x o-3,3 \mathrm{a}, 4,7$ a-tetrahydrofuro[3,2-c]pyran-3carboxylate, 340 .

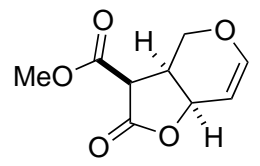

The reaction was performed with $0.040 \mathrm{~g}(0.277 \mathrm{mmol})$ of Meldrum's acid according to Method A. The crude product was purified by flash column chromatography (25\% EtOAc/ pet. ether) to give the title compound as a clear oil $(0.0214 \mathrm{~g}, 39 \%$ yield $)$ along with the bis-adduct $(0.0418 \mathrm{~g}, 53 \%$ yield). The desired product co-eluted, in part, with the remaining MeldrumÕs acid and, as a result, the isolated yield was determined by ${ }^{1} \mathrm{H}$ NMR spectroscopy. An analytical sample was obtained by careful chromatography, using $p$-anisaldehyde dip to distinguish the title compound (stained black) and Meldrum's acid (stained pink). This provided the title compound, which also contained a small quantity (ca. 14:1 ratio) of a 
diastereoisomer, presumably differing at the doubly $\alpha$-position (C-3). The stereochemistry of the major isomer is tentatively assigned on the basis of NOE correlations as shown on the diagram (see NOESY spectrum). $\mathbf{R}_{\mathbf{f}}=0.24$ (30\% EtOAc/pet. ether). ${ }^{\mathbf{1}} \mathbf{H}-\mathbf{N M R}(500 \mathrm{MHz}$, $\left.\mathrm{CDCl}_{3}\right): \delta 6.62(\mathrm{~d}, J=6.2 \mathrm{~Hz}, 1 \mathrm{H}, \mathrm{HC}=), 5.11(\mathrm{dd}, J=7.0,3.2 \mathrm{~Hz}, 1 \mathrm{H}, \mathrm{OCH}), 5.01$ (dd, $J=5.7,3.9 \mathrm{~Hz}, 1 \mathrm{H}, \mathrm{HC}=), 4.11\left(\mathrm{dd}, J=11.8,3.5 \mathrm{~Hz}, 1 \mathrm{H}\right.$, one of $\mathrm{CH}_{2} \mathrm{OR}$ ), 3.88 (partially obs. dd, $J=11.8,6.1 \mathrm{~Hz}, 1 \mathrm{H}$, one of $\left.\mathrm{CH}_{2} \mathrm{OR}\right), 3.85\left(\mathrm{~s}, 3 \mathrm{H}, \mathrm{CO}_{2} \mathrm{CH}_{3}\right), 3.56(\mathrm{~d}, J=7.7 \mathrm{~Hz}$, $1 \mathrm{H}, \mathrm{CHCO}_{2} \mathrm{Me}$ ), 3.21 (app. tdd, $\left.J=7.2,6.6,3.5 \mathrm{~Hz} 1 \mathrm{H}, \mathrm{CHCH}_{2} \mathrm{OR}\right) .{ }^{13} \mathbf{C}-\mathrm{NMR}(126 \mathrm{MHz}$, $\left.\mathrm{CDCl}_{3}\right): \delta 170.6,167.2,148.9,99.6,70.8,63.6,53.3,47.6,37.7$ ppm. IR (film): 2958, 2880, 1774, 1732, 1642, 1240, 1143, 1080, $970 \mathrm{~cm}^{-1}$. HRMS (ESI) calculated for $\mathrm{C}_{9} \mathrm{H}_{10} \mathrm{O}_{5} \mathrm{Na}^{+}[\mathrm{M}$ $+\mathrm{Na}]^{+}: 221.0426$, found 221.0422 .

5,5-Bis-(cis-6-((tert-Butyldimethylsilyl)oxy)-3,6-dihydro-2H-pyran-3-yl)-2,2dimethyl-1,3-dioxane-4,6-dione, 341.

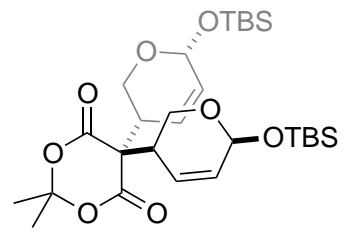

This material was a mixture of two diastereoisomers (ca. 1.2:1 ratio). $\mathbf{R}_{\mathbf{f}}=0.71(30 \%$ EtOAc / pet. ether). ${ }^{1} \mathbf{H}-\mathbf{N M R}\left(500 \mathrm{MHz}, \mathrm{CDCl}_{3}\right): \delta 5.94(\mathrm{~d}, J=10.5 \mathrm{~Hz}, 2 \times 0.55 \mathrm{H}), 5.88$ $(\mathrm{d}, J=10.4 \mathrm{~Hz}, 2 \times 0.45 \mathrm{H}), 5.85-5.83(\mathrm{~m}, 2 \times 1 \mathrm{H}), 5.25(\mathrm{~s}, 2 \times 1 \mathrm{H}), 4.05$ (app. t,$J=10.5$ $\mathrm{Hz}, 2 \times 0.45 \mathrm{H}), 3.97$ (app. t, $J=10.4 \mathrm{~Hz}, 2 \times 0.55 \mathrm{H}), 3.70(\mathrm{~m}, 2 \times 1 \mathrm{H}), 3.30-3.25(\mathrm{~m}, 2 \times 1 \mathrm{H})$, $1.76(\mathrm{~s}, 2.7 \mathrm{H}), 1.75(\mathrm{~s}, 3.3 \mathrm{H}), 0.901(\mathrm{~s}, 8.1 \mathrm{H}), 0.898(\mathrm{~s}, 9.9 \mathrm{H}), 0.11-0.10($ complex m, 12H) ${ }^{13} \mathrm{C}-\mathrm{NMR}\left(126 \mathrm{MHz}, \mathrm{CDCl}_{3}\right): \delta 165.58,131.13,130.85,125.25,125.20,106.24,106.14,88.83$, $88.75,58.91,58.66,55.40,55.08,38.52,38.50,29.91,29.77,25.69,17.99,17.98,-4.48,-5.10$, -5.11. HRMS (ESI) calculated for $\mathrm{C}_{28} \mathrm{H}_{48} \mathrm{O}_{8} \mathrm{Si}_{2} \mathrm{Na}^{+}[\mathrm{M}+\mathrm{Na}]^{+}: 591.2785$, found 591.2794 . Melting point $=55-60{ }^{\circ} \mathrm{C}$.

Bis-2,2-(((cis)-6-((tert-butyldimethylsilyl)oxy)-3,6-dihydro-2 $H$-pyran-3-yl)-1 Hindene-1,3(2H)-dione, 352.

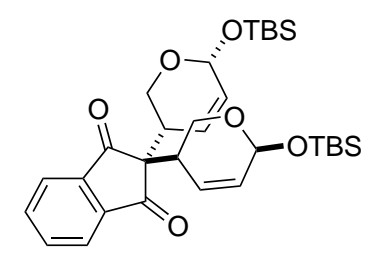

The reaction was performed with $1 H$-indene-1,3(2H)-dione (0.029 g, $0.2 \mathrm{mmol}, 1.0$ equiv) according to method B. The crude product was purified by flash column chromatography $(10 \%$ EtOAc/Pet. Ether) to give the title compound as a clear film (0.0115 g, $37 \%$ yield $) . \mathbf{R}_{\mathbf{f}}=0.44$ $\left(15 \%\right.$ EtOAc/Pet.) ${ }^{\mathbf{1}} \mathbf{H}$ NMR $\left(500 \mathrm{MHz}, \mathrm{CDCl}_{3}\right) \delta 7.97(\mathrm{dt}, J=6.0,3.0 \mathrm{~Hz}, 2 \mathrm{H}), 7.89-7.85$ $(\mathrm{m}, 2 \mathrm{H}), 5.75-5.68(\mathrm{~m}, 2 \mathrm{H}), 5.64(\mathrm{dt}, J=10.3,2.8 \mathrm{~Hz}, 1 \mathrm{H}), 5.53-5.49(\mathrm{~m}, 1 \mathrm{H}), 5.19(\mathrm{~d}, J=$ $1.5 \mathrm{~Hz}, 2 \mathrm{H}), 4.20-4.11(\mathrm{~m}, 1 \mathrm{H}), 3.91(\mathrm{t}, J=10.6 \mathrm{~Hz}, 1 \mathrm{H}), 3.78(\mathrm{~s}, 0 \mathrm{H}), 3.74$ (ddd, $J=11.1$, $5.2,1.7 \mathrm{~Hz}, 1 \mathrm{H}), 3.64(\mathrm{t}, J=6.2 \mathrm{~Hz}, 0 \mathrm{H}), 3.61-3.54(\mathrm{~m}, 1 \mathrm{H}), 3.16-3.05(\mathrm{~m}, 2 \mathrm{H}), 0.90(\mathrm{~s}, 10 \mathrm{H})$, $0.88(\mathrm{~s}, 10 \mathrm{H}), 0.11(\mathrm{~s}, 2 \mathrm{H}), 0.10(\mathrm{~s}, 3 \mathrm{H}), 0.07(\mathrm{~s}, 3 \mathrm{H}), 0.06(\mathrm{~s}, 3 \mathrm{H}) .{ }^{13} \mathrm{C}$ NMR $(126 \mathrm{MHz}$, $\left.\mathrm{CDCl}_{3}\right) \delta 201.40,201.37,200.97,142.07,142.05,141.92,136.06,136.03,135.98,130.71,130.70$, $126.00,125.83,123.26,123.15,122.90,88.71,88.53,58.26,57.91,57.86,57.72,36.39,36.32$, 
$25.74,25.72,25.64,18.01,-4.49,-5.05,-5.06$.

Methyl (((1S,4aS,9bS)-7-Methyl-9-oxo-1,4a,9,9b-tetrahydrofuro[3,2-c:4,5-c']dipyran-1-yl)methyl) carbonate, 354 .<smiles>COCC1OC=CC2(COc3cc(C)oc(=O)c32)O1</smiles>

The reaction was performed with 4-hydroxy-6-methyl- $\alpha$-pyrone $(0.017 \mathrm{~g}, 0.133 \mathrm{mmol}, 1.0$ equiv), methyl-(2R,3S,6R)-6-((tert-butyldimethylsilyl)oxy)-2-(hydroxymethyl)-3,6-dihydro-2Hpyran-3-ol dicarbonate $\left(0.05 \mathrm{~g}, 0.133 \mathrm{mmol}, 1.0\right.$ equiv) and $\mathrm{Pd}\left(\mathrm{PPh}_{3}\right)_{4}(0.023 \mathrm{~g}, 0.020$ mmol, $15 \mathrm{~mol} \%$ ) according to Method B. The crude product was purified by flash column chromatography (40\% EtOAc/Pet. Ether) to give the title compound as a yellow solid $(0.0299$ g, $77 \%$ yield). $\mathbf{R}_{\mathbf{f}}=0.21$ (40\% EtOAc/Pet. Ether). ${ }^{1} \mathbf{H} \mathbf{~ N M R ~}\left(500 \mathrm{MHz}, \mathrm{CDCl}_{3}\right) \delta 6.81$ (d, $J=6.2 \mathrm{~Hz}, 1 \mathrm{H}), 5.96(\mathrm{~s}, 1 \mathrm{H}), 5.30(\mathrm{dd}, J=6.2,4.6 \mathrm{~Hz}, 1 \mathrm{H}), 5.08(\mathrm{ddd}, J=7.4,4.8,1.4$ $\mathrm{Hz}, 1 \mathrm{H}), 4.75(\mathrm{dd}, J=12.4,2.0 \mathrm{~Hz}, 1 \mathrm{H}), 4.43(\mathrm{dd}, J=12.3,6.6 \mathrm{~Hz}, 1 \mathrm{H}), 3.80(\mathrm{~s}, 3 \mathrm{H}), 3.64$ $(\mathrm{ddd}, \mathrm{J}=11.2,6.6,1.9 \mathrm{~Hz}, 1 \mathrm{H}), 3.24(\mathrm{dd}, J=11.2,7.5 \mathrm{~Hz}, 1 \mathrm{H}), 2.28(\mathrm{~s}, 3 \mathrm{H}) .{ }^{13} \mathbf{C}$ NMR $\left(126 \mathrm{MHz}, \mathrm{CDCl}_{3}\right) \delta 172.58,166.70,161.92,155.42,149.46,99.59,97.91,95.91,79.42,74.64$, $67.72,54.93,37.13,20.56 .[\alpha]_{\mathbf{D}^{25}}{ }^{25}-209.9^{\circ}\left(c=0.5, \mathrm{CH}_{2} \mathrm{Cl}_{2}\right)$. IR (film): 2362, 2341, 1750, 1717, 1633, 1577, 1447, $1255 \mathrm{~cm}^{-1}$. HRMS (ESI) calculated for $\mathrm{C}_{14} \mathrm{H}_{14} \mathrm{O}_{7} \mathrm{Na}^{+}[\mathrm{M}+\mathrm{Na}]^{+}$: 317.0632, found 317.0633. Melting point $=153-156{ }^{\circ} \mathrm{C}$.

Methyl (((6bS,7S,10a $S)-6-O x o-6,6 \mathrm{~b}, 7,10 \mathrm{a}-$ tetrahydropyrano[3',4':4,5]furo[3,2-c]chromen-7-yl)methyl) carbonate, 355.<smiles>COCC1OC=CC2Oc3c(c(=O)oc4ccccc34)C21</smiles>

The reaction was performed with 4-hydroxycoumarin (0.022 g, $0.133 \mathrm{mmol}, 1.0$ equiv), methyl-(2R,3S,6R)-6-((tert-butyldimethylsilyl)oxy)-2-(hydroxymethyl)-3,6-dihydro-2H-pyran-3ol dicarbonate (0.05 g, $0.133 \mathrm{mmol}, 1.0$ equiv) and $\mathrm{Pd}\left(\mathrm{PPh}_{3}\right)_{4}(0.023 \mathrm{~g}, 0.020 \mathrm{mmol}, 15 \mathrm{~mol} \%)$ according to Method B. The crude product was purified by flash column chromatography $(20 \%$ EtOAc/Pet. Ether) to give the title compound as an off-white solid $(0.0393 \mathrm{~g}, 90 \%$ yield $)$. $\mathbf{R}_{\mathbf{f}}$ $=0.46\left(40 \%\right.$ EtOAc/Pet. Ether). ${ }^{1} \mathbf{H}$ NMR $\left(500 \mathrm{MHz}, \mathrm{CDCl}_{3}\right) \delta 7.69(\mathrm{dd}, J=7.9,1.7 \mathrm{~Hz}$, $1 \mathrm{H}), 7.61(\mathrm{t}, J=7.9 \mathrm{~Hz}, 1 \mathrm{H}), 7.40(\mathrm{~d}, J=8.4 \mathrm{~Hz}, 1 \mathrm{H}), 7.31(\mathrm{t}, J=7.6 \mathrm{~Hz}, 1 \mathrm{H}), 6.88(\mathrm{~d}$, $J=6.2 \mathrm{~Hz}, 1 \mathrm{H}), 5.43(\mathrm{dd}, J=6.2,4.6 \mathrm{~Hz}, 1 \mathrm{H}), 5.30-5.24(\mathrm{~m}, 1 \mathrm{H}), 4.79(\mathrm{dd}, J=12.4,2.1$ $\mathrm{Hz}, 1 \mathrm{H}), 4.48(\mathrm{dd}, J=12.4,6.5 \mathrm{~Hz}, 1 \mathrm{H}), 3.84-3.73(\mathrm{~m}, 4 \mathrm{H}), 3.43(\mathrm{dd}, J=11.2,7.5 \mathrm{~Hz}, 1 \mathrm{H})$, $1.56(\mathrm{~s}, 2 \mathrm{H}) .{ }^{13} \mathrm{C}$ NMR $\left(126 \mathrm{MHz}, \mathrm{CDCl}_{3}\right) \delta 168.32,160.49,155.43,155.16,149.66,133.21$, 124.13, 123.18, 117.11, 112.26, 102.36, 97.91, 80.18, 74.54, 67.64, 54.96, 38.07. IR (film): 2360, $1753,1718,1634,1499,1366,1263,1193,1109,910 \mathrm{~cm}^{-1} \cdot[\alpha]_{\mathbf{D}}{ }^{25}=-153.5^{\circ}\left(c=1.0, \mathrm{CH}_{2} \mathrm{Cl}_{2}\right)$. HRMS (ESI) calculated for $\mathrm{C}_{17} \mathrm{H}_{14} \mathrm{O}_{7} \mathrm{Na}^{+}[\mathrm{M}+\mathrm{Na}]^{+}: 353.0632$, found 353.0635. Melting point $=159-162{ }^{\circ} \mathrm{C}$. 


\section{Electrophilic Substrate Preparation}

Preparation of $c i s-332$

\section{( \pm )-6-Hydroxy-3,6-dihydro-2H-pyran-3-one}<smiles>O=C1C=CC(O)CO1</smiles>

To a solution of furfuryl alcohol $(4.0 \mathrm{~g}, 40.8 \mathrm{mmol}, 1.0$ eq.) in dichloromethane (98 $\mathrm{mL})$ at $0{ }^{\circ} \mathrm{C}$ was added $m$ CPBA (11.0 g, $48.9 \mathrm{mmol}, 1.2 \mathrm{eq}$.) in 3 portions over 15 minutes. The reaction mixture was allowed to slowly warm to ambient temperature and stirring was continued overnight. The reaction was then cooled to $-78{ }^{\circ} \mathrm{C}$ and stirred for 15 minutes before removal of insoluble $\mathrm{m}$-chlorobenzoic acid (white precipitate) by filtration. The filtrate was concentrated in vacuo and purified by flash column chromatography (30\% EtOAc/Pet. Ether). The title compound was isolated as a white crystalline solid ( $3.6 \mathrm{~g}, 77 \%$ yield). $\mathrm{Rf}=0.18$ (30\% EtOAc/ Pet. Ether). ${ }^{1} \mathbf{H}-\mathbf{N M R}\left(500 \mathrm{MHz} \mathrm{CDCl}_{3}\right): \delta 6.96(\mathrm{dd}, J=10.4,3.1 \mathrm{~Hz}, 1 \mathrm{H}, \mathrm{HC}=)$, $6.17(\mathrm{~d}, J=10.4 \mathrm{~Hz}, 1 \mathrm{H}, \mathrm{HC}=), 5.65(\mathrm{dd}, J=4.7,3.1 \mathrm{~Hz}, 1 \mathrm{H}, \mathrm{CHOH}), 4.58(\mathrm{~d}, J=16.9 \mathrm{~Hz}$, $1 \mathrm{H}$, one of $\left.\mathrm{CH}_{2} \mathrm{OR}\right), 4.14\left(\mathrm{~d}, J=16.9 \mathrm{~Hz}, 1 \mathrm{H}\right.$, one of $\left.\mathrm{CH}_{2} \mathrm{OR}\right), 3.08(\mathrm{~d}, J=5.2 \mathrm{~Hz}, 1 \mathrm{H}, \mathrm{OH})$. ${ }^{13}$ C-NMR $\left(126 \mathrm{MHz}, \mathrm{CDCl}_{3}\right): \delta 194.4,145.5,128.0,88.2,66.6$. Melting point $=51-53{ }^{\circ} \mathrm{C}$ (lit. $\mathrm{mp}=55-57{ }^{\circ} \mathrm{C}$ ). These data match values previously reported. ${ }^{26}$

\section{( \pm )-6-[(tert-Butyldimethylsilyl)oxy]-3,6-dihydro-2H-pyran-3-one}<smiles>O=C1C=CC(O)OC1</smiles>

To a solution of 6-hydroxy-3,6-dihydro-2H-pyran-3-one $(1.0 \mathrm{~g}, 8.76 \mathrm{mmol}, 1.0 \mathrm{eq}$. $)$ in THF $(60 \mathrm{~mL})$ was added $\mathrm{AgNO}_{3}(1.8 \mathrm{~g}, 10.5 \mathrm{mmol}, 1.2$ eq.) and pyridine $(3.15 \mathrm{~mL}, 38.9 \mathrm{mmol}, 4.44$ eq.). The suspension was stirred for 20 minutes to allow the dissolution of any large lumps of solid. TBSCl (1.72 g, $11.4 \mathrm{mmol}, 1.3$ eq.) was added at room temperature and precipitation of a white solid resulted. The reaction was stirred overnight, after which the reaction mixture was filtered and concentrated in vacuo. The resulting crude product was subjected to flash column chromatography (5\% EtOAc / Pet. Ether) to afford the desired product as a white crystalline solid (1.76 g, 88\% yield). $\mathbf{R}_{\mathbf{f}}=0.34$ (5\% EtOAc / Pet. Ether). ${ }^{\mathbf{1}} \mathbf{H}-\mathbf{N M R}$ (500 $\left.\mathrm{MHz}, \mathrm{CDCl}_{3}\right): \delta 6.86(\mathrm{dd}, J=10.3,3.1 \mathrm{~Hz}, 1 \mathrm{H}, \mathrm{HC}=), 6.08(\mathrm{~d}, J=10.3 \mathrm{~Hz}, 1 \mathrm{H}, \mathrm{HC}=)$, $5.53(\mathrm{~d}, J=3.1 \mathrm{~Hz}, 1 \mathrm{H}, \mathrm{CHOTBS}), 4.50\left(\mathrm{~d}, J=16.8 \mathrm{~Hz}, 1 \mathrm{H}\right.$, one of $\left.\mathrm{CH}_{2} \mathrm{OR}\right), 4.08(\mathrm{~d}, J=$ $16.8 \mathrm{~Hz}, 1 \mathrm{H}$, one of $\left.\mathrm{CH}_{2} \mathrm{OR}\right), 0.92\left(\mathrm{~s}, 9 \mathrm{H}, \mathrm{C}\left(\mathrm{CH}_{3}\right) 3\right), 0.17$ (s, 6H, $\left.\mathrm{Si}\left(\mathrm{CH}_{3}\right) 2\right) .{ }^{\mathbf{1 3}} \mathbf{C}-\mathbf{N M R}(126$ $\left.\mathrm{MHz}, \mathrm{CDCl}_{3}\right): \delta 195.0,147.2,126.5,88.4,66.5,25.6,18.1,-4.5,-5.4$. IR (film): 2931, 2858, $1706,1256,1105,1040,994,876,836,780 \mathrm{~cm}^{-1}$. Melting point $=28-31{ }^{\circ} \mathrm{C}$. These data match values previously reported. ${ }^{2 \mathrm{~b}}$ 
cis-(土)-6-[(tert-Butyldimethylsilyl)oxy]-3,6-dihydro-2H-pyran-3-ol, 333.<smiles>Oc1ccc(O)cc1</smiles>

To a solution of ( \pm )-6-[(tert-butyldimethylsilyl)oxy]-3,6-dihydro-2H-pyran-3-one (2.0 g, $8.76 \mathrm{mmol}, 1.0$ eq.) in $60 \mathrm{~mL}$ of methanol was added $\mathrm{CeCl}_{3} 7 \mathrm{H}_{2} \mathrm{O}(3.92 \mathrm{~g}, 10.5 \mathrm{mmol}, 1.2$ eq.). The reaction mixture was cooled to $-20{ }^{\circ}$ Cand sodium borohydride $(0.40 \mathrm{~g}, 10.5 \mathrm{mmol}$, 1.2 eq.) was added. The reaction was stirred at $-20{ }^{\circ} \mathrm{C}$ for 30 minutes before quenching with $8 \mathrm{~mL}$ of acetone. The reaction mixture was warmed to room temperature, concentrated to approximately $1 / 4$ the solvent volume, diluted with water, extracted with dichloromethane, dried over $\mathrm{MgSO}_{4}$, filtered and concentrated in vacuo. The crude product was purified by flash column chromatography (15\% EtOAc / Pet. Ether). The product was isolated as a clear colorless oil (1.45 g, $72 \%$ yield). $\mathbf{R}_{\mathbf{f}}=0.33$ (20\% EtOAc / Pet. Ether). ${ }^{\mathbf{1}} \mathbf{H}-\mathbf{N M R}$ $\left(500 \mathrm{MHz}, \mathrm{CDCl}_{3}\right): \delta 5.95(\mathrm{dd}, J=10.2,2.7 \mathrm{~Hz}, 1 \mathrm{H}, \mathrm{HC}=), 5.75(\mathrm{dt}, J=10.2,1.9 \mathrm{~Hz}, 1 \mathrm{H}$, $\mathrm{HC}=), 5.25(\mathrm{~m}, 1 \mathrm{H}, \mathrm{CHOTBS}), 4.15(\mathrm{~m}, 1 \mathrm{H}, \mathrm{CHOH}), 3.77\left(\mathrm{~m}, 2 \mathrm{H}, \mathrm{CH}_{2} \mathrm{OR}\right), 1.65(\mathrm{br} \mathrm{m}, 1 \mathrm{H}$, $\mathrm{OH}), 0.91$ (s, 9H, C( $\left.\left.\mathrm{CH}_{3}\right) 3\right), 0.14\left(\mathrm{~s}, 3 \mathrm{H}, \mathrm{SiCH}_{3}\right), 0.13$ (s, 3H, $\left.\mathrm{SiCH}_{3}\right) .{ }^{\mathbf{1 3}} \mathbf{C}-\mathbf{N M R}(126 \mathrm{MHz}$, $\left.\mathrm{CDCl}_{3}\right): \delta 130.9,130.8,89.9,64.6,63.1,25.7,18.1,-3.6,-4.3$. IR (film): 3344, 2955, 2930, $2858,1253,1028,989,866,835,778, \mathrm{~cm}-1$. These data match values previously reported in the literature. ${ }^{2 \mathrm{~b}}$ The minor trans-diastereomer was also isolated as a clear colorless oil $(0.062$ g, $3 \%$ yield, see below for full characterization).

cis-( \pm$)-6$-[( tert-Butyldimethylsilyl)oxy]-3,6-dihydro-2H-pyran-3-yl Methyl Carbonate, 332.<smiles>CO[C@H]1C=C[C@H](O[SnH3])OC1</smiles>

To a solution of cis-( \pm )-6-[(tert-butyldimethylsilyl)oxy]-3,6-dihydro-2H-pyran-3-ol (1.34 g, $5.83 \mathrm{mmol}, 1.0$ eq.) in $70 \mathrm{~mL}$ of freshly distilled $\mathrm{CH}_{2} \mathrm{Cl}_{2}$ was added DMAP (0.89 g, 7.29 mmol, 1.25 eq.), followed by methyl chloroformate $(0.563 \mathrm{~mL}, 0.689 \mathrm{~g}, 7.29 \mathrm{mmol}, 1.25$ eq.). The reaction was stirred overnight at room temperature before concentration in vacuo and purification by flash column chromatography (5\% EtOAc / Pet. Ether). The desired product was isolated as a clear colorless oil $(1.41 \mathrm{~g}, 84 \%$ yield $) . \mathbf{R}_{\mathbf{f}}=0.31$ (5\% EtOAc/Pet. Ether). ${ }^{1} \mathbf{H}-\mathbf{N M R}\left(500 \mathrm{MHz}, \mathrm{CDCl}_{3}\right): \delta 5.92(\mathrm{ddt}, J=10.3,2.2,1.0 \mathrm{~Hz}, 1 \mathrm{H}, \mathrm{HC}=), 5.85$ (ddd, $J$ $=10.3,2.2,1.8 \mathrm{~Hz}, 1 \mathrm{H}, \mathrm{HC}=), 5.28(\mathrm{~m}, 1 \mathrm{H}, \mathrm{CHOTBS}), 5.13(\mathrm{ddq}, J=7.6,5.8,1.9 \mathrm{~Hz}, 1 \mathrm{H}$, $\mathrm{CHOCO}_{2} \mathrm{Me}$ ), $3.90\left(\mathrm{~m}, 2 \mathrm{H}, \mathrm{CH}_{2} \mathrm{OR}\right), 3.80\left(\mathrm{~s}, 3 \mathrm{H}, \mathrm{CO}_{2} \mathrm{CH}_{3}\right), 0.91$ (s, 9H, $\left.\mathrm{C}\left(\mathrm{CH}_{3}\right) 3\right), 0.13$ (s, $\left.3 \mathrm{H}, \mathrm{SiCH}_{3}\right), 0.13\left(\mathrm{~s}, 3 \mathrm{H}, \mathrm{SiCH}_{3}\right) .{ }^{13} \mathrm{C}-\mathrm{NMR}\left(126 \mathrm{MHz}, \mathrm{CDCl}_{3}\right): \delta 155.3,132.2,126.4,89.2$, 68.4, 60.1, 54.9, 25.7, 18.0, -4.4, -5.2. IR (film): 2956, 2931, 2856, 1749, 1258, 1034, 837, 779 $\mathrm{cm}^{-1}$. HRMS (ESI) calculated for $\mathrm{C}_{13} \mathrm{H}_{24} \mathrm{O}_{5} \mathrm{SiNa}^{+}[\mathrm{M}+\mathrm{Na}]^{+}: 311.1291$, found: 311.1293 . 


\title{
Preparation of trans-332
}

\author{
trans-( \pm$)-6$-[(tert-Butyldimethylsilyl)oxy]-3,6-dihydro-2H-pyran-3-yl Benzoate
}<smiles>CCCCCCCCCCOc1ccccc1</smiles>

To a solution of cis- $( \pm)-6$-[(tert-butyldimethylsilyl)oxy]-3,6-dihydro-2H-pyran-3-ol (0.7243 g, $3.14 \mathrm{mmol}$, 1.0 eq.), triphenylphosphine (0.907 g, $3.46 \mathrm{mmol}, 1.1$ eq.) and benzoic acid $\left(0.461 \mathrm{~g}, 3.77 \mathrm{mmol}, 1.2\right.$ eq.) in $18.8 \mathrm{~mL}$ of THF, at $0{ }^{\circ} \mathrm{C}$, was added $0.67 \mathrm{~mL}$ of diisopropylazodicarboxylate (DIAD, $0.699 \mathrm{~g}, 3.46 \mathrm{mmol}, 1.1 \mathrm{eq}$.$) . The reaction was stirred at$ $0{ }^{\circ} \mathrm{C}$ for 30 minutes before warming to room temperature and quenching with water. The reaction mixture was extracted with EtOAc, dried over $\mathrm{MgSO}_{4}$, filtered and concentrated in vacuo. The crude product was purified by flash column chromatography ( $10 \%$ EtOAc / Pet. Ether). The desired product was isolated as a clear yellow oil (1.039 g, 99\% yield). $\mathbf{R f}=0.15$ (20\% EtOAc / Pet. Ether). ${ }^{1} \mathbf{H}-\mathbf{N M R}\left(500 \mathrm{MHz}, \mathrm{CDCl}_{3}\right): \delta 8.07-8.05(\mathrm{~m}, 2 \mathrm{H}, \mathrm{ArH}), 7.56$ $(\mathrm{m}, 1 \mathrm{H}, \mathrm{ArH}), 7.44-7.41(\mathrm{~m}, 2 \mathrm{H}, \mathrm{ArH}), 6.12(\mathrm{dd}, J=10.0,5.1 \mathrm{~Hz}, 1 \mathrm{H}, \mathrm{HC}=), 6.04(\mathrm{dd}, J=$ 10.0, 3.0 Hz, 1H, HC=), 5.39 (d, $J=3.0 \mathrm{~Hz}, 1 \mathrm{H}, \mathrm{CHOTBS}), 5.19(\mathrm{~m}, 1 \mathrm{H}, \mathrm{CHOBz}), 4.31$ (dd, $J=13.0,2.9 \mathrm{~Hz}, 1 \mathrm{H}$, one of $\left.\mathrm{CH}_{2} \mathrm{OR}\right), 3.97\left(\mathrm{dt}, J=13.0,1.3 \mathrm{~Hz}, 1 \mathrm{H}\right.$, one of $\left.\mathrm{CH}_{2} \mathrm{OR}\right), 0.92(\mathrm{~s}$, 9H, $\left.\mathrm{C}\left(\mathrm{CH}_{3}\right) 3\right), 0.15$ (s, $\left.6 \mathrm{H}, \mathrm{Si}\left(\mathrm{CH}_{3}\right) 2\right) .{ }^{13} \mathrm{C}-\mathrm{NMR}\left(126 \mathrm{MHz}, \mathrm{CDCl}_{3}\right): \delta 166.3,133.4,133.1$, 130.0, 129.8, 128.3, 123.3, 88.0, 64.0, 61.4, 25.7, 21.6, 18.1, -4.5, -5.3. IR (film): 2930, 2858, $1776,1716,1265,1108,1022,836,779,710 \mathrm{~cm}-1$. HRMS (ESI) calculated for $\mathrm{C}_{18} \mathrm{H}_{26} \mathrm{O}_{4} \mathrm{SiNa}+$ $[\mathrm{M}+\mathrm{Na}]+:$ 357.1498, found: 357.1493 . These data match values previously reported. ${ }^{2 \mathrm{~b}}$

trans-( \pm )-6-[(tert-Butyldimethylsilyl)oxy]-3,6-dihydro-2 H-pyran-3-ol, 333.<smiles>Oc1ccc(O)cc1</smiles>

To a solution of trans-( \pm -6-[(tert-butyldimethylsilyl)oxy]-3,6-dihydro- $2 H$-pyran-3-yl benzoate $(1.039 \mathrm{~g}, 3.106 \mathrm{mmol}, 1.0$ eq.) in $21 \mathrm{~mL}$ of methanol was added potassium carbonate (0.644 g, $4.66 \mathrm{mmol}, 1.5 \mathrm{eq}$.). The yellow solution quickly became colorless and was stirred for an hour at room temperature. The reaction mixture was concentrated in vacuo to half the solvent volume, diluted with EtOAc and water, extracted with EtOAc, dried over $\mathrm{MgSO}_{4}$, filtered and concentrated in vacuo. The crude product was purified by flash column chromatography (20\% EtOAc/Pet. Ether). The desired product was isolated as a white crystalline solid (0.483 g, 68\% yield). Rf $=0.29$ (25\% EtOAc / Pet. Ether). ${ }^{1} \mathbf{H}-\mathbf{N M R}(500$ $\left.\mathrm{MHz}, \mathrm{CDCl}_{3}\right): \delta 6.06(\mathrm{dd}, J=10.0,5.3 \mathrm{~Hz}, 1 \mathrm{H}, \mathrm{HC}=), 5.85(\mathrm{dd}, J=10.0,3.0 \mathrm{~Hz}, 1 \mathrm{H}, \mathrm{HC}=)$, $5.29(\mathrm{~d}, J=3.0 \mathrm{~Hz}, 1 \mathrm{H}, \mathrm{CHOTBS}), 4.16\left(\mathrm{dd}, J=12.2,2.6 \mathrm{~Hz}, 1 \mathrm{H}\right.$, one of $\left.\mathrm{CH}_{2} \mathrm{OR}\right), 3.83(\mathrm{~m}$, $1 \mathrm{H}, \mathrm{CHOH}), 3.77\left(\mathrm{dt}, J=12.2,1.3 \mathrm{~Hz}, 1 \mathrm{H}\right.$, one of $\left.\mathrm{CH}_{2} \mathrm{OR}\right), 1.85(\mathrm{~d}, J=9.2 \mathrm{~Hz}, 1 \mathrm{H}, \mathrm{OH})$, 0.91 (s, 9H, C( $\left.\left.\mathrm{CH}_{3}\right) 3\right), 0.14$ (s, 6H, $\left.\mathrm{Si}\left(\mathrm{CH}_{3}\right) 2\right) .{ }^{13} \mathrm{C}-\mathrm{NMR}\left(126 \mathrm{MHz}, \mathrm{CDCl}_{3}\right): \delta 131.0,127.5$, 88.2, 64.3, 61.6, 25.7, 18.1, -4.5, -5.3. IR (film): 3379, 2929, 2858, 1251, 1105, 1024, 977, 871, $836,777 \mathrm{~cm}^{-1}$. Melting point $=50-52{ }^{\circ} \mathrm{C}$ (lit. $\left.\mathrm{mp}=39-43{ }^{\circ} \mathrm{C}\right)$. These data match values previously reported. ${ }^{2 \mathrm{~b}}$ 
trans-( \pm )-6-[(tert-Butyldimethylsilyl)oxy]-3,6-dihydro-2 $H$-pyran-3-yl Methyl Carbonate, 332 .<smiles></smiles>

To a solution of trans- $( \pm)$-6-[(tert-butyldimethylsilyl)oxy]-3,6-dihydro- $2 H$-pyran-3-ol (0.472 $\mathrm{g}, 2.05 \mathrm{mmol}, 1.0$ eq. $)$ in dichloromethane $(40 \mathrm{~mL})$ was added DMAP $(0.313 \mathrm{~g}, 2.56 \mathrm{mmol}, 1.25$ eq.), followed by methyl chloroformate $(0.198 \mathrm{~mL}, 0.242 \mathrm{~g}, 2.56 \mathrm{mmol}, 1.25$ eq. $)$. The reaction was stirred overnight at room temperature before concentration in vacuo and purification by flash column chromatography ( $5 \%$ EtOAc / Pet. Ether). The desired product was isolated as a clear colorless oil $(0.321 \mathrm{~g}, 54 \%$ yield, $70 \% \mathrm{brsm}) . \mathbf{R}_{\mathbf{f}}=0.25$ (5\% EtOAc / Pet. Ether). ${ }^{1} \mathbf{H}-\mathbf{N M R}\left(500 \mathrm{MHz}, \mathrm{CDCl}_{3}\right): \delta 6.03(\mathrm{ddd}, J=10.0,4.5,1.0 \mathrm{~Hz}, 1 \mathrm{H}, \mathrm{HC}=), 6.00(\mathrm{dd}, J$ $=10.0,2.5 \mathrm{~Hz}, 1 \mathrm{H}, \mathrm{HC}=), 5.33(\mathrm{~d}, J=2.4 \mathrm{~Hz}, 1 \mathrm{H}, \mathrm{CHOTBS}), 4.80$ (ddd, $J=4.4,2.9$, $1.4 \mathrm{~Hz}, 1 \mathrm{H}, \mathrm{CHOCO}_{2} \mathrm{Me}$ ), $4.20\left(\mathrm{dd}, J=13.1,2.8 \mathrm{~Hz}, 1 \mathrm{H}\right.$, one of $\mathrm{CH}_{2} \mathrm{OR}$ ), 3.90 (dt, $J=$ 13.1, $1.2 \mathrm{~Hz}, 1 \mathrm{H}$, one of $\left.\mathrm{CH}_{2} \mathrm{OR}\right), 3.78\left(\mathrm{~s}, 3 \mathrm{H}, \mathrm{CO}_{2} \mathrm{CH}_{3}\right), 0.90\left(\mathrm{~s}, 9 \mathrm{H}, \mathrm{C}\left(\mathrm{CH}_{3}\right) 3\right), 0.13(\mathrm{~s}, 3 \mathrm{H}$, $\left.\mathrm{SiCH}_{3}\right), 0.13\left(\mathrm{~s}, 3 \mathrm{H}, \mathrm{SiCH}_{3}\right) .{ }^{13} \mathrm{C}-\mathrm{NMR}\left(126 \mathrm{MHz}, \mathrm{CDCl}_{3}\right): \delta 155.4,133.9,122.5,87.9,66.9$, 61.0, 54.8, 25.7, 18.1, -4.5, -5.3. IR (film): 2956, 2930, 2858, 1743, 1256, 1025, 944, 869, 837, $779 \mathrm{~cm}^{-1}$. HRMS (ESI) calculated for $\mathrm{C}_{13} \mathrm{H}_{24} \mathrm{O}_{5} \mathrm{SiNa}^{+}[\mathrm{M}+\mathrm{Na}]^{+}:$311.1291, found: 311.1294.

Preparation and Reaction of Hemiacetal Substrate 6-Hydroxy-3,6-dihydro-2H-pyran-3-yl Methyl Carbonate<smiles>CO[C@H]1C=C[C@@H](OC(C)=O)OC1</smiles>

To a solution of cis- $( \pm)-6$-[(tert-butyldimethylsilyl)oxy]-3,6-dihydro-2 $H$-pyran-3-yl methyl carbonate $(0.096 \mathrm{~g}, 0.33 \mathrm{mmol}, 1.0$ eq. $)$ in acetonitrile $(8 \mathrm{~mL})$, at room temperature, was added HFěpyridine $(0.240 \mathrm{~mL}, 0.266 \mathrm{~g}, 13.3 \mathrm{mmol}, 40 \mathrm{eq}$.$) . The reaction was stirred overnight$ before being quenched with saturated aqueous sodium bicarbonate, extracted with EtOAc, dried over $\mathrm{MgSO}_{4}$, filtered and concentrated in vacuo. The crude product was purified by flash column chromatography (50\% EtOAc / Pet. Ether). The desired product was isolated as a 3:1 mixture of the two possible diastereomers ( $0.018 \mathrm{~g}, 31 \%$ yield). $\mathbf{R}_{\mathbf{f}}=0.23$ ( $40 \%$ EtOAc / Pet. Ether). ${ }^{1} \mathbf{H}-\mathbf{N M R}\left(500 \mathrm{MHz}, \mathrm{CDCl}_{3}\right): \delta$ MAJOR $6.14(\mathrm{~m}, 1 \mathrm{H}, \mathrm{HC}=), 6.10(\mathrm{dd}, J$ $=10.1,2.6 \mathrm{~Hz}, 1 \mathrm{H}, \mathrm{HC}=), 5.42(\mathrm{dd}, J=4.8,2.5 \mathrm{~Hz}, 1 \mathrm{H}, \mathrm{CHOH}), 4.84(\mathrm{ddd}, J=4.6,3.0$, $1.6 \mathrm{~Hz}, 1 \mathrm{H}, \mathrm{CHOCO}_{2} \mathrm{Me}$ ), $4.28\left(\mathrm{dd}, J=13.1,2.9 \mathrm{~Hz}, 1 \mathrm{H}\right.$, one of $\left.\mathrm{CH}_{2} \mathrm{OR}\right), 3.95(\mathrm{~m}, 1 \mathrm{H}$, one of $\mathrm{CH}_{2} \mathrm{OR}$ ), $3.805\left(\mathrm{~s}, 3 \mathrm{H}, \mathrm{OCO}_{2} \mathrm{CH}_{3}\right), 2.77(\mathrm{~d}, J=4.2 \mathrm{~Hz}, 1 \mathrm{H}, \mathrm{OH})$. MINOR $6.04(\mathrm{dd}, J=$ $10.3,2.8 \mathrm{~Hz}, 1 \mathrm{H}, \mathrm{HC}=), 5.96(\mathrm{dt}, J=10.3,1.8 \mathrm{~Hz}, 1 \mathrm{H}, \mathrm{HC}=), 5.33(\mathrm{~m}, 1 \mathrm{H}, \mathrm{CHOH}), 5.10$ (dddt, $J=6.6,5.0,3.1,1.7 \mathrm{~Hz}, 1 \mathrm{H}, \mathrm{CHOCO}_{2} \mathrm{Me}$ ), 3.98 (dd, $J=11.5,6.5 \mathrm{~Hz}$, one of $\mathrm{CH}_{2} \mathrm{OR}$ ), 3.93 (ddd, $J=11.5,5.0,1.0 \mathrm{~Hz}$, one of $\mathrm{CH}_{2} \mathrm{OR}$ ), $3.812\left(\mathrm{~s}, 3 \mathrm{H}, \mathrm{OCO}_{2} \mathrm{CH}_{3}\right), 2.90$ (d, $J=6.4$ $\mathrm{Hz}, 1 \mathrm{H}, \mathrm{OH}) .{ }^{13} \mathrm{C}-\mathrm{NMR}\left(126 \mathrm{MHz}, \mathrm{CDCl}_{3}\right): \delta 155.3,132.0,131.0,127.9,124.5,89.3,87.7$, 68.0, 66.5, 61.1, 61.0, 55.0, 54.9. IR (film): 3413, 2959, 2925, 1740, 1443, 1252, 1061, 975, 937 $\mathrm{cm}^{-1}$. HRMS (ESI) calculated for $\mathrm{C}_{7} \mathrm{H}_{10} \mathrm{O}_{5} \mathrm{Na}^{+}[\mathrm{M}+\mathrm{Na}]^{+}:$197.0426, found: 197.0422. 


\section{Attempted Pd-AA Reaction with Hemiacetal}

The reaction vessel was charged with 6-hydroxy-3,6-dihydro- $2 H$-pyran-3-yl methyl carbonate (0.102 mmol, $0.018 \mathrm{~g}, 1.0$ eq.), 4-hydroxy-6-methyl- $\alpha$-pyrone $(0.102 \mathrm{mmol}, 0.013 \mathrm{~g}$, 1.0 eq.) and $\mathrm{Pd}\left(\mathrm{PPh}_{3}\right)_{4}(0.006 \mathrm{~g}, 0.005 \mathrm{mmol}, 5 \mathrm{~mol} \%)$, evacuated and back-filled with argon. Toluene ( $2 \mathrm{~mL}$, degassed by sparging with argon for approximately 20 minutes) was added to the reaction vessel. The reaction was stirred overnight at ambient temperature before concentration in vacuo and purification via flash column chromatography. The reaction produced a complex mixture of undesired products.

\section{Preparation of trans-Cyclohexene Substrate}

\section{trans-( \pm$)-4-($ Acetoxy $)$ cyclohex-2-en-1-yl Acetate}<smiles>COC(=O)OC1CCC(OC(C)=O)CC1</smiles>

To a solution of palladium acetate $(0.21 \mathrm{~g}, 0.94 \mathrm{mmol}, 15 \mathrm{~mol} \%), \mathrm{LiOAc} \cdot 2 \mathrm{H}_{2} \mathrm{O}(0.702 \mathrm{~g}$, $6.86 \mathrm{mmol}, 1.1 \mathrm{eq}$.$) , and p-benzoquinone (0.19 g, 1.75 \mathrm{mmol}, 28 \mathrm{~mol} \%)$ in $10 \mathrm{~mL}$ of acetic acid was added manganese dioxide $(0.651 \mathrm{~g}, 7.49 \mathrm{mmol}, 1.2 \mathrm{eq}$.$) , followed by a solution of 1,3-$ cyclohexadiene ( $0.50 \mathrm{~g}, 6.24 \mathrm{mmol}, 1 \mathrm{eq}$.) in $20 \mathrm{~mL}$ of pentane. The biphasic reaction mixture was stirred at room temperature overnight. The pentane layer was separated from the acetic acid layer. The acetic acid layer was subsequently diluted with $10 \mathrm{~mL}$ of brine and extracted twice with pentane, then twice with 1:1 pentane/ethyl acetate. The combined extracts were washed three times with brine, three times with water and three times with $2 \mathrm{M}$ aqueous $\mathrm{NaOH}$. The resulting solution was dried with magnesium sulfate, filtered and concentrated in vacuo. The desired product was isolated as a yellow crystalline solid $(1.014 \mathrm{~g}, 82 \%$ yield, 86:14 ratio of trans:cis diastereomers as determined by $1 \mathrm{H}-\mathrm{NMR}$ spectroscopy). ${ }^{\mathbf{1}} \mathbf{H}-\mathbf{N M R}(500 \mathrm{MHz}$, $\left.\mathrm{CDCl}_{3}\right): \delta$ MAJOR $5.89(\mathrm{~d}, J=1.4 \mathrm{~Hz}, 2 \mathrm{H}, 2 \times \mathrm{HC}=), 5.31(\mathrm{dtd}, J=5.5,2.8,1.4 \mathrm{~Hz}, 2 \mathrm{H}, 2$ x CHOAc), 2.14-2.10 (m, 2H, 2 x one of $\mathrm{CH} 2), 2.05$ (s, 6H, $\left.2 \mathrm{x} \mathrm{CH}_{3}\right), 1.72-1.67$ (m, $2 \mathrm{H}, 2 \mathrm{x}$ one of $\mathrm{CH}_{2}$ ). MINOR $5.91(\mathrm{~d}, J=1.5 \mathrm{~Hz}, 2 \mathrm{H}, 2 \times \mathrm{HC}=), 5.22(\mathrm{~m}, 2 \mathrm{H}, 2 \times \mathrm{CHOAc}), 2.07(\mathrm{~s}, 6 \mathrm{H}$, $\left.2 \times \mathrm{CH}_{3}\right), 1.92-1.84$ (complex m, $\left.4 \mathrm{H}, 2 \times \mathrm{CH}_{2}\right) \cdot{ }^{13} \mathrm{C}-\mathrm{NMR}\left(126 \mathrm{MHz}, \mathrm{CDCl}_{3}\right): \delta$ MAJOR $170.6,130.3,67.6,25.7,21.2$. These data match values previously reported. ${ }^{27}$

\section{trans-( \pm$)-4-[($ Methoxycarbonyl)oxy $]$ cyclohex-2-en-1-yl Methyl Carbonate}<smiles>COC(OC)OC1CCC(OC(C)=O)CC1</smiles>

The 86:14 mixture of trans- and cis- $( \pm)-4$-(acetoxy)cyclohex-2-en-1-yl acetate $(0.500 \mathrm{~g}$, $2.52 \mathrm{mmol}, 1$ eq.) was dissolved in $12 \mathrm{~mL}$ of methanol and $3 \mathrm{~mL}$ of $2 \mathrm{M}$ aqueous $\mathrm{NaOH}$ was added. The reaction was stirred at reflux for 15 minutes and then concentrated in vacuo to approximately $5 \mathrm{~mL}$. The aqueous layer was saturated with $\mathrm{NaOH}$ and extracted twice with ethyl acetate. The combined organics were dried over magnesium sulfate, filtered and concentrated in vacuo to give a white crystalline solid, presumed to consist mainly of trans- 
( \pm )-cyclohex-2-ene-1,4-diol (0.267 g).

To a solution of this material (0.178 g, $1.56 \mathrm{mmol}, 1$ eq.) and DMAP (0.762 g, $6.24 \mathrm{mmol}$, 4 eq.) in $14 \mathrm{~mL}$ of dichloromethane was added methyl chloroformate $(0.482 \mathrm{~mL}, 0.59 \mathrm{~g}, 6.24$ mmol, 4 eq.) at $0 \mathrm{oC}$. The reaction mixture was slowly warmed to room temperature and stirred overnight. The reaction was diluted with water and extracted twice with dichloromethane. The combined organic extracts were dried over magnesium sulfate, filtered and concentrated in vacuo. The crude reaction mixture was purified using flash column chromatography $(15 \%$ EtOAc / Pet. Ether). The desired product trans- 8 was isolated as a white solid $(0.221 \mathrm{~g}, 58 \%$ yield over 2 steps), which contained ca. $10 \%$ of the cis-isomer. $\mathrm{Rf}=0.29$ (15\% EtOAc / Pet. Ether). ${ }^{1} \mathbf{H}-\mathbf{N M R}\left(500 \mathrm{MHz}, \mathrm{CDCl}_{3}\right): \delta 5.99(\mathrm{~d}, J=1.6 \mathrm{~Hz}, 2 \mathrm{H}, 2 \times \mathrm{HC}=), 5.18$ (dtd, $J$ $\left.=5.3,2.7,1.3 \mathrm{~Hz}, 2 \mathrm{H}, 2 \times \mathrm{CHOCO}_{2} \mathrm{Me}\right), 3.79\left(\mathrm{~s}, 6 \mathrm{H}, 2 \times \mathrm{CO}_{2} \mathrm{CH}_{3}\right), 2.21-2.17(\mathrm{~m}, 2 \mathrm{H}, 2 \mathrm{x}$ one of $\mathrm{CH}_{2}$ ), 1.79 (ddt, $J=8.6,4.3,2.2 \mathrm{~Hz}, 2 \mathrm{H}, 2 \mathrm{x}$ one of $\mathrm{CH}_{2}$ ). ${ }^{13} \mathbf{C}-\mathbf{N M R}(126 \mathrm{MHz}$, $\left.\mathrm{CDCl}_{3}\right): \delta 155.3,130.0,71.0,54.8,25.4$. IR (film): 2959, 1738, 1446, 1402, 1318, 1268, 1010, 936, 901, 784, $761 \mathrm{~cm}^{-1}$. HRMS (ESI) calculated for $\mathrm{C}_{10} \mathrm{H}_{14} \mathrm{O}_{6} \mathrm{Na}+[\mathrm{M}+\mathrm{Na}]^{+}: 253.0688$, found 253.0686. Melting point $=92-95{ }^{\circ} \mathrm{C}$.

\section{Nucleophilic Substrate Preparation}

\section{Preparation of 4-hydroxy-7-methoxy-2H-chromen-2-one}
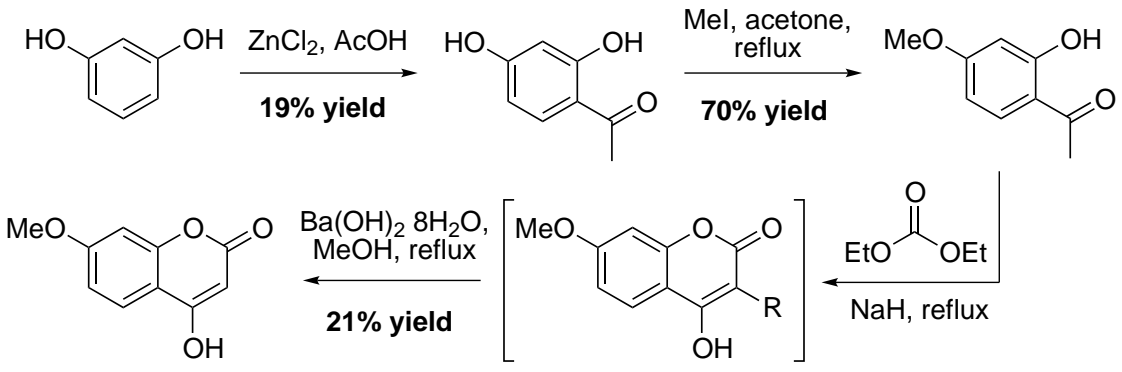

$\mathrm{R}=\mathrm{H}, \mathrm{R}=\mathrm{CO}_{2} \mathrm{Et}$

\section{1-(2,4-Dihydroxyphenyl)ethanone}<smiles>CC(=O)c1ccc(O)cc1O</smiles>

To a solution of resorcinol $(2.2 \mathrm{~g}, 20 \mathrm{mmol}, 1.0$ eq. $)$ in glacial acetic acid $(6.3 \mathrm{~mL})$ was added anhydrous zinc chloride $(2.8 \mathrm{~g}, 20 \mathrm{mmol}, 1.0$ eq. $)$. The reaction was stirred at reflux for 5 hours before quenching with ice-water $(150 \mathrm{~mL})$. The solvent was gradually evaporated under a stream of air, producing a red precipitate which was subsequently dried in a vacuum desiccator over $\mathrm{P}_{2} \mathrm{O}_{5}$. The title compound was obtained as a red solid $(0.577 \mathrm{~g}, 19 \%$ yield - unoptimized). ${ }^{1} \mathbf{H}-\mathbf{N M R}\left(500 \mathrm{MHz}, \mathrm{CDCl}_{3}\right): \delta 12.69(\mathrm{~s}, 1 \mathrm{H}), 7.65(\mathrm{~d}, J=8.6 \mathrm{~Hz}, 1 \mathrm{H})$, 6.41-6.38 (m, 2H), 5.36 (br s, 1H), $2.57(\mathrm{~s}, 3 \mathrm{H}) .{ }^{13} \mathbf{C}-\mathrm{NMR}\left(126 \mathrm{MHz}, \mathrm{CDCl}_{3}\right): \delta 202.6,165.1$, $162.4,133.0,114.4,107.6,103.5,26.3$. These data match values previously reported. ${ }^{28}$

\section{1-(2-Hydroxy-4-methoxyphenyl)ethanone}

To a solution of 1-(2,4-dihydroxyphenyl)ethanone ( $0.577 \mathrm{~g}, 3.79 \mathrm{mmol}, 1.0$ eq.) and $\mathrm{K}_{2} \mathrm{CO}_{3}$ $(0.524 \mathrm{~g}, 3.79 \mathrm{mmol})$ in acetone $(15 \mathrm{~mL})$ was added iodomethane $(0.236 \mathrm{~mL}, 3.79 \mathrm{mmol})$. 


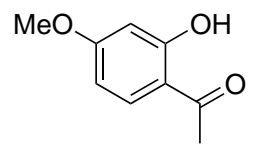

The reaction was stirred at reflux overnight before quenching with water. The acetone was subsequently removed in vacuo and resultant reaction mixture was extracted twice with EtOAc. The combined organic layers were dried over $\mathrm{MgSO}_{4}$, filtered and concentrated in vacuo. The crude product was purified by flash column chromatography (10\% EtOAc / pet. ether) to give the title compound as a yellow oil (0.443 g, $70 \%$ yield). $\mathbf{R}_{\mathbf{f}}=0.5$ (20\% EtOAc / pet. ether). ${ }^{1} \mathbf{H}-\mathbf{N M R}\left(500 \mathrm{MHz}, \mathrm{CDCl}_{3}\right): \delta 12.76(\mathrm{~s}, 1 \mathrm{H}), 7.64(\mathrm{~d}, J=8.8 \mathrm{~Hz}, 1 \mathrm{H}), 6.46-6.43(\mathrm{~m}, 2 \mathrm{H})$, $3.85(\mathrm{~s}, 3 \mathrm{H}), 2.57(\mathrm{~s}, 3 \mathrm{H}) .{ }^{13} \mathrm{C}-\mathrm{NMR}\left(126 \mathrm{MHz}, \mathrm{CDCl}_{3}\right): \delta 202.6,166.1,165.3,132.3,113.9$, $107.7,100.8,55.6,26.2$. These data match values previously reported. ${ }^{29}$

\section{4-Hydroxy-7-methoxy-2H-chromen-2-one}<smiles>COc1ccc2c(O)cc(=O)oc2c1</smiles>

To a suspension of $\mathrm{NaH}(0.534 \mathrm{~g}-60 \%$ dispersion in mineral oil, $13.4 \mathrm{mmol}, 5$ eq.) in diethyl carbonate $(2.2 \mathrm{~mL})$ was added a solution of 1-(2-hydroxy-4-methoxyphenyl)ethanone $(0.443 \mathrm{~g}$, $2.67 \mathrm{mmol})$ in diethyl carbonate $(1.5 \mathrm{~mL})$. The reaction was stirred at reflux overnight before quenching with water. The reaction mixture was $\mathrm{Et}_{2} \mathrm{O}$ three times to remove excess diethyl carbonate. The aqueous layer was then acidified using $1 \mathrm{~N} \mathrm{HCl}$. The resulting cream precipitate was removed via filtration and washed with water, then pet. ether and dried in a vacuum desiccator over $\mathrm{P}_{2} \mathrm{O}_{5}$. The isolated cream solid $(0.279 \mathrm{~g})$ was contaminated with significant amounts of the undesired ethyl ester and was therefore suspended in methanol and stirred at reflux for 2 hours in the presence of $\mathrm{Ba}(\mathrm{OH})_{2} \cdot 8 \mathrm{H}_{2} \mathrm{O}$. After cooling to room temperature the reaction mixture was washed with $\mathrm{Et}_{2} \mathrm{O}$, then acidified with $1 \mathrm{~N} \mathrm{HCl}$ to produce a white precipitate. The precipitate was filtered off and washed with water and dried in a vacuum desiccator over $\mathrm{P}_{2} \mathrm{O}_{5}$. The title compound was obtained as a white solid $(0.041 \mathrm{~g}, 21 \%$ yield). ${ }^{1}$ H-NMR $\left(500 \mathrm{MHz}\right.$, DMSO-d $\left._{6}\right): \delta 12.35(\mathrm{~s}, 1 \mathrm{H}), 7.71(\mathrm{~d}, J=8.7 \mathrm{~Hz}, 1 \mathrm{H}), 6.95-6.91(\mathrm{~m}$, $2 \mathrm{H}), 5.44(\mathrm{~s}, 1 \mathrm{H}), 3.84(\mathrm{~s}, 3 \mathrm{H})$. Melting point $=260-263{ }^{\circ} \mathrm{C}\left(\right.$ lit. $\left.8 \mathrm{mp}=258-260{ }^{\circ} \mathrm{C}\right)$. These data match values previously reported. ${ }^{30}$

\section{Preparation of $\beta$-Dicarbonyl Compounds from Methyl Acetoacetate}

General Procedure for the Aldol Reaction of an Aldehyde/Ketone with Methyl Acetoacetate and Subsequent Lactonization. To a suspension of $\mathrm{NaH}$ (1.96 eq.) in THF $(1 \mathrm{M})$ at $0{ }^{\circ} \mathrm{C}$, was added methyl acetoacetate (1.96 eq.). After visible gas evolution had ceased, the reaction was stirred for 15 minutes at $0{ }^{\circ} \mathrm{C}$. $n \mathrm{BuLi}$ (1.96 eq., $2 \mathrm{M}$ in cyclohexane) was then added and the reaction mixture stirred for another 15 minutes before the addition of the ketone or aldehyde ( 1 eq.). The reaction was stirred for 60 minutes at $0{ }^{\circ} \mathrm{C}$ before quenching with saturated aqueous $\mathrm{NH}_{4} \mathrm{Cl}$. The reaction mixture was extracted twice with EtOAc and concentrated in vacuo. The crude product was redissolved in THF ( $\tilde{1} 5 \mathrm{~mL}$ ) and a $0.1 \mathrm{M}$ solution of $\mathrm{NaOH}(\tilde{5} 0 \mathrm{~mL})$ was added. The reaction was stirred for 3 hours at room temperature. The reaction mixture was washed with EtOAc, acidified to $p \mathrm{H} 3$ with $1 \mathrm{M} \mathrm{HCl}$ and extracted twice with $\mathrm{CH}_{2} \mathrm{Cl}_{2}$. The combined organic layers were dried over $\mathrm{MgSO}_{4}$, filtered 
and concentrated in vacuo. The crude product was purified by flash column chromatography.

1-Oxaspiro[5.5] undecane-2,4-dione<smiles>O=C1CC(=O)OC2(CCCCC2)C1</smiles>

The reaction was performed with $2.42 \mathrm{~mL}(22.4 \mathrm{mmol})$ of methyl acetoacetate, $0.9 \mathrm{~g}$ $(22.4 \mathrm{mmol})$ of $\mathrm{NaH}(60 \%$ dispersion in mineral oil $), 11.2 \mathrm{~mL}(22.4 \mathrm{mmol})$ of $n \mathrm{BuLi}(2 \mathrm{M}$ in cyclohexane) and $1.18 \mathrm{~mL}(11.4 \mathrm{mmol})$ of cyclohexanone in a total of $25 \mathrm{~mL}$ of THF. The crude product was purified by flash column chromatography (40\% EtOAc / pet. ether) to give the title compound as a cream solid $(0.56 \mathrm{~g}, 27 \%$ yield - unoptimized). $\mathrm{Rf}=0.22(40 \%$ EtOAc / pet. ether). ${ }^{1} \mathbf{H}-\mathrm{NMR}\left(500 \mathrm{MHz}, \mathrm{CDCl}_{3}\right): \delta$ MAJOR (DIKETO-) 3.37 (d, $J=$ $\left.3.1 \mathrm{~Hz}, 2 \mathrm{H}, \mathrm{CH}_{2} \mathrm{CO}_{2} \mathrm{R}\right), 2.63\left(\mathrm{~d}, J=2.6 \mathrm{~Hz}, 2 \mathrm{H}, \mathrm{CH}_{2} \mathrm{C}=\mathrm{O}\right), 1.82-1.27$ (m, 10H, cyclohexyl$\left.\mathrm{C}_{6} \mathrm{H}_{10}\right)$. MINOR (ENOL-) $5.17(\mathrm{~s}, 1 \mathrm{H}, \mathrm{HC}=), 2.43\left(\mathrm{~s}, 2 \mathrm{H}, \mathrm{CH}_{2} \mathrm{C}=\right), 1.94-1.91(\mathrm{~m}, 2 \mathrm{H}$, one cyclohexyl- $\left.\mathrm{CH}_{2}\right), 1.82-1.27\left(\mathrm{~m}, 6 \mathrm{H}, 3 \mathrm{x}\right.$ cyclohexyl- $\left.\mathrm{CH}_{2}\right), 0.86(\mathrm{dt}, J=6.5,3.4 \mathrm{~Hz}, 2 \mathrm{H}$, one cyclohexyl- $\left.\mathrm{CH}_{2}\right) .{ }^{13} \mathrm{C}-\mathrm{NMR}\left(126 \mathrm{MHz}, \mathrm{CDCl}_{3}\right): \delta$ MAJOR 200.9, 167.6, 80.5, 49.3, 44.4, $36.8,24.6,21.5$. Melting point $=117-119{ }^{\circ} \mathrm{C}$ (lit. $\mathrm{mp}=118-120{ }^{\circ} \mathrm{C}$ ). These data match values previously reported. ${ }^{31}$

\section{( \pm )-6-(4-Bromophenyl)oxane-2,4-dione}<smiles>O=C1CC(=O)OC(c2ccc(Br)cc2)C1</smiles>

The reaction was performed with $1.71 \mathrm{~g}(15.9 \mathrm{mmol})$ of methyl acetoacetate, $0.636 \mathrm{~g}$ (15.9 mmol) of $\mathrm{NaH}(60 \%$ dispersion in mineral oil), $7.95 \mathrm{~mL}(22.4 \mathrm{mmol})$ of $n \mathrm{BuLi}(2 \mathrm{M}$ in cyclohexane) and $1.5 \mathrm{~g}(8.1 \mathrm{mmol})$ of 4-bromobenzaldehyde in a total of $20 \mathrm{~mL}$ of THF. The crude product was purified by flash column chromatography ( $40 \%$ EtOAc / pet. ether) to give the title compound as a cream solid $\left(0.37 \mathrm{~g}, 17 \%\right.$ yield - unoptimized). $\mathbf{R}_{\mathbf{f}}=0.22(40 \%$ EtOAc / pet. ether). ${ }^{1} \mathbf{H}-\mathbf{N M R}\left(500 \mathrm{MHz} \mathrm{CDCl}_{3}\right): \delta 7.59-7.58(\mathrm{~m}, 2 \mathrm{H}, \mathrm{ArH}), 7.30-7.29$ $(\mathrm{m}, 2 \mathrm{H}, \mathrm{ArH}), 5.67(\mathrm{dd}, J=10.8,3.1 \mathrm{~Hz}, 1 \mathrm{H}, \mathrm{ArCHOR}), 3.69(\mathrm{dd}, J=19.1,1.2 \mathrm{~Hz}, 1 \mathrm{H}$, one of $\left.\mathrm{RO}_{2} \mathrm{CCH}_{2}\right), 3.51\left(\mathrm{dd}, J=19.1,0.7 \mathrm{~Hz}, 1 \mathrm{H}\right.$, one of $\left.\mathrm{RO}_{2} \mathrm{CCH}_{2}\right), 2.95$ (ddd, $J=18.3$, $3.2,1.3 \mathrm{~Hz}, 1 \mathrm{H}$, one of $\left.\mathrm{CH}_{2} \mathrm{C}=\mathrm{O}\right), 2.83\left(\mathrm{ddd}, J=18.3,10.8,0.8 \mathrm{~Hz}, 1 \mathrm{H}\right.$, one of $\left.\mathrm{CH}_{2} \mathrm{C}=\mathrm{O}\right)$. ${ }^{13}$ C-NMR $\left(126 \mathrm{MHz}, \mathrm{CDCl}_{3}\right): \delta 198.8,166.5,135.5,132.3,127.5,123.4,75.8,47.0,45.1$. IR (KBr): 2923, 1746, 1718, 1635, 1490, 1385, 1289, 1062, $1010 \mathrm{~cm}^{-1}$. HRMS (ESI) calculated for $\mathrm{C}_{11} \mathrm{H}_{9} \mathrm{O}_{3} \mathrm{BrNa}^{+}[\mathrm{M}+\mathrm{Na}]^{+}:$290.9633, found 290.9642. Melting point $=127-130{ }^{\circ} \mathrm{C}$. These data match values previously reported. ${ }^{32}$ 


\section{Derivatization of Pd-AA Cascade Products}

Preparation of $\left(5 \mathrm{a} S^{*}, 6 R^{*}, 9 \mathrm{a} S^{*}\right)$-6-Bromo-7-hydroxy-3-methyl-6,7,9,9atetrahydrofuro[3,2-c:4,5-c']dipyran-1(5aH)-one, 357.<smiles>Cc1cc2c(c(=O)o1)C1COC(O)C(Br)C1O2</smiles>

To a solution of cis-( \pm -3-methyl-9,9a-dihydrofuro[3,2-c:4,5-c']dipyran-1(5a $H)$-one $(0.025$ g, $0.121 \mathrm{mmol}, 1.0$ equiv) in $\mathrm{CH}_{3} \mathrm{CN} / \mathrm{H}_{2} \mathrm{O}(1: 1,1.6 \mathrm{~mL})$ was added NBS $(0.026 \mathrm{~g}, 0.145 \mathrm{mmol}$, 1.2 equiv) at room temperature. The reaction was stirred for 3 hours before being concentrated in vacuo and purified by flash column chromatography (50\% EtOAc/Pet. Ether). The title compound was isolated as a white amorphous foam $(0.0159 \mathrm{~g}, 43 \%$ yield, $1.3: 1 \mathrm{dr}, 19 \%$ yield of the epi-Br diastereomer $\left(\mathrm{R}_{\mathrm{f}}=0.21(60 \%\right.$ EtOAc/Pet. Ether $)$ ) was also isolated from the column). $\mathbf{R}_{\mathbf{f}}=0.28$ (60\% EtOAc/Pet. Ether). ${ }^{\mathbf{1}} \mathbf{H}$ NMR $\left(500 \mathrm{MHz}, \mathrm{CDCl}_{3}\right)$ MAJOR: $\delta$ $6.02(\mathrm{~s}, 1 \mathrm{H}), 5.33(\mathrm{dd}, J=8.7,6.9 \mathrm{~Hz}, 1 \mathrm{H}), 5.20(\mathrm{~d}, J=2.7 \mathrm{~Hz}, 1 \mathrm{H}), 4.37(\mathrm{dd}, J=12.1$, $5.2 \mathrm{~Hz}, 1 \mathrm{H}), 4.18(\mathrm{dd}, J=6.9,2.9 \mathrm{~Hz}, 1 \mathrm{H}), 4.17(\mathrm{dd}, J=12.1,4.4 \mathrm{~Hz}, 1 \mathrm{H}), 3.66(\mathrm{dt}, J=$ 8.9, $4.5 \mathrm{~Hz}, 1 \mathrm{H}), 2.29(\mathrm{~s}, 3 \mathrm{H})$. MINOR: $\delta 6.03(\mathrm{~s}, 1 \mathrm{H}), 5.20(\mathrm{dd}, J=9.4,8.5 \mathrm{~Hz}, 1 \mathrm{H}), 5.16$ $(\mathrm{d}, J=6.9 \mathrm{~Hz}, 1 \mathrm{H}), 4.33(\mathrm{dd}, J=12.3,6.4 \mathrm{~Hz}, 1 \mathrm{H}), 4.09(\mathrm{dd}, J=12.4,5.8 \mathrm{~Hz}, 1 \mathrm{H}), 3.94$ $(\mathrm{dd}, J=8.3,7.0 \mathrm{~Hz}, 1 \mathrm{H}), 3.73(\mathrm{dt}, J=9.6,6.1 \mathrm{~Hz}, 1 \mathrm{H}), 2.29(\mathrm{~s}, 3 \mathrm{H}) \cdot{ }^{13} \mathrm{C}$ NMR $(126 \mathrm{MHz}$, $\left.\mathrm{CDCl}_{3}\right)$ MAJOR: $\delta 171.38,166.56,161.58,100.62,95.96,91.20,86.33,60.41,49.16,38.26$, 20.58. MINOR: $\delta$ 171.41, 166.65, 161.49, 99.75, 95.90, 95.36, 87.62, 58.48, 50.20, 39.86, 20.57 . IR (film): 3442, 2988, 1699, 1634, 1578, 1455, 1384, $1078 \mathrm{~cm}^{-1}$. HRMS (ESI) calculated for $\mathrm{C}_{11} \mathrm{H}_{12} \mathrm{O}_{5} \mathrm{Br}^{+}[\mathrm{M}+\mathrm{H}]^{+}:$302.9868, found 302.9861 .

epi-Br Diastereomer $-{ }^{1} \mathbf{H}$ NMR $\left(500 \mathrm{MHz}, \mathrm{CDCl}_{3}\right) \delta 6.03(\mathrm{~s}, 1 \mathrm{H}), 5.34(\mathrm{~d}, J=7.2$ $\mathrm{Hz}, 1 \mathrm{H}), 5.25(\mathrm{dd}, J=9.5,3.0 \mathrm{~Hz}, 1 \mathrm{H}), 4.20(\mathrm{dd}, J=7.1,3.0 \mathrm{~Hz}, 1 \mathrm{H}), 3.99(\mathrm{dd}, J=12.4$, $3.6 \mathrm{~Hz}, 1 \mathrm{H}), 3.80(\mathrm{dd}, J=12.4,1.5 \mathrm{~Hz}, 1 \mathrm{H}), 3.58(\mathrm{dd}, J=9.4,2.4 \mathrm{~Hz}, 1 \mathrm{H}), 2.29(\mathrm{~s}, 3 \mathrm{H})$.

(3aS,8bS)-3-(Hydroxymethyl)-6-methyl-3,3a-dihydro-1H-furo[3', 4':4,5] furo[3,2c]pyran-8(8bH)-one, 359 .

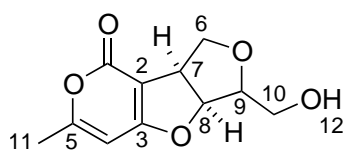

To a solution of ( $\left.5 \mathrm{a} S^{*}, 6 R^{*}, 9 \mathrm{a} S^{*}\right)$-6-bromo-7-hydroxy-3-methyl-6,7,9,9a-tetrahydrofuro[3,2c:4,5-c']dipyran-1 $(5 \mathrm{aH})$-one $(0.027 \mathrm{~g}, 0.089 \mathrm{mmol}, 1.0$ equiv) in $\mathrm{MeOH}(1 \mathrm{~mL})$ was added $\mathrm{NaBH}_{4}\left(0.005 \mathrm{~g}, 1.2\right.$ equiv) at $0{ }^{\circ} \mathrm{C}$. The reaction was stirred at $0{ }^{\circ} \mathrm{C}$ for 30 minutes before quenching with acetone and warming to room temperature. The solvent was removed in vacuo and the crude product purified by preparative thin layer chromatography $\left(5 \% \mathrm{MeOH} / \mathrm{CH}_{2} \mathrm{Cl}_{2}\right)$. The title compound was isolated as a clear film $\left(0.020 \mathrm{~g}\right.$, quantitative yield). $\mathbf{R}_{\mathbf{f}}=0.57$ (10\% $\mathrm{MeOH} / \mathrm{CH}_{2} \mathrm{Cl}_{2}$ ). IR (film): 3412, 2950, 2883, 1700, 1637, 1581, 1454, 1423, 1242, 1042, 982 $\mathrm{cm}^{-1}$. HRMS (ESI) calculated for $\mathrm{C}_{11} \mathrm{H}_{12} \mathrm{O}_{5} \mathrm{Na}^{+}[\mathrm{M}+\mathrm{Na}]^{+}: 247.0582$, found 247.0583. 


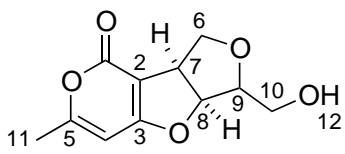

\begin{tabular}{|c|c|c|c|c|}
\hline \multicolumn{5}{|c|}{${ }^{1} \mathrm{H} 500 \mathrm{MHz},{ }^{13} \mathrm{C} 125 \mathrm{MHz}, \mathrm{CDCl}_{3}$} \\
\hline Position & $\delta_{\mathrm{C}}$, mult & $\delta_{\mathrm{H}}(J, \mathrm{~Hz})$ & COSY & $\mathrm{HMBC}$ \\
\hline 1 & $161.9, \mathrm{C}$ & - & - & - \\
\hline 2 & $101.5, \mathrm{C}$ & - & - & $5.95,4.22,4.01,2.27$ \\
\hline 3 & $171.2, \mathrm{C}$ & - & - & 5.95 \\
\hline 4 & $95.6, \mathrm{CH}$ & $5.95, \mathrm{~s}$ & 2.27 & 2.27 \\
\hline 5 & $166.0, \mathrm{C}$ & - & - & $5.95,2.27$ \\
\hline 6 & $72.4, \mathrm{CH}_{2}$ & $4.22 / 4.01, \mathrm{~m}$ & $4.01,4.22$ & 4.19 (weak) \\
\hline 7 & $44.9, \mathrm{CH}$ & $4.00, \mathrm{~m}$ & 5.32 & 4.01(weak) \\
\hline 8 & $91.1, \mathrm{CH}$ & 5.32, dd $(8.7,2.5)$ & 4.0 & 3.78 \\
\hline 9 & $85.2, \mathrm{CH}$ & $4.19, \mathrm{~m}$ & 3.78 & $4.22,4.01$ (weak), 4.00 \\
\hline 10 & $61.4, \mathrm{CH}_{2}$ & $3.78, \mathrm{~m}$ & 4.19 & - \\
\hline 11 & $20.5, \mathrm{CH}_{3}$ & $2.27, \mathrm{~s}$ & 5.95 & 5.95 \\
\hline
\end{tabular}

\begin{tabular}{ccc}
\hline \multicolumn{3}{c}{${ }^{\mathbf{1}} \mathbf{H} \mathbf{5 0 0} \mathbf{~ M H z}, \mathbf{C}_{\mathbf{6}} \mathbf{D}_{\mathbf{6}}$} \\
\hline Position & $\delta_{\mathrm{H}}(J, \mathrm{~Hz})$ & COSY \\
\hline 1 & - & - \\
2 & - & - \\
3 & - & 1.44 \\
4 & $5.16, \mathrm{~s}$ & - \\
5 & - & $3.78,3.44$ \\
6 & 3.99, dd $(9.2,3.0)$ & $3.99,3.44$ \\
$6 \mathrm{~b}$ & 3.78, dd $(9.2,6.6)$ & $4.70,3.99,3.78$ \\
7 & 3.44, ddd $(8.9,6.8,3.1)$ & $3.44,3.91$ \\
8 & 4.70, dd $(8.8,2.1)$ & $4.70,3.12$ \\
9 & 3.91, td $(5.6,2.1)$ & $3.91,1.21$ \\
10 & 3.12, app. s & 5.17 \\
11 & $1.44, \mathrm{~s}$ & 3.12 \\
\hline 12 & $1.21, \mathrm{~s}$ &
\end{tabular}


((3aS,8b $S)$-6-Methyl-8-oxo-3,3a,8,8b-tetrahydro-1H-furo[3',4':4,5]furo[3,2-c]pyran-3-yl)methyl Acetate, 360.

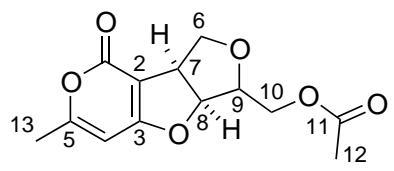

To a solution of (3a $S, 8 \mathrm{~b} S$ )-3-(hydroxymethyl)-6-methyl-3,3a-dihydro-1H-furo[3',4':4,5] furo[3,2c]- pyran-8(8bH)-one (0.0094 g, $0.0309 \mathrm{mmol}, 1.0$ equiv) in pyridine $(0.5 \mathrm{~mL})$ was added acetic anhydride $(0.5 \mathrm{~mL})$ at room temperature. The reaction was stirred overnight before cyclic loading onto HP20ss beads. The resin was washed five times with $\mathrm{H}_{2} \mathrm{O}$ and the product was subsequently eluted with acetone. After removal of the solvent in vacuo, the title compound was isolated as an off-white solid (0.0085 g, 76\% yield). HRMS (ESI) calculated for $\mathrm{C}_{13} \mathrm{H}_{15} \mathrm{O}_{6}{ }^{+}[\mathrm{M}+\mathrm{H}]^{+}: 276.0869$, found 267.0872. Melting point $=120-121{ }^{\circ} \mathrm{C}$.

\begin{tabular}{|c|c|c|c|c|}
\hline \multicolumn{5}{|c|}{${ }^{1} \mathrm{H} 500 \mathrm{MHz},{ }^{13} \mathrm{C} 125 \mathrm{MHz}, \mathrm{CDCl}_{3}$} \\
\hline Position & $\delta_{\mathrm{C}}$, mult & $\delta_{\mathrm{H}}(J, \mathrm{~Hz})$ & COSY & HMBC \\
\hline 1 & $161.9, \mathrm{C}$ & - & - & - \\
\hline 2 & 101.6, C & - & - & $5.95,4.16,4.05,4.00$ \\
\hline 3 & $171.2, \mathrm{C}$ & - & - & 5.95 \\
\hline 4 & $95.6, \mathrm{CH}$ & $5.95, \mathrm{~d}(1.2)$ & 2.28 & 2.28 \\
\hline 5 & $166.2, \mathrm{C}$ & - & - & $5.95,2.28$ \\
\hline 6 & 72.7, $\mathrm{CH}_{2}$ & $4.16, \mathrm{~m}$ & $4.05,4.00$ & $5.28,4.34$ \\
\hline $6 \mathrm{~b}$ & - & $4.05, \mathrm{dd},(9.3,3.1)$ & $4.16,4.00$ & $5.28,4.34$ \\
\hline 7 & $44.9, \mathrm{CH}$ & 4.00 , ddd $(9.5,6.7,3.1)$ & $5.28,4.16,4.05$ & - \\
\hline 8 & 91.7, CH & $5.28, \mathrm{dd}(8.7,2.1)$ & $4.05,4.00$ & $4.34,4.26,4.22$ \\
\hline 9 & $82.7, \mathrm{CH}$ & $4.34, \mathrm{~m}$ & $4.25,4.19$ & $5.28,4.26,4.22,4.16,4.05$ \\
\hline 10 & $63.1, \mathrm{CH}_{2}$ & 4.26 , dd $(11.8,6.0)$ & $4.22,4.34$ & 4.34 \\
\hline $10 \mathrm{~b}$ & - & $4.22, \mathrm{~m}$ & $4.26,4.34$ & - \\
\hline 11 & 170.7, C & - & - & $4.26,4.22,2.12$ \\
\hline 12 & $21.0, \mathrm{CH}_{3}$ & $2.12, \mathrm{~s}$ & - & - \\
\hline 13 & $20.6, \mathrm{CH}_{3}$ & $2.28, \mathrm{~s}$ & 5.95 & - \\
\hline
\end{tabular}




\subsubsection{Spectra}

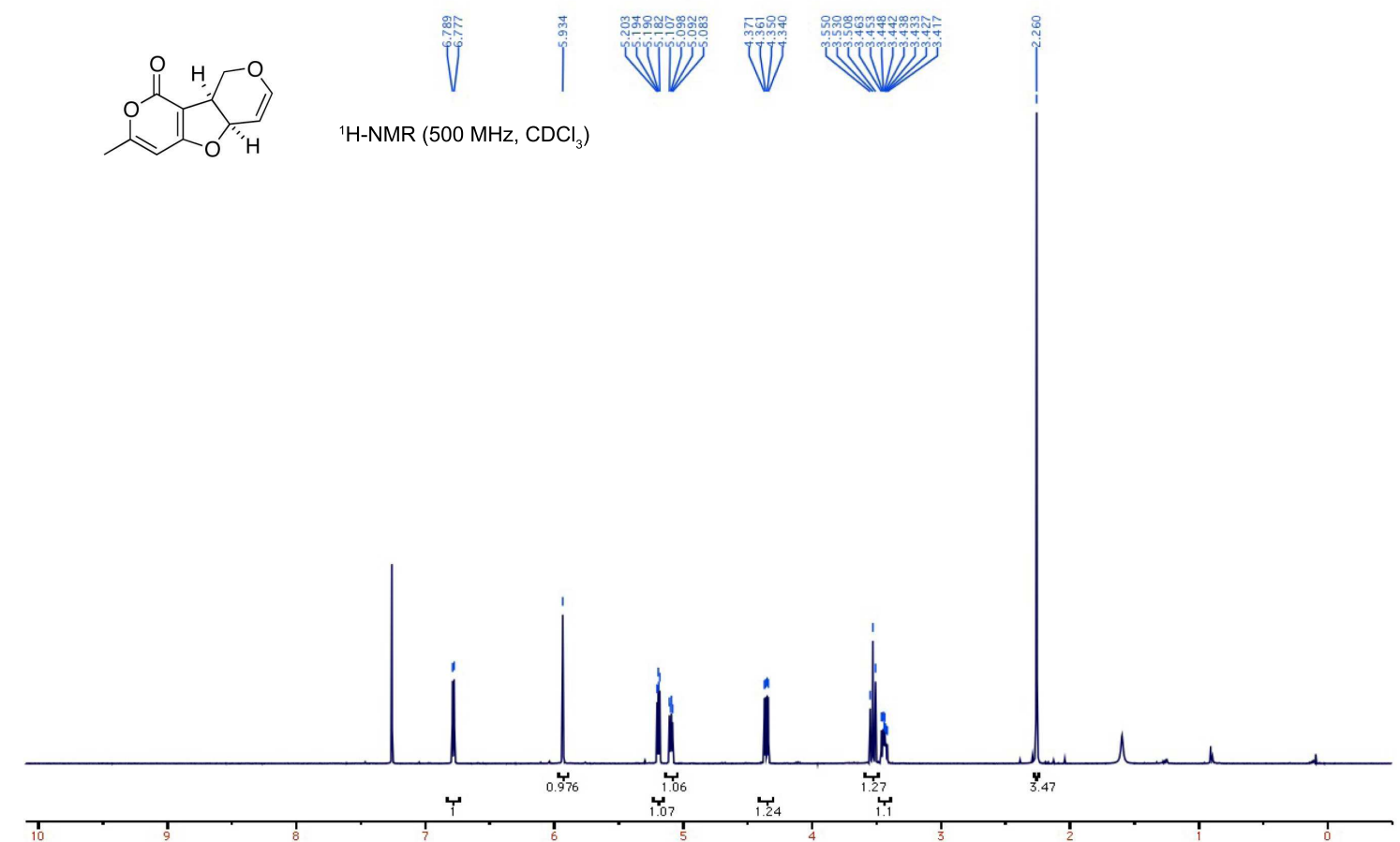

${ }^{13} \mathrm{C}-\mathrm{NMR}\left(126 \mathrm{MHz}, \mathrm{CDCl}_{3}\right)$

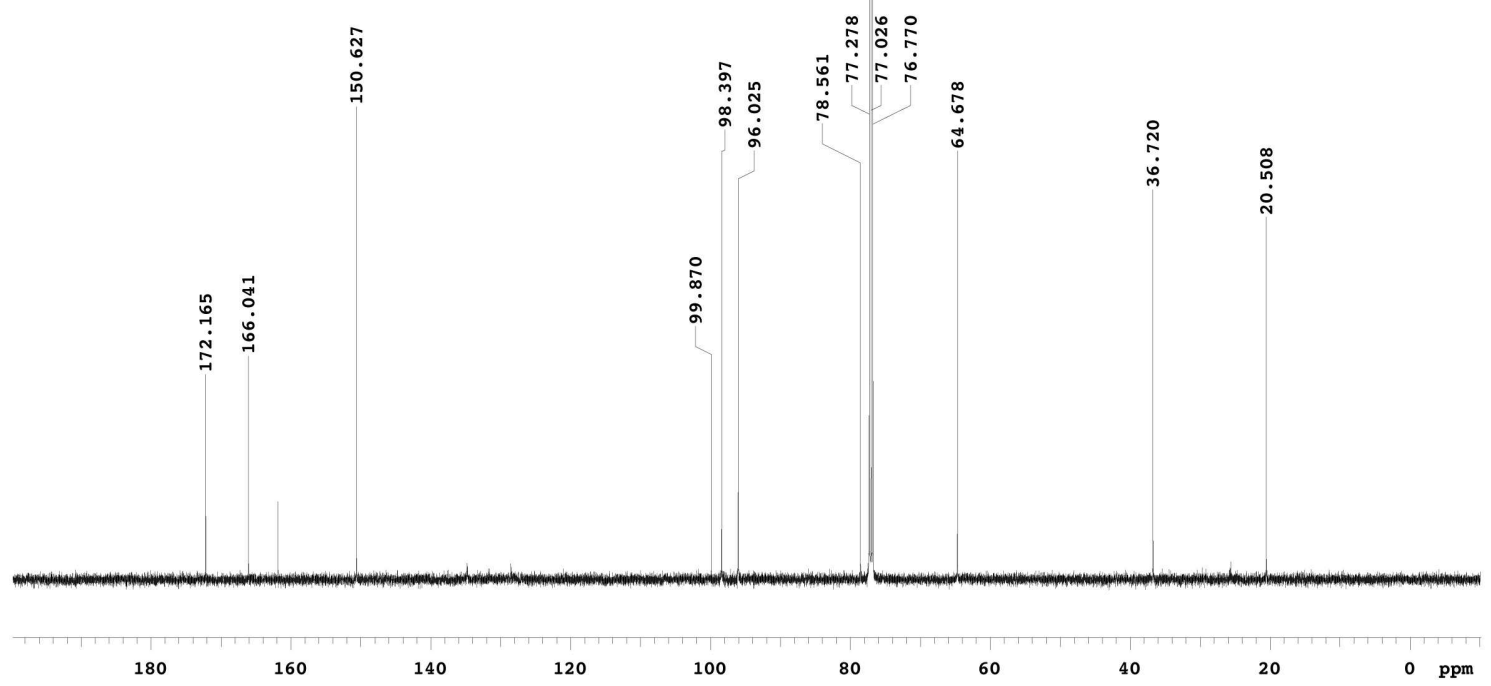



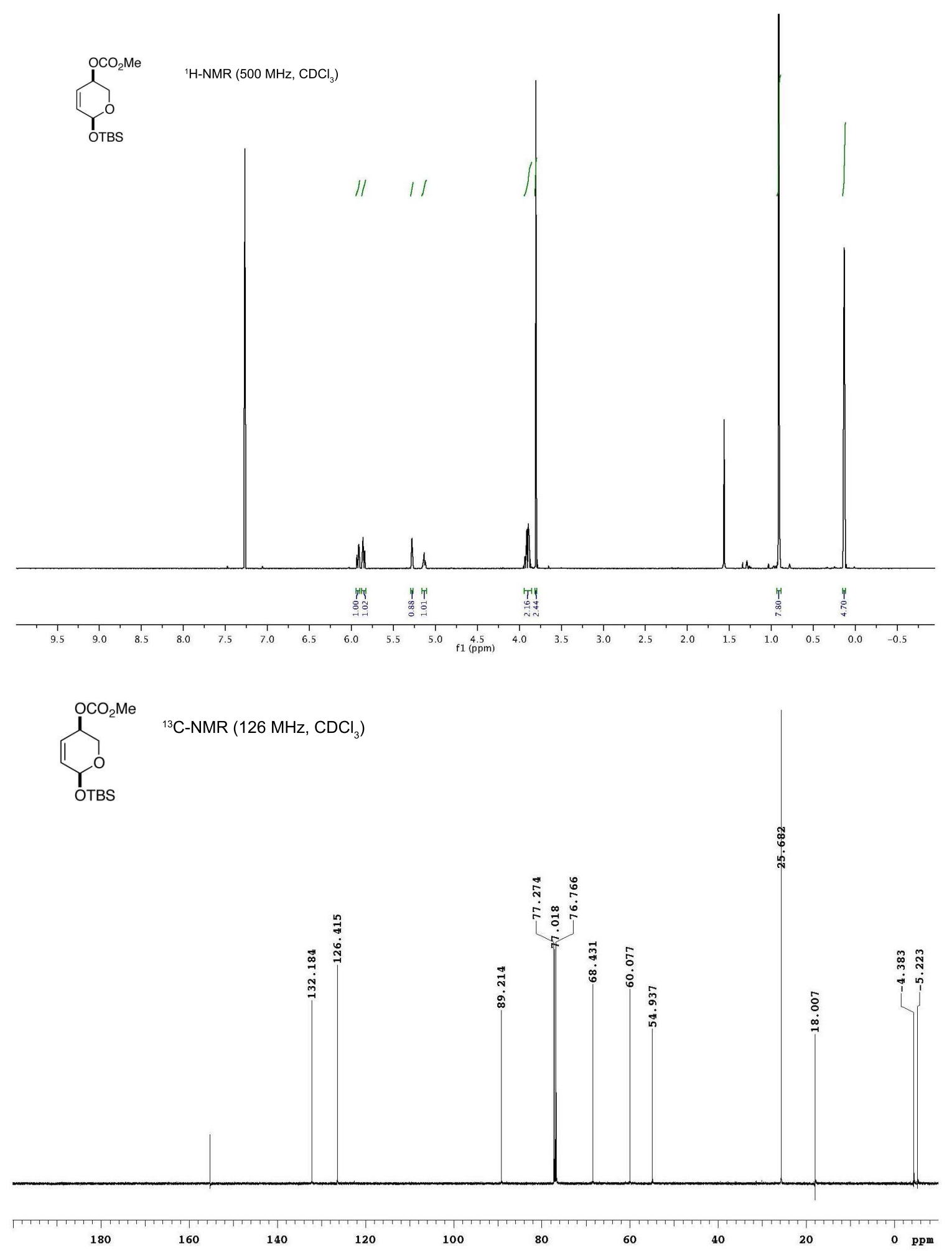

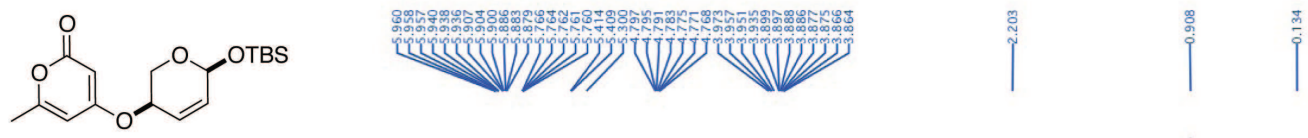

${ }^{1} \mathrm{H}-\mathrm{NMR}\left(500 \mathrm{MHz}, \mathrm{CDCl}_{3}\right)$

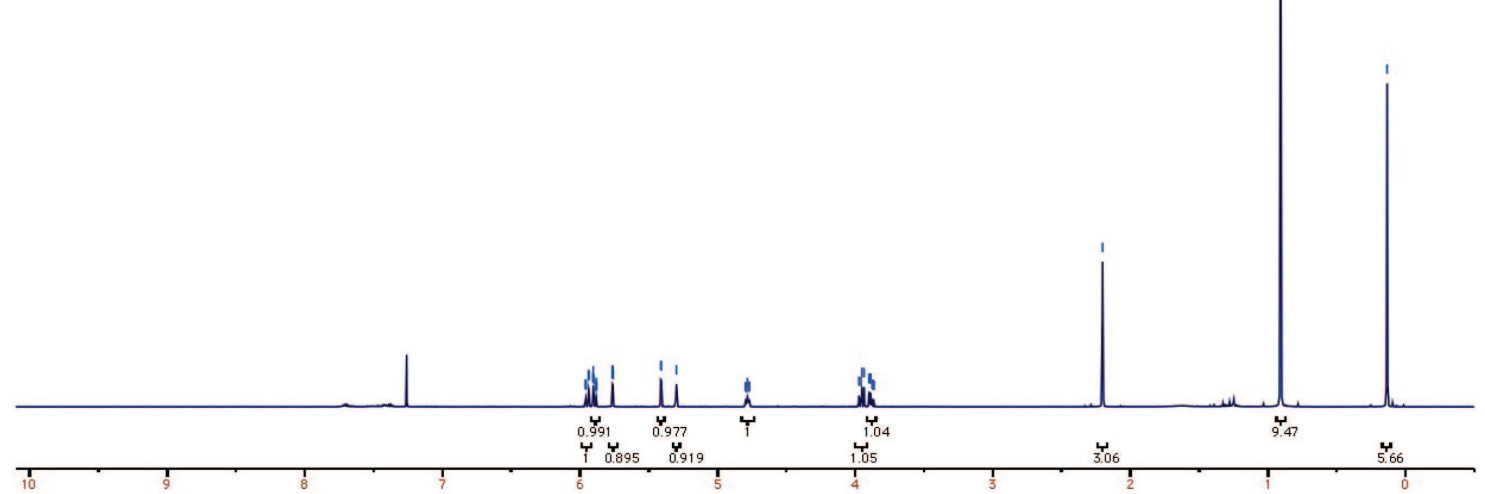

舀-

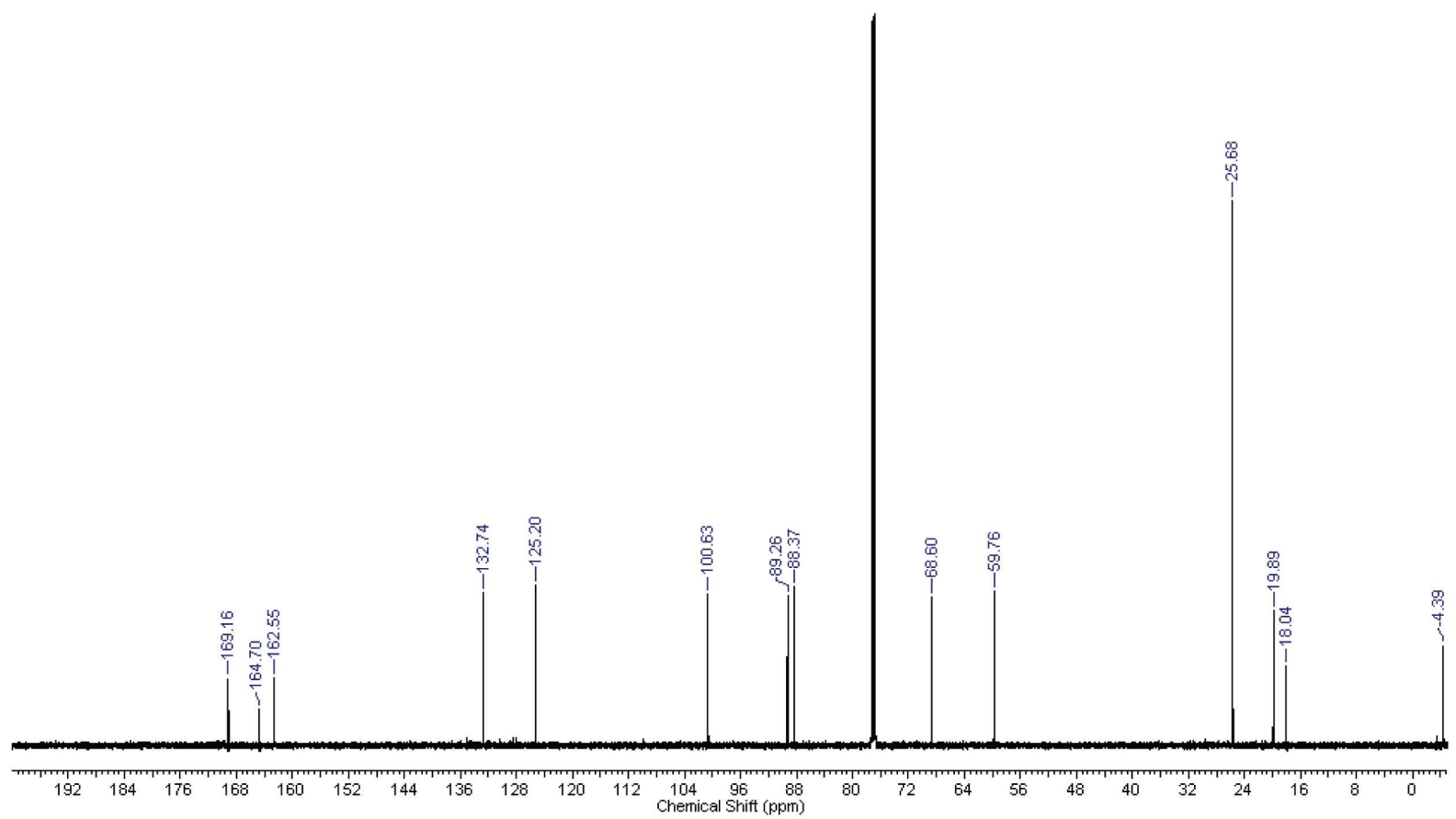



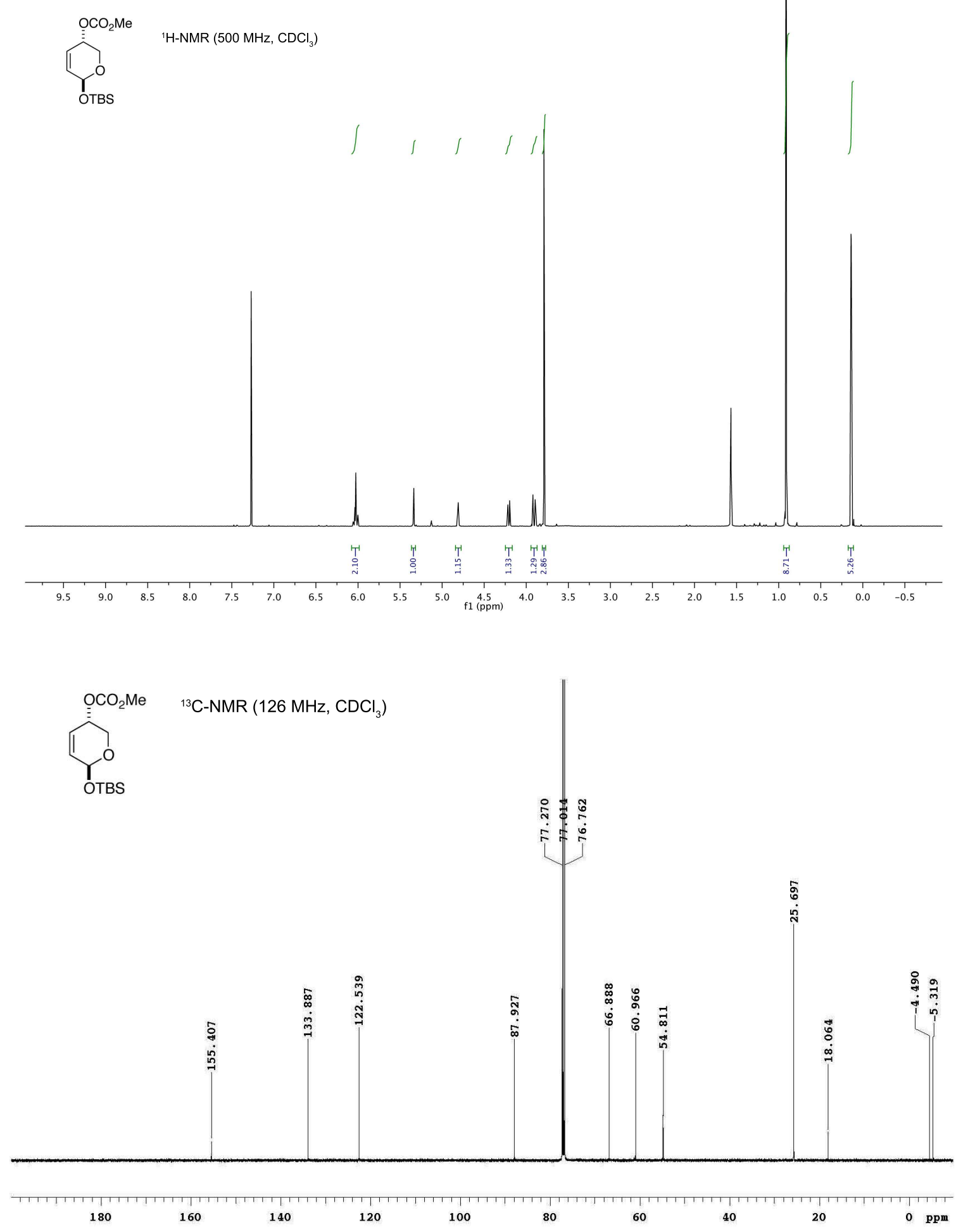

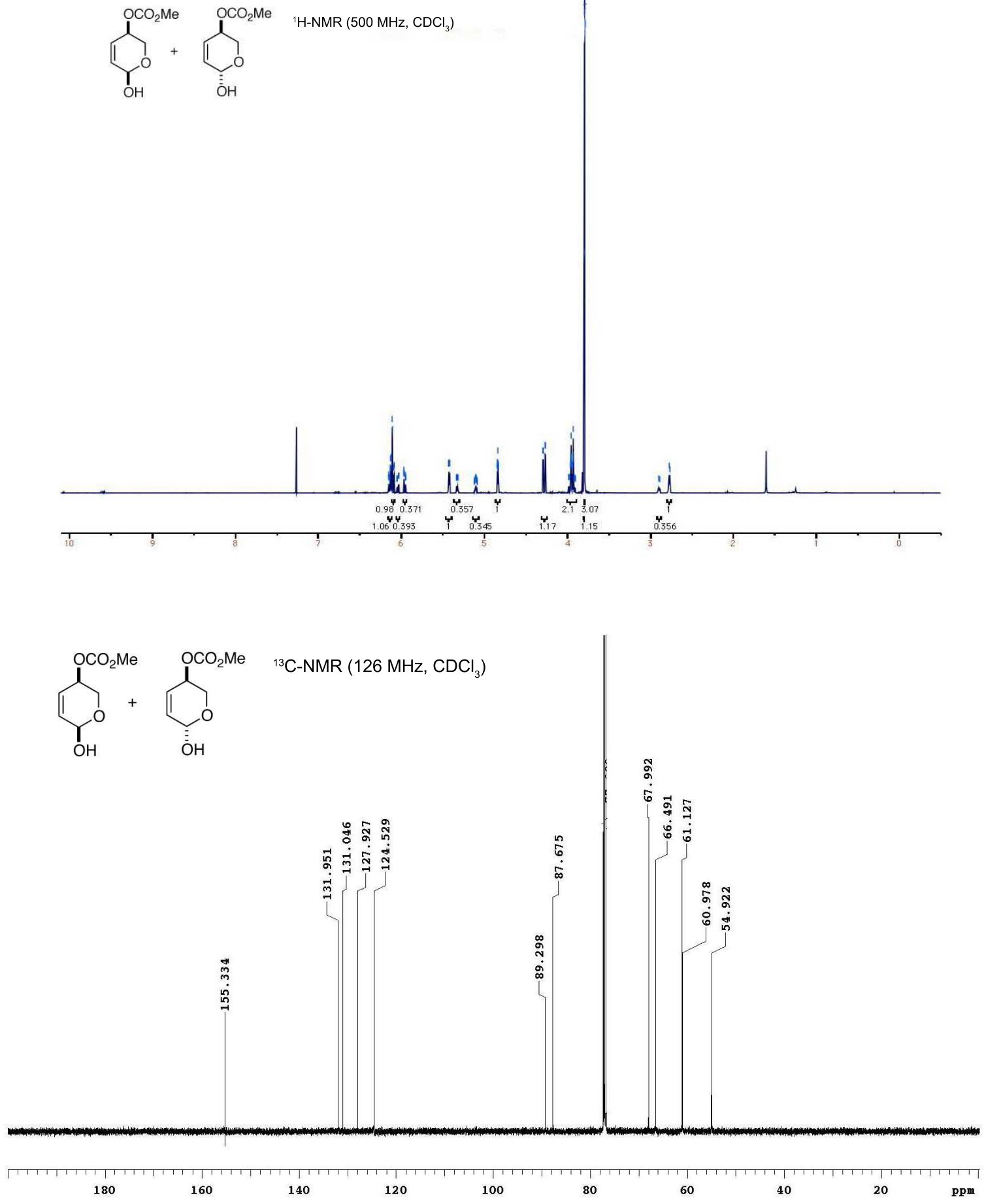

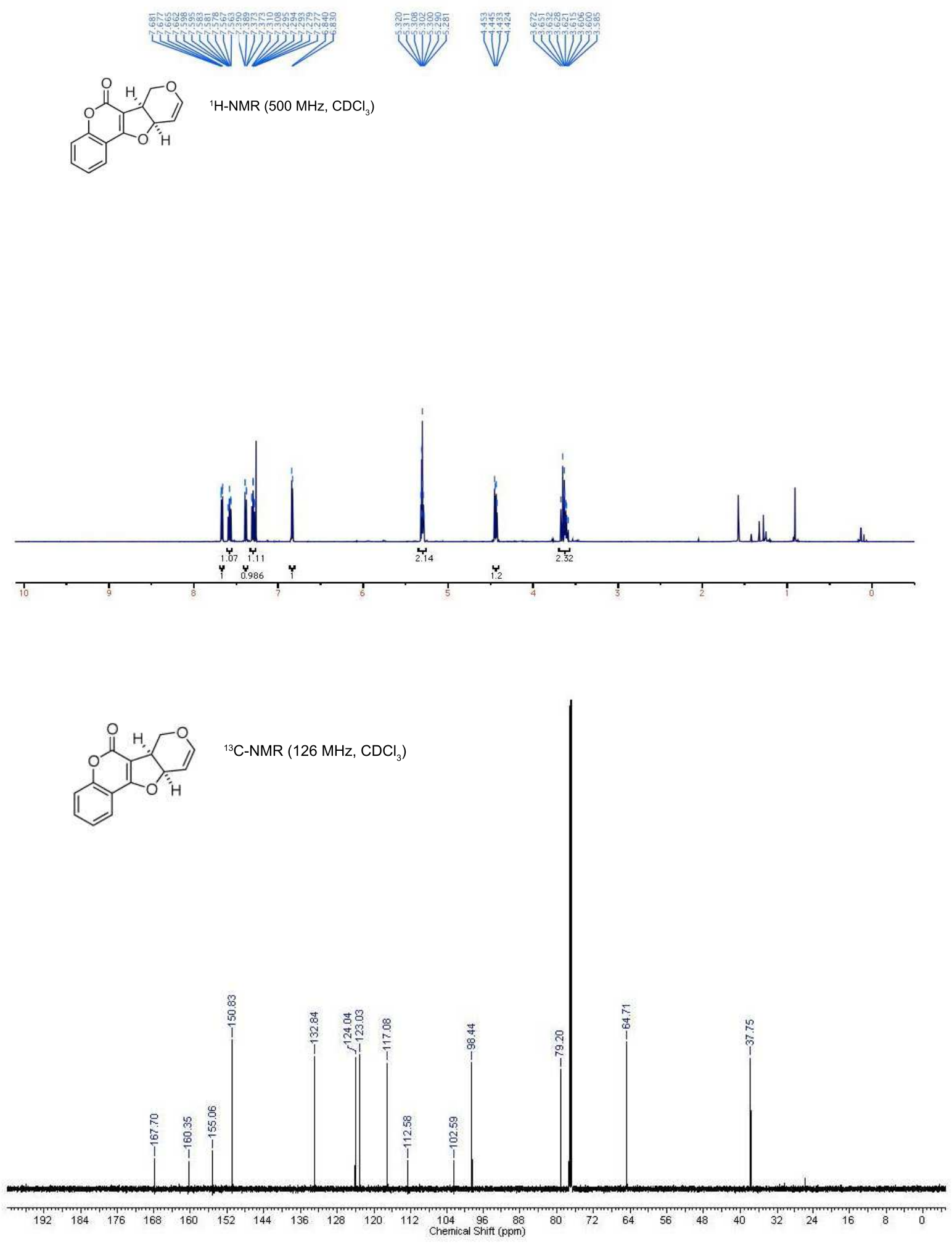

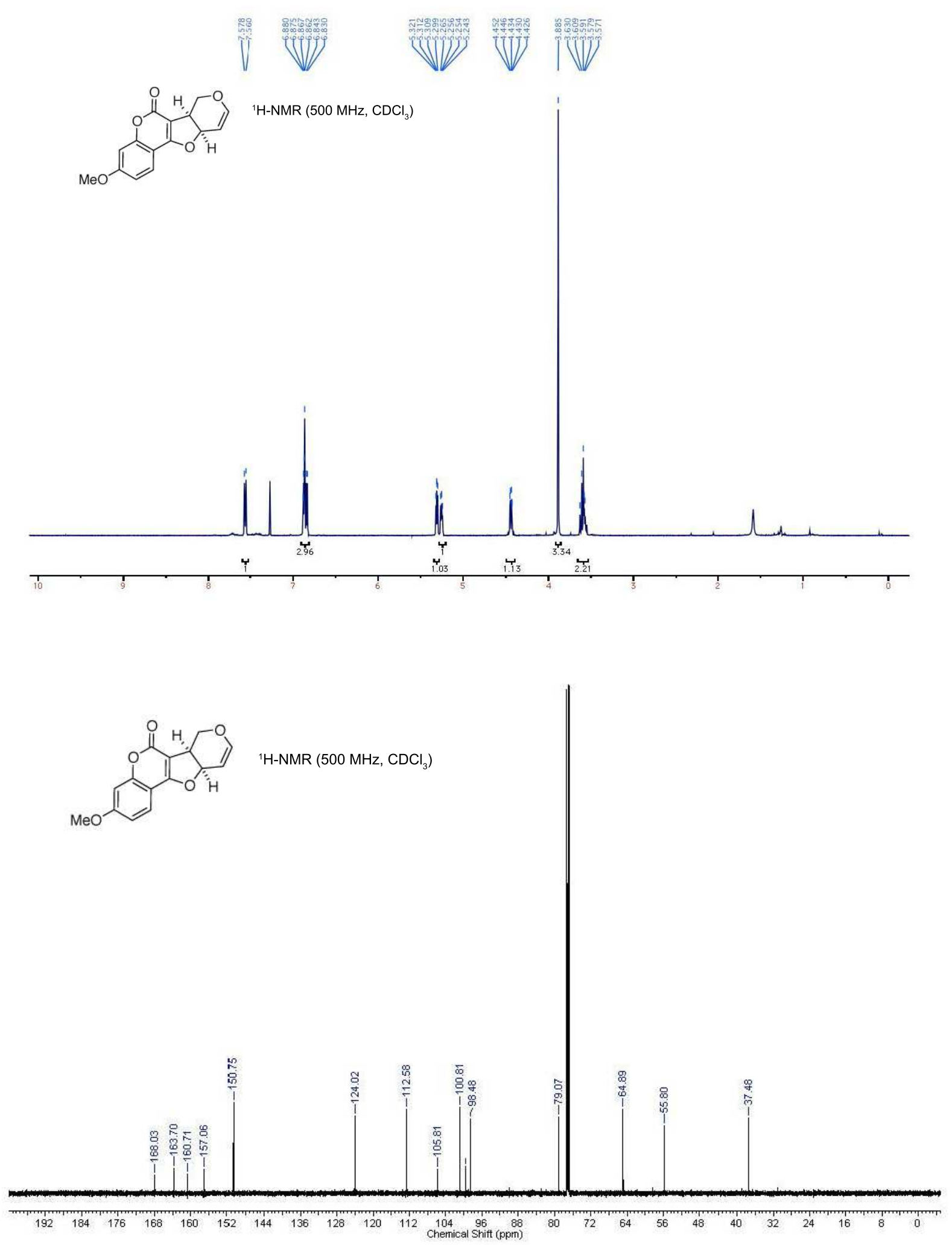

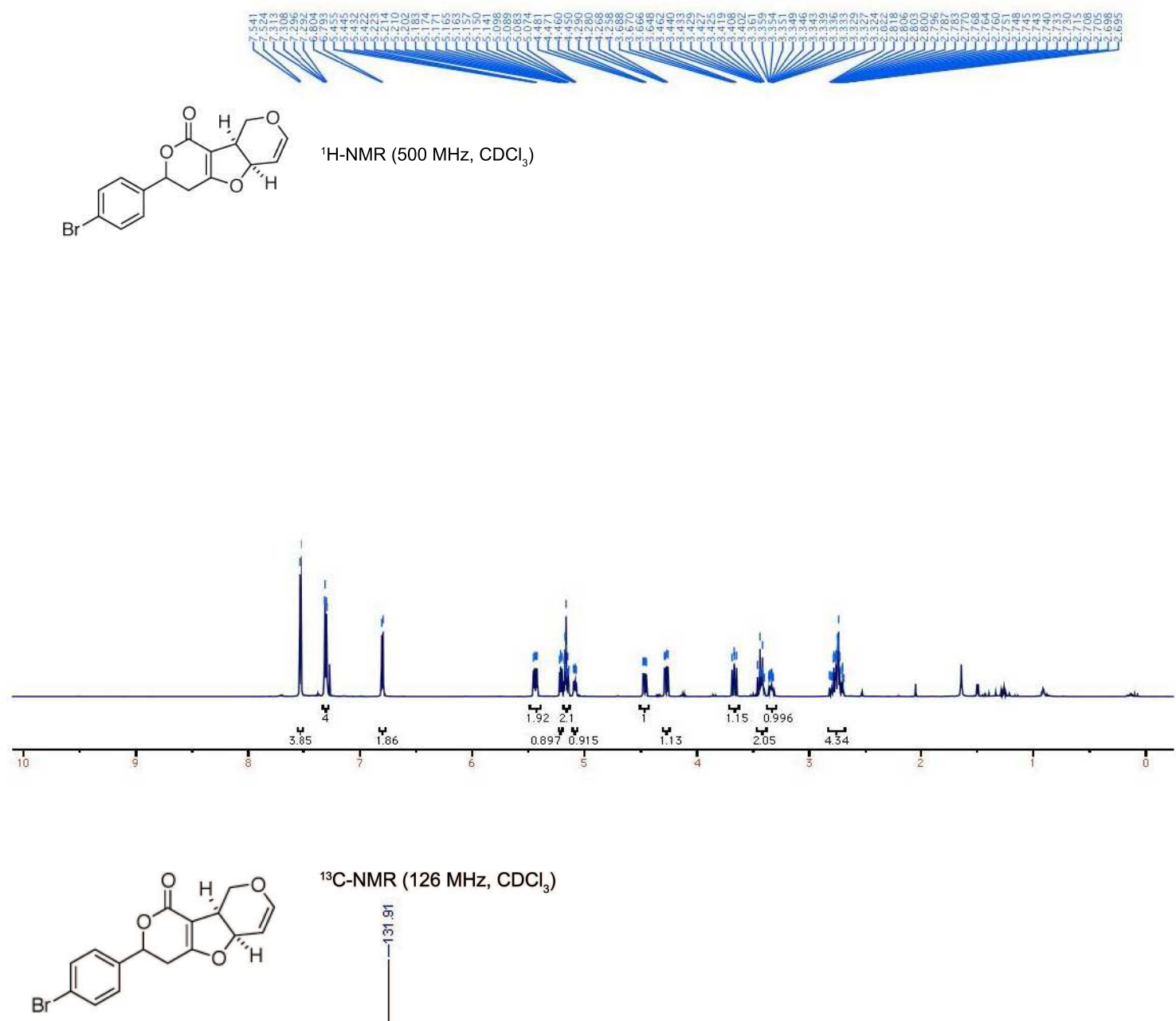

${ }^{13} \mathrm{C}-\mathrm{NMR}\left(126 \mathrm{MHz}, \mathrm{CDCl}_{3}\right.$ )

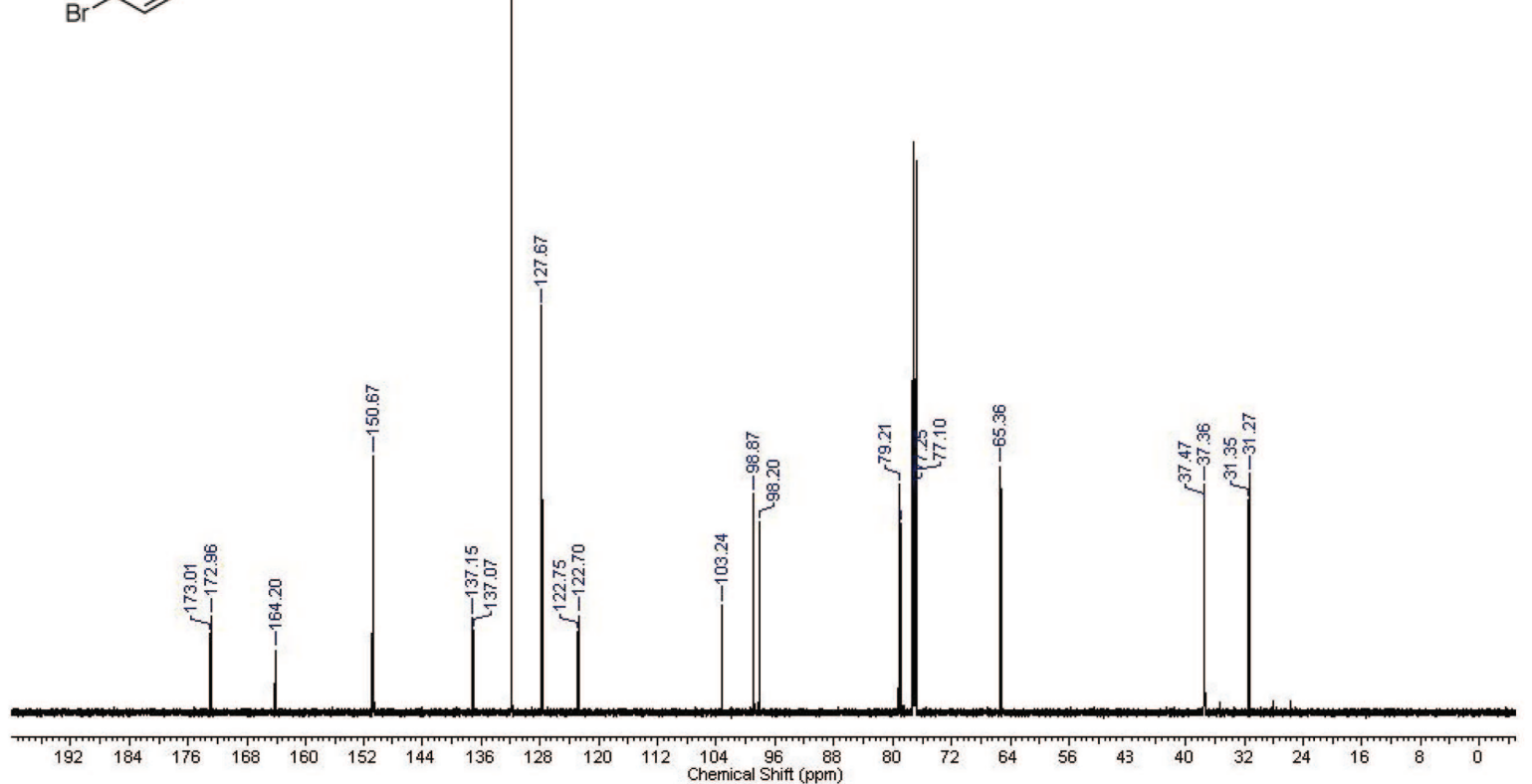



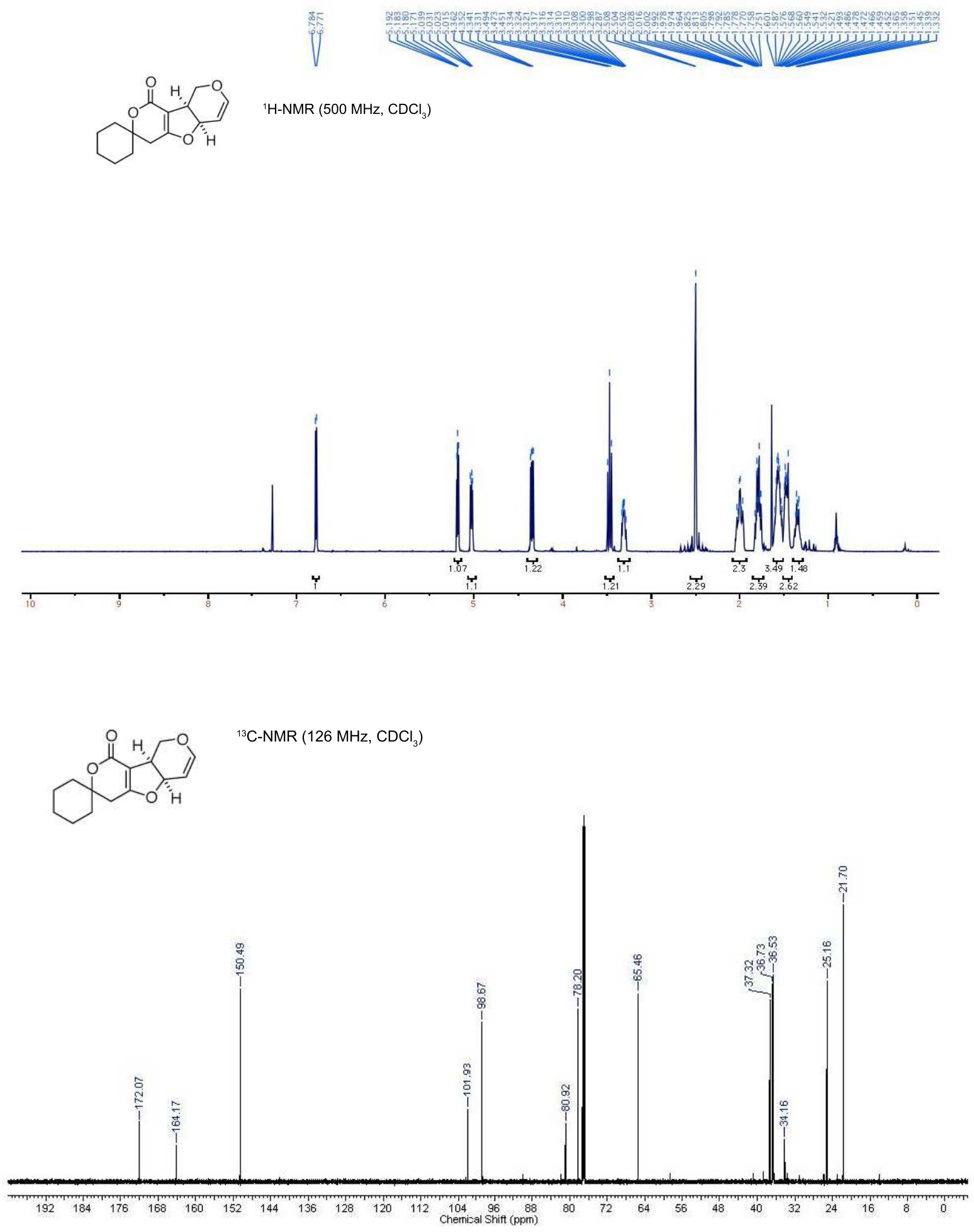

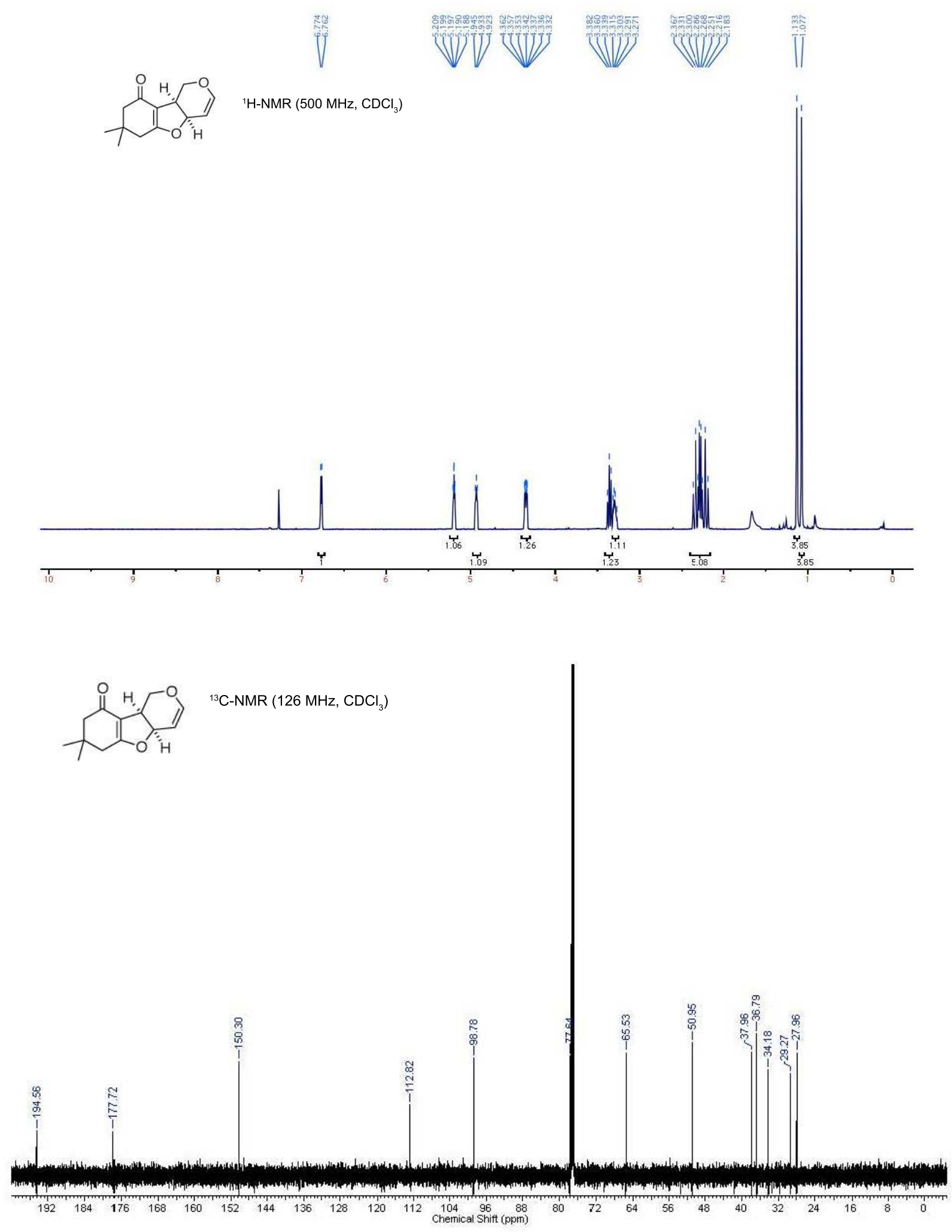

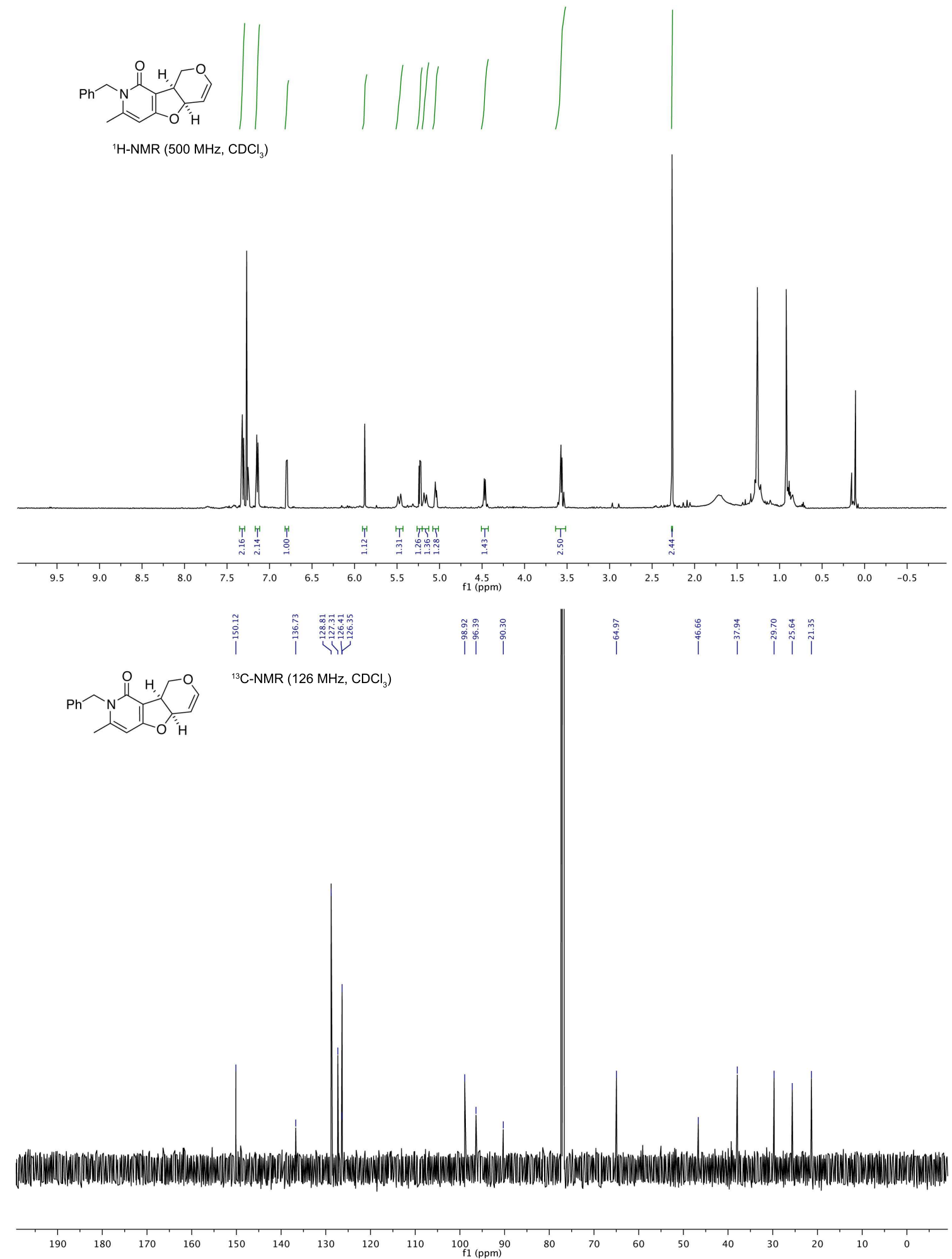

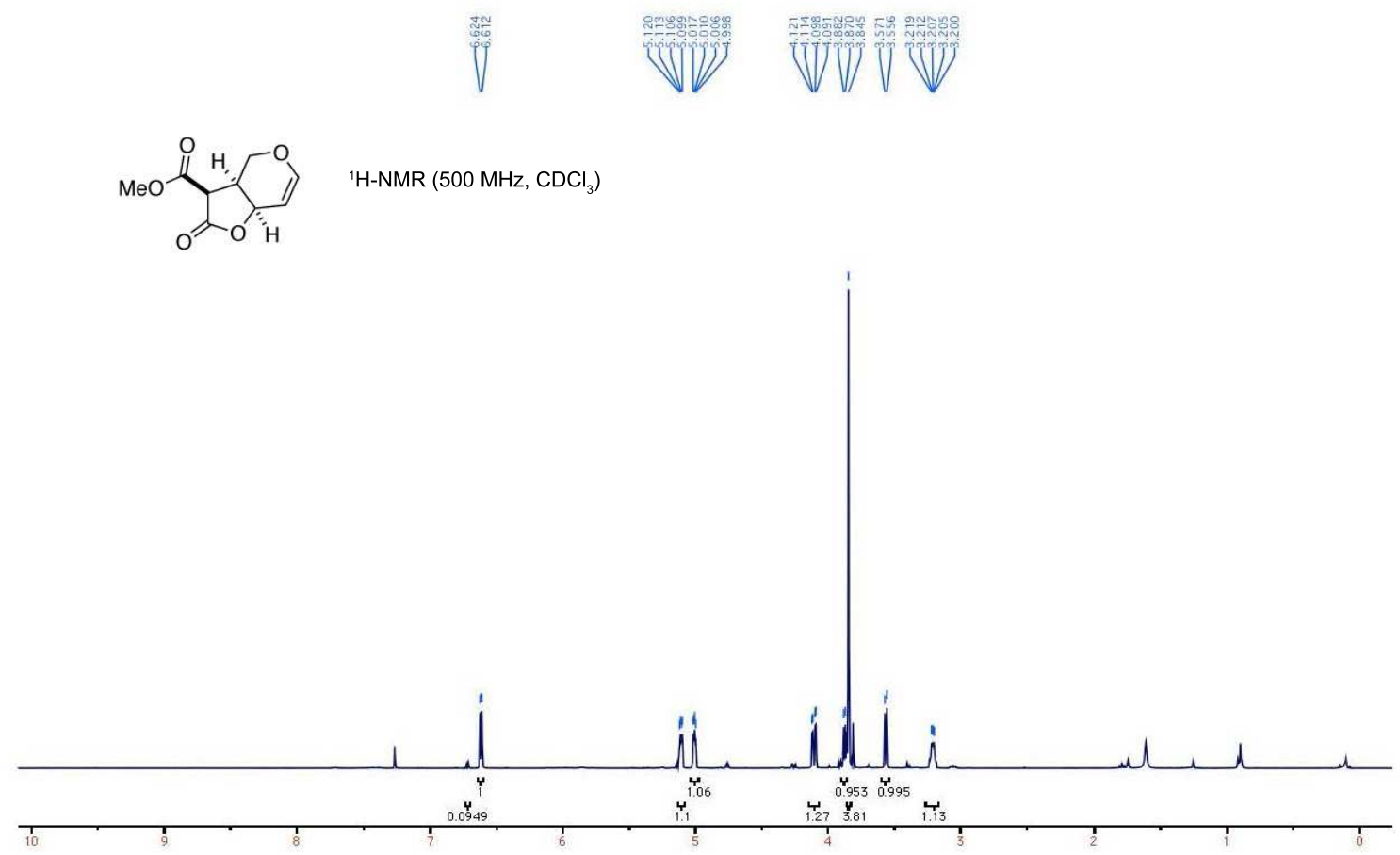

政

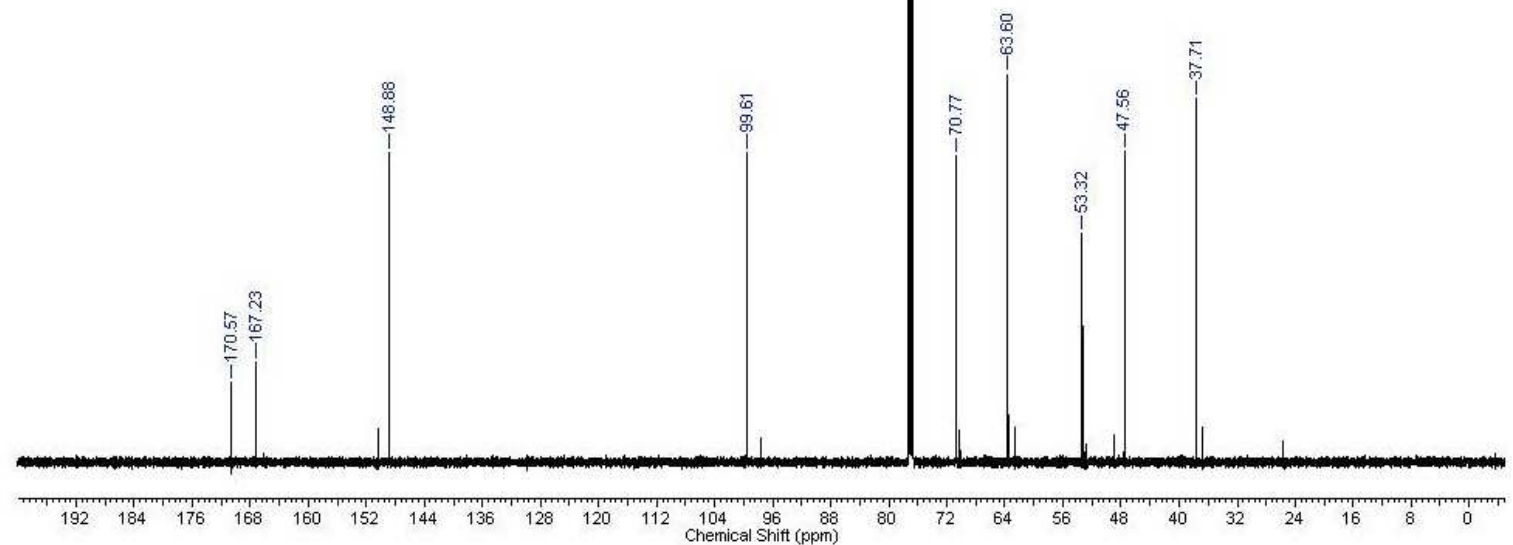



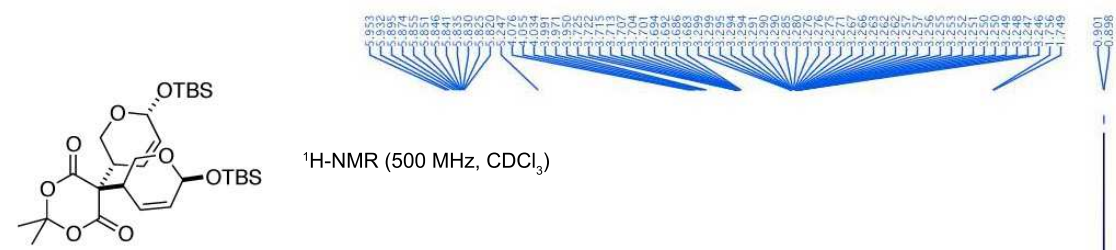

${ }^{1} \mathrm{H}-\mathrm{NMR}\left(500 \mathrm{MHz}, \mathrm{CDCl}_{3}\right)$
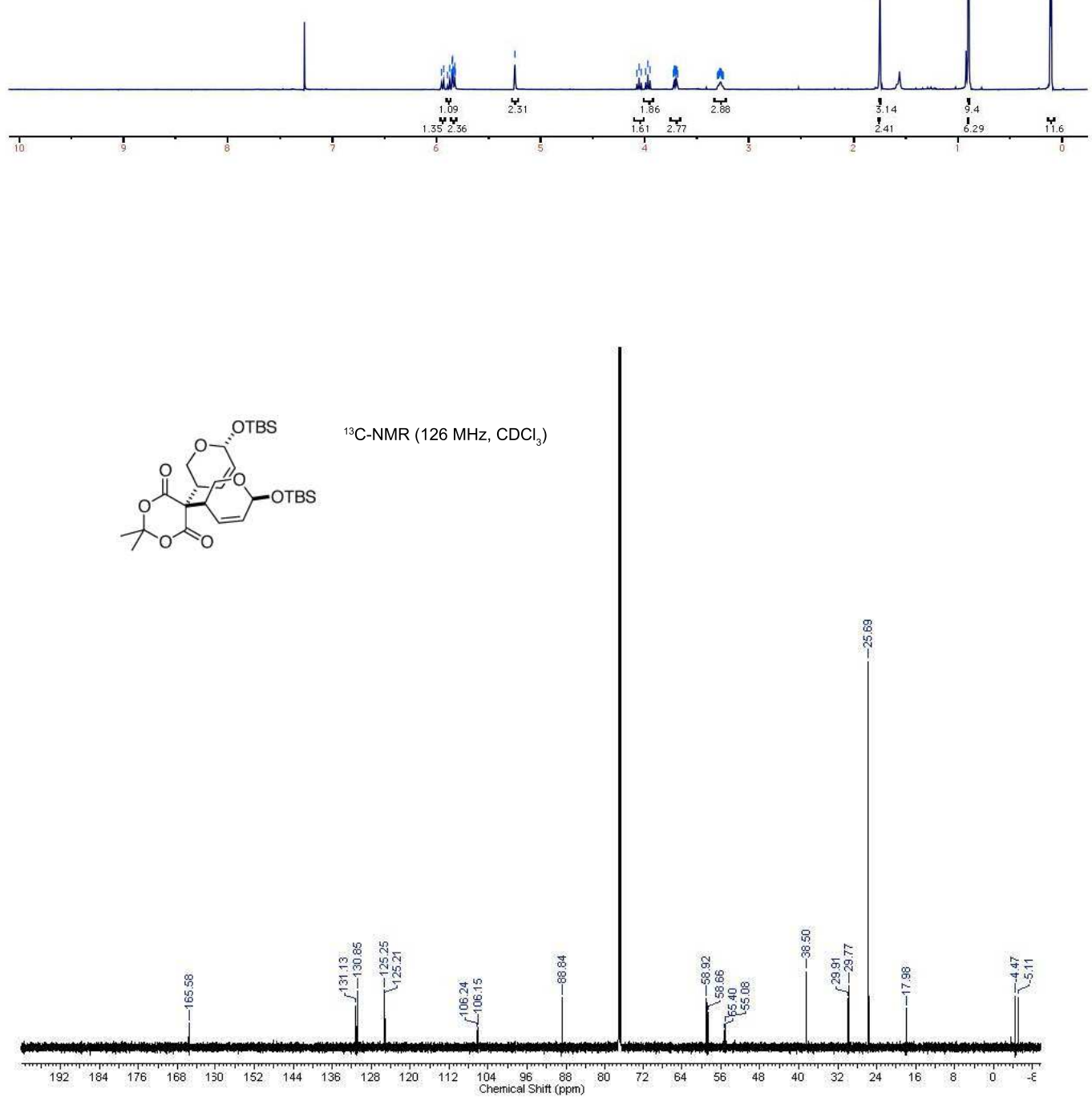

${ }^{13} \mathrm{C}-\mathrm{NMR}\left(126 \mathrm{MHz}, \mathrm{CDCl}_{3}\right.$ ) 

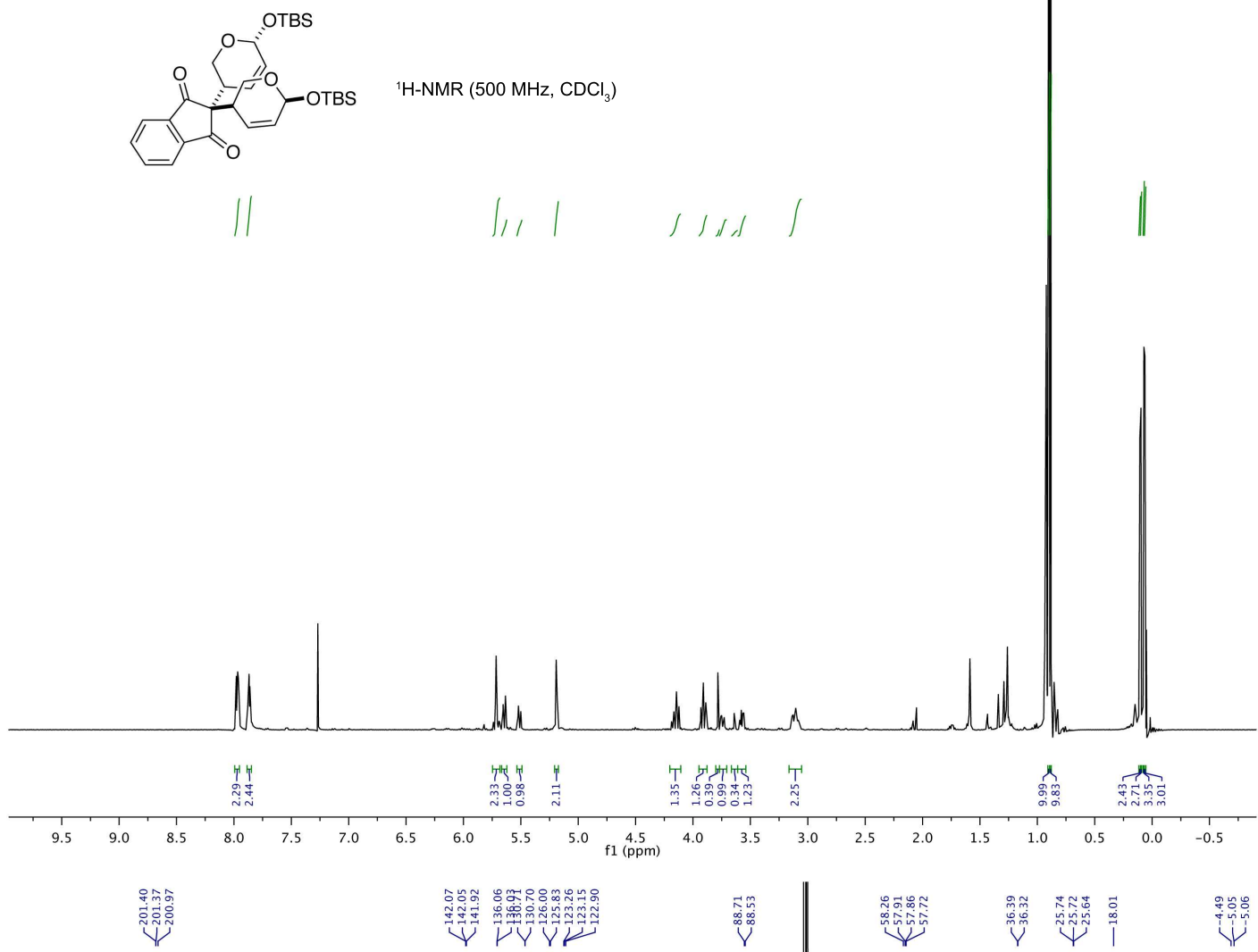

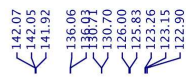

$\overbrace{\substack{n \\ \infty}}^{\substack{\infty \\ \infty}}$

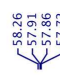

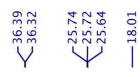

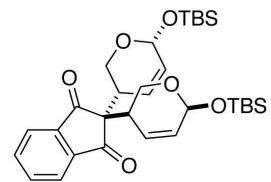

${ }^{13} \mathrm{C}-\mathrm{NMR}\left(126 \mathrm{MHz}, \mathrm{CDCl}_{3}\right)$

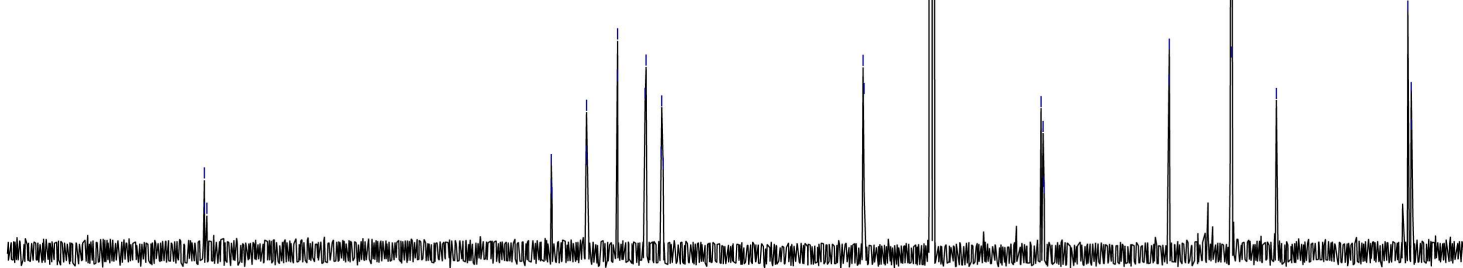

$\begin{array}{llllllllllllllllllllllllll}230 & 220 & 210 & 200 & 190 & 180 & 170 & 160 & 150 & 140 & 130 & 120 & \begin{array}{c}\mathrm{f} 1 \\ (10 \mathrm{ppm})\end{array} & 100 & 90 & 80 & 70 & 60 & 50 & 40 & 30 & 20 & 10 & 0 & -10\end{array}$ 

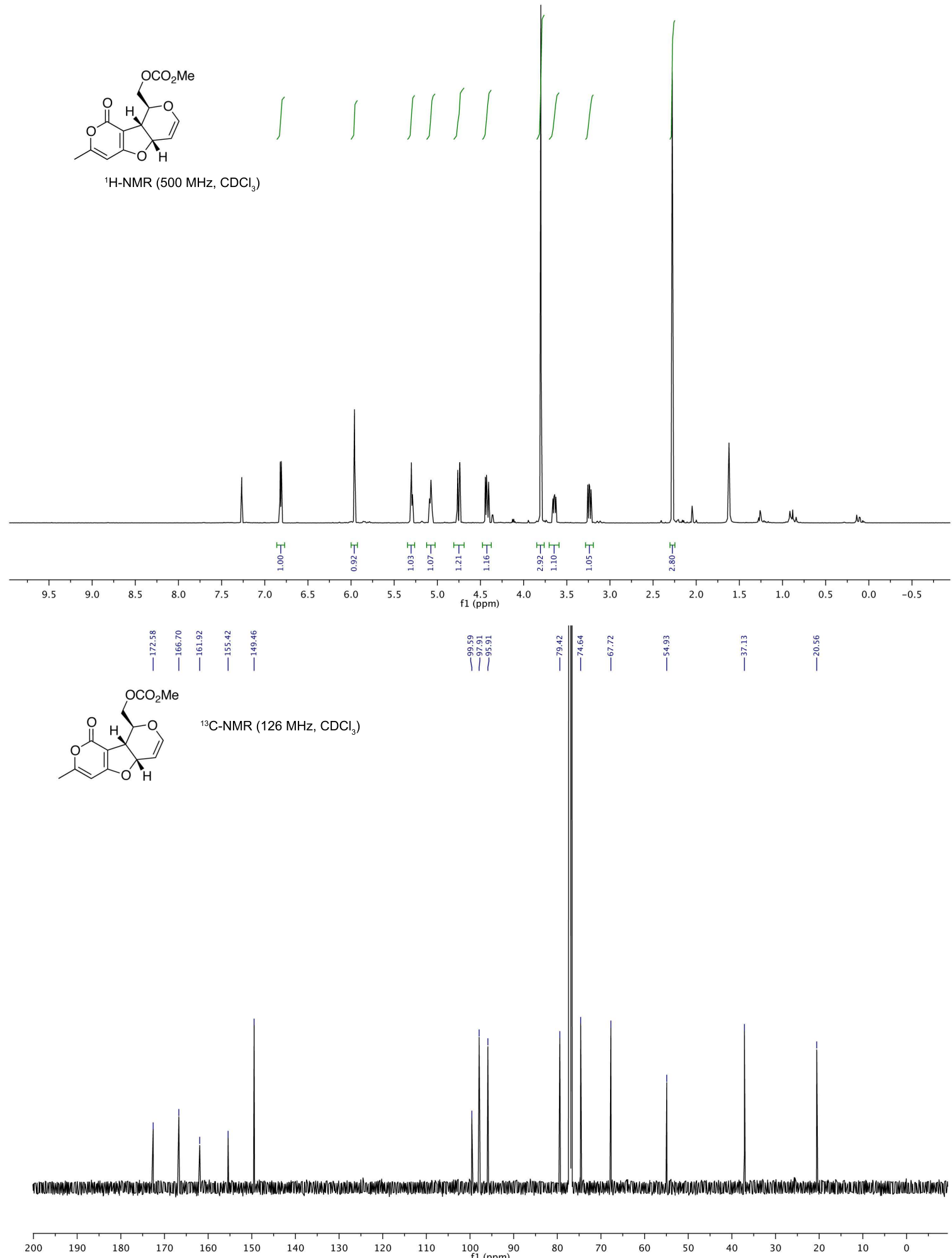

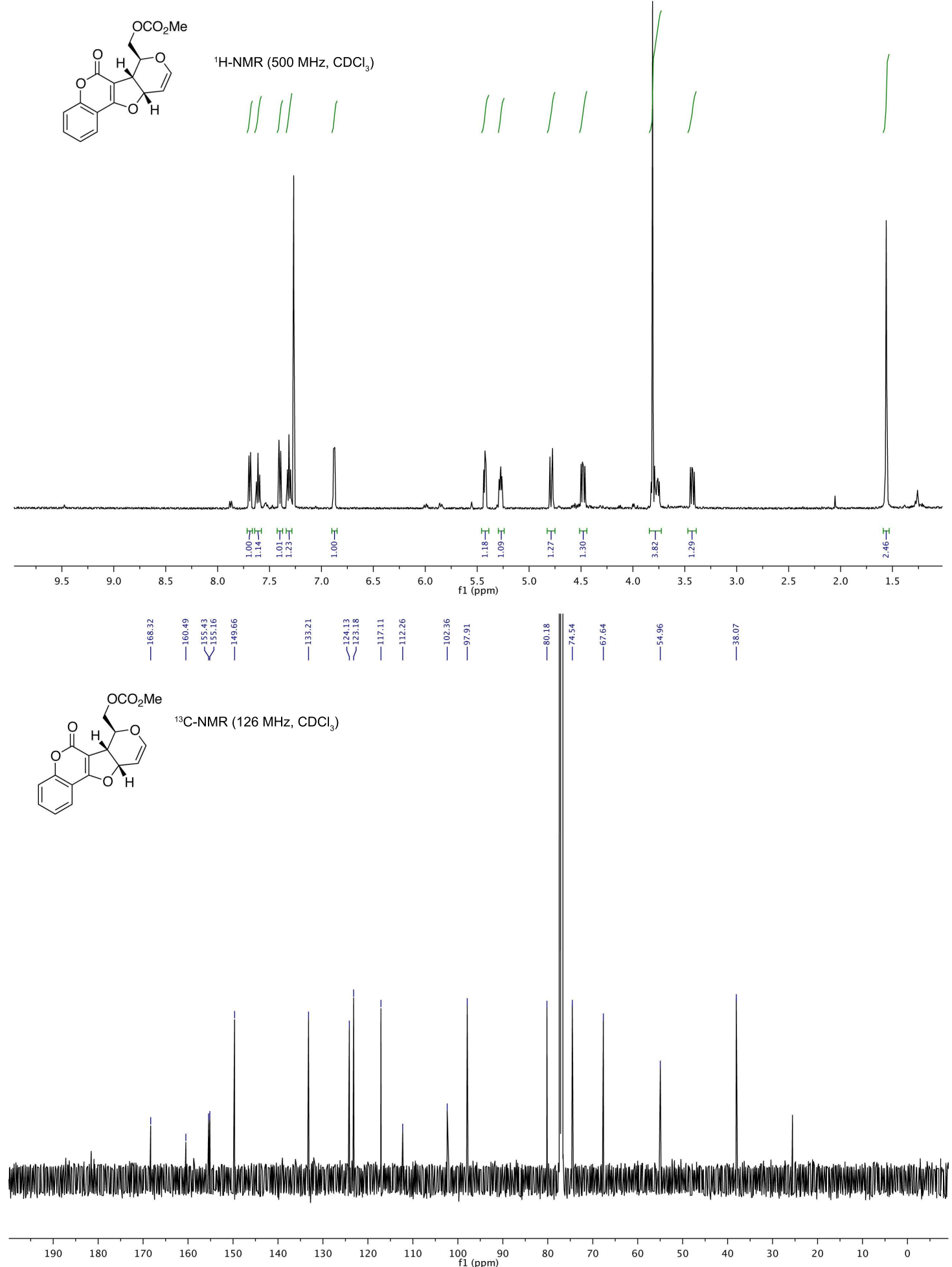
Sample Name:
mark $\mathrm{K}^{2}$ NOES

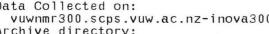

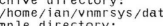

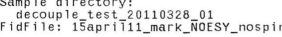
Pulse Sequence: NoESY
Solventiacdci3

ant: cdc13
collected on: Apr 152011

operator: ian

Relax: delay 3.000 sec

Width
20 width 2420.7 H
19.

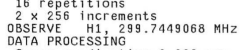

Gauss apodization $0.069 \mathrm{sec}$
F1 DATA PROCESSING

Gauss apodization 0.038
FT size $1024 \times 1024.038$

(1)

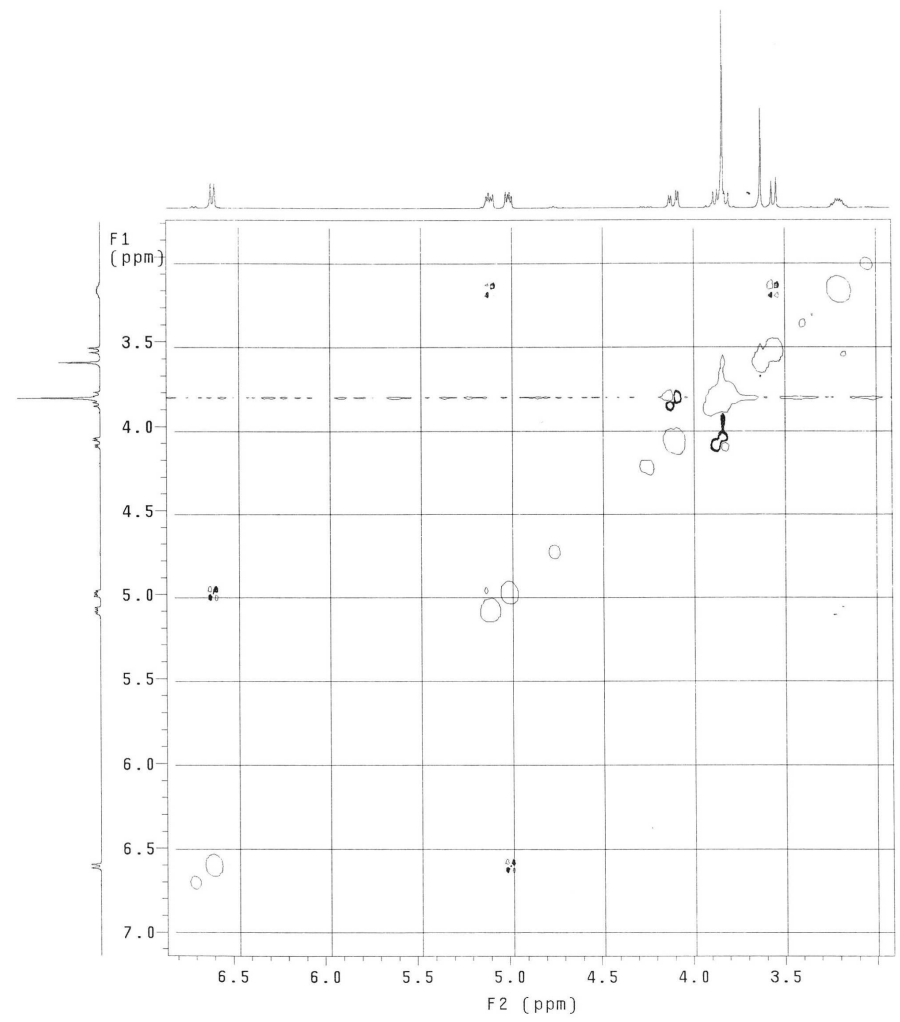

noesy

Data collected on:

Archive directory:
/home/ian/ $y$ nnrsys/data

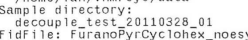

Pul se Sequence: NOESY
Solvent
bat chl 13

operator: ian

Re lax de day $2.000 \mathrm{sec}$
Aca. $t$ ime $0.150 \mathrm{sec}$

Width $2131.3 \mathrm{~Hz}$

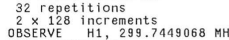

DATA PROCESSING
Gauss apodization 0.055 sec

F1 DATA PROCESSING

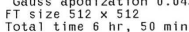
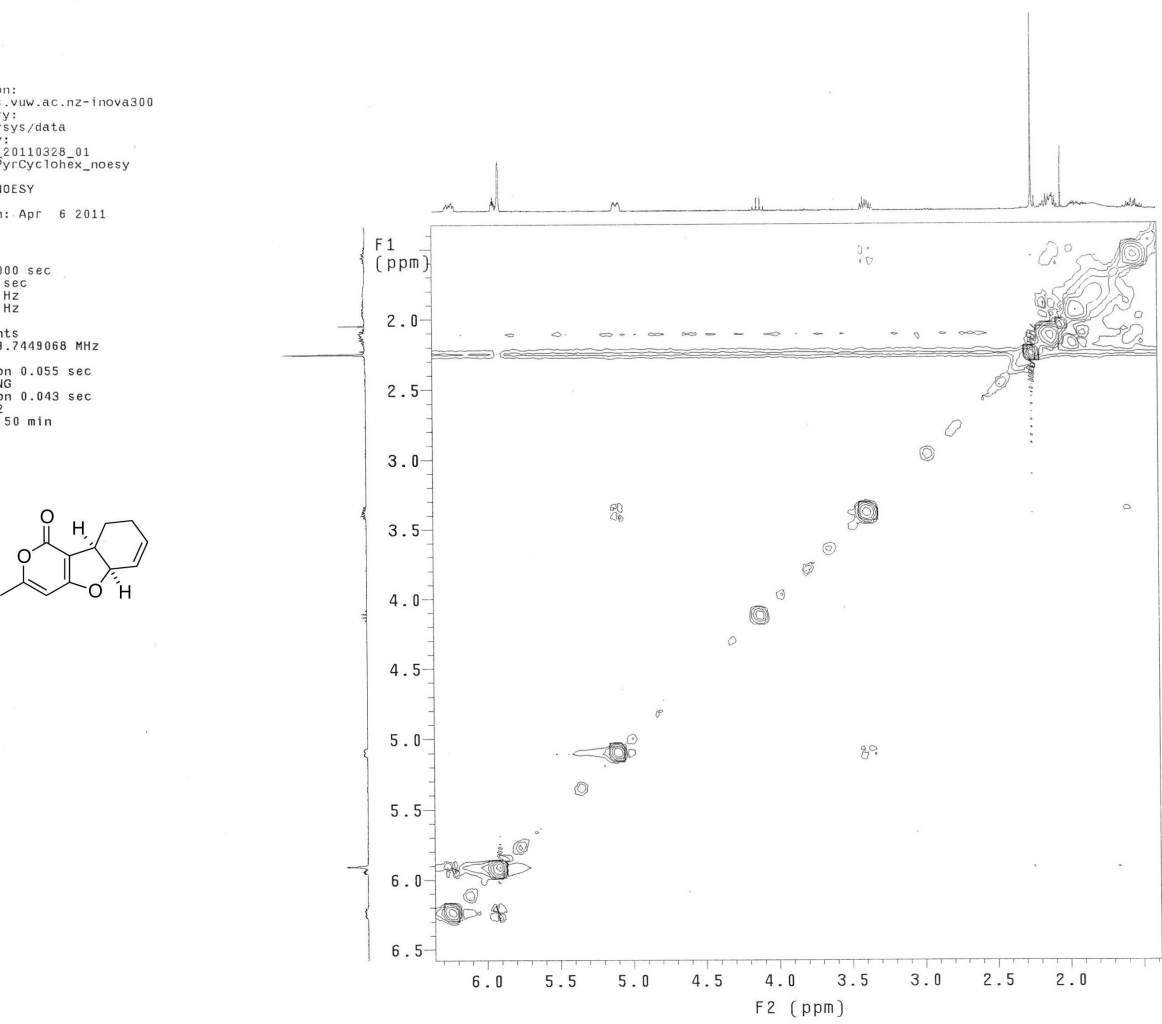
$300 \mathrm{MHz}$ Homonuclear Decoupling Spectra

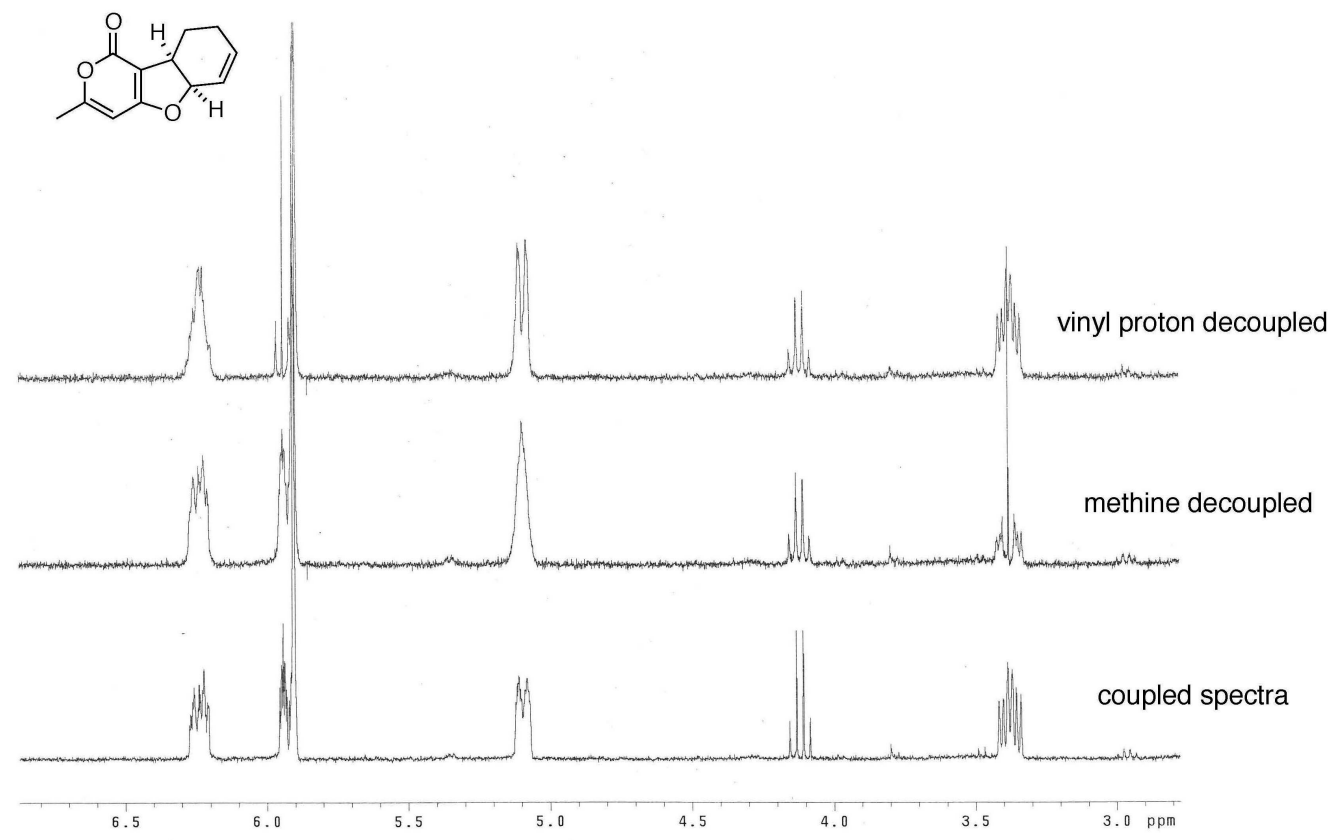



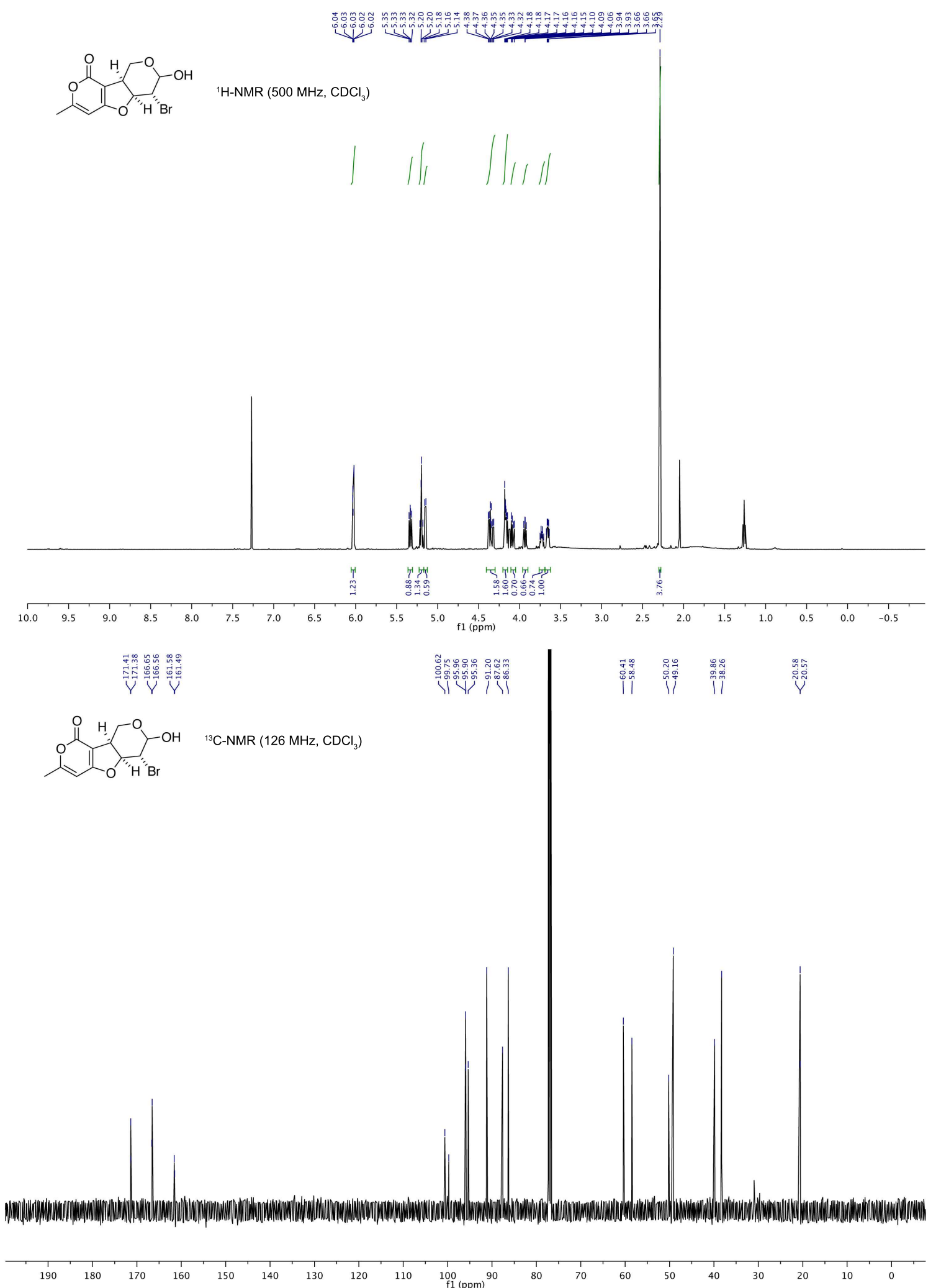
7.5. EXPERIMENTAL DATA FOR CHAPTER 7

293

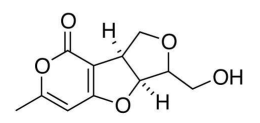

${ }^{1} \mathrm{H}-\mathrm{NMR}\left(500 \mathrm{MHz}, \mathrm{CDCl}_{3}\right.$ )
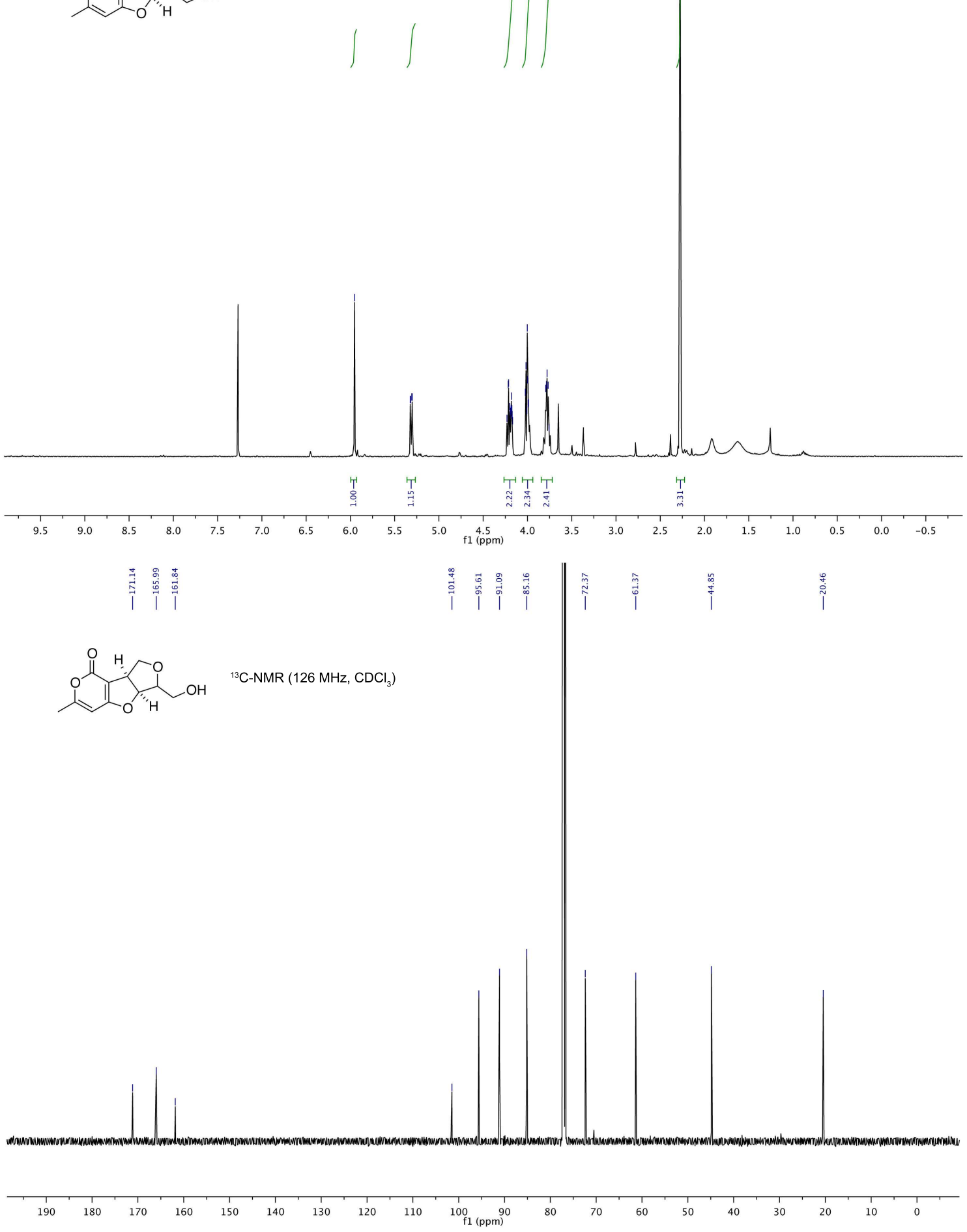


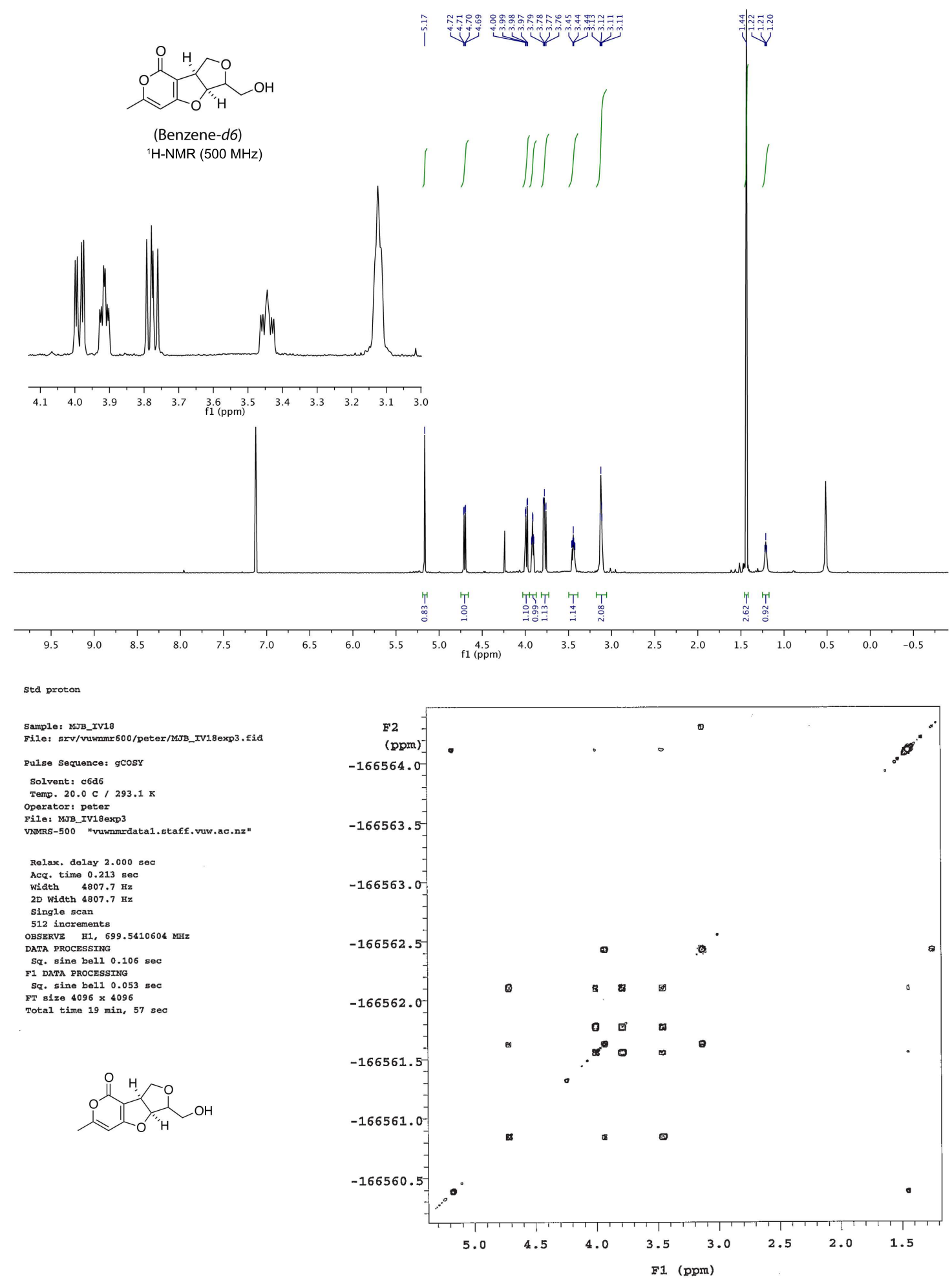




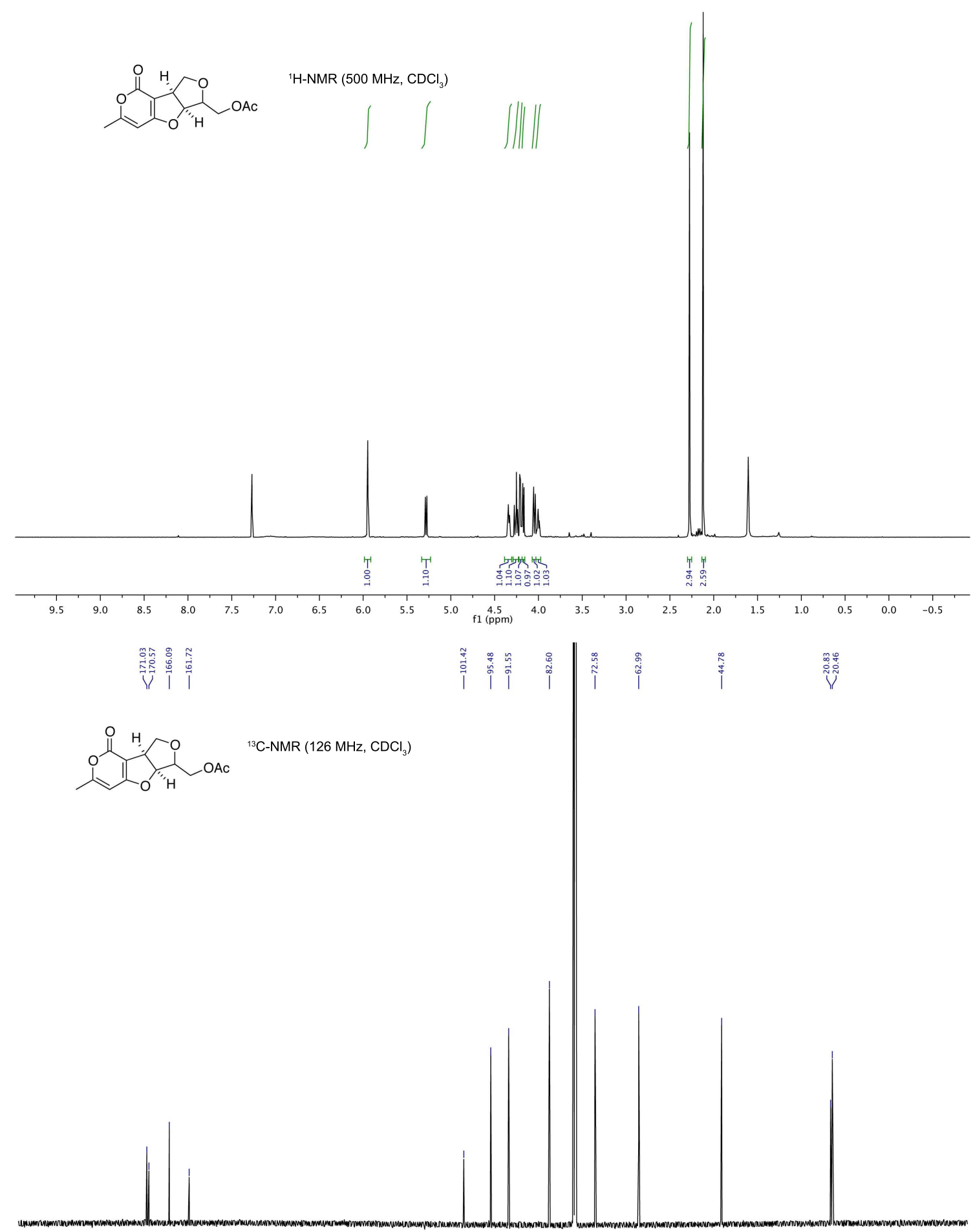

$\begin{array}{llllllllll}190 & 180 & 170 & 160 & 150 & 140 & 130 & 120 & 110 & 100 \\ \mathrm{f} 1(\mathrm{ppm}) & 90\end{array}$ 


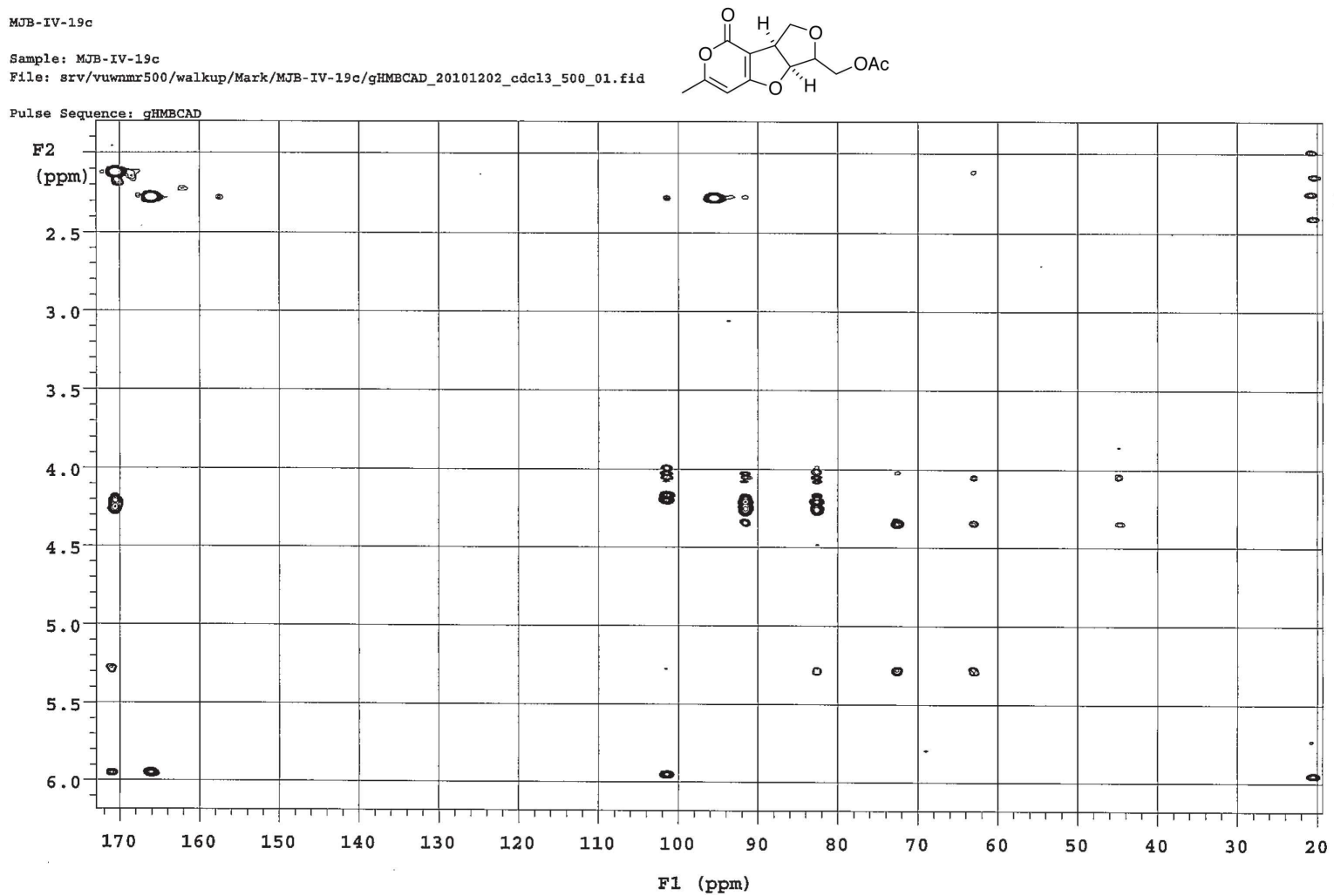




\section{References for Chapter 7}

[1] Trost, B. M.; Patterson, D. E.; Hembre, E. J. J. Am. Chem. Soc. 1999, 121, 10834. Trost, B. M.; Hembre, E. J. Tetrahedron Lett. 1999, 40, 219.

[2] a) Achmatowicz Jr., O.; Buckowski, P.; Szechner, B.; Zierchowska, A.; Zamojski, A. Tetrahedron 1971, 27 , 1973. b) Sugawara, K.; Imanishi, Y.; Hashiyama, T. Tetrahedron: Asymm. 2000, 11, 4529.

[3] In contrast, the analogous hemiacetal substrate produced a complex mixture of undesired products. See Section 7.5 for details.

[4] Amatore, C.; Jutand, A. Coordination Chem. Rev. 1998, 178-180, 511.

[5] Brescia, M.-C.; Shimshock, Y. C.; DeShong, P. J. Org. Chem. 1997, 62, 1257.

[6] Trost, B. M.; Verhoeven,T. R. J. Org. Chem. 1976, 41, 3215.

[7] Crystal structure solved using SHELX: Dolomanov, O. V.; Bourhis, L. J.; Gildea, R. J.; Howard, J. A. K.; Puschmann, H. J. Appl. Cryst. 2009, 42, 339. Sheldrick, G. M. Acta Cryst. 2008, A64, 112. Graphical representation created using ORTEP-3: Farrugia, L. J. J. Appl. Cryst. 1997, 30, 565.

[8] For examples of strain energy in 5,6-ring systems see Velluz, L.; Valls, J.; Nominé, G. Angew. Chem. Int. Ed. 1965, 4, 181.

[9] The isomerization of $\pi$-allyl-Pd complexes by $\operatorname{Pd}(0)$ has been previously implicated in the loss of stereospecificity in Pd-AA reactions: Takahashi, T.; Jinbo, Y.; Kitamura, K.; Tsuji, J. Tetrahedron Lett. 1984, 25, 5921. Granberg, K. L.; Bäckvall, J. E. J. Am. Chem. Soc. 1992, 114, 6858.

[10] Tan, S. F.; Ang, K. P.; Jayachandran, H. J. Chem. Soc., Perkin Trans. 1 1983, 471. The $p K_{a}$ of 6-methyl4-hydroxy- $\alpha$-pyrone in $\mathrm{H}_{2} \mathrm{O}$ is 4.94: Ang, K.; Tan, S. J. Chem. Soc., Perkin Trans. 1 1979, 1525.

[11] Moreno-Mañas and coworkers have shown this experimentally, by reacting a 4-allyloxy-6-methyl- $\alpha$-pyrone with a $\operatorname{Pd}(0)$ catalyst to obtain a C3 alkylated 4-hydroxy- $\alpha$-pyrone: Moreno-Mañas, M.; Ribas, J.; Virgili, A. J. Org. Chem. 1988, 53, 5328 .

[12] Related processes have been noted previously: Fillion, E.; Carret, S.; Mercier, L. G.; Trépanier, V. É. Org. Lett. 2008, 10, 437. Basson, M. M.; Holzapfel, C. W.; Verdoorn, G. H. Heterocycles, 1989, 29, 2261.

[13] Kitson, R. R. A.; Millemaggi, A.; Taylor, R. J. K. Angew. Chem. Int. Ed. 2009, 48, 9426.

[14] For discussion of solvent effects in enolate alkylation, see: Trost, B. M.; Xu, J. J. Org. Chem. 2007, 72, 9372 and references cited therein.

[15] Compound $\mathbf{3 5 3}$ was kindly provided by Claire Turner. The details of the synthesis of $\mathbf{3 5 3}$ will be reported in due course.

[16] Silverman, R. B. The Organic Chemistry of Drug Design and Action; Elsevier Academic Press, 2004.

[17] Rosengren, A. M.; Karlsson, B. C. G.; Nicholls, I. A. ACS Med. Chem. Lett. 2012, 3, 650.

[18] Thaisrivongs, S.; Romero, D.; Tommasi, R.; Janakiraman, M.; Strohbach, J.; Turner, S.; Biles, C.; Morge, R.; Johnson, P.; Aristoff, P.; Tomich, P.; Lynn, J.; Horng, M.; Chong, K.; Hinshaw, R.; Howe, W.; Finzel, B.; Watenpaugh, K. J. Med. Chem. 1996, 39, 4630.

[19] Jung, M.; Min, S. Tetrahedron 2007, 63, 3682.

[20] Kohno, J.; Hirano, N.; Sugawara, K.; Nishio, M.; Hashiyama, T.; Nakanishi, N.; Komatsubara, S. Tetrahedron 2001, 57, 1731. F urstner, A.; Feyen, F.; Prinz, H.; Waldmann, H. Tetrahedron 2004, 60, 9543.

[21] Vyvyan, J. Tetrahedron 2002, 58, 1631.

[22] Kraus, G.; Zhang, N. J. Org. Chem. 2000, 65, 5644. 
[23] For an example, see: O’Malley, S. J.; Tan, K. L.; Watzke, A.; Bergman, R. G.; Ellman, J. A. J. Am. Chem. Soc. 2005, 12\%, 13496.

[24] Basson, M. M.; Holzapfel, C. W.; Verdoorn, G. H. Heterocycles 1989, 29, 2261.

[25] Krohn, K.; Ludewig, K.; Aust, H. J.; Draeger, S.; Schulz, B. J. Antibiot. 1994, 47, 113.

[26] Mohammed, A. A.; Bhogal, N.; Findlay, J. B. C.; Fishwick, C. W. G. J. Med. Chem. 2005, 48, 5655

[27] Bäckvall, J.-E.; Byström, S. E.; Nordberg, R. E. J. Org. Chem. 1984, 49, 4619

[28] Xiao, Z-. P.; Shi, D-. H.; Li, H-. Q.; Zhang, L-. N.; Xu, C.; Zhu, H-. L. Bioorg. Med. Chem. 2007, $15,3707$.

[29] Lau, C. K.; Belanger, P. C.; Scheigetz, D. J. J. Org. Chem. 1987, 52, 1670.

[30] Buckle, D. R.; Cantello, B. C. C.; Smith, H.; Spicer, B. A. J. Med. Chem. 1975, 4, 391.

[31] Thaisrivongs, S.; Gammill, R. B.; Skulnick, H. I.; Zhang, Q.; Tommasi, R. A.; Piper, R. C.; Johnson, P. D.; Aristoff, P. A.; Strohbach, J. W.; Turner, S. R.; Skaletzki, L. L.; Romero, D. L.; Yang, C.- P.; Pyran-2ones and 5,6-dihydropyran-2-ones useful for treating hyperplasia and other diseases. European Patent EP 1203770, May 8, 2002. Thaisrivongs, S.; Romero, D. L.; Tommasi, R. A.; Janakiraman, M. N.; Strohbach, J. W.; Turner, S. R.; Biles, C.; Morge, R. R.; Johnson, P. D.; Aristoff, P. A.; Tomich, P. K.; Lynn, J. C.; Horng, M.- M.; Chong, K.- T.; Hinshaw, R. R.; Howe, W.; J.; Finzel, B. C.; Watenpaugh, K. D. J. Med. Chem. 1996, 39, 4630 .

[32] Tummatorn, J.; Dudley, G. B. J. Am. Chem. Soc. 2008, 130, 5050.

[33] Schmid, C. R.; Bryant, J. D.; Dowlatzedah, M.; Phillips, J. L.; Prather, D. E.; Schantz, R. D.; Sear, N. L.; Vianco, C. S. J. Org. Chem. 1991, 56, 4056. 
Chapter 8

${ }^{13}$ C NMR Analysis of 3,6-Dihydro-2 $H$-pyrans:

Assignment of Remote

Stereochemistry using Axial

Shielding Effects 


\subsection{Introduction}

Relative stereochemical relationships have a profound effect on the chemical and physical properties of a compound. ${ }^{1}$ Complex molecules often exhibit remarkable differences in biological activity, reactivity and catalysis with the inversion of just a single stereocenter. The assignment of atom connectivity and spatial arrangement is, therefore, of fundamental importance, and NMR spectroscopy has emerged as a powerful tool for structure elucidation. ${ }^{2}$ However, a number of structural motifs still prove to be particularly challenging and laborious to assign. ${ }^{3}$ The distinctive structural properties of 3,6-dihydro- $2 H$-pyrans (i.e. 370) represent just such a challenge, ${ }^{4}$ and new methods for NMR-based stereochemical assignment are needed to facilitate the use of these compounds in chemical research. ${ }^{5}$ The preparation and use of various 3,6-dihydro-2 $H$-pyrans as substrates for Pd-catalyzed allylic alkylation cascades (see Section 7.2) led us to this challenge and we ultimately discovered that the analysis of axial deshielding effects could be used to assign the remote relative stereochemistry across the pyran ring system.

The utility of 3,6-dihydro- $2 H$-pyrans stems from their facile preparation in enantioenriched form and a wide range of potential reactivity (Figure 8.1). Enantioenriched 3,6-dihydro$2 \mathrm{H}$-pyrans can be prepared from a variety of sources, including chiral allylic sulfoxides $(\mathbf{3 7 1}),{ }^{6}$ furfuryl alcohols $(\mathbf{3 7 2}),{ }^{7}$ glycals $(\mathbf{3 7 3}),{ }^{8}$ and gem-dichlorocyclopropanes $(\mathbf{3 7 4}),{ }^{9}$ as well as by enzymatic kinetic resolution. ${ }^{10}$ This heterocyclic motif is present in a number

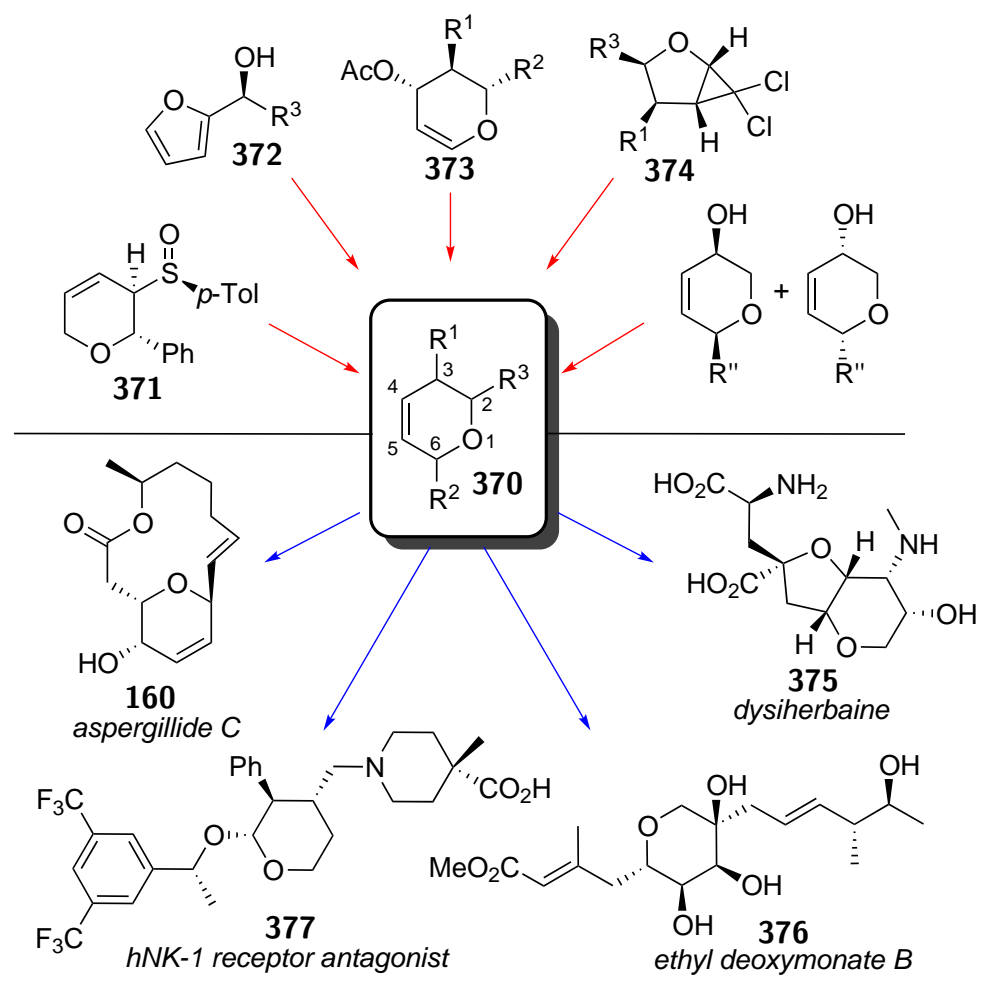

Figure 8.1 Synthesis and Utility of 3,6-dihydro-2H-pyrans. 
of natural products, such as aspergillide $\mathrm{C}(\mathbf{1 6 0}),{ }^{11}$ and also serves as a robust synthetic intermediate. ${ }^{12}$ The versatility of 3,6-dihydro- $2 H$-pyrans has enabled the synthesis of a number of important synthetic targets such as dysiherbaine $(\mathbf{3 7 5}),{ }^{13}$ ethyl deoxymonate B (376), ${ }^{14}$ and the hNK-1 receptor antagonist $377 .{ }^{15}$ However, the utility of 3,6-dihydro- $2 H$-pyrans is often impeded by the challenging task of assigning the relative stereochemistry of two substituents located on opposite sides of the ring system. Previous stereochemical assignments have relied on derivatization and, in specific cases, the use of NOE correlations and ${ }^{3} J_{\mathrm{HH}}$ coupling constants. ${ }^{16}$

Research into the total synthesis of oxylipin natural products (i.e. labillarides E-H) led us to prepare dihydropyran 332 as a synthetic intermediate (Scheme 8.1). Preliminary ${ }^{1} \mathrm{H}-$

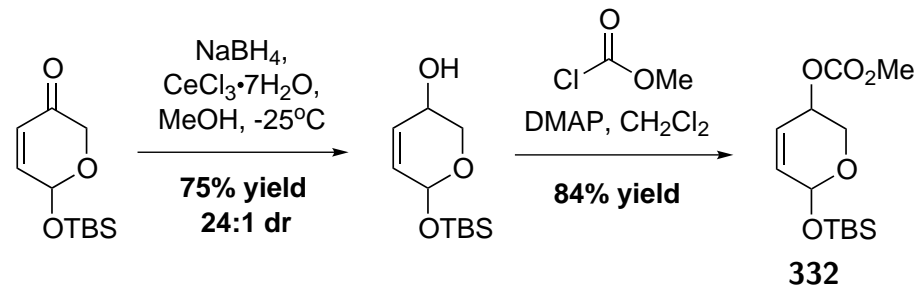

Scheme 8.1 Preparation of Dihydropyran 332.

NMR analysis of this compound did not provide sufficient evidence to deduce the relative stereochemistry at C3 and C6, as the alkene and ring oxygen limit what could be inferred from ${ }^{3} J_{\mathrm{HH}}$ coupling. Additionally, the divalent oxygen and planarity of the alkene remove a number of potential 1,3-diaxial interactions and, therefore, the effectiveness of NOE correlation is limited in compounds having substitution patterns that cause bias towards the ${ }^{2} \mathrm{C}_{\mathrm{O}}$ cis-332 or ${ }^{\mathrm{O}} \mathrm{C}_{2}$ trans-332 conformers (Figure 8.2). ${ }^{\dagger}$ These conformations are predominant in compounds with an electronegative atom bound to $\mathrm{C} 6$, due to the anomeric effect. ${ }^{17}$ To overcome these challenges we sought to develop a facile method for the assignment of relative stereochemistry by analyzing the shielding effects of axial substituents on the ${ }^{13} \mathrm{C}$ NMR resonances of the 3,6-dihydro- $2 H$-pyran ring system.

\subsection{Conformational Analysis and ${ }^{13} \mathrm{C}$ NMR Shielding Effects}

${ }^{13} \mathrm{C}$ NMR spectroscopy has been used previously to deduce the stereochemistry of highly substituted ring systems, such as carbohydrates and inositols. ${ }^{20}$ Comparing the spectra of two inositol diastereomers, scyllo- (378) and myo-inositol (379), illustrates the shielding effects of an axial hydroxyl group on the carbons within the ring system of $\mathbf{3 7 9}$ (Figure 8.3). The $\gamma$-position experiences the largest shielding and is shifted upfield by $2.6 \mathrm{ppm}$. Additionally, the $\alpha$ - and $\beta$-positions experience moderate shielding and the $\delta$-position actually becomes slightly deshielded and experiences a downfield shift of $0.6 \mathrm{ppm}$. In carbohydrates and inositols, these observations have been referred to as the $\gamma$-substituent effect. ${ }^{21}{ }^{13} \mathrm{C}$ NMR resonances are

\footnotetext{
${ }^{\dagger}$ A weak NOE correlation to both $\mathrm{H} 2 \mathrm{a}$ and $\mathrm{H} 2 \mathrm{~b}$ was observed upon irradiation of $\mathrm{H} 6$ in $\mathbf{3 3 2}$
} 


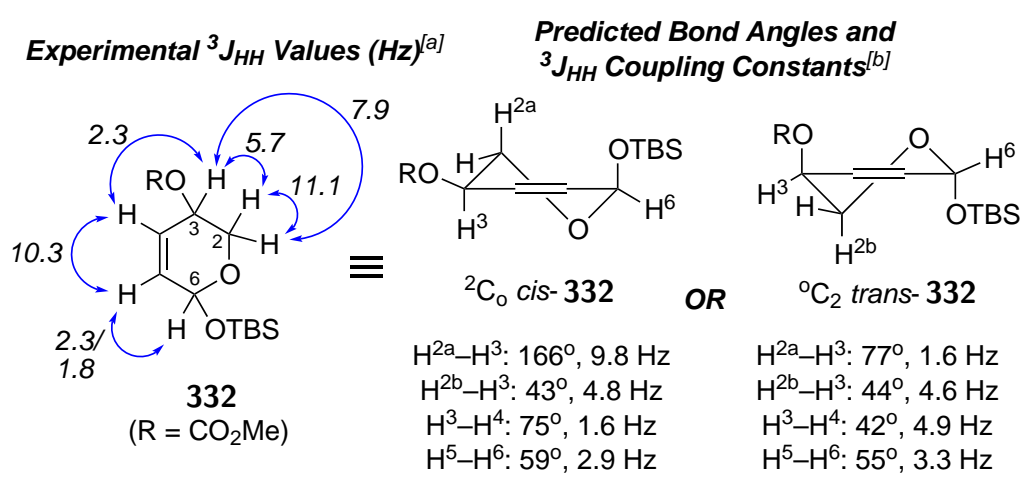

[a] ${ }^{1} \mathrm{H}$ NMR (500 MHz, $\mathrm{CDCl}_{3}$ ) [b] Geometry optimization was performed on the two lowest energy half-chair conformers using Gaussian '09 ${ }^{18}$ (mPW1PW91, TZVP) and ${ }^{3} J_{\mathrm{HH}}$ values were calculated using a modified Karplus equation. ${ }^{19}$ [c] The compound described was ultimately proven to be cis-332.

Figure 8.2 Challenges in the Structural Assignment of Dihydropyran 332.

\begin{tabular}{|c|c|c|c|}
\hline & & & \\
\hline $\mathrm{OH}$ & $\mathrm{HO} \beta\left[\begin{array}{l}\mathrm{OH} \\
\operatorname{lor}\end{array}\right.$ & $\delta_{C}(p p m)$ & $\delta_{c}($ scyllo $)-\delta_{c}(m y o)$ \\
\hline & & $\alpha: 73.2$ & 1.5 \\
\hline 'OH & U & $\beta: 73.4$ & 1.3 \\
\hline & $\mathrm{OH}$ & $\gamma: 72.1$ & 2.6 \\
\hline $\begin{array}{c}378 \\
\text { scyllo-Inositol }\end{array}$ & $\begin{array}{c}379 \\
\text { myo-Inositol }\end{array}$ & $\delta: 75.3$ & -0.6 \\
\hline $\mathrm{OCO}_{2} \mathrm{Me}$ & $\mathrm{OCO}_{2} \mathrm{Me}$ & $\delta_{\mathrm{C}}($ trans $)$ & $\delta_{\mathrm{C}}($ cis $)-\delta_{\mathrm{C}}($ trans $)$ \\
\hline & & $\beta^{\prime}: 61.0$ & -1.0 \\
\hline & & $\alpha: 66.9$ & 1.5 \\
\hline & & $\beta: 122.5$ & 3.9 \\
\hline ÖTBS & ÖTBS & $\gamma: 133.9$ & -1.7 \\
\hline cis- 332 & trans- 332 & $\delta: 87.9$ & 1.3 \\
\hline
\end{tabular}

Figure 8.3 Axial Shielding Effects in Inositol and 3,6-Dihydro-2H-pyran Diastereomers.

influenced by the complex interplay of steric effects, hyperconjugation and electronic motion present in a given molecule, and therefore the distorted geometry and polarization of a 3,6dihydro- $2 \mathrm{H}$-pyran ring results in significantly different axial shielding effects, relative to that observed with the inositol diastereomers. ${ }^{25}$ To interpret the ${ }^{13} \mathrm{C}$ shielding effects of pseudo-axial substituents on a 3,6-dihydro- $2 H$-pyran ring, one must first understand the conformational bias inherent to the ring system. ${ }^{22}$

The planar alkene and divalent oxygen leave only the $\mathrm{C} 2$ and $\mathrm{C} 6$ substituents with a potential 1,3-diaxial interaction (Figure 8.4). However, electronegative substituents at the allylic position of an unsaturated ring have been shown to have a preference for axial orientation - a phenomenon referred to as the allylic effect. ${ }^{23}$ This effect is analogous to the anomeric effect and is a result of stereoelectronic stabilization via delocalization of electron density into the $\sigma^{*}$ orbital of the exocyclic $\mathrm{C}-\mathrm{X}$ bond. Considering anomeric and allylic effects, along with the reduced number of 1,3-diaxial interactions, led us to postulate that the 3,6-trans diastereomer should prefer the diaxial conformer trans-A with a high degree of conformational homogeneity. 

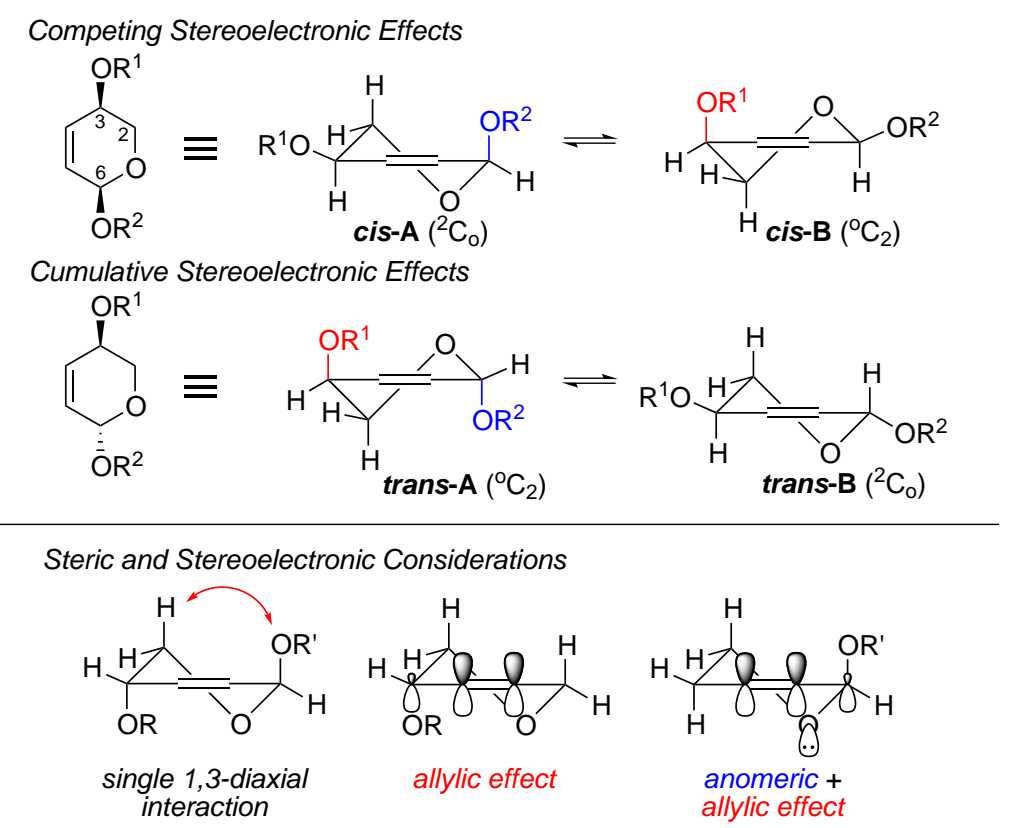

Figure 8.4 Anomeric and Allylic Effects in the Conformational Preference of 3,6 -Dihydro- $2 H$-pyrans.

Competition between allylic and anomeric effects in the 3,6-cis diastereomer is likely to result in a greater amount of conformational heterogeniety, and significant population of both cis-A and cis-B conformers. In most cases, the stereoelectronic benefits experienced by an axial C6 electronegative substituent should dictate the major conformer. This conformational hypothesis is supported by the ${ }^{1} \mathrm{H}-\mathrm{NMR}$ spectra of the diastereomeric pyrans, cis-332 and trans-332 (Figure 8.5). The 3,6-trans diastereomer contains diastereotopic protons at C2 that are significantly differentiated from each other. This is presumably a result of one proton residing primarily in the sterically congested axial position. In contrast, the ${ }^{1} \mathrm{H}-\mathrm{NMR}$

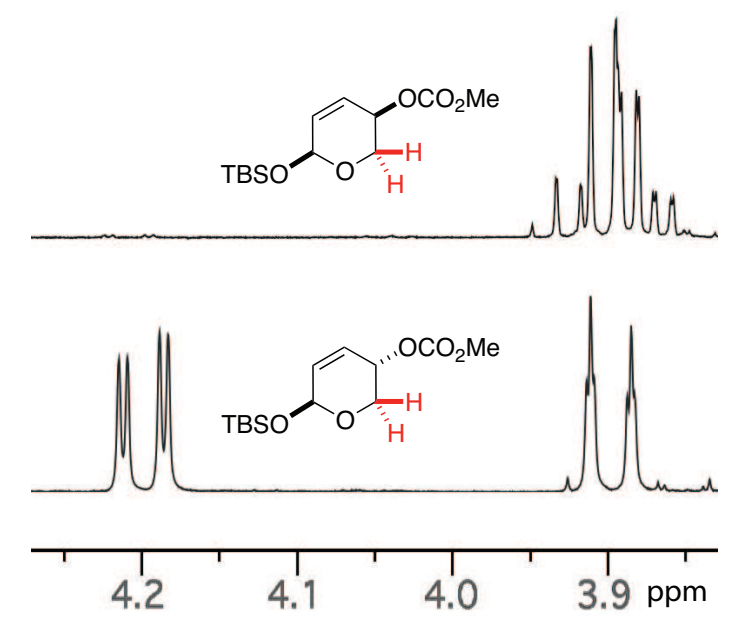

Figure 8.5 Conformational Insights from ${ }^{1} \mathrm{H}-\mathrm{NMR}$. 
spectrum of the 3,6-cis diastereomer shows the oxymethylene peaks have significant overlap. Their appearance as an overlapping multiplet suggests a higher degree of conformational heterogeneity, although additional conformational differences may also be a contributing factor. ${ }^{24}$

Ultimately, the observed ${ }^{13} \mathrm{C}$ chemical shifts represent a weighted average of conformers, and therefore, the magnitude of axial shielding is dependent on the conformational distribution of a compound. Dihydropyran trans-332, with a strong preference for the diaxial conformation (trans-A), should experience ${ }^{13} \mathrm{C}$ shielding effects from both substituents. However, cis332, with a greater amount of conformational heterogeneity and a single axial substituent in each conformer, should experience significantly less shielding of the ring carbons relative to trans-332. To evaluate the magnitude of axial shielding on each ring carbon the inductive influences of the substituents need to be removed. This can be achieved, to a certain extent, by subtracting the chemical shifts of the two diastereomers (i.e. Figure 8.3). The difference in the ${ }^{13} \mathrm{C}$ chemical shifts of cis-332 and trans-332 show a clear difference in shielding around the 3,6-dihydro- $2 \mathrm{H}$-pyran ring and provides a diagnostic shielding pattern for the assignment of relative stereochemistry in similar compounds (Figure 8.6).

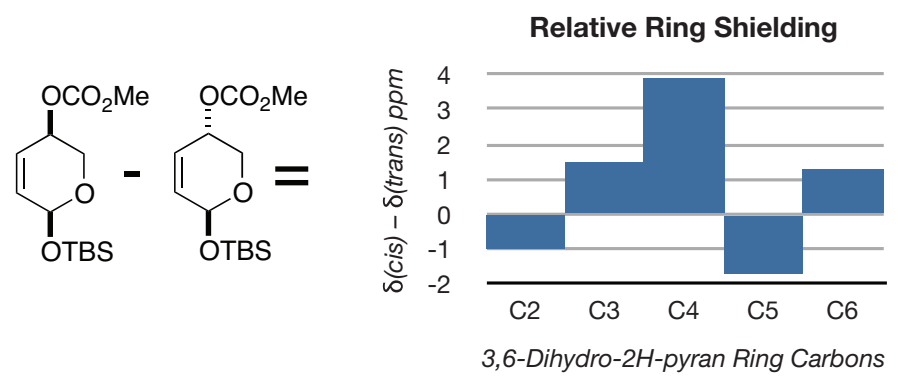

Figure 8.6 A Diagnostic Axial Shielding Pattern in 3,6-Dihydro- $2 H$-pyrans: $\delta_{\mathrm{C}}($ cis-332) $\delta_{\mathrm{C}}($ trans-332).

\subsection{Assignment of Relative Stereochemistry using a ${ }^{13} \mathrm{C}$ NMR Shielding Template}

To evaluate the utility of a shielding template in the assignment of relative stereochemistry in 3,6-dihydro- $2 H$-pyrans, a range of diastereomeric pairs were investigated (Table 8.1 ). ${ }^{* *}$ In general, substrates with oxygen-tethered substituents at C3 and C6 fit all five positions of the template very well. The largest deviation from the average shielding values are seen at $\mathrm{C} 2$ and C5, which have root-mean-squared (RMS) differences of 0.43 and $0.29 \mathrm{ppm}$, respectively. Interestingly, the varying inductive and steric effects of different substituents, $\mathrm{R}^{1}$ and $\mathrm{R}^{2}$, caused significant changes in the ${ }^{13} \mathrm{C}$ chemical shifts of the respective ring carbons and, in some cases, even caused $\mathrm{C} 4$ to have a higher chemical shift than C5. This meant that no reliable

\footnotetext{
${ }^{* *}$ Details of the synthesis of these compounds can be found in Section 8.6
} 
Table 8.1 Development of a ${ }^{13} \mathrm{C}$ NMR Shielding Template.

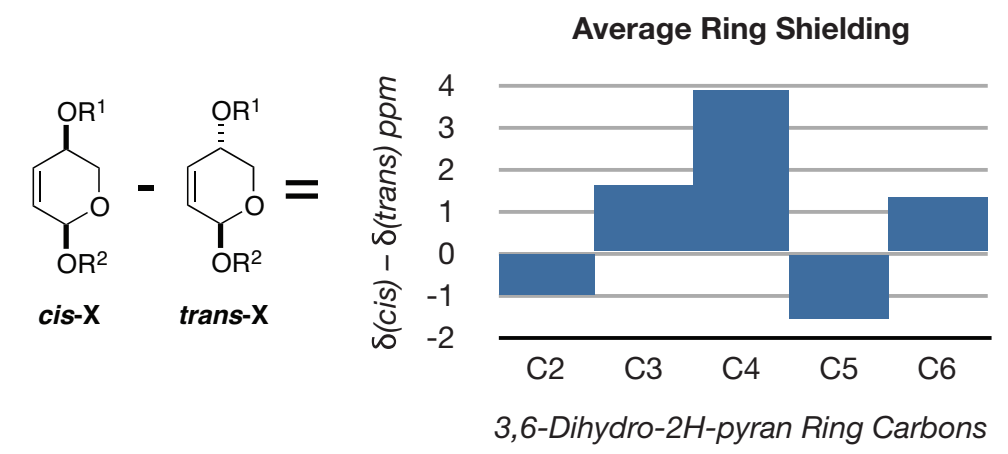

\begin{tabular}{|c|c|c|c|c|c|c|c|}
\hline \multirow{2}{*}{$\mathrm{X}$} & \multicolumn{2}{|c|}{ Substrates } & \multicolumn{5}{|c|}{$\delta($ cis $)-\delta($ trans $)$ in $\mathrm{ppm}^{[\mathrm{a}]}$} \\
\hline & $\mathrm{R}^{1}$ & $\mathrm{R}^{2}$ & $\mathrm{C} 2$ & C3 & $\mathrm{C} 4$ & $\mathrm{C} 5$ & $\mathrm{C} 6$ \\
\hline 380 & $\mathrm{CO}_{2} \mathrm{Me}$ & TBS & -1.0 & 1.5 & 3.9 & -1.7 & 1.3 \\
\hline 381 & $\mathrm{Ac}$ & TBS & -0.6 & 1.5 & 3.7 & -1.2 & 1.5 \\
\hline $382^{[\mathrm{b}]}$ & Ac & $i \operatorname{Pr}$ & -1.3 & 1.6 & 3.9 & -1.8 & 1.2 \\
\hline 383 & Ac & $n \operatorname{Pr}$ & -1.1 & 1.6 & 3.8 & -1.6 & 1.4 \\
\hline 384 & Ac & Allyl & -1.2 & 1.7 & 3.9 & -1.7 & 1.3 \\
\hline 385 & Ac & $\mathrm{CH}_{2} \mathrm{CCH}$ & -1.5 & 1.8 & 4.2 & -1.9 & 1.0 \\
\hline 386 & Ac & $\mathrm{CH}_{2} \mathrm{Ph}$ & -1.3 & 1.7 & 4.2 & -1.6 & 1.3 \\
\hline \multirow[t]{2}{*}{$387^{[\mathrm{b}]}$} & $\mathrm{CO}_{2} \mathrm{Me}$ & $\mathrm{H}$ & -0.1 & 1.5 & 3.4 & -1.0 & 1.6 \\
\hline & \multicolumn{2}{|c|}{ RMS error $^{[c]}$} & 0.43 & 0.1 & 0.24 & 0.29 & 0.17 \\
\hline
\end{tabular}

\begin{abstract}
[a] Standard Conditions: ${ }^{13} \mathrm{C}$ NMR $\left(126 \mathrm{MHz}, \mathrm{CDCl}_{3}\right)$ chemical shifts were measured relative to $\mathrm{CDCl}_{3}$ and reported to 1 decimal place. All carbon assignments are based on ${ }^{1} \mathrm{H} /{ }^{1} \mathrm{H}$ COSY and ${ }^{1} \mathrm{H} /{ }^{13} \mathrm{C}$ HSQC spectra. A full table of ${ }^{1} \mathrm{H}$ and ${ }^{13} \mathrm{C}$ NMR assignments can be found in Section 8.6.3. Initial stereochemical assignments were based on literature data and reaction precedent. [b] Diastereomers were not separated prior to NMR analysis. [c] RMS error relative to the average ${ }^{13} \mathrm{C}$ shielding values.
\end{abstract}

conclusions could be drawn from the magnitude of isolated ${ }^{13} \mathrm{C}$ NMR shifts. Despite the variance in net chemical shift, the relative difference used to evaluate axial shielding remained relatively constant. The ${ }^{13} \mathrm{C}$ NMR shielding template, shown in Table 8.1, provides a facile method by which the relative stereochemistry of disubstituted 3,6 -dihydro- $2 H$-pyrans can be assigned.

This approach is particularly useful in assigning the stereochemical outcomes of transformations such as the Ferrier reaction, shown in Figure 8.7. In this case, a 3:1 mixture of diastereomers was obtained and the ${ }^{13} \mathrm{C}$ NMR resonances for the ring carbons of each diastereomer were readily assigned from the ${ }^{1} \mathrm{H} /{ }^{13} \mathrm{C}$ HSQC spectrum. Subtracting the ${ }^{13} \mathrm{C}$ resonances of each ring carbon from their diastereomeric counterpart reveals the magnitude of axial shielding. These five values were then compared to, and found to match, the shielding template from Table 8.1, to ultimately reveal the major product from this reaction to be trans-382. 


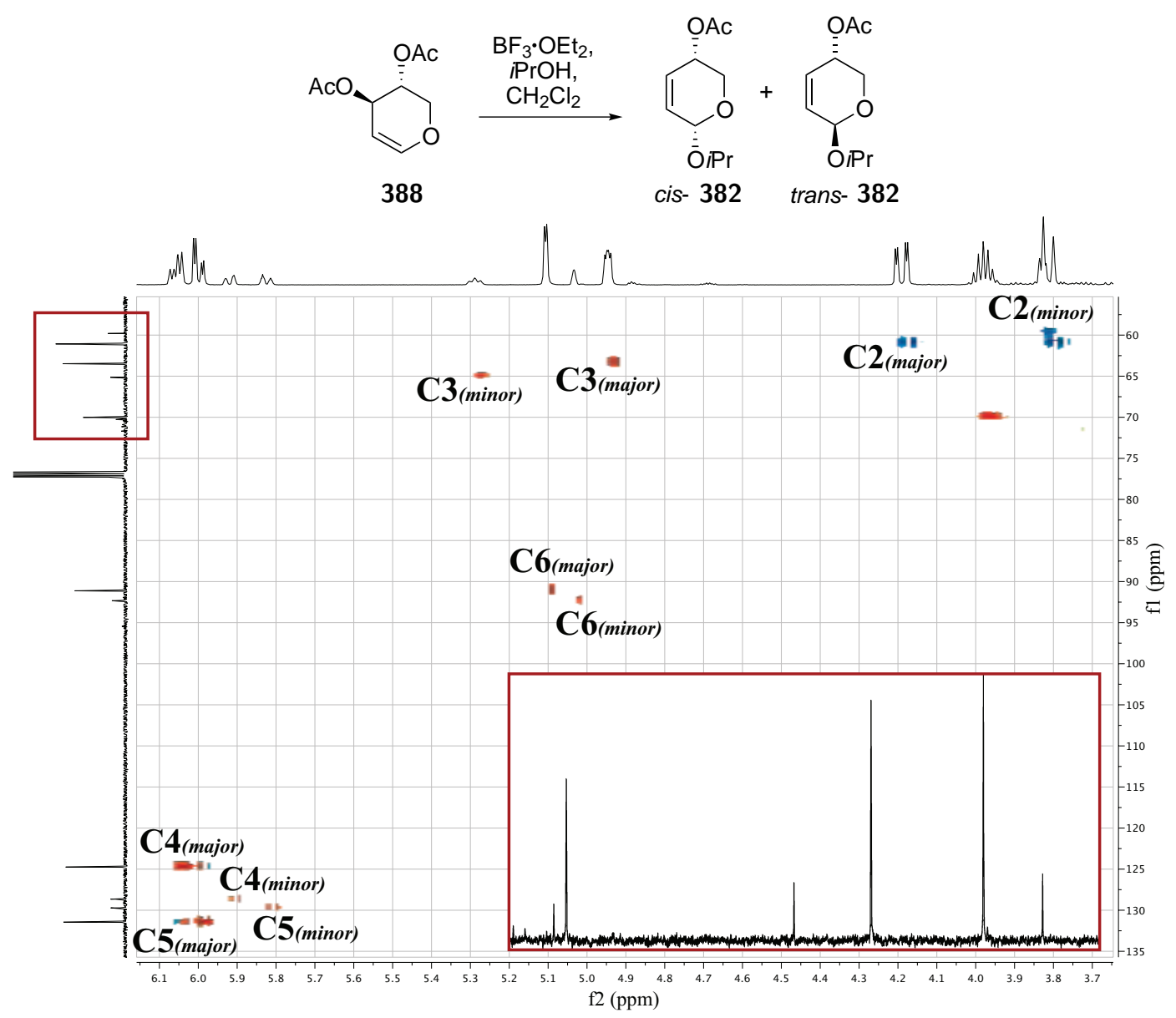

Relative Ring Shielding

\begin{tabular}{|c|cc|r|}
\hline \multirow{2}{*}{ Ring Carbon } & \multicolumn{2}{|c|}{$\delta_{\mathbf{C}}(\mathbf{p p m})$} & \multirow{2}{*}{ Minor } \\
& Major & \\
\hline C2 & 59.8 & 61.1 & -1.3 \\
C3 & 65.1 & 63.5 & 1.6 \\
C4 & 128.7 & 124.8 & 3.9 \\
C5 & 129.7 & 131.5 & -1.8 \\
C6 & 92.3 & 91.1 & 1.2 \\
\hline
\end{tabular}

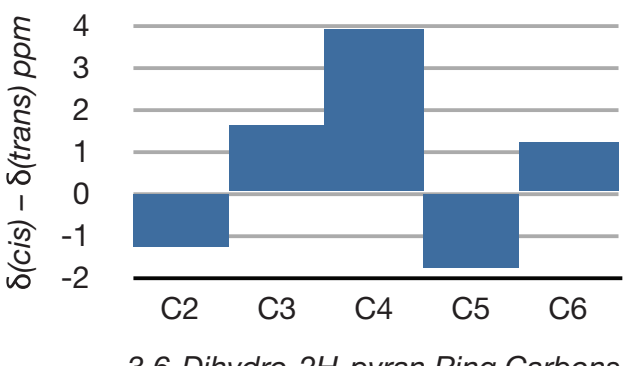

Figure 8.7 Assignment of Relative Stereochemistry using the Axial Shielding Template: A Worked Example. 
Given the propensity of certain carbons in the dihydropyran ring to vary more than others, we propose the use of the RMS error, from Table 8.1, for each carbon to evaluate how well a given comparison matches the shielding template (Table 8.2). The diastereomeric pairs of 381-386 provide a very good match to all positions of the shielding template. The hemiacetal $\mathbf{3 8 7}$ displays some variance in ring shielding, but still has a very good match with the template at $\mathrm{C} 3$ and $\mathrm{C} 6$, and a good match at $\mathrm{C} 2, \mathrm{C} 4$, and $\mathrm{C} 5$, and therefore still provides strong evidence for the proposed relative stereochemical configuration.

Table 8.2 Using RMS Error to Evaluate Template Matching.

\begin{tabular}{c|ccccc|c}
\hline Ring Carbon & C2 & C3 & C4 & C5 & C6 & \multirow{2}{*}{ Template Match } \\
\cline { 1 - 5 } Template Value (ppm) & -1.0 & 1.6 & 3.9 & -1.6 & 1.3 & \\
\hline 2 x (RMS error) & \pm 0.86 & \pm 0.2 & \pm 0.48 & \pm 0.58 & \pm 0.34 & VERY GOOD \\
3 x (RMS error) & \pm 1.29 & \pm 0.3 & \pm 0.72 & \pm 0.87 & \pm 0.51 & GOOD \\
4 x (RMS error) & \pm 1.72 & \pm 0.4 & \pm 0.96 & \pm 1.16 & \pm 0.68 & OK
\end{tabular}

Additionally, the analysis of five discrete shielding values serves to both reinforce any stereochemical conclusions made with this method and enable the rapid detection of anomalies and inconsistencies. To a certain extent, this provides a safeguard against the misassignment of relative stereochemistry. One such anomaly was observed in compounds containing a C3 hydroxyl group (Table 8.3). The ring carbons from this series of compounds were a very good fit for the shielding template with the exception of $\mathrm{C} 2$ and $\mathrm{C} 5$, which provided a good and ok match, respectively.

Table 8.3 ${ }^{13} \mathrm{C}$ NMR Shielding Effects in C3-Hydroxy Compounds.

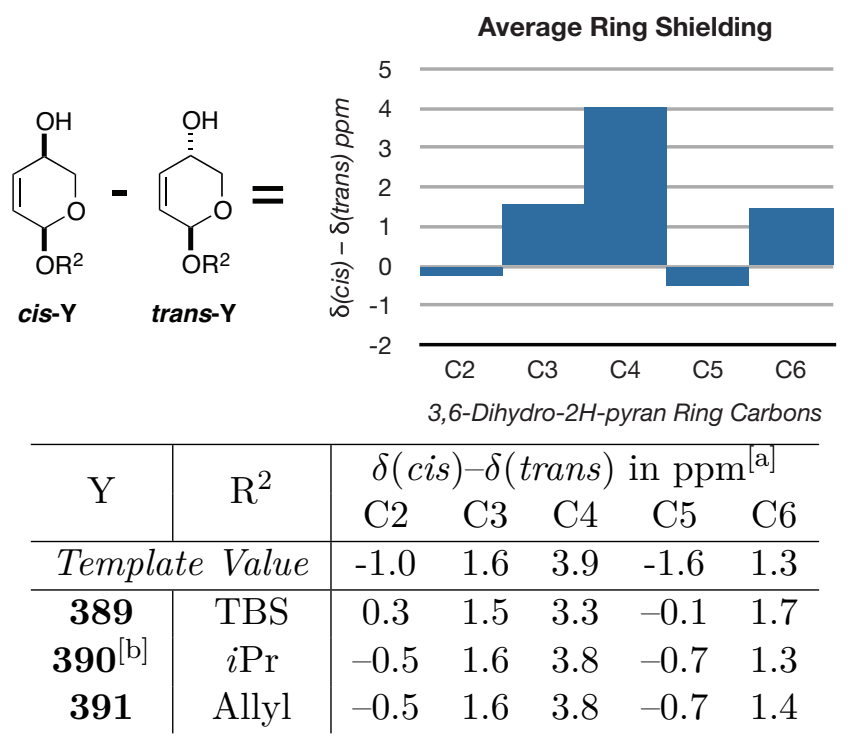

\footnotetext{
${ }^{[a]}$ Standard Conditions from Table 8.1 apply. ${ }^{1} \mathrm{H}$ and ${ }^{13} \mathrm{C}$ NMR assignments can be found in Section 8.6.3. ${ }^{[\mathrm{b}]}$ Diastereomers were not separated prior to NMR analysis.
} 
To explore the limitations of this method, a number of 3,6-dihydro- $2 \mathrm{H}$-pyrans lacking certain fundamental axial shielding properties were investigated (Table 8.4). The axial or

Table 8.4 Scope and Limitations of the ${ }^{13} \mathrm{C}$ NMR Shielding Template.

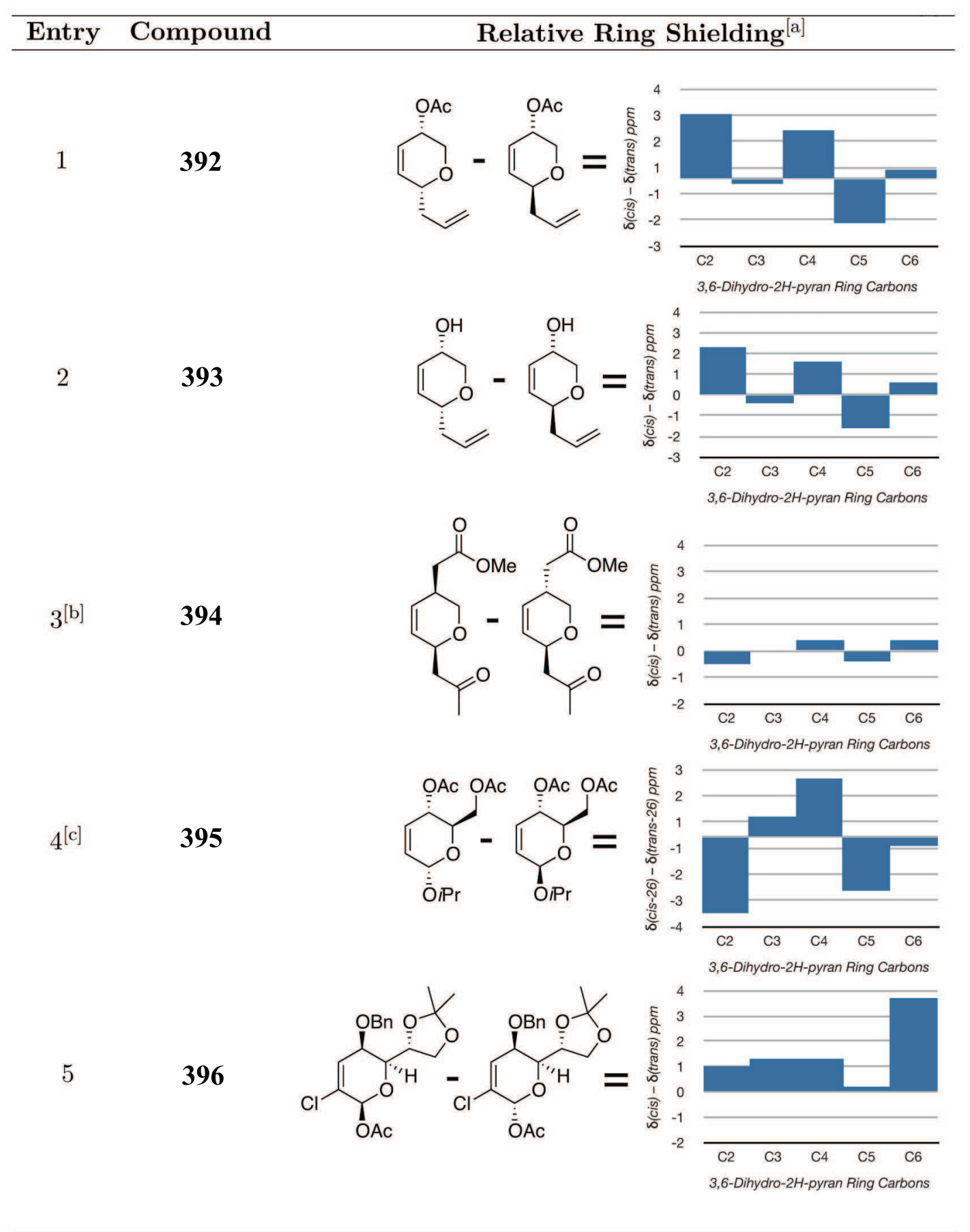

[a] Standard Conditions from Table 8.1 apply. [b] ${ }^{13} \mathrm{C}$ NMR data for cis- and trans-399 was taken from the literature. ${ }^{26}[\mathrm{c}]$ Diastereomers were not separated prior to NMR analysis. 
pseudo-axial orientation of an electronegative atom (i.e. oxygen) is a prerequisite to the observation of shielding effects, and therefore replacing one or both of these atoms with carbon not only reduces shielding effects but also removes the stereoelectronic conformational bias described previously. ${ }^{13} \mathrm{C}$ NMR analysis of 392 and 393 illustrates the significant change in the shielding pattern around the ring upon removal of the oxygen atom bound to $\mathrm{C} 6$ (Entries $1 \& 2$ ). To further this point, Entry 3 shows a drastic reduction in shielding effects when both electronegative atoms connected to the ring system are removed. In fact, the ${ }^{13} \mathrm{C}$ resonances of the diastereomers trans-394 and cis-394 show very little difference in chemical shift. Aditionally, highly substituted ring systems, such as 395 and 396, present a significantly more complex situation and therefore do not fit the current shielding template.

\subsection{Complimentary Calculations of Dihydro- $2 \mathrm{H}$-pyran ${ }^{13} \mathrm{C}$ NMR Resonances}

To extend the utility of this methodology, the use of complimentary computational methods was investigated with the aim of enabling stereochemical assignment using ${ }^{13} \mathrm{C}$ NMR data from a single diastereomer. ${ }^{27}$ Given the relatively simple nature of the 3,6-dihydro- $2 \mathrm{H}$-pyran ring system, we envisioned that density functional calculations could be used to accurately calculate ${ }^{13} \mathrm{C}$ NMR resonances of the ring carbons and distinguish the shielding effects that characterize each diastereomer. A preliminary evaluation of this method was performed by attempting to accurately calculate the ${ }^{13} \mathrm{C}$ NMR resonances of the unsubstituted parent ring system, 3,6-dihydro- $2 H$-pyran. ${ }^{28}$ GIAO NMR calculations were performed with a range of functionals and basis sets to ultimately reveal that the use of the mPW1PW91 functional ${ }^{29}$ and decontracted TZVP basis set (decTZVP), ${ }^{30}$ in conjunction with a multistandard (MSTD) referencing method, ${ }^{31}$ yielded the most accurate results (Table 8.5). ${ }^{32}$ Adding substituents to the ring system (i.e. cis- and trans-332) requires consideration and analysis of a number of possible conformers. ${ }^{33}$ Using an energy threshold of $1.5 \mathrm{kcal} / \mathrm{mol}$ above the global minimum, only the two half-chair ring conformers, ${ }^{2} \mathrm{C}_{\mathrm{o}}$ and ${ }^{\circ} \mathrm{C}_{2}$, were considered (Figure 8.8). The substituents of these ring conformers can exist in three different staggered conformations about each exocyclic $\mathrm{C}-\mathrm{O}$ bond. Thus, conformers $\mathrm{A}, \mathrm{B}$ and $\mathrm{C}$ represent the three possible staggered conformations about the $\mathrm{C} 3-\mathrm{O}$ bond, while keeping the $\mathrm{C} 6-\mathrm{O}$ bond constant. Ultimately, 4 out of a possible 18 conformers of cis-332 were found to meet the selected energy threshold. ${ }^{34}$ GIAO NMR calculations for each conformer and subsequent Boltzmann-weighted averaging provided calculated ${ }^{13} \mathrm{C}$ chemical shifts for the five carbons of the dihydropyran ring system. The calculated spectra for each diastereomer, cis-332 and trans-332, were in good agreement with the experimental data, providing CP3 and DP4 assignment probabilities in excess of $95 \%{ }^{35}$ Additionally, a root-mean-squared error of less than $2.5 \mathrm{ppm}$ was obtained for both cis- and trans-332. Interestingly, a large percentage of this error stems from the $\mathrm{sp}^{2}$ carbons and very consistent differences between calculated and experimental values were observed across a range of dihydropyrans. This observation prompted analysis of the difference between calculated 
Table 8.5 Evaluating Calculated ${ }^{13} \mathrm{C}$ NMR Chemical Shifts for Dihydro-2H-pyrans.

Calculated and Experimental ${ }^{13} \mathrm{C}$ NMR Chemical Shifts ${ }^{[a]}$

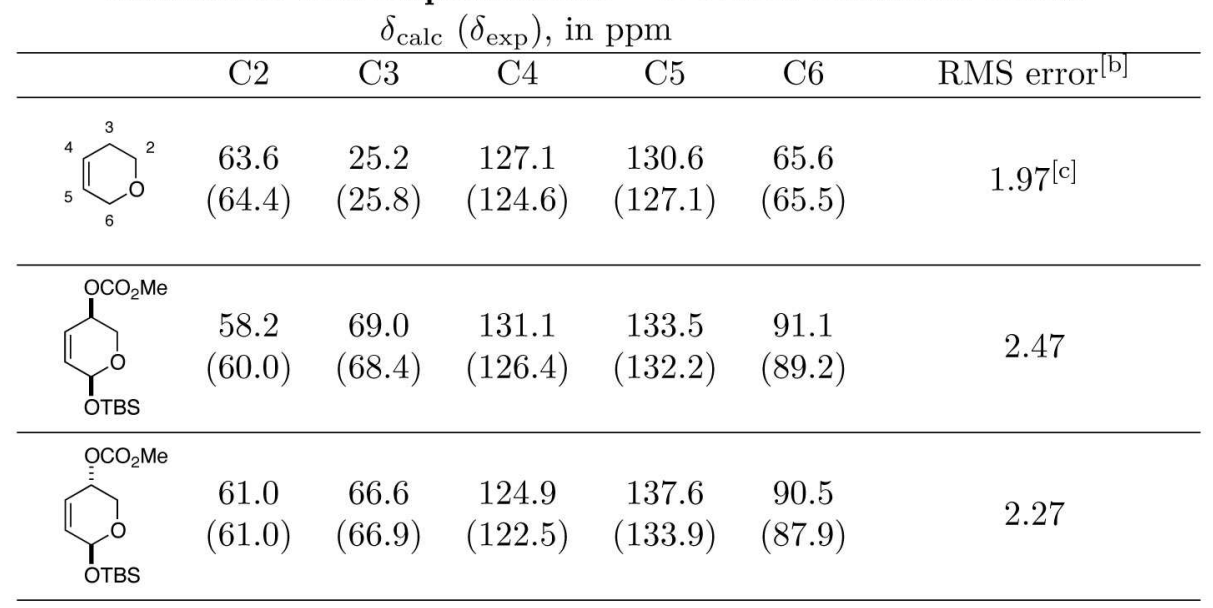

Average Difference Between Calculated and Experimental Data ${ }^{[\mathrm{d}]}$

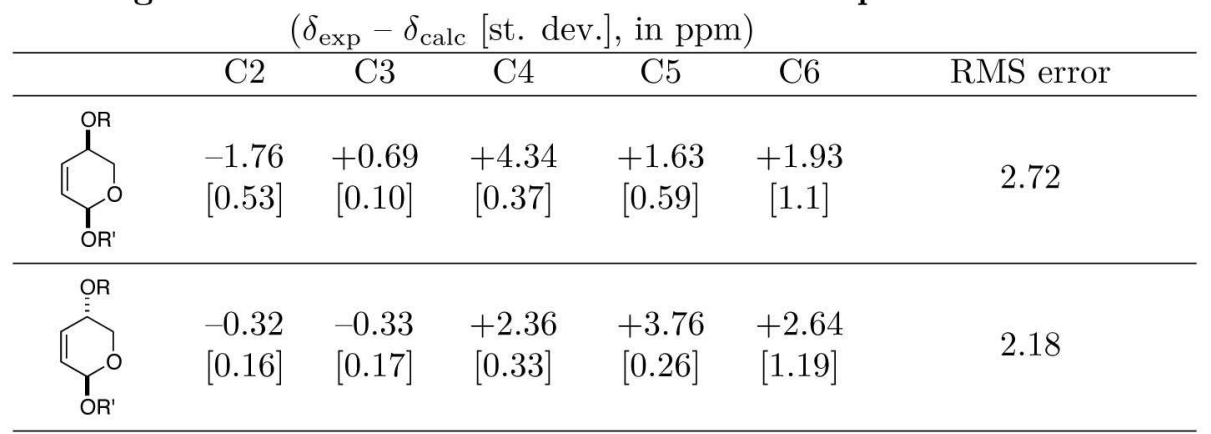

[a] GIAO ${ }^{13} \mathrm{C}$ NMR calculations were performed using Gaussian '09 at the mPW1PW91/TZVP//mPW1PW91/decTZVP level on conformers within $1.5 \mathrm{kcal} / \mathrm{mol}$ of the global minima (4 conformers for each cis-diastereomer and 2 conformers for each transdiastereomer). These data were subjected to Boltzmann averaging, where appropriate, to provide the results shown. See Appendix A for full details of the computational methodology used. [b] Average RMS error between experimental and calculated ${ }^{13} \mathrm{C}$ chemical shifts. $[\mathrm{c}]{ }^{13} \mathrm{C}$ NMR data for 3,6-dihydro- $2 H$-pyran was taken from the literature. ${ }^{28}$ [d] $\mathrm{R}$ and $\mathrm{R}$ ' represent the cis- and trans-distereomers from Table 8.1 (i.e. compounds 332, 381-387). 
and experimental data for all 16 dihydropyrans reported in Table 8.1. These data enabled the calculation of a correction constant for each position of the 3,6-dihydro- $2 \mathrm{H}$-pyran ring, thus enabling highly accurate ${ }^{13} \mathrm{C}$ NMR calculations.

The 3,6-dihydro- $2 H$-pyrans 397,398 , and 399 were obtained as single diastereomers during research into Pd-catalyzed allylic alkylation cascades (for additional details, see Chapter 7). With characterization data from only one diastereomer, shielding effects could not be evaluated by comparison of ${ }^{13} \mathrm{C}$ NMR resonances from the two possible diastereomers, as shown previously. To overcome this problem the aforementioned computational methodology was used to predict the absolute chemical shifts of both the cis- and trans-diastereomers. The differential nature of axial shielding on the ring carbons provides the opportunity to match experimental and calculated ${ }^{13} \mathrm{C}$ resonances with a high degree of confidence (Table 8.6). Computational predictions of the ${ }^{13} \mathrm{C}$ chemical shifts for the dihydropyran ring were found to be in good agreement with experimental data, and provided a DP4 probability in excess of $95 \%$ for all three compounds. This statistical analysis differs from more traditional error calculations (e.g. RMS error) in that a larger weighting is placed on calculated values with a greater difference from experimental values. Thus, data pertaining to $\mathrm{C} 4$ and $\mathrm{C} 5$, which have the greatest difference between experimental and calculated values, are considered the most important points of comparison for compounds 397-399. ${ }^{36}$ Additionally, by applying the appropriate set of correction constants for either the cis- or trans- diastereomer (from Table 8.5) a good match (in most cases $<1 \mathrm{ppm}$ difference) with the experimental data could also be obtained.

Overall, by combining the direct comparison of ${ }^{13} \mathrm{C}$ NMR calculations with an awareness of how axial shielding differentiates the ring carbons of the cis- and trans-3,6-dihydro- $2 \mathrm{H}$-pyran diastereomers, robust evidence to support the assignment of relative stereochemistry can be obtained with experimental data from just one diastereomer. 


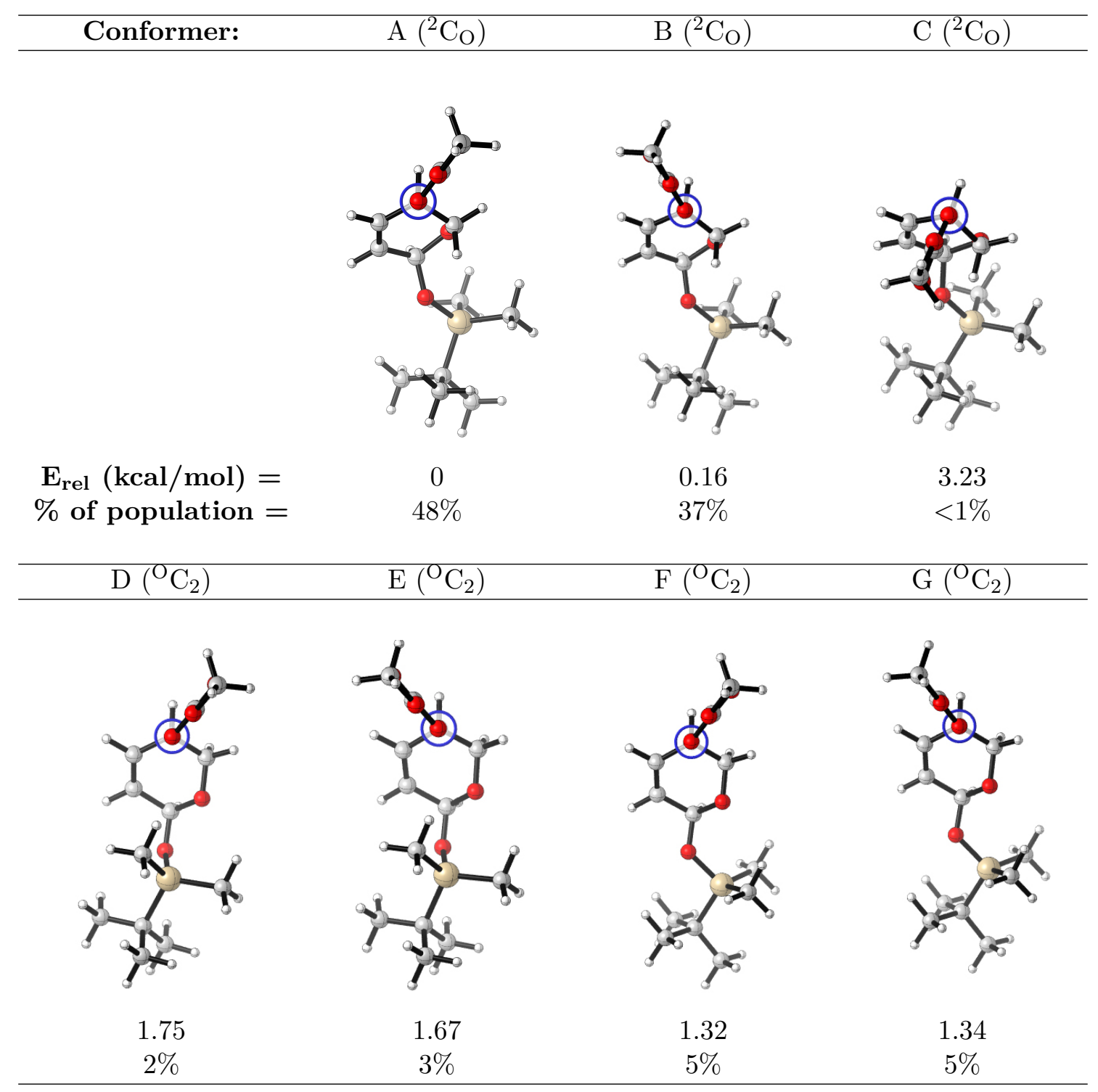

Figure 8.8 Newman Projections for the Lowest Energy Conformers of cis-332. 
Table 8.6 Assignment of Relative Stereochemistry with NMR Data from a Single Diastereomer.

\begin{tabular}{|c|c|c|c|c|c|c|c|}
\hline Entry & Substrate & & Exp. ${ }^{[a]}$ & Calc. ${ }^{[b]}$ & Corr. ${ }^{[c]}$ & Other $^{[\mathrm{b}]}$ & $\mathrm{DP}_{4}{ }^{[\mathrm{d}]}$ \\
\hline 1 & $\mathrm{OBz}$ & $\begin{array}{l}\mathrm{C} 2 \\
\mathrm{C} 3 \\
\mathrm{C} 4 \\
\mathrm{C} 5 \\
\mathrm{C} 6\end{array}$ & $\begin{array}{c}61.4 \\
64.0 \\
123.3 \\
133.4 \\
88.0\end{array}$ & $\begin{array}{c}61.3 \\
63.6 \\
125.6 \\
137.2 \\
90.4\end{array}$ & $\begin{array}{c}61.6 \\
63.9 \\
123.2 \\
133.4 \\
87.7\end{array}$ & $\begin{array}{c}59.6 \\
65.8 \\
130.7 \\
134.7 \\
91.9\end{array}$ & $98.9 \%$ \\
\hline 2 & & $\begin{array}{l}\mathrm{C} 2 \\
\mathrm{C} 3 \\
\mathrm{C} 4 \\
\mathrm{C} 5 \\
\mathrm{C} 6\end{array}$ & $\begin{array}{c}60.0 \\
68.5 \\
126.4 \\
132.2 \\
89.2\end{array}$ & $\begin{array}{c}59.2 \\
68.5 \\
130.4 \\
134.5 \\
91.7\end{array}$ & $\begin{array}{c}61.0 \\
67.8 \\
126.1 \\
132.9 \\
89.8\end{array}$ & $\begin{array}{c}60.9 \\
66.3 \\
124.8 \\
137.6 \\
90.3\end{array}$ & $97.4 \%$ \\
\hline $3^{[\mathrm{e}]}$ & OTBS & $\begin{array}{l}\mathrm{C} 2 \\
\mathrm{C} 3 \\
\mathrm{C} 4 \\
\mathrm{C} 5 \\
\mathrm{C} 6\end{array}$ & $\begin{array}{c}59.8 \\
68.6 \\
125.2 \\
132.7 \\
89.3\end{array}$ & $\begin{array}{c}58.9 \\
68.5 \\
127.8 \\
134.8 \\
91.4\end{array}$ & $\begin{array}{c}60.7 \\
67.8 \\
123.5 \\
133.2 \\
89.5\end{array}$ & $\begin{array}{c}59.7 \\
66.0 \\
123.0 \\
138.6 \\
90.1\end{array}$ & 98.9 \\
\hline
\end{tabular}

KEY: Exp. = Experimental ${ }^{13} \mathrm{C}$ NMR resonacnes, Calc. = Calculated ${ }^{13} \mathrm{C}$ NMR resonances, Corr. = Corrected Calculated ${ }^{13} \mathrm{C}$ NMR resonances, Other $=$ Calculated ${ }^{13} \mathrm{C}$ NMR resonances for the other possible diastereomer, DP4 $=\mathrm{DP} 4$ probability that the calculated ${ }^{13} \mathrm{C}$ NMR resonances match the experimental data.

[a] All carbon assignments are based on ${ }^{1} \mathrm{H} /{ }^{1} \mathrm{H}$ COSY and ${ }^{1} \mathrm{H} /{ }^{13} \mathrm{C}$ HSQC spectra. A full table of ${ }^{1} \mathrm{H}$ and ${ }^{13} \mathrm{C}$ NMR assignments can be found in Section 8.6.3. [b] GIAO ${ }^{13} \mathrm{C}$ NMR calculations were performed using Gaussian '09 at the mPW1PW91/TZVP//mPW1PW91/decTZVP level on conformers within $1.5 \mathrm{kcal} / \mathrm{mol}$ of the global minima. These data were subjected to Boltzmann averaging, where appropriate, to provide the results shown. See Appendix A for full details of the computational methodology used. [c] cis-Correction constants $(\mathrm{ppm}): \mathrm{C} 2=1.76, \mathrm{C} 3=-0.69, \mathrm{C} 4=-$ 4.34, $\mathrm{C} 5=-1.63, \mathrm{C} 6=-1.93$. trans-Correction constants $(\mathrm{ppm}): \mathrm{C} 2=0.32, \mathrm{C} 3=0.33, \mathrm{C} 4=-2.36, \mathrm{C} 5$ $=-3.76, \mathrm{C} 6=-2.64$. [d] The DP4 probability of the uncorrected calculated ${ }^{13} \mathrm{C}$ NMR resonances was calculated using the web-based applet found at http://www-jmg.ch.cam.ac.uk/tools/nmr/DP4/. ${ }^{35}$ [e] This pyrone containing 3,6-dihydro- $2 H$-pyran required consideration of only one cis-conformer as the others were outside of the $1.5 \mathrm{kcal} / \mathrm{mol}$ energy threshold. 


\subsection{Summary}

In conclusion, the use of axial shielding magnitudes in the assignment of remote relative stereochemistry across 3,6-dihydro- $2 H$-pyran ring systems has been demonstrated. This approach enables the expeditious characterization of products from stereoselective reactions such as the Ferrier reaction. Computational analysis has provided a rigorous understanding of the conformational distribution of these compounds and enabled the accurate prediction of ${ }^{13} \mathrm{C}$ chemical shifts within the pyran ring. Consequently, reliable stereochemical assignments were made with experimental data from a single diastereomer. With the accumulation of ${ }^{1} \mathrm{H} /{ }^{13} \mathrm{C}$ HSQC data on a wider range of cyclic compounds, we ultimately hope to be able to extend the principles of this methodology to the stereochemical assignment of dihydropyran rings with a wider range of substitution patterns. 


\subsection{Experimental Data for Chapter 8}

\subsubsection{General Experimental Methods}

Unless otherwise noted, the following conditions apply. All reactions were performed in flamedried septum-sealed glassware with magnetic stirring under an atmosphere of argon. Moistureand oxygen-sensitive liquids and solutions were transferred using a stainless steel syringe or cannula. Before use, solvents were refluxed over the appropriate drying agent and distilled under argon: tetrahydrofuran from sodium benzophenone ketyl radical; dichloromethane, acetonitrile and triethylamine from calcium hydride; methanol and toluene from sodium. Furfuryl alcohol and $\mathrm{BF}_{3} \cdot \mathrm{OEt}_{2}$ were distilled under reduced pressure before use. All other commercially available chemicals were used as received, without further purification. Analytical thin layer chromatography (TLC) was performed using plastic-backed pre-coated silica TLC plates (Polygram SilG/UV 254 ). Visualization was achieved by UV irradiation $(254 \mathrm{~nm})$ or by heating after treatment with a potassium permanganate dip $\left(1.5 \mathrm{~g} \mathrm{KMnO}_{4}, 10 \mathrm{~g} \mathrm{~K}_{2} \mathrm{CO}_{3}\right.$, $1.25 \mathrm{~mL}$ of $10 \%$ aqueous $\mathrm{NaOH}$ solution and $200 \mathrm{~mL}$ of water) or $p$-anisaldehyde dip (0.7 $\mathrm{mL} p$-anisaldehyde, $9.5 \mathrm{~mL}$ conc. $\mathrm{H}_{2} \mathrm{SO}_{4}, 2.7 \mathrm{~mL}$ of acetic acid and $250 \mathrm{~mL}$ of EtOH). The purification of products by flash column chromatography (FCC) was conducted using silica gel 60 (220-240 mesh) with the solvent systems indicated. ${ }^{1} \mathrm{H}$ NMR spectra were recorded on either: a Varian Unity Inova 500 spectrometer at $500 \mathrm{MHz}$, or a Varian Inova 300 at $300 \mathrm{MHz}$. Data are listed as chemical shift in ppm using $\mathrm{CDCl}_{3}$ as internal standard $(7.26 \mathrm{ppm}) .{ }^{13} \mathrm{C}$ NMR spectra were recorded on a Varian Unity Inova 500 spectrometer at $125 \mathrm{MHz}$ and the data are listed as chemical shift in ppm using $\mathrm{CDCl}_{3}$ as internal standard $(77 \mathrm{ppm})$. All ${ }^{13} \mathrm{C}$ experiments were ${ }^{1} \mathrm{H}$ decoupled. The assignment of atom connectivity and spatial relationships are exclusively based on 2D NMR correlations $\left({ }^{1} \mathrm{H} /{ }^{1} \mathrm{H}-\mathrm{COSY},{ }^{1} \mathrm{H} /{ }^{13} \mathrm{C}-\mathrm{HMBC}\right.$ and ${ }^{1} \mathrm{H} /{ }^{13} \mathrm{C}-$ HSQC). IR bands were measured as a thin film on a Bruker FT-IR Tensor 27 spectrometer with ATR sampling accessory. High-resolution mass spectrometry (HRMS) was performed on a Waters QTOF Premier Tandem mass spectrometer.

\subsubsection{Experimental Data}

A number of the compounds reported in Chapter 8 were prepared as substrates for palladiumcatalyzed allylic alkylation cascades, or obtained as side products in these reactions. Full experimental details and characterization data for the following compounds can be found in Chapter 7:

- cis-( \pm )-6-[(tert-Butyldimethylsilyl)oxy]-3,6-dihydro-2H-pyran-3-yl methyl carbonate (cis380) •trans- $( \pm)-6$ - $[($ tert-Butyldimethylsilyl)oxy $]-3,6$-dihydro- $2 H$-pyran-3-yl methyl carbonate (trans-380) • cis- $( \pm)-6$-((tert-Butyldimethylsilyl)oxy-3,6-dihydro-2 $H$-pyran-3-ol (cis-389)

-trans- $( \pm)-6-(($ tert-Butyldimethylsilyl)oxy-3,6-dihydro-2 $H$-pyran-3-ol (trans-389) •trans( \pm )-6-((tert-Butyldimethylsilyl)oxy-3,6-dihydro-2 $H$-pyran-3-yl benzoate (trans-400) • cis- $( \pm)$ 6-[(tert-Butyl- dimethylsilyl)oxy]-3,6-dihydro-2H-pyran-3-yl benzyl carbonate (cis-401) •4(cis-( \pm )-6-[(tert-Butyldimethylsilyl)oxy]-3,6-dihydro-2 $H$-pyran-3-yl)-6-methyl-2H-pyran-2-one (cis-402) 


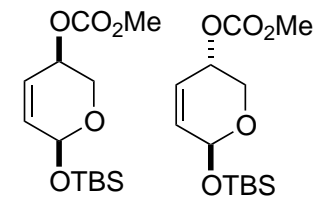<smiles>Oc1ccc(O)cc1</smiles><smiles>O=C(O)C1C=CC(O[18O]c2ccccc2)CO1</smiles><smiles>Cc1cc(OC2C=CC(O)OC2)cc(=O)o1</smiles>

cis-380 trans- $\mathbf{3 8 0}$ cis-389 trans-389

trans- $\mathbf{4 0 0}$ cis- $\mathbf{4 0 1}$

cis-402

Ultimately, these compounds are derived from furfuryl alcohol, as shown below for the case of cis-380, trans-380 and cis-389.
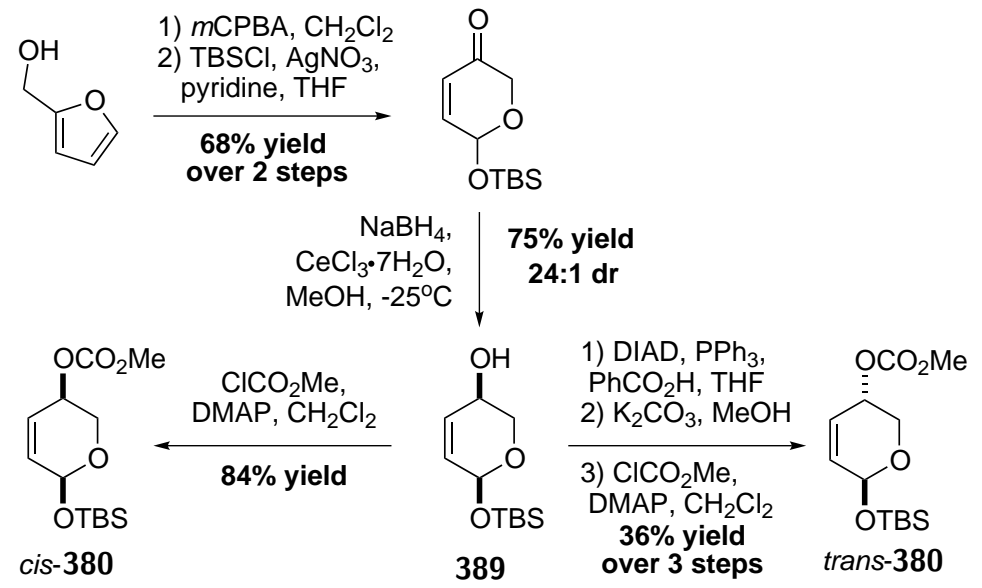

Full ${ }^{1} \mathbf{H}$ and ${ }^{13} \mathbf{C}$ NMR assignments for all compounds in Chapter 8 can be found in Section 8.6.3.

Preparation of (3R,6S)-6-((tert-Butyldimethylsilyl)oxy)-3,6-dihydro-2H-pyran3-yl acetate, cis-381.<smiles>CC(=O)OC1C=CC(O)OC1</smiles>

381

To a solution of cis-( \pm -6-((tert-butyldimethylsilyl)oxy)-3,6-dihydro-2H-pyran-3-ol (cis333, $0.33 \mathrm{~g}, 1.43 \mathrm{mmol}, 1.0$ equiv), $4 \mathrm{~A}$ molecular sieves and PS-C lipase (0.066 g) in methyl tert-butyl ether $(15 \mathrm{~mL})$ was added vinyl acetate $(0.26 \mathrm{~mL}, 0.25 \mathrm{~g}, 2.86 \mathrm{mmol}, 2.0$ equiv) at room temperature. The reaction was stirred at room temperature for 72 hours before being filtered through celite and concentrated in vacuo. The crude product was purified by flash column chromatography (15\% EtOAc/Pet. ether). The title compound was isolated as a clear oil (0.244 g, 63\% yield). $\mathbf{R}_{\mathbf{f}}=0.50$ (15\% EtOAc/PE). ${ }^{\mathbf{1}} \mathbf{H}$ and ${ }^{\mathbf{1 3}} \mathbf{C} \mathbf{~ N M R}$ data can be found in Section 8.6.3. $[\alpha]_{\mathrm{D}}{ }^{25}=+42.7^{\circ}\left(c=1.0, \mathrm{CHCl}_{3}\right)$. Enantioenriched starting material was also recovered $(0.094 \mathrm{~g}, 29 \%$ yield $) \cdot[\alpha]_{\mathrm{D}}{ }^{25}=+21.9^{\circ}\left(c=0.44, \mathrm{CHCl}_{3}\right)$. Characterization data matches the literature. ${ }^{37}$ 
Preparation of trans-( \pm )-6-((tert-butyldimethylsilyl)oxy)-3,6-dihydro-2Hpyran-3-yl acetate, trans-381.

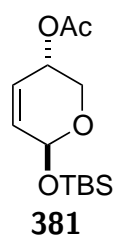

To a solution of $( \pm)$-trans-6-((tert-butyldimethylsilyl)oxy-3,6-dihydro- $2 \mathrm{H}$-pyran-3-ol (trans332, $0.0064 \mathrm{~g}, 0.028 \mathrm{mmol}, 1.0$ equiv) in pyridine $(0.5 \mathrm{~mL})$ was added acetic anhydride $(0.5$ $\mathrm{mL})$. The reaction was stirred overnight at room temperature before quenching with $3 \mathrm{~mL}$ of $\mathrm{H}_{2} \mathrm{O}$. The reaction mixture was then cyclic loaded onto HP20 resin with $\mathrm{MeOH}(1 \mathrm{~mL}$ increments up to $5 \mathrm{~mL}$ of $\mathrm{MeOH}$ ). The resin was then washed three times with $\mathrm{H}_{2} \mathrm{O}$, and the product was subsequently eluted with acetone. The volatiles were removed in vacuo, the product was redissolved in EtOAc, washed with $\mathrm{CuSO}_{4}$, brine, dried over $\mathrm{MgSO}_{4}$, filtered, and concentrated in vacuo. The title compound was isolated as a clear film $(0.007 \mathrm{~g}, 92 \%$ yield). $\mathbf{R}_{\mathbf{f}}=0.29$ (10\% EtOAc/PE). ${ }^{\mathbf{1}} \mathbf{H}$ and ${ }^{\mathbf{1 3}} \mathbf{C}$ NMR data can be found in Section 8.6.3. IR (film): 2955, 2931, 2858, 1739, 1372, 1237, 1109, 1031, 960, 870, $838 \mathrm{~cm}^{-1}$. HRMS - EI $(\mathrm{m} / z)$ : calculated for $\mathrm{C}_{13} \mathrm{H}_{24} \mathrm{O}_{4} \mathrm{SiNa}[\mathrm{M}+\mathrm{Na}]^{+}: 295.1342$, found: 295.1345, $1.0 \mathrm{ppm}$.

\section{General Procedure for the Ferrier Reaction of D- $O$-diacetyl xylal.}

Preparation of $(3 S, 6 R)$-6-propoxy-3,6-dihydro- $2 H$-pyran-3-yl acetate and $(3 S, 6 S)-6$-propoxy-3,6-dihydro- $2 H$-pyran-3-yl acetate, cis- and trans-383.

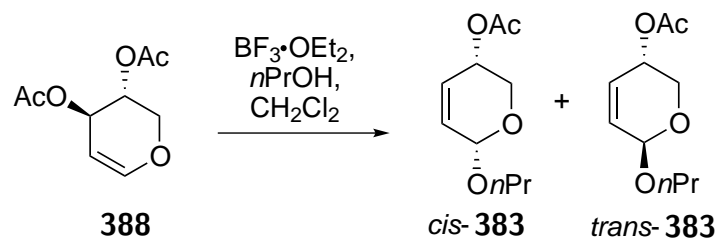

To a solution of 3,4-di- $O$-acetyl-D-xylal $(0.100 \mathrm{~g}, 0.5 \mathrm{mmol}, 1$ equiv) and $n$-propanol (0.045 $\mathrm{mL}, 0.6 \mathrm{mmol}, 1.2$ equiv) in $\mathrm{CH}_{2} \mathrm{Cl}_{2}(5 \mathrm{~mL})$ was added $\mathrm{BF}_{3} \cdot \mathrm{OEt}_{2}(0.012 \mathrm{~mL}, 0.014 \mathrm{~g}, 0.6$ mmol, 1.2 equiv) at $0{ }^{\circ} \mathrm{C}$. The reaction was slowly warmed to room temperature and stirred for an hour before being diluted with $\mathrm{CH}_{2} \mathrm{Cl}_{2}$ and quenched with water. The reaction mixture was subsequently extracted with $\mathrm{CH}_{2} \mathrm{Cl}_{2}$ and the combined organic layers were dried over $\mathrm{MgSO}_{4}$, filtered and concentrated in vacuo. The crude product was purified by silica gel chromatography (10\% EtOAc/ pet. ether). The title compounds were isolated separately; trans-383 was isolated as a clear, colorless oil (0.0216 g, 22\% yield), cis-383 was isolated as a clear colorless oil (0.0094 g, $9 \%$ yield). Characterization data for trans-383: $\mathbf{R}_{\mathbf{f}}=0.29(10 \%$ EtOAc/ pet. ether). ${ }^{\mathbf{1}} \mathbf{H}$ and ${ }^{\mathbf{1}^{\mathbf{3}} \mathbf{C}} \mathbf{N M R}$ data can be found in Section 8.6.3. IR (film): 2964, $2878,1731,1371,1233,1189,1104,1016,956,897,845 \mathrm{~cm}^{-1} \cdot[\alpha]_{\mathbf{D}}{ }^{25}=+120.7{ }^{\circ}(c=1.08$, $\mathrm{CHCl}_{3}$ ). Characterization data for cis-383: $\mathbf{R}_{\mathbf{f}}=0.35$ (10\% EtOAc/ pet. ether). ${ }^{1} \mathbf{H}$ and ${ }^{13}$ C NMR data can be found in Section 8.6.3. IR (film): 2964, 2880, 1736, 1371, 1231, 1099, 1030, 960, $895 \mathrm{~cm}^{-1} \cdot[\alpha]_{\mathbf{D}}{ }^{25}=+107.7{ }^{\circ}\left(c=0.47, \mathrm{CHCl}_{3}\right)$. HRMS - EI $(m / z)$ : calculated for $\mathrm{C}_{10} \mathrm{H}_{16} \mathrm{O}_{4} \mathrm{Na}[\mathrm{M}+\mathrm{Na}]^{+}:$:223.0946, found: 223.0951, $2.2 \mathrm{ppm}$. 
Preparation of $(3 S, 6 S)$-6-isopropoxy-3,6-dihydro-2H-pyran-3-yl acetate and (3S,6R)-6-isopropoxy-3,6-dihydro-2H-pyran-3-yl acetate, cis- and trans-382.

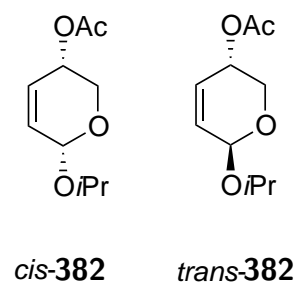

Using the general procedure for the Ferrier reaction, 3,4-di- $O$-acetyl-D-xylal $(0.100 \mathrm{~g}, 0.5$ mmol, 1 equiv), isopropanol (0.046 mL, $0.036 \mathrm{~g}, 0.6 \mathrm{mmol}, 1.2$ equiv) and $\mathrm{BF}_{3} \cdot \mathrm{OEt}_{2}(0.012$ $\mathrm{mL}, 0.6 \mathrm{mmol}, 1.2$ equiv) were added to the reaction. The crude product was purified by silica gel chromatography $(10 \% \mathrm{EtOAc} / \mathrm{PE})$ and the title compounds were isolated together as a clear, colorless oil (0.047 g, $46 \%$ yield). ${ }^{\mathbf{1}} \mathbf{H}$ and ${ }^{\mathbf{1 3}} \mathbf{C}$ NMR data for both trans- and cis-382 can be found in Section 8.6.3. Characterization data matches the literature. ${ }^{39}$

Preparation of $(3 S, 6 S)-6$-(allyloxy)-3,6-dihydro- $2 H$-pyran-3-yl acetate and $(3 S, 6 R)$-6-(allyloxy)-3,6-dihydro- $2 H$-pyran-3-yl acetate, cis- and trans-384.<smiles>C=CCOC1C=CC(OC(C)=O)CC1</smiles>

Using the general procedure for the Ferrier reaction, 3,4-di-O-acetyl-D-xylal (0.100 g, 0.5 mmol, 1 equiv), allyl alcohol (0.041 mL, $0.035 \mathrm{~g}, 0.6 \mathrm{mmol}, 1.2$ equiv) and $\mathrm{BF}_{3} \cdot \mathrm{OEt}_{2}(0.012 \mathrm{~mL}$, $0.014 \mathrm{~g}, 0.6 \mathrm{mmol}, 1.2$ equiv) were added to the reaction. The crude product was purified by silica gel chromatography (10\% EtOAc/PE) and the title compounds were isolated separately; trans-384 was isolated as a clear, colorless oil (0.036 g, $16 \%$ yield), cis-384 was isolated as a clear colorless oil $(0.016 \mathrm{~g}, 37 \%$ yield $)$. Characterization data for trans-384: $\mathbf{R}_{\mathbf{f}}=0.16$ $\left(10 \%\right.$ EtOAc/PE) ${ }^{\mathbf{1}} \mathbf{H}$ and ${ }^{{ }^{13} \mathbf{C}}$ NMR data can be found in Section 8.6.3. IR (film): 2984, 2920, 1730, 1371, 1232, 1036, 1014, $956 \mathrm{~cm}^{-1}$. HRMS - EI $(\mathrm{m} / z)$ : calculated for $\mathrm{C}_{10} \mathrm{H}_{14} \mathrm{O}_{4} \mathrm{Na}[\mathrm{M}+\mathrm{Na}]^{+}:$221.0790, found: 221.0790, $0.0 \mathrm{ppm} .[\alpha]_{\mathrm{D}}{ }^{25}=+86.1{ }^{\circ}(c=1.05$, $\left.\mathrm{CHCl}_{3}\right)$. Characterization data for $\mathrm{cis}-\mathbf{3 8 4}: \mathbf{R}_{\mathbf{f}}=0.24(10 \% \mathrm{EtOAc} / \mathrm{PE}){ }^{\mathbf{1}} \mathbf{H}$ and ${ }^{{ }^{13} \mathbf{C}} \mathrm{NMR}$ data can be found in Section 8.6.3. IR (film): 2886, 1736, 1371, 1230, 1094, 1033, $959 \mathrm{~cm}^{-1}$. HRMS - EI $(m / z)$ : calculated for $\mathrm{C}_{10} \mathrm{H}_{12} \mathrm{O}_{4} \mathrm{Na}[\mathrm{M}+\mathrm{Na}]^{+}: 221.0790$, found: 221.0792, 0.9 ppm. $[\alpha]_{\mathrm{D}}{ }^{25}=+56.66^{\circ}\left(\mathrm{CHCl}_{3}, c=0.8\right)$.

Preparation of (3S,6R)-6-(prop-2-yn-1-yloxy)-3,6-dihydro-2H-pyran-3-yl acetate and $(3 S, 6 S)$-6-(prop-2-yn-1-yloxy)-3,6-dihydro- $2 H$-pyran-3-yl acetate, cisand trans-385.

Using the general procedure for the Ferrier reaction, 3,4-di- $O$-acetyl-D-xylal $(0.100 \mathrm{~g}, 0.5$ mmol, 1 equiv), propargyl alcohol $\left(0.034 \mathrm{~g}, 0.6 \mathrm{mmol}, 1.2\right.$ equiv) and $\mathrm{BF}_{3} \cdot \mathrm{OEt}_{2}(0.012 \mathrm{~mL}$, $0.014 \mathrm{~g}, 0.6 \mathrm{mmol}, 1.2$ equiv) were added to the reaction. The crude product was purified by silica gel chromatography $(10 \% \mathrm{EtOAc} / \mathrm{PE})$. The title compounds were isolated separately; trans-385 was isolated as a clear, colorless oil (0.021 g, $22 \%$ yield), cis-385 was isolated as a clear colorless oil (0.019 g, $20 \%$ yield). Characterization data for trans-385: $\mathbf{R}_{\mathbf{f}}=0.30(10 \%$ 


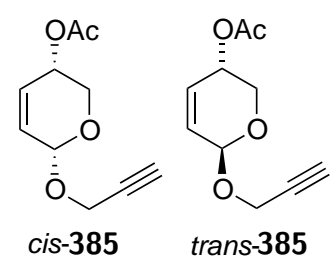

EtOAc/PE). ${ }^{\mathbf{1}} \mathbf{H}$ and ${ }^{\mathbf{1 3}^{\mathbf{3}} \mathbf{C}}$ NMR data can be found in Section 8.6.3. HRMS - EI $(\mathrm{m} / \mathrm{z})$ : calculated for $\mathrm{C}_{10} \mathrm{H}_{12} \mathrm{O}_{4} \mathrm{Na}[\mathrm{M}+\mathrm{Na}]^{+}: 219.0633$, found: $219.0630,-1.4 \mathrm{ppm}$. $[\alpha]_{\mathrm{D}}{ }^{25}=+55.3^{\circ}$ $\left(c=1.05, \mathrm{CHCl}_{3}\right)$. Characterization data for cis-385: $\mathbf{R}_{\mathbf{f}}=0.24(10 \% \mathrm{EtOAc} / \mathrm{PE}) .{ }^{\mathbf{1}} \mathbf{H}$ and ${ }^{13} \mathrm{C}$ NMR data can be found in Section 8.6.3. HRMS - EI $(m / z)$ : calculated for $\mathrm{C}_{10} \mathrm{H}_{12} \mathrm{O}_{4} \mathrm{Na}$ $[\mathrm{M}+\mathrm{Na}]^{+}: 219.0633$, found: $219.0635,0.9 \mathrm{ppm} .[\alpha]_{\mathrm{D}}{ }^{25}=+23.6^{\circ}\left(c=0.97, \mathrm{CHCl}_{3}\right)$.

Preparation of (3S,6R)-6-(benzyloxy)-3,6-dihydro- $2 H$-pyran-3-yl acetate and (3S,6S)-6-(benzyloxy)-3,6-dihydro- $2 H$-pyran-3-yl acetate, cis- and trans-386.

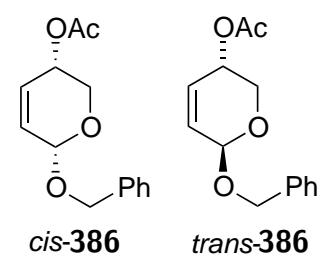

Using the general procedure for the Ferrier reaction, 3,4-di- $O$-acetyl-D-xylal $(0.100 \mathrm{~g}, 0.5$ mmol, 1 equiv), benzyl alcohol $\left(0.062 \mathrm{~mL}, 0.6 \mathrm{mmol}, 1.2\right.$ equiv) and $\mathrm{BF}_{3} \cdot \mathrm{OEt}_{2}(0.012 \mathrm{~mL}$, $0.014 \mathrm{~g}, 0.6 \mathrm{mmol}, 1.2$ equiv) were added to the reaction. The crude product was purified by silica gel chromatography $(10 \% \mathrm{EtOAc} / \mathrm{PE})$ and the title compounds were isolated together $\left(\mathbf{R}_{\mathbf{f}}=0.28\right.$ and 0.20 .) as a clear, colorless oil $\left(0.099 \mathrm{~g}, 80 \%\right.$ yield, ca. $3: 1$ trans: cis ratio). ${ }^{1} \mathbf{H}$ and ${ }^{13} \mathbf{C}$ NMR data for cis-385 and trans-385 can be found in Section 8.6.3. Characterization data matches the literature. ${ }^{39}$

Preparation of trans-(3S,6S)-6-allyl-3,6-dihydro- $2 H$-pyran-3-yl acetate, trans404.

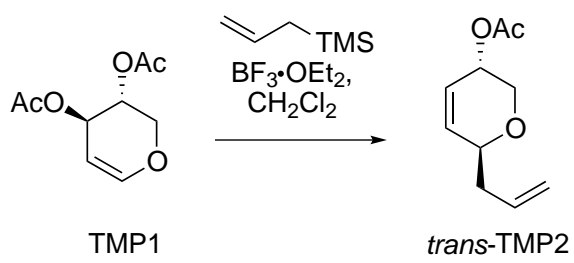

Using the general procedure for the Ferrier reaction, 3,4-di- $O$-acetyl-D-xylal $(0.200 \mathrm{~g}, 1.0$ mmol, 1 equiv), allyl trimethylsilane $(0.190 \mathrm{~mL}, 1.2 \mathrm{mmol}, 1.2$ equiv $)$ and $\mathrm{BF}_{3} \cdot \mathrm{OEt}_{2}(0.13 \mathrm{~mL}$, $1.2 \mathrm{mmol}, 1.2$ equiv) were added to the reaction. The crude product was purified by silica gel chromatography (10\% EtOAc/PE) and the title compounds was isolated as a clear, colorless oil (0.130 g, $72 \%$ yield). $\mathbf{R}_{\mathbf{f}}=0.32\left(10 \%\right.$ EtOAc/PE). ${ }^{\mathbf{1}} \mathbf{H}$ and ${ }^{\mathbf{1 3}} \mathbf{C} \mathbf{~ N M R}$ data can be found in Section 8.6.3. Trace amounts $(<10 \%)$ of the cis-diastereomer were observed by ${ }^{1} \mathrm{H}$ and ${ }^{13} \mathrm{C}$ NMR. Characterization data matches the literature. ${ }^{37}$ 
Preparation of 'cis'-(2R,3S,6S)- and 'trans'-(2R,3S,6R)-3-Acetoxy-6isopropoxy-3,6-dihydro-2H-pyran-2-yl)methyl acetate, 'cis'- and 'trans'-405.

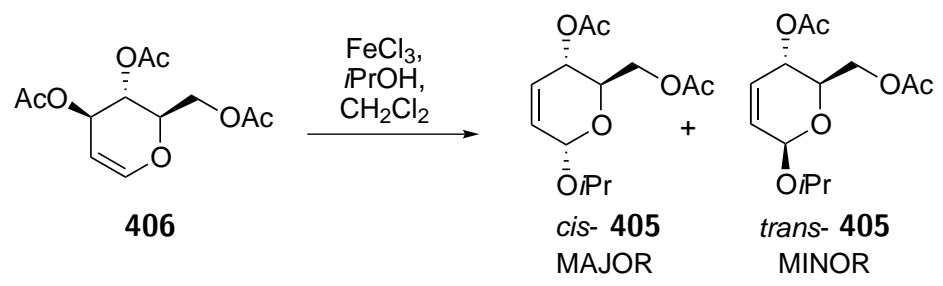

To a solution of 3,4,6-tri- $O$-acetyl-D-glucal $(0.100 \mathrm{~g}, 0.367 \mathrm{mmol}, 1$ equiv) and 2-propanol (0.031 mL, $0.404 \mathrm{mmol}, 1.1$ equiv) was added $\mathrm{FeCl}_{3}\left(0.082 \mathrm{~mL}\right.$ of a $0.1 \mathrm{M}$ solution in $\mathrm{CH}_{2} \mathrm{Cl}_{2}$, $0.008 \mathrm{mmol}, 22 \mathrm{~mol} \%)$. The crude product was purified by silica gel chromatography $(10 \%$ EtOAc/PE) and the title compounds were isolated as a clear, colorless oil (0.031 g, 31\% yield, 7.1:1 dr, 'cis':'trans'). $\mathbf{R}_{\mathbf{f}}=0.19\left(10 \%\right.$ EtOAc/PE). ${ }^{\mathbf{1}} \mathbf{H}$ and ${ }^{\mathbf{1 3}} \mathbf{C} \mathbf{N M R}$ data can be found in Section 8.6.3. Characterization data matches the literature. ${ }^{38}$

Preparation of ( \pm )-6-Hydroxy-3,6-dihydro-2H-pyran-3-yl methyl carbonate, cis-, and trans-387.<smiles></smiles>

To a solution of 380 (0.096 g, $0.33 \mathrm{mmol}, 1.0$ equiv) in acetonitrile $(8 \mathrm{~mL})$, at room temperature, was added HF.pyridine $(0.240 \mathrm{~mL}, 0.266 \mathrm{~g}, 13.3 \mathrm{mmol}, 40$ equiv). The reaction was stirred overnight before being quenched with saturated aqueous sodium bicarbonate, extracted with EtOAc, dried over MgSO4, filtered and concentrated in vacuo. The crude product was purified by silica gel chromatography (50\% EtOAc / PE). The desired product was isolated as a $c a$. 3:1 mixture of trans- and cis-diastereomers, respectively. $(0.018 \mathrm{~g}, 31 \%$ yield). $\mathbf{R}_{\mathbf{f}}=0.23$ (40\% EtOAc / Pet. Ether). ${ }^{\mathbf{1}} \mathbf{H}$ and ${ }^{{ }^{\mathbf{1 3}} \mathbf{C}} \mathbf{~ N M R}$ data can be found in Section 8.6.3. IR (film) 3413, 2959, 2925, 1740, 1443, 1252, 1061, 975, $937 \mathrm{~cm}^{-1}$. HRMS EI $(m / z)$ calculated for $\mathrm{C}_{7} \mathrm{H}_{10} \mathrm{O}_{5} \mathrm{Na}^{+}[\mathrm{M}+\mathrm{Na}]^{+}:$197.0426, found: 197.0422 .

Representative Procedure for the Saponification of 3,6-Dihydro-2H-pyran-3-yl acetates.

Preparation of cis- $(S, R)$ - and trans- $(S, S)$-6-Isopropoxy-3,6-dihydro-2 $H$-pyran3-ol, cis- and trans-390.

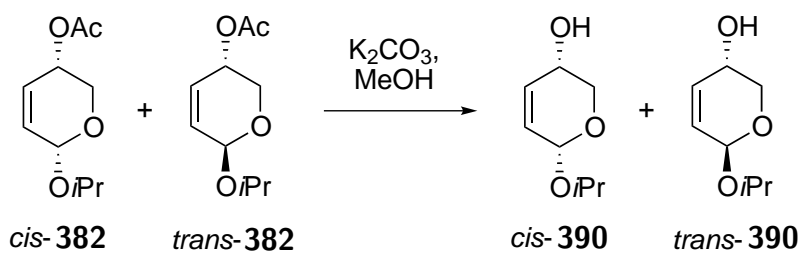

To a solution of cis- $(S, R)$ - and trans- $(S, S)$-6-isopropoxy-3,6-dihydro- $2 H$-pyran-3 (382, $0.047 \mathrm{~g}, 0.232 \mathrm{mmol}, 1.0$ equiv) in methanol $(2.5 \mathrm{~mL})$ was added $\mathrm{K}_{2} \mathrm{CO}_{3}(0.160 \mathrm{~g}, 1.16 \mathrm{mmol}$, 
5.0 equiv). The reaction mixture was stirred at room temperature for 3 hours before being quenched with $\mathrm{H}_{2} \mathrm{O}$ and extracted twice with EtOAc. The combined organic layers were dried over $\mathrm{MgSO}_{4}$, filtered, and concentrated in vacuo. The title compound was isolated as a clear colorless oil. Characterization data matches the literature. ${ }^{39}$

Preparation of trans-(3S,6S)-6-Allyloxy-3,6-dihydro-2 $H$-pyran-3-ol, trans-391.<smiles>C=COc1ccc(O)cc1</smiles>

trans-391

Using the general procedure for acetate saponification, trans-(3S,6S)-6-allyloxy-3,6-dihydro$2 \mathrm{H}$-pyran-3-acetate ( $0.036 \mathrm{~g}, 0.18 \mathrm{mmol}, 1.0$ equiv) and $\mathrm{K}_{2} \mathrm{CO}_{3}(0.124 \mathrm{~g}, 0.90 \mathrm{mmol}, 5.0$ equiv) were added to the reaction. The title compound was obtained as a clear colorless oil $(0.010$ g, $36 \%$ yield). ${ }^{\mathbf{1}} \mathbf{H}$ and ${ }^{{ }^{\mathbf{1 3}} \mathbf{C}} \mathbf{~ N M R}$ data can be found in Section 8.6.3. IR (film): 3398, $2922,1399,1319,1260,1188,1099,1077,1033,964,930,833 \mathrm{~cm}^{-1} \cdot[\alpha]_{\mathbf{D}}{ }^{25}=+68.3^{\circ}(c=0.5$, $\left.\mathrm{CHCl}_{3}\right)$. HRMS - EI $(\mathrm{m} / z)$ calculated for $\mathrm{C}_{8} \mathrm{H}_{12} \mathrm{O}_{3} \mathrm{Na}^{+}[\mathrm{M}+\mathrm{Na}]^{+}:$179.0684, found: 179.0688 .

\section{Preparation of cis-(3S,6R)-6-Allyloxy-3,6-dihydro-2H-pyran-3-ol, cis-391.}

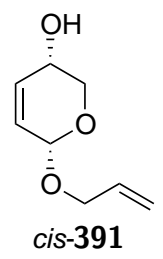

Using the general procedure for acetate saponification, cis-(3S,6R)-6-allyloxy-3,6-dihydro$2 \mathrm{H}$-pyran-3-acetate $\left(0.016 \mathrm{~g}, 0.081 \mathrm{mmol}, 1.0\right.$ equiv) and $\mathrm{K}_{2} \mathrm{CO}_{3}(0.056 \mathrm{~g}, 0.40 \mathrm{mmol}, 5.0$ equiv) were added to the reaction. The title compound was obtained as a clear colorless oil (0.004 g, 32\% yield). ${ }^{\mathbf{1}} \mathbf{H}$ and ${ }^{\mathbf{1 3}} \mathbf{C}$ NMR data can be found in Section 8.6.3. IR (film): $3397,2919,1395,1276,1261,1186,1032,935,884 \mathrm{~cm}^{-1} .[\alpha]_{\mathrm{D}}{ }^{25}=+34.7^{\circ}\left(c=0.19, \mathrm{CHCl}_{3}\right)$. HRMS - EI $(m / z)$ calculated for $\mathrm{C}_{8} \mathrm{H}_{12} \mathrm{O}_{3} \mathrm{Na}^{+}[\mathrm{M}+\mathrm{Na}]^{+}:$179.0684, found: 179.0681 .

Preparation of trans-(3S,6S)-6-allyl-3,6-dihydro- $2 H$-pyran-3-ol, trans-407.<smiles>C=CCC1C=CC(O)CO1</smiles>

trans-407

Using the general procedure for acetate saponification, trans-(3S,6S)-6-allyl-3,6-dihydro2H-pyran-3-ol (trans-407, $0.130 \mathrm{~g}, 0.716 \mathrm{mmol}, 1.0$ equiv) and $\mathrm{K}_{2} \mathrm{CO}_{3}$ (0.495 g, $3.58 \mathrm{mmol}$, 5.0 equiv) were added to the reaction. The title compound was obtained as a clear colorless

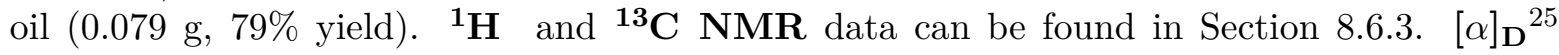
$=+98.6^{\circ}\left(c=0.52, \mathrm{CHCl}_{3}\right)$. HRMS - EI $(m / z)$ calculated for $\mathrm{C}_{8} \mathrm{H}_{12} \mathrm{O}_{2} \mathrm{Na}^{+}[\mathrm{M}+\mathrm{Na}]^{+}$: 
163.0735, found: 163.0730 . Characterization data matches the literature. ${ }^{41}$

Preparation of cis-(3S,6S)-6-allyl-3,6-dihydro- $2 H$-pyran-3-yl acetate, cis-404.

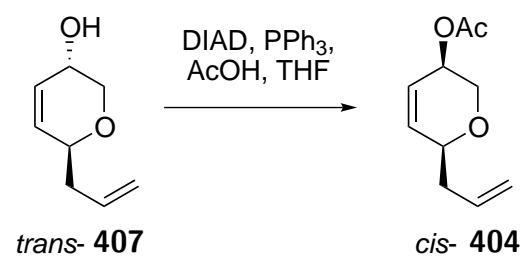

To a solution of trans-(3S,6S)-6-allyl-3,6-dihydro-2H-pyran-3-ol (trans-407, $0.0688 \mathrm{~g}, 0.49$ mmol, 1.0 equiv), $\mathrm{PPh}_{3}$ (0.142 g, $0.54 \mathrm{mmol}, 1.1$ equiv), and acetic acid ( $0.034 \mathrm{~mL}, 0.59 \mathrm{mmol}$, 1.2 equiv) in THF $(5 \mathrm{~mL})$ at $0{ }^{\circ} \mathrm{C}$ was added DIAD $(0.105 \mathrm{~mL}, 0.54 \mathrm{mmol}, 1.1$ equiv). The reaction was stirred at $0{ }^{\circ} \mathrm{C}$ for 30 minutes before warming to room temperature, stirring for an additional hour, and quenching with $\mathrm{H}_{2} \mathrm{O}$. The reaction mixture was subsequently extracted with EtOAc and the combined organics were dried over $\mathrm{MgSO}_{4}$, filtered, and concentrated in vacuo. The crude product was purified by silica gel chromatography (10\% EtOAc/PE) and the title compound was isolates as a clear colorless oil $(0.0464 \mathrm{~g}, 52 \%$ yield $) . \mathbf{R}_{\mathbf{f}}=0.2(10 \%$ EtOAc/PE). ${ }^{1} \mathbf{H}$ and ${ }^{13} \mathbf{C} \mathbf{~ N M R}$ data can be found in Section 8.6.3. $[\alpha]_{\mathbf{D}}{ }^{25}=-90.6^{\circ}(c=$ $\left.0.42, \mathrm{CHCl}_{3}\right)$. HRMS - EI $(\mathrm{m} / z)$ calculated for $\mathrm{C}_{10} \mathrm{H}_{14} \mathrm{O}_{3} \mathrm{Na}^{+}[\mathrm{M}+\mathrm{Na}]^{+}: 205.0841$, found: 205.0840. Characterization data matches the literature. ${ }^{41}$ 


\subsubsection{NMR Data and Assignments}

cis-( \pm )-6-[(tert-Butyldimethylsilyl)oxy]-3,6-dihydro2H-pyran-3-yl methyl carbonate, cis-380.<smiles>COC(=O)OC1C=CC(O[Si](C)(C)C(C)(C)C)OC1</smiles>

NMR Assignments

\begin{tabular}{c|c|c|c}
\hline $\mathrm{C}$ & $\delta_{\mathrm{C}}, \mathrm{mult}$ & $\delta_{\mathrm{H}}(J, \mathrm{~Hz})$ & $\mathrm{COSY}$ \\
\hline $2 \mathrm{a}$ & $60.1, \mathrm{CH}_{2}$ & $3.91, \mathrm{ddd}(11.1,7.9,0.5)$ & $2 \mathrm{~b}, 3$ \\
$2 \mathrm{~b}$ & - & $3.87, \mathrm{ddd}(11.1,5.7,0.9)$ & $2 \mathrm{a}, 3$ \\
3 & $68.4, \mathrm{CH}$ & $5.13, \mathrm{~m}$ & $2 \mathrm{a}, 2 \mathrm{~b}, 4$ \\
4 & $126.4, \mathrm{CH}$ & $5.92, \mathrm{ddt}(10.3,2.3,1.0)$ & 3,5 \\
5 & $132.2, \mathrm{CH}$ & $5.85, \mathrm{ddd}(10.3,2.3,1.8)$ & 4,6 \\
6 & $89.2, \mathrm{CH}$ & $5.28, \mathrm{~m}$ & 5 \\
7 & $155.3, \mathrm{C}$ & - & - \\
8 & $54.9, \mathrm{CH}_{3}$ & $3.80, \mathrm{~s}$ & - \\
9 & $-4.4, \mathrm{CH}_{3}$ & $0.133, \mathrm{~s}$ & - \\
10 & $-5.2, \mathrm{CH}_{3}$ & $0.130, \mathrm{~s}$ & - \\
11 & $18.0, \mathrm{C}$ & - & - \\
12 & $25.7, \mathrm{CH}_{3}$ & $0.91, \mathrm{~s}$ & -
\end{tabular}

trans- $( \pm)-6-[($ tert-Butyldimethylsilyl)oxy $]-3,6-$ dihydro-2H-pyran-3-yl methyl carbonate, trans-408.<smiles>COC(=O)O[C@H]1C=C[C@@H](O[Si](C)(C)C(C)(C)C)O[C@@H]1O</smiles>

NMR Assignments

\begin{tabular}{c|c|c|c}
\hline $\mathrm{C}$ & $\delta_{\mathrm{C}}, \mathrm{mult}$ & $\delta_{\mathrm{H}}(J, \mathrm{~Hz})$ & $\mathrm{COSY}$ \\
\hline $2 \mathrm{a}$ & $61.0, \mathrm{CH}_{2}$ & $4.20, \mathrm{dd}(13.1,2.8)$ & $2 \mathrm{~b}, 3$ \\
$2 \mathrm{~b}$ & - & $3.90, \mathrm{dt}(13.1,1.2)$ & $2 \mathrm{a}$ \\
3 & $66.9, \mathrm{CH}$ & $4.8, \mathrm{ddd}(4.4,2.9,1.4)$ & $2 \mathrm{a}, 4$ \\
4 & $122.5, \mathrm{CH}$ & $6.03, \mathrm{ddd}(10.1,4.3,0.8)$ & 3,5 \\
5 & $133.9, \mathrm{CH}$ & $6.00, \mathrm{dt}(10.0,2.6)$ & 4,6 \\
6 & $87.9, \mathrm{CH}$ & $5.33, \mathrm{~d}(2.4)$ & 5 \\
7 & $155.4, \mathrm{C}$ & - & - \\
8 & $54.8, \mathrm{CH}_{3}$ & $3.78, \mathrm{~s}$ & - \\
9 & $-4.5, \mathrm{CH}_{3}$ & $0.13, \mathrm{~s}$ & - \\
10 & $-5.3, \mathrm{CH}_{3}$ & $0.13, \mathrm{~s}$ & - \\
11 & $18.1, \mathrm{C}$ & - & - \\
12 & $25.7, \mathrm{CH}_{3}$ & $0.91, \mathrm{~s}$ & -
\end{tabular}


(3R,6S)-6-((tert-butyldimethylsilyl)oxy)-3,6-dihydro2H-pyran-3-yl acetate, cis-381.<smiles>CC(=O)OC1CCC(O[Si](C)(C)C(C)(C)C)CC1</smiles>

NMR Assignments

\begin{tabular}{c|c|c|c}
\hline $\mathrm{C}$ & $\delta_{\mathrm{C}}$, mult & $\delta_{\mathrm{H}}(J, \mathrm{~Hz})$ & $\mathrm{COSY}$ \\
\hline $2 \mathrm{a}$ & $60.6, \mathrm{CH}_{2}$ & $3.86, \mathrm{dd}(9.5,5.4)$ & $2 \mathrm{~b}, 3$ \\
$2 \mathrm{~b}$ & - & $3.83, \mathrm{dd}(9.5,4.0)$ & $2 \mathrm{a}, 3$ \\
3 & $65.0, \mathrm{CH}$ & $5.24, \mathrm{~m}$ & $2 \mathrm{a}, 2 \mathrm{~b}, 4$ \\
4 & $126.9, \mathrm{CH}$ & $5.88, \mathrm{dd}(10.3,2.1)$ & 3,5 \\
5 & $132.1, \mathrm{CH}$ & $5.84, \mathrm{dt}(10.3,1.7)$ & 5,6 \\
6 & $89.5, \mathrm{CH}$ & 5.28, app. s & 5 \\
7 & $170.61, \mathrm{C}$ & - & - \\
8 & $21.1, \mathrm{CH}_{3}$ & $2.08, \mathrm{~s}$ & - \\
9 & $-4.4, \mathrm{CH}_{3}$ & $0.141, \mathrm{~s}$ & - \\
10 & $-5.2, \mathrm{CH}_{3}$ & $0.139, \mathrm{~s}$ & - \\
11 & $25.7, \mathrm{C}$ & - & - \\
12 & $18.1, \mathrm{CH}_{3}$ & $0.92, \mathrm{~s}$ & -
\end{tabular}

(3R,6S)-6-((tert-butyldimethylsilyl)oxy)-3,6-dihydro2H-pyran-3-yl acetate, trans-381.<smiles>CC(=O)O[C@H]1C=C[C@@H](O[Si](C)(C)C(C)(C)C)OC1</smiles>

NMR Assignments

\begin{tabular}{c|c|c|c}
\hline $\mathrm{C}$ & $\delta_{\mathrm{C}}$, mult & $\delta_{\mathrm{H}}(J, \mathrm{~Hz})$ & $\mathrm{COSY}$ \\
\hline $2 \mathrm{a}$ & $61.2, \mathrm{CH}_{2}$ & $4.2, \mathrm{dd}(13.0,2.8)$ & $2 \mathrm{~b}, 3$ \\
$2 \mathrm{~b}$ & - & $3.82, \mathrm{dd}(13.0,1.2)$ & $2 \mathrm{a}, 3$ \\
3 & $63.5, \mathrm{CH}$ & $4.95, \mathrm{~m}$ & $2 \mathrm{a}, 2 \mathrm{~b}, 4$ \\
4 & $123.2, \mathrm{CH}$ & $6.01, \mathrm{~m}$ & 3,5 \\
5 & $133.2, \mathrm{CH}$ & $6.00, \mathrm{~m}$ & 5,6 \\
6 & $87.9, \mathrm{CH}$ & $5.35, \mathrm{~d}(1.9)$ & 5 \\
7 & $170.7, \mathrm{C}$ & - & - \\
8 & $21.13, \mathrm{CH}_{3}$ & $2.09, \mathrm{~s}$ & - \\
9 & $-4.5, \mathrm{CH}_{3}$ & $0.140, \mathrm{~s}$ & - \\
10 & $-5.32, \mathrm{CH}_{3}$ & $0.136, \mathrm{~s}$ & - \\
11 & $25.7, \mathrm{C}$ & - & - \\
12 & $18.07, \mathrm{CH}_{3}$ & $0.91, \mathrm{~s}$ & -
\end{tabular}

(3S,6S)-6-propoxy-3,6-dihydro-2H-pyran-3-yl acetate (trans-), trans-382.<smiles>CCCOC1C=CC(OC=O)CC1</smiles>

NMR Assignments

\begin{tabular}{c|c|c|c}
\hline $\mathrm{C}$ & $\delta_{\mathrm{C}}, \mathrm{mult}$ & $\delta_{\mathrm{H}}(J, \mathrm{~Hz})$ & $\mathrm{COSY}$ \\
\hline $2 \mathrm{a}$ & $61.2, \mathrm{CH}_{2}$ & $4.17, \mathrm{dd}(13.0,2.9)$ & $5,2 \mathrm{~b}$ \\
$2 \mathrm{~b}$ & - & $3.83, \mathrm{dd}(13.0,1.2)$ & $2 \mathrm{a}$ \\
3 & $63.4, \mathrm{CH}$ & $4.95, \mathrm{dt}(2.9,1.6)$ & $4,2 \mathrm{a}$ \\
4 & $124.9, \mathrm{CH}$ & $6.08-6.03, \mathrm{~m}$ & $2,3,5$ \\
5 & $131.0, \mathrm{CH}$ & $6.08-6.03, \mathrm{~m}$ & $2,4,5$ \\
6 & $92.9, \mathrm{CH}$ & $5.00, \mathrm{~d}(2.3)$ & 3 \\
$7 \mathrm{a}$ & $70.2, \mathrm{CH}_{2}$ & $3.73, \mathrm{dt}(9.5,6.8)$ & $7 \mathrm{~b}, 8$ \\
$7 \mathrm{~b}$ & - & $3.45, \mathrm{dt}(9.5,6.7)$ & $7 \mathrm{a}, 8$ \\
8 & $22.9, \mathrm{CH}_{2}$ & $1.63, \mathrm{~m}$ & $7 \mathrm{a}, 7 \mathrm{~b}, 9$ \\
9 & $10.6, \mathrm{CH}_{3}$ & $0.94, \mathrm{t}(7.4)$ & 8 \\
10 & $170.6, \mathrm{C}$ & - & - \\
11 & $21.1, \mathrm{CH}_{3}$ & $2.10, \mathrm{~s}$ & -
\end{tabular}

(3S,6R)-6-propoxy-3,6-dihydro-2H-pyran-3-yl acetate (cis-), cis-382.<smiles>CCCOC1C=C[C@H](OC=O)CO1</smiles>

NMR Assignments

\begin{tabular}{c|c|c|c}
\hline $\mathrm{C}$ & $\delta_{\mathrm{C}}, \mathrm{mult}$ & $\delta_{\mathrm{H}}(J, \mathrm{~Hz})$ & $\mathrm{COSY}$ \\
\hline $2 \mathrm{a}$ & $60.1, \mathrm{CH}_{2}$ & $3.86, \mathrm{dd}(11.1,5.7)$ & $2 \mathrm{~b}, 5$ \\
$2 \mathrm{~b}$ & - & $3.82, \mathrm{dd}(11.1,7.9)$ & $2 \mathrm{a}, 5$ \\
3 & $65.0, \mathrm{CH}$ & $5.29, \mathrm{~m}$ & $3,4,6 \mathrm{a}, 6 \mathrm{~b}$ \\
4 & $128.7, \mathrm{CH}$ & $5.95, \mathrm{~m}$ & $2,3,5$ \\
5 & $129.4, \mathrm{CH}$ & $5.88, \mathrm{dt}(10.3,2)$ & $2,4,5$ \\
6 & $94.3, \mathrm{CH}$ & 4.95, app. d & 3,4 \\
$7 \mathrm{a}$ & $70.5, \mathrm{CH}_{2}$ & $3.75, \mathrm{dt}(9.4,6.8)$ & $7 \mathrm{~b}, 8$ \\
$7 \mathrm{~b}$ & - & $3.46, \mathrm{dt}(9.4,6.7)$ & $7 \mathrm{a}, 8$ \\
8 & $23.0, \mathrm{CH}_{2}$ & $1.64, \mathrm{sextet}(7.2)$ & $7 \mathrm{a}, 7 \mathrm{~b}, 9$ \\
9 & $10.6, \mathrm{CH}_{3}$ & $0.95, \mathrm{t}(7.4)$ & 8 \\
10 & $170.6, \mathrm{C}$ & - & - \\
11 & $21.0, \mathrm{CH}_{3}$ & $2.08, \mathrm{~s}$ & -
\end{tabular}


(3S,6R)-6-isopropoxy-3,6-dihydro-2H-pyran-3-yl acetate, trans-383.<smiles>CC(C)OC1C=CC(OC=O)CC1</smiles>

NMR Assignments

\begin{tabular}{c|c|c|c}
\hline $\mathrm{C}$ & $\delta_{\mathrm{C}}, \mathrm{mult}$ & $\delta_{\mathrm{H}}(J, \mathrm{~Hz})$ & $\mathrm{COSY}$ \\
\hline $2 \mathrm{a}$ & $61.1, \mathrm{CH}_{2}$ & $4.19, \mathrm{dd}(13.0,2.8)$ & $2 \mathrm{~b}, 3$ \\
$2 \mathrm{~b}$ & - & $3.84-3.80, \mathrm{~m}$ & $2 \mathrm{a}, 3 \mathrm{a}$ \\
3 & $63.5, \mathrm{CH}$ & $4.95, \mathrm{dt}(3.6,1.9)$ & $2 \mathrm{a}, 2 \mathrm{ba}, 4$ \\
4 & $124.8, \mathrm{CH}$ & $6.06, \mathrm{dd}(10.1,5.1)$ & 3,5 \\
5 & $131.5, \mathrm{CH}$ & $6.00, \mathrm{dd}(10.1,2.9)$ & 4,6 \\
6 & $91.1, \mathrm{CH}$ & $5.11, \mathrm{~d}(3.0)$ & 5 \\
7 & $70.0, \mathrm{CH}$ & $3.98,7(6.0)$ & 8,9 \\
8 & $23.6, \mathrm{CH}_{3}$ & $1.23, \mathrm{~d}(6.2)$ & 7 \\
9 & $21.8, \mathrm{CH}_{3}$ & $1.18, \mathrm{~d}(6.1)$ & 7 \\
10 & $170.7, \mathrm{C}$ & - & - \\
11 & $21.1, \mathrm{CH}_{3}$ & $2.09, \mathrm{~s}$ & -
\end{tabular}

(3S,6R)-6-isopropoxy-3,6-dihydro-2H-pyran-3-yl acetate, cis-383.

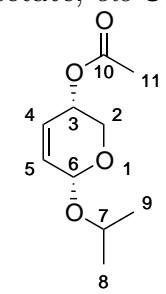

NMR Assignments

\begin{tabular}{c|c|c|c}
\hline $\mathrm{C}$ & $\delta_{\mathrm{C}}, \mathrm{mult}$ & $\delta_{\mathrm{H}}(J, \mathrm{~Hz})$ & COSY \\
\hline 2 & $59.8, \mathrm{CH}_{2}$ & $3.83, \mathrm{~m}$ & 3 \\
3 & $65.1, \mathrm{CH}$ & 5.29, app. $\mathrm{t}(7.2)$ & - \\
4 & $128.7, \mathrm{CH}$ & 5.92, dd $(10.3,1.1)$ & 5 \\
5 & $129.7, \mathrm{CH}$ & $5.84-5.81, \mathrm{~m}$ & 4 \\
6 & $92.3, \mathrm{CH}$ & 5.03, app. s & - \\
7 & $70.3, \mathrm{CH}_{2}$ & $3.98,7(6.0)$ & 8,9 \\
8 & $23.6, \mathrm{CH}_{2}$ & $1.24, \mathrm{~m}$ & 7 \\
9 & $21.8, \mathrm{CH}_{3}$ & $1.18, \mathrm{~m}$ & 7 \\
10 & $170.7, \mathrm{C}$ & - & - \\
11 & $21.1, \mathrm{CH}_{3}$ & $2.06, \mathrm{~s}$ & -
\end{tabular}

(3S,6R)-6-(allyloxy)-3,6-dihydro-2H-pyran-3-yl acetate, trans-384.<smiles>CC(=O)OC1CCCCC1OC=O</smiles>

NMR Assignments

\begin{tabular}{c|c|c|c}
\hline $\mathrm{C}$ & $\delta_{\mathrm{C}}, \mathrm{mult}$ & $\delta_{\mathrm{H}}(J, \mathrm{~Hz})$ & $\mathrm{COSY}$ \\
\hline $2 \mathrm{a}$ & $61.3, \mathrm{CH}_{2}$ & $4.17, \mathrm{dd}(13.0,2.7)$ & $2 \mathrm{~b}, 3$ \\
$2 \mathrm{~b}$ & - & $3.83, \mathrm{~d}(13.1)$ & $2 \mathrm{a}, 3$ \\
3 & $63.3, \mathrm{CH}$ & $4.95, \mathrm{dd}(4.4,2.5)$ & $2 \mathrm{a}, 2 \mathrm{~b}, 4$ \\
4 & $125.1, \mathrm{CH}$ & $6.08, \mathrm{dd}(10.0,5.0)$ & 3,5 \\
5 & $130.8, \mathrm{CH}$ & $6.03, \mathrm{dd}(10.1,2.8)$ & 4,6 \\
6 & $92.1, \mathrm{CH}$ & $5.05, \mathrm{~d}(2.4)$ & 5 \\
$7 \mathrm{a}$ & $68.9, \mathrm{CH}_{2}$ & $4.25, \mathrm{ddt}(12.7,5.2,1.4)$ & $7 \mathrm{~b}, 8$ \\
$7 \mathrm{~b}$ & - & $4.06, \mathrm{ddt}(12.7,6.4,1.1)$ & $7 \mathrm{a}, 8$ \\
8 & $134.1, \mathrm{CH}$ & $5.92, \mathrm{dddd}$ & $7 \mathrm{a}, 7 \mathrm{~b}$, \\
& & $(17.1,10.5,6.4,5.3)$ & $9 \mathrm{a}, 9 \mathrm{~b}$ \\
$9 \mathrm{a}$ & $117.7, \mathrm{CH}_{2}$ & $5.30, \mathrm{dd}(17.2,1.6)$ & $8,9 \mathrm{~b}$ \\
$9 \mathrm{~b}$ & - & $5.20, \mathrm{dd}(10.3,1.3)$ & $8,9 \mathrm{a}$ \\
10 & $170.6, \mathrm{C}$ & - & - \\
11 & $21.1, \mathrm{CH}_{3}$ & $2.08, \mathrm{~s}$ & -
\end{tabular}

(3S,6S)-6-(allyloxy)-3,6-dihydro-2H-pyran-3-yl acetate, cis-384.<smiles>CC(=O)OC1CCCCC1OC=O</smiles>

NMR Assignments

\begin{tabular}{c|c|c|c}
\hline $\mathrm{C}$ & $\delta_{\mathrm{C}, \mathrm{mult}}$ & $\delta_{\mathrm{H}}(J, \mathrm{~Hz})$ & $\mathrm{COSY}$ \\
\hline $2 \mathrm{a}$ & $60.1, \mathrm{CH}_{2}$ & $3.85, \mathrm{dd}(11.0,5.7)$ & $2 \mathrm{~b}, 3$ \\
$2 \mathrm{~b}$ & - & $3.81, \mathrm{dd}(11.0,8.0)$ & $2 \mathrm{a}, 3$ \\
3 & $65.0, \mathrm{CH}$ & $5.32-5.27, \mathrm{~m}$ & $2 \mathrm{a}, 2 \mathrm{~b}, 4$ \\
4 & $129.0, \mathrm{CH}$ & $5.97-5.89, \mathrm{~m}$ & 3,5 \\
5 & $129.1, \mathrm{CH}$ & $5.87, \mathrm{dt}(10.3,2.1)$ & 4,6 \\
6 & $93.4, \mathrm{CH}$ & $4.99, \mathrm{~s}$ & 4,5 \\
$7 \mathrm{a}$ & $69.1, \mathrm{CH}_{2}$ & $4.27, \mathrm{ddt}(12.8,5.2,1.4)$ & $7 \mathrm{~b}, 8$ \\
$7 \mathrm{~b}$ & - & $4.06, \mathrm{ddt}(12.8,6.3,1.2)$ & $7 \mathrm{a}, 8$ \\
8 & $134.2, \mathrm{CH}$ & $5.97-5.89, \mathrm{~m}$ & $7 \mathrm{a}, 7 \mathrm{~b}$ \\
& & & $9 \mathrm{a}, 9 \mathrm{~b}$ \\
$9 \mathrm{a}$ & 117.5 & $5.32-5.27, \mathrm{~m}$ & $8,9 \mathrm{~b}$ \\
$9 \mathrm{~b}$ & - & $5.20, \mathrm{dd}(10.4,1.3)$ & $8,9 \mathrm{a}$ \\
10 & $170.6, \mathrm{C}$ & - & - \\
11 & $21.0, \mathrm{CH}_{3}$ & $2.06, \mathrm{~s}$ & -
\end{tabular}


(3S,6R)-6-(prop-2-yn-1-yloxy)-3,6-dihydro-2H-pyran3-yl acetate,

$$
\text { trans-385. }
$$<smiles>[13CH3]C(=O)[18OH]</smiles><smiles>C#CCOC1C=CCCC1OC</smiles>

NMR Assignments

\begin{tabular}{c|c|c|c}
\hline $\mathrm{C}$ & $\delta_{\mathrm{C}}, \mathrm{mult}$ & $\delta_{\mathrm{H}}(J, \mathrm{~Hz})$ & $\mathrm{COSY}$ \\
\hline $2 \mathrm{a}$ & $61.5, \mathrm{CH}_{2}$ & $4.12, \mathrm{dd}(13.0,2.7)$ & $2 \mathrm{~b}, 3$ \\
$2 \mathrm{~b}$ & - & $3.84, \mathrm{~d}(13.0)$ & $2 \mathrm{a}, 3$ \\
3 & $63.1, \mathrm{CH}$ & $4.94, \mathrm{dd}(4.8,2.7)$ & $2 \mathrm{a}, 2 \mathrm{~b}, 4$ \\
4 & $125.4, \mathrm{CH}$ & $6.10, \mathrm{dd}(10.1,5.2)$ & 3,5 \\
5 & $130.3, \mathrm{CH}$ & $6.03, \mathrm{dd}(10.1,3.0)$ & 4,6 \\
6 & $91.1, \mathrm{CH}$ & $5.21, \mathrm{~d}(2.8)$ & 5 \\
7 & 54.8 & $4.29, \mathrm{~d}(2.3)$ & 9 \\
8 & - & - & - \\
9 & $74.7, \mathrm{CH}$ & $2.44, \mathrm{t}(2.2)$ & 7 \\
10 & - & - & - \\
11 & $21.1, \mathrm{CH}_{3}$ & $2.08, \mathrm{~s}$ & -
\end{tabular}

(3S,6S)-6-(prop-2-yn-1-yloxy)-3,6-dihydro-2H-pyran3 -yl acetate,

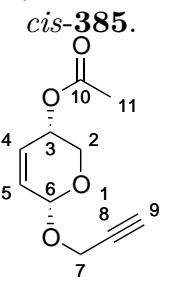

NMR Assignments

\begin{tabular}{c|c|c|c}
\hline $\mathrm{C}$ & $\delta_{\mathrm{C}, \mathrm{mult}}$ & $\delta_{\mathrm{H}}(J, \mathrm{~Hz})$ & $\mathrm{COSY}$ \\
\hline $2 \mathrm{a}$ & $60.0, \mathrm{CH}_{2}$ & $3.87, \mathrm{dd}(10.9,5.7)$ & $2 \mathrm{~b}, 3$ \\
$2 \mathrm{~b}$ & - & $3.77, \mathrm{dd}(10.9,8.5)$ & $2 \mathrm{a}, 3$ \\
3 & $64.9, \mathrm{CH}$ & $5.33-5.30, \mathrm{~m}$ & $2 \mathrm{a}, 2 \mathrm{~b}, 4,5$ \\
4 & $129.6, \mathrm{CH}$ & $5.97, \mathrm{dd}(10.3,1.0)$ & $3,5,6$ \\
5 & $128.4, \mathrm{CH}$ & $5.86, \mathrm{~d}(10.3)$ & $3,4,6$ \\
6 & $92.3, \mathrm{CH}$ & $5.16, \mathrm{~s}$ & 4,5 \\
7 & $54.9, \mathrm{CH}_{2}$ & $4.31, \mathrm{~s}$ & 9 \\
8 & $79.1, \mathrm{C}$ & - & - \\
9 & $74.7, \mathrm{CH}$ & $2.44, \mathrm{~s}$ & 7 \\
10 & $170.5, \mathrm{C}$ & - & - \\
11 & $21.0, \mathrm{CH}_{3}$ & $2.07, \mathrm{~s}$ & -
\end{tabular}

(3S,6R)-6-(benzyloxy)-3,6-dihydro-2H-pyran-3-yl acetate, trans-386.<smiles>CCOC1CCC(OC(C)=O)CC1</smiles>

NMR Assignments

\begin{tabular}{c|c|c|c}
\hline $\mathrm{C}$ & $\delta_{\mathrm{C}}$, mult & $\delta_{\mathrm{H}}(J, \mathrm{~Hz})$ & $\mathrm{COSY}$ \\
\hline $2 \mathrm{a}$ & $61.4, \mathrm{CH}_{2}$ & $4.21, \mathrm{dd}$ & $2 \mathrm{~b}, 3$ \\
& & $(13.1,2.8)$ & \\
$2 \mathrm{~b}$ & - & $3.87, \mathrm{~m}$ & $2 \mathrm{a}, 3$ \\
3 & $63.3, \mathrm{CH}$ & $4.96, \mathrm{~m}$ & $2 \mathrm{a}, 2 \mathrm{~b}, 4$ \\
4 & $124.9, \mathrm{CH}$ & $6.09, \mathrm{~m}$ & 3,5 \\
5 & $130.7, \mathrm{CH}$ & $6.04, \mathrm{dd}$ & 4,6 \\
& & $(10.1,2.9)$ & \\
6 & $92.0, \mathrm{CH}$ & $5.10, \mathrm{dt}$ & 5 \\
& & $(2.9,0.6)$ & \\
$7 \mathrm{a}$ & $69.9, \mathrm{CH}_{2}$ & $4.79, \mathrm{~d}(11.7)$ & $7 \mathrm{~b}$ \\
$7 \mathrm{~b}$ & - & $4.59, \mathrm{~d}(11.7)$ & $7 \mathrm{a}$ \\
8 & $137.5, \mathrm{C} ;$ & $7.38-7.27, \mathrm{~m}$ & 8 \\
& $128.5-127.4,5 \mathrm{xCH}$ & & - \\
9 & $170.6, \mathrm{C}$ & - & -
\end{tabular}

(3S,6S)-6-(benzyloxy)-3,6-dihydro-2H-pyran-3-yl acetate, cis-386.<smiles>CC(=O)OC1CCCCC1Oc1ccccc1</smiles>

NMR Assignments

\begin{tabular}{c|c|c|c}
\hline $\mathrm{C}$ & $\delta_{\mathrm{C}}, \mathrm{mult}$ & $\delta_{\mathrm{H}}(J, \mathrm{~Hz})$ & $\mathrm{COSY}$ \\
\hline 2 & $60.1, \mathrm{CH}_{2}$ & $3.92, \mathrm{~m}$ & 3 \\
3 & $65.0, \mathrm{CH}$ & $5.32, \mathrm{~m}$ & 2,4 \\
4 & $129.1, \mathrm{CH}$ & $5.94, \mathrm{ddt}$ & 3,5 \\
& & $(10.3,2.2,1.0)$ & \\
5 & $129.1, \mathrm{CH}$ & $5.88, \mathrm{ddd}$ & 4,6 \\
& & $(10.3,2.4,1.8)$ & \\
6 & $93.3, \mathrm{CH}$ & $5.04, \mathrm{dd}$ & 5 \\
& & $(2.4,1.3)$ & \\
$7 \mathrm{a}$ & $69.3, \mathrm{CH}_{2}$ & $4.75, \mathrm{~d}(11.9)$ & $7 \mathrm{~b}$ \\
$7 \mathrm{~b}$ & - & $4.47, \mathrm{~d}(11.9)$ & $7 \mathrm{a}$ \\
8 & $138.4, \mathrm{C} ;$ & $7.38-7.28, \mathrm{~m}$ & 8 \\
& $128.5-127.4,5 \mathrm{xCH}$ & & \\
9 & $170.3, \mathrm{C}$ & - & - \\
10 & $21.1, \mathrm{CH}_{3}$ & $2.05, \mathrm{~s}$ & -
\end{tabular}


cis-( \pm )-6-Hydroxy-3,6-dihydro-2H-pyran-3-yl methyl carbonate, cis-387.<smiles>COC(=O)O[C@H]1C=C[C@@H](O)[C@H](O)O1</smiles>

NMR Assignments

\begin{tabular}{c|c|c|c}
\hline $\mathrm{C}$ & $\delta_{\mathrm{C}}, \mathrm{mult}$ & $\delta_{\mathrm{H}}(J, \mathrm{~Hz})$ & $\mathrm{COSY}$ \\
\hline 2 & $61.0, \mathrm{CH}_{2}$ & $3.95, \mathrm{~m}$ & 3 \\
3 & $68.0, \mathrm{CH}$ & $5.10, \mathrm{dddt}$ & 2,4 \\
& & $(6.6,5.0,3.1,1.7)$ & \\
4 & $127.9, \mathrm{CH}$ & $6.04, \mathrm{dd}(10.3,2.8)$ & 3,5 \\
5 & $131.0, \mathrm{CH}$ & $5.96, \mathrm{dt}(10.3,1.8)$ & 4 \\
6 & $89.3, \mathrm{CH}$ & $5.33, \mathrm{~m}$ & 9 \\
7 & $155.3, \mathrm{C}$ & - & - \\
8 & $54.9, \mathrm{CH}_{3} ;$ & $3.80, \mathrm{~s}$ & - \\
9 & - & $2.90, \mathrm{~d}(6.4)$ & 6
\end{tabular}

trans-( \pm )-6-Hydroxy-3,6-dihydro-2H-pyran-3-yl methyl carbonate, trans-387.<smiles>COC(=O)OC1C=CC(O)OC1</smiles>

NMR Assignments

\begin{tabular}{c|c|c|c}
\hline $\mathrm{C}$ & $\delta_{\mathrm{C}}, \mathrm{mult}$ & $\delta_{\mathrm{H}}(J, \mathrm{~Hz})$ & $\mathrm{COSY}$ \\
\hline $2 \mathrm{a}$ & $61.1, \mathrm{CH}_{2}$ & $4.28, \mathrm{dd}(13.1,2.9)$ & $2 \mathrm{~b}, 4$ \\
$2 \mathrm{~b}$ & - & $3.95, \mathrm{~m}$ & $2 \mathrm{a}$ \\
3 & $66.5, \mathrm{CH}$ & $4.84, \mathrm{ddd}$ & $2 \mathrm{a}, 4$ \\
& & $(4.6,3.0,1.6)$ & \\
4 & $124.5, \mathrm{CH}$ & $6.14, \mathrm{~m}$ & 3,5 \\
5 & $132.0, \mathrm{CH}$ & $6.10, \mathrm{dd}(10.1,2.6)$ & 4,6 \\
6 & $87.7, \mathrm{CH}$ & $5.42, \mathrm{dd}(4.8,2.5)$ & 5,9 \\
7 & $155.3, \mathrm{C}$ & - & - \\
8 & $55.0, \mathrm{CH}_{3} ;$ & $3.80, \mathrm{~s}$ & - \\
9 & - & $2.77, \mathrm{~d}(4.2)$ & 6
\end{tabular}

(3S,6R)-6-(allyloxy)-3,6-dihydro-2H-pyran-3-ol, trans-391.<smiles>C=CCOC1C=CC(O)CC1O</smiles>

NMR Assignments

\begin{tabular}{c|c|c|c}
\hline $\mathrm{C}$ & $\delta_{\mathrm{C}}$, mult & $\delta_{\mathrm{H}}(J, \mathrm{~Hz})$ & $\mathrm{COSY}$ \\
\hline $2 \mathrm{a}$ & $64.30, \mathrm{CH}_{2}$ & $4.13, \mathrm{dd}(12.2,2.5)$ & $2 \mathrm{~b}, 3$ \\
$2 \mathrm{~b}$ & - & $3.79, \mathrm{dt}(12.2,1.3)$ & $2 \mathrm{a}, 3$ \\
3 & $61.54, \mathrm{CH}$ & $3.93, \mathrm{app} . \mathrm{s}$ & $2 \mathrm{a}, 2 \mathrm{~b}$ \\
& & & 4,10 \\
4 & $129.22, \mathrm{CH}$ & $6.14, \mathrm{ddt}$ & 3,5 \\
& & $(10.0,5.3,1.1)$ & \\
5 & $128.47, \mathrm{CH}$ & $5.90, \mathrm{dd}(10.0,3.3)$ & 4,6 \\
6 & $92.31, \mathrm{CH}$ & $4.99, \mathrm{~d}(3.1)$ & 5 \\
$7 \mathrm{a}$ & $68.5, \mathrm{CH}_{2}$ & $4.26, \mathrm{ddt}$ & $7 \mathrm{~b}, 8$ \\
& & $(12.7,5.2,1.4)$ & \\
$7 \mathrm{~b}$ & - & $4.06, \mathrm{ddt}$ & $7 \mathrm{a}, 8$ \\
& & $(12.7,6.3,1.2)$ & \\
8 & $134.19, \mathrm{CH}$ & $5.95, \mathrm{~m}$ & $7 \mathrm{a}, 7 \mathrm{~b}$, \\
& & & $9 \mathrm{a}, 9 \mathrm{~b}$ \\
$9 \mathrm{a}$ & $117.56, \mathrm{CH}_{2}$ & $5.30, \mathrm{ddd}$ & $8,9 \mathrm{~b}$ \\
& & $(17.2,3.6,2.0)$ & \\
$9 \mathrm{~b}$ & - & $5.21, \mathrm{~m}$ & $8,9 \mathrm{a}$ \\
10 & - & $2.00, \mathrm{~d}(8.9)$ & 3
\end{tabular}

(3S,6S)-6-(allyloxy)-3,6-dihydro-2H-pyran-3-ol,<smiles>C=CCOC1C=CC(OCC)CO1</smiles>

NMR Assignments

\begin{tabular}{c|c|c|c}
\hline $\mathrm{C}$ & $\delta_{\mathrm{C}}$, mult & $\delta_{\mathrm{H}}(J, \mathrm{~Hz})$ & COSY \\
\hline $2 \mathrm{a}$ & $63.81, \mathrm{CH}_{2}$ & $3.82, \mathrm{dd}(11.0,5.3)$ & $2 \mathrm{~b}, 3$ \\
$2 \mathrm{~b}$ & - & $3.72, \mathrm{dd}(11.0,8.0)$ & $2 \mathrm{a}, 3$ \\
3 & $63.18, \mathrm{CH}$ & $4.25, \mathrm{~m}$ & $2 \mathrm{a}, 2 \mathrm{~b}, 4$ \\
4 & $133.00, \mathrm{CH}$ & $6.04, \mathrm{~m}$ & 3,5 \\
5 & $127.78, \mathrm{CH}$ & $5.80, \mathrm{dt}(10.3,2.0)$ & 4,6 \\
6 & $93.66, \mathrm{CH}$ & $4.98, \mathrm{~m}$ & 4,5 \\
$7 \mathrm{a}$ & $69.15, \mathrm{CH}_{2}$ & $4.28, \mathrm{~m}$ & $7 \mathrm{~b}, 8$ \\
$7 \mathrm{~b}$ & - & $4.07, \mathrm{dd}(12.7,6.3)$ & $7 \mathrm{a}, 8$ \\
8 & $134.22, \mathrm{CH}$ & $5.95, \mathrm{~m}$ & $7 \mathrm{a}, 7 \mathrm{~b}$ \\
& & & $9 \mathrm{a}, 9 \mathrm{~b}$ \\
$9 \mathrm{a}$ & 117.49 & $5.31, \mathrm{~m}$ & $8,9 \mathrm{~b}$ \\
$9 \mathrm{~b}$ & - & $5.21, \mathrm{~m}$ & $8,9 \mathrm{a}$ \\
10 & - & $1.56, \mathrm{br} . \mathrm{s}$ & 3
\end{tabular}


(3S,6R)-6-(isopropoxy)-3,6-dihydro-2H-pyran-3-ol,<smiles>CC(C)OC1C=CC(O)CC1O</smiles>

NMR Assignments

\begin{tabular}{c|c|c|c}
\hline $\mathrm{C}$ & $\delta_{\mathrm{C}}$, mult & $\delta_{\mathrm{H}}(J, \mathrm{~Hz})$ & $\mathrm{COSY}$ \\
\hline $2 \mathrm{a}$ & $64.11, \mathrm{CH}_{2}$ & $4.14, \mathrm{dd}(12.3,2.6)$ & $2 \mathrm{~b}, 3$ \\
$2 \mathrm{~b}$ & - & $3.77, \mathrm{~d}(12.3)$ & $2 \mathrm{a}, 3$ \\
3 & $61.63, \mathrm{CH}$ & 3.81, app. s & $2 \mathrm{a}, 2 \mathrm{~b}$, \\
& & & 4,10 \\
4 & $128.95, \mathrm{CH}$ & $6.11, \mathrm{dd}$ & 3,5 \\
& & $(10.0,5.3)$ & \\
5 & $129.07, \mathrm{CH}$ & $5.85, \mathrm{dd}(10.0,3.1)$ & 4,6 \\
6 & $91.31, \mathrm{CH}$ & $5.04, \mathrm{~d}(3.0)$ & 5 \\
7 & $69.82, \mathrm{CH}$ & $3.98, \mathrm{~m}$ & 8,9 \\
8 & $23.53, \mathrm{CH} 3$ & $1.23, \mathrm{~d}(6.4)$ & 7 \\
9 & $21.82, \mathrm{CH}_{3}$ & $1.17, \mathrm{~d}(6.1)$ & 7 \\
10 & - & $2.11, \mathrm{br} . \mathrm{s}$ & 3
\end{tabular}

(3S,6S)-6-(isopropoxy)-3,6-dihydro-2H-pyran-3-ol,

$$
\text { cis-390. }
$$

NMR Assignments

\begin{tabular}{c|c|c|c}
\hline $\mathrm{C}$ & $\delta_{\mathrm{C}}, \mathrm{mult}$ & $\delta_{\mathrm{H}}(J, \mathrm{~Hz})$ & COSY \\
\hline 2 & $63.64, \mathrm{CH}_{2}$ & $3.79-3.69, \mathrm{~m}$ & 3 \\
3 & $63.208, \mathrm{CH}$ & 4.22, app. s & 2,4 \\
4 & $132.75, \mathrm{CH}$ & $6.00, \mathrm{~m}$ & 3,5 \\
5 & $128.38, \mathrm{CH}$ & $5.74, \mathrm{dt}(10.2,2.1)$ & 4,6 \\
6 & $92.66, \mathrm{CH}$ & 5.01, app. s & 5 \\
7 & $70.28, \mathrm{CH}$ & $3.98, \mathrm{~m}$ & 8,9 \\
8 & $23.63, \mathrm{CH}_{3}$ & $1.24, \mathrm{~d}(7.0)$ & 7 \\
9 & $21.88, \mathrm{CH}_{3}$ & $1.17, \mathrm{~d}(6.1)$ & 7 \\
10 & - & 1.84, br. s & 3
\end{tabular}

4-[cis-士-6[(tert-butyldimethylsilyl)oxy]]-3,6-dihydro2H-pyran-3-yl $]$ oxy-6-methyl-2H-pyran-2-one,

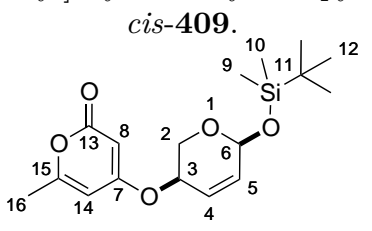

NMR Assignments

\begin{tabular}{c|c|c|c}
\hline $\mathrm{C}$ & $\delta_{\mathrm{C}}, \mathrm{mult}$ & $\delta_{\mathrm{H}}(J, \mathrm{~Hz})$ & $\mathrm{COSY}$ \\
\hline $2 \mathrm{a}$ & $59.8, \mathrm{CH}_{2}$ & $3.95, \mathrm{dd}(11.1,8.0)$ & $2 \mathrm{~b}, 3$ \\
$2 \mathrm{~b}$ & - & $3.88, \mathrm{ddd}(11.1,5.4,0.9)$ & $2 \mathrm{a}, 3$ \\
3 & $68.6, \mathrm{CH}$ & $4.79, \mathrm{~m}$ & $2 \mathrm{a}, 2 \mathrm{~b}, 4$ \\
4 & $125.2, \mathrm{CH}$ & 5.95, app. dt $(10.3,0.9)$ & 3,5 \\
5 & $132.7, \mathrm{CH}$ & 5.89, app. dt $(10.3,1.9)$ & 4,6 \\
6 & $89.3, \mathrm{CH}$ & $5.41, \mathrm{~d}(2.1)$ & 5 \\
7 & $169.2, \mathrm{C}$ & - & - \\
8 & $88.4, \mathrm{CH}$ & $5.3, \mathrm{~s}$ & 14 \\
9 & $-5.3, \mathrm{CH}_{3}$ & $0.13, \mathrm{~s}$ & - \\
10 & $-4.4, \mathrm{CH}_{3}$ & $0.13, \mathrm{~s}$ & - \\
11 & $18.0, \mathrm{C}$ & - & - \\
12 & $25.7, \mathrm{CH}_{3}$ & $0.91, \mathrm{~s}$ & - \\
13 & $164.7, \mathrm{C}$ & - & - \\
14 & $100.6, \mathrm{CH}$ & $5.77, \mathrm{~s}$ & 8,16 \\
15 & $162.6, \mathrm{C}$ & - & - \\
16 & $19.9, \mathrm{CH}_{3}$ & $2.2, \mathrm{~s}$ & 14
\end{tabular}

trans-( \pm$)-6(($ tert-butyldimethylsilyl)oxy)-3,6-dihydro2H-pyran-3-yl benzoate,

\begin{tabular}{|c|c|c|c|}
\hline $\mathrm{C}$ & $\delta_{\mathrm{C}}$, mult & $\delta_{\mathrm{H}}(J, \mathrm{~Hz})$ & COSY \\
\hline $2 a$ & $61.4, \mathrm{CH}_{2}$ & $\begin{array}{c}4.31, \mathrm{dd} \\
(13.0,2.9)\end{array}$ & $2 \mathrm{~b}, 3$ \\
\hline $2 \mathrm{~b}$ & - & $\begin{array}{c}3.97, \mathrm{dt} \\
(13.0,1.3)\end{array}$ & $2 \mathrm{a}, 3$ \\
\hline 3 & $64.0, \mathrm{CH}$ & $5.19, \mathrm{~m}$ & $2 \mathrm{a}, 2 \mathrm{~b}, 4$ \\
\hline 4 & $123.3, \mathrm{CH}$ & $\begin{array}{c}6.12, \mathrm{dd} \\
(10.0,5.1)\end{array}$ & 3,5 \\
\hline 5 & $133.4, \mathrm{CH}$ & $\begin{array}{c}6.04, \mathrm{dd} \\
(10.0,3.0)\end{array}$ & 4,6 \\
\hline 6 & $88.0, \mathrm{CH}$ & $5.39, \mathrm{~d}(3.0)$ & 5 \\
\hline 7 & $166.3, \mathrm{C}$ & - & - \\
\hline 8 & $\begin{array}{c}133.1,130.0 \\
129.8,128.3, \\
\mathrm{Ph}-\mathrm{CH}\end{array}$ & $\begin{array}{c}8.06,7.56 \\
7.43, \mathrm{~m}\end{array}$ & - \\
\hline 9 & $-5.3, \mathrm{CH}_{3}$ & $0.15, \mathrm{~s}$ & - \\
\hline 10 & $-4.5, \mathrm{CH}_{3}$ & $0.15, \mathrm{~s}$ & - \\
\hline 11 & 18.1, C & - & - \\
\hline 12 & $25.7, \mathrm{CH}_{3}$ & $0.92, \mathrm{~s}$ & - \\
\hline
\end{tabular}<smiles>CC(C)(C)[Si](C)(C)OC1C=C[C@@H](OC(=O)c2ccccc2)CO1</smiles>

NMR Assignments 
trans-( \pm$)-6(($ tert-butyldimethylsilyl)oxy)-3,6-dihydro2H-pyran-3-ol, trans-411.<smiles>CC(C)(C)[Si](C)(C)OC1C=CCCC1</smiles>

NMR Assignments

\begin{tabular}{c|c|c|c}
\multicolumn{4}{|c}{ NMR Assignments } \\
\hline $\mathrm{C}$ & $\delta_{\mathrm{C}}, \mathrm{mult}$ & $\delta_{\mathrm{H}}(J, \mathrm{~Hz})$ & $\mathrm{COSY}$ \\
\hline $2 \mathrm{a}$ & $64.3, \mathrm{CH}_{2}$ & $4.16, \mathrm{dd}$ & $2 \mathrm{~b}, 3$ \\
& & $(12.2,2.6)$ & \\
& & $3.77, \mathrm{dt}$ & $2 \mathrm{a}, 3$ \\
& - & $(12.2,1.3)$ & \\
& & $3.83, \mathrm{~m}$ & $2 \mathrm{a}, 2 \mathrm{~b}, 4$ \\
3 & $61.6, \mathrm{CH}$ & $6.06, \mathrm{dd}$ & 3,5 \\
4 & $127.5, \mathrm{CH}$ & $(10,5.3)$ & \\
& & $5.85, \mathrm{dd}$ & 4,6 \\
5 & $131.0, \mathrm{CH}$ & $(10,3.0)$ & \\
& & $5.29, \mathrm{~d}(3.0)$ & 5 \\
6 & $88.2, \mathrm{CH}$ & $-85, \mathrm{~d}(9.2)$ & 3 \\
7 & - & $0.14, \mathrm{~s}$ & - \\
9 & $-5.3, \mathrm{CH}_{3}$ & $0.14, \mathrm{~s}$ & - \\
10 & $-4.5, \mathrm{CH}_{3}$ & - & - \\
11 & $18.1, \mathrm{C}$ & $0.91, \mathrm{~s}$ & -
\end{tabular}

cis-(土)-6((tert-butyldimethylsilyl)oxy)-3,6-dihydro2H-pyran-3-ol,

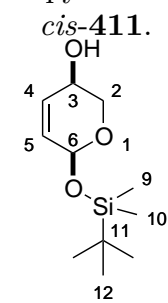

NMR Assignments

\begin{tabular}{c|c|c|c}
\hline $\mathrm{C}$ & $\delta_{\mathrm{C}}, \mathrm{mult}$ & $\delta_{\mathrm{H}}(J, \mathrm{~Hz})$ & $\mathrm{COSY}$ \\
\hline 2 & $64.6, \mathrm{CH}_{2}$ & $3.77, \mathrm{~m}$ & 3 \\
3 & $63.1, \mathrm{CH}$ & $4.15, \mathrm{~m}$ & 2,4 \\
4 & $130.8, \mathrm{CH}$ & $5.95, \mathrm{dd}$ & 3,5 \\
& & $(10,2.7)$ & \\
5 & $130.9, \mathrm{CH}$ & $5.75, \mathrm{dd}$ & 4,6 \\
& & $(10.2,1.9)$ & \\
6 & $89.9, \mathrm{CH}$ & $5.25, \mathrm{~m}$ & 5 \\
7 & - & $1.65, \mathrm{~m}$ & 3 \\
9 & $-4.3, \mathrm{CH}_{3}$ & $0.14, \mathrm{~s}$ & - \\
10 & $-3.6, \mathrm{CH}_{3}$ & $0.13, \mathrm{~s}$ & - \\
11 & $18.1, \mathrm{C}$ & - & - \\
12 & $25.7, \mathrm{CH}_{3}$ & $0.91, \mathrm{~s}$ & -
\end{tabular}

(3S,6R)-6-(allyl)-3,6-dihydro-2H-pyran-3-yl acetate, trans-392.<smiles>O=C(O)[13OH]</smiles><smiles>C=CC1C=CCCO1</smiles>

NMR Assignments

\begin{tabular}{c|c|c|c}
\hline $\mathrm{C}$ & $\delta_{\mathrm{C}}$, mult & $\delta_{\mathrm{H}}(J, \mathrm{~Hz})$ & $\mathrm{COSY}$ \\
\hline $2 \mathrm{a}$ & $65.1, \mathrm{CH}_{2}$ & $4.12, \mathrm{dd}(11.4,5.0)$ & $2 \mathrm{~b}, 3$ \\
$2 \mathrm{~b}$ & - & $3.53, \mathrm{dd}(11.4,6.8)$ & $2 \mathrm{a}, 3$ \\
3 & $64.9, \mathrm{CH}$ & $5.24, \mathrm{~m}$ & $2 \mathrm{a}, 2 \mathrm{~b}, 4$ \\
4 & $124.43, \mathrm{CH}$ & $5.85-5.78, \mathrm{~m}$ & 3,5 \\
5 & $133.52, \mathrm{CH}$ & $5.91, \mathrm{app} . \mathrm{d}(10.4)$ & 4,6 \\
6 & $73.1, \mathrm{CH}$ & $4.18, \mathrm{~m}$ & $5,7 \mathrm{a}, 7 \mathrm{~b}$ \\
$7 \mathrm{a}$ & $38.64, \mathrm{CH}$ & $4.25, \mathrm{ddt}(12.7,5.2,1.4)$ & $6,7 \mathrm{~b}, 8$ \\
$7 \mathrm{~b}$ & - & $4.06, \mathrm{ddt}(12.7,6.4,1.1)$ & $6,7 \mathrm{a}, 8$ \\
8 & $133.86, \mathrm{CH}$ & $5.85-5.78, \mathrm{~m}$ & $7 \mathrm{a}, 7 \mathrm{~b}, 9$ \\
9 & $117.6, \mathrm{CH}$ & $5.16-5.06, \mathrm{~m}$ & $8,9 \mathrm{~b}$ \\
10 & $170.6, \mathrm{C}$ & - & - \\
11 & $21.09, \mathrm{CH}_{3}$ & $2.06, \mathrm{~s}$ & -
\end{tabular}

(3S,6S)-6-(allyloxy)-3,6-dihydro-2H-pyran-3-yl acetate, cis-392.<smiles>C=CCC1C=CC(OC=O)CC1</smiles>

NMR Assignments

\begin{tabular}{c|c|c|c}
\hline $\mathrm{C}$ & $\delta_{\mathrm{C}, \mathrm{mult}}$ & $\delta_{\mathrm{H}}(J, \mathrm{~Hz})$ & $\mathrm{COSY}$ \\
\hline $2 \mathrm{a}$ & $67.98, \mathrm{CH}_{2}$ & $4.06-4.08, \mathrm{~m}$ & $2 \mathrm{~b}, 3$ \\
$2 \mathrm{~b}$ & - & $3.76, \mathrm{dd}(12.9,2.6)$ & $2 \mathrm{a}, 3$ \\
3 & $64.67, \mathrm{CH}$ & 5.00, app. s & $2 \mathrm{a}, 2 \mathrm{~b}, 4$ \\
4 & $122.45, \mathrm{CH}$ & $5.94, \mathrm{~m}$ & 3,5 \\
5 & $135.7, \mathrm{CH}$ & 6.02, app. d $(10.3)$ & 4,6 \\
6 & $73.5, \mathrm{CH}$ & $4.08-4.06, \mathrm{~m}$ & 4,5 \\
$7 \mathrm{a}$ & $39.18, \mathrm{CH}_{2}$ & $2.43, \mathrm{dt}(14.0,7.4)$ & $7 \mathrm{~b}, 8$ \\
$7 \mathrm{~b}$ & - & $2.33, \mathrm{dt}(14.3,6.7)$ & $7 \mathrm{a}, 8$ \\
8 & $133.83, \mathrm{CH}$ & $5.86, \mathrm{ddt}(17.3,10.2,7.0)$ & $7 \mathrm{a}, 7 \mathrm{~b}$, \\
& & & $9 \mathrm{a}, 9 \mathrm{~b}$ \\
9 & 117.69 & $5.17-5.12, \mathrm{~m}$ & $8,9 \mathrm{~b}$ \\
10 & $170.92, \mathrm{C}$ & - & - \\
11 & $21.28, \mathrm{CH}_{3}$ & $2.10, \mathrm{~s}$ & -
\end{tabular}


(3S,6R)-6-(allyl)-3,6-dihydro-2H-pyran-3-ol, trans-393.<smiles>C=CCC1C=CC(O)CC1O</smiles>

NMR Assignments

\begin{tabular}{|c|c|c|c|}
\hline $\mathrm{C}$ & $\delta_{\mathrm{C}}$, mult & $\delta_{\mathrm{H}}(J, \mathrm{~Hz})$ & COSY \\
\hline $2 \mathrm{a}$ & $68.72, \mathrm{CH}_{2}$ & $\begin{array}{c}4.08, \mathrm{dd} \\
(11.2,4.8)\end{array}$ & $2 \mathrm{~b}, 3$ \\
\hline $2 b$ & - & $\begin{array}{c}3.44, \mathrm{dd} \\
(11.2,6.9)\end{array}$ & $2 \mathrm{a}, 3$ \\
\hline 3 & $62.96, \mathrm{CH}$ & 4.20 , app. s & $2 \mathrm{a}, 2 \mathrm{~b}, 4,10$ \\
\hline 4 & $131.92, \mathrm{CH}$ & $5.87-5.79, \mathrm{~m}$ & 3,5 \\
\hline 5 & $128.62, \mathrm{CH}$ & $\begin{array}{c}5.90, \mathrm{dt} \\
(10.4,2.5)\end{array}$ & 4,6 \\
\hline 6 & 73.34, CH & $\begin{array}{c}4.15, \mathrm{ddt} \\
(8.0,4.3,2.1)\end{array}$ & $5,7 \mathrm{a}, 7 \mathrm{~b}$ \\
\hline $7 \mathrm{a}$ & $38.91, \mathrm{CH}_{2}$ & $2.37-2.25, \mathrm{~m}$ & $6,7 \mathrm{~b}, 8$ \\
\hline $7 \mathrm{~b}$ & - & $2.37-2.25, \mathrm{~m}$ & $6,7 \mathrm{a}, 8$ \\
\hline 8 & $134.21, \mathrm{CH}$ & $5.87-5.79, \mathrm{~m}$ & $7 \mathrm{a}, 7 \mathrm{~b}, 9$ \\
\hline 9 & $117.63, \mathrm{CH}_{2}$ & $5.15-5.09, \mathrm{~m}$ & 8 \\
\hline 10 & - & $1.73, \mathrm{~d}(7.6)$ & 3 \\
\hline
\end{tabular}

(3S,6S)-6-(allyl)-3,6-dihydro-2H-pyran-3-ol, cis-393.<smiles>C=CC1C=CC(O)CO1</smiles>

\begin{tabular}{c|c}
\multicolumn{2}{c}{ NMR Assignments } \\
\hline $\mathrm{C}$ & $\delta_{\mathrm{C}}, \mathrm{mult}$ \\
\hline 2 & $71.04, \mathrm{CH}_{2}$ \\
3 & $62.6, \mathrm{CH}$ \\
4 & $133.52, \mathrm{CH}$ \\
5 & $126.99, \mathrm{CH}$ \\
6 & $73.97, \mathrm{CH}$ \\
7 & $39.46, \mathrm{CH}_{2}$ \\
8 & $134.21, \mathrm{CH}$ \\
9 & $117.94, \mathrm{CH}_{2}$ \\
10 & -
\end{tabular}

cis-393 was produced as a side product of the original Ferrier reaction and comprises less than $10 \%$ of the trans-393 sample. The small amount of material prevented full characterization but did provide a set of ${ }^{13} \mathrm{C}$ NMR data for comparison.
((2R,3S,6S)-3-acetoxy-6-isopropoxy-3,6-dihydro- $2 H$ pyran-3-yl)methyl acetate,

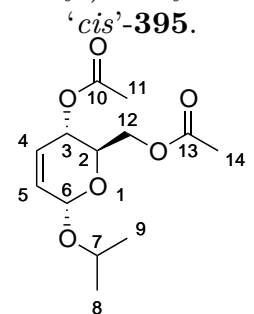

NMR Assignments

\begin{tabular}{c|c|c|c}
\hline $\mathrm{C}$ & $\delta_{\mathrm{C}}, \mathrm{mult}$ & $\delta_{\mathrm{H}}(J, \mathrm{~Hz})$ & $\mathrm{COSY}$ \\
\hline 2 & $66.74, \mathrm{CH}$ & $4.14, \mathrm{~m}$ & $2 \mathrm{~b}, 3,12$ \\
3 & $65.39, \mathrm{CH}$ & $5.29, \mathrm{dd}$ & $2 \mathrm{a}, 2 \mathrm{ba}, 4$ \\
& & $(9.5,1.5)$ & \\
4 & $128.45, \mathrm{CH}$ & 5.86, app. d $(10.2)$ & 3,5 \\
5 & $128.8, \mathrm{CH}$ & $5.80, \mathrm{dt}$ & 4,6 \\
& & $(10.2,2.3)$ & \\
6 & $92.82, \mathrm{CH}$ & $5.13, \mathrm{~m}$ & 5 \\
7 & $70.75, \mathrm{CH}$ & $3.98,7(6.2)$ & 8,9 \\
8 & $23.51, \mathrm{CH}_{3}$ & $1.25, \mathrm{~d}(6.2)$ & 7 \\
9 & $21.99, \mathrm{CH}_{3}$ & $1.18, \mathrm{~d}(6.1)$ & 7 \\
10 & $170.78, \mathrm{C}$ & - & - \\
11 & $20.97, \mathrm{CH}_{3}$ & $2.09, \mathrm{~s}$ & - \\
$12 \mathrm{a}$ & $63.12, \mathrm{CH}_{3}$ & $4.23, \mathrm{dd}$ & $12 \mathrm{~b}, 2$ \\
& & $(11.8,5.5)$ & \\
$12 \mathrm{~b}$ & - & $4.16, \mathrm{~m}$ & $12 \mathrm{a}, 2$ \\
13 & $170.31, \mathrm{C}$ & - & - \\
14 & $20.76, \mathrm{CH}_{3}$ & $2.09, \mathrm{~s}$ & -
\end{tabular}


((2R,3S,6R)-3-acetoxy-6-isopropoxy-3,6-dihydro-2Hpyran-3-yl)methyl acetate,

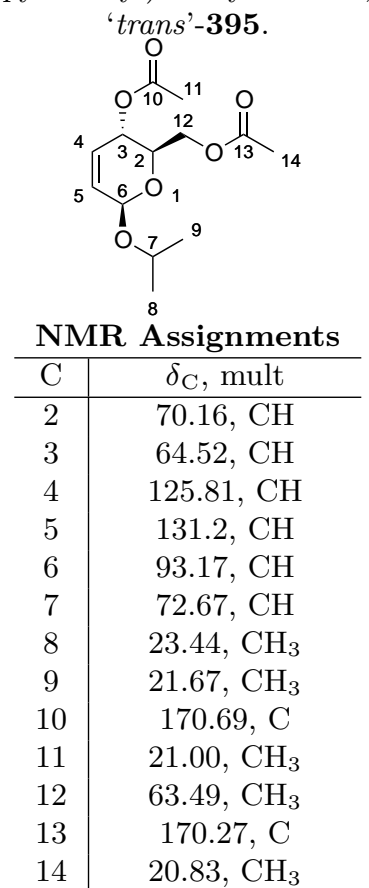

'trans'-412 was produced as a side product of the original Ferrier reaction and comprises less than $10 \%$ of the 'cis'-412 sample. The small amount of material prevented full characterization but did provide a set of ${ }^{13} \mathrm{C}$ NMR data for comparison. 


\subsubsection{Spectra}

${ }^{1} \mathrm{H}-\mathrm{NMR}\left(500 \mathrm{MHz}, \mathrm{CDCl}_{3}\right)$

$\overbrace{\text { OTBS }}^{\text {OAC }}$
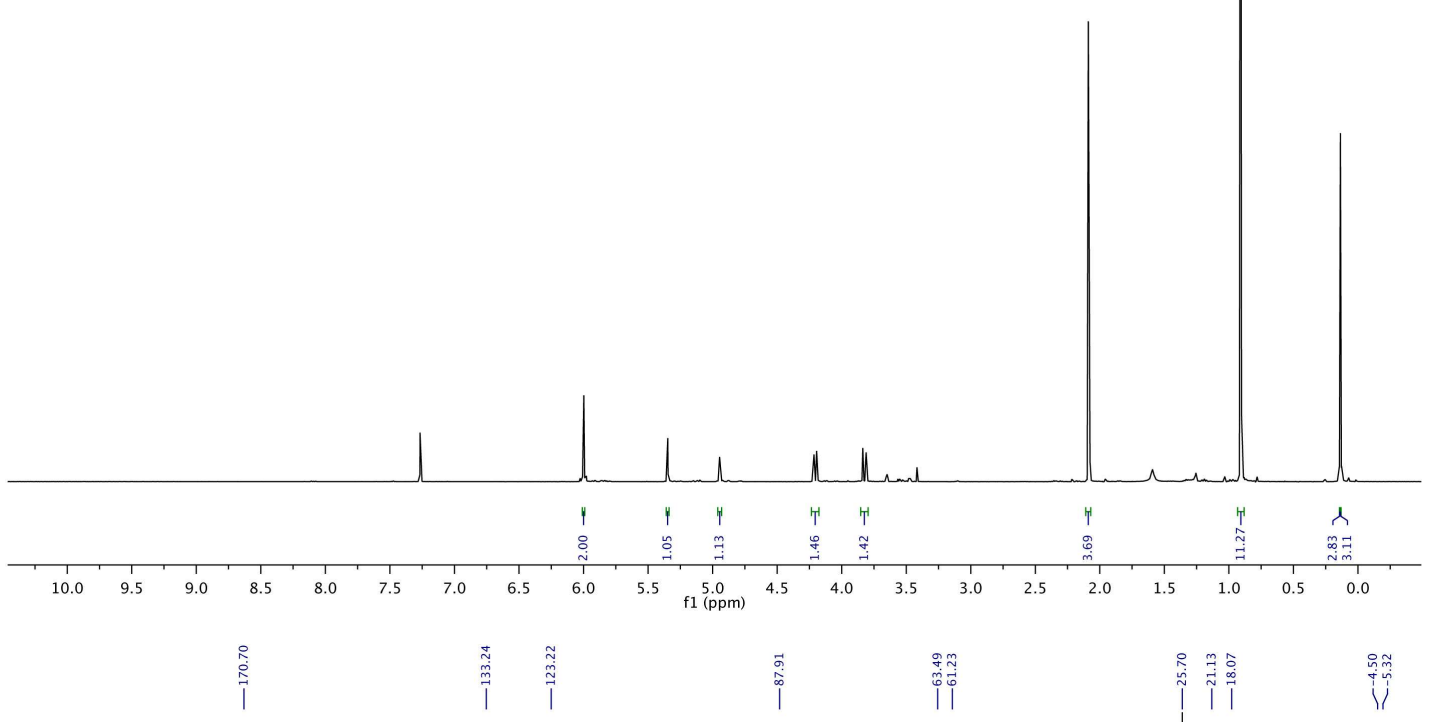

$\vec{\sigma}$
$\substack{0 \\ \infty}$

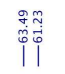

${ }^{13} \mathrm{C}-\mathrm{NMR}\left(126 \mathrm{MHz}, \mathrm{CDCl}_{3}\right)$

$\overbrace{\text { OTBS }}^{\text {OAC }}$

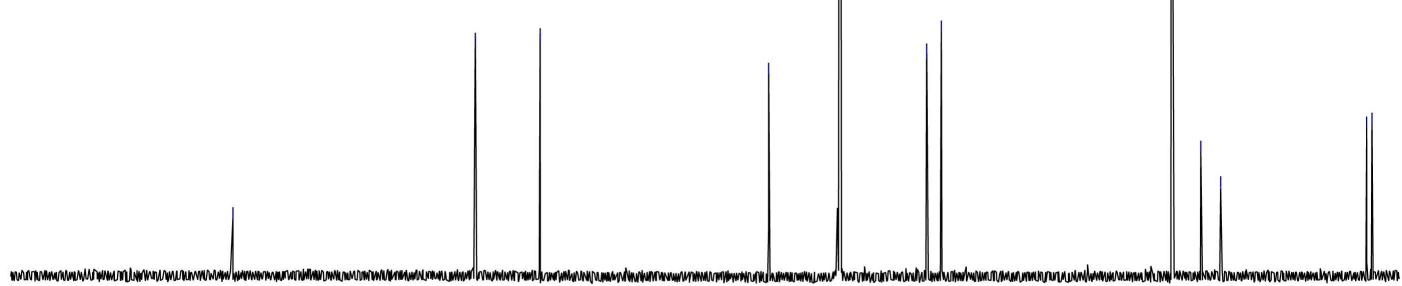

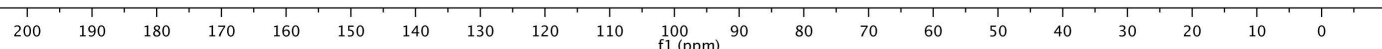



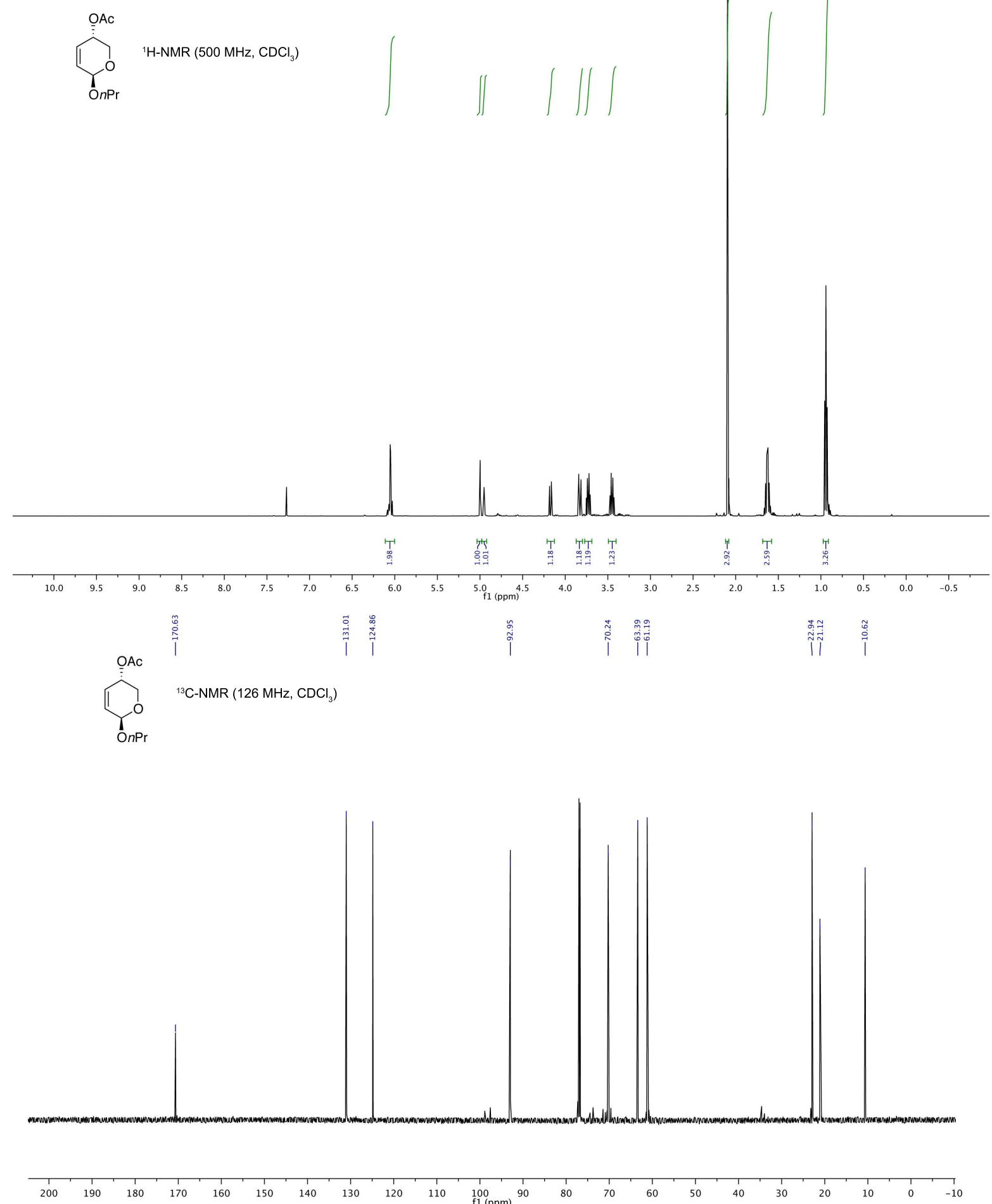

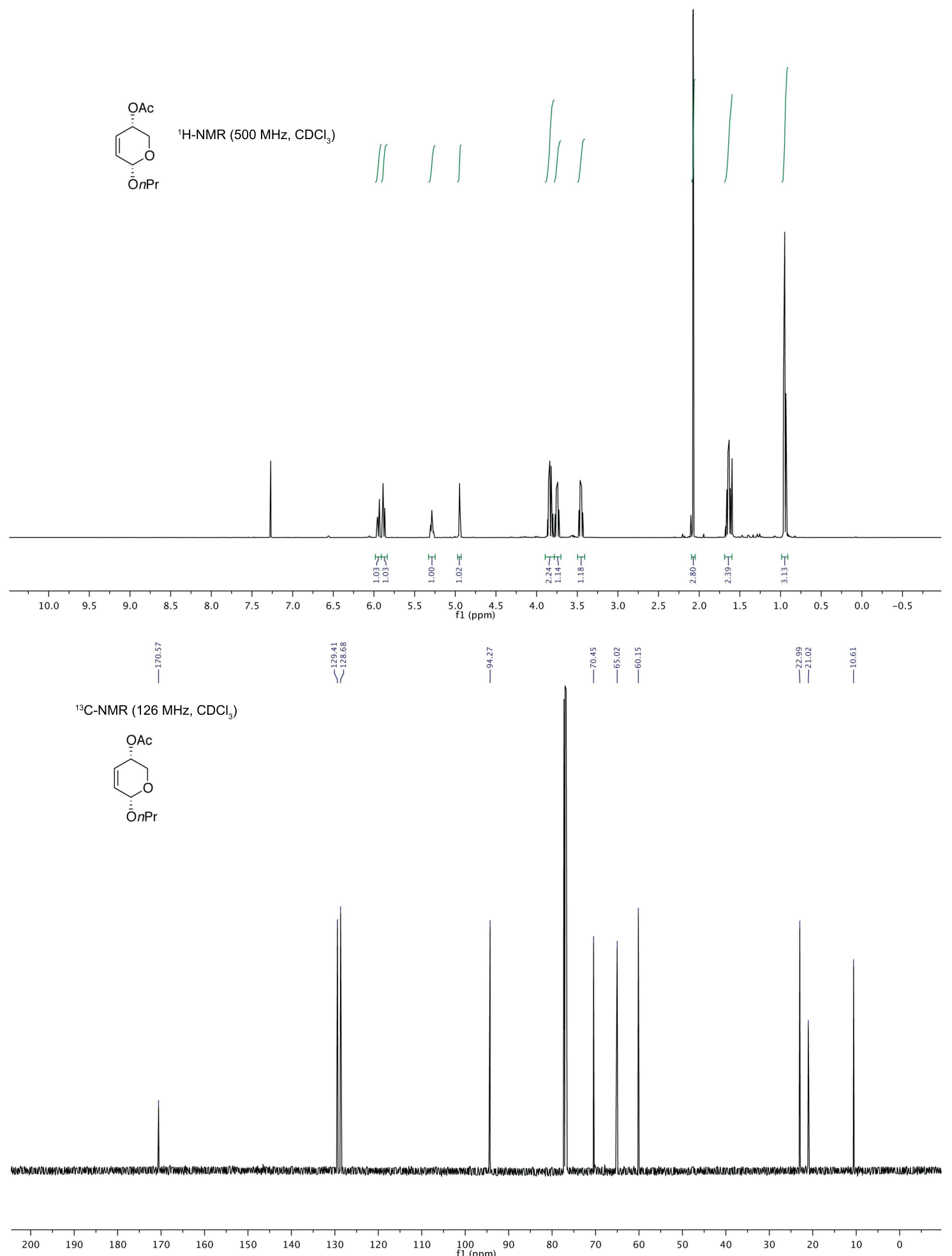


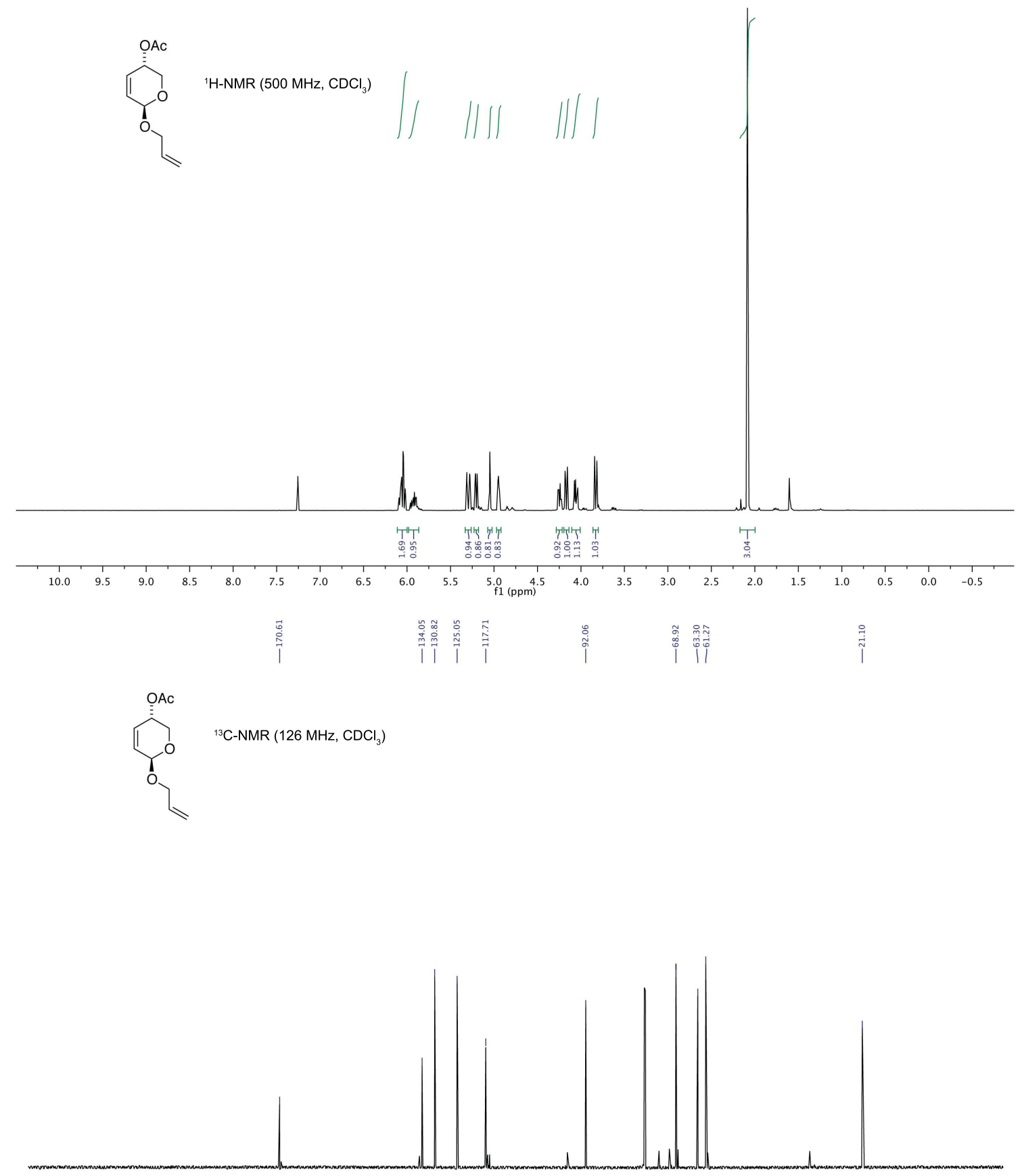

$\begin{array}{rllllllllllllllllllllllllll}1 & 230 & 220 & 210 & 200 & 190 & 180 & 170 & 160 & 150 & 140 & 130 & 120 & \begin{array}{c}110 \\ \mathrm{f} 1(\mathrm{ppm})\end{array} & 100 & 90 & 80 & 70 & 60 & 50 & 40 & 30 & 20 & 10 & 0 & -10\end{array}$ 

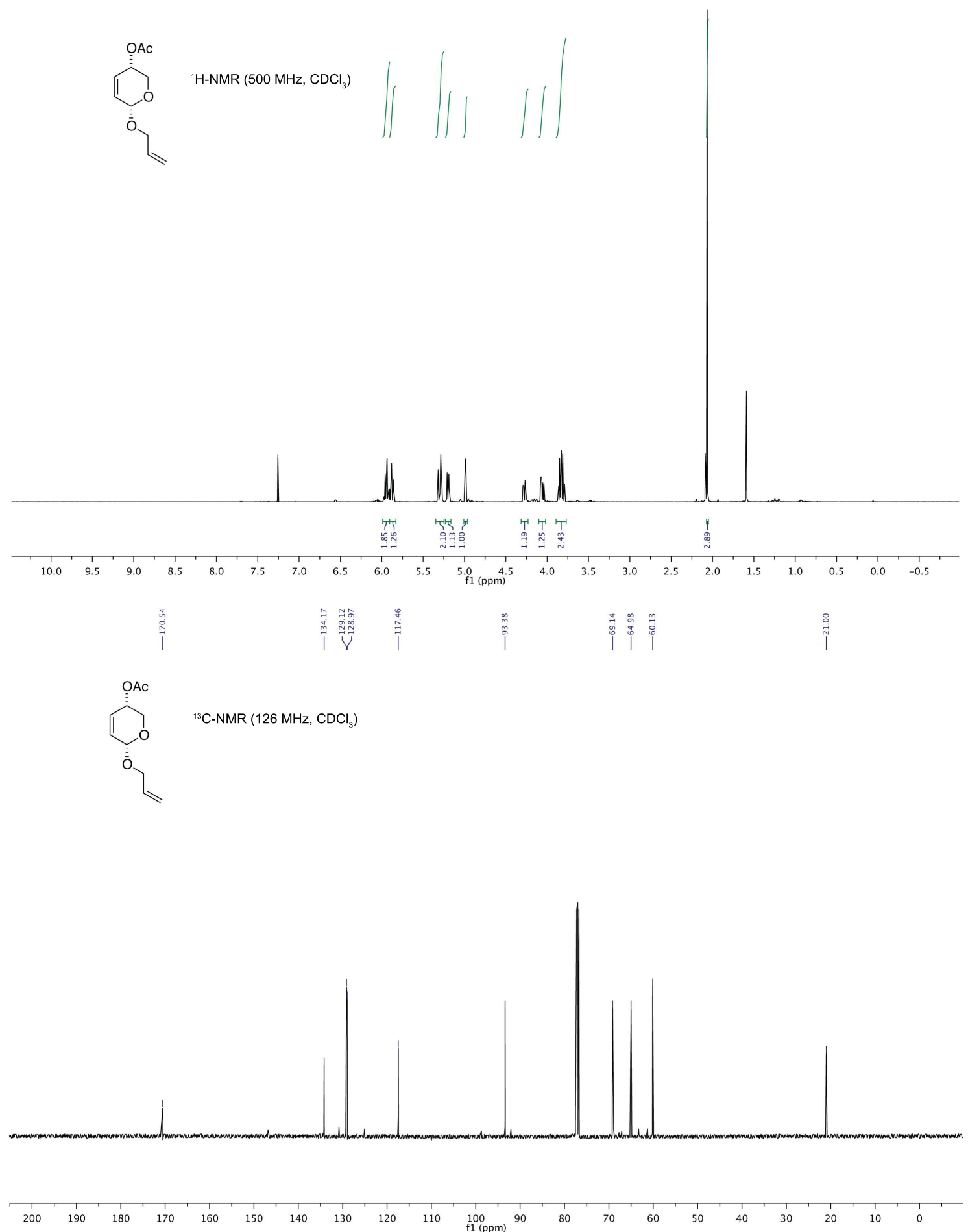


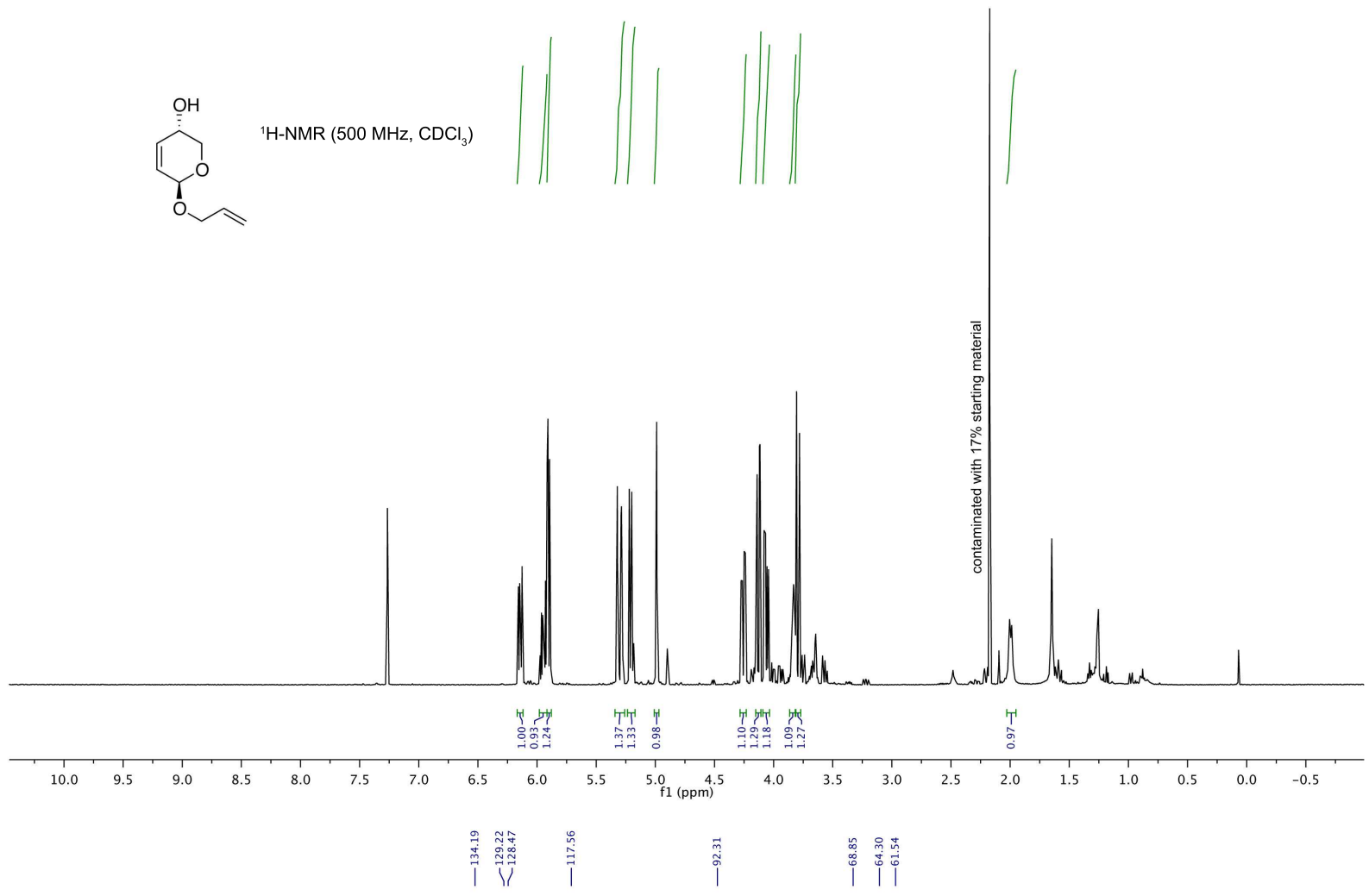

${ }^{13} \mathrm{C}-\mathrm{NMR}\left(126 \mathrm{MHz}, \mathrm{CDCl}_{3}\right.$ )<smiles>C=CCOC1C=CC(O)CO1</smiles> 


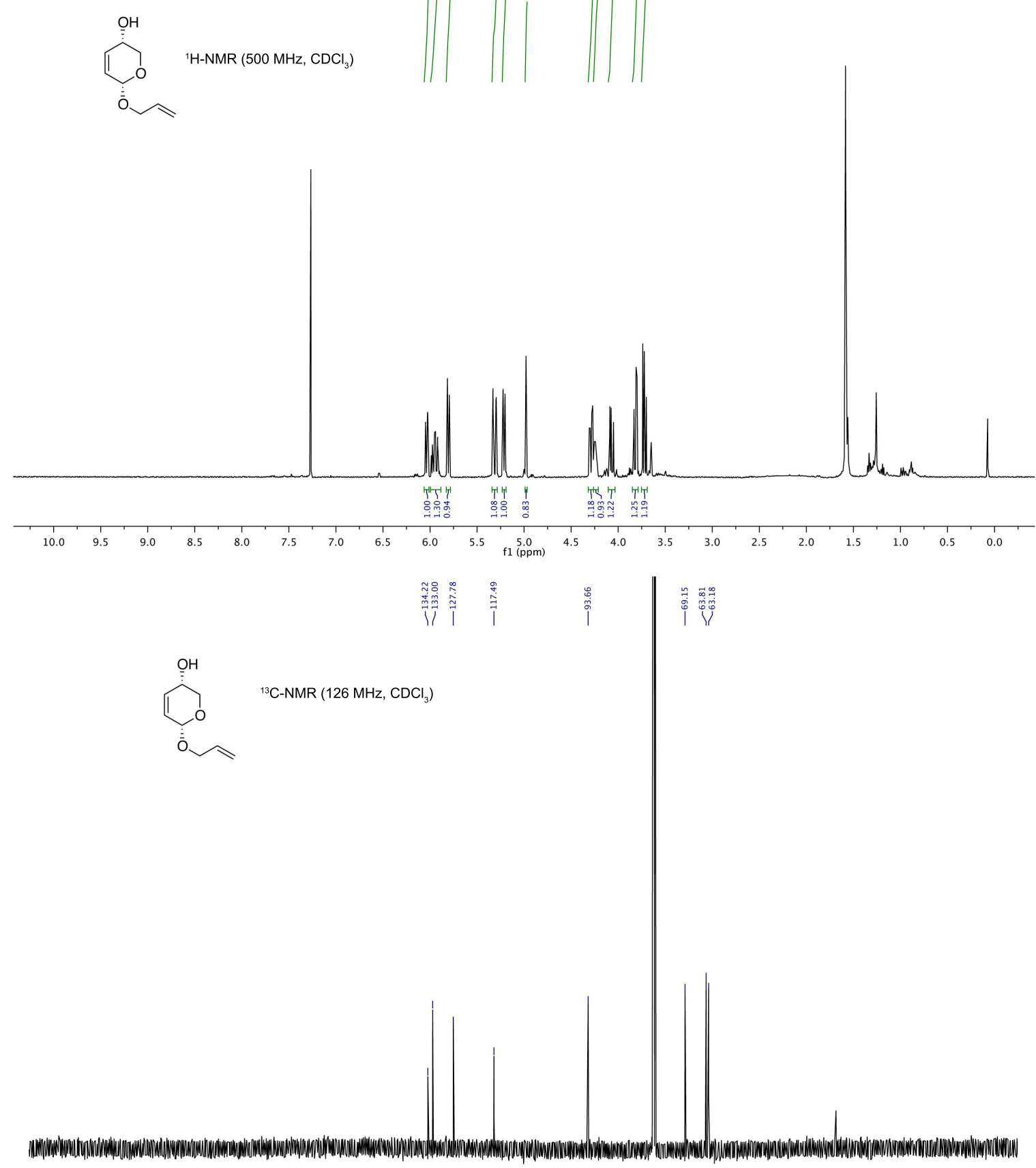

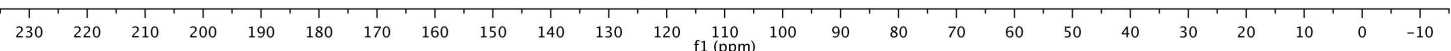




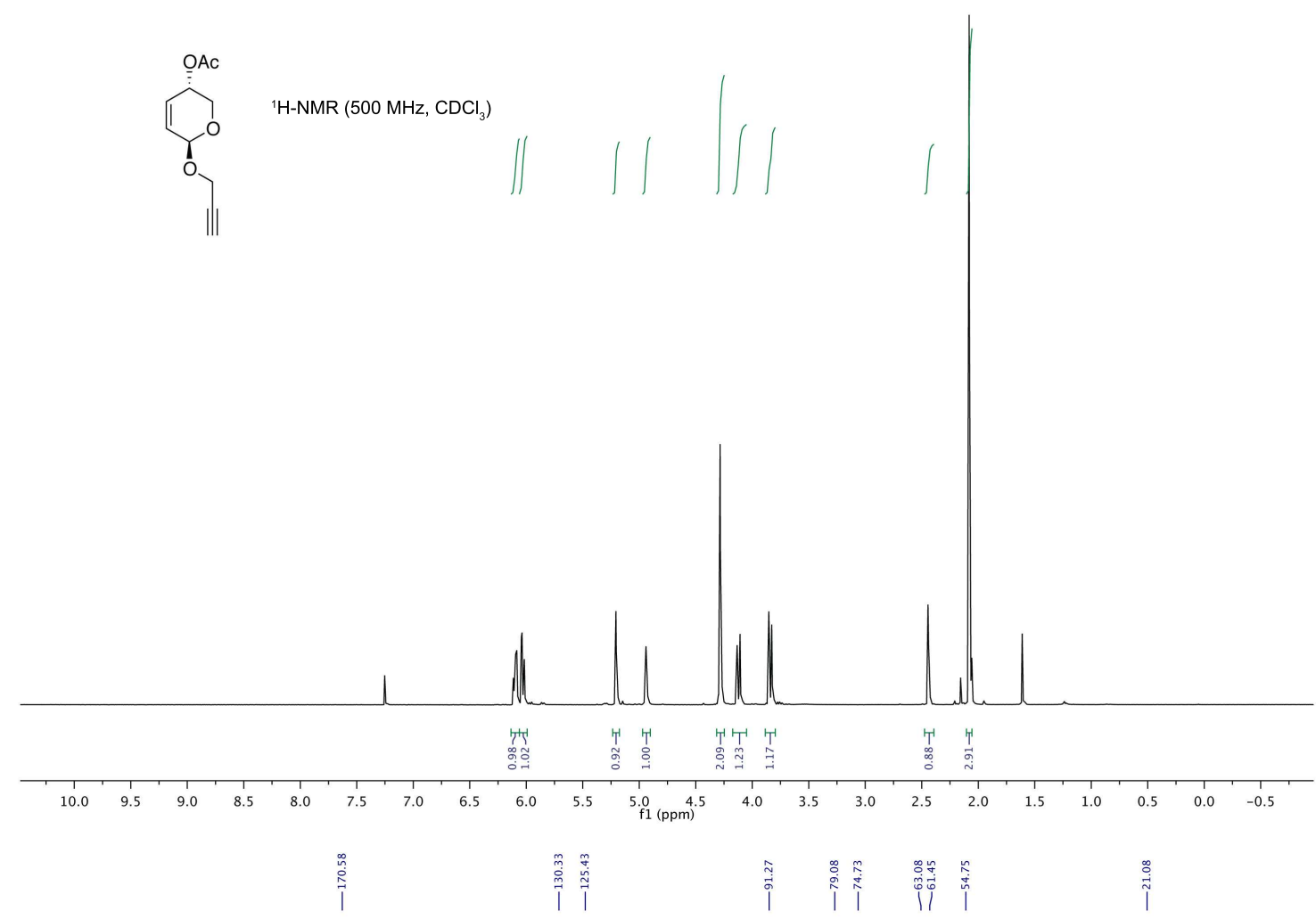

${ }^{13} \mathrm{C}-\mathrm{NMR}\left(126 \mathrm{MHz}, \mathrm{CDCl}_{3}\right)$

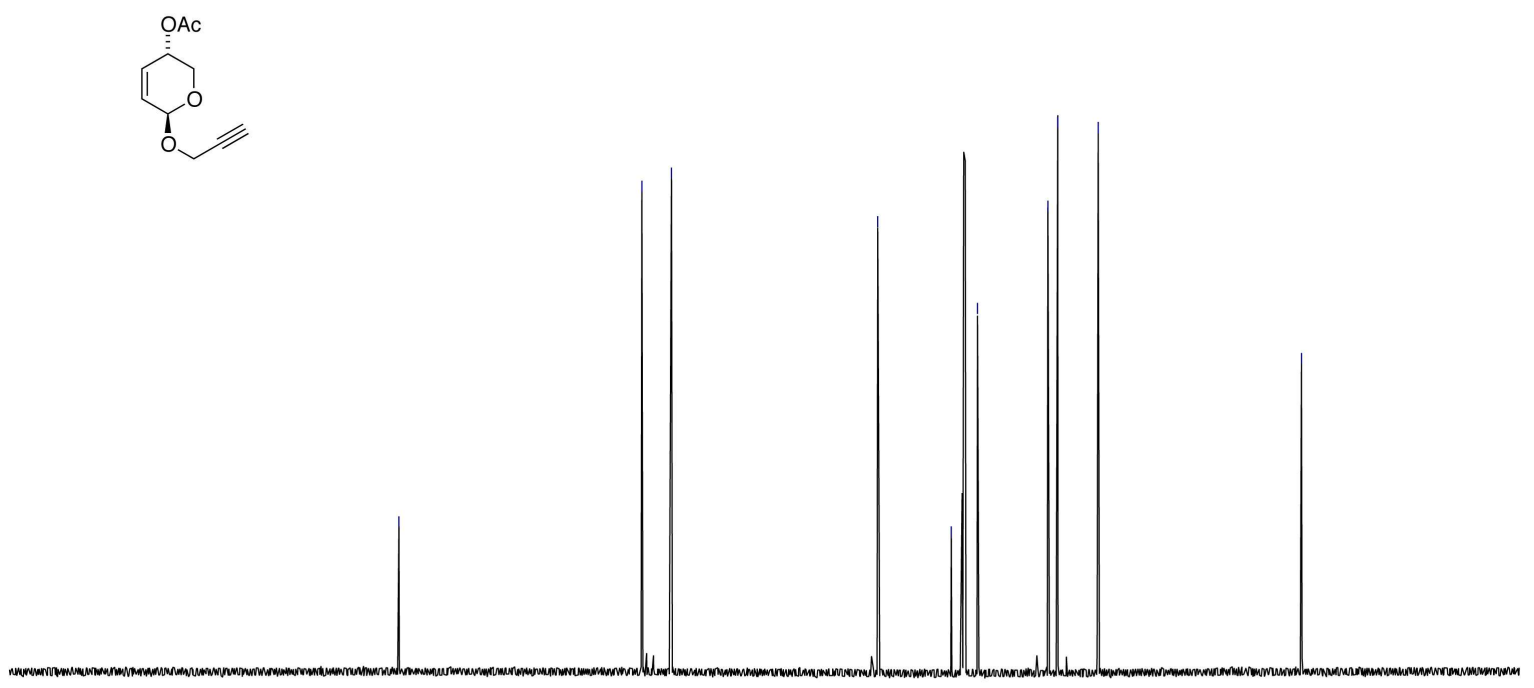

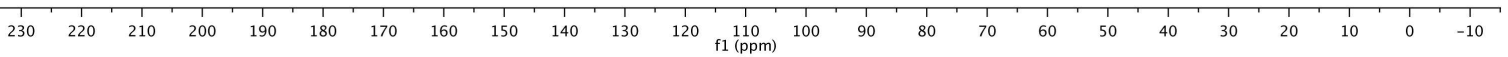




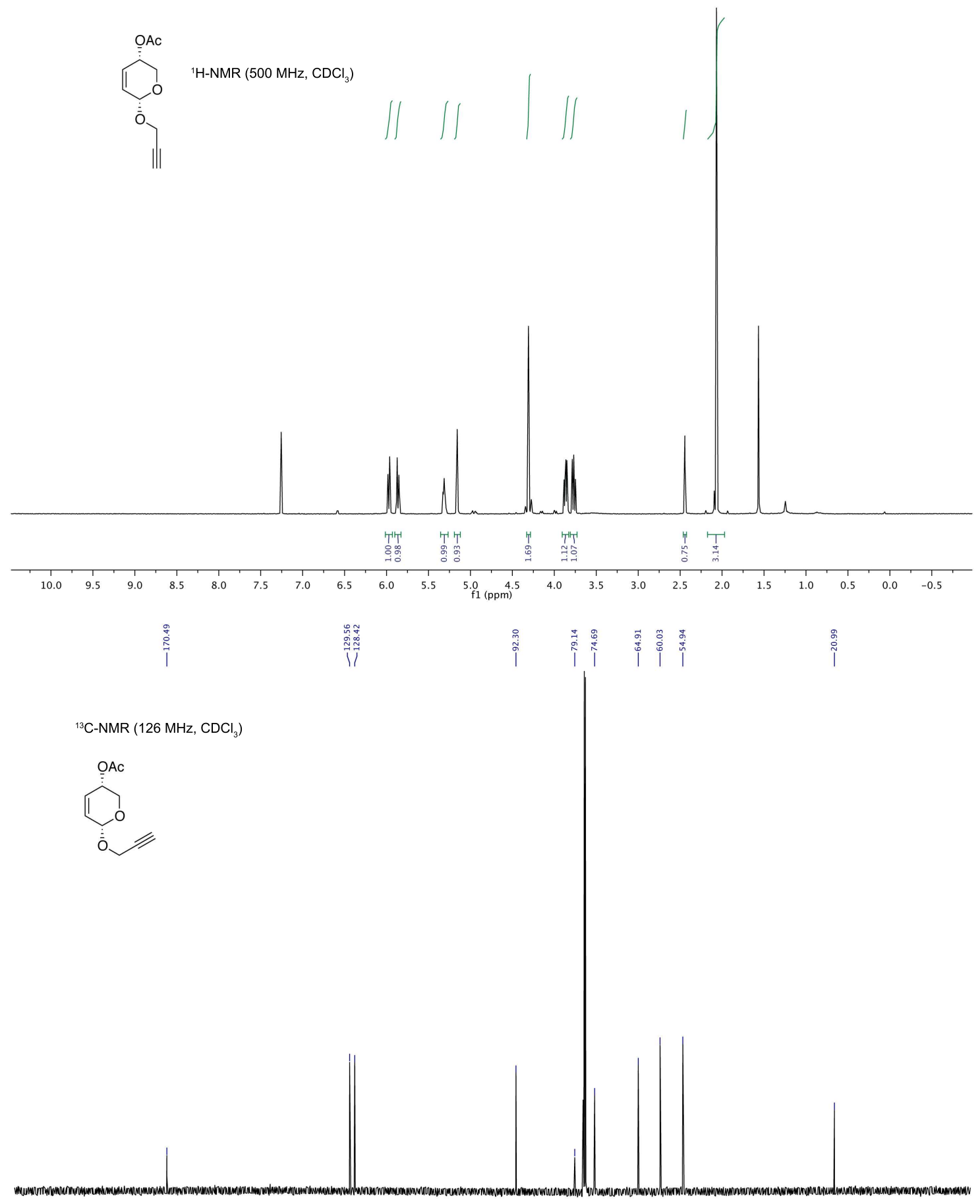

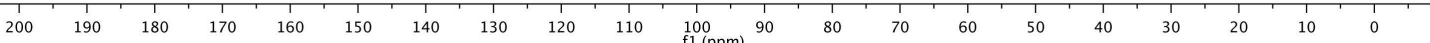




\section{References for Chapter 8}

[1] For selected examples of how the structure of chiral compounds relates to function, see: Cheng, R. P.; Gellman, S. H.; DeGrado, W. F. Chem. Rev. 2001, 101, 3219. Reist, M.; Carrupt, P.-A.; Francotte, E.; Testa, B. Chem. Res. Toxicol. 1998, 11, 1521. Schlatter, A.; Woggon, W.-D. Adv. Synth. Catal. 2008, 350, 995

[2] Seco, J. M.; Quiñoá, E.; Riguera, R. Chem. Rev. 2004, 104, 17. Duus, J. Ø.; Gotfredsen, C. H.; Bock, K. Chem. Rev. 2000, 100, 4589. Berger, S.; Sicker, D. Classics in Spectroscopy: Isolation and Structure Elucidation of Natural Products; Wiley-VCH: Weinheim, 2009.

[3] Maier, M. Nat. Prod. Rep. 2009, 26, 1005. Nicolaou, K. C.; Snyder, S. A. Angew. Chem. Int. Ed. 2005, 44, 1012. Blunt, J. W.; Copp, B. R.; Keyzers, R. A.; Munro, M. H. G.; Prinsep, M. R. Nat. Prod. Rep. 2012, 29, 144 .

[4] For examples of the stereochemical reassignment of 3,6-dihydro-2H-pyran ring systems, see: Hande, S. M.; Uenishi, J. Tetrahedron Lett. 2009, 50, 189. Nicolaou, K. C.; Koftis, T. V.; Vyskocil, S.; Petrovic, G.; Tang, W.; Frederick, M. O.; Chen, D. Y.-K.; Li, Y.; Ling, T.; Yamada, Y. M. A. J. Am. Chem. Soc. 2006, 128, 2859. Buchgraber, P.; Snaddon, T. N.; Wirtz, C.; Mynott, R.; Goddard, R.; Fürstner, A. Angew. Chem. Int. Ed. 2008, 47, 8450 .

[5] For recent examples of new methods for NMR-based stereochemical assignment, see: Napolitano, J. G.; Gavín, J. A.; García, C.; Norte, M.; Fernández, J. J.; Hernández Daranas, A. Chem. J. Eur. 2011, 17, 6338. Schmidt, Y.; Lehr, K.; Colas, L.; Breit, B. Chem. Eur. J. 2012, 18, 7071. Hong, S.-p.; McIntosh, M. C. Tetrahedron 2001, 57, 5055. Fleming, F. F.; Wei, G. J. Org. Chem. 2009, 74, 3551. Nilewski, C.; Geisser, R. W.; Ebert, M.-O.; Carreira, E. M. J. Am. Chem. Soc. 2009, 131, 15866.

[6] De la Pradilla, R. F.; Lwoff, N.; del Águila, M. A.; Tortosa, M.; Viso, A. J. Org. Chem. 2008, $73,8929$.

[7] Jackson, K. L.; Henderson, J. A.; Morris, J. C.; Motoyoshi, H.; Phillips, A. J. Tetrahedron Lett. 2008, 49, 2939. Harris, J. M.; Keranen, M. D.; O’Doherty, G. A. J. Org. Chem. 1999, 64, 2982.

[8] Srihari, P.; Sridhar, Y. Eur. J. Org. Chem. 2011, 6690. Panarese, J. D.; Waters, S. P. Org. Lett. 2009, 11, 5086. Ferrier, R. J.; Zubkov, O. A. Organic Reactions, 2003, 62, 569.

[9] Hewitt, R. J.; Harvey, J. E. Org. Biomol. Chem. 2011, 9, 998.

[10] Sugawara, K.; Imanishi, Y.; Hashiyama, T. Tetrahedron: Asymmetry 2000, 11, 4529.

[11] Kito, K.; Ookura, S. Y.; Namikoshi, M.; Ooi, T.; Kusumi, T. Org. Lett. 2008, 10, 225.

[12] For an additional example of the synthetic utility of 3,6-dihydro-2H-pyrans, see: Babu, R. S.; O'Doherty, G. A. J. Am. Chem. Soc. 2003, 125, 12406 .

[13] Sasaki, M.; Tsubone, K.; Aoki, K.; Akiyama, N.; Shoji, M.; Oikawa, M.; Sakai, R.; Shimamoto, K. J. Org. Chem. 2008, 73, 264

[14] De la Pradilla, R. F.; Lwoff, N. Tetrahedron Lett. 2008, 49, 4167. De la Pradilla, R. F.; Lwoff, N.; Viso, A. Eur. J. Org. Chem. 2009, 2312.

[15] Sugawara, K.; Hashiyama, T. Tetrahedron Lett. 2007, 48, 3723.

[16] For selected examples of approaches previously employed for the assignment of relative stereochemistry across a 3,6-dihydro- $2 H$-pyran ring, see: assignment by derivatization - Cook, M. J.; Fletcher, M. J. E.; Gray, D.; Lovell, P. J.; Gallagher, T. Tetrahedron 2004, 60, 5085. Hosokawa, S.; Kirschbaum, B.; Isobe, M. Tetrahedron: Lett. 1998, 39, 1917. Oshida, M.; Ono, M.; Nakazaki, A.; Kobayashi, S. Heterocycles 2010, 80, 313. Assignment using NOE correlation $+{ }^{3} \boldsymbol{J}_{\mathbf{H H}}$ coupling - Saeeng, R.; Isobe, M. Org. Lett. 2005, 7, 1585. Schmidt, B.; Nave, S. J. Org. Chem. 2006, and reference ${ }^{15}$. Assignment based on ${ }^{3} \boldsymbol{J}_{\mathbf{H H}}$ coupling alone - Panek, J. S.; Sparks, M. A. Tetrahedron Lett. 1988, 29, 4517. Dunkerton, L. V.; Euske, J. M.; Serino, A. J. Carbohydr. Res. 1987, 171, 89. Sarabia, F.; García-Castro, M.; Chammaa, S.; Sánchez-Ruiz, A. J. Carbohydrate Chem. 2006, 25, 267. X-ray crystallography - Ookura, R.; Kito, K.; Saito, Y.; Kusumi, T.; Ooi, T. Chem. Lett. 2009, 38, 384. Doboszewski, B.; Blaton, N.; Herdewijn, P. J. Org. Chem. 1995, 60, 7909. de Lerma, J. L.; Martínez-Carrera, S.; García-Blanco, S. Acta Cryst. 1973, B29, 537 . 
[17] Ansyln, E. V.; Dougherty, D. A. Modern Physical Organic Chemistry; University Science Books: Sausalito, 2004, pp 259-292.

[18] Gaussian 09, Revision A.1, Frisch, M. J.; Trucks, G. W.; Schlegel, H. B.; Scuseria, G. E.; Robb, M. A.; Cheeseman, J. R.; Scalmani, G.; Barone, V.; Mennucci, B.; Petersson, G. A.; Nakatsuji, H.; Caricato, M.; Li, X.; Hratchian, H. P.; Izmaylov, A. F.; Bloino, J.; Zheng, G.; Sonnenberg, J. L.; Hada, M.; Ehara, M.; Toyota, K.; Fukuda, R.; Hasegawa, J.; Ishida, M.; Nakajima, T.; Honda, Y.; Kitao, O.; Nakai, H.; Vreven, T.; Montgomery, Jr., J. A.; Peralta, J. E.; Ogliaro, F.; Bearpark, M.; Heyd, J. J.; Brothers, E.; Kudin, K. N.; Staroverov, V. N.; Kobayashi, R.; Normand, J.; Raghavachari, K.; Rendell, A.; Burant, J. C.; Iyengar, S. S.; Tomasi, J.; Cossi, M.; Rega, N.; Millam, J. M.; Klene, M.; Knox, J. E.; Cross, J. B.; Bakken, V.; Adamo, C.; Jaramillo, J.; Gomperts, R.; Stratmann, R. E.; Yazyev, O.; Austin, A. J.; Cammi, R.; Pomelli, C.; Ochterski, J. W.; Martin, R. L.; Morokuma, K.; Zakrzewski, V. G.; Voth, G. A.; Salvador, P.; Dannenberg, J. J.; Dapprich, S.; Daniels, A. D.; Farkas, Ě.; Foresman, J. B.; Ortiz, J. V.; Cioslowski, J.; Fox, D. J. Gaussian, Inc., Wallingford CT, 2009.

[19] Balacco, G. J. Chem. Inf. Comput. Sci. 1996, 36 885. Haasnoot, C. A. G.; de Leeuw, F. A. A. M.; Altona, C. Tetrahedron, 1981, 36, 2783. Donders, L. A.; de Leeuw, Altona, C. Magn. Reson. Chem. 1989, $27,556$. Karplus, M. J. Am. Chem. Soc. 1963, 852870.

[20] Dorman, D. E.; Angyal, S. J.; Roberts, J. D. J. Am. Chem. Soc. 1970, 92, 1351. Dorman, D. E.; Roberts, J. D. J. Am. Chem. Soc. 1970, 92, 1355

[21] Also referred to as the $\gamma$ - or $\gamma$-gauche effect. Stothers, J. B. Carbon-13 NMR Spectroscopy; Academic Press: New York, 1972; Chapter 3. Ayer, W. A.; Browne, L. M.; Fung, S.; Stothers, J. B. Org. Magn. Resonance 1978, 11, 73. Angyal, S. J.; Odier, L. Carbohydrate Res. 1982, 100, 43. Brakta, M.; Lhoste, P.; Sinou, D. J. Org. Chem. 1989, 54, 1890. Dawe, R. D.; Fraser-Reid, B. J. Org. Chem. 1984, 49, 522.

[22] For discussion on the comformation of dihydropyran derivatives, see: Achmatowicz, O., Jr.; Jurczak, J.; Konowal, A.; Zamojski, A. Org. Magn. Resonance 1970, 2, 55. Achmatowicz, O., Jr.; Chmielewski, M.; Jurczak, J.; Kozerski, L.; Zamojski, A. Org. Magn. Resonance 1972, 4, 537. Jankowaki, K. Org. Magn. Resonance 1980, 13, 380.

[23] Ferrier, R. J.; Sankey, G. H. J. Chem. Soc. 1966, 2345. Anet, E. F. L. J. Carbohydr. Res. 1966, 1, 348. Angerbauer, R.; Schmidt, R. R. Carbohydr. Res. 1981, 89, 193.

[24] Conformer searching revealed that cis-332 appears to have a larger number of relevant conformers, and more rotational freedom in the ring substituents, relative to the trans-diastereomer. See Figure 8.8 for additional details.

[25] Jacobsen, N. E. NMR Spectroscopy Explained: Simplified Theory, Applications and Examples for Organic Chemistry and Structural Biology; VCH: Weinheim, 2007.

$[26]{ }^{13} \mathrm{C}$ NMR data for $\mathbf{3 9 4}$ was taken from the literature and assignments supported by calculated NMR spectra. White, J. D.; Theramongkol, P.; Kuraoda, C.; Engebrecht, J. R. J. Org. Chem. 1988, 53, 5909.

[27] For reviews on the computational prediction of NMR chemical shifts, see: Lodewyk, M. W.; Siebert, M. R.; Tantillo, D. J. Chem. Rev. 2012, 112, 1839. Bifulco, G.; Dambruoso, P.; Gomez-Paloma, L.; Riccio, R. Chem Rev. 2007, 107, 3744.

[28] Eliel, E. L.; Manoharan, M.; Pietrusiewicz, K. M.; Hargrave, K. D. Org. Magn. Reson. 1983, $21,1983$.

[29] Adamo, C.; Barone, V. J. Chem. Phys. 1998, 108, 664. Perdew, J. P.; Chevary, J. A.; Vosko, S. H.; Jackson, K. A.; Pederson, M. R.; Singh, D. J.; Fiolhais, C. Phys. Rev. B, 1992, 46, 6671. Perdew, J. P.; Wang, Y. Phys. Rev. B, 1992, 45, 13244.

[30] Schaefer, A.; Horn, H.; Ahlrichs, R. J. Chem. Phys. 1992, 97, 2571. Schaefer, A.; Huber, C.; Ahlrichs, R. J. Chem. Phys. 1994, 100, 5829 .

[31] Sarotti, A. M.; Pellegrinet, S. C. J. Org. Chem. 2009, 74, 7254.

[32] A full description of the screening of computational basis sets and functionals can be found in Appendix A. 
[33] For leading references on Boltzmann-averaged GIAO ${ }^{13} \mathrm{C}$ NMR chemical shift calculations, see: Barone, G.; Duca, D.; Silvestri, A.; Gomez-Paloma, L.; Riccio, R.; Bifulco, G. Chem. Eur. J. 2002, 8, 3240. Smith, S. G.; Paton, R. S.; Burton, J. W.; Goodman, J. M. J. Org. Chem. 2008, 73, 4053. Sebag, A. B.; Forsyth, D. A.; Plante, M. A. J. Org. Chem. 2001, 66, 7967.

[34] For cis-332 and trans-332, MM2 and molecular force field calculations were performed on the two possible pseudo-chair ring conformers and their respective staggered conformers for each ring substituent (18 total). From these results, conformers representing energy minima within $4 \mathrm{kcal} / \mathrm{mol}$ of the global minima were subjected to DFT geometry optimization (mPW1PW91/TZVP), and it is from this second set of calculations that the 4 relevant cis-conformers and 2 relevant trans-conformers were identified to be within $1.5 \mathrm{kcal} / \mathrm{mol}$ of their respective global minima. It was assumed that these conformers were also a suitable representation of the conformer distribution for compounds $\mathbf{3 8 1 - 3 8 7}$, and these structures were reoptimized, at the DFT level, for the various substituents.

[35] Smith, S. G.; Goodman, J. M. J. Am. Chem. Soc. 2010, 132, 12946. Smith, S. G.; Goodman, J. M. J. Org. Chem. 2009, 74, 4597.

[36] Calculation of DP4 probability also requires one to calculate the NMR spectra of the opposite diastereomer.

[37] Sugawara, K.; Imanishi, Y.; Hashiyama, T. Tetrahedron: Asymmetry 2000, 11, 4529.

[38] Sarabia, F.; García-Castro, M.; Chammaa, S.; Sánchez-Ruiz, A. J. Carbohydrate Chem. 2006, $25,267$.

[39] Fava, C.; Galeazzi, R.; Mobbili, G.; Orena, M. Tetrahedron: Asymmetry 2001, 12, 2731.

[40] Das, S. K.; Reddy, K. A.; Roy, J. Synlett, 2003, 1607.

[41] Balog, A.; Yu, M. S.; Curran, D. P.; Yu, G.; Carcanague, D. R.; Shue, Y.-K. Synth. Commun. 1996, 26, 935. 
Appendix A

Computational Data 


\section{A.1 Computational Investigations of Labillarides E-H}
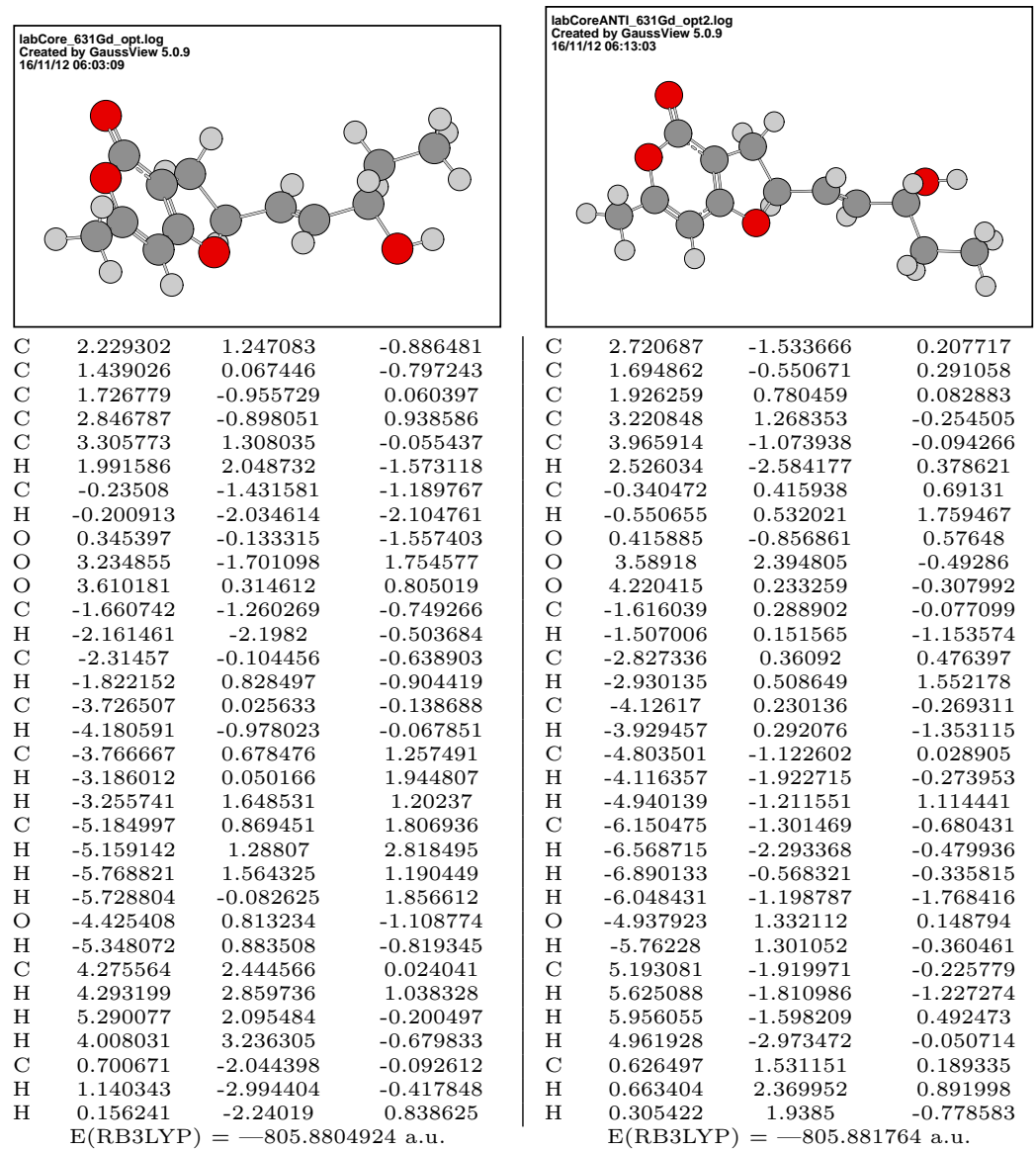


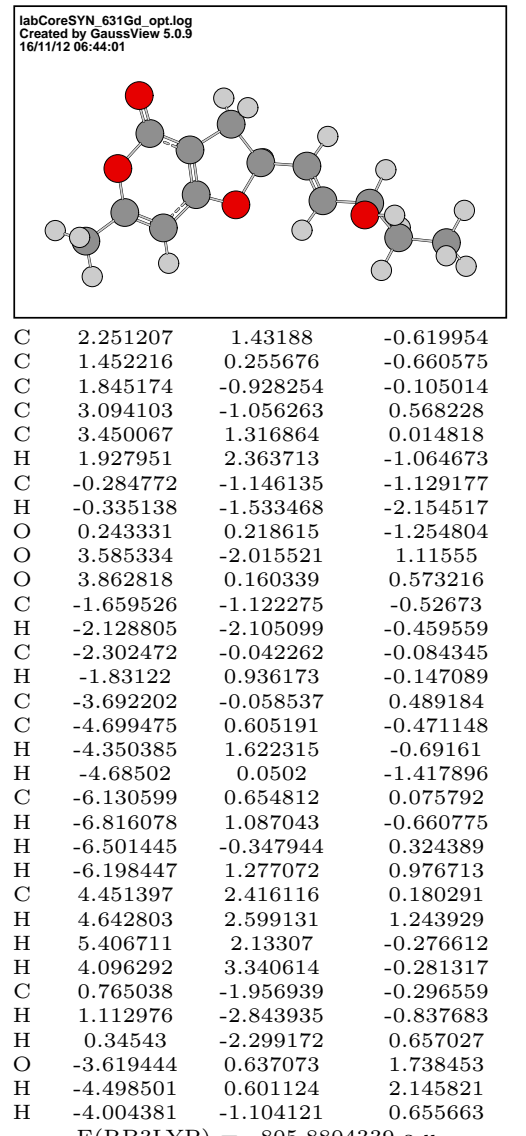

\begin{tabular}{|c|c|c|c|}
\hline \multicolumn{4}{|c|}{$\begin{array}{l}\text { labCoreSYN 631Gd oopt2.log } \\
\text { Created by Gaussview 5.0.9 } \\
\text { 16/11/12 06:52:39 }\end{array}$} \\
\hline $\mathrm{C}$ & -2.953827 & -1.443564 & 0.106537 \\
\hline $\mathrm{C}$ & -1.7993 & -0.658592 & -0.169229 \\
\hline $\mathrm{C}$ & -1.831754 & 0.70646 & -0.238185 \\
\hline $\mathrm{C}$ & -3.033731 & 1.436321 & -0.015205 \\
\hline $\mathrm{C}$ & -4.112342 & -0.755258 & 0.299582 \\
\hline $\mathrm{H}$ & -2.916842 & -2.523803 & 0.154869 \\
\hline $\mathrm{C}$ & 0.342678 & -0.10224 & -0.747548 \\
\hline $\mathrm{H}$ & 0.539409 & -0.234368 & -1.816494 \\
\hline $\mathrm{O}$ & -0.585188 & -1.199102 & -0.379089 \\
\hline $\mathrm{O}$ & -3.228904 & 2.629531 & -0.012786 \\
\hline $\mathrm{O}$ & -4.17088 & 0.591187 & 0.240073 \\
\hline $\mathrm{C}$ & 1.606006 & -0.257187 & 0.035534 \\
\hline $\mathrm{H}$ & 1.495298 & -0.220239 & 1.120064 \\
\hline $\mathrm{C}$ & 2.811557 & -0.397659 & -0.517297 \\
\hline $\mathrm{H}$ & 2.915928 & -0.448014 & -1.601861 \\
\hline $\mathrm{C}$ & 4.105286 & -0.46158 & 0.245792 \\
\hline $\mathrm{C}$ & 4.892395 & 0.859672 & 0.126822 \\
\hline $\mathrm{H}$ & 5.046939 & 1.077922 & -0.937873 \\
\hline $\mathrm{H}$ & 4.268879 & 1.666775 & 0.531969 \\
\hline C & 6.242175 & 0.835263 & 0.852723 \\
\hline $\mathrm{H}$ & 6.739901 & 1.808206 & 0.784647 \\
\hline $\mathrm{H}$ & 6.121635 & 0.598635 & 1.917696 \\
\hline $\mathrm{H}$ & 6.924598 & 0.097198 & 0.413543 \\
\hline $\mathrm{C}$ & -5.445286 & -1.368973 & 0.591994 \\
\hline $\mathrm{H}$ & -5.835297 & -0.995895 & 1.546044 \\
\hline $\mathrm{H}$ & -6.169504 & -1.093767 & -0.183465 \\
\hline $\mathrm{H}$ & -5.371737 & -2.458144 & 0.639097 \\
\hline $\mathrm{C}$ & -0.44029 & 1.221761 & -0.486446 \\
\hline $\mathrm{H}$ & -0.372147 & 1.898751 & -1.34432 \\
\hline $\mathrm{H}$ & -0.040662 & 1.762809 & 0.381585 \\
\hline $\mathrm{O}$ & 4.836439 & -1.562091 & -0.302811 \\
\hline $\mathrm{H}$ & 5.654344 & -1.655554 & 0.209037 \\
\hline $\mathrm{H}$ & 3.886118 & -0.64326 & 1.311921 \\
\hline
\end{tabular}




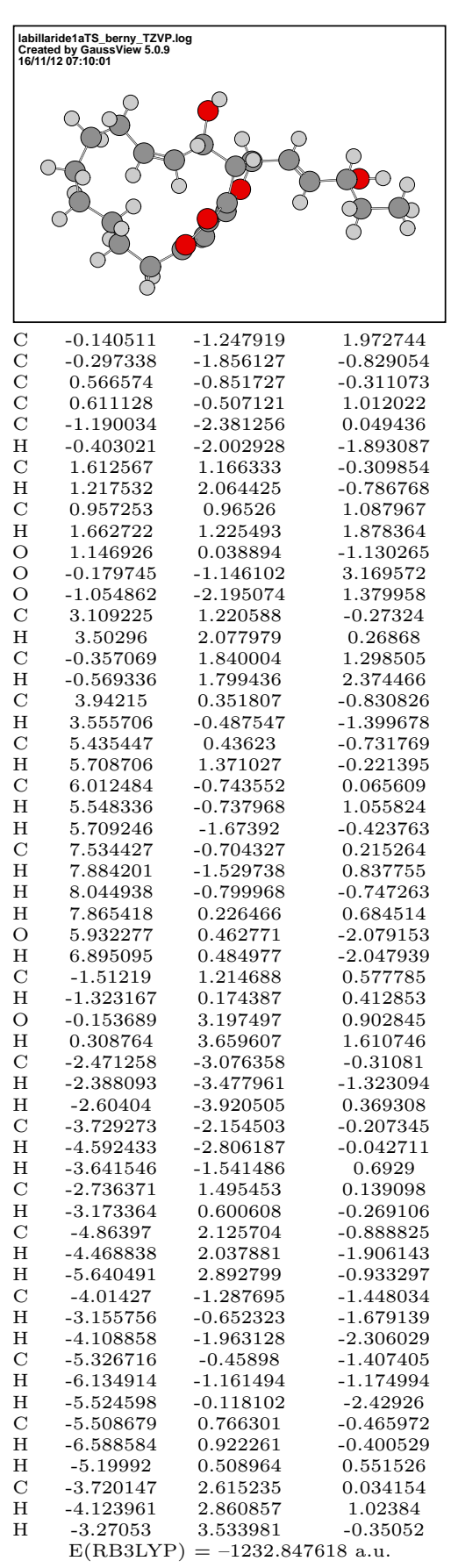

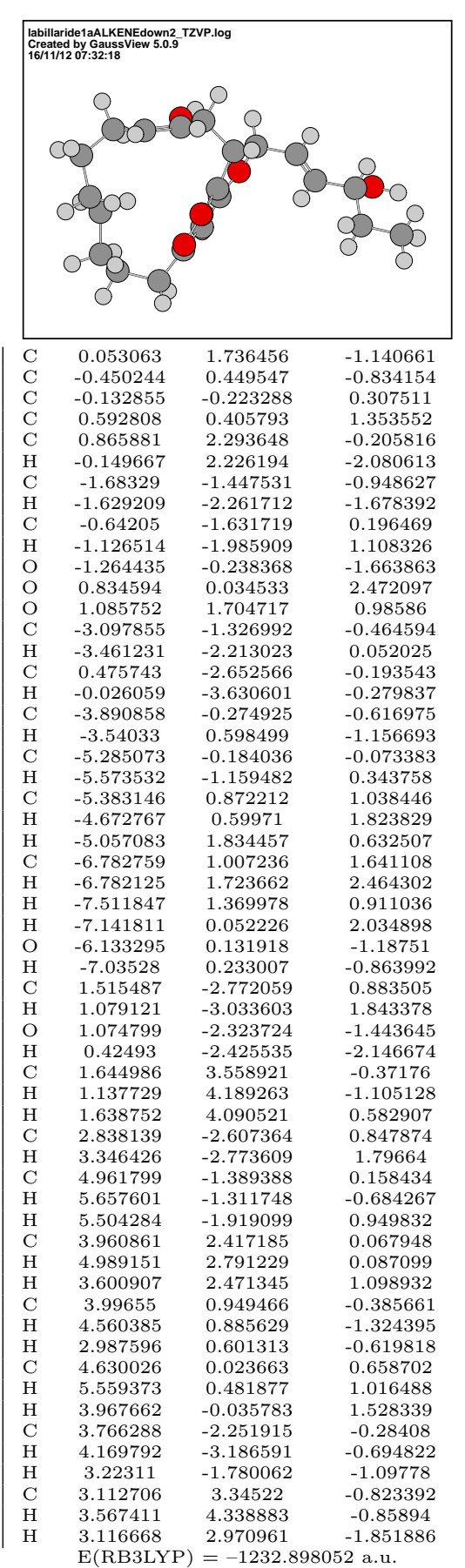




\section{A.2 ${ }^{13} \mathrm{C}$ NMR Analysis of 3,6-Dihydro-2H-pyrans}

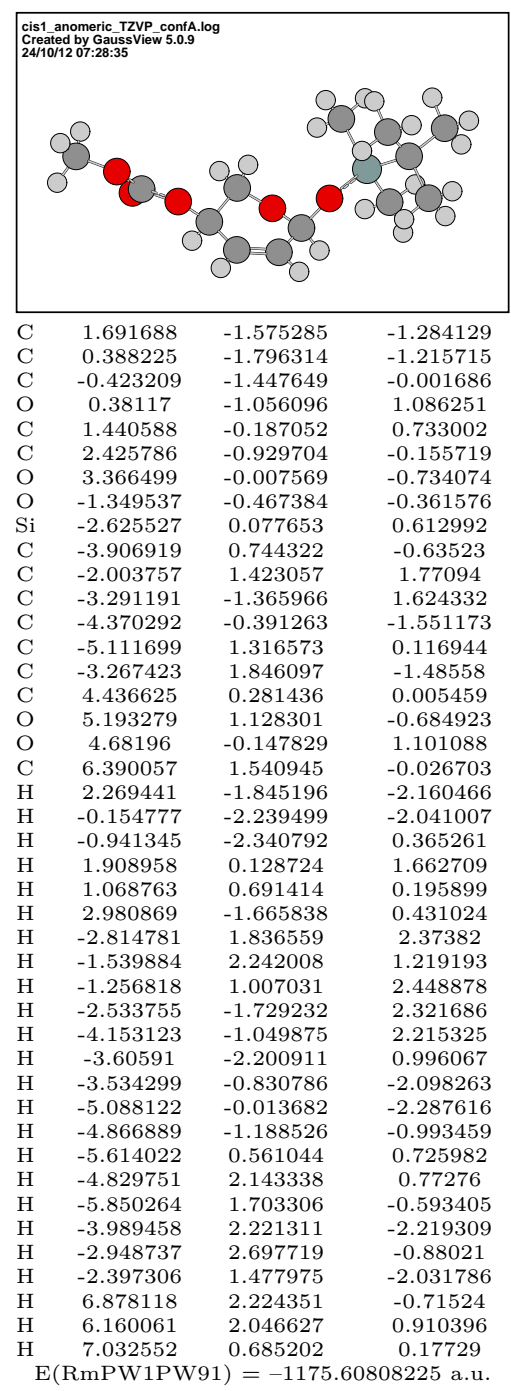

\begin{tabular}{|c|c|c|c|}
\hline \multicolumn{4}{|c|}{\begin{tabular}{|l} 
cis1_anomeric TZVP 20-4.12.log \\
Creaeded by Gausssview 5.0.9 \\
24/10/12 07:40:49
\end{tabular}} \\
\hline $\mathrm{C}$ & 1.909971 & -0.496415 & -1.354611 \\
\hline C & 0.633325 & -0.672466 & -1.659151 \\
\hline $\mathrm{C}$ & -0.375655 & -1.150246 & -0.655377 \\
\hline $\mathrm{O}$ & 0.225225 & -1.555148 & 0.55365 \\
\hline $\mathrm{C}$ & 1.238345 & -0.678973 & 1.006334 \\
\hline $\mathrm{C}$ & 2.406581 & -0.720209 & 0.039025 \\
\hline $\mathrm{O}$ & 3.294258 & 0.334661 & 0.450493 \\
\hline $\mathrm{O}$ & -1.325427 & -0.144362 & -0.468846 \\
\hline $\mathrm{Si}$ & -2.762618 & -0.319057 & 0.41527 \\
\hline $\mathrm{C}$ & -3.91208 & 1.027508 & -0.296428 \\
\hline $\mathrm{C}$ & -2.412094 & -0.048619 & 2.242861 \\
\hline C & -3.44143 & -2.056895 & 0.153261 \\
\hline $\mathrm{C}$ & -4.143769 & 0.774652 & -1.78843 \\
\hline $\mathrm{C}$ & -5.255639 & 0.999913 & 0.438551 \\
\hline $\mathrm{C}$ & -3.2626 & 2.402825 & -0.115383 \\
\hline $\mathrm{C}$ & 4.560311 & 0.225907 & 0.053137 \\
\hline $\mathrm{O}$ & 5.231471 & 1.269309 & 0.530705 \\
\hline $\mathrm{O}$ & 5.021205 & -0.659264 & -0.617031 \\
\hline $\mathrm{C}$ & 6.61709 & 1.299053 & 0.191931 \\
\hline $\mathrm{H}$ & 2.633471 & -0.192807 & -2.101768 \\
\hline $\mathrm{H}$ & 0.258693 & -0.486032 & -2.658035 \\
\hline $\mathrm{H}$ & -0.858054 & -2.062134 & -1.024789 \\
\hline $\mathrm{H}$ & 1.533122 & -1.021282 & 1.996546 \\
\hline $\mathrm{H}$ & 0.868928 & 0.348071 & 1.081242 \\
\hline $\mathrm{H}$ & 2.942087 & -1.670018 & 0.105843 \\
\hline $\mathrm{H}$ & -3.324721 & -0.115065 & 2.838396 \\
\hline $\mathrm{H}$ & -1.959878 & 0.927769 & 2.422935 \\
\hline $\mathrm{H}$ & -1.719695 & -0.813096 & 2.598522 \\
\hline $\mathrm{H}$ & -2.763004 & -2.803657 & 0.57046 \\
\hline $\mathrm{H}$ & -4.401459 & -2.169736 & 0.660985 \\
\hline $\mathrm{H}$ & -3.593107 & -2.28546 & -0.9029 \\
\hline $\mathrm{H}$ & -3.205482 & 0.781648 & -2.34598 \\
\hline $\mathrm{H}$ & -4.785635 & 1.557208 & -2.207696 \\
\hline $\mathrm{H}$ & -4.639019 & -0.182106 & -1.969935 \\
\hline $\mathrm{H}$ & -5.770192 & 0.043 & 0.320855 \\
\hline $\mathrm{H}$ & -5.142806 & 1.191904 & 1.5081 \\
\hline $\mathrm{H}$ & -5.918456 & 1.774755 & 0.038497 \\
\hline $\mathrm{H}$ & -3.904626 & 3.180578 & -0.543379 \\
\hline $\mathrm{H}$ & -3.114393 & 2.648273 & 0.938675 \\
\hline $\mathrm{H}$ & -2.293359 & 2.458331 & -0.613822 \\
\hline $\mathrm{H}$ & 7.007425 & 2.204093 & 0.647841 \\
\hline $\mathrm{H}$ & 7.129619 & 0.423357 & 0.588455 \\
\hline $\mathrm{H}$ & 6.749211 & 1.331413 & -0.888913 \\
\hline
\end{tabular}

$\mathrm{E}(\mathrm{RmPW} 1 \mathrm{PW} 91)=-1175.60782525$ a.u.

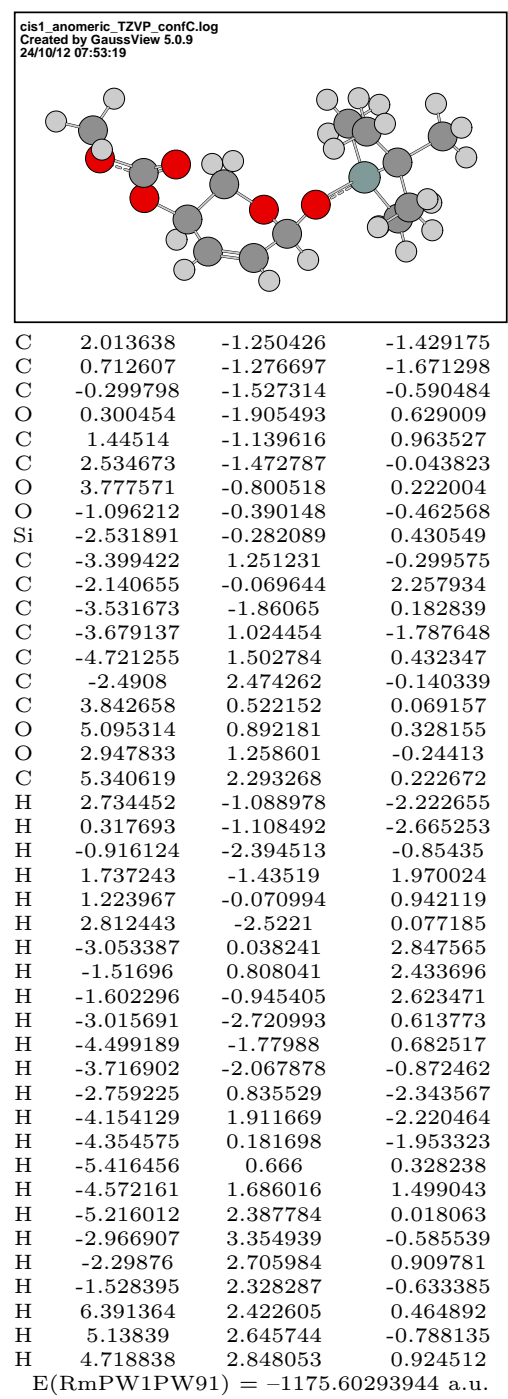




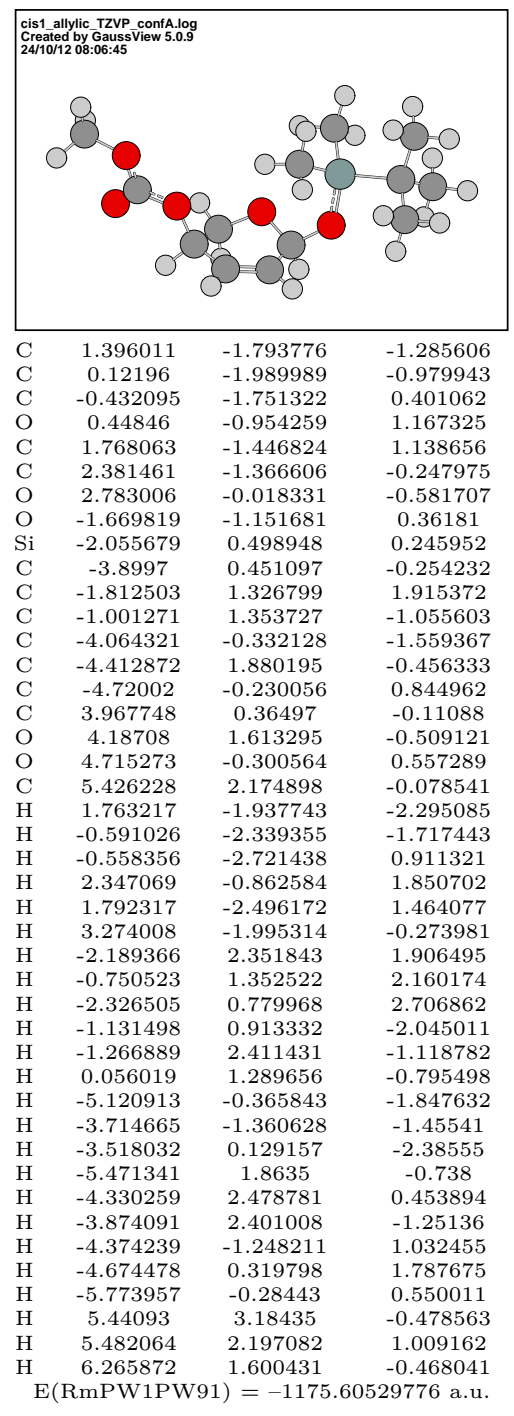

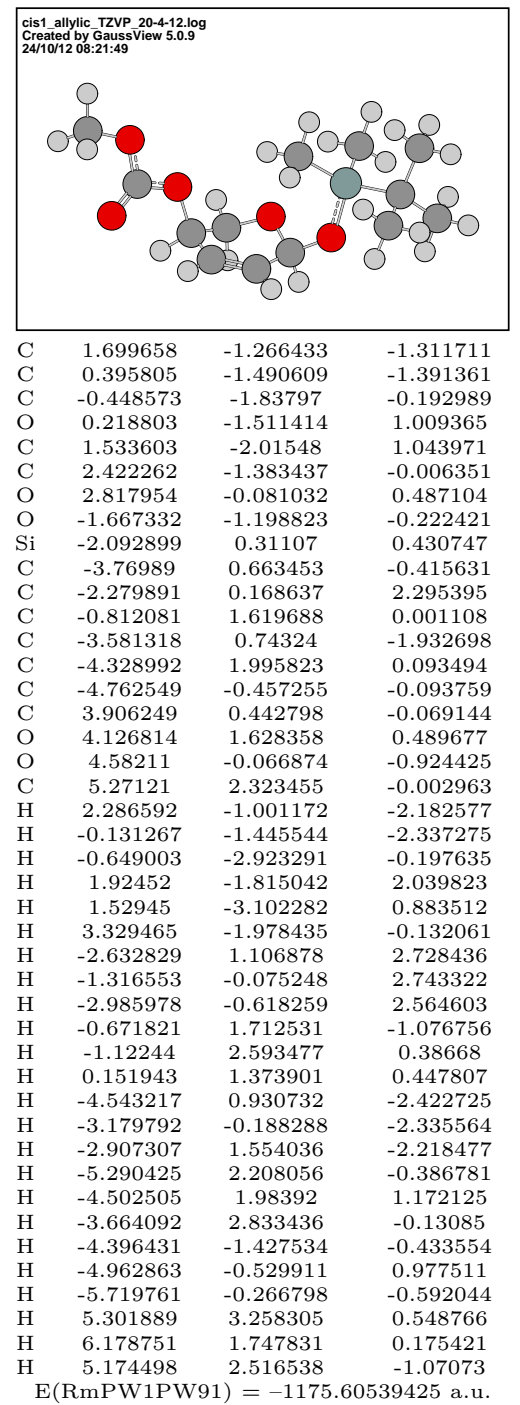




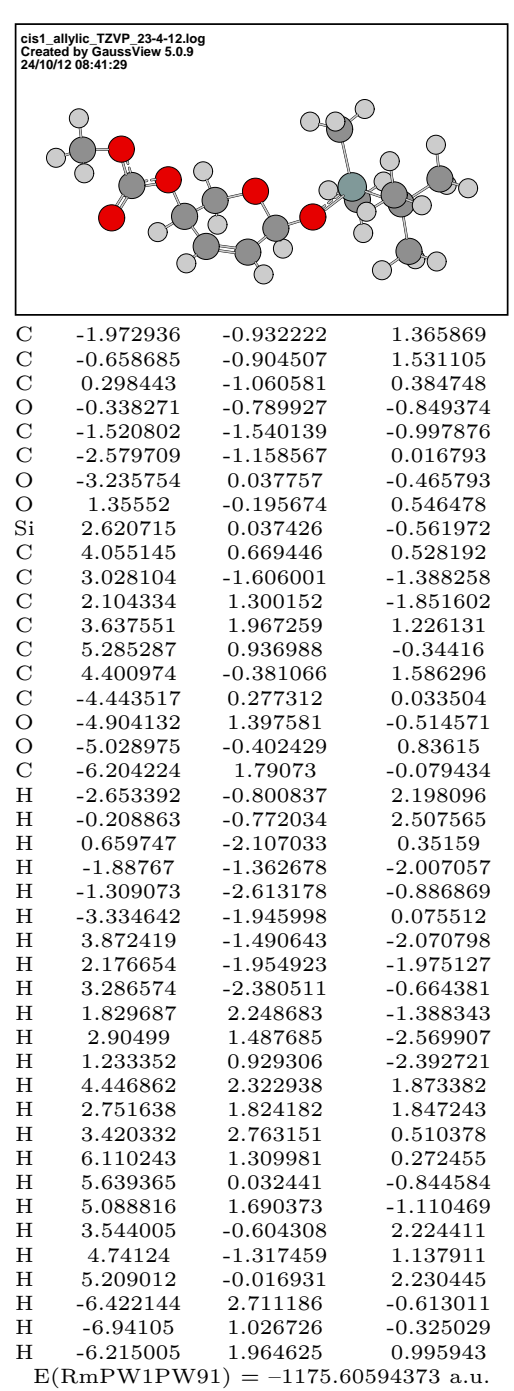

\begin{tabular}{|c|c|c|c|}
\hline \multicolumn{4}{|c|}{ 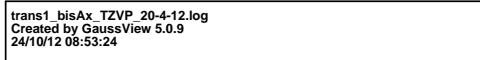 } \\
\hline $\mathrm{C}$ & -1.851585 & 1.327318 & 0.294397 \\
\hline C & -0.700249 & 1.197355 & 0.936109 \\
\hline $\mathrm{C}$ & 0.162915 & -0.024468 & 0.818995 \\
\hline $\mathrm{O}$ & -0.492287 & -1.084757 & 0.175074 \\
\hline $\mathrm{C}$ & -1.204851 & -0.693009 & -0.981606 \\
\hline $\mathrm{C}$ & -2.31761 & 0.280276 & -0.667 \\
\hline $\mathrm{O}$ & -3.430864 & -0.471827 & -0.128196 \\
\hline $\mathrm{O}$ & 1.353606 & 0.339193 & 0.1817 \\
\hline $\mathrm{Si}$ & 2.748691 & -0.619866 & 0.08736 \\
\hline C & 4.154179 & 0.647223 & -0.16208 \\
\hline $\mathrm{C}$ & 2.937585 & -1.599646 & 1.685402 \\
\hline $\mathrm{C}$ & 2.599637 & -1.808746 & -1.362485 \\
\hline $\mathrm{C}$ & 3.874613 & 1.474158 & -1.420484 \\
\hline C & 5.493009 & -0.079319 & -0.318445 \\
\hline $\mathrm{C}$ & 4.218893 & 1.581562 & 1.048792 \\
\hline $\mathrm{C}$ & -4.614431 & 0.128496 & -0.184832 \\
\hline $\mathrm{O}$ & -5.519325 & -0.68598 & 0.350438 \\
\hline $\mathrm{O}$ & -4.838741 & 1.217377 & -0.646491 \\
\hline $\mathrm{C}$ & -6.853103 & -0.181172 & 0.359099 \\
\hline $\mathrm{H}$ & -2.47743 & 2.202042 & 0.419219 \\
\hline $\mathrm{H}$ & -0.327129 & 1.978306 & 1.587766 \\
\hline $\mathrm{H}$ & 0.373838 & -0.41514 & 1.820582 \\
\hline $\mathrm{H}$ & -0.531585 & -0.222863 & -1.706058 \\
\hline $\mathrm{H}$ & -1.611449 & -1.60371 & -1.417234 \\
\hline $\mathrm{H}$ & -2.655138 & 0.755838 & -1.590655 \\
\hline $\mathrm{H}$ & 2.940835 & -0.956204 & 2.566699 \\
\hline $\mathrm{H}$ & 2.127486 & -2.323402 & 1.794623 \\
\hline $\mathrm{H}$ & 3.875008 & -2.159543 & 1.677994 \\
\hline $\mathrm{H}$ & 1.753678 & -2.478576 & -1.199694 \\
\hline $\mathrm{H}$ & 3.498245 & -2.419194 & -1.471321 \\
\hline $\mathrm{H}$ & 2.434547 & -1.278222 & -2.301426 \\
\hline $\mathrm{H}$ & 3.852292 & 0.854325 & -2.319779 \\
\hline $\mathrm{H}$ & 4.66195 & 2.222929 & -1.561163 \\
\hline $\mathrm{H}$ & 2.921529 & 2.000982 & -1.350628 \\
\hline $\mathrm{H}$ & 5.499138 & -0.746519 & -1.18344 \\
\hline $\mathrm{H}$ & 6.300289 & 0.646521 & -0.463905 \\
\hline $\mathrm{H}$ & 5.746411 & -0.671187 & 0.564474 \\
\hline $\mathrm{H}$ & 4.458236 & 1.043127 & 1.968772 \\
\hline $\mathrm{H}$ & 4.998376 & 2.337257 & 0.902031 \\
\hline $\mathrm{H}$ & 3.273494 & 2.105658 & 1.200012 \\
\hline $\mathrm{H}$ & -7.451968 & -0.952948 & 0.833658 \\
\hline $\mathrm{H}$ & -6.911057 & 0.74655 & 0.927282 \\
\hline $\mathrm{H}$ & -7.204387 & -0.000131 & -0.656213 \\
\hline
\end{tabular}

\begin{tabular}{|c|c|c|c|}
\hline \multicolumn{4}{|c|}{$\begin{array}{l}\text { trans1 bisAx_ZZVP confB.log } \\
\text { Created by GaussVView 5.0.9 } \\
\text { 24/10/12 10:33:27 }\end{array}$} \\
\hline $\mathrm{C}$ & -1.635627 & 2.031526 & -0.132451 \\
\hline $\mathrm{C}$ & -0.45511 & 1.984989 & 0.465419 \\
\hline $\mathrm{C}$ & 0.219384 & 0.694814 & 0.828765 \\
\hline $\mathrm{O}$ & -0.633358 & -0.410982 & 0.69204 \\
\hline $\mathrm{C}$ & -1.381076 & -0.40084 & -0.508081 \\
\hline $\mathrm{C}$ & -2.322827 & 0.784628 & -0.581131 \\
\hline $\mathrm{O}$ & -3.48915 & 0.617149 & 0.255798 \\
\hline $\mathrm{O}$ & 1.3852 & 0.572804 & 0.065915 \\
\hline $\mathrm{Si}$ & 2.573248 & -0.615477 & 0.295234 \\
\hline $\mathrm{C}$ & 4.154104 & 0.168559 & -0.430207 \\
\hline $\mathrm{C}$ & 2.731775 & -0.98603 & 2.135407 \\
\hline $\mathrm{C}$ & 2.081952 & -2.182333 & -0.622305 \\
\hline $\mathrm{C}$ & 3.916577 & 0.532443 & -1.899138 \\
\hline C & 5.31694 & -0.823494 & -0.334809 \\
\hline $\mathrm{C}$ & 4.504302 & 1.43893 & 0.34946 \\
\hline C & -4.471089 & -0.115796 & -0.261117 \\
\hline $\mathrm{O}$ & -5.459314 & -0.169044 & 0.626501 \\
\hline $\mathrm{O}$ & -4.474146 & -0.639246 & -1.345015 \\
\hline C & -6.595882 & -0.927904 & 0.218095 \\
\hline $\mathrm{H}$ & -2.12849 & 2.975491 & -0.332668 \\
\hline $\mathrm{H}$ & 0.075094 & 2.889599 & 0.73723 \\
\hline $\mathrm{H}$ & 0.465429 & 0.707506 & 1.896507 \\
\hline $\mathrm{H}$ & -0.715076 & -0.360126 & -1.376656 \\
\hline $\mathrm{H}$ & -1.933239 & -1.337258 & -0.543823 \\
\hline $\mathrm{H}$ & -2.664351 & 0.886062 & -1.61351 \\
\hline $\mathrm{H}$ & 2.951122 & -0.090958 & 2.719694 \\
\hline $\mathrm{H}$ & 1.810641 & -1.427553 & 2.520941 \\
\hline $\mathrm{H}$ & 3.535518 & -1.704119 & 2.310243 \\
\hline $\mathrm{H}$ & 1.148177 & -2.570584 & -0.212608 \\
\hline $\mathrm{H}$ & 2.843637 & -2.957887 & -0.519875 \\
\hline $\mathrm{H}$ & 1.932043 & -1.994011 & -1.686548 \\
\hline $\mathrm{H}$ & 3.707777 & -0.349105 & -2.50956 \\
\hline $\mathrm{H}$ & 4.808922 & 1.012417 & -2.315873 \\
\hline $\mathrm{H}$ & 3.08133 & 1.225787 & -2.011817 \\
\hline $\mathrm{H}$ & 5.119021 & -1.742695 & -0.89093 \\
\hline $\mathrm{H}$ & 6.225625 & -0.379423 & -0.755382 \\
\hline $\mathrm{H}$ & 5.540448 & -1.097123 & 0.699233 \\
\hline $\mathrm{H}$ & 4.717564 & 1.229668 & 1.40036 \\
\hline $\mathrm{H}$ & 5.398312 & 1.907418 & -0.076351 \\
\hline $\mathrm{H}$ & 3.695227 & 2.170531 & 0.307672 \\
\hline $\mathrm{H}$ & -7.291501 & -0.878532 & 1.05062 \\
\hline $\mathrm{H}$ & -7.045186 & -0.498877 & -0.676901 \\
\hline $\mathrm{H}$ & -6.3179 & -1.961957 & 0.017364 \\
\hline
\end{tabular}




\begin{tabular}{|c|c|c|c|}
\hline \multicolumn{4}{|c|}{ 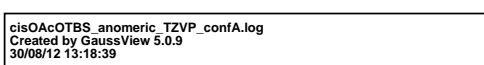 } \\
\hline $\mathrm{C}$ & -2.132021 & -1.05748 & 908 \\
\hline C & -0.84373 & -1.362516 & 1.573031 \\
\hline $\mathrm{C}$ & 0.004263 & -1.358178 & 0.333978 \\
\hline $\mathrm{O}$ & -0.760182 & -1.195025 & -0.837173 \\
\hline $\mathrm{C}$ & -1.76785 & -0.206859 & -0.730928 \\
\hline C & -2.809973 & -0.652075 & 0.282935 \\
\hline $\mathrm{O}$ & -3.692251 & 0.43965 & 0.597433 \\
\hline $\mathrm{O}$ & 0.983467 & -0.372377 & 0.47508 \\
\hline $\mathrm{Si}$ & 2.278009 & -0.125136 & -0.592257 \\
\hline $\mathrm{C}$ & 3.583642 & 0.766439 & 0.475039 \\
\hline $\mathrm{C}$ & 1.713695 & 0.934493 & -2.039779 \\
\hline $\mathrm{C}$ & 2.879162 & -1.792067 & -1.231768 \\
\hline $\mathrm{C}$ & 3.996587 & -0.131048 & 1.644752 \\
\hline $\mathrm{C}$ & 4.816148 & 1.09345 & -0.373338 \\
\hline $\mathrm{C}$ & 2.988967 & 2.065541 & 1.027664 \\
\hline $\mathrm{C}$ & -4.754546 & 0.620314 & -0.211403 \\
\hline $\mathrm{O}$ & -4.990524 & -0.068663 & -1.166673 \\
\hline $\mathrm{H}$ & $\begin{array}{l}-2.736547 \\
\end{array}$ & -1.083101 & 2.449318 \\
\hline $\mathrm{H}$ & -0.341298 & -1.629727 & 2.494244 \\
\hline $\mathrm{H}$ & 0.473466 & -2.339995 & 0.204449 \\
\hline $\mathrm{H}$ & -2.204295 & -0.097329 & -1.721232 \\
\hline $\mathrm{H}$ & -1.346499 & 0.751654 & -0.410763 \\
\hline $\mathrm{H}$ & -3.407955 & -1.468808 & -0.129084 \\
\hline $\mathrm{H}$ & 2.534936 & 1.126288 & -2.733216 \\
\hline $\mathrm{H}$ & 1.320542 & 1.895772 & -1.705646 \\
\hline $\mathrm{H}$ & 0.924105 & 0.417096 & -2.58675 \\
\hline $\mathrm{H}$ & 2.109234 & -2.270137 & -1.840525 \\
\hline $\mathrm{H}$ & 3.760145 & -1.662478 & -1.863684 \\
\hline $\mathrm{H}$ & 3.146371 & -2.473107 & -0.422172 \\
\hline $\mathrm{H}$ & 3.140283 & -0.396108 & 2.267554 \\
\hline $\mathrm{H}$ & 4.722232 & 0.3888 & 2.279767 \\
\hline $\mathrm{H}$ & 4.467923 & -1.056343 & 1.305229 \\
\hline $\mathrm{H}$ & 5.287599 & 0.194558 & -0.778147 \\
\hline $\mathrm{H}$ & 4.576942 & 1.753824 & -1.210197 \\
\hline $\mathrm{H}$ & 5.567351 & 1.605136 & 0.237958 \\
\hline $\mathrm{H}$ & 3.722995 & 2.573719 & 1.66263 \\
\hline $\mathrm{H}$ & 2.713201 & 2.760423 & 0.23106 \\
\hline $\mathrm{H}$ & 2.099783 & 1.875729 & 1.631317 \\
\hline $\mathrm{C}$ & -5.579095 & 1.785075 & 0.247349 \\
\hline $\mathrm{H}$ & -6.46143 & 1.872669 & -0.379501 \\
\hline $\mathrm{H}$ & -5.868047 & 1.654195 & 1.289999 \\
\hline $\mathrm{H}$ & -4.988735 & 2.699973 & 0.185009 \\
\hline
\end{tabular}

\begin{tabular}{|c|c|c|c|}
\hline \multicolumn{4}{|c|}{$\begin{array}{l}\text { cisOAcOTBS_anomeric _TZVP_confB.log } \\
\text { Creaeted by Gaussview 5.0.9 } \\
\text { 30/08/12 14:10:26 }\end{array}$} \\
\hline $\mathrm{C}$ & 2.285976 & -0.372005 & -1.349275 \\
\hline $\mathrm{C}$ & 1.01853 & -0.611695 & -1.649262 \\
\hline $\mathrm{C}$ & 0.032496 & -1.114364 & -0.63508 \\
\hline $\mathrm{O}$ & 0.651302 & -1.472054 & 0.579254 \\
\hline $\mathrm{C}$ & 1.624718 & -0.54371 & 1.015524 \\
\hline C & 2.793695 & -0.542195 & 0.048218 \\
\hline $\mathrm{O}$ & 3.628072 & 0.564434 & 0.433432 \\
\hline $\mathrm{O}$ & -0.95938 & -0.1462 & -0.463416 \\
\hline $\mathrm{Si}$ & -2.394845 & -0.368444 & 0.411472 \\
\hline $\mathrm{C}$ & -3.580677 & 0.942681 & -0.307383 \\
\hline $\mathrm{C}$ & -2.066398 & -0.091789 & 2.242407 \\
\hline $\mathrm{C}$ & -3.01849 & -2.125963 & 0.142689 \\
\hline $\mathrm{C}$ & -3.794583 & 0.682365 & -1.800721 \\
\hline $\mathrm{C}$ & -4.927466 & 0.8759 & 0.419044 \\
\hline $\mathrm{C}$ & -2.973868 & 2.33693 & -0.122947 \\
\hline $\mathrm{C}$ & 4.922666 & 0.513442 & 0.068017 \\
\hline $\mathrm{O}$ & 5.401725 & -0.390829 & -0.561383 \\
\hline $\mathrm{H}$ & 2.994638 & -0.051725 & -2.103632 \\
\hline $\mathrm{H}$ & 0.635995 & -0.463565 & -2.651595 \\
\hline $\mathrm{H}$ & -0.411739 & -2.051867 & -0.987828 \\
\hline $\mathrm{H}$ & 1.935553 & -0.857725 & 2.010396 \\
\hline $\mathrm{H}$ & 1.206138 & 0.465445 & 1.076995 \\
\hline $\mathrm{H}$ & 3.378472 & -1.461317 & 0.129842 \\
\hline $\mathrm{H}$ & -2.979564 & -0.195585 & 2.831743 \\
\hline $\mathrm{H}$ & -1.652688 & 0.900205 & 2.429763 \\
\hline $\mathrm{H}$ & -1.347615 & -0.831148 & 2.598947 \\
\hline $\mathrm{H}$ & -2.323815 & -2.853382 & 0.567222 \\
\hline H & -3.980249 & -2.266966 & 0.64009 \\
\hline H & -3.152052 & -2.358772 & -0.915002 \\
\hline $\mathrm{H}$ & -2.853462 & 0.720895 & -2.35219 \\
\hline $\mathrm{H}$ & -4.459922 & 1.442673 & -2.224284 \\
\hline $\mathrm{H}$ & -4.255594 & -0.290796 & -1.98515 \\
\hline $\mathrm{H}$ & -5.414411 & -0.094749 & 0.296844 \\
\hline $\mathrm{H}$ & -4.826772 & 1.069036 & 1.489596 \\
\hline $\mathrm{H}$ & -5.609351 & 1.632463 & 0.016057 \\
\hline $\mathrm{H}$ & -3.635182 & 3.094897 & -0.557014 \\
\hline $\mathrm{H}$ & -2.841832 & 2.587579 & 0.93202 \\
\hline $\mathrm{H}$ & -2.002639 & 2.420841 & -0.613521 \\
\hline $\mathrm{C}$ & 5.65955 & 1.727613 & 0.54741 \\
\hline $\mathrm{H}$ & 5.530596 & 1.846997 & 1.622982 \\
\hline $\mathrm{H}$ & 6.71324 & 1.630938 & 0.303323 \\
\hline $\mathrm{H}$ & 5.249454 & 2.617789 & 0.068853 \\
\hline
\end{tabular}

\begin{tabular}{|c|c|c|c|}
\hline \multicolumn{4}{|c|}{ 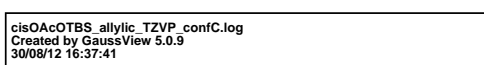 } \\
\hline $\mathrm{C}$ & 2.179004 & -1.860338 & -0.71968 \\
\hline $\mathrm{C}$ & 0.859185 & -1.920151 & -0.624963 \\
\hline $\mathrm{C}$ & 0.085173 & -1.064892 & 0.336564 \\
\hline $\mathrm{O}$ & 0.854004 & 0.043423 & 0.763266 \\
\hline $\mathrm{C}$ & 2.112933 & -0.358702 & 1.253077 \\
\hline $\mathrm{C}$ & 2.983731 & -0.965813 & 0.164855 \\
\hline $\mathrm{O}$ & 3.573363 & 0.044861 & -0.681338 \\
\hline $\mathrm{O}$ & -1.060647 & -0.611788 & -0.276072 \\
\hline $\mathrm{Si}$ & -2.190144 & 0.457195 & 0.40452 \\
\hline $\mathrm{C}$ & -3.797562 & 0.072013 & -0.551073 \\
\hline $\mathrm{C}$ & -2.351576 & 0.093963 & 2.246203 \\
\hline $\mathrm{C}$ & -1.621109 & 2.227899 & 0.143687 \\
\hline $\mathrm{C}$ & -3.563816 & 0.273251 & -2.051161 \\
\hline $\mathrm{C}$ & -4.913065 & 1.010873 & -0.082116 \\
\hline $\mathrm{C}$ & -4.214316 & -1.378558 & -0.296438 \\
\hline $\mathrm{C}$ & 4.687859 & 0.639413 & -0.222139 \\
\hline $\mathrm{O}$ & 5.195484 & 0.377356 & 0.836786 \\
\hline $\mathrm{H}$ & 2.717185 & -2.458732 & -1.445614 \\
\hline $\mathrm{H}$ & 0.271722 & -2.588346 & -1.24254 \\
\hline $\mathrm{H}$ & -0.169577 & -1.660187 & 1.234723 \\
\hline $\mathrm{H}$ & 2.589594 & 0.519495 & 1.682461 \\
\hline $\mathrm{H}$ & 1.993627 & -1.104089 & 2.052432 \\
\hline $\mathrm{H}$ & 3.79897 & -1.51601 & 0.640071 \\
\hline $\mathrm{H}$ & -3.131316 & 0.714987 & 2.691829 \\
\hline $\mathrm{H}$ & -1.416626 & 0.321456 & 2.761441 \\
\hline $\mathrm{H}$ & -2.604053 & -0.949672 & 2.440295 \\
\hline $\mathrm{H}$ & -1.485246 & 2.448747 & -0.915964 \\
\hline $\mathrm{H}$ & -2.33725 & 2.942366 & 0.554525 \\
\hline $\mathrm{H}$ & -0.662136 & 2.374191 & 0.641623 \\
\hline $\mathrm{H}$ & -4.476682 & 0.036647 & -2.608902 \\
\hline $\mathrm{H}$ & -2.766794 & -0.371842 & -2.424419 \\
\hline $\mathrm{H}$ & -3.297577 & 1.305968 & -2.286644 \\
\hline $\mathrm{H}$ & -5.837625 & 0.800312 & -0.63049 \\
\hline $\mathrm{H}$ & -5.133625 & 0.889463 & 0.981262 \\
\hline $\mathrm{H}$ & -4.665653 & 2.060473 & -0.256614 \\
\hline $\mathrm{H}$ & -3.438905 & -2.079977 & -0.610016 \\
\hline $\mathrm{H}$ & -4.429398 & -1.56192 & 0.758799 \\
\hline $\mathrm{H}$ & -5.123842 & -1.614778 & -0.859663 \\
\hline $\mathrm{C}$ & 5.194178 & 1.655627 & -1.200545 \\
\hline $\mathrm{H}$ & 4.436893 & 2.424335 & -1.357485 \\
\hline $\mathrm{H}$ & 6.106176 & 2.10434 & -0.818238 \\
\hline $\mathrm{H}$ & 5.382819 & 1.183714 & -2.164904 \\
\hline
\end{tabular}




\begin{tabular}{|c|c|c|c|}
\hline \multicolumn{4}{|c|}{$\begin{array}{l}\text { cisOAcOCTBS_allylic_TZVP_confD.log } \\
\text { Created by Gaussview 5.0.9 } \\
\text { 30/08/12 17:14:59 }\end{array}$} \\
\hline C & -2.359827 & -0.880009 & 1.299126 \\
\hline C & -1.045911 & -0.955474 & 1.453023 \\
\hline $\mathrm{C}$ & -0.102615 & -1.040378 & 0.287737 \\
\hline $\mathrm{O}$ & -0.726713 & -0.611978 & -0.907828 \\
\hline $\mathrm{C}$ & -1.957686 & -1.26506 & -1.1159 \\
\hline C & -2.983578 & -0.904727 & -0.061588 \\
\hline $\mathrm{O}$ & -3.529098 & 0.390799 & -0.401887 \\
\hline $\mathrm{O}$ & 0.998578 & -0.250972 & 0.528496 \\
\hline $\mathrm{Si}$ & 2.27098 & 0.027932 & -0.560584 \\
\hline C & 3.731098 & 0.497483 & 0.574676 \\
\hline C & 2.601608 & -1.546436 & -1.540757 \\
\hline C & 1.808977 & 1.426432 & -1.725487 \\
\hline C & 3.348917 & 1.715549 & 1.42143 \\
\hline C & 4.959159 & 0.836269 & -0.275476 \\
\hline C & 4.064729 & -0.672741 & 1.50416 \\
\hline $\mathrm{C}$ & -4.73244 & 0.694098 & 0.110116 \\
\hline $\mathrm{O}$ & -5.365941 & -0.050196 & 0.811842 \\
\hline $\mathrm{H}$ & -3.027143 & -0.801381 & 2.148511 \\
\hline $\mathrm{H}$ & -0.586012 & -0.966341 & 2.433748 \\
\hline $\mathrm{H}$ & 0.205665 & -2.094296 & 0.144588 \\
\hline $\mathrm{H}$ & -2.31147 & -0.971361 & -2.102595 \\
\hline $\mathrm{H}$ & -1.818694 & -2.355441 & -1.106247 \\
\hline $\mathrm{H}$ & -3.805766 & -1.623743 & -0.080504 \\
\hline $\mathrm{H}$ & 3.452376 & -1.407612 & -2.210944 \\
\hline $\mathrm{H}$ & 1.735474 & -1.795855 & -2.156248 \\
\hline $\mathrm{H}$ & 2.820019 & -2.397947 & -0.894466 \\
\hline $\mathrm{H}$ & 1.568596 & 2.336471 & -1.174095 \\
\hline $\mathrm{H}$ & 2.619686 & 1.651138 & -2.421577 \\
\hline $\mathrm{H}$ & 0.927236 & 1.142806 & -2.300932 \\
\hline $\mathrm{H}$ & 4.171827 & 1.976438 & 2.096078 \\
\hline $\mathrm{H}$ & 2.465622 & 1.520211 & 2.031709 \\
\hline $\mathrm{H}$ & 3.143386 & 2.5935 & 0.804958 \\
\hline $\mathrm{H}$ & 5.800371 & 1.108782 & 0.370972 \\
\hline $\mathrm{H}$ & 5.282687 & -0.00954 & -0.887208 \\
\hline $\mathrm{H}$ & 4.775634 & 1.682482 & -0.941439 \\
\hline $\mathrm{H}$ & 3.207123 & -0.956615 & 2.117024 \\
\hline $\mathrm{H}$ & 4.391957 & -1.555901 & 0.950421 \\
\hline $\mathrm{H}$ & 4.879312 & -0.394418 & 2.181755 \\
\hline $\mathrm{C}$ & -5.158306 & 2.071624 & -0.30027 \\
\hline $\mathrm{H}$ & -5.011811 & 2.216947 & -1.369693 \\
\hline $\mathrm{H}$ & -6.199969 & 2.22376 & -0.033117 \\
\hline $\mathrm{H}$ & -4.539185 & 2.80586 & 0.217746 \\
\hline & & $=-1100$ & 9185 a.u. \\
\hline
\end{tabular}

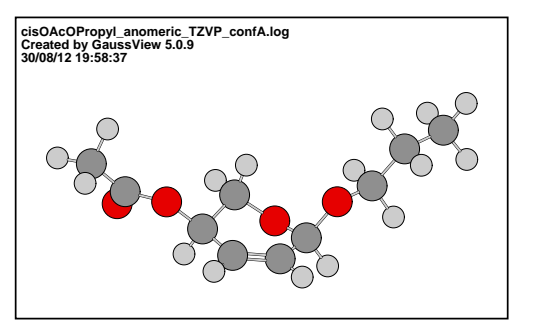

$\begin{array}{|llll|}\mathrm{C} & -1.01654 & 1.707276 & -0.525053 \\ \mathrm{C} & 0.285669 & 1.937985 & -0.458842 \\ \mathrm{C} & 1.194569 & 1.126293 & 0.414656 \\ \mathrm{O} & 0.493719 & 0.253674 & 1.266778\end{array}$

$\begin{array}{llll}\mathrm{O} & 0.493719 & 0.253674 & 0.414656\end{array}$

$\begin{array}{llll}\text { C } & -0.579843 & -0.418103 & 0.635182\end{array}$

C $\quad-1.645883 \quad 0.592646 \quad 0.243427$

O $\quad-2.624527 \quad-0.023165 \quad-0.611433$

$\begin{array}{llll}\text { O } & 2.095202 & 0.459299 & -0.422355\end{array}$

C $\quad-3.654663 \quad-0.649095 \quad-0.008863$

O $-3.786268 \quad-0.721453 \quad 1.182843$

$\begin{array}{llll}\mathrm{H} & -1.668339 & 2.314542 & -1.142546\end{array}$

$\begin{array}{llll}\mathrm{H} & 0.752612 & 2.727456 & -1.033898\end{array}$

$\begin{array}{llll}\mathrm{H} & 1.746806 & 1.781407 & 1.101186 \\ \mathrm{H} & -0.964649 & -1.13772 & 1.354469\end{array}$

\begin{tabular}{llll} 
& -0.964649 & -1.13772 & 1.354469 \\
\hline & -0.239533 & -0.945434 & -0.261959
\end{tabular}

\begin{tabular}{llll} 
& -0.239533 & -0.945434 & -0.261959 \\
\hline & -2.154777 & 0.962964 & 1.136927
\end{tabular}

C $-4.590483-1.230321-1.136927$

$\begin{array}{llll}\mathrm{C} & -4.590483 & -1.230321 & -1.025306\end{array}$

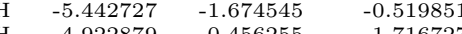

$\begin{array}{llll}\mathrm{H} & -4.922879 & -0.456255 & -1.716727 \\ \mathrm{H} & -4.070846 & -1.989439 & -1.611093\end{array}$

$\begin{array}{llll}\mathrm{H} & -4.070846 & -1.989439 & -1.611093\end{array}$

$\begin{array}{llll}\text { C } & 3.199081 & -0.114761 & 0.261663\end{array}$

$\begin{array}{llll}\mathrm{H} & 2.847897 & -0.862026 & 0.980793\end{array}$

$\begin{array}{llll}\mathrm{H} & 3.726078 & 0.667268 & 0.82678\end{array}$

$\begin{array}{llll}\text { C } & 4.125981 & -0.745643 & -0.752357\end{array}$

$\begin{array}{llll}\mathrm{H} & 4.434628 & 0.018222 & -1.470073\end{array}$

$\mathrm{H} \quad 3.567792 \quad-1.497416 \quad-1.315514$

C $\quad 5.344643 \quad-1.377565 \quad-0.096778$

$\mathrm{H} \quad 5.996926 \quad-1.834827 \quad-0.841522$

$\begin{array}{llll}\mathrm{H} & 5.934576 & -0.636762 & 0.447551 \\ \mathrm{H} & 5.056252 & -2.15654 & 0.612439\end{array}$

0.612439

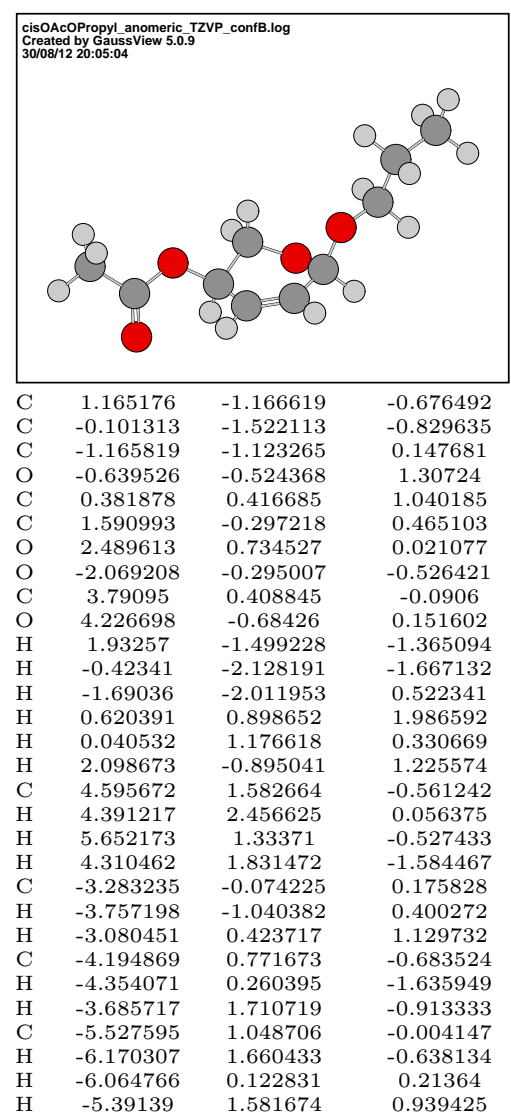

$\mathrm{E}(\mathrm{RmPW} 1 \mathrm{PW} 91)=-691.64355783$ a.u.
$\mathrm{E}(\mathrm{RmPW} 1 \mathrm{PW} 91)=-691.64333656$ a.u. 

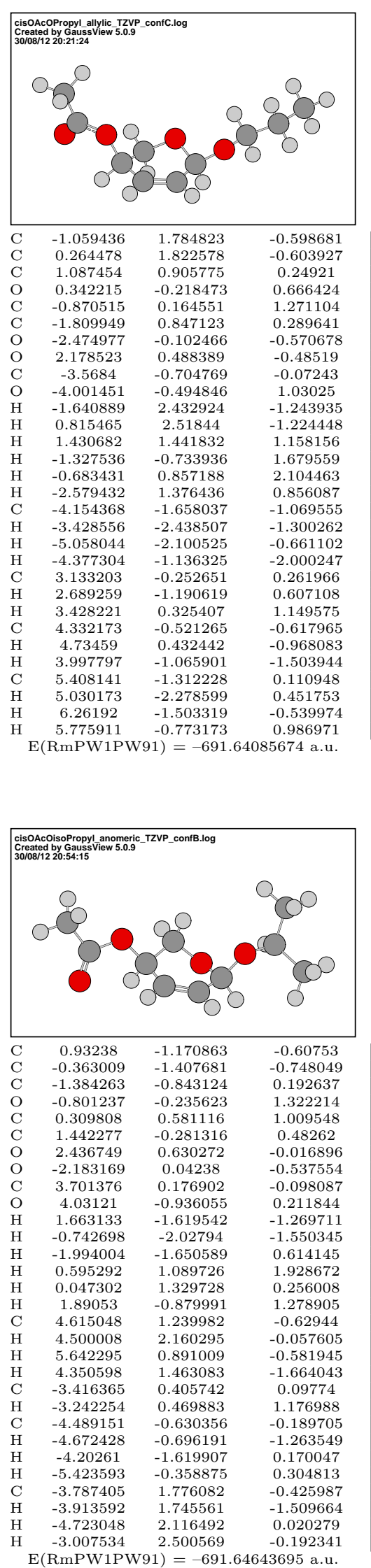
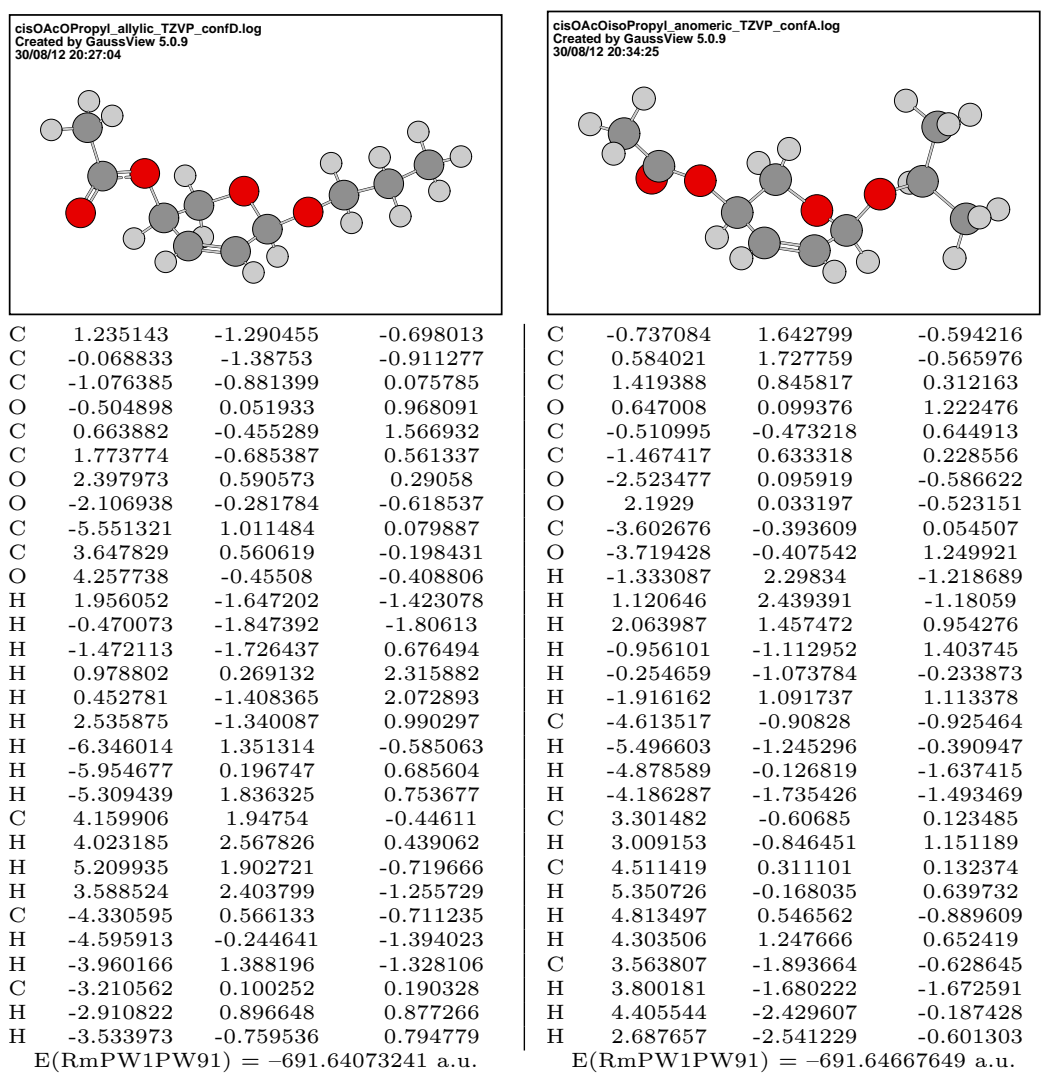

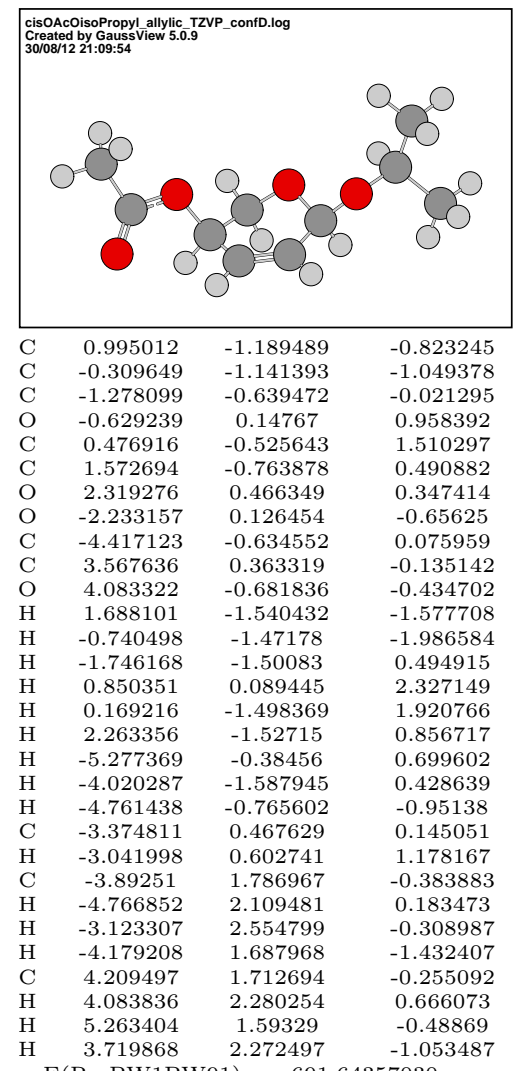
$\mathrm{E}(\mathrm{RmPW} 1 \mathrm{PW} 91)=-691.64357030 \mathrm{a} \cdot \mathrm{u}$ 


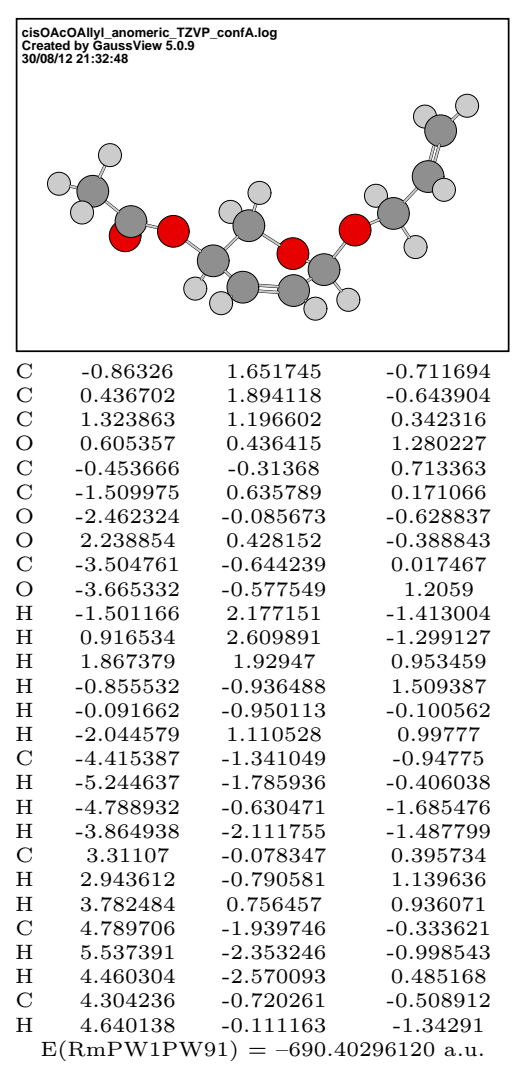

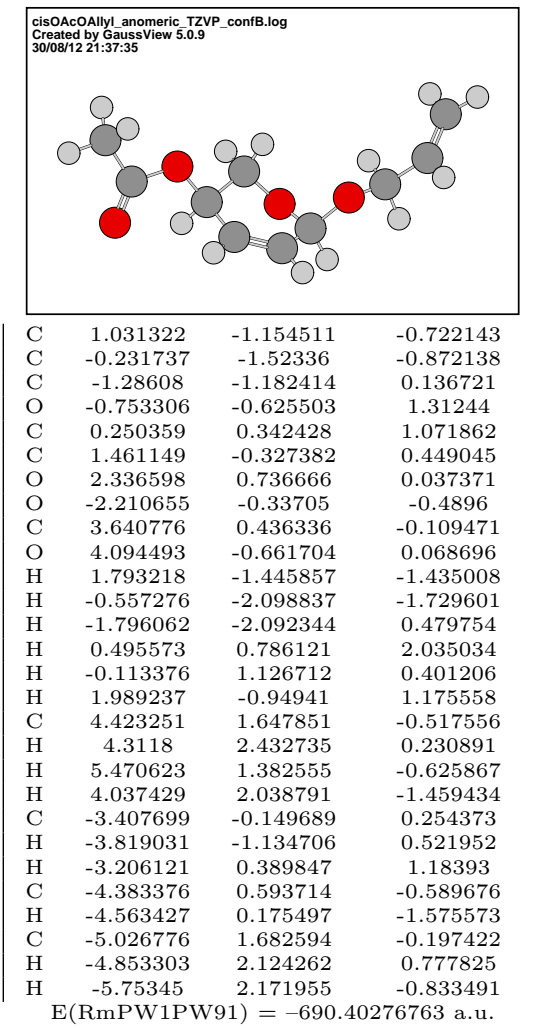

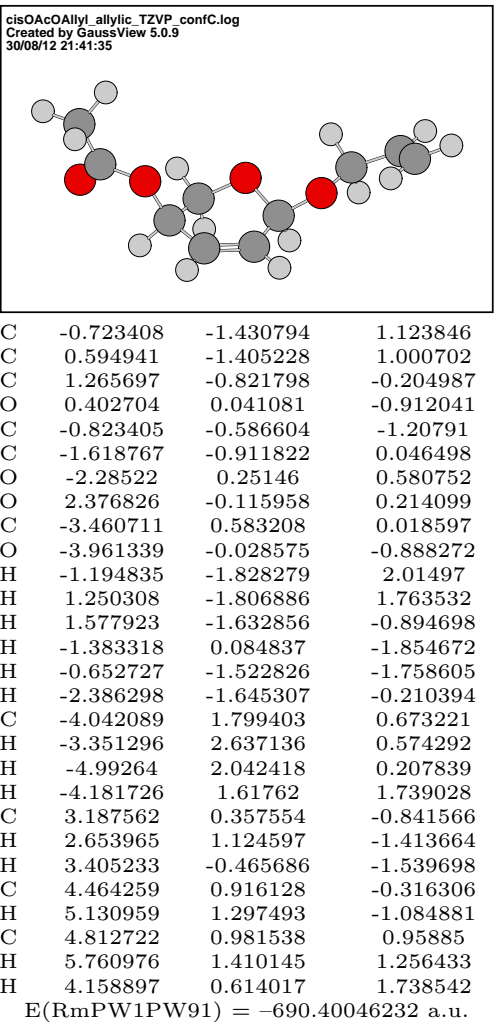

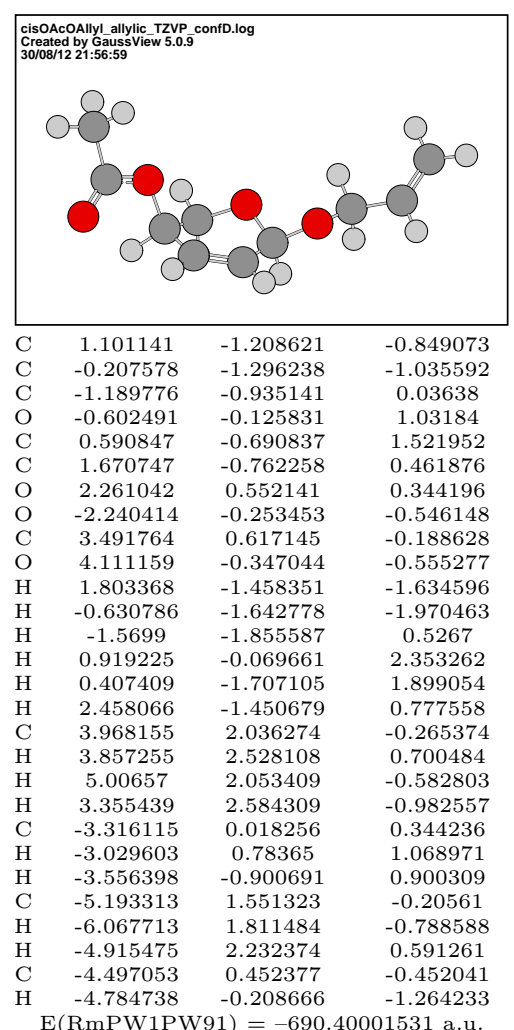

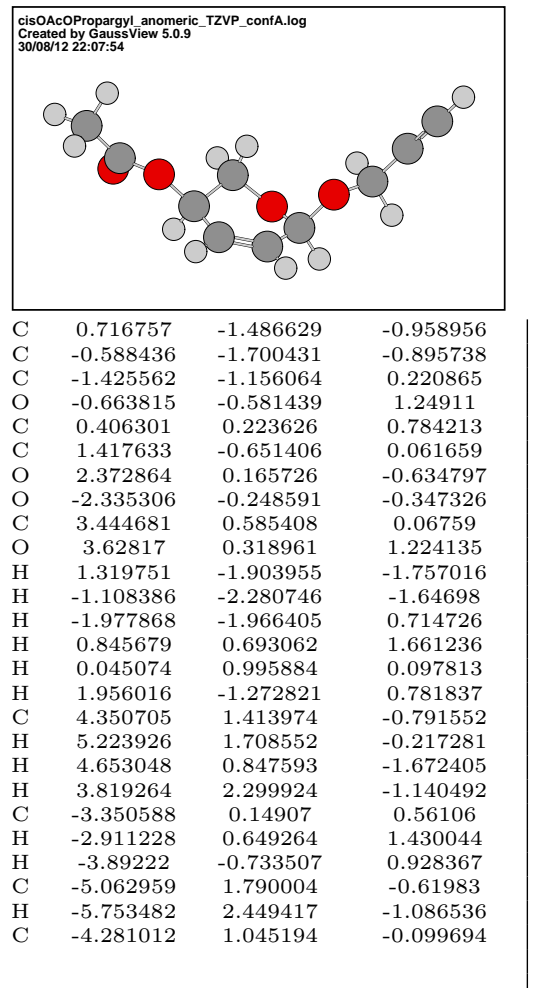

$\mathrm{E}(\mathrm{RmPW} 1 \mathrm{PW} 91)=-689.14310459$ a.u.

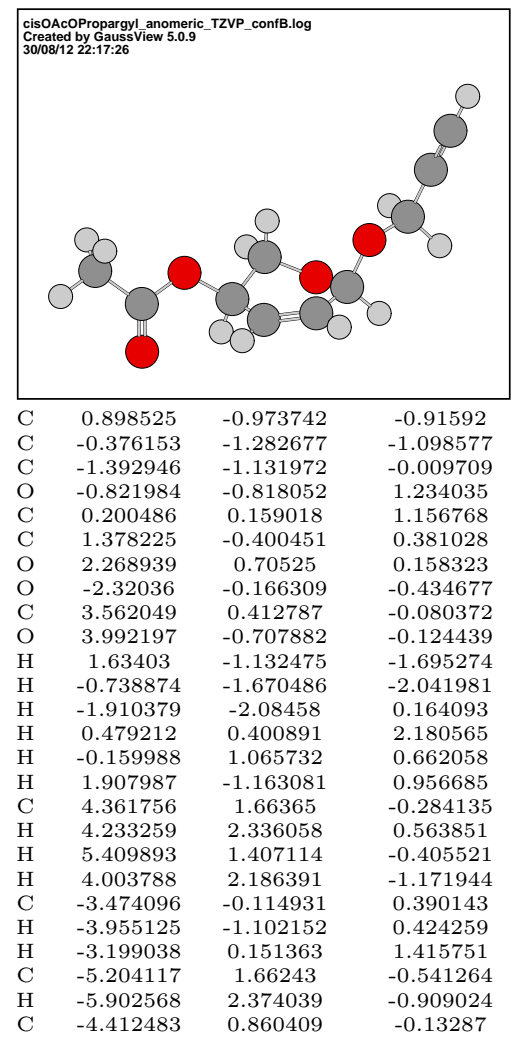

$\mathrm{E}(\mathrm{RmPW} 1 \mathrm{PW} 91)=-689.14283053$ a.u. 


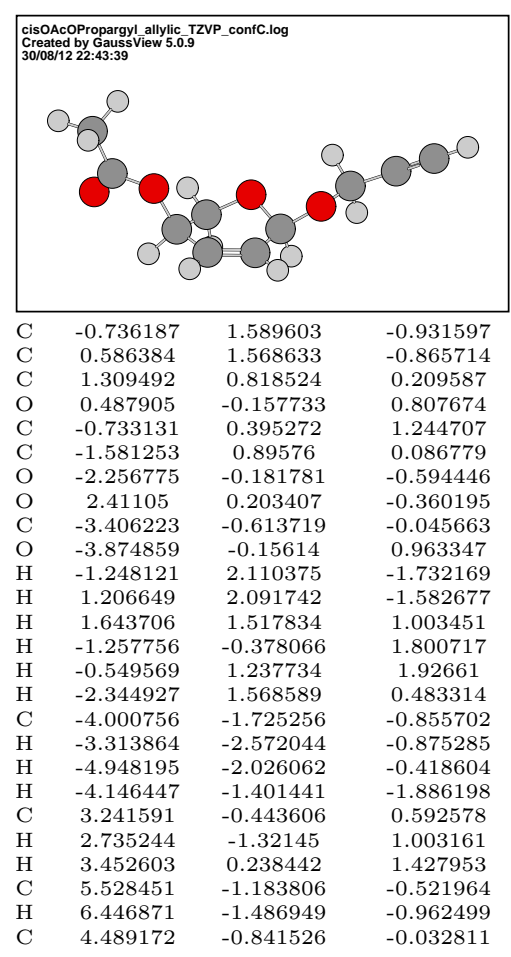

$\mathrm{E}(\mathrm{RmPW} 1 \mathrm{PW} 91)=-689.14014340$ a.u.

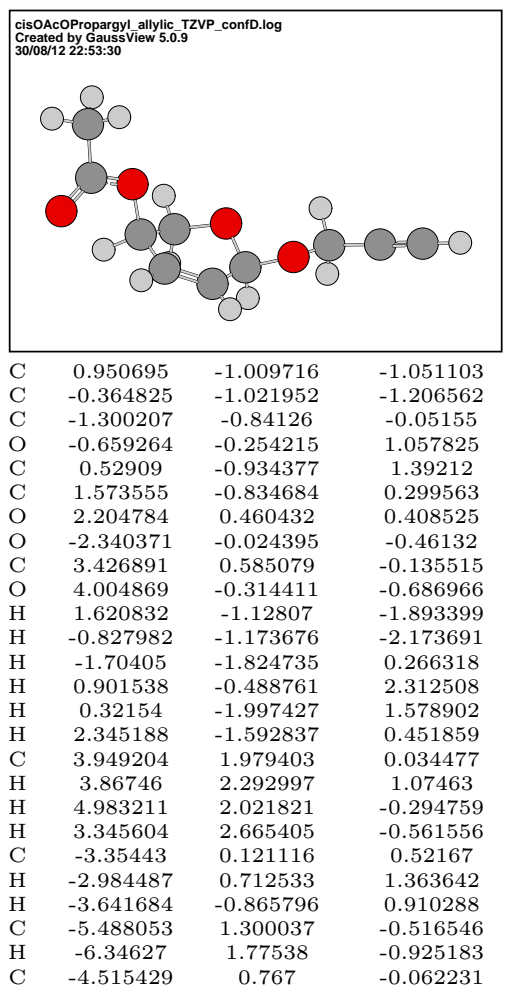

$\mathrm{E}(\mathrm{RmPW}$ 1PW91 $)=-689.13997361$ a.u.

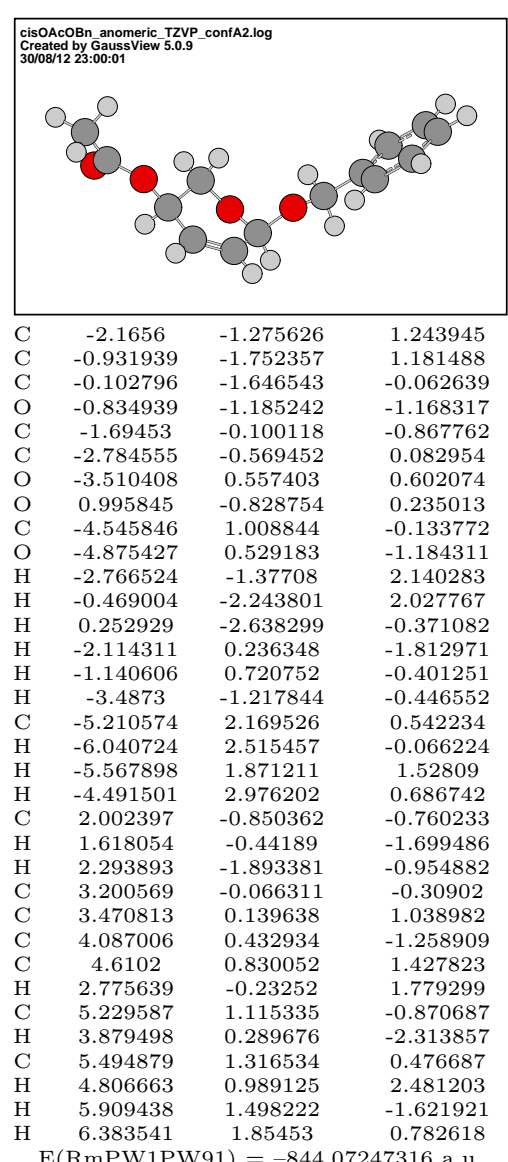




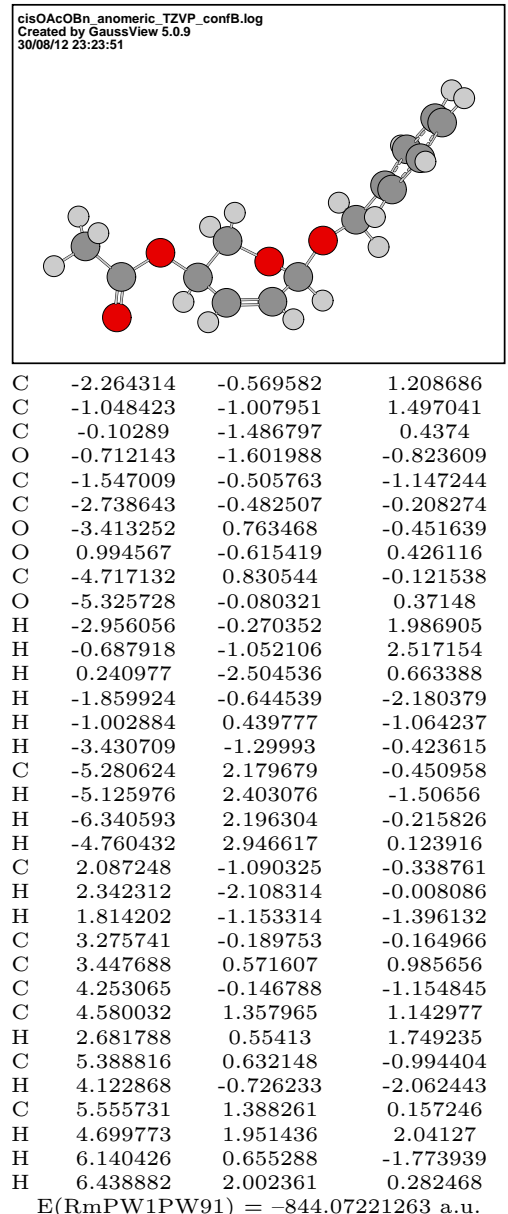

$\mathrm{E}(\mathrm{RmPW} 1 \mathrm{PW} 91)=-844.07221263 \mathrm{a.u}$.
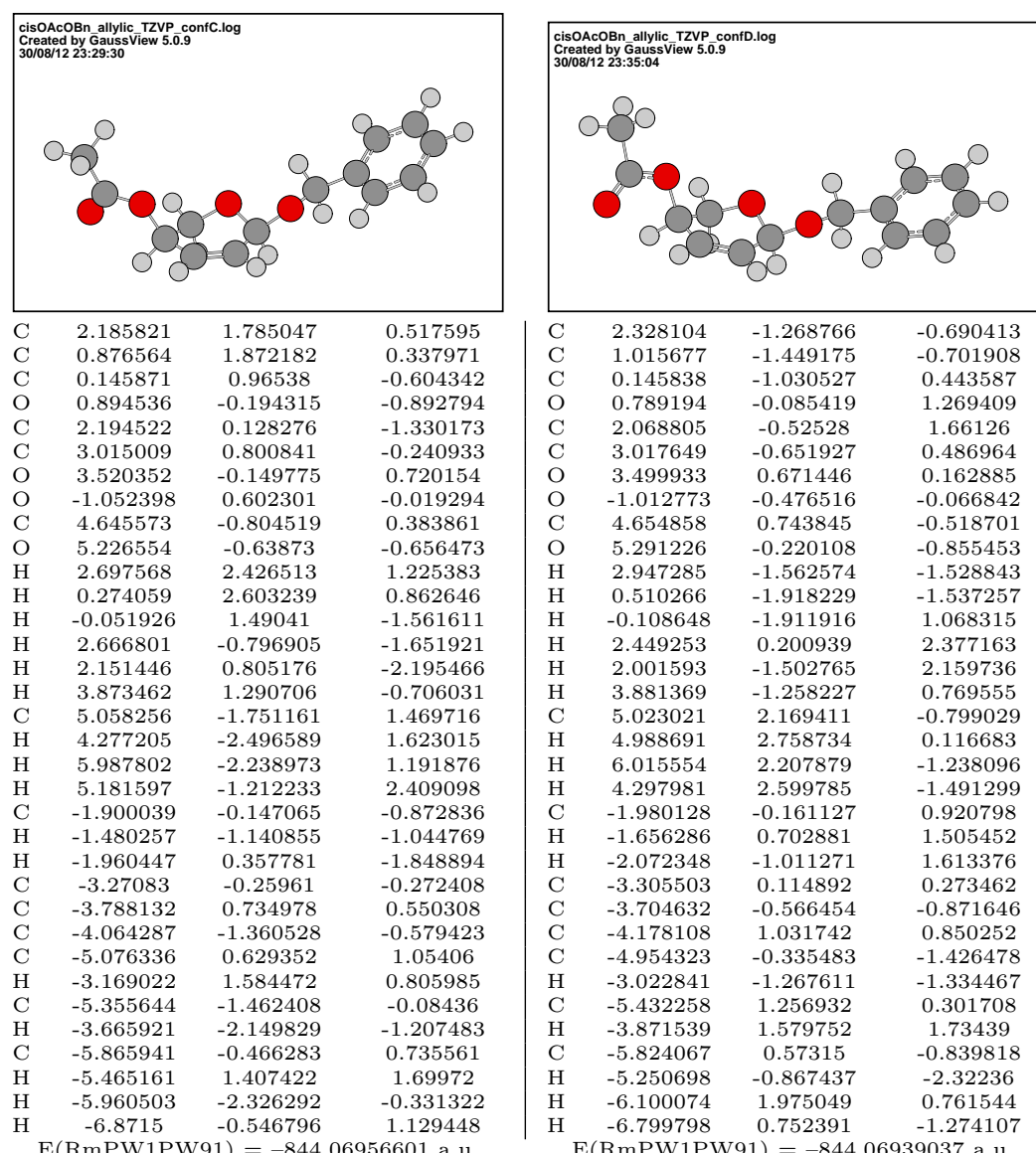

$\mathrm{E}(\mathrm{RmPW} 1 \mathrm{PW} 91)=-844.06956601 \mathrm{a} . \mathrm{u}$. 


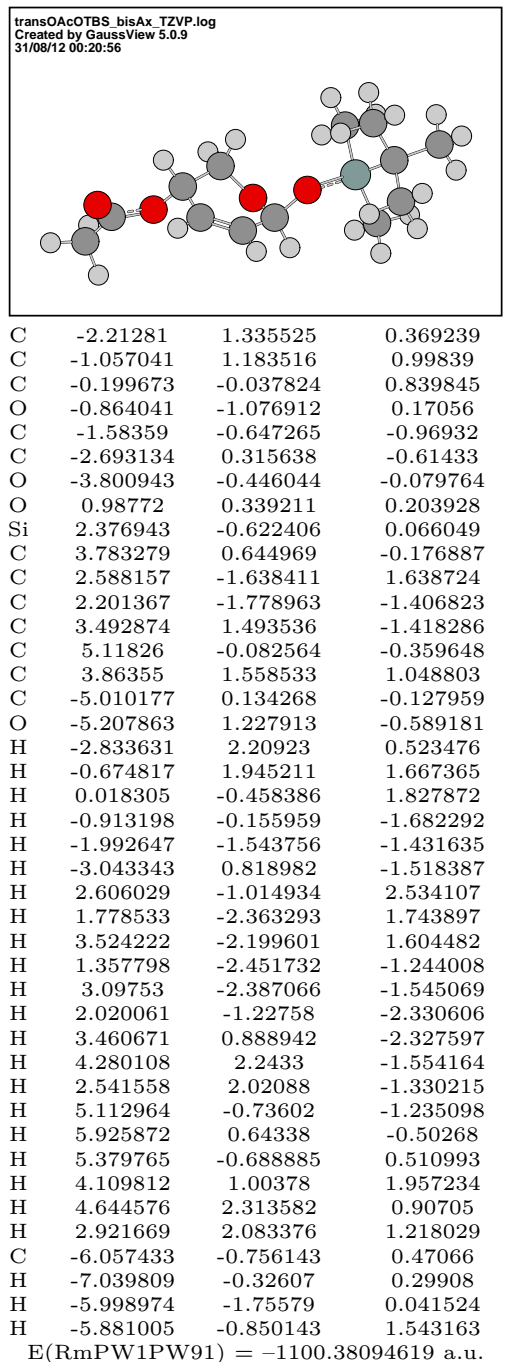

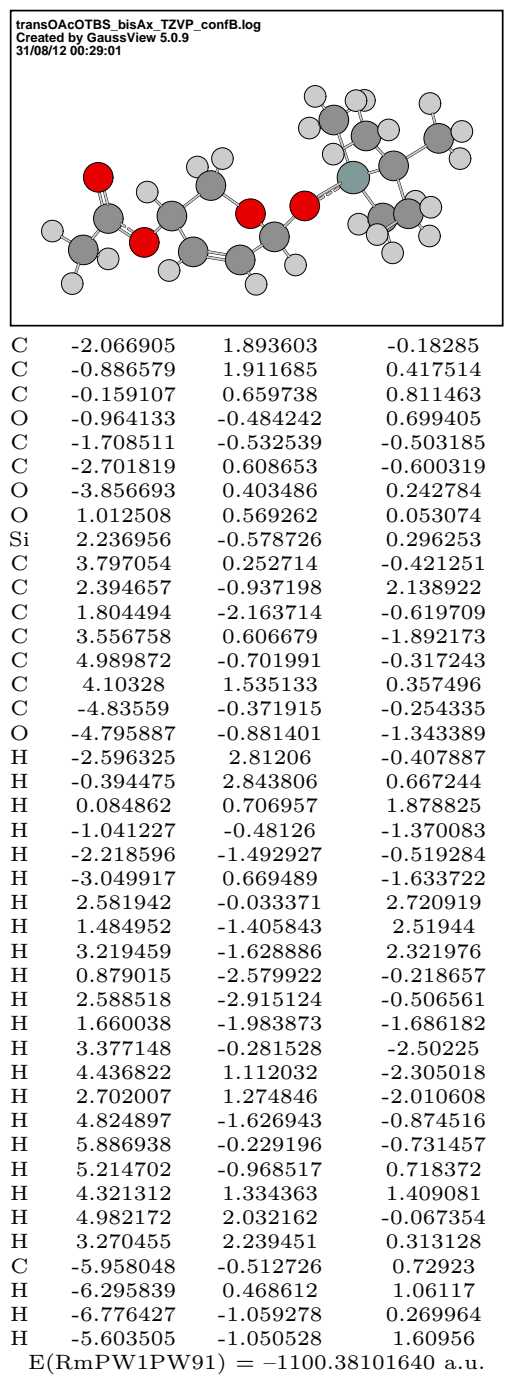

\begin{tabular}{|c|c|c|c|}
\hline \multicolumn{4}{|c|}{ 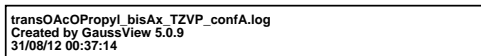 } \\
\hline $\mathrm{C}$ & -1.116876 & 1.361331 & 0.003152 \\
\hline $\mathrm{C}$ & 0.041816 & 1.472529 & 0.635448 \\
\hline $\mathrm{C}$ & 0.986547 & 0.320555 & 0.79551 \\
\hline $\mathrm{O}$ & 0.412695 & -0.907606 & 0.437149 \\
\hline $\mathrm{C}$ & -0.323433 & -0.850773 & -0.769758 \\
\hline $\mathrm{C}$ & -1.507656 & 0.083251 & -0.669822 \\
\hline $\mathrm{O}$ & -2.556274 & -0.593061 & 0.061835 \\
\hline $\mathrm{O}$ & 2.141222 & 0.617908 & 0.060037 \\
\hline $\mathrm{C}$ & -3.807486 & -0.143387 & -0.12162 \\
\hline $\mathrm{O}$ & -4.087723 & 0.771505 & -0.851123 \\
\hline $\mathrm{H}$ & -1.806138 & 2.193345 & -0.069469 \\
\hline $\mathrm{H}$ & 0.358196 & 2.410706 & 1.074739 \\
\hline $\mathrm{H}$ & 1.246925 & 0.195261 & 1.854637 \\
\hline $\mathrm{H}$ & 0.316698 & -0.513674 & -1.59186 \\
\hline $\mathrm{H}$ & -0.65686 & -1.866186 & -0.975847 \\
\hline $\mathrm{H}$ & -1.888476 & 0.301408 & -1.670159 \\
\hline $\mathrm{C}$ & -4.785072 & -0.92675 & 0.702119 \\
\hline $\mathrm{H}$ & -5.796988 & -0.626956 & 0.446101 \\
\hline $\mathrm{H}$ & -4.651656 & -1.995347 & 0.536699 \\
\hline $\mathrm{H}$ & -4.601858 & -0.73498 & 1.760298 \\
\hline $\mathrm{C}$ & 4.432775 & 0.221316 & -0.465227 \\
\hline $\mathrm{H}$ & 4.165308 & 0.178651 & -1.523811 \\
\hline $\mathrm{H}$ & 4.640418 & 1.269457 & -0.236836 \\
\hline $\mathrm{C}$ & 5.664656 & -0.630917 & -0.199585 \\
\hline $\mathrm{H}$ & 6.510533 & -0.294055 & -0.799771 \\
\hline $\mathrm{H}$ & 5.96504 & -0.582329 & 0.849509 \\
\hline $\mathrm{H}$ & 5.484044 & -1.680164 & -0.443412 \\
\hline $\mathrm{C}$ & 3.243332 & -0.228097 & 0.352409 \\
\hline $\mathrm{H}$ & 2.990046 & -1.268793 & 0.124476 \\
\hline $\mathrm{H}$ & 3.473786 & -0.170655 & 1.42577 \\
\hline
\end{tabular}

$\mathrm{E}(\mathrm{RmPW} 1 \mathrm{PW} 91)=-691.64431257$ a.u. 

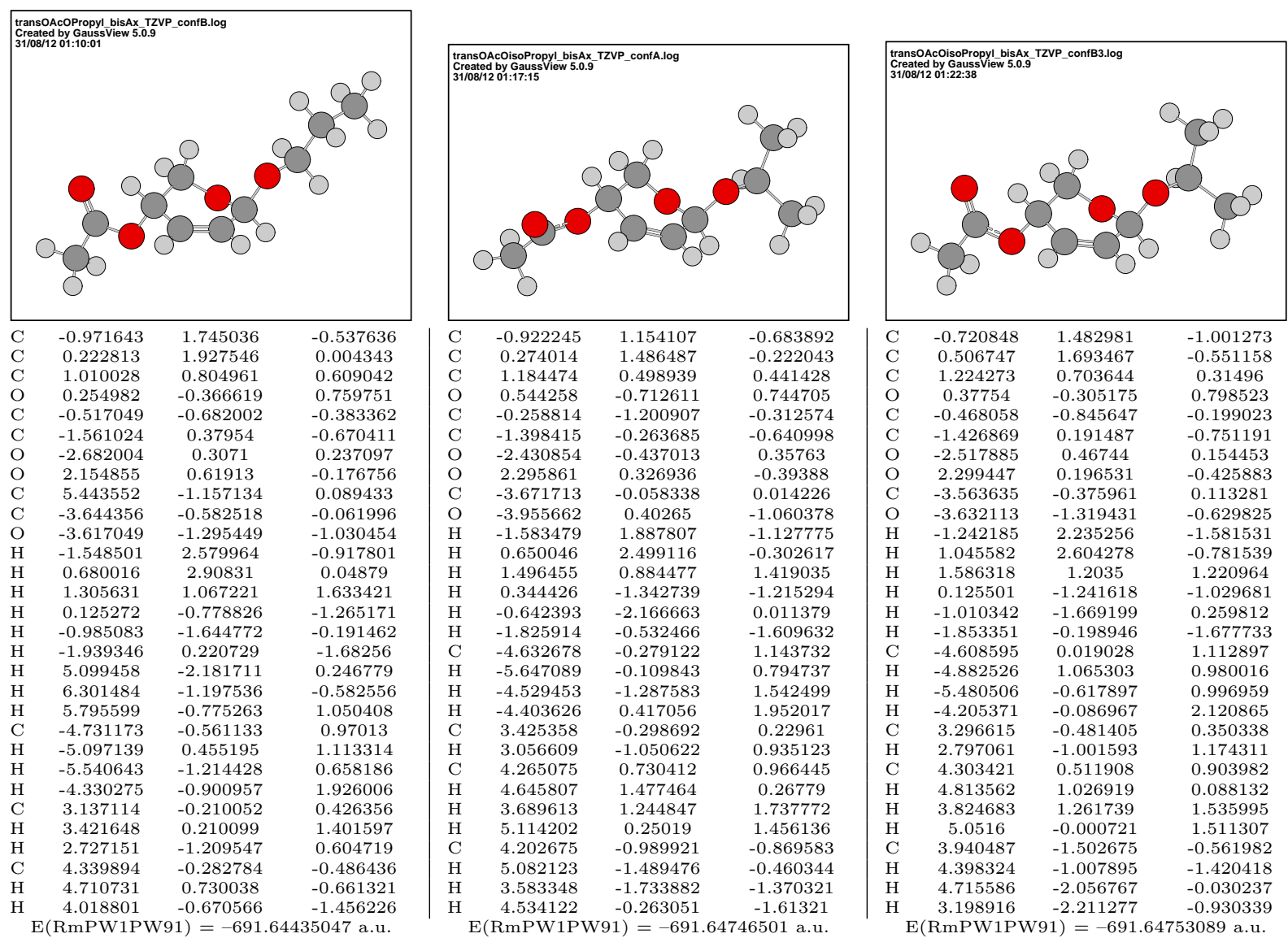


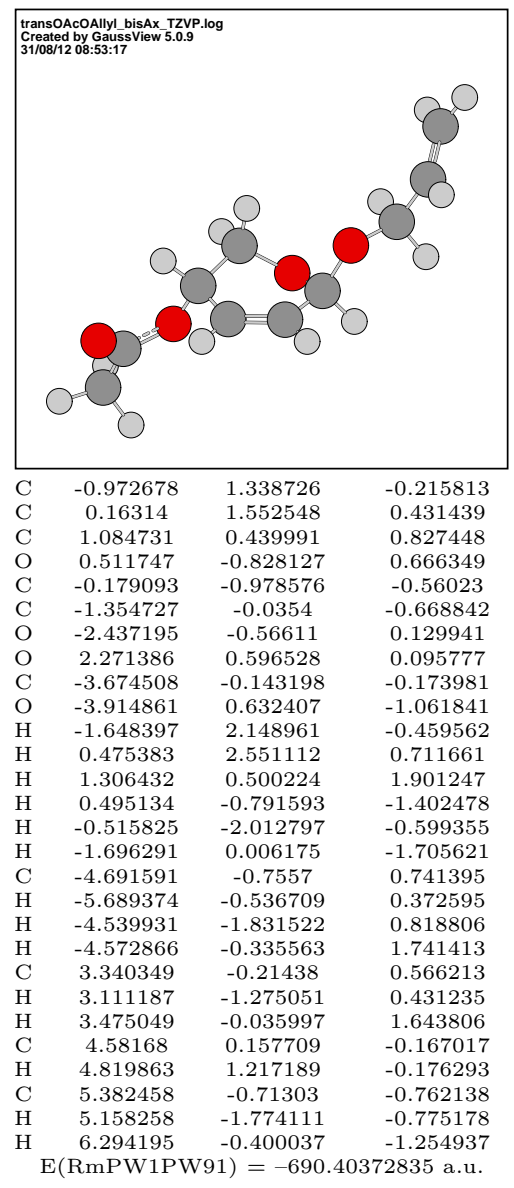

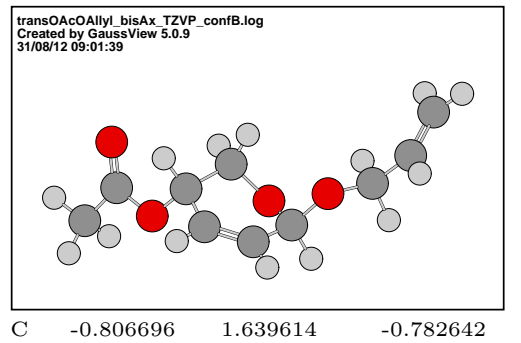

\begin{tabular}{|cccc}
\hline $\mathrm{C}$ & -0.806696 & 1.639614 & -0.782642 \\
$\mathrm{C}$ & 0.365396 & 1.908956 & -0.228228 \\
$\mathrm{C}$ & 1.117758 & 0.89995 & 0.584211 \\
$\mathrm{O}$ & 0.348494 & -0.227214 & 0.89894 \\
$\mathrm{C}$ & -0.379496 & -0.729609 & -0.2069 \\
$\mathrm{C}$ & -1.39966 & 0.271049 & -0.713647 \\
$\mathrm{O}$ & -2.561784 & 0.350527 & 0.139963 \\
$\mathrm{O}$ & 2.288456 & 0.580806 & -0.11942 \\
$\mathrm{C}$ & -3.518662 & -0.573231 & -0.057107 \\
$\mathrm{O}$ & -3.452648 & -1.434063 & -0.894554 \\
$\mathrm{H}$ & -1.361842 & 2.400596 & -1.318383 \\
$\mathrm{H}$ & 0.827494 & 2.883485 & -0.32614 \\
$\mathrm{H}$ & 1.382169 & 1.327427 & 1.560543 \\
$\mathrm{H}$ & 0.29909 & -0.976673 & -1.030069 \\
$\mathrm{H}$ & -0.865178 & -1.643943 & 0.125834 \\
$\mathrm{H}$ & -1.732485 & -0.053761 & -1.701837 \\
$\mathrm{C}$ & -4.652905 & -0.381222 & 0.903817 \\
$\mathrm{H}$ & -5.01671 & 0.644962 & 0.85916 \\
$\mathrm{H}$ & -5.452164 & -1.076618 & 0.665168 \\
$\mathrm{H}$ & -4.301055 & -0.558365 & 1.921012 \\
$\mathrm{C}$ & 3.230385 & -0.157497 & 0.648622 \\
$\mathrm{H}$ & 3.429643 & 0.385031 & 1.585143 \\
$\mathrm{H}$ & 2.830242 & -1.140214 & 0.912802 \\
$\mathrm{C}$ & 4.488126 & -0.291307 & -0.136954 \\
$\mathrm{H}$ & 4.897967 & 0.635586 & -0.527088 \\
$\mathrm{C}$ & 5.110407 & -1.44036 & -0.351602 \\
$\mathrm{H}$ & 4.712823 & -2.379148 & 0.018374 \\
$\mathrm{H}$ & 6.041931 & -1.486874 & -0.901375 \\
$\mathrm{E}(\mathrm{RmPW} 1 \mathrm{PW} 91)$ & $=-690.40380280 \mathrm{a} . \mathrm{u}$.
\end{tabular}




\begin{tabular}{|c|c|c|c|}
\hline \multicolumn{4}{|c|}{\begin{tabular}{|l} 
transoAcOPPropargy! bisAx_TZVP_confB.log \\
Craeted by GaussvView 5.0.9 \\
31/08/12 09:18:48
\end{tabular}} \\
\hline $\mathrm{C}$ & -0.656016 & 1.600882 & -0.786201 \\
\hline C & 0.517974 & 1.826209 & -0.216668 \\
\hline $\mathrm{C}$ & 1.213773 & 0.792782 & 0.6135 \\
\hline $\mathrm{O}$ & 0.403189 & -0.306211 & 0.91529 \\
\hline $\mathrm{C}$ & -0.331556 & -0.781572 & -0.199781 \\
\hline $\mathrm{C}$ & -1.30515 & 0.257955 & -0.718847 \\
\hline $\mathrm{O}$ & -2.470936 & 0.387989 & 0.122304 \\
\hline $\mathrm{O}$ & 2.388354 & 0.429729 & -0.070471 \\
\hline C & -3.462291 & -0.49836 & -0.080913 \\
\hline $\mathrm{O}$ & -3.420741 & -1.365054 & -0.913583 \\
\hline $\mathrm{H}$ & -1.171948 & 2.380895 & -1.33347 \\
\hline $\mathrm{H}$ & 1.022159 & 2.779633 & -0.312348 \\
\hline $\mathrm{H}$ & 1.476089 & 1.214744 & 1.592612 \\
\hline $\mathrm{H}$ & 0.346548 & -1.055976 & -1.014378 \\
\hline $\mathrm{H}$ & -0.856012 & -1.675513 & 0.129016 \\
\hline $\mathrm{H}$ & -1.640021 & -0.056407 & -1.709721 \\
\hline $\mathrm{C}$ & -4.597924 & -0.256723 & 0.866668 \\
\hline $\mathrm{H}$ & -4.917293 & 0.783888 & 0.816993 \\
\hline $\mathrm{H}$ & -5.423108 & -0.918095 & 0.619758 \\
\hline $\mathrm{H}$ & -4.265396 & -0.447351 & 1.887941 \\
\hline $\mathrm{C}$ & 3.266458 & -0.35059 & 0.726119 \\
\hline $\mathrm{H}$ & 3.532638 & 0.199137 & 1.639476 \\
\hline $\mathrm{H}$ & 2.778802 & -1.281057 & 1.033975 \\
\hline $\mathrm{C}$ & 4.471808 & -0.655264 & -0.02194 \\
\hline $\mathrm{C}$ & 5.475426 & -0.924657 & -0.619358 \\
\hline $\mathrm{H}$ & 6.363608 & -1.159482 & -1.153656 \\
\hline
\end{tabular}

$\mathrm{E}(\mathrm{RmPW} 1 \mathrm{PW} 91)=-689.14390951$ a.u.

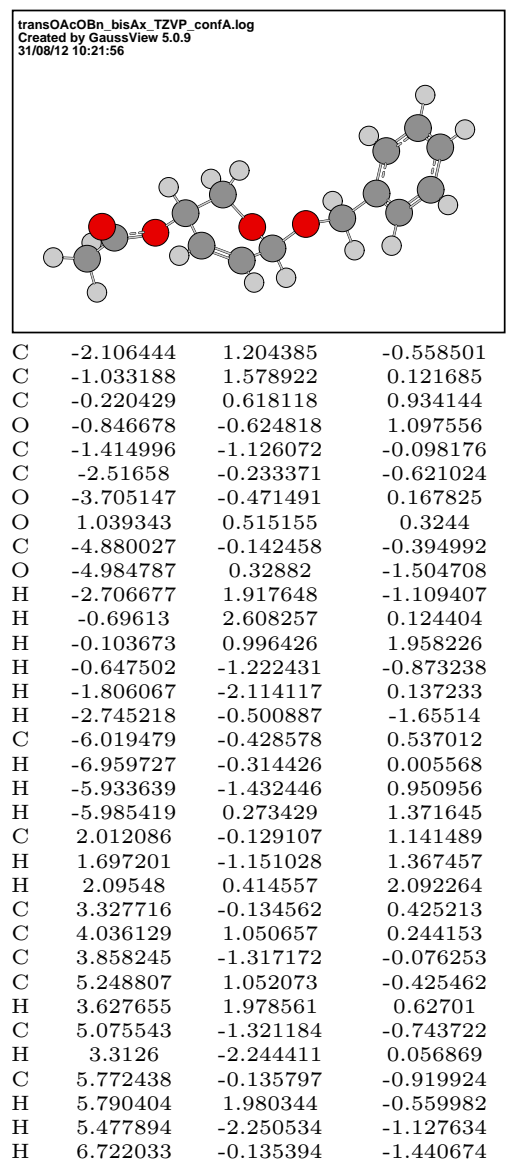

$\mathrm{E}(\mathrm{RmPW} 1 \mathrm{PW} 91)=-844.07287251 \mathrm{a} . \mathrm{u}$

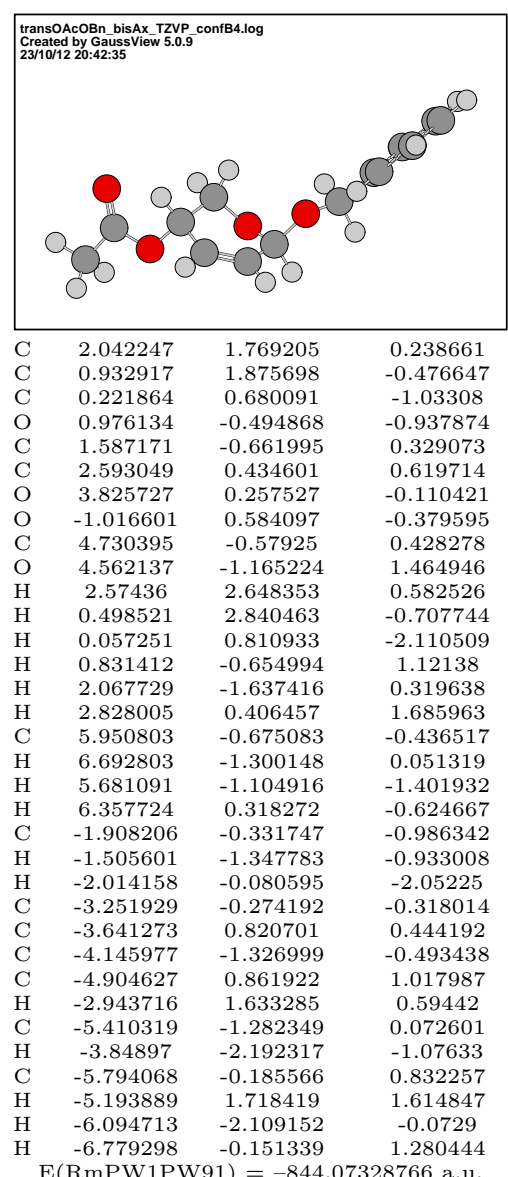

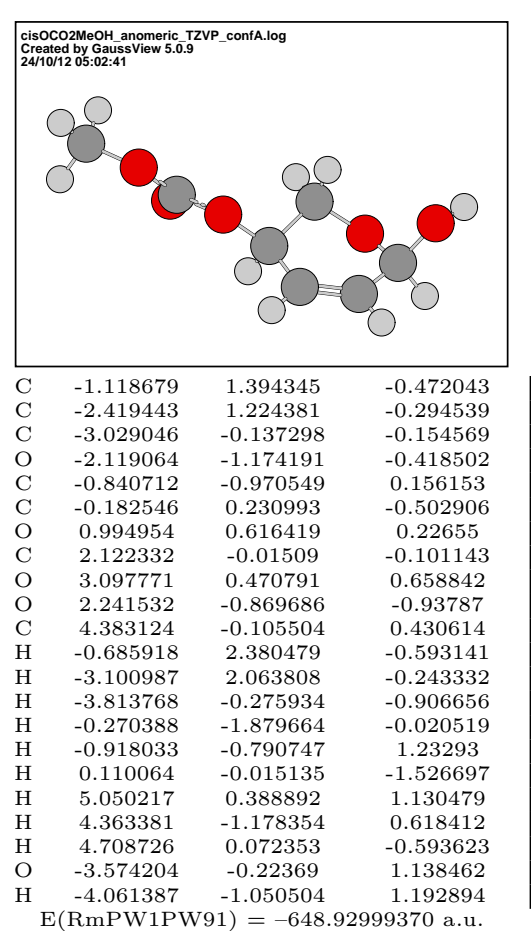

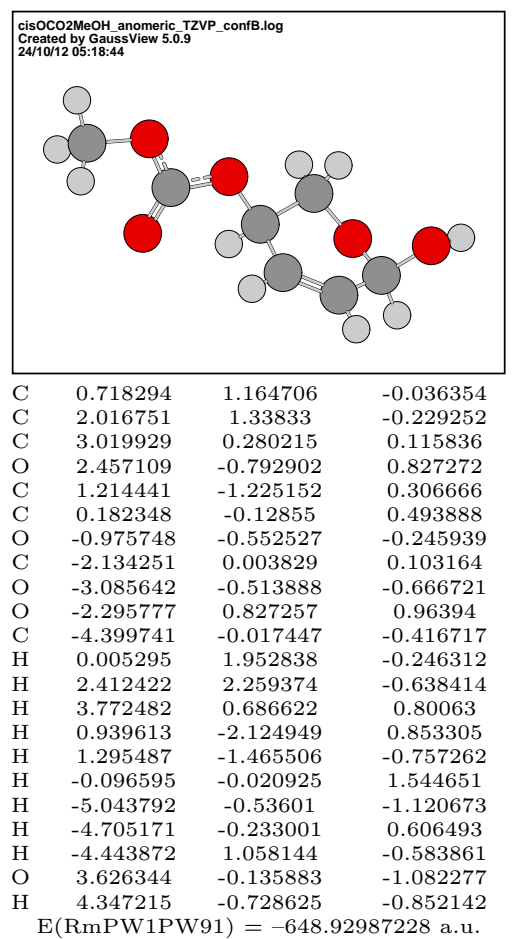

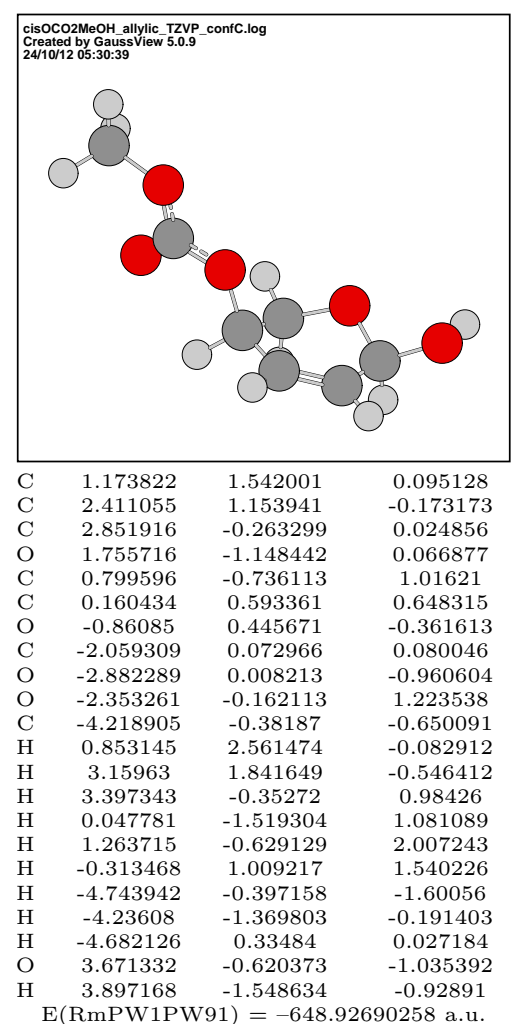



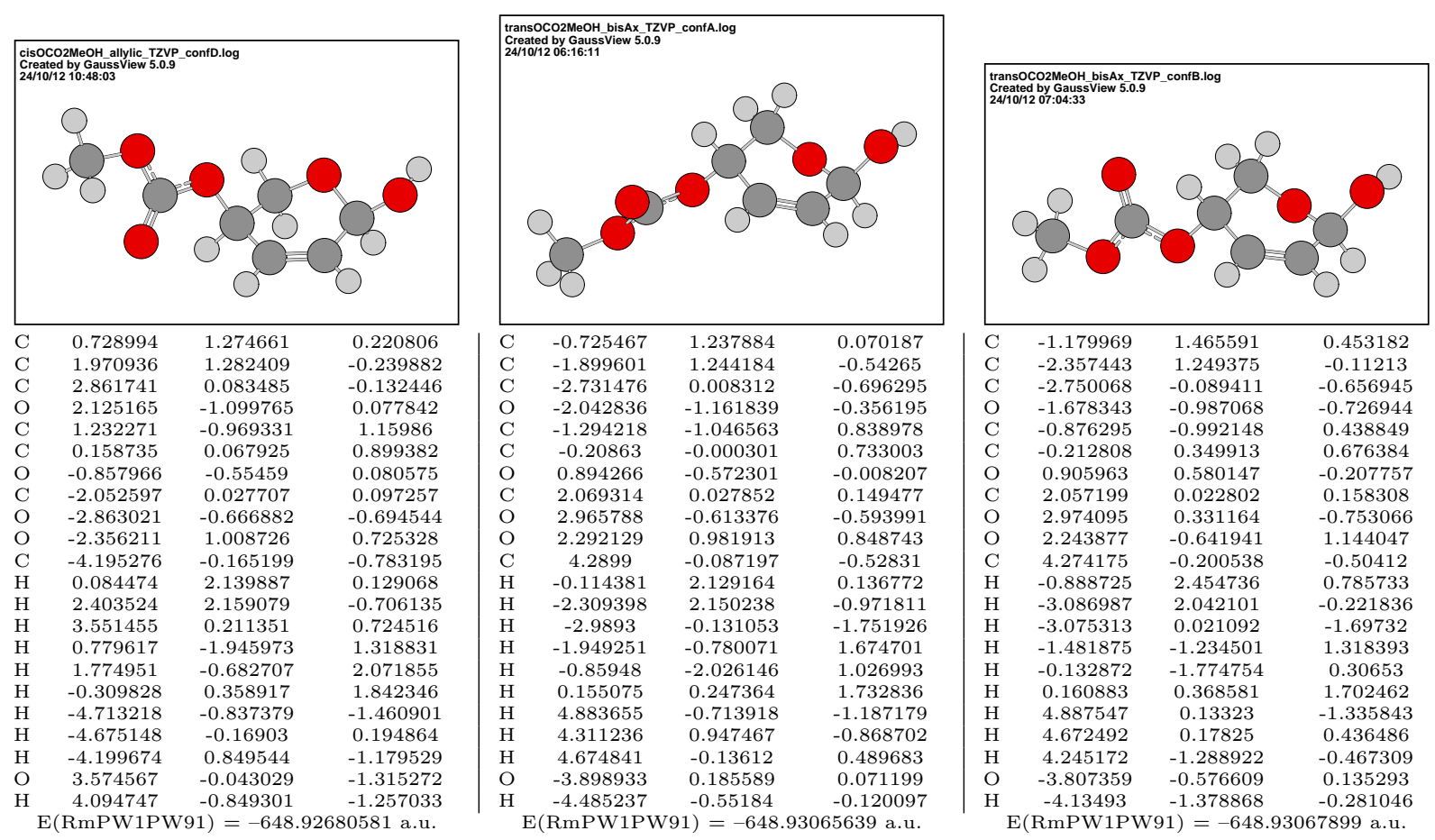


\begin{tabular}{|c|c|c|c|}
\hline \multicolumn{4}{|c|}{ 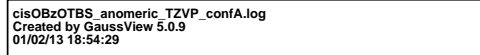 } \\
\hline $\mathrm{C}$ & -0.618402 & -1.219474 & 1.899724 \\
\hline $\mathrm{C}$ & 0.703545 & -1.201799 & 1.974474 \\
\hline $\mathrm{C}$ & 1.578429 & -1.399103 & 0.770427 \\
\hline $\mathrm{O}$ & 0.845031 & -1.780934 & -0.369266 \\
\hline $\mathrm{C}$ & -0.363391 & -1.064905 & -0.543392 \\
\hline $\mathrm{C}$ & -1.316669 & -1.400835 & 0.592675 \\
\hline $\mathrm{O}$ & -2.440994 & -0.505534 & 0.575878 \\
\hline $\mathrm{O}$ & 2.315739 & -0.23089 & 0.565614 \\
\hline $\mathrm{Si}$ & 3.545255 & -0.050504 & -0.58867 \\
\hline $\mathrm{C}$ & 4.651485 & 1.327105 & 0.132718 \\
\hline $\mathrm{C}$ & 2.792026 & 0.448823 & -2.237855 \\
\hline $\mathrm{C}$ & 4.465913 & -1.682835 & -0.780564 \\
\hline $\mathrm{C}$ & 5.262089 & 0.853807 & 1.454435 \\
\hline C & 5.77254 & 1.666663 & -0.853475 \\
\hline $\mathrm{C}$ & 3.805965 & 2.577929 & 0.391693 \\
\hline $\mathrm{C}$ & -3.476878 & -0.847278 & -0.211751 \\
\hline $\mathrm{O}$ & -3.494506 & -1.84248 & -0.890544 \\
\hline $\mathrm{H}$ & -1.237991 & -1.098578 & 2.780946 \\
\hline $\mathrm{H}$ & 1.215874 & -1.045251 & 2.915458 \\
\hline $\mathrm{H}$ & 2.253857 & -2.245155 & 0.940746 \\
\hline $\mathrm{H}$ & -0.772813 & -1.370776 & -1.503487 \\
\hline $\mathrm{H}$ & -0.183577 & 0.015028 & -0.552141 \\
\hline $\mathrm{H}$ & -1.689185 & -2.42169 & 0.477559 \\
\hline $\mathrm{H}$ & 3.562683 & 0.599788 & -2.996408 \\
\hline $\mathrm{H}$ & 2.216152 & 1.371649 & -2.152419 \\
\hline $\mathrm{H}$ & 2.12275 & -0.337554 & -2.590252 \\
\hline $\mathrm{H}$ & 3.806083 & -2.451864 & -1.186679 \\
\hline $\mathrm{H}$ & 5.300125 & -1.56828 & -1.475883 \\
\hline $\mathrm{H}$ & 4.869896 & -2.044128 & 0.166662 \\
\hline $\mathrm{H}$ & 4.490581 & 0.586861 & 2.179293 \\
\hline $\mathrm{H}$ & 5.871 & 1.65052 & 1.895555 \\
\hline $\mathrm{H}$ & 5.912167 & -0.013439 & 1.317427 \\
\hline $\mathrm{H}$ & 6.414533 & 0.806802 & -1.06022 \\
\hline $\mathrm{H}$ & 5.383415 & 2.032084 & -1.806489 \\
\hline $\mathrm{H}$ & 6.411565 & 2.454665 & -0.440484 \\
\hline $\mathrm{H}$ & 4.426738 & 3.366484 & 0.831388 \\
\hline $\mathrm{H}$ & 3.3762 & 2.978791 & -0.529153 \\
\hline $\mathrm{H}$ & 2.986932 & 2.372822 & 1.083075 \\
\hline $\mathrm{C}$ & -4.586665 & 0.13671 & -0.141776 \\
\hline $\mathrm{C}$ & -4.518474 & 1.284096 & 0.64545 \\
\hline $\mathrm{C}$ & -5.726408 & -0.117274 & -0.900949 \\
\hline $\mathrm{C}$ & -5.585299 & 2.167834 & 0.670056 \\
\hline $\mathrm{H}$ & -3.630764 & 1.477454 & 1.231319 \\
\hline $\mathrm{C}$ & -6.790069 & 0.767895 & -0.872413 \\
\hline $\mathrm{H}$ & -5.758713 & -1.013374 & -1.506627 \\
\hline $\mathrm{C}$ & -6.720426 & 1.911168 & -0.086949 \\
\hline $\mathrm{H}$ & -5.531476 & 3.059672 & 1.28167 \\
\hline $\mathrm{H}$ & -7.675091 & 0.567903 & -1.463123 \\
\hline $\mathrm{H}$ & -7.552528 & 2.604163 & -0.064964 \\
\hline
\end{tabular}

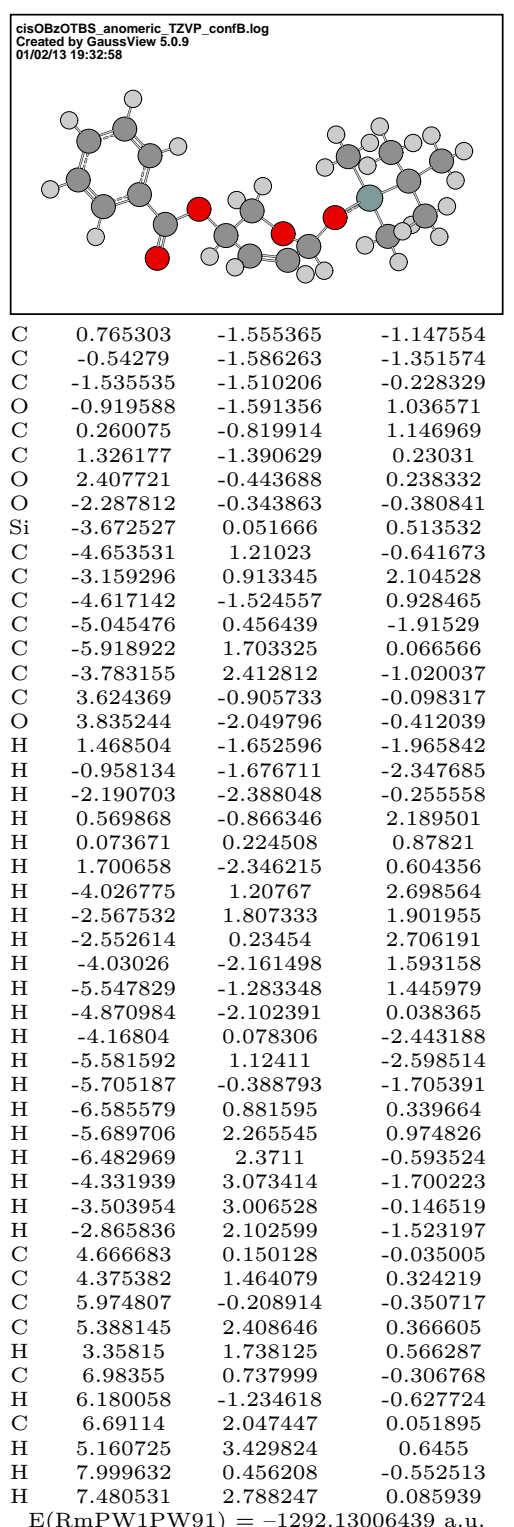

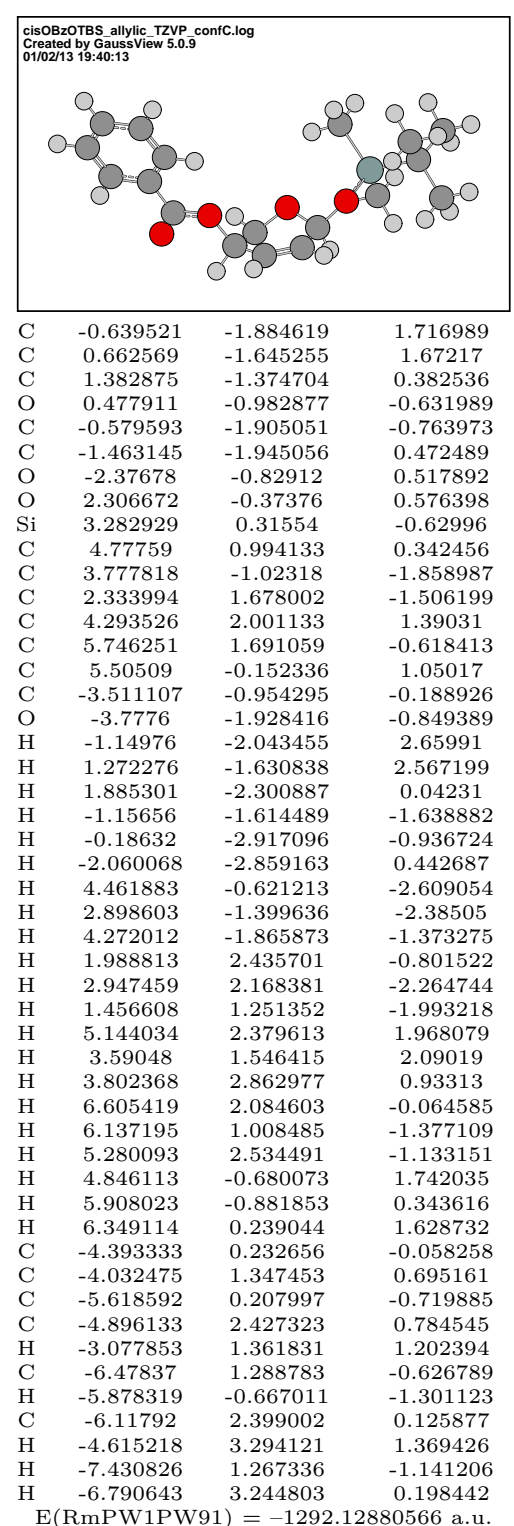




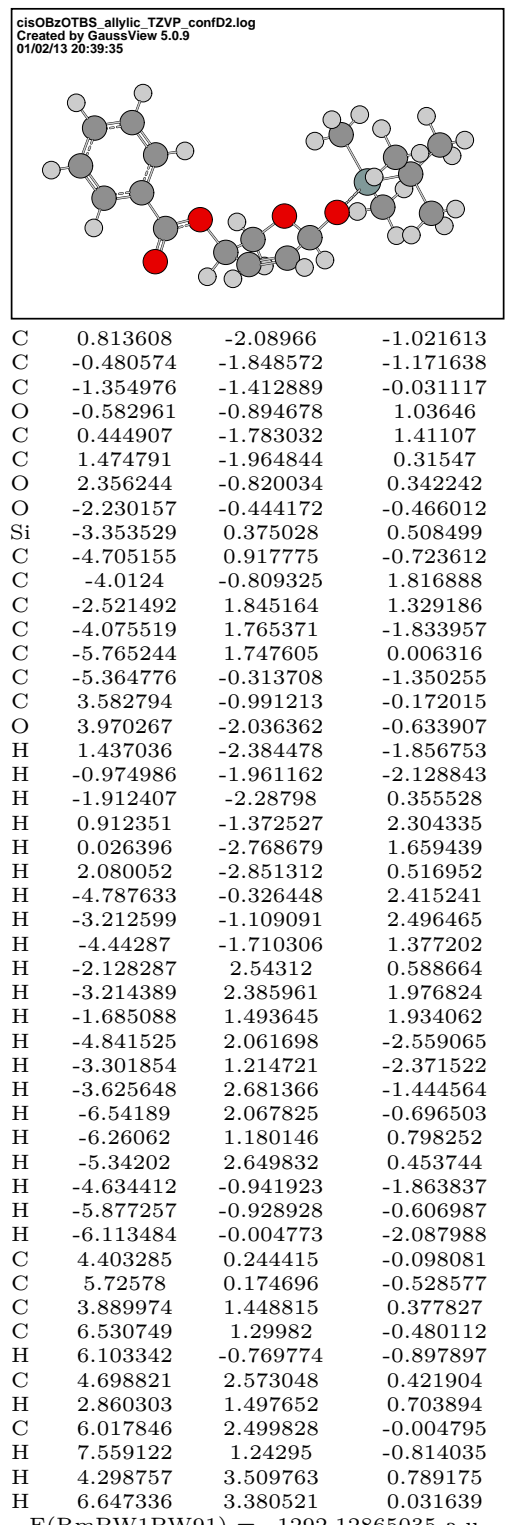

\begin{tabular}{|c|c|c|c|}
\hline \multicolumn{4}{|c|}{$\begin{array}{l}\text { transOBzOTBS_bisAx TZVYP_confA.log } \\
\text { Created by GaussView 5.0.9 } \\
\text { o2:02/13 07:34:58 }\end{array}$} \\
\hline C & -0.711424 & -1.595301 & -0.738878 \\
\hline $\mathrm{C}$ & 0.365578 & -1.0876 & -1.31946 \\
\hline $\mathrm{C}$ & 1.091718 & 0.109967 & -0.78069 \\
\hline $\mathrm{O}$ & 0.369478 & 0.765564 & 0.229201 \\
\hline $\mathrm{C}$ & -0.197317 & -0.118232 & 1.177597 \\
\hline $\mathrm{C}$ & -1.219776 & -1.040419 & 0.554507 \\
\hline $\mathrm{O}$ & -2.440067 & -0.29182 & 0.354711 \\
\hline $\mathrm{O}$ & 2.35892 & -0.299874 & -0.353272 \\
\hline $\mathrm{Si}$ & 3.629745 & 0.736353 & 0.077461 \\
\hline C & 5.179062 & -0.370989 & -0.043283 \\
\hline $\mathrm{C}$ & 3.681207 & 2.183681 & -1.128527 \\
\hline C & 3.362447 & 1.385971 & 1.822316 \\
\hline C & 5.003057 & -1.592381 & 0.864098 \\
\hline C & 6.416538 & 0.415528 & 0.398257 \\
\hline $\mathrm{C}$ & 5.365002 & -0.841542 & -1.488313 \\
\hline C & -3.574728 & -1.000296 & 0.271272 \\
\hline $\mathrm{O}$ & -3.615819 & -2.203247 & 0.35537 \\
\hline $\mathrm{H}$ & -1.243353 & -2.437583 & -1.163334 \\
\hline $\mathrm{H}$ & 0.774401 & -1.519425 & -2.225112 \\
\hline $\mathrm{H}$ & 1.183597 & 0.863553 & -1.571015 \\
\hline $\mathrm{H}$ & 0.582465 & -0.729917 & 1.64313 \\
\hline $\mathrm{H}$ & -0.660817 & 0.502685 & 1.941976 \\
\hline $\mathrm{H}$ & -1.441639 & -1.859708 & 1.241878 \\
\hline $\mathrm{H}$ & 3.730975 & 1.851342 & -2.166697 \\
\hline $\mathrm{H}$ & 2.798513 & 2.816091 & -1.014526 \\
\hline $\mathrm{H}$ & 4.554927 & 2.80969 & -0.935663 \\
\hline $\mathrm{H}$ & 2.438898 & 1.965714 & 1.859941 \\
\hline $\mathrm{H}$ & 4.183072 & 2.035207 & 2.13399 \\
\hline $\mathrm{H}$ & 3.280356 & 0.573132 & 2.54552 \\
\hline $\mathrm{H}$ & 4.901325 & -1.309671 & 1.914425 \\
\hline $\mathrm{H}$ & 5.878257 & -2.2471 & 0.787753 \\
\hline $\mathrm{H}$ & 4.12425 & -2.176285 & 0.585804 \\
\hline $\mathrm{H}$ & 6.343203 & 0.747752 & 1.436264 \\
\hline $\mathrm{H}$ & 7.308984 & -0.214788 & 0.321801 \\
\hline $\mathrm{H}$ & 6.589194 & 1.295992 & -0.225567 \\
\hline $\mathrm{H}$ & 5.537917 & -0.006134 & -2.170695 \\
\hline $\mathrm{H}$ & 6.233838 & -1.50483 & -1.56003 \\
\hline $\mathrm{H}$ & 4.494758 & -1.395531 & -1.845072 \\
\hline $\mathrm{C}$ & -4.766423 & -0.135752 & 0.07405 \\
\hline C & -4.664815 & 1.246605 & -0.06477 \\
\hline $\mathrm{C}$ & -6.016164 & -0.748273 & 0.026894 \\
\hline C & -5.808531 & 2.006957 & -0.24844 \\
\hline $\mathrm{H}$ & -3.691072 & 1.715178 & -0.031623 \\
\hline C & -7.156224 & 0.015672 & -0.15493 \\
\hline $\mathrm{H}$ & -6.072598 & -1.823575 & 0.134374 \\
\hline C & -7.053341 & 1.393827 & -0.292746 \\
\hline $\mathrm{H}$ & -5.728872 & 3.081254 & -0.357685 \\
\hline $\mathrm{H}$ & -8.126887 & -0.46272 & -0.190341 \\
\hline $\mathrm{H}$ & -7.945434 & 1.991231 & -0.435755 \\
\hline
\end{tabular}

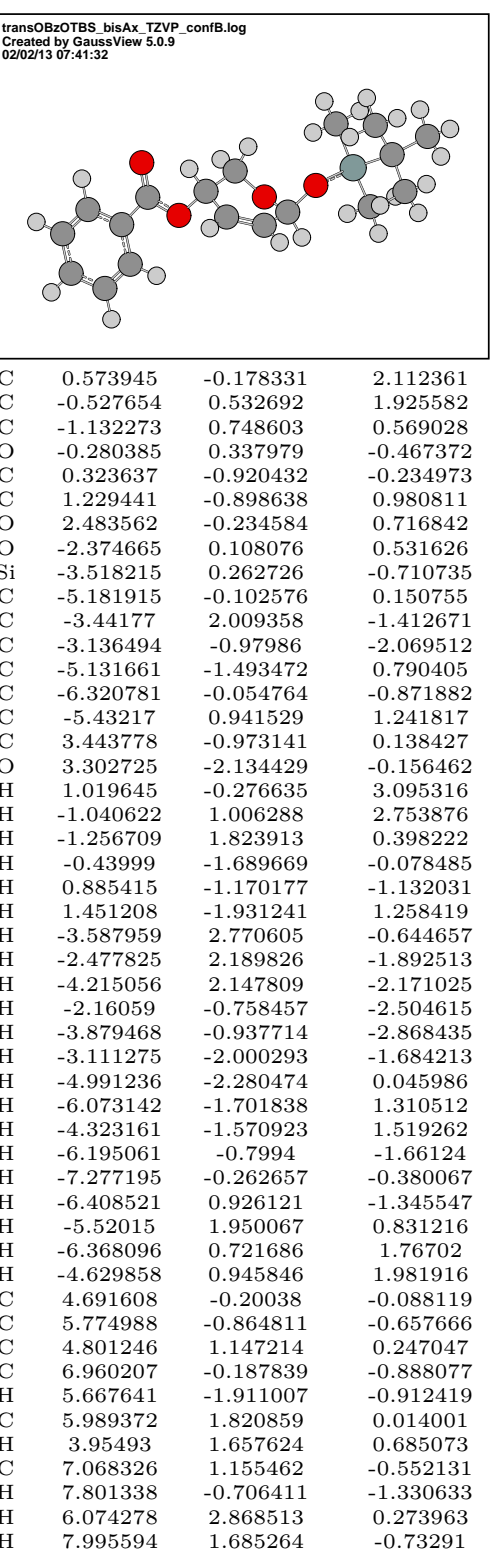

$\mathrm{E}(\mathrm{RmPW}$ 1PW91 $)=-1292.13119315$ 


\begin{tabular}{|c|c|c|c|}
\hline \multicolumn{4}{|c|}{ 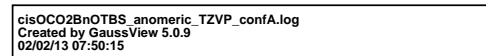 } \\
\hline $\mathrm{C}$ & 0.317579 & -1.6896 & 1.689149 \\
\hline $\mathrm{C}$ & 1.633702 & -1.571559 & 1.769179 \\
\hline $\mathrm{C}$ & 2.508605 & -1.498034 & 0.551292 \\
\hline $\mathrm{O}$ & 1.796589 & -1.757186 & -0.635795 \\
\hline $\mathrm{C}$ & 0.532247 & -1.123956 & -0.696078 \\
\hline $\mathrm{C}$ & -0.376713 & -1.71869 & 0.367503 \\
\hline $\mathrm{O}$ & -1.572583 & -0.930572 & 0.501438 \\
\hline $\mathrm{O}$ & 3.136049 & -0.251071 & 0.536335 \\
\hline $\mathrm{Si}$ & 4.387866 & 0.199643 & -0.515007 \\
\hline $\mathrm{C}$ & 5.254317 & 1.632743 & 0.399515 \\
\hline $\mathrm{C}$ & 3.66725 & 0.748808 & -2.163097 \\
\hline $\mathrm{C}$ & 5.527096 & -1.275551 & -0.788366 \\
\hline $\mathrm{C}$ & 5.809046 & 1.130625 & 1.73546 \\
\hline $\mathrm{C}$ & 6.401992 & 2.177857 & -0.455203 \\
\hline $\mathrm{C}$ & 4.241368 & 2.750243 & 0.66636 \\
\hline $\mathrm{C}$ & -2.570962 & -1.227259 & -0.331196 \\
\hline $\mathrm{O}$ & -3.591679 & -0.425768 & -0.046263 \\
\hline $\mathrm{O}$ & -2.554952 & -2.073785 & -1.185234 \\
\hline $\mathrm{C}$ & -4.764822 & -0.620808 & -0.863491 \\
\hline $\mathrm{H}$ & -0.302618 & -1.757821 & 2.575298 \\
\hline $\mathrm{H}$ & 2.140588 & -1.521082 & 2.724625 \\
\hline $\mathrm{H}$ & 3.257106 & -2.297734 & 0.58669 \\
\hline $\mathrm{H}$ & 0.140003 & -1.302539 & -1.694888 \\
\hline $\mathrm{H}$ & 0.621996 & -0.046147 & -0.526946 \\
\hline $\mathrm{H}$ & -0.662925 & -2.736294 & 0.090251 \\
\hline $\mathrm{H}$ & 4.450673 & 1.079409 & -2.848019 \\
\hline $\mathrm{H}$ & 2.958464 & 1.56871 & -2.038222 \\
\hline $\mathrm{H}$ & 3.140521 & -0.085084 & -2.629656 \\
\hline $\mathrm{H}$ & 5.005911 & -2.074224 & -1.31985 \\
\hline $\mathrm{H}$ & 6.384406 & -0.984745 & -1.398841 \\
\hline $\mathrm{H}$ & 5.907598 & -1.682221 & 0.149985 \\
\hline $\mathrm{H}$ & 5.019199 & 0.722633 & 2.368738 \\
\hline $\mathrm{H}$ & 6.27994 & 1.955436 & 2.281347 \\
\hline $\mathrm{H}$ & 6.568148 & 0.356501 & 1.600307 \\
\hline $\mathrm{H}$ & 7.162327 & 1.419099 & -0.656152 \\
\hline $\mathrm{H}$ & 6.052281 & 2.565409 & -1.414897 \\
\hline $\mathrm{H}$ & 6.899662 & 3.003587 & 0.064841 \\
\hline $\mathrm{H}$ & 4.717716 & 3.565245 & 1.222409 \\
\hline $\mathrm{H}$ & 3.84928 & 3.17571 & -0.260171 \\
\hline $\mathrm{H}$ & 3.396091 & 2.392688 & 1.256717 \\
\hline $\mathrm{H}$ & -4.50603 & -0.411813 & -1.902033 \\
\hline $\mathrm{H}$ & -5.07036 & -1.664999 & -0.793145 \\
\hline $\mathrm{C}$ & -5.834334 & 0.299466 & -0.368239 \\
\hline C & -5.98491 & 1.568821 & -0.916424 \\
\hline C & -6.687339 & -0.099551 & 0.656063 \\
\hline $\mathrm{C}$ & -6.967869 & 2.427443 & -0.447753 \\
\hline $\mathrm{H}$ & -5.325553 & 1.887116 & -1.715711 \\
\hline $\mathrm{C}$ & -7.670331 & 0.75633 & 1.128031 \\
\hline $\mathrm{H}$ & -6.578759 & -1.088114 & 1.087229 \\
\hline $\mathrm{C}$ & -7.81189 & 2.021867 & 0.576057 \\
\hline $\mathrm{H}$ & -7.076647 & 3.412975 & -0.883103 \\
\hline $\mathrm{H}$ & -8.329341 & 0.434881 & 1.924937 \\
\hline $\mathrm{H}$ & -8.581394 & 2.690307 & 0.941944 \\
\hline
\end{tabular}

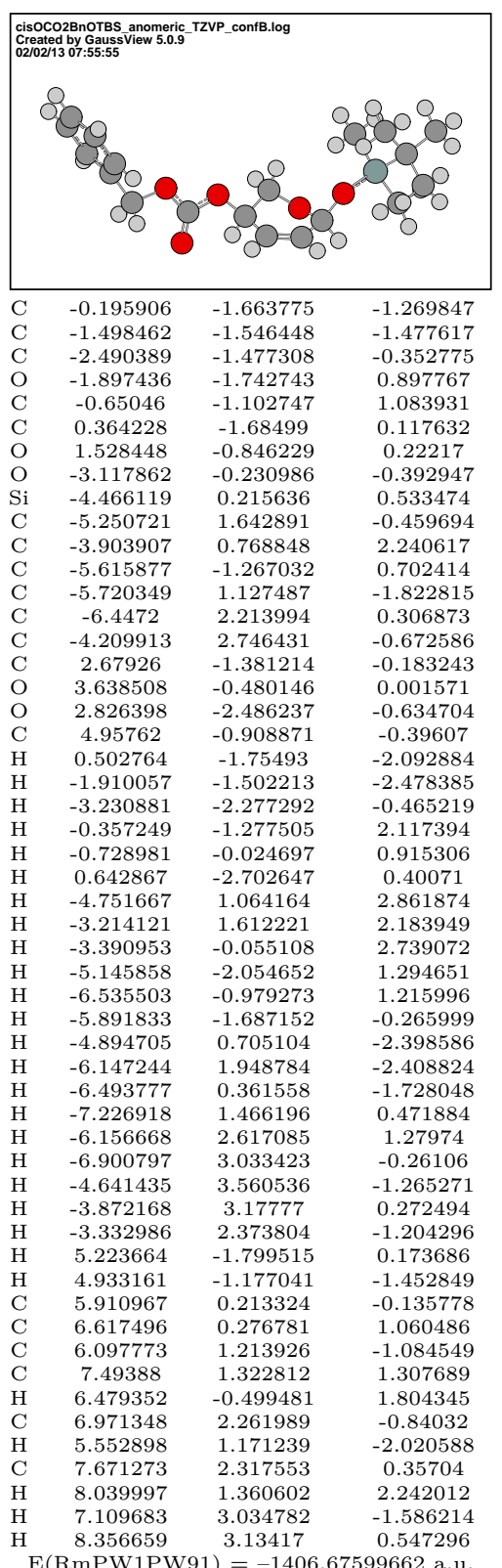

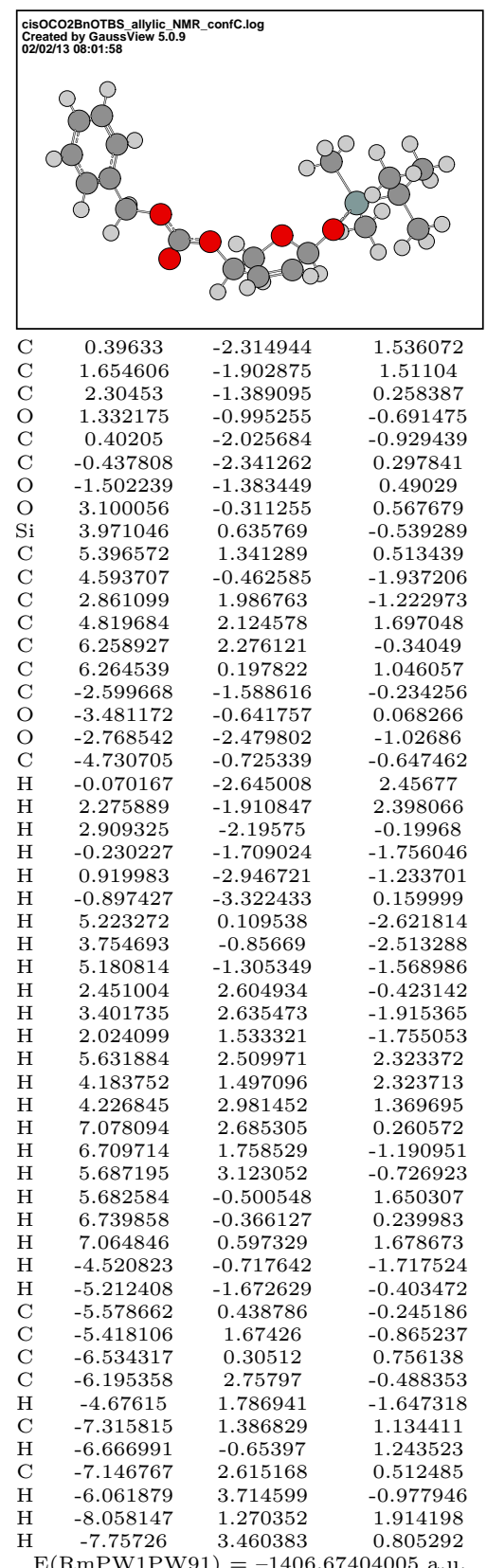




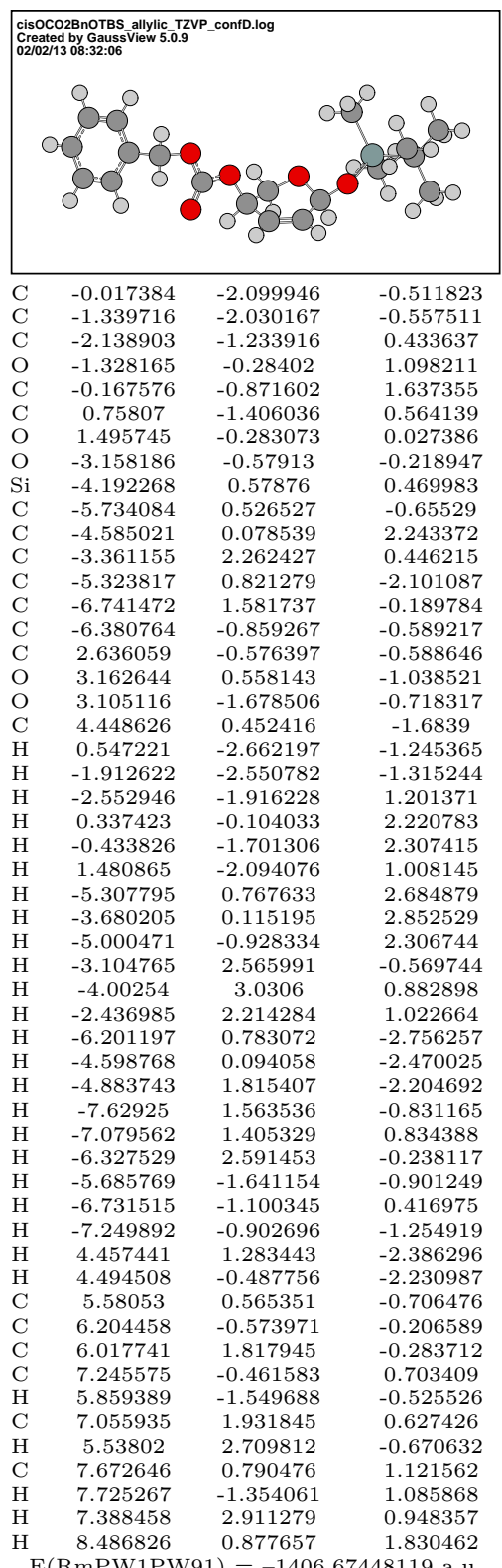

\begin{tabular}{|c|c|c|c|}
\hline \multicolumn{4}{|c|}{\begin{tabular}{|l} 
transoco28noTBS bisAx TZVP_confA.log \\
Created by GaussView 5.0.9. \\
02/02/13 08:40:38
\end{tabular}} \\
\hline $\mathrm{C}$ & 0.185351 & -1.615449 & -0.821714 \\
\hline $\mathrm{C}$ & 1.233552 & -1.015804 & -1.365919 \\
\hline $\mathrm{C}$ & 1.916068 & 0.160935 & -0.733048 \\
\hline $\mathrm{O}$ & 1.183557 & 0.694204 & 0.338477 \\
\hline $\mathrm{C}$ & 0.661761 & -0.287634 & 1.211929 \\
\hline $\mathrm{C}$ & -0.32538 & -1.200826 & 0.521832 \\
\hline $\mathrm{O}$ & -1.585778 & -0.497229 & 0.415876 \\
\hline $\mathrm{O}$ & 3.207771 & -0.226405 & -0.361196 \\
\hline $\mathrm{Si}$ & 4.411168 & 0.825659 & 0.204795 \\
\hline $\mathrm{C}$ & 6.040872 & -0.087367 & -0.187573 \\
\hline $\mathrm{C}$ & 4.273116 & 2.474418 & -0.696838 \\
\hline $\mathrm{C}$ & 4.189496 & 1.103021 & 2.052065 \\
\hline $\mathrm{C}$ & 6.041071 & -1.451917 & 0.508202 \\
\hline $\mathrm{C}$ & 7.231191 & 0.737036 & 0.310779 \\
\hline $\mathrm{C}$ & 6.162022 & -0.294896 & -1.699356 \\
\hline $\mathrm{C}$ & -2.659176 & -1.258567 & 0.229493 \\
\hline $\mathrm{O}$ & -3.724626 & -0.465817 & 0.163128 \\
\hline $\mathrm{O}$ & -2.670348 & -2.459782 & 0.143625 \\
\hline $\mathrm{C}$ & -4.977426 & -1.149375 & -0.042693 \\
\hline $\mathrm{H}$ & -0.316079 & -2.44054 & -1.311952 \\
\hline $\mathrm{H}$ & 1.647672 & -1.350446 & -2.309448 \\
\hline $\mathrm{H}$ & 1.961519 & 0.983548 & -1.455461 \\
\hline $\mathrm{H}$ & 1.469382 & -0.902287 & 1.62312 \\
\hline $\mathrm{H}$ & 0.176471 & 0.248394 & 2.025319 \\
\hline $\mathrm{H}$ & -0.49132 & -2.087218 & 1.138317 \\
\hline $\mathrm{H}$ & 4.341162 & 2.362771 & -1.78021 \\
\hline $\mathrm{H}$ & 3.325427 & 2.963136 & -0.462316 \\
\hline $\mathrm{H}$ & 5.072934 & 3.146717 & -0.379734 \\
\hline $\mathrm{H}$ & 3.234474 & 1.597432 & 2.236668 \\
\hline $\mathrm{H}$ & 4.981078 & 1.737167 & 2.456245 \\
\hline $\mathrm{H}$ & 4.195948 & 0.161647 & 2.603616 \\
\hline $\mathrm{H}$ & 5.993854 & -1.355342 & 1.595276 \\
\hline $\mathrm{H}$ & 6.962079 & -1.99554 & 0.270034 \\
\hline $\mathrm{H}$ & 5.198483 & -2.066847 & 0.187422 \\
\hline $\mathrm{H}$ & 7.200183 & 0.890647 & 1.391938 \\
\hline $\mathrm{H}$ & 8.169106 & 0.218672 & 0.084219 \\
\hline $\mathrm{H}$ & 7.281322 & 1.71862 & -0.166807 \\
\hline $\mathrm{H}$ & 6.196869 & 0.653488 & -2.240332 \\
\hline $\mathrm{H}$ & 7.084629 & -0.837061 & -1.933516 \\
\hline $\mathrm{H}$ & 5.3262 & -0.877177 & -2.091458 \\
\hline $\mathrm{H}$ & -4.917276 & -1.713459 & -0.974352 \\
\hline $\mathrm{H}$ & -5.131577 & -1.85721 & 0.772041 \\
\hline $\mathrm{C}$ & -6.065241 & -0.124192 & -0.089428 \\
\hline $\mathrm{C}$ & -6.331174 & 0.568355 & -1.266902 \\
\hline $\mathrm{C}$ & -6.820017 & 0.157144 & 1.044043 \\
\hline $\mathrm{C}$ & -7.32964 & 1.528481 & -1.309244 \\
\hline $\mathrm{H}$ & -5.748092 & 0.354787 & -2.155275 \\
\hline $\mathrm{C}$ & -7.82232 & 1.115253 & 1.004789 \\
\hline $\mathrm{H}$ & -6.620392 & -0.378479 & 1.965073 \\
\hline $\mathrm{C}$ & -8.077773 & 1.802984 & -0.172413 \\
\hline $\mathrm{H}$ & -7.527755 & 2.06128 & -2.230976 \\
\hline $\mathrm{H}$ & -8.404672 & 1.32422 & 1.893557 \\
\hline $\mathrm{H}$ & -8.860703 & 2.550478 & -0.205455 \\
\hline
\end{tabular}

\begin{tabular}{|c|c|c|c|}
\hline \multicolumn{4}{|c|}{$\begin{array}{l}\text { transoco2BnoTBS bisAx_TZVP_confB.log } \\
\text { Created by Gausssiew 5.0.9 } \\
\text { 02/02/13 08:45:44 }\end{array}$} \\
\hline $\mathrm{C}$ & 0.334522 & -1.890076 & -1.172412 \\
\hline $\mathrm{C}$ & 1.40142 & -1.256687 & -1.634556 \\
\hline $\mathrm{C}$ & 1.950575 & -0.018122 & -0.988832 \\
\hline $\mathrm{O}$ & 1.074611 & 0.519847 & -0.034276 \\
\hline $\mathrm{C}$ & 0.522815 & -0.45098 & 0.833111 \\
\hline $\mathrm{C}$ & -0.332764 & -1.458714 & 0.09132 \\
\hline $\mathrm{O}$ & -1.62612 & -0.926944 & -0.273211 \\
\hline $\mathrm{O}$ & 3.208509 & -0.319274 & -0.457161 \\
\hline $\mathrm{Si}$ & 4.310709 & 0.81569 & 0.15217 \\
\hline $\mathrm{C}$ & 6.00843 & -0.025972 & -0.075141 \\
\hline $\mathrm{C}$ & 4.160244 & 2.418045 & -0.827655 \\
\hline $\mathrm{C}$ & 3.930073 & 1.152325 & 1.962951 \\
\hline $\mathrm{C}$ & 6.012075 & -1.370218 & 0.659675 \\
\hline $\mathrm{C}$ & 7.114712 & 0.866989 & 0.493765 \\
\hline $\mathrm{C}$ & 6.265937 & -0.269453 & -1.564344 \\
\hline $\mathrm{C}$ & -2.554573 & -0.958333 & 0.6797 \\
\hline $\mathrm{O}$ & -3.677337 & -0.45039 & 0.181753 \\
\hline $\mathrm{O}$ & -2.408607 & -1.378364 & 1.798857 \\
\hline $\mathrm{C}$ & -4.787191 & -0.414606 & 1.102417 \\
\hline $\mathrm{H}$ & -0.075899 & -2.751069 & -1.686547 \\
\hline $\mathrm{H}$ & 1.923852 & -1.602584 & -2.518105 \\
\hline $\mathrm{H}$ & 2.037775 & 0.772637 & -1.742168 \\
\hline $\mathrm{H}$ & 1.317353 & -0.996061 & 1.35396 \\
\hline $\mathrm{H}$ & -0.066015 & 0.085943 & 1.573521 \\
\hline $\mathrm{H}$ & -0.497241 & -2.314933 & 0.748965 \\
\hline $\mathrm{H}$ & 4.313322 & 2.267534 & -1.897655 \\
\hline $\mathrm{H}$ & 3.174671 & 2.865021 & -0.68325 \\
\hline $\mathrm{H}$ & 4.900883 & 3.143017 & -0.483798 \\
\hline $\mathrm{H}$ & 2.936355 & 1.593829 & 2.054132 \\
\hline $\mathrm{H}$ & 4.649142 & 1.850251 & 2.39642 \\
\hline $\mathrm{H}$ & 3.948559 & 0.236277 & 2.55523 \\
\hline $\mathrm{H}$ & 5.87284 & -1.248856 & 1.736364 \\
\hline $\mathrm{H}$ & 6.972477 & -1.876426 & 0.511795 \\
\hline $\mathrm{H}$ & 5.225873 & -2.031111 & 0.29084 \\
\hline $\mathrm{H}$ & 6.983779 & 1.052271 & 1.562399 \\
\hline $\mathrm{H}$ & 8.090174 & 0.385531 & 0.365644 \\
\hline $\mathrm{H}$ & 7.163904 & 1.834612 & -0.011656 \\
\hline $\mathrm{H}$ & 6.308444 & 0.664281 & -2.129616 \\
\hline $\mathrm{H}$ & 7.226244 & -0.778125 & -1.702589 \\
\hline $\mathrm{H}$ & 5.491267 & -0.898285 & -2.007133 \\
\hline $\mathrm{H}$ & -4.988891 & -1.429045 & 1.44756 \\
\hline $\mathrm{H}$ & -4.505238 & 0.187819 & 1.966607 \\
\hline $\mathrm{C}$ & -5.966141 & 0.166411 & 0.389306 \\
\hline $\mathrm{C}$ & -6.855657 & -0.658737 & -0.29114 \\
\hline $\mathrm{C}$ & -6.184719 & 1.540273 & 0.389549 \\
\hline $\mathrm{C}$ & -7.942694 & -0.121233 & -0.963344 \\
\hline $\mathrm{H}$ & -6.694087 & -1.73057 & -0.294407 \\
\hline $\mathrm{C}$ & -7.27039 & 2.081561 & -0.281668 \\
\hline $\mathrm{H}$ & -5.497385 & 2.190255 & 0.918697 \\
\hline $\mathrm{C}$ & -8.151398 & 1.250604 & -0.959289 \\
\hline $\mathrm{H}$ & -8.629384 & -0.77336 & -1.488593 \\
\hline $\mathrm{H}$ & -7.431118 & 3.152427 & -0.273775 \\
\hline $\mathrm{H}$ & -9.001684 & 1.67161 & -1.481406 \\
\hline
\end{tabular}




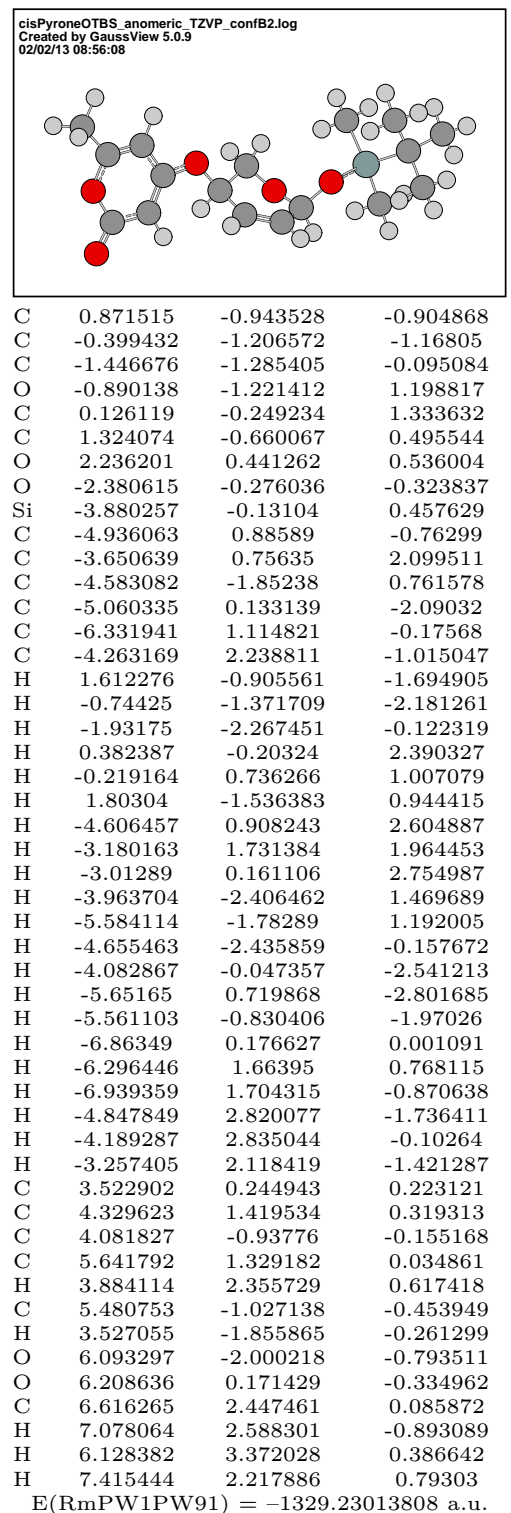

\begin{tabular}{|c|c|c|c|}
\hline \multicolumn{4}{|c|}{ 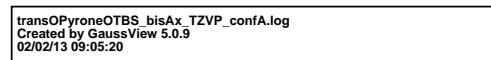 } \\
\hline C & 0.830639 & 1.022065 & -0.65639 \\
\hline $\mathrm{C}$ & -0.327116 & 0.764059 & -1.246212 \\
\hline $\mathrm{C}$ & -1.247002 & -0.331126 & -0.793106 \\
\hline $\mathrm{O}$ & -0.632707 & -1.195723 & 0.127242 \\
\hline C & 0.094082 & -0.521062 & 1.132986 \\
\hline C & 1.254378 & 0.27165 & 0.568946 \\
\hline $\mathrm{O}$ & 2.323781 & -0.648956 & 0.285624 \\
\hline $\mathrm{O}$ & -2.411047 & 0.253149 & -0.289332 \\
\hline $\mathrm{Si}$ & -3.831181 & -0.583552 & 0.116045 \\
\hline $\mathrm{C}$ & -5.207839 & 0.722294 & -0.082599 \\
\hline C & -4.041909 & -2.045529 & -1.052714 \\
\hline $\mathrm{C}$ & -3.700712 & -1.207461 & 1.885569 \\
\hline $\mathrm{C}$ & -4.923298 & 1.910524 & 0.841417 \\
\hline $\mathrm{C}$ & -6.562445 & 0.108383 & 0.282926 \\
\hline C & -5.245654 & 1.21216 & -1.532472 \\
\hline $\mathrm{H}$ & 1.487942 & 1.792734 & -1.040001 \\
\hline $\mathrm{H}$ & -0.662424 & 1.340188 & -2.100144 \\
\hline $\mathrm{H}$ & -1.483332 & -0.978981 & -1.644398 \\
\hline $\mathrm{H}$ & -0.558068 & 0.165563 & 1.68271 \\
\hline $\mathrm{H}$ & 0.460285 & -1.284021 & 1.817298 \\
\hline $\mathrm{H}$ & 1.595173 & 0.965966 & 1.34344 \\
\hline $\mathrm{H}$ & -4.058932 & -1.741211 & -2.10052 \\
\hline $\mathrm{H}$ & -3.231367 & -2.764561 & -0.918522 \\
\hline $\mathrm{H}$ & -4.977831 & -2.567048 & -0.842269 \\
\hline $\mathrm{H}$ & -2.89982 & -1.94508 & 1.958958 \\
\hline $\mathrm{H}$ & -4.627986 & -1.684374 & 2.20912 \\
\hline $\mathrm{H}$ & -3.478263 & -0.395723 & 2.579747 \\
\hline $\mathrm{H}$ & -4.918513 & 1.618279 & 1.894045 \\
\hline $\mathrm{H}$ & -5.699203 & 2.674275 & 0.719998 \\
\hline $\mathrm{H}$ & -3.961341 & 2.37419 & 0.616085 \\
\hline $\mathrm{H}$ & -6.589211 & -0.24128 & 1.317563 \\
\hline $\mathrm{H}$ & -7.355498 & 0.855839 & 0.173731 \\
\hline $\mathrm{H}$ & -6.821153 & -0.733509 & -0.363819 \\
\hline $\mathrm{H}$ & -5.483677 & 0.406326 & -2.230581 \\
\hline $\mathrm{H}$ & -6.015768 & 1.98211 & -1.650198 \\
\hline $\mathrm{H}$ & -4.291471 & 1.649006 & -1.832438 \\
\hline C & 3.576342 & -0.197129 & 0.178165 \\
\hline $\mathrm{C}$ & 3.97954 & 1.09623 & 0.323441 \\
\hline C & 4.538474 & -1.215272 & -0.103492 \\
\hline $\mathrm{C}$ & 5.360873 & 1.455664 & 0.2042 \\
\hline $\mathrm{H}$ & 3.307984 & 1.915189 & 0.524194 \\
\hline $\mathrm{C}$ & 5.833932 & -0.870571 & -0.218277 \\
\hline $\mathrm{H}$ & 4.217636 & -2.238599 & -0.220929 \\
\hline $\mathrm{C}$ & 6.953699 & -1.800352 & -0.509272 \\
\hline $\mathrm{H}$ & 6.590664 & -2.819642 & -0.621298 \\
\hline $\mathrm{H}$ & 7.463259 & -1.499169 & -1.426415 \\
\hline $\mathrm{H}$ & 7.688481 & -1.768438 & 0.297261 \\
\hline $\mathrm{O}$ & 5.842186 & 2.549066 & 0.312404 \\
\hline $\mathrm{O}$ & 6.245669 & 0.398032 & -0.074181 \\
\hline
\end{tabular}

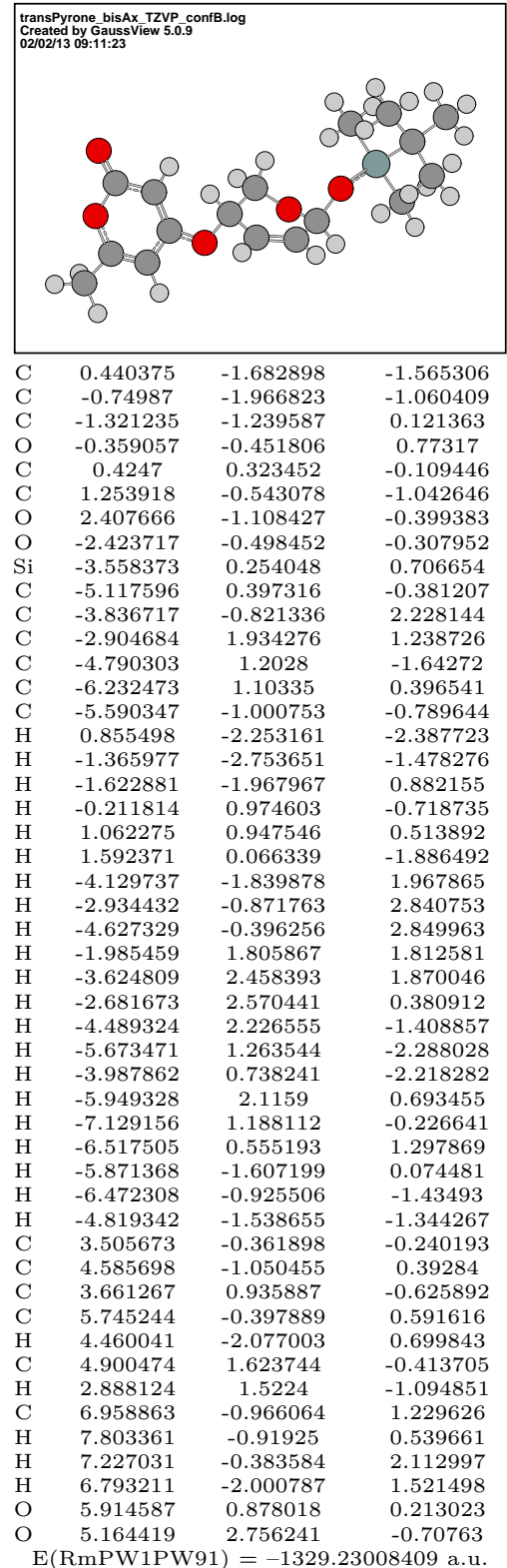


Appendix B

Crystallographic Information 


\section{B.1 Furopyrone X-Ray Crystal Structure}

Table B.1 Crystal Data and Structure Refinement for MJBA

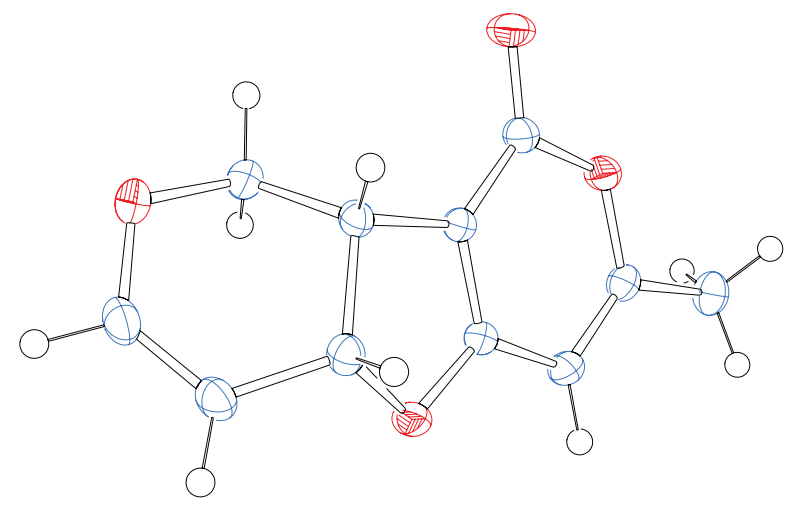

Identification code

Empirical formula

Formula weight

Temperature / K

Crystal system

Space group

$\mathrm{a} / \AA, \mathrm{b} / \AA, \mathrm{c} / \AA$

$\alpha /{ }^{\circ} \mathrm{C}, \beta /{ }^{\circ} \mathrm{C}, \gamma /{ }^{\circ} \mathrm{C}$

Volume / $\AA^{3}$

$\mathrm{Z}$

$\rho_{\text {calc }} / \mathrm{mg} \mathrm{mm}^{-3}$

$\mu / \mathrm{mm}^{? 1}$

$\mathrm{F}(000)$

Crystal size / $\mathrm{mm}^{3}$

Theta range for data collection

Index ranges

Reflections collected

Independent reflections

Data/restraints/parameters

Goodness-of-fit on F2

Final $\mathrm{R}$ indexes $[\mathrm{I}>2 \theta(\mathrm{I})]$

Final $\mathrm{R}$ indexes [all data]

Largest diff. peak/hole / e $\AA^{-3}$

$$
\begin{gathered}
\text { MJBA } \\
\mathrm{C}_{11} \mathrm{H}_{10} \mathrm{O}_{4} \\
206.19 \\
296.0 \\
\text { monoclinic }
\end{gathered}
$$

$\mathrm{P} 2{ }_{1} / \mathrm{c}$

7.7627(3), 11.0574(4), 11.2765(4)

$90.00,103.614(2), 90.00$

$940.73(6)$

4

1.456

0.112

432

$0.54 \times 0.44 \times 0.35$

2.62 to $68.96{ }^{\circ} \mathrm{C}$

$-11 \leq \mathrm{h} \leq 12,-16 \leq \mathrm{k} \leq 17,-17 \leq \mathrm{l} \leq 16$

24388

$3438[\mathrm{R}(\mathrm{int})=0.0302]$

$3438 / 0 / 176$

1.152

$\mathrm{R}_{1}=0.0505, \mathrm{wR}_{2}=0.1202$

$\mathrm{R}_{1}=0.0568, \mathrm{wR}_{2}=0.1233$

$0.483 /-0.216$ 
Table B.2 Fractional Atomic Coordinates $\left(\mathrm{x} 10^{4}\right)$ and Equivalent Isotropic Displacement Parameters $\left(\AA^{2} \times 10^{3}\right)$ for MJBA.

$\mathrm{U}_{\text {eq }}$ is defined as $1 / 3$ of of the trace of the orthogonalised $\mathrm{U}_{\mathrm{IJ}}$ tensor.

\begin{tabular}{ccccc}
\hline Atom & $\mathbf{x}$ & $\mathbf{y}$ & $\mathbf{z}$ & $\mathbf{U}(\mathbf{e q})$ \\
\hline O1 & $1639.0(11)$ & $2951.8(7)$ & $399.7(7)$ & $20.97(17)$ \\
C2 & $2953.8(14)$ & $4656.8(9)$ & $1541.5(9)$ & $17.26(18)$ \\
O & $2899.7(13)$ & $7999.5(7)$ & $2121.0(8)$ & $27.09(19)$ \\
O4 & $3322.7(11)$ & $4714.5(7)$ & $3603.7(7)$ & $21.14(17)$ \\
O5 & $2370.8(15)$ & $4514.7(8)$ & $-619.5(8)$ & $32.7(2)$ \\
C6 & $2021.9(15)$ & $2900(1)$ & $2549.1(10)$ & $20.2(2)$ \\
C7 & $2747.9(13)$ & $4079.6(9)$ & $2567.3(9)$ & $17.18(18)$ \\
C8 & $4341.5(14)$ & $5758.7(9)$ & $3270.8(10)$ & $19.6(2)$ \\
C9 & $1514.7(14)$ & $2371.1(9)$ & $1444.7(10)$ & $18.81(19)$ \\
C10 & $3735.4(14)$ & $5886.0(9)$ & $1875.2(9)$ & $17.89(19)$ \\
C11 & $2351.9(15)$ & $4116.9(10)$ & $382.4(10)$ & $20.9(2)$ \\
C12 & $2324.7(16)$ & $6863.6(10)$ & $1539.2(10)$ & $22.0(2)$ \\
C13 & $4080.4(16)$ & $6872.5(10)$ & $3941.1(10)$ & $23.4(2)$ \\
C14 & $768.3(16)$ & $1132.6(10)$ & $1188.0(12)$ & $24.3(2)$ \\
C15 & $3498.5(16)$ & $7892.6(10)$ & $3353.8(11)$ & $25.0(2)$ \\
\hline
\end{tabular}

Table B.3 Hydrogen Atom Coordinates $\left(\AA \times 10^{4}\right)$ and Isotropic Displacement Parameters $\left(\AA^{2} \times 10^{3}\right)$ for MJBA.

\begin{tabular}{ccccc}
\hline Atom & $\mathbf{x}$ & $\mathbf{y}$ & $\mathbf{z}$ & $\mathbf{U}(\mathbf{e q})$ \\
\hline H15 & $3470.0(2)$ & $8665(15)$ & $3758(15)$ & $27(4)$ \\
H8 & $5560.0(2)$ & $5490(14)$ & $3486(13)$ & $22(4)$ \\
H6 & $1900.0(2)$ & $2508(15)$ & $3251(15)$ & $27(4)$ \\
H10 & $4750.0(2)$ & $6065(15)$ & $1543(15)$ & $29(4)$ \\
H12B & $2000.0(2)$ & $7018(14)$ & $660(15)$ & $28(4)$ \\
H14C & $950.0(2)$ & $658(17)$ & $1902(17)$ & $39(5)$ \\
H14A & $1320.0(2)$ & $734(16)$ & $650(16)$ & $35(4)$ \\
H12A & $1249(19)$ & $6626(14)$ & $1818(14)$ & $20(3)$ \\
H14B & $-460.0(2)$ & $1191(15)$ & $812(16)$ & $32(4)$ \\
H13 & $4480.0(2)$ & $6882(15)$ & $4793(17)$ & $32(4)$ \\
\hline
\end{tabular}


Table B.4 Anisotropic Displacement Parameters $\left(\AA^{2} \times 10^{3}\right)$ for MJBA.

The Anisotropic displacement factor exponent takes the form: $-2 \pi 2\left[\mathrm{~h}^{2} \mathrm{a}^{* 2} \mathrm{U}_{11}+\ldots+2 \mathrm{hka} \mathrm{x}\right.$ b x $\left.\mathrm{U}_{12}\right]$

\begin{tabular}{ccccccc}
\hline Atom & $\mathbf{U}_{\mathbf{1 1}}$ & $\mathbf{U}_{\mathbf{2 2}}$ & $\mathbf{U}_{\mathbf{3 3}}$ & $\mathbf{U}_{\mathbf{2 3}}$ & $\mathbf{U}_{\mathbf{1 3}}$ & $\mathbf{U}_{\mathbf{1 2}}$ \\
\hline $\mathrm{O} 1$ & $28.2(4)$ & $17.4(3)$ & $17.0(3)$ & $-1.8(3)$ & $4.5(3)$ & $-3.9(3)$ \\
C2 & $21.5(4)$ & $14.6(4)$ & $16.3(4)$ & $-0.2(3)$ & $5.6(3)$ & $-0.7(3)$ \\
O & $38.8(5)$ & $15.8(4)$ & $27.7(4)$ & $-0.3(3)$ & $9.7(4)$ & $1.3(3)$ \\
O4 & $28.9(4)$ & $18.6(4)$ & $15.1(3)$ & $-0.8(3)$ & $3.6(3)$ & $-3.4(3)$ \\
O5 & $57.8(6)$ & $24.9(4)$ & $16.9(4)$ & $-0.7(3)$ & $11.5(4)$ & $-8.8(4)$ \\
C6 & $25.3(5)$ & $17.3(4)$ & $18.5(4)$ & $2.2(3)$ & $6.2(4)$ & $-1.3(4)$ \\
C7 & $19.9(4)$ & $16.2(4)$ & $15.2(4)$ & $0.0(3)$ & $3.7(3)$ & $0.6(3)$ \\
C8 & $19.4(4)$ & $17.7(4)$ & $20.9(5)$ & $-1.8(3)$ & $2.9(4)$ & $-1.8(3)$ \\
C9 & $19.3(4)$ & $16.0(4)$ & $21.3(5)$ & $1.2(3)$ & $4.9(4)$ & $-0.8(3)$ \\
C10 & $20.9(4)$ & $15.5(4)$ & $18.5(4)$ & $-1.2(3)$ & $7.1(4)$ & $-1.7(3)$ \\
C11 & $28.2(5)$ & $17.0(4)$ & $18.3(4)$ & $-1.3(3)$ & $6.8(4)$ & $-2.4(4)$ \\
C12 & $26.8(5)$ & $17.2(4)$ & $21.5(5)$ & $0.7(3)$ & $4.9(4)$ & $0.9(4)$ \\
C13 & $27.3(5)$ & $21.7(5)$ & $21.0(5)$ & $-5.5(4)$ & $5.1(4)$ & $-3.8(4)$ \\
C14 & $24.6(5)$ & $17.2(5)$ & $30.5(5)$ & $-2.2(4)$ & $5.3(4)$ & $-3.7(4)$ \\
C15 & $30.3(5)$ & $18.9(5)$ & $28.0(5)$ & $-5.8(4)$ & $11.4(4)$ & $-3.1(4)$ \\
\hline
\end{tabular}

Table B.5 Bond Lengths for MJBA.

\begin{tabular}{ccc|ccc}
\hline Atom & Atom & Length/ & Atom & Atom & Length/ \\
\hline O1 & C9 & $1.3652(12)$ & O5 & C11 & $1.2158(13)$ \\
O1 & C11 & $1.4040(13)$ & C6 & C7 & $1.4191(14)$ \\
C2 & C7 & $1.3631(13)$ & C6 & C9 & $1.3480(15)$ \\
C2 & C10 & $1.4999(14)$ & C8 & C10 & $1.5393(15)$ \\
C2 & C11 & $1.4126(14)$ & C8 & C13 & $1.4838(15)$ \\
O & C12 & $1.4385(14)$ & C9 & C14 & $1.4886(15)$ \\
O & C15 & $1.3632(15)$ & C10 & C12 & $1.5218(15)$ \\
O4 & C7 & $1.3462(12)$ & C13 & C15 & $1.3319(17)$ \\
O4 & C8 & $1.4963(13)$ & & & \\
\hline
\end{tabular}

Table B.6 Bond Angles for MJBA.

\begin{tabular}{cccc|cccc}
\hline Atom & Atom & Atom & Angle $^{\circ}$ & Atom & Atom & Atom & Angle $^{\circ}$ \\
\hline O1 & C9 & C14 & $111.60(9)$ & C7 & C2 & C10 & $109.43(9)$ \\
O1 & C11 & C2 & $114.87(9)$ & C7 & C2 & C11 & $120.53(9)$ \\
C2 & C7 & C6 & $122.93(9)$ & C7 & O4 & C8 & $105.75(8)$ \\
C2 & C10 & C8 & $100.48(8)$ & C9 & O1 & C11 & $123.48(8)$ \\
C2 & C10 & C12 & $110.87(9)$ & C9 & C6 & C7 & $116.15(9)$ \\
O & C12 & C10 & $112.26(9)$ & C11 & C2 & C10 & $129.89(9)$ \\
O4 & C7 & C2 & $114.29(9)$ & C12 & C10 & C8 & $110.39(8)$ \\
O4 & C7 & C6 & $122.77(9)$ & C13 & C8 & O4 & $111.65(9)$ \\
O4 & C8 & C10 & $105.94(8)$ & C13 & C8 & C10 & $113.54(9)$ \\
O5 & C11 & O1 & $115.84(9)$ & C13 & C15 & O & $125.09(10)$ \\
O5 & C11 & C2 & $129.29(10)$ & C15 & O & C12 & $112.59(9)$ \\
C6 & C9 & O1 & $121.92(9)$ & C15 & C13 & C8 & $121.37(10)$ \\
C6 & C9 & C14 & $126.47(10)$ & & & & \\
\hline
\end{tabular}

